

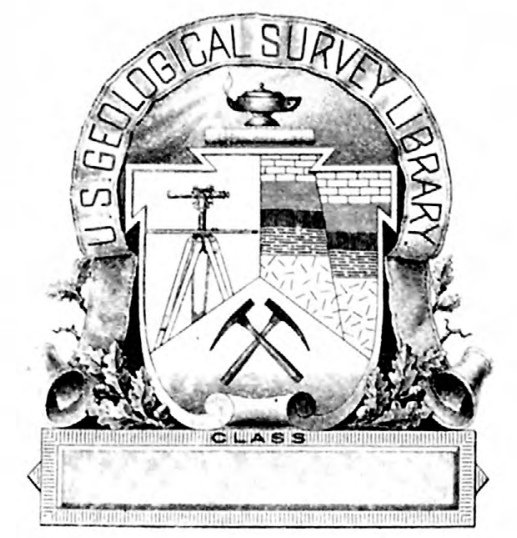





\section{ANNUAL AND SEASONAL LOW-FLOW CHARACTERISTICS OF IOWA STREAMS}

IOWA NATURAL RESOURCES COUNCIL

BULLETIN NO. 13

Prepared by the

U.S. GEOLOGICAL SURVEY

in cooperation with the

IOWA NATURAL RESOURCES COUNCIL and the

IOWA DEPARTMENT OF ENVIRONMENTAL QUALITY
US Gaglogleal Survoy

JUN $7-2002$

Denvor Library
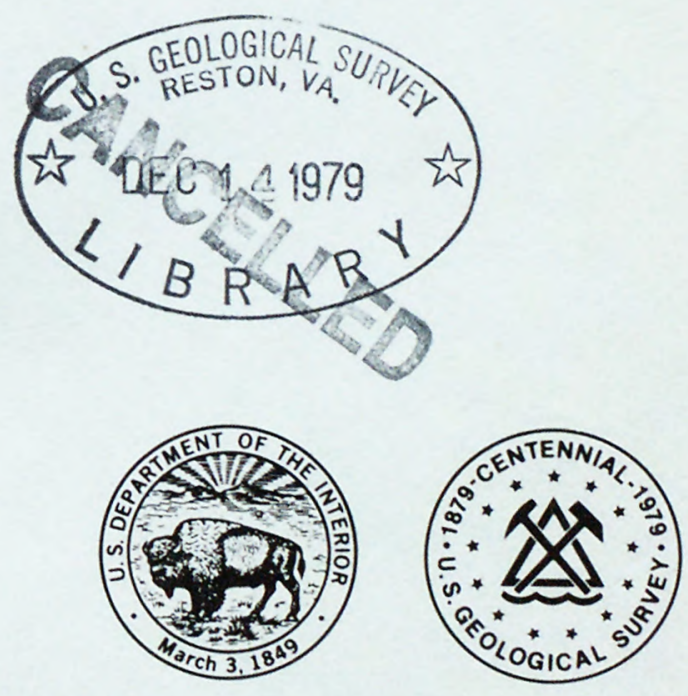


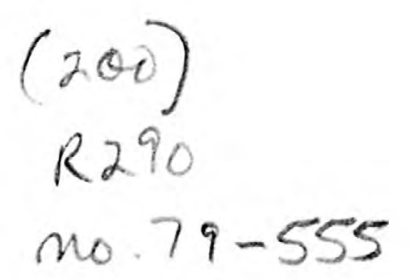

\section{ANNUAL AND SEASONAL LOW-FLOW CHARACTERISTICS OF IOWA STREAMS}

U.S. GEOLOGICAL SURVEY,

OPEN-FILE REPORT 79-555

Prepared in cooperation with the IOWA NATURAL RESOURCES COUNCIL and the IOWA DEPARTMENT OF ENVIRONMENTAL QUALITY
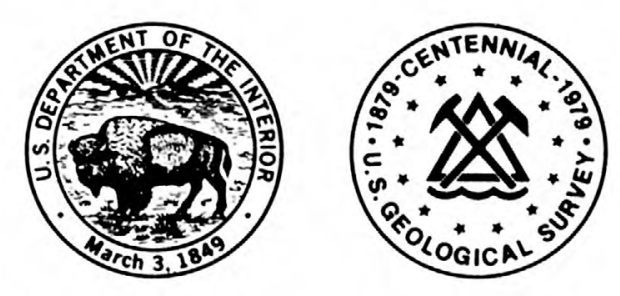
DEPARTMENT OF ENVIRONMENTAL QUALITY FROM THE UNITED STATES ENVIRONMENTAL PROTECTION AGENCY. 



\section{UNITED STATES \\ DEPARTMENT OF THE INTERIOR}

GEOLOGICAL SURVEY

ANNUAL AND SEASONAL LOW-FLOW CHARACTERISTICS OF IOWA STREAMS

By Oscar G. Lara

Open-File Report 79-555

Prepared in cooperation with the IOWA NATURAL RESOURCES COUNCIL

IOWA DEPARTMENT OF ENVIRONMENTAL QUALITY

Iowa City, Iowa

MARCH 1979 



\section{CONTENTS}

Glossary.....................

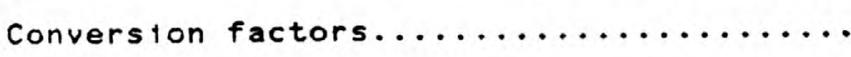

Abstract

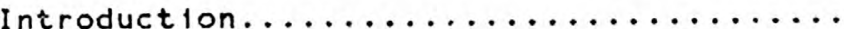

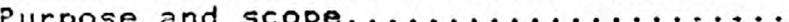

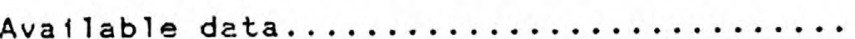

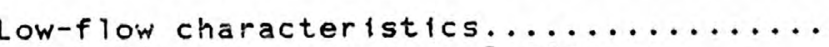

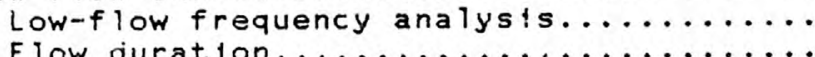

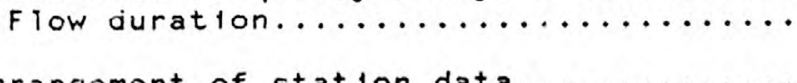

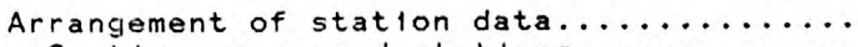

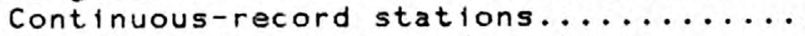

Low-flow partial-record stations........

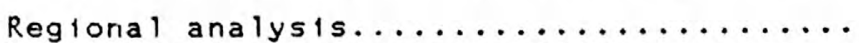

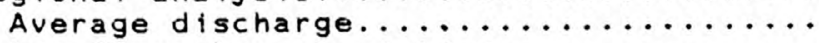

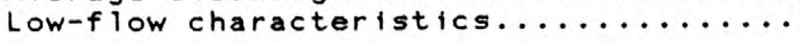

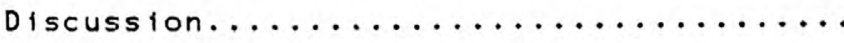

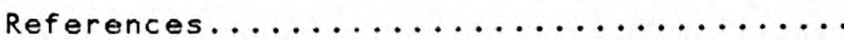

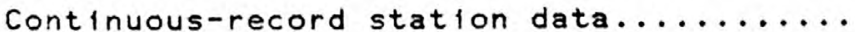

Low-fiow partial-record station data.....

Index of continuous-record stations......

Index for partial-record stations........

\section{ILLUSTRATIONS}

Page

VI

1

2

2

9

9
10

11

11

13

13

15

349

493

499

Figure 1. Bar graph showing perlod of

Page record of continuous-record gaging stations.............. 3

2. Low-flow frequency curves for gaging station 05421000

Wapsipinicon River at

Independence, Jowa...........

3. Flow-duration curve for gaging station 05484000 South Raccoon River at Redfleld, Iowa......... g

4. Regional equations for computing the average discharge and Iowa normal annual precipitation for

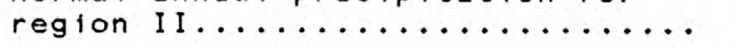

Plate 1. Map of I owa showing location of continuous-record gaging stations..Back cover

2. Map of lowa showing location of lowflow partial-record stations........ Back cover

3. Map showing 7-day $Q 2$ and 7 -day Q10 low flow for main stems of

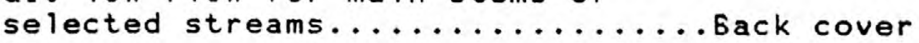

4. Map showing 7-day 02 and 7-day Q10 low flow for all stream points other than the main stems shown in Plate $3 \ldots \ldots \ldots \ldots$......... cover 
CONVERSION FACTORS

The following factors may be used to convert inch-pound unlts given herein to the International System of Units (SI).

Multiply inch-pound units

Length

\author{
1nches $(\ln )$ \\ feet $(f t)$ \\ miles ( $m 1$ ) \\ square miles $\left(m t^{2}\right)$
}

cublc feet ( $\left.f t^{2}\right)$

cublc feet/second ( $f t^{3 / s} / \mathrm{s}$ )

$\begin{array}{ll} & 0.02832 \\ \text { cuble feet per second per } & 0.01093\end{array}$

25.4

$$
\begin{aligned}
& 0.0254 \\
& 0.3048
\end{aligned}
$$$$
1.609
$$

Area

2.590

Volume

28.32

0.02832

Flow

$28 \cdot 32$

0.01093
To obtain SI units

$$
\begin{aligned}
& \text { millimeters (mm) } \\
& \text { meters (m) } \\
& \text { meters (m) } \\
& \text { kilometers ( } \mathrm{km})
\end{aligned}
$$

square $\mathrm{kllometers}\left(\mathrm{km}^{2}\right)$

cublc decimeters ( $\left.\mathrm{dm}^{2}\right)$

cublc meters $\left(\mathrm{m}^{\mathrm{s}}\right)$

1 ters per second ( $\mathrm{L} / \mathrm{s}$ )

cubic decimeters per second $\left(\mathrm{dm}^{2} / \mathrm{s}\right)$

cubic meters per second $\left(\mathrm{m}^{3} / \mathrm{s}\right)$

cublc meters per second per square kilometer $\left(\left(\mathrm{m}^{2} / \mathrm{s}\right) / \mathrm{km}^{2}\right)$ 
The low-flow characteristics of Iowa streams are described by annual and seasonal low-flow frequency and duration data. Tabulated in this report are lowfiow data collected at 135 gaging stations on Iowa streams, three on the Mississippi River, four on the Missouri River, and 426 partial-record sites.

The information contained in this report is based on all of the dally discharge records collected by the U.S. Geological Survey through the 1976 water year. Consideration is given to the regional aspects of low-flow characteristics by including regresston equations to estimate the average discharge and generallzed maps to estimate the 7-day, 2-year and the 7-day, 10-year discharges at ungaged sites.

\section{INTRODUCTION}

\section{Purpose and Scope}

It is significant that the title of this report should refer again to low flows. This is the third time within the last 20 years that state agencles and the U.S. Geological Survey have cooperated in the preparation of a report dealing with the low-flow characteristics of Iowa Streams. Each time, these reports have been made avallable in response to continually increasing demand for water-related data, from state and city officials, farmers, industry, private citizens, and others. If this continually increasing demand for water is an indication of economic growth, then Iowa is growing and developing at a rapid rate. With the prospect of continuing growth making demands upon the water supplies and the multitude of water problems that need to be resolved, it is clear that we must continue to find out more it is clear that we must continue to find out more this report is to make updated information on the water supply characteristics of Iowa streams
avaliabie to planners, engineers, water managers, and avaliabie to planners, engineers, water managers, and characteristics of Iowa streams were by Schwob (1958) characteristics of Iowa streams were by Schwob (1958)
and Heinitz (1970); the latter report included streamiflow data collecied through 1956. This report extends the 1970 study by the addition of 10 more years of record. Furthermore, this report also includes:

a. Low-flow b. Annual low-flow discharges, corresponding to selected perlods of consecutive days, for each climatic year of record.

c. Annual and seasonal low-flow characteristics at each gaging station having 10 or more years of record.

d. A complete list of discharge measurements made at each of 426 low-flow partlal-record stations. e. Selected low-flow parameters estimated for each partial record site.

$f$. Reglonal equations for estimating the average discharge at ungaged sites.

g. Maps that show resional trends of selected low-flow parameters.

This report, prepared by the U.S. Geological Survey, is the result of a 2-year cooperative Environmental Quality, the Iowa Natural Resources Council, and the U.S. Geologica! Survey. 


\section{AVAILABLE DATA}

The bastc streanflow data presented in this report have been complied from the U.S. Geological Survey flles. The data collection sites consist of 143 continuous-record gaging stations and 425 partici-record stations. The locations of these stations are shown in plates 1 and 2 . The continuous-record gaging stations provide a chronological record of the streamflow at these sites. The perlod of record of these stations is shown in the bar graph, figure 1.

which Low-flow partial-record stations are sites at systematically in order to define adequate relations with conct

\section{LOW-FLOW CHARACTERISTICS}

The low-flow characteristics of streams at gaging stations are described in tables at the end of this report. These tables show the frequency of annual and seasonal minimum flows, and annual and seasonal duration of dally discharges.

Methods for preparing low-flow and duration data, and the advantages of relating low flows to recurrence intervals, or probabilities of occurrence are generally understood by most users. These methods are well documented in publications by Riggs (1972), Chow (1964), and Linsley (1975) among others and are discussed only brlefly in this report.

\section{Low-flow Frequency Analysis}

A low-flow frequency analysis consists of a study of past records of flow and an estimate of the frequency of occurrence of future flows. Following is a summary of the methods of analysis used for this is a summary of the methods of analysis used for this study:

1. Complle annual low flows by searching the record of dally flows for the lowest average discharge corresponding to a selected perlod, within each climatic year. The perlods selected for this study were $3,7,14,30,60,120$, arid 183 consecut lve days. (These data are tabulated for each continuousrecord gaging station immediately following the station description).
2. Compute low-flow frequency values using as basic data the arrays of annual low-flow, computed in step 1. Two methods of fitting low-flow frequency curves were used: For stations with complete and continuous record (no missing records, or zero values) the log-pearson distribution function was selected; for the remaining stations, a graphical (distribution-free) method was used.

Fitting a log-Pearson type III frequency curve is done by substituting in

where:

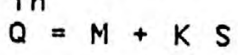

Q is a discharge on the frequency curve and is expressed in log units.

$M$ is the mean of the data expressed in log units.

$S$ is the standard deviation of the data expressed in log units.

$K$ is a frequency variate corresponding to the skew of the data and a selected recurrence interval. Values of $K$ are read from tables.

For a more detalled explanation the reader is referred to Riggs (1968, 1972).

Low-flow frequency curves using a graphical method are fitted as follows:

1. Arrange the array of low-flow discharges in order of magnitude and assign ranks beginning with the smallest as number 1 .

2. Compute the recurrence interval (RI) of each value using the formula:

Where:

$$
R I=(n+1) / m
$$

is the number of years of record. idata (tems)

3. is the rank. plot each value versus its computed recurrence interval using log-probability coordinates.

4. Draw a smooth curve to fit the plotted data. A typical family of low-flow frequency curves is shown in figure 2 .

Seasonal low-flow characteristics were obtained by repeating the above procedure using dally discharge data for each calendar quarter of a year. 
YEARS OF RECORD

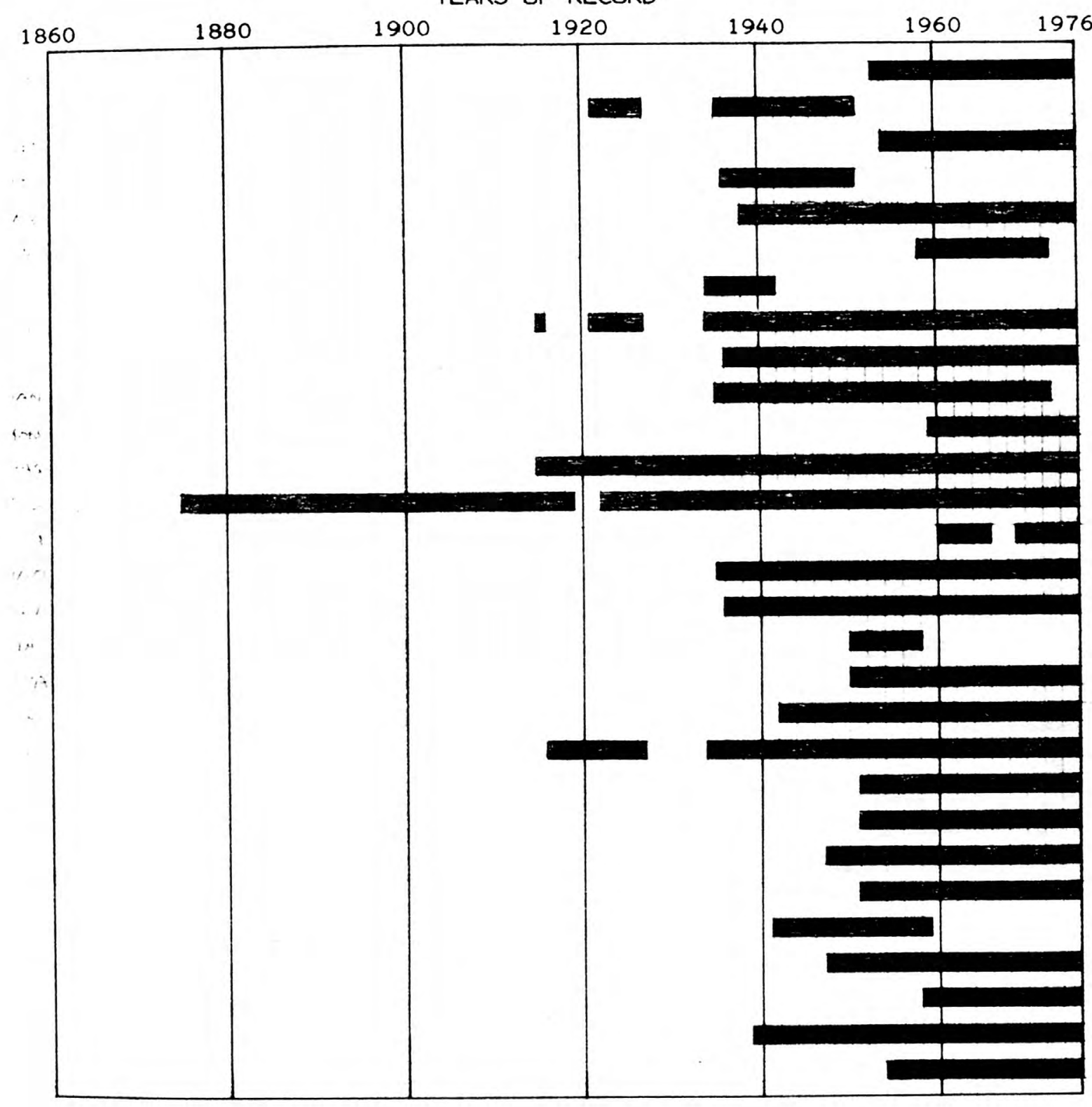

GAGING STATIONS

UPPER IOWA RIVER AT DECORAH

UPPER IOWA RIVER NEAR DECORAH

PAINT CREEK AT WATERVILLE

YELLOW RIVER AT ION

MISSISSIPPI RIVER AT MCGREGOR

TURKEY RIVER AT SPILLVILLE

TURKEY RIVER AT ELKADER

TURKEY RIVER AT GARBER

LITTLE MAQUOKETA RIVER NEAR DURANGO MAQUOKETA RIVER NEAR MANCHESTER BEAR CREEK NEAR MONFOUTH

MAQUOKETA RIVER NEAR MAQUOKETA MISSISSIPPI RIVER AT CLINTON WAPSIPINICON RIVER NEAR ELMA WAPSIPINICON RIVER AT INDEPENDENCE WAPSIPINICON RIVER NEAR DEWITT WEST BRANCH IOWA RIVER NEAR KLEMME EAST BRANCH IOWA RIVER NEAR KLEMME IOWA RIVER NEAR ROWAN

IOWA RIVER AT MARSHALLTOWN TIMBER CREEK NEAR MARSHALLTOWN RICHLAND CREEK NEAR HAVEN

SALT CREEK NEAR ELBERON WALNUT CREEK NEAR HARTWICK IOWA RIVER NEAR BELLE PLAINE BIG BEAR CREEK AT LADORA IOWA RIVER AT MARENGO

RAPID CREEK NEAR IOWA CITY CLEAR CREEK NEAR CORAL VILLE

Figure 1. Perlod of record of continuous-record gaging stations. 


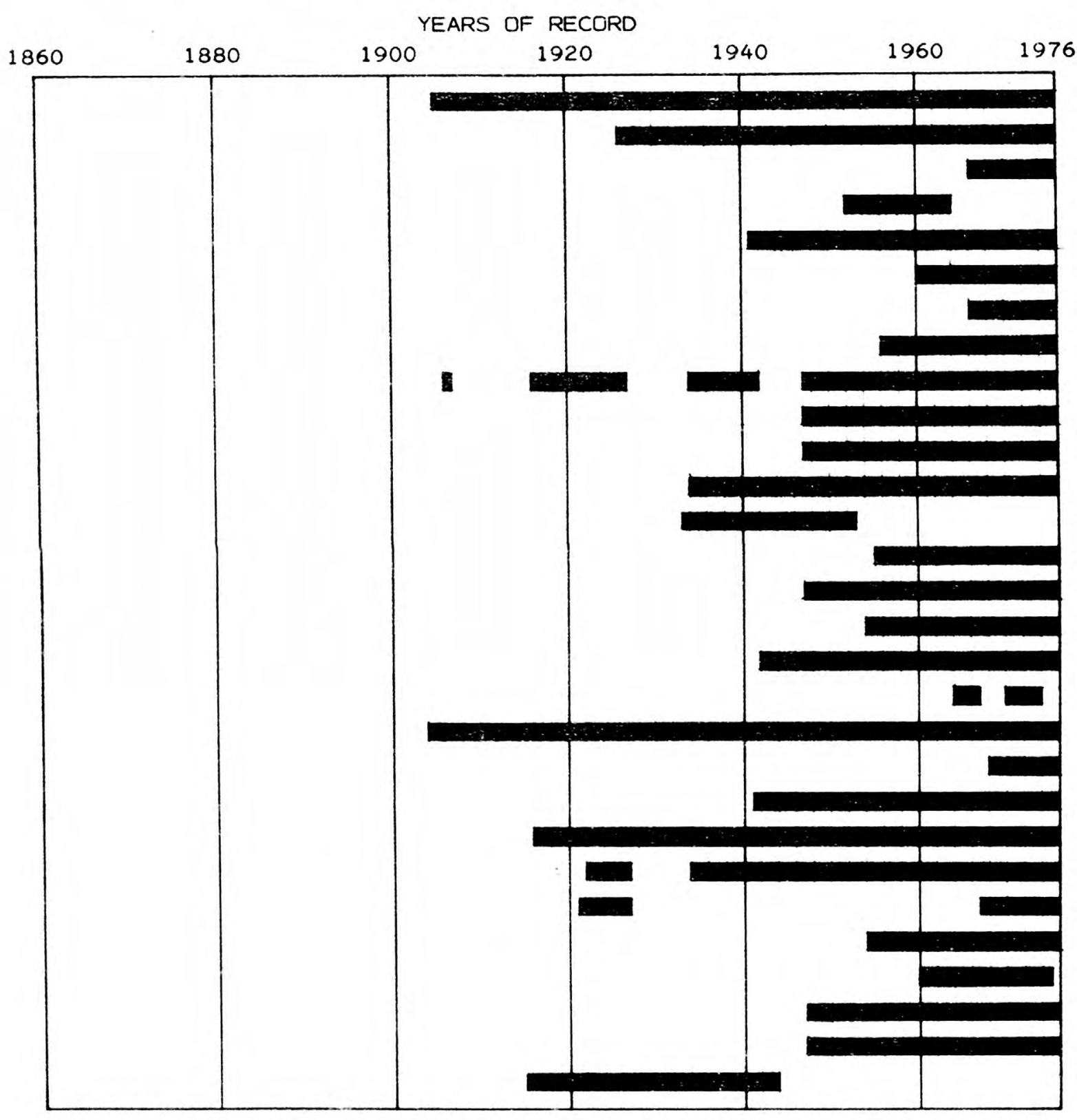

GAGING STATIONS

IOWA RIVER AT IOWA CITY

RALSTON CREEK AT IOWA CITY

SOUTH BRANCH RALSTON CREEK AT IOWA CITY

OLD MANS CREEK NEAR IOWA CITY

ENGLISH RIVER AT KALONA

IOWA RIVER NEAR LONE TREE

CEDAR RIVER AT CHARLES CITY

LITTLE CEDAR RIVER NEAR IONIA

CEDAR RIVER AT JANESVILLE

WEST FORK CEDAR RIVER AT FINCHFORD

SHELL ROCK RIVER NEAR NORTHWOOD

WINNEBAGO RIVER AT MASON CITY

SHELL ROCK RIVER AT MARBLE ROCK

SHELL ROCK RIVER AT SHELL ROCK

BEAVER CREEK AT NEW HARTFORD

BLACK HAWK CREEK AT HUDSON

CEDAR RIVER AT WATERLOO

HALF MILE CREEK NEAR GLADBROOK

CEDAR RIVER AT CEDAR RAPIDS

PRAIRIE CREEK AT FAIRFAX

CEDAR RIVER NEAR CONESVILLE

IOWA RIVER AT WAPELLO

SOUTH SKUNK RIVER NEAR AMES

SQUAW CREEK AT AMES

SOUTH SKUNK RIVER BELOW SQUAW CR NR AMES

INDIAN CREEK NEAR MINGO

SOUTH SKUNK RIVER NEAR OSKALOOSA

NORTH SKUNK RIVER NEAR SIGOURNEY

SKUNK RIVER AT COPPOCK

Figure 1. Perlod of record of continuous-record gaging stations--continued. 


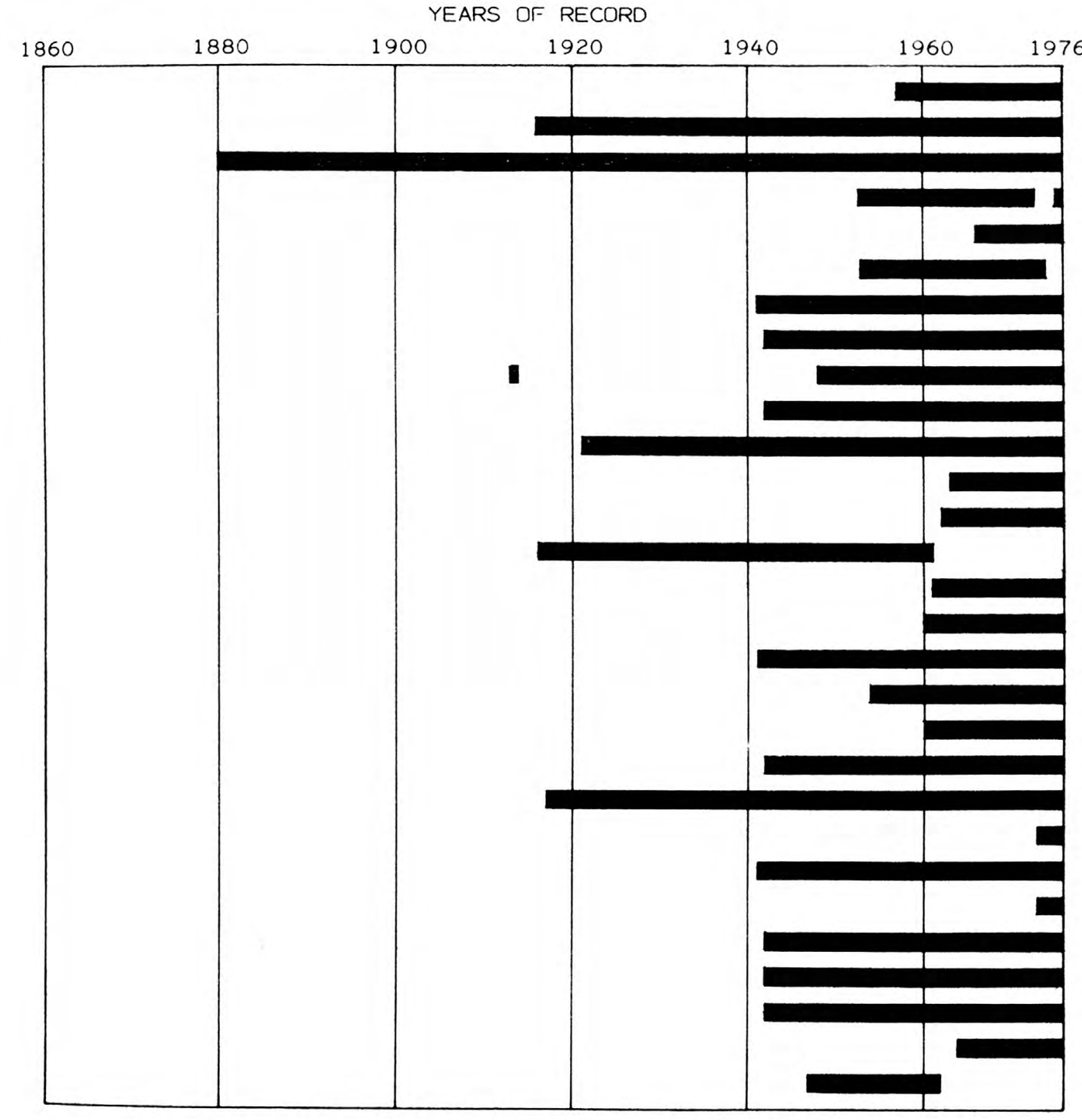

GAGING STATIONS

BIG CREEK NEAR MOUNT PLEASANT

SKUNK RIVER AT AUGUSTA

MISSISSIPPI RIVER AT KEOKUK

DES MOINES RIVER AT ESTHERVILLE

DES MOINES RIVER AT HUMBOLDT

EAST FORK DES MOINES RIVER NEAR BURT

EAST FORK DES MOINES RIVER AT DAKOTA CITY

LIZARD CREEK NEAR CLARE

DES MOINES RIVER AT FORT DODGE

BOONE RIVER NEAR WEBSTER CITY

DES MOINES RIVER NEAR STRATFORD

DES MOINES RIVER NEAR SAYLORVILLE

BEAVER CREEK NEAR GRIMES

DES MOINES RIVER AT DES MOINES

BIG CEDAR CREEK NEAR VARINA

NORTH RACCOON RIVER NEAR SAC CITY

NORTH RACCOON RIVER NEAR JEFFERSON

EAST FORK HARDIN CREEK NEAR CHURDAN

MIDDLE RACCOON RIVER AT PANORA

SOUTH RACCOON RIVER AT REDFIELD

RACCOON RIVER AT VAN METER

WALNUT CREEK AT DES MOINES

DES MOINES R BELOW RACCOON R AT DES MOINES FOURMILE CREEK AT DES MOINES

NORTH RIVER NEAR NORWALK

MIDOLE RIVER NEAR INDIANOLA

SOUTH RIVER NEAR ACKWORTH

WHITE BREAST CREEK NEAR DALLAS

WHITE BREAST CREEK NEAR KNOXVILLE

Figure 1. Period of record of continuous-record gaging stations--continued. 


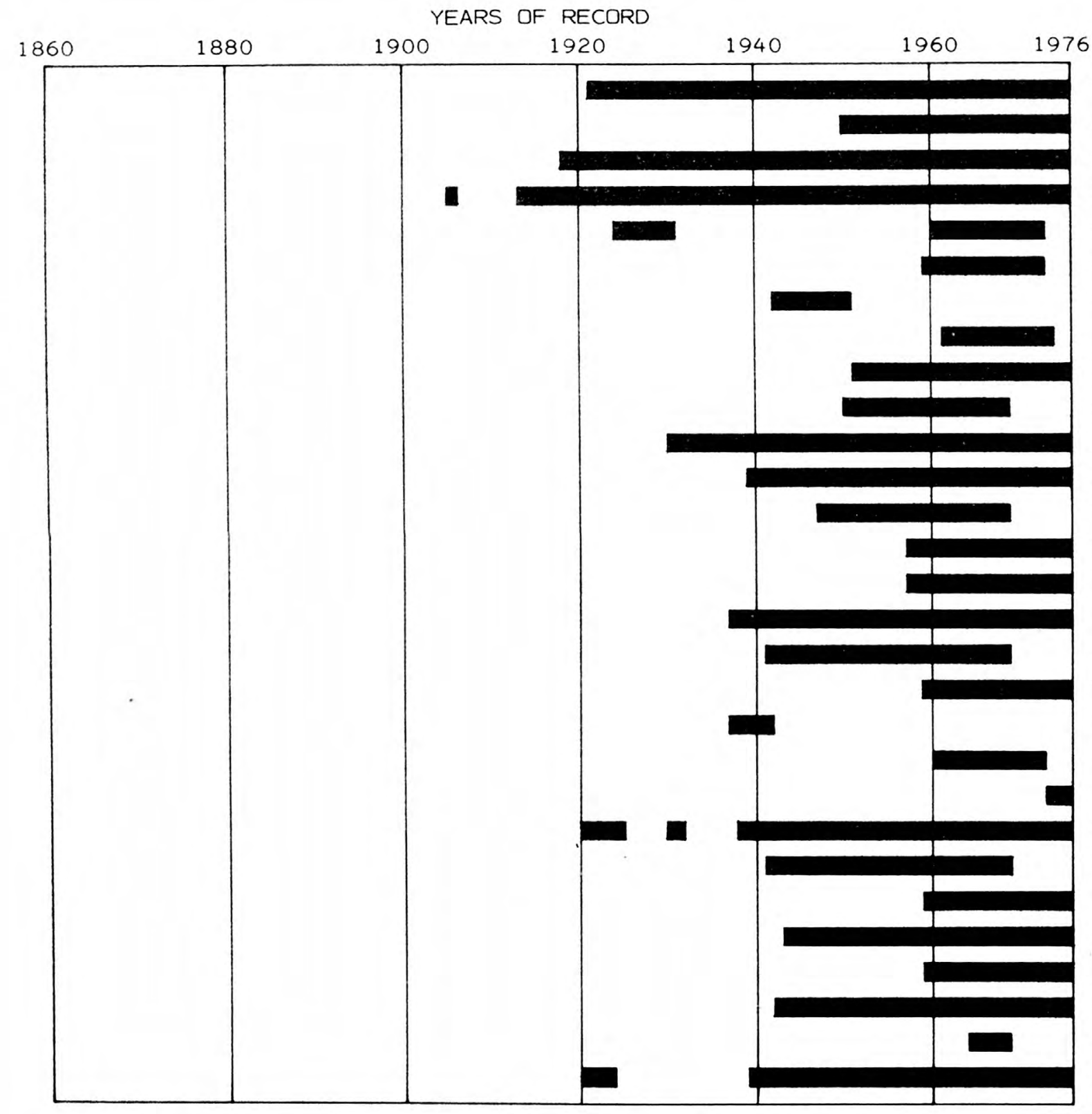

GAGING STATIONS

DES MOINES RIVER NEAR TRACY

CEDAR CREEK NEAR BUSSEY

DES MOINES RIVER AT OTTUMWA

DES MOINES RIVER AT KEOSAUQUA

SUGAR CREEK NEAR KEOKUK

FOX RIVER AT BLOOMFIELD

FOX RIVER AT CANTRIL

ROCK RIVER AT ROCK RAPIDS

ROCK RIVER NEAR ROCK VALLEY

DRY CREEK AT HAWARDEN

BIG SIOUX RIVER AT AKRON

MISSOURI RIVER AT SIOUX CITY

PERRY CREEK AT 38TH STREET, SIOUX CITY

FLOYD RIVER AT ALTON

WEST BRANCH FLOYD RIVER NEAR STRUBLE

FLOYD RIVER AT JAMES

WEST FORK DITCH AT HOLLY SPRINGS

MONONA-HARRISON DITCH NEAR TURIN

LITTLE SIOUX RIVER AT SPENCER

LITTLE SIOUX RIVER AT GILLETT GROVE

LITTLE SIOUX RIVER AT LINN GROVE

LITTLE SIOUX RIVER AT CORRECTIONVILLE

LITTLE SIOUX RIVER NEAR KENNEBEC

ODEBOLT CREEK NEAR ARTHUR

MAPLE RIVER AT MAPLETON

LITTLE SIOUX RIVER NEAR TURIN

SOLDIER RIVER AT PISGAH

STEER CREEK NEAR MAGNOL IA

BOYER RIVER AT LOGAN

Figure 1. Period of record of continuous-record gaging stations--continued. 


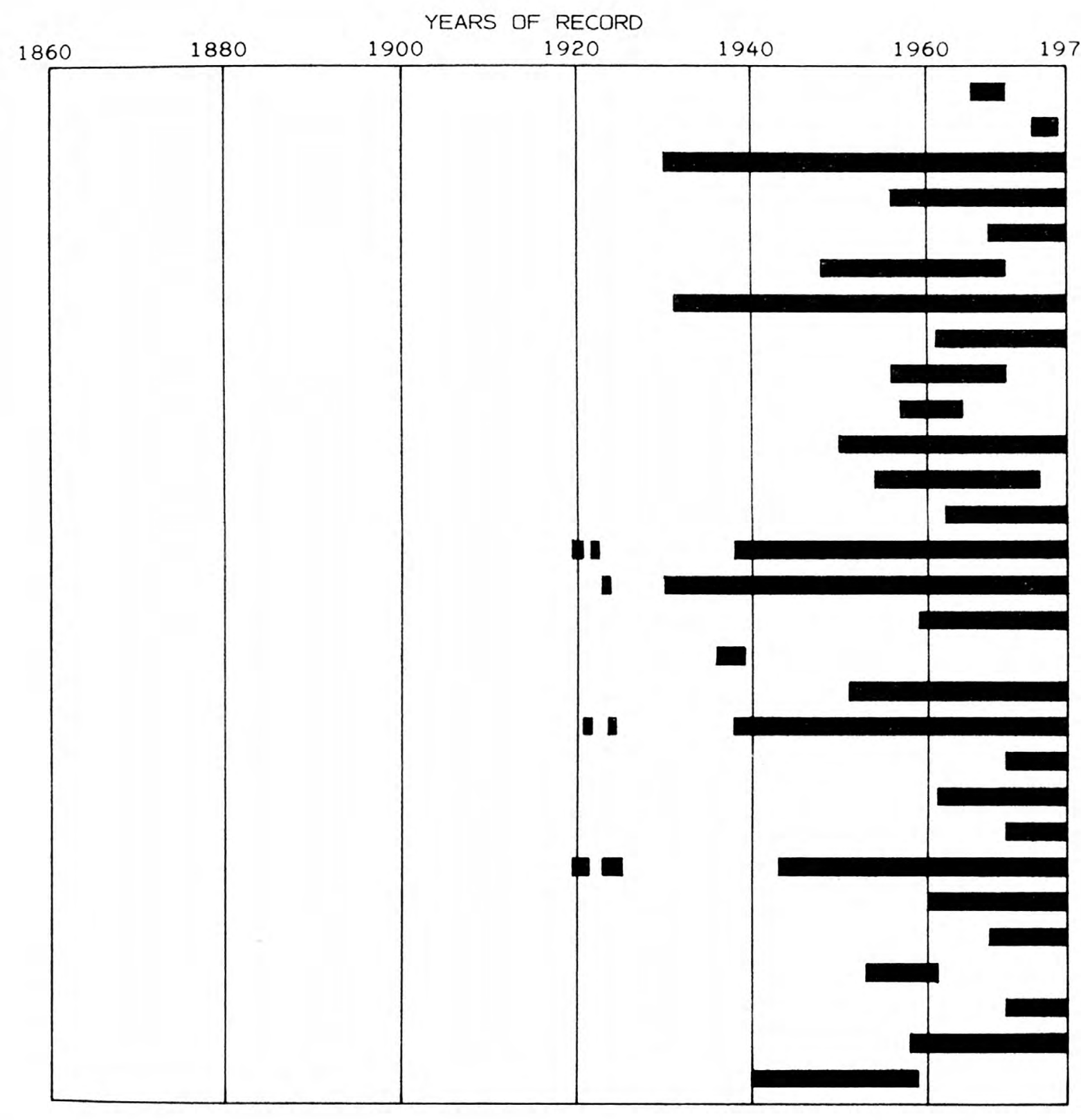

GAGING STATIONS

THOMPSON CREEK NEAR WOODBINE

WILLOW CREEK NEAR LOGAN

MISSOURI RIVER AT OMAHA, NB

INDIAN CREEK AT COUNCIL BLUFFS

MOSQUITO CREEK NEAR EARLING

WAUBONSIE CREEK NEAR BARTLETT

MISSOURI RIVER AT NEBRASKA CITY, NB

WEST NISHNABOTNA RIVER AT HANCOCK

MULE CREEK NEAR MALVERN

SPRING VALLEY CREEK NEAR TABOR

WEST NISHNABOTNA RIVER AT RANDOLPH

DAVIDS CREEK NEAR HAMLIN

EAST NISHNABOTNA RIVER NEAR ATLANTIC

EAST NISHNABOTNA RIVER AT RED OAK

NISHNABOTNA RIVER ABOVE HAMBURG

TARKIO RIVER AT STANTON

TARKIO RIVER AT BLANCHARD

MISSOURI RIVER AT RULO, NB

NODAWAY RIVER AT CLARINDA

PLATTE RIVER NEAR DIAGONAL

E F ONE HUNDRED AND TWO RIVER NR BEDFORD

ELK CREEK NEAR DECATUR CITY

THOMPSON RIVER AT DAVIS CITY

WELDON RIVER NEAR LEON

CHARITON RIVER NEAR CHARITON

HONEY CREEK NEAR RUSSELL

$S$ F CHARITON RIVER NEAR PROMISE CITY

CHARITON RIVER NEAR RATHBUN

CHARITON RIVER NEAR CENTERVILLE

Figure 1. Period of record of continuous-record gaging stations--continued. 


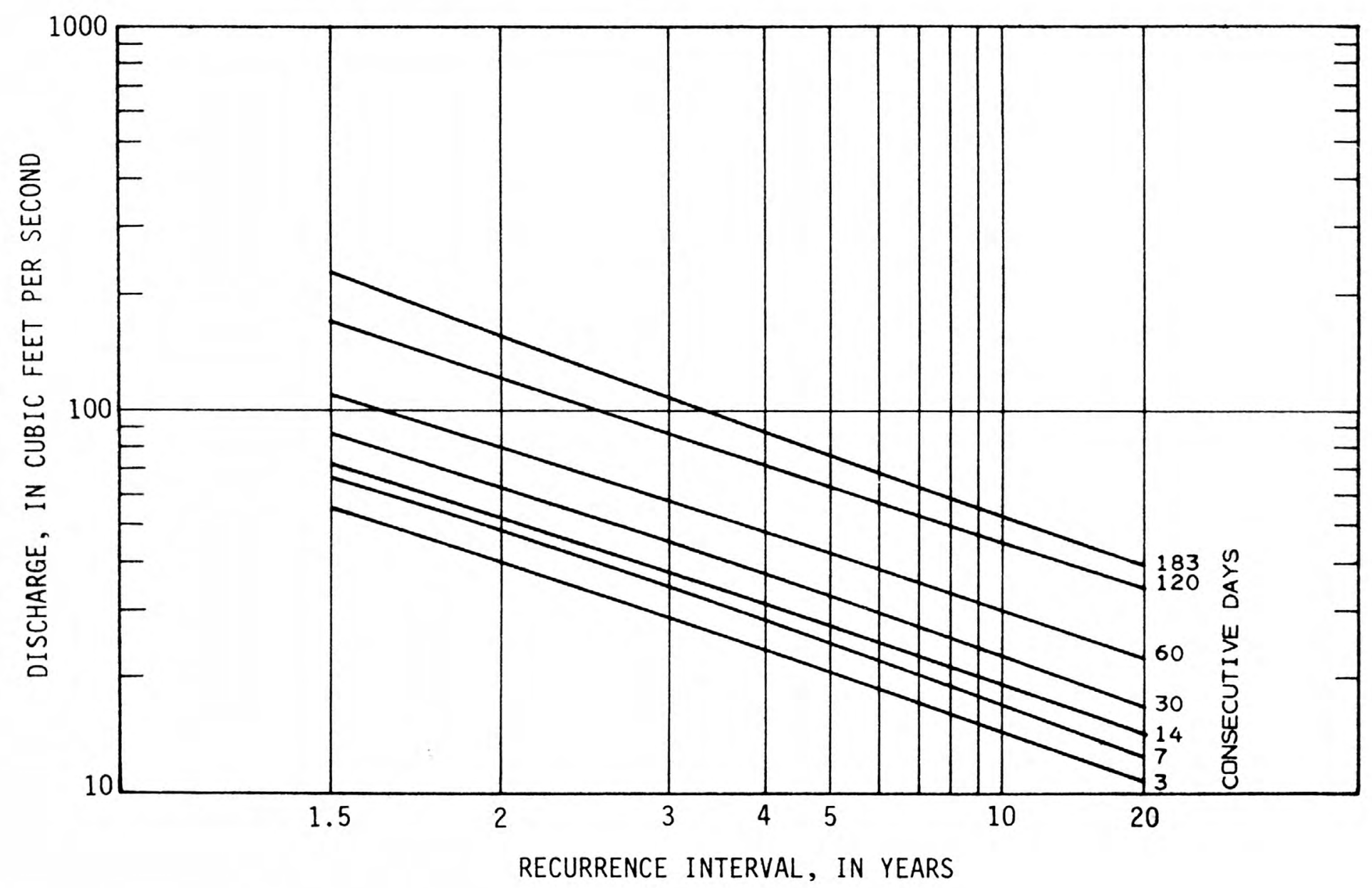

Figure 2. Low-flow frequency curves for gaging station 05421000 Wapsipinicon River at Independence, Iowa. 
Flow Duration

Flow- duration is a familiar tool to those concerned with water supply problems, power development, dilution and disposal of sewage and industrial waste materials. It is also useful to hydrologists to compare basin characteristics.

The flow-duration data indicate the percent of time within a given period during which glven rates time within a glven period durlng which glven rates for flow were exceeded. for a given gaging station, the dally mean discharges were arranged In class intervals, depending upon their magnitudes. Determination was then made of the percent of time during which the flow was equal or greater than the lower limit of each class. An anriual and two seasonal duration data values were prepared. The first is based on the daily discharges for the entire water year. A typlcal flow duration curve is shown in figure 3 . The second tabulation Is based on the inlly discharges between Aprti 1 and based on the dally discharges between Aprl 1 and September 30 of each water year. The third is based on dally discharges during the months of July and August.

\section{ARRANGEMENT OF STATION DATA}

Continuous-record Stations

Data for each continuous-record station are presented in three parts, the first part is a paragraph describing the location of the station, the second is a table issting the basic low-flow data collected at this station, the third part is a collected at this station, the third part is a summary of the annual and season.

Most of the data presented for each gaging station are self explanatory. However, a statement is needed concerning stream flows which are regulated by man-made structures. In this instance, a "Remarks" paragraph has been added to explain the point in time when regulation started, and also the type and extent of regulation. If adequate periods of record are avallable at a station for both natural and regulated flows, two sets of data are presented

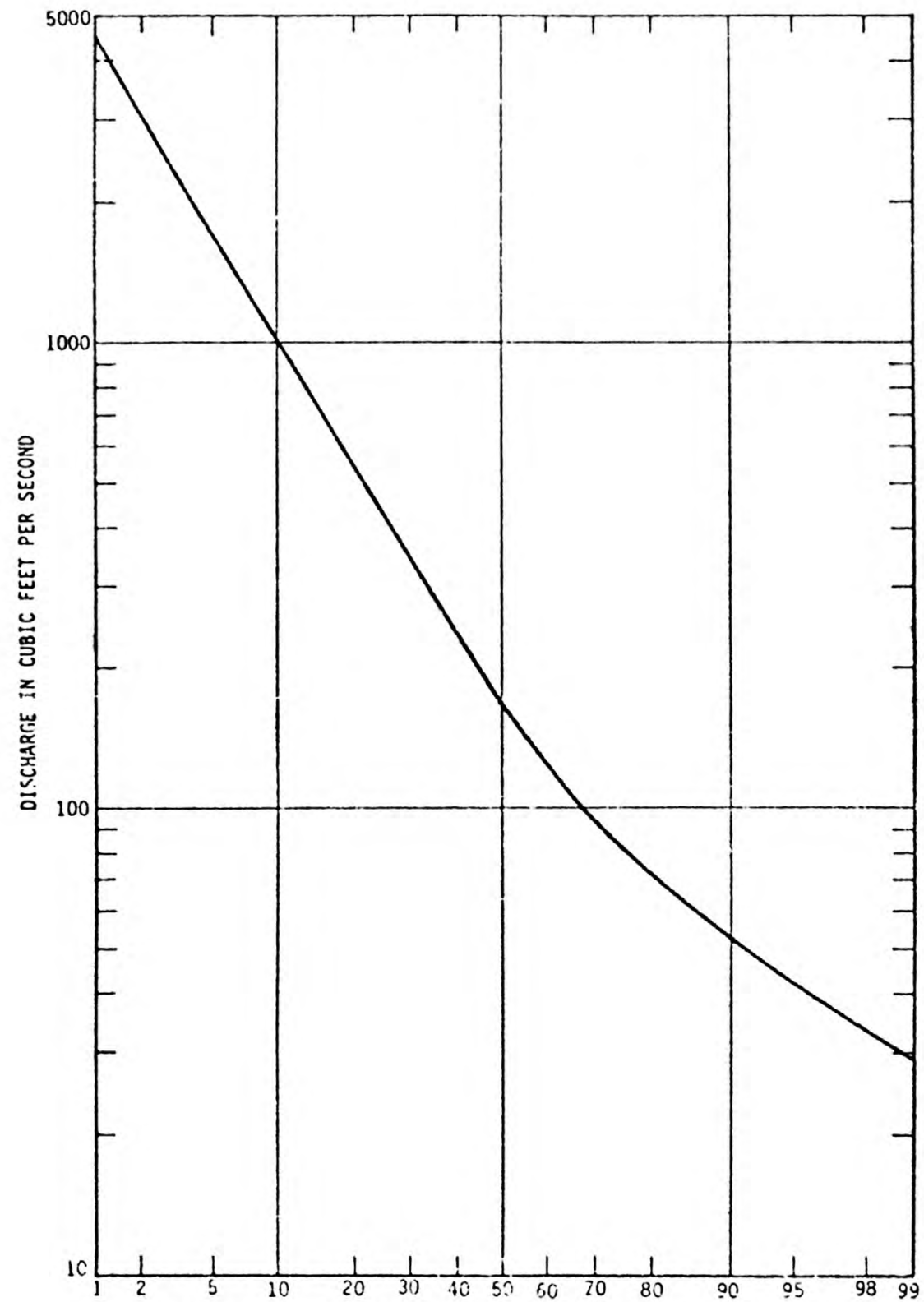

PERCENT OF TIME DISCHARGE EYUALEL OR EXCEEDEd THAT SHOWN

Figure 3. Flow-duration curve for gaging station $054840 C 0$ South Raccoon River at Redfield, lowa. 
for this station, one based on the natural river flows and the other based on the regulated flows. For example, see the data for Station 5-454500 Iowa River at Iowa City.

$$
\text { A statemerit is also required concerning the }
$$
perlod of record in years listed for each station. This figure indicates the number of complete water years of record. This number does not necessarily agree with the number of climatic years shown in the tabulations of low-flow discharges. Generally, for a given station record, the number of climatic years is 1 less than the number of water years. The reason for this difference is the manner in which they are defined. The water year is a 12-month period beginring Oct. 1 and ending sept. 30, while the climatic year is a 12 -month perlod beginning 6 months later on April 1 and ending on March 31 . In both cases the years are designated by the calendar year in which they end. Thus, if the record of a given station started October 1, 1929, the first complete water year will end September 30, 1930, and the year 15 called the 1930 water year. The first complete climatic year will start 6 months later on April 1. 1930, and end on March 31, 1931, and w111 be called the $1931 \mathrm{climatic}$ year. For this reason $n$ complete will yleld $(n-1)$ climatic Water years of lecord will yield ( $n$-l) years. record, the number of water years and the number of climatic years will be the same.

Gaging stations are listed in downstream order. the same as in water-supply papers published by the U.S. Geological Survey.

For stations having less than 10 years of record, only the station description and the basic low-flow data are tabulated. However, selected lowflow values have been estimated and are included at the bottom of the basic data tables. Low-flow values for short-record stations were estimated on the bas 1 s of a relation developed between the short-term record and the concurrent $r \in c o r d$ of a nearby long-term station.
Low-flow Partial-record Stations

Data for low-flow partial-record stations listed at the end of the report include the following:

1. Station description

2. Size of dralnage area, $\ln \mathrm{m}^{2}$

. Discharge measurements and the dates when made, l1sted in chronological order

4. $Q(84)$ discharge which corresponds to the 84 percent of time ordinate of a seasona percent of time ordinate of a seasonai
duration curve prepared by using dally discharges from April 1 through Sept. 30.

5. 702 seven-day low-flow with a 2-year recurrence interval.

E. 7010 seven-day low-flow with a 10-year recurrence interval.

For estimating low-flow characteristics at a partial-record site, the base-flow measurements at the site are correlated with concurrent flows at nearby gaging stations where continuous records are avaliable. Based on these correlations the flow characteristics at the gaging station are used to make low-flow frequency estimates for the partialrecord site.

Measurements at partial-record stations were made during perlods of base flow when streamflow is primarlly from ground-water sources and in accordance with a predetermined plan. To obtain satisfactory correlations, measurments should be made only after substantial lapse of time following the last precipitation. The exact length of eiapsed time appears to be a function of the characteristics of the basins, and may be in some instances as great as 10 days. Because of these $11 \mathrm{mitat}$ ions measurements often of these ilmitatlons, sultable measurements often cannot be made each year. This explains why more than one measurement was made in certain years and none in others. 
Estimates of streamflow values often are required at points remote from gaging stations. consequently. methods are needed to transfer gagingstation information to ungaged sites. Regional station information to ungaged sites. Regional discussion of methods of regional arialysis is beyond the scope of this report. Readers who are interested in this subject are referred to Riggs (1973). The followirg paragraphs describe the results of regional aralysis studies. conducted to provlde the capabrlity a f ingaged areas.

\section{Average Discharge}

The average discharge is an important and usefu streamflow parameter because it defines the total water avallabie from the stream at a given location.

Using standard regional analysis procedures the state was divided into three hydrologic regions (figure 4 ).

Within each region the following equations were developed:

$$
\begin{array}{ll}
\text { Region I } & Q_{a}=0.54 \mathrm{~A}^{1.02} \\
\text { Region II } & Q_{a}=0.19 \mathrm{~A}^{0.98}(P-25)^{0.57}
\end{array}
$$

Region
where:

Qa is the average discharge, in cubic feet per second.

A is the size of the drainage area in square miles.

$P$ is the normal annual precipitation, in inches, determined from map (f igure 4 ).

The standard errors of these equations are approximately 17 percent.

The average discharge ( $Q$ a) shown for the gaging stations with short-term record and for the partialrecord stations was estimated by using the equations shown in figure 4 .
Although the principles of regional analysis apply quite well to average flows, they could not be applied successfully to low flows. This is because low flows are closeiy related to geologic parameters, which at present are not clearly identifled and cannot easily be quantifled or described by simple indexes. Therefore, low-flow characteristics at ungaged sites in Iowa cannot, at the present time, be reliably estimated by regional regression equations. The use of base-flow measurements at the site as explained elsewhere in the report, remains the recommended procedure for the recommended characteristics at ungaged sites.

There are times, however, when there is a need for making quick low-flow estimates, especially in certain phases of the planning stage, where time is of essence and where certain facts need not be accurate but reasonable.

$$
\text { For users who need to make this type of }
$$

estimate, two maps have been prepared (see plates 3 and 4). These maps show areal trends of two often used low-flow parameters, the 7 -day, 2 -year $(702)$ and the 7-day, 10-year (7Q10) discharges. These trends were delineated on maps using the information collected at the gaging stations and at the partialrecord sites. The data given on plate 4 is applicable to all stream points except to those points on the main stem of selected streams shown on plate 3. To use these maps proceed as follows.

1. Determine the size of the drainage area in square miles.

2. Determine the location of the stream point using elther plate 3 or plate 4.

3 . Compute the low-flow parameter in $\mathrm{ft}^{3} / \mathrm{s}$ by multiplying the size of the dralnage area by the map number. Use the upper figure to estimate the 702 . and the lower figure to estimate the 7010 . 


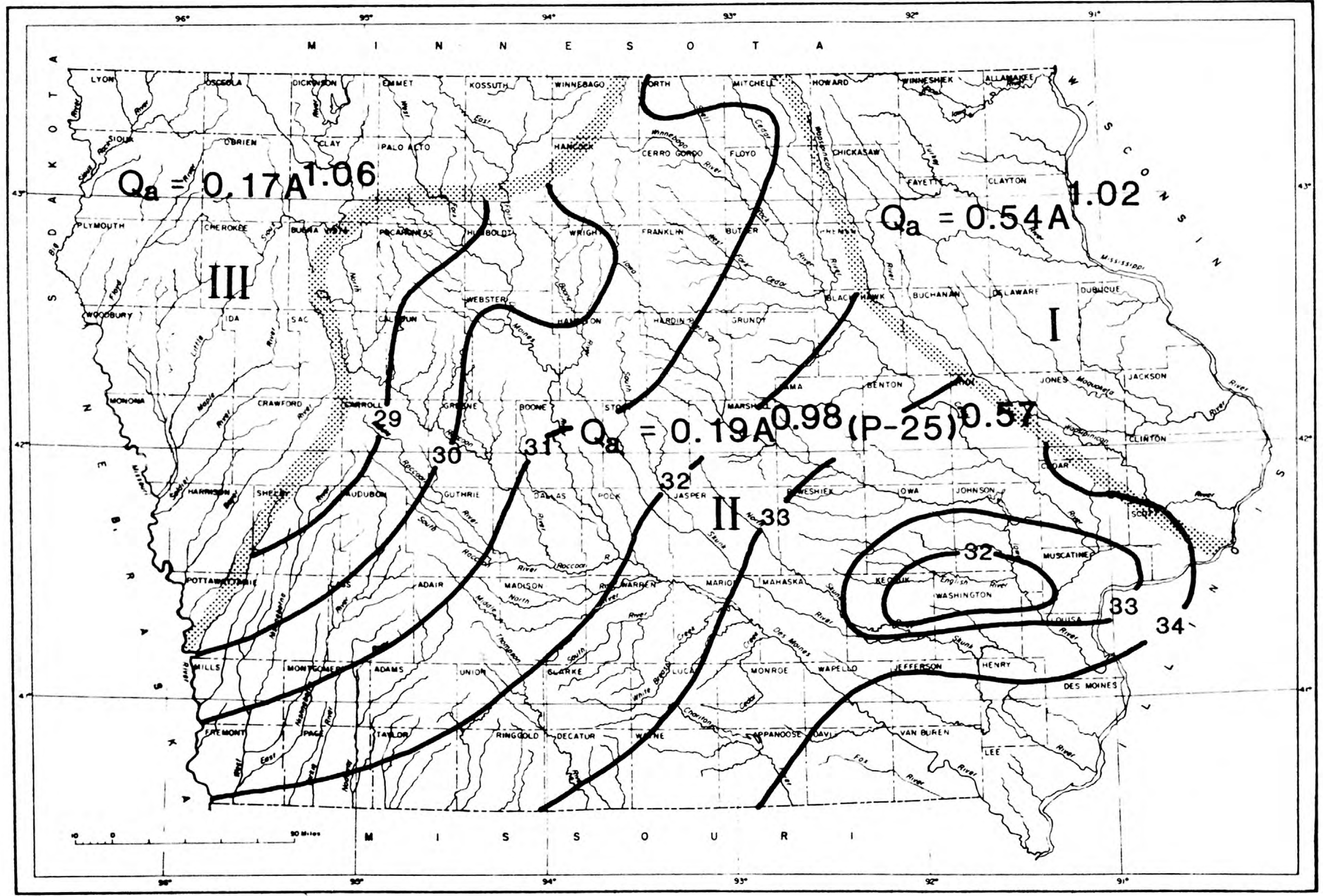

Flgure 4. Regional equations to compute the average discharge, and lowa normal annual precipitation for region II. 


\section{DISCUSSION}

In this report discussion has been directed toward an understanding of the data presented and the procedures used in compiling and analyzing these data.

Further comment should be made concerning rellablitity and limitations of these data. It must be recognized that any record of streamflow is but a sample from an infinitely large population of flow regimes. The longer the record the better the prediction of future hydrologic behavior of a stream. Similarly the shorter the record the greater the risk of making an erroneous decision, or a biased est imate.

A cursory examination of the low-flow baslc data tables presented for each gaging station will show that the low-flow discharges in the perlod 1969-1975 are substantially higher than those of previous perlods. Climatological records for lowa show that precipitation during this period was significantly higher than normal. For example, Eurmeister (1973), reports that during an 18-month perlod, March 1972 to August 1973, the monthly runoffs at selected stations throughout the state were 6 to 10 times higher than the average. Therefore, it is reasonable to expect that the low-flow frequency data for stations with short pertods of record which include this period of high perlods of record which include this perlod of high runoff will be slanificantly Computations have shown that the magnitude of this "climatic bias" varies with the length of record, the geographlc location of the basin, and the type of low-flow parameter. For this reason it is highly recommended that readers of this report who need to use information at a station with a short period of record determine the extent to which this station data may be biased.

A generally accepted method of computing this factor is to use the records of a nearby long-term station and compare the results based on the longterm record with the results based on the concurrent short-term record. The ratio between these values is the factor by which the short-term values should be adjusted.

To Hllustrate this procedure the reader is stations on the Cedar River. The upstream station is at Charles City where the dralnage area $151054 \mathrm{~m}^{2}$; the period of record at this station $1 \mathrm{~s} 11$ years (1966-1976). The second station $1 \mathrm{~s}$ downstream at Janesville, where data have been collected for 56 years. Now compare any low-flow frequency index listed for Charles City with an equivalent index at Janesville. This comparison will show that the Charles city indices are conslderably higher than those at Janesvilie and are affected by some factor of climatic bias.

To compute this factor for the 7-day Q2, proceed as follows

1. Read the 7-day Q2 11sted in the table for Cedar River at Janesville, which is $136 \mathrm{ft}^{3} / \mathrm{s}$.

2. Using the low-flow discharges listed in the table for Janesviile, compute the 7-day Q2 based on the concurrent short perlod (1966-1976). The result from this computation is $228 \mathrm{ft}^{3} / \mathrm{s}$.

The ratio between these values.

$$
136 / 228=0.60
$$

is the factor by wich the value 11 sted for Charles City should be adjusted or $169 \times .60=101 \mathrm{ft}^{\mathrm{3}} / \mathrm{s}$ Similar computation for the 7 -day 010 yielded a factor equal to:

$$
68 / 152=0.45
$$

The adjusted value for the 7 -day $Q 10$ at Charles City is then $113 \times .45=51 \mathrm{ft}^{3} / \mathrm{s}$.

Similar procedures could be used to adjust other low-flow parameters.

\section{REFERENCES}

Burmelster, I. L., 1973, Signifleant Hydrologic Events March 1972 to August 1973, Supplement to speech given at Annual Meeting, Iowa Section ASCE, Des Molnes, Iowa, $29 \mathrm{p}$.

Chow, V. T., 1964, Handbook of applled hydrology. McGrawH 111 Co., New York, Pp 8-2, 8-37, and $1,18-19$. 
Heinitz, A. J., 1966, Low-flow characteristics of lowa streams through 1966: Iowa Natural Resources Council Bul1. No. 10, $176 \mathrm{p}$

Langbein. W. B. and Iser1, K. T., 1960, General introduction and hydrologic definitions: U.S. Geol. Survey Water-Supply Paper 1541-A., $29 \mathrm{p}$.

Linsley, R. K., Kohler, M. A., Paulhus, J. L., 1975, Hydrology for engineers, McGraw-Hill Co., New York.

Riggs, H. C., 1968, Frequency curves: U.S. Geol. Survey Techniques Water-Resources Inv., book 4 , chap. A2, $15 \mathrm{p}$.

-1969, Some statistical tools in hydrology: U.S. Geol. Survey Techniques Water-Resources Inv. book 4, chap. Al, 39 P.

1972, Low-flow investigations: U.S. Geol. Survey Technicues Water-Resources Inv., book 4 , chap. $81,18 \mathrm{p}$

- 1973, Regional analyses of streamflow characteristics: TWR.I Book 4, chap. B3, $15 \mathrm{p}$.

Schiwob, H. H., 1958, Low-flow characteristles of Iowa streams: lowa Natural Resources Council Bull. No. 9, $111 \mathrm{p}$. 
CONTINUOUS-RECORD

STATION DATA 

Location.--Lat $43^{\circ} 18^{\prime} 19^{\prime \prime}$, long, $91^{\circ} 47^{\prime} 48^{\prime \prime}$, in NE1/4 SW1/4 sec.16, T.98 N., R.8 W. Winneshiek County, on right bank $1,200 \mathrm{ft}$ upstream from bridge on U.S. Highway 52 (city route) in Decorah, 1,500 ft downstream from Dry Run cutof $f$, and 3.0 miles upstream from Trout Run. LOW FLOW DISCHARGES, IN YEAR. ENDING MARCH 31

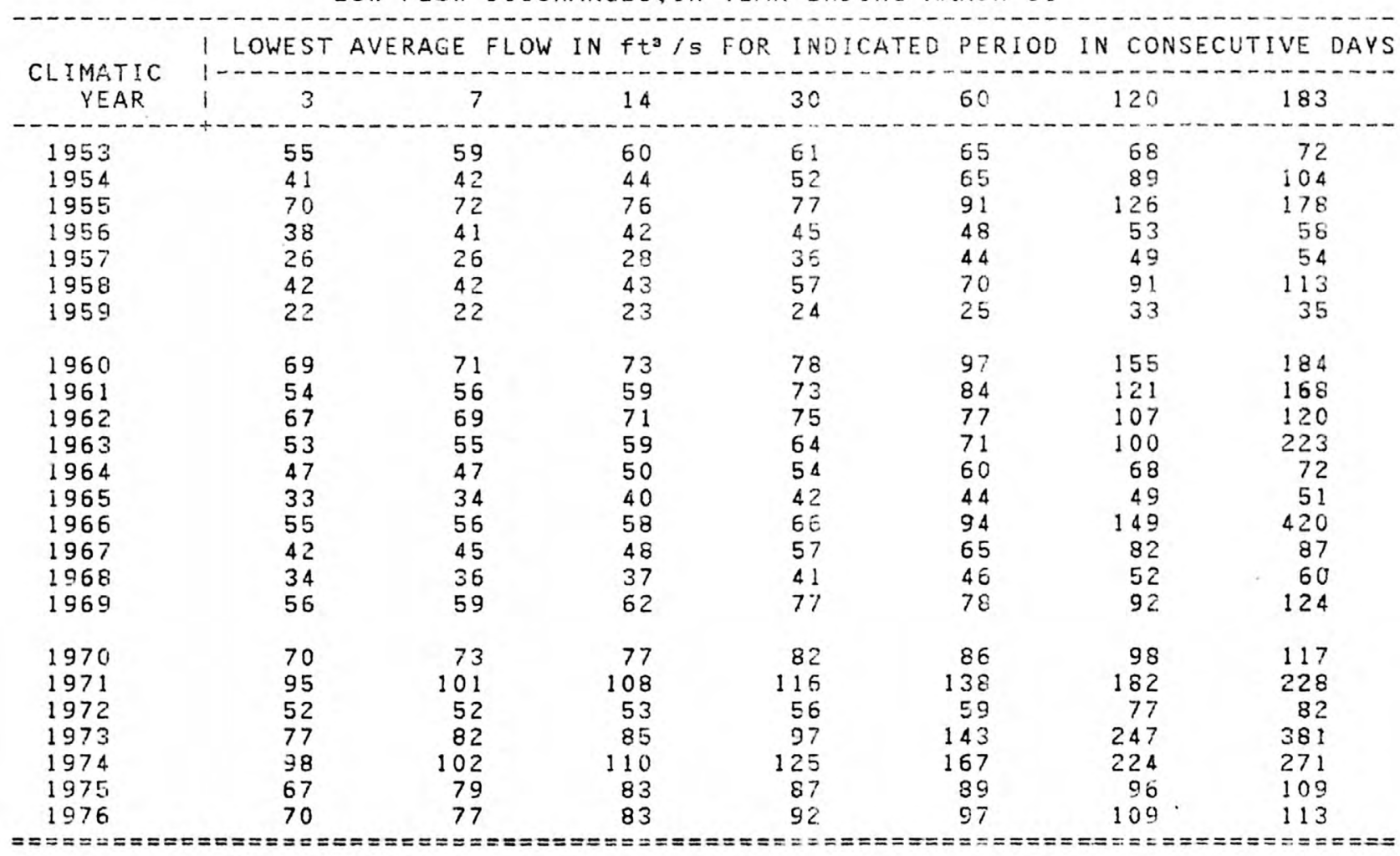


MINIMUM AVERAGE FLOWS FOR PERIOD OF RECORD

\begin{tabular}{|c|c|c|c|c|c|c|c|}
\hline PERIOD OF CONSECUTIVE DAYS I & 3 & 7 & 14 & 30 & 60 & 120 & 183 \\
\hline $\begin{array}{l}\text { ARGE, IN } \mathrm{ft}^{3} / \mathrm{s} \\
\text { IC YEAR }\end{array}$ & $\begin{array}{l}22 \\
1959\end{array}$ & $\begin{array}{l}22 \\
1959\end{array}$ & $\begin{array}{l}23 \\
1959\end{array}$ & $\begin{array}{l}24 \\
1959\end{array}$ & $\begin{array}{l}25 \\
1959\end{array}$ & $\begin{array}{l}33 \\
1959\end{array}$ & \\
\hline
\end{tabular}

MAGNITUDE AND FREQUENCY OF ANNUAL. LOW FLOWS

RECURRENCE I LOWEST AVERAGE FLOW, IN $\mathrm{ft}^{3} / \mathrm{s}, \mathrm{FOR}$ INDICATED PERIOD IN CONSECUTIVE DAYS

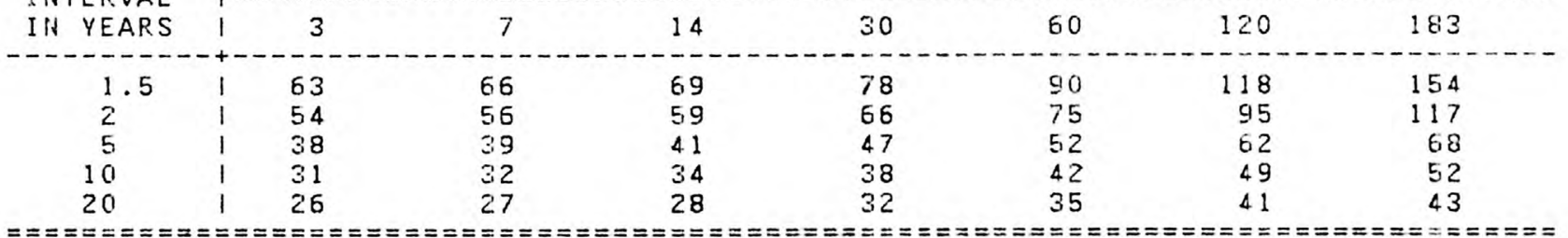

MAGNITUDE AND FREQUENCY OF SEASONAL LOW FLOWS

LOWEST AVERAGE FLOW, IN $\mathrm{ft}^{3} / \mathrm{s}, F O R$ THE INDICATED PERIOD IN CONSECUTIVE DAYS WITHIN EACH WATER-YEAR QUARTER

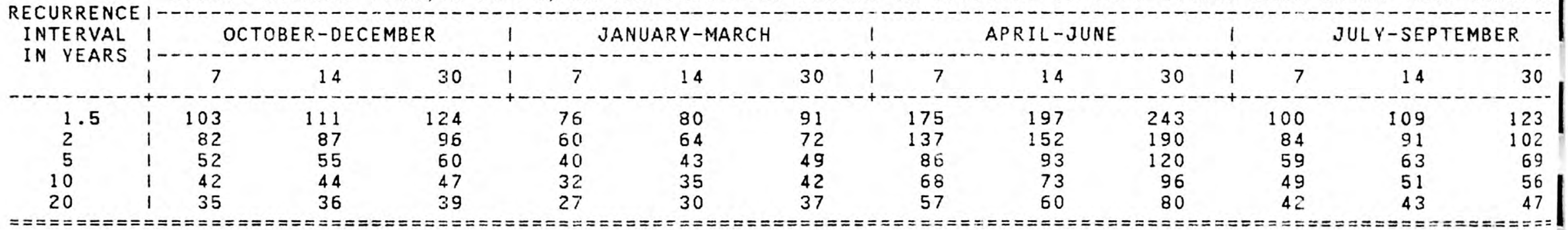

DURATION OF DAILY DISCHARGES FOR ANNUAL AND SEASONAL PERIODS

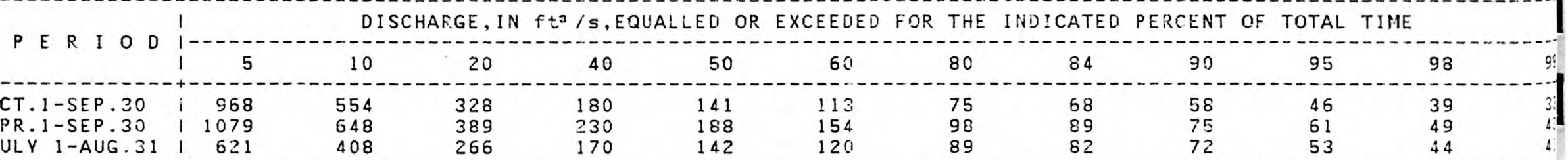


Location.--Lat $43^{\circ} 18^{\prime} 20^{\prime \prime}$, long $91^{\circ} 44^{\prime} 50^{\prime \prime}$, In NE1/4 SE1/4 sec.14, T.98 N., R.8 W. Winneshiek County, on left bank $500 \mathrm{ft}$ upstream from county highway Dridge in Freeport, 1.2 miles downstream from Trout Creek, and 3 miles downstream from Decorah.

Remarks.--Discontinued September 1951.

LOW FLOW DISCHARGES, IN YEAR. ENDING MARCH 31

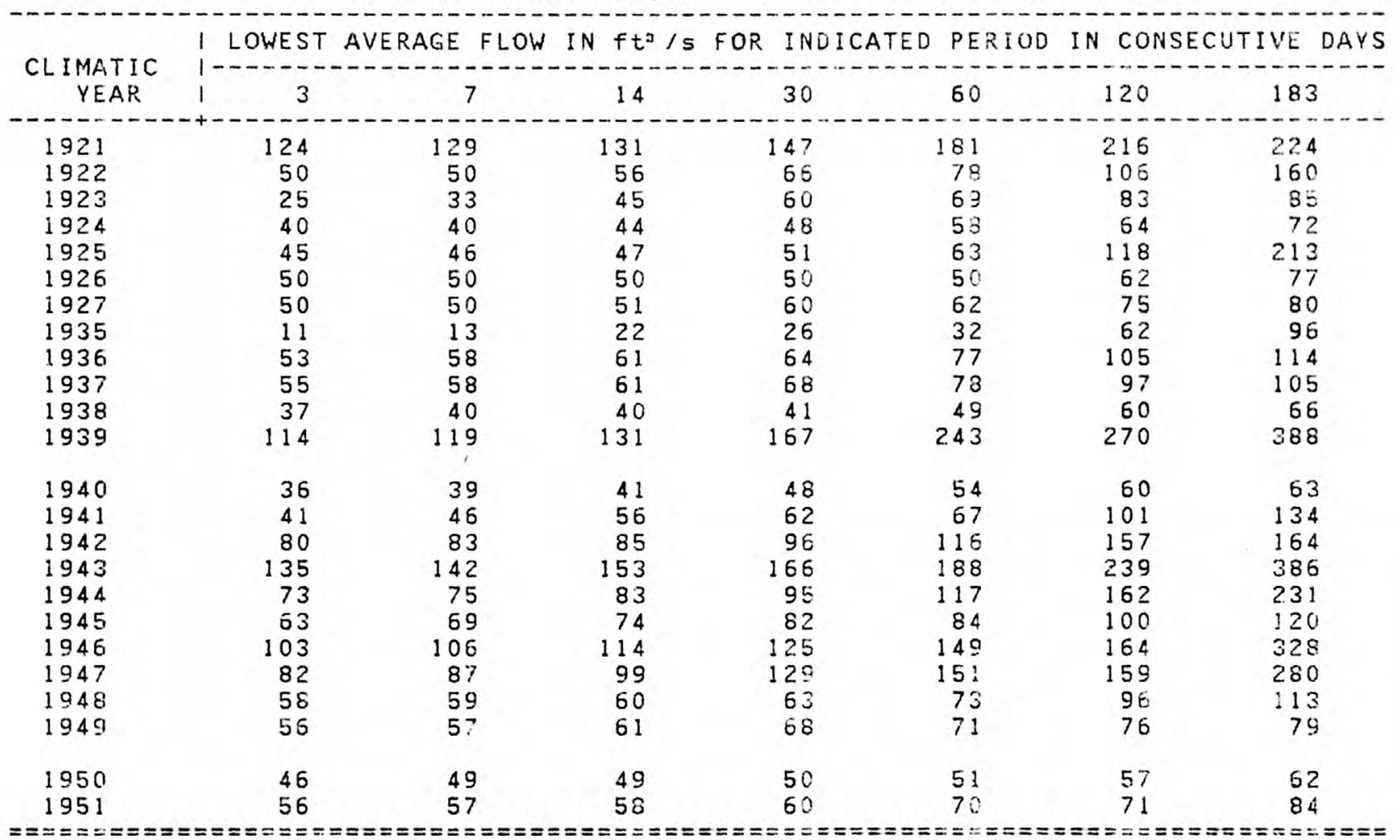


MINIMUM AVERAGE FLOWS FOR PERIOD OF RECORD

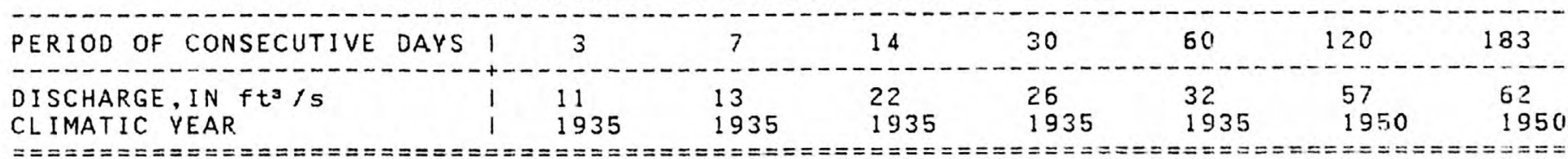

MAGNITUDE AND FREQUENCY OF ANNUAL LOW FLOWS

RECURRENCE I LOWEST AVERAGE FLOW, IN $\mathrm{ft}^{3} / 5, \mathrm{FOR}$ INDICATED PERIOD IN CONSECUTIVE DAYS

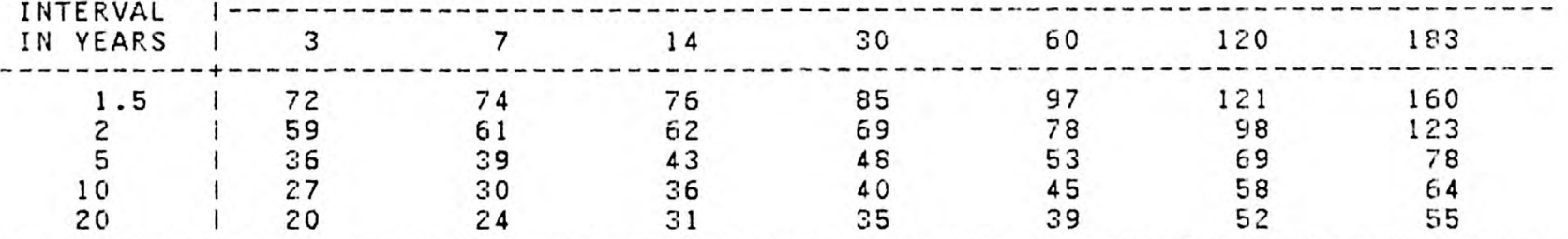

MAGNITUDE AND FREQUENCY OF SEASONAL LOW FLOW'S

LOWEST AVERAGE FLOW, IN $\mathrm{ft}^{3} / \mathrm{s}, \mathrm{FOR}$ THE INDICATED PERIOD IN CONSECUTIVE DAYS WITHIN EACH WATER-YEAR QUARTER

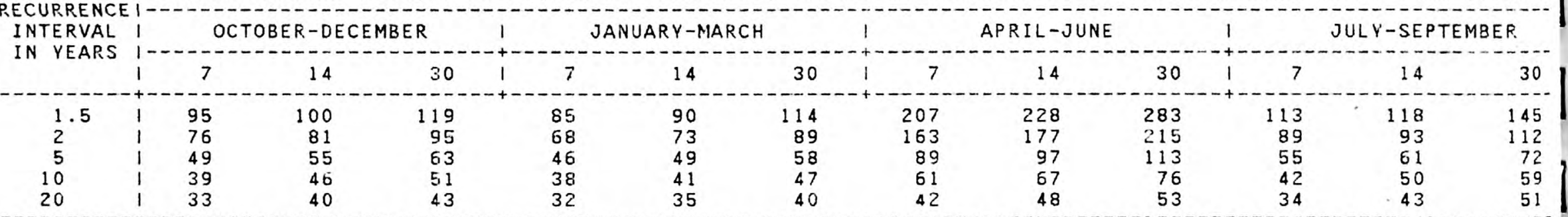

DURATION OF DAILY DISCHARGES FOR ANNUAL AND SEASONAL FERIODS DISCHARGE, IN $\mathrm{ft}^{3}$ is, EQUALLED OR EXCEEDED FOR THE INDICATED PERCENT OF TOTAL TIME 
Location.--Lat $43^{\circ} 12 \cdot 35^{\prime \prime}$, long $91^{\circ} 18^{\prime} 20^{\prime \prime}$, in NWl/4 NWi/4 sec. 22, T.97 N., R.4 W. on rlght bank $100 \mathrm{ft}$ downstream from highway bridge, $0.5 \mathrm{miles}$ northwest of Waterville and io miles upstrean from mouth.

LOW FLOW DISCHARGES, IN YEAR ENDING MARCH 31

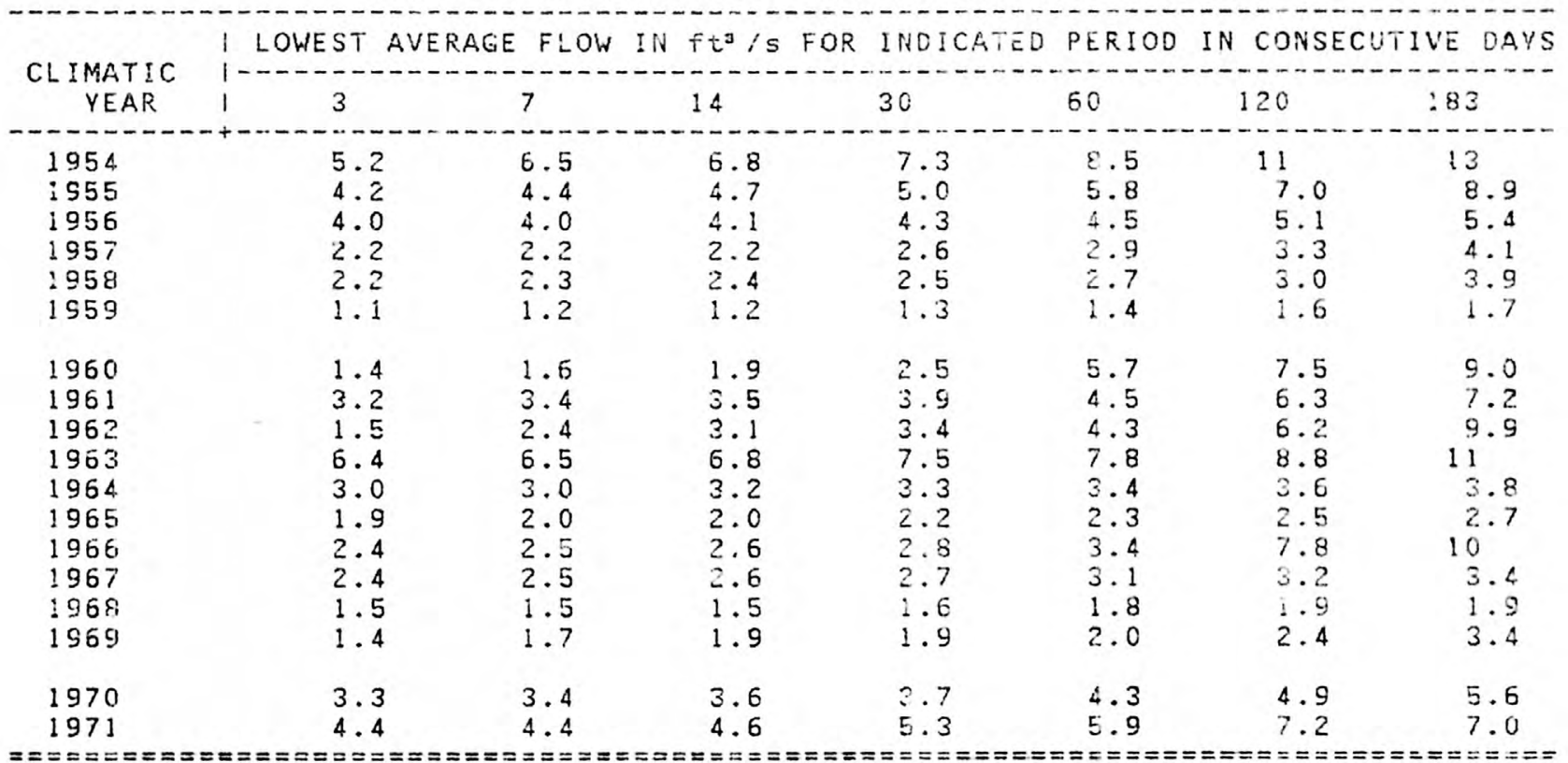




DRAINAGE AREA: $42.8 \mathrm{~m}^{2}$ PERIOD OF RECORD: 21 YEARS AVERAGE DISCHARGE: 15.9 ft ${ }^{3} / 5$

MINIMUM AVERAGE FLOWS FOR PERIOD OF RECORD

\begin{tabular}{|c|c|c|c|c|c|c|c|}
\hline PERIDD OF CONSECUTIVE DAYS & 1 & 7 & 14 & 30 & 60 & 120 & 183 \\
\hline $\begin{array}{l}\text { DISCHARGE, IN } \mathrm{ft}^{3} / \mathrm{s} \\
\text { CLIMATIC YEAR }\end{array}$ & $\begin{array}{r}1.1 \\
1959\end{array}$ & $\begin{array}{r}1.2 \\
1959\end{array}$ & $\begin{array}{r}1.2 \\
1959\end{array}$ & $\begin{array}{r}1.3 \\
1959\end{array}$ & $\begin{array}{r}1.4 \\
1959\end{array}$ & $\begin{array}{r}1.6 \\
1959\end{array}$ & $\begin{array}{r}1.7 \\
1959\end{array}$ \\
\hline
\end{tabular}

MAGNITUDE AND FREQUENCY OF ANNUAL LOW FLOWS

RECURRENCE I LOWEST AVERAGE FLOW, IN $\mathrm{ft}^{3} / \mathrm{s}$, FOR INDICATED PERIOD IN CONSECUTIVE DAYS

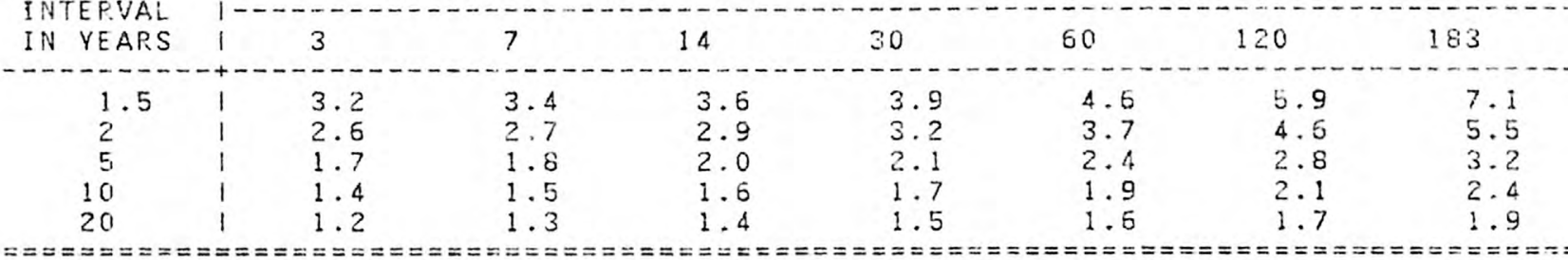

MAGNITUDE AND FREQUENCY OF SEASONAL LOW FLOWS

I LOWEST AVERAGE FLOW, IN $\mathrm{ft}^{3} / \mathrm{s}, \mathrm{FOR}$ THE INOICATED PERIOD IN CONSECUTIVE DAYS WITHIN EACH WATER-YEAR QUARTER

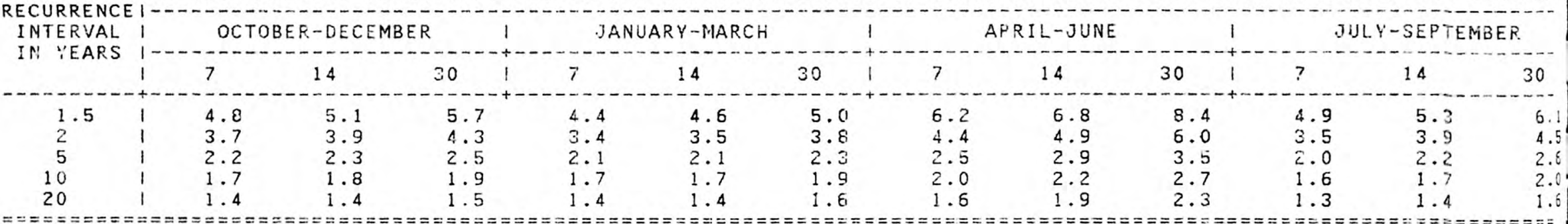

DURATION OF DAILY DISCHARGES FOR FINNUAL AND SEASONAL FERIODS

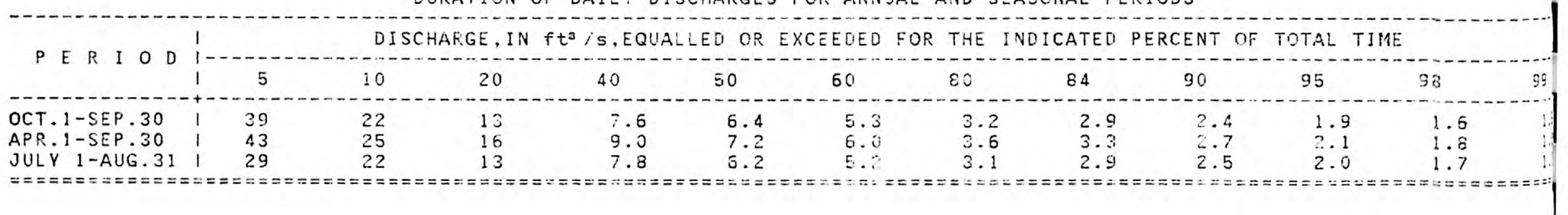




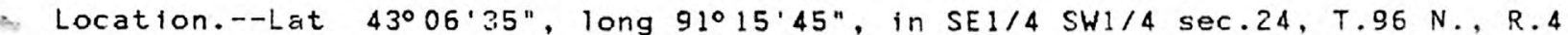
W. Allamakee County, on downstream side of county highway bridge at Ion, miles northwest of McGregor, and 8 miles upstream from mouth.

Remarks.--Disconitinued Sept. 30, 1951.

LOW FLOW DISCHARGES, IN YEAR ENDING MARCH 31

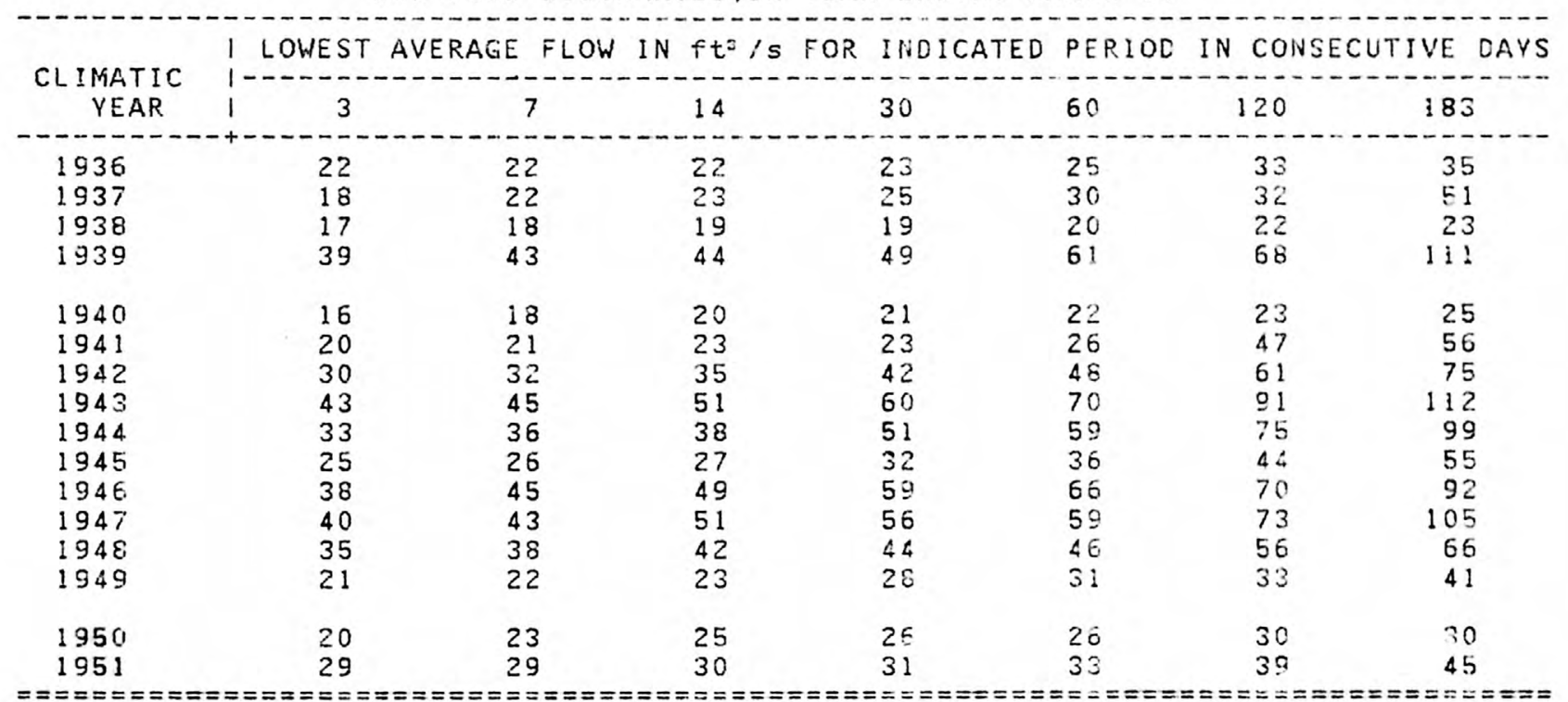




\section{DRAINAGE AREA: $221 \mathrm{~m}^{2}$ PERIOD OF RECORD: 17 YEARS AVERAGE DISCHARGE: $140 \mathrm{ft} \mathrm{t}^{2} / \mathrm{s}$}

MINIMUM AVERAGE FLOWS FOR PERIOD OF RECORD

\begin{tabular}{|c|c|c|c|c|c|c|c|}
\hline PERIOD OF CONSECUTIVE DAYS & 3 & 7 & 14 & 30 & 60 & 120 & 183 \\
\hline $\begin{array}{l}\text { DISCHARGE, IN } \mathrm{ft}^{3} / \mathrm{s} \\
\text { CLIMATIC YEAR }\end{array}$ & $\begin{array}{l}16 \\
1940\end{array}$ & $\begin{array}{l}18 \\
1938\end{array}$ & $\begin{array}{l}19 \\
1938\end{array}$ & $\begin{array}{l}19 \\
1938\end{array}$ & $\begin{array}{l}20 \\
1938\end{array}$ & $\begin{array}{l}22 \\
1538\end{array}$ & $\begin{array}{l}23 \\
1938\end{array}$ \\
\hline
\end{tabular}

MAGNITUDE AND FREQUENCY OF ANNUAL LOW FLOWS

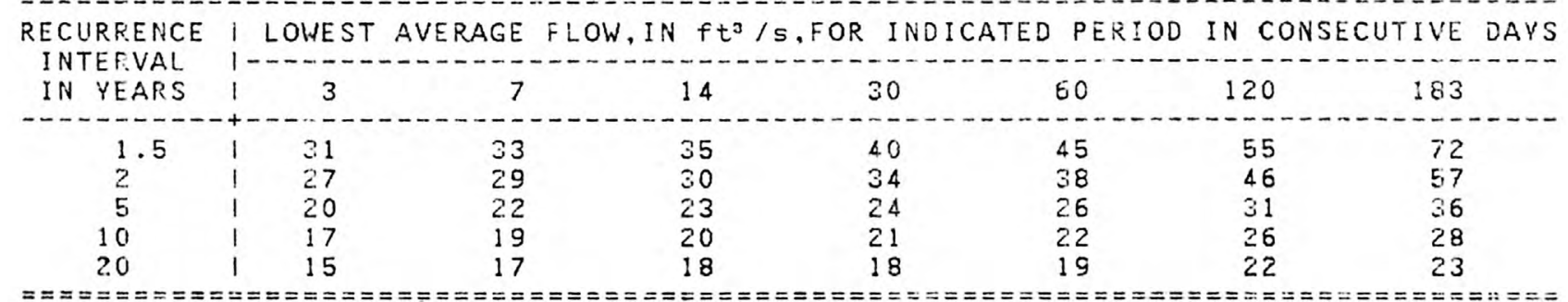

MAGNITUDE AND FREQUENCY OF SEASONAL LOW FLOWS

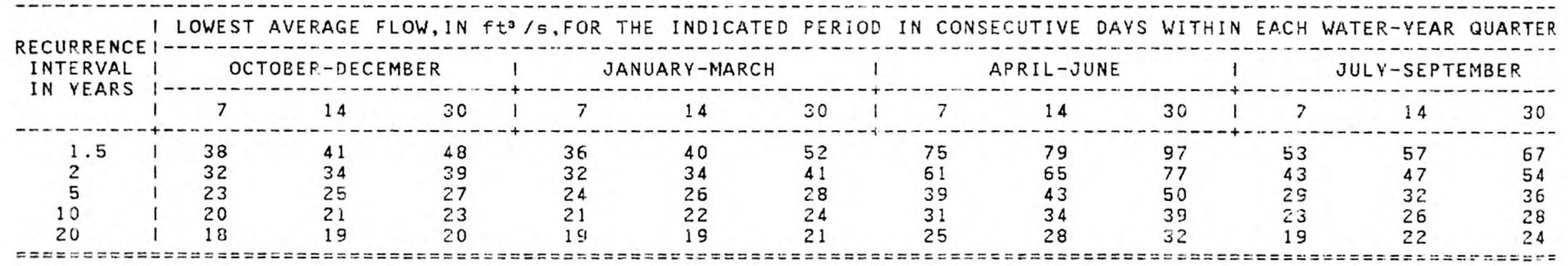

DURATION OF DAILY DISCHARGES FOR ANUUAL AND SEASONAL PERIOOS

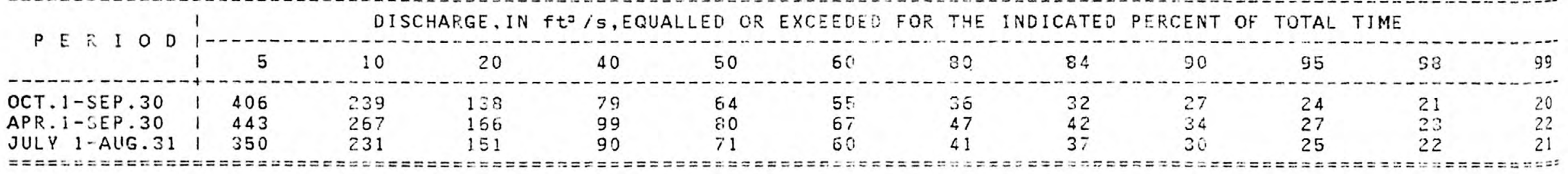


Location.--Lat 43001'29", Tong 91010'21", in SE1/4 SE1/4 sec.22, T.95 N., R.3 W., Clayton County, on right bank in city park at east end of Main street in McGregor, 2.6 miles upstream from Wisconsin River, 4.3 miles downstream from Yellow River, and at mile 633.4 upstream from ohio River.

Remarks.--Flow regulated by navigation dams.

LOW FLOW DISCHARGES, IN YEAR ENDING MARCH 31

\begin{tabular}{|c|c|c|c|c|c|c|c|}
\hline \multirow{2}{*}{$\begin{array}{c}\text { CLIMATIC } \\
\text { YEAR }\end{array}$} & I LOWEST & GE FLOW & $N f t^{3} / s$ & $R$ INDICATED & \multicolumn{3}{|c|}{ PERIOD IN CONSECUTIVE DAYS } \\
\hline & 13 & 7 & 14 & 30 & 60 & 120 & 183 \\
\hline $\begin{array}{l}1938 \\
1939\end{array}$ & $\begin{array}{l}8130 \\
9970\end{array}$ & $\begin{array}{r}8370 \\
11300\end{array}$ & $\begin{array}{r}8700 \\
13400\end{array}$ & $\begin{array}{r}9430 \\
15200\end{array}$ & $\begin{array}{r}9640 \\
17400\end{array}$ & $\begin{array}{l}10200 \\
18900\end{array}$ & $\begin{array}{l}10700 \\
23400\end{array}$ \\
\hline $\begin{array}{l}1940 \\
1941 \\
1942 \\
1943 \\
1944 \\
1945 \\
1946 \\
1947 \\
1948 \\
1949\end{array}$ & $\begin{array}{r}6700 \\
9180 \\
9680 \\
14400 \\
12900 \\
13200 \\
14100 \\
12600 \\
12400 \\
9000\end{array}$ & $\begin{array}{r}6740 \\
9600 \\
10200 \\
14700 \\
15000 \\
14500 \\
14400 \\
12800 \\
12700 \\
9150\end{array}$ & $\begin{array}{r}6910 \\
10000 \\
10800 \\
15800 \\
16500 \\
15200 \\
16400 \\
13500 \\
13000 \\
9860\end{array}$ & $\begin{array}{r}7590 \\
10300 \\
12400 \\
17700 \\
17300 \\
15300 \\
19500 \\
15000 \\
13300 \\
10700\end{array}$ & $\begin{array}{r}8490 \\
11900 \\
16000 \\
19800 \\
19900 \\
16600 \\
21900 \\
19500 \\
14400 \\
11000\end{array}$ & $\begin{array}{r}9900 \\
12600 \\
19400 \\
20800 \\
21000 \\
17700 \\
22500 \\
21500 \\
16200 \\
11900\end{array}$ & $\begin{array}{l}10900 \\
14100 \\
29200 \\
27000 \\
21400 \\
18400 \\
23600 \\
25400 \\
16600 \\
12200\end{array}$ \\
\hline $\begin{array}{l}1950 \\
1951 \\
1952 \\
1953 \\
1954 \\
1955 \\
1956 \\
1957 \\
1958 \\
1959\end{array}$ & $\begin{array}{r}8970 \\
9930 \\
21100 \\
13600 \\
14100 \\
15000 \\
12900 \\
10700 \\
12800 \\
9030\end{array}$ & $\begin{array}{r}9280 \\
11500 \\
22100 \\
14500 \\
14800 \\
15100 \\
13200 \\
10900 \\
12900 \\
9440\end{array}$ & $\begin{array}{r}10400 \\
12800 \\
22700 \\
15200 \\
15800 \\
15400 \\
13700 \\
11400 \\
13300 \\
9740\end{array}$ & $\begin{array}{r}12000 \\
13600 \\
23300 \\
15700 \\
16900 \\
15700 \\
14200 \\
12000 \\
14700 \\
9870\end{array}$ & $\begin{array}{l}12600 \\
14200 \\
24700 \\
16000 \\
17800 \\
17100 \\
14400 \\
13100 \\
15700 \\
10300\end{array}$ & $\begin{array}{l}14400 \\
14400 \\
28800 \\
16400 \\
18600 \\
19400 \\
15800 \\
14700 \\
18900 \\
12000\end{array}$ & $\begin{array}{l}14400 \\
14700 \\
34000 \\
17300 \\
19500 \\
24100 \\
16300 \\
14700 \\
21100 \\
12900\end{array}$ \\
\hline $\begin{array}{l}1960 \\
1961 \\
1962 \\
1963 \\
1964 \\
1965 \\
1966 \\
1967 \\
1968 \\
1969\end{array}$ & $\begin{array}{r}9810 \\
10600 \\
9100 \\
12000 \\
10800 \\
7400 \\
15000 \\
12000 \\
9830 \\
15000\end{array}$ & $\begin{array}{r}9980 \\
10600 \\
10200 \\
12000 \\
10900 \\
8020 \\
16000 \\
13100 \\
10400 \\
17600\end{array}$ & $\begin{array}{l}12300 \\
11100 \\
11000 \\
12200 \\
11200 \\
8290 \\
17300 \\
14400 \\
10500 \\
19700\end{array}$ & $\begin{array}{l}13800 \\
12000 \\
11800 \\
12500 \\
12000 \\
9340 \\
18000 \\
15400 \\
11200 \\
23000\end{array}$ & $\begin{array}{l}14600 \\
12600 \\
12600 \\
13100 \\
12300 \\
11800 \\
21100 \\
16300 \\
12100 \\
24500\end{array}$ & $\begin{array}{l}17500 \\
16000 \\
14100 \\
15000 \\
12900 \\
14500 \\
32300 \\
18000 \\
12800 \\
27000\end{array}$ & $\begin{array}{l}21600 \\
17400 \\
14600 \\
19100 \\
13400 \\
16800 \\
32700 \\
18000 \\
13200 \\
36401\end{array}$ \\
\hline $\begin{array}{l}1970 \\
1971 \\
1972 \\
1973\end{array}$ & $\begin{array}{l}11200 \\
10200 \\
11400 \\
13500\end{array}$ & $\begin{array}{l}12600 \\
11000 \\
12700 \\
14900\end{array}$ & $\begin{array}{l}14400 \\
11500 \\
14900 \\
18300\end{array}$ & $\begin{array}{l}11900 \\
12600 \\
17100 \\
24100\end{array}$ & $\begin{array}{l}16400 \\
14300 \\
18100 \\
28000\end{array}$ & $\begin{array}{l}18400 \\
18600 \\
23300 \\
33800\end{array}$ & $\begin{array}{l}18300 \\
25300 \\
28500 \\
38500\end{array}$ \\
\hline
\end{tabular}


MISSISSIPPI RIVER AT MCGREGOR--Cont inued

LOW FLOW DISCHARGES, IN YEAR ENDING MARCH 31--Continued

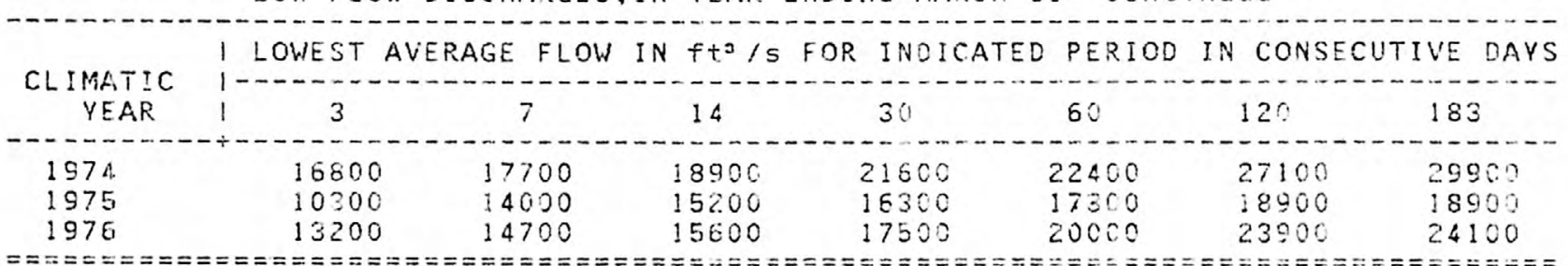


DRAINAGE AREA: $67,500 \mathrm{mi}^{2}$ PERIOD OF RECORD: 40 YEARS AVERAGE DISCHARGE: 33,720 ft $3 / 5$

MINIMUM AVERAGE FLOWS FOR PERIOD OF RECORD

\begin{tabular}{|c|c|c|c|c|c|c|c|}
\hline DERIOD OF CONSECUTIVE DAYS ! & 3 & 7 & 14 & 30 & 60 & 120 & 183 \\
\hline $\begin{array}{l}\text { RGE, IN } f t=/ s \\
\text { IC YEAR }\end{array}$ & $\begin{array}{r}6700 \\
1940 \\
\end{array}$ & $\begin{array}{r}6740 \\
1940\end{array}$ & $\begin{array}{l}6910 \\
1940 \\
\end{array}$ & $\begin{array}{r}7590 \\
1940\end{array}$ & $\begin{array}{r}8490 \\
1940\end{array}$ & $\begin{array}{l}9900 \\
1940\end{array}$ & \\
\hline
\end{tabular}

MAGNITUDE AND FREQUENCY OF ANNUAL LOW FLOWS

\begin{tabular}{|c|c|c|c|c|c|c|c|}
\hline \multirow{2}{*}{$\begin{array}{l}\text { RECURRENCE } \\
\text { INTERVAL } \\
\text { IN YEARS }\end{array}$} & LOWEST & $\mathrm{GE}$ & $f t^{2} 1$ & IND I & $\begin{array}{l}\text { D PERIO } \\
-\end{array}$ & IN CONS & TIVE DAYS \\
\hline & 13 & 7 & 14 & 30 & 60 & 120 & 183 \\
\hline $\begin{array}{l}1.5 \\
2 \\
5 \\
10 \\
20\end{array}$ & $\begin{array}{r}10300 \\
11000 \\
11500 \\
8680 \\
5440\end{array}$ & $\begin{array}{r}13600 \\
12300 \\
9970 \\
8940 \\
8160\end{array}$ & $\begin{array}{r}14800 \\
13300 \\
10600 \\
9430 \\
8500\end{array}$ & $\begin{array}{l}16000 \\
14300 \\
11400 \\
10100 \\
9200\end{array}$ & $\begin{array}{l}17500 \\
15600 \\
12400 \\
11000 \\
10000\end{array}$ & $\begin{array}{l}19000 \\
17500 \\
13300 \\
12200 \\
11200\end{array}$ & $\begin{array}{l}22500 \\
19500 \\
14700 \\
12800 \\
11500\end{array}$ \\
\hline
\end{tabular}

MAGNITUDE AND FREQUENCY OF SEASONAL LOW FLOWS

I LOWEST AVERAGE FLOW, IN $\mathrm{ft}^{3} / \mathrm{s}, \mathrm{FOR}$ THE INDICATED PENIOD IN CONSECUTIVE DAVS WITHIN EACH WATER-YEAR QUARTER

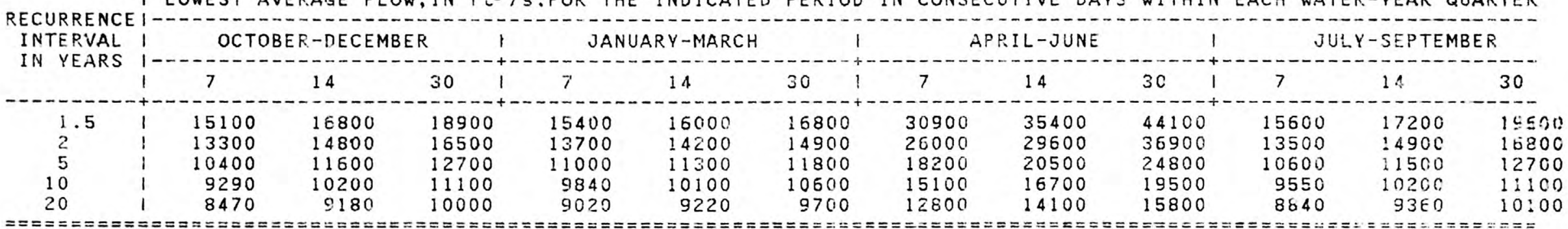

DURATION OF DAILY DISCHARGES FOR ANNUAL AND SEASONAL PERIODS

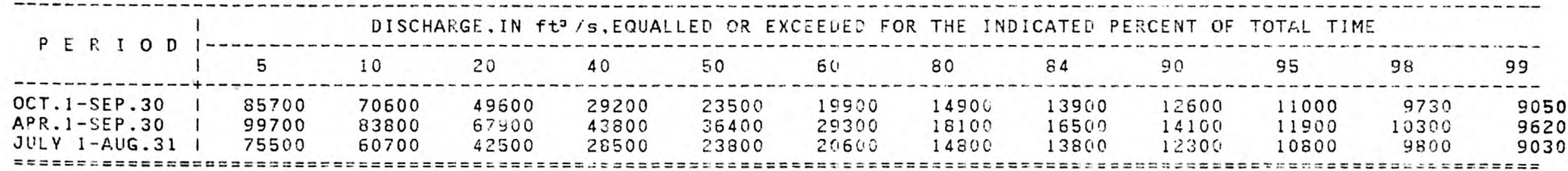




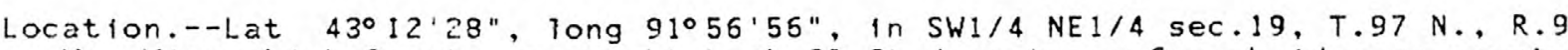
W. Winneshiek County, on right bank $60 \mathrm{ft}$ downstream from bridge on county highway W14 at north edge of Spilluille, $150 \mathrm{ft}$ downstream from old mijl dam, $0.6 \mathrm{mlle}$ upstream from Wonder Creek and at mile 98.5 .

Remarks.--Discontinued September 1973.

LOW FLOW DISCHARGES, IN YEAR ENDING MARCH 31

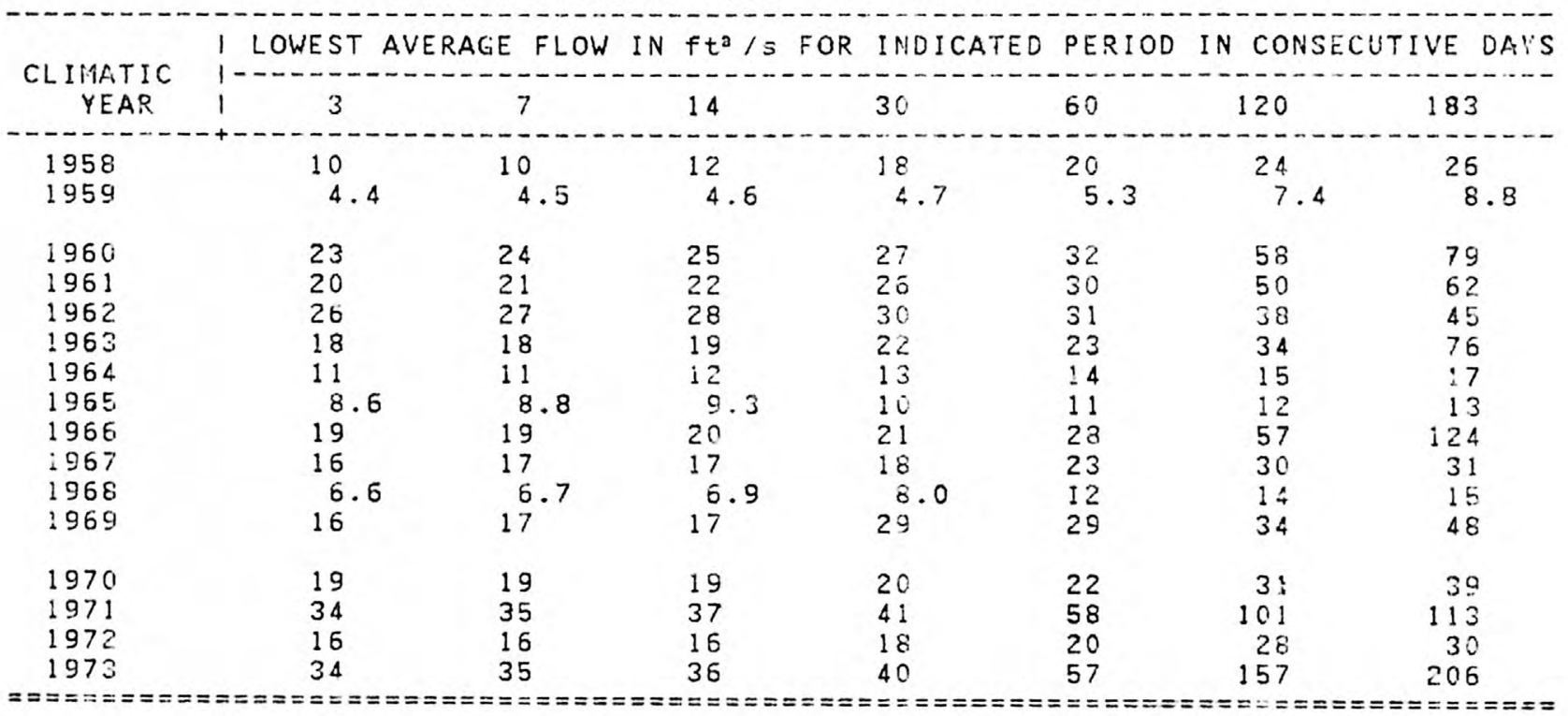




\section{DRAINAGE AREA: $177 \mathrm{mi}^{2}$ PERIOD OF RECORD: 17 YEARS AVERAGE DISCHARGE: 109 ft3 /S}

MINIMUM AVERAGE FLOWS FOR PERIOD OF RECORD

\begin{tabular}{|c|c|c|c|c|c|c|c|}
\hline PERIOD OF CONSECUTIVE DAYS & 1 & 7 & 14 & 30 & 60 & 120 & 183 \\
\hline $\begin{array}{l}\text { DISCHARGE, IN } \mathrm{ft}^{3} / \mathrm{s} \\
\text { CLIMATIC YEAR }\end{array}$ & $\begin{array}{r}4.4 \\
1959\end{array}$ & $\begin{array}{r}4.5 \\
1959\end{array}$ & $\begin{array}{r}4.6 \\
1959\end{array}$ & $\begin{array}{r}4.7 \\
1959\end{array}$ & $\begin{array}{r}5.3 \\
1959\end{array}$ & $\begin{array}{r}7.4 \\
1959\end{array}$ & $\begin{array}{r}8.8 \\
1959\end{array}$ \\
\hline
\end{tabular}

MAGNITUDE AND FREQUENCY OF ANNUAL LOW FLOWS

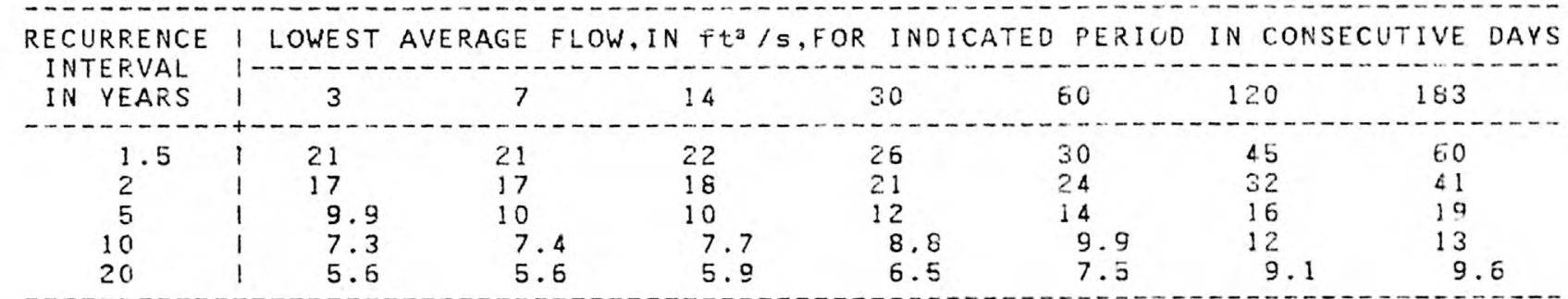

MAGNITUDE AND FREQUENCY OF SEASONAL LOW FLOWS

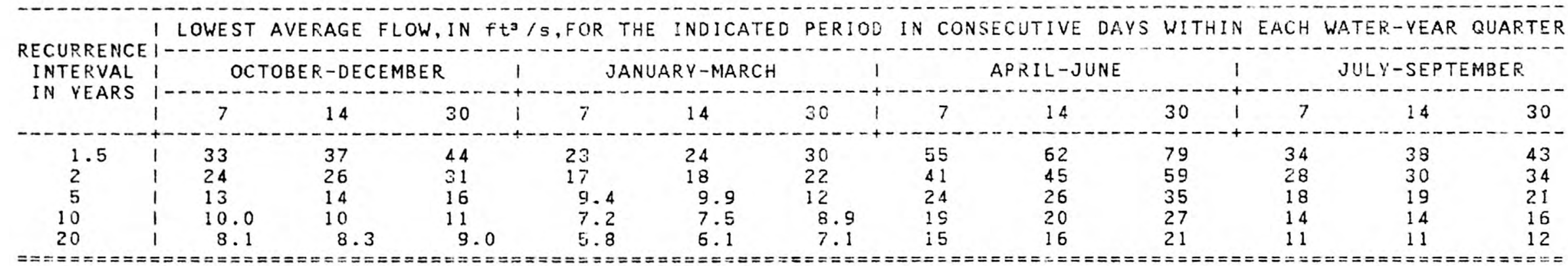

DURATION OF DAILY DISCHARGES FOR ANNUAL AND SEASONAL PERIODS

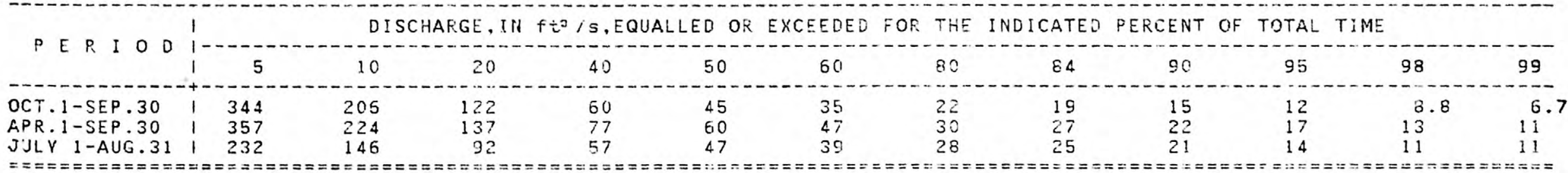


Location.--Lat $42^{\circ} 51^{\prime} 05^{\prime \prime}$, long $91^{\circ} 24^{\prime} 15^{n}$, in NW1/4 SE1/4 sec.23, T.93 N., R.5 W. Clayton County, in tallrace of Central States Power and Light Corporation's hydroelectric plant in Elkader, 2.7 miles upstream from Roberts Creek and at mile 37.0

Remarks.--Flow regivated by powerplant. Discontinued September i 942 .

LOW FLOW DISCHARGES, IN YEAR. ENDING MARCH 31

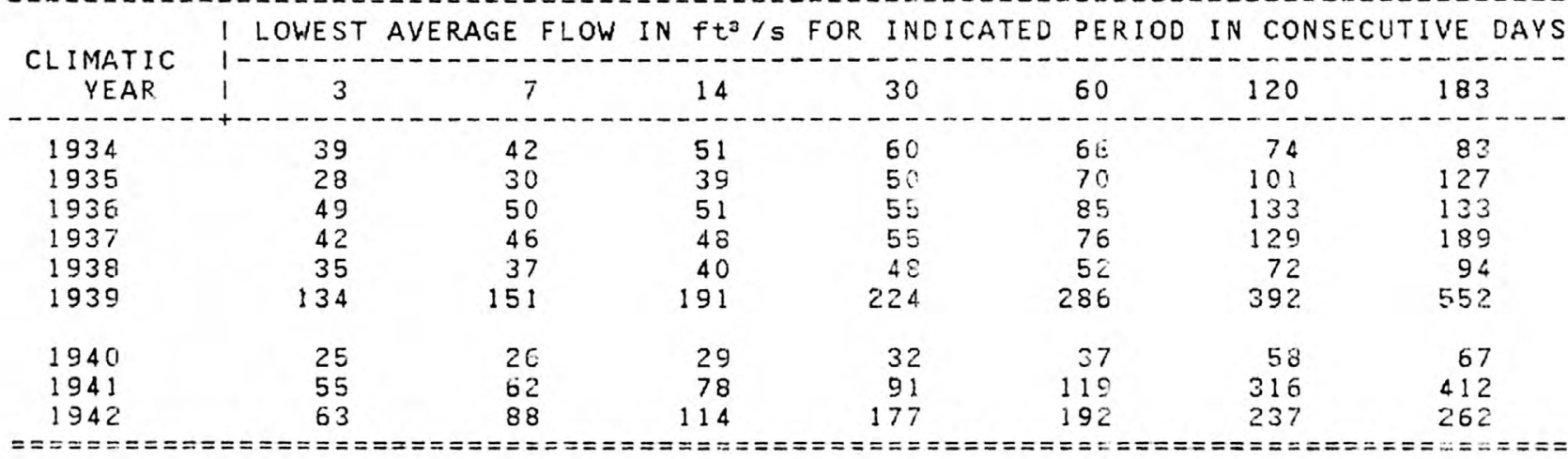




\section{DRAINAGE AREA: $891 \mathrm{~m}^{2}$ PERIOD OF RECORD: 10 YEARS AVERAGE D:SCHARGE: $487 \mathrm{ft} 3 / \mathrm{s}$}

MINIMUM AVERAGE FLOWS FOR PERIOD OF RECORD

\begin{tabular}{|c|c|c|c|c|c|c|c|}
\hline PERIOD OF CONSECUTIVE DAYS & 3 & 7 & 14 & 30 & 60 & 120 & 183 \\
\hline $\begin{array}{l}\text { SCHARGE, IN ft3 } / \mathrm{s} \\
\text { IMATIC YEAR }\end{array}$ & $\begin{array}{l}25 \\
1940\end{array}$ & $\begin{array}{l}26 \\
1940\end{array}$ & $\begin{array}{l}29 \\
1940\end{array}$ & $\begin{array}{l}32 \\
1940\end{array}$ & $\begin{array}{l}37 \\
1940\end{array}$ & $\begin{array}{l}58 \\
1940\end{array}$ & \\
\hline
\end{tabular}

MAGNITUDE AND FREQUENCY OF ANNUAL LOW FLOWS

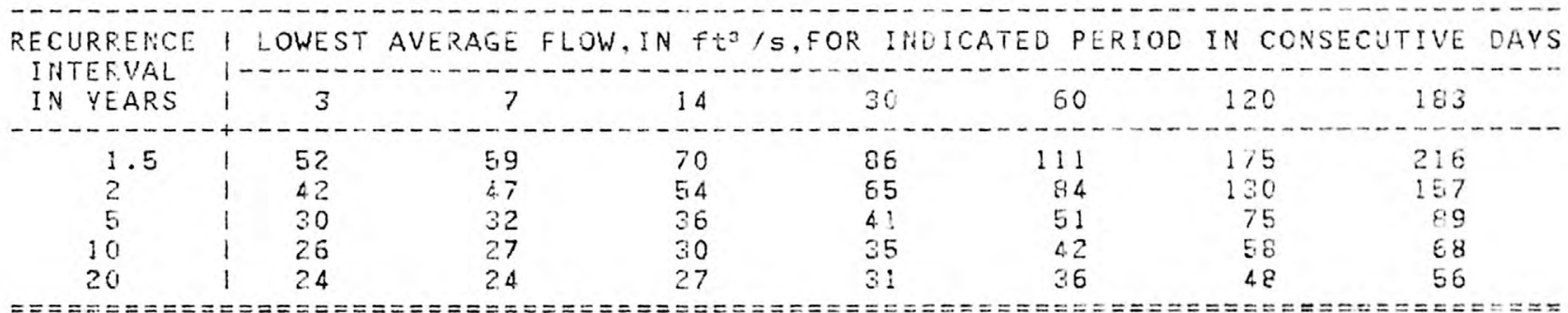

MAGNITUDE AND FREQUENCY OF SEASONAL LOW FLOWS

I LOWEST AVERAGE FLOW, IN $\mathrm{ft}^{3} / 5$ FOR THE INDICATED PERIOD IN CONSECUTIVE DAYS WITHIN EACH WATER-YEAR OUARTER RECURRENCE I- LOWEST AVERAGE ELOW, IN FTJ/S,FOR THEINDICATED PERIOD IN CONSECUTIVE DAVS WITHIN EACH WATER-YEAR QUARTER

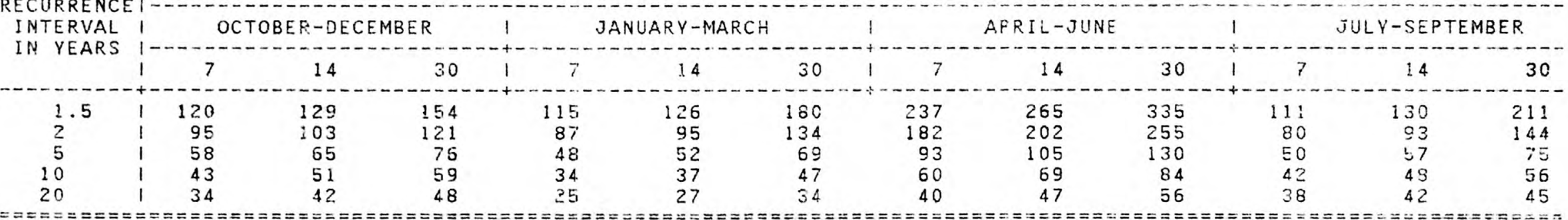

DURATION OF DAILY DISCHARGES FOR ANNUAL AND SEASONAL FERIODS

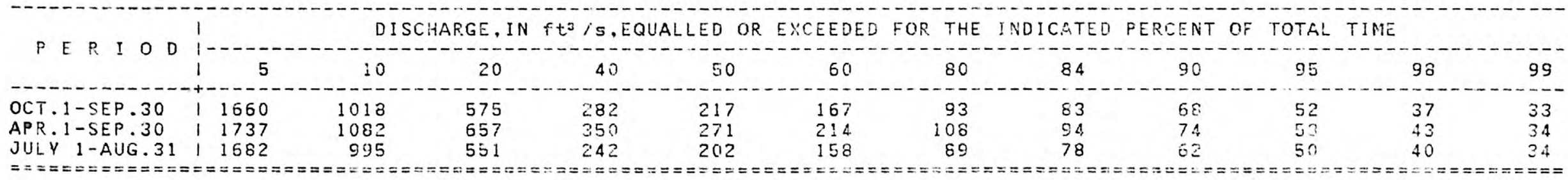


Location.--Lat $42^{\circ} 44^{\prime} 24^{\prime \prime}$, long $91^{\circ} 15^{\prime} 42^{\prime \prime}$, in SE1/4 NW1/4 sec.36, T.92 N., R.4 W. , Clayton County, on left bank 10 feet downstream from bridge on county highway $\mathrm{C} 43,800 \mathrm{ft}$ upstream from Wayman Creek, $1,000 \mathrm{ft}$ southeast of Garber, 2,000 ft downstream from Elk Creek, 1 mlie downstream from Volga River, and $19.8 \mathrm{miles}$ upstrean from mouth.

Remarks.--Sight diurnal fluctuation caused by powerplant at Eikader to 1963. LOW FLOW DISCHARGES, IN YEAR ENDING MARCH 31

\begin{tabular}{|c|c|c|c|c|c|c|c|}
\hline \multirow{2}{*}{$\begin{array}{c}\text { CLIMATIC } \\
\text { YEAR }\end{array}$} & I LOWEST & RAGE F & $N f t^{2}$ & IND & $D$ PER & IN CON & IVE \\
\hline & 1 & 7 & 14 & 30 & 60 & 120 & 183 \\
\hline $\begin{array}{l}1915 \\
1915 \\
1921 \\
1922 \\
1923 \\
1924 \\
1925 \\
1926 \\
1927 \\
1934 \\
1935 \\
1936 \\
1937 \\
1938 \\
1939\end{array}$ & $\begin{array}{r}140 \\
280 \\
297 \\
315 \\
88 \\
176 \\
209 \\
163 \\
180 \\
92 \\
61 \\
87 \\
83 \\
70 \\
236\end{array}$ & $\begin{array}{r}140 \\
280 \\
307 \\
335 \\
123 \\
180 \\
223 \\
169 \\
183 \\
93 \\
63 \\
89 \\
86 \\
74 \\
259\end{array}$ & $\begin{array}{r}140 \\
280 \\
324 \\
365 \\
155 \\
180 \\
236 \\
177 \\
213 \\
94 \\
72 \\
92 \\
90 \\
82 \\
320\end{array}$ & $\begin{array}{r}140 \\
377 \\
412 \\
381 \\
199 \\
180 \\
255 \\
209 \\
265 \\
102 \\
84 \\
105 \\
102 \\
101 \\
400\end{array}$ & $\begin{array}{r}169 \\
440 \\
539 \\
437 \\
230 \\
208 \\
272 \\
250 \\
339 \\
110 \\
95 \\
151 \\
125 \\
120 \\
507\end{array}$ & $\begin{array}{l}337 \\
671 \\
561 \\
549 \\
331 \\
225 \\
318 \\
330 \\
430 \\
126 \\
181 \\
235 \\
218 \\
132 \\
622\end{array}$ & $\begin{array}{r}341 \\
1050 \\
575 \\
768 \\
313 \\
254 \\
441 \\
369 \\
627 \\
135 \\
216 \\
227 \\
318 \\
152 \\
820\end{array}$ \\
\hline $\begin{array}{l}1940 \\
1941 \\
1942 \\
1943 \\
1944 \\
1945 \\
1946 \\
1947 \\
1948 \\
1949\end{array}$ & $\begin{array}{r}49 \\
93 \\
209 \\
333 \\
187 \\
127 \\
250 \\
113 \\
230 \\
108\end{array}$ & $\begin{array}{r}51 \\
113 \\
215 \\
347 \\
191 \\
137 \\
256 \\
166 \\
234 \\
113\end{array}$ & $\begin{array}{r}54 \\
138 \\
227 \\
392 \\
207 \\
144 \\
274 \\
199 \\
241 \\
120\end{array}$ & $\begin{array}{r}59 \\
166 \\
240 \\
402 \\
257 \\
150 \\
334 \\
229 \\
251 \\
125\end{array}$ & $\begin{array}{r}70 \\
199 \\
327 \\
491 \\
317 \\
166 \\
382 \\
270 \\
292 \\
138\end{array}$ & $\begin{array}{r}98 \\
562 \\
466 \\
637 \\
391 \\
218 \\
399 \\
327 \\
359 \\
154\end{array}$ & $\begin{array}{l}105 \\
670 \\
537 \\
862 \\
472 \\
276 \\
591 \\
469 \\
379 \\
185\end{array}$ \\
\hline $\begin{array}{l}1950 \\
1951 \\
1952 \\
1953 \\
1954 \\
1955 \\
1956 \\
1957 \\
1958 \\
1959\end{array}$ & $\begin{array}{r}84 \\
110 \\
390 \\
165 \\
98 \\
138 \\
85 \\
100 \\
94 \\
56\end{array}$ & $\begin{array}{r}85 \\
111 \\
404 \\
173 \\
98 \\
146 \\
94 \\
100 \\
94 \\
56\end{array}$ & $\begin{array}{r}87 \\
115 \\
445 \\
180 \\
99 \\
154 \\
96 \\
108 \\
95 \\
58\end{array}$ & $\begin{array}{r}88 \\
124 \\
526 \\
188 \\
112 \\
158 \\
101 \\
133 \\
115 \\
60\end{array}$ & $\begin{array}{r}89 \\
136 \\
647 \\
212 \\
157 \\
198 \\
111 \\
157 \\
127 \\
65\end{array}$ & $\begin{array}{r}101 \\
166 \\
732 \\
223 \\
212 \\
252 \\
125 \\
170 \\
158 \\
89\end{array}$ & $\begin{array}{r}130 \\
244 \\
899 \\
305 \\
237 \\
336 \\
141 \\
207 \\
162 \\
98\end{array}$ \\
\hline 1960 & 236 & 2.43 & 264 & 315 & 397 & 604 & 759 \\
\hline
\end{tabular}


LOW FLOW DISCHARGES, IN YEAR ENDING MARCH 31--Continued

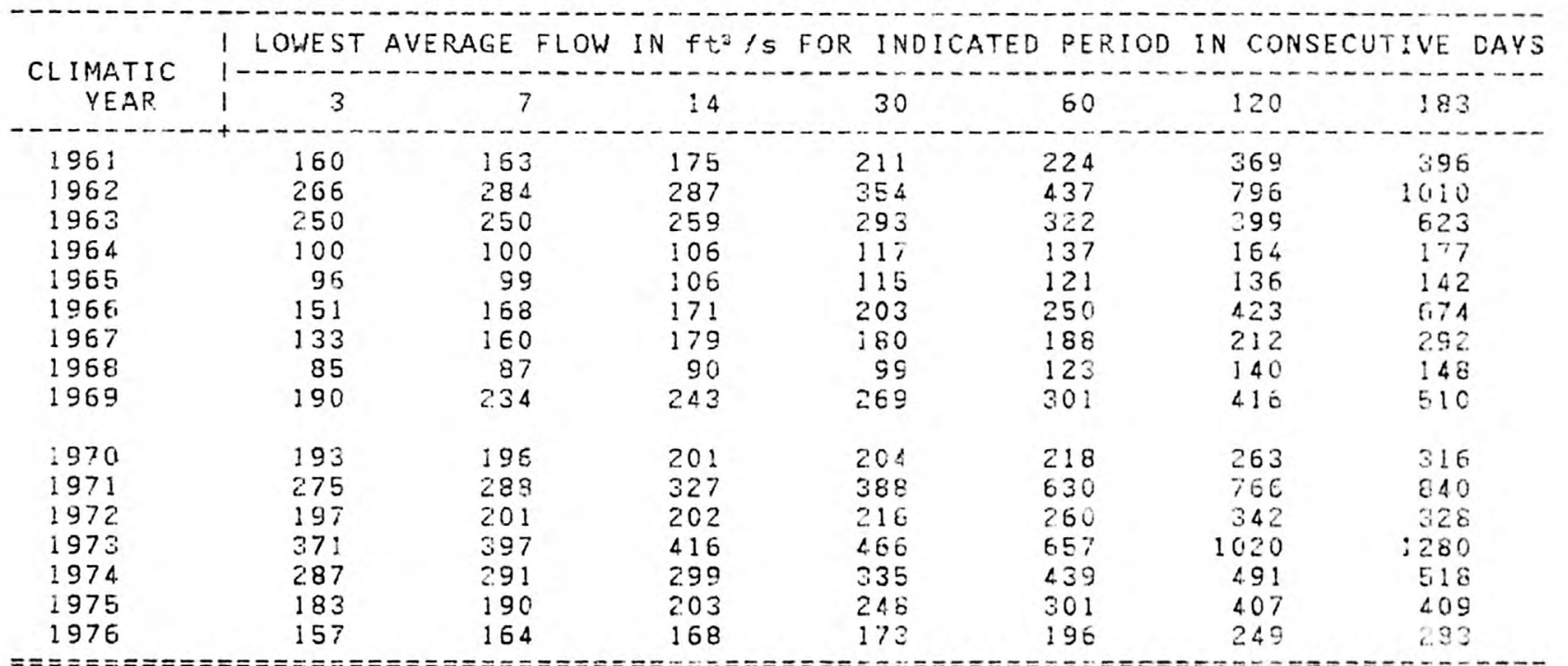




DRAINAGE AREA: $1545 \mathrm{miz}$ PERIOD OF RECORD: 56 VEARS AVERAGE DISCHARGE: 905 ft $/ 5$
MINIMUM AVERAGE FLOWS FOR PERIOD OF RECORD
PERIOD OF CONSECUTIVE DAYS I

MAGNITUDE AND FREQUENCY OF ANNUAL LOW FLOWS

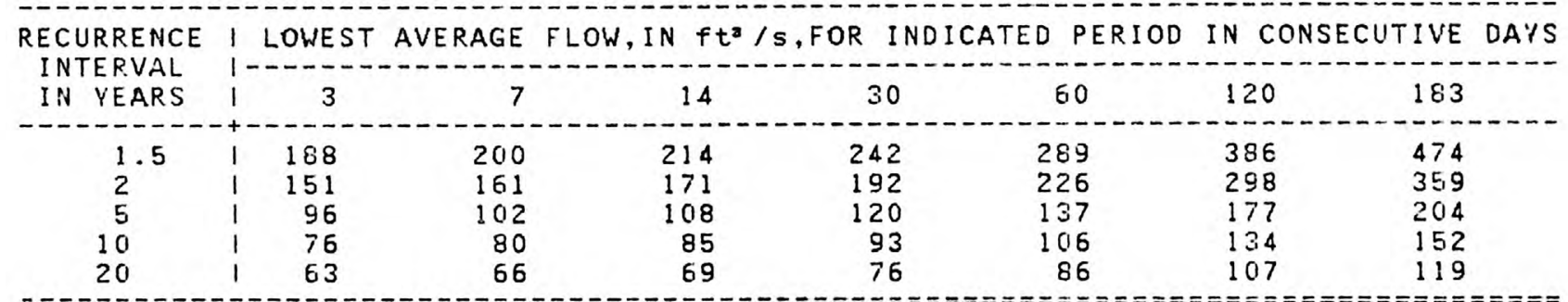

MAGNITUDE AND FREQUENCY OF SEASONAL LOW FLOWS

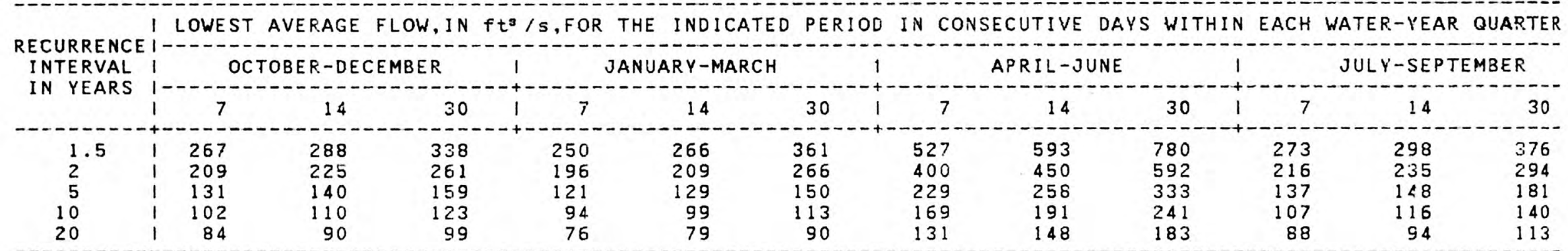

DURATION OF DAILY DISCHARGES FOR ANNUAL AND SEASONAL PERIODS

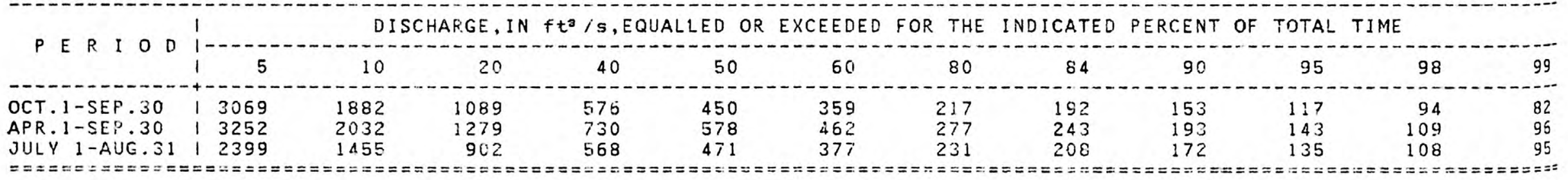


Location.--Lat $42033^{\prime 18 ", ~ l o n g ~} 90044146^{\prime \prime}$, in NW1/4 NE1/4 sec.5, T.89 N., R.2 E., Dubuque County, on left bank 10 ft upstream from bridge on county highway, $300 \mathrm{ft}$ upstream from Cloie Branch, $1.7 \mathrm{miles}$ east of Durango, 5.6 miles northwest of courthouse at Dubuque, and 6.4 miles upstream from mouth.

LOW FLOW DISCHARGES, IN YEAR ENDING MARCH 31

\begin{tabular}{|c|c|c|c|c|c|c|c|c|}
\hline \multirow{2}{*}{$\begin{array}{c}\text { CLIMATIC } \\
\text { YEAR }\end{array}$} & \multicolumn{2}{|c|}{1 LOWEST AVERAGE FLOW } & $f t^{3} / S$ FOR & INDICATED & $\begin{array}{l}\text { PERIOD } \\
-\end{array}$ & \multicolumn{3}{|c|}{ N CONSECUTIVE DAYS } \\
\hline & i 3 & 7 & 14 & 30 & 60 & 120 & 183 & \\
\hline $\begin{array}{l}1936 \\
1937 \\
1938 \\
1939\end{array}$ & $\begin{array}{l}10 \\
5.2 \\
8.8 \\
12\end{array}$ & $\begin{array}{r}10 \\
5.8 \\
9.9 \\
14\end{array}$ & $\begin{array}{l}11 \\
6.0 \\
11 \\
16\end{array}$ & $\begin{array}{l}12 \\
6.6 \\
12 \\
28\end{array}$ & $\begin{array}{l}12 \\
7.3 \\
13 \\
42\end{array}$ & $\begin{array}{l}16 \\
16 \\
14 \\
50\end{array}$ & $\begin{array}{l}16 \\
20 \\
15 \\
78\end{array}$ & \\
\hline $\begin{array}{l}1940 \\
1941 \\
1942 \\
1943 \\
1944 \\
1945 \\
1946 \\
1947 \\
1948 \\
1949\end{array}$ & $\begin{array}{l}8.7 \\
9.7 \\
11 \\
19 \\
13 \\
15 \\
31 \\
14 \\
18 \\
13\end{array}$ & $\begin{array}{l}9.3 \\
11 \\
11 \\
19 \\
17 \\
16 \\
36 \\
15 \\
19 \\
14\end{array}$ & $\begin{array}{l}9.9 \\
15 \\
11 \\
21 \\
19 \\
18 \\
40 \\
17 \\
20 \\
15\end{array}$ & $\begin{array}{l}11 \\
18 \\
13 \\
24 \\
20 \\
21 \\
43 \\
24 \\
20 \\
19\end{array}$ & $\begin{array}{l}12 \\
22 \\
17 \\
32 \\
24 \\
24 \\
63 \\
30 \\
24 \\
22\end{array}$ & $\begin{array}{l}14 \\
39 \\
30 \\
38 \\
32 \\
30 \\
69 \\
37 \\
32 \\
24\end{array}$ & $\begin{array}{l}15 \\
45 \\
46 \\
39 \\
36 \\
36 \\
72 \\
45 \\
36 \\
26\end{array}$ & \\
\hline $\begin{array}{l}1950 \\
1951 \\
1952 \\
1953 \\
1954 \\
1955 \\
1956 \\
1957 \\
1958 \\
1959\end{array}$ & $\begin{array}{l}11 \\
12 \\
26 \\
18 \\
11 \\
12 \\
7.3 \\
6.0 \\
6.8 \\
5.6\end{array}$ & $\begin{array}{l}12 \\
12 \\
28 \\
18 \\
11 \\
13 \\
7.6 \\
6.5 \\
7.4 \\
6.6\end{array}$ & $\begin{array}{r}12 \\
13 \\
28 \\
20 \\
11 \\
14 \\
8.1 \\
6.6 \\
7.5 \\
6.9\end{array}$ & $\begin{array}{l}13 \\
13 \\
36 \\
20 \\
13 \\
14 \\
8.5 \\
8.1 \\
9.3 \\
7.4\end{array}$ & $\begin{array}{r}14 \\
14 \\
47 \\
21 \\
17 \\
16 \\
9.6 \\
9.2 \\
11 \\
9.9\end{array}$ & $\begin{array}{l}15 \\
15 \\
89 \\
26 \\
19 \\
17 \\
11 \\
12 \\
14 \\
11\end{array}$ & $\begin{array}{l}20 \\
16 \\
88 \\
38 \\
20 \\
21 \\
12 \\
15 \\
16 \\
13\end{array}$ & \\
\hline $\begin{array}{l}1960 \\
1961 \\
1962 \\
1963 \\
1964 \\
1965 \\
1966 \\
1967 \\
1968 \\
1969\end{array}$ & $\begin{array}{r}13 \\
12 \\
14 \\
26 \\
13 \\
8.4 \\
5.6 \\
11 \\
6.4 \\
17\end{array}$ & $\begin{array}{r}13 \\
13 \\
17 \\
26 \\
13 \\
9.5 \\
5.7 \\
1 \frac{1}{7.0} \\
19\end{array}$ & $\begin{array}{l}13 \\
15 \\
21 \\
28 \\
14 \\
12 \\
6.7 \\
12 \\
7.9 \\
20\end{array}$ & $\begin{array}{l}20 \\
18 \\
27 \\
29 \\
16 \\
14 \\
9.2 \\
13 \\
5.7 \\
25\end{array}$ & $\begin{array}{l}33 \\
20 \\
35 \\
30 \\
18 \\
15 \\
12 \\
17 \\
12 \\
25\end{array}$ & $\begin{array}{l}41 \\
27 \\
40 \\
36 \\
15 \\
16 \\
21 \\
18 \\
21 \\
31\end{array}$ & $\begin{array}{l}55 \\
31 \\
66 \\
46 \\
20 \\
18 \\
44 \\
22 \\
34 \\
46\end{array}$ & \\
\hline $\begin{array}{l}1970 \\
1971 \\
1972 \\
1973\end{array}$ & $\begin{array}{l}21 \\
18 \\
22 \\
36\end{array}$ & $\begin{array}{l}21 \\
18 \\
22 \\
38\end{array}$ & $\begin{array}{l}23 \\
19 \\
23 \\
39\end{array}$ & $\begin{array}{l}24 \\
21 \\
26 \\
42\end{array}$ & $\begin{array}{l}26 \\
25 \\
34 \\
62\end{array}$ & $\begin{array}{r}28 \\
34 \\
53 \\
121\end{array}$ & $\begin{array}{r}32 \\
49 \\
57 \\
195\end{array}$ & \\
\hline
\end{tabular}


LOW FLOW DISCHARGES, IN YEARं ENDING MARCH 31--Continued

\begin{tabular}{|c|c|c|c|c|c|c|c|}
\hline CLIMATIC & LOWES & SE & $\mathrm{ft}^{\mathrm{j}}$ & Ino & PE & 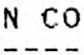 & VE \\
\hline YEAR & 13 & 7 & 14 & 30 & 60 & 120 & 183 \\
\hline $\begin{array}{l}1974 \\
1975 \\
1976\end{array}$ & $\begin{array}{l}33 \\
30 \\
13\end{array}$ & $\begin{array}{l}35 \\
32 \\
14\end{array}$ & $\begin{array}{l}35 \\
34 \\
14\end{array}$ & $\begin{array}{l}45 \\
35 \\
15\end{array}$ & $\begin{array}{l}53 \\
38 \\
17\end{array}$ & $\begin{array}{l}60 \\
43 \\
28\end{array}$ & $\begin{array}{l}62 \\
45 \\
3 .\end{array}$ \\
\hline
\end{tabular}


05-4145.00 LITTLE MAQUOKETA RIVER NEAR DURANGO--Continued

DRAINAGE AREA: $130 \mathrm{~m}^{2}$ PERIOD OF RECORD: 42 YEARS AVERAGE DISCHARGE: 87.0 ft3/s

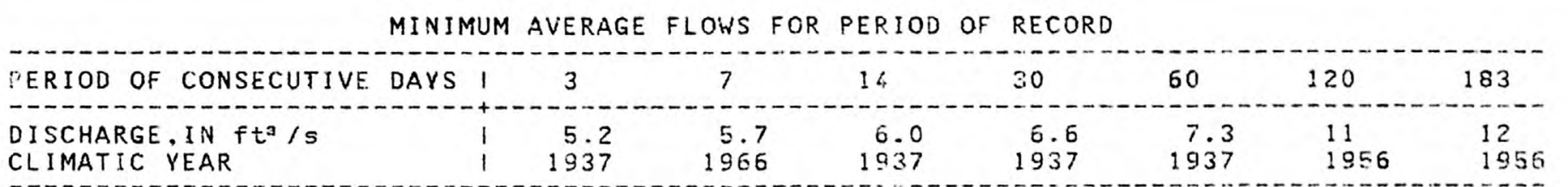

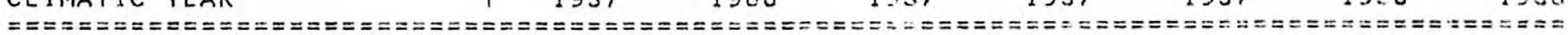

MAGNITUDE AND FREQUENCY OF ANNUAL LOW FLOWS

RECURRENCE I LOWEST AVERAGE FLOW, IN $\mathrm{ft}^{3} / \mathrm{s}$, FOR INDICATED PERIOD IN CONSECUTIVE DAYS

INTERVAL 1

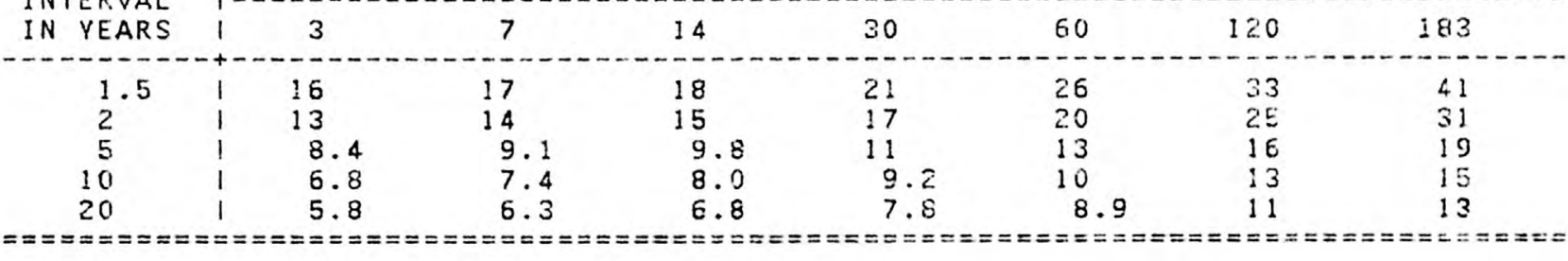

MAGNITUDE AND FREQUENCY OF SEASONAL LOW FLOWS

I LOWEST AVERAGE FLOW, IN $\mathrm{ft}^{3} / \mathrm{s}$,FOR THE INDICATED PERIOD IN CONSECUTIVE DAYS WITHIN EACH WATER-VEAR QUARTER

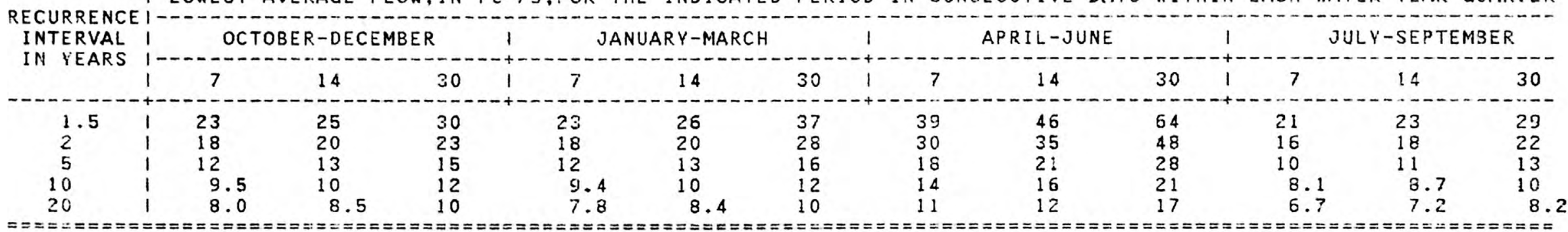

DURATION OF DAILY DISCHARGES FOR ANHUAL FND SEASONAL PERIODS

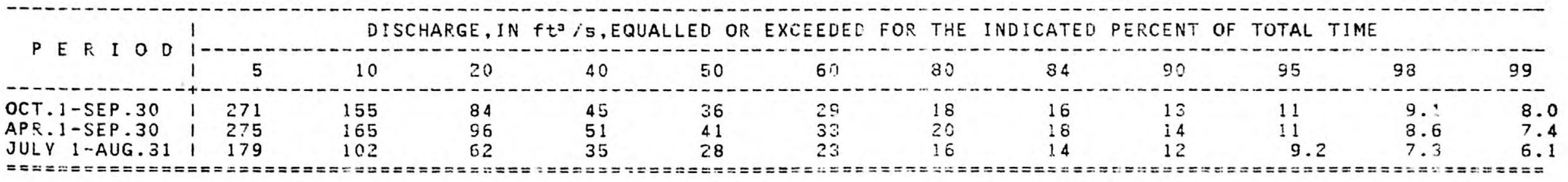


Location.--Lat $42^{\circ} 27^{\prime} 22^{\prime \prime}$, long $91^{\circ} 25^{\prime} 56^{\prime \prime}$, in NWi/4 NE1/4 sec.9, T.88 N.. R.5 W. Delaware County, on left bank $0.6 \mathrm{miles}$ downstream from Sand Creek, 1.5 miles upstream from Spring Branch, 2.3 miles southeast from dam on Mcquoketa River in Manchester, and at mile 100.5.

Remarks.--Diurnal fiuctuation caused by powerplant 2 miles upstream. Disconcontinued September 1973.

LOW FLOW DISCHARGES, IN YEAR ENDING MARCH 31

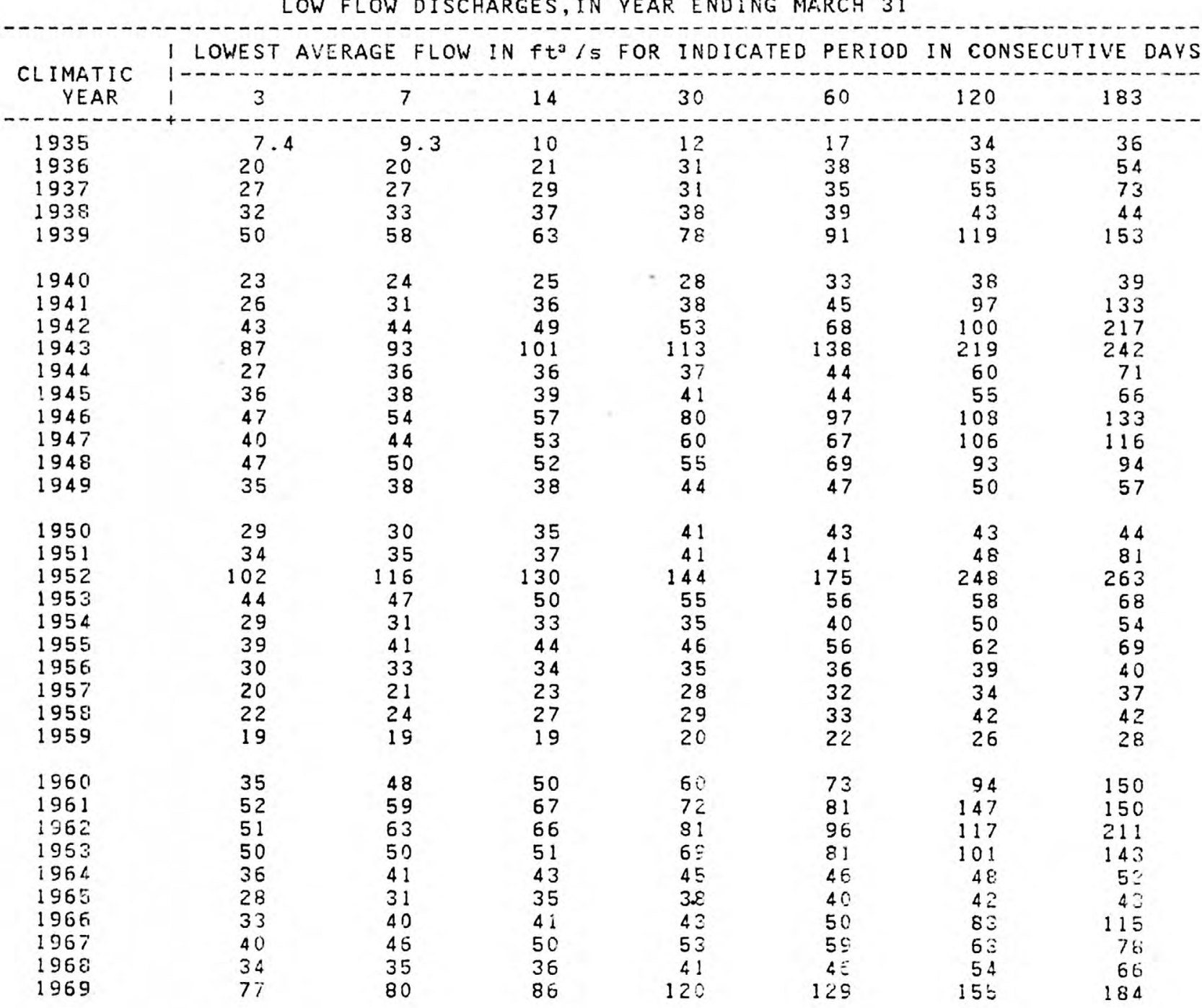


LOW FLOW DISCHARGES, IN YEAR ENDING MARCH 31--Cont inued

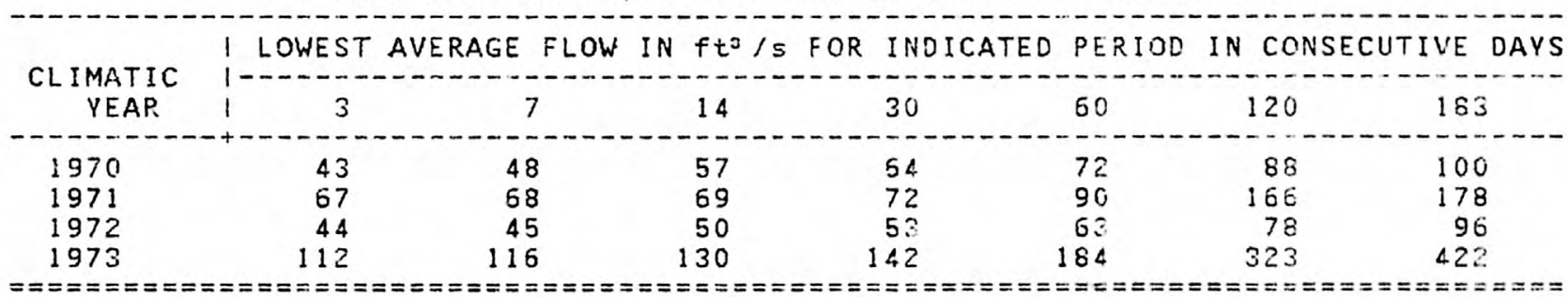




DRAINAGE AREA: $305 \mathrm{~m}^{2} \quad$ PERIOD OF RECORD: 40 YEARS AVERAGE DISCHARGE: $20 \mathrm{f}$ ft $/ \mathrm{s}$
MINIMUM AVERAGE FLOWS FOR PERIOD OF RECORD
PERIOD OF CONSECUTIVE DAYS I

MAENITUDE AND FREQUENCY OF ANNUAL LOW FLOWS

\begin{tabular}{l|c|c|ccc} 
RECURRENCE \\
INTERVAL
\end{tabular}

MAGNITUDE AND FREQUENCY OF SEASONAL LOW FLOWS

RECURRENCE
INTERVAL

DURATION OF DAILY DISCHARGES FOR ANNUAL AND SEASONAL PERIODS

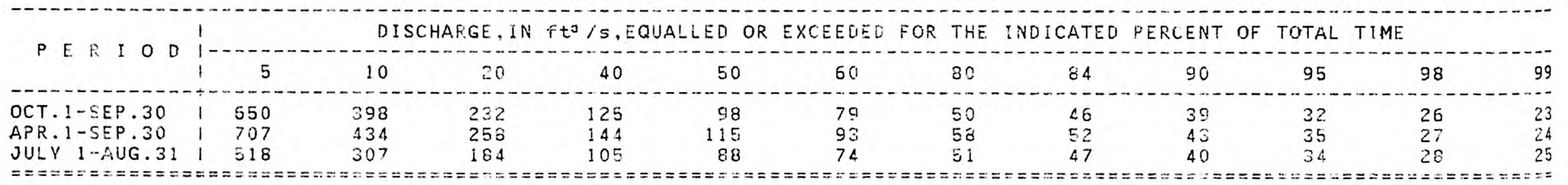


Location.--Lat $42^{\circ} 02^{\prime} 18^{\prime \prime}$, long $90^{\circ} 52^{\prime} 59^{\prime \prime}$, In NE1/4 SE1/4 sec.31, T.84 N., R.1 E., Jackson County, on right bank $15 \mathrm{ft}$ downstream from bridge on county highway, 1.6 miles upstream from Rat Run, $2.8 \mathrm{miles}$ south of Monmouth, and 8.2 miles upstream from mouth.

Remarks.--Discontinued September 1976.

LOW FLOW DISCHARGES, IN YEAR. ENDING MARCH 31

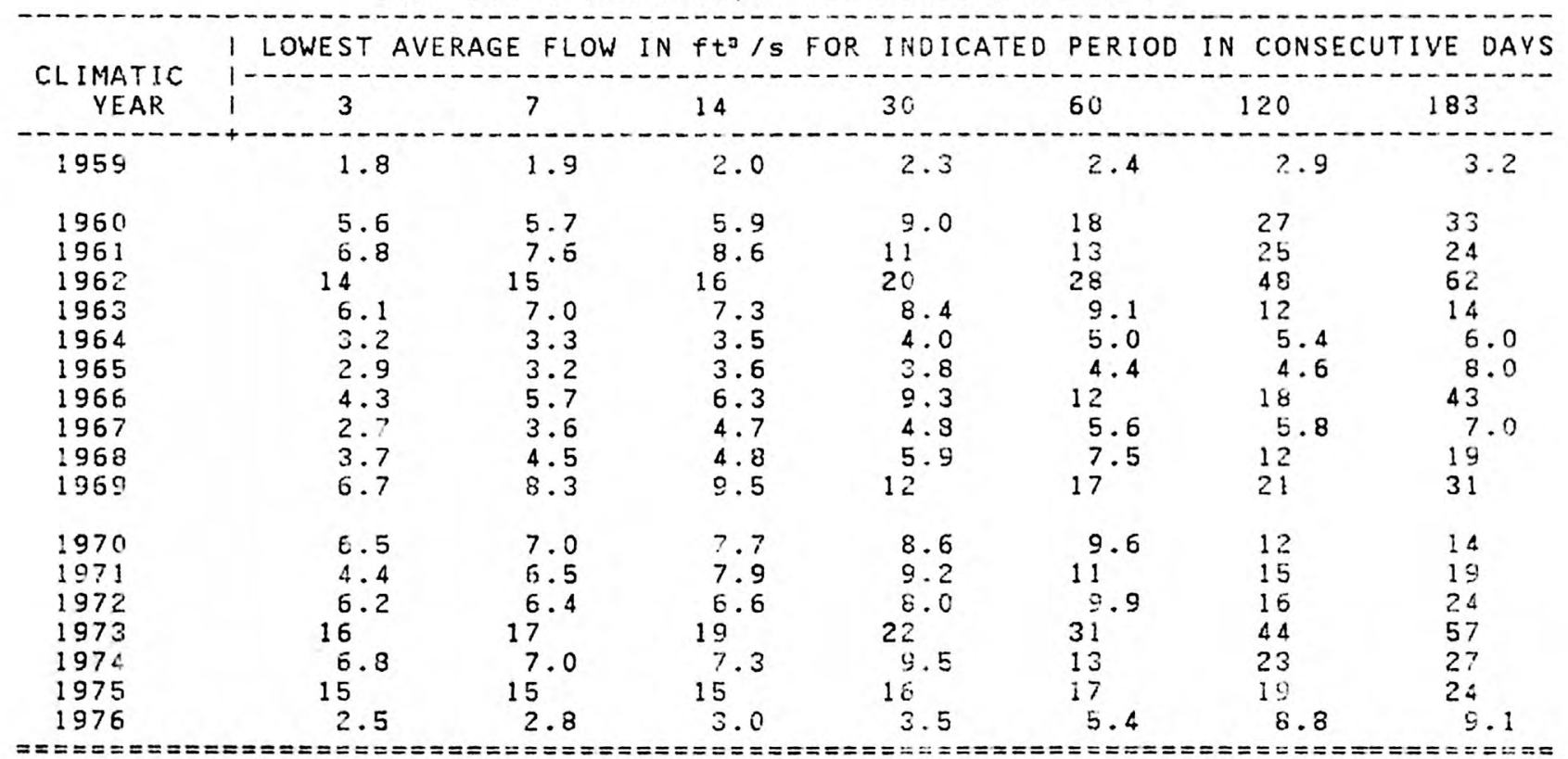




DRAINAGE AREA: $61.3 \mathrm{~m}^{2}$ PERIOB OF RECORD: 19 VEARS AVERAGE DISCHARGE: $44.9 \mathrm{ft}^{3} / \mathrm{s}$

MINIMUM AVERAGE FLOWS FOR PERIOD OF RECORD

\begin{tabular}{|c|c|c|c|c|c|c|c|}
\hline PERIOD OF CONSECUTIVE DAYS & 1 & 7 & 14 & 30 & 60 & 120 & 183 \\
\hline $\begin{array}{l}\text { DISCHARGE, IN } \mathrm{Ft}^{3} / \mathrm{s} \\
\text { CLIMATIC YEAR }\end{array}$ & $\begin{array}{r}1.8 \\
1959\end{array}$ & $\begin{array}{r}1.9 \\
1959\end{array}$ & $\begin{array}{r}2.0 \\
1959\end{array}$ & $\begin{array}{r}2.3 \\
1959\end{array}$ & $\begin{array}{r}2.4 \\
1959\end{array}$ & $\begin{array}{r}2.9 \\
1959\end{array}$ & $\begin{array}{r}3.2 \\
1959\end{array}$ \\
\hline
\end{tabular}

MAGNITUDE AND FREQUENCY OF ANNUAL LOW FLOWS

\begin{tabular}{l|l|l} 
RECURRENCE \\
INTERVAL \\
IN INEARS
\end{tabular}

MAGNITUDE AND FREQUENCY OF SEASONAL LOW FLOWS

I LOWEST AVERAGE FLOW, IN $\mathrm{ft}^{3} / \mathrm{s}, \mathrm{FOR}$ THE INDICATED PERIOD IN CONSECUTIVE DAYS WITHIN EACH WATER-YEAR QUARTER

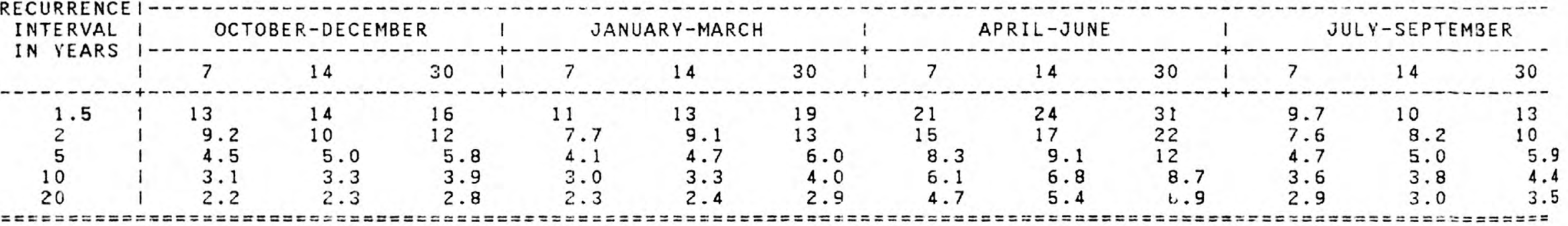

DURATION OF DAILY DISCHARGES FOR ANNUAL AND SEASONAL PERIODS

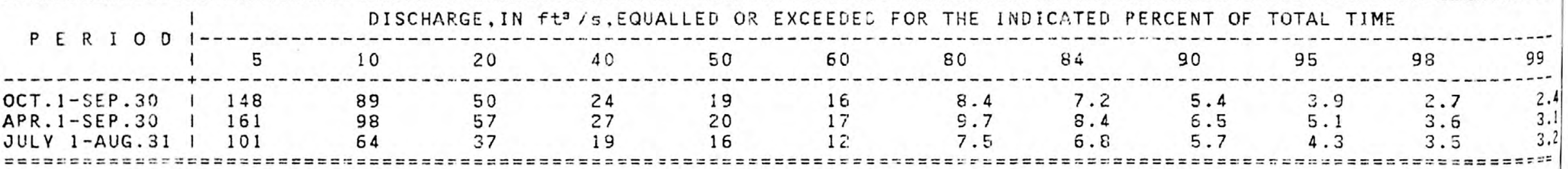


Location.--Lat $42^{\circ} 05^{\prime} \mathrm{1}^{\prime \prime}$, long $90^{\circ} 38^{\prime} 04^{\prime \prime}$, In SWl/4 NE1/4 sec.17, T.84 N., R.3 E. Jackson County, on rlght bank $500 \mathrm{ft}$ upstream from bridge on State 62, 1,200 ft upstream from Pra!rie Creek, $2.0 \mathrm{miles}$ northeas from mouth.

Remarks.--Diurnal fiuctuation caused by powerplant 4 miles upst.ream. Published as "below North Fork Maquoketa River nr Maquoketa" prior t. 1940. LOW FLOW DISCHARGES, IN YEAR ENDING MARCH 31

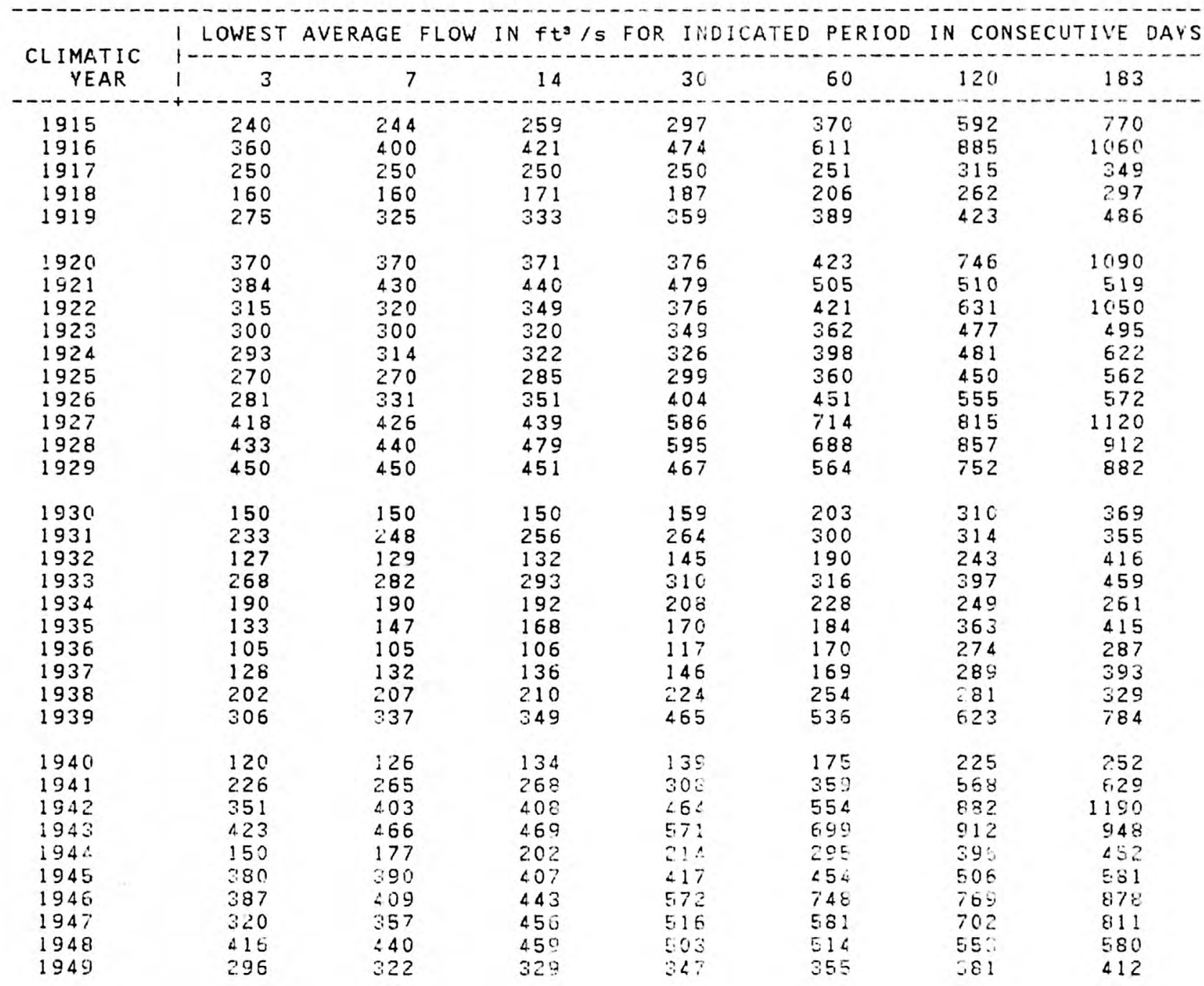


LOW FLOW DISCHARGES, IN YEAR ENDING MARCH 31--Continued

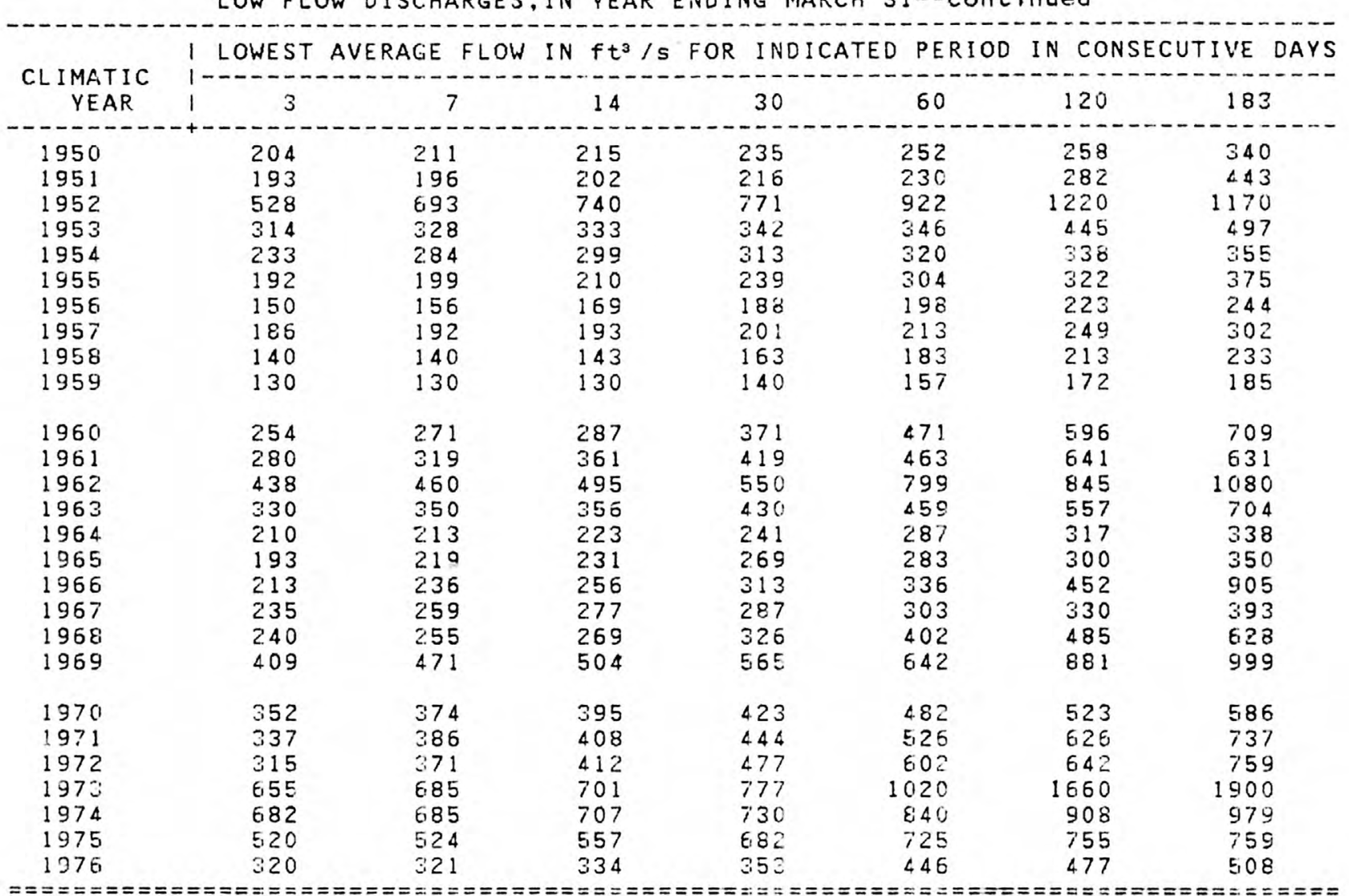


MINIMUM AVERAGE FLOWS FOR PERIOD OF RECORD

\begin{tabular}{|c|c|c|c|c|c|c|c|}
\hline PERIOD OF CONSECUTIVE DAYS & 3 & 7 & 14 & 30 & 60 & 120 & 183 \\
\hline $\begin{array}{l}\text { DISCHARGE, IN } \mathrm{ft}^{3} / \mathrm{s} \\
\text { CLIMATIC YEAR }\end{array}$ & $\begin{array}{l}105 \\
1936\end{array}$ & $\begin{array}{l}105 \\
1936\end{array}$ & $\begin{array}{l}106 \\
1936\end{array}$ & $\begin{array}{l}117 \\
1936\end{array}$ & $\begin{array}{l}157 \\
1959\end{array}$ & $\begin{array}{l}172 \\
1959\end{array}$ & $\begin{array}{l}185 \\
1959\end{array}$ \\
\hline
\end{tabular}

MAGNITUDE AND FREQUENCY OF ANNUAL LOW FLOWS

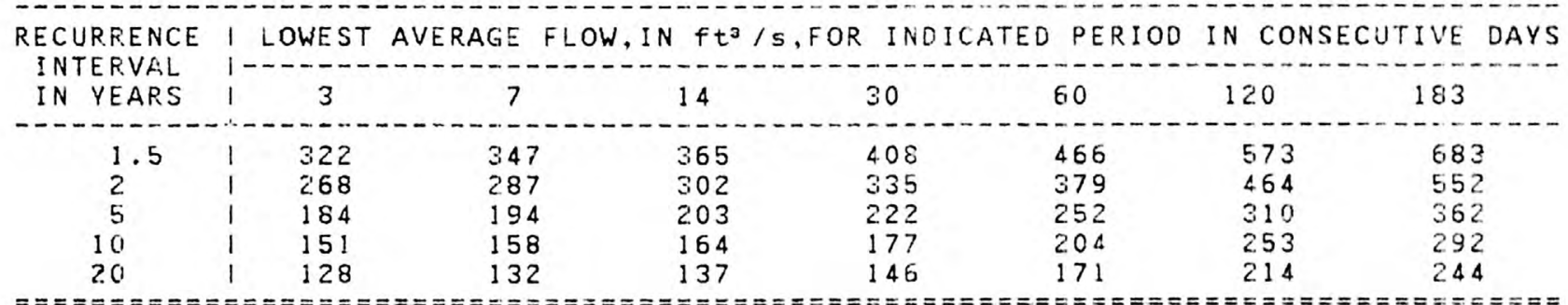

MAGNITUDE AND FREQUENCY OF SEASONAL LOW FLOWS

1 LOWEST AVERAGE FLOW, IN $\mathrm{ft}^{3} / \mathrm{s}$, FOR THE INDICATED PERIOD IN CONSECUTIVE DAYS WITHIN EACH WATER-VEAR QUARTER

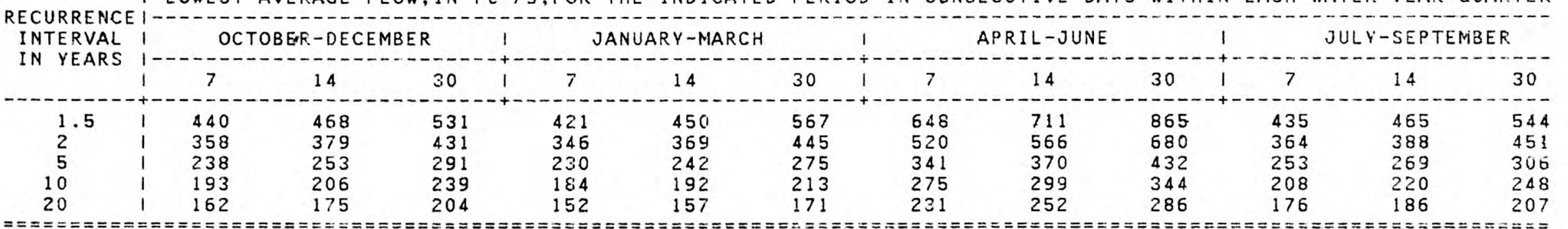

DURATION OF DAILY DISCHARGES FOR ANNUAL AND SEASONAL PERIODS

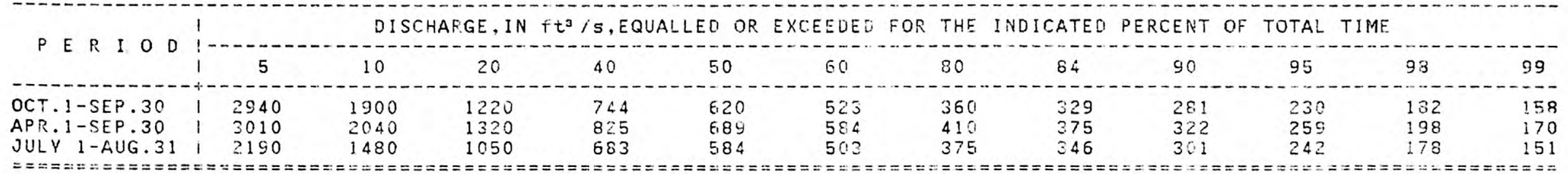


Location.--Lat $41^{\circ} 46^{\prime} 53^{\prime \prime}$, long $90^{\circ} 15^{\prime} 04^{\prime \prime}$, in NW1/4 sec.34, T.81 N., R.6 W. Clinton County, on right bank at foot of Seventh Avenue in Camanche, $5.0 \mathrm{mi}$ upstream from Wapsipinicon River, $6.4 \mathrm{~m}$ i downstream from clinton, $10.6 \mathrm{mi}$ downstream from Dam 13, and at mile 511.8 upstream from Ohto River. Prior to June 6, 1969, at site $400 \mathrm{ft}$ downstream.

Remarks.--Fiow regulated by navigation dams. LOW FLOW DISCHARGES, IN YEAR ENDING MARCH 31

\begin{tabular}{|c|c|c|c|c|c|c|c|}
\hline \multirow{2}{*}{$\begin{array}{c}\text { CLIMATIC } \\
\text { YEAR }\end{array}$} & LOWEST & GE FLO & $N f t^{3} / s$ & R INDICATED & PERIOD & \multicolumn{2}{|c|}{ IN CONSECUTIVE DAYS } \\
\hline & 3 & 7 & 14 & 30 & 60 & 120 & 183 \\
\hline $\begin{array}{l}1875 \\
1876 \\
1877 \\
1878 \\
1879\end{array}$ & $\begin{array}{l}18000 \\
14000 \\
16300 \\
23000 \\
11100\end{array}$ & $\begin{array}{l}19400 \\
14300 \\
15900 \\
24400 \\
12900\end{array}$ & $\begin{array}{l}22900 \\
16300 \\
19100 \\
26800 \\
14900\end{array}$ & $\begin{array}{l}25200 \\
21000 \\
24100 \\
27500 \\
19100\end{array}$ & $\begin{array}{l}26200 \\
26200 \\
25100 \\
28600 \\
19600\end{array}$ & $\begin{array}{l}27000 \\
29900 \\
30800 \\
32700 \\
23400\end{array}$ & $\begin{array}{l}35400 \\
37500 \\
35500 \\
33800 \\
29400\end{array}$ \\
\hline $\begin{array}{l}1880 \\
1881 \\
1882 \\
1883 \\
1884 \\
1885 \\
1886 \\
1887 \\
1888 \\
1889\end{array}$ & $\begin{array}{l}20300 \\
16000 \\
24700 \\
15000 \\
14000 \\
18000 \\
15000 \\
12000 \\
12000 \\
12300\end{array}$ & $\begin{array}{l}20900 \\
16600 \\
25500 \\
15900 \\
14700 \\
19000 \\
15400 \\
12600 \\
12100 \\
13100\end{array}$ & $\begin{array}{l}24000 \\
18700 \\
28300 \\
18400 \\
17400 \\
24400 \\
16400 \\
14100 \\
14100 \\
15500\end{array}$ & $\begin{array}{l}29900 \\
21100 \\
32700 \\
25500 \\
22400 \\
30100 \\
23300 \\
18700 \\
17900 \\
17100\end{array}$ & $\begin{array}{l}31800 \\
21600 \\
37900 \\
28500 \\
23000 \\
35700 \\
25100 \\
18900 \\
18800 \\
19300\end{array}$ & $\begin{array}{l}35100 \\
23500 \\
55300 \\
38300 \\
26700 \\
36300 \\
28500 \\
25500 \\
20300 \\
23100\end{array}$ & $\begin{array}{l}34500 \\
28700 \\
87200 \\
44500 \\
31200 \\
57000 \\
34700 \\
30900 \\
25500 \\
26100\end{array}$ \\
\hline $\begin{array}{l}1890 \\
1891 \\
1892 \\
1893 \\
1894 \\
1895 \\
1896 \\
1897 \\
1898 \\
1899\end{array}$ & $\begin{array}{r}8000 \\
14000 \\
10000 \\
13000 \\
13000 \\
9670 \\
8000 \\
11700 \\
10000 \\
9000\end{array}$ & $\begin{array}{r}8290 \\
14000 \\
10400 \\
13700 \\
13700 \\
10100 \\
8570 \\
12400 \\
10600 \\
9290\end{array}$ & $\begin{array}{r}9640 \\
14100 \\
11600 \\
14000 \\
14000 \\
11900 \\
10100 \\
15100 \\
12700 \\
10600\end{array}$ & $\begin{array}{l}11300 \\
14500 \\
14100 \\
15100 \\
14100 \\
13400 \\
13300 \\
16900 \\
15700 \\
14000\end{array}$ & $\begin{array}{l}12400 \\
15200 \\
17600 \\
14800 \\
14800 \\
14200 \\
14400 \\
18600 \\
16600 \\
16000\end{array}$ & $\begin{array}{l}15100 \\
19500 \\
18800 \\
17200 \\
17200 \\
19000 \\
16700 \\
25200 \\
20000 \\
19100\end{array}$ & $\begin{array}{l}18300 \\
29100 \\
19900 \\
23000 \\
23000 \\
19300 \\
20000 \\
24900 \\
24200 \\
20800\end{array}$ \\
\hline $\begin{array}{l}1900 \\
1901 \\
1902 \\
1903 \\
1904 \\
1905 \\
1906 \\
1907 \\
1908 \\
1909\end{array}$ & $\begin{array}{r}14000 \\
16000 \\
9000 \\
15000 \\
16000 \\
14000 \\
21700 \\
26000 \\
13300 \\
11000\end{array}$ & $\begin{array}{r}14300 \\
16000 \\
9140 \\
15600 \\
16000 \\
14600 \\
22700 \\
26400 \\
14100 \\
11300\end{array}$ & $\begin{array}{l}16500 \\
16200 \\
10900 \\
18100 \\
17000 \\
16400 \\
24002 \\
28900 \\
16700 \\
14000\end{array}$ & $\begin{array}{l}20300 \\
17200 \\
14500 \\
24800 \\
20000 \\
29500 \\
25200 \\
38000 \\
18500 \\
16750\end{array}$ & $\begin{array}{l}21200 \\
19200 \\
16200 \\
28400 \\
21700 \\
20700 \\
31700 \\
38200 \\
15100 \\
19800\end{array}$ & $\begin{array}{l}25300 \\
27200 \\
20300 \\
32800 \\
26300 \\
28700 \\
38500 \\
47100 \\
27300 \\
22900\end{array}$ & $\begin{array}{l}30600 \\
48600 \\
23700 \\
31700 \\
57500 \\
38700 \\
46500 \\
50000 \\
37400 \\
26020\end{array}$ \\
\hline
\end{tabular}


LOW FLOW DISCHARGES, IN YEAR ENDING MARCH 31--Cont inued

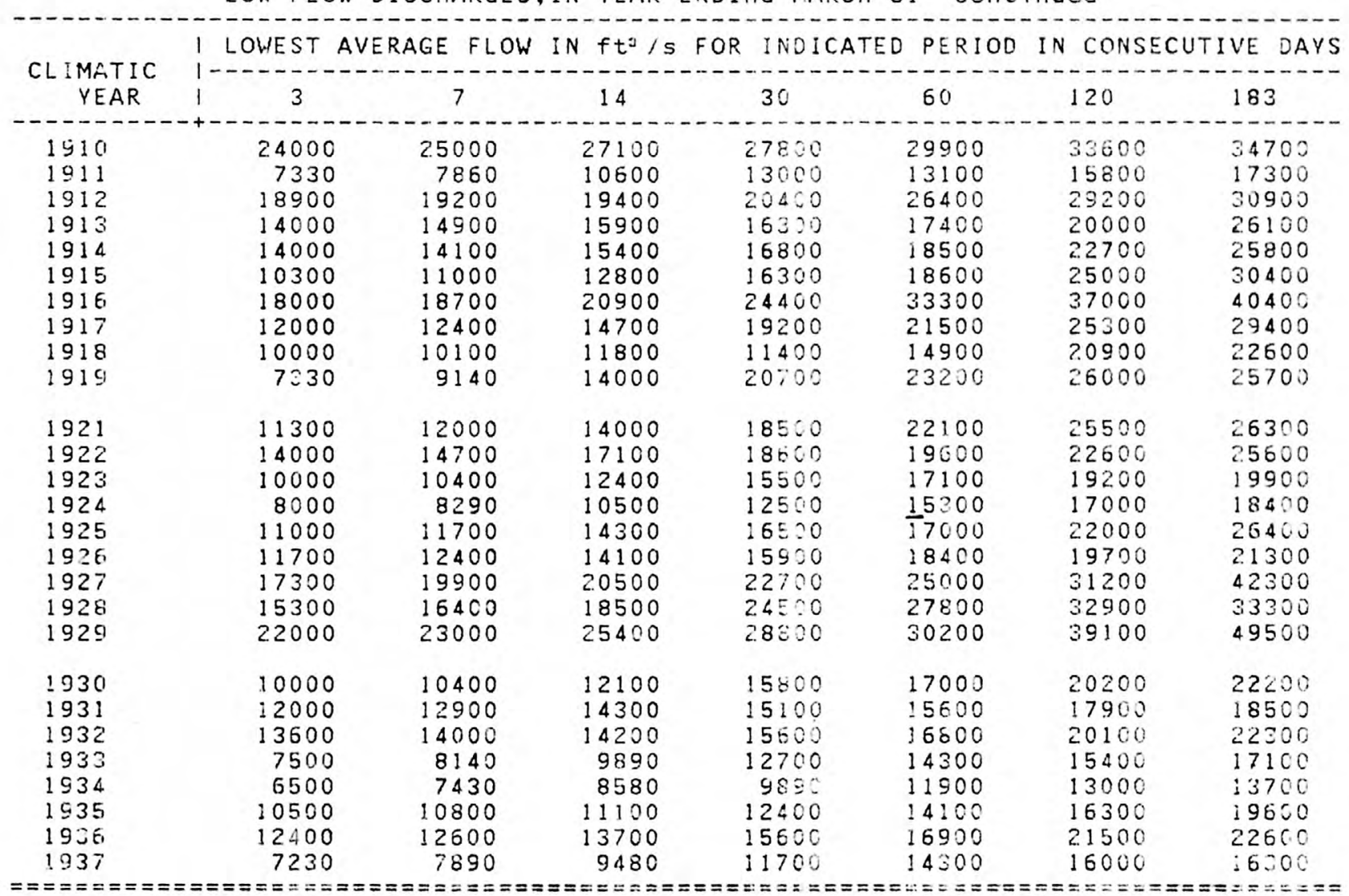


DRAINAGE AREA: $85,600 \mathrm{~m}^{2}$ PERIOD OF RECORD: 63 YEARS AVERAGE DISCHARGE: 47,800 ft 3 /s

MINIMUM AVERAGE FLOWS FOR PERIOD OF RECORD

\begin{tabular}{|c|c|c|c|c|c|c|c|}
\hline PERIOD OF CONSECUTIVE DAYS & 3 & 7 & 14 & 30 & 60 & 120 & 183 \\
\hline $\begin{array}{l}\text { ISCHARGE, IN } \mathrm{ft}^{3} / \mathrm{s} \\
\text { LIMATIC YEAR }\end{array}$ & $\begin{array}{l}6500 \\
1934\end{array}$ & $\begin{array}{l}7430 \\
1934\end{array}$ & $\begin{array}{r}8580 \\
1934\end{array}$ & $\begin{array}{l}9890 \\
1934\end{array}$ & $\begin{array}{l}11900 \\
1934\end{array}$ & $\begin{array}{l}13000 \\
1934\end{array}$ & \\
\hline
\end{tabular}

MAGNITUDE AND FREQUENCY OF ANNUAL LOW FLOWS

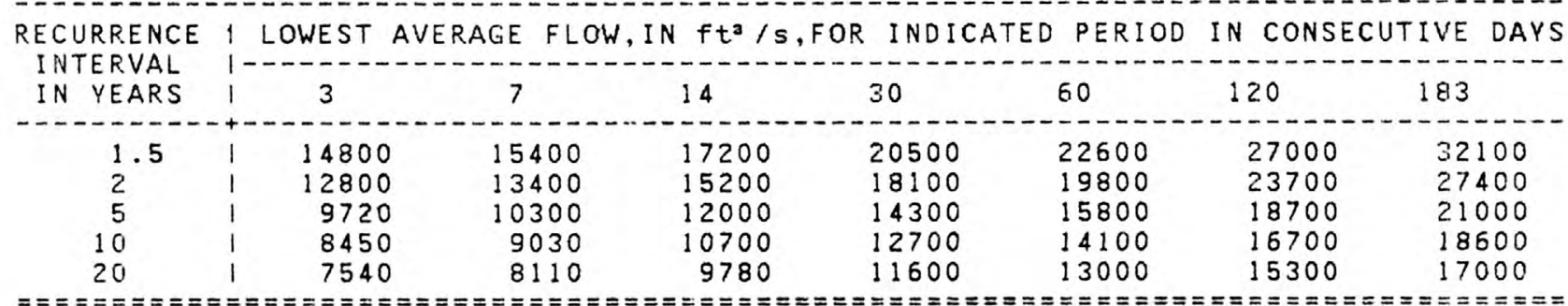

MAGNITUDE AND FREQUENCY OF SEASONAL LOW FLOWS

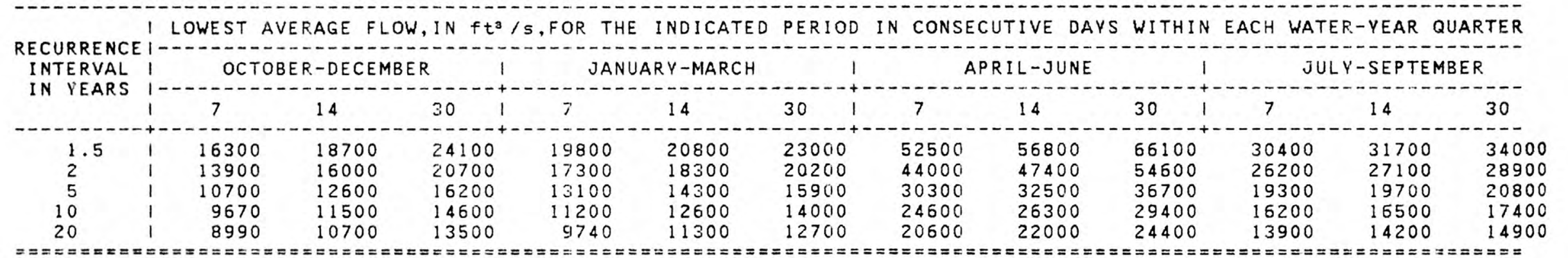

DURATION OF DAILY DISCHARGES FOR ANNUAL AND SEASONAL PERIODS

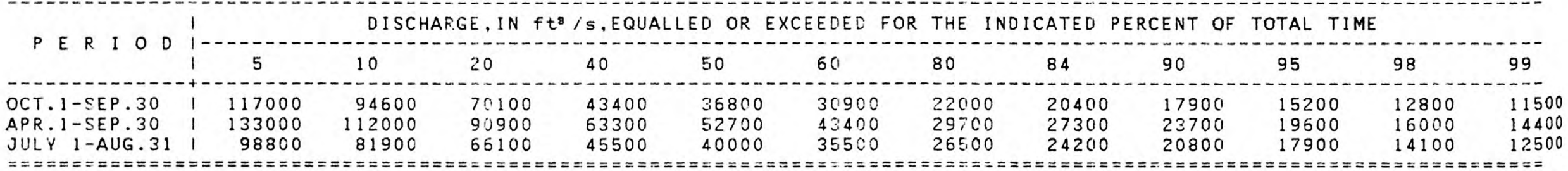


LOW FLOW DISCHARGES, IN YEAR. ENDING MARCH 31

\begin{tabular}{|c|c|c|c|c|c|c|c|}
\hline \multirow{2}{*}{$\begin{array}{c}\text { CLIMATIC } \\
\text { YEAR }\end{array}$} & I LOWEST & GE FLOW & N fto $/ \mathrm{s}$ & $R$ INDICATED & PERIO & \multicolumn{2}{|c|}{$\begin{array}{l}\text { N CONSECUTIVE DAYS } \\
\end{array}$} \\
\hline & 13 & 7 & 14 & 30 & 60 & 120 & 183 \\
\hline $\begin{array}{l}1938 \\
1939\end{array}$ & $\begin{array}{l}11200 \\
13000\end{array}$ & $\begin{array}{l}11500 \\
14700\end{array}$ & $\begin{array}{l}11900 \\
17300\end{array}$ & $\begin{array}{l}13800 \\
22400\end{array}$ & $\begin{array}{l}15000 \\
27700\end{array}$ & $\begin{array}{l}16500 \\
32400\end{array}$ & $\begin{array}{l}16900 \\
39600\end{array}$ \\
\hline $\begin{array}{l}1940 \\
1941 \\
1942 \\
1943 \\
1944 \\
1945 \\
1946 \\
1947 \\
1948 \\
1949\end{array}$ & $\begin{array}{l}13000 \\
16900 \\
15100 \\
22400 \\
17100 \\
19700 \\
21900 \\
20400 \\
17600 \\
13100\end{array}$ & $\begin{array}{l}12300 \\
17400 \\
15700 \\
22600 \\
18200 \\
19800 \\
22600 \\
20600 \\
17800 \\
14000\end{array}$ & $\begin{array}{l}12600 \\
18200 \\
16500 \\
23400 \\
21400 \\
20100 \\
26000 \\
20900 \\
18100 \\
14700\end{array}$ & $\begin{array}{l}13000 \\
19200 \\
18200 \\
26600 \\
22700 \\
20800 \\
29800 \\
23800 \\
19100 \\
15000\end{array}$ & $\begin{array}{l}13600 \\
19700 \\
22200 \\
30500 \\
27900 \\
22600 \\
33700 \\
28900 \\
21100 \\
16100\end{array}$ & $\begin{array}{l}16100 \\
23500 \\
29500 \\
33100 \\
29900 \\
24200 \\
35200 \\
41900 \\
23400 \\
17200\end{array}$ & $\begin{array}{l}17200 \\
24500 \\
43500 \\
42900 \\
30400 \\
25700 \\
36500 \\
43400 \\
26300 \\
17800\end{array}$ \\
\hline $\begin{array}{l}1950 \\
1951 \\
1952 \\
1953 \\
1954 \\
1955 \\
1956 \\
1957 \\
1958 \\
1959\end{array}$ & $\begin{array}{l}14400 \\
14100 \\
24100 \\
20100 \\
21400 \\
20000 \\
18100 \\
13800 \\
17000 \\
17000\end{array}$ & $\begin{array}{l}15400 \\
14300 \\
26900 \\
20600 \\
21800 \\
20400 \\
18800 \\
14100 \\
17000 \\
17000\end{array}$ & $\begin{array}{l}15700 \\
16600 \\
29400 \\
21000 \\
22300 \\
21000 \\
19900 \\
15400 \\
17600 \\
17600\end{array}$ & $\begin{array}{l}17500 \\
18600 \\
34000 \\
22100 \\
23100 \\
21400 \\
20700 \\
17100 \\
19700 \\
19700\end{array}$ & $\begin{array}{l}18300 \\
20100 \\
36000 \\
23700 \\
24200 \\
25400 \\
21400 \\
19000 \\
20600 \\
20600\end{array}$ & $\begin{array}{l}19400 \\
20200 \\
42800 \\
24500 \\
25000 \\
29100 \\
22100 \\
20800 \\
24900 \\
24900\end{array}$ & $\begin{array}{l}19900 \\
20900 \\
49800 \\
26500 \\
26200 \\
35300 \\
22400 \\
20800 \\
26600 \\
26600\end{array}$ \\
\hline $\begin{array}{l}1960 \\
1961 \\
1962 \\
1963 \\
1964 \\
1965 \\
1966 \\
1967 \\
1968 \\
1969\end{array}$ & $\begin{array}{l}16000 \\
15000 \\
15300 \\
17200 \\
15200 \\
11400 \\
22300 \\
16800 \\
16900 \\
22000\end{array}$ & $\begin{array}{l}16200 \\
15200 \\
16100 \\
17400 \\
15600 \\
11900 \\
24300 \\
17800 \\
17100 \\
24000\end{array}$ & $\begin{array}{l}18400 \\
15500 \\
16800 \\
17700 \\
15900 \\
12000 \\
26000 \\
19400 \\
17600 \\
26400\end{array}$ & $\begin{array}{l}20600 \\
16700 \\
19000 \\
18500 \\
16500 \\
13800 \\
28200 \\
20400 \\
18200 \\
29700\end{array}$ & $\begin{array}{l}25700 \\
18500 \\
20800 \\
19900 \\
17300 \\
15800 \\
31300 \\
21600 \\
20100 \\
32500\end{array}$ & $\begin{array}{l}31600 \\
24300 \\
25000 \\
23100 \\
18200 \\
21100 \\
46100 \\
23700 \\
21300 \\
37200\end{array}$ & $\begin{array}{l}37700 \\
27000 \\
28800 \\
29300 \\
18700 \\
22300 \\
45700 \\
25000 \\
22500 \\
45400\end{array}$ \\
\hline $\begin{array}{l}1970 \\
1971 \\
1972 \\
1973 \\
1974 \\
1975 \\
1976\end{array}$ & $\begin{array}{l}18200 \\
17600 \\
18100 \\
21200 \\
23200 \\
18500 \\
19200\end{array}$ & $\begin{array}{l}19900 \\
18100 \\
19900 \\
21600 \\
23700 \\
18000 \\
20900\end{array}$ & $\begin{array}{l}21300 \\
18920 \\
21200 \\
24100 \\
25100 \\
19600 \\
22900\end{array}$ & $\begin{array}{l}22600 \\
20200 \\
23400 \\
32000 \\
28800 \\
20200 \\
24102\end{array}$ & $\begin{array}{l}23600 \\
23400 \\
24900 \\
43400 \\
30500 \\
21220 \\
25700\end{array}$ & $\begin{array}{l}25900 \\
26900 \\
31400 \\
50200 \\
36160 \\
23500 \\
32100\end{array}$ & $\begin{array}{l}25700 \\
34100 \\
35400 \\
58400 \\
59000 \\
24900 \\
32500\end{array}$ \\
\hline
\end{tabular}


DRAINAGE AREA: $85,600 \mathrm{~m}^{2}$ PERIOD OF RECORD: 39 YEARS AVERAGE DISCHARGE: 46,400 ft $/ \mathrm{s}$

MINIMUM AVERAGE FLOWS FOR PERIOD OF RECORD

\begin{tabular}{|c|c|c|c|c|c|c|c|}
\hline PERIOD OF CONSECUTIVE DAYS I & 3 & 7 & 14 & 30 & 60 & 120 & 183 \\
\hline $\begin{array}{l}\text { RGE, IN } f t^{3} / s \\
\text { IC YEAR }\end{array}$ & $\begin{array}{c}11200 \\
1938 \\
=====\end{array}$ & $\begin{array}{l}11500 \\
1938 \\
======\end{array}$ & $\begin{array}{l}11900 \\
1938 \\
====\end{array}$ & $\begin{array}{l}13000 \\
1940 \\
=====\end{array}$ & $\begin{array}{l}13600 \\
1940 \\
====\end{array}$ & $\begin{array}{l}16100 \\
1940 \\
====\end{array}$ & $\begin{aligned} & 16900 \\
& 1938 \\
&====\end{aligned}$ \\
\hline
\end{tabular}

MAGNITUDE AND FREQUENCY OF ANNUAL LOW FLOWS

\begin{tabular}{|c|c|c|c|c|c|c|c|c|}
\hline $\begin{array}{l}\text { RECURRENCE } \\
\text { INTERVAL }\end{array}$ & 1 & WWE ST & $G E$ & 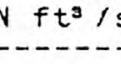 & INDIC & PERIC & IN CONS & IVE DAYS \\
\hline IN YEARS & 1 & 3 & 7 & 14 & 30 & 60 & 120 & 183 \\
\hline $\begin{array}{l}1.5 \\
2 \\
5 \\
10 \\
20\end{array}$ & $\begin{array}{l}1 \\
\vdots \\
1 \\
1\end{array}$ & $\begin{array}{l}18900 \\
17500 \\
14700 \\
13400 \\
12300\end{array}$ & $\begin{array}{l}19700 \\
18100 \\
15200 \\
13800 \\
12700\end{array}$ & $\begin{array}{l}20900 \\
19100 \\
15900 \\
14400 \\
13200\end{array}$ & $\begin{array}{l}22800 \\
20700 \\
17200 \\
15700 \\
14500\end{array}$ & $\begin{array}{l}25300 \\
22700 \\
18600 \\
16800 \\
15600\end{array}$ & $\begin{array}{l}29400 \\
26000 \\
20900 \\
18800 \\
17300\end{array}$ & $\begin{array}{l}33100 \\
28800 \\
22300 \\
19600 \\
17700\end{array}$ \\
\hline
\end{tabular}

MAGNITUDE AND FREQUENCY OF SEASONAL LOW FLOWS

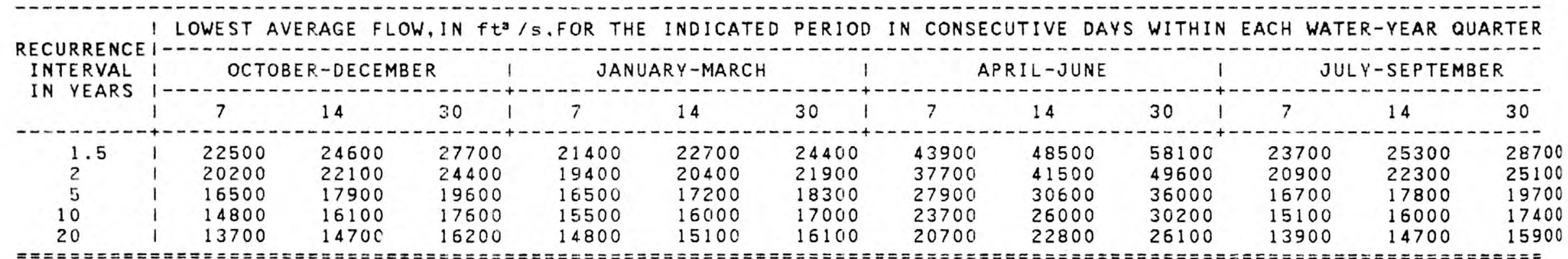

DURATION OF DAILY DISCHARGES FOR ANNUAL AND SEASONAL PERIODS

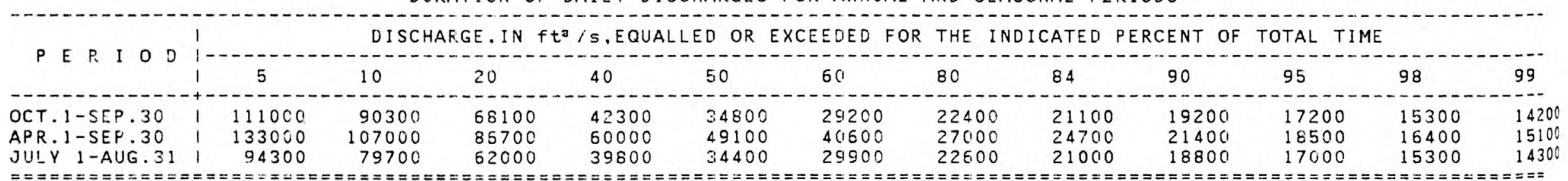


Location.--Lat $43^{\circ} 14^{\prime} 34^{\prime \prime}$, long $92^{\circ} 31.48^{\prime \prime}$, In NW1/4 NW1/4 sec.8, T.97 N.. R.14 W. Howard County, on right bank $10 \mathrm{ft}$ downsiream from bridge on county highway E17, 0.2 mile downstream from smali left-bank tributary, 4.8 miles west of Elma, and at milewisti.

LOW FLOW DISCHARGES, IN YEAR. ENDING MARCH 31

\begin{tabular}{|c|c|c|c|c|c|c|c|}
\hline \multirow{2}{*}{$\begin{array}{c}\text { CL IMAT IC } \\
\text { YEAR }\end{array}$} & LOWEST & GE FLC & $f t^{3} / 5$ & IND I & PERIO & IN CONS & IVE DAYS \\
\hline & 13 & 7 & 14 & 30 & 60 & 120 & 183 \\
\hline $\begin{array}{l}1960 \\
1961 \\
1962 \\
1963 \\
1964 \\
1965 \\
1966 \\
1969\end{array}$ & $\begin{array}{l}4.2 \\
4.6 \\
7.1 \\
7.1 \\
4.7 \\
3.6 \\
5.7 \\
8.4\end{array}$ & $\begin{array}{l}4.5 \\
4.7 \\
7.6 \\
7.3 \\
4.8 \\
3.9 \\
5.9 \\
3.8\end{array}$ & $\begin{array}{l}4.6 \\
4.9 \\
7.8 \\
7.7 \\
4.9 \\
4.1 \\
6.0 \\
9.4\end{array}$ & $\begin{array}{r}4.9 \\
6.4 \\
8.4 \\
8.8 \\
5.4 \\
4.3 \\
6.4 \\
12\end{array}$ & $\begin{array}{r}8.0 \\
7.8 \\
9.9 \\
9.7 \\
6.3 \\
5.4 \\
15 \\
14\end{array}$ & $\begin{array}{l}15 \\
11 \\
15 \\
12 \\
7.6 \\
6.5 \\
22 \\
17\end{array}$ & $\begin{array}{l}21 \\
15 \\
19 \\
39 \\
7.7 \\
6.9 \\
76 \\
37\end{array}$ \\
\hline $\begin{array}{l}1970 \\
1971 \\
1972 \\
1973 \\
1974 \\
1975 \\
1976\end{array}$ & $\begin{array}{r}9.9 \\
9.0 \\
6.7 \\
9.7 \\
11 \\
9.7 \\
7.9\end{array}$ & $\begin{array}{r}10 \\
9.3 \\
6.7 \\
9.9 \\
12 \\
10 \\
8.1\end{array}$ & $\begin{array}{l}11 \\
10 \\
6.8 \\
11 \\
16 \\
10 \\
8.4\end{array}$ & $\begin{array}{l}11 \\
13 \\
7.0 \\
13 \\
18 \\
11 \\
8.7\end{array}$ & $\begin{array}{l}11 \\
16 \\
8.4 \\
27 \\
18 \\
11 \\
9.0\end{array}$ & $\begin{array}{l}12 \\
23 \\
10 \\
41 \\
36 \\
12 \\
12\end{array}$ & $\begin{array}{l}14 \\
36 \\
11 \\
90 \\
58 \\
12 \\
12\end{array}$ \\
\hline
\end{tabular}


05-4205.60 WAPSIPINICON RIVER NEAR ELMA--Continued

DRAINAGE AREA: $95.2 \mathrm{~m}^{2}$ PERIOD OF RECORD: 18 YEARS AVERAGE DISCHARGE: 60.0 ft3 /S

MINIMUM AVERAGE FLOWS FOR PERIOD OF RECORD

\begin{tabular}{|c|c|c|c|c|c|c|c|}
\hline PERIOD OF CONSECUTIVE DAYS I & 3 & 7 & 14 & 30 & 60 & 20 & 183 \\
\hline $\begin{array}{l}\text { DISCHARGE, IN } \mathrm{ft}^{3} / \mathrm{s} \\
\text { CLIMATIC YEAR }\end{array}$ & $\begin{array}{r}3.6 \\
1965\end{array}$ & $\begin{array}{r}3.9 \\
1965\end{array}$ & $\begin{array}{r}4.1 \\
1965\end{array}$ & $\begin{array}{r}4.3 \\
1965\end{array}$ & $\begin{array}{r}5.4 \\
1965\end{array}$ & $\begin{array}{r}6.5 \\
1965\end{array}$ & $\begin{array}{r}6.9 \\
1965\end{array}$ \\
\hline
\end{tabular}

MAGNITUDE AND FREQUENCY OF ANNUAL LOW FLOWS

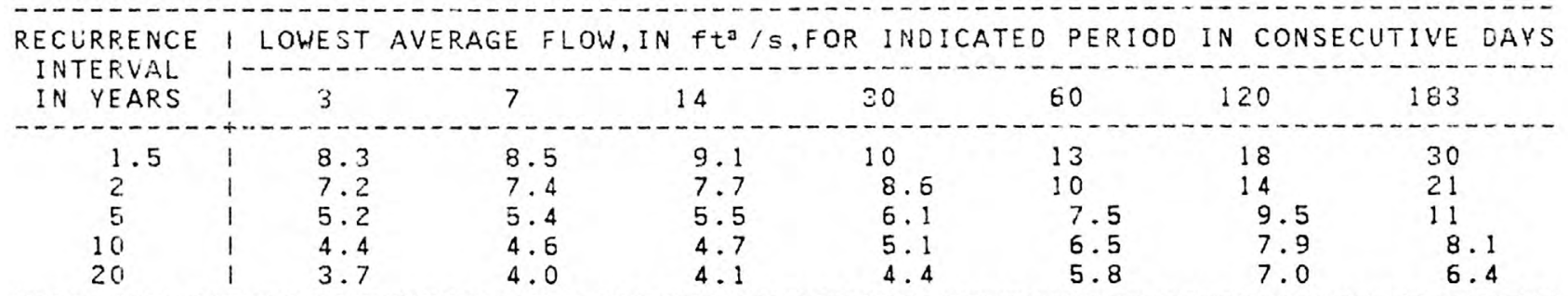

MAGNITUDE AND FREQUENCY OF SEASONAL LOW FLOWS

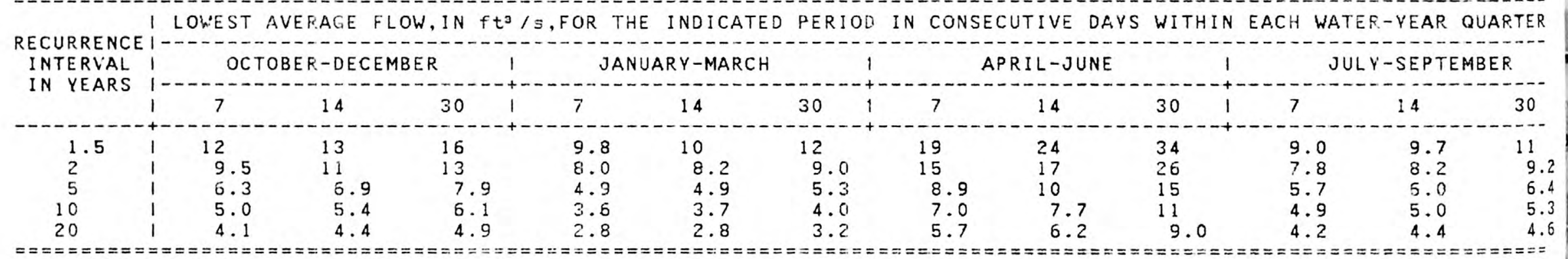

DURATION OF DAILY DISCHARGES FOR ANNUAL AND SEASONAL PERIODS

\begin{tabular}{|c|c|c|c|c|c|c|c|c|c|c|c|c|}
\hline$P E R$ I & 1 & 10 & 20 & 40 & 50 & 60 & $\begin{array}{l}\text { THE } \\
80\end{array}$ & $\begin{array}{l}\text { ATED } \\
84\end{array}$ & $\begin{array}{l}\text { ENT } \\
90\end{array}$ & $\frac{T A L}{95}$ & 98 & 99 \\
\hline $\begin{array}{l}\text { OCT. } 1 \text {-SEP. } 30 \\
\text { APR. } 1-S E P .30 \\
\text { JULY } 1 \text { - } 1 \text {-AUG. } 31\end{array}$ & $\begin{array}{l}211 \\
233 \\
101\end{array}$ & $\begin{array}{r}113 \\
128 \\
53\end{array}$ & $\begin{array}{l}54 \\
66 \\
30\end{array}$ & $\begin{array}{l}23 \\
31 \\
15\end{array}$ & $\begin{array}{l}16 \\
2.3 \\
12\end{array}$ & $\begin{array}{l}12 \\
16 \\
11\end{array}$ & $\begin{array}{l}8.8 \\
9.6 \\
7.8\end{array}$ & $\begin{array}{l}8.0 \\
8.8 \\
7.2\end{array}$ & $\begin{array}{l}6.8 \\
7.5 \\
6.3\end{array}$ & $\begin{array}{l}5.4 \\
6.2 \\
5.2\end{array}$ & $\begin{array}{l}4 \cdot 1 \\
5 \cdot 1 \\
4 \cdot 4\end{array}$ & $\begin{array}{l}2.8 \\
4.5 \\
4.6\end{array}$ \\
\hline
\end{tabular}

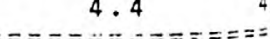




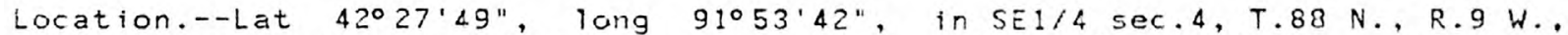
Buchanan county, on $r i g h t$ bank at $51 \times t h$ Street in independence, 1,800 ft downstream from dam at abandoned hyciroelectric piant, $4.9 \mathrm{miles}$ downstream from Otter Creek, 9.7 miles upstream from "ine creek, and at mile 142.5 .

Remarks.--Diurnal fluctuation caused by poverpiant at Independence prior to April 1958.

LOW FLOW DISCHARGES, IN YEAR. ENDING MARCH 31

\begin{tabular}{|c|c|c|c|c|c|c|c|}
\hline \multirow{2}{*}{$\begin{array}{c}\text { CLIMATIC } \\
\text { YEAR }\end{array}$} & \multicolumn{7}{|c|}{ I LOWEST AVERAGE FLOW IN $\mathrm{ft}^{3} / \mathrm{s}$ FOR INOICATED PERIOD IN CONSECUTIVE } \\
\hline & 1. 3 & 7 & 14 & 30 & 60 & 120 & 183 \\
\hline $\begin{array}{l}1935 \\
1936 \\
1937 \\
1938 \\
1939\end{array}$ & $\begin{array}{l}8.0 \\
26 \\
9.0 \\
13 \\
95\end{array}$ & $\begin{array}{r}3.0 \\
31 \\
9.0 \\
19 \\
128\end{array}$ & $\begin{array}{c}12 \\
32 \\
9.0 \\
21 \\
143\end{array}$ & $\begin{array}{c}12 \\
37 \\
0.9 \\
24 \\
204\end{array}$ & $\begin{array}{r}20 \\
54 \\
17 \\
31 \\
233\end{array}$ & $\begin{array}{r}28 \\
77 \\
103 \\
36 \\
336\end{array}$ & $\begin{array}{r}55 \\
83 \\
226 \\
40 \\
480\end{array}$ \\
\hline $\begin{array}{l}1940 \\
1941 \\
1942 \\
1943 \\
1944 \\
1945 \\
1946 \\
1947 \\
1948 \\
1949\end{array}$ & $\begin{array}{r}14 \\
10 \\
36 \\
165 \\
43 \\
32 \\
42 \\
35 \\
20 \\
32\end{array}$ & $\begin{array}{r}16 \\
17 \\
48 \\
174 \\
64 \\
58 \\
79 \\
55 \\
33 \\
41\end{array}$ & $\begin{array}{r}16 \\
25 \\
53 \\
177 \\
67 \\
60 \\
85 \\
63 \\
36 \\
48\end{array}$ & $\begin{array}{r}20 \\
41 \\
67 \\
187 \\
74 \\
61 \\
112 \\
82 \\
44 \\
58\end{array}$ & $\begin{array}{r}22 \\
56 \\
91 \\
275 \\
128 \\
65 \\
125 \\
109 \\
71 \\
83\end{array}$ & $\begin{array}{r}37 \\
154 \\
418 \\
357 \\
174 \\
93 \\
146 \\
190 \\
139 \\
91\end{array}$ & $\begin{array}{r}39 \\
268 \\
547 \\
543 \\
226 \\
131 \\
203 \\
249 \\
132 \\
103\end{array}$ \\
\hline $\begin{array}{l}1950 \\
1951 \\
1952 \\
1953 \\
1954 \\
1955 \\
1956 \\
1957 \\
1958 \\
1959\end{array}$ & $\begin{array}{l}18 \\
33 \\
85 \\
44 \\
20 \\
42 \\
17 \\
16 \\
31 \\
29\end{array}$ & $\begin{array}{r}23 \\
48 \\
121 \\
49 \\
22 \\
50 \\
17 \\
17 \\
32 \\
29\end{array}$ & $\begin{array}{r}27 \\
49 \\
148 \\
55 \\
24 \\
56 \\
18 \\
22 \\
43 \\
29\end{array}$ & $\begin{array}{r}37 \\
52 \\
162 \\
60 \\
33 \\
65 \\
18 \\
44 \\
60 \\
30\end{array}$ & $\begin{array}{r}40 \\
58 \\
253 \\
67 \\
52 \\
93 \\
18 \\
55 \\
70 \\
36\end{array}$ & $\begin{array}{r}45 \\
68 \\
386 \\
75 \\
71 \\
146 \\
29 \\
65 \\
85 \\
61\end{array}$ & $\begin{array}{r}48 \\
102 \\
454 \\
108 \\
76 \\
203 \\
36 \\
74 \\
106 \\
65\end{array}$ \\
\hline $\begin{array}{l}1960 \\
1961 \\
1962 \\
1963 \\
1964 \\
1965 \\
1966 \\
1967 \\
1968 \\
1969\end{array}$ & $\begin{array}{r}88 \\
39 \\
123 \\
69 \\
43 \\
26 \\
63 \\
53 \\
34 \\
122\end{array}$ & $\begin{array}{r}92 \\
40 \\
130 \\
71 \\
45 \\
23 \\
65 \\
56 \\
34 \\
141\end{array}$ & $\begin{array}{r}100 \\
41 \\
135 \\
73 \\
48 \\
33 \\
66 \\
50 \\
35 \\
173\end{array}$ & $\begin{array}{r}135 \\
65 \\
152 \\
85 \\
45 \\
3 \\
75 \\
65 \\
41 \\
152\end{array}$ & $\begin{array}{r}180 \\
80 \\
181 \\
99 \\
5 \\
4 \\
157 \\
76 \\
60 \\
201\end{array}$ & $\begin{array}{r}26 ! \\
185 \\
369 \\
147 \\
68 \\
55 \\
324 \\
86 \\
75 \\
262\end{array}$ & $\begin{array}{r}359 \\
184 \\
545 \\
293 \\
76 \\
65 \\
826 \\
134 \\
76 \\
399\end{array}$ \\
\hline
\end{tabular}


WAPSIPINICON RIVER AT INDEPENDENCE--Cont inued

LOW FLOW DISCHARGES, IN YEAR ENDING MARCH 31--Cont inued

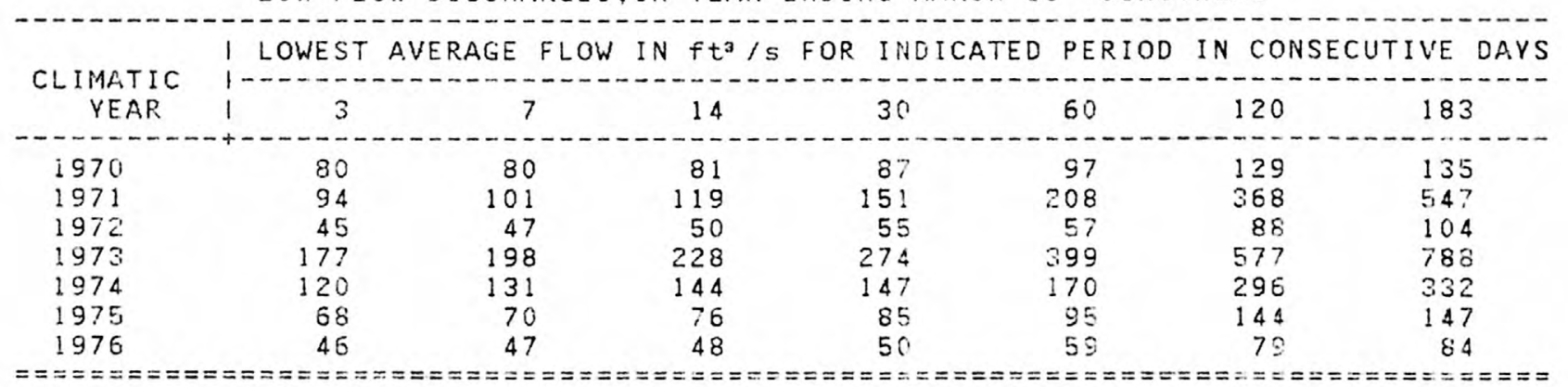




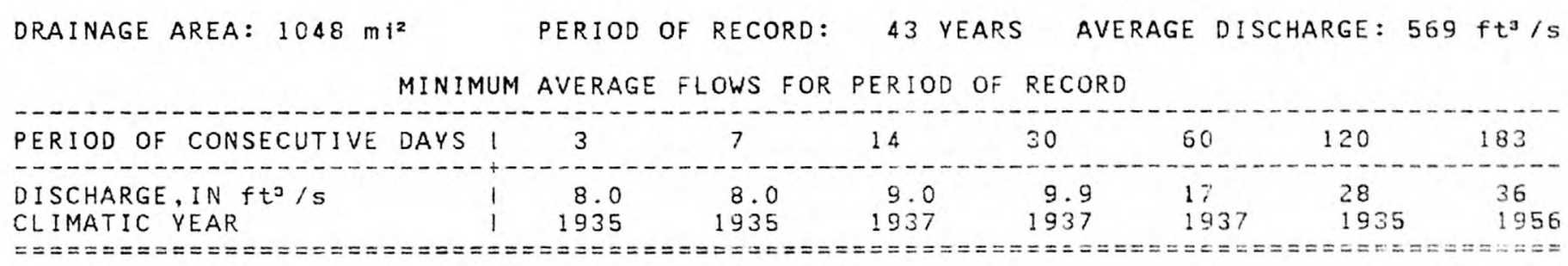

MAGNITUDE AND FREQUENCY OF ANNUAL LOW FLOWS

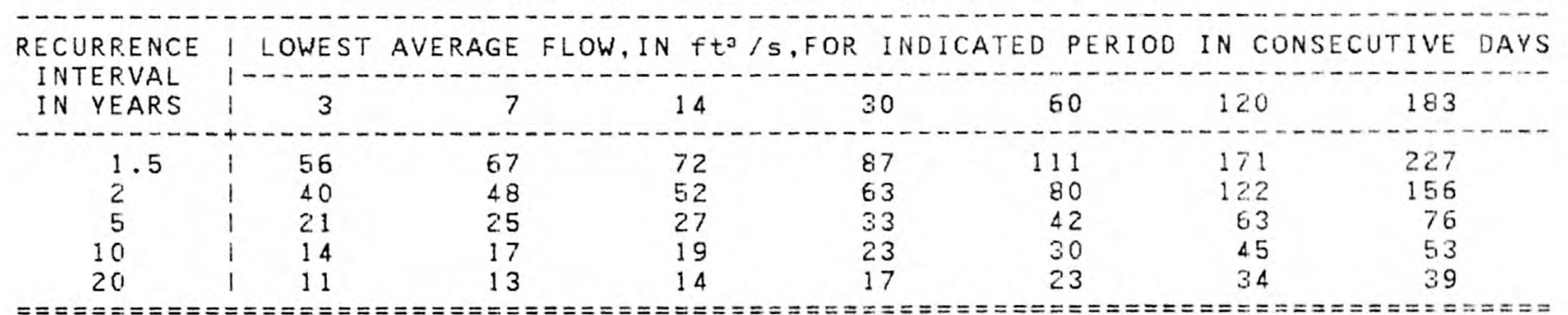

MAGNITUDE AND FREQUENCY OF SEASONAL LOW FLOWS

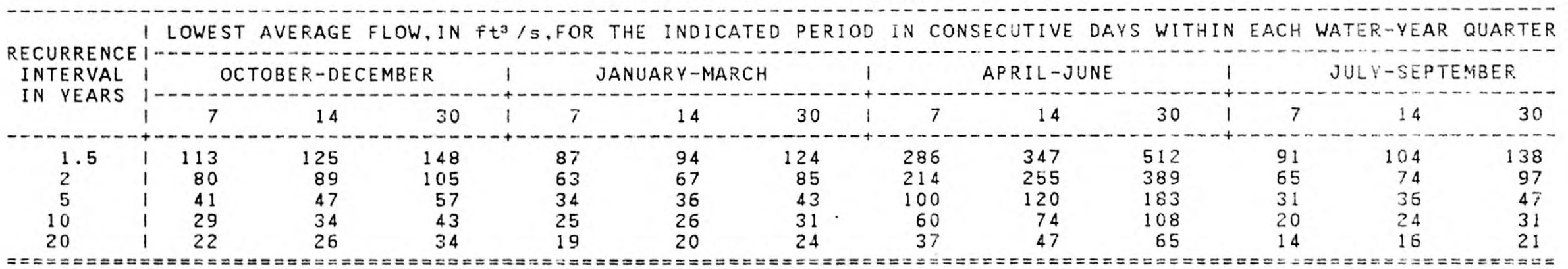

DURATION OF DAILY DISCHARGES FOR ANNUAL AND SEASONAL PERIODS

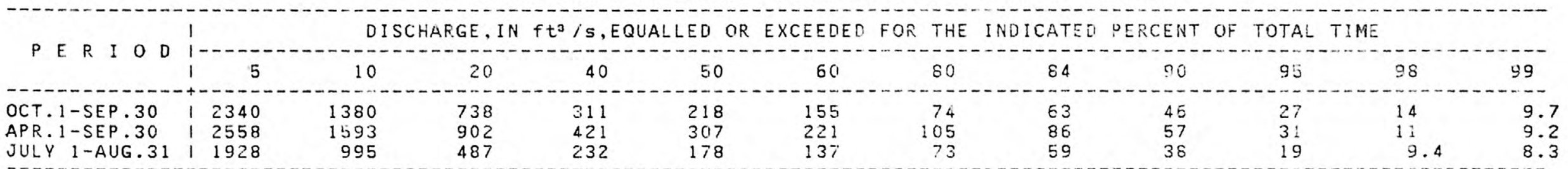


Location.--Lat 41046'01", long 90032.05", in SW1/4 NE1/4 sec.6, T.80 N., R.4 E., Cilinton County, on left bank $5 \mathrm{ft}$ upstream from bridge on U.S. Highway 61 , 0.9 mile downstream from Silver Creek, 4.0 miles south of water tower in De Witt, 6.2 miles upstream from Brophy Creek, and 18.2 miles upstream from mouth.

LOW FLOW DISCHARGES, IN YEAR. ENDING MARCH 31

\begin{tabular}{|c|c|c|c|c|c|c|c|}
\hline \multirow{2}{*}{$\begin{array}{c}\text { CLIMATIC } \\
\text { YEAR }\end{array}$} & I LOWEST & AGE $F$ & $N f t^{2}$ & IN & $D P E F$ & \multicolumn{2}{|c|}{ IN CONSECUTIVE } \\
\hline & 13 & 7 & 14 & 30 & 60 & 120 & 183 \\
\hline $\begin{array}{l}1936 \\
1937 \\
1938 \\
1939\end{array}$ & $\begin{array}{r}136 \\
86 \\
122 \\
434\end{array}$ & $\begin{array}{r}137 \\
88 \\
123 \\
443\end{array}$ & $\begin{array}{r}138 \\
92 \\
125 \\
457\end{array}$ & $\begin{array}{r}155 \\
99 \\
131 \\
519\end{array}$ & $\begin{array}{l}212 \\
129 \\
150 \\
593\end{array}$ & $\begin{array}{l}356 \\
356 \\
167 \\
869\end{array}$ & $\begin{array}{r}328 \\
461 \\
188 \\
1110\end{array}$ \\
\hline $\begin{array}{l}1940 \\
1941 \\
1942 \\
1943 \\
1944 \\
1945 \\
1946 \\
1947 \\
1948 \\
1949\end{array}$ & $\begin{array}{r}70 \\
154 \\
119 \\
564 \\
187 \\
203 \\
303 \\
287 \\
150 \\
172\end{array}$ & $\begin{array}{r}70 \\
164 \\
122 \\
594 \\
196 \\
209 \\
319 \\
300 \\
151 \\
175\end{array}$ & $\begin{array}{r}73 \\
204 \\
139 \\
631 \\
201 \\
216 \\
352 \\
342 \\
156 \\
203\end{array}$ & $\begin{array}{r}80 \\
270 \\
160 \\
771 \\
236 \\
223 \\
398 \\
416 \\
168 \\
235\end{array}$ & $\begin{array}{r}90 \\
366 \\
280 \\
932 \\
333 \\
239 \\
531 \\
515 \\
284 \\
244\end{array}$ & $\begin{array}{r}117 \\
551 \\
1030 \\
1400 \\
427 \\
342 \\
564 \\
810 \\
443 \\
270\end{array}$ & $\begin{array}{r}137 \\
586 \\
1550 \\
1490 \\
547 \\
416 \\
696 \\
954 \\
426 \\
313\end{array}$ \\
\hline $\begin{array}{l}1950 \\
1951 \\
1952 \\
1953 \\
1954 \\
1955 \\
1956 \\
1957 \\
1958 \\
1959\end{array}$ & $\begin{array}{r}20 \\
150 \\
530 \\
2111 \\
100 \\
240 \\
90 \\
121 \\
147 \\
100\end{array}$ & $\begin{array}{r}208 \\
153 \\
565 \\
217 \\
101 \\
241 \\
90 \\
123 \\
152 \\
100\end{array}$ & $\begin{array}{r}209 \\
154 \\
606 \\
220 \\
102 \\
248 \\
93 \\
124 \\
158 \\
101\end{array}$ & $\begin{array}{lll}2 & 1 & 7 \\
1 & 6 & 2 \\
7 & 0 & 2 \\
2 & 23 \\
1 & 24 \\
3 & 22 \\
1 & 0 & 1 \\
1 & 37 \\
1 & 78 \\
1 & 14\end{array}$ & $\begin{array}{r}226 \\
181 \\
1100 \\
236 \\
180 \\
432 \\
101 \\
154 \\
211 \\
130\end{array}$ & $\begin{array}{r}270 \\
234 \\
1300 \\
378 \\
199 \\
525 \\
130 \\
167 \\
276 \\
164\end{array}$ & $\begin{array}{r}384 \\
318 \\
1480 \\
439 \\
219 \\
645 \\
141 \\
209 \\
306 \\
185\end{array}$ \\
\hline $\begin{array}{l}1960 \\
1961 \\
1962 \\
1963 \\
1964 \\
1965 \\
1968 \\
1967 \\
1968 \\
1969\end{array}$ & $\begin{array}{l}281 \\
280 \\
426 \\
208 \\
130 \\
105 \\
283 \\
207 \\
257 \\
439\end{array}$ & $\begin{array}{l}288 \\
283 \\
474 \\
245 \\
130 \\
109 \\
289 \\
234 \\
271 \\
551\end{array}$ & $\begin{array}{l}304 \\
290 \\
542 \\
299 \\
134 \\
121 \\
313 \\
239 \\
304 \\
586\end{array}$ & $\begin{array}{l}372 \\
334 \\
752 \\
335 \\
147 \\
129 \\
387 \\
257 \\
460 \\
721\end{array}$ & $\begin{array}{l}486 \\
392 \\
849 \\
344 \\
180 \\
142 \\
521 \\
283 \\
526 \\
84 ?\end{array}$ & $\begin{array}{r}809 \\
704 \\
1220 \\
454 \\
207 \\
167 \\
803 \\
304 \\
623 \\
983\end{array}$ & $\begin{array}{r}1020 \\
669 \\
1600 \\
699 \\
233 \\
240 \\
1500 \\
444 \\
888 \\
1100\end{array}$ \\
\hline $\begin{array}{l}1970 \\
1971 \\
1972\end{array}$ & $\begin{array}{l}283 \\
397 \\
211\end{array}$ & $\begin{array}{l}287 \\
403 \\
218\end{array}$ & $\begin{array}{l}294 \\
418 \\
226\end{array}$ & $\begin{array}{l}310 \\
260 \\
237\end{array}$ & $\begin{array}{l}544 \\
599 \\
270\end{array}$ & $\begin{array}{r}429 \\
1200 \\
561\end{array}$ & $\begin{array}{r}480 \\
1280 \\
537\end{array}$ \\
\hline
\end{tabular}


WAPSIPINICON RIVER NEAR DE WITT--Continued

LOW FLOW DISCHARGES, IN YEAR ENDING MARCH 31--Cont1nued

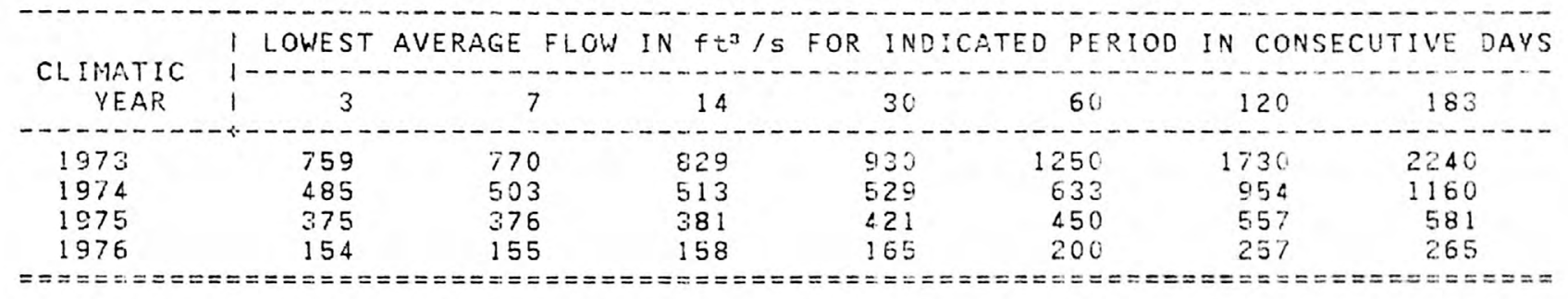


MINIMUM AVERAGE FLOWS FOR FERIOD OF RECORD

\begin{tabular}{|c|c|c|c|c|c|c|c|}
\hline PERIOD OF CONSECUTIVE DAYS & 3 & 7 & 14 & 30 & 60 & 120 & 183 \\
\hline $\begin{array}{l}\text { DISCHARGE, IN } \mathrm{ft}^{3} \text { is } \\
\text { CLIMATIC YEAR }\end{array}$ & $\begin{array}{l}70 \\
1940\end{array}$ & $\begin{array}{l}70 \\
1940\end{array}$ & $\begin{array}{c}73 \\
1940\end{array}$ & $\begin{array}{l}80 \\
1940\end{array}$ & $\begin{array}{l}90 \\
1940\end{array}$ & $\begin{array}{l}117 \\
1940\end{array}$ & $\begin{array}{l}137 \\
1940\end{array}$ \\
\hline
\end{tabular}

MAGNITUDE AND FREQUENCY OF ANNUAL LOW FLOWS

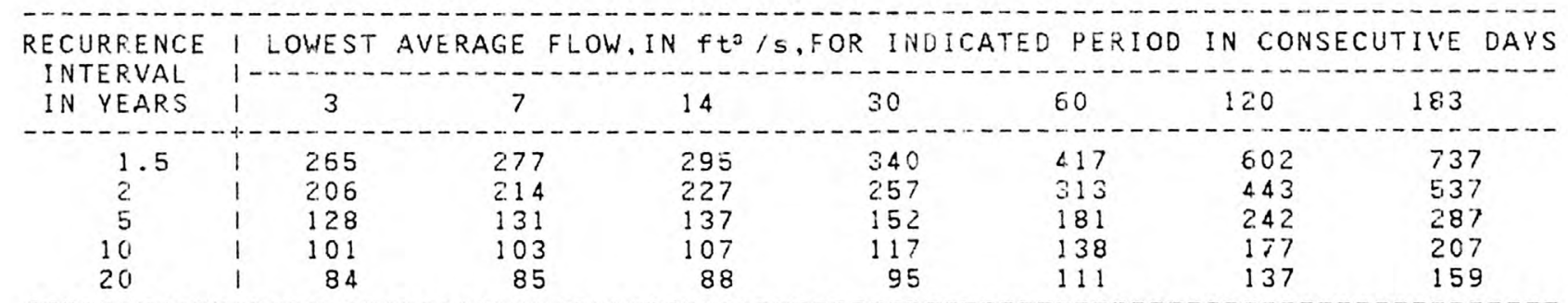

MAGNITUDE AND FREQUENCY OF SEASONAL LOW FLOWS

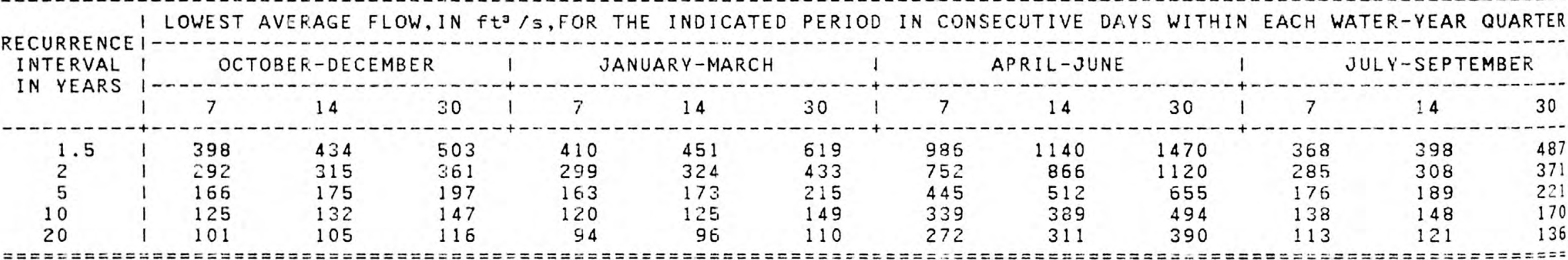

DURATIDN OF DAILY DISCHARGES FOR ANNUAL AND SEASONAL PERIODS

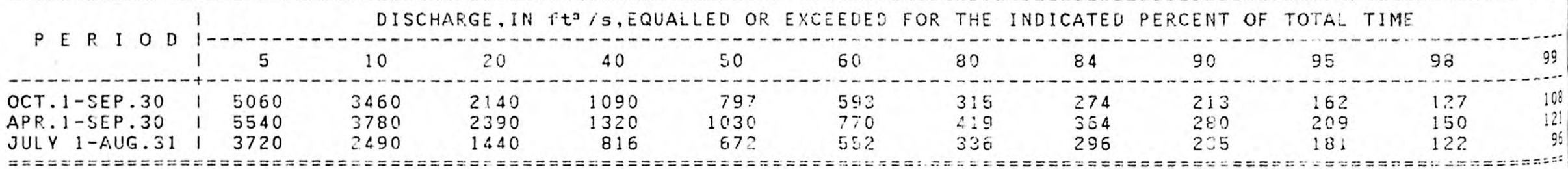


Location.--Lat $42^{\circ} 57^{\prime} 50^{\prime \prime}$, long $93^{\circ} 42^{\prime} 20^{\prime \prime}$, in NE1/4 NW1/4 sec.17, T.94 N., R.24 W. Hancock County, on downstream side of highway bridge 6 miles southwest of Klemme, 12.4 mi ies upstream from confluence with East Branch Iowa River. and at mile 338.9 above mouth of Iowa River. Remarks.--Published as "West fork Iowa River near Klemme" $1948-58$.
Discontinued Sept. 30, 1958.

LOW FLOW DISCHARGES, IN YEAR ENDING MARCH 31

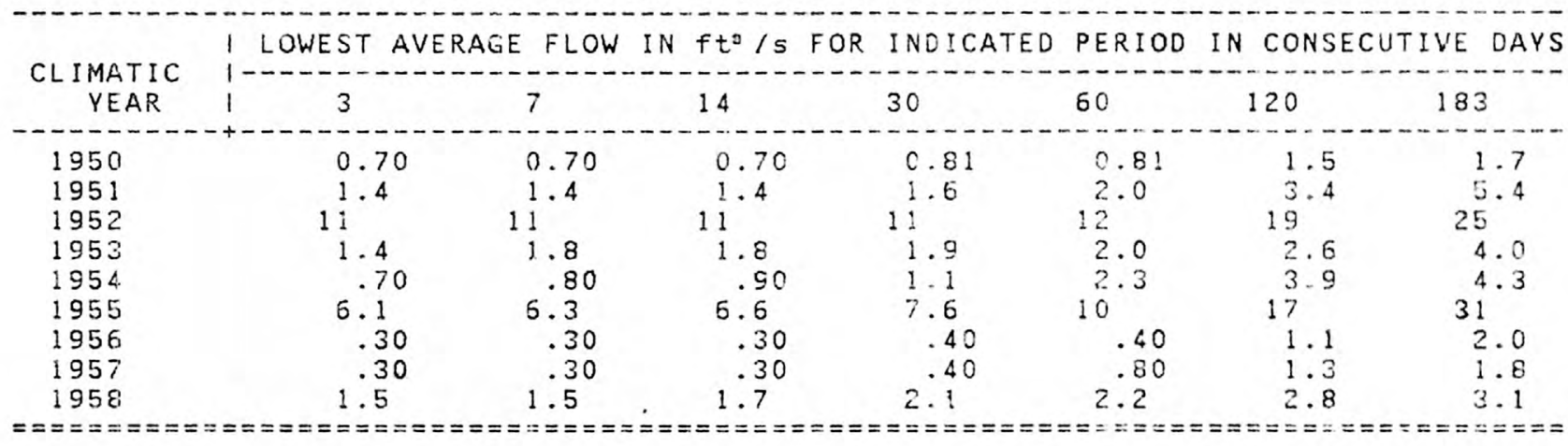




DRAINAGE AREA: $112 \mathrm{mi}^{2} \quad$ PERIOD OF RECORD: 10 YEARS AVERAGE DISCHARGE: $38.2 \mathrm{ft} 3 / \mathrm{s}$
MINIMUM AVERAGE FLOWS FOR PERIOD OF RECORD

MAGNITUDE AND FREQUENCY OF ANNUAL LOW FLOWS

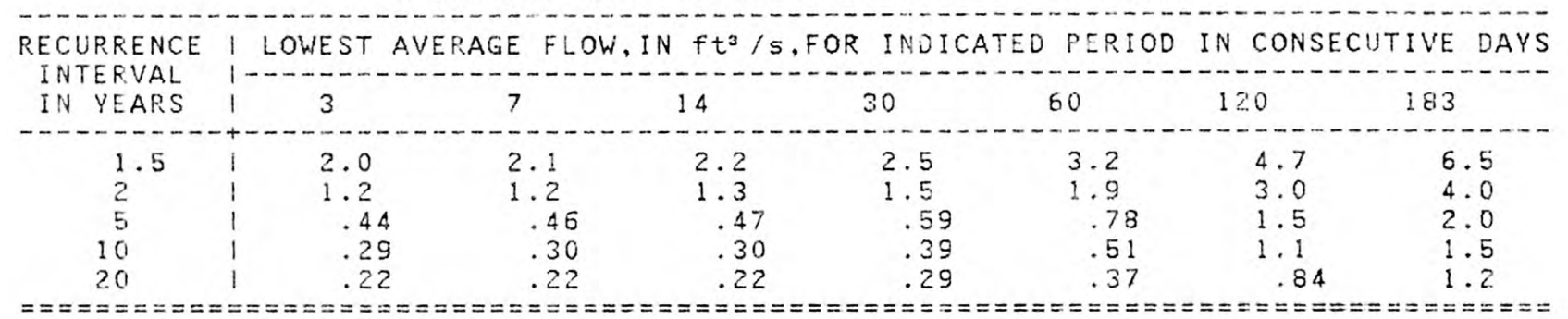

MAGNITUDE AND FREQUENCY OF SEASONAL LOW FLOWS

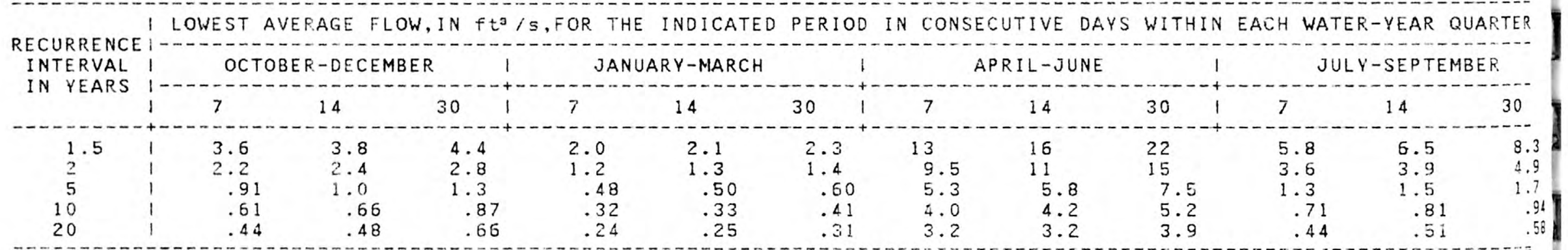

DURATION OF DAILY DISCHARGES FOR ANNUAL AND SEASONAL PERIODS

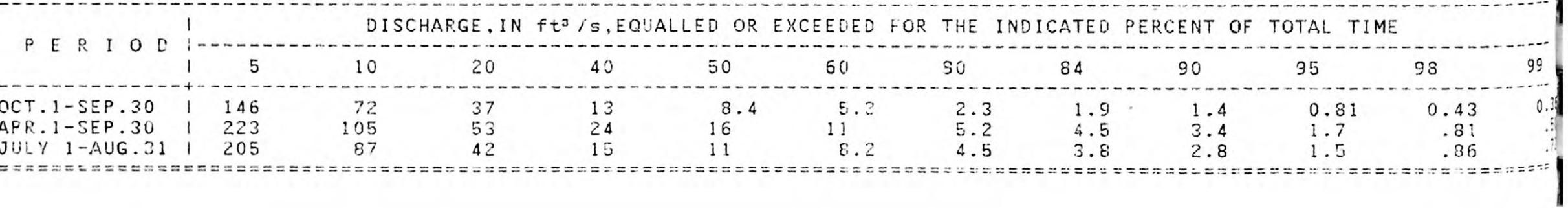


Location.--lat $43^{\circ} 00^{\prime} 31^{\prime \prime}$, long $93^{\circ} 37^{\prime} 42^{\prime \prime}$, in NE1/4 NW1/4 sec.36, T.95 N., R.24 W. Hancock County, on left bank $15 \mathrm{ft}$ downstream from bridge on county highway E55, 1.2 mile west of Chicago. Rock Island and Paciflc Railroad crossing in Klemme, 1.5 miles upstream from Drainage ditch $9,18.2$ miles upstream from confiuence with West Branch lowa River, and at mile 341.0 .

Remarks.--Published as "East Fork lowa River near Klemme" $1948-58$. Discontinued Sept. $30,1976$.

LOW FLOW DISCHARGES, IN YEAR. ENDING MARCH 31

\begin{tabular}{|c|c|c|c|c|c|c|c|}
\hline \multirow{2}{*}{$\begin{array}{c}\text { CLIMAT I C } \\
\text { YEAR }\end{array}$} & LOWEST A & GE FLOI & $f t^{3} / s$ & INDIC & PERIOD & $N$ CONSE & IVE DAYS \\
\hline & 13 & 7 & 14 & 30 & 60 & 120 & 183 \\
\hline $\begin{array}{l}1950 \\
1951 \\
1952 \\
1953 \\
1954 \\
1955 \\
1956 \\
1957 \\
1958 \\
1959\end{array}$ & $\begin{array}{c}1.0 \\
2.0 \\
14 \\
1.6 \\
2.8 \\
7.1 \\
1.3 \\
1.6 \\
1.5 \\
.20\end{array}$ & $\begin{array}{r}1.1 \\
2.0 \\
11 \\
2.2 \\
2.8 \\
7.4 \\
1.3 \\
1.6 \\
1.6 \\
.23\end{array}$ & $\begin{array}{c}1.1 \\
2.1 \\
14 \\
2.6 \\
2.9 \\
7.8 \\
1.4 \\
1.7 \\
1.8 \\
.26\end{array}$ & $\begin{array}{r}1.2 \\
2.6 \\
15 \\
2.7 \\
3.2 \\
8.6 \\
1.4 \\
1.7 \\
2.4 \\
.28\end{array}$ & $\begin{array}{c}1.3 \\
3.4 \\
18 \\
3.1 \\
4.9 \\
12 \\
1.7 \\
2.5 \\
2.9 \\
.32\end{array}$ & $\begin{array}{c}2.8 \\
4.8 \\
33 \\
4.5 \\
6.3 \\
18 \\
2.5 \\
3.6 \\
4.6 \\
.68\end{array}$ & $\begin{array}{r}3.3 \\
6.6 \\
41 \\
6.2 \\
7.3 \\
29 \\
3.5 \\
4.3 \\
5.1 \\
1.6\end{array}$ \\
\hline $\begin{array}{l}1960 \\
1961 \\
1962 \\
1963 \\
1964 \\
1965 \\
1966 \\
1967 \\
1968 \\
1969\end{array}$ & $\begin{array}{c}3.7 \\
.60 \\
4.7 \\
4.1 \\
4.9 \\
3.5 \\
12 . \\
2.8 \\
.38 \\
2.4\end{array}$ & $\begin{array}{c}4.0 \\
.60 \\
5.2 \\
4.2 \\
5.0 \\
3.6 \\
12 \\
3.0 \\
.40 \\
2.6\end{array}$ & $\begin{array}{c}4.4 \\
.63 \\
5.2 \\
4.5 \\
5.1 \\
4.2 \\
13 . \\
3.3 \\
.48 \\
2.9\end{array}$ & $\begin{array}{c}4.8 \\
.94 \\
5.7 \\
5.6 \\
5.7 \\
7.1 \\
14 . \\
3.4 \\
.91 \\
3.4\end{array}$ & $\begin{array}{r}6.1 \\
1.5 \\
5.6 \\
7.3 \\
6.9 \\
9.1 \\
30 \\
4.1 \\
1.5 \\
3.6\end{array}$ & $\begin{array}{r}7.1 \\
2.3 \\
13 \\
12 \\
9.2 \\
14 \\
69 \\
5.0 \\
2.8 \\
8.0\end{array}$ & $\begin{array}{c}9.4 \\
3.1 \\
20 \\
34 \\
10 \\
22 \\
109 \\
6.1 \\
4.0 \\
19\end{array}$ \\
\hline $\begin{array}{l}1970 \\
1971 \\
1972 \\
1973 \\
1974 \\
1975 \\
1975\end{array}$ & $\begin{array}{r}5.6 \\
3.0 \\
3.7 \\
13 \\
5.4 \\
2.6 \\
2.7\end{array}$ & $\begin{array}{r}5.7 \\
3.1 \\
3.8 \\
15 \\
5.5 \\
2.6 \\
2.7\end{array}$ & $\begin{array}{r}5.0 \\
3.3 \\
3.9 \\
15 \\
5.7 \\
2.6 \\
2.9\end{array}$ & $\begin{array}{r}6.3 \\
3.9 \\
4.1 \\
20 \\
6.9 \\
2.6 \\
2.5\end{array}$ & $\begin{array}{r}6.8 \\
6.6 \\
5.8 \\
26 \\
8.9 \\
2.7 \\
4.2\end{array}$ & $\begin{array}{r}9.3 \\
9.8 \\
9.3 \\
64 \\
15 \\
3.6 \\
6.1\end{array}$ & $\begin{array}{r}11 \\
13 \\
16 \\
73 \\
15 \\
5.5 \\
7.0\end{array}$ \\
\hline
\end{tabular}


MINIMUM AVERAGE FLOWS FOR PERIOD OF RECORD

\begin{tabular}{|c|c|c|c|c|c|c|c|}
\hline PERIOD OF CONSECUTIVE DAYS & 3 & 7 & 14 & 30 & 60 & 120 & 183 \\
\hline $\begin{array}{l}\text { DISCHARGE, IN } \mathrm{ft}^{3} / \mathrm{s} \\
\text { CLIMATIC YEAR }\end{array}$ & $\begin{array}{l}0.20 \\
1959\end{array}$ & $\begin{array}{l}0.24 \\
1959\end{array}$ & $\begin{array}{l}0.26 \\
1959\end{array}$ & $\begin{array}{c}0.28 \\
1959\end{array}$ & $\begin{array}{c}0.32 \\
1959\end{array}$ & $\begin{array}{c}0.69 \\
1959\end{array}$ & $\begin{array}{r}1.6 \\
1959\end{array}$ \\
\hline
\end{tabular}

MAGNITUDE AND FREQUENCY OF ANNUAL LOW FLOWS

RECURRENCE I LOWEST AVERAGE FLOW, IN $f t^{3} / \mathrm{s}$, FOR INDICATED PERIOD IN CONSECUTIVE DAYS

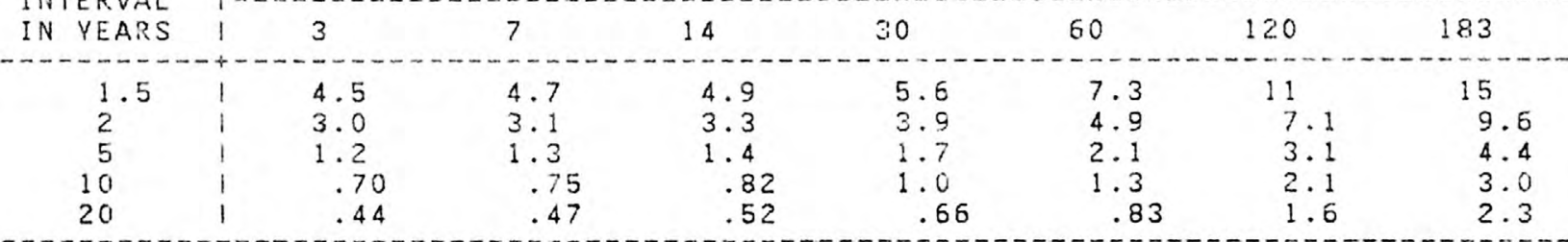

MAGNITUDE AND FREQUENCY OF SEASONAL LOW FLOWS

L LWEST AVERAGE FLOW, IN $\mathrm{ft}^{3} / \mathrm{s}, F O R$ THE INDICATED PERIOD IN CONSECUTIVE DAYS WITHIN EACH WATER-YEAR QUARTER

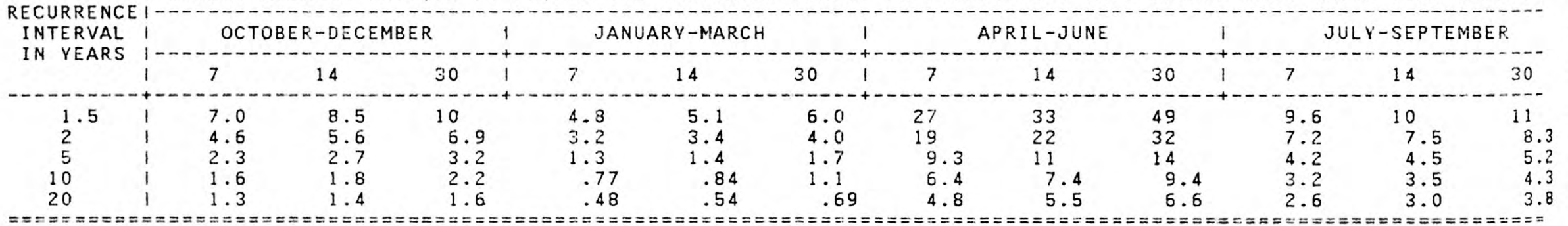

DURATION OF DAILY DISCHAREES FOR ANNUAL ANO SEASONAL PERIODS

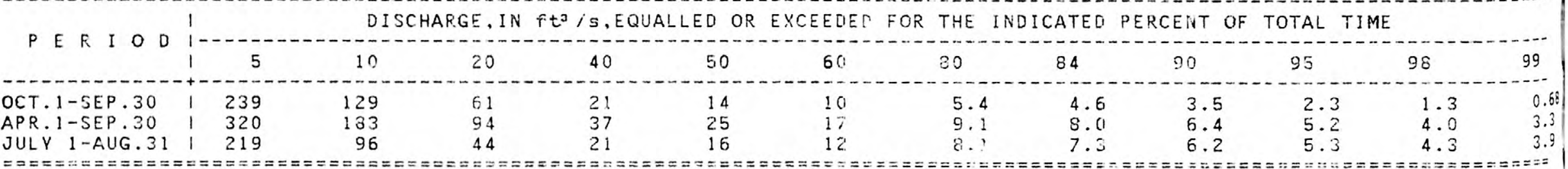


Location.--Lat $42^{\circ} 45^{\prime} 36^{\prime \prime}$, long $93^{\circ} 37^{\prime} 23^{\prime \prime}$, in NW1/4 NE1/4 sec.25, T.92 N., R.24 W. Wright County, on left bank $10 \mathrm{ft}$ downstream from bridge on county highway $\mathrm{c} 38$, 0.9 mile downstream from Drainage ditch $123,3.8 \mathrm{miles}$ northwest of Rowan, $10.7 \mathrm{~m} f$ les downstream from confiuence of East and West Branches, and at mile 316.4

LOW FLOW DISCHARGES, IN YEAR ENDING MARCH 31

\begin{tabular}{|c|c|c|c|c|c|c|c|}
\hline \multirow{2}{*}{$\begin{array}{c}\text { CLIMATIC } \\
\text { YEAR }\end{array}$} & I LOWEST AVERAGE & FLOW & $f t^{3} / s$ FOR & INDICATED & PERIOD & \multicolumn{2}{|c|}{ IN CONSECUTIVE DAYS } \\
\hline & 13 & 7 & 14 & 30 & 60 & 120 & 183 \\
\hline $\begin{array}{l}1942 \\
1943 \\
1944 \\
1945 \\
1946 \\
1947 \\
1948 \\
1949\end{array}$ & $\begin{array}{r}24 \\
11 \\
19 \\
13 \\
16 \\
25 \\
8.7 \\
4.5\end{array}$ & $\begin{array}{r}25 \\
12 \\
19 \\
13 \\
17 \\
27 \\
9.6 \\
5.5\end{array}$ & $\begin{array}{l}25 \\
12 \\
20 \\
14 \\
18 \\
29 \\
10 \\
6.6\end{array}$ & $\begin{array}{l}27 \\
13 \\
23 \\
16 \\
23 \\
34 \\
12 \\
8.5\end{array}$ & $\begin{array}{l}49 \\
17 \\
34 \\
18 \\
33 \\
48 \\
20 \\
11\end{array}$ & $\begin{array}{r}161 \\
23 \\
47 \\
25 \\
46 \\
79 \\
30 \\
14\end{array}$ & $\begin{array}{r}223 \\
28 \\
54 \\
33 \\
79 \\
100 \\
28 \\
17\end{array}$ \\
\hline $\begin{array}{l}1950 \\
1951 \\
1952 \\
1953 \\
1954 \\
1955 \\
1956 \\
1957 \\
1958 \\
1959\end{array}$ & $\begin{array}{r}4.3 \\
6.7 \\
51 \\
11 \\
5.9 \\
25 \\
6.4 \\
6.0 \\
7.9 \\
2.9\end{array}$ & $\begin{array}{c}4.4 \\
6.7 \\
51 \\
14 \\
6.0 \\
26 \\
6.6 \\
6.0 \\
8.1 \\
3.0\end{array}$ & $\begin{array}{c}4.6 \\
7.1 \\
52 \\
19 \\
6.4 \\
29 \\
6.8 \\
6.2 \\
9.2 \\
3.1\end{array}$ & $\begin{array}{r}5.1 \\
9.5 \\
53 \\
20 \\
9.2 \\
31 \\
7.0 \\
6.5 \\
13 \\
3.2\end{array}$ & $\begin{array}{l}6.2 \\
12 \\
59 \\
20 \\
15 \\
40 \\
7.2 \\
9.8 \\
14 \\
3.7\end{array}$ & $\begin{array}{r}11 \\
18 \\
103 \\
23 \\
20 \\
63 \\
10.0 \\
13 \\
18 \\
6.0\end{array}$ & $\begin{array}{r}12 \\
26 \\
145 \\
28 \\
22 \\
105 \\
12 \\
13 \\
18 \\
7.2\end{array}$ \\
\hline $\begin{array}{l}1960 \\
1961 \\
1962 \\
1963 \\
1964 \\
1965 \\
1966 \\
1967 \\
1968 \\
1969\end{array}$ & $\begin{array}{l}15 \\
5.2 \\
13 \\
20 \\
14 \\
14 \\
55 \\
11 \\
5.5 \\
22\end{array}$ & $\begin{array}{l}15 \\
5.3 \\
13 \\
20 \\
15 \\
15 \\
56 \\
12 \\
5.6 \\
22\end{array}$ & $\begin{array}{l}17 \\
5.7 \\
14 \\
21 \\
15 \\
16 \\
61 \\
13 \\
5.9 \\
24\end{array}$ & $\begin{array}{l}21 \\
8.5 \\
16 \\
24 \\
17 \\
24 \\
74 \\
13 \\
7.6 \\
26\end{array}$ & $\begin{array}{r}29 \\
13 \\
21 \\
28 \\
21 \\
35 \\
136 \\
17 \\
11 \\
27\end{array}$ & $\begin{array}{r}36 \\
15 \\
40 \\
42 \\
27 \\
46 \\
217 \\
21 \\
14 \\
41\end{array}$ & $\begin{array}{r}48 \\
16 \\
60 \\
99 \\
30 \\
75 \\
328 \\
24 \\
17 \\
85\end{array}$ \\
\hline $\begin{array}{l}1970 \\
1971 \\
1972 \\
1973 \\
1974 \\
1975 \\
1976\end{array}$ & $\begin{array}{l}23 \\
16 \\
21 \\
52 \\
37 \\
22 \\
15\end{array}$ & $\begin{array}{l}23 \\
17 \\
21 \\
54 \\
40 \\
22 \\
15\end{array}$ & $\begin{array}{l}24 \\
18 \\
22 \\
58 \\
40 \\
22 \\
16\end{array}$ & $\begin{array}{l}25 \\
25 \\
24 \\
65 \\
41 \\
22 \\
18\end{array}$ & $\begin{array}{l}27 \\
27 \\
31 \\
89 \\
51 \\
22 \\
19\end{array}$ & $\begin{array}{r}37 \\
41 \\
52 \\
218 \\
86 \\
25 \\
24\end{array}$ & $\begin{array}{r}43 \\
51 \\
76 \\
267 \\
91 \\
28 \\
26\end{array}$ \\
\hline
\end{tabular}




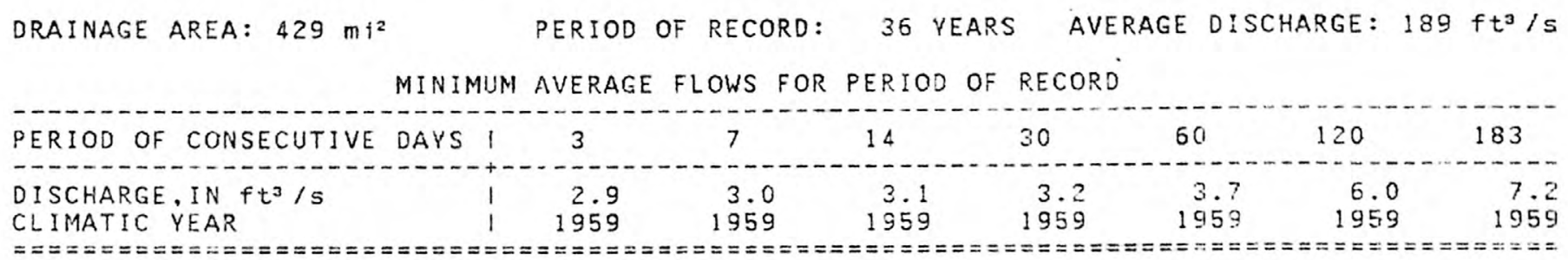

MAGNITUDE AND FREQUENCY OF ANNUAL LOW FLOWS

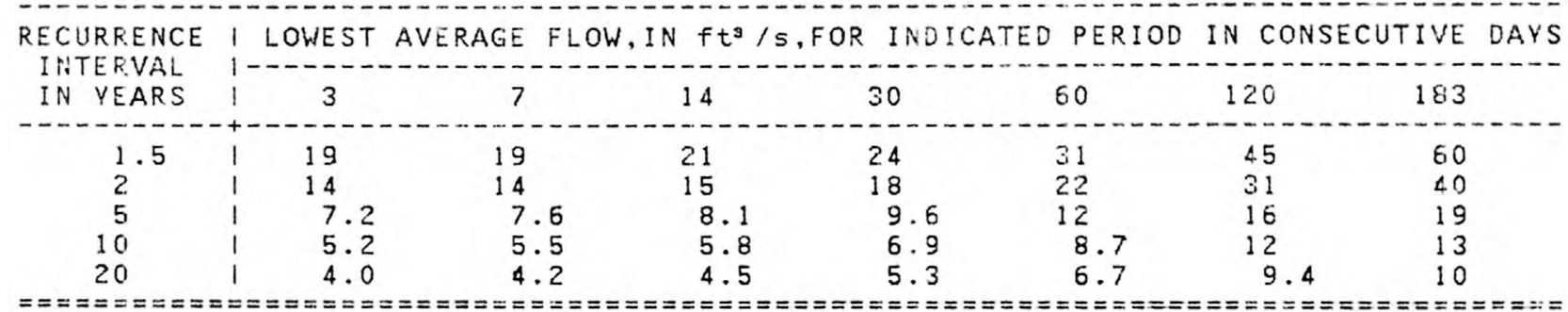

MAGNITUDE AND FREQUENCY OF SEASONAL LOW FLOWS

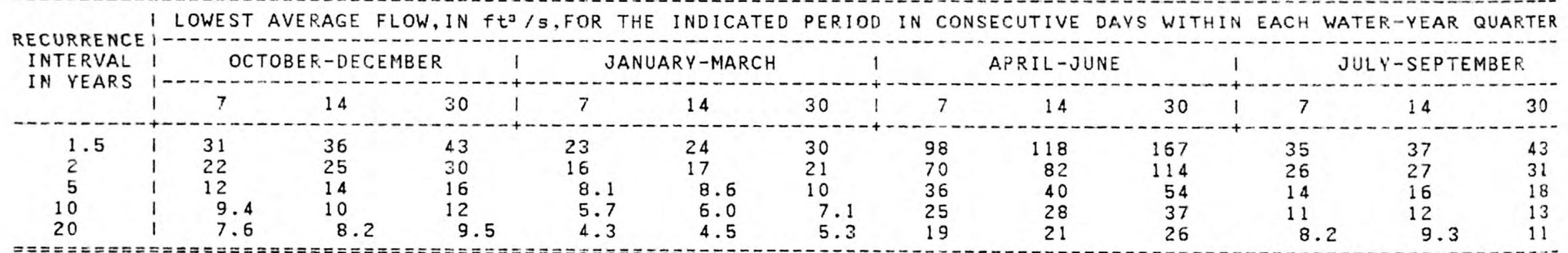

DURATION OF DAILY DISCHARGES FOR ANNUAL AND SEASONAL PERIODS

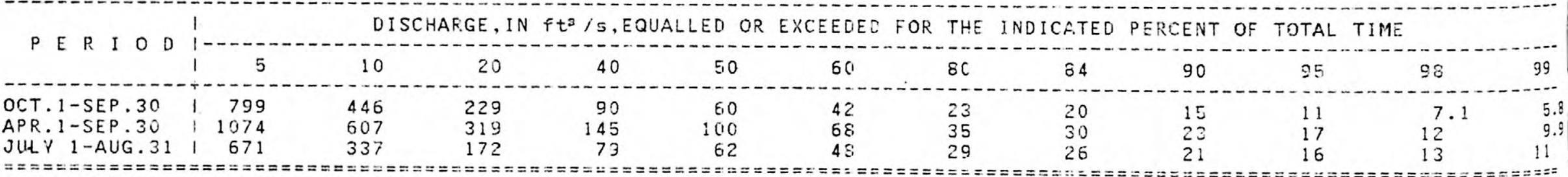




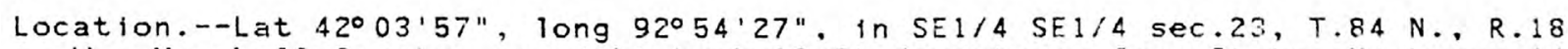
W. Marshal 1 County, on right bank $10 \mathrm{ft}$ downstream from State Highway 14 bridge, 1,500 ft upstream from Burnett Creek, 2.2 miles upstream froli Linn Creek, and at mile 222.8 .

Remarks.--Slight diurnal fluctuation caused by powerplant at Iowa Fails. LOW FLOW DISCHARGES, IN YEAR ENDING MIARCH 31

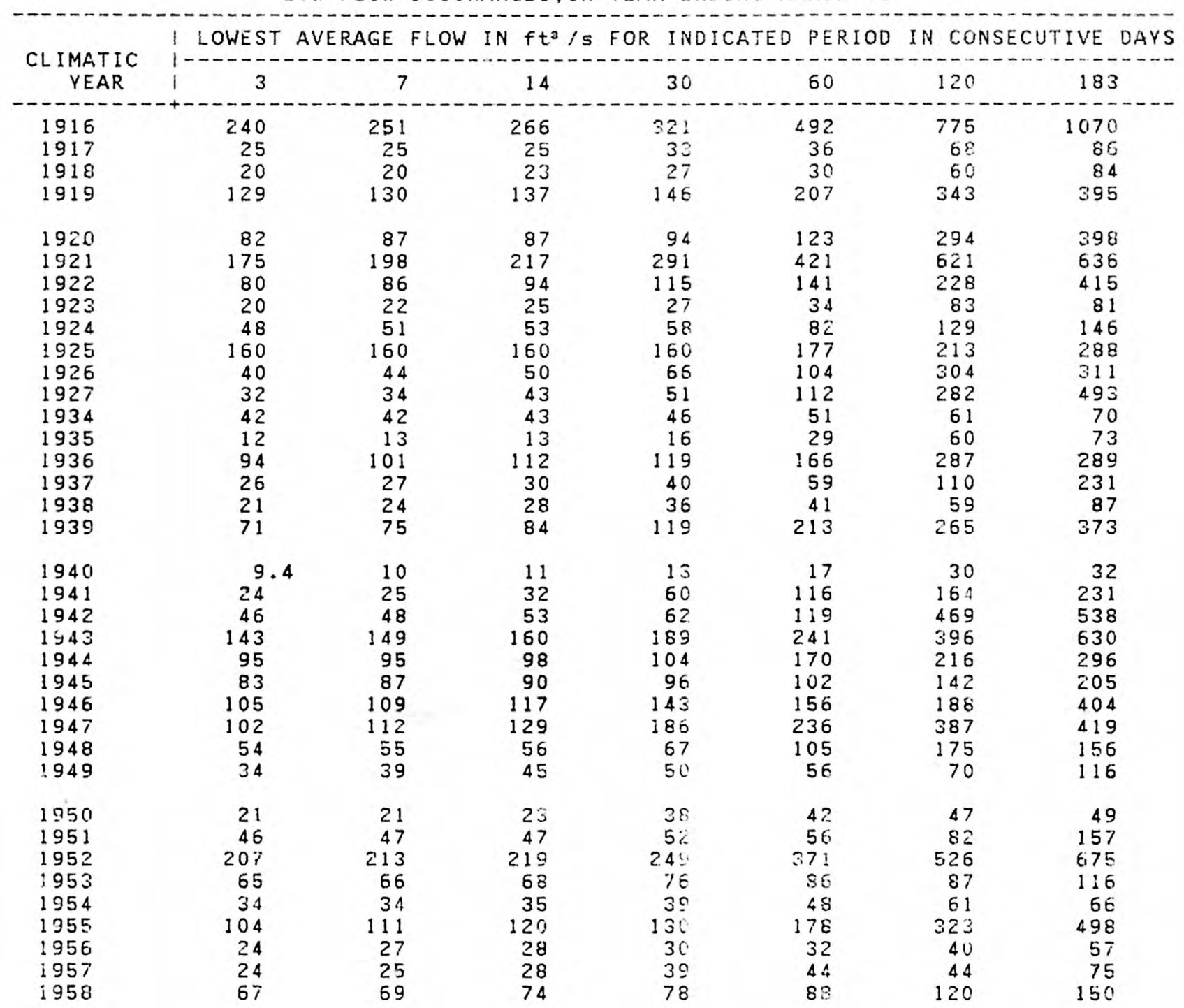


LOW FLOW DISCHARGES, IN YEAR ENDING MARCH 31--Cont 1nued

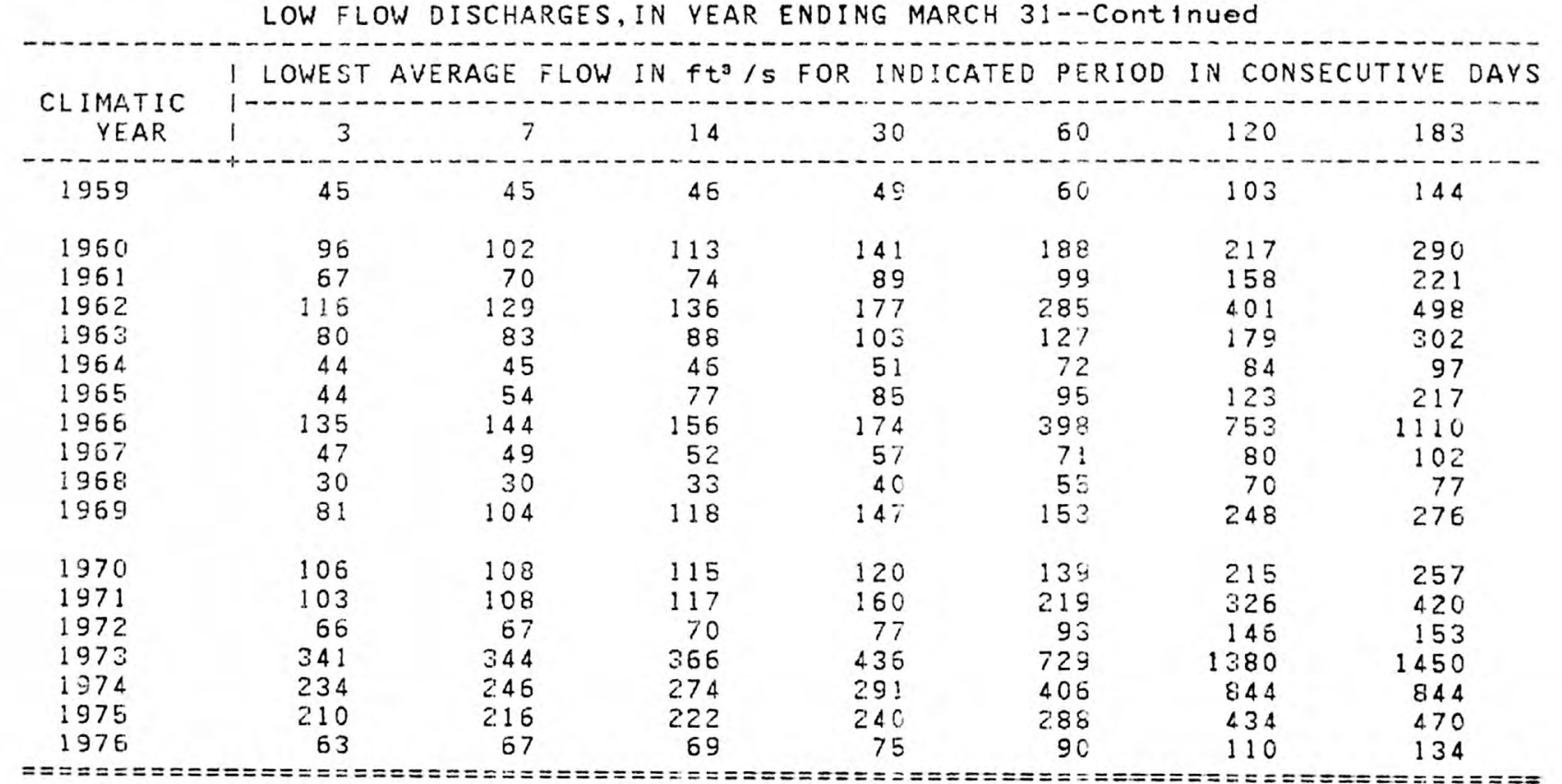


05-4515.00 IOWA RIVER AT MARSHALLTOWN--Continued

DRAINAGE AREA: $1564 \mathrm{mi}^{2} \quad$ PERIOD OF RECORD: 58 YEARS AVERAGE DISCHARGE: 776 ft /S

MINIMUM AVERAGE FLOWS FOR PERIOD OF RECORD
PERIOD OF CONSECUTIVE DAYS I

MAGNITUDE AND FREQUENCY OF ANNUAL LON FLOWS

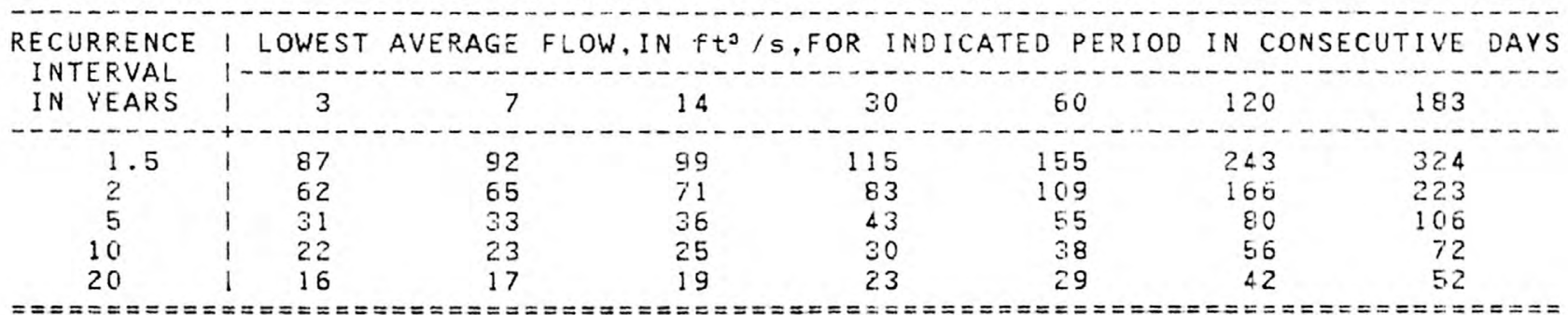

MAGNITUIDE AND FREQUENCY OF SEASONAL LOW FLOWS

I LOWEST AVERAGE FLOW, IN $\mathrm{ft}^{3} / \mathrm{s}, \mathrm{FOR}$ THE INDICATED PERIOD IN CONSECUTIVE DAYS WITHIN EACH WATER-YEAR QUARTER

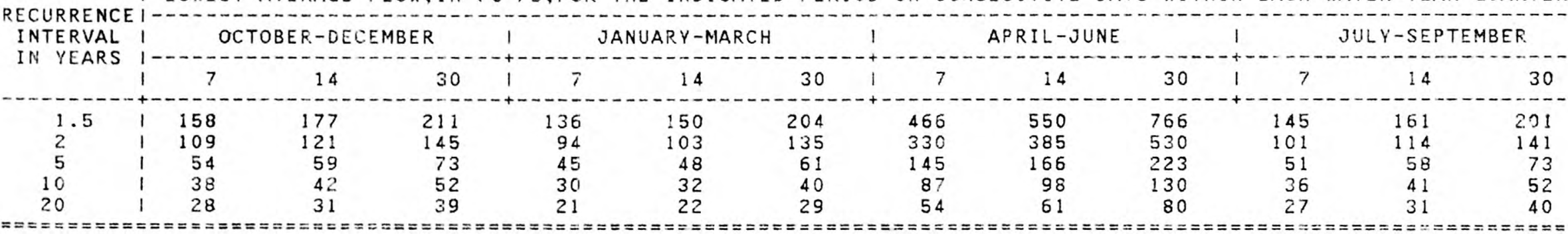

DURATION OF DAILY DISCHARGES FOR ANNUAL ANO SEASONAL PERIODS

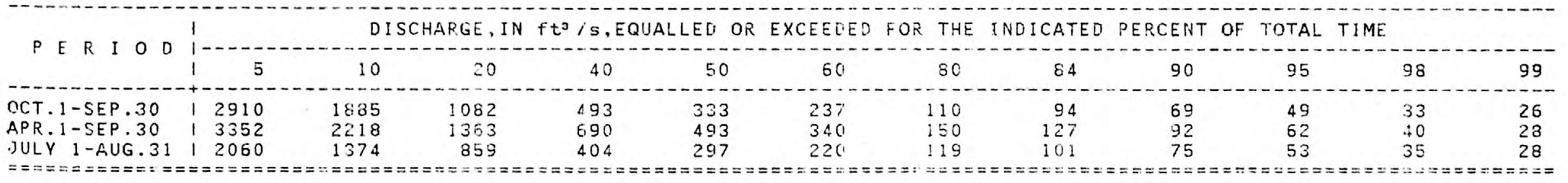


TIMBER CREEK NEAR MARSHALLTOWN

Location.--Lat $42000125^{\prime \prime}$, long 92051 15", in SE1/4 SW1/4 sec.8, T.83 N., R.17 W. Marshal; County, on left bank 20 feet downstream from bridge on U.S. Highway $30,3.5$ miles upstream from mouth, and 4.1 miles southeast of court house in Marshalitown.

LOW FLOW DISCHARGES, IN YEAR ENDING MARCH 31

\begin{tabular}{|c|c|c|c|c|c|c|c|}
\hline CLIMATIC & $\mid$ LOWEST A & E. FLOh & $f t^{3} / s$ & INDICA & PERIOD & IN CONSE & IVE DAYS \\
\hline YEAR & 13 & 7 & 14 & 30 & 60 & 120 & 183 \\
\hline $\begin{array}{l}1951 \\
1952 \\
1953 \\
1954 \\
1955 \\
1956 \\
1957 \\
1958 \\
1959\end{array}$ & $\begin{array}{l}0.40 \\
16 \\
2.5 \\
.40 \\
3.6 \\
.30 \\
0 \\
2.1 \\
2.5\end{array}$ & $\begin{array}{c}0.44 \\
16 \\
2.5 \\
.40 \\
4.0 \\
.30 \\
0 \\
2.5 \\
2.6\end{array}$ & $\begin{array}{c}0.48 \\
17 \\
2.7 \\
.40 \\
5.1 \\
.30 \\
.07 \\
2.7 \\
2.7\end{array}$ & $\begin{array}{c}0.51 \\
20 \\
3.0 \\
.40 \\
8.5 \\
.33 \\
.37 \\
3.6 \\
2.9\end{array}$ & $\begin{array}{c}0.71 \\
32 \\
3.1 \\
.69 \\
13 \\
.41 \\
.85 \\
4.8 \\
4.0\end{array}$ & $\begin{array}{c}0.82 \\
37 \\
5.8 \\
1.5 \\
21 . \\
.73 \\
1.0 \\
6.9 \\
6.3\end{array}$ & $\begin{array}{r}1.1 \\
43 \\
9.5 \\
2.0 \\
27 \\
1.1 \\
1.5 \\
7.5 \\
8.2\end{array}$ \\
\hline $\begin{array}{l}1960 \\
1961 \\
1962 \\
1963 \\
1964 \\
1965 \\
1966 \\
1967 \\
1968 \\
1969\end{array}$ & $\begin{array}{c}3.4 \\
3.7 \\
11 \\
6.2 \\
2.2 \\
2.8 \\
14 \\
1.8 \\
.21 \\
1.2\end{array}$ & $\begin{array}{c}3.6 \\
3.9 \\
13 \\
5.8 \\
2.3 \\
3.4 \\
15 \\
2.0 \\
.21 \\
1.3\end{array}$ & $\begin{array}{c}3.7 \\
4.1 \\
14 \\
7.4 \\
2.4 \\
3.5 \\
15 \\
2.3 \\
.27 \\
1.7\end{array}$ & $\begin{array}{c}4.5 \\
5.3 \\
18 \\
8.4 \\
2.8 \\
5.4 \\
19 \\
2.5 \\
.67 \\
2.9\end{array}$ & $\begin{array}{r}6.1 \\
7.0 \\
31 . \\
8.7 \\
4.4 \\
5.7 \\
33 \\
3.3 \\
1.5 \\
3.5\end{array}$ & $\begin{array}{r}11 \\
11 \\
39 \\
12 \\
5.2 \\
8.1 \\
69 \\
3.9 \\
2.4 \\
4.3\end{array}$ & $\begin{array}{l}14 \\
14 \\
47 \\
18 \\
6.4 \\
11 \\
73 \\
5.7 \\
3.3 \\
6.6\end{array}$ \\
\hline $\begin{array}{l}1970 \\
1971 \\
1972 \\
1973 \\
1974 \\
1975 \\
1976\end{array}$ & $\begin{array}{l}6.5 \\
14 \\
2.3 \\
13 \\
13 \\
23 \\
3.6\end{array}$ & $\begin{array}{l}5.6 \\
15 \\
2.4 \\
13 \\
22 \\
23 \\
4.4\end{array}$ & $\begin{array}{l}7.1 \\
17 \\
2.5 \\
15 \\
24 \\
24 \\
5.0\end{array}$ & $\begin{array}{l}7 \cdot 2 \\
23 \\
5 \cdot 1 \\
25 \\
27 \\
26 \\
E .1\end{array}$ & $\begin{array}{c}8.4 \\
35 \\
4.2 \\
48 \\
36 \\
30 \\
7.0\end{array}$ & $\begin{array}{c}13 \\
58 \\
5.4 \\
106 \\
41 \\
47 \\
5.8\end{array}$ & $\begin{array}{c}15 \\
68 \\
5.6 \\
116 \\
45 \\
50 \\
12\end{array}$ \\
\hline
\end{tabular}


DRAINAGE AREA: $118 \mathrm{mi}^{2}$ PERIOD OF RECORD: 27 VEARS AVERAGE DISCHARGE: 56.7 ft 3 /S

MINIMUM AVERAGE FLOWS FOR PERIOD OF RECORD

\begin{tabular}{|c|c|c|c|c|c|c|c|}
\hline PERIOD OF CONSECUTIVE DAYS & 3 & 7 & 14 & 30 & 60 & 120 & 183 \\
\hline $\begin{array}{l}\text { DISCHARGE, IN } \mathrm{ft}^{3} / \mathrm{s} \\
\text { CLIMATIC YEAR }\end{array}$ & $\begin{array}{c}0 \\
1957\end{array}$ & $\begin{array}{c}0 \\
1957\end{array}$ & $\begin{array}{l}0.07 \\
1957\end{array}$ & $\begin{array}{l}0.33 \\
1956\end{array}$ & $\begin{array}{c}0.42 \\
1956\end{array}$ & $\begin{array}{l}0.74 \\
1956\end{array}$ & $\begin{array}{r}1.1 \\
1951\end{array}$ \\
\hline
\end{tabular}

MAGNITUDE AND FREQUENCY OF ANNUAL LOW FLOWS

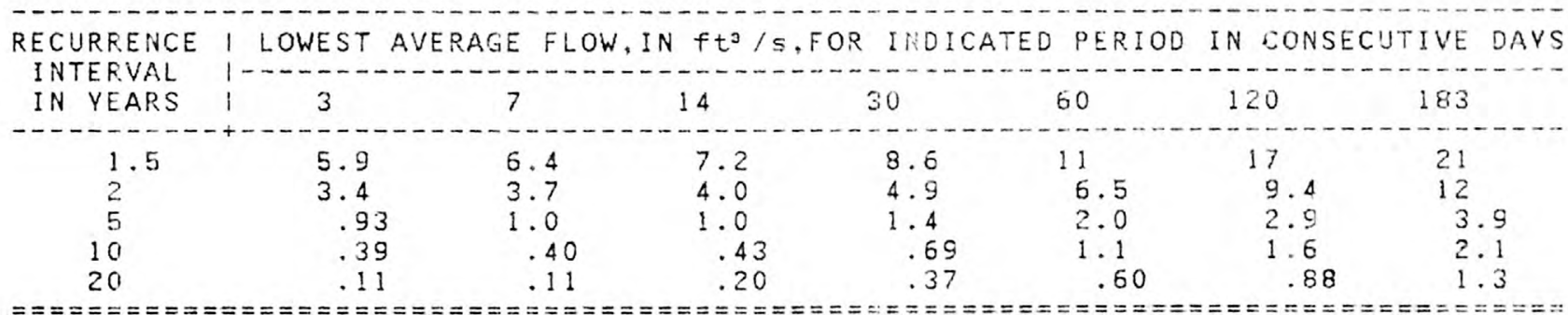

MAGNITUDE AND FREQUENCY OF SEASONAL LOW FLOWS

I LOWEST AVERAGE FLOW, IN $\mathrm{ft}^{3} / \mathrm{s}, \mathrm{FOR}$ THE INDICATED PERIOD IN CONSECUTIVE DAYS WITHIN EACH WATER-YEAR QUARTER

\begin{tabular}{|c|c|c|c|c|c|c|c|c|c|c|c|c|}
\hline \multirow{2}{*}{$\begin{array}{l}\text { INTERVAL } \\
\text { IN YEARS }\end{array}$} & OCT & - DECEI & & JA & Y-MARCH & & 1 & APRIL-JUNE & & 1 & -SEPTE & \\
\hline & 7 & 14 & 30 & 7 & 14 & 30 & 7 & 14 & 30 & 7 & 14 & 30 \\
\hline $\begin{array}{l}1.5 \\
2 \\
5 \\
10 \\
20\end{array}$ & $\begin{array}{l}9.6 \\
5.0 \\
1.4 \\
.61 \\
.23\end{array}$ & $\begin{array}{c}10 \\
5.6 \\
1.7 \\
.87 \\
.52\end{array}$ & $\begin{array}{c}13 \\
6.9 \\
2.2 \\
1.2 \\
.71\end{array}$ & $\begin{array}{l}9.6 \\
4.8 \\
1.2 \\
.56 \\
.29\end{array}$ & $\begin{array}{c}11 \\
5.7 \\
1.4 \\
.65 \\
.33\end{array}$ & $\begin{array}{l}20 \\
11 \\
2.8 \\
1.3 \\
.65\end{array}$ & $\begin{array}{r}42 \\
26 \\
7.7 \\
3.4 \\
1.6\end{array}$ & $\begin{array}{r}47 \\
29 \\
9.0 \\
4.3 \\
2.1\end{array}$ & $\begin{array}{l}64 \\
40 \\
13 \\
6.5 \\
3.4\end{array}$ & $\begin{array}{l}10 \\
6.1 \\
2.0 \\
1.0 \\
.54\end{array}$ & $\begin{array}{c}11 \\
6.6 \\
2.4 \\
1.3 \\
\quad .78\end{array}$ & $\begin{array}{r}13 \\
8.4 \\
3.2 \\
1.9 \\
1.2\end{array}$ \\
\hline
\end{tabular}

DURATION OF DAILY DISCHARGES FOR ANNUAL AND SEASONAL PERIODS

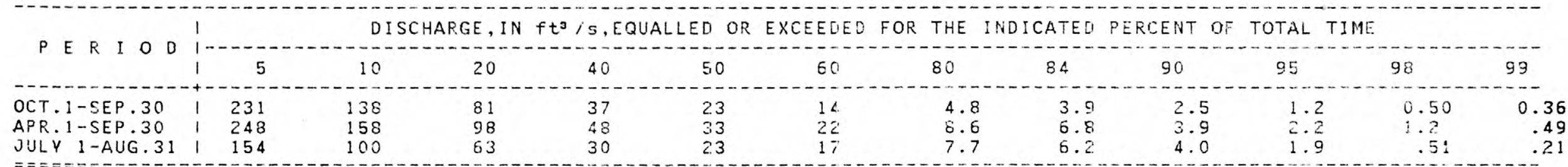


Location.--Lat 41053'58", long 92028'27", in SE1/4 NE1/4 sec.21, T.82 N., R.14 W. Tama County, on right bank 5 ft upstream from bridge on county highway.

0.6 mile northeast of Haven, and $2.8 \mathrm{miles}$ upstream from mouth.

LOW FLOW DISCHARGES, IN YEAR. ENDING MARCH 31

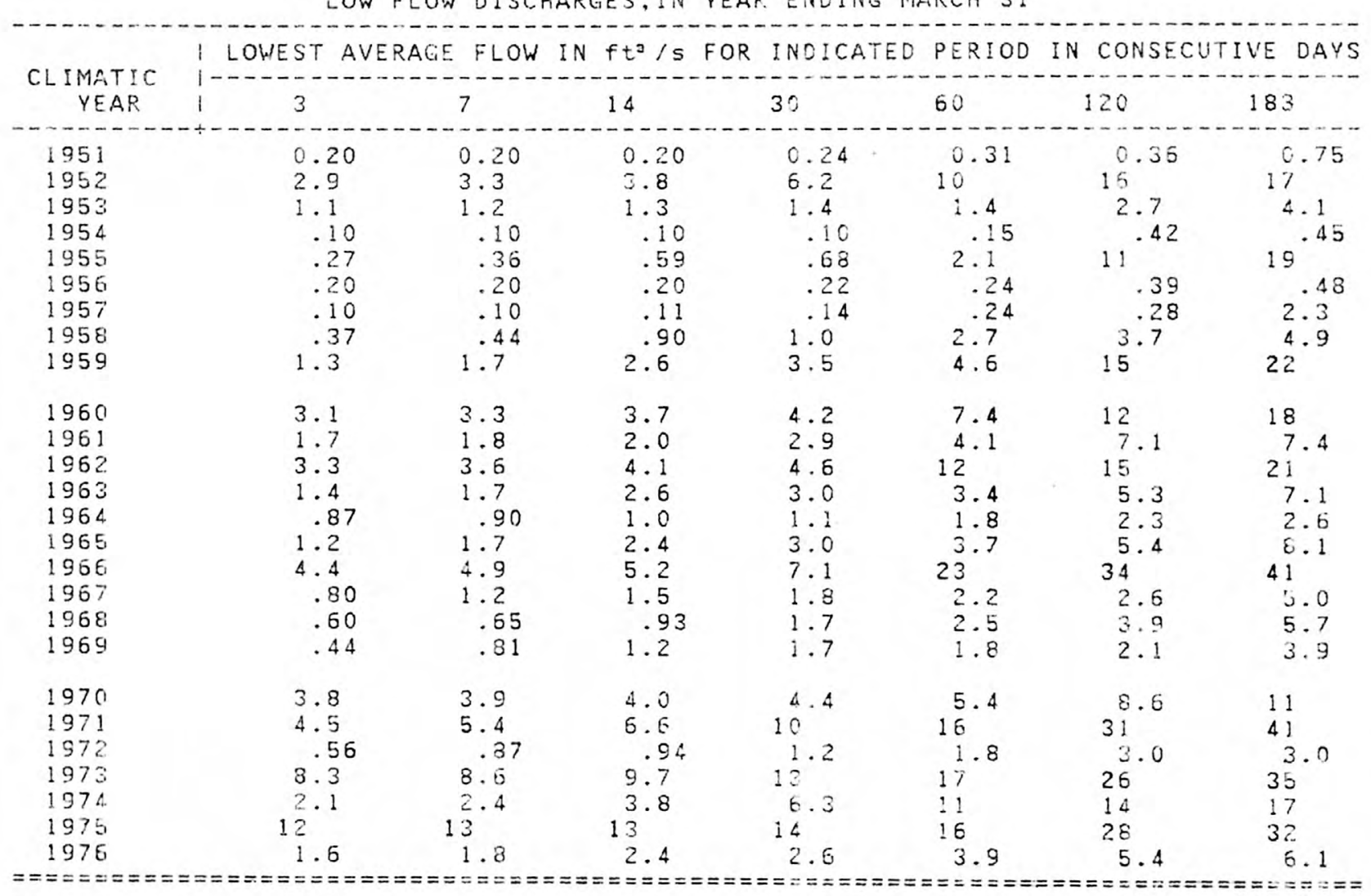


DRAINAGE AREA: $56.1 \mathrm{mi}^{2}$ PERIOD OF RECORD: 27 YEARS AVERAGE DISCHARGE: 33.3 ft?/S

MINIMUM AVERAGE FLOWS FOR PERIOD OF RECORD

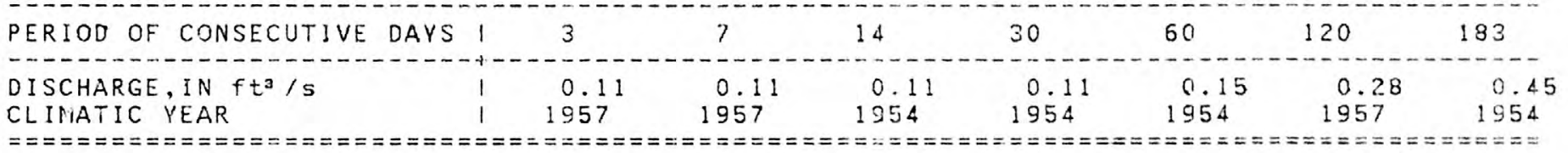

MAGNITUDE AND FREQUENCY OF ANNUAL LOW FLOWS

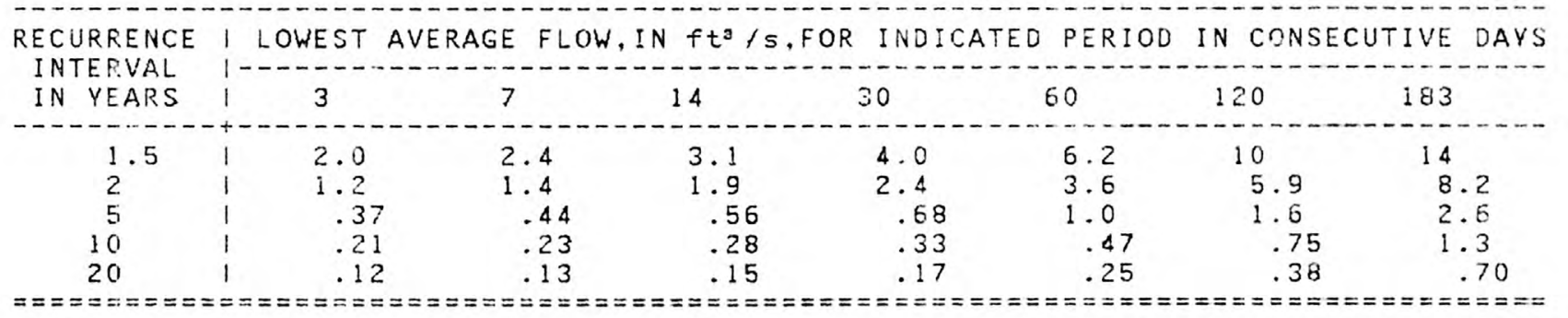

MAGNITUDE AND FREQUENCY OF SEASONAL LOW FLOWS

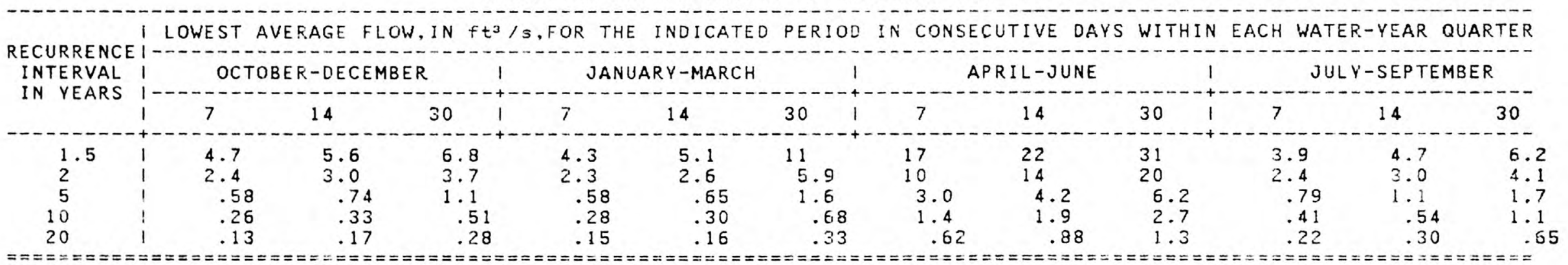

DURATION OF DAILY DISCHARGES FOR ANNUAL AND SEASONAL FERIODS

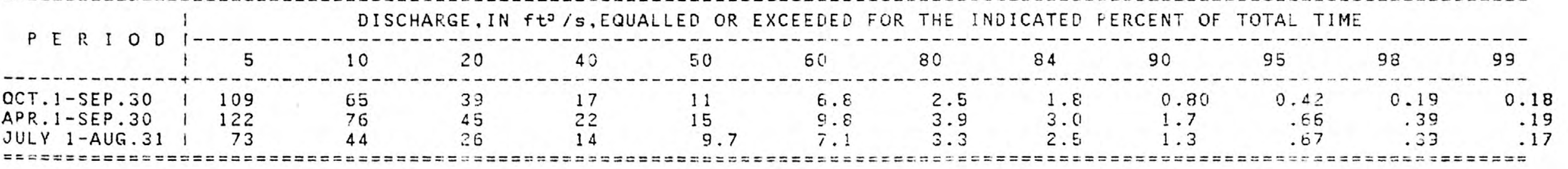




\section{SALT CREEK NEAR ELBERON}

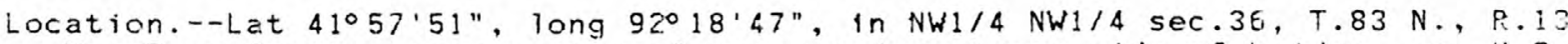
W. Tama County, near center of span on downstream side of bridge on U.S. Highway 30 , 2.0 miles upstream from Hog Run, $3.0 \mathrm{miles}$ south of Elberon, and 9.0 miles upstream from mouth.

LOW FLOW DISCHARGES, IN YEAR ENDING MARCH 31

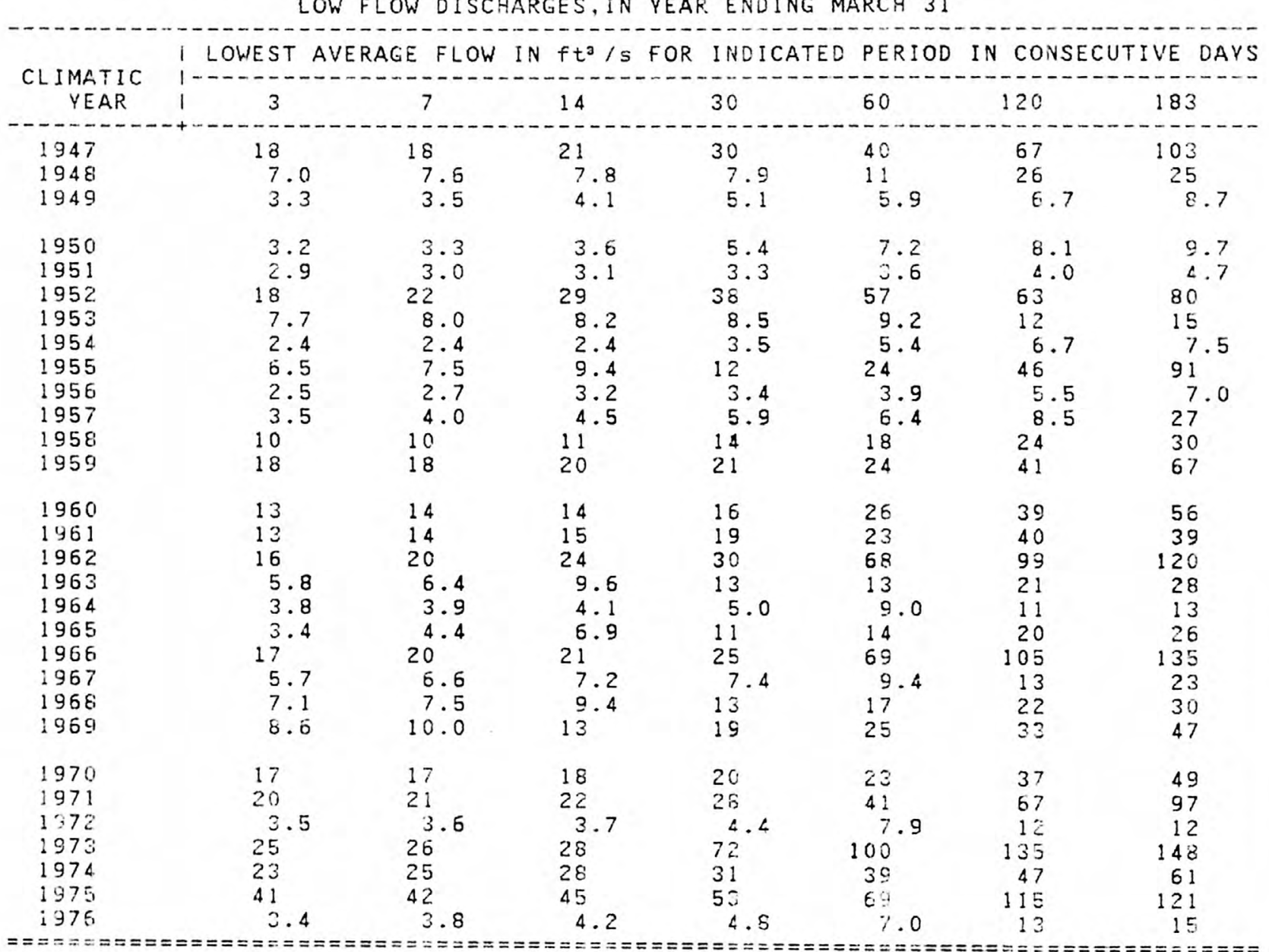




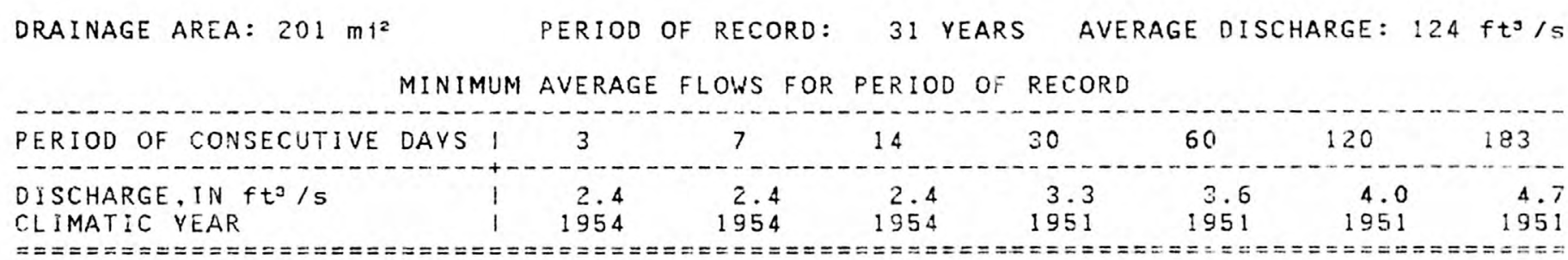

MAGNITUDE AND FREQUENCY OF ANNUAL LOW FLOWS

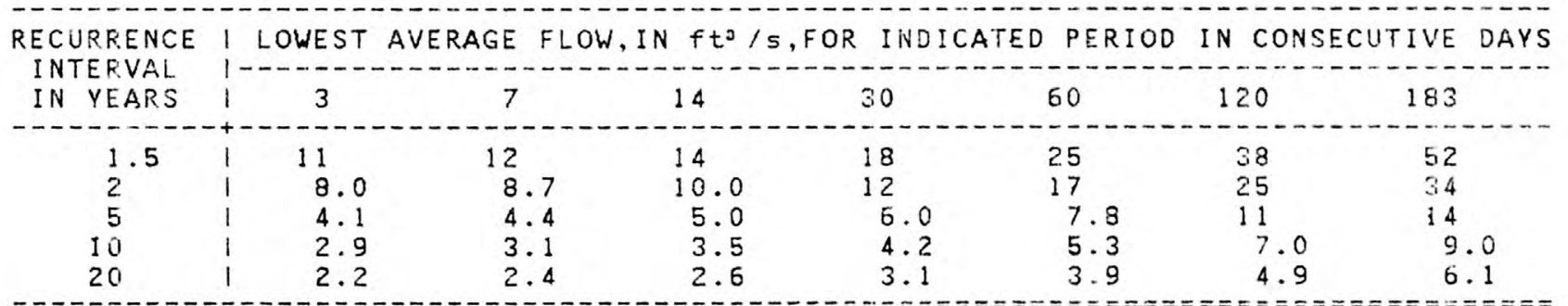

MAGNITUDE AND FREQUENCY OF SEASONAL LOW FLOWS

LOWEST AVERAGE FLOW, IN $\mathrm{ft}^{3} / \mathrm{s}, \mathrm{FOR}$ THE INDICATED PERIOD IN CONSECUTIVE DAYS WITHIN EACH WATER-YEAR QUARTER RECURRENCE I-

INTERVAL I OCTOBER-DECEMBER I JANUARY-MARCH I I I I I I S I JUNE

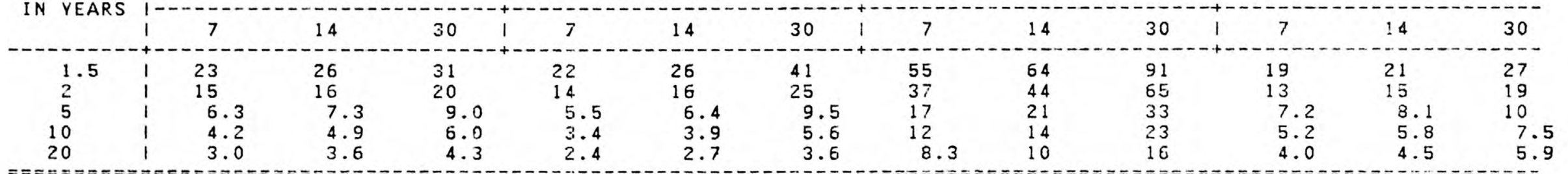

DURATION OF DAILY DISCHARGES FOR ANNUAL AND SEASONAL FERIODS

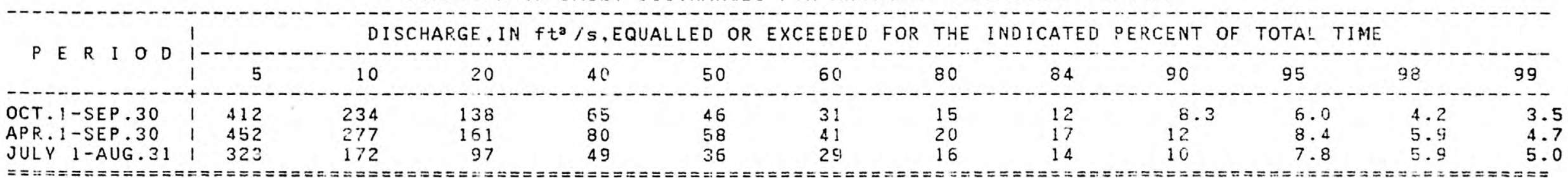


Location.--Lat $41^{\circ} 50^{\prime} 06^{\prime \prime}$, long $92^{\circ} 23^{\prime} 10^{\prime \prime}$, in SE1/4 SW1/4 sec.8, T.8! N., R.13 W. Poweshilek County, on left bank $5 \mathrm{ft}$ upstream from bridge on county highway V2l, 1.2 miles downstream from North Walnut Creek, 4.0 miles northwest of Hartwick, and $6.5 \mathrm{miles}$ upstream from mouth.

LOW FLOW DISCHARGES, IN YEAR. ENDING MARCH 31

\begin{tabular}{|c|c|c|c|c|c|c|c|}
\hline \multirow{2}{*}{$\begin{array}{c}\text { CLIMAT IC } \\
\text { YEAR }\end{array}$} & LOWEST A & E FLOW & $f t^{3} / s$ & INDICA & PERIOD & $N$ CONSE & VE DAYS \\
\hline & 13 & 7 & 14 & 30 & 60 & 120 & 183 \\
\hline $\begin{array}{l}1951 \\
1952 \\
1954 \\
1954 \\
1955 \\
1956 \\
1957 \\
1958 \\
1959\end{array}$ & $\begin{array}{l}0.11 \\
3.0 \\
.27 \\
.10 \\
0 \\
0 \\
0 \\
0 \\
1.6\end{array}$ & $\begin{array}{l}0.11 \\
3.4 \\
.57 \\
.10 \\
.04 \\
0 \\
0 \\
0 \\
1.9\end{array}$ & $\begin{array}{l}0.11 \\
4.0 \\
.72 \\
.10 \\
.31 \\
0 \\
0 \\
.01 \\
2.1\end{array}$ & $\begin{array}{l}0.12 \\
4.5 \\
.82 \\
.10 \\
.61 \\
0 \\
0 \\
.22 \\
2.7\end{array}$ & $\begin{array}{l}0.36 \\
8.5 \\
1.1 \\
.15 \\
1.4 \\
.04 \\
.01 \\
1.1 \\
5.0\end{array}$ & $\begin{array}{c}0.49 \\
18 \\
3.0 \\
. .36 \\
11 . \\
.14 \\
. .27 \\
1.6 \\
11 .\end{array}$ & $\begin{array}{c}1.2 \\
22^{4} \\
4.0 \\
.36 \\
24 . \\
.61 \\
3.3 \\
2.3 \\
28\end{array}$ \\
\hline $\begin{array}{l}1960 \\
1961 \\
1562 \\
1963 \\
1964 \\
1965 \\
1966 \\
1967 \\
1968 \\
1969\end{array}$ & $\begin{array}{l}3.0 \\
1.2 \\
3.0 \\
2.7 \\
.50 \\
1.5 \\
3.5 \\
.11 \\
2.3 \\
.51\end{array}$ & $\begin{array}{l}3.1 \\
1.2 \\
3.2 \\
2.9 \\
.60 \\
2.0 \\
4.0 \\
.16 \\
2.6 \\
.77\end{array}$ & $\begin{array}{l}3.3 \\
1.3 \\
3.7 \\
3.5 \\
.61 \\
2.1 \\
4.4 \\
.19 \\
3.0 \\
1.2\end{array}$ & $\begin{array}{l}3.8 \\
1.8 \\
4.5 \\
3.9 \\
.73 \\
3.3 \\
6.3 \\
.33 \\
4.5 \\
1.6\end{array}$ & $\begin{array}{r}7.0 \\
2.8 \\
13 \\
4.0 \\
1.4 \\
3.8 \\
22 \\
1.2 \\
5.6 \\
1.7\end{array}$ & $\begin{array}{r}12 \\
7.2 \\
16 \\
6.3 \\
1.6 \\
7.8 \\
41 \\
1.9 \\
8.2 \\
2.5\end{array}$ & $\begin{array}{l}28 \\
6.7 \\
20 \\
9.4 \\
2.1 \\
10 \\
48 \\
5.2 \\
9.9 \\
3.7\end{array}$ \\
\hline $\begin{array}{l}1970 \\
1971 \\
1972 \\
1973 \\
1974 \\
1975 \\
1976\end{array}$ & $\begin{array}{l}4.8 \\
4.3 \\
.87 \\
8.5 \\
4.1 \\
11 . \\
1.9\end{array}$ & $\begin{array}{c}4.9 \\
4.9 \\
.92 \\
9.2 \\
4.2 \\
1: \\
2.0\end{array}$ & $\begin{array}{r}5.0 \\
5.5 \\
1.0 \\
11 \\
4.8 \\
11 \\
2.3\end{array}$ & $\begin{array}{r}5.2 \\
8.7 \\
1.1 \\
18 \\
5.8 \\
12.0 \\
3.0\end{array}$ & $\begin{array}{c}6.6 \\
14 \\
1.3 \\
28 \\
7.3 \\
15 . \\
4.0\end{array}$ & $\begin{array}{c}9.9 \\
34 \\
2.1 \\
43 \\
7.6 \\
25 . \\
6.9\end{array}$ & $\begin{array}{l}12 \\
56 \\
2.9 \\
53 \\
14 \\
30 \\
7.1\end{array}$ \\
\hline
\end{tabular}


05-4522.00 WALNUT CREEK NEAR HARTWICK--Continued
DRAINAGE AREA:
$70.9 \mathrm{mi}^{2}$
PERIOD OF RECORD:
27 YEARS
AVERAGE DISCHARGE:
$41.8 \mathrm{ft}^{3} / \mathrm{s}$

MINIMUM AVERAGE FLOWS FOR PERIOD OF RECORD

\begin{tabular}{|c|c|c|c|c|c|c|c|}
\hline PERIOD OF CONSECUTIVE DAYS I & 3 & 7 & 14 & 30 & 60 & 120 & 183 \\
\hline $\begin{array}{l}\text { DISCHARGE, IN } \mathrm{ft}^{3} / \mathrm{s} \\
\text { CLIMATIC YEAR }\end{array}$ & $\begin{array}{l}0 \\
1958\end{array}$ & $\begin{array}{l}0 \\
1958\end{array}$ & $\begin{array}{l}0 \\
1957\end{array}$ & $\begin{array}{l}0 \\
1957\end{array}$ & $\begin{array}{l}0.01 \\
1957\end{array}$ & $\begin{array}{l}0.14 \\
1956\end{array}$ & $\begin{array}{l}0.37 \\
1954\end{array}$ \\
\hline
\end{tabular}

MAGNITUDE AND FREQUENCY OF ANNUAL LOW FLOWS

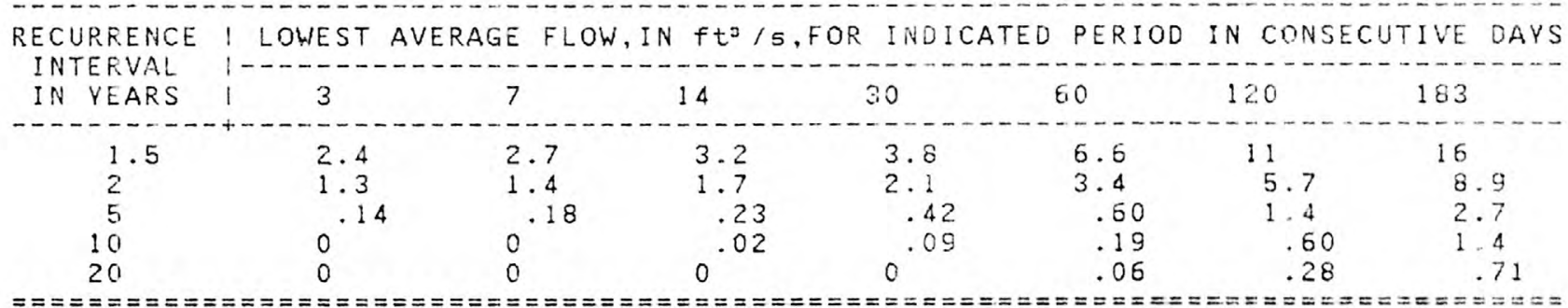

MAGNITUDE AND FREQUENCY OF SEASONAL LOW FLOWS

LOWEST AVERAGE FLOW IN FEJ TFOR THE INDICATED PERIOD IN CONSECUTIVE DAYS WITHIN EACH WATER-YEAR QUARTER

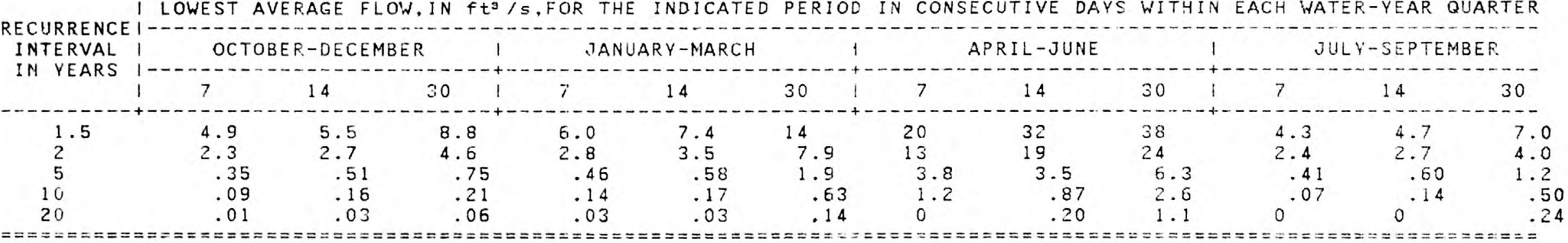

DURATION OF DAILY DISCHARGES FOR ANNUAL ANO SEASONAL FERIODS

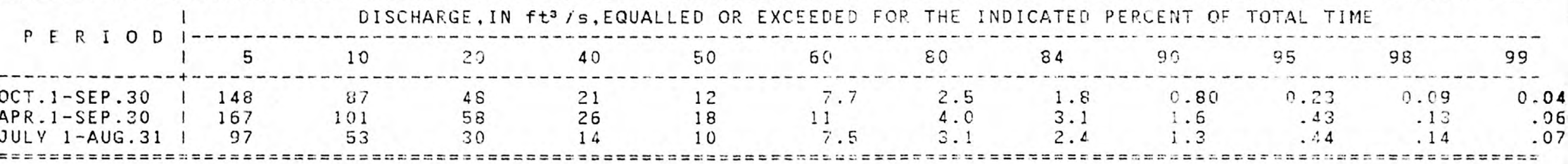


Location.--Lat $41^{\circ} 51 \cdot 20^{\prime \prime}$, long $92^{\circ} 14.20 "$, in NW1/4 sec.5, T.81 N., R.12 W., Io'ra County, on right bank $5 \mathrm{ft}$ downstream from State Highway 212 bridge, $0.5 \mathrm{mile}$ downstream from Wainut Creek, 2.7 miles south of Eelle Plaine and at $\operatorname{mile} 159.0$.

Remarks.--Discontinued Sept. 30, 1959.

LOW FLOW DISCHARGES, IN YEAR ENDING MARCH 31

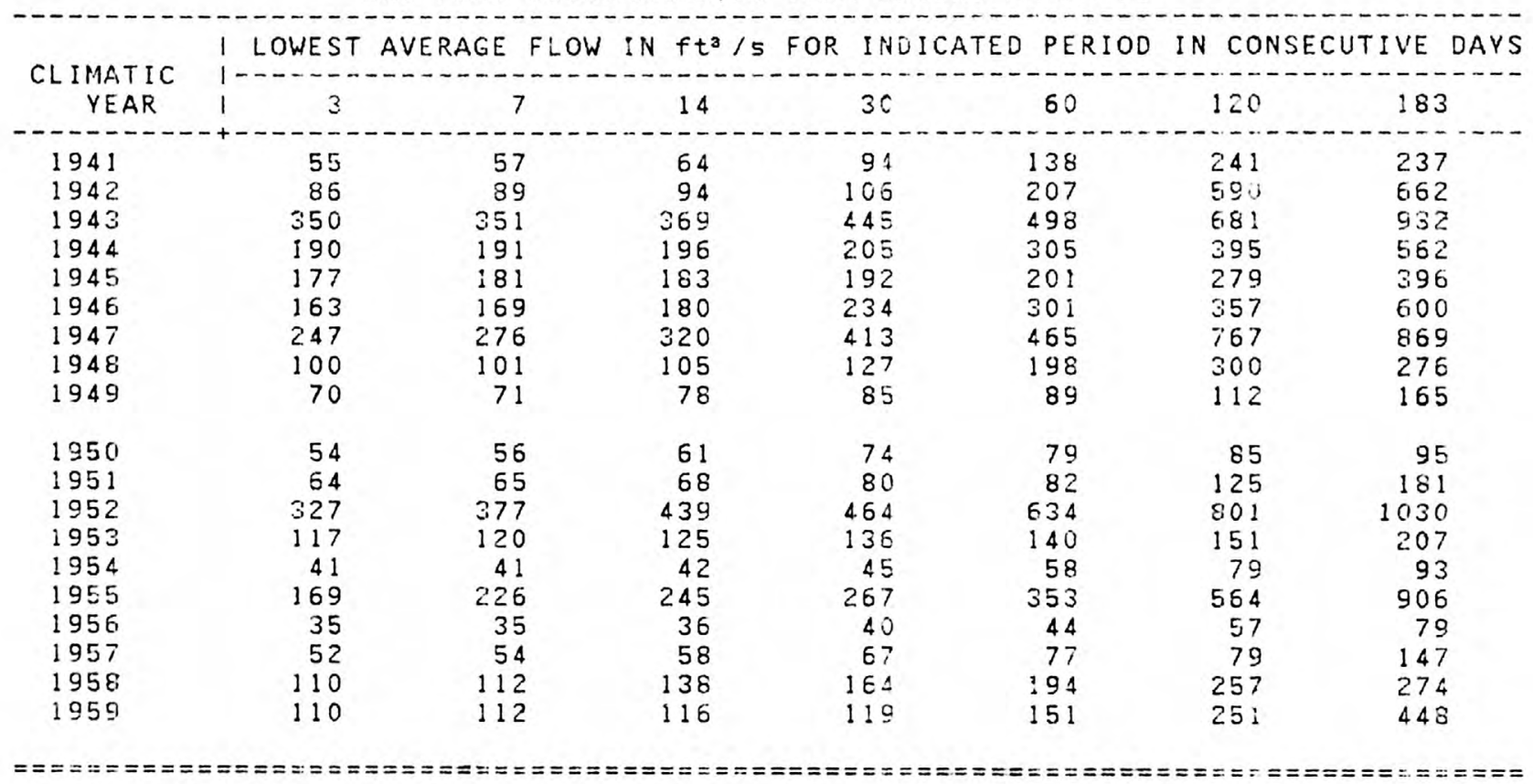




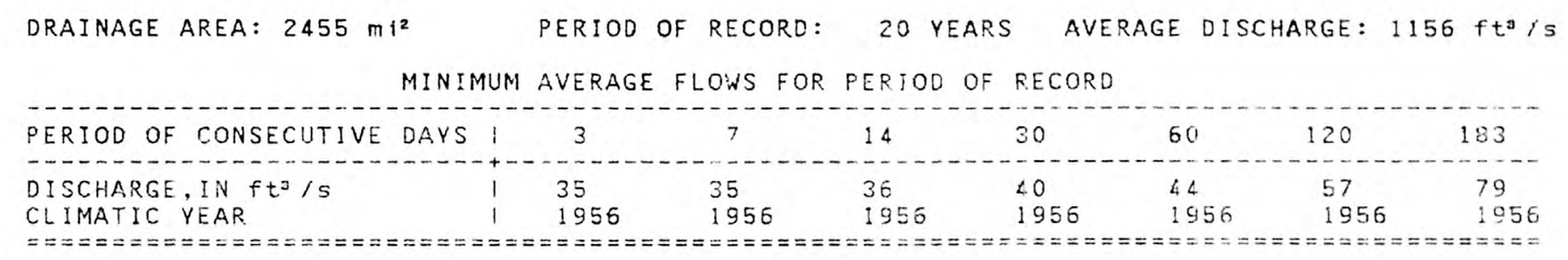

MAGNITUDE AND FREQUENCY OF ANNUAL LOW FLOWS

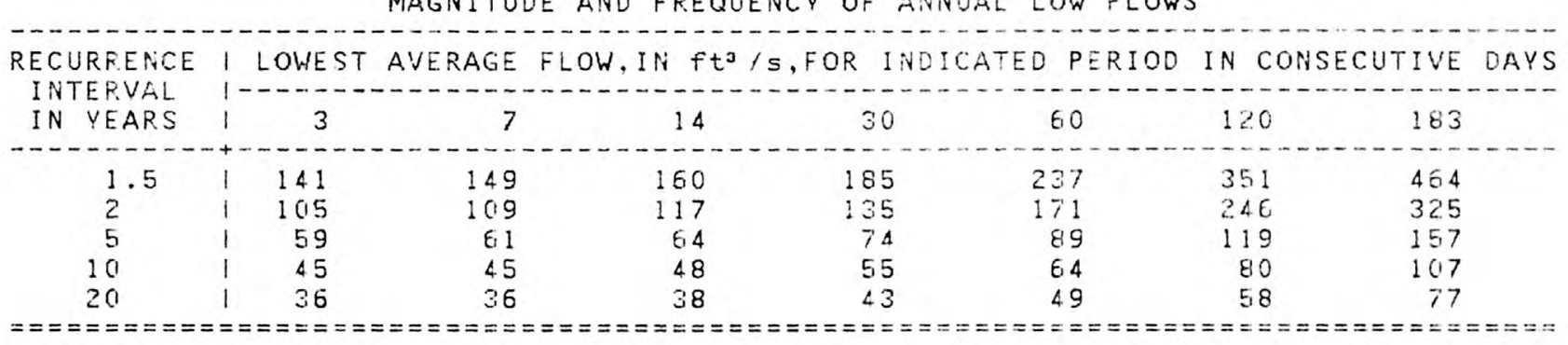

MAGNITUDE AND FREQUENCY OF SEASONAL LOW FLOWS

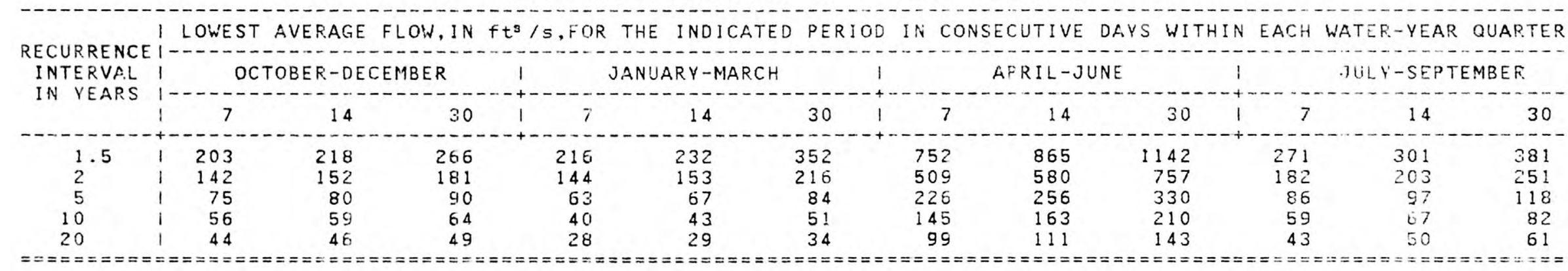

DURATION OF DAILY DISCHARGES FOR ANNUAL ANO SEASONAL FERIODS

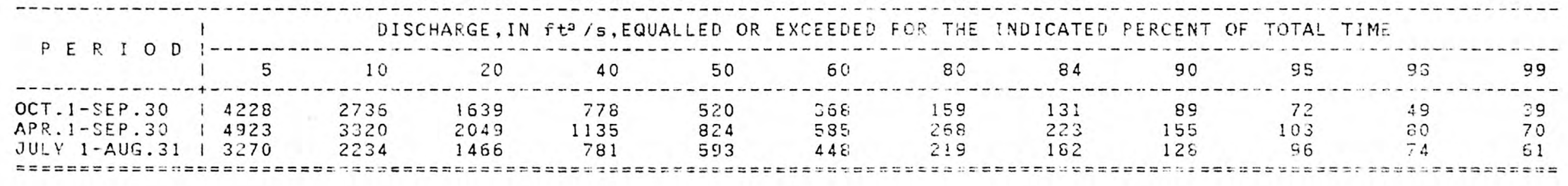


Location.--Lat $41^{\circ} 44^{\prime} 58^{\prime \prime}$, long $92^{\circ} 10^{\circ} 55^{\prime \prime}$, In SWl/4 SW1/4 sec.T, T.80 N., R.11 W. Iowa county, or left bank $10 \mathrm{ft}$. downstream from bridge on county Creek, 2.8 miles upstream from Little Bear Creek, and 8.1 miles upstream from mouth.

Remarks.--Prior to October 1966, published as Bear Creek at Ladora.

LOW FLOW DISCHARGES, IN YEAR. ENDING MARCH 31

\begin{tabular}{|c|c|c|c|c|c|c|c|}
\hline & LOWEST A & E FLO & $f t^{3} / s$ & INDIC, & PERIO & IN CONSE & IVE DAYS \\
\hline YEAR & 3 & 7 & 14 & 30 & 60 & 120 & 183 \\
\hline $\begin{array}{l}1947 \\
1948 \\
1949\end{array}$ & $\begin{array}{r}23 \\
1.0 \\
2.8\end{array}$ & $\begin{array}{r}24 \\
1.3 \\
2.9\end{array}$ & $\begin{array}{r}30 \\
1.7 \\
3.8\end{array}$ & $\begin{array}{l}38 \\
2.1 \\
5.2\end{array}$ & $\begin{array}{r}45 \\
7.8 \\
6.1\end{array}$ & $\begin{array}{r}102 \\
17 \\
8.7\end{array}$ & $\begin{array}{r}125 \\
15 \\
28\end{array}$ \\
\hline $\begin{array}{l}1950 \\
1951 \\
1952 \\
1953 \\
1954 \\
1955 \\
1956 \\
1957 \\
1959 \\
1959\end{array}$ & $\begin{array}{c}2.0 \\
.60 \\
16 \\
3.7 \\
1.3 \\
2.5 \\
0 \\
.10 \\
1.7 \\
7.7\end{array}$ & $\begin{array}{c}2.1 \\
.60 \\
17 \\
3.9 \\
1.3 \\
2.7 \\
0 \\
.10 \\
1.8 \\
9.0\end{array}$ & $\begin{array}{l}2.3 \\
.61 \\
18.2 \\
4.2 \\
1.3 \\
2.9 \\
0 \\
.15 \\
3.5 \\
12\end{array}$ & $\begin{array}{c}3.2 \\
.65 \\
20 \\
4.5 \\
1.4 \\
5.3 \\
.06 \\
.25 \\
4.2 \\
15\end{array}$ & $\begin{array}{r}6.1 \\
1.0 \\
28 \\
4.5 \\
2.4 \\
7.8 \\
.17 \\
.59 \\
7.3 \\
20\end{array}$ & $\begin{array}{c}6.6 \\
2.7 \\
66 \\
8.7 \\
3.1 \\
22 \\
.66 \\
1.1 \\
11 \\
33\end{array}$ & $\begin{array}{l}8.9 \\
3.9 \\
74 \\
12 \\
3.9 \\
44 \\
1.8 \\
4.7 \\
11 \\
69\end{array}$ \\
\hline $\begin{array}{l}1960 \\
1961 \\
1962 \\
1963 \\
1964 \\
1965 \\
1966 \\
1967 \\
1968 \\
1969\end{array}$ & $\begin{array}{l}5.7 \\
5.0 \\
13 \\
10 \\
.60 \\
2.4 \\
11 \\
2.8 \\
6.7 \\
2.4\end{array}$ & $\begin{array}{c}6.2 \\
5.1 \\
15 \\
10 \\
.66 \\
2.7 \\
12 . \\
2.9 \\
7.1 \\
2.7\end{array}$ & $\begin{array}{l}7.0 \\
5.5 \\
16 \\
10 \\
.68 \\
3.3 \\
12 \\
3.0 \\
7.7 \\
3.8\end{array}$ & $\begin{array}{c}8.9 \\
7.2 \\
20 \\
12 \\
.87 \\
4.8 \\
17 \\
3.0 \\
9.6 \\
6.3\end{array}$ & $\begin{array}{c}22 \\
9.0 \\
52 \\
12 \\
1.4 \\
6.8 \\
60 \\
5.9 \\
10.9 \\
6.9\end{array}$ & $\begin{array}{l}41 \\
19 \\
54 \\
20 \\
5.5 \\
10 \\
87 \\
7.6 \\
15 \\
9.4\end{array}$ & $\begin{array}{c}74 \\
19 \\
75 \\
27 \\
7.1 \\
15 \\
127 \\
16 \\
10 \\
11\end{array}$ \\
\hline $\begin{array}{l}1970 \\
1971 \\
1972 \\
1973 \\
1972 \\
1975 \\
1976\end{array}$ & $\begin{array}{c}15 \\
16 \\
4.0 \\
36 \\
12 \\
25 \\
5.7\end{array}$ & $\begin{array}{c}15 \\
19 \\
4.8 \\
38 \\
15 \\
26 \\
5.2\end{array}$ & $\begin{array}{c}15 \\
24 \\
5.5 \\
43 \\
17 \\
27 \\
7.1\end{array}$ & $\begin{array}{l}1 E \\
46 \\
6.9 \\
6 \% \\
20 \\
2 \% \\
5.0\end{array}$ & $\begin{array}{l}22 \\
57 \\
8.2 \\
91 \\
26 \\
34 \\
11\end{array}$ & $\begin{array}{r}32 \\
111 \\
12 \\
125 \\
29 \\
55 \\
17\end{array}$ & $\begin{array}{r}44 \\
158 \\
17 \\
164 \\
47 \\
64 \\
20\end{array}$ \\
\hline
\end{tabular}


DRAINAGE AREA: $189 \mathrm{mi}^{2} \quad$ PERIOD OF RECORD: 31 YEARS AVERAGE DISCHARGE: 117 Ft $3 / 5$

MINIMUM AVERAGE FLOWS FOR PERIOD OF RECORD

\begin{tabular}{|c|c|c|c|c|c|c|c|}
\hline FERIOD OF CONSECUTIVE DAYS & 3 & 7 & 14 & 30 & 60 & 120 & 183 \\
\hline $\begin{array}{l}\text { ISCHARGE, IN } \mathrm{ft}^{3} / \mathrm{s} \\
\text { IMATIC YEAR }\end{array}$ & $\begin{array}{l}0 \\
1956\end{array}$ & $\begin{array}{l}0 \\
1956\end{array}$ & 1556 & $\begin{array}{l}0.06 \\
1956\end{array}$ & $\begin{array}{l}0.18 \\
1956\end{array}$ & $\begin{array}{l}0.67 \\
1956\end{array}$ & $\begin{array}{r}1.8 \\
1956\end{array}$ \\
\hline
\end{tabular}

MAGNITUDE AND FREQUENCY OF ANNUAL LOW FLOWS

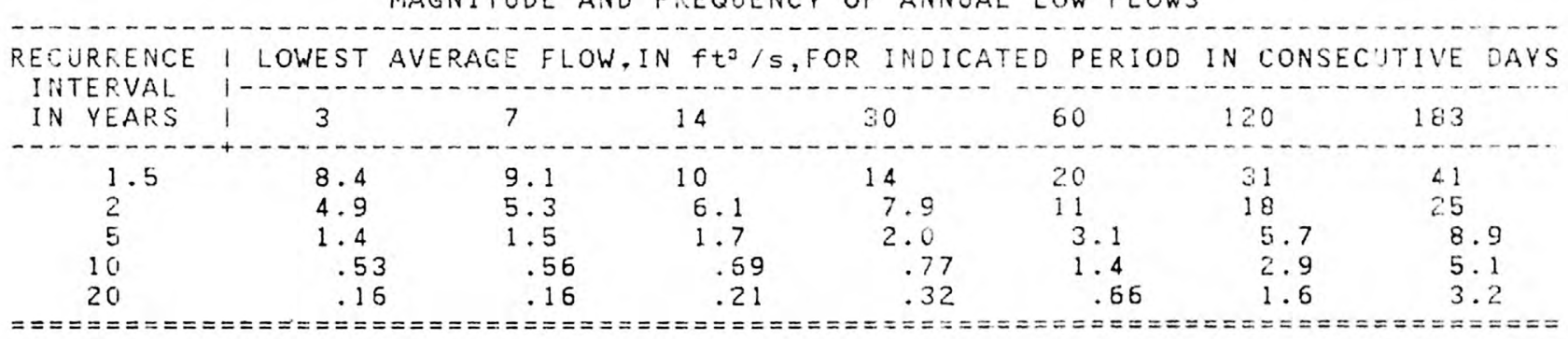

MAGNITUDE AND FREQUENCY OF SEASONAL LOW FLOWS

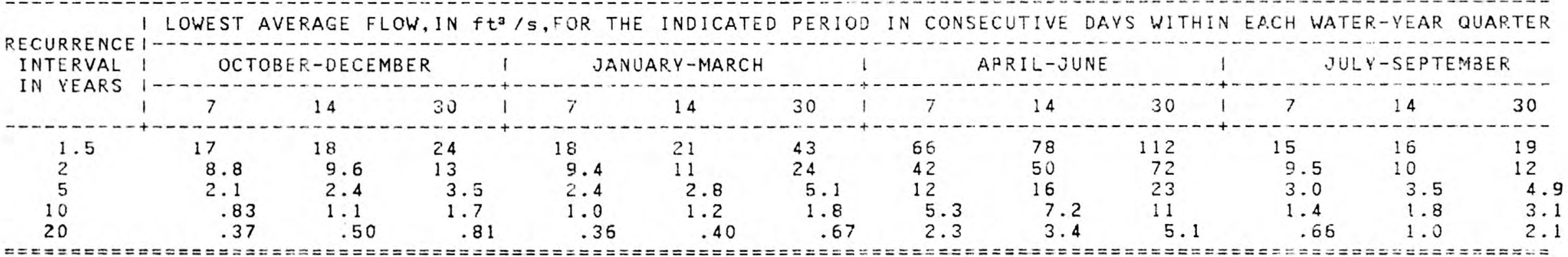

DURATION OF DAILY DISCHARGES FOR ANHUAL AND SEASONAL FERIODS

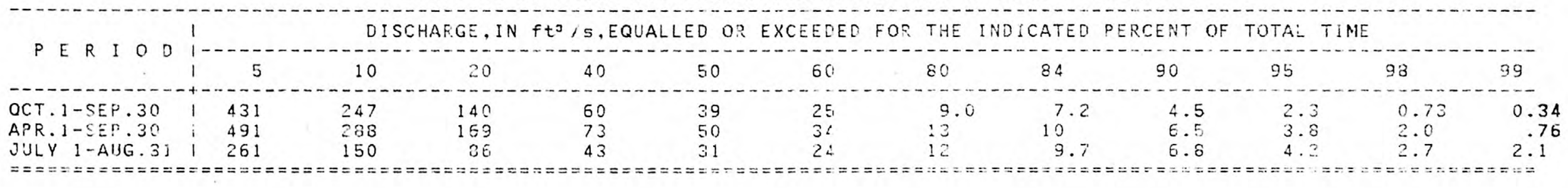




\section{IOWA RIVER AT MARENGO}

Location.--Lat 41048.41", long $92^{\circ} 03^{\prime} 42^{\prime \prime}$, in SW1/4 NE1/4 sec.24, T.81 N., R.1 W., Iowa County, on right bank $10 \mathrm{ft}$ downstream from abandoned highwa bridge, $0.7 \mathrm{mile}$ downstream from Big Bear Creek, $0.5 \mathrm{mlle}$ north of Marengo.

4.9 miles upstream from Hilton Creek. arid at mile 139.4 .

LOW FLOW DISCHARGES, IN YEAR. ENDING MARCH 31

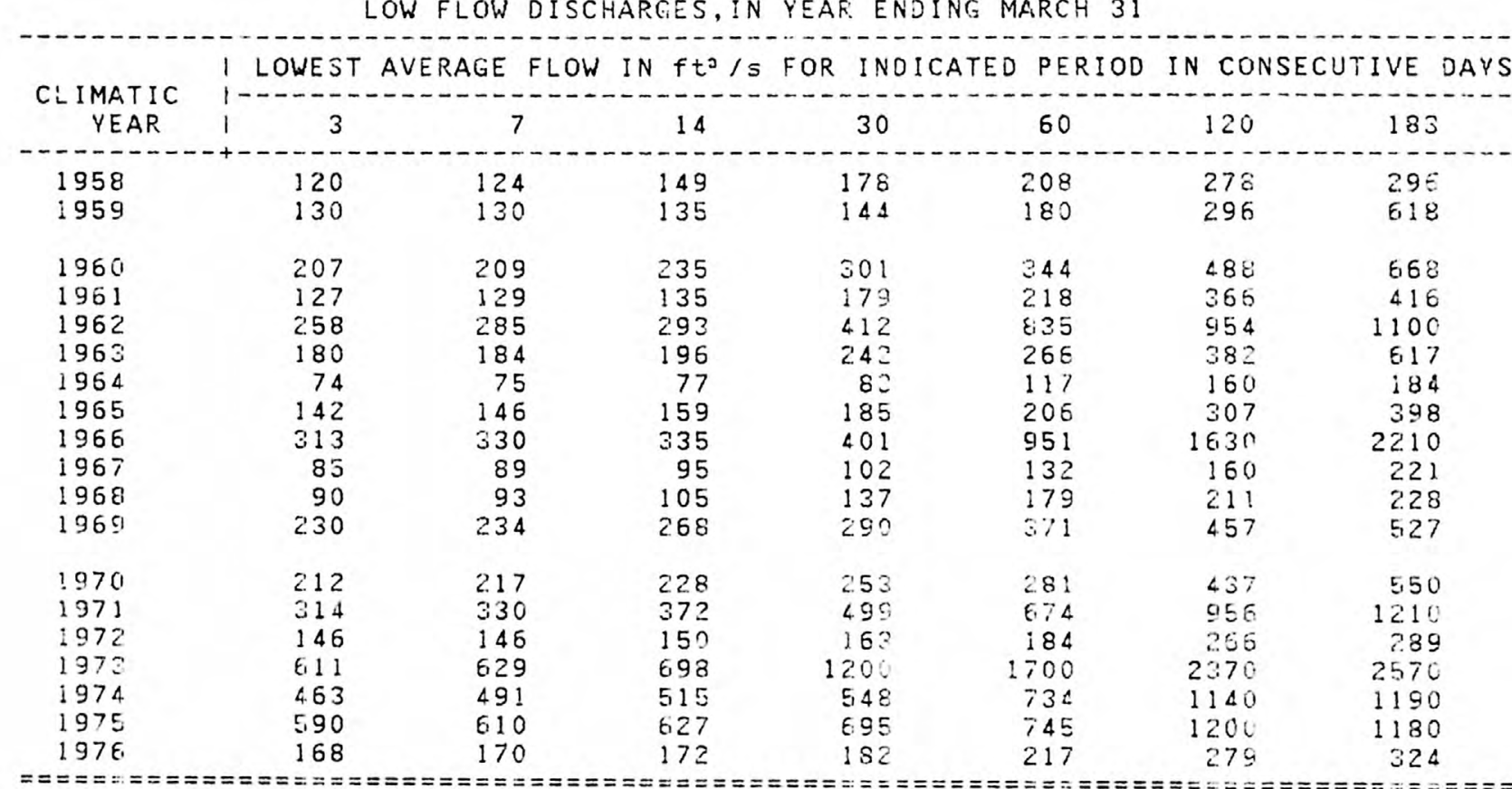




\begin{tabular}{l} 
DRAINAGE AREA: $2794 \mathrm{~m}^{2} \quad$ PERIOD OF RECORD: 20 VEARS AVERAGE DISCHARGE: $1724 \mathrm{ft}^{3} / \mathrm{S}$ \\
MINIMUN AVERAGE FLOWS FOR PERIOD OF RECORD \\
\hline PERIOD OF CONSECUTIVE DAYS I
\end{tabular}

MAGNITUDE AND FREQUENCY OF AYNUAL LOW FLOWS

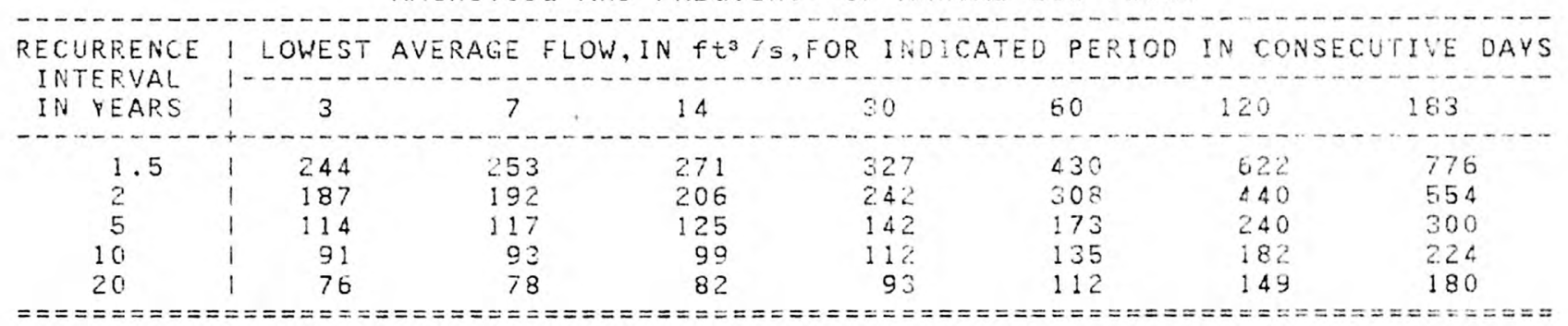

MAGNITUDE AND FREQUENCY OF SEASONAL LOW FLOWS

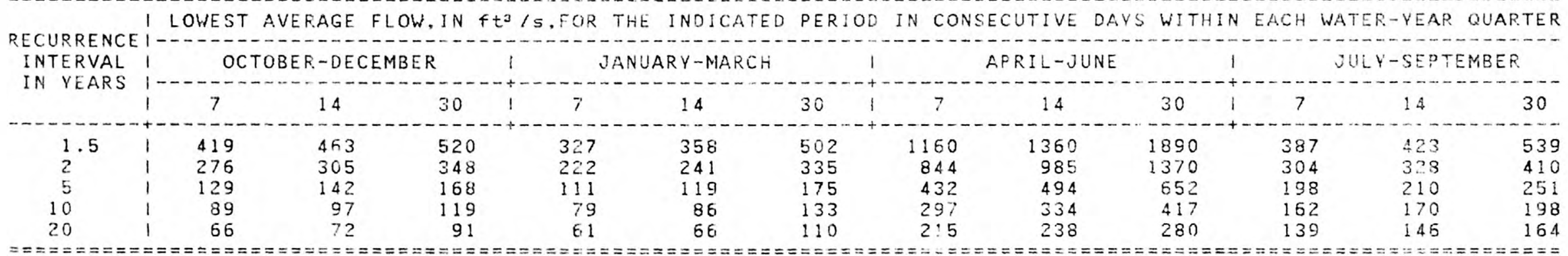

DURATION OF DAILY DISCHARGES FOR ANNUAL AND SEASONAL FERIODS

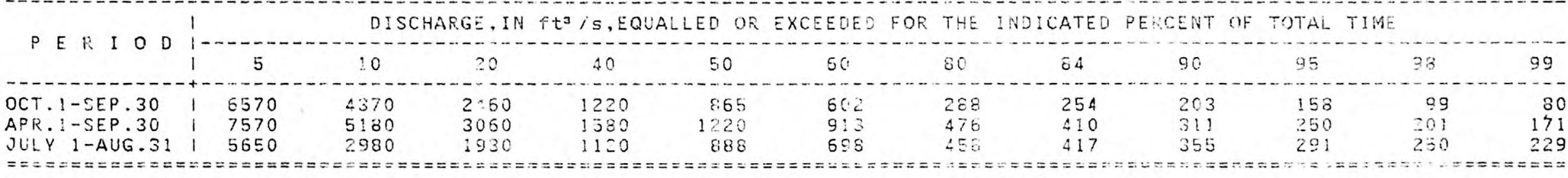


Location.--Lat 41041'19", long 91029'15", in NE1/4 NE1/4 sec.36. T.80 N., R.6 W. Johnson County, on left bank $80 \mathrm{ft}$ upstream from bridge on State Highway $1,3.5$ miles northeast of lowa City, and 4.7 miles upstream from moutr: .

LOW FLOW DISCHARGES, IN VEAR ENDING MARCH 31

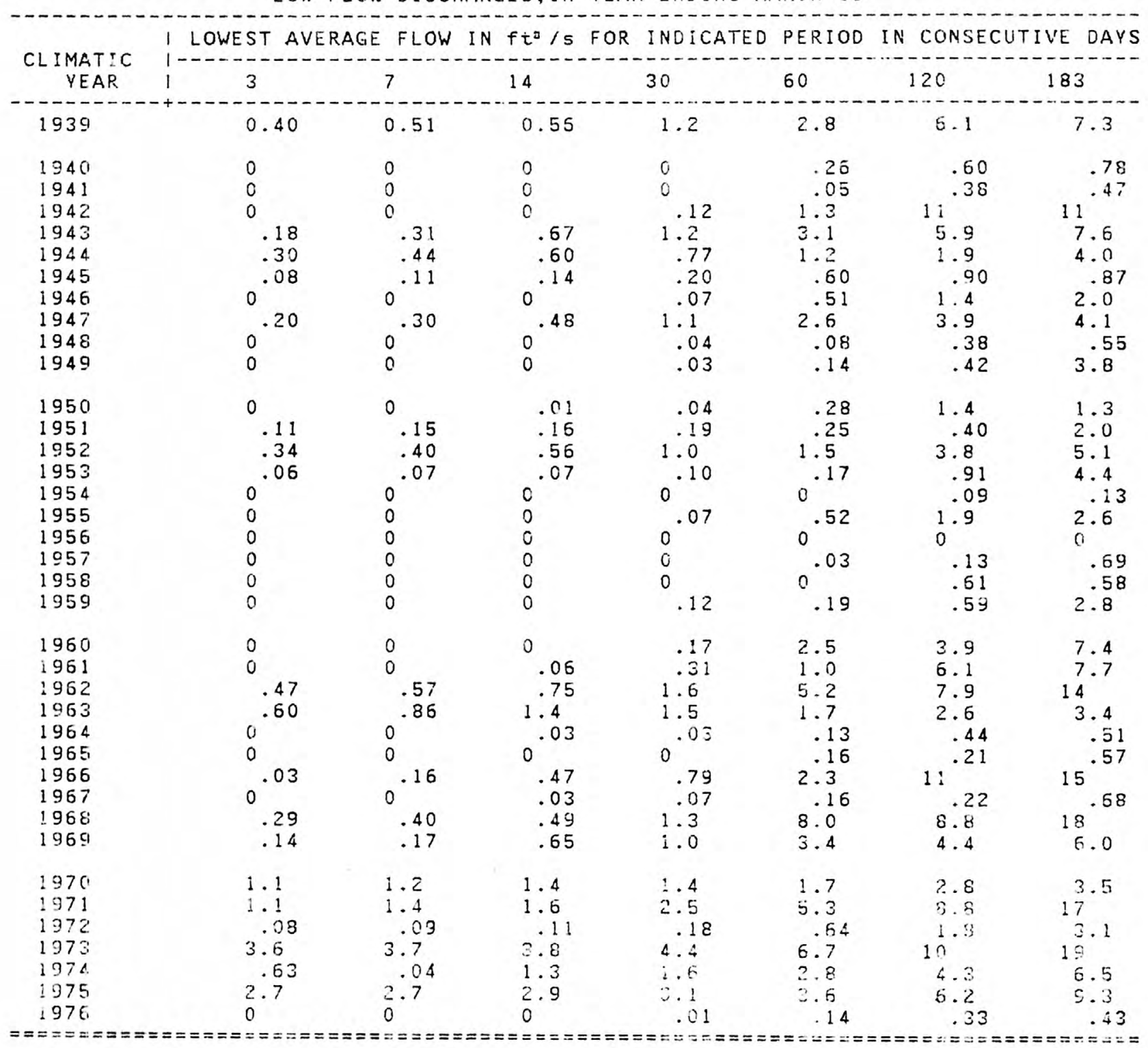


DRAINAGE AREA: $25.3 \mathrm{~m}^{2}$ PERIOD OF RECORD: 39 VEARS AVERAGE DISCHARGE: 15.4 ft $3 / \mathrm{S}$

MINIMUM AVEPAGE FLOWS FOR PERIOD OF RECORD

\begin{tabular}{|c|c|c|c|c|c|c|c|}
\hline PEPIOD OF CONSECUTIVE DAYS I & 3 & 7 & 14 & 30 & 60 & 120 & 183 \\
\hline $\begin{array}{l}\text { ISCHARGE, IN } \mathrm{ft}^{\circ} / \mathrm{s} \\
\text { LIMATIC YEAR }\end{array}$ & $\begin{array}{l}0 \\
1976\end{array}$ & $\begin{array}{c}0 \\
1976\end{array}$ & $\begin{array}{l}0 \\
1976\end{array}$ & $\begin{array}{c}0 \\
1965\end{array}$ & $\begin{array}{c}0 \\
1958\end{array}$ & $\begin{array}{c}0 \\
1956\end{array}$ & $\begin{array}{l}0 \\
1956\end{array}$ \\
\hline
\end{tabular}

MAGNITUDE AND FREQUENCY OF ANNUAL LOW FLOWS

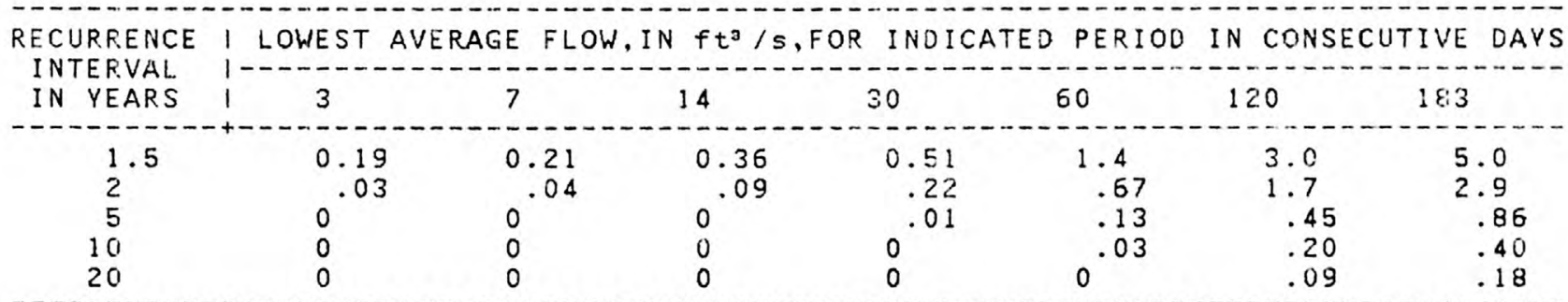

MAGNITUDE AND FREQUENCY OF SEASONAL LOW FLOWS

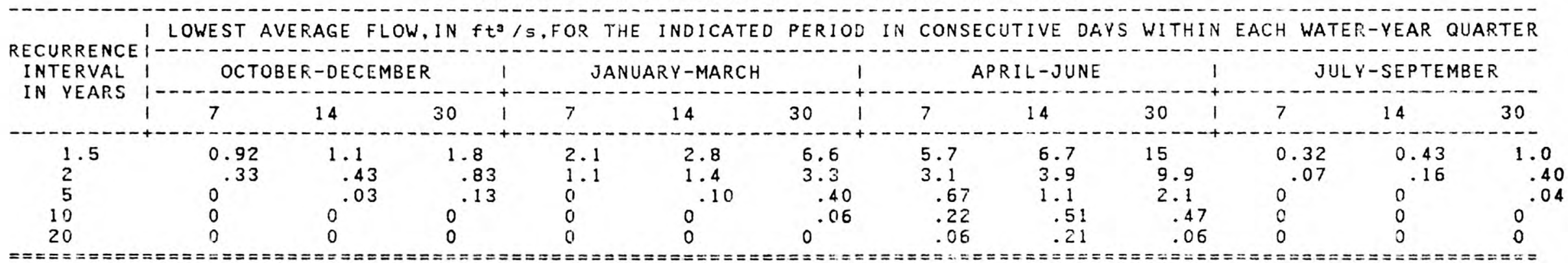

DURATION OF DAILY DISCHARGES FOR ANNIAL AND SEASONAL PERIODS

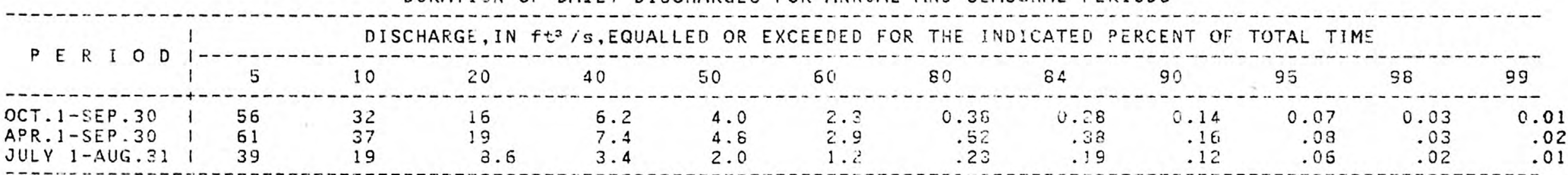


Location.--Lat 41040'36", long 91035'55", in NE1/4 SE1/4 sec.1, T.79 N., R.7 W. Johnson County, on left bank about $50 \mathrm{ft}$ upstream from bridge on county
highway, 1.i miles west of Post office in Coralville, 1.5 miles downstream from Deer Creek and 2.7 milies upstream from mouth.

LOW FLOW DISCHARGES, IN YEAR. ENDING MARCH 31

\begin{tabular}{|c|c|c|c|c|c|c|c|}
\hline \multirow{2}{*}{$\begin{array}{c}\text { CLIMATI IC } \\
\text { YEAR }\end{array}$} & I LOWEST AL & E FLOV & $f t^{3} / s$ & I $P: D I \subseteq A$ & PER IOD & IN CONS & WVE DAYS \\
\hline & $i$ & 7 & 14 & 30 & 60 & 120 & 183 \\
\hline $\begin{array}{l}1954 \\
1955 \\
1956 \\
1957 \\
1958 \\
1959\end{array}$ & $\begin{array}{r}0.31 \\
.57 \\
.30 \\
.17 \\
.20 \\
2.4\end{array}$ & $\begin{array}{r}0.31 \\
.84 \\
.30 \\
.31 \\
.31 \\
2.9\end{array}$ & $\begin{array}{c}0.31 \\
1.2 \\
.30 \\
.44 \\
.40 \\
3.4\end{array}$ & $\begin{array}{l}0.32 \\
1.7 \\
.41 \\
.72 \\
.49 \\
4.2\end{array}$ & $\begin{array}{c}0.40 \\
2.0 \\
.55 \\
1.4 \\
.71 \\
4.7\end{array}$ & $\begin{array}{r}1.7 \\
2.5 \\
.71 \\
1.6 \\
1.7 \\
9.3\end{array}$ & $\begin{array}{r}1.4 \\
6.9 \\
1.0 \\
6.2 \\
1.6 \\
25\end{array}$ \\
\hline $\begin{array}{l}1960 \\
1961 \\
1962 \\
1963 \\
1964 \\
1965 \\
1966 \\
1967 \\
1968 \\
1969\end{array}$ & $\begin{array}{l}2.6 \\
4.3 \\
1.6 \\
7.4 \\
.70 \\
2.1 \\
7.4 \\
2.1 \\
1.6 \\
1.5\end{array}$ & $\begin{array}{l}2.6 \\
4.5 \\
2.4 \\
7.7 \\
.70 \\
2.4 \\
9.9 \\
2.5 \\
1.7 \\
2.1\end{array}$ & $\begin{array}{c}2.8 \\
4.9 \\
2.7 \\
8.1 \\
.71 \\
3.2 \\
12 . \\
3.0 \\
2.1 \\
2.9\end{array}$ & $\begin{array}{r}3.8 \\
6.6 \\
4.7 \\
9.4 \\
1.0 \\
4.2 \\
15 \\
3.2 \\
5.9 \\
3.1\end{array}$ & $\begin{array}{l}10 \\
7.2 \\
12 \\
10.0 \\
2.2 \\
4.9 \\
37 \\
4.9 \\
13 \\
4.7\end{array}$ & $\begin{array}{c}22 . \\
23 \\
17 \\
15 \\
3.4 \\
6.5 \\
53 \\
5.2 \\
23 \\
6.2\end{array}$ & $\begin{array}{c}37 \\
19 \\
26 \\
18 \\
3.9 \\
12 \\
74 \\
9.0 \\
33 \\
8.4\end{array}$ \\
\hline $\begin{array}{l}1970 \\
1971 \\
1972 \\
1973 \\
1974 \\
1975 \\
1976\end{array}$ & $\begin{array}{c}10 \\
8.1 \\
1.6 \\
16 \\
11 \\
12 . \\
2.3\end{array}$ & $\begin{array}{c}10 \\
9.5 \\
1.8 \\
16 \\
12 \\
12 \\
2.7\end{array}$ & $\begin{array}{r}11 \\
9.9 \\
1.9 \\
18 \\
17 \\
12 \\
3.3\end{array}$ & $\begin{array}{l}12 \\
12 \\
2 . E \\
22 \\
20 \\
13 \\
2.8\end{array}$ & $\begin{array}{l}14 \\
21 \\
3.9 \\
32 \\
26 \\
21 \\
5.0\end{array}$ & $\begin{array}{c}22 \\
45 \\
6.6 \\
51 \\
39 \\
37 \\
7.3\end{array}$ & $\begin{array}{l}28 \\
57 \\
12 \\
91 \\
50 \\
35 \\
10\end{array}$ \\
\hline
\end{tabular}


DRAINAGE AREA: $98.1 \mathrm{~m}^{2}$ PERIOD OF RECORD: 24 YEARS AVERAGE DISCHARGE: 63.4 ft3/s

MINIMUM AVERAGE FLOWS FOR PERIOD OF RECORD

\begin{tabular}{|c|c|c|c|c|c|c|c|}
\hline IOD OF CONSECUTIVE DAYS & 3 & 7 & $1 t_{*}$ & 30 & 60 & 120 & 183 \\
\hline $\begin{array}{l}\text { HARGE, IN } \mathrm{ft}^{3} / \mathrm{s} \\
\text { ATIC YEAR }\end{array}$ & $\begin{array}{r}0.18 \\
1957^{8}\end{array}$ & $\begin{array}{l}0.31 \\
1956\end{array}$ & $\begin{array}{l}0.31 \\
1956\end{array}$ & $\begin{array}{l}0.32 \\
1954\end{array}$ & $\begin{array}{l}0.40 \\
1954\end{array}$ & $\begin{array}{l}0.71 \\
1956\end{array}$ & $\begin{array}{r}1.0 \\
1956\end{array}$ \\
\hline
\end{tabular}

MAGNITUDE AND FREQUENCY OF ANNUAL LOW FLOWS

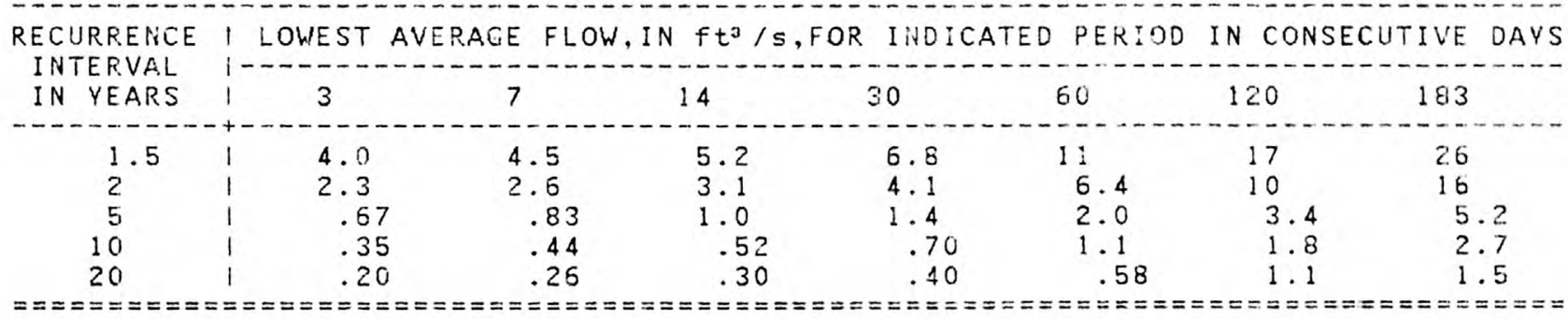

MAGNITUDE AND FREQUENCY OF SEASONAL LOW FLOWS

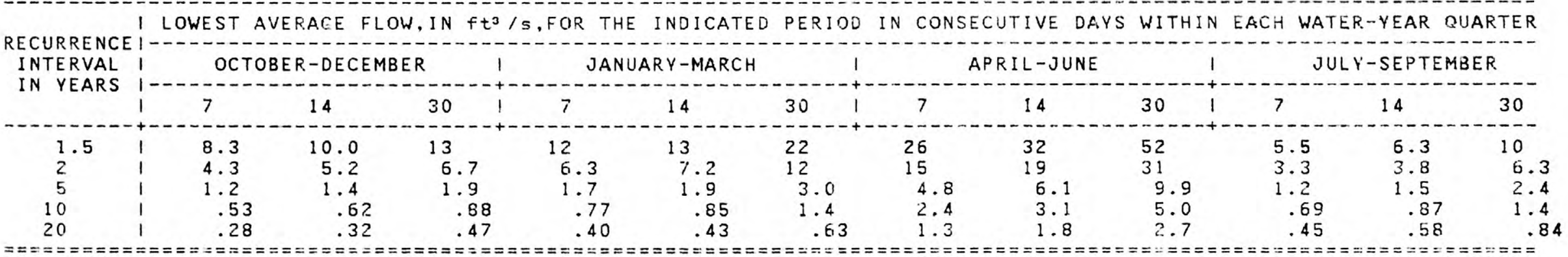

DURATION OF DAILY DISCHARGES FOR ANNUAL AND SEASONAL FERIODS

I DISCHARGE, IN ft3/S, EQUALLEQ OR EXCEEUEO FOR THE INDICATED PERCENT OF TOTAL TIME

\begin{tabular}{|c|c|c|c|c|c|c|c|c|c|c|c|c|}
\hline . & 5 & 10 & 20 & 40 & 50 & 60 & 80 & 64 & 90 & 95 & 98 & 99 \\
\hline $\begin{array}{l}\text { OCT.1-SEP. } 30 \\
\text { APR. } 1-S E P .30 \\
\text { JUL } 1 \text { 1-AUG.31 }\end{array}$ & $\begin{array}{l}230 \\
1 \quad 261 \\
1 \quad 188\end{array}$ & $\begin{array}{r}133 \\
161 \\
96\end{array}$ & $\begin{array}{l}73 \\
88 \\
49\end{array}$ & $\begin{array}{l}32 \\
36 \\
21\end{array}$ & $\begin{array}{l}22 \\
2.5 \\
14\end{array}$ & $\begin{array}{l}13 \\
16 \\
9.8\end{array}$ & $\begin{array}{l}4.1 \\
5.1 \\
4.2\end{array}$ & $\begin{array}{l}3.3 \\
4.1 \\
3.3\end{array}$ & $\begin{array}{l}1.9 \\
2.5 \\
2.1\end{array}$ & $\begin{array}{l}1.0 \\
1.3 \\
1.3\end{array}$ & $\begin{array}{r}0.53 \\
.70 \\
.74\end{array}$ & $\begin{array}{r}0.34 \\
.54 \\
.62\end{array}$ \\
\hline
\end{tabular}


Location.--Lat $41039.24^{\mathrm{n}}$, long $91032127^{\mathrm{n}}$, in SE1/4 SE1/4 sec.9, T.79 N., R.6 W. Johnson County, on right bank $25 \mathrm{ft}$ downstream from Hydraulics Laboratory of University of Iowa in Iowa City, $175 \mathrm{ft}$ downstream from University Dam, $0.8 \mathrm{mile}$ upstream from Ralston Creek, $3.6 \mathrm{mfles}$ downstream from Clear Creek, and at $\mathrm{mile} 74.2$.

Remarks.--Slight fluctuation at low stages caused by powerplant at Iowa City. Flow regulated by Coralville Lake, 9.1 miles upstream, since Sept. 17. 1958.

LOW FLOW DISCHARGES, IN YEAR. ENDING MARCH 31

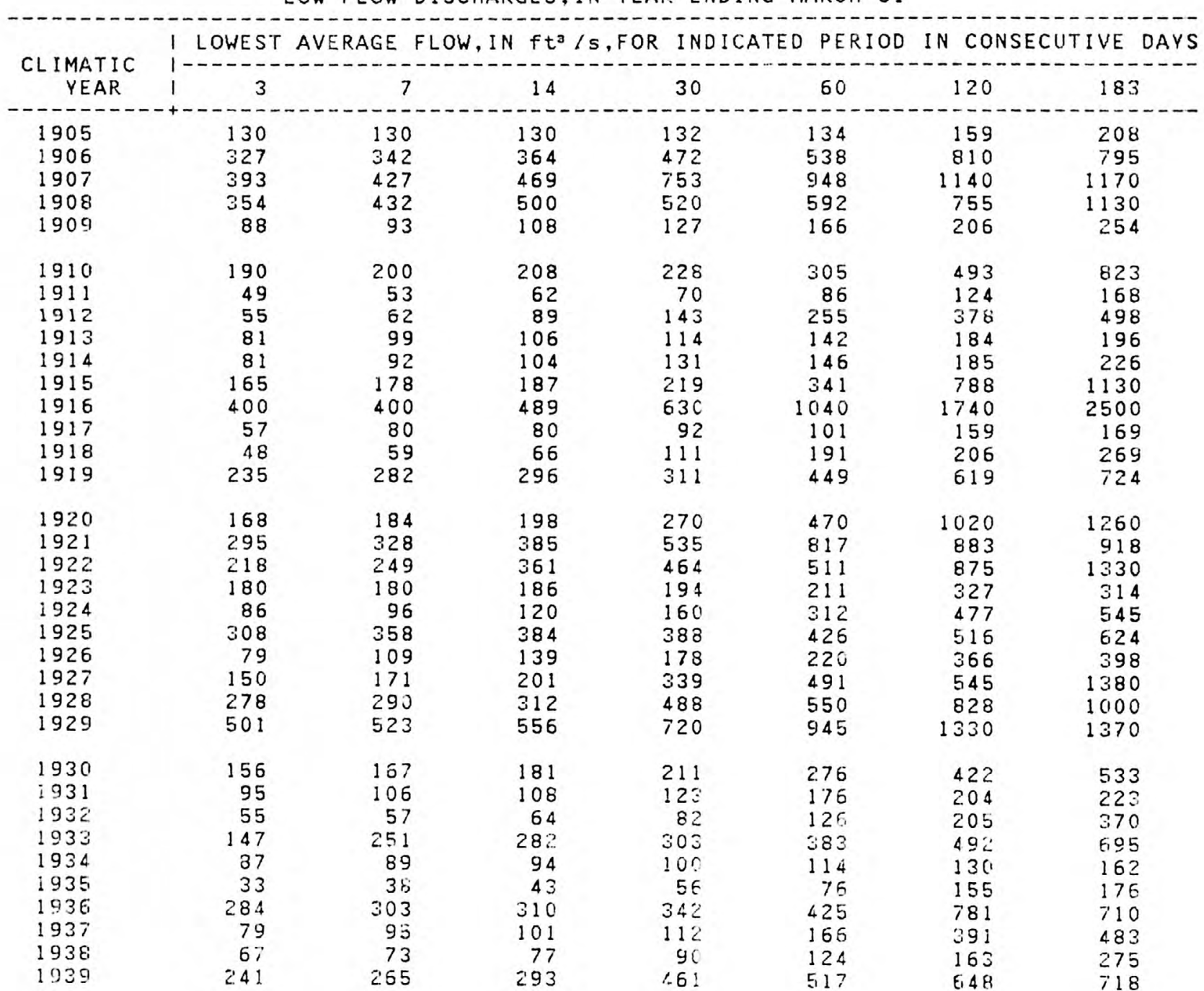


IOWA RIVER AT IOWA CITY--Continued

LOW FLOW DISCHARGES, IN YEAR ENDING MARCH 31--Continued

\begin{tabular}{|c|c|c|c|c|c|c|c|c|}
\hline \multirow{2}{*}{$\begin{array}{c}\text { CLIMATIC } \\
\text { YEAR }\end{array}$} & I LOWEST & $A G E$ & $f t^{2}$ & $R$ IN & $E D P E$ & IN C & \multicolumn{2}{|c|}{ IVE DAYS } \\
\hline & 3 & 7 & 14 & 30 & 60 & 120 & 183 & \\
\hline $\begin{array}{l}1940 \\
1941 \\
1942 \\
1943 \\
1944 \\
1945 \\
1946 \\
1947 \\
1948 \\
1949\end{array}$ & $\begin{array}{r}34 \\
91 \\
137 \\
441 \\
260 \\
235 \\
262 \\
360 \\
148 \\
120\end{array}$ & $\begin{array}{r}34 \\
94 \\
138 \\
519 \\
273 \\
242 \\
284 \\
427 \\
151 \\
124\end{array}$ & $\begin{array}{r}38 \\
101 \\
154 \\
543 \\
287 \\
246 \\
299 \\
473 \\
155 \\
134\end{array}$ & $\begin{array}{r}39 \\
141 \\
192 \\
654 \\
329 \\
257 \\
399 \\
604 \\
182 \\
153\end{array}$ & $\begin{array}{r}49 \\
182 \\
388 \\
784 \\
470 \\
276 \\
506 \\
676 \\
280 \\
174\end{array}$ & $\begin{array}{r}92 \\
2.80 \\
862 \\
998 \\
602 \\
362 \\
527 \\
1120 \\
400 \\
205\end{array}$ & $\begin{array}{r}105 \\
286 \\
1040 \\
1200 \\
85 \\
51 \\
781 \\
1220 \\
37 \\
338\end{array}$ & \\
\hline $\begin{array}{l}1950 \\
1951 \\
1952 \\
1953 \\
1954 \\
1955 \\
1956 \\
1957 \\
1958\end{array}$ & $\begin{array}{r}93 \\
89 \\
410 \\
138 \\
65 \\
216 \\
44 \\
59 \\
142\end{array}$ & $\begin{array}{r}105 \\
91 \\
552 \\
148 \\
66 \\
292 \\
45 \\
70 \\
146\end{array}$ & $\begin{array}{r}115 \\
93 \\
717 \\
154 \\
66 \\
321 \\
46 \\
76 \\
170\end{array}$ & $\begin{array}{r}132 \\
96 \\
745 \\
163 \\
71 \\
346 \\
52 \\
94 \\
203\end{array}$ & $\begin{array}{r}149 \\
107 \\
1060 \\
176 \\
83 \\
482 \\
57 \\
102 \\
236\end{array}$ & $\begin{array}{r}190 \\
146 \\
1330 \\
251 \\
109 \\
714 \\
78 \\
111 \\
301\end{array}$ & $\begin{array}{r}210 \\
205 \\
1510 \\
320 \\
130 \\
1140 \\
100 \\
22 \\
322\end{array}$ & \\
\hline
\end{tabular}




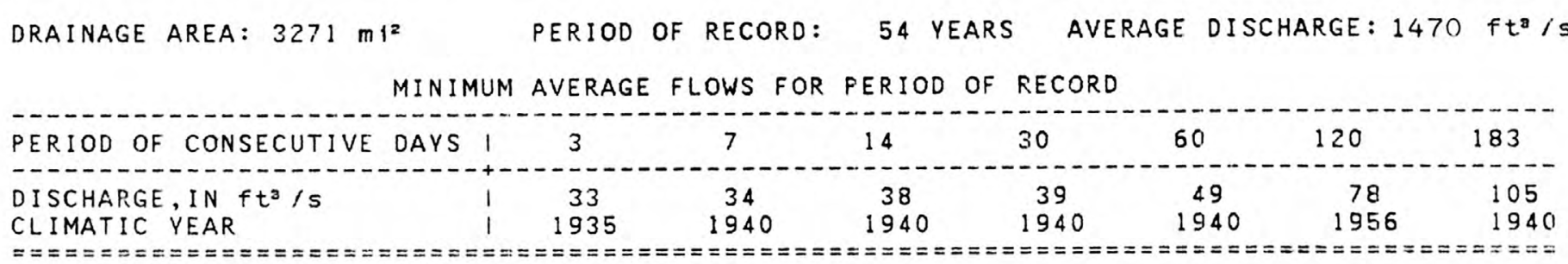

MAGNITUDE AND FREQUENCY OF ANNUAL LOW FLOWS

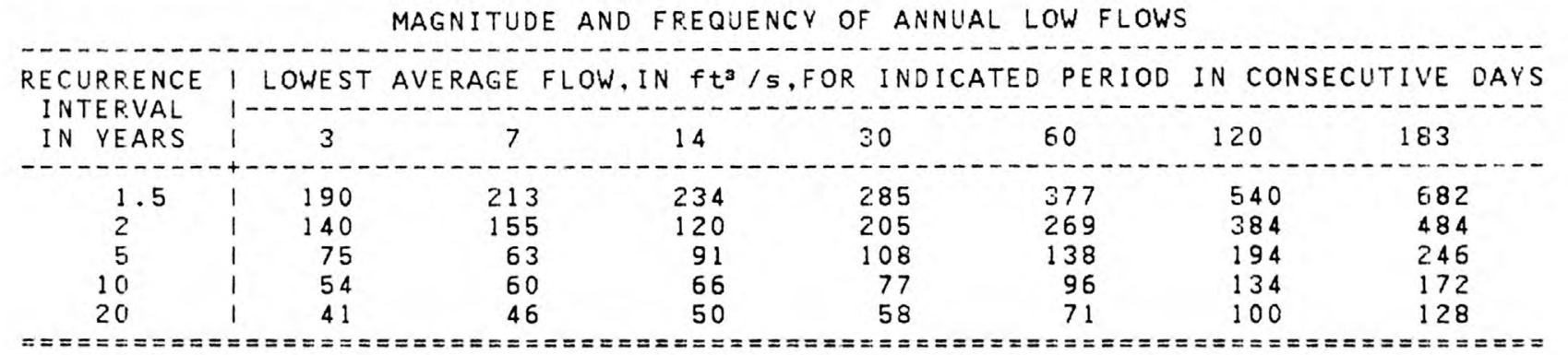

MAGNITUDE AND FREQUENCY OF SEASONAL LOW FLOWS

LOWEST AVERAGE FLOW, IN $\mathrm{ft}^{3} / \mathrm{s}, \mathrm{FOR}$ THE INDICATED PERIOD IN CONSECUTIVE DAYS WITHIN EACH WATER-YEAR QUARTER

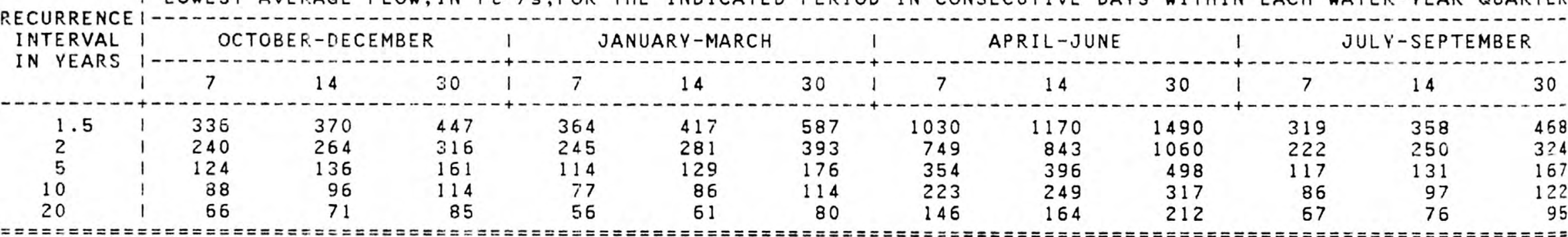

DURATION OF DAILY DISCHARGES FOR ANNUAL AND SEASONAL PERIODS

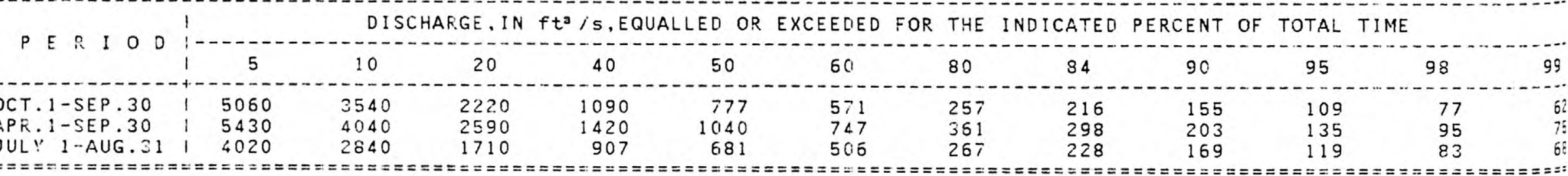


IOWA RIVER AT IOWA CITY--Continued, regulated period

LOW FLOW DISCHARGES, IN YEAR. ENDING MARCH 31

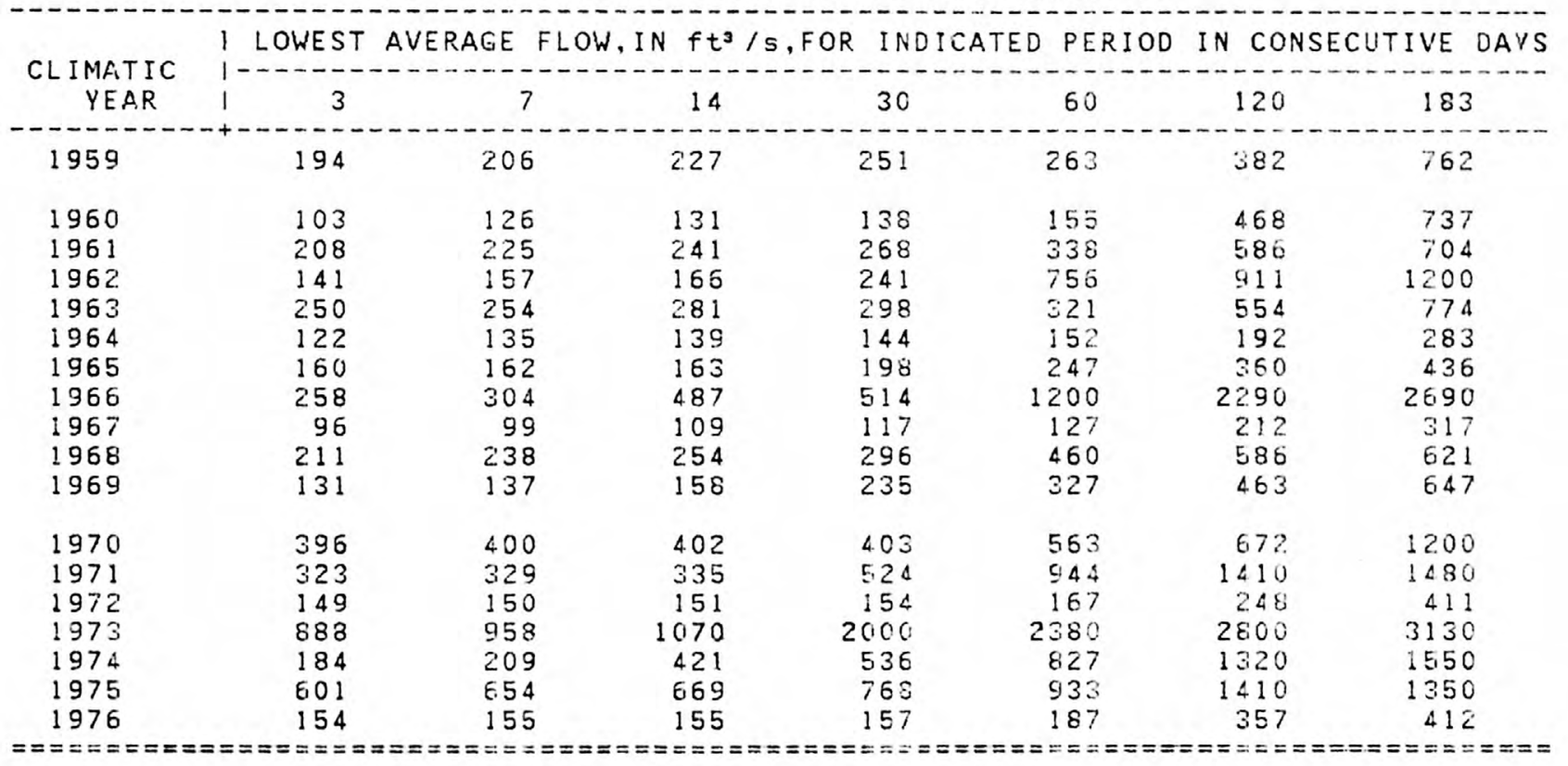




DRAINAGE AREA: $3271 \mathrm{~m}^{2} \quad$ PERIOD OF RECORD: 18 YEARS AVERAGE DISCHARGE: 2160 ft is
MINIMUM AVERAGE FLOWS FOR FERIOD OF RECORD

MAGNITUDE AND FREQUENCY OF ANNUAL LOW FLOWS

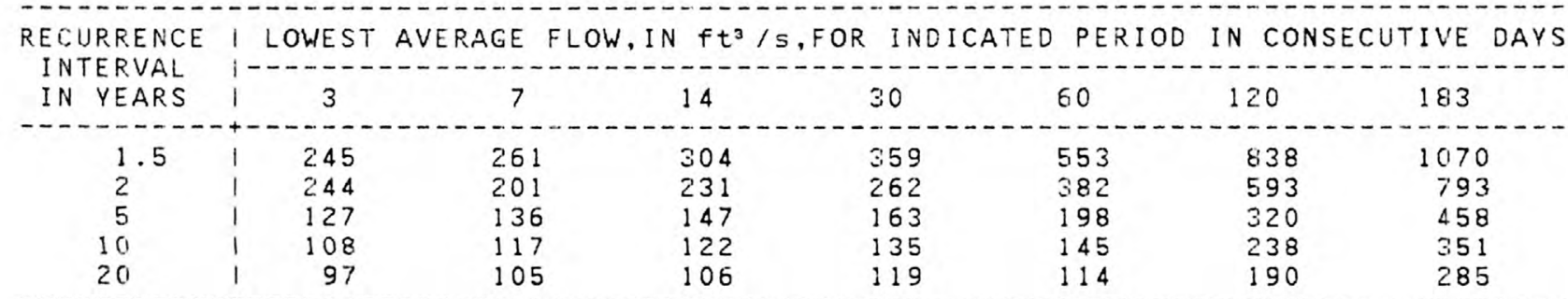

MAGNITUDE AND FREQUENCY OF SEASONAL LOW FLOWS

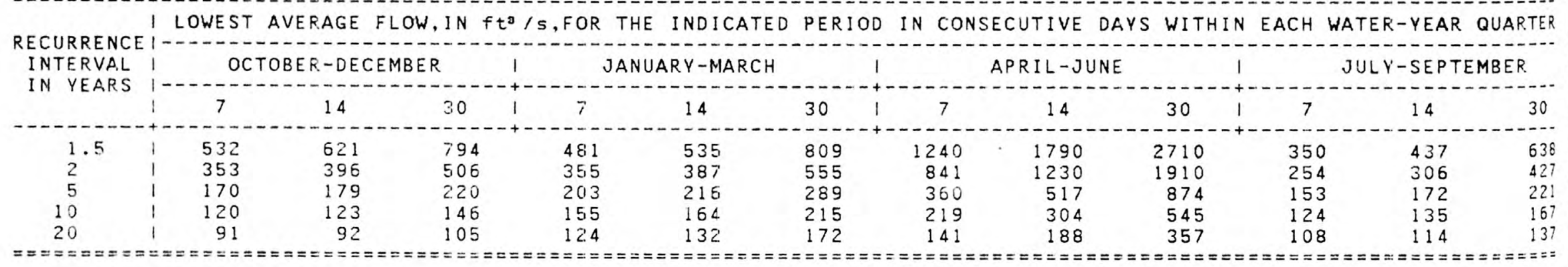

DURATION OF DAILY DISCHARGES FOR ANNUAL AND SEASONAL PERIODS

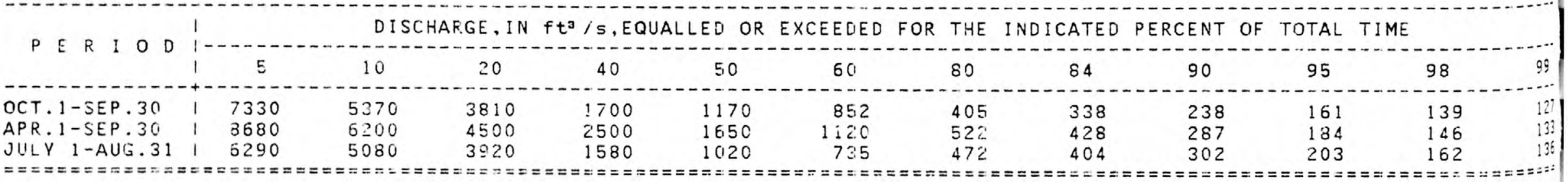


Location.--Lat 41039.50", long 91030'48", in SEl/4 NWl/4 sec.11, T.79 N., R.6 W. Johnson County, on left bank $10 \mathrm{ft}$ upstream from bridge on Rochester Avenue, $1.0 \mathrm{mile}$ northeast of post office in Iowa city and $2.2 \mathrm{miles}$ upstream from mouth.

LOW FLOW DISCHARGES, IN YEAR ENDING MARCH 31

\begin{tabular}{|c|c|c|c|c|c|c|c|}
\hline \multirow{2}{*}{$\underset{\text { CLEAR }}{\text { CLMATIC }}$} & 1 LOWEST A & FLOV & $f t^{3} / s$, & INDICA & PER IOD & IN CONSE & E DAYS \\
\hline & 13 & 7 & 14 & 30 & 60 & 120 & 83 \\
\hline $\begin{array}{l}1926 \\
1927 \\
1928 \\
1929\end{array}$ & $\begin{array}{l}0 \\
0 \\
.02 \\
.06\end{array}$ & $\begin{array}{l}0 \\
0 \\
.02 \\
.07\end{array}$ & $\begin{array}{l}0 \\
0 \\
.02 \\
.11\end{array}$ & $\begin{array}{r}0.02 \\
.07 \\
.04 \\
.30\end{array}$ & $\begin{array}{r}0.04 \\
.48 \\
.09 \\
.47\end{array}$ & $\begin{array}{r}0.25 \\
.89 \\
1.2 \\
1.9\end{array}$ & $\begin{array}{l}0.38 \\
2.0 \\
1.5 \\
1.7\end{array}$ \\
\hline $\begin{array}{l}1930 \\
1931 \\
1932 \\
1933 \\
1934 \\
1935 \\
1936 \\
1937 \\
1938 \\
1939\end{array}$ & $\begin{array}{l}0 \\
0 \\
0 \\
0 \\
0 \\
0 \\
.02 \\
0 \\
0 \\
.02\end{array}$ & $\begin{array}{l}0 \\
0 \\
0 \\
0 \\
0 \\
0 \\
.02 \\
0 \\
0 \\
0.02\end{array}$ & $\begin{array}{l}0 \\
0 \\
0 \\
0 \\
0 \\
0 \\
.03 \\
0 \\
0 \\
0.03 \\
.03\end{array}$ & $\begin{array}{l}0^{.05} \\
.01 \\
.07 \\
0 \\
0 \\
0^{.04} \\
0 \\
.09\end{array}$ & $\begin{array}{l}.18 \\
.01 \\
.10 \\
.28 \\
0 \\
0 \\
.13 \\
0.04 \\
.29\end{array}$ & $\begin{array}{l}.44 \\
.38 \\
.20 \\
.35 \\
.01 \\
.08 \\
.42 \\
.63 \\
.07 \\
.72\end{array}$ & $\begin{array}{l}.41 \\
.49 \\
.37 \\
.70 \\
.05 \\
.23 \\
.35 \\
.85 \\
.32 \\
.74\end{array}$ \\
\hline $\begin{array}{l}1940 \\
1941 \\
1942 \\
1943 \\
1944 \\
1945 \\
1946 \\
1947 \\
1948 \\
1949\end{array}$ & $\begin{array}{l}0 \\
0 \\
0 \\
0 \\
.01 \\
.01 \\
0.01 \\
.0 \\
0\end{array}$ & $\begin{array}{l}0 \\
0 \\
0 \\
0 \\
.02 \\
.01 \\
.0 \begin{array}{l}.01 \\
0.03 \\
0 \\
0\end{array}\end{array}$ & 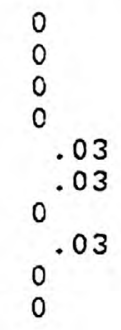 & $\begin{array}{l}0 \\
0 \\
0 \\
0 \\
.04 \\
.03 \\
.01 \\
.11 \\
0 \\
0\end{array}$ & $\begin{array}{l}0 \\
0 \\
.11 \\
.09 \\
.13 \\
.07 \\
.07 \\
.29 \\
.01 \\
.03\end{array}$ & $\begin{array}{r}.04 \\
.01 \\
1.3 \\
.61 \\
.20 \\
.12 \\
.24 \\
.42 \\
.06 \\
.06\end{array}$ & $\begin{array}{r}.05 \\
.06 \\
1.7 \\
.82 \\
.47 \\
.12 \\
.33 \\
.54 \\
.03 \\
.52\end{array}$ \\
\hline $\begin{array}{l}1950 \\
1951 \\
1952 \\
1953 \\
1954 \\
1955 \\
1956 \\
1957 \\
1959 \\
1959\end{array}$ & $\begin{array}{ll}0 & \\
& .01 \\
.05 \\
.01 \\
0 & \\
0 & \\
0 & \\
0 & \\
0 & \\
0 & \end{array}$ & $\begin{array}{ll}0 & \\
& .01 \\
.06 \\
.01 \\
0 & .01 \\
0 & \\
0 & \\
0 & \\
0 & \\
0 & \end{array}$ & $\begin{array}{ll}0 & \\
& .01 \\
& .09 \\
& .01 \\
0 & \\
0 & \\
0 & \\
0 & \\
0 & \\
0 & \end{array}$ & $\begin{array}{ll}0 & \\
.02 \\
.16 \\
.01 \\
0 \\
0 \\
0 \\
0 \\
0 \\
0 \\
\\
.01\end{array}$ & $\begin{array}{l}.02 \\
.07 \\
.24 \\
.03 \\
0.03 \\
0 \\
0 \\
0 \\
0 \\
.03\end{array}$ & $\begin{array}{r}.14 \\
.10 \\
.43 \\
.14 \\
.01 \\
.15 \\
0.01 \\
.01 \\
.04 \\
.09\end{array}$ & $\begin{array}{r}.11 \\
.30 \\
.56 \\
.68 \\
.01 \\
.23 \\
0.08 \\
.08 \\
.05 \\
.51\end{array}$ \\
\hline $\begin{array}{l}1260 \\
1961 \\
1962 \\
1963\end{array}$ & $\begin{array}{l}0 \\
.02 \\
.10 \\
.10\end{array}$ & $\begin{array}{r}0 \\
.02 \\
.10 \\
.14\end{array}$ & $\begin{array}{l}.01 \\
.03 \\
.12 \\
.22\end{array}$ & $\begin{array}{l}.07 \\
.08 \\
.39 \\
.23\end{array}$ & $\begin{array}{l}.59 \\
.21 \\
.65 \\
.33\end{array}$ & $\begin{array}{r}.70 \\
.80 \\
1.8 \\
.39\end{array}$ & $\begin{array}{r}1.1 \\
1.0 \\
1.8 \\
.46\end{array}$ \\
\hline
\end{tabular}


$05-4550.00$ (1) 
05-4550.00 RALSTON CREEK AT IOWA CITY--Continued

DRAINAGE AREA: $3.01 \mathrm{~m} \mathbf{1}^{2} \quad$ PERIOD OF RECORD: 52 YEARS AVERAGE DISCHARGE: $1.7 \mathrm{ft}$ I
MINIMUM AVERAGE FLOWS FOR PERIOD OF RECORD

MAGNITUDE AND FREQUENCY OF ANNUAL LOW FLOWS

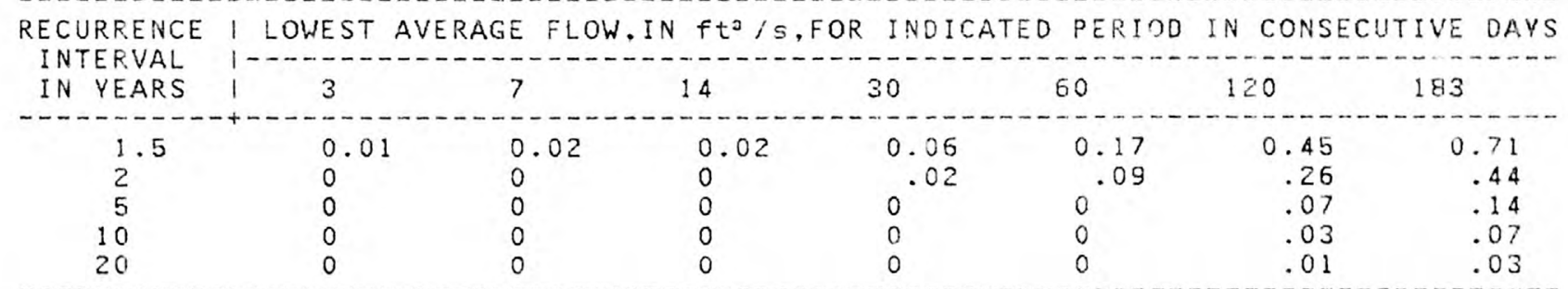

MAGNITUDE AND FREQUENCY OF SEASONAL LOW FLOWS

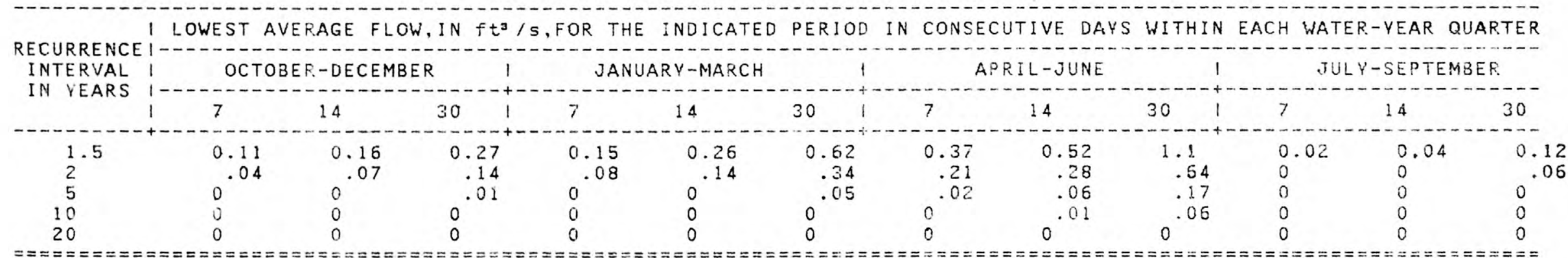

DURATION OF DAILY DISCHARGES FOR ANNUAL AND SEASONAL PERIODS

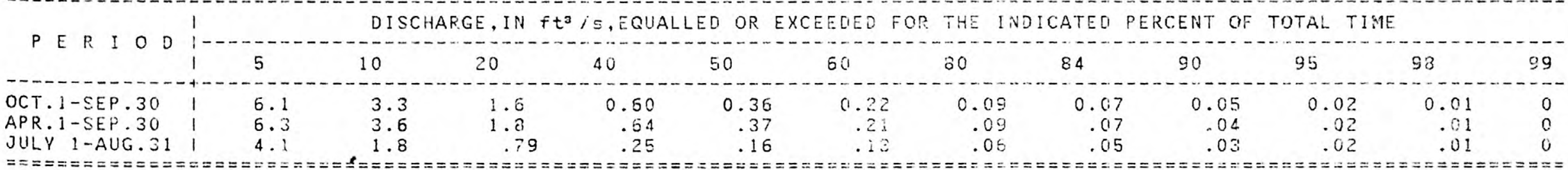


Location.--Lat 41039'05", long 92030'27", in SW1/4 NE1/4 sec.14, T.79 N., R.6 W., Johnson County, on right bank $60 \mathrm{ft}$ downstream from bridge on Muscatine Avenue in Iowa City, and 1.2 miles upstream from mouth.

LOW FLOW DISCHARGES, IN YEAR ENDING MARCH 31

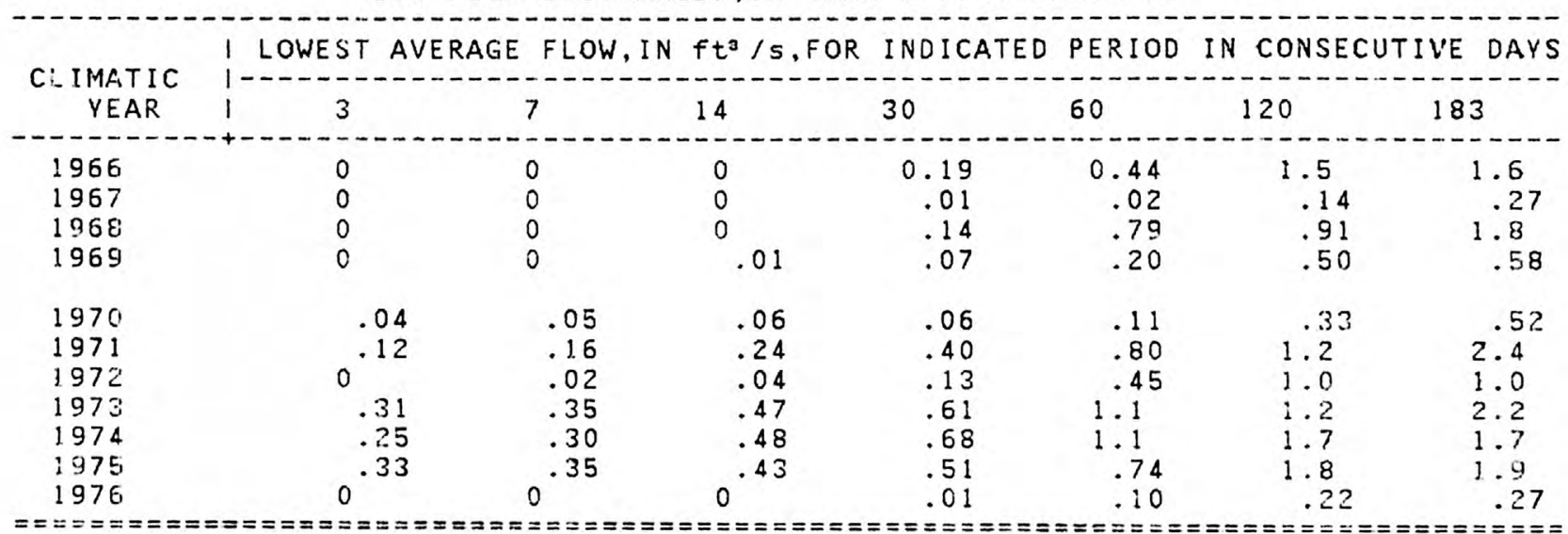


05-4550.10 SOUTH BRANCH RALSTON CREEK AT IOWA CITY--Continued

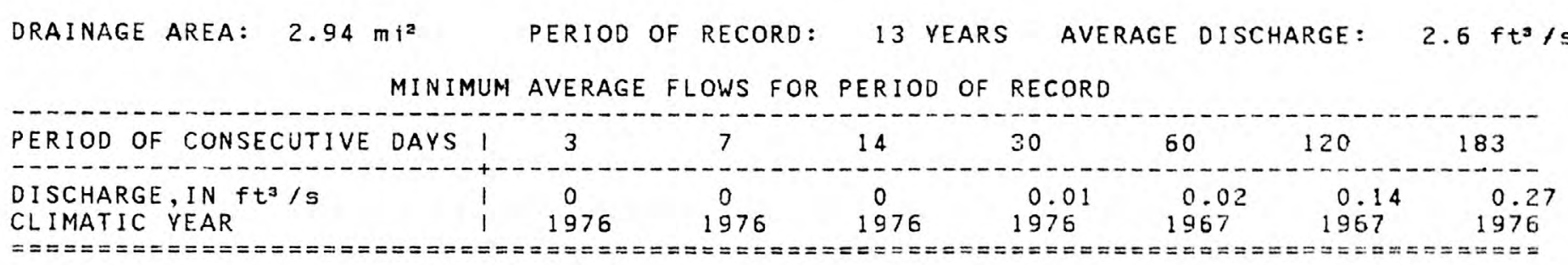

MAGNITUDE AND FREQUENCY OF ANNUAL LOW FLOWS

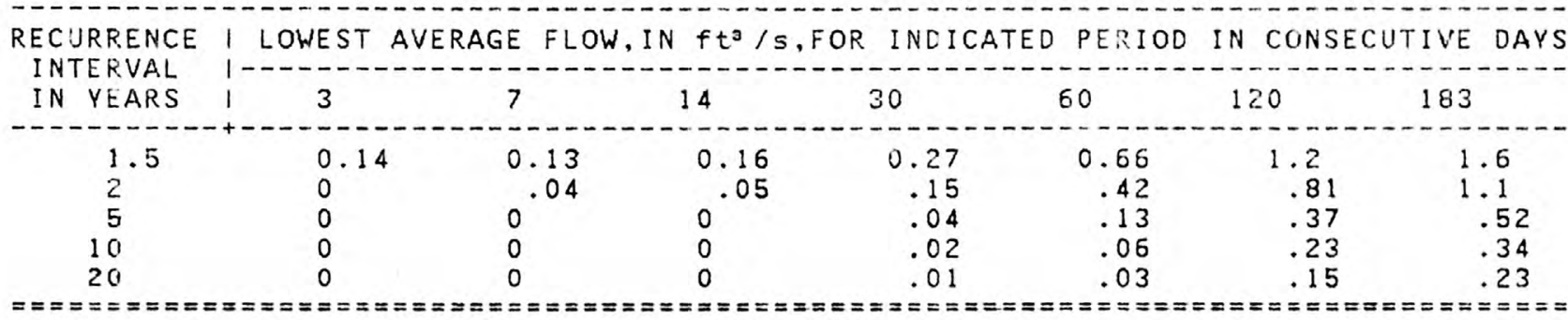

MAGNITUDE AND FREQUENCY OF SEASONAL LOW FLOWS

LOWEST AVERAGE FLOW, IN $\mathrm{ft}^{\circ} / \mathrm{s}, F O R$ THE INDICATED PERIOD IN CONSECUTIVE DAYS WITHIN EACH WATER-YEAR QUARTER

RECURRENCE
INTERVAL
IN YEARS

DURATION OF DAILY DISCHARGES FOR ANNUAL AND SEASONAL PERIODS

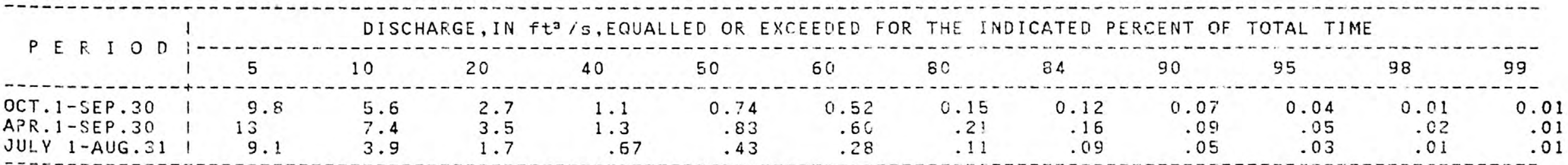

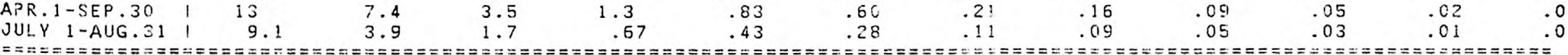


OLD MANS CREEK NEAR IOWA CITY

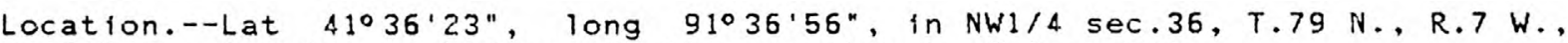
Johnson County, at bridge, 3 mfles southwest of Iowa City.

LOW FLOW DISCHARGES, IN YEAR. ENDING MARCH 31

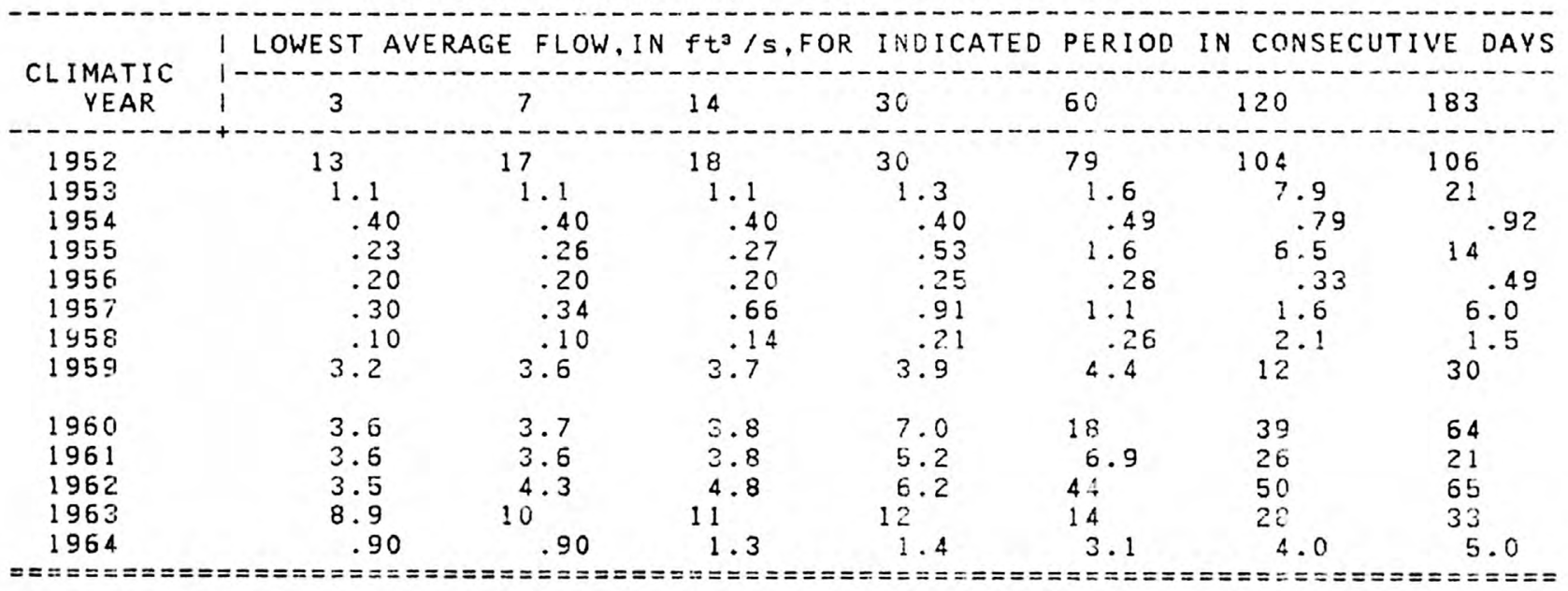


05-4551.00 OLD MANS CREEK NEAR IOWA CITY--Continued

DRAINAGE AREA: $201 \mathrm{~m}^{2}$ PERIOD OF RECORD: 14 YEARS AVERAGE DISCHARGE: 97.6 ft3/s

MINIMUM AVERAGE FLOWS FOR PERIOD OF RECORD

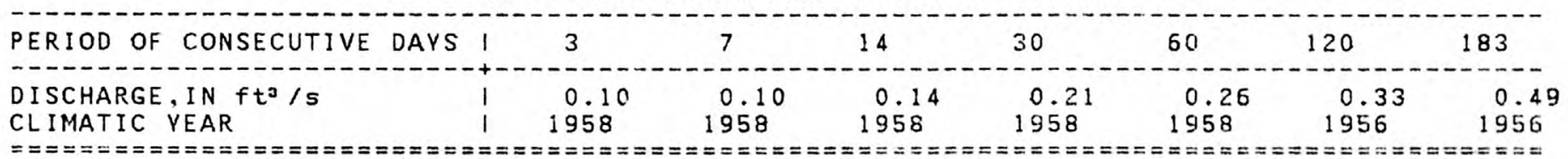

MAGNITUDE AND FREQUENCY OF ANNUAL LOW FLOWS

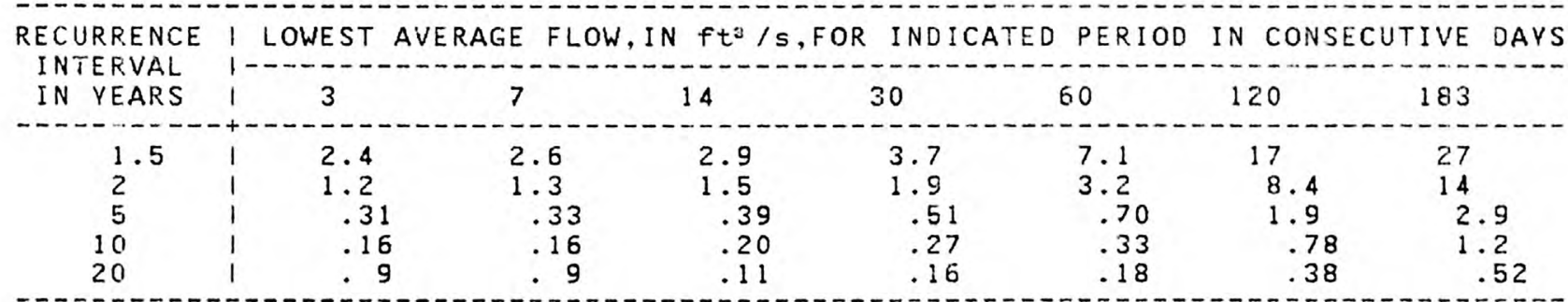

MAGNITUDE AND FREQUENCY OF SEASONAL LOW FLOWS

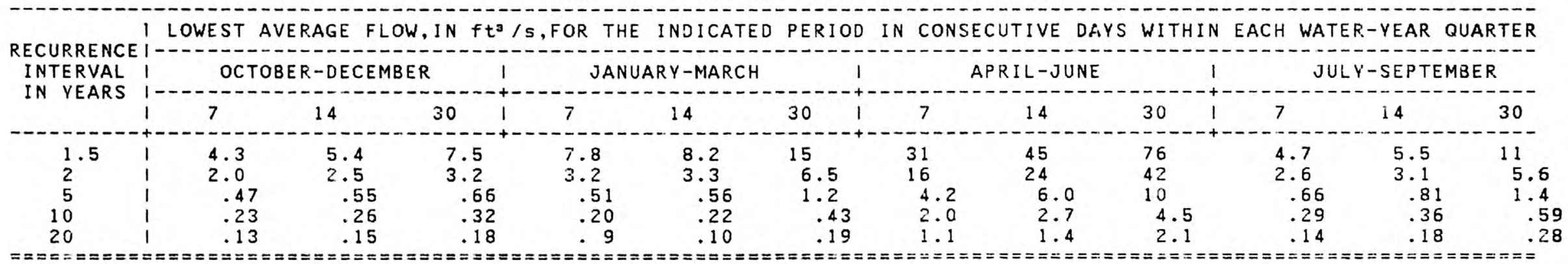

DURATION OF DAILY DISCHARGES FOR FNNUAL AND SEASONAL FERIONS

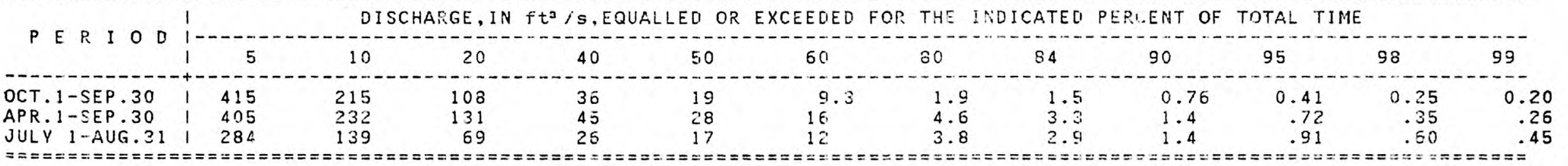


Location.--Lat $41027.59 "$, long $91042.56 "$, In SE1/4 SE1/4 $\mathrm{sec} .13$, T.77 N., R.8 W., Washington Cisunty, on right bank $30 \mathrm{ft}$ upstream from bridge on State Highway $1,0.8 \mathrm{mile}$ south of Kalona, $1.1 \mathrm{mile}$ upstream from Camp Creek, 4.5 miles downstream from Sinith Creek, and 14.5 miles upstream from mouth.

LOW FLOW DISCHARGES, IN YEAR ENDING MARCH 31

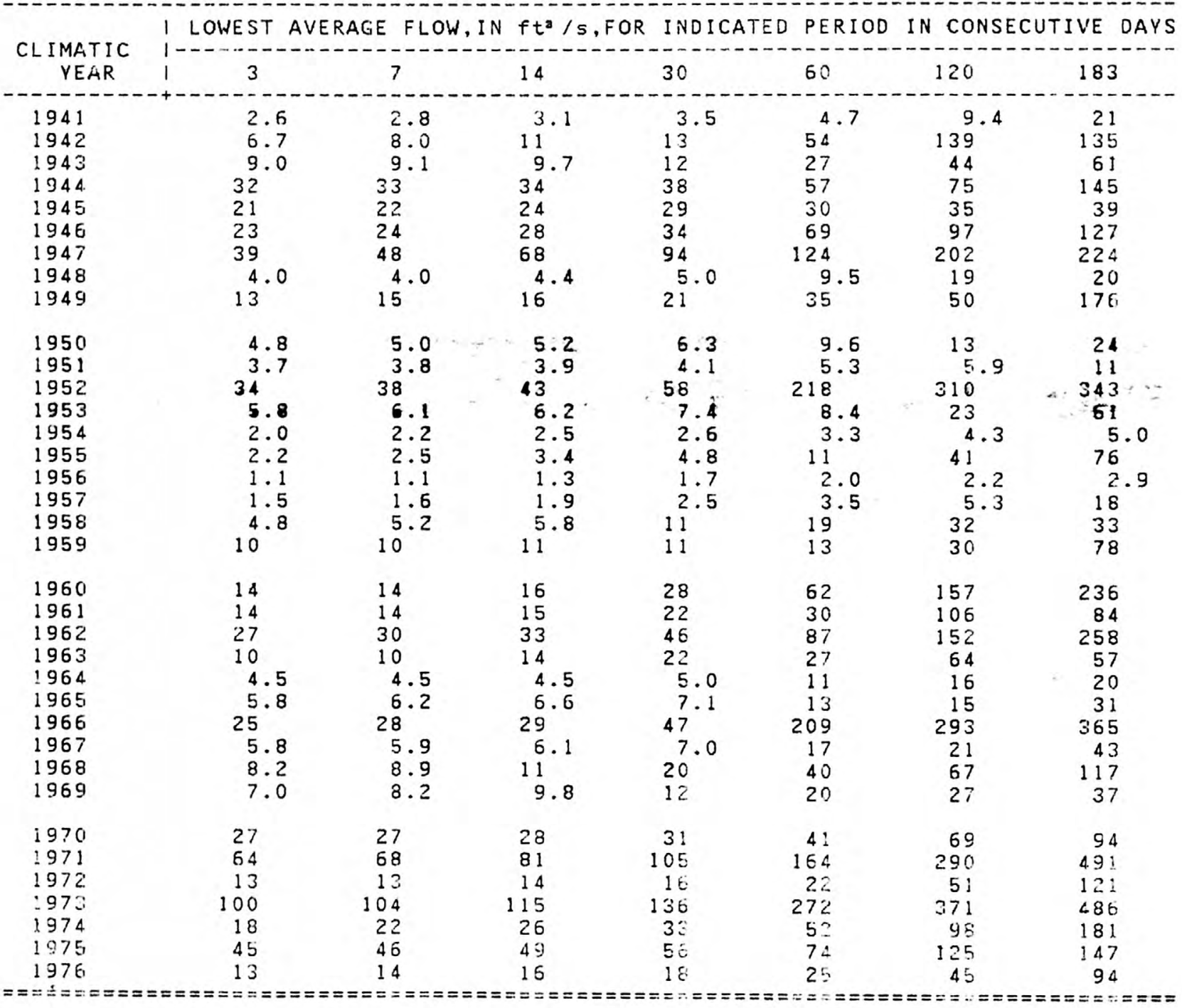


05-4555.00 ENGLISH RIVER AT KALONA--Continued

DRAINAGE AREA: $573 \mathrm{~m}^{2} \quad$ PERIOD OF RECORD: 37 YEARS AVERAGE DISCHARGE: 367 ft 3 /S
MINIMUM AVERAGE FLOWS FOR PERIOD OF RECORD

MAGNITUDE AND FREQUENCY OF ANNUAL LOW FLOWS

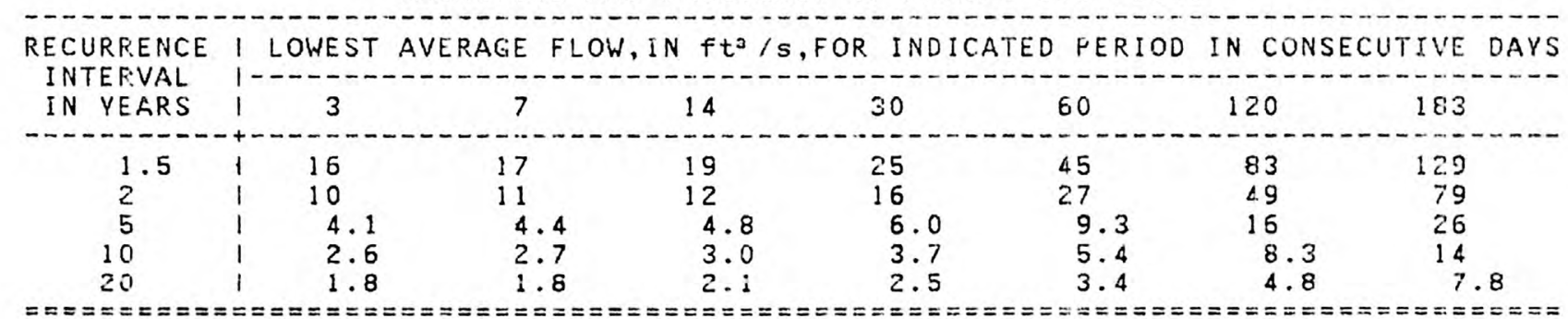

MAGNITUDE AND FREQUENCY OF SEASONAL LOW FLOWS

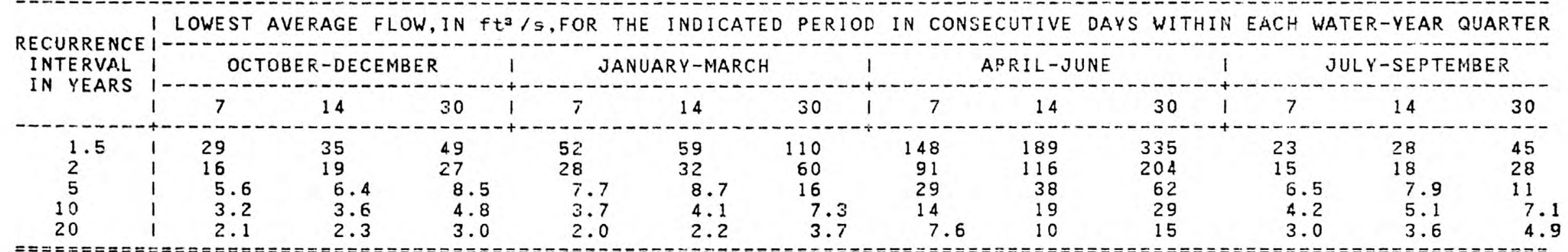

DURATION OF DAILY DISCHARGES FOR ANNUAL AND SEASONAL PERIODS

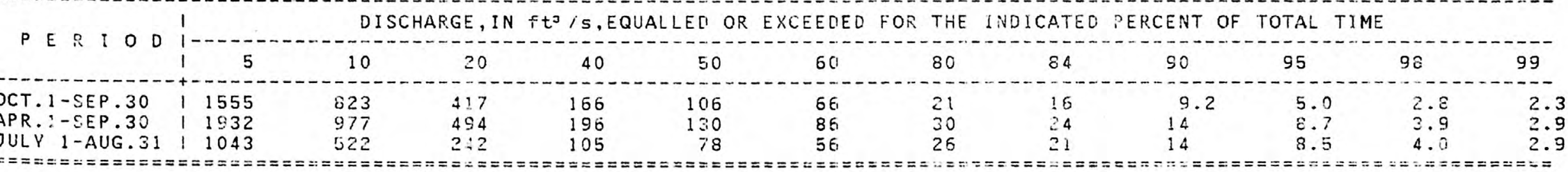


Location.--Lat 41025'15", long 91028'25", In NW1/4 NE1/4 sec.6, T.76 N., R.5 W. Louisa County, on left bank $10 \mathrm{ft}$ downstream from bridge on county highway W66, 5 miles southwest of Lone Tree, $6.2 \mathrm{~m} f$ les downstream from English River, and at mile 47.2 .

Remarks.--Flow regulated by Coralville Lake, 36.1 miles upstream, since Sept. 17. 1958 .

LOW FLOW DISCHARGES, IN YEAR. ENDING MARCH 31

\begin{tabular}{|c|c|c|c|c|c|c|c|c|}
\hline \multirow{2}{*}{$\begin{array}{c}\text { CLIMATIC } \\
\text { YEAR }\end{array}$} & LOWEST & $\mathrm{AGE}$ & $N f^{3}$ & $R$ IN & $D P E$ & In C & IVE & DAYS \\
\hline & 3 & 7 & 14 & 30 & 60 & 120 & 183 & \\
\hline $\begin{array}{l}1960 \\
1961 \\
1962 \\
1963 \\
1964 \\
1965 \\
1966 \\
1967 \\
1968 \\
1969\end{array}$ & $\begin{array}{l}155 \\
210 \\
315 \\
340 \\
162 \\
194 \\
624 \\
119 \\
254 \\
231\end{array}$ & $\begin{array}{l}172 \\
245 \\
349 \\
364 \\
164 \\
198 \\
651 \\
128 \\
281 \\
235\end{array}$ & $\begin{array}{l}176 \\
308 \\
398 \\
370 \\
167 \\
205 \\
684 \\
150 \\
335 \\
245\end{array}$ & $\begin{array}{l}218 \\
361 \\
576 \\
393 \\
190 \\
246 \\
722 \\
175 \\
505 \\
365\end{array}$ & $\begin{array}{r}328 \\
454 \\
1110 \\
421 \\
202 \\
302 \\
2260 \\
193 \\
608 \\
431\end{array}$ & $\begin{array}{r}799 \\
821 \\
1250 \\
727 \\
240 \\
427 \\
3300 \\
286 \\
852 \\
562\end{array}$ & $\begin{array}{r}1240 \\
901 \\
1670 \\
970 \\
354 \\
563 \\
3680 \\
466 \\
926 \\
804\end{array}$ & \\
\hline $\begin{array}{l}1970 \\
1971 \\
1972 \\
1973 \\
1974 \\
1975 \\
1976\end{array}$ & $\begin{array}{r}433 \\
509 \\
202 \\
1370 \\
345 \\
786 \\
209\end{array}$ & $\begin{array}{r}500 \\
540 \\
205 \\
1480 \\
384 \\
801 \\
210\end{array}$ & $\begin{array}{r}501 \\
622 \\
208 \\
1690 \\
566 \\
823 \\
211\end{array}$ & $\begin{array}{r}508 \\
980 \\
217 \\
2460 \\
658 \\
904 \\
210\end{array}$ & $\begin{array}{r}679 \\
1350 \\
239 \\
2970 \\
1040 \\
1300 \\
266\end{array}$ & $\begin{array}{r}873 \\
2060 \\
378 \\
3510 \\
1560 \\
1870 \\
490\end{array}$ & $\begin{array}{r}1510 \\
2430 \\
720 \\
4140 \\
2640 \\
1730 \\
589\end{array}$ & \\
\hline
\end{tabular}


MINIMUM AVERAGE FLOWS FOR PERIOD OF R.ECORD

\begin{tabular}{|c|c|c|c|c|c|c|c|c|}
\hline PERIOD OF CONSECUTIVE DAYS & 1 & 3 & 7 & 14 & 30 & 60 & 120 & 183 \\
\hline $\begin{array}{l}\text { DISCHARGE, IN } \mathrm{ft}^{\mathrm{o}} / \mathrm{s} \\
\text { CLIMATIC YEAR }\end{array}$ & 1 & $\begin{array}{l}119 \\
1967\end{array}$ & $\begin{array}{l}128 \\
1957\end{array}$ & $\begin{array}{l}150 \\
1967\end{array}$ & $\begin{array}{l}175 \\
1967\end{array}$ & $\begin{array}{l}193 \\
1967\end{array}$ & $\begin{array}{l}240 \\
1964\end{array}$ & $\begin{array}{l}354 \\
1964\end{array}$ \\
\hline
\end{tabular}

MAGNITUDE AND FREQUENCY OF ANNUAL LOW FLOWS

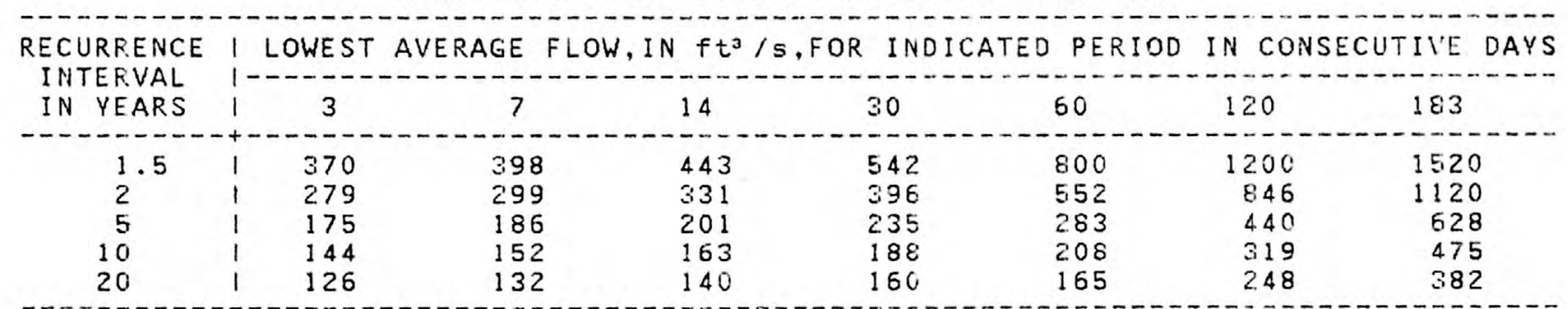

MAGNITUDE AND FREQUENCY OF SEASONAL LOW FLOW'S

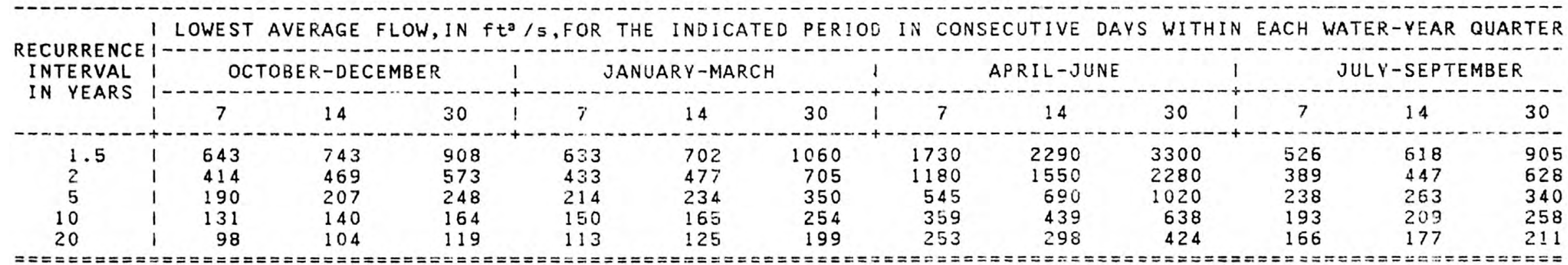

DURATION OF DAILY DISCHARGES FOR ANINIAL AND SEASONAL PERIODS

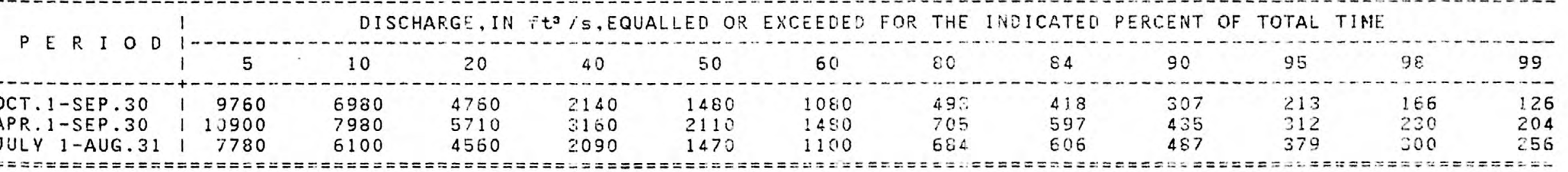


$05-4577.00$

CEDAR RIVER AT CHARLES CITY

Location.--Lat $43^{\circ} 03^{\prime} 45^{\prime \prime}$, long $92^{\circ} 40.23^{\prime \prime}$, In SE1/4 NE1/4, sec.12, T.95 N., R.16 W., Floyd County, on $r$ ight bank $800 \mathrm{ft}$ downstream from bridge on U.S. Highway 18 (Brantingham Street) in Charles City, 10.6 miles upstream from Gizzard Creek, and at mile 252.9 upstream from mouth of Iowa River.

Remarks.--Occasional minor regulation by dam $0.2 \mathrm{mlie}$ upstream of gage.

LOW FLOW DISCHARGES, IN YEAR. ENDING MARCH 31

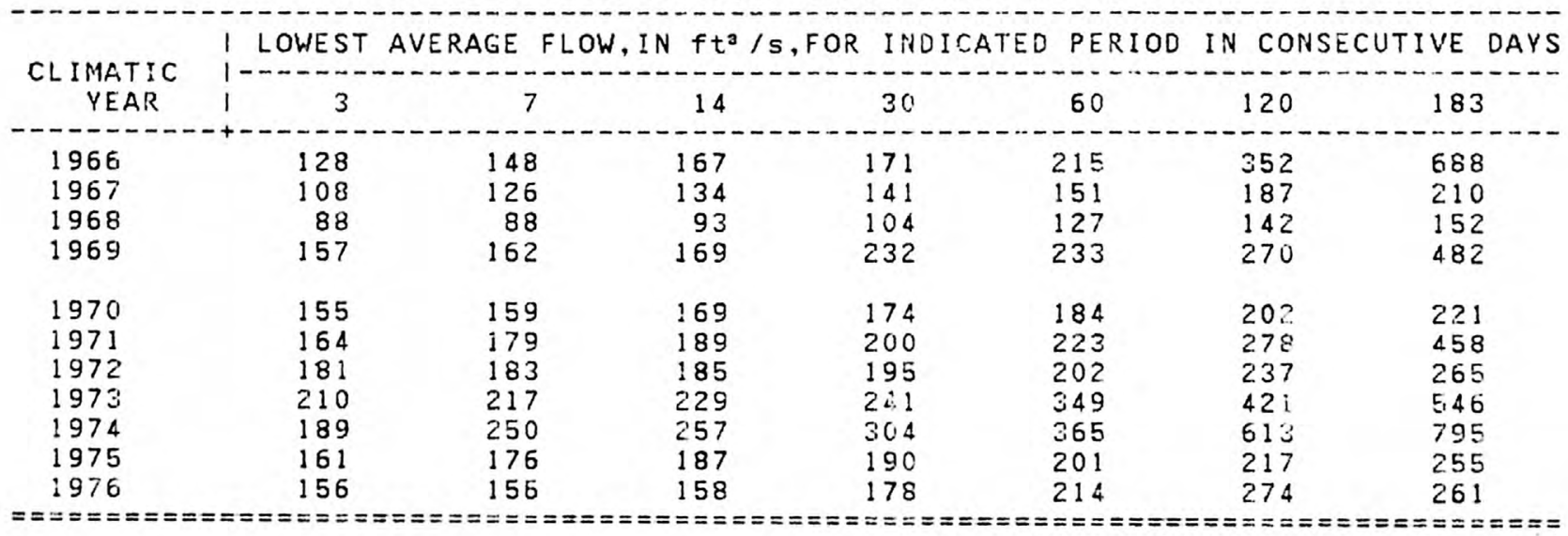


DRAINAGE AREA: $1054 \mathrm{~m}^{2}$ PERIOD OF RECORD: 12 VEARS AVERAGE DISCHARGE: 687 ft3/S

MINIMUM AVERAGE FLOWS FOR PERIOD OF RECORD

\begin{tabular}{|c|c|c|c|c|c|c|c|}
\hline ERIOD OF CONSECUTIVE DAYS I & 3 & 7 & 14 & 30 & 60 & 120 & 183 \\
\hline $\begin{array}{l}\text { ARGE IN } \mathrm{ft}^{3} / \mathrm{s} \\
\text { TIC YEAR }\end{array}$ & $\begin{array}{r}88 \\
1968 \\
\end{array}$ & $\begin{array}{l}88 \\
1968\end{array}$ & $\begin{array}{c}93 \\
1968\end{array}$ & $\begin{array}{l}104 \\
1968\end{array}$ & $\begin{array}{l}127 \\
1968\end{array}$ & $\begin{array}{l}142 . \\
1968 \\
==-=\end{array}$ & \\
\hline
\end{tabular}

MAGNITUDE AND FREQUENCY OF ANNUAL LOW FLOWS

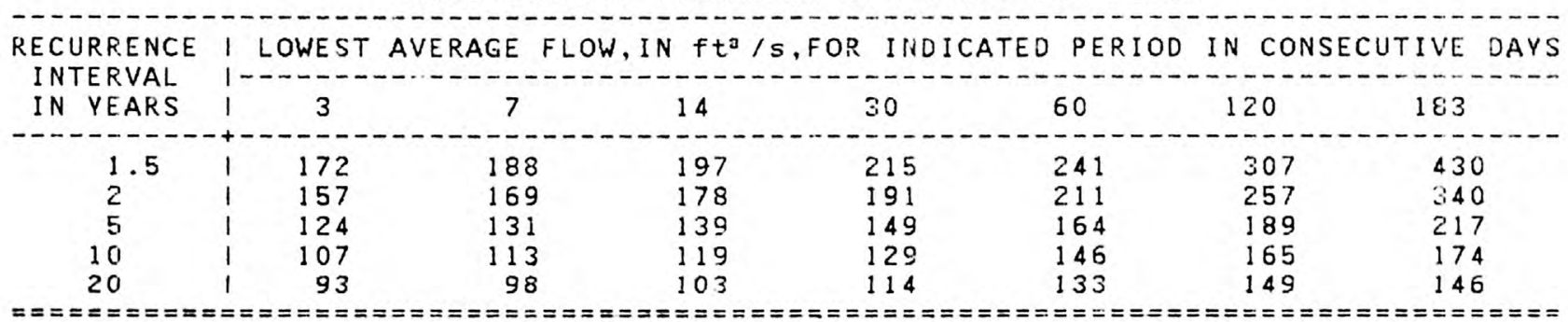

MAGNITUDE AND FREQUENCY OF SEASONAL LOW FLOWS

I LOWEST AVERAGE FLOW, IN $\mathrm{ft}^{3} / \mathrm{s}$, FOR THE INDICATED PERIOD IN CONSECUTIVE DAYS WITHIN EACH WATER-YEAR QUARTER

$\begin{aligned} & \text { RECURRENCE } \\ & \text { INTERVAL }\end{aligned}$
IN VEARS

DURATION OF DAILY DISCHARGES FOR ANNUAL AND SEASONAL PERIODS

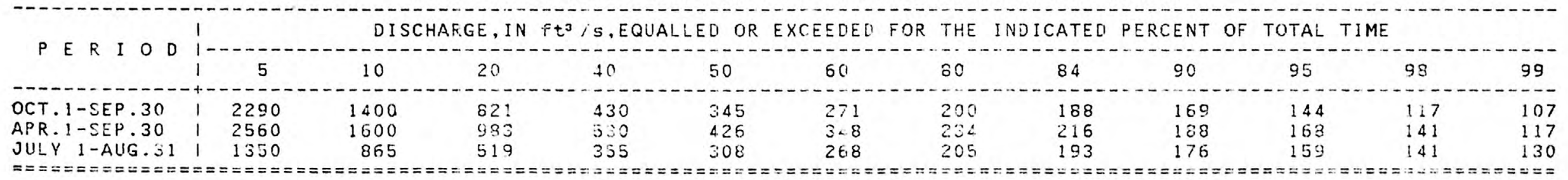




\section{LITTLE CEDAR RIVER NEAR IONIA}

Location.--Lat $43^{\circ} 02^{\prime} 05^{\prime \prime}$, long $92^{\circ} 30^{\prime} 05^{\prime \prime}$, In SW1/4 NE1/4 sec.21, T.95 N., R.14 W. , Chlckasaw County, on left bank $12 \mathrm{ft}$ downstream from bridge on county highway $857,2.4 \mathrm{~m} 1$ les west of Ionfa, 6.4 miles upstream from mouth, and

7.6 miles downstream from Beaver Creek.

LOW FLOW DISCHARGES, IN YEAR ENDING MARCH 31

\begin{tabular}{|c|c|c|c|c|c|c|c|}
\hline \multirow{2}{*}{$\begin{array}{c}\text { CLIMATIC } \\
\text { YEAR }\end{array}$} & I LOWEST & E FLOW, IN & $f t^{3} / s, F O R$ & INDICATED & PERIOL & \multicolumn{2}{|c|}{ IN CONSECUTIVE DAYS } \\
\hline & 13 & 7 & 14 & 30 & 60 & 120 & 183 \\
\hline $\begin{array}{l}1956 \\
1957 \\
1958 \\
1959\end{array}$ & $\begin{array}{l}5.6 \\
10 \\
10 \\
3.0\end{array}$ & $\begin{array}{l}5.7 \\
10 \\
10 \\
3.1\end{array}$ & $\begin{array}{l}5.9 \\
10 \\
11 \\
3.1\end{array}$ & $\begin{array}{l}6.0 \\
11 \\
16 \\
3.3\end{array}$ & $\begin{array}{l}6.2 \\
15 \\
20 \\
3.8\end{array}$ & $\begin{array}{l}8.7 \\
19 \\
28 \\
6.1\end{array}$ & $\begin{array}{l}11 \\
23 \\
28 \\
8.9\end{array}$ \\
\hline $\begin{array}{l}1960 \\
1961 \\
1962 \\
1963 \\
1964 \\
1965 \\
1966 \\
1967 \\
1968 \\
1969\end{array}$ & $\begin{array}{c}22 \\
13 \\
26 \\
24 \\
12 \\
7.4 \\
25 \\
13 \\
6.0 \\
26\end{array}$ & $\begin{array}{c}23 \\
13 \\
27 \\
25 \\
12 \\
7.6 \\
25 \\
13 \\
6.2 \\
27\end{array}$ & $\begin{array}{c}23 \\
14 \\
29 \\
27 \\
12 \\
8.1 \\
26 \\
14 \\
6.6 \\
30\end{array}$ & $\begin{array}{c}25 \\
17 \\
31 \\
30 \\
13 \\
8.8 \\
29 \\
15 \\
8.0 \\
42\end{array}$ & $\begin{array}{l}31 \\
21 \\
31 \\
32 \\
16 \\
13 \\
59 \\
19 \\
15 \\
43\end{array}$ & $\begin{array}{l}56 \\
32 \\
45 \\
47 \\
21 \\
16 \\
92 \\
27 \\
18 \\
54\end{array}$ & $\begin{array}{r}70 \\
56 \\
61 \\
119 \\
23 \\
19 \\
245 \\
31 \\
20 \\
114\end{array}$ \\
\hline $\begin{array}{l}1970 \\
1971 \\
1972 \\
1973 \\
1974 \\
1975 \\
1976\end{array}$ & $\begin{array}{l}24 \\
32 \\
21 \\
36 \\
51 \\
23 \\
16\end{array}$ & $\begin{array}{l}25 \\
37 \\
21 \\
37 \\
54 \\
23 \\
16\end{array}$ & $\begin{array}{l}27 \\
38 \\
22 \\
40 \\
58 \\
24 \\
16\end{array}$ & $\begin{array}{l}28 \\
43 \\
22 \\
46 \\
64 \\
25 \\
18\end{array}$ & $\begin{array}{l}29 \\
63 \\
24 \\
77 \\
70 \\
26 \\
25\end{array}$ & $\begin{array}{r}36 \\
87 \\
43 \\
108 \\
119 \\
29 \\
35\end{array}$ & $\begin{array}{r}43 \\
139 \\
43 \\
203 \\
165 \\
34 \\
35\end{array}$ \\
\hline
\end{tabular}


05-4580.00 LITTLE CEDAR RIVER NEAR IONIA--Cont inued

DRAINAGE AREA: $306 \mathrm{~m}^{2}$ PERIOD OF RECORD: 22 VEARS AVERAGE OISCHARGE: $156 \mathrm{ft}^{3} / \mathrm{s}$ MINIMUM AVERAGE FLOWS FOR PERIOD OF RECORD

\begin{tabular}{|c|c|c|c|c|c|c|c|}
\hline PERIOD OF CONSECUTIVE DAYS & 3 & 7 & 14 & 30 & 60 & 20 & 183 \\
\hline $\begin{array}{l}\text { ISCHARGE, IN } \mathrm{ft}^{3} / \mathrm{s} \\
\text { LIMATIC YEAR }\end{array}$ & $\begin{array}{r}3.0 \\
1959\end{array}$ & $\begin{array}{r}3.1 \\
1959\end{array}$ & $\begin{array}{r}3.1 \\
1959\end{array}$ & $\begin{array}{r}3.3 \\
1959\end{array}$ & $\begin{array}{r}3.8 \\
1959\end{array}$ & $\begin{array}{r}6.1 \\
1959\end{array}$ & \\
\hline
\end{tabular}

MAGNITUDE AND FREQUENCY OF ANNUAL LOW FLOWS

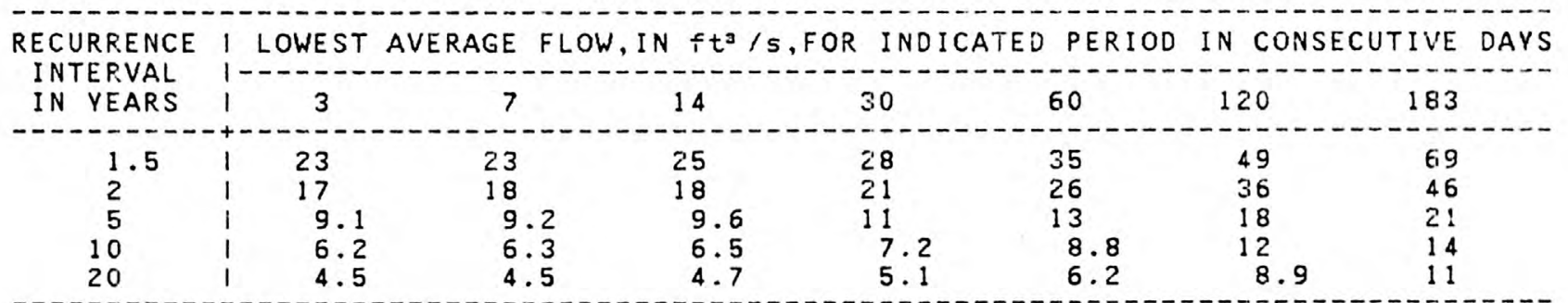

MAGNITUDE AND FREQUENCY OF SEASONAL LOW FLOWS

LOWEST AVERAGE FLOW, IN $f t^{\mathrm{a}} / \mathrm{s}$,FOR THE INDICATED PERIOD IN CONSECUTIVE DAYS WITHIN EACH WATER-YEAR QUARTER

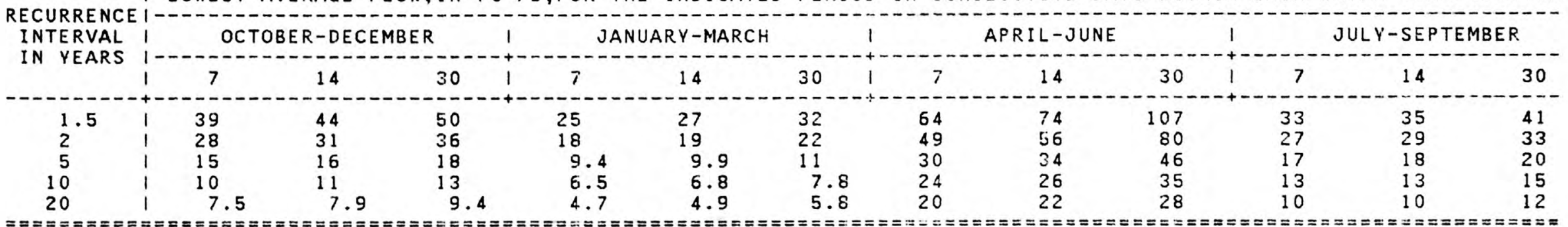

DURATION OF DAILY DISCHARGES FOR ANNUAL AND SEASONAL FERIODS

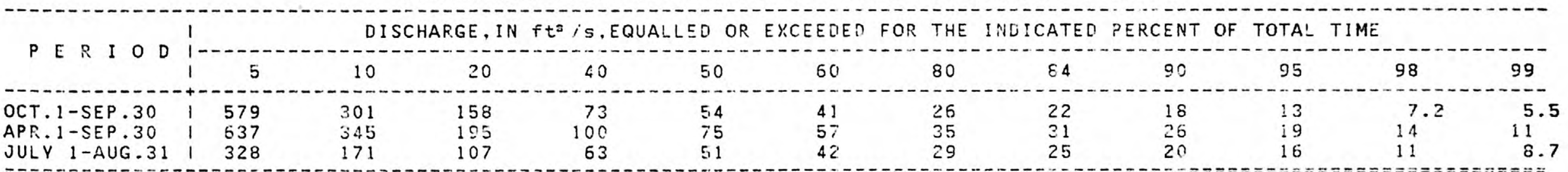


$05-4585.00$

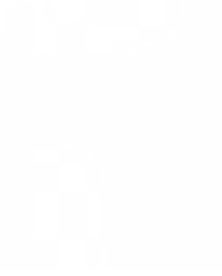

CEDAR RIVER AT JANESVILLE

Location.--Lat $42038.54^{\prime \prime}$, long $92027154^{\prime \prime}$, in NE1/4 SWl/4 sec. 35 , T.91 N., R.14 W. Bremer County, on left bank $300 \mathrm{ft}$ downstream from bridge on county highway at Janesvilie, $3.6 \mathrm{~m}$ tles upstream from West Fork Cedar River, and at mile 207.7 upsiream from mouth of Iowa River.

Remarks.--Diurnal fluctuation caused by powerplant at Waverly, $10 \mathrm{miles}$ upstream.

LOW FLOW DISCHARGES, IN YEAR ENDING MARCH 31

\begin{tabular}{|c|c|c|c|c|c|c|c|}
\hline \multirow{2}{*}{$\begin{array}{c}\text { CLIMATIC } \\
\text { YEAR }\end{array}$} & I LOWEST & $A G E$ A $F$ & $f t^{3}$ & 2 IND & PER & $\mathrm{NCO}$ & VE \\
\hline & 13 & 7 & 14 & 30 & 60 & 120 & 183 \\
\hline $\begin{array}{l}1906 \\
1916 \\
1917 \\
1918 \\
1919\end{array}$ & $\begin{array}{r}190 \\
140 \\
60 \\
50 \\
250\end{array}$ & $\begin{array}{r}197 \\
140 \\
60 \\
50 \\
250\end{array}$ & $\begin{array}{r}200 \\
163 \\
62 \\
60 \\
253\end{array}$ & $\begin{array}{r}230 \\
183 \\
68 \\
81 \\
300\end{array}$ & $\begin{array}{r}267 \\
271 \\
74 \\
132 \\
415\end{array}$ & $\begin{array}{l}316 \\
444 \\
114 \\
190 \\
704\end{array}$ & $\begin{array}{l}352 \\
553 \\
143 \\
255 \\
724\end{array}$ \\
\hline $\begin{array}{l}1920 \\
1921 \\
1922 \\
1923 \\
1924 \\
1925 \\
1926 \\
1927 \\
1934 \\
1935 \\
1936 \\
1937 \\
1938 \\
1939\end{array}$ & $\begin{array}{r}120 \\
118 \\
140 \\
60 \\
89 \\
90 \\
59 \\
114 \\
60 \\
51 \\
83 \\
97 \\
74 \\
252\end{array}$ & $\begin{array}{r}120 \\
142 \\
140 \\
60 \\
89 \\
91 \\
70 \\
123 \\
61 \\
57 \\
87 \\
100 \\
80 \\
269\end{array}$ & $\begin{array}{r}129 \\
223 \\
140 \\
65 \\
92 \\
96 \\
83 \\
139 \\
66 \\
64 \\
89 \\
105 \\
82 \\
298\end{array}$ & $\begin{array}{r}141 \\
251 \\
158 \\
77 \\
100 \\
101 \\
107 \\
185 \\
73 \\
77 \\
101 \\
128 \\
87 \\
379\end{array}$ & $\begin{array}{r}175 \\
344 \\
165 \\
89 \\
162 \\
116 \\
141 \\
197 \\
92 \\
81 \\
117 \\
138 \\
96 \\
463\end{array}$ & $\begin{array}{r}318 \\
407 \\
223 \\
125 \\
216 \\
193 \\
168 \\
251 \\
103 \\
85 \\
165 \\
227 \\
145 \\
509\end{array}$ & $\begin{array}{r}318 \\
437 \\
291 \\
154 \\
247 \\
322 \\
167 \\
299 \\
115 \\
93 \\
183 \\
319 \\
185 \\
744\end{array}$ \\
\hline $\begin{array}{l}1940 \\
1941 \\
1942 \\
1947 \\
1948 \\
1949\end{array}$ & $\begin{array}{r}90 \\
71 \\
190 \\
181 \\
162 \\
117\end{array}$ & $\begin{array}{r}92 \\
77 \\
213 \\
192 \\
164 \\
130\end{array}$ & $\begin{array}{r}98 \\
92 \\
226 \\
204 \\
167 \\
145\end{array}$ & $\begin{array}{l}108 \\
106 \\
240 \\
224 \\
171 \\
157\end{array}$ & $\begin{array}{l}122 \\
146 \\
122 \\
234 \\
198 \\
175\end{array}$ & $\begin{array}{l}141 \\
218 \\
474 \\
367 \\
252 \\
191\end{array}$ & $\begin{array}{l}149 \\
331 \\
599 \\
566 \\
285 \\
202\end{array}$ \\
\hline $\begin{array}{l}1950 \\
1951 \\
1952 \\
1953 \\
1954 \\
1955 \\
1956 \\
1957 \\
1958 \\
1959\end{array}$ & $\begin{array}{r}79 \\
100 \\
307 \\
127 \\
110 \\
187 \\
77 \\
84 \\
94 \\
52\end{array}$ & $\begin{array}{r}83 \\
100 \\
350 \\
144 \\
112 \\
193 \\
77 \\
87 \\
103 \\
55\end{array}$ & $\begin{array}{r}90 \\
101 \\
417 \\
174 \\
115 \\
195 \\
82 \\
95 \\
115 \\
56\end{array}$ & $\begin{array}{r}114 \\
114 \\
440 \\
184 \\
125 \\
202 \\
88 \\
112 \\
141 \\
62\end{array}$ & $\begin{array}{r}128 \\
124 \\
545 \\
193 \\
154 \\
243 \\
93 \\
126 \\
160 \\
74\end{array}$ & $\begin{array}{r}143 \\
138 \\
641 \\
203 \\
200 \\
335 \\
115 \\
147 \\
182 \\
94\end{array}$ & $\begin{array}{l}150 \\
158 \\
702 \\
254 \\
2.25 \\
431 \\
130 \\
176 \\
186 \\
107\end{array}$ \\
\hline
\end{tabular}


CEDAR RIVER AT JANESVILLE--Cont Inued

LOW FLOW OISCHARGES, IN YEAR ENDING MARCH 31--Cont1nued

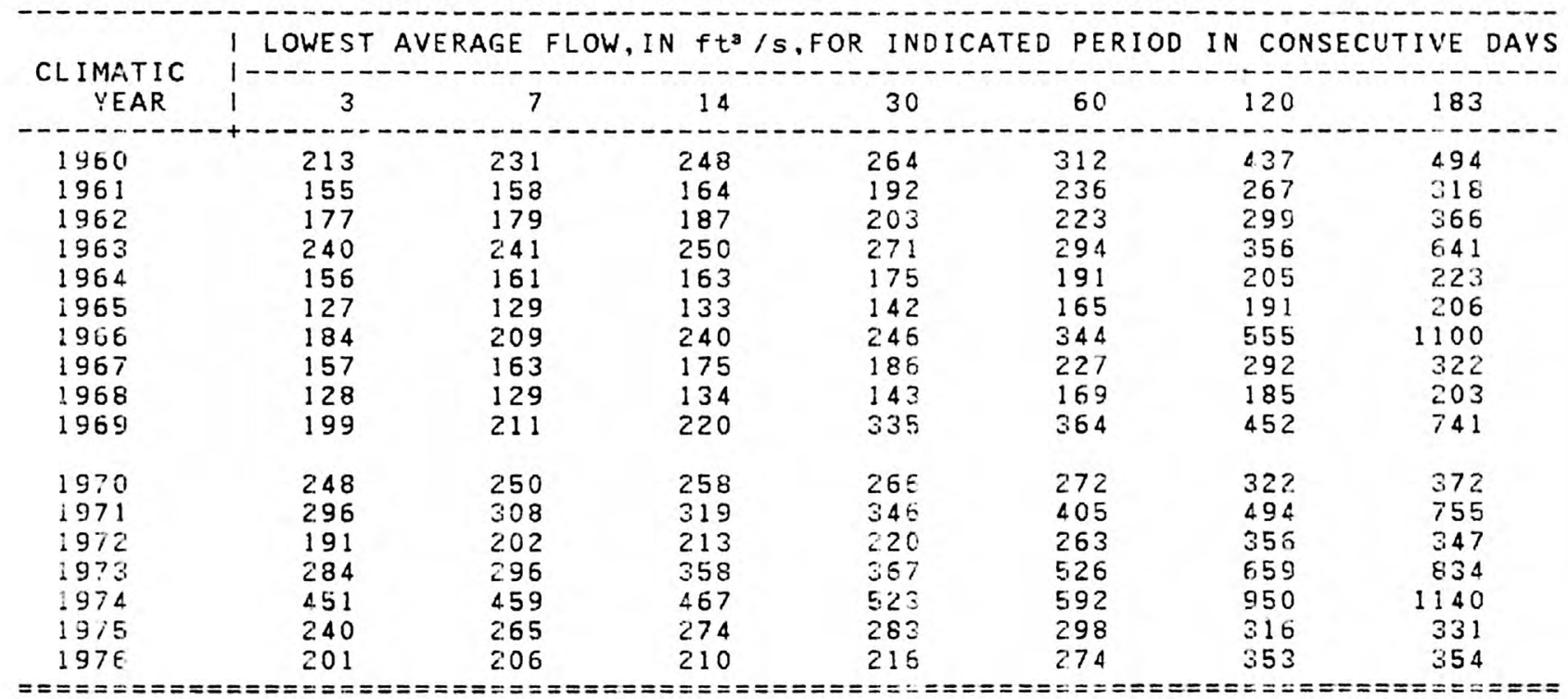




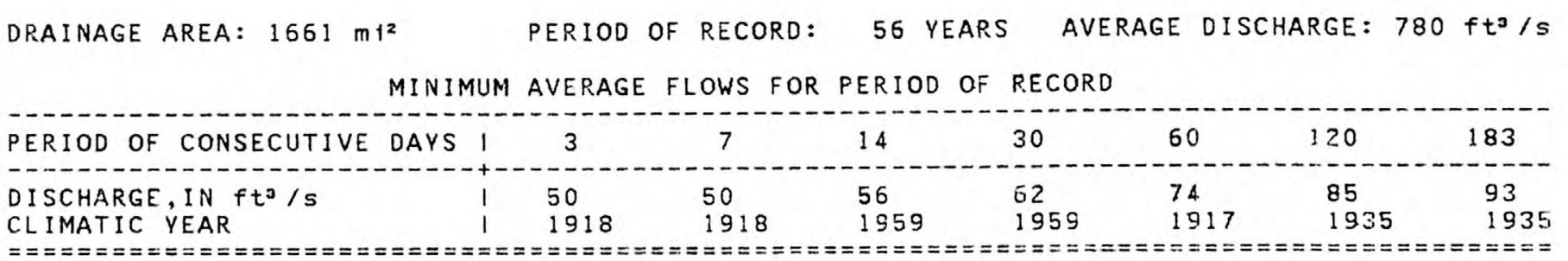

MAGNITUDE AND FREQUENCY OF ANNUAL LOW FLOWS

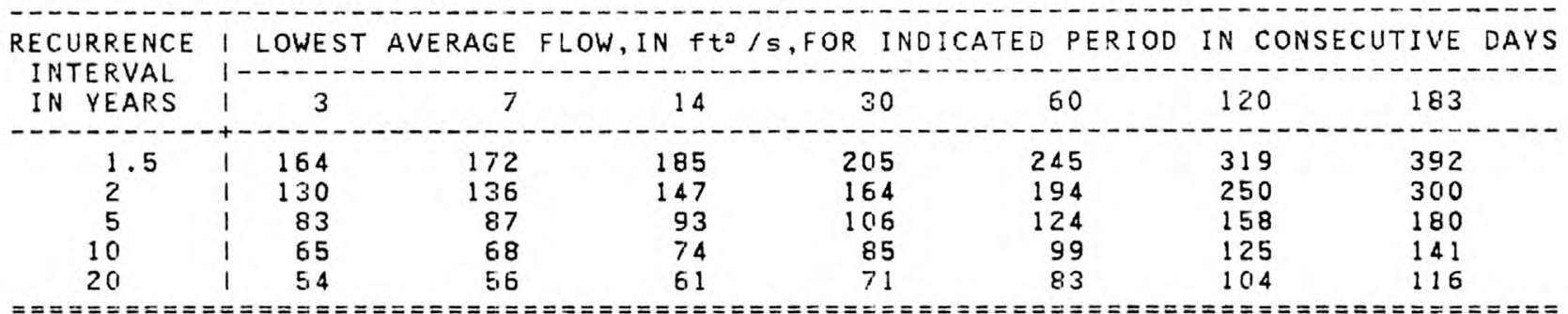

MAGNITUDE AND FREQUENCY OF SEASONAL LOW FLOWS

I LOWEST AVERAGE FLOW, IN $\mathrm{ft}^{\mathrm{J}} / \mathrm{s}, \mathrm{FOR}$ THE INDICATED PERIOD IN CONSECUTIVE DAYS WITHIN EACH WATER-YEAR QUARTER

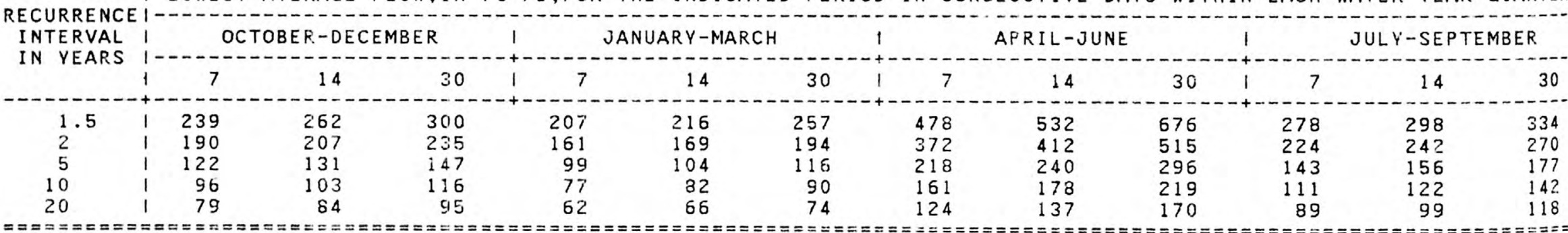

DURATION OF DAILY DISCHARGES FOR ANNUAL AND SEASONAL PERIODS

\begin{tabular}{|c|c|c|c|c|c|c|c|c|c|c|c|c|}
\hline . & 1 & D I & $A R G E, I$ & $/ 5, E$ & $E D O$ & $E E D E$ & R THE & CATE & CENT & TOTAL & & \\
\hline & 5 & 10 & 20 & 40 & 50 & 60 & 80 & 84 & 90 & 95 & 98 & 99 \\
\hline $\begin{array}{l}\text { OCT.1-SEP. } 30 \\
\text { APR.1-SEP. } 30 \\
\text { JUL } Y 1 \text {-AUG. } 31\end{array}$ & $\begin{array}{l}2701 \\
12934 \\
1892\end{array}$ & $\begin{array}{l}1629 \\
1856 \\
1214\end{array}$ & $\begin{array}{r}945 \\
1126 \\
797\end{array}$ & $\begin{array}{l}505 \\
657 \\
475\end{array}$ & $\begin{array}{l}389 \\
520 \\
386\end{array}$ & $\begin{array}{l}313 \\
409 \\
319\end{array}$ & $\begin{array}{l}198 \\
250 \\
211\end{array}$ & $\begin{array}{l}177 \\
228 \\
190\end{array}$ & $\begin{array}{l}146 \\
186 \\
158\end{array}$ & $\begin{array}{l}112 \\
145 \\
127\end{array}$ & $\begin{array}{r}85 \\
110 \\
94\end{array}$ & $\begin{array}{l}73 \\
82 \\
76\end{array}$ \\
\hline
\end{tabular}


$05-4589.00$

WEST FORK CEDAR RIVER AT FINCHFOR.D

Location.--Lat $42^{\circ} 37^{\prime} 50^{\prime \prime}$, long $92^{\circ} 32^{\prime} 24^{\prime \prime}$, in SWi/4 SE $1 / 4 \mathrm{sec} .6$, T.90 N.. R.14 W. B lack Hawk County. on left bank $100 \mathrm{ft}$ downstream from bridge on county highway $C 55$ at Finchiford, $3.2 \mathrm{miles}$ upstream from Shell Rock River, and 5.0 miles upstream from mouth.

Remarks.--An authorized diversion 15 made into Big Marsh, 16 miles upstream from gage, of 2,100 acre-ft each year between Sept. 1 and Nov. 15 . Net effect on daily flows at gage is unknown.

LOW FLOW DISCHARGES, IN YEAR. ENDING MARCH 31

\begin{tabular}{|c|c|c|c|c|c|c|c|}
\hline \multirow{2}{*}{$\begin{array}{c}\text { CLIMATIC } \\
\text { YEAR }\end{array}$} & I LOWEST & AGE FL & $\begin{array}{l}V \\
f t^{3} / \mathrm{s}\end{array}$ & $\begin{array}{l}R \text { INDIC } \\
-\end{array}$ & $\begin{array}{l}\text { D PERIO } \\
-\end{array}$ & IN $\mathrm{CO}$ & IVE DAYS \\
\hline & 13 & 7 & 14 & 30 & 60 & 120 & 183 \\
\hline $\begin{array}{l}1947 \\
1948 \\
1949\end{array}$ & $\begin{array}{l}67 \\
36 \\
25\end{array}$ & $\begin{array}{l}72 \\
36 \\
26\end{array}$ & $\begin{array}{l}83 \\
37 \\
27\end{array}$ & $\begin{array}{r}105 \\
40 \\
28\end{array}$ & $\begin{array}{r}113 \\
59 \\
33\end{array}$ & $\begin{array}{r}162 \\
93 \\
42\end{array}$ & $\begin{array}{r}179 \\
96 \\
48\end{array}$ \\
\hline $\begin{array}{l}1950 \\
1951 \\
1952 \\
1953 \\
1954 \\
1955 \\
1956 \\
1957 \\
1958 \\
1959\end{array}$ & $\begin{array}{r}16 \\
21 \\
140 \\
50 \\
17 \\
37 \\
8.0 \\
11 \\
18 \\
6.0\end{array}$ & $\begin{array}{c}17 \\
21 \\
142 \\
51 \\
18 \\
40 \\
8.0 \\
11 \\
20 \\
6.1\end{array}$ & $\begin{array}{c}18 \\
21 \\
146 \\
53 \\
18 \\
43 \\
8.4 \\
12 \\
25 \\
6.4\end{array}$ & $\begin{array}{c}21 \\
26 \\
167 \\
54 \\
26 \\
49 \\
9.0 \\
13 \\
33 \\
6.4\end{array}$ & $\begin{array}{r}22 \\
32 \\
227 \\
55 \\
36 \\
83 \\
10 \\
17 \\
35 \\
7.9\end{array}$ & $\begin{array}{r}30 \\
49 \\
308 \\
59 \\
50 \\
146 \\
21 \\
21 \\
42 \\
13\end{array}$ & $\begin{array}{r}35 \\
73 \\
390 \\
67 \\
56 \\
216 \\
27 \\
21 \\
53 \\
18\end{array}$ \\
\hline $\begin{array}{l}1960 \\
1961 \\
1962 \\
1963 \\
1964 \\
1965 \\
1966 \\
1967 \\
1968 \\
1969\end{array}$ & $\begin{array}{l}78 \\
42 \\
30 \\
64 \\
48 \\
38 \\
89 \\
37 \\
22 \\
61\end{array}$ & $\begin{array}{l}89 \\
43 \\
32 \\
64 \\
49 \\
38 \\
96 \\
39 \\
22 \\
66\end{array}$ & $\begin{array}{r}101 \\
45 \\
37 \\
66 \\
50 \\
41 \\
107 \\
41 \\
23 \\
79\end{array}$ & $\begin{array}{r}127 \\
57 \\
42 \\
70 \\
51 \\
48 \\
116 \\
45 \\
30 \\
98\end{array}$ & $\begin{array}{r}146 \\
71 \\
53 \\
76 \\
56 \\
74 \\
214 \\
58 \\
48 \\
140\end{array}$ & $\begin{array}{r}224 \\
110 \\
75 \\
118 \\
69 \\
94 \\
417 \\
64 \\
57 \\
222\end{array}$ & $\begin{array}{r}251 \\
142 \\
82 \\
241 \\
76 \\
150 \\
694 \\
73 \\
60 \\
312\end{array}$ \\
\hline $\begin{array}{l}1970 \\
1971 \\
972 \\
1973 \\
1974 \\
1975 \\
1976\end{array}$ & $\begin{array}{r}100 \\
65 \\
36 \\
109 \\
121 \\
90 \\
43\end{array}$ & $\begin{array}{r}101 \\
67 \\
36 \\
112 \\
125 \\
91 \\
44\end{array}$ & $\begin{array}{r}103 \\
70 \\
37 \\
132 \\
152 \\
94 \\
45\end{array}$ & $\begin{array}{r}104 \\
81 \\
36 \\
156 \\
176 \\
102 \\
47\end{array}$ & $\begin{array}{r}111 \\
95 \\
44 \\
255 \\
21 \% \\
110 \\
55\end{array}$ & $\begin{array}{r}145 \\
154 \\
98 \\
394 \\
417 \\
14 ! \\
83\end{array}$ & $\begin{array}{r}167 \\
219 \\
115 \\
453 \\
500 \\
148 \\
91\end{array}$ \\
\hline
\end{tabular}


05-4589.00 WEST FORK CEDAR RIVER AT FINCHFORD--Continued

DRAINAGE AREA: $846 \mathrm{mi}^{2} \quad$ PERIOD OF RECORD: 31 VEARS AVERAGE DISCHARGE: 450 ft $3 \mathrm{~s}$
MINIMUM AVERAGE FLOWS FOR PERIOD OF RECORD

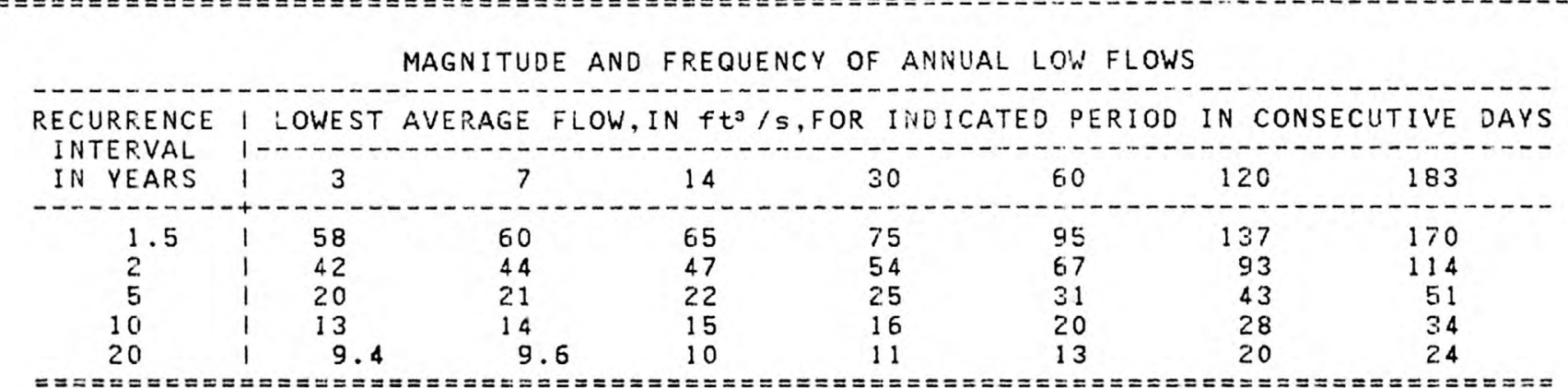

MAGNITUDE AND FREQUENCY OF SEASONAL LOW FLOWS

I LOWEST AVERAGE FLOW, IN $\mathrm{ft}^{3} / \mathrm{s}$, FOR THE INDICATED PERIOD IN CONSECUTIVE DAYS WITHIN EACH WATER-YEAR QUARTER

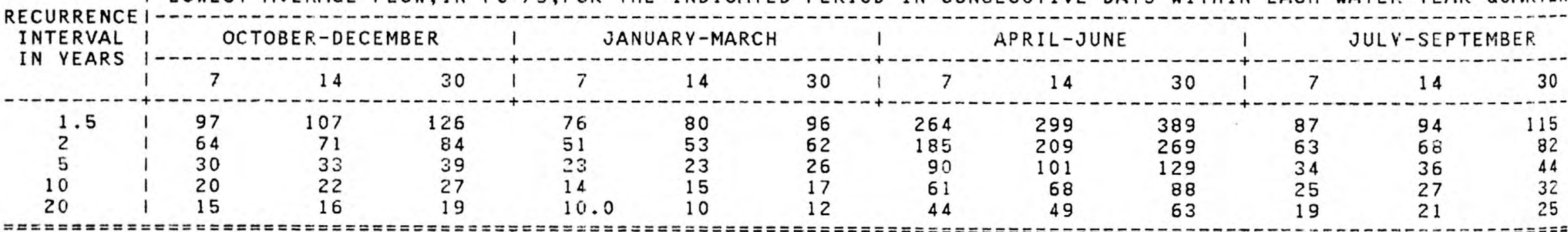

DURATION OF DAILY DISCHARGES FOR ANNUAL ANO SEASONAL PERIODS

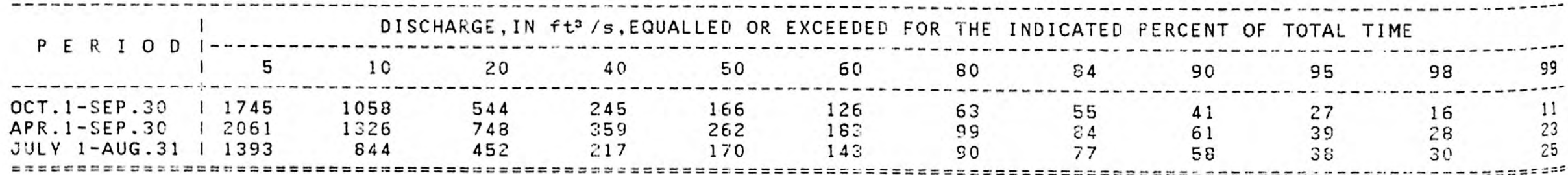


$05-4590.00$

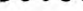

\section{SHELL ROCK RIVER NEAR NORTHWOOD}

Location.--Lat $43^{\circ} 24^{\prime} 51^{\prime \prime}$, long $93^{\circ} 13^{\prime} 14^{\prime \prime}$, in NW1/4 NW1/4 sec.9, T.99 N., R.20 W. Worth County, on right bank $50 \mathrm{ft}$ downstream from bridge on county highway A27, 1.3 miles downstream from Drainage ditch $2,2.0 \mathrm{miles} 50 u t h$ of Northwood, 3.7 miles upstream from Eik Creek, and $84.5 \mathrm{miles}$ upstream from mouth.

LOW FLOW DISCHARGES, IN YEAR. ENDING MARCH 31

\begin{tabular}{|c|c|c|c|c|c|c|c|}
\hline \multirow{2}{*}{$\begin{array}{c}\text { CLIMATIC } \\
\text { YEAR }\end{array}$} & I LOWEST AVERAGE & GE FLO & $f t^{3} / 5$, & IND ICA & PERIOD & \multicolumn{2}{|c|}{ N CONSECUTIVE DAYS } \\
\hline & 13 & 7 & 14 & 30 & 60 & 120 & 183 \\
\hline $\begin{array}{l}1947 \\
1948 \\
1949\end{array}$ & $\begin{array}{l}11 \\
11 \\
17\end{array}$ & $\begin{array}{l}12 \\
11 \\
17\end{array}$ & $\begin{array}{l}14 \\
11 \\
18\end{array}$ & $\begin{array}{l}18 \\
13 \\
23\end{array}$ & $\begin{array}{l}24 \\
23 \\
31\end{array}$ & $\begin{array}{l}77 \\
33 \\
32\end{array}$ & $\begin{array}{l}92 \\
34 \\
33\end{array}$ \\
\hline $\begin{array}{l}1950 \\
1951 \\
1952 \\
1953 \\
1954 \\
1955 \\
1956 \\
1957 \\
1958 \\
1959\end{array}$ & $\begin{array}{l}11 \\
13 \\
36 \\
9.0 \\
10 \\
20 \\
9.3 \\
7.0 \\
9.4 \\
.30\end{array}$ & $\begin{array}{l}11 \\
13 \\
44 \\
11 \\
11 \\
21 \\
11 \\
7.3 \\
9.5 \\
.30\end{array}$ & $\begin{array}{l}12 \\
14 \\
45 \\
14 \\
12 \\
23 \\
12 \\
7.8 \\
10.33\end{array}$ & $\begin{array}{l}14 \\
15 \\
49 \\
18 \\
15 \\
24 \\
13 \\
8.1 \\
16.41\end{array}$ & $\begin{array}{l}17 \\
18 \\
54 \\
19 \\
20 \\
30 \\
14 \\
12 \\
20 \\
.75\end{array}$ & $\begin{array}{l}22 \\
22 \\
76 \\
21 \\
23 \\
43 \\
15 \\
16 \\
27 \\
2.3\end{array}$ & $\begin{array}{l}23 \\
22 \\
93 \\
23 \\
26 \\
56 \\
15 \\
17 \\
26 \\
5.6\end{array}$ \\
\hline $\begin{array}{l}1960 \\
1961 \\
1962 \\
1963 \\
1964 \\
1965 \\
1966 \\
1967 \\
1968 \\
1969\end{array}$ & $\begin{array}{l}11 \\
10 \\
17 \\
20 \\
11 \\
3.1 \\
22 \\
16 \\
2.7 \\
23\end{array}$ & $\begin{array}{l}13 \\
11 \\
18 \\
21 \\
12 \\
3.3 \\
23 \\
17 \\
2.8 \\
24\end{array}$ & $\begin{array}{l}15 \\
11 \\
19 \\
22 \\
12 \\
3.8 \\
23 \\
17 \\
3.4 \\
27\end{array}$ & $\begin{array}{c}29 \\
14 \\
24 \\
25 \\
13 \\
4.1 \\
27 \\
18 \\
4.9 \\
60\end{array}$ & $\begin{array}{l}34 \\
18 \\
30 \\
26 \\
15 \\
7.3 \\
45 \\
26 \\
8.3 \\
61\end{array}$ & $\begin{array}{r}62 \\
23 \\
45 \\
37 \\
17 \\
15 \\
105 \\
36 \\
13 \\
85\end{array}$ & $\begin{array}{r}85 \\
24 \\
62 \\
91 \\
19 \\
18 \\
162 \\
37 \\
14 \\
207\end{array}$ \\
\hline $\begin{array}{l}1970 \\
1971 \\
1972 \\
1973 \\
1974 \\
1975 \\
1976\end{array}$ & $\begin{array}{l}21 \\
17 \\
17 \\
38 \\
36 \\
27 \\
22\end{array}$ & $\begin{array}{l}21 \\
18 \\
19 \\
42 \\
38 \\
27 \\
23\end{array}$ & $\begin{array}{l}22 \\
20 \\
19 \\
44 \\
41 \\
27 \\
23\end{array}$ & $\begin{array}{l}24 \\
24 \\
19 \\
47 \\
46 \\
28 \\
28\end{array}$ & $\begin{array}{l}20 \\
33 \\
21 \\
82 \\
50 \\
31 \\
32\end{array}$ & $\begin{array}{r}34 \\
51 \\
3 \% \\
103 \\
124 \\
33 \\
50\end{array}$ & $\begin{array}{r}35 \\
101 \\
47 \\
130 \\
195 \\
35 \\
45\end{array}$ \\
\hline
\end{tabular}




DRAINAGE AREA: $300 \mathrm{~m}^{2}=4$ PERIOD OF RECORD: 31 VEARS AVERAGE DISCHARGE: $144 \mathrm{ft} / \mathrm{s}$
MINIMUM AVERAGE FLOWS FOR PERIOD OF RECORD

MAGNITUDE AND FREQUENCY OF ANNUAL LOW FLOWS

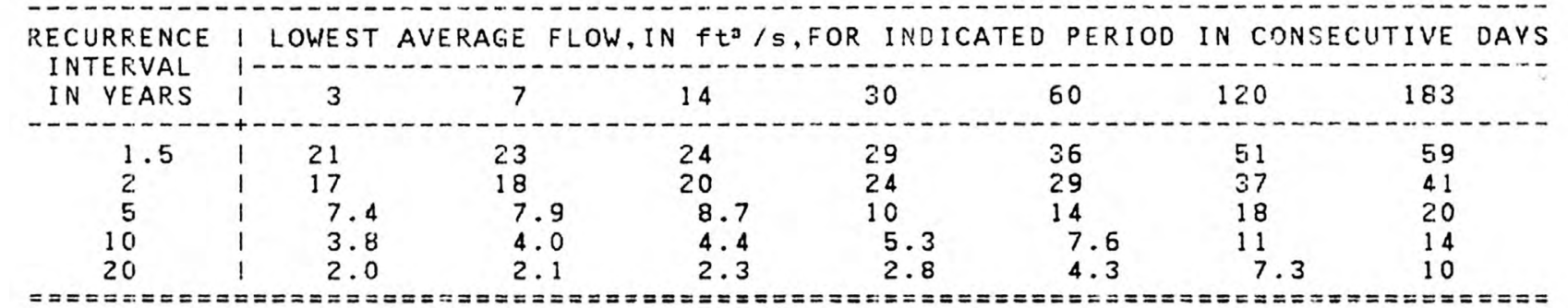

MAGNITUDE ANO FREQUENCY OF SEASONAL LOW FLOWS

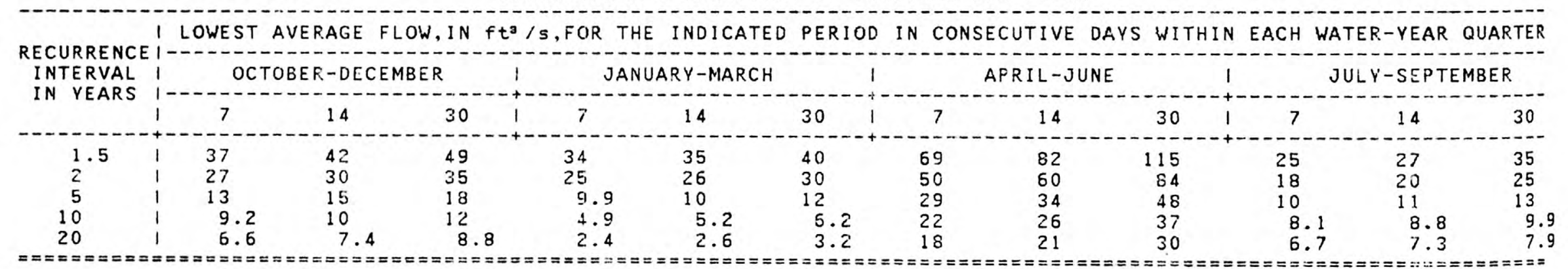

DURATION OF DAILY DISCHARGES FOR ANNUAL AND SEASONAL PERIODS

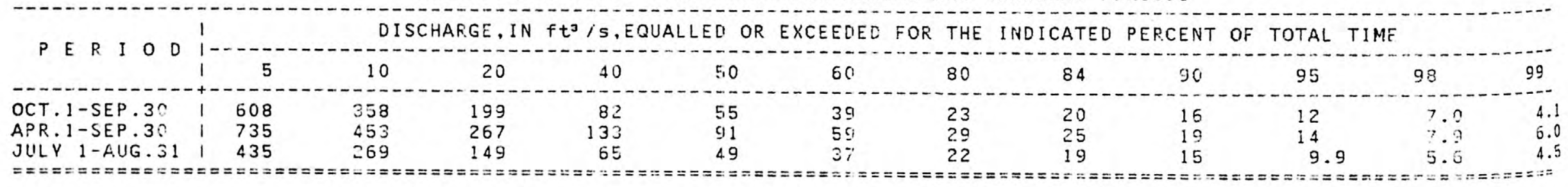




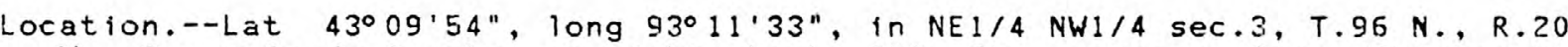
W., Cerro Gordo County, on right bank $650 \mathrm{ft}$ upstream from Thirteenth Street Bridge in Mason City, 0.1 mile downstream from Calmus Creek, and 1.0 mile upstream from Willow Creek.

LOW FLOW DISCHARGES, IN YEAR. ENDING MARCH 31

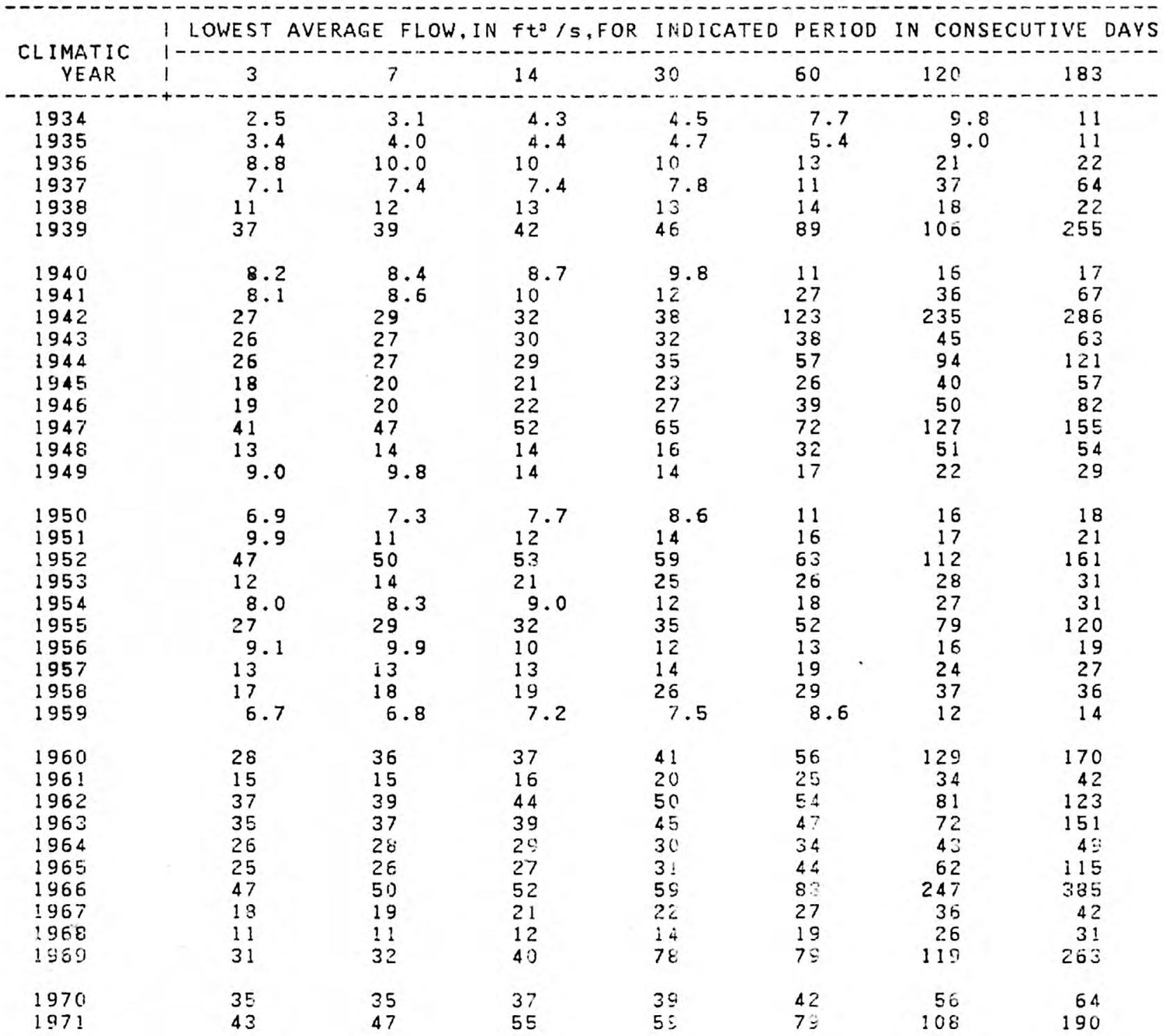


LOW FLOW DISCHARGES, IN YEAR ENDING MARCH 31--Continued

\begin{tabular}{|c|c|c|c|c|c|c|c|c|}
\hline \multirow{2}{*}{$\begin{array}{c}\text { CL IMA.T IC } \\
\text { YEAR }\end{array}$} & 1 LOWES & GE & $f t^{3}$ & INS & $\begin{array}{l}\mathrm{PE} \\
0\end{array}$ & IN CO & IVE & DAYS \\
\hline & 13 & 7 & 14 & 30 & 60 & 120 & 183 & \\
\hline $\begin{array}{l}1972 \\
1973 \\
1974 \\
1975 \\
1976\end{array}$ & $\begin{array}{l}31 \\
71 \\
58 \\
23 \\
25\end{array}$ & $\begin{array}{l}34 \\
75 \\
63 \\
23 \\
26\end{array}$ & $\begin{array}{l}35 \\
82 \\
63 \\
23 \\
27\end{array}$ & $\begin{array}{l}37 \\
88 \\
E 6 \\
24 \\
31\end{array}$ & $\begin{array}{r}52 \\
172 \\
73 \\
25 \\
42\end{array}$ & $\begin{array}{r}87 \\
237 \\
185 \\
31 \\
67\end{array}$ & $\begin{array}{r}105 \\
297 \\
270 \\
44 \\
67\end{array}$ & \\
\hline
\end{tabular}




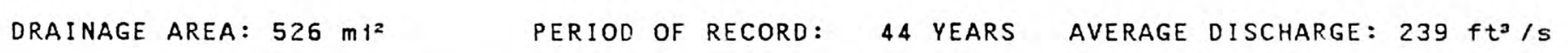

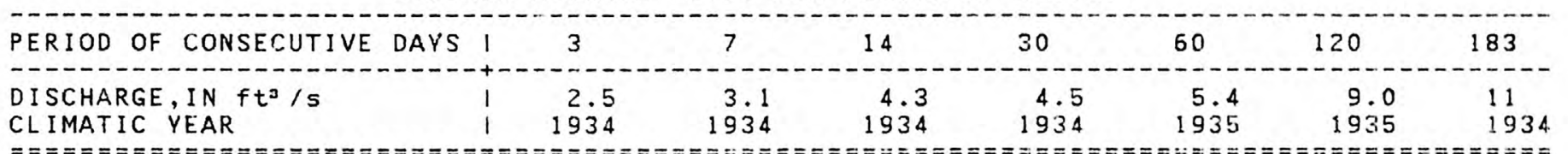

MAGNITUDE AND FREQUENCY OF ANNUAL LOW FLOWS

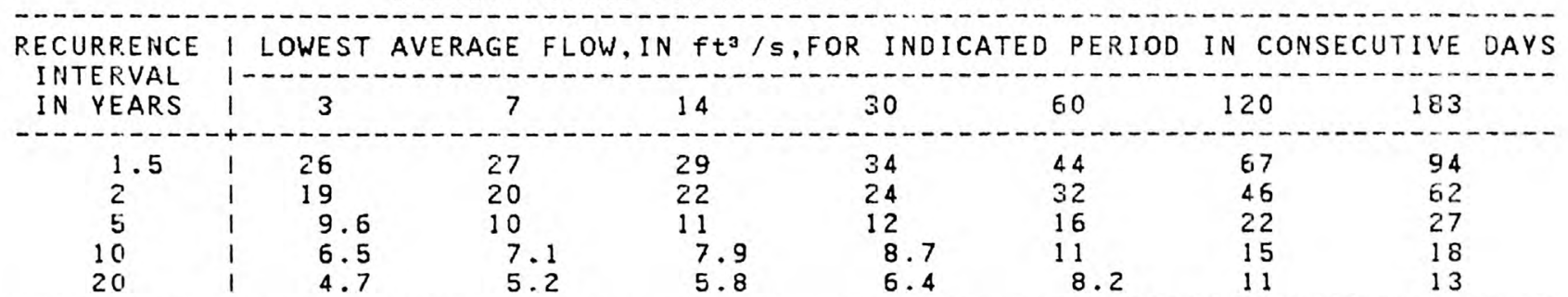

MAGNITUDE AND FREQUENCY OF SEASONAL LOW FLOWS

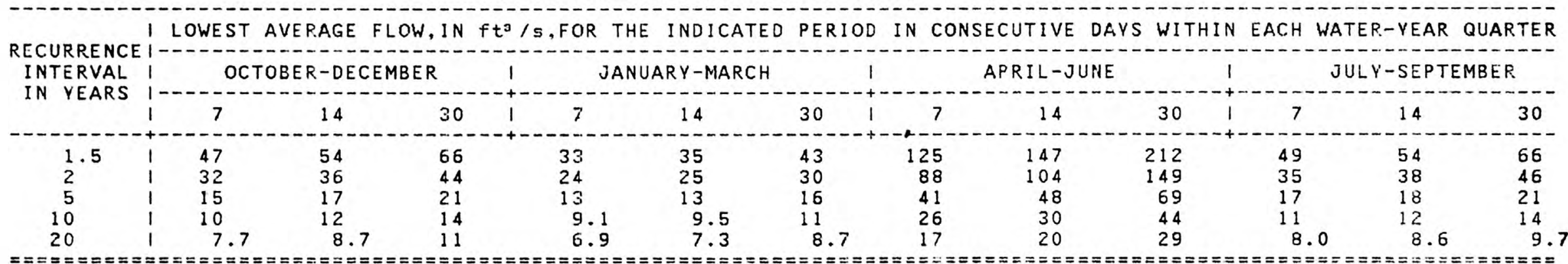

DURATION OF DAILY DISCHARGES FOR ANNUAL AND SEASONAL FERIODS

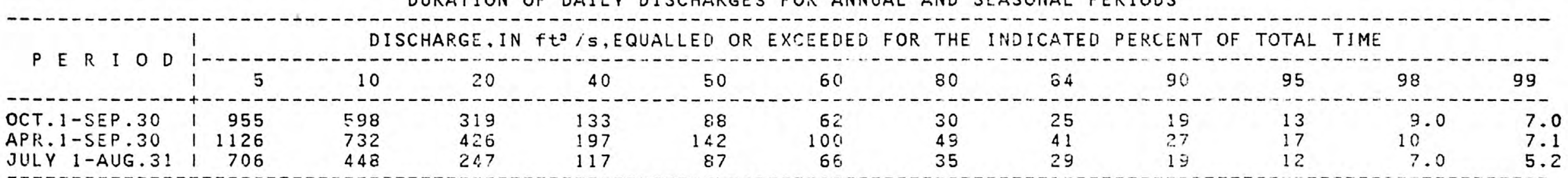


Location.--Lat $42^{\circ} 58^{\prime} 00^{\prime \prime}$, long $92^{\circ} 52^{\prime} 15^{\prime \prime}$, in SE1/4 SE1/4 sec.8, T.94 N., R.17 W. Floyd County, on left bank $20 \mathrm{ft}$ above dam at Marble Rock, i.1 mile upstream from Ackley Creek, $9.5 \mathrm{miles}$ downstream from Winnebago River, and at mile 247.1 above mouth of lowa River.

Remarks.--Flow regulated by powerplant at station prior to Oct. $1,1942$. Published as "at Greene", 1933-42. Discontinued September 1953.

LOW FLOW DISCHARGES, IN YEAR. ENDING MARCH 31

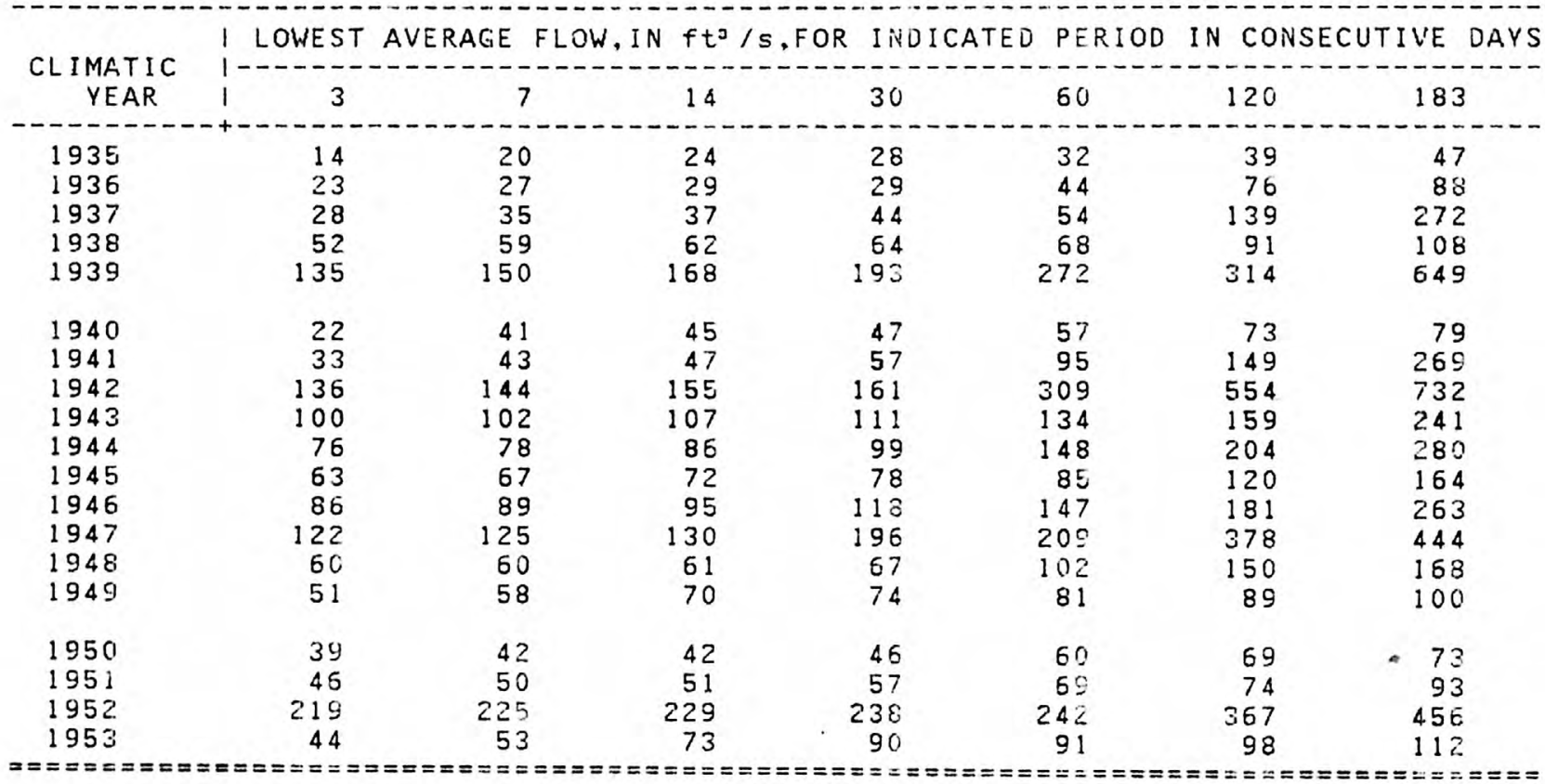


05-4605.00 SHELL ROCK RIVER AT MARBLE ROCK--Cont 1nued
DRAINAGE AREA: $1318 \mathrm{mi}^{2}$
PERIOD OF RECORD:
20 YEARS
AVERAGE DISCHARGE: $612 \mathrm{ft}^{3} / \mathrm{s}$

MINIMUM AVERAGE FLOWS FOR PERIOD OF RECORD

\begin{tabular}{|c|c|c|c|c|c|c|c|}
\hline PERIOD OF CONSECUTIVE DAYS & 1 & 7 & 14 & 30 & 60 & 120 & 183 \\
\hline $\begin{array}{l}\text { ISCHARGE, IN } \mathrm{ft}^{3} / \mathrm{s} \\
\text { LIMATIC YEAR }\end{array}$ & $\begin{array}{l}14 \\
1935\end{array}$ & $\begin{array}{l}20 \\
1935\end{array}$ & $\begin{array}{l}24 \\
1935\end{array}$ & $\begin{array}{l}28 \\
1935\end{array}$ & $\begin{array}{l}32 \\
1935\end{array}$ & $\begin{array}{l}39 \\
1935\end{array}$ & $\begin{array}{l}47 \\
1935\end{array}$ \\
\hline
\end{tabular}

MAGNITUDE AND FREQUENCY OF ANNUAL LOW FLOWS

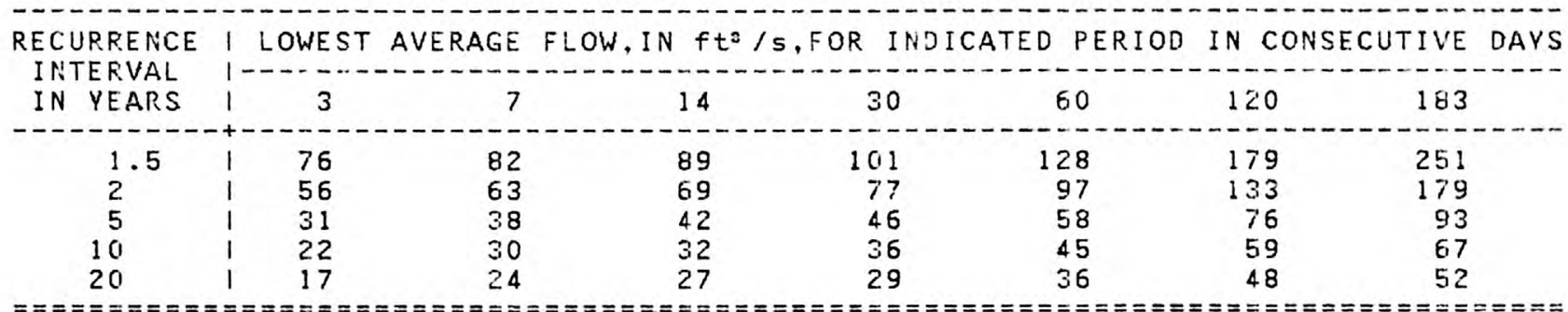

MAGNITUDE AND FREQUENCY OF SEASONAL LOW FLOWS

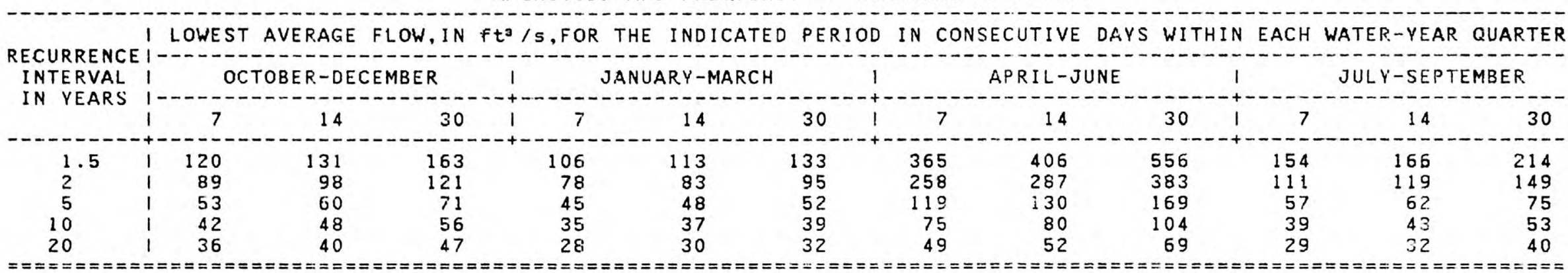

DURATION OF DAILY DISCHARGES FOR ANMUAL AND SEASONAL PERIODS

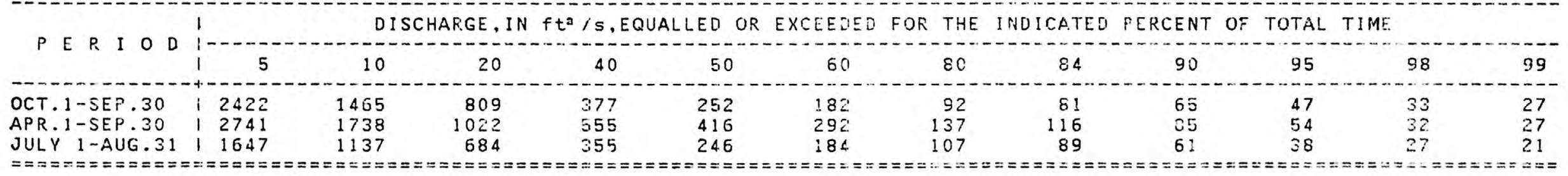


$05-4620.00$

\begin{tabular}{|c|c|c|c|c|c|c|c|c|}
\hline \multirow{2}{*}{$\begin{array}{c}\text { CLIMATIC } \\
\text { YEAR }\end{array}$} & LOWEST & AGE $F$ & 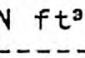 & R INL & D PER & \multicolumn{3}{|c|}{ IN CONSECUTIVE DAYS } \\
\hline & 13 & 7 & 14 & 30 & 60 & 120 & 183 & \\
\hline $\begin{array}{l}1955 \\
1956 \\
1957 \\
1958 \\
1959\end{array}$ & $\begin{array}{r}181 \\
79 \\
71 \\
100 \\
39\end{array}$ & $\begin{array}{r}184 \\
81 \\
73 \\
102 \\
39\end{array}$ & $\begin{array}{r}189 \\
82 \\
83 \\
113 \\
40\end{array}$ & $\begin{array}{r}194 \\
87 \\
88 \\
130 \\
42\end{array}$ & $\begin{array}{r}247 \\
90 \\
95 \\
149 \\
45\end{array}$ & $\begin{array}{r}354 \\
102 \\
110 \\
172 \\
61\end{array}$ & $\begin{array}{l}472 \\
112 \\
122 \\
171 \\
73\end{array}$ & \\
\hline $\begin{array}{l}1960 \\
1961 \\
1962 \\
1963 \\
1964 \\
1965 \\
1966 \\
1967 \\
1968 \\
1969\end{array}$ & $\begin{array}{r}164 \\
105 \\
147 \\
196 \\
118 \\
96 \\
216 \\
108 \\
50 \\
132\end{array}$ & $\begin{array}{r}173 \\
108 \\
170 \\
203 \\
136 \\
98 \\
244 \\
128 \\
53 \\
154\end{array}$ & $\begin{array}{r}183 \\
113 \\
184 \\
207 \\
146 \\
106 \\
245 \\
145 \\
59 \\
172\end{array}$ & $\begin{array}{r}232 \\
134 \\
187 \\
231 \\
149 \\
120 \\
256 \\
163 \\
76 \\
315\end{array}$ & $\begin{array}{l}281 \\
157 \\
198 \\
243 \\
160 \\
153 \\
359 \\
215 \\
109 \\
339\end{array}$ & $\begin{array}{l}455 \\
204 \\
282 \\
331 \\
179 \\
194 \\
817 \\
243 \\
124 \\
454\end{array}$ & $\begin{array}{r}562 \\
271 \\
367 \\
633 \\
214 \\
276 \\
1200 \\
248 \\
138 \\
859\end{array}$ & \\
\hline $\begin{array}{l}1970 \\
1971 \\
1972 \\
1973 \\
1974 \\
1975 \\
1976\end{array}$ & $\begin{array}{l}193 \\
256 \\
175 \\
274 \\
313 \\
133 \\
150\end{array}$ & $\begin{array}{l}208 \\
264 \\
176 \\
282 \\
330 \\
137 \\
160\end{array}$ & $\begin{array}{l}217 \\
295 \\
179 \\
302 \\
378 \\
150 \\
176\end{array}$ & $\begin{array}{l}222 \\
321 \\
189 \\
333 \\
396 \\
175 \\
190\end{array}$ & $\begin{array}{l}233 \\
391 \\
222 \\
503 \\
437 \\
198 \\
232\end{array}$ & $\begin{array}{l}291 \\
490 \\
323 \\
5.69 \\
843 \\
230 \\
310\end{array}$ & $\begin{array}{l}341 \\
787 \\
349 \\
843 \\
915 \\
264 \\
302\end{array}$ & \\
\hline
\end{tabular}

SHELL ROCK RIVER AT SHEL! ROCK

ocation.--Lat $42^{\circ} 39^{\prime} 10^{\prime \prime}$, long $92^{\circ} 35^{\prime} 46^{\prime \prime}$, in NE1/4 NW1/4 sec.11, T.91 N., R.15

1.. Butler County, on right bank $400 \mathrm{ft}$ uostream from bridge on county

highway C45 in Shell Rock. 2.2 miles downstream from Curry Creek, and 10.4

miles upstream froin mouth.

Remarks.--Diurnal fluctuation caused by powerplant 24 mfles upstream at Greene.

LOW FLOW DISCHARGE 
MINIMUM AVERAGE FLOWS FOR PERIOD OF RECORD

\begin{tabular}{|c|c|c|c|c|c|c|c|}
\hline PERIOD OF CONSECUTIVE DAYS & 3 & 7 & 14 & 30 & 60 & 120 & 183 \\
\hline $\begin{array}{l}\text { DISCHARGE, IN } \mathrm{ft}^{3} / \mathrm{s} \\
\text { CLIMATIC VEAR }\end{array}$ & $\begin{array}{l}39 \\
1959\end{array}$ & $\begin{array}{l}39 \\
1959\end{array}$ & $\begin{array}{l}40 \\
1959\end{array}$ & $\begin{array}{l}42 \\
1959\end{array}$ & $\begin{array}{l}45 \\
1959\end{array}$ & $\begin{array}{l}61 \\
1959\end{array}$ & $\begin{array}{l}73 \\
1959\end{array}$ \\
\hline
\end{tabular}

MAGNITUDE AND FREQUENCY OF ANNUAL LOW FLOWS

\begin{tabular}{l|ccccc} 
RECURRENCE \\
INTERVAL \\
IN YOWEARS
\end{tabular}

MAGNITUDE AND FREQUENCY OF SEASONAL LOW FLOWS

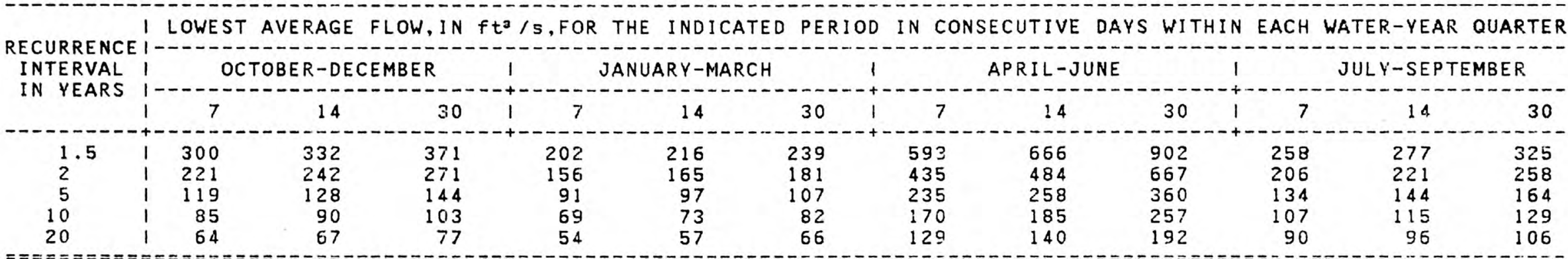

DURATION OF DAILY DISCHARGES FOR ANNUAL AND SEASONAL PERIODS

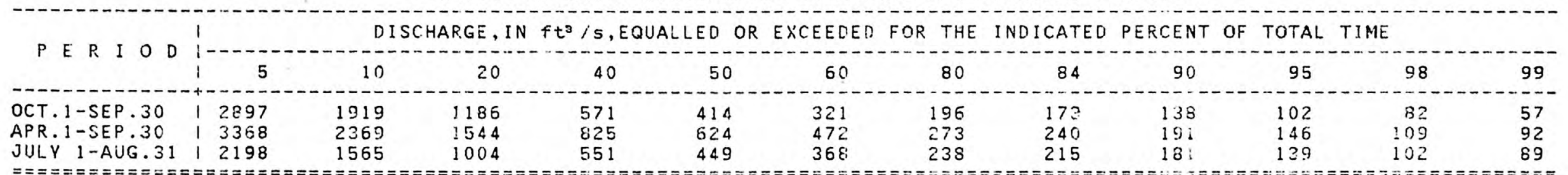


Location.--Lat $42^{\circ} 30^{\prime} 50^{\prime \prime}$, long $92^{\circ} 37^{\prime} 55^{\prime \prime}$, in SE1/4 SE1/4 sec.28, T.90 N., R.15 W. , Butler County, on downstream side of center bridge pier of bridge on county highway T55, $0.2 \mathrm{mile}$ north of New Hartford, and $8 \mathrm{miles}$ upstream from mouth.

LOW FLOW DISCHARGES, IN YEAR. ENDING MARCH 31

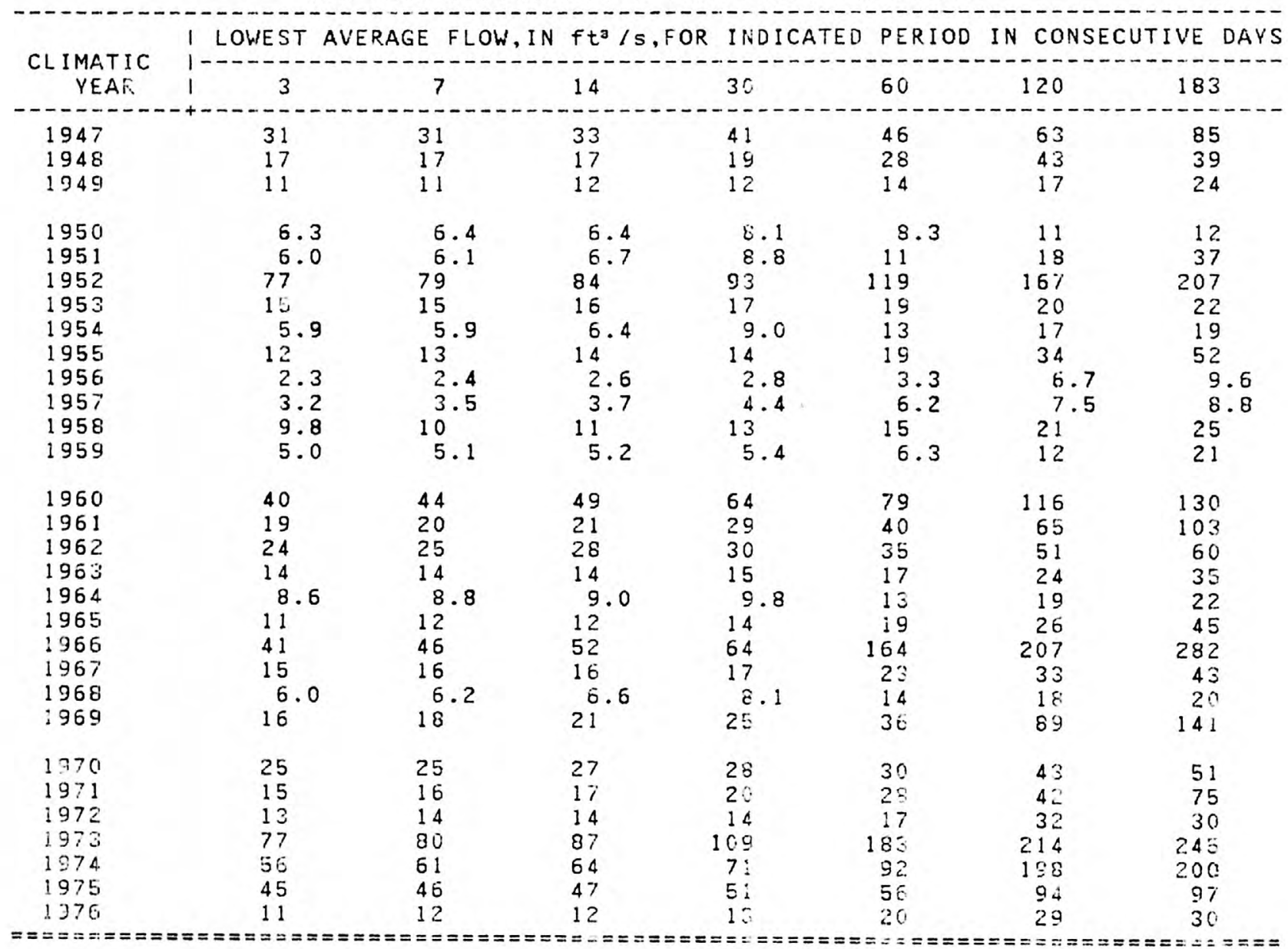


DRAINAGE AREA: $347 \mathrm{~m}^{2} \quad$ PERIOD OF RECORD: 31 YEARS AVERAGE DISCHARGE: 188 fT3/S MINIMUM AVERAGE FLOWS FOR PERIOD OF RECORD

\begin{tabular}{|c|c|c|c|c|c|c|c|}
\hline PERIOD OF CONSECUTIVE DAYS I & 3 & 7 & 14 & 30 & 60 & 120 & 183 \\
\hline $\begin{array}{l}\text { I SCHARGE, IN } \mathrm{ft}^{3} / \mathrm{s} \\
\text { LIMATIC VEAR }\end{array}$ & $\begin{array}{r}2.3 \\
1956\end{array}$ & $\begin{array}{r}2.4 \\
1955\end{array}$ & $\begin{array}{r}2.6 \\
1956\end{array}$ & $\begin{array}{r}2.8 \\
1956\end{array}$ & $\begin{array}{r}3.3 \\
1956\end{array}$ & $\begin{array}{r}6.7 \\
1956\end{array}$ & $\begin{array}{r}8.8 \\
1957\end{array}$ \\
\hline
\end{tabular}

MAGNITUDE AND FREQUENCY OF ANNUAL LOW FLOWS

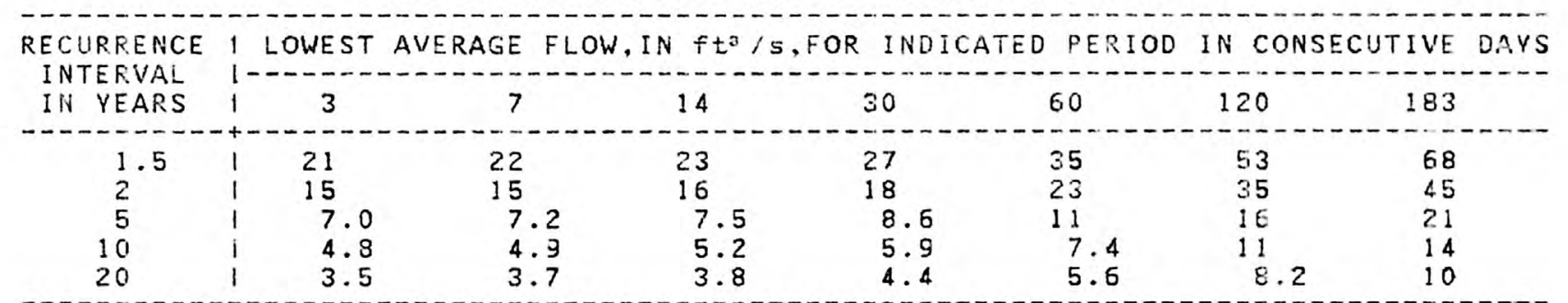

MAGNITUDE AND FREQUENCY OF SEASONAL LOW FLOWS

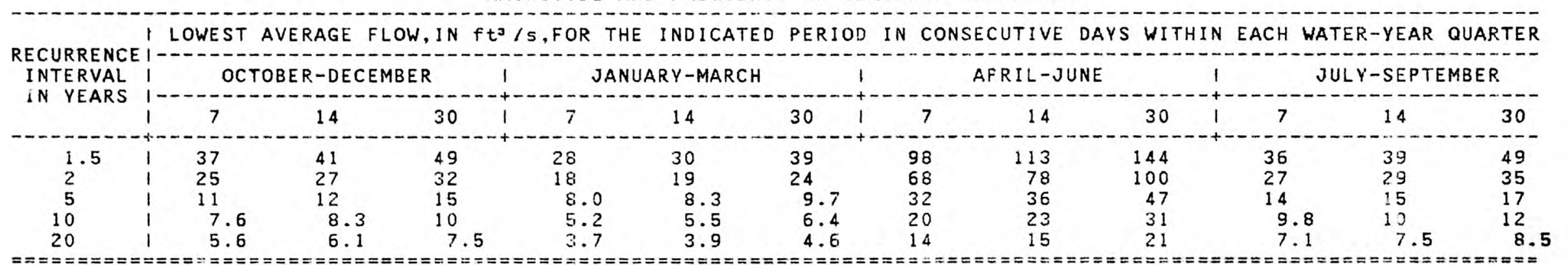

DURATION OF DAILY DISCHARGES FOR ANNUAL AND SEASONAL FERIODS

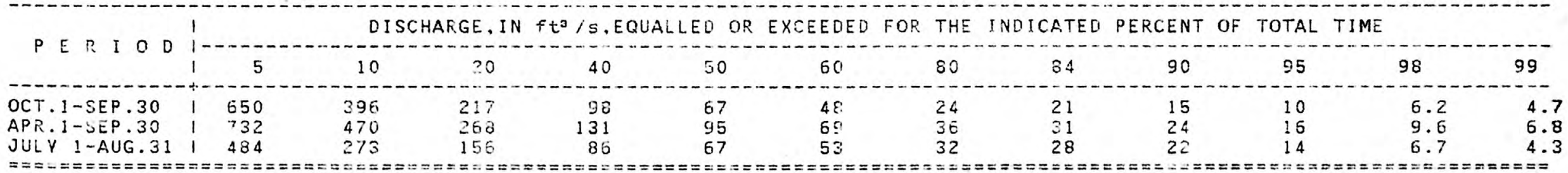


$05-4635.00$

Location.--Lat $42^{\circ} 24^{\prime} 28^{\prime \prime}$, long $92^{\circ} 27^{\prime} 47^{\prime \prime}$, In SW1/4 NE1/4 sec.27, T.88 N., R.14 W. B B lack Hawk County, on left bank $35 \mathrm{ft}$ downstream from bridge on State Highway $58,0.2 \mathrm{mile}$ northwest of Chlcago Great Western Raliway tracks at the west edge of Hudson, $4.5 \mathrm{miles}$ upstream from Frescotts Creek, and 9.6 miles upstream from mouth.

LOW FLOW DISCHARGES, IN YEAR ENDING MARCH 31

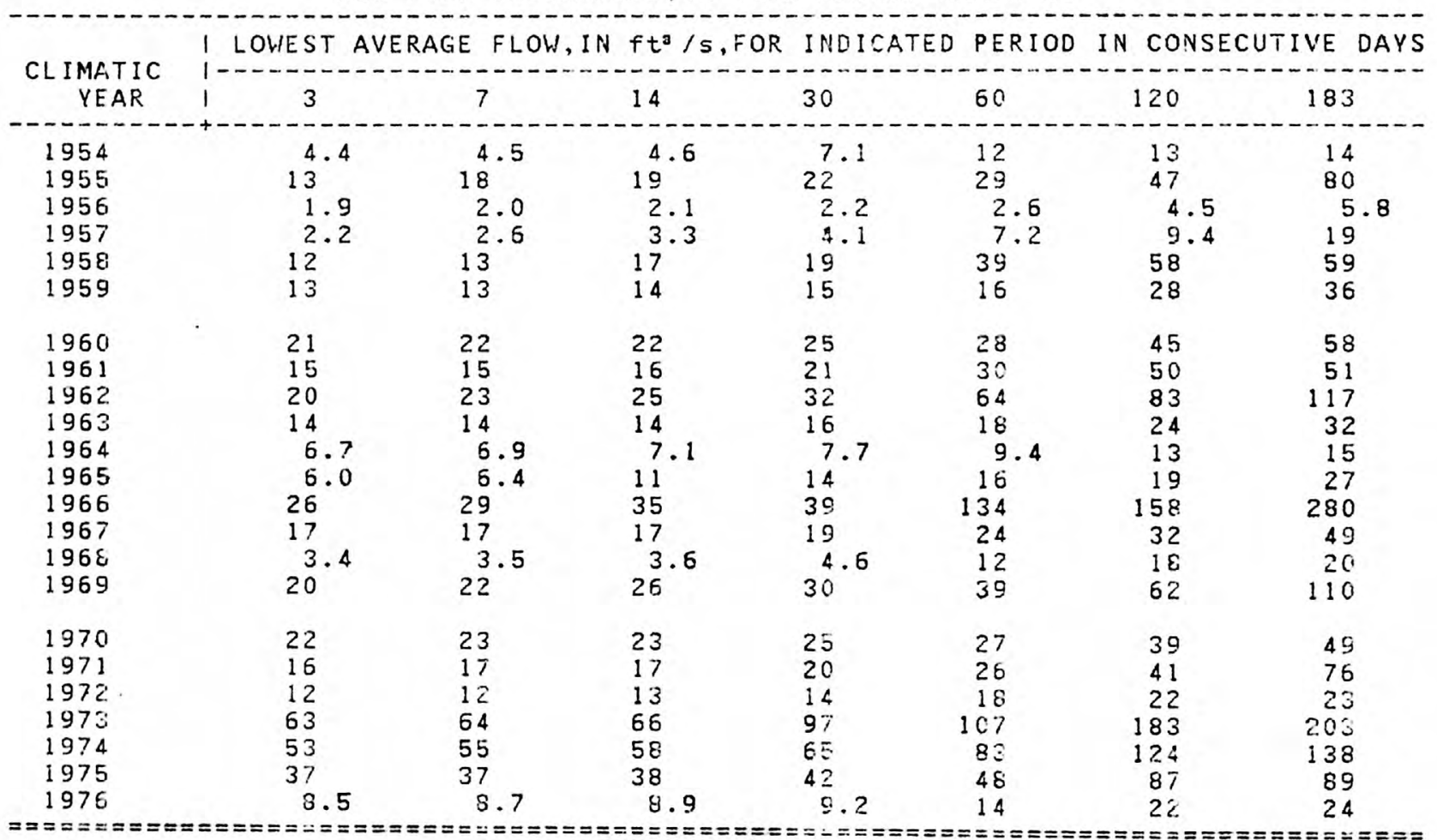


05-4635.00 BLACK HAWK CREEK AT HUDSON--Continued

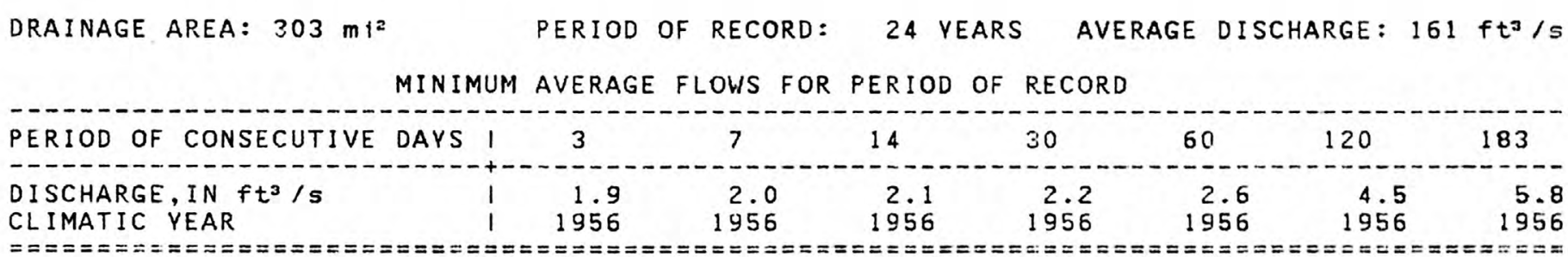

MAGNITUDE AND FREQUENCY OF ANNUAL LOW FLOWS

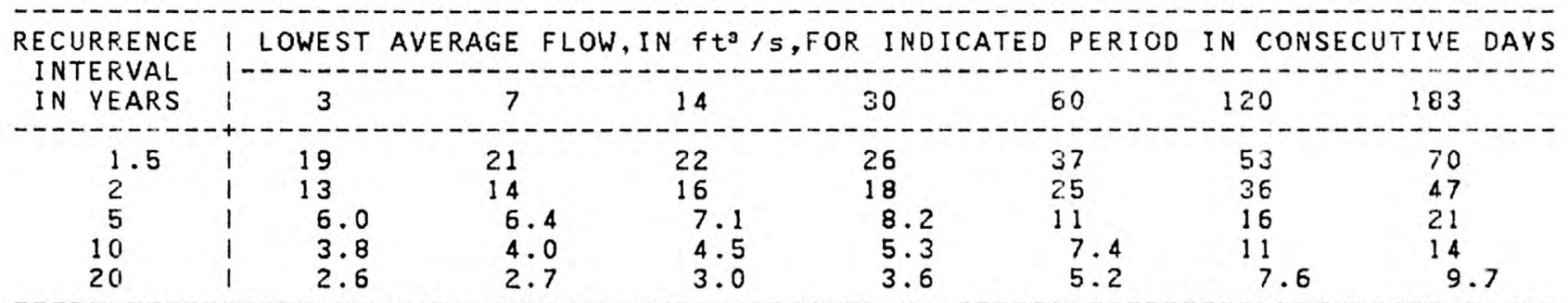

MAGNITUDE AND FREQUENCY OF SEASONAL LOW FLOWS

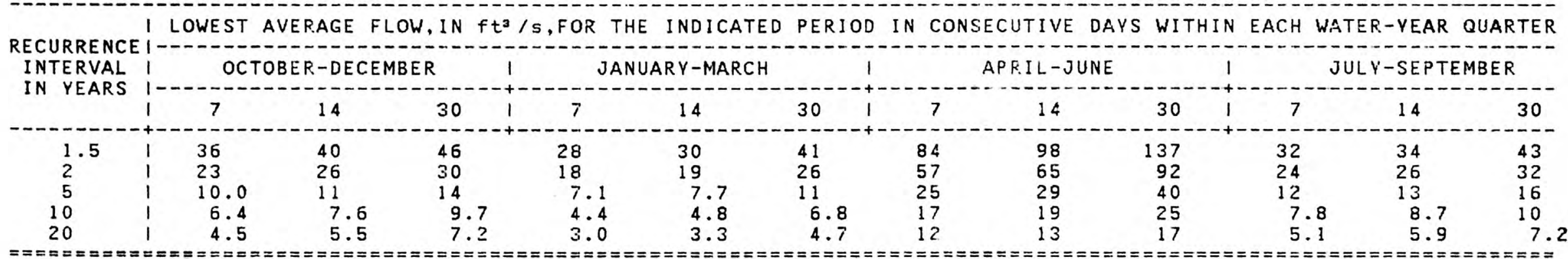

DURATION OF DAILY DISCHARGES FOR FINUUAL AND SEASONAL PERIODS

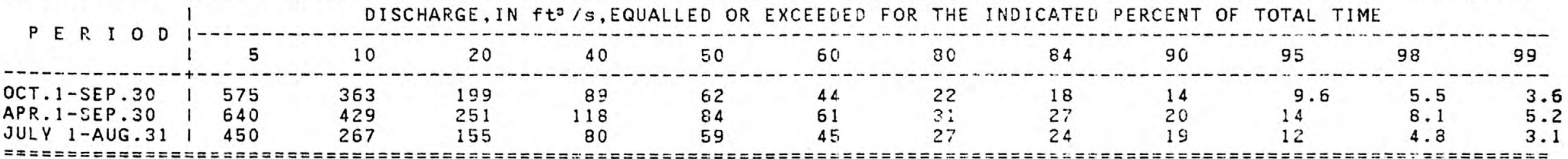


Location.--Lat $42^{\circ} 29^{\prime} 44^{\prime \prime}$, long 92020.03", in NW1/4 NW1/4 sec.25, T.89 N., R.13 W. , B lack Hawk County, on left bank at foot of East Seventh Street, 0.3 mile upstream from Eleventh Avenue Bridge in Water loo, 1.1 mile downstream from Black Hawk Creek, and at mile 187.9 above mouth of Iowa River.

Remarks.--Slight diurnal fiuctuation caused by powerplant upstream of station. LOW FLOW DISCHARGES, IN YEAR ENDING MARCH 31

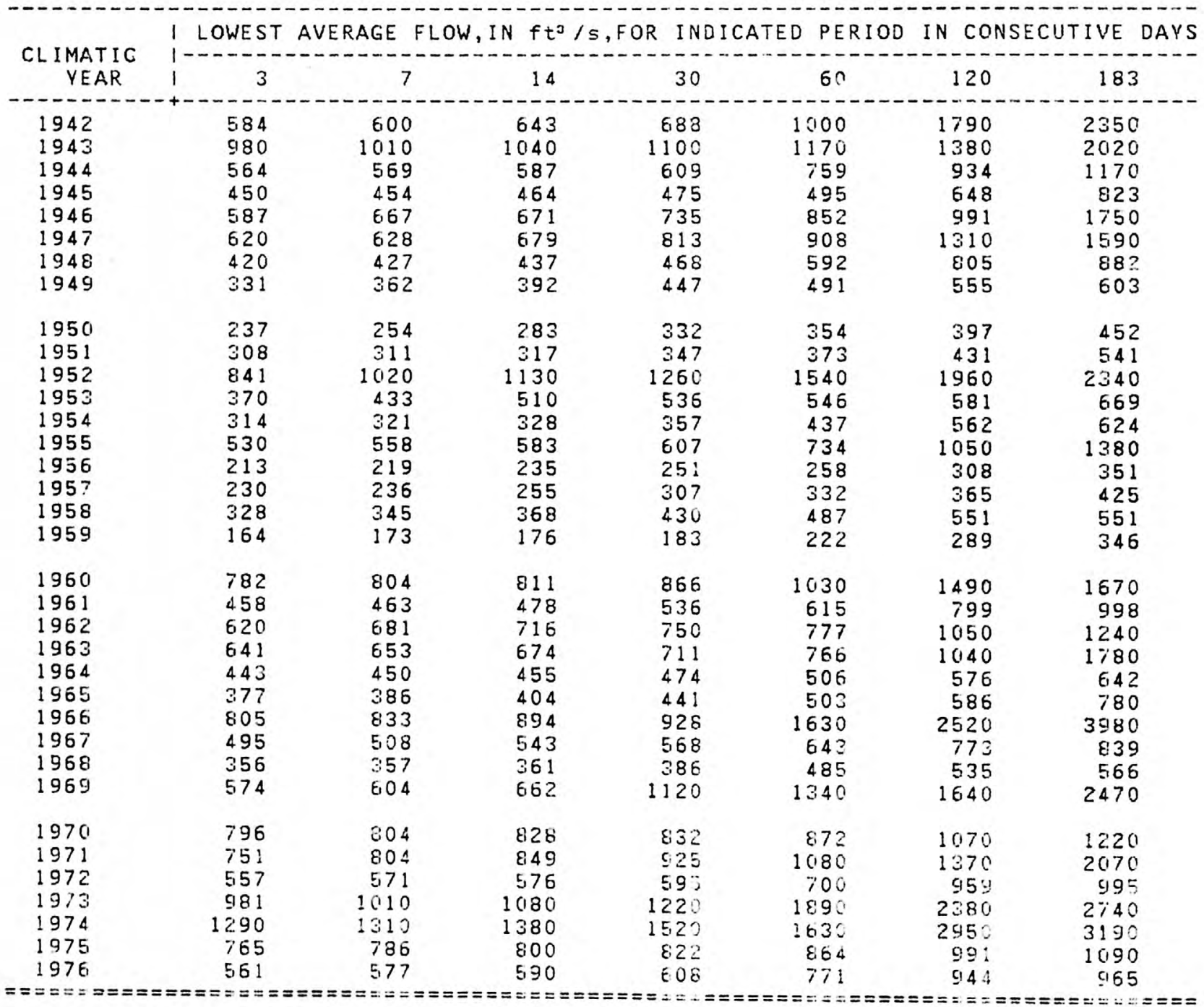


05-4640.00 CEDAR RIVER AT WATERLOO--Continued

DRAINAGE AREA: $5146 \mathrm{ml}^{2} \quad$ PERIOD OF RECORD: 36 VEARS AVERAGE DISCHARGE: $2772 \mathrm{ft}^{3} / \mathrm{S}$

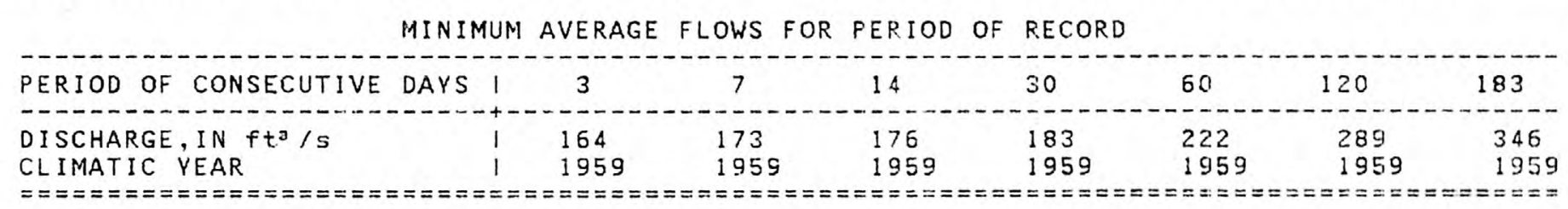

MAGNITUDE AND FREQUENCY OF ANNUAL LOW FLOWS

RECURRENCE I LOWEST AVERAGE FLOW, IN $\mathrm{ft}^{2} / \mathrm{s}, \mathrm{FOR}$ INDICATED PERIOD IN CONSECUTIVE UAYS INTERVAL

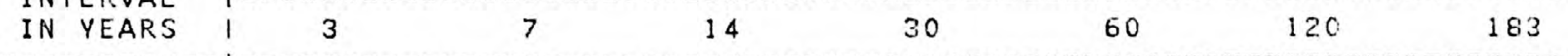

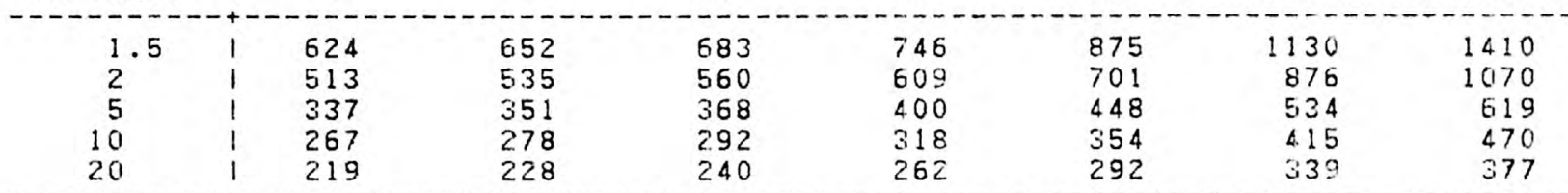

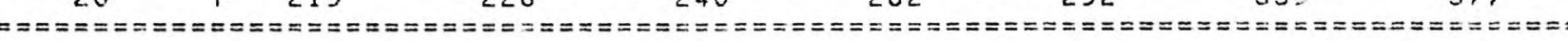

MAGNITUDE AND FREQUENCY OF SEASONAL LOW FLOWS

I LOWEST AVERAGE FLOW, IN $\mathrm{ft}^{\circ} / \mathrm{s}, F O R$ THE INDICATED PERIOD IN CONSECUTIVE DAYS WITHIN EACH WATER-YEAR QUARTER RECURRENCE 1 LOWEST AVERAGE FLOW, IN

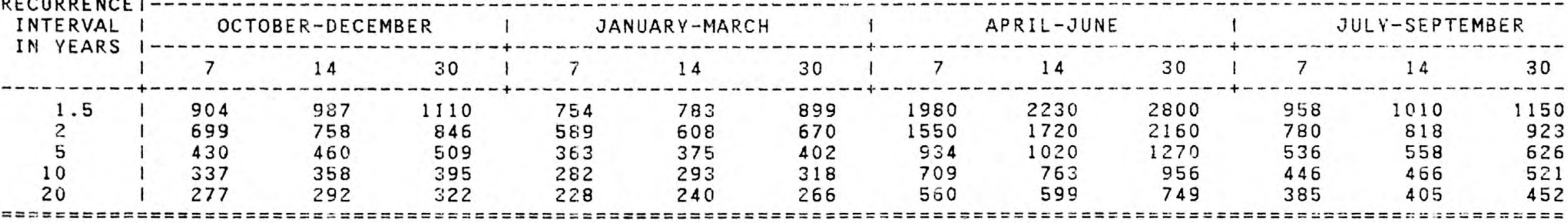

DURATION OF DAILY DISCHARGES FOR ANNUAL AND SEASONAL FERIODS

\begin{tabular}{|c|c|c|c|c|c|c|c|c|c|c|c|c|}
\hline \multirow{2}{*}{$\begin{array}{llllll}P & E & R & I & O & D\end{array}$} & 1 & \multicolumn{2}{|c|}{ DISCHARGE, IN } & \multicolumn{2}{|c|}{3 is, EQUALLED } & OR EXCEELIED & THE & INDICA.TEO & \multicolumn{3}{|c|}{ PERCENT OF TOTAL TIME } & $-\ldots$ \\
\hline & $i$ & 10 & 20 & 40 & 50 & 60 & 80 & 84 & 90 & 95 & 98 & 99 \\
\hline $\begin{array}{l}\text { OCT. 1-SEP. } 30 \\
\text { APR. I-SEP. } 30 \\
\text { JULY } 1 \text {-AUG. } 31\end{array}$ & $\begin{array}{r}9510 \\
10600 \\
17330\end{array}$ & $\begin{array}{l}5050 \\
7270 \\
4760\end{array}$ & $\begin{array}{l}3610 \\
4520 \\
3130\end{array}$ & $\begin{array}{l}1940 \\
2620 \\
1860\end{array}$ & $\begin{array}{l}1440 \\
2070 \\
1490\end{array}$ & $\begin{array}{l}1120 \\
1610 \\
1230\end{array}$ & $\begin{array}{l}702 \\
95 ! \\
858\end{array}$ & $\begin{array}{l}631 \\
878 \\
783\end{array}$ & $\begin{array}{l}521 \\
722 \\
669\end{array}$ & $\begin{array}{l}4: 1 \\
574 \\
557\end{array}$ & $\begin{array}{l}319 \\
176 \\
48 !\end{array}$ & $\begin{array}{l}273 \\
425 \\
445\end{array}$ \\
\hline
\end{tabular}


HALF MILE CREEK NEAR GLADBROOK

Location - Lat $42^{\circ} 12^{\prime 40 "}$ " long 92036'39". In SW1/4 SWl/4 sec.33, T.86 N., R.15 W. Tama County, on right bank $10 \mathrm{ft}$ downstream from bridge on councy highway, $0.8 \mathrm{mile}$ upstream from mouth, and 5.3 miles northeast of Gladbrook.

Remarks.--Discontinued September 1974.

LOW FLOW DISCHARGES, IN YEAR. ENDING MARCH 31

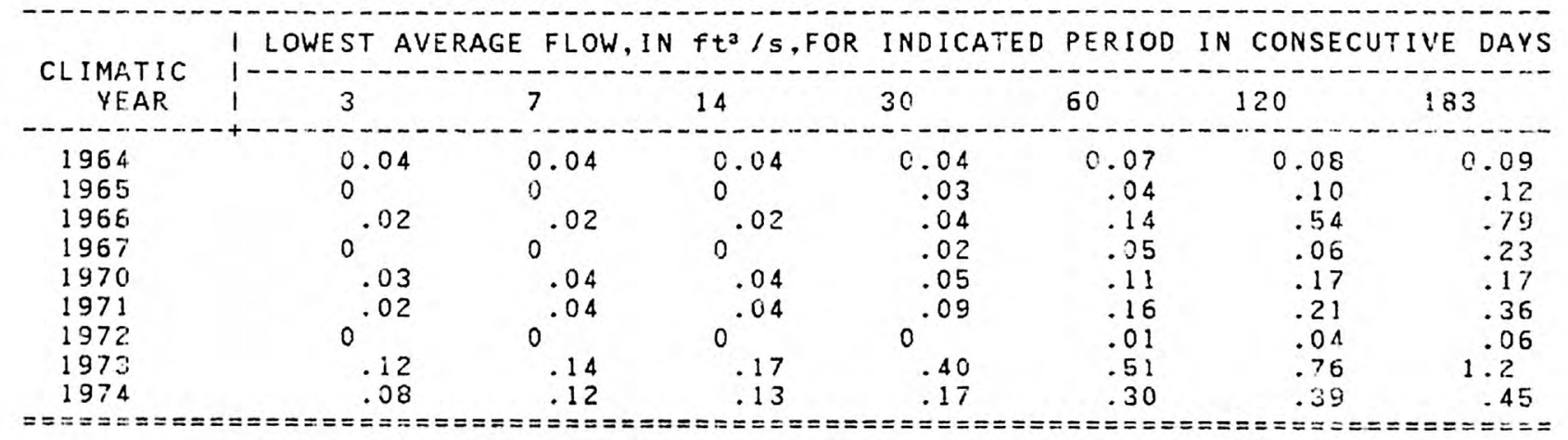




\section{5-4641.33 HALF MILE CREEK NEAR GLADBROOK--Continued}
DRAINAGE AREA:
$1.33 \mathrm{mi}$
PERIOD OF RECORD:
10 VEARS
AVERAGE DISCHARGE :
$0.8 \mathrm{ft}^{3} / \mathrm{s}$

MINIMUM AVERAGE FLOWS FOR PERIOD OF RECORD

\begin{tabular}{|c|c|c|c|c|c|c|c|}
\hline PERIOD OF CONSECUTIVE DAYS & 3 & 7 & 14 & 30 & 60 & 120 & 183 \\
\hline $\begin{array}{l}\text { HARGE, IN } \mathrm{ft}^{3} / \mathrm{s} \\
\text { ATIC YEAR }\end{array}$ & $\begin{array}{l}0 \\
1972\end{array}$ & $\begin{array}{l}0 \\
1972\end{array}$ & $\begin{array}{l}0 \\
1972\end{array}$ & $\begin{array}{l}0 \\
1972\end{array}$ & $\begin{array}{l}0.01 \\
1972\end{array}$ & $\begin{array}{l}0.04 \\
1972\end{array}$ & $\begin{array}{l}0.06 \\
1972\end{array}$ \\
\hline
\end{tabular}

MAGNITUDE AND FREQUENCY OF ANNUAL LOW FLOWS

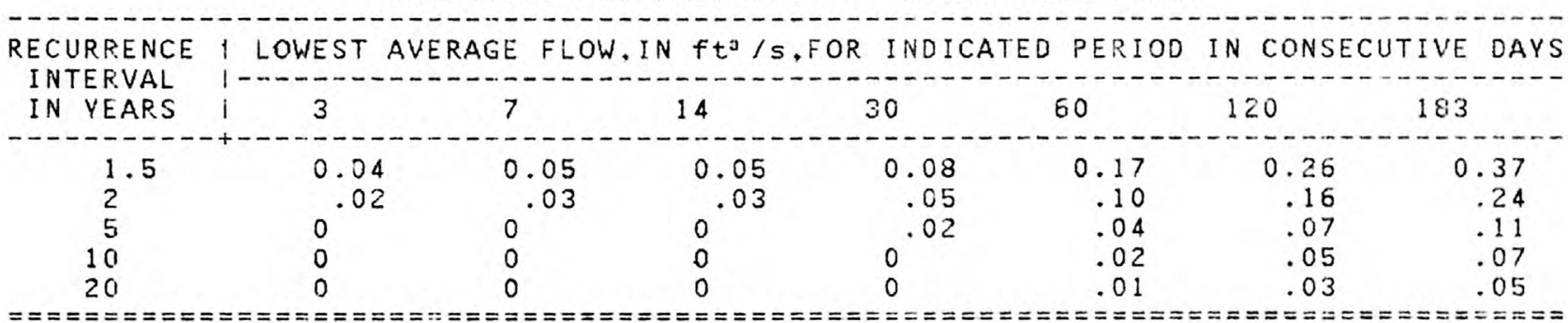

MAGNITUDE AND FREQUENCY OF SEASONAL LOW FLOWS

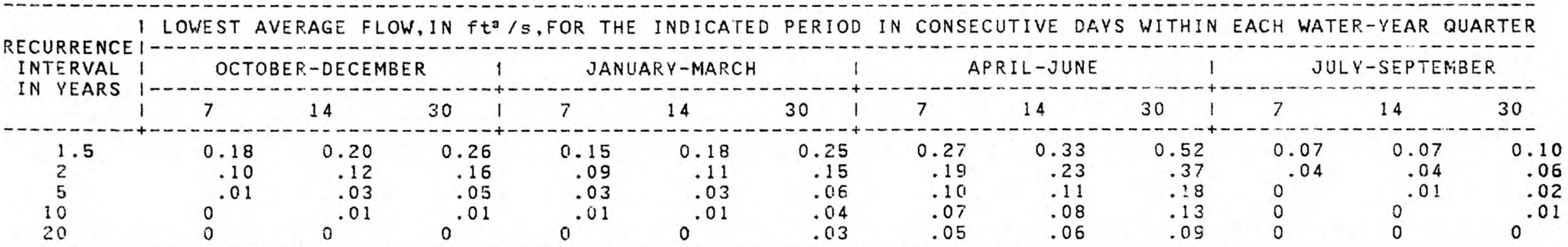

DURATION OF DAILY DISCHARGES FOR ANNUAL AND SEASONAL FERIODS

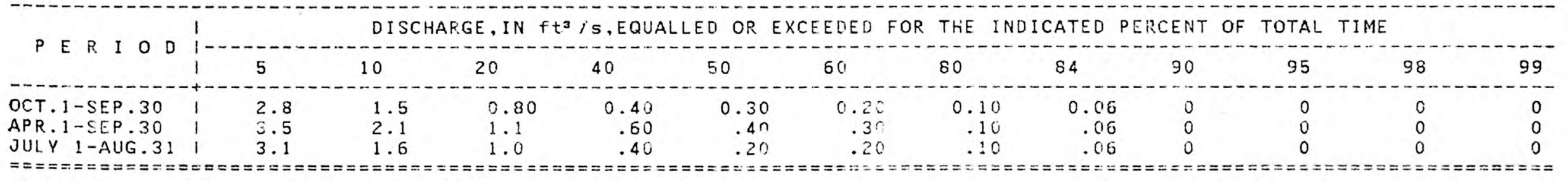


$05-4645.00$

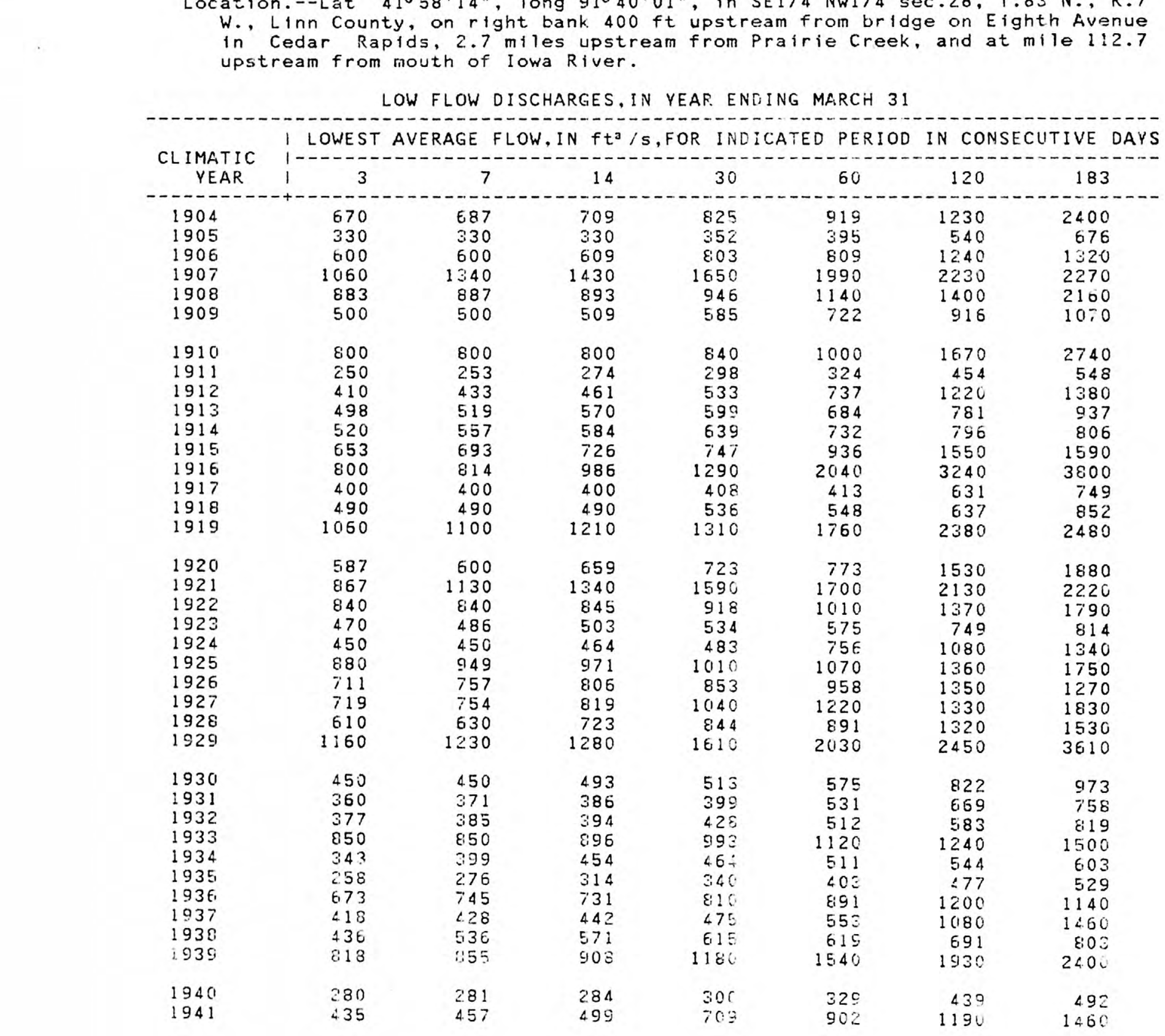

CEDAR RIVER AT CEDAR RAPIDS

ation.--Lat $41^{\circ} 58^{\prime 1} 14^{\prime \prime}$, long $91040.01^{\prime \prime}$, in SE1/4 NW1/4 sec.28, T.83 N., R.7 W., Linn County, an right bank $400 \mathrm{ft}$ upstream from bridge on Eighth Avenue in Cedar Rapids, 2.7 miles upst

LW FLOW DISCHARGES, IN YEAR ENGING MARCH 31 
LOW FLOW DISCHARGES, IN YEAR ENDING MARCH 31--Cont inued

\begin{tabular}{|c|c|c|c|c|c|c|c|}
\hline \multirow{2}{*}{$\begin{array}{c}\text { CLIMATIC } \\
\text { YEAR }\end{array}$} & I LOWEST & AGE & $N f t^{3}$ & OR IND & ED PER & IN $\mathrm{CO}$ & IVE DAYS \\
\hline & 1 & 7 & 14 & 30 & 60 & 120 & 183 \\
\hline $\begin{array}{l}1942 \\
1943 \\
1944 \\
1945 \\
1946 \\
1947 \\
1948 \\
1949\end{array}$ & $\begin{array}{r}682 \\
1240 \\
590 \\
620 \\
687 \\
836 \\
573 \\
460\end{array}$ & $\begin{array}{r}698 \\
1470 \\
678 \\
629 \\
914 \\
985 \\
579 \\
564\end{array}$ & $\begin{array}{r}729 \\
1530 \\
700 \\
637 \\
937 \\
1020 \\
585 \\
626\end{array}$ & $\begin{array}{r}768 \\
1590 \\
729 \\
651 \\
1060 \\
1150 \\
632 \\
657\end{array}$ & $\begin{array}{r}1160 \\
1750 \\
934 \\
680 \\
1310 \\
1370 \\
822 \\
706\end{array}$ & $\begin{array}{r}2520 \\
2130 \\
1140 \\
993 \\
1430 \\
1980 \\
1110 \\
749\end{array}$ & $\begin{array}{r}3030 \\
2830 \\
1450 \\
1150 \\
2350 \\
2490 \\
1180 \\
833\end{array}$ \\
\hline $\begin{array}{l}1950 \\
1951 \\
1952 \\
1953 \\
1954 \\
1955 \\
1956 \\
1957 \\
1958 \\
1959\end{array}$ & $\begin{array}{l}268 \\
330 \\
946 \\
483 \\
347 \\
643 \\
280 \\
309 \\
410 \\
250\end{array}$ & $\begin{array}{r}301 \\
404 \\
1260 \\
554 \\
394 \\
680 \\
281 \\
317 \\
417 \\
250\end{array}$ & $\begin{array}{r}371 \\
406 \\
1550 \\
607 \\
401 \\
742 \\
299 \\
344 \\
443 \\
258\end{array}$ & $\begin{array}{r}434 \\
430 \\
1690 \\
651 \\
443 \\
777 \\
318 \\
401 \\
539 \\
280\end{array}$ & $\begin{array}{r}446 \\
477 \\
2190 \\
711 \\
519 \\
977 \\
328 \\
425 \\
625 \\
321\end{array}$ & $\begin{array}{r}508 \\
552 \\
2800 \\
739 \\
641 \\
1350 \\
386 \\
474 \\
732 \\
421\end{array}$ & $\begin{array}{r}539 \\
768 \\
3310 \\
873 \\
699 \\
1810 \\
449 \\
583 \\
750 \\
534\end{array}$ \\
\hline $\begin{array}{l}1960 \\
1961 \\
1962 \\
1963 \\
1964 \\
1965 \\
1966 \\
1967 \\
1968 \\
1969\end{array}$ & $\begin{array}{r}1060 \\
590 \\
800 \\
673 \\
420 \\
352 \\
937 \\
437 \\
543 \\
945\end{array}$ & $\begin{array}{r}1120 \\
601 \\
811 \\
691 \\
500 \\
491 \\
999 \\
528 \\
558 \\
1200\end{array}$ & $\begin{array}{r}1220 \\
633 \\
844 \\
716 \\
507 \\
604 \\
1100 \\
625 \\
577 \\
1330\end{array}$ & $\begin{array}{r}1470 \\
730 \\
935 \\
783 \\
549 \\
638 \\
1180 \\
657 \\
659 \\
1680\end{array}$ & $\begin{array}{r}1940 \\
833 \\
1180 \\
878 \\
610 \\
666 \\
2110 \\
808 \\
718 \\
1800\end{array}$ & $\begin{array}{r}2250 \\
1190 \\
1940 \\
1200 \\
714 \\
773 \\
3170 \\
995 \\
808 \\
2160\end{array}$ & $\begin{array}{r}2410 \\
1360 \\
2620 \\
2020 \\
787 \\
915 \\
4920 \\
1160 \\
851 \\
2840\end{array}$ \\
\hline $\begin{array}{l}1970 \\
1971 \\
1972 \\
1973 \\
1974 \\
1975 \\
1976\end{array}$ & $\begin{array}{r}887 \\
863 \\
763 \\
1960 \\
1990 \\
967 \\
562\end{array}$ & $\begin{array}{r}970 \\
929 \\
790 \\
2010 \\
2060 \\
1160 \\
661\end{array}$ & $\begin{array}{r}988 \\
980 \\
817 \\
2190 \\
2150 \\
1210 \\
677\end{array}$ & $\begin{array}{r}1050 \\
1130 \\
893 \\
2370 \\
2190 \\
1260 \\
697\end{array}$ & $\begin{array}{r}1090 \\
1440 \\
942 \\
3590 \\
2390 \\
1400 \\
982\end{array}$ & $\begin{array}{l}1310 \\
1930 \\
1240 \\
3710 \\
3820 \\
1610 \\
1050\end{array}$ & $\begin{array}{l}1570 \\
2540 \\
1310 \\
3990 \\
4050 \\
1700 \\
1110\end{array}$ \\
\hline
\end{tabular}




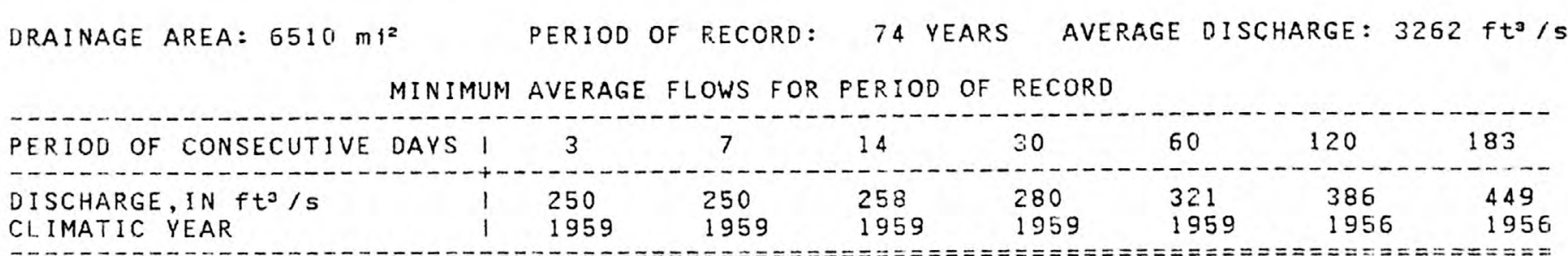

MAGNITUDE AND FREQUENCY OF ANNUAL LOW FLOWS

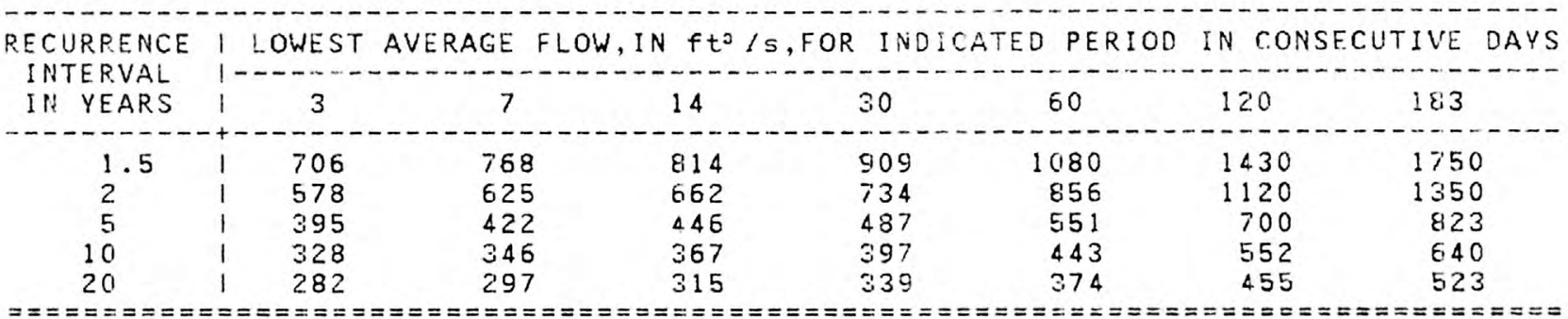

MAGNITUDE AND FREQUENCY OF SEASONAL LOW FLOWS

I LOWEST AVERAGE FLOW. IN $\mathrm{ft}^{\mathrm{J}} / \mathrm{s}, \mathrm{FOR}$ THE INOICATED PERIOD IN CONSECUTIVE DAYS WITHIN EACH WATER-YEAR QUARTER

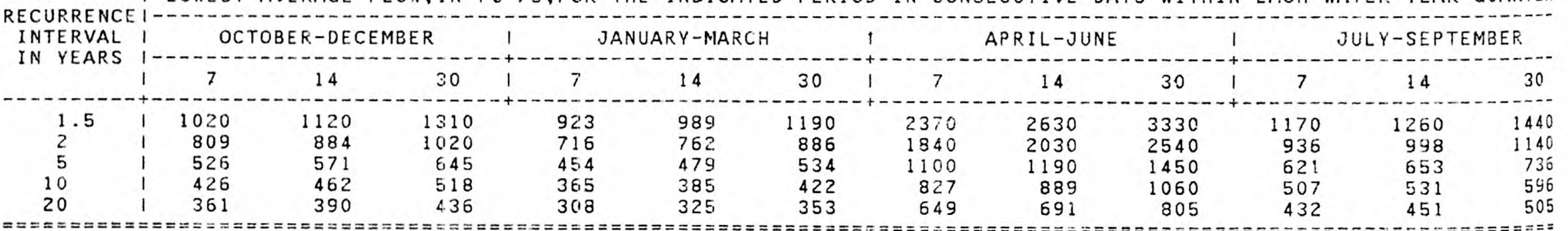

DURATION OF DAILY DISCHARGES FOR AN UUAL AND SEASONAL PERIODS

$P E R$ I $O$ DISCHARSE, IN $\mathrm{ft}^{3} / 5$, EQUALLEG OR EXCEEDED FOR THE INDICATED PERCENT OF TOTAL TIME

\begin{tabular}{|c|c|c|c|c|c|c|c|c|c|c|c|c|}
\hline & 5 & 10 & 20 & 40 & 50 & 60 & 80 & 84 & 90 & 95 & 38 & 99 \\
\hline $\begin{array}{l}\text { OCT.1-SEP. } 30 \\
\text { AFR. } 1-S E P .30 \\
\text { JULY } 1 \text {-AUG. } 31\end{array}$ & $\begin{array}{r}10400 \\
11200 \\
17910\end{array}$ & $\begin{array}{l}7240 \\
8100 \\
5640\end{array}$ & $\begin{array}{l}4590 \\
5440 \\
3730\end{array}$ & $\begin{array}{l}2480 \\
3150 \\
2210\end{array}$ & $\begin{array}{l}1870 \\
2480 \\
1770\end{array}$ & $\begin{array}{l}1460 \\
1950 \\
1490\end{array}$ & $\begin{array}{l}833 \\
1190 \\
1010\end{array}$ & $\begin{array}{r}787 \\
1060 \\
897\end{array}$ & $\begin{array}{l}642 \\
858 \\
733\end{array}$ & $\begin{array}{l}513 \\
66: \\
580\end{array}$ & $\begin{array}{l}396 \\
5: 4 \\
458\end{array}$ & $\begin{array}{l}339 \\
439 \\
407\end{array}$ \\
\hline
\end{tabular}


Location.-- Lat 41055'30", long 91046'55", in SW1/4SE1/4 sec.9, T.82 N., R.8 W. , on right bank $12 \mathrm{ft}$ upstream from bridge on State Highway 149 at west side of $F$ a irfax, and 10.7 miles upstream from mouth.

LOW FLOW DISCHARGES, IN YEAR. ENDING MARCH 31

\begin{tabular}{|c|c|c|c|c|c|c|c|c|}
\hline \multirow{2}{*}{$\begin{array}{c}\text { CLIMATIC } \\
\text { YEAR }\end{array}$} & I LOWEST & E FLOW, IN & $f t^{3} / s, F O R$ & INDICATED & PERIO & \multicolumn{3}{|c|}{ IN CONSECUTIVE DAYS } \\
\hline & 13 & 7 & 14 & 30 & 60 & 120 & 183 & \\
\hline $\begin{array}{l}1958 \\
1969\end{array}$ & $\begin{array}{l}11 \\
10\end{array}$ & $\begin{array}{l}12 \\
11\end{array}$ & $\begin{array}{l}13 \\
14\end{array}$ & $\begin{array}{l}18 \\
22\end{array}$ & $\begin{array}{l}20 \\
23\end{array}$ & $\begin{array}{l}28 \\
29\end{array}$ & $\begin{array}{l}42 \\
43\end{array}$ & \\
\hline $\begin{array}{l}1970 \\
1971 \\
1972 \\
1973 \\
1974 \\
1975 \\
1976\end{array}$ & $\begin{array}{c}19 \\
14 \\
6.9 \\
39 \\
15 \\
33 \\
4.8\end{array}$ & $\begin{array}{r}20 \\
15 \\
7.5 \\
41 \\
16 \\
33 \\
5.0\end{array}$ & $\begin{array}{c}22 \\
15 \\
7.9 \\
43 \\
17 \\
35 \\
5.4\end{array}$ & $\begin{array}{r}22 \\
20 \\
8.3 \\
48 \\
20 \\
39 \\
5.9\end{array}$ & $\begin{array}{c}27 \\
34 \\
9.4 \\
77 \\
30 \\
43 \\
8.4\end{array}$ & $\begin{array}{r}45 \\
74 \\
12 \\
116 \\
45 \\
54 \\
9\end{array}$ & $\begin{array}{r}53 \\
98 \\
13 \\
13 \\
6 \\
58\end{array}$ & 8 \\
\hline
\end{tabular}


05-4646.40 PRAIRIE CREEK AT FAIRFAX--Continued

DRAINAGE AREA: $178 \mathrm{~m}^{2}$ PERIOD OF RECORD: 10 YEARS AVERAGE DISCHARGE: 136 Ft $/ \mathrm{s}$

MINIMUM AVERAGE FLOWS FOR PERIOD OF RECORD

\begin{tabular}{|c|c|c|c|c|c|c|c|}
\hline PERIOD OF CONSECUTIVE DAYS I & 3 & 7 & 14 & 30 & 60 & $: 20$ & 183 \\
\hline $\begin{array}{l}\text { DISCHARGE, IN } \mathrm{ft}^{3} / \mathrm{s} \\
\text { CLIMATIC YEAR }\end{array}$ & $\begin{array}{r}4.8 \\
1976\end{array}$ & $\begin{array}{r}5.0 \\
1976\end{array}$ & $\begin{array}{r}5.4 \\
1976\end{array}$ & $\begin{array}{r}5.9 \\
1976\end{array}$ & $\begin{array}{r}8.4 \\
1975\end{array}$ & $\begin{array}{r}9.2 \\
1976\end{array}$ & $\begin{array}{r}9.8 \\
1976\end{array}$ \\
\hline
\end{tabular}

MAGNITUDE AND FREQUENCY OF ANNUAL LOW FLOWS

RECURRENCE I LOWEST AVERAGE FLOW, IN $\mathrm{ft} / \mathrm{s} / \mathrm{s}$, FOR INDICATED PERIOD IN CONSECUTIVE DAYS

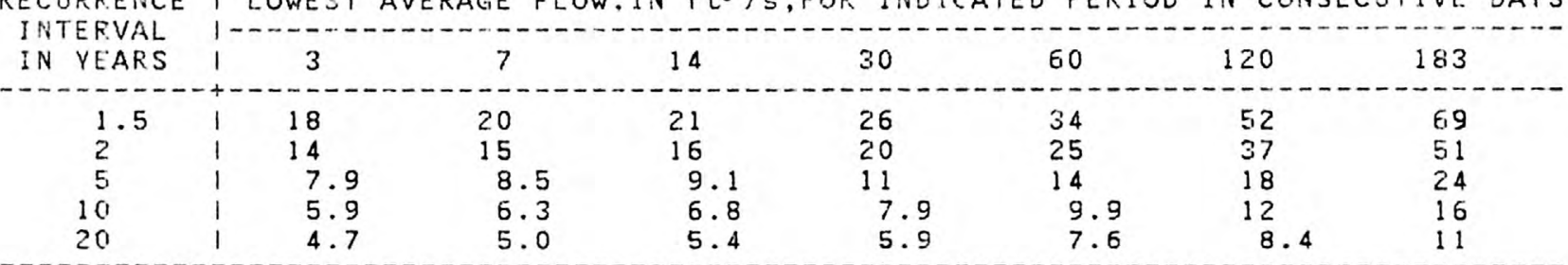

MAGNITUDE AND FREQUENCY OF SEASONAL LOW FLOWS

LOWEST AVERAGE FLOW, IN $\mathrm{ft}^{3} / \mathrm{s}$, FOR THE INDICATED PERIOD IN CONSECUTIVE DAYS WITHIN EACH WATER-YEAR QUARTER

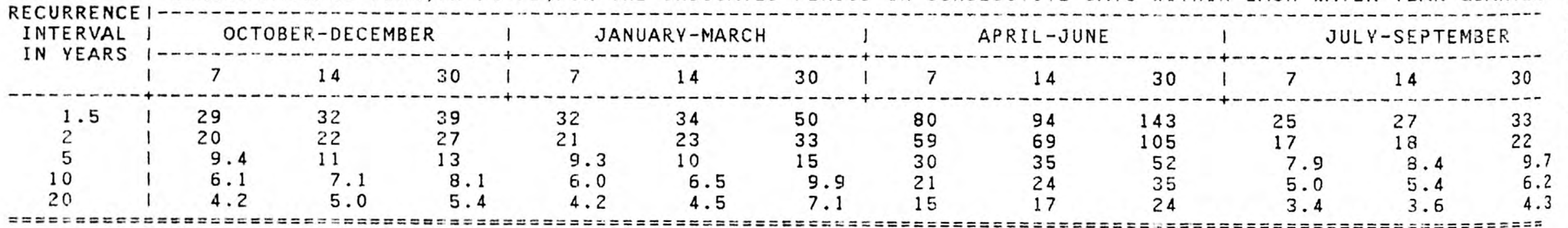

DURATION OF DAILY DISCHARGES FOR ANNUAL ANO SEASONAL PERIODS

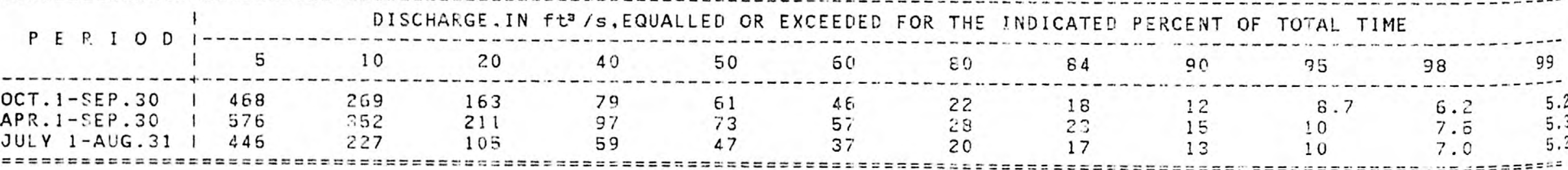


$05-4650.00$

CEDAR RIVER NEAR CONESVILLE

Location.--Lat 41024'36", long 91017.06", in SWl/4 SWl/4 sec.2, T.76 N., R.4 W., Muscatine County, on right bank $10 \mathrm{ft}$ downstream from bridge on county highway $\mathrm{G} 28,3.4$ miles northeast of Conesville. 5.2 miles downstrean from Wapsinonoc Creek, 10.7 miles upstream from mouth, and at mile 39.8 upstream

from mouth of Iowa River.

LOW FLOW DISCHARGES, IN YEAR. ENDING MARCH 3

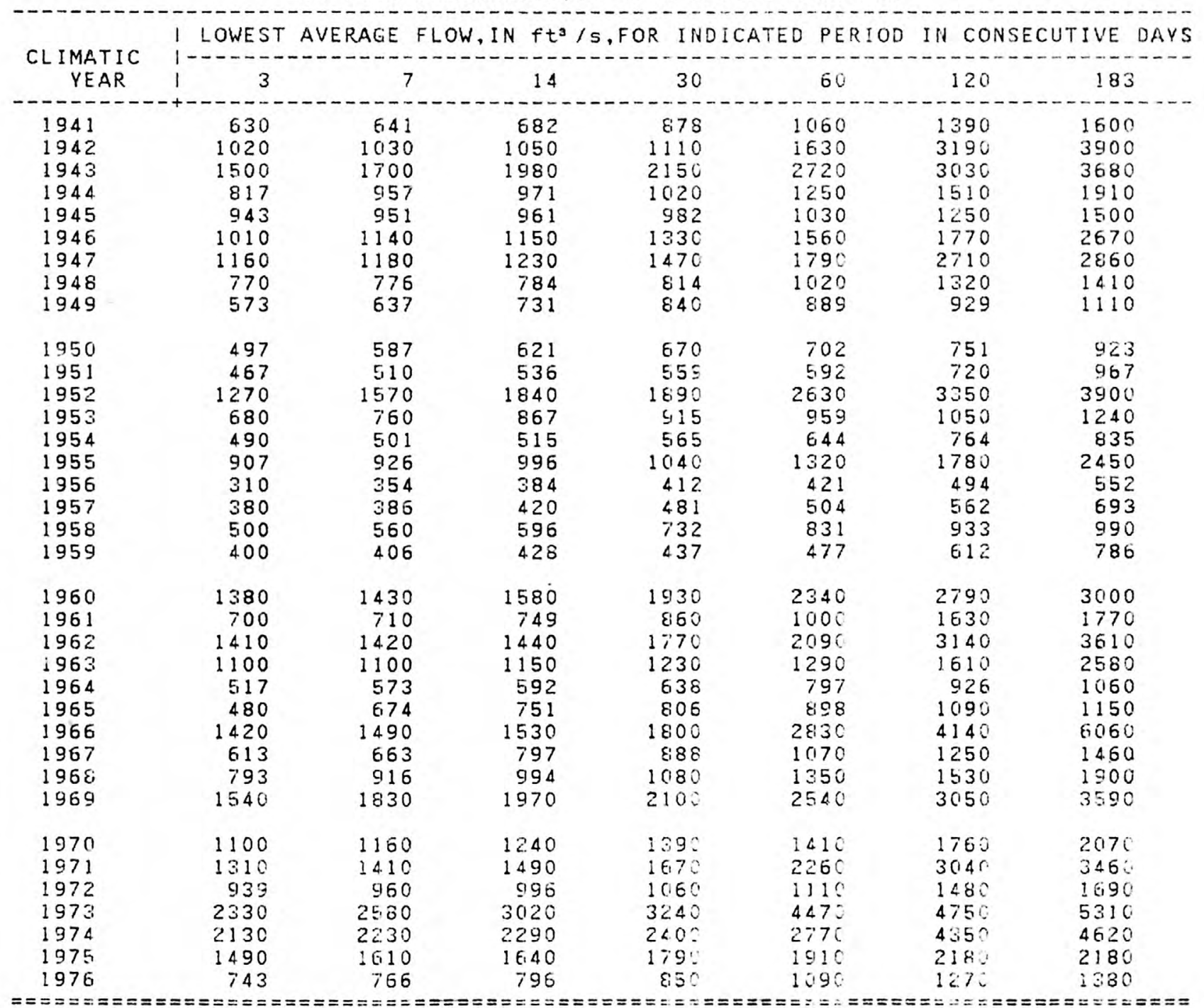




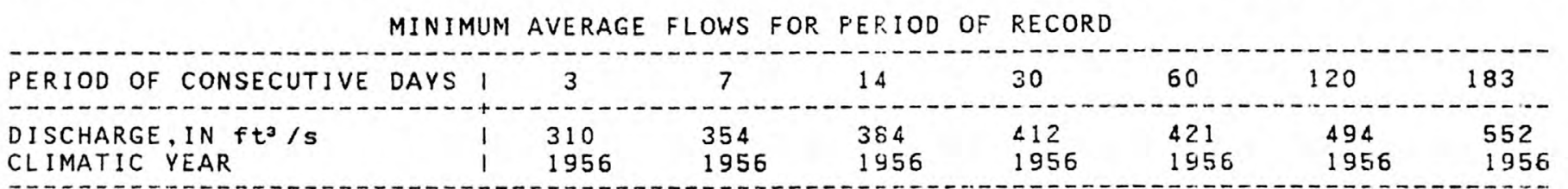

MAGNITUDE AND FREQUENCY OF ANNUAL LOW FLOWS

\begin{tabular}{|c|c|c|c|c|c|c|c|c|c|}
\hline $\begin{array}{l}\text { ECURRENCE } \\
\text { INTERVAL. }\end{array}$ & 1 & OWE ST & $\begin{array}{ll}A G E \\
A G\end{array}$ & $N f t^{3}$ & R INL & $\begin{array}{l}D \quad P E \\
--D-1\end{array}$ & IN CO & IVE & DAYS \\
\hline IN YEARS & 1 & 3 & 7 & 14 & 30 & 60 & 120 & 183 & \\
\hline $\begin{array}{l}1.5 \\
2 \\
5 \\
10 \\
20\end{array}$ & $\begin{array}{l}1 \\
1 \\
1 \\
1\end{array}$ & $\begin{array}{r}1050 \\
847 \\
554 \\
444 \\
371\end{array}$ & $\begin{array}{r}1130 \\
912 \\
600 \\
484 \\
407\end{array}$ & $\begin{array}{r}1200 \\
966 \\
636 \\
516 \\
437\end{array}$ & $\begin{array}{r}1330 \\
1070 \\
699 \\
564 \\
473\end{array}$ & $\begin{array}{r}1600 \\
1260 \\
783 \\
616 \\
506\end{array}$ & $\begin{array}{r}2060 \\
1590 \\
945 \\
723 \\
580\end{array}$ & $\begin{array}{r}2440 \\
1880 \\
1110 \\
851 \\
682\end{array}$ & \\
\hline
\end{tabular}

MAGNITUDE AND FREQUENCY OF SEASONAL LOW FLOWS

1 LOWEST AVERAGE FLOW, IN $\mathrm{ft}^{3} / \mathrm{s}, \mathrm{FOR}$ THE INDICATED PERIOD IN CONSECUTIVE DAYS WITHIN EACH WATER-YEAR QUARTER

\begin{tabular}{c|ccccccc} 
RECURRENCE \\
INTERVAL \\
IN YEARS
\end{tabular}

DURATION OF DAILY DISCHARGES FOR ANIUUAL AND SEASONAL PERIODS

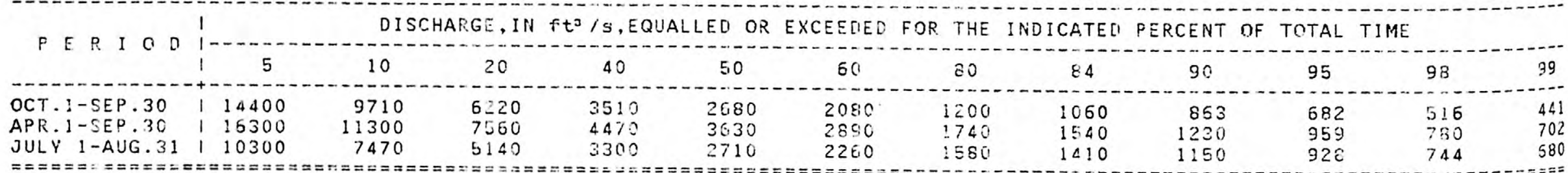


Location.--Lat $41^{\circ} 10^{\prime} 48^{\prime \prime}$, long 91010.57", in NW1/4 SE1/4 sec.27, T.74 N., R.3 W. Louisa County, on right bank $30 \mathrm{ft}$ downstream from bridge on State Highway 99 at east edge of Wapel10, 13.0 miles downstream from Cedar River, and at mile 16.0 .

Remarks. - Flow regulated by Coralville Lake, 67.3 miles upstream, since Sept. 17,1958 .

LOW FLOW DISCHARGES, IN YEAR ENDING MARCH 31

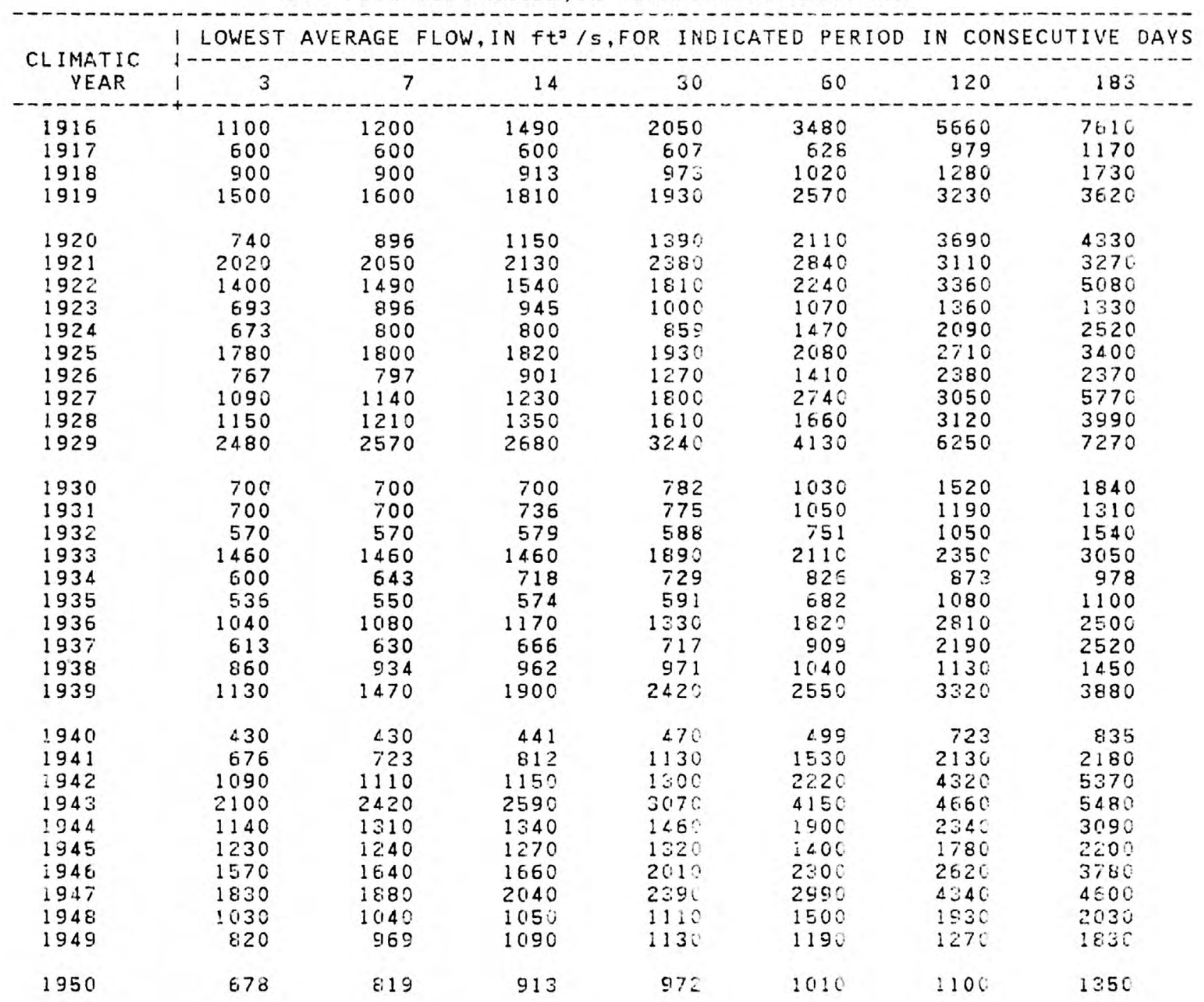


LOW FLOW DISCHARGES, IN YEAR ENDIMG MARCH 31--Continued

\begin{tabular}{|c|c|c|c|c|c|c|c|c|}
\hline \multirow{2}{*}{$\begin{array}{c}\text { CLIMATIC } \\
\text { YEAR }\end{array}$} & LOWEST & RAGE $F$ & $N f t^{3}$ & OR INL & $=0$ PEF & \multicolumn{3}{|c|}{ IN CONSECUTIVE DAYS } \\
\hline & i & 7 & 14 & 30 & 60 & 120 & 183 & \\
\hline $\begin{array}{l}1951 \\
1952 \\
1953 \\
1954 \\
1955 \\
1956 \\
1957 \\
1958\end{array}$ & $\begin{array}{r}593 \\
2030 \\
1100 \\
620 \\
1300 \\
400 \\
540 \\
790\end{array}$ & $\begin{array}{r}617 \\
2510 \\
1230 \\
521 \\
1350 \\
401 \\
543 \\
797\end{array}$ & $\begin{array}{r}641 \\
3140 \\
1280 \\
641 \\
1470 \\
434 \\
581 \\
839\end{array}$ & $\begin{array}{r}666 \\
3220 \\
1280 \\
700 \\
1540 \\
450 \\
671 \\
986\end{array}$ & $\begin{array}{r}765 \\
4440 \\
1330 \\
801 \\
2000 \\
504 \\
722 \\
1110\end{array}$ & $\begin{array}{r}933 \\
5590 \\
1580 \\
942 \\
2720 \\
622 \\
762 \\
1360\end{array}$ & $\begin{array}{l}1230 \\
6560 \\
1810 \\
1020 \\
3910 \\
720 \\
1110 \\
1430\end{array}$ & \\
\hline
\end{tabular}


DRAINAGE AREA: $12,499 \mathrm{mi}^{2}$ PERIOD OF RECORD: 44 VEARS AVERAGE DISCHARGE: 5950 ft3 /s

MINIMUM AVERAGE FLOWS FOR FERIOD OF RECORD

\begin{tabular}{|c|c|c|c|c|c|c|c|c|}
\hline PERIOD OF CONSECUTIVE DAYS & 1 & 3 & 7 & 14 & 30 & 60 & 120 & 183 \\
\hline $\begin{array}{l}\text { DISCHARGE, IN } \mathrm{ft}^{3} / \mathrm{s} \\
\text { CL IMATIC YEAR }\end{array}$ & 1 & $\begin{array}{l}400 \\
1956\end{array}$ & $\begin{array}{l}401 \\
1956\end{array}$ & $\begin{array}{l}434 \\
1956\end{array}$ & $\begin{array}{l}459 \\
1956\end{array}$ & $\begin{array}{l}499 \\
1940\end{array}$ & $\begin{array}{l}622 \\
1956\end{array}$ & $\begin{array}{l}722 \\
1956\end{array}$ \\
\hline
\end{tabular}

MAGNITUDE AND FREQUENCY OF ANNUAL LOW FLOWS

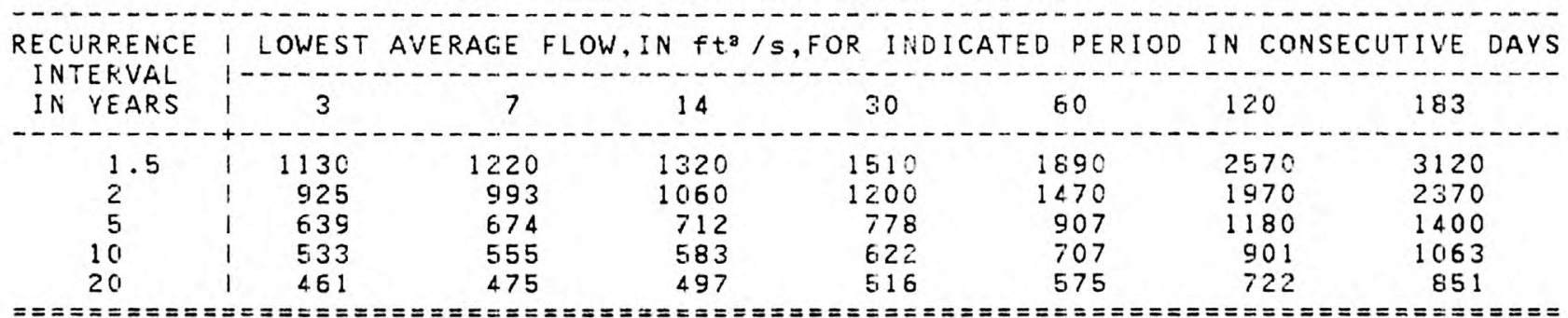

MAGNITUDE AND FREQUENCY OF SEASONAL LOW FLOWS

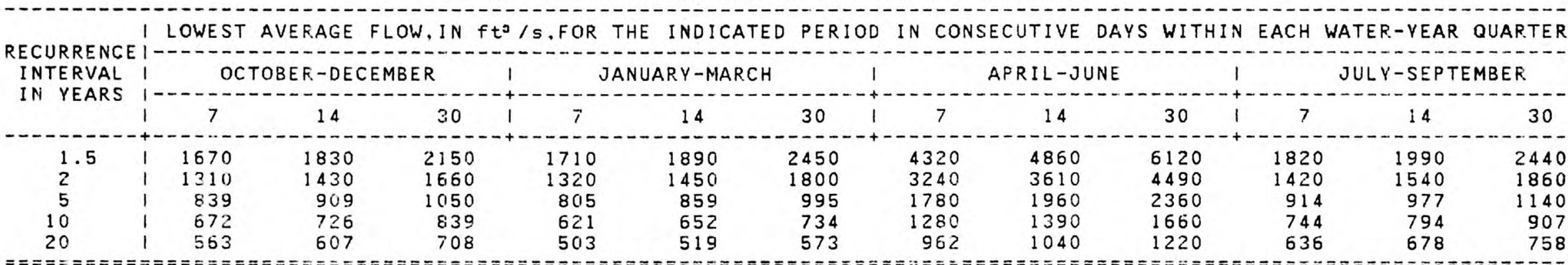

DURATION OF DAILY DISCHARGES FOR ANNUAL AND SEASONAL PERIODS

\begin{tabular}{|c|c|c|c|c|c|c|c|c|c|c|c|c|}
\hline \multirow{2}{*}{$P E \therefore I O O D$} & 1 & DI & GE. I & is, E & $\begin{array}{lll}E & 0 & C \\
- & 0\end{array}$ & $E E[E$ & R THE & CATE & CENT & OTAL. & & \\
\hline & 5 & 10 & 20 & 40 & 50 & 60 & 80 & 84 & 90 & 95 & 98 & 99 \\
\hline $\begin{array}{l}\text { OCT. } 1 \text {-SEP. } 30 \\
\text { APR. } 1 \text {-SEP. } 30 \\
\text { JULY } 1 \text {-AUG. } 31\end{array}$ & $\begin{array}{l}19500 \\
121500 \\
113500\end{array}$ & $\begin{array}{r}13300 \\
13400 \\
9780\end{array}$ & $\begin{array}{l}6460 \\
9060 \\
5440\end{array}$ & $\begin{array}{l}4530 \\
5810 \\
4100\end{array}$ & $\begin{array}{l}3490 \\
4490 \\
3410\end{array}$ & $\begin{array}{l}2640 \\
3480 \\
2780\end{array}$ & $\begin{array}{l}1480 \\
2020 \\
1090\end{array}$ & $\begin{array}{l}1310 \\
1760 \\
1490\end{array}$ & $\begin{array}{l}1050 \\
1360 \\
1190\end{array}$ & $\begin{array}{r}830 \\
1010 \\
879\end{array}$ & $\begin{array}{l}643 \\
772 \\
665\end{array}$ & $\begin{array}{l}556 \\
552 \\
569\end{array}$ \\
\hline
\end{tabular}


$05-4655.00$

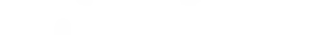

IOWA RIVER AT WAPELLO--Continued, regulated period

LOW FLOW DISCHARGES, IN VEAF. ENIING MARCH 31

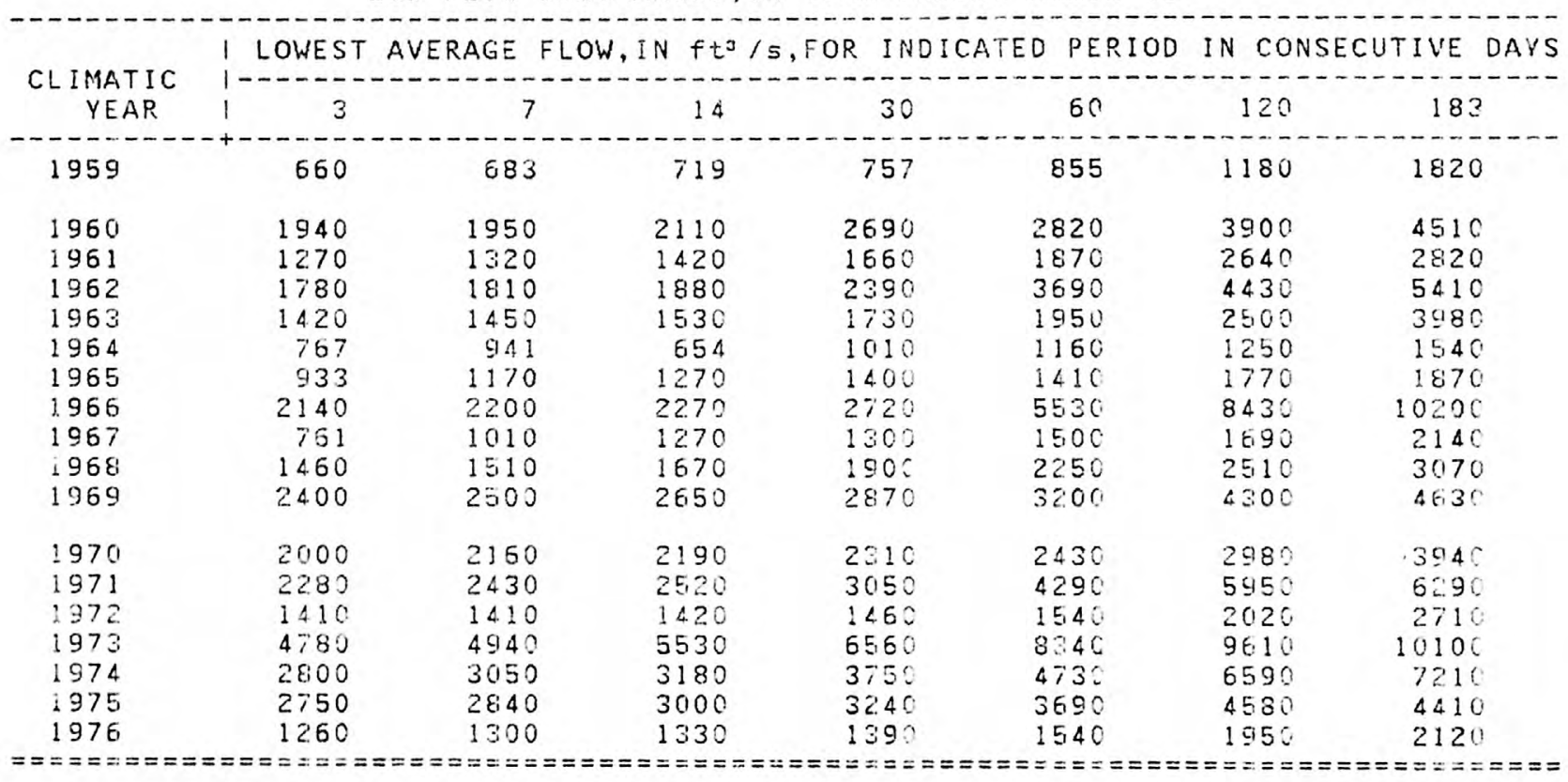


MINIMUM AVERAGE FLOWS FOR FERIOD OF RECORD

\begin{tabular}{|c|c|c|c|c|c|c|c|}
\hline ERIOD OF CONSECUTIVE DAYS & 3 & 7 & 14 & 30 & 50 & 120 & 183 \\
\hline $\begin{array}{l}\text { I SCHARGE, IN } \mathrm{ft}^{3} / \mathrm{s} \\
\text { LIMATIC YEAR }\end{array}$ & $\begin{array}{l}660 \\
1959\end{array}$ & $\begin{array}{l}683 \\
1959\end{array}$ & $\begin{array}{l}654 \\
1964\end{array}$ & $\begin{array}{l}757 \\
1959\end{array}$ & $\begin{array}{l}855 \\
1959\end{array}$ & $\begin{array}{l}1180 \\
1959\end{array}$ & \\
\hline
\end{tabular}

MAGNITUDE AND FREQUENCY OF ANNUAL LOW FLOWS

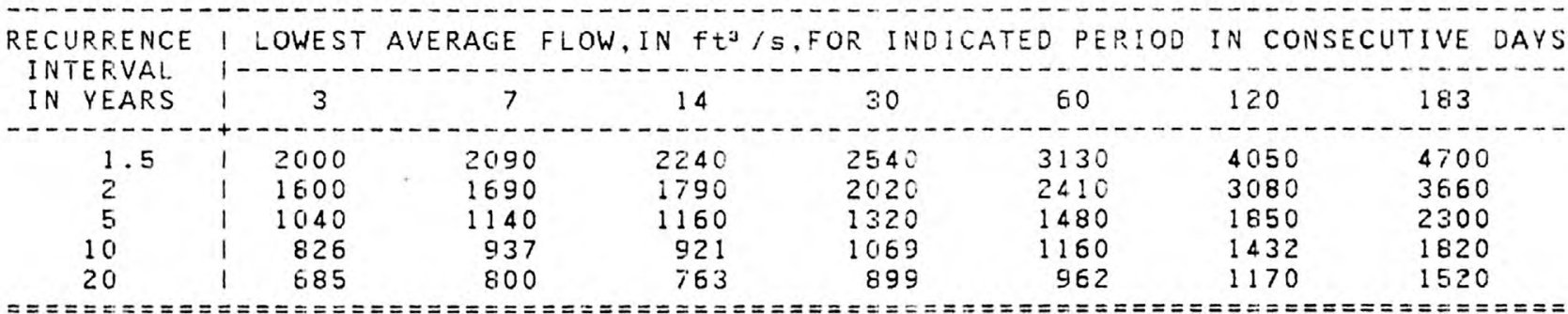

MAGNITUDE AND FREQUENCY OF SEASONAL LOW FLOWS

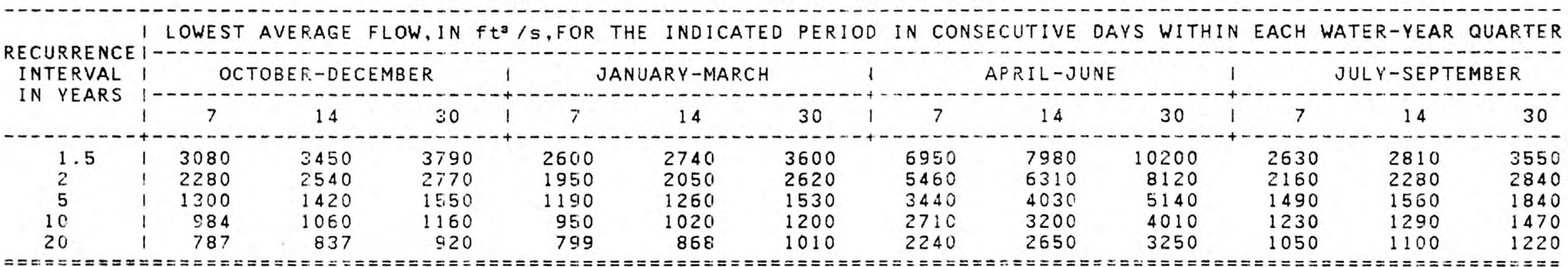

DURATION OF DAILY DISCHARGES FOR ANNUAL ANO SEASONAL PERIODS

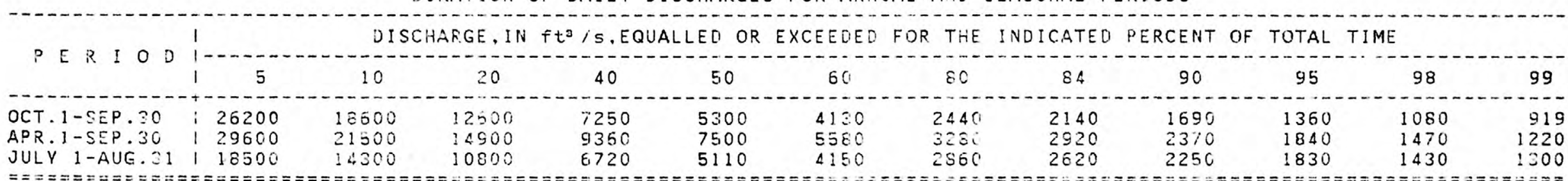


Location.--Lat 42004'05", long 93037'02", in NW1/4 SW1/4 sec.23, T.84 N., R.24 W. Story County, on left bank 2.5 miles north of Ames, 3.5 miles downstream from Keigley Branch, 5.2 miles upstream from Squaw Creek, and at mile 228.1 upstream from mouth ö́ skunk River.

Remarks.--Several diversions for irrigation upstream of station. Published as Skunk River near Ames prior to October 1966. LOW FLOW DISCHARGES, IN YEAR. ENDING MARCH 31

\begin{tabular}{|c|c|c|c|c|c|c|c|}
\hline \multirow{2}{*}{$\begin{array}{c}\text { CLIMAT IC } \\
\text { YEAR }\end{array}$} & LOWEST AI & SE FLOW & $f t^{3} / 5$, & IND ICA & PER IOL & IN CONSEC & IVE DAYS \\
\hline & 13 & 7 & 14 & 30 & 60 & 120 & 183 \\
\hline $\begin{array}{l}1922 \\
1923 \\
1924 \\
1925 \\
1926 \\
1927 \\
1934 \\
1935 \\
1936 \\
1937 \\
1938 \\
1939\end{array}$ & $\begin{array}{l}1.6 \\
4.0 \\
2.0 \\
7.0 \\
2.0 \\
1.0 \\
.43 \\
0 \\
6.4 \\
.20 \\
.18 \\
3.0\end{array}$ & $\begin{array}{l}1.6 \\
4.6 \\
2.0 \\
1.0 \\
2.0 \\
1.3 \\
.47 \\
0 \\
7.4 \\
.24 \\
.19 \\
3.4\end{array}$ & $\begin{array}{r}1.9 \\
6.2 \\
3.0 \\
7.0 \\
2.5 \\
1.5 \\
.49 \\
.01 \\
11 . \\
.26 \\
.22 \\
4.6\end{array}$ & $\begin{array}{c}3.7 \\
10 \\
5.5 \\
9.1 \\
2.9 \\
3.1 \\
1.1 \\
.04 \\
13.04 \\
.34 \\
11.39\end{array}$ & $\begin{array}{r}16 \\
11 \\
9.8 \\
14 \\
9.9 \\
5.9 \\
2.4 \\
.21 \\
16.78 \\
.78 \\
23^{.46}\end{array}$ & $\begin{array}{r}41 \\
28 \\
34 \\
25 \\
25 \\
15 \\
3.2 \\
2.0 \\
58 \\
4.6 \\
28^{.85}\end{array}$ & $\begin{array}{r}108 \\
24 \\
72 \\
41 \\
26 \\
116 \\
8.0 \\
5.8 \\
67 \\
4.5 \\
3.1 \\
45\end{array}$ \\
\hline $\begin{array}{l}1940 \\
1941 \\
1942 \\
1943 \\
1944 \\
1945 \\
1946 \\
1947 \\
1948 \\
1949\end{array}$ & $\begin{array}{c}.10 \\
.46 \\
2.0 \\
22 \\
14 \\
9.4 \\
2.2 \\
4.8 \\
1.5 \\
.30\end{array}$ & $\begin{array}{c}.10 \\
.62 \\
2.4 \\
25 \\
15 \\
9.8 \\
2.4 \\
5.7 \\
1.6 \\
.34\end{array}$ & $\begin{array}{c}.10 \\
.82 \\
3.4 \\
27 \\
17 \\
10 \\
2.7 \\
10 \\
1.8 \\
.59\end{array}$ & $\begin{array}{c}.12 \\
2.0 \\
4.5 \\
33 \\
21 \\
11 \\
4.2 \\
19 \\
2.3 \\
.69\end{array}$ & $\begin{array}{l}11^{.28} \\
12 \\
43 \\
54 \\
12 \\
7.4 \\
28 \\
4.1 \\
1.3\end{array}$ & $\begin{array}{l}32^{.89} \\
86 \\
78 \\
72 \\
19 \\
8.3 \\
52 \\
12 \\
3.7\end{array}$ & $\begin{array}{r}57^{.81} \\
106 \\
129 \\
92 \\
40 \\
34 \\
51 \\
13 \\
6.5\end{array}$ \\
\hline $\begin{array}{l}1950 \\
1951 \\
1952 \\
1953 \\
1954 \\
1955 \\
1950 \\
1957 \\
1958 \\
1959\end{array}$ & 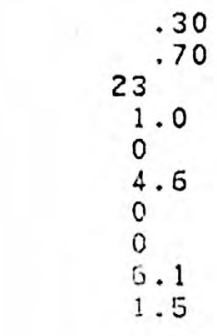 & 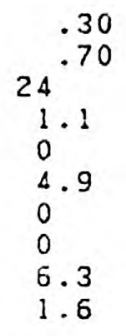 & $\begin{array}{l}.36 \\
26 \\
.74 \\
1.4 \\
0 \\
5.2 \\
0 \\
0 \\
7.1 \\
1.6\end{array}$ & $\begin{array}{l}1.40 \\
32.2 \\
1.7 \\
10.04 \\
0 \\
0 \\
9.1 \\
1.7\end{array}$ & $\begin{array}{r}1.54 \\
1.5 \\
49 \\
2.3 \\
27^{.20} \\
.03 \\
14^{.24} \\
2.1\end{array}$ & $\begin{array}{c}5.89 \\
74 \\
3.0 \\
.45 \\
62 \\
.07 \\
5.4 \\
24 \\
4.3\end{array}$ & $\begin{array}{c}1.1 \\
14 \\
92 \\
4.9 \\
.55 \\
108.20 \\
6.5 \\
44 \\
9.8\end{array}$ \\
\hline $\begin{array}{l}1960 \\
1961 \\
1982 \\
1963 \\
1064 \\
1965\end{array}$ & $\begin{array}{r}7.3 \\
3.7 \\
13 \\
3.7 \\
.20 \\
.60\end{array}$ & $\begin{array}{l}13 \\
3.8 \\
10 \\
3.7 \\
.26 \\
.00\end{array}$ & $\begin{array}{r}14 \\
4.0 \\
17 \\
3.9 \\
.29 \\
.67\end{array}$ & $\begin{array}{r}22 \\
6.7 \\
25 \\
5.2 \\
.85 \\
.81\end{array}$ & $\begin{array}{r}47 \\
11 \\
62 \\
E .9 \\
2.3 \\
1.9\end{array}$ & $\begin{array}{r}55 \\
23 \\
110 \\
17 \\
2.9 \\
2.3\end{array}$ & $\begin{array}{r}79 \\
39 \\
127 \\
30 \\
4.3 \\
3.3\end{array}$ \\
\hline
\end{tabular}


LOW FLOW DISCHARGES, IN YEAR ENDING MARCH 31--Continued

\begin{tabular}{|c|c|c|c|c|c|c|c|}
\hline \multirow{2}{*}{$\begin{array}{c}\text { CLIMATIC } \\
\text { YEAR }\end{array}$} & LOWEST A & GE FLOW & $f t^{3} / s, F O R$ & IND ICATE & PERIOD & IN CONSE & IVE DAYS \\
\hline & 13 & 7 & 14 & 30 & 60 & 120 & 183 \\
\hline $\begin{array}{l}1966 \\
1967 \\
1968 \\
1969\end{array}$ & $\begin{array}{l}1.3 \\
.13 \\
.74 \\
4.0\end{array}$ & $\begin{array}{l}1.6 \\
.26 \\
.77 \\
5.2\end{array}$ & $\begin{array}{l}2.0 \\
.53 \\
1.0 \\
7.4\end{array}$ & $\begin{array}{l}5.2 \\
1.0 \\
1.6 \\
12\end{array}$ & $\begin{array}{l}23 \\
1.9 \\
3.4 \\
18\end{array}$ & $\begin{array}{r}141 \\
2.2 \\
4.2 \\
40\end{array}$ & $\begin{array}{r}175 \\
2.7 \\
4.2 \\
42\end{array}$ \\
\hline $\begin{array}{l}1970 \\
1971 \\
1972 \\
1973 \\
1974 \\
1975 \\
1976\end{array}$ & $\begin{array}{c}11 \\
5.0 \\
1.1 \\
32 \\
20 \\
8.4 \\
.46\end{array}$ & $\begin{array}{c}11 \\
5.7 \\
1.2 \\
34 \\
22 \\
8.6 \\
.63\end{array}$ & $\begin{array}{c}12 \\
7.1 \\
1.2 \\
39 \\
27 \\
9.0 \\
.89\end{array}$ & $\begin{array}{l}12 \\
14 \\
1.4 \\
57 \\
37 \\
20 \\
1.0\end{array}$ & $\begin{array}{c}17 \\
33 \\
2.7 \\
124 \\
88 \\
33 \\
4.2\end{array}$ & $\begin{array}{c}36 \\
47 \\
7.5 \\
186 \\
188 \\
72 \\
12\end{array}$ & $\begin{array}{c}43 \\
78 \\
7.4 \\
26 \% \\
276 \\
89 \\
14\end{array}$ \\
\hline
\end{tabular}




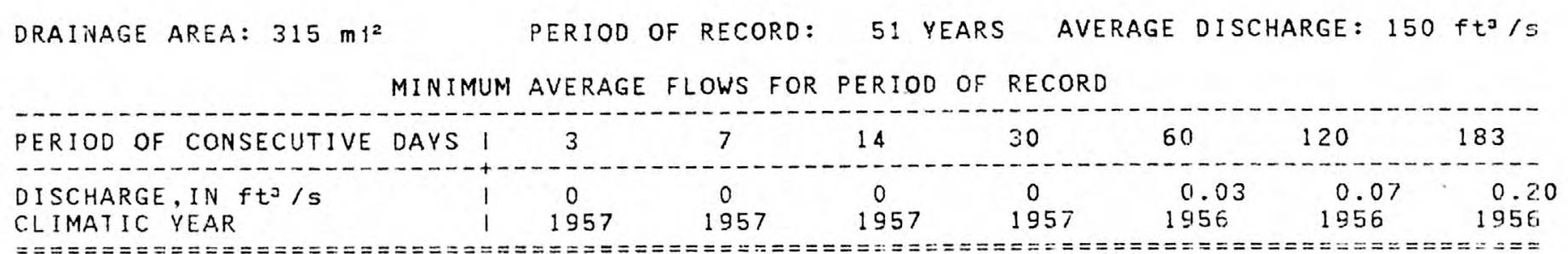

MAGNITUDE AND FREQUENCY OF ANNUAL LOW FLOWS

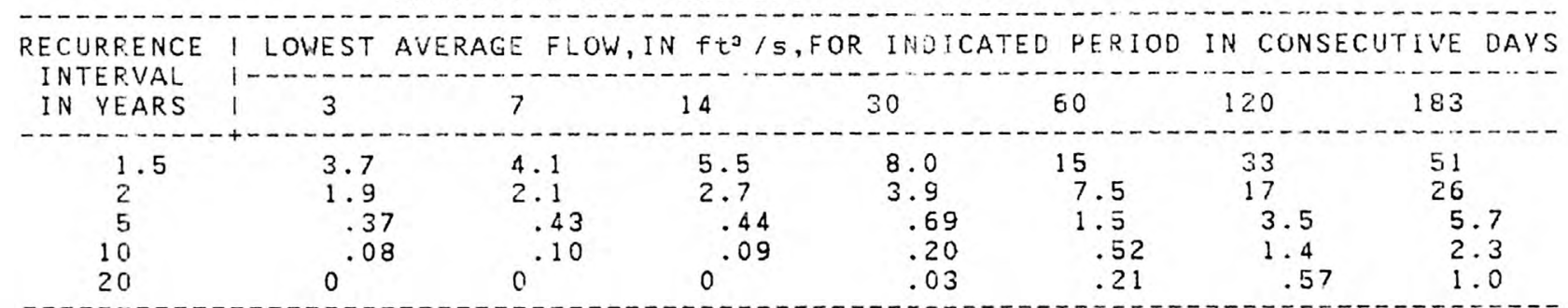

MAGNITUDE AND FREQUENCY OF SEASONAL LOW FLOWS

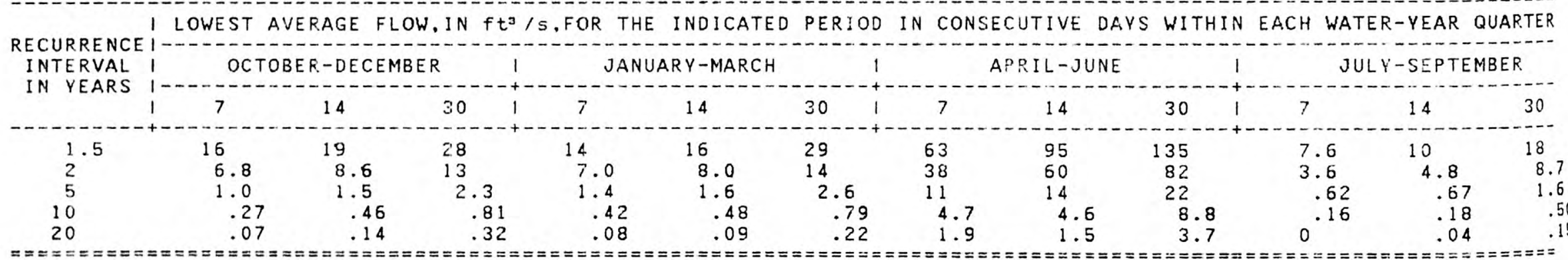

DURATIOIN OF DAILY DISCHARGES FOR ANNUAL AND SEASONAL PERIODS

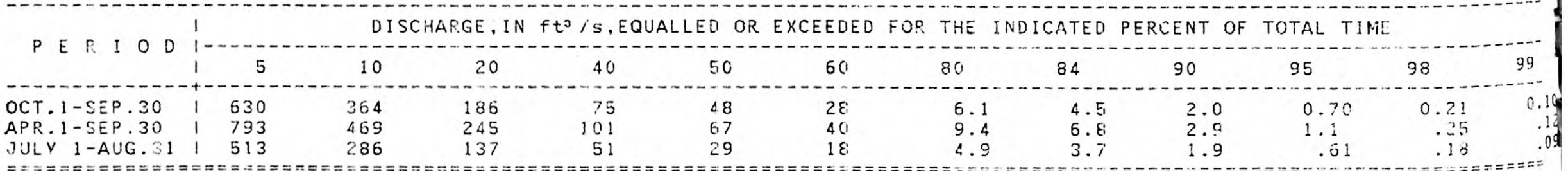


Location.--Lat $42^{\circ} 01^{\prime} 21^{\prime \prime}$, long 93037:45", in NE1/4 NW1/4 sec.10, T.83 N., R.24 W. Story County, on ieft bank. $65 \mathrm{ft}$ downstream from Lincolr Way Bridge in Ames, 0.1 mile downitream from College Creek, and $1.8 \mathrm{miles}$ upstream from mouth.

LOW FLOW DISCHARGES, IN YEAR. ENDING MARCH 31

\begin{tabular}{|c|c|c|c|c|c|c|c|}
\hline \multirow{2}{*}{$\begin{array}{c}\text { CLIMATIC } \\
\text { YEAR }\end{array}$} & I LOWEST AVERAGE & GE FLO & $f t^{3} / s$ & INDIC & PERIOD & \multicolumn{2}{|c|}{ IN CONSECUTIVE DAYS } \\
\hline & 13 & 7 & 14 & 30 & 60 & 120 & 183 \\
\hline $\begin{array}{l}1921 \\
1922 \\
1923 \\
1924 \\
1925 \\
1926 \\
1927 \\
1967 \\
1968 \\
1969\end{array}$ & $\begin{array}{l}3.1 \\
4.0 \\
6.0 \\
1.0 \\
4.0 \\
0 \\
.50 \\
.13 \\
.14 \\
2.7\end{array}$ & $\begin{array}{l}3.8 \\
5.2 \\
6.0 \\
2.5 \\
4.0 \\
.14 \\
.50 \\
.15 \\
.14 \\
3.2\end{array}$ & $\begin{array}{l}5.0 \\
7.0 \\
6.0 \\
3.2 \\
4.0 \\
.79 \\
.64 \\
.19 \\
.17 \\
4.0\end{array}$ & $\begin{array}{l}13 \\
14 \\
8.4 \\
5.1 \\
5.5 \\
1.6 \\
1.6 \\
.21 \\
.28 \\
7.7\end{array}$ & $\begin{array}{l}30 \\
21 \\
12 \\
8.4 \\
9.1 \\
6.1 \\
2.6 \\
.29 \\
.75 \\
11\end{array}$ & $\begin{array}{l}66 \\
28 \\
23 \\
29 \\
16 \\
17 \\
7.6 \\
.43 \\
1.2 \\
30\end{array}$ & $\begin{array}{l}71 \\
54 \\
21 \\
65 \\
29 \\
18 \\
93 \\
.52 \\
1.4 \\
32\end{array}$ \\
\hline $\begin{array}{l}1970 \\
1971 \\
1972 \\
1973 \\
1974 \\
1975 \\
1976\end{array}$ & $\begin{array}{l}7.5 \\
3.5 \\
0 \\
14 \\
18 \\
4.5 \\
.07\end{array}$ & $\begin{array}{r}7.8 \\
4.7 \\
0 \\
15 \\
22 \\
4.8 \\
.14\end{array}$ & $\begin{array}{l}8.1 \\
7.6 \\
0 \\
17 \\
32 \\
5.6 \\
.26\end{array}$ & $\begin{array}{ll}9.1 & 9.1 \\
15 & .07 \\
29 & \\
41 & \\
10 & \\
& .59\end{array}$ & $\begin{array}{l}13 \\
26 \\
67.26 \\
78 \\
12 \\
2.4\end{array}$ & $\begin{array}{r}27 \\
48 \\
2.3 \\
92 \\
167 \\
39 \\
5.0\end{array}$ & $\begin{array}{c}28 \\
58 \\
2.1 \\
125 \\
221 \\
40 \\
5.4\end{array}$ \\
\hline
\end{tabular}




DRAINAGE AREA: $204 \mathrm{mi}^{2} \quad$ PERIOD OF RECORD: 19 VEARS AVERAGE DISCHARGE: $120 \mathrm{ft} 3 / \mathrm{s}$
MINIMUM AVERAGE FLOWS FOR PERIOD OF RECORD

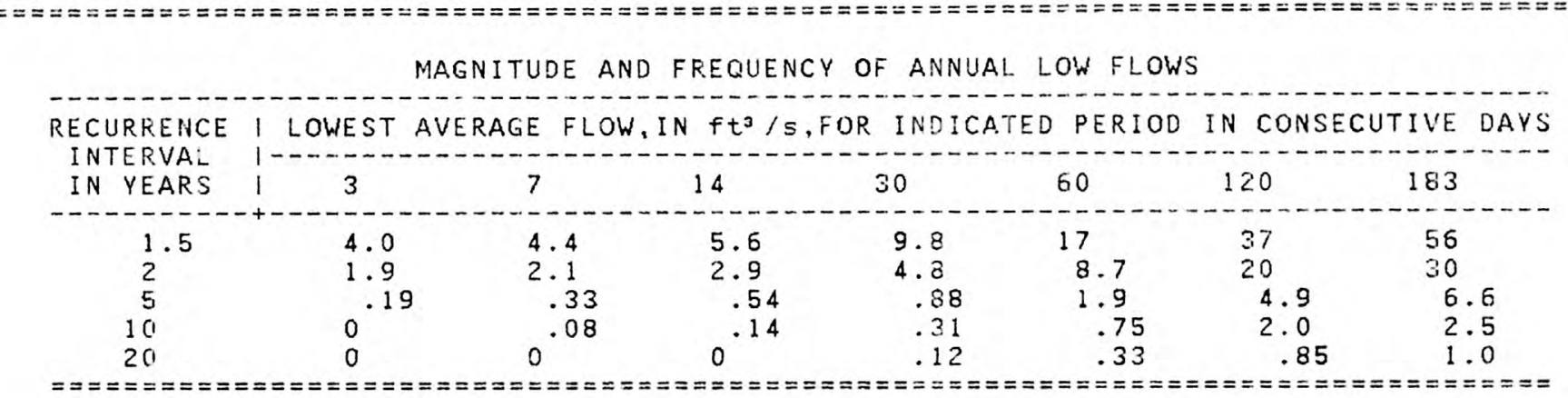

MAGNITUDE AND FREQUENCY OF SEASONAL LOW FLOWS

I LOWEST AVERAGE FLOW, IN $\mathrm{ft}^{\mathrm{s}} / \mathrm{s}, \mathrm{FOR}$ THE INDICATED PERIOD IN CONSECUTIVE DAYS WITHIN EACH WATER-YEAR QUARTER

\begin{tabular}{|c|c|c|c|c|c|c|c|c|c|c|c|c|}
\hline \multirow{2}{*}{$\begin{array}{l}\text { INTERVAL } \\
\text { IN YEARS }\end{array}$} & OCT & -DECEM & & JA & RV-MARC & & & {$[L-J U N E$} & & $\mathrm{J}$ & -SEPTEI & \\
\hline & 7 & 14 & 30 & 7 & 14 & 30 & 7 & 14 & 30 & 7 & 14 & 30 \\
\hline $\begin{array}{l}1.5 \\
2 \\
5 \\
10 \\
20\end{array}$ & $\begin{array}{l}26 \\
13 \\
2.1 \\
.42 \\
0\end{array}$ & $\begin{array}{r}34 \\
16 \\
2.4 \\
.61 \\
.16\end{array}$ & $\begin{array}{l}41 \\
2.2 \\
4.8 \\
1.8 \\
.72\end{array}$ & $\begin{array}{c}18 \\
8.3 \\
1.1 \\
.19 \\
0\end{array}$ & $\begin{array}{l}19 \\
9.0 \\
1.3 \\
.24 \\
0\end{array}$ & $\begin{array}{l}25 \\
11 \\
1.9 \\
.67 \\
.26\end{array}$ & $\begin{array}{l}50 \\
27 \\
6.2 \\
2.5 \\
1.1\end{array}$ & $\begin{array}{l}62 \\
35 \\
9.6 \\
4.4 \\
2.2\end{array}$ & $\begin{array}{l}97 \\
56 \\
16 \\
7.6 \\
3.8\end{array}$ & $\begin{array}{l}5.5 \\
2.4 \\
.33 \\
.08 \\
0\end{array}$ & $\begin{array}{r}7.0 \\
3.1 \\
.49 \\
.17 \\
.06\end{array}$ & $\begin{array}{r}13 \\
6.5 \\
1.2 \\
.43 \\
.17\end{array}$ \\
\hline
\end{tabular}

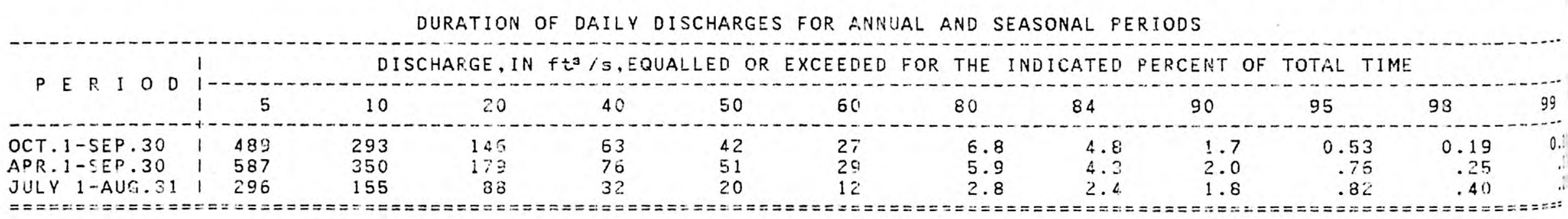


SOUTH SKUNK RIVER BELOW SQUAW CREEK NEAR AMES

Location.--Lat $42^{\circ} 00^{\prime} 31^{\prime \prime}$, long 93035'37", In NE1/4 NWI/4 sec.13, T.83 N., R.24 W. Story County, on right bank $15 \mathrm{ft}$ downstream from bridge on county highway, $0.2 \mathrm{mi}$ downstream from Squaw Creek, $0.2 \mathrm{mi}$ upstream from bridge on U.S. Highway $30,2 \mathrm{~m} 1$ southeast of Ames, and at mile 222.6 upstream from mouth of Skunk River.

Remarks.--Low flows are affected by pumpage by city of Ames from surficial aquifer and do not represent the natural flow of the stream. Prior to October 1966, published as Skunk River belaw Squaw Creek near Ames.

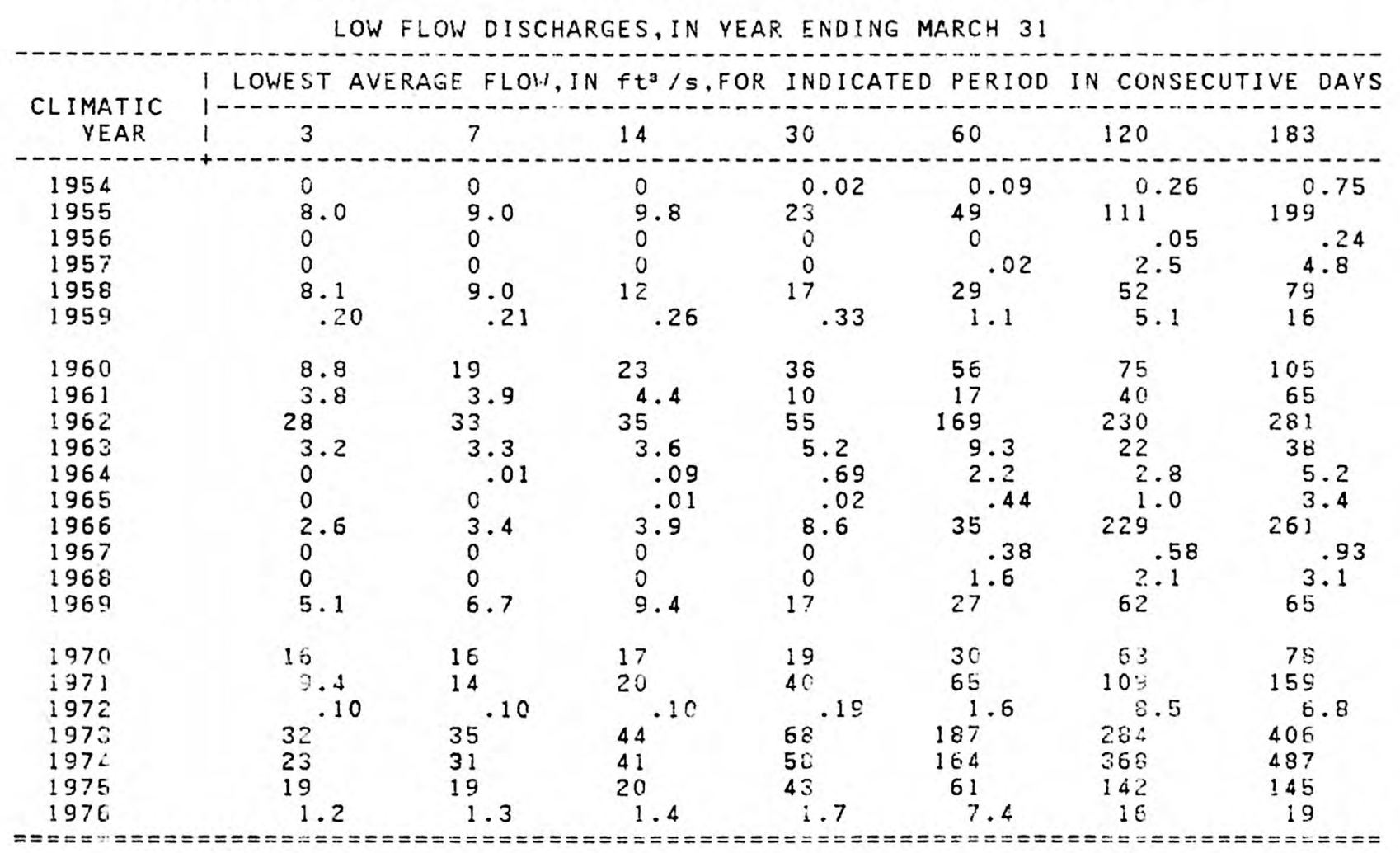


05-4710.00 SOUTH SKUNK RIVER BELOW SQUAW CREEK NEAR AMES--ContInUEd

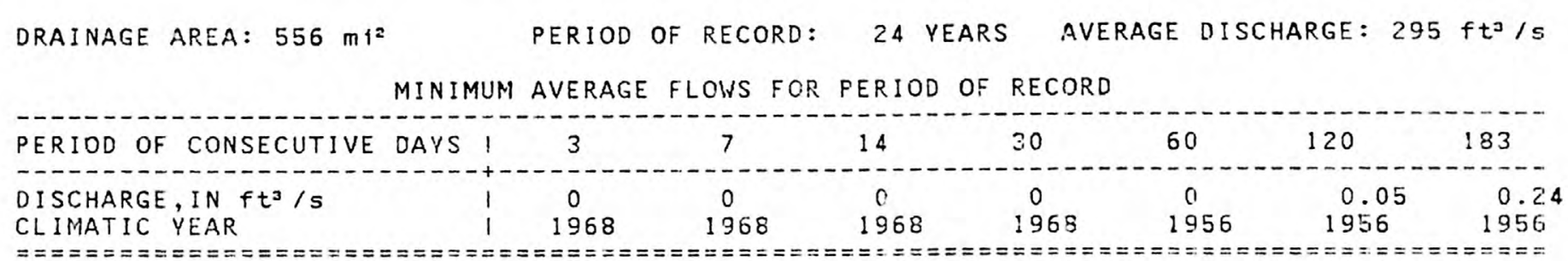

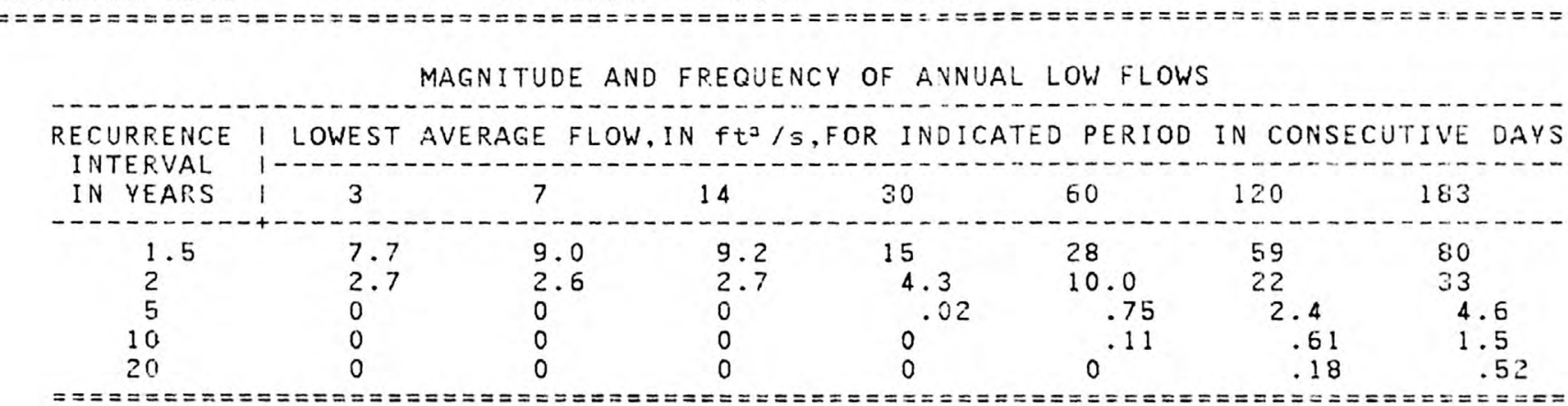

MAGNITUDE AND FREQUENCY OF SEASONAL LOW FLOWS

1 LOWEST AVERAGE FLOW, IN $\mathrm{ft}^{3} / \mathrm{s}$, FOR THE INDICATED PERIOD IN CONSECUTIVE DAYS WITHIN EACH WATER-VEAR QUARTER RECURRENCE

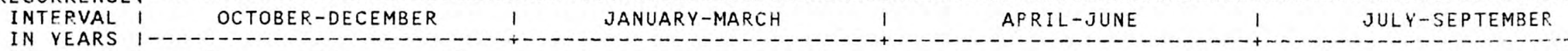

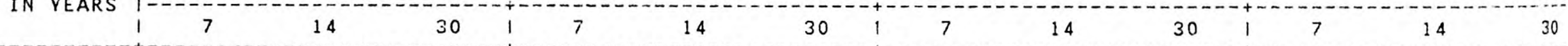

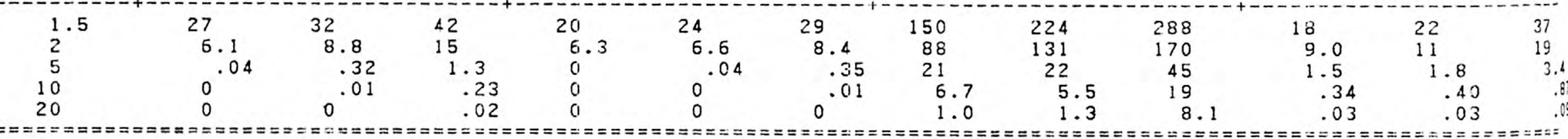

DURATION OF DAILY DISCHARGES FOR FNNUAL ANO SEASONAL PERIODS

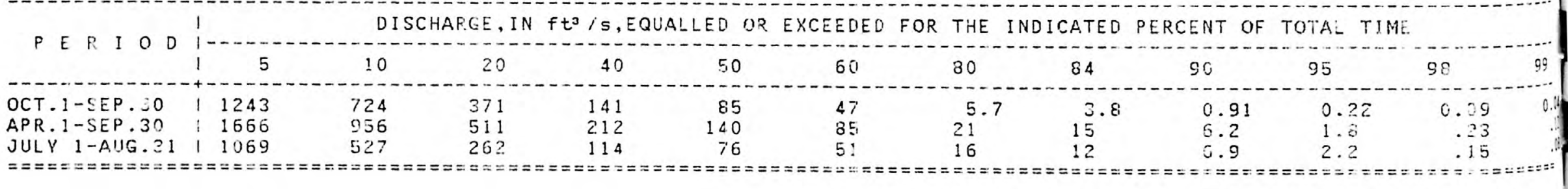


Location.--Lat 4i०48'17", long 93018'26", In NW1/4 NW1/4 sec.28, T.81 N., R.21 W. Jasper County, on right bank $30 \mathrm{ft}$ downstream from bridge on staie Highway 117 , 0.7 mile downstream from Wolf Creek, $2.9 \mathrm{miles}$ northwest of Mingo, and $3.3 \mathrm{miles}$ upstream from Clear Creek.

Remarks.--Discontinued September 1975.

LOW FLOW DISCHARGES, IN YEAR. ENDING MARCH 31

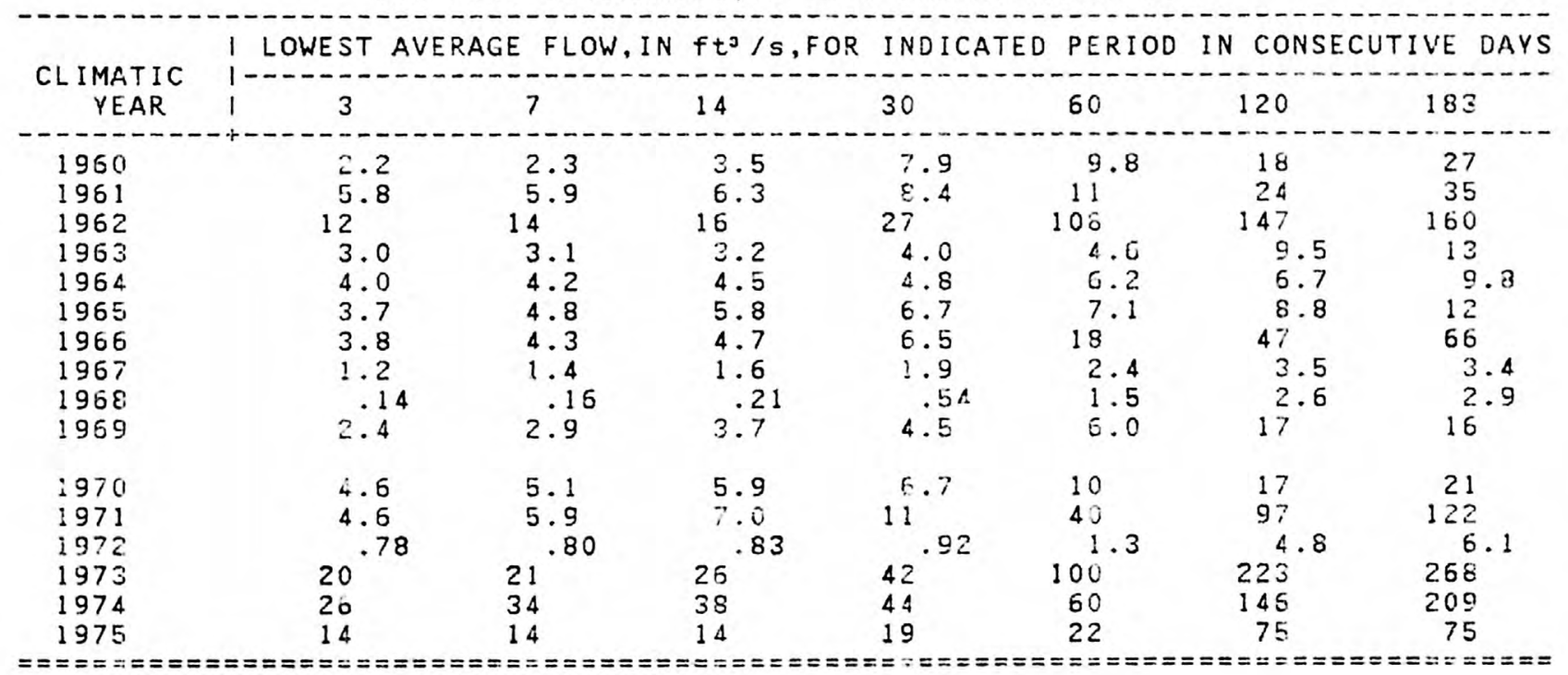




DRAINAGE AREA: $276 \mathrm{~m}^{2} \quad$ PERIOD OF RECORD: 17 YEARS AVERAGE DISCHARGE: $182 \mathrm{ft} / \mathrm{s}$
MINIMUM AVERAGE FLOWS FOR PERIOD OF RECORD
PERIOD OF CONSECUTIVE DAYS I

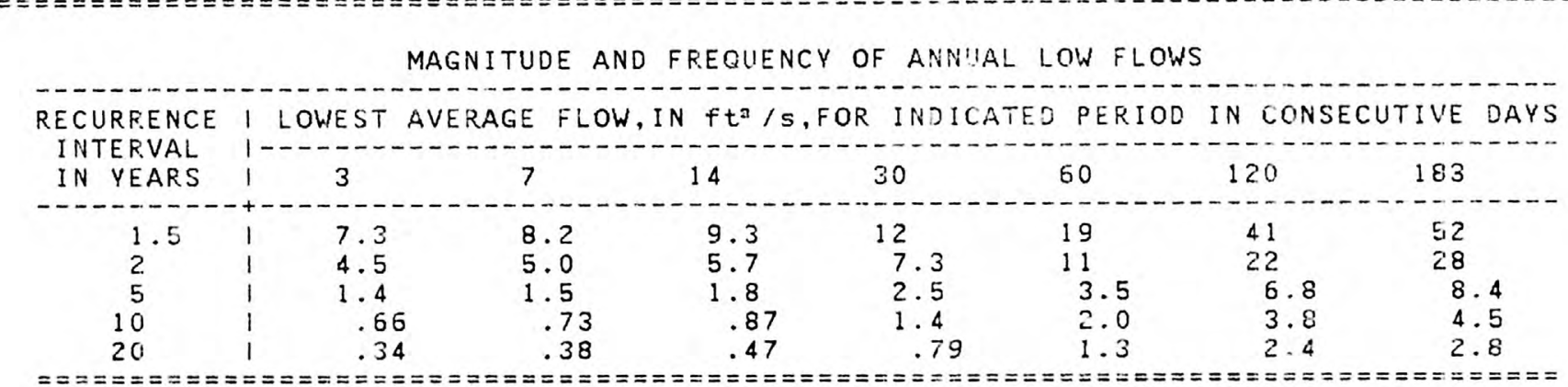

MAGNITUDE AND FREQUENCY OF SEASONAL LOW FLOW'S

I LOWEST AVERAGE FLOW, IN $\mathrm{ft}^{3} / \mathrm{s}$, FOR THE INDICATED PERIOD IN CONSECUTIVE DAYS WITHIN EACH WATER-YEAR OUARTER

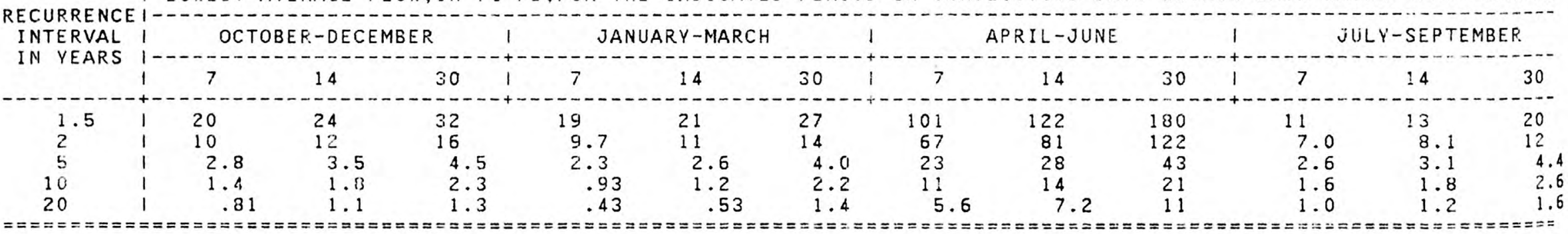

DURATION OF DAILY DISCHARGES FOR AINNUAL SINO SEASONAL PERIDOS

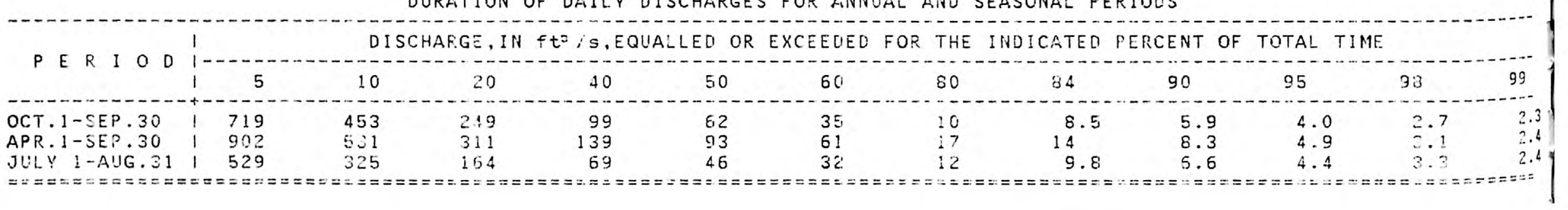




\section{SOUTH SKUNK RIVER NEAR OSKALOOSA}

Location.--Lat 41021'19", long 92039'31", in NW1/4 SW1/4 sec.25, T.76 N., R.16 W. , Mahaska County, on right bank $400 \mathrm{ft}$ upstream from bridge on U.S. Highway $63,0.3$ mile downstream from painter Creek, $4.0 \mathrm{miles}$ north of Oskaloosa, 53.7 miles upstream from confluence with North Skunk River, and at mile 147.3 upstream from mouth of Sklink River.

Remarks.--Prior to October 1966 published as "Skunk River near Oskaloosa."

LOW FLOW DISCHARGES, IN YEAR ENDING MARCH 31

\begin{tabular}{|c|c|c|c|c|c|c|c|}
\hline \multirow{2}{*}{$\begin{array}{c}\text { CLIMATIC } \\
\text { YEAR }\end{array}$} & I LOWEST & $A G E F L$ & $N f t^{3} /$ & $\begin{array}{l}R \text { IND: } \\
-1\end{array}$ & D PERI & \multicolumn{2}{|c|}{ IN CONSECUTIVE DAYS } \\
\hline & 3 & 7 & 14 & 30 & 60 & 120 & 183 \\
\hline $\begin{array}{l}1947 \\
1948 \\
1949\end{array}$ & $\begin{array}{r}152 \\
37 \\
29\end{array}$ & $\begin{array}{r}156 \\
37 \\
31\end{array}$ & $\begin{array}{r}184 \\
45 \\
32\end{array}$ & $\begin{array}{r}303 \\
64 \\
32\end{array}$ & $\begin{array}{r}321 \\
107 \\
52\end{array}$ & $\begin{array}{r}550 \\
179 \\
79\end{array}$ & $\begin{array}{l}565 \\
168 \\
141\end{array}$ \\
\hline $\begin{array}{l}1950 \\
1951 \\
1952 \\
1953 \\
1954 \\
1955 \\
1956 \\
1957 \\
1958 \\
1959\end{array}$ & $\begin{array}{c}28 \\
7.6 \\
170 \\
56 \\
14 \\
58 \\
4.4 \\
1.8 \\
52 \\
27\end{array}$ & $\begin{array}{c}29 \\
7.6 \\
174 \\
57 \\
14 \\
64 \\
4.6 \\
2.0 \\
54 \\
27\end{array}$ & $\begin{array}{c}33 \\
7.9 \\
184 \\
57 \\
14 \\
68 \\
4.7 \\
3.4 \\
57 \\
27\end{array}$ & $\begin{array}{c}50 \\
8.9 \\
226 \\
58 \\
15 \\
130 \\
4.9 \\
7.5 \\
63 \\
30\end{array}$ & $\begin{array}{c}51 \\
11 \\
357 \\
63 \\
19 \\
198 \\
5.6 \\
9.6 \\
86 \\
38\end{array}$ & $\begin{array}{r}57 \\
26 \\
462 \\
110 \\
27 \\
373 \\
12 \\
10 \\
122 \\
87\end{array}$ & $\begin{array}{r}72 \\
33 \\
512 \\
159 \\
30 \\
670 \\
23 \\
26 \\
149 \\
145\end{array}$ \\
\hline $\begin{array}{l}1960 \\
1961 \\
1962 \\
1963 \\
1964 \\
1965 \\
1966 \\
1967 \\
1968 \\
1969\end{array}$ & $\begin{array}{r}98 \\
75 \\
155 \\
47 \\
25 \\
33 \\
93 \\
23 \\
23 \\
53\end{array}$ & $\begin{array}{r}106 \\
77 \\
176 \\
51 \\
26 \\
37 \\
101 \\
26 \\
23 \\
56\end{array}$ & $\begin{array}{r}108 \\
78 \\
191 \\
65 \\
27 \\
41 \\
104 \\
29 \\
25 \\
61\end{array}$ & $\begin{array}{r}151 \\
93 \\
267 \\
83 \\
31 \\
44 \\
127 \\
32 \\
34 \\
67\end{array}$ & $\begin{array}{r}199 \\
106 \\
653 \\
84 \\
50 \\
54 \\
183 \\
4 \% \\
45 \\
86\end{array}$ & $\begin{array}{r}276 \\
194 \\
696 \\
130 \\
66 \\
77 \\
57 \% \\
52 \\
5 i \\
130\end{array}$ & $\begin{array}{r}339 \\
255 \\
866 \\
162 \\
86 \\
110 \\
572 \\
69 \\
56 \\
163\end{array}$ \\
\hline $\begin{array}{l}1970 \\
1971 \\
1972 \\
1973 \\
1974 \\
1975 \\
1976\end{array}$ & $\begin{array}{r}52 \\
125 \\
36 \\
263 \\
184 \\
158 \\
61\end{array}$ & $\begin{array}{r}96 \\
135 \\
36 \\
274 \\
200 \\
165 \\
67\end{array}$ & $\begin{array}{r}99 \\
159 \\
38 \\
308 \\
221 \\
178 \\
70\end{array}$ & $\begin{array}{r}103 \\
290 \\
45 \\
454 \\
278 \\
138 \\
72\end{array}$ & $\begin{array}{r}132 \\
276 \\
52 \\
736 \\
406 \\
219 \\
86\end{array}$ & $\begin{array}{r}218 \\
505 \\
80 \\
1090 \\
697 \\
412 \\
143\end{array}$ & $\begin{array}{r}262 \\
012 \\
82 \\
1190 \\
2140 \\
447 \\
183\end{array}$ \\
\hline
\end{tabular}




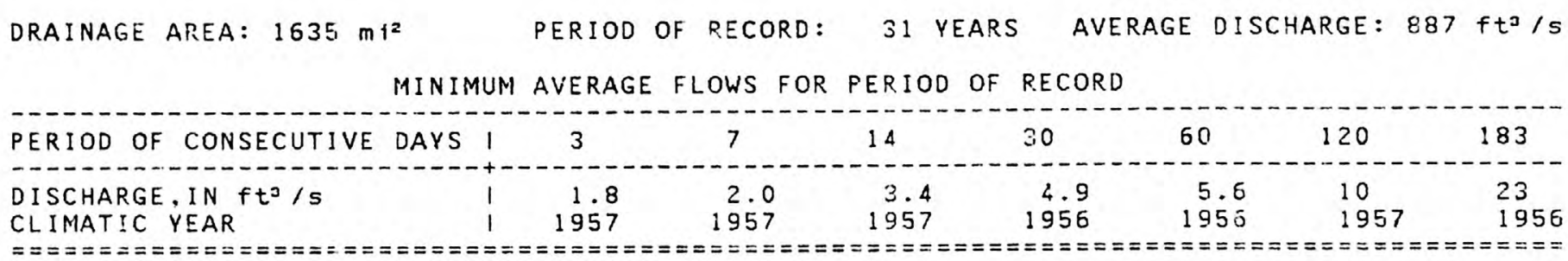

MAGNITUDE AND FREQUENCY OF ANNUAL LOW FLOWS

\begin{tabular}{|c|c|c|c|c|c|c|c|c|}
\hline $\begin{array}{l}\text { RECURRENCE } \\
\text { INTERVAL }\end{array}$ & 1. & WW ST & $A G E$ & $N f t^{3}$ & DR INID & ED PL & IN C & TIVE \\
\hline IN YEARS & i & 3 & 7 & 14 & 30 & 60 & 120 & 183 \\
\hline $\begin{array}{l}1.5 \\
2 \\
5 \\
10 \\
20\end{array}$ & $\begin{array}{l}1 \\
1 \\
1 \\
1\end{array}$ & $\begin{array}{l}84 \\
55 \\
19 \\
10 \\
5.6\end{array}$ & $\begin{array}{l}88 \\
58 \\
20 \\
10 \\
5.9\end{array}$ & $\begin{array}{l}93 \\
60 \\
2.2 \\
12 \\
7.1\end{array}$ & $\begin{array}{r}114 \\
72 \\
2.6 \\
15 \\
9.0\end{array}$ & $\begin{array}{r}153 \\
94 \\
33 \\
18 \\
11\end{array}$ & $\begin{array}{r}246 \\
148 \\
50 \\
28 \\
17\end{array}$ & $\begin{array}{r}296 \\
184 \\
70 \\
42 \\
28\end{array}$ \\
\hline
\end{tabular}

MAGNITUDE AND FREQUENCY OF SEASONAL LOW FLOWS

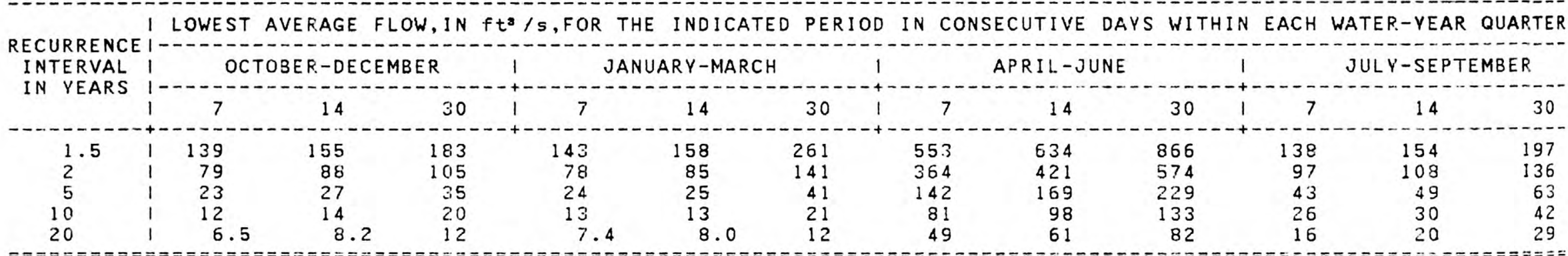

DURATION OF DAILY DISCHARGES FOR ANNUAL AND SEASONAL PERIODS DISCHARGE, IN $\mathrm{ft}^{3} / \mathrm{s}$, EQUALLED OR EXCEEDEO FOR. THE INOICATED PERCENT OF TOTAL TIME

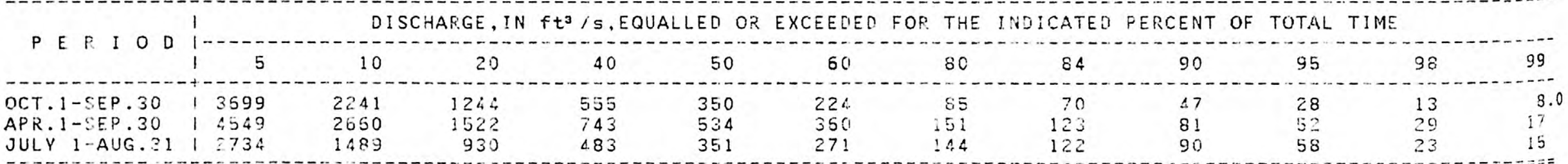


Location.--Lat 41018.03", long 92012'16", In NEi/4 SE1/4 sec.14, T.75 N., R. 22 W., Keokuk County, on right bank $20 \mathrm{ft}$ downstream from bridge on State Highway 149, 1.2 miles downstream from Cedar Creek, 2.2 miles south of Sigourney, 4.0 miles upstream from Bridge Creek, and 16.2 miles upstream from confiuence with South Skunk River.

LOW FLOW DISCHARGES, IN YEAR: ENDING MARCH 31

\begin{tabular}{|c|c|c|c|c|c|c|c|}
\hline \multirow{2}{*}{$\begin{array}{c}\text { CLIMATIC } \\
\text { YEAR }\end{array}$} & LOWEST & GE FL & $f t^{3} /$ & I NOD I & PERIO & IN CON & IVE DAY \\
\hline & 13 & 7 & 14 & 30 & 50 & 120 & 183 \\
\hline $\begin{array}{l}1947 \\
1948 \\
1949\end{array}$ & $\begin{array}{l}81 \\
4.4 \\
11\end{array}$ & $\begin{array}{c}83 \\
6.3 \\
11\end{array}$ & $\begin{array}{c}105 \\
8.7 \\
13\end{array}$ & $\begin{array}{r}147 \\
21 \\
14\end{array}$ & $\begin{array}{r}166 \\
41 \\
27\end{array}$ & $\begin{array}{r}312 \\
54 \\
44\end{array}$ & $\begin{array}{r}289 \\
57 \\
75\end{array}$ \\
\hline $\begin{array}{l}1950 \\
1951 \\
1952 \\
1953 \\
1954 \\
1955 \\
1956 \\
1957 \\
1958 \\
1959\end{array}$ & $\begin{array}{c}7.2 \\
3.3 \\
48 \\
11 \\
1.4 \\
12 \\
.63 \\
.10 \\
7.4 \\
18\end{array}$ & $\begin{array}{l}7.5 \\
3.4 \\
53 \\
13 \\
1.5 \\
14 \\
.93 \\
.10 \\
8.0 \\
18\end{array}$ & $\begin{array}{c}8.1 \\
3.5 \\
72 \\
13 \\
1.5 \\
18 \\
1.1 \\
.10 \\
8.4 \\
18\end{array}$ & $\begin{array}{l}11 \\
3.7 \\
91 \\
14 \\
2.0 \\
22 \\
2.3 \\
.10 \\
13 \\
19\end{array}$ & $\begin{array}{c}15 \\
4.5 \\
171 \\
19 \\
2.9 \\
57 \\
2.4 \\
2.26 \\
25 \\
23\end{array}$ & $\begin{array}{c}23 \\
5.7 \\
300 \\
72 \\
3.7 \\
92 \\
3.8 \\
2.3 \\
36 \\
58\end{array}$ & $\begin{array}{c}35 \\
8.8 \\
304 \\
113 \\
4.8 \\
152 \\
13 \\
16 \\
35 \\
100\end{array}$ \\
\hline $\begin{array}{l}1960 \\
1961 \\
1962 \\
1963 \\
1964 \\
1965 \\
1966 \\
1967 \\
1968 \\
1969\end{array}$ & $\begin{array}{l}19 \\
31 \\
57 \\
25 \\
10 \\
14 \\
44 \\
10 \\
12 \\
9.2\end{array}$ & $\begin{array}{l}21 \\
32 \\
64 \\
25 \\
10 \\
15 \\
45 \\
11 \\
14 \\
12\end{array}$ & $\begin{array}{l}31 \\
32 \\
69 \\
26 \\
10 \\
16 \\
47 \\
13 \\
16 \\
12\end{array}$ & $\begin{array}{l}51 \\
38 \\
84 \\
29 \\
12 \\
19 \\
56 \\
14 \\
23 \\
15\end{array}$ & $\begin{array}{r}115 \\
46 \\
145 \\
32 \\
22 \\
23 \\
274 \\
19 \\
26 \\
16\end{array}$ & $\begin{array}{r}199 \\
103 \\
277 \\
61 \\
27 \\
31 \\
372 \\
21 \\
44 \\
24\end{array}$ & $\begin{array}{r}284 \\
99 \\
415 \\
69 \\
37 \\
48 \\
433 \\
34 \\
45 \\
32\end{array}$ \\
\hline $\begin{array}{l}1970 \\
1971 \\
1972 \\
1973 \\
1974 \\
1975 \\
1976\end{array}$ & $\begin{array}{r}36 \\
43 \\
13 \\
129 \\
69 \\
63 \\
33\end{array}$ & $\begin{array}{r}37 \\
45 \\
14 \\
133 \\
78 \\
64 \\
39\end{array}$ & $\begin{array}{r}38 \\
54 \\
14 \\
155 \\
87 \\
66 \\
40\end{array}$ & $\begin{array}{r}38 \\
76 \\
10 \\
266 \\
97 \\
70 \\
41\end{array}$ & $\begin{array}{r}48 \\
180 \\
10 \\
402 \\
132 \\
83 \\
43\end{array}$ & $\begin{array}{r}82 \\
400 \\
36 \\
530 \\
246 \\
151 \\
77\end{array}$ & $\begin{array}{r}10 \% \\
581 \\
55 \\
566 \\
310 \\
196 \\
120\end{array}$ \\
\hline
\end{tabular}


05-4725.00 NORTH SKUNK. RIVER NEAR SIGOURNEY--Cont inued

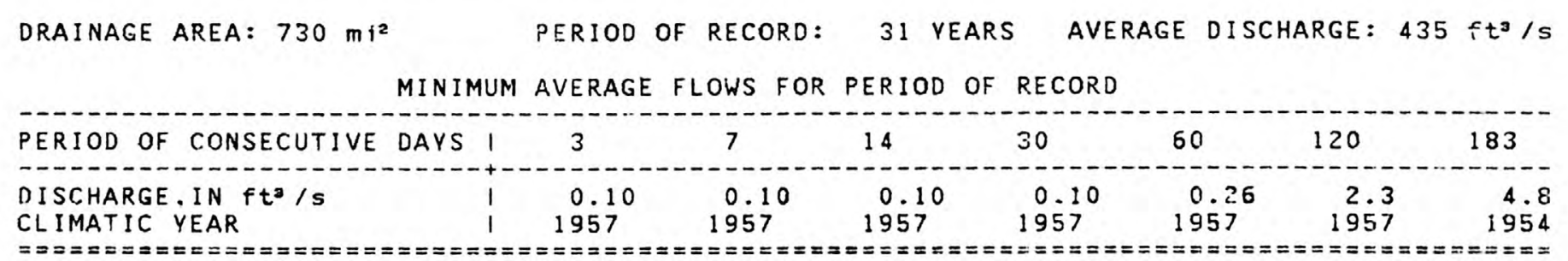

MAGNITUDE AND FREQUENCY OF ANNUAL LOW FLOWS

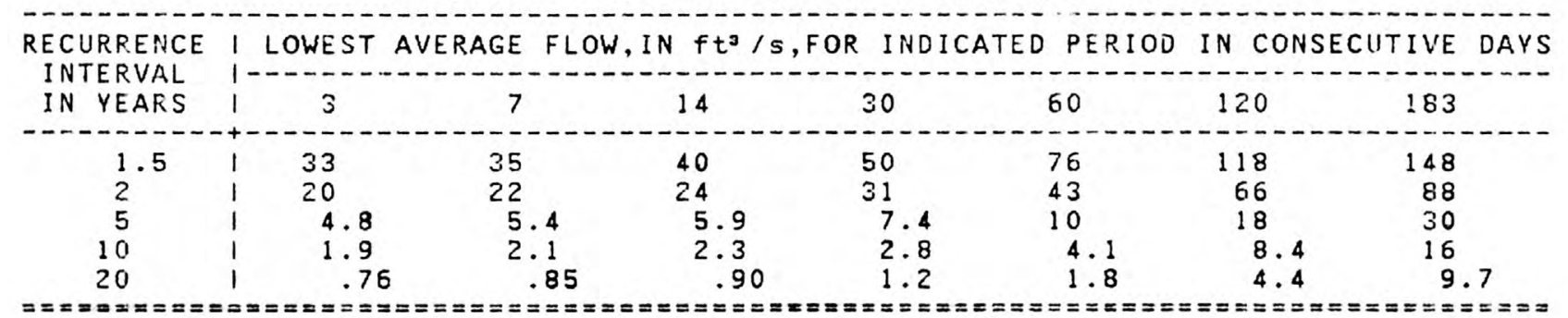

MAGNITUDE AND FREQUENCY OF SEASONAL LOW FLOWS

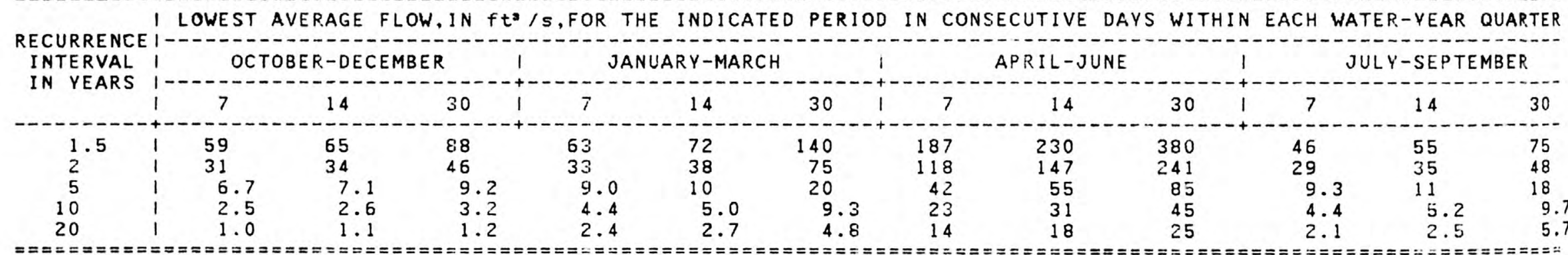

DURATION OF DAILY DISCHARGES FOR ANNUAL AND SEASONAL PERIODS

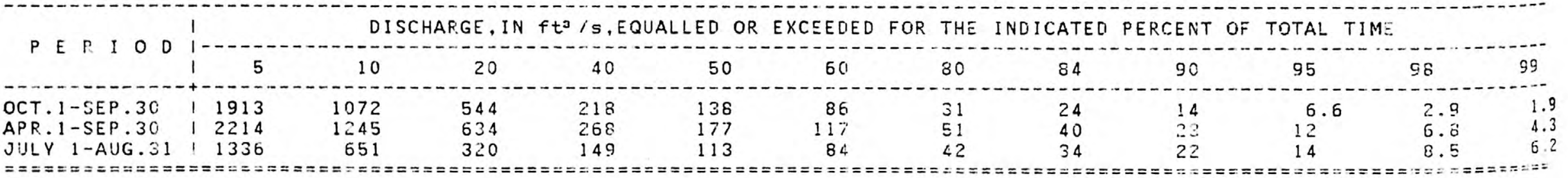


Location.--Lat 41009.50", long 91043.05", in NE1/4 NE1/4 sec.1, T.73 N., R.8 W. Jefferson County, at bridge on State Highway $7 \varepsilon, 0.5 \mathrm{mile}$ west of Coppock, $3 / 4 \mathrm{mile}$ upstream from Crooked Creek, and $66 \mathrm{miles}$ upstream from mouth of skunk River.

Remarks.--Station operation discontinued Sept. 30, 1944. Peak data collected by observer unti? 1951 .

LOW FLOW DISCHARGES. IN YEAR. ENDING MARCH 31

\begin{tabular}{|c|c|c|c|c|c|c|c|}
\hline \multirow{2}{*}{$\begin{array}{c}\text { CLIMATIC } \\
\text { YEAR }\end{array}$} & \multicolumn{2}{|c|}{ I LOWEST AVERAGE } & $N f t^{3}$ & $R$ IN & $D P E$ & \multicolumn{2}{|c|}{ IN CONSECUTIVE DAYS } \\
\hline & 3 & 7 & 14 & 30 & 60 & 120 & 183 \\
\hline $\begin{array}{l}1915 \\
1916 \\
1917 \\
1918 \\
1919\end{array}$ & $\begin{array}{r}33 \\
400 \\
55 \\
30 \\
88\end{array}$ & $\begin{array}{r}39 \\
429 \\
67 \\
30 \\
91\end{array}$ & $\begin{array}{r}49 \\
500 \\
80 \\
31 \\
95\end{array}$ & $\begin{array}{r}57 \\
723 \\
117 \\
35 \\
100\end{array}$ & $\begin{array}{r}101 \\
961 \\
148 \\
50 \\
137\end{array}$ & $\begin{array}{r}302 \\
1520 \\
169 \\
77 \\
173\end{array}$ & $\begin{array}{r}644 \\
2410 \\
175 \\
133 \\
235\end{array}$ \\
\hline $\begin{array}{l}1920 \\
1921 \\
1922 \\
1923 \\
1924 \\
1925 \\
1926 \\
1927 \\
1928 \\
1929\end{array}$ & $\begin{array}{r}84 \\
185 \\
187 \\
150 \\
168 \\
120 \\
82 \\
157 \\
78 \\
311\end{array}$ & $\begin{array}{r}86 \\
193 \\
203 \\
156 \\
180 \\
120 \\
87 \\
173 \\
83 \\
327\end{array}$ & $\begin{array}{r}99 \\
203 \\
236 \\
179 \\
206 \\
123 \\
100 \\
208 \\
88 \\
334\end{array}$ & $\begin{array}{r}133 \\
261 \\
368 \\
207 \\
298 \\
150 \\
160 \\
273 \\
99 \\
417\end{array}$ & $\begin{array}{l}278 \\
349 \\
554 \\
236 \\
355 \\
239 \\
228 \\
391 \\
183 \\
613\end{array}$ & $\begin{array}{r}772 \\
382 \\
742 \\
328 \\
573 \\
346 \\
325 \\
997 \\
445 \\
1650\end{array}$ & $\begin{array}{r}1170 \\
406 \\
1130 \\
340 \\
708 \\
568 \\
375 \\
2000 \\
399 \\
1630\end{array}$ \\
\hline $\begin{array}{l}1930 \\
1931 \\
1932 \\
1933 \\
1934 \\
1935 \\
1936 \\
1937 \\
1938 \\
1939\end{array}$ & $\begin{array}{r}200 \\
20 \\
66 \\
206 \\
20 \\
19 \\
50 \\
50 \\
15 \\
107\end{array}$ & $\begin{array}{r}200 \\
20 \\
83 \\
219 \\
22 \\
19 \\
50 \\
52 \\
16 \\
116\end{array}$ & $\begin{array}{r}200 \\
20 \\
122 \\
248 \\
36 \\
19 \\
50 \\
54 \\
20 \\
134\end{array}$ & $\begin{array}{r}235 \\
20 \\
150 \\
272 \\
35 \\
22 \\
80 \\
64 \\
27 \\
166\end{array}$ & $\begin{array}{r}290 \\
102 \\
196 \\
358 \\
54 \\
32 \\
217 \\
87 \\
38 \\
202\end{array}$ & $\begin{array}{r}398 \\
126 \\
269 \\
531 \\
77 \\
49 \\
524 \\
241 \\
55 \\
306\end{array}$ & $\begin{array}{r}428 \\
145 \\
453 \\
690 \\
112 \\
83 \\
485 \\
235 \\
143 \\
342\end{array}$ \\
\hline $\begin{array}{l}1240 \\
1941 \\
1942 \\
1943 \\
1941\end{array}$ & $\begin{array}{r}8.3 \\
47 \\
105 \\
183 \\
180\end{array}$ & $\begin{array}{r}65 \\
114 \\
227 \\
183\end{array}$ & $\begin{array}{r}79 \\
75 \\
133 \\
261 \\
187\end{array}$ & $\begin{array}{r}11 \\
8 c \\
17 E \\
296 \\
218\end{array}$ & $\begin{array}{l}21 \\
152 \\
301 \\
402 \\
310\end{array}$ & $\begin{array}{r}46 \\
247 \\
811 \\
618 \\
432\end{array}$ & $\begin{array}{r}66 \\
459 \\
828 \\
6160 \\
6181\end{array}$ \\
\hline
\end{tabular}




DRAINAGE AREA: $2916 \mathrm{~m}^{2}=$ PERIOD OF RECORD: 31 YEARS AVERAGE DISCHARGE: 1350 ft /
MINIMUM AVERAGE FLOWS FOR PERIOD OF RECORD

MAGNITUDE AND FREQUENCY OF ANNUAL LOW FLOWS

\begin{tabular}{l|cccccc} 
RECURRENCE \\
INTERVAL
\end{tabular}

MAGNITUDE AND FREQUENCY OF SEASONAL LOW FLOWS

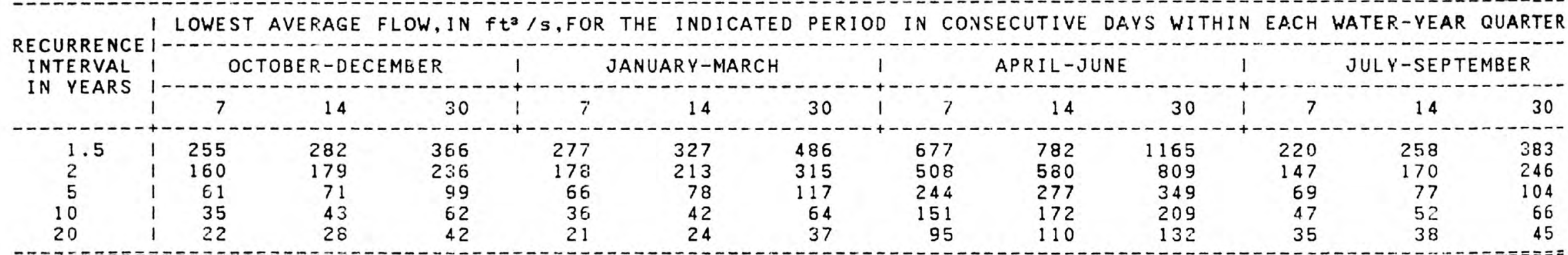

DURATION OF DAILY DISCHARGES FOR ANNUAL AND SEASONAL PERIODS

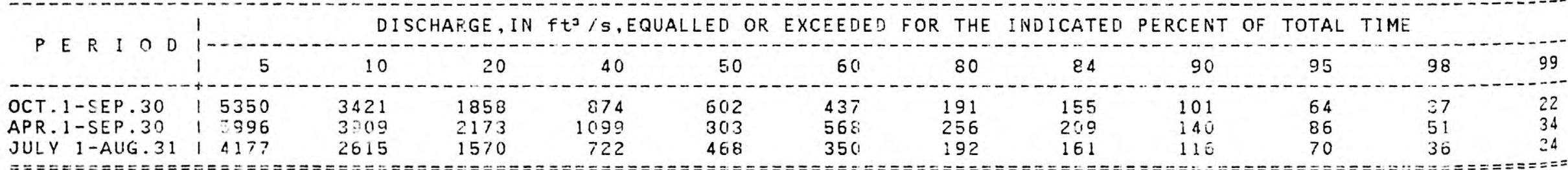


Location.--Lat $41^{\circ} 00^{\prime} 52^{\prime \prime}$, long $91^{\circ} 34^{\prime 4} 49^{\prime \prime}$, in NW1/4 NW1/4 sec.29, T.72 N., R.6 W., Henry County, on left bank $12 \mathrm{ft}$ downstream from bridge on county highway, $100 \mathrm{ft}$ downstream from Lynn Creek, $0.7 \mathrm{mile}$ downstream from Brandywine Creek, arid 3.7 miles northwest of courthouse at Mount pleasant.

LOW FLOW DISCHARGES, IN VEAR. ENDING MARCH 31

\begin{tabular}{|c|c|c|c|c|c|c|c|}
\hline \multirow{2}{*}{$\begin{array}{c}\text { CLIMATIC } \\
\text { YEAR }\end{array}$} & \multirow{2}{*}{\multicolumn{2}{|c|}{ I LOWEST AVERAGE FLOW }} & \multirow{2}{*}{$\mathrm{fto}^{2} / \mathrm{s}$} & IND I CATED & \multicolumn{3}{|c|}{$\begin{array}{l}\text { PERIOD IN CONSECUTIVE DAYS } \\
\text { PES }\end{array}$} \\
\hline & & 7 & & 30 & 60 & 120 & 183 \\
\hline $\begin{array}{l}1957 \\
1958 \\
1959\end{array}$ & $\begin{array}{l}0 \\
0 \\
.80\end{array}$ & $\begin{array}{l}0 \\
0 \\
1.3\end{array}$ & $\begin{array}{l}0 \\
0 \\
1.5\end{array}$ & $\begin{array}{l}0 \\
1.10\end{array}$ & $\begin{array}{l}0 \\
.20 \\
3.1\end{array}$ & $\begin{array}{l}0 \\
1.2 \\
7.6\end{array}$ & $\begin{array}{l}0.40 \\
2.4 \\
3.0\end{array}$ \\
\hline $\begin{array}{l}1960 \\
1961 \\
1962 \\
1963 \\
1964 \\
1965 \\
1966 \\
1967 \\
1968 \\
1969\end{array}$ & $\begin{array}{l}0 \\
.10 \\
.30 \\
0 \\
0 \\
0 \\
0.10 \\
0.06 \\
.0 .66\end{array}$ & $\begin{array}{l}.10 \\
.10 \\
.30 \\
0 \\
0 \\
0 \\
.110 \\
0.77 \\
0.0\end{array}$ & $\begin{array}{l}.40 \\
.10 \\
.40 \\
0 \\
0 \\
0 \\
.20 \\
0.20 \\
1.2 \\
.18\end{array}$ & $\begin{array}{l}.90 \\
.20 \\
.60 \\
.30 \\
0 \\
0 \\
1.2 \\
0.2 \\
6.6 \\
.72\end{array}$ & $\begin{array}{c}5.3 \\
.40 \\
2.6 \\
.90 \\
.10 \\
.70 \\
16 \\
0 \\
10 \\
4.1\end{array}$ & $\begin{array}{c}32 \\
2.8 \\
18 \\
5.9 \\
.80 \\
1.3 \\
23 \\
1.3 \\
20 \\
9.1\end{array}$ & $\begin{array}{r}55 \\
2.4 \\
44 \\
5.3 \\
2.8 \\
2.4 \\
47 \\
1.6 \\
33 \\
12\end{array}$ \\
\hline $\begin{array}{l}1970 \\
1971 \\
1972 \\
1973 \\
1974 \\
1975 \\
1976\end{array}$ & $\begin{array}{l}.22 \\
1.4 \\
0 \\
.50 \\
3.4 \\
.66 \\
0.66\end{array}$ & $\begin{array}{c}.37 \\
4.7 \\
.01 \\
.62 \\
3.8 \\
.73 \\
0.73\end{array}$ & $\begin{array}{l}1.2 \\
7.2 \\
.05 \\
1.1 \\
5.4 \\
.88 \\
6\end{array}$ & $\begin{array}{c}1.6 \\
13.42 \\
1.5 \\
14.5 \\
1.5 \\
.45\end{array}$ & $\begin{array}{r}4.7 \\
24 \\
1.2 \\
2.1 \\
44 \\
2.2 \\
4.5\end{array}$ & $\begin{array}{l}12 \\
36 \\
1.7 \\
12 \\
99 \\
9.0 \\
20\end{array}$ & $\begin{array}{c}13 \\
64 \\
5.2 \\
19 \\
131 \\
15 \\
23\end{array}$ \\
\hline
\end{tabular}


DRAINAGE AREA: $106 \mathrm{~m}^{2} \quad$ PERIOD OF RECORD: 21 YEARS AVERAGE DISCHARGE: 66.5 ft ${ }^{3} / \mathrm{s}$ MINIMUM AVERAGE FLOWS FOR PERIOD OF RECORD

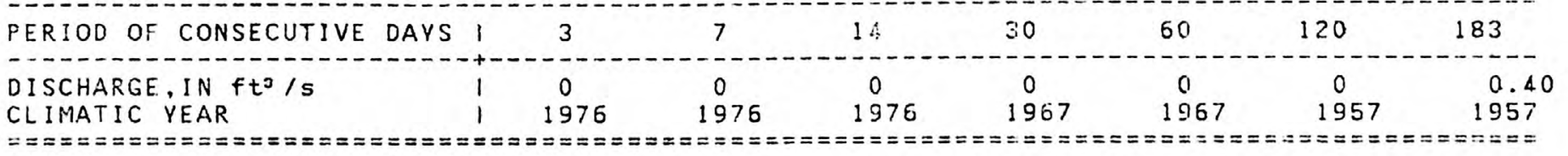

MAGNITUDE AND FREQUENCY OF ANIUAL LOW FLOWS

RECURRENCE
INTERVAL
IN YNEARS

MAGNITUDE AND FREQUENCY OF SEASONAL LOW FLOWS

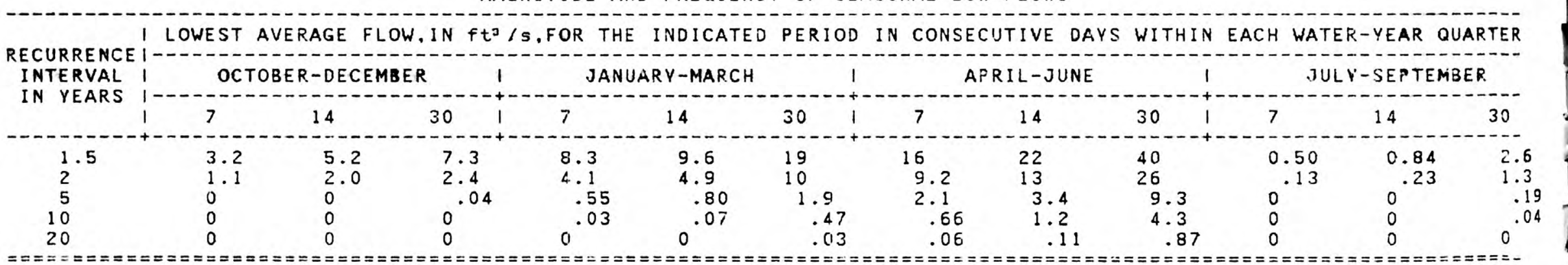

DURATION OF DAILY DISCHARGES FOR ANNUAL ANJ SEASONAL PERIODS

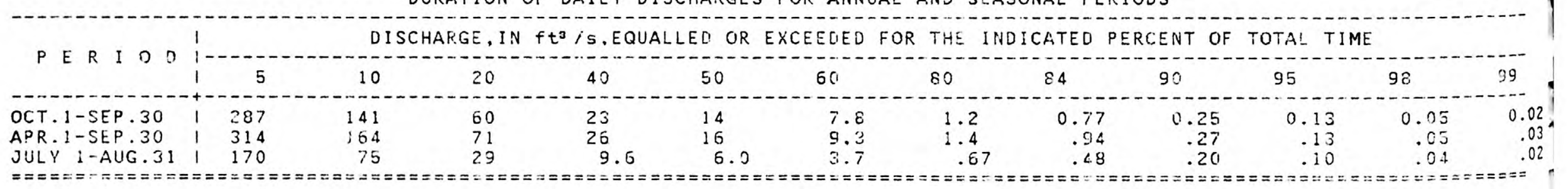


Location.--Lat $40^{\circ} 45^{\prime} 13^{\prime \prime}$, long $91^{\circ} 16^{\prime} 40^{\prime \prime}$, in NE1/4 NE1/4 sec.26, T.69 N.. R.4 W. Des Molnes County, on left bank $300^{\circ} \mathrm{ft}$ upstream from bridge on State Highway 394 at Augusta, 2.0 miles upstream from Long Creek, and at mile 12.5 .

LOW FLOW DISCHARGES, IN YEAR. ENDING MARCH 31

\begin{tabular}{|c|c|c|c|c|c|c|c|c|}
\hline \multirow{2}{*}{$\underset{\text { YEAR }}{\text { CLIMATIC }}$} & LOWEST & EE FLOW & $N$ & 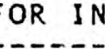 & E $\mathrm{DPE}$ & \multicolumn{3}{|c|}{ IN CONSECUTIVE DAYS } \\
\hline & $i$ & 7 & 14 & 30 & 60 & 120 & 183 & \\
\hline $\begin{array}{l}1916 \\
1917 \\
1918 \\
1919\end{array}$ & $\begin{array}{r}800 \\
64 \\
40 \\
150\end{array}$ & $\begin{array}{r}800 \\
73 \\
40 \\
161\end{array}$ & $\begin{array}{r}800 \\
86 \\
40 \\
168\end{array}$ & $\begin{array}{r}1020 \\
151 \\
43 \\
179\end{array}$ & $\begin{array}{r}1490 \\
193 \\
62 \\
221\end{array}$ & $\begin{array}{r}2550 \\
236 \\
101 \\
271\end{array}$ & $\begin{array}{r}3330 \\
259 \\
197 \\
454\end{array}$ & \\
\hline $\begin{array}{l}1920 \\
1921 \\
1922 \\
1923 \\
1924 \\
1925 \\
1926 \\
1927 \\
1928 \\
1929\end{array}$ & $\begin{array}{r}72 \\
127 \\
267 \\
107 \\
118 \\
170 \\
81 \\
200 \\
88 \\
325\end{array}$ & $\begin{array}{l}120 \\
181 \\
272 \\
130 \\
170 \\
179 \\
109 \\
283 \\
95 \\
334\end{array}$ & $\begin{array}{l}133 \\
195 \\
303 \\
171 \\
250 \\
184 \\
127 \\
339 \\
131 \\
370\end{array}$ & $\begin{array}{l}178 \\
262 \\
503 \\
189 \\
340 \\
208 \\
358 \\
385 \\
173 \\
670\end{array}$ & $\begin{array}{l}410 \\
367 \\
793 \\
251 \\
444 \\
407 \\
433 \\
622 \\
288 \\
838\end{array}$ & $\begin{array}{r}1150 \\
392 \\
1060 \\
384 \\
717 \\
509 \\
936 \\
1760 \\
801 \\
2560\end{array}$ & $\begin{array}{r}1610 \\
433 \\
1770 \\
390 \\
849 \\
847 \\
956 \\
3580 \\
717 \\
2630\end{array}$ & \\
\hline $\begin{array}{l}1930 \\
1931 \\
1932 \\
1933 \\
1934 \\
1935 \\
1936 \\
1937 \\
1938 \\
1939\end{array}$ & $\begin{array}{r}249 \\
27 \\
56 \\
270 \\
20 \\
7.0 \\
100 \\
55 \\
18 \\
137\end{array}$ & $\begin{array}{r}250 \\
29 \\
69 \\
270 \\
23 \\
7.5 \\
100 \\
64 \\
18 \\
143\end{array}$ & $\begin{array}{r}250 \\
29 \\
139 \\
281 \\
35 \\
14 \\
100 \\
71 \\
25 \\
176\end{array}$ & $\begin{array}{r}278 \\
30 \\
186 \\
480 \\
40 \\
23 \\
152 \\
83 \\
35 \\
211\end{array}$ & $\begin{array}{r}370 \\
129 \\
532 \\
512 \\
57 \\
64 \\
291 \\
110 \\
49 \\
247\end{array}$ & $\begin{array}{l}647 \\
166 \\
867 \\
726 \\
100 \\
101 \\
733 \\
487 \\
71 \\
434\end{array}$ & $\begin{array}{r}636 \\
182 \\
1130 \\
1460 \\
136 \\
130 \\
692 \\
566 \\
212 \\
460\end{array}$ & \\
\hline $\begin{array}{l}1940 \\
1941 \\
1942 \\
1943 \\
1944 \\
1945 \\
1946 \\
1947 \\
1948 \\
1949\end{array}$ & $\begin{array}{r}14 \\
66 \\
120 \\
244 \\
210 \\
340 \\
262 \\
397 \\
87 \\
69\end{array}$ & $\begin{array}{r}15 \\
73 \\
140 \\
263 \\
221 \\
344 \\
336 \\
407 \\
99 \\
87\end{array}$ & $\begin{array}{r}15 \\
88 \\
167 \\
295 \\
227 \\
351 \\
352 \\
428 \\
113 \\
96\end{array}$ & $\begin{array}{l}18 \\
158 \\
197 \\
376 \\
264 \\
357 \\
418 \\
563 \\
148 \\
124\end{array}$ & $\begin{array}{l}30 \\
191 \\
354 \\
627 \\
385 \\
437 \\
561 \\
731 \\
263 \\
210\end{array}$ & $\begin{array}{r}63 \\
329 \\
1020 \\
759 \\
535 \\
726 \\
869 \\
1190 \\
380 \\
260\end{array}$ & $\begin{array}{r}88 \\
662 \\
1060 \\
918 \\
808 \\
983 \\
942 \\
1110 \\
440 \\
426\end{array}$ & \\
\hline $\begin{array}{l}1950 \\
1951 \\
1952 \\
1953 \\
1954\end{array}$ & $\begin{array}{r}65 \\
36 \\
422 \\
88 \\
16\end{array}$ & $\begin{array}{r}73 \\
37 \\
447 \\
107 \\
16\end{array}$ & $\begin{array}{r}83 \\
43 \\
508 \\
109 \\
17\end{array}$ & $\begin{array}{r}103 \\
45 \\
6181 \\
112 \\
22\end{array}$ & $\begin{array}{r}116 \\
64 \\
1140 \\
141 \\
29\end{array}$ & $\begin{array}{r}168 \\
81 \\
1420 \\
352 \\
42\end{array}$ & $\begin{array}{r}288 \\
108 \\
1910 \\
541 \\
53\end{array}$ & \\
\hline
\end{tabular}


LOW FLOW DISCHARGES, IN YEAR ENDING MARCH 31--Continued

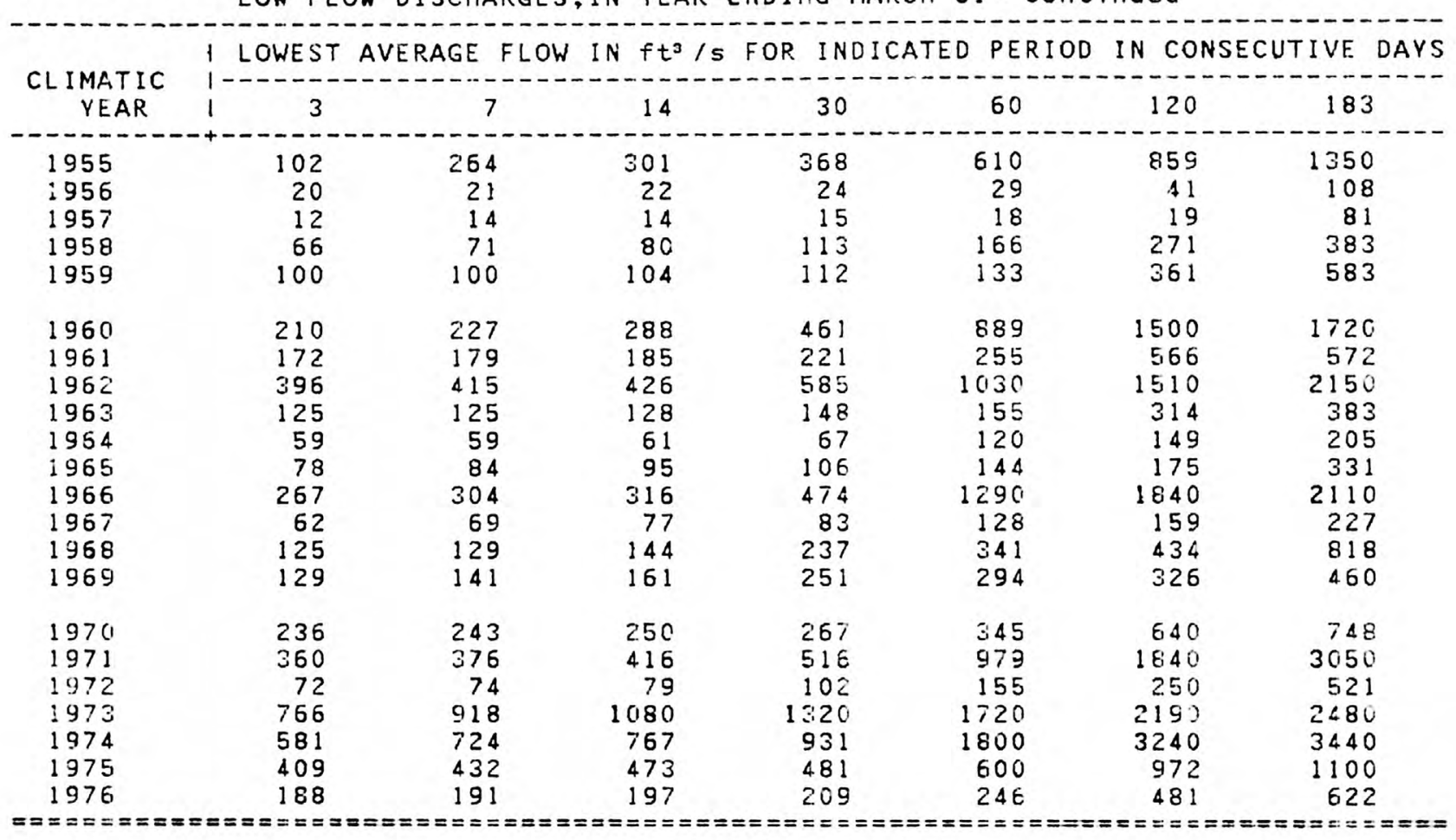




\section{DRAINAGE AREA: $4303 \mathrm{~m}^{2}$ PERIOD OF RECORD: 62 YEARS AVERAGE DISCHARGE: 2350 ft? /s}

MINIMUM AVERAGE FLOWS FOR PERIOD OF RECORD

PERIOD OF CONSECUTIVE DAVS I
DISCHARGE, IN f $\mathrm{ft}^{3} / \mathrm{s}$

MAGNITUDE AND FREQUENCY OF ANINUAL LOW FLOWS

\begin{tabular}{|c|c|c|c|c|c|c|c|c|}
\hline $\begin{array}{l}\text { RECURRENCE } \\
\text { INTERVAL }\end{array}$ & $i$ & OWES & $R A G E$ & $\begin{array}{ll}N & f \\
0\end{array}$ & $D R$ & $=0 \quad P$ & IN $\mathrm{C}$ & \multirow{2}{*}{$\begin{array}{l}\text { IVE DAYS } \\
183\end{array}$} \\
\hline IN YEARS & i & 3 & 7 & 14 & 30 & 60 & 120 & \\
\hline $\begin{array}{l}1.5 \\
2 \\
5 \\
10 \\
20\end{array}$ & $\begin{array}{l}1 \\
1 \\
1 \\
1\end{array}$ & $\begin{array}{r}182 \\
118 \\
46 \\
27 \\
17\end{array}$ & $\begin{array}{r}207 \\
135 \\
52 \\
30 \\
19\end{array}$ & $\begin{array}{r}229 \\
151 \\
60 \\
35 \\
22\end{array}$ & $\begin{array}{r}299 \\
195 \\
75 \\
44 \\
27\end{array}$ & $\begin{array}{r}448 \\
290 \\
111 \\
65 \\
40\end{array}$ & $\begin{array}{r}770 \\
490 \\
178 \\
99 \\
59\end{array}$ & $\begin{array}{l}975 \\
642 \\
264 \\
162 \\
107\end{array}$ \\
\hline
\end{tabular}

MAGNITUDE AND FREQUENCY OF SEASONAL LOW FLOWS

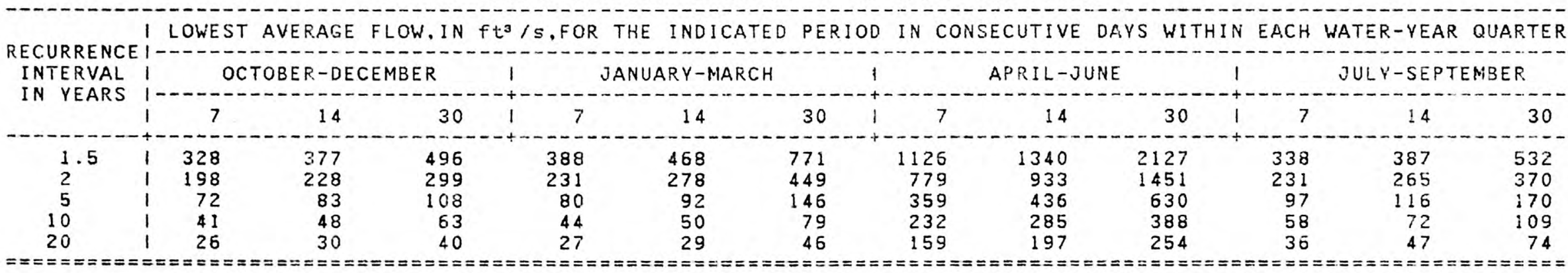

DURATION OF DAILY DISCHARGES FOR ANNUAL AND SEASONAL PERIODS

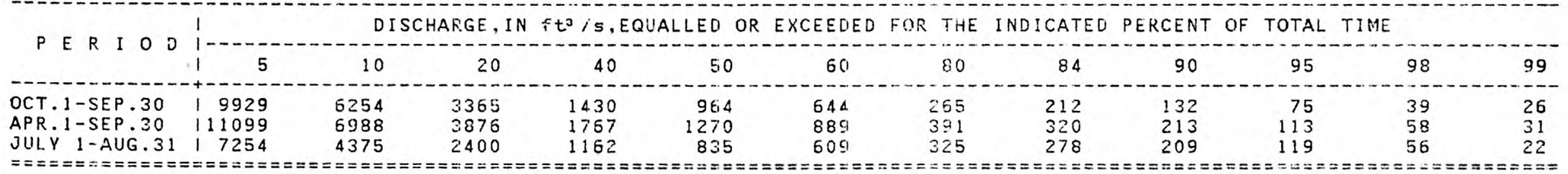


Location.--Lat 40023'37", Tong 91022'27", in SE1/4 SW1/4 sec.30, T.65 N., R.4 W., Lee County, near right bank in taliwater of dam and powerplant of Union Electric Co. at Keokuk, $0.2 \mathrm{mile}$ upstream from bridge on U.S. Highway 136, 2.7 mfles upstream from Des Moines River, and at mile 364.2 upstream from Ohio River.

Remarks.--Flow regulated by powerplant upstream of station since 1913 , and reservolrs and navigation dams upstream of station since about 1935.

LOW FLOW DISCHARGES, IN YEAR ENDING MARCH 31

\begin{tabular}{|c|c|c|c|c|c|c|c|}
\hline \multirow{2}{*}{$\begin{array}{c}\text { CLIMATIC } \\
\text { YEAR }\end{array}$} & I LOWEST & \multicolumn{2}{|c|}{ GE FLOW IN $\mathrm{fi}^{3} / \mathrm{s}$} & INDI & PERIC & \multicolumn{2}{|c|}{ N CONSECUTIVE DAY } \\
\hline & 3 & 7 & 14 & 30 & 60 & 120 & 183 \\
\hline $\begin{array}{l}1880 \\
1881 \\
1882 \\
1883 \\
1884 \\
1885 \\
1886 \\
1887 \\
1888 \\
1889\end{array}$ & $\begin{array}{l}20700 \\
18000 \\
38000 \\
18300 \\
18000 \\
24300 \\
19000 \\
14000 \\
14300 \\
16700\end{array}$ & $\begin{array}{l}22300 \\
19100 \\
38400 \\
19400 \\
18700 \\
26300 \\
19600 \\
14600 \\
16100 \\
17900\end{array}$ & $\begin{array}{l}27300 \\
22000 \\
40400 \\
226100 \\
21200 \\
32100 \\
21500 \\
16800 \\
19000 \\
18600\end{array}$ & $\begin{array}{l}31700 \\
23000 \\
42000 \\
30800 \\
26500 \\
37500 \\
30300 \\
21500 \\
19400 \\
19500\end{array}$ & $\begin{array}{l}33300 \\
23900 \\
49000 \\
33700 \\
27900 \\
41400 \\
34300 \\
21700 \\
20700 \\
22000\end{array}$ & $\begin{array}{l}38800 \\
27000 \\
77600 \\
46400 \\
32800 \\
46600 \\
38800 \\
32400 \\
24200 \\
27100\end{array}$ & $\begin{array}{r}41700 \\
34300 \\
119000 \\
51200 \\
37900 \\
72100 \\
49000 \\
34300 \\
29200 \\
30000\end{array}$ \\
\hline $\begin{array}{l}1890 \\
1891 \\
1892 \\
1893 \\
1894 \\
1895 \\
1896 \\
1897 \\
1898 \\
1899\end{array}$ & $\begin{array}{r}9330 \\
18700 \\
15300 \\
15000 \\
11300 \\
10700 \\
9000 \\
19000 \\
11000 \\
10000\end{array}$ & $\begin{array}{r}10900 \\
19700 \\
16000 \\
15700 \\
12600 \\
11000 \\
9430 \\
19700 \\
11600 \\
10100\end{array}$ & $\begin{array}{l}13200 \\
20600 \\
17000 \\
16000 \\
14400 \\
13100 \\
10600 \\
21700 \\
13500 \\
11700\end{array}$ & $\begin{array}{l}15000 \\
21600 \\
17700 \\
16100 \\
18800 \\
15000 \\
14500 \\
25800 \\
16500 \\
15300\end{array}$ & $\begin{array}{l}19500 \\
22600 \\
22500 \\
17400 \\
23500 \\
15800 \\
15800 \\
27500 \\
18200 \\
17600\end{array}$ & $\begin{array}{l}21800 \\
26300 \\
23200 \\
20900 \\
24700 \\
21300 \\
18100 \\
30700 \\
23100 \\
21600\end{array}$ & $\begin{array}{l}24100 \\
33800 \\
23600 \\
26400 \\
25900 \\
22500 \\
21100 \\
32900 \\
27300 \\
23400\end{array}$ \\
\hline $\begin{array}{l}1900 \\
1901 \\
1902 \\
1903 \\
1904 \\
1905 \\
1906 \\
1907 \\
1908 \\
1909\end{array}$ & $\begin{array}{l}14000 \\
17000 \\
24400 \\
25000 \\
16700 \\
14000 \\
38700 \\
28000 \\
20700 \\
14000\end{array}$ & $\begin{array}{l}14600 \\
17000 \\
24800 \\
25000 \\
17000 \\
14100 \\
40000 \\
28300 \\
20900 \\
14700\end{array}$ & $\begin{array}{l}16600 \\
17600 \\
25500 \\
26300 \\
19000 \\
15600 \\
42100 \\
30900 \\
22100 \\
17300\end{array}$ & $\begin{array}{l}20900 \\
18700 \\
26700 \\
28100 \\
22300 \\
20700 \\
43600 \\
46100 \\
23100 \\
21400\end{array}$ & $\begin{array}{l}23900 \\
20700 \\
31200 \\
32100 \\
25000 \\
22700 \\
41900 \\
52500 \\
26100 \\
23300\end{array}$ & $\begin{array}{l}29700 \\
35800 \\
41400 \\
43200 \\
31400 \\
33500 \\
55700 \\
57200 \\
34600 \\
27200\end{array}$ & $\begin{array}{l}32700 \\
53400 \\
43800 \\
54600 \\
64600 \\
42700 \\
60200 \\
59000 \\
47300 \\
32700\end{array}$ \\
\hline $\begin{array}{l}1910 \\
1911 \\
1912 \\
1913 \\
1914 \\
1915 \\
1916\end{array}$ & $\begin{array}{r}28000 \\
9000 \\
21800 \\
16700 \\
16000 \\
12000 \\
22000\end{array}$ & $\begin{array}{r}30300 \\
9660 \\
21900 \\
13200 \\
17000 \\
13200 \\
23600\end{array}$ & $\begin{array}{l}31600 \\
12000 \\
22700 \\
21000 \\
17800 \\
15000 \\
26400\end{array}$ & $\begin{array}{l}32400 \\
14100 \\
24000 \\
22000 \\
20200 \\
18500 \\
36500\end{array}$ & $\begin{array}{l}37700 \\
15700 \\
32000 \\
22300 \\
21500 \\
24300 \\
51100\end{array}$ & $\begin{array}{l}44600 \\
19000 \\
39100 \\
27400 \\
27700 \\
34100 \\
59900\end{array}$ & $\begin{array}{l}46000 \\
20800 \\
41200 \\
36200 \\
30100 \\
39500 \\
63600\end{array}$ \\
\hline
\end{tabular}


LOW FLOW DISCHARGES, IN YEAR ENDING MARCH 31--Cont inued

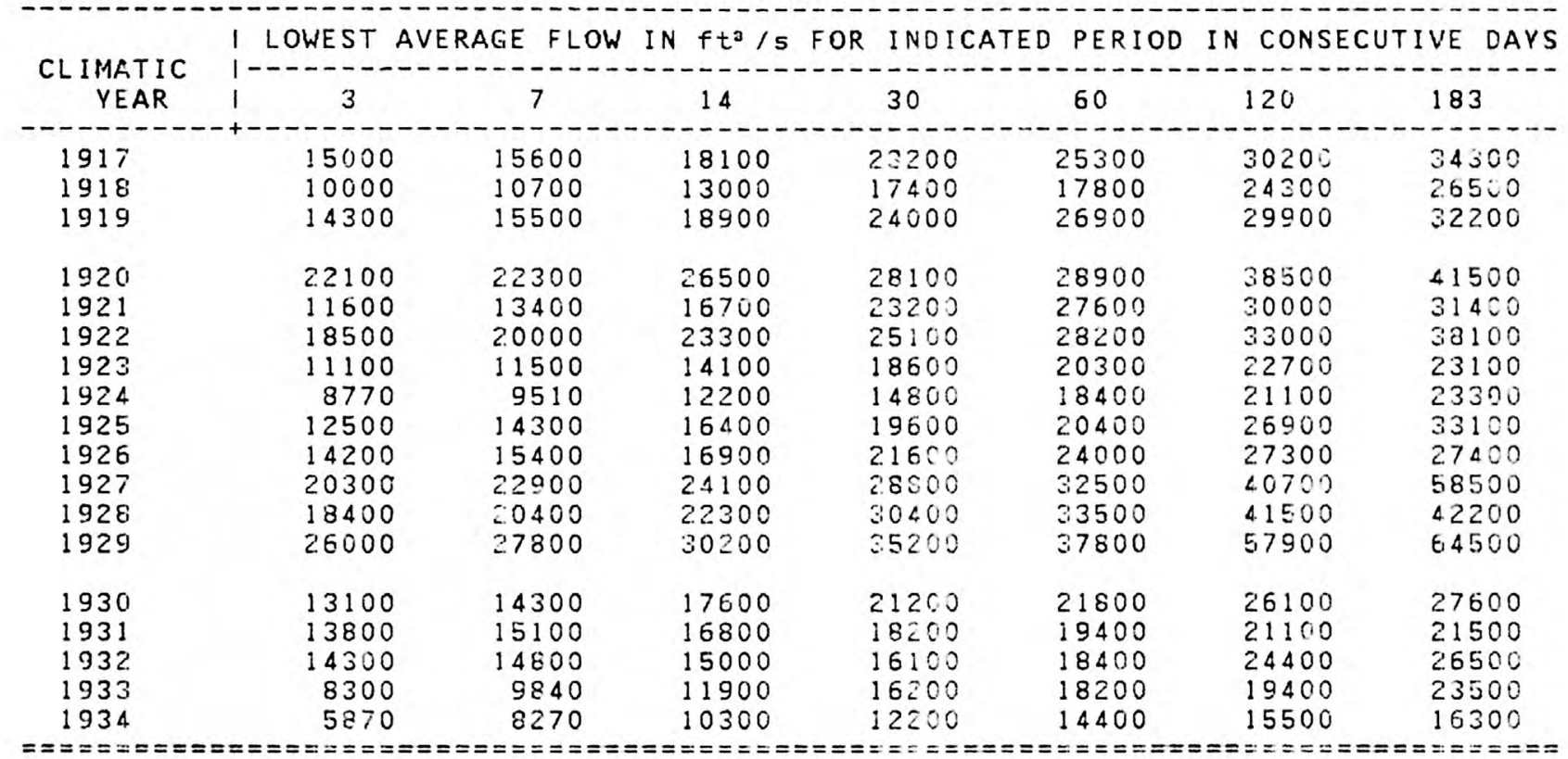


MINIMUM AVERAGE FLOWS FOR PERIOD OF RECORD

\begin{tabular}{|c|c|c|c|c|c|c|c|}
\hline PERIOD OF CONSECUTIVE DAYS & 3 & 7 & 14 & 30 & 50 & 120 & 183 \\
\hline $\begin{array}{l}\text { DISCHARGE, IN } \mathrm{ft}^{3} / \mathrm{s} \\
\text { CLIMATIC YEAR }\end{array}$ & $\begin{array}{l}5870 \\
1934\end{array}$ & $\begin{array}{l}82 \% 0 \\
1934\end{array}$ & $\begin{array}{l}\text { i0300 } \\
1934\end{array}$ & $\begin{array}{l}12200 \\
1934\end{array}$ & $\begin{array}{l}: 2400 \\
: 934\end{array}$ & $\begin{array}{l}15500 \\
1934\end{array}$ & $\begin{array}{l}16300 \\
1934\end{array}$ \\
\hline
\end{tabular}

MAENITUDE AND FREQUENCY OF ANIVUAL LOW FLCWS

RECLRRENCE i LOWEST AVERAGE FLOW, IN $\mathrm{ft}^{\mathrm{a}} / \mathrm{s}, \mathrm{FOR}$ INDICATED PERIOD :S CONSECUTIVE DAYS

\begin{tabular}{|c|c|c|c|c|c|c|c|c|}
\hline IN YEARS & i & 3 & 7 & 14 & 20 & 60 & 20 & 183 \\
\hline $\begin{array}{l}1.5 \\
2 \\
5 \\
10 \\
20\end{array}$ & $\begin{array}{l}1 \\
1 \\
1 \\
1 \\
1\end{array}$ & $\begin{array}{r}18500 \\
15700 \\
11400 \\
9670 \\
8440\end{array}$ & $\begin{array}{r}19300 \\
16500 \\
12400 \\
10800 \\
9620\end{array}$ & $\begin{array}{l}21400 \\
18600 \\
14400 \\
12700 \\
11500\end{array}$ & $\begin{array}{l}25100 \\
21900 \\
17200 \\
15400 \\
14100\end{array}$ & $\begin{array}{l}27900 \\
24300 \\
19200 \\
17200 \\
15900\end{array}$ & $\begin{array}{l}34700 \\
25900 \\
23000 \\
20400 \\
18600\end{array}$ & $\begin{array}{l}40600 \\
34300 \\
25600 \\
23800 \\
20200\end{array}$ \\
\hline
\end{tabular}

MAGNITUDE AND FREGUENCY OF SEASONAL LCW FLOWS

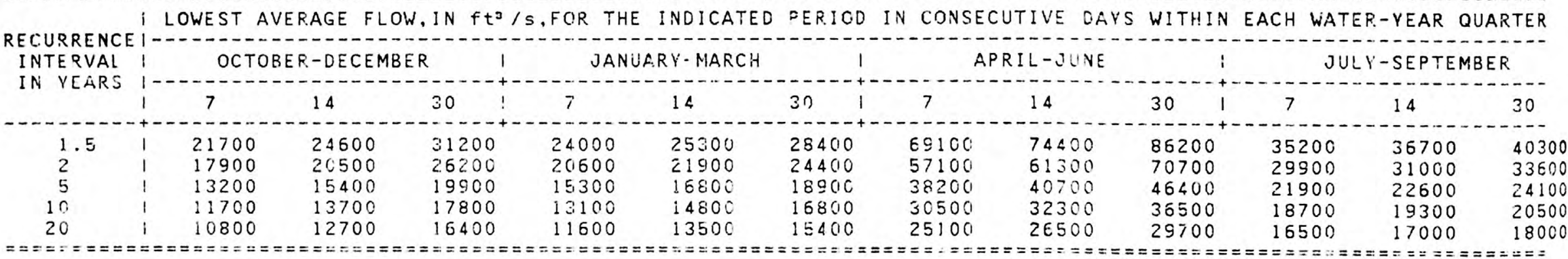

DURATION OF DAILY DISCHARGES FOR RNNUAL AND SEASONAL FERIODS

DISCHARGE, I II $\mathrm{ft}^{3}$ is, EQUALLEQ OR EXCEEDEC FOR THE INDICATEO PERCENT OF TOTAL TIME

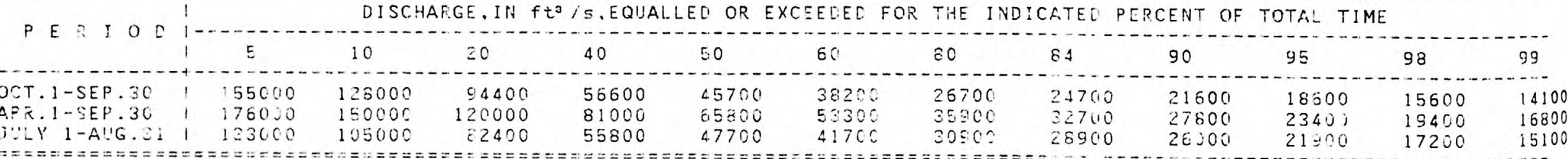


LOW FLOW DISCHARGES, IN YEAR. ENDING MARCH 31

\begin{tabular}{|c|c|c|c|c|c|c|c|}
\hline \multirow{2}{*}{$\begin{array}{c}\text { CLIMATIC } \\
\text { YEAR }\end{array}$} & \multicolumn{7}{|c|}{ I LOWEST AVERAGE FLOW IN $\mathrm{ft}^{\circ} / \mathrm{s}$ FOR INDICATED PERIOD IN CONSECUTIVE DAYS } \\
\hline & 13 & 7 & 14 & 30 & 60 & 120 & 183 \\
\hline $\begin{array}{l}1935 \\
1936 \\
1937 \\
1938 \\
1939\end{array}$ & $\begin{array}{l}11700 \\
16000 \\
10400 \\
10900 \\
17800\end{array}$ & $\begin{array}{l}12000 \\
16300 \\
10700 \\
12600 \\
20700\end{array}$ & $\begin{array}{l}12900 \\
17600 \\
11900 \\
14200 \\
23600\end{array}$ & $\begin{array}{l}14100 \\
21000 \\
12600 \\
16500 \\
30300\end{array}$ & $\begin{array}{l}16200 \\
22000 \\
15600 \\
18200 \\
35000\end{array}$ & $\begin{array}{l}18400 \\
28300 \\
23800 \\
18900 \\
45800\end{array}$ & $\begin{array}{l}21600 \\
28800 \\
23100 \\
20000 \\
57300\end{array}$ \\
\hline $\begin{array}{l}1940 \\
1941 \\
1942 \\
1943 \\
1944 \\
1945 \\
1946 \\
1947 \\
1948 \\
1949\end{array}$ & $\begin{array}{l}10700 \\
18400 \\
14800 \\
29100 \\
25700 \\
22500 \\
29200 \\
20700 \\
18400 \\
12600\end{array}$ & $\begin{array}{l}13000 \\
19300 \\
16500 \\
30200 \\
26800 \\
25000 \\
30400 \\
22200 \\
19700 \\
14500\end{array}$ & $\begin{array}{l}14300 \\
22000 \\
17700 \\
30300 \\
28500 \\
25300 \\
33300 \\
23900 \\
20300 \\
15500\end{array}$ & $\begin{array}{l}14500 \\
23300 \\
20700 \\
34200 \\
30400 \\
25400 \\
36200 \\
29700 \\
21900 \\
17500\end{array}$ & $\begin{array}{l}15800 \\
23800 \\
27100 \\
44700 \\
33500 \\
27800 \\
42300 \\
36200 \\
26900 \\
18000\end{array}$ & $\begin{array}{l}19800 \\
30100 \\
47900 \\
49400 \\
36600 \\
30100 \\
44500 \\
42300 \\
30400 \\
20600\end{array}$ & $\begin{array}{l}20500 \\
32400 \\
64400 \\
57800 \\
38100 \\
32900 \\
49100 \\
44700 \\
31000 \\
23000\end{array}$ \\
\hline $\begin{array}{l}1950 \\
1951 \\
1952 \\
1953 \\
1954 \\
1955 \\
1956 \\
1957 \\
1958 \\
1959\end{array}$ & $\begin{array}{l}13800 \\
17100 \\
28300 \\
23700 \\
22600 \\
26900 \\
20100 \\
14200 \\
20000 \\
15400\end{array}$ & $\begin{array}{l}15600 \\
17300 \\
34100 \\
24600 \\
24300 \\
28300 \\
20600 \\
15200 \\
22000 \\
16400\end{array}$ & $\begin{array}{l}16200 \\
20000 \\
39600 \\
25500 \\
25000 \\
29300 \\
20700 \\
16400 \\
22500 \\
17000\end{array}$ & $\begin{array}{l}19700 \\
21000 \\
46600 \\
26400 \\
25200 \\
30600 \\
23800 \\
17700 \\
24700 \\
17800\end{array}$ & $\begin{array}{l}20800 \\
22600 \\
57600 \\
27800 \\
26400 \\
38900 \\
24700 \\
20700 \\
26600 \\
19400\end{array}$ & $\begin{array}{l}22400 \\
24000 \\
65400 \\
31000 \\
28500 \\
42500 \\
25600 \\
23200 \\
30700 \\
21900\end{array}$ & $\begin{array}{l}25500 \\
25900 \\
68500 \\
36600 \\
29500 \\
48500 \\
25800 \\
24500 \\
32200 \\
23100\end{array}$ \\
\hline $\begin{array}{l}1960 \\
1961 \\
1962 \\
1963 \\
1964 \\
1965 \\
1966 \\
1967 \\
1968 \\
1969\end{array}$ & $\begin{array}{l}20800 \\
22000 \\
17700 \\
21600 \\
17700 \\
10400 \\
24100 \\
19700 \\
19400 \\
26800\end{array}$ & $\begin{array}{l}22300 \\
23500 \\
20200 \\
23100 \\
18500 \\
12100 \\
30400 \\
20300 \\
19800 \\
28700\end{array}$ & $\begin{array}{l}25300 \\
24500 \\
21300 \\
23600 \\
19200 \\
13200 \\
32300 \\
20700 \\
22800 \\
31900\end{array}$ & $\begin{array}{l}29600 \\
25200 \\
24000 \\
25000 \\
20000 \\
16300 \\
34400 \\
23200 \\
24500 \\
40500\end{array}$ & $\begin{array}{l}35900 \\
27100 \\
31900 \\
27100 \\
21100 \\
20100 \\
45000 \\
20400 \\
26800 \\
44400\end{array}$ & $\begin{array}{l}46200 \\
34600 \\
40000 \\
30100 \\
21600 \\
26900 \\
66300 \\
28700 \\
30000 \\
55600\end{array}$ & $\begin{array}{l}51700 \\
37000 \\
49200 \\
38600 \\
22100 \\
26800 \\
71600 \\
30700 \\
31700 \\
58700\end{array}$ \\
\hline $\begin{array}{l}1970 \\
1971 \\
1972 \\
1970 \\
1974 \\
1975 \\
1976\end{array}$ & $\begin{array}{l}25000 \\
19500 \\
19100 \\
32600 \\
35600 \\
24200 \\
24300\end{array}$ & $\begin{array}{l}25200 \\
20000 \\
21800 \\
33400 \\
40600 \\
28400 \\
25500\end{array}$ & $\begin{array}{l}27100 \\
22700 \\
23200 \\
37000 \\
23600 \\
28600 \\
27500\end{array}$ & $\begin{array}{l}29: 00 \\
27060 \\
26200 \\
50100 \\
47200 \\
31500 \\
29260\end{array}$ & $\begin{array}{l}29800 \\
35220 \\
28100 \\
70200 \\
49400 \\
336.20 \\
33620\end{array}$ & $\begin{array}{l}32600 \\
47200 \\
46500 \\
85700 \\
61200 \\
59200 \\
39500\end{array}$ & $\begin{array}{l}53500 \\
54400 \\
43900 \\
89200 \\
81400 \\
39700 \\
40900\end{array}$ \\
\hline
\end{tabular}




\section{DRAINAGE AREA: $119,000 \mathrm{~m}^{2}=$ PERIOD OF RECORD: 42 YEARS AVERAGE OISCHARGE: 64,200 $\mathrm{ft}^{3} / \mathrm{s}$}

MINIMUM AVERAGE FLOWS FOR PERIOD OF FECORD

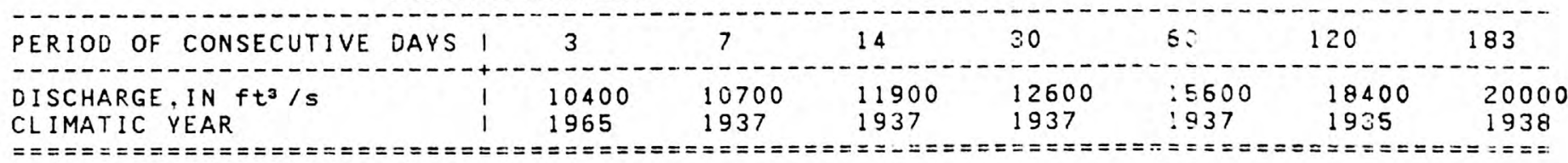

MAGNITUDE AND FREQUENCY OF ANNUAL LOW FLOWS

RECURRENCE I LOWEST AVERAGE FLOW, IN $f t^{3} / 5, F O R$ INDICATED PERIOD IN CONSECUTIVE OAYS

\begin{tabular}{|c|c|c|c|c|c|c|c|c|}
\hline IN YEARS & i & 3 & 7 & 14 & 30 & 60 & 120 & 183 \\
\hline $\begin{array}{l}1.5 \\
2 \\
5 \\
10 \\
20\end{array}$ & $\begin{array}{l}1 \\
1 \\
1 \\
1\end{array}$ & $\begin{array}{l}22500 \\
19800 \\
15000 \\
12800 \\
11200\end{array}$ & $\begin{array}{l}24200 \\
21200 \\
16200 \\
14000 \\
12400\end{array}$ & $\begin{array}{l}25800 \\
22600 \\
17400 \\
15100 \\
13500\end{array}$ & $\begin{array}{l}28700 \\
24900 \\
19100 \\
16700 \\
14900\end{array}$ & $\begin{array}{l}32600 \\
28000 \\
21200 \\
18600 \\
16800\end{array}$ & $\begin{array}{l}38800 \\
33000 \\
24600 \\
21400 \\
19300\end{array}$ & $\begin{array}{l}42700 \\
36000 \\
26400 \\
22700 \\
20100\end{array}$ \\
\hline
\end{tabular}

MAGNITUDE AND FREQUENCY OF SEASONAL LOW FLOWS

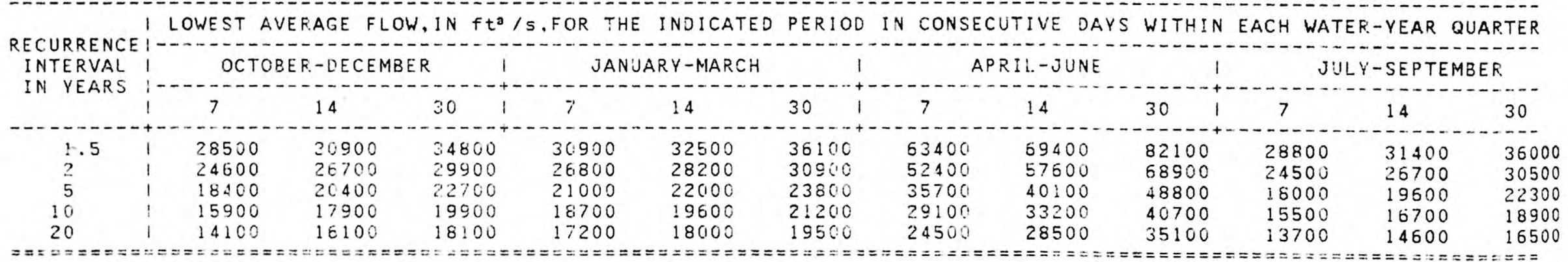

DURA.TION OF DAILY DISCHARGES FOR AINUAL AND SEASONAL FERIODS

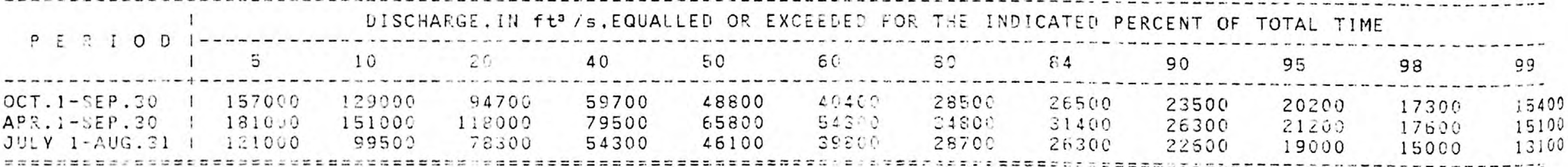




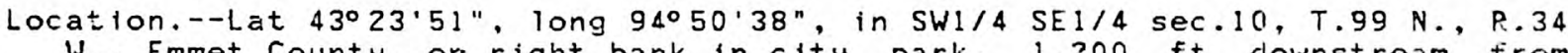
W., Emmet County, or right bank in city park, 1,200 ft downstream from bridge on State Highway 9 at Estherville, 0.1 mile upstream from School Creek, $2.3 \mathrm{miles}$ upstream from Brown Creek, and at mile 404.2.

Remarks.--Diurnal fluctuation at low flow caused by powerplant $0.3 \mathrm{mile}$ upstream from station which discharges $\varepsilon_{1}$ average dally flow of about 0.5 $\mathrm{ft}^{\mathrm{a}} / \mathrm{s}$ into river from subterranean wells. Prior to 1971 published as West Fork Des Moines Rlver at Estherville, Iowa.

LOW FLOW DISCHARGES, IN YEAR. ENDING MARCH 31

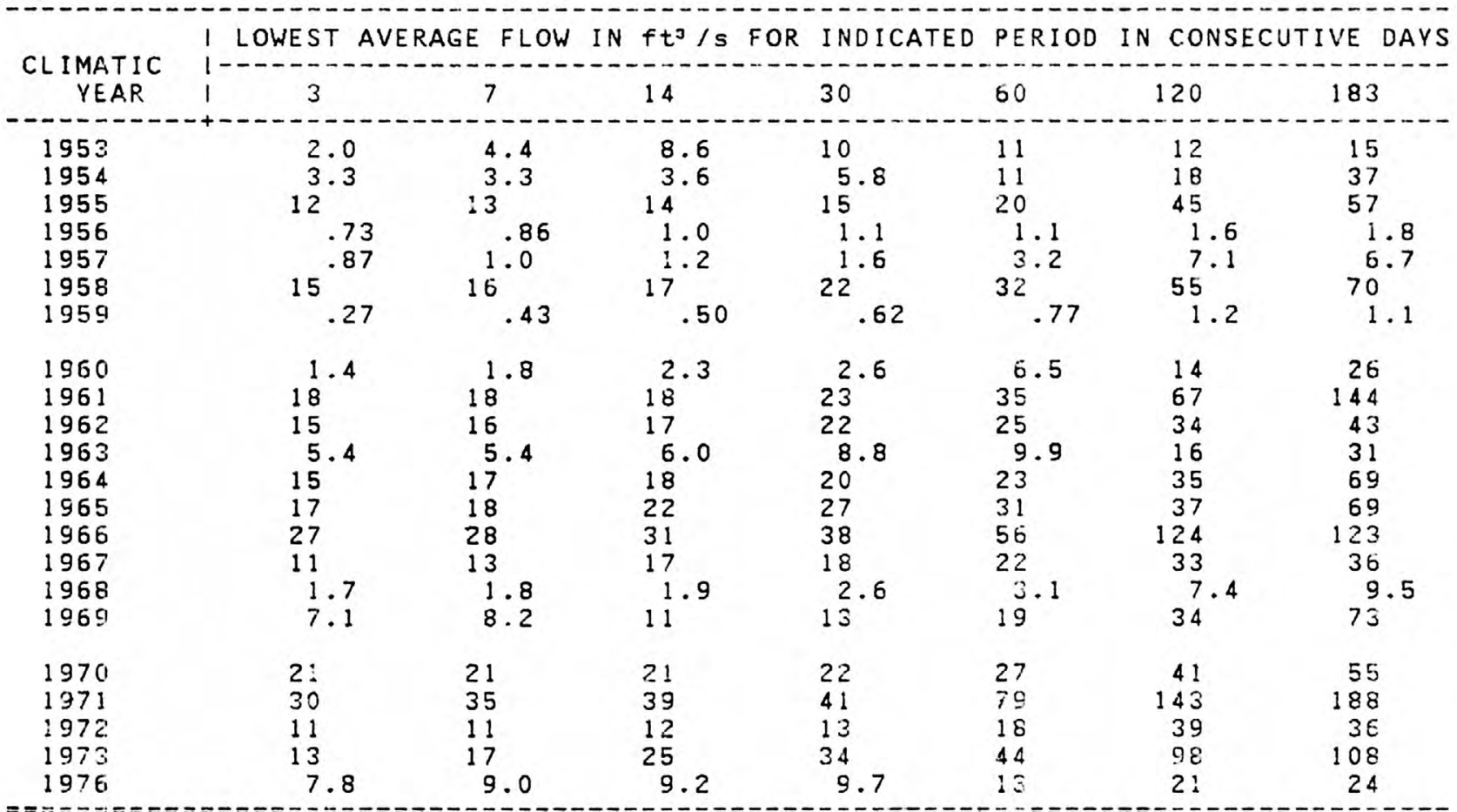




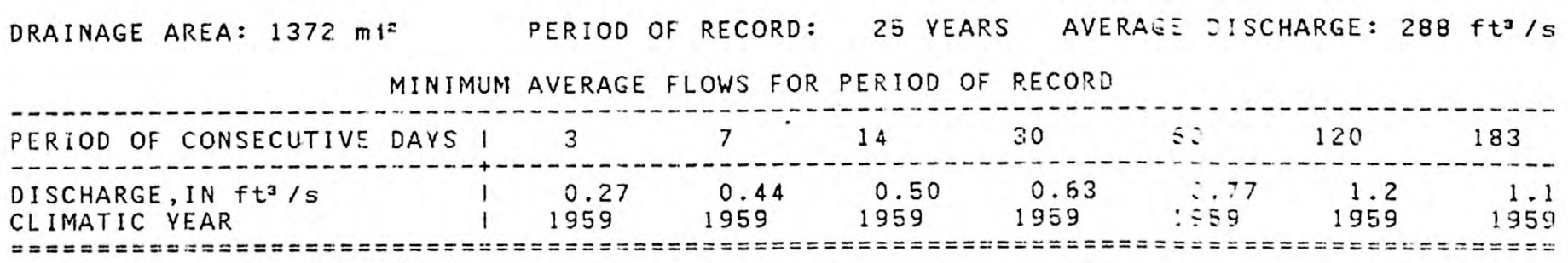

MAGNITUDE AND FREQUENCY OF ANHUAL LOW FLOWS

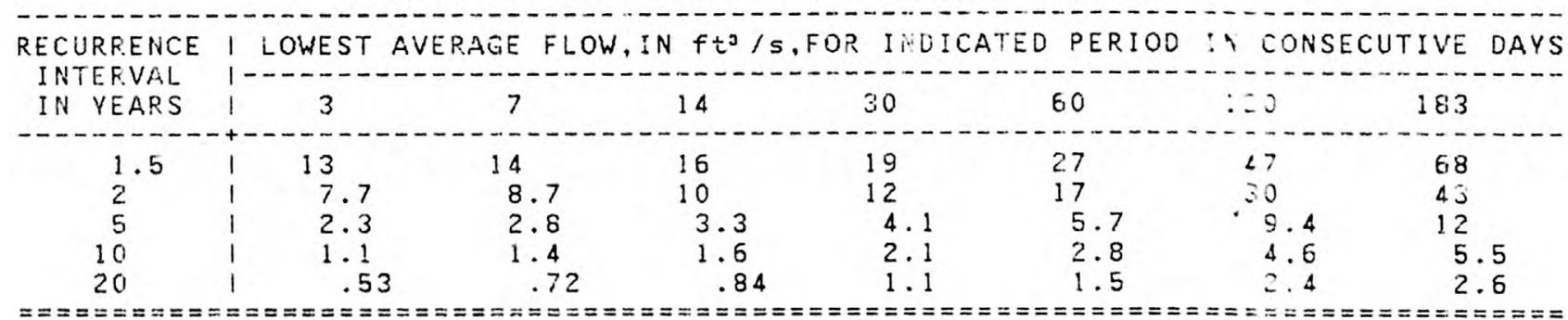

MAGNITUDE AND FREQUENCY OF SEASONAL LOW FLOWS

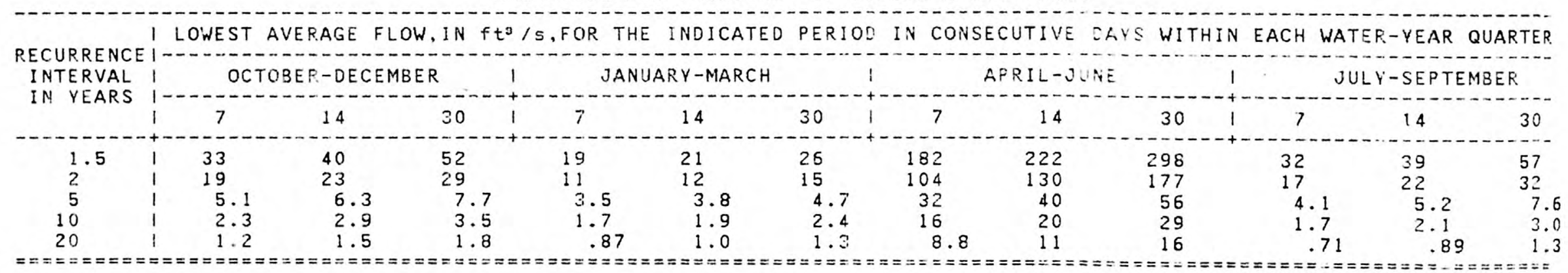

DURATION OF DAILY DISCHARGES FOR ANMUAL ANO SEASONAL FERIODS

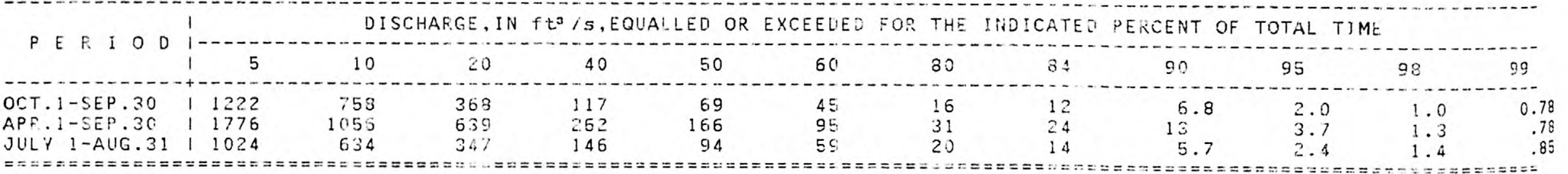


Location.--Lat $42^{\circ} 43^{\prime} 12^{\prime \prime}$, long $94^{\circ} 13^{\prime} 06^{\prime \prime}$, In SE1/4 SW1/4 sec.1, T.91 N., R.29 W. Humboldt County, on left bank $5 \mathrm{ft}$ downstream from First Avenue bridge In clty of Humboldt, about $700 \mathrm{ft}$ below dam. $3.2 \mathrm{miles}$ upstream from Indian Creek, 3.9 miles upstream from East Fork. Des Hoines River, and at mile 334.3 .

Remarks.--Lov-flow discharges occasionally affected by minor regulation. Prior to October 1970 published as West Fork Des Moines River at Humbolt, Iowa.

LOW FLOW DISCHARGES, IN VEAR ENOING MARCH 31

\begin{tabular}{|c|c|c|c|c|c|c|c|c|}
\hline \multirow{2}{*}{$\begin{array}{c}\text { CLIMATIC } \\
\text { YEAR }\end{array}$} & I LOWEST & AGE & \multicolumn{2}{|c|}{$V f t^{3} / s$ FOR IN } & D PERIOD & \multicolumn{3}{|c|}{ IVE DAYS } \\
\hline & $i \quad 3$ & 7 & 14 & 30 & 60 & 120 & 183 & \\
\hline $\begin{array}{l}1966 \\
1967 \\
1968 \\
1969\end{array}$ & $\begin{array}{l}98 \\
42 \\
20 \\
49\end{array}$ & $\begin{array}{r}102 \\
44 \\
21 \\
57\end{array}$ & $\begin{array}{r}111 \\
46 \\
23 \\
62\end{array}$ & $\begin{array}{r}143 \\
49 \\
27 \\
68\end{array}$ & $\begin{array}{r}220 \\
50 \\
33 \\
85\end{array}$ & $\begin{array}{r}364 \\
69 \\
43 \\
117\end{array}$ & & $\begin{array}{l}5 \\
5 \\
4 \\
9\end{array}$ \\
\hline $\begin{array}{l}1970 \\
1971 \\
1972 \\
1973 \\
1974 \\
1975 \\
1976\end{array}$ & $\begin{array}{r}88 \\
79 \\
57 \\
166 \\
114 \\
27 \\
37\end{array}$ & $\begin{array}{r}90 \\
80 \\
68 \\
172 \\
117 \\
30 \\
39\end{array}$ & $\begin{array}{r}98 \\
86 \\
78 \\
192 \\
124 \\
31 \\
39\end{array}$ & $\begin{array}{r}103 \\
114 \\
81 \\
224 \\
130 \\
32 \\
41\end{array}$ & $\begin{array}{r}112 \\
162 \\
94 \\
349 \\
167 \\
37 \\
54\end{array}$ & $\begin{array}{r}167 \\
250 \\
174 \\
497 \\
452 \\
45 \\
78\end{array}$ & $\begin{array}{r}233 \\
289 \\
155 \\
473 \\
598 \\
60 \\
97\end{array}$ & $\begin{array}{l}33 \\
39 \\
55 \\
73 \\
98 \\
50 \\
97\end{array}$ \\
\hline
\end{tabular}


DRAINAGE AREA: $2256 \mathrm{mi}^{2}$ PERIOD OF RECORD: 12 YEARS AVERAGE DISCHARGE: 758 ft3/s MINIMUM AVERAGE FLOWS FOR PERIOD OF RECORD

\begin{tabular}{|c|c|c|c|c|c|c|c|}
\hline PERIOD OF CONSECUTIVE DAYS & 3 & 7 & 14 & 30 & $\leq 0$ & 120 & 183 \\
\hline $\begin{array}{l}\text { DISCHARGE, IN } \mathrm{ft}^{\circ} / \mathrm{s} \\
\text { CLIMATIC YEAR }\end{array}$ & $\begin{array}{l}20 \\
1968\end{array}$ & $\begin{array}{l}21 \\
1968\end{array}$ & $\begin{array}{l}23 \\
1968\end{array}$ & $\begin{array}{l}27 \\
1968\end{array}$ & $\begin{array}{l}33 \\
\vdots 968\end{array}$ & $\begin{array}{l}43 \\
1968\end{array}$ & $\begin{array}{l}54 \\
1968\end{array}$ \\
\hline
\end{tabular}

MAGNITUDE AND FREQUENCY OF ANNUAL LOW FLOWS

RECURRENCE I LOWEST AVERAGE FLOW, IN $\mathrm{ft}^{3} / \mathrm{s}, \mathrm{FOR}$ INDICATED PERIOD : V CONSECUTIVE DAYS

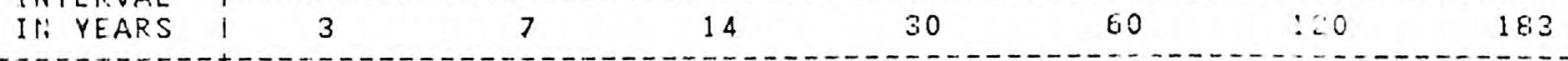

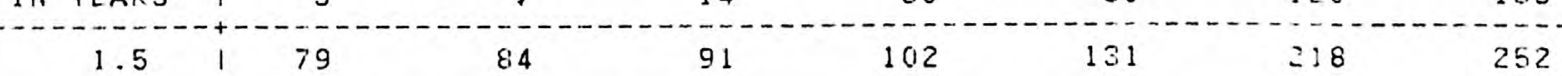

$\begin{array}{rrrrrrrr}1.5 & 79 & 84 & 91 & 102 & 131 & 218 & 252 \\ 2 & 60 & 65 & 69 & 77 & 94 & 29 & 175\end{array}$

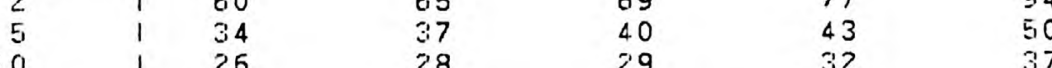

$20 \quad 1 \quad 20$

2829

32

MAGNITUDE AND FREQUENCY OF SEASONAL LOW FLOWS

I LOWEST AVER.AGE FLOW, IN $\mathrm{ft}^{3} / \mathrm{s}, F O R$ THE INDICATEO PERIOD IN CONSECUTIVE DAYS WITHIN EACH WATER-YEAR QUARTER

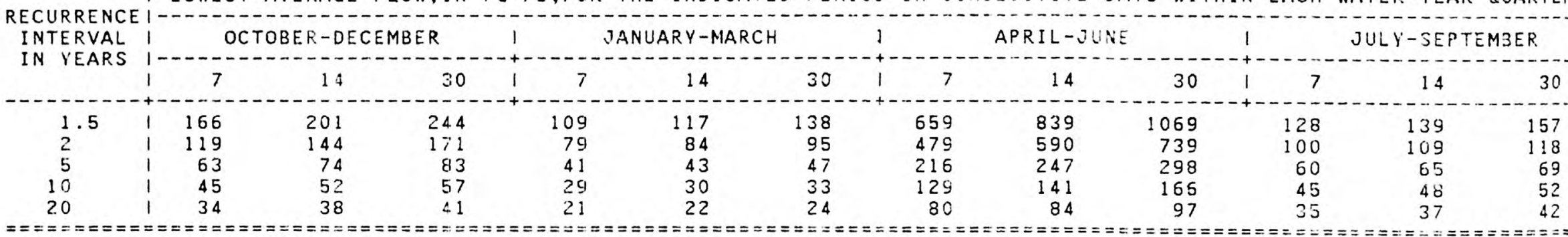

DURATION OF DAILY DISCHARGES FOR ANYUAL AND SEASONAL PERIODS

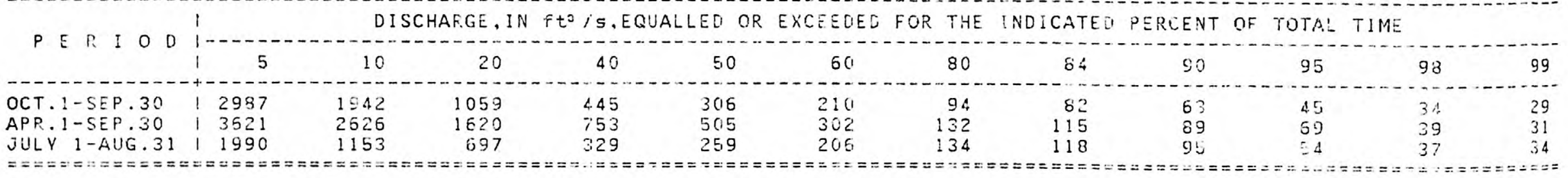




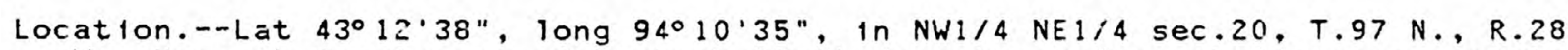
W., Kossuth County, on $r$ ght bank $30 \mathrm{ft}$ downstream from or idge on county highway, $0.8 \mathrm{~m} 1 \mathrm{le}$ upstream from Buffalo Creek, $2.2 \mathrm{~m} 1 \mathrm{les}$ northeast of Burt, $4.7 \mathrm{miles}$ downstream from Mud Creek, and at mile 389.7 upstream from mouth of Des Moines River.

Remarks.--Discontinued September 1974.

LOW FLOW DISCHARGES, IN YEAR. ENDING MARCH 31

\begin{tabular}{|c|c|c|c|c|c|c|c|}
\hline CLIMATIC & 1 LOWEST & E FLOV & $f t^{3} / s$ & INDIC & PERIOD & IN CONSE & $\begin{array}{l}\text { IVE DAYS } \\
\end{array}$ \\
\hline YEAR & 13 & 7 & 14 & 30 & 60 & 120 & 183 \\
\hline $\begin{array}{l}1953 \\
1954 \\
1955 \\
1956 \\
1957 \\
1958 \\
1959\end{array}$ & $\begin{array}{c}2.4 \\
.40 \\
5.4 \\
.13 \\
.20 \\
.90 \\
0\end{array}$ & $\begin{array}{l}2.8 \\
.40 \\
5.6 \\
.20 \\
.23 \\
.93 \\
0\end{array}$ & $\begin{array}{l}3.4 \\
.40 \\
5.9 \\
.24 \\
.28 \\
1.2 \\
0\end{array}$ & $\begin{array}{c}3.6 \\
.63 \\
6.3 \\
.47 \\
.48 \\
1.7 \\
0\end{array}$ & $\begin{array}{c}4.1 \\
1.9 \\
9.1 \\
.67 \\
.88 \\
2.7 \\
.08\end{array}$ & $\begin{array}{c}4.5 \\
3.7 \\
23 \\
.74 \\
1.7 \\
5.5 \\
.41\end{array}$ & $\begin{array}{c}8.1 \\
4.2 \\
47 \\
.76 \\
1.9 \\
5.1 \\
.53\end{array}$ \\
\hline $\begin{array}{l}1960 \\
1961 \\
1962 \\
1963 \\
1964 \\
1965 \\
1966 \\
1967 \\
1968 \\
1969\end{array}$ & $\begin{array}{r}1.3 \\
1.7 \\
5.9 \\
5.7 \\
2.3 \\
11 \\
4.0 \\
2.0 \\
1.6 \\
3.9\end{array}$ & $\begin{array}{r}1.7 \\
1.7 \\
6.0 \\
5.8 \\
2.3 \\
14 \\
4.8 \\
2.3 \\
1.6 \\
4.5\end{array}$ & $\begin{array}{r}2.3 \\
1.9 \\
6.3 \\
6.3 \\
2.4 \\
15 \\
5.5 \\
2.7 \\
1.7 \\
5.4\end{array}$ & $\begin{array}{r}3.4 \\
2.7 \\
8.4 \\
8.3 \\
2.8 \\
18 \\
6.1 \\
2.8 \\
2.0 \\
6.5\end{array}$ & $\begin{array}{c}12 \\
3.6 \\
10 \\
10 \\
3.2 \\
21 \\
12 \\
3.4 \\
2.8 \\
33\end{array}$ & $\begin{array}{c}16 \\
4.3 \\
14 \\
18 \\
4.6 \\
30 \\
44 \\
4.1 \\
4.4 \\
71\end{array}$ & $\begin{array}{c}24 \\
6.7 \\
23 \\
55 \\
9.2 \\
69 \\
52 \\
4.5 \\
6.5 \\
74\end{array}$ \\
\hline $\begin{array}{l}1970 \\
1971 \\
1972 \\
1973 \\
1974\end{array}$ & $\begin{array}{l}6.9 \\
1.4 \\
.33 \\
8.9 \\
3.3\end{array}$ & $\begin{array}{c}7.2 \\
1.7 \\
.36 \\
10^{\circ} \\
3.7\end{array}$ & $\begin{array}{c}8.0 \\
2.0 \\
.52 \\
11 . \\
4.4\end{array}$ & $\begin{array}{c}8.6 \\
2.6 \\
.83 \\
13.8 \\
4.3\end{array}$ & $\begin{array}{l}10 \\
6.4 \\
1.0 \\
3 ? \\
10\end{array}$ & $\begin{array}{l}16 \\
25 \\
12 \\
61 \\
52\end{array}$ & $\begin{array}{r}22 \\
60 \\
14 \\
85 \\
106\end{array}$ \\
\hline
\end{tabular}




\section{DRAINAGE AREA: $462 \mathrm{~m}^{2}$

MINIMUM AVERAGE FLOWS FOR PERIOD OF RECORD

\begin{tabular}{|c|c|c|c|c|c|c|c|}
\hline PERIOD OF CONSECUTIVE DAYS & 3 & 7 & 14 & 30 & 60 & 120 & 183 \\
\hline $\begin{array}{l}\text { ISCHARGE, IN } \mathrm{ft}^{3} / \mathrm{s} \\
\text { LIMATIC YEAR }\end{array}$ & $\begin{array}{l}0 \\
1959\end{array}$ & $\begin{array}{l}0 \\
1959\end{array}$ & $\begin{array}{c}0 \\
1959\end{array}$ & $\begin{array}{l}0 \\
1959\end{array}$ & $\begin{array}{r}0.08 \\
1959\end{array}$ & $\begin{array}{l}0.42 \\
1959\end{array}$ & $\begin{array}{l}0.53 \\
1959\end{array}$ \\
\hline
\end{tabular}

MAGNITUDE AND FREQUENCY OF ANNUAL LOW FLOWS

\begin{tabular}{l|cccccc} 
RECURRENCE \\
INTERVAL \\
IN Y LEARS
\end{tabular}

MAGNITUDE AND FREQUENCY OF SEASONAL LOW FLOW'S

I LOWEST AVERAGE FLOW, IN $\mathrm{ft}^{3} / \mathrm{s}, \mathrm{FOR}$ THE INDICATED PERIOD IN CONSECUTIVE DAYS WITHIN EACH WATER-YEAR QUARTER

$\begin{aligned} & \text { RECURRENCE } \\ & \text { INTERVAL }\end{aligned}$
IN YEARS

DURATION OF DAILY DISCHARGES FOR ANNUAL AND SEASONAL PERIODS

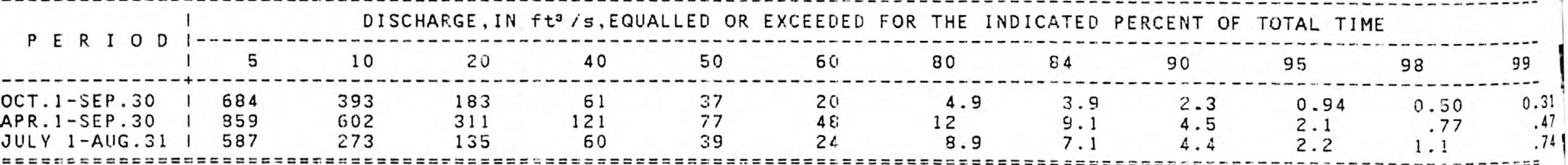


Location.--Lat $42^{\circ} 43^{\prime} 26^{\prime \prime}$, long $94^{\circ} 11^{\prime} 30^{\prime \prime}$, In NW1/4 SE1/4 sec.6, T.91 N., R.28 W. Humboldt County, on right bank $50 \mathrm{ft}$ upstream from old $\mathrm{m} f 11$ dam, in city park at east edge of Dakota City, $500 \mathrm{ft}$ upstream from bridge on county highway P56, 0.6 mile downstream from bridge on State Highway $3,3.4$ miles upstream from confluence with Des Moines River, and at mile 333.8 upstream from mouth of Des Moines River.

Remarks.--Published as "near Hardy", 1940-54.

LOW FLOW DISCHARGES, IN YEAR. ENDING MARCH 31

\begin{tabular}{|c|c|c|c|c|c|c|c|}
\hline \multirow{2}{*}{$\begin{array}{c}\text { CLIMATIC } \\
\text { YEAR }\end{array}$} & \multicolumn{3}{|c|}{ 1 LUWES I AVERAGE FLUW IN TE- IS } & \multicolumn{2}{|c|}{$\begin{array}{l}\text { INDICATED PERIOD } \\
-\end{array}$} & \multicolumn{2}{|l|}{$\mathrm{N} C \mathrm{O}$} \\
\hline & 13 & 7 & 14 & 30 & 60 & 120 & 183 \\
\hline $\begin{array}{l}1941 \\
1942 \\
1943 \\
1944 \\
1945 \\
1946 \\
1947 \\
1948 \\
1949\end{array}$ & $\begin{array}{r}18 \\
38 \\
21 \\
48 \\
27 \\
32 \\
33 \\
15 \\
5.8\end{array}$ & $\begin{array}{c}19 \\
40 \\
23 \\
49 \\
27 \\
33 \\
34 \\
15 \\
6.8\end{array}$ & $\begin{array}{c}21 \\
45 \\
25 \\
51 \\
29 \\
33 \\
37 \\
16 \\
7.9\end{array}$ & $\begin{array}{l}25 \\
60 \\
32 \\
61 \\
29 \\
42 \\
54 \\
17 \\
9.3\end{array}$ & $\begin{array}{r}56 \\
171 \\
39 \\
96 \\
34 \\
49 \\
87 \\
25 \\
14\end{array}$ & $\begin{array}{r}69 \\
568 \\
70 \\
151 \\
71 \\
59 \\
173 \\
35 \\
21\end{array}$ & $\begin{array}{r}81 \\
678 \\
107 \\
197 \\
164 \\
159 \\
206 \\
34 \\
28\end{array}$ \\
\hline $\begin{array}{l}1950 \\
1951 \\
1952 \\
1953 \\
1954 \\
1955 \\
1956 \\
1957 \\
1958 \\
1959\end{array}$ & $\begin{array}{l}6.4 \\
16 \\
72 \\
18 \\
14 \\
34 \\
11 \\
6.9 \\
13.9 \\
7.9\end{array}$ & $\begin{array}{l}7.0 \\
16 \\
73 \\
19 \\
14 \\
35 \\
11 \\
7.9 \\
13.9 \\
8.4\end{array}$ & $\begin{array}{l}8.5 \\
15 \\
75 \\
19 \\
15 \\
38 \\
12 \\
8.8 \\
14 \\
8.5\end{array}$ & $\begin{array}{l}12 \\
18 \\
80 \\
20 \\
17 \\
42 \\
14 \\
9.8 \\
16 \\
8.7\end{array}$ & $\begin{array}{r}15 \\
20 \\
111 \\
22 \\
20 \\
61 \\
15 \\
14 \\
18 \\
9.7\end{array}$ & $\begin{array}{r}18 \\
24 \\
206 \\
25 \\
23 \\
116 \\
15 \\
17 \\
24 \\
11\end{array}$ & $\begin{array}{r}18 \\
41 \\
323 \\
35 \\
30 \\
197 \\
16 \\
18 \\
24 \\
11\end{array}$ \\
\hline $\begin{array}{l}1960 \\
1961 \\
1962 \\
1963 \\
1964 \\
1965 \\
1966 \\
1967 \\
1968 \\
1969\end{array}$ & $\begin{array}{l}27 \\
14 \\
54 \\
37 \\
18 \\
47 \\
41 \\
17 \\
16 \\
26\end{array}$ & $\begin{array}{l}29 \\
15 \\
58 \\
38 \\
19 \\
48 \\
43 \\
17 \\
17 \\
30\end{array}$ & $\begin{array}{l}32 \\
16 \\
60 \\
41 \\
21 \\
53 \\
45 \\
18 \\
18 \\
33\end{array}$ & $\begin{array}{l}39 \\
18 \\
77 \\
50 \\
23 \\
68 \\
53 \\
19 \\
21 \\
40\end{array}$ & $\begin{array}{r}88 \\
21 \\
91 \\
58 \\
26 \\
100 \\
106 \\
20 \\
22 \\
92\end{array}$ & $\begin{array}{r}128 \\
24 \\
116 \\
98 \\
34 \\
158 \\
320 \\
24 \\
26 \\
181\end{array}$ & $\begin{array}{r}154 \\
30 \\
162 \\
235 \\
40 \\
295 \\
488 \\
26 \\
30 \\
227\end{array}$ \\
\hline $\begin{array}{l}1970 \\
1971 \\
1972 \\
1973 \\
1974 \\
1975 \\
1976\end{array}$ & $\begin{array}{l}26 \\
17 \\
15 \\
73 \\
39 \\
15 \\
23\end{array}$ & $\begin{array}{l}27 \\
18 \\
15 \\
78 \\
41 \\
17 \\
23\end{array}$ & $\begin{array}{l}31 \\
19 \\
17 \\
85 \\
42 \\
20 \\
24\end{array}$ & $\begin{array}{l}32 \\
23 \\
18 \\
90 \\
45 \\
21 \\
25\end{array}$ & $\begin{array}{r}35 \\
25 \\
26 \\
220 \\
82 \\
23 \\
28\end{array}$ & $\begin{array}{r}62 \\
61 \\
60 \\
412 \\
369 \\
25 \\
37\end{array}$ & $\begin{array}{r}78 \\
115 \\
71 \\
466 \\
587 \\
28 \\
38\end{array}$ \\
\hline
\end{tabular}




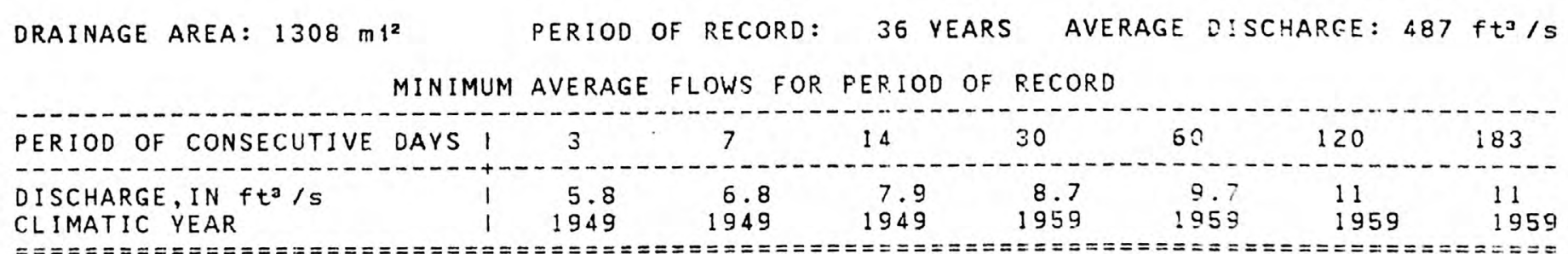

MAGNITUDE AND FREQUENCY OF ANNUAL LOW FLOWS

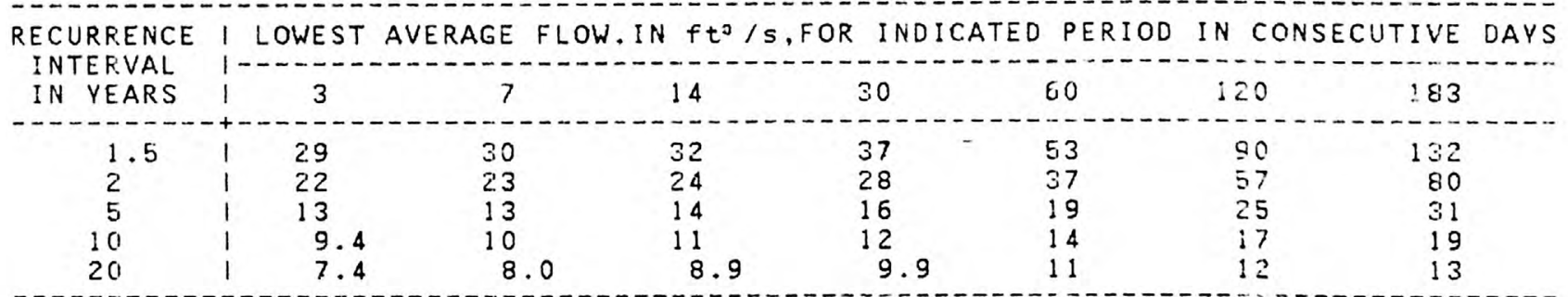

MAGNITUDE AND FREQUENCY OF SEASONAL LOW FLOWS

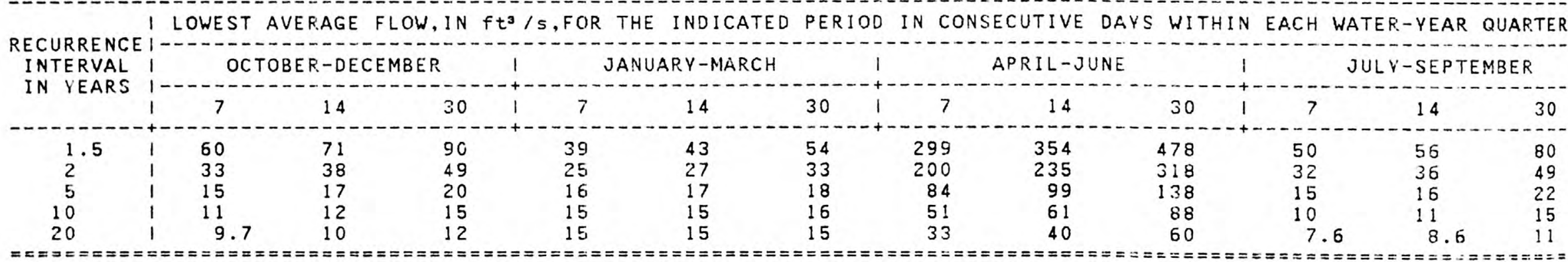

DURATION OF DAILY DISCHARGES FOR ANNUAL AND SEASONAL PERIODS

\begin{tabular}{|c|c|c|c|c|c|c|c|c|c|c|c|c|}
\hline \multirow{2}{*}{$P E R I O O$} & 1 & $D$ & ARGE, & is, E & ED & EEOE & THE & ATE & [EN] & OTAL & & \\
\hline & 15 & 10 & 20 & 40 & 50 & 60 & 80 & 84 & 90 & 95 & 98 & 99 \\
\hline $\begin{array}{l}O C T .1 \text {-SEP. } 30 \\
A P R \cdot 1-S E P .30 \\
\text { JULV } 1 \text {-AUG. } 31\end{array}$ & $\begin{array}{l}12039 \\
12493 \\
1 \quad 1882\end{array}$ & $\begin{array}{l}1315 \\
1765 \\
1146\end{array}$ & $\begin{array}{r}659 \\
1050 \\
603\end{array}$ & $\begin{array}{l}2.65 \\
4.49 \\
2.53\end{array}$ & $\begin{array}{l}156 \\
307 \\
169\end{array}$ & $\begin{array}{l}94 \\
191 \\
114\end{array}$ & $\begin{array}{l}33 \\
70 \\
56\end{array}$ & $\begin{array}{l}2.8 \\
56 \\
48\end{array}$ & $\begin{array}{l}21 \\
35 \\
36\end{array}$ & $\begin{array}{l}16 \\
22 \\
25\end{array}$ & $\begin{array}{l}12 \\
14 \\
18\end{array}$ & $\begin{array}{l}9.8 \\
10 \\
15\end{array}$ \\
\hline
\end{tabular}


Location.--Lat $42^{\circ} 32^{\prime} 35^{\prime \prime}$, Tong $94^{\circ} 20^{\prime} 45^{\prime \prime}$, in NE1/4 NE1/4 sec.11, T.89 N., R.30 W. Webster County, on $r i g h t$ bank $20 \mathrm{ft}$ downstream from bridge on county highway, 2.3 miles downstream from Drainage ditch $3,3.0 \mathrm{miles}$ south of Clare, and 8.2 miles upstream from South Lizard Creek.

Remarks.--Published as "North Lizard Creek near Clare", 1940-54.

LOW FLOW DISCHARGES, IN YEAR. ENDING MARCH 31

\begin{tabular}{|c|c|c|c|c|c|c|c|}
\hline CI IMATIC & LOWEST A & GE FLO & $f t^{2} / s$ & INDIC & PERIOD & IN CONSE & IVE DAVS \\
\hline YEAR & 13 & 7 & 14 & 30 & 60 & 120 & 183 \\
\hline $\begin{array}{l}1942 \\
1943 \\
1944 \\
1945 \\
1946 \\
1947 \\
1948 \\
1949\end{array}$ & $\begin{array}{l}1.1 \\
4.0 \\
.10 \\
2.7 \\
1.0 \\
.50 \\
1.0 \\
.30\end{array}$ & $\begin{array}{l}1.3 \\
4.5 \\
.13 \\
2.9 \\
1.0 \\
.67 \\
1.3 \\
.33\end{array}$ & $\begin{array}{l}1.5 \\
5.3 \\
.25 \\
3.0 \\
1.3 \\
1.4 \\
1.5 \\
.36\end{array}$ & $\begin{array}{l}2.0 \\
8.0 \\
.29 \\
3.8 \\
2.6 \\
3.1 \\
2.2 \\
.41\end{array}$ & $\begin{array}{r}4.0 \\
11 . \\
1.2 \\
4.6 \\
6.5 \\
4.6 \\
2.7 \\
.70\end{array}$ & $\begin{array}{l}38 \\
16 \\
2.1 \\
9.6 \\
7.2 \\
7.6 \\
6.4 \\
3.3\end{array}$ & $\begin{array}{l}45 \\
23 \\
2.3 \\
16 \\
15 \\
6.9 \\
6.5 \\
5.2\end{array}$ \\
\hline $\begin{array}{l}1950 \\
1951 \\
1952 \\
1953 \\
1954 \\
1955 \\
1956 \\
1957 \\
1958 \\
1959\end{array}$ & $\begin{array}{r}1.27 \\
23 \\
2.3 \\
1.9 \\
1.0 \\
5.5 \\
.10 \\
0.50 \\
.50 \\
.10\end{array}$ & $\begin{aligned} & .30 \\
& 1.3 \\
& 23 \\
& 3.3 \\
& 1.0 \\
& 5.5 \\
& .10 \\
& .06 \\
& .67 \\
& .10\end{aligned}$ & $\begin{array}{c}1.30 \\
1.4 \\
25 \\
3.9 \\
1.1 \\
5.8 \\
.12 \\
.08 \\
.93 \\
.10\end{array}$ & $\begin{array}{c}.30 \\
1.8 \\
35 \\
4.5 \\
1.2 \\
7.6 \\
.18 \\
.11 \\
2.3 \\
.11\end{array}$ & $\begin{array}{c}. .42 \\
3.1 \\
42 \\
5.2 \\
1.7 \\
18 . \\
.30 \\
.25 \\
5.8 \\
.27\end{array}$ & $\begin{array}{r}1.3 \\
6.2 \\
58 \\
5.3 \\
3.5 \\
39 \\
.73 \\
.46 \\
7.0 \\
1.1\end{array}$ & $\begin{array}{c}2.6 \\
16 \\
89 \\
7.8 \\
4.3 \\
73 \\
1.1 \\
.71 \\
12 . \\
1.8\end{array}$ \\
\hline $\begin{array}{l}1960 \\
1961 \\
1962 \\
1963 \\
1964 \\
1965 \\
1966 \\
1967 \\
1968 \\
1969\end{array}$ & $\begin{array}{l}3.6 \\
.40 \\
7.7 \\
6.6 \\
.60 \\
1.2 \\
3.5 \\
.55 \\
.04 \\
.92\end{array}$ & $\begin{array}{l}3.9 \\
.40 \\
8.7 \\
6.8 \\
.60 \\
1.3 \\
4.1 \\
.68 \\
.12 \\
1.6\end{array}$ & $\begin{array}{l}5.2 \\
.44 \\
9.1 \\
7.4 \\
.62 \\
1.8 \\
4.5 \\
.80 \\
.24 \\
2.5\end{array}$ & $\begin{array}{l}10 \\
12.75 \\
11 \\
.71 \\
2.0 \\
5.7 \\
.93 \\
.57 \\
3.9\end{array}$ & $\begin{array}{l}13 \\
1.3 \\
13 \\
14 \\
1.1 \\
2.9 \\
9.5 \\
1.0 \\
1.2 \\
5.1\end{array}$ & $\begin{array}{r}19 \\
2.9 \\
16 \\
24 \\
2.9 \\
6.4 \\
55 \\
1.5 \\
2.0 \\
14\end{array}$ & $\begin{array}{l}26 \\
5.0 \\
16 \\
61 \\
3.7 \\
13 \\
95 \\
2.0 \\
2.9 \\
14\end{array}$ \\
\hline $\begin{array}{l}1970 \\
1971 \\
1972 \\
1973 \\
1974 \\
1975 \\
1978\end{array}$ & $\begin{array}{r}4.1 \\
.76 \\
.87 \\
37 \\
17 \\
.77 \\
.33\end{array}$ & $\begin{array}{r}4.3 \\
1.3 \\
1.1 \\
39 \\
19 \\
.94 \\
.40\end{array}$ & $\begin{array}{c}4.5 \\
1.5 \\
1.2 \\
40 \\
19 \\
1.3 \\
.84\end{array}$ & $\begin{array}{l}5.2 \\
2.9 \\
1.8 \\
50 \\
25 \\
1.5 \\
1.2\end{array}$ & $\begin{array}{l}6.0 \\
4.9 \\
2.4 \\
63 \\
4 ! \\
1.6 \\
2.3\end{array}$ & $\begin{array}{r}10 \\
12 \\
14 \\
132 \\
133 \\
2.0 \\
4.4\end{array}$ & $\begin{array}{r}15 \\
14 \\
15 \\
130 \\
225 \\
2.7 \\
4.7\end{array}$ \\
\hline
\end{tabular}


MINIMUM AVERAGE FLOWS FOR PERIOD OF RECORD

\begin{tabular}{|c|c|c|c|c|c|c|c|}
\hline PERIOD OF CONSECUTIVE DAYS & 3 & 7 & 14 & 30 & so & 120 & 183 \\
\hline $\begin{array}{l}\text { HARGE, IN } \mathrm{ft}^{3} / \mathrm{s} \\
\text { ATIC YEAR }\end{array}$ & $\begin{array}{c}0 \\
1957\end{array}$ & $\begin{array}{l}0.06 \\
1957\end{array}$ & $\begin{array}{c}0.08 \\
1957\end{array}$ & $\begin{array}{c}0.12 \\
1959\end{array}$ & $: 0.25$ & $\begin{array}{l}0.46 \\
1957\end{array}$ & $\begin{array}{c}0.71 \\
1957\end{array}$ \\
\hline
\end{tabular}

MAGNITUDE AND FREQUENCY OF ANNUAL LOW FLOWS

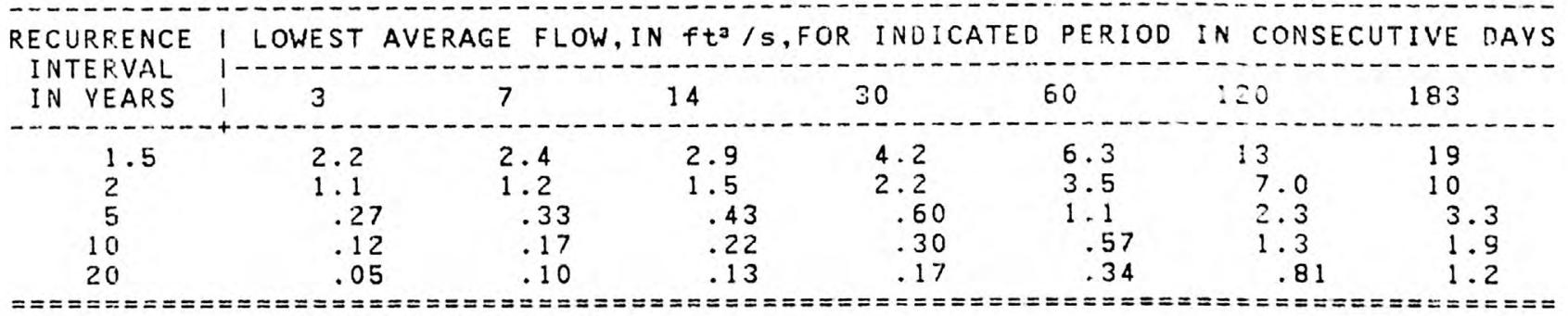

MAGNITUDE AND FREQUENCY OF SEASONAL LOW FLOWS

LOWEST AVER.AGE FLOW, IN $\mathrm{ft}^{3} / \mathrm{s}, F O R$ THE INDICATED PERIOD IN CONSECUTIVE OAYS WITHIN EACH WATER-YEAR QUARTER

\begin{tabular}{l|ccccccc} 
RECURRENCEE \\
INTERVAL \\
IN YEARS
\end{tabular}

DURATION OF DAILY DISCHARGES FOR ANNUAL AND SEASONAL PERIODS

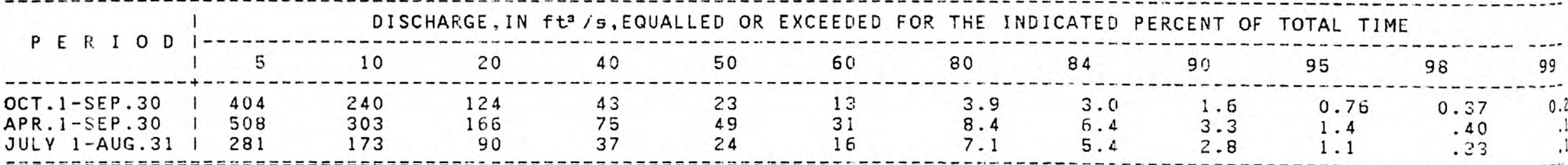


Location.--Lat $42^{\circ} 30^{\prime} 22^{\prime \prime}$, long $94^{\circ} 12^{\prime} 04^{\prime \prime}$, in NW1/4 SW1/4 sec.19. T.89 N.. R.28 W. Webster County, on right bank $400 \mathrm{ft}$ upstream from Soldier Creek, 1,800 ft downstream from Illinols Central Railraod bridge in Fort Dodge, $2,000 \mathrm{ft}$ downstream from Lizard Creek, and at mile 314.6 .

Remarks.--Diurnal fluctuation caused by powerplant upstream from station from 1918 to 1971, occasional minor regulation caused by dam since 1971.

LOW FLOW DISCHARGES, IN YEAR. ENDING MARCH 31

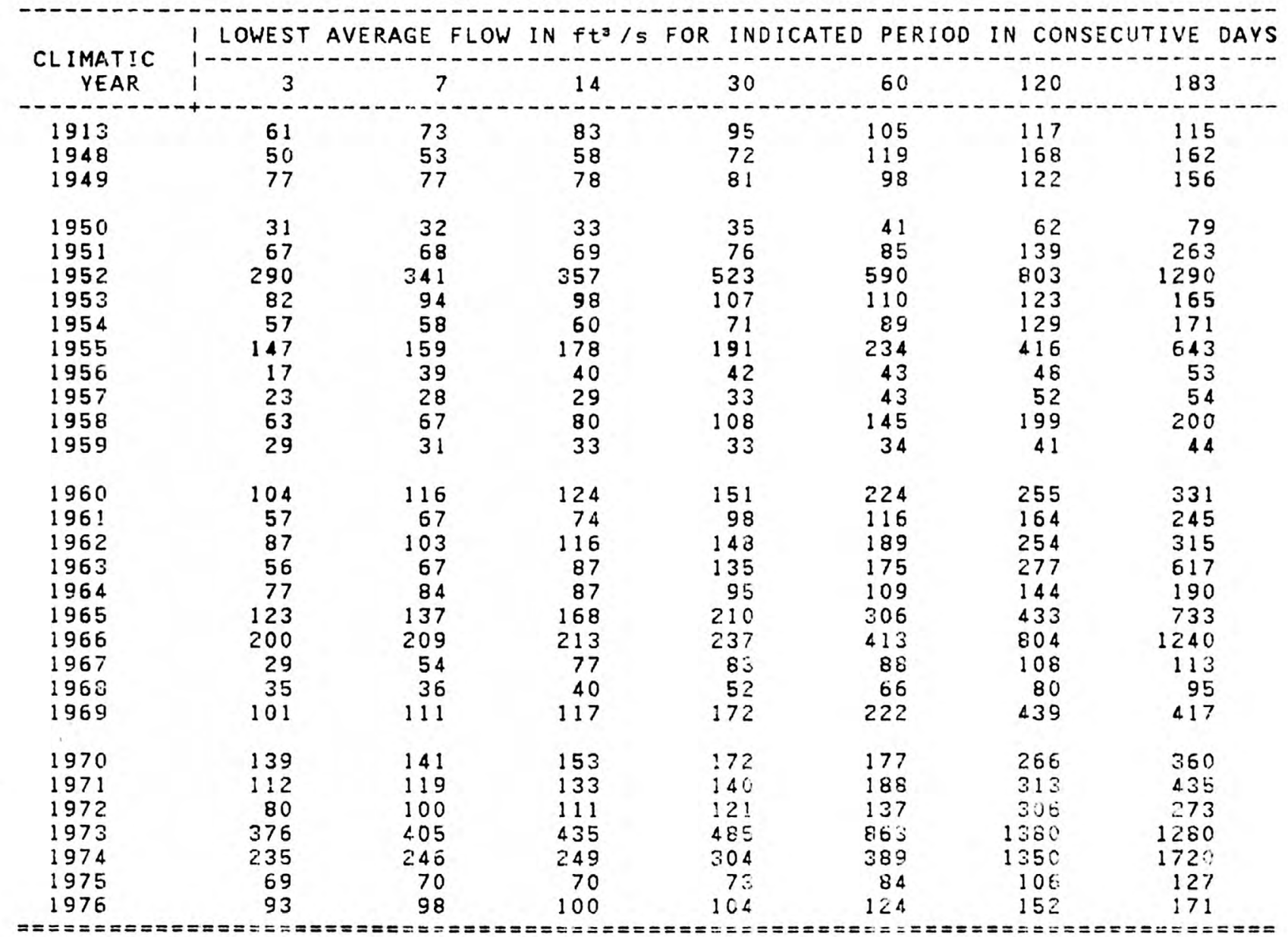


MINIMUM AVERAGE FLOWS FOR PERIOD OF RECORD

\begin{tabular}{|c|c|c|c|c|c|c|c|}
\hline RIOD OF CONSECUTIVE DAYS & 3 & 7 & 14 & 30 & 60 & 120 & 183 \\
\hline $\begin{array}{l}\text { AARGE, IN } \mathrm{ft}^{3} / \mathrm{s} \\
\text { TIC YEAR }\end{array}$ & $\begin{array}{l}17 \\
1956\end{array}$ & $\begin{array}{l}28 \\
1957\end{array}$ & $\begin{array}{l}29 \\
1957\end{array}$ & $\begin{array}{l}33 \\
1959 \\
=====\end{array}$ & $\begin{array}{l}34 \\
: 959 \\
====\end{array}$ & $\begin{array}{l}41 \\
1959\end{array}$ & $\begin{array}{l}44 \\
1959\end{array}$ \\
\hline
\end{tabular}

MAGNITUDE AND FREQUENCY OF ANNUAL. LOW FLOW'S

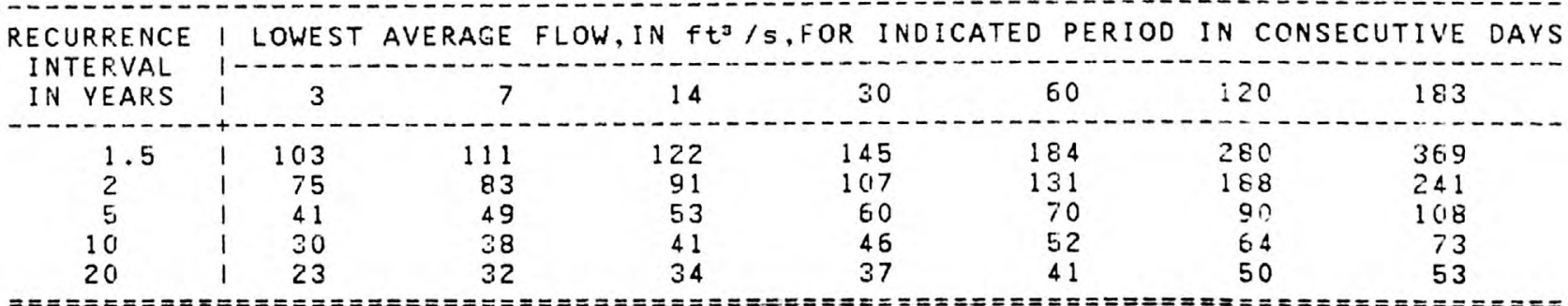

MAGNITUDE AND FREQUENCY OF SEASONAL LOW FLOWS

I LOWEST AVERAGE FLOW, IN $\mathrm{ft}^{3} / \mathrm{s}, \mathrm{FOR}$ THE INDICATED PERIOD IN CONSECUTIVE DAYS WITHIN EACH WATER-YEAR QUARTER

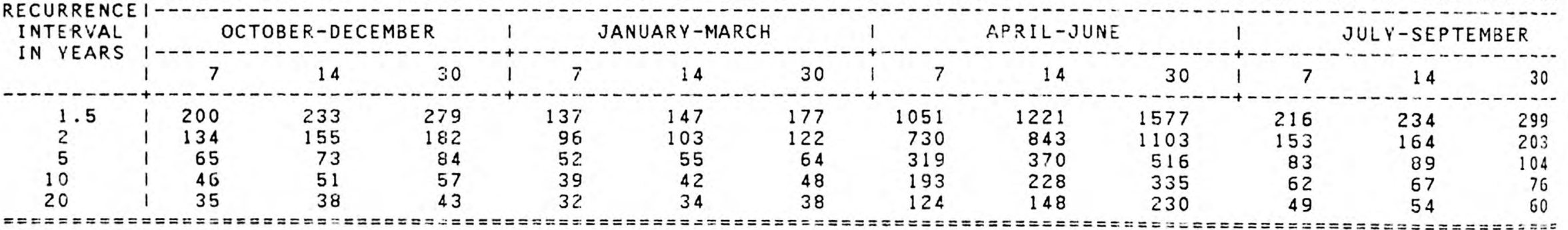

DURATION OF DAILY DISCHARGES FOR ANNUAL AND SEASONAL FERIODS

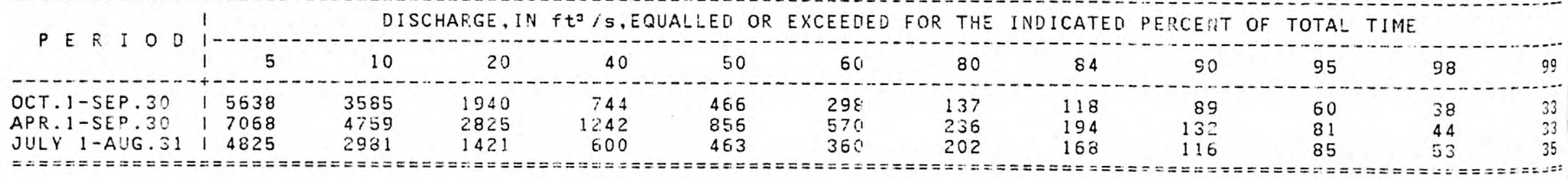


Location. - - Lat $42^{\circ} 26^{\prime} 01^{\prime \prime}$, long $93^{\circ} 48^{\prime} 12^{\prime \prime}$, In NW1/4 SEl/4 sec.18. T.88 N., R.25 W. Hamition County, on right bank $100^{\circ} \mathrm{ft}$ upstream from bridge on State Highway 17, 1.0 miles southeast of junction of U.S. Highway 20 and State Highway 17 in Webster City, and 3.2 miles downstream from Brewers Creek.

LOW FLOW DISCHARGES, IN YEAR. ENDING MARCH 31

\begin{tabular}{|c|c|c|c|c|c|c|c|}
\hline \multirow{2}{*}{$\begin{array}{c}\text { CLIMATIC } \\
\text { YEAR }\end{array}$} & I LOWEST & GE FL & $f t^{3} /$ & INDIC & PERIOD & \multicolumn{2}{|c|}{ IN CONSECUTIVE DAYS } \\
\hline & 13 & 7 & 14 & 30 & 60 & 120 & 183 \\
\hline $\begin{array}{l}1942 \\
1943 \\
1944 \\
1945 \\
1946 \\
1947 \\
1948 \\
1949\end{array}$ & $\begin{array}{l}5.3 \\
18 \\
18 \\
13 \\
22 \\
13 \\
6.0 \\
4.9\end{array}$ & $\begin{array}{l}5.5 \\
19 \\
18 \\
13 \\
22 \\
13 \\
6.0 \\
5.7\end{array}$ & $\begin{array}{l}6.1 \\
21 \\
20 \\
14 \\
24 \\
15 \\
6.8 \\
6.6\end{array}$ & $\begin{array}{l}8.6 \\
26 \\
29 \\
15 \\
30 \\
22 \\
8.0 \\
7.5\end{array}$ & $\begin{array}{c}24 \\
33 \\
47 \\
17 \\
35 \\
42 \\
15 \\
9.0\end{array}$ & $\begin{array}{r}169 \\
58 \\
68 \\
25 \\
46 \\
85 \\
24 \\
16\end{array}$ & $\begin{array}{r}233 \\
81 \\
82 \\
47 \\
198 \\
83 \\
22 \\
24\end{array}$ \\
\hline $\begin{array}{l}1950 \\
1951 \\
1952 \\
1953 \\
1954 \\
1955 \\
1956 \\
1957 \\
1958 \\
1959\end{array}$ & $\begin{array}{c}2.8 \\
8.0 \\
57 \\
12 \\
5.5 \\
20 \\
5.8 \\
1.7 \\
6.6 \\
5.0\end{array}$ & $\begin{array}{l}2.8 \\
8.1 \\
70 \\
14 \\
6.1 \\
21 \\
6.2 \\
2.0 \\
7.3 \\
5.1\end{array}$ & $\begin{array}{r}3.0 \\
8.4 \\
74 \\
14 \\
7.1 \\
23 \\
6.5 \\
2.2 \\
8.2 \\
5.2\end{array}$ & $\begin{array}{r}3.6 \\
10 \\
114 \\
15 \\
9.0 \\
26 \\
6.9 \\
3.1 \\
12 \\
5.3\end{array}$ & $\begin{array}{c}4.4 \\
12 \\
118 \\
16 \\
11 \\
51 \\
7.1 \\
7.4 \\
15 \\
6.3\end{array}$ & $\begin{array}{c}7.9 \\
21 \\
159 \\
19 \\
14 \\
97 \\
9.6 \\
10 \\
19 \\
9.5\end{array}$ & $\begin{array}{c}7.7 \\
46 \\
252 \\
23 \\
17 \\
187 \\
15 \\
11 \\
27 \\
13\end{array}$ \\
\hline $\begin{array}{l}1960 \\
1961 \\
1962 \\
1963 \\
1964 \\
1965 \\
1966 \\
1967 \\
1968 \\
1969\end{array}$ & $\begin{array}{l}15 \\
7.4 \\
44 \\
16 \\
12 \\
15 \\
29 \\
7.7 \\
3.5 \\
21\end{array}$ & $\begin{array}{l}15 \\
7.6 \\
48 \\
17 \\
12 \\
15 \\
30 \\
9.0 \\
3.6 \\
24\end{array}$ & $\begin{array}{l}18 \\
7.8 \\
56 \\
18 \\
13 \\
18 \\
30 \\
9.4 \\
3.8 \\
27\end{array}$ & $\begin{array}{l}28 \\
8.6 \\
64 \\
22 \\
17 \\
19 \\
38 \\
9.8 \\
5.8 \\
41\end{array}$ & $\begin{array}{c}53 \\
9.5 \\
83 \\
29 \\
22 \\
31 \\
103 \\
12 \\
12 \\
59\end{array}$ & $\begin{array}{r}63 \\
13 \\
113 \\
53 \\
29 \\
49 \\
251 \\
14 \\
15 \\
105\end{array}$ & $\begin{array}{r}93 \\
18 \\
154 \\
141 \\
32 \\
116 \\
490 \\
14 \\
16 \\
201\end{array}$ \\
\hline $\begin{array}{l}1970 \\
1971 \\
1972 \\
1973 \\
1974 \\
1975 \\
1976\end{array}$ & $\begin{array}{r}25 \\
11 \\
13 \\
103 \\
34 \\
16 \\
5.7\end{array}$ & $\begin{array}{r}26 \\
12 \\
14 \\
114 \\
37 \\
17 \\
7.3\end{array}$ & $\begin{array}{r}29 \\
13 \\
16 \\
125 \\
39 \\
21 \\
8.8\end{array}$ & $\begin{array}{r}31 \\
18 \\
21 \\
181 \\
5 ! \\
26 \\
15\end{array}$ & $\begin{array}{r}35 \\
25 \\
21 \\
365 \\
72 \\
34 \\
20\end{array}$ & $\begin{array}{r}61 \\
55 \\
65 \\
509 \\
440 \\
42 \\
36\end{array}$ & $\begin{array}{r}73 \\
79 \\
78 \\
546 \\
504 \\
42 \\
35\end{array}$ \\
\hline
\end{tabular}




\section{DRAINAGE AREA : $844 \mathrm{~m}^{2}$ PERIOD OF RECORD: 36 VEARS AVERAGE DISCHARGE: 380 ft $3 / 5$}

MINIMUM AVERAGE FLOWS FOR PERIOD OF RECORD

\begin{tabular}{|c|c|c|c|c|c|c|c|}
\hline PERIOD OF CONSECUTIVE DAYS & 3 & 7 & 14 & 30 & EO & 120 & 183 \\
\hline $\begin{array}{l}\text { ARGE, IN f } \mathrm{ft}^{3} / \mathrm{s} \\
\text { TIC YEAR }\end{array}$ & $\begin{array}{r}1.7 \\
1957\end{array}$ & $\begin{array}{r}2.0 \\
1957\end{array}$ & $\begin{array}{r}2.2 \\
1957\end{array}$ & $\begin{array}{r}3.1 \\
1957\end{array}$ & $: 8.4$ & $\begin{array}{r}7.9 \\
1950\end{array}$ & \\
\hline
\end{tabular}

MAGNITUDE AND FREQUENCY OF ANNUAL LOW FLOWS

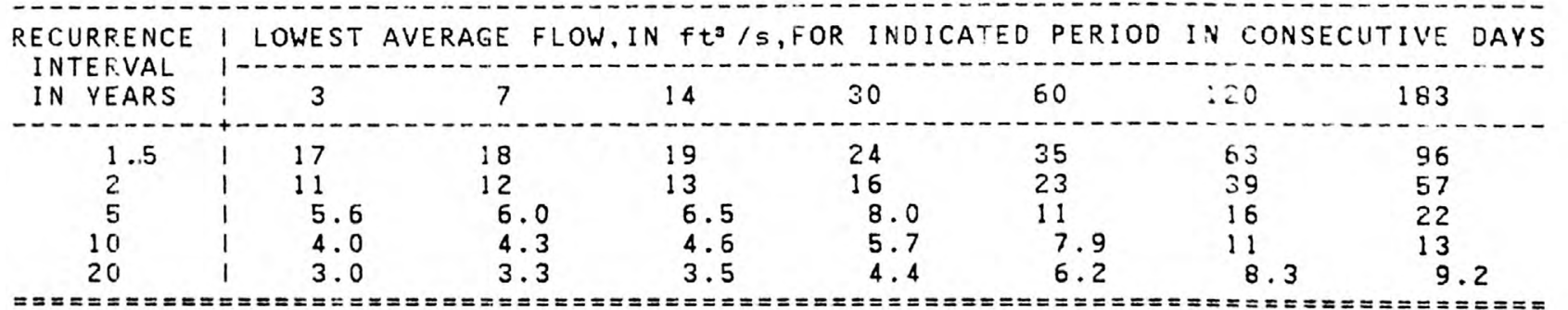

MAGNITUDE AND FREQUENCY OF SEASONAL LOW FLOWS

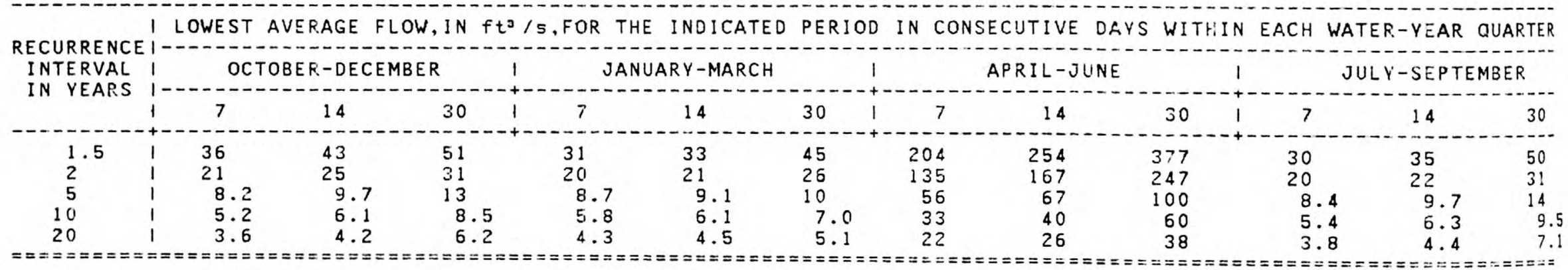

DURATION OF DAILY DISCHAREES FOR ANNUAL AND SEASONAL PERIODS

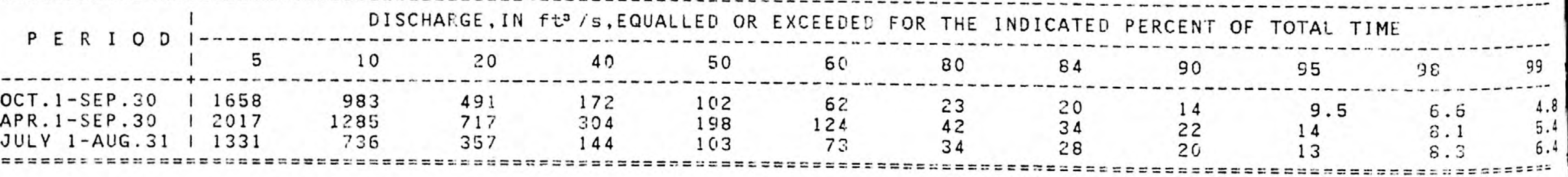


Location.--Lat $42^{\circ} 15^{\prime} 04^{\prime \prime}$, long 93059.52", In NW1/4 NE1/4 sec.21, T.86 N., R.27 W. Webster County, on $r$ lght bank $6 \mathrm{ft}$ downstream from bridge on State Highway $175,0.1 \mathrm{mile}$ downstream from Sk 11 let Creek, 4.0 miles southwest of Stratford, $7.3 \mathrm{miles}$ downstream from Boone River and at m1le 276.7 .

Remarks.--Sight diurnal fluctuation caused by powerplant at Fort Docige to 1971. Since 1971 occasional minor regulation caused by dam at Fort Dodge. Published as 05481500 Des Moines River near Boone, Iowa, 1920-67

LOW FLOW DISCHARGES, IN YEAR ENDING MARCH 31

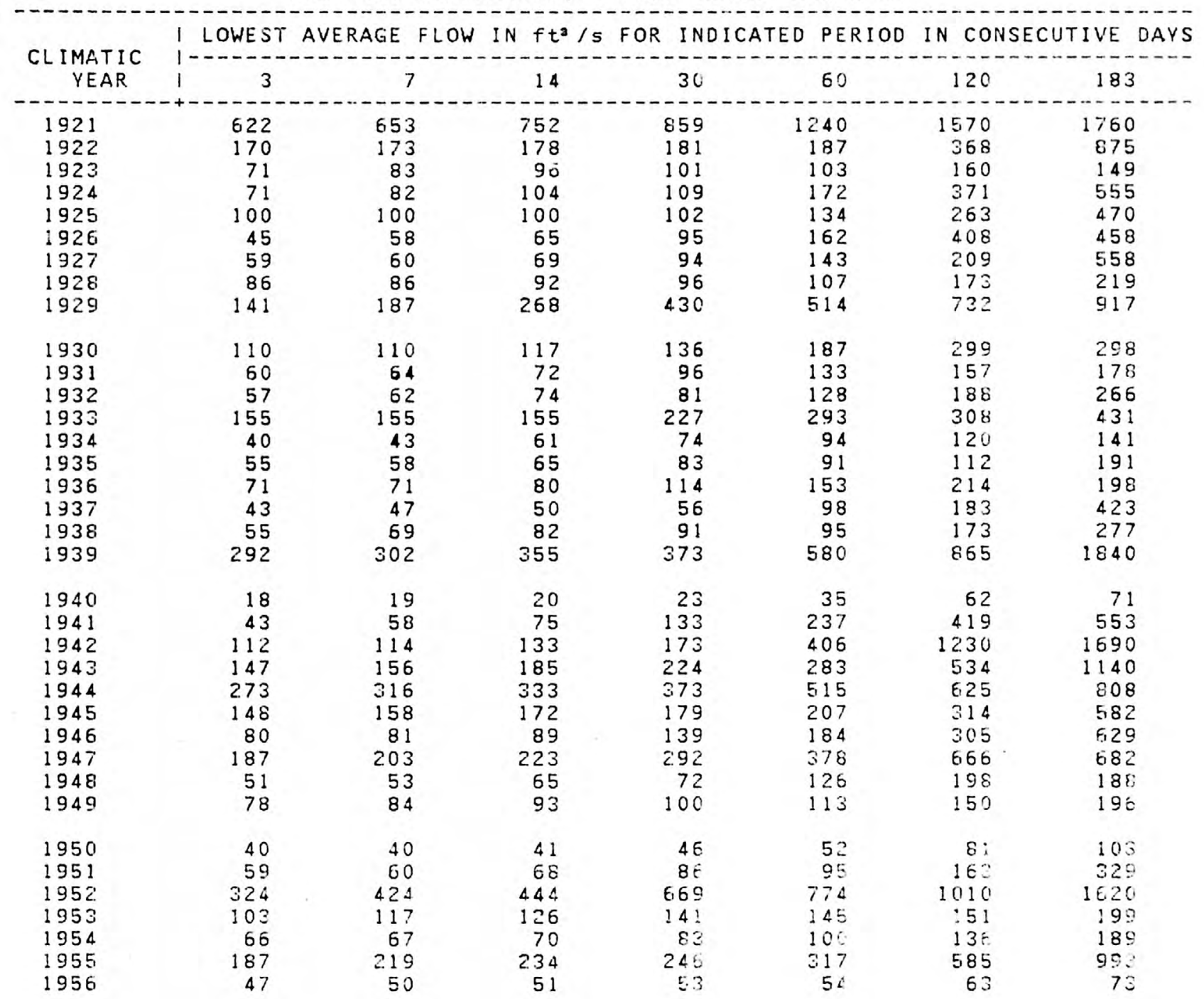


DES MOINES RIVER NEAR STRATFORD--Cont inued

LOW FLOW DISCHARGES, IN YEAR ENDING MARCH 31--Continued

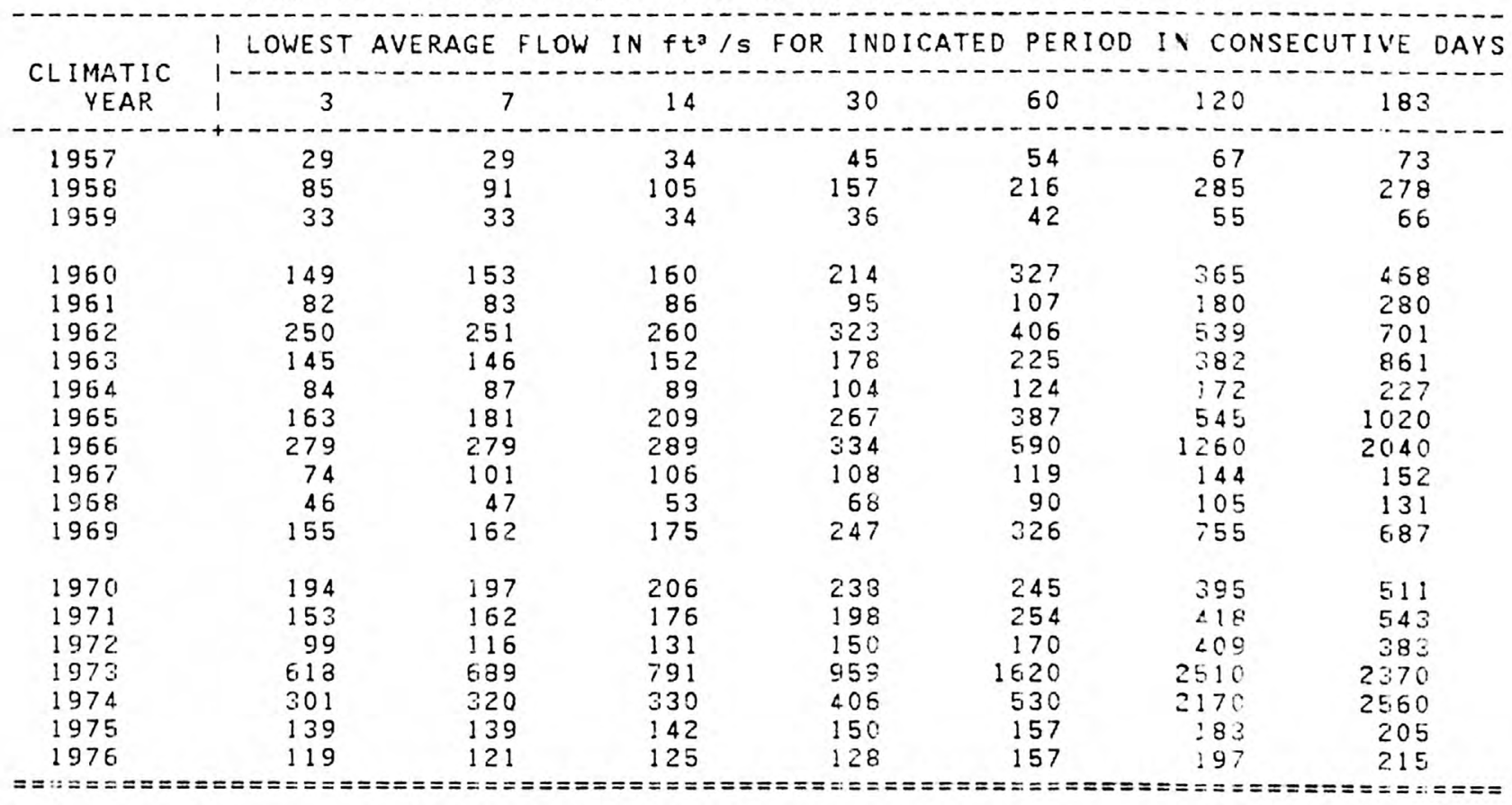


05-4813.00 DES MOINES RIVER NEAR STRATFORD--Continued

DRAINAGE AREA: $5452 \mathrm{~m}^{2}$ PERIOD OF RECORD: 56 YEARS AVERAGE DISCHARGE: 1760 ft is

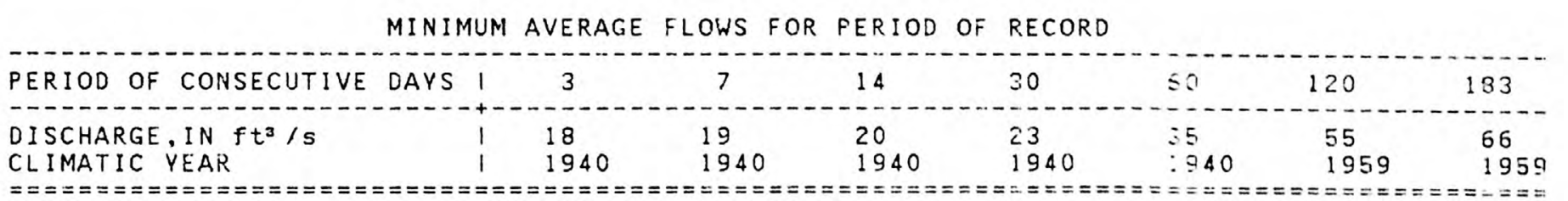

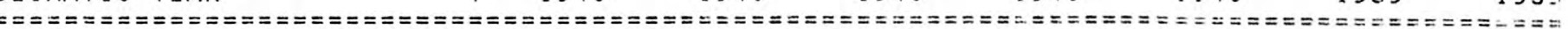

MAGNITUDE AND FREQUENCY OF ANNUAL LOW FLOWS

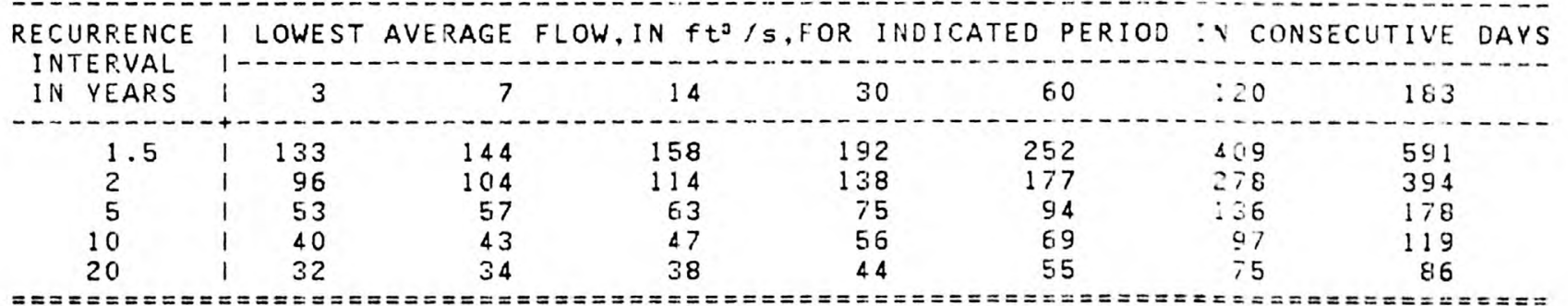

MAGNITUDE AND FREQUENCY OF SEASONAL LOW FLOWS

LOWEST AVERAGE FLOW, IN $\mathrm{ft}^{2} / \mathrm{s}, F O R$ THE INDICATED PERIOD IN CONSECUTIVE DAYS WITHIN EACH WATER-VEAR QUARTER

\begin{tabular}{l}
$\begin{array}{l}\text { RECURRENCE } \\
\text { INTERVAL } \\
\text { IN }\end{array}$ YEARS \\
\hline
\end{tabular}

DURATION OF DAILY DISCHARGES FOR ANNUAL AND SEASONAL PERIODS

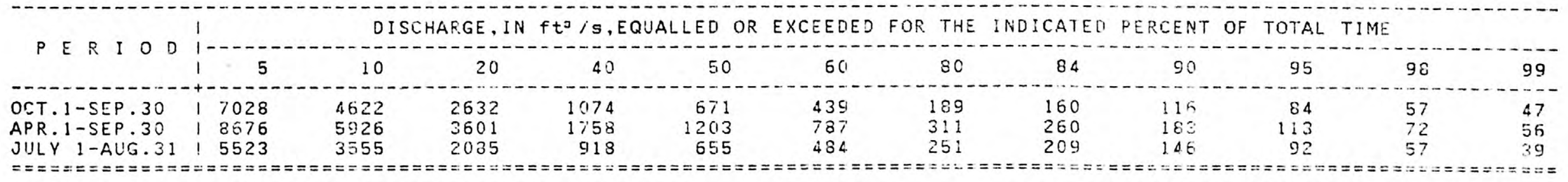


Location.--Lat $41^{\circ} 40^{\prime} 50^{\prime \prime}$, long $93^{\circ} 40^{\prime 07 "}$, near center of sec.5, T.79 N., R.24 W., Polk County, near center of span on downstream side of bridge on county highway F42,2.0 miles west of Saylorvilie, 2.1 miles downstream from Rock Creek, 2.4 miles upstream from Beaver Creek, and at mile : : 1.6 .

LOW FLOW DISCHARGES, IN VEAP. ENDING MARCH 31

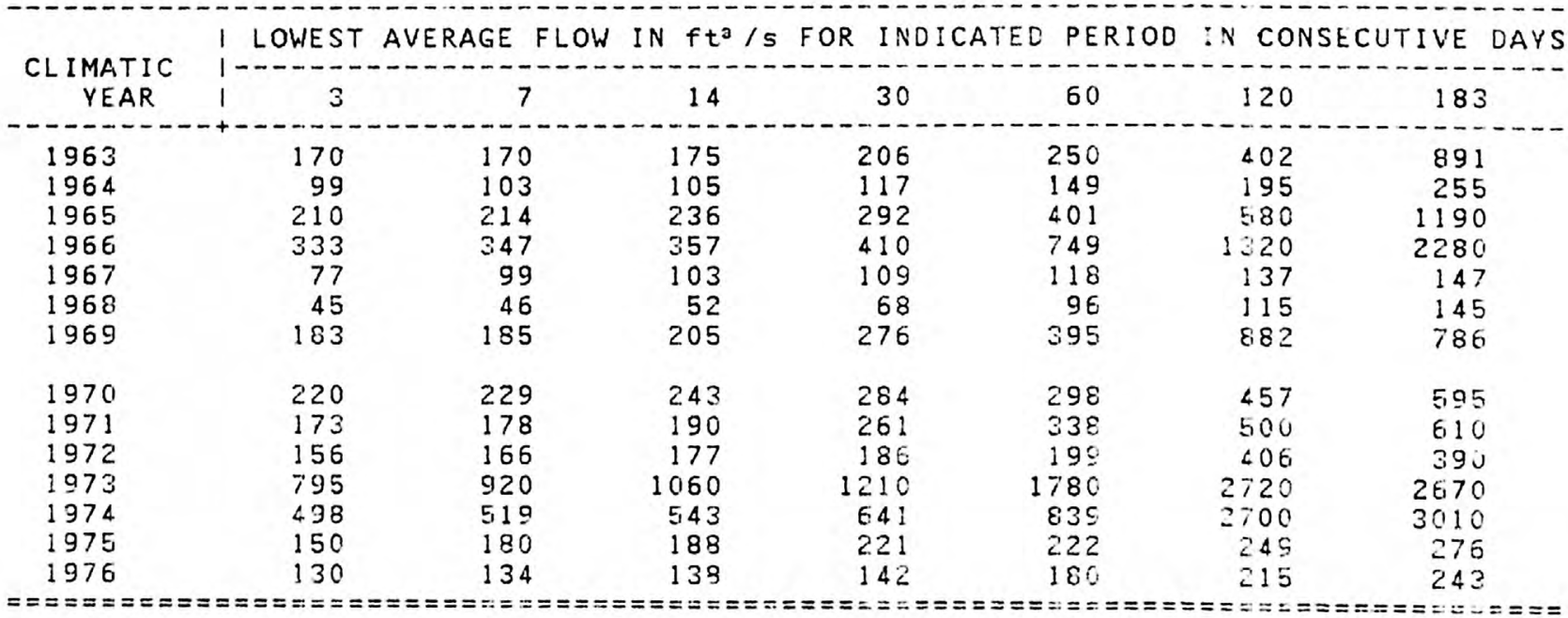




\section{DRAINAGE AREA: $5841 \mathrm{mi}^{2}$ PERIOD OF RECORD: 15 YEARS AVERAGE DISCHARGE: 2546 ft3/S}

MINIMUM AVERAGE FLOWS FOR PERIOD OF RECORD

\begin{tabular}{|c|c|c|c|c|c|c|c|}
\hline PERIOD OF CONSECUTIVE DAYS & 3 & 7 & 14 & 30 & 50 & 120 & 183 \\
\hline $\begin{array}{l}\text { ISCHARGE, IN } \mathrm{ft}^{3} / \mathrm{s} \\
\text { LIMATIC YEAR }\end{array}$ & $\begin{array}{r}45 \\
1968 \\
\end{array}$ & $\begin{array}{l}46 \\
1968\end{array}$ & $\begin{array}{r}52 \\
1968 \\
\end{array}$ & $\begin{array}{l}68 \\
19 \in 8\end{array}$ & $\stackrel{96}{: 968}$ & $\begin{array}{l}115 \\
1968\end{array}$ & $\begin{array}{l}145 \\
1968\end{array}$ \\
\hline
\end{tabular}

MAGNITUDE AND FREQUENCY OF ANNUAL LOW FLOWS

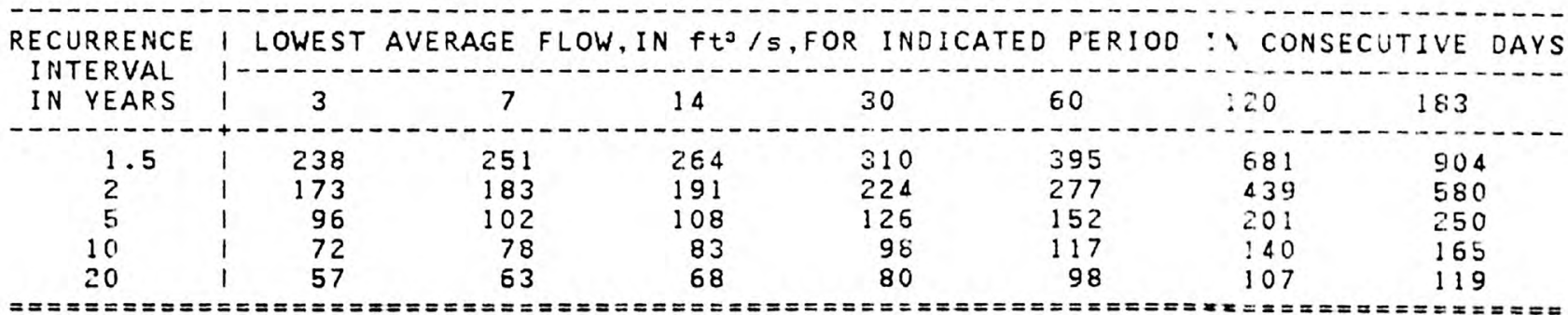

MAGNITUDE AND FREQUENCY OF SEASONAL LOW FLOWS

LOWEST AVERAGE FLOW, IN ft $/ 5$, FOR THE INDICATED PERIOD IN CONSECUTIVE OAYS WITHIN EACH WATER-YEAR QUARTER

RECURRENCEI-

INTERVAL I OCTOBER-DECEMBER I JANUARY-MARCH I I I I S ILPTINE

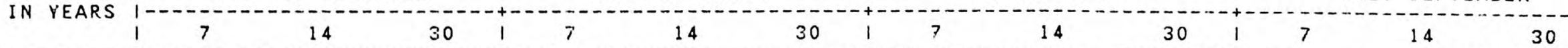

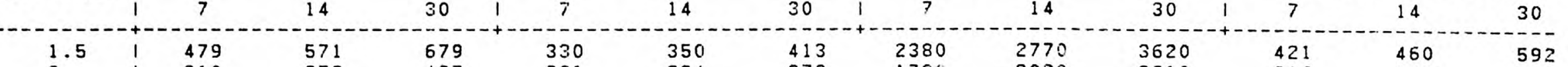

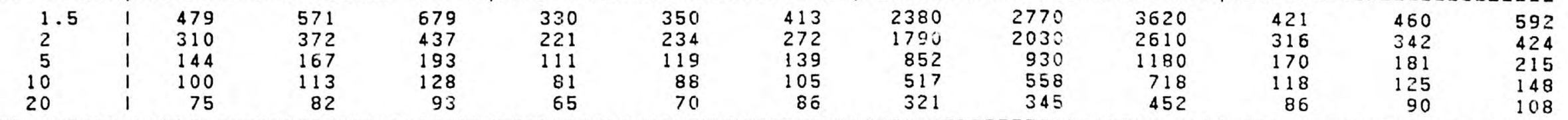

DURATION OF DAILY DISCHARGES FOR ANNUAL ANO SEASONAL PERIODS

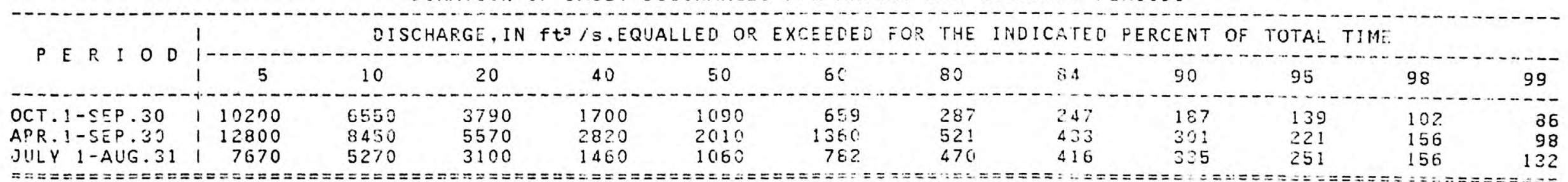


Location.--Lat $41^{\circ} 41^{\prime} 18^{\prime \prime}$, long $93^{\circ} 44^{\prime} 08^{\prime \prime}$, in SW1/4 SW1/4 sec. 35 . T.80 N., R.25 W. Polk County, on right bank $6 \mathrm{ft}$ upstream from Northwest 70 th Avenue Bridge, $0.5 \mathrm{mile}$ downstream from Little Beaver Creek. $=5 \mathrm{miles}$ east of Grimes and $6 \mathrm{miles}$ upstream from mouth.

LOW FLOW DISCHARGES, IN YEAR. ENDING MARCH 31

\begin{tabular}{|c|c|c|c|c|c|c|c|}
\hline \multirow{2}{*}{$\begin{array}{c}\text { CLIMATIC } \\
\text { YEAR }\end{array}$} & \multicolumn{2}{|c|}{$1-\cdots+\cdots-\cdots+\cdots$} & $f t^{3} / s$ & R INUICA & D PERIOD & \multicolumn{2}{|c|}{ I CONSECUTIVE DAYS } \\
\hline & 13 & 7 & 14 & 30 & 60 & 120 & 183 \\
\hline $\begin{array}{l}1962 \\
1963 \\
1964 \\
1965 \\
1966 \\
1967 \\
1958 \\
1969\end{array}$ & $\begin{array}{l}8.7 \\
.57 \\
1.2 \\
3.0 \\
.70 \\
.10 \\
.28 \\
2.9\end{array}$ & $\begin{array}{c}10 \\
.64 \\
2.0 \\
3.1 \\
.74 \\
.10 \\
.34 \\
3.6\end{array}$ & $\begin{array}{c}12 \\
.69 \\
2.1 \\
3.7 \\
.83 \\
.10 \\
.48 \\
5.1\end{array}$ & $\begin{array}{l}35 \\
.75 \\
2.5 \\
6.4 \\
3.1 \\
.15 \\
1.0 \\
6.3\end{array}$ & $\begin{array}{c}139 \\
.88 \\
3.9 \\
8.5 \\
21 \\
.28 \\
1.4 \\
8.7\end{array}$ & $\begin{array}{r}188 \\
5.1 \\
4.6 \\
12 \\
52 \\
.50 \\
2.3 \\
42\end{array}$ & $\begin{array}{c}236 \\
6.1 \\
7.5 \\
15 \\
69 \\
.65 \\
2.3 \\
45\end{array}$ \\
\hline $\begin{array}{l}1970 \\
1971 \\
1972 \\
1973 \\
1974 \\
1975 \\
1976\end{array}$ & $\begin{array}{l}7.6 \\
0 \\
0 \\
2.0 \\
31 \\
4.8 \\
1.6\end{array}$ & $\begin{array}{c}12 \\
0.08 \\
5.0 \\
46 \\
5.2 \\
1.8\end{array}$ & $\begin{array}{r}14 \\
.20 \\
.01 \\
18 \\
80 \\
6.6 \\
1.9\end{array}$ & $\begin{array}{r}15 \\
2.5 \\
.19 \\
47 \\
101 \\
7.6 \\
2.1\end{array}$ & $\begin{array}{c}19 \\
6.0 \\
.26 \\
72 \\
146 \\
9.7 \\
4.1\end{array}$ & $\begin{array}{r}37 \\
21 \\
3.8 \\
156 \\
291 \\
28 \\
12\end{array}$ & $\begin{array}{c}36 \\
32 \\
4.7 \\
193 \\
339 \\
32 \\
16\end{array}$ \\
\hline
\end{tabular}


DRAINAGE AREA: $358 \mathrm{mi}^{2}$ PERIOD OF RECORD: 16 YEARS AVERAGE DISCHARGE: 203 ft3/s

MINIMUM AVERAGE FLOWS FOR PERIOD OF RECORD

\begin{tabular}{|c|c|c|c|c|c|c|c|}
\hline PERIOD OF CONSECUTIVE DAYS & 3 & 7 & 14 & 30 & 50 & 120 & 183 \\
\hline $\begin{array}{l}\text { HARGE, IN } \mathrm{ft}^{3} / \mathrm{s} \\
\text { ATIC YEAR }\end{array}$ & $\begin{array}{l}0 \\
1972\end{array}$ & $\begin{array}{l}0 \\
1972\end{array}$ & $\begin{array}{l}0.01 \\
1972\end{array}$ & $\begin{array}{l}0.15 \\
1957\end{array}$ & $\begin{array}{l}9.26 \\
1972\end{array}$ & $\begin{array}{r}0.50 \\
1967\end{array}$ & $\begin{array}{l}0.65 \\
1967\end{array}$ \\
\hline
\end{tabular}

MAGNITUDE AND FREQUENCY OF SNNUAL LOW FLOWS

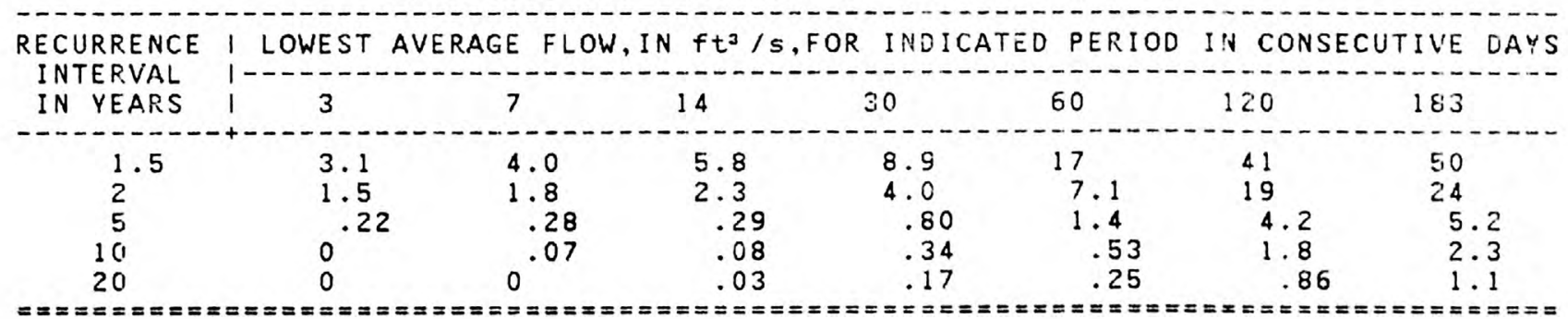

MAGNITUDE AND FREQUENCY OF SEASONAL LOW FLOWS

I LOWEST AVERAGE FLOW, IN $\mathrm{ft}^{\mathrm{S}} / \mathrm{s}, \mathrm{FOR}$ THE INDICATED PERIOD IN CONSECUTIVE DAYS WITHIN EACH WATER-YEAR QUARTER

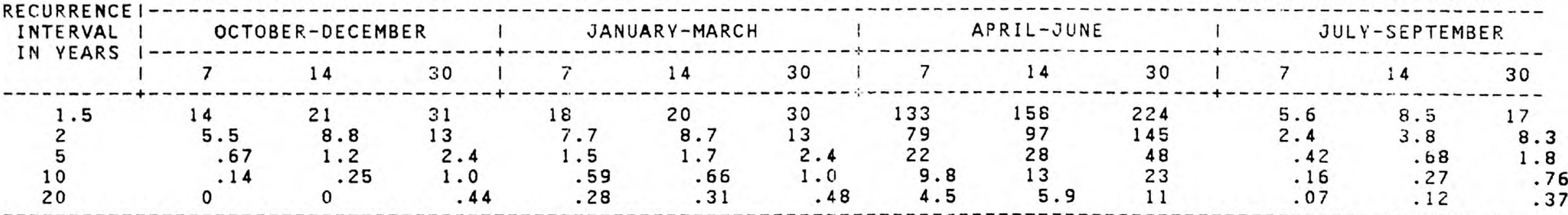

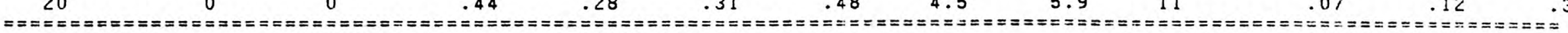

DURATION OF DAILY DISCHARGES FOR FNNUAL ANO SEASONAL PERIODS

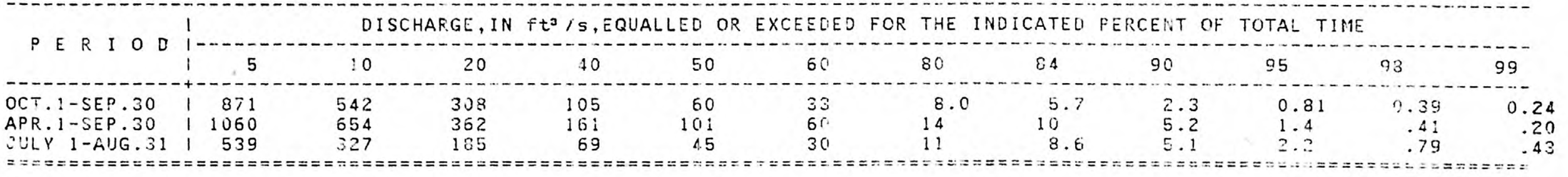


Location.--Lat $41^{\circ} 36^{\prime 4} 45^{\prime \prime}$, long 93037'15", in NE1/4 NE1/4 sec.34, T.79 N., R. 24 W. Polk County, on right bank $5 \mathrm{ft}$ upstream from Seco-d Avenue bridge in Des Molnes, i.8 miles upstream from Center Street dam, $2.8 \mathrm{miles}$ upstream from Raccoon River, $4.5 \mathrm{miles}$ downstream from Beaver Creek. and at mile 204.3 upstream from mouth.

Remarks.--Discontinued Sept. 30, 1961.

LOW FLOW DISCHARGES, IN YEAR ENDING MARCH 31

\begin{tabular}{|c|c|c|c|c|c|c|c|}
\hline \multirow{2}{*}{$\begin{array}{c}\text { CLIMATIC } \\
\text { YEAR }\end{array}$} & I LOWEST & AGE & $\begin{array}{l}n \\
-\end{array}$ & R IN & D PE & IN CO & IVE \\
\hline & 13 & 7 & 14 & 30 & 60 & 120 & 183 \\
\hline $\begin{array}{l}1916 \\
1917 \\
1918 \\
1919\end{array}$ & $\begin{array}{r}580 \\
100 \\
70 \\
288\end{array}$ & $\begin{array}{r}580 \\
100 \\
70 \\
324\end{array}$ & $\begin{array}{r}698 \\
100 \\
76 \\
340\end{array}$ & $\begin{array}{r}985 \\
100 \\
83 \\
401\end{array}$ & $\begin{array}{r}1820 \\
111 \\
106 \\
647\end{array}$ & $\begin{array}{r}2720 \\
201 \\
155 \\
888\end{array}$ & $\begin{array}{r}3440 \\
246 \\
21 \% \\
1220\end{array}$ \\
\hline $\begin{array}{l}1920 \\
1921 \\
1922 \\
1923 \\
1924 \\
1925 \\
1926 \\
1927 \\
1928 \\
1929\end{array}$ & $\begin{array}{r}355 \\
935 \\
200 \\
53 \\
51 \\
110 \\
105 \\
73 \\
93 \\
325\end{array}$ & $\begin{array}{r}367 \\
1000 \\
201 \\
81 \\
89 \\
113 \\
106 \\
77 \\
93 \\
445\end{array}$ & $\begin{array}{r}375 \\
1000 \\
203 \\
111 \\
120 \\
120 \\
124 \\
91 \\
96 \\
505\end{array}$ & $\begin{array}{r}435 \\
1150 \\
208 \\
158 \\
124 \\
125 \\
134 \\
136 \\
98 \\
553\end{array}$ & $\begin{array}{r}470 \\
1290 \\
238 \\
176 \\
233 \\
180 \\
195 \\
253 \\
114 \\
672\end{array}$ & $\begin{array}{r}837 \\
1780 \\
515 \\
256 \\
471 \\
349 \\
436 \\
371 \\
247 \\
1230\end{array}$ & $\begin{array}{r}905 \\
1990 \\
1180 \\
259 \\
733 \\
566 \\
497 \\
1050 \\
300 \\
1420\end{array}$ \\
\hline $\begin{array}{l}1930 \\
1931 \\
1932 \\
1933 \\
1934 \\
1935 \\
1936 \\
1937 \\
1938 \\
1939\end{array}$ & $\begin{array}{r}129 \\
41 \\
41 \\
179 \\
60 \\
59 \\
80 \\
67 \\
80 \\
332\end{array}$ & $\begin{array}{r}142 \\
41 \\
44 \\
185 \\
61 \\
62 \\
82 \\
68 \\
83 \\
345\end{array}$ & $\begin{array}{r}145 \\
46 \\
50 \\
188 \\
79 \\
70 \\
98 \\
68 \\
97 \\
393\end{array}$ & $\begin{array}{r}168 \\
53 \\
60 \\
259 \\
90 \\
95 \\
134 \\
72 \\
124 \\
404\end{array}$ & $\begin{array}{r}236 \\
75 \\
68 \\
341 \\
118 \\
112 \\
195 \\
133 \\
129 \\
617\end{array}$ & $\begin{array}{r}391 \\
92 \\
134 \\
363 \\
149 \\
142 \\
365 \\
209 \\
194 \\
911\end{array}$ & $\begin{array}{r}436 \\
91 \\
209 \\
488 \\
183 \\
218 \\
322 \\
428 \\
297 \\
1960\end{array}$ \\
\hline $\begin{array}{l}1940 \\
1941 \\
1942 \\
1943 \\
1944 \\
1945 \\
1946 \\
1947 \\
1948 \\
1949\end{array}$ & $\begin{array}{r}24 \\
72 \\
154 \\
228 \\
287 \\
183 \\
90 \\
280 \\
100 \\
102\end{array}$ & $\begin{array}{r}24 \\
84 \\
172 \\
241 \\
351 \\
189 \\
91 \\
287 \\
106 \\
106\end{array}$ & $\begin{array}{r}26 \\
98 \\
207 \\
266 \\
361 \\
199 \\
101 \\
372 \\
110 \\
112\end{array}$ & $\begin{array}{l}30 \\
171 \\
263 \\
320 \\
398 \\
210 \\
162 \\
472 \\
130 \\
125\end{array}$ & $\begin{array}{l}44 \\
294 \\
483 \\
403 \\
555 \\
250 \\
205 \\
592 \\
200 \\
136\end{array}$ & $\begin{array}{r}71 \\
541 \\
1430 \\
721 \\
710 \\
397 \\
153 \\
963 \\
290 \\
179\end{array}$ & $\begin{array}{r}80 \\
849 \\
2040 \\
1420 \\
926 \\
766 \\
772 \\
914 \\
271 \\
257\end{array}$ \\
\hline $\begin{array}{l}1950 \\
1951\end{array}$ & $\begin{array}{l}32 \\
58\end{array}$ & $\begin{array}{l}37 \\
62\end{array}$ & $\begin{array}{l}42 \\
71\end{array}$ & $\begin{array}{l}55 \\
9 .\end{array}$ & $\begin{array}{r}84 \\
104\end{array}$ & $\begin{array}{l}117 \\
173\end{array}$ & $\begin{array}{l}150 \\
324\end{array}$ \\
\hline
\end{tabular}


LOW FLOW DISCHARGES, IN YEAR ENDING MARCH 31--Continued

\begin{tabular}{|c|c|c|c|c|c|c|c|c|}
\hline \multirow{2}{*}{$\begin{array}{c}\text { CLIMATIC } \\
\text { YEAR }\end{array}$} & I LOWEST & $\begin{array}{l}\text { GE } F \\
-\end{array}$ & $f t^{3}$ & IN & $\begin{array}{r}\text { PE } \\
--\end{array}$ & \multicolumn{2}{|c|}{ N CONSECUTIVE } & DAYS \\
\hline & 13 & 7 & 14 & 30 & 60 & 120 & 183 & \\
\hline $\begin{array}{l}1952 \\
1953 \\
1954 \\
1955 \\
1956 \\
1957 \\
1958 \\
1959\end{array}$ & $\begin{array}{r}490 \\
100 \\
72 \\
280 \\
50 \\
54 \\
92 \\
45\end{array}$ & $\begin{array}{r}508 \\
129 \\
72 \\
2.83 \\
51 \\
54 \\
98 \\
46\end{array}$ & $\begin{array}{r}531 \\
155 \\
74 \\
293 \\
54 \\
56 \\
118 \\
48\end{array}$ & $\begin{array}{r}812 \\
166 \\
86 \\
328 \\
57 \\
50 \\
181 \\
48\end{array}$ & $\begin{array}{r}951 \\
175 \\
111 \\
490 \\
59 \\
60 \\
249 \\
59\end{array}$ & $\begin{array}{r}1250 \\
182 \\
161 \\
833 \\
65 \\
84 \\
312 \\
96\end{array}$ & $\begin{array}{r}1910 \\
303 \\
213 \\
1380 \\
77 \\
95 \\
312 \\
145\end{array}$ & \\
\hline $\begin{array}{l}1960 \\
1961\end{array}$ & $\begin{array}{l}213 \\
120\end{array}$ & $\begin{array}{l}2.22 \\
121\end{array}$ & $\begin{array}{l}2.47 \\
126\end{array}$ & $\begin{array}{l}319 \\
134\end{array}$ & $\begin{array}{l}414 \\
15:\end{array}$ & $\begin{array}{l}46 \% \\
305\end{array}$ & $\begin{array}{l}579 \\
455\end{array}$ & \\
\hline
\end{tabular}


DRAINAGE AREA: $6245 \mathrm{mi}^{2} \quad$ PERIOD OF RECORD: 46 YEARS AVERAGE DISCHARGE: $1984 \mathrm{ft}^{3} / \mathrm{S}$

MINIMUM AVERAGE FLOWS FOR PERIOD OF RECORD

\begin{tabular}{|c|c|c|c|c|c|c|c|}
\hline PERIOD OF CONSECUTIVE DAYS I & 3 & 7 & 14 & 30 & 50 & 120 & 183 \\
\hline $\begin{array}{l}\text { ARGE, IN } \mathrm{ft}^{3} / \mathrm{s} \\
\text { TIC YEAR }\end{array}$ & $\begin{array}{l}24 \\
1940\end{array}$ & $\begin{array}{l}24 \\
1940\end{array}$ & $\begin{array}{l}26 \\
1940\end{array}$ & $\begin{array}{l}30 \\
1940\end{array}$ & $\begin{array}{l}42 \\
: 980\end{array}$ & $\begin{array}{l}65 \\
1956\end{array}$ & $\begin{array}{l}77 \\
1956\end{array}$ \\
\hline
\end{tabular}

MAGNITUDE AND FREQUENCY OF ANNUAL LOW FLOWS

\begin{tabular}{l|cccccc} 
RECURRENCE \\
INTERVAL \\
IN YNEARS
\end{tabular}

$0=0$

MAGNITUDE AND FREQUENCY OF SEASONAL LOW FLOW'S

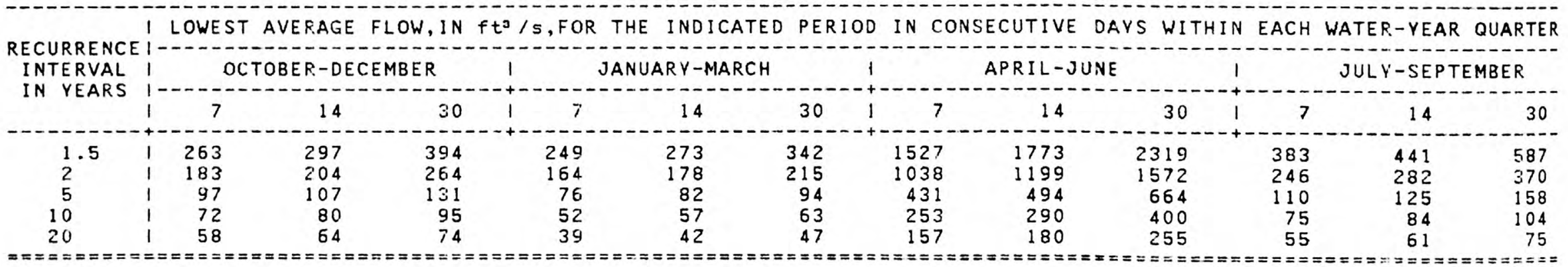

DURATION OF DAILY DISCHARGES FOR ANNUAL AND SEASONAL FERIODS

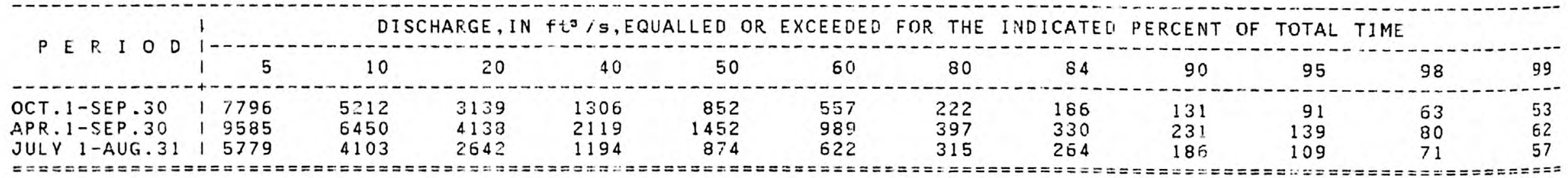


Location.--Lat $42^{\circ} 41^{\prime} 16^{\prime \prime}$, long $94^{\circ} 47^{\prime} 52^{\prime \prime}$, in NE1/4 NE1/4 sec.24, T.91 N.. R.34 W. , Pochahontas County, on left bank $5 \mathrm{ft}$ downstream from bridge on county highway $\mathrm{N3}, 2.0$ miles downstream from Dralnage di:ch $21,3.5 \mathrm{miles}$ upstream from Drainage ditch 74 , and $5.5 \mathrm{miles}$ northeast on Varina.

LOW FLOW DISCHARGES, IN YEAR. ENDING MA.RCH 31

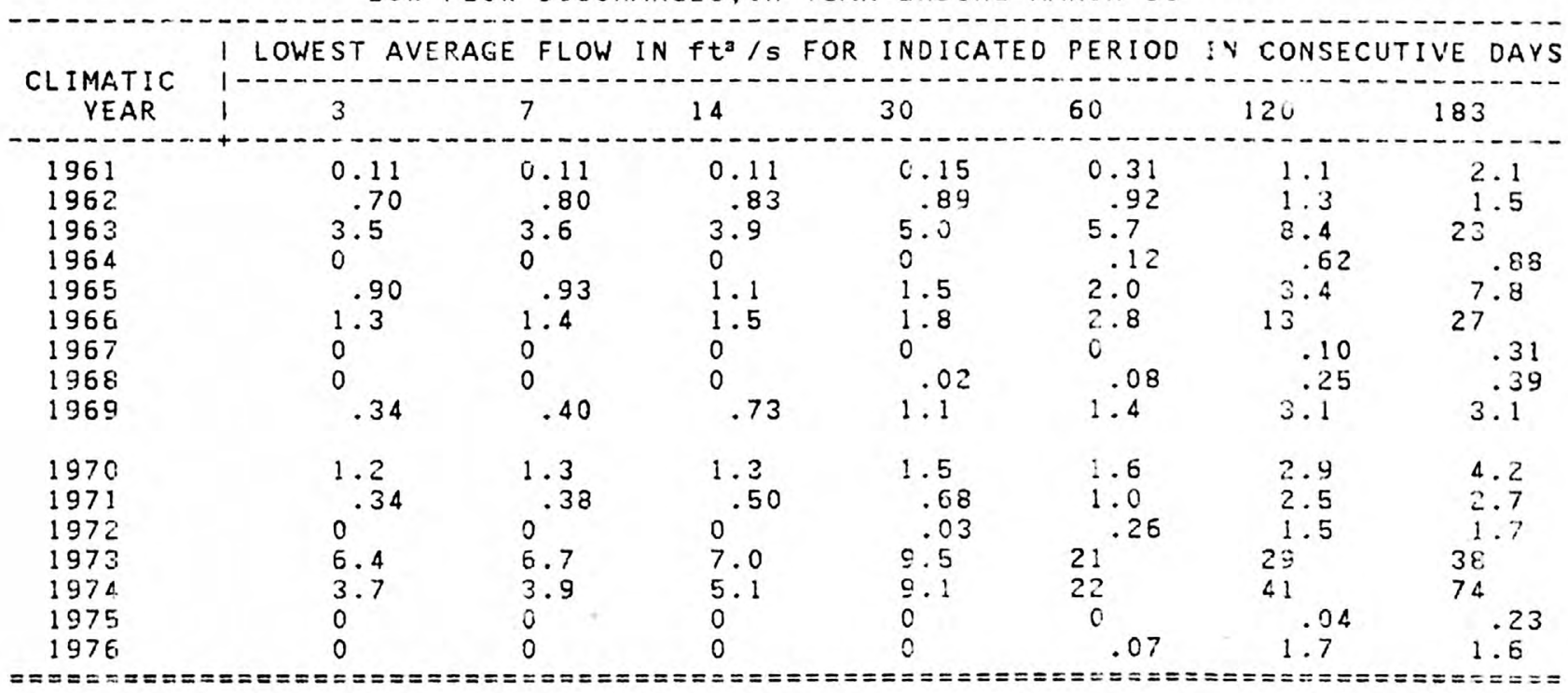




DRAINAGE AREA: $80.0 \mathrm{~m}^{2} \quad$ PERIOD OF RECORD: 17 YEARS AVERAGE DISCHARGE: $34.6 \mathrm{ft} / \mathrm{s} / \mathrm{S}$
MINIMUN AVERAGE FLOWS FOR PERIOD OF RECORD

MAGNITUDE AND FREQUENCY OF ANNUAL LOW FLOWS

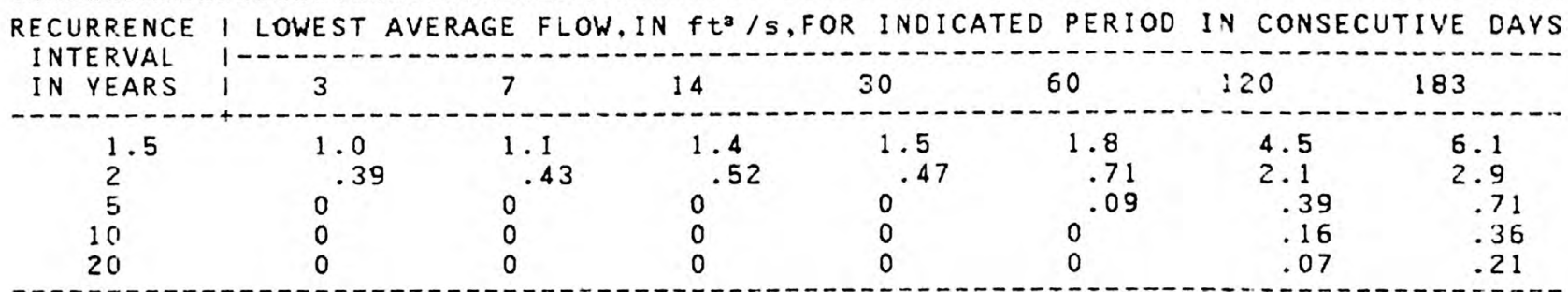

MAGNITUDE AND FREQUENCY OF SEASONAL LOW FLOWS

LOWEST AVERAGE FLOW, IN $\mathrm{ft} / \mathrm{s}$,FOR THE INDICATED PERIOD IN CONSECUTIVE OAYS WITHIN EACH WATER-YEAR QUARTER

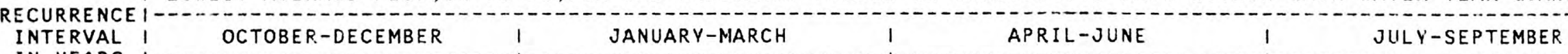

$\begin{array}{llllllllllllll}1 & 7 & 14 & 30 & 1 & 7 & 14 & 30 & 1 & 7 & 14 & 30 & 14 & 7\end{array}$

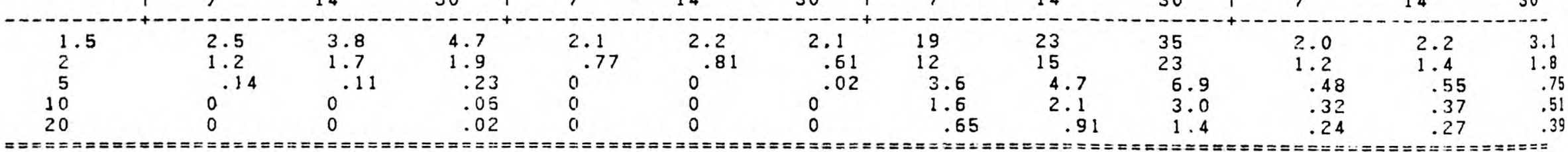

DURATION OF DAILY DISCHARGES FOR ANNUAL AND SEASONAL FERIODS

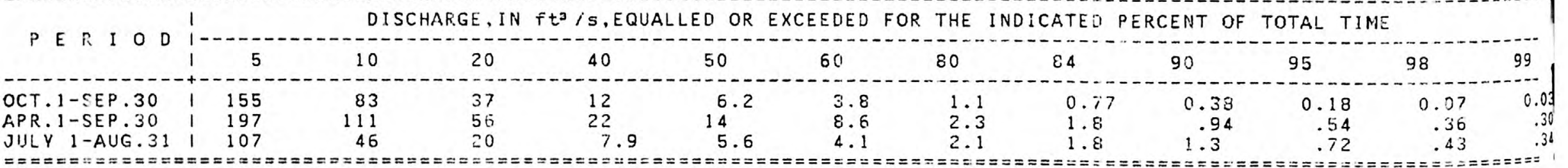




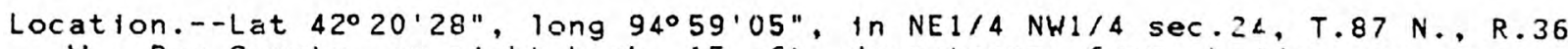
W. Sac County, on right bank $15 \mathrm{ft}$ downstream from is idge on county highway, 0.2 mile upstream from Indian Creek. 0.9 mi le downstreail froin Drainage ditch 73 , and 5.6 miles south of Sac Citj.

LOW FLOW DISCHARGES, IN YEAR ENDING MARCH 31

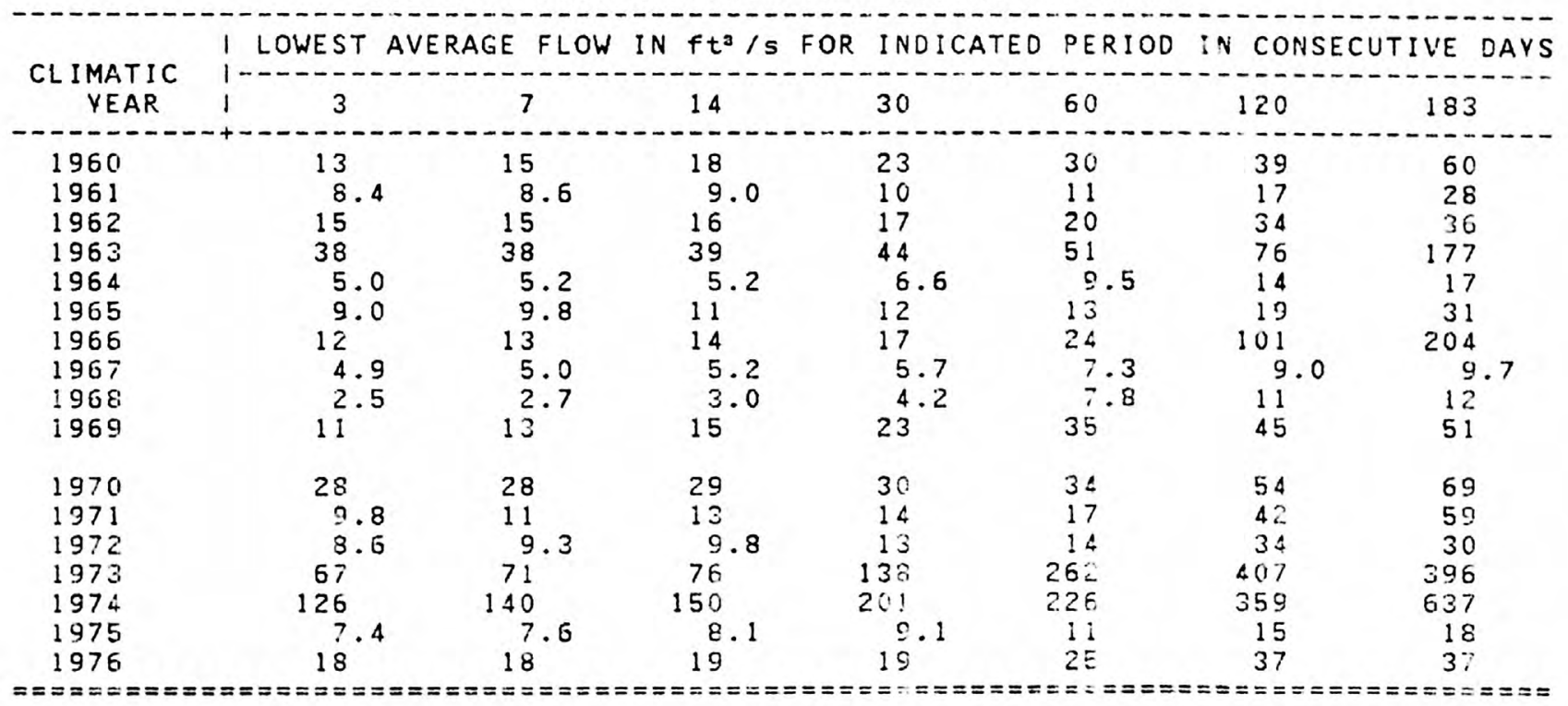




\section{DRAINAGE AREA: $713 \mathrm{mi}^{2}$ PERIOD OF RECORD: 18 YEARS AVERAGE 2:SCHARGE: 288 ft $3 / \mathrm{s}$}

MINIMUM AVERAGE FLOWS FOR PERIOD OF RECORD

\begin{tabular}{|c|c|c|c|c|c|c|c|}
\hline PERIDD OF CONSECUTIVE DAYS I & 3 & 7 & 14 & 30 & 56 & 120 & 183 \\
\hline $\begin{array}{l}\text { HARGE, IN } \mathrm{ft}^{3} / \mathrm{s} \\
\text { ATIC YEAR } \\
=============\end{array}$ & $\begin{array}{r}2.5 \\
1968 \\
\end{array}$ & $\begin{array}{r}2.7 \\
1968 \\
====\end{array}$ & $\begin{aligned} & 3.0 \\
1968 & \\
== & ===\end{aligned}$ & $\begin{aligned} & 4.2 \\
& 1968 \\
&=====\end{aligned}$ & $\begin{array}{l}\quad .3 \\
i \leq 57 \\
====\end{array}$ & $\begin{array}{r}9.0 \\
1967 \\
======\end{array}$ & $\begin{aligned} & 9.7 \\
& 1967 \\
&====\end{aligned}$ \\
\hline
\end{tabular}

MAGNITUDE AND FREQUENCY OF ANNUAL LOW FLOWS

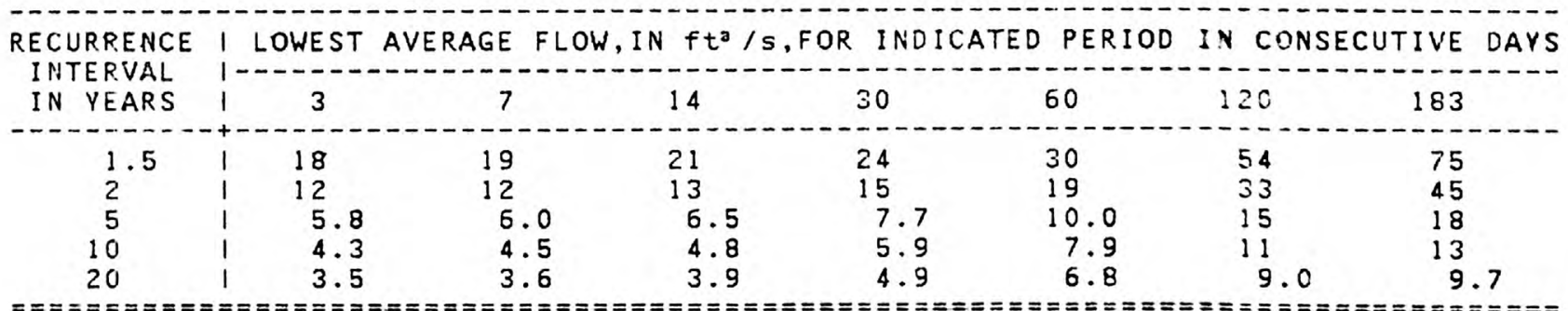

MAGNITUDE AND FREQUENCY OF SEASONAL LOW FLOW'S

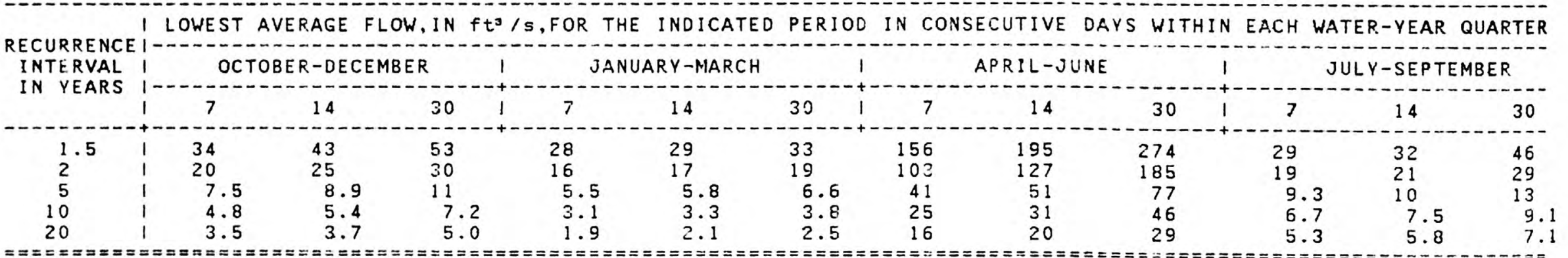

DURATION OF DAILY DISCHARGES FOR ANNUAL AND SEASONAL FERIODS

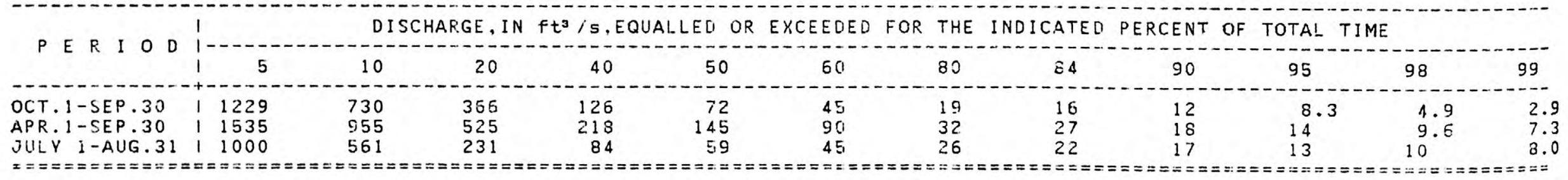


Location.--Lat 41059'17", long 94022'36", in SW1/4 NW1/4 sec.20, T.83 N., R.30 W., Greene County, on right bank $5 \mathrm{ft}$ downstream from bridge on State Highway 4, 0.1 mile downstream from Dralnage ditches 33 , and $40,2.9$ miles south of Jefferson, and 4.2 miles upstream from Hardin Creek.

Remarks.--Published as "Raccoon River near Jefferson", 1940-55.

LOW FLOW DISCHARGES, IN YEAR ENDING MARCH 31

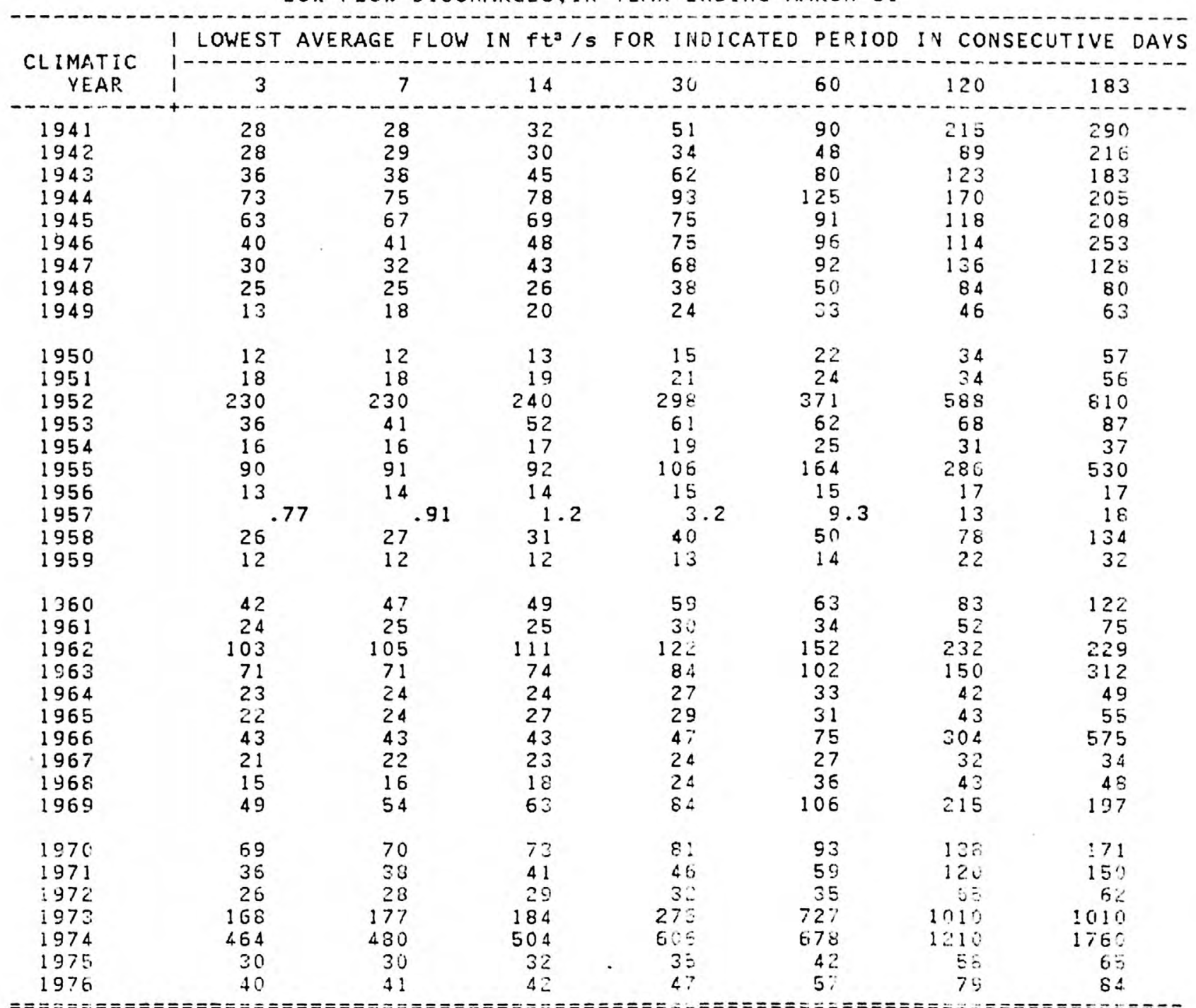


DRAINAGE AREA: $1619 \mathrm{mi}^{2}$ PERIOD OF RECORD: 36 VEARS AVERAGE $2: S C H A R G E: 671 \mathrm{ft}^{3} / \mathrm{s}$

MINIMUM AVERAGE FLOWS FOR PERIOD OF RECORD

\begin{tabular}{|c|c|c|c|c|c|c|c|c|}
\hline PERIOD OF CONSECUTIVE DAYS & 1 & 3 & 7 & 14 & 30 & 53 & 120 & 183 \\
\hline $\begin{array}{l}\text { DISCHARGE, IN } \mathrm{ft}^{3} / \mathrm{s} \\
\text { CL IMATIC YEAR }\end{array}$ & 1 & $\begin{array}{l}0.77 \\
1957\end{array}$ & $\begin{array}{l}0.92 \\
1957\end{array}$ & $\begin{array}{r}1.2 \\
1957\end{array}$ & $\begin{array}{r}3.2 \\
1957\end{array}$ & $\stackrel{5.3}{195}$ & $\begin{array}{l}13 \\
1957\end{array}$ & $\begin{array}{l}17 \\
1956\end{array}$ \\
\hline
\end{tabular}

MAGNITUDE AND FREQUENCY OF ANNUAL LOW FLOWS

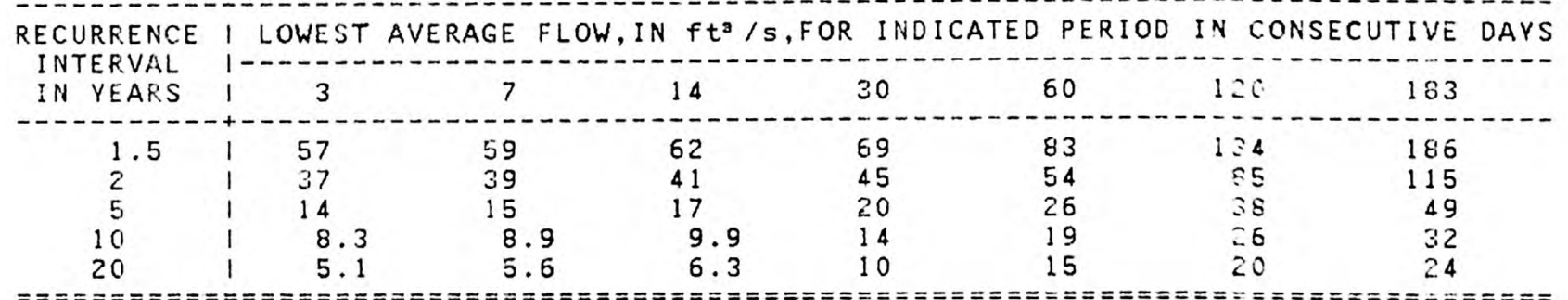

MRGNITUDE AND FREQUENCY OF SEASONAL LOW FLOWS

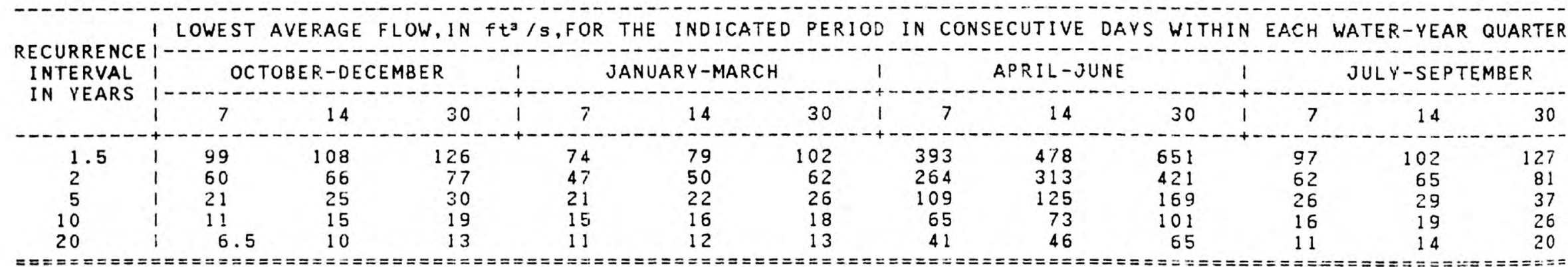

DURATION OF DAILY DISCHARGES FOR FNNUAL AND SEASONAL PERIODS

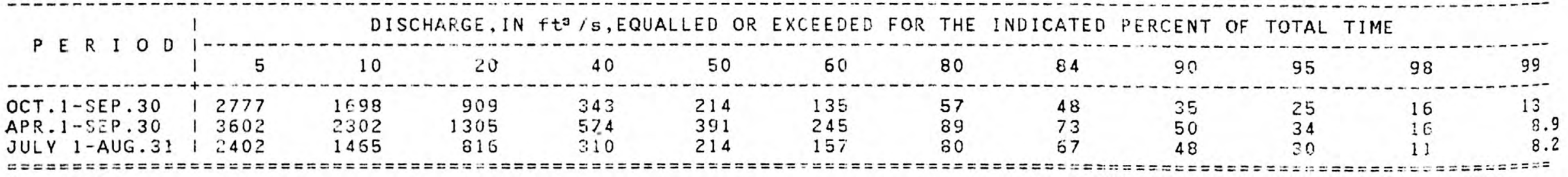




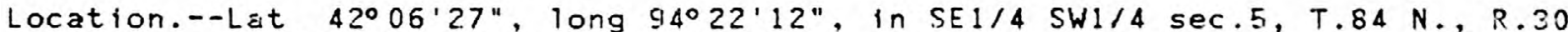
W., Greene County, on left bank $35 \mathrm{ft}$ upstream from bridge on couniy highway E26, 1.6 nities upstream from small left-bank tributary. 4.4 miles upstream from mouth, and $6.5 \mathrm{miles}$ southeast of Churdan.

LOW FLOW DISCHARGES, IN YEAR. ENDING MARCH 31

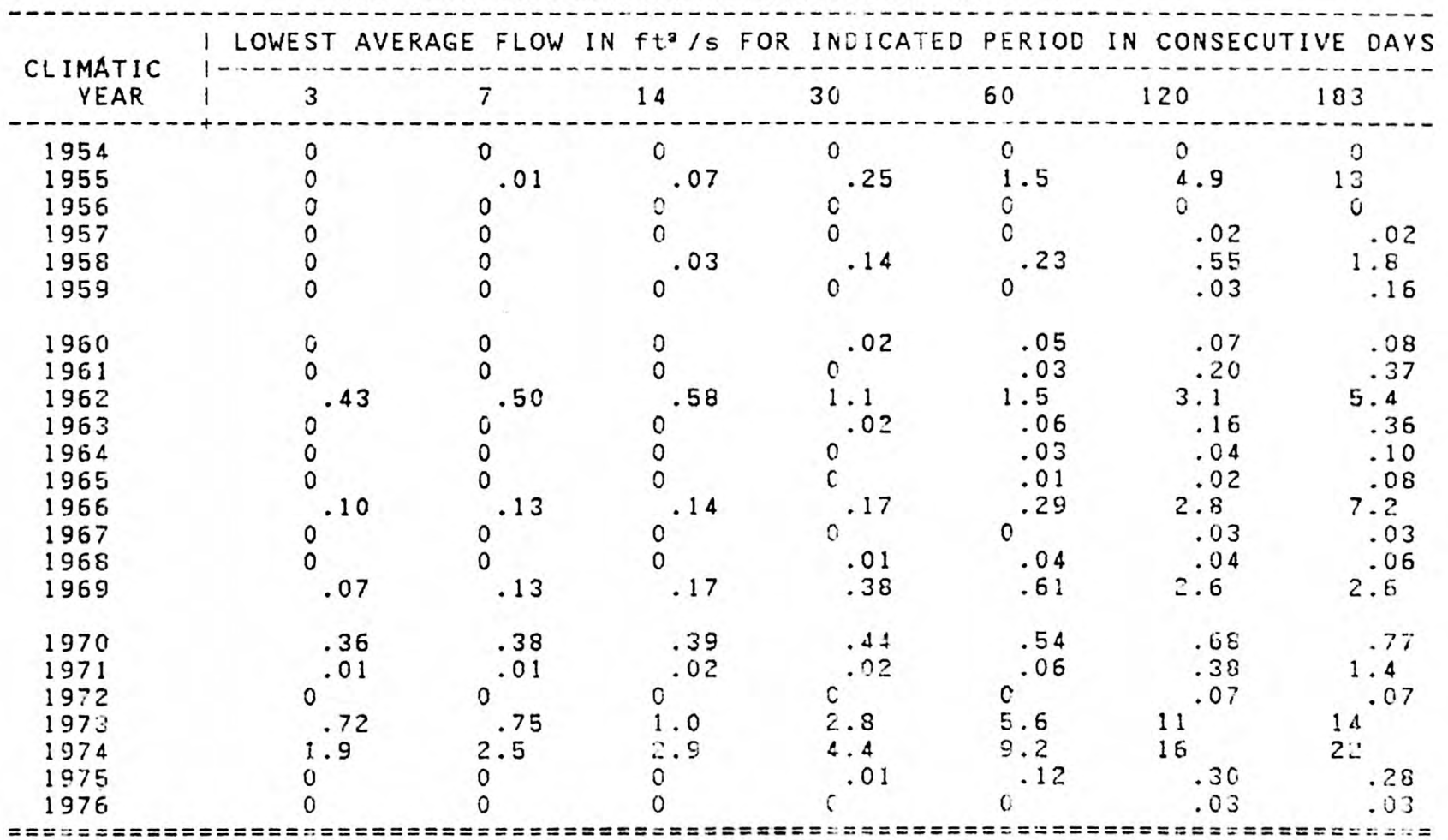




\section{DRAINAGE AREA: $24.0 \mathrm{~m}^{2} \quad$ PERIOD OF RECORD: 24 VEARS AVERAGE $0:$ SCHARGE: 9.6 ft3 $/ \mathrm{s}$}

MINIMUM AVERAGE FLOWS FOR PERIOD OF RECORD

PERIOD OF CONSECUTIVE DAYS I
DISCHARGE, IN $\mathrm{ft}^{\mathrm{s}} / \mathrm{s}$

MAGNITUDE AND FREQUENCY OF ANNUAL LOW FLOWS

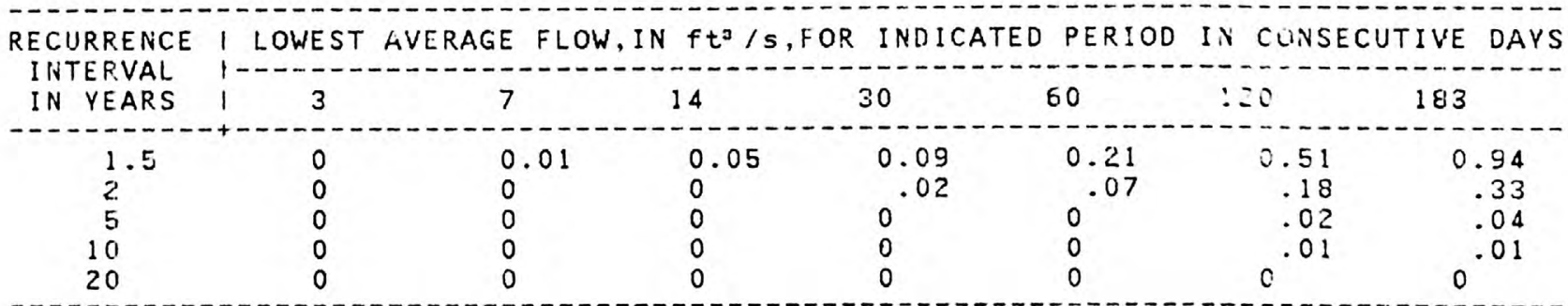

MAGNITUDE AND FREQUENCY OF SEASONAL LOW FLOWS

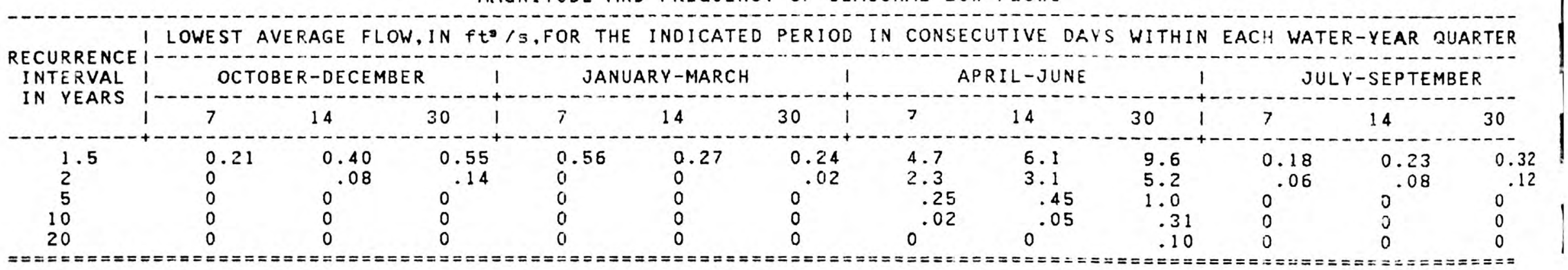

DURATION OF DAILY DISCHARGES FOR ANIUAL AND SEASONAL FERIODS

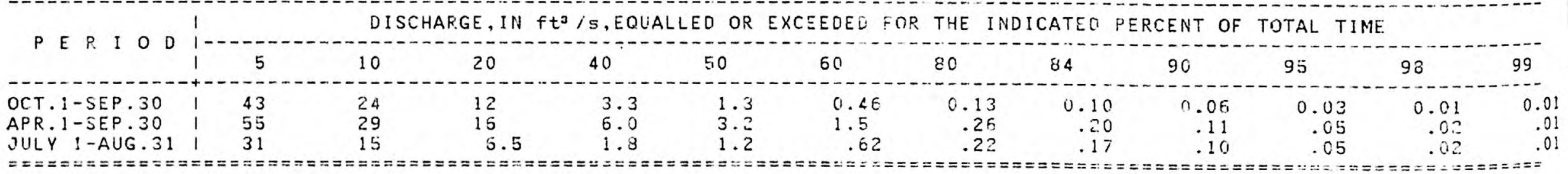


Location.--Lat $41^{\circ} 41^{\prime} 14^{\prime \prime}$, long $94^{\circ} 22^{\prime} 15^{\prime \prime}$, In NE1/4 NW1/4 sec.5, T.79 N., R.30 W., Guthrle County, on left bank $15 \mathrm{ft}$ downstream from bridge on county highway, 0.2 miles southwest of Panora, $1.5 \mathrm{miles}$ upstream from Andy's Branch, and 1.7 miles downstream from Lake Panorama.

Remarks.--City of Panora diverts approximately 100 acre-ft/yr upstream of station. Flow regulated by dam on Lake Panora since August 1970 .

LOW FLOW DISCHARGES, IN YEAR ENDING MARCH 31

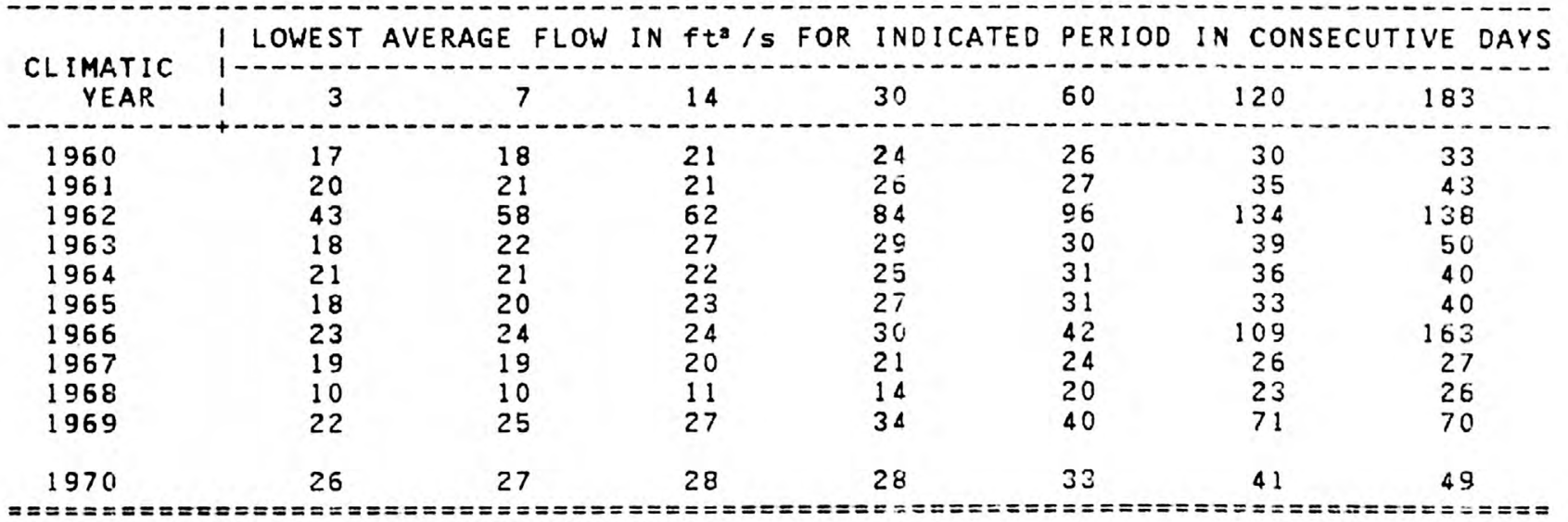




\section{DRAINAGE AREA: $440 \mathrm{~m}^{2}$ PERIOD OF RECORD: 12 YEARS AVERAGE DISCHARGE: 180ft3/S} MINIMUM AVERAGE FLOWS FOR PERIOD OF RECORD

\begin{tabular}{|c|c|c|c|c|c|c|c|}
\hline PERIOD OF CONSECUTIVE DAYS I & 3 & 7 & 14 & 30 & 50 & 120 & 183 \\
\hline $\begin{array}{l}\text { ISCHARGE, IN } \mathrm{ft}^{2} / \mathrm{s} \\
\text { LIMATIC YEAR }\end{array}$ & $\begin{array}{l}10 \\
1968\end{array}$ & $\begin{array}{l}10 \\
1968\end{array}$ & $\begin{array}{l}11 \\
1968\end{array}$ & $\begin{array}{l}14 \\
1968\end{array}$ & $\begin{array}{l}20 \\
1968\end{array}$ & $\begin{array}{l}23 \\
1968\end{array}$ & $\begin{array}{l}26 \\
196\end{array}$ \\
\hline
\end{tabular}

MAGNITUDE AND FREQUENCY OF ANNUAL LOW FLOWS

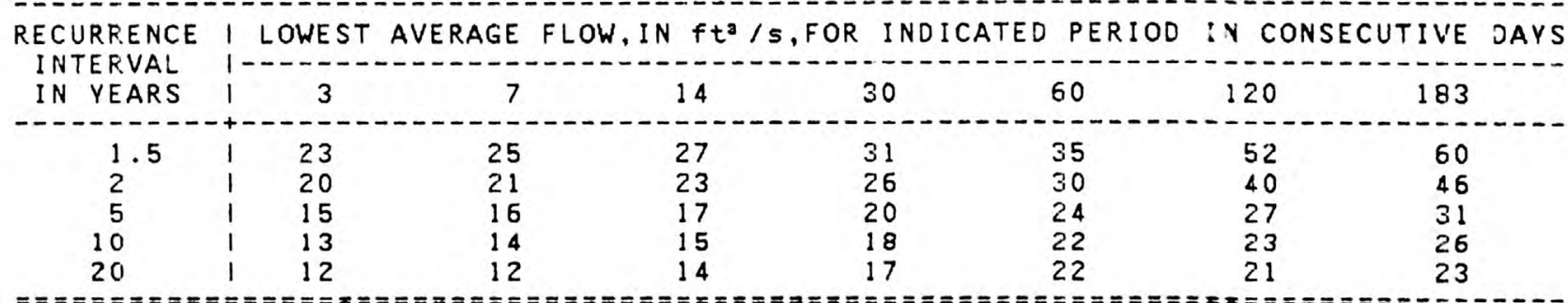

MAGNITUIDE AND FREQUENCY OF SEASONAL LOW FLOWS

LOWEST AVERAGE FLOW, IN $\mathrm{ft}^{3} / \mathrm{s}, \mathrm{FOR}$ THE INDICATED PERIOD IN CONSECUTIVE DAYS WITHIN EACH WATER-YEAR OUARTER

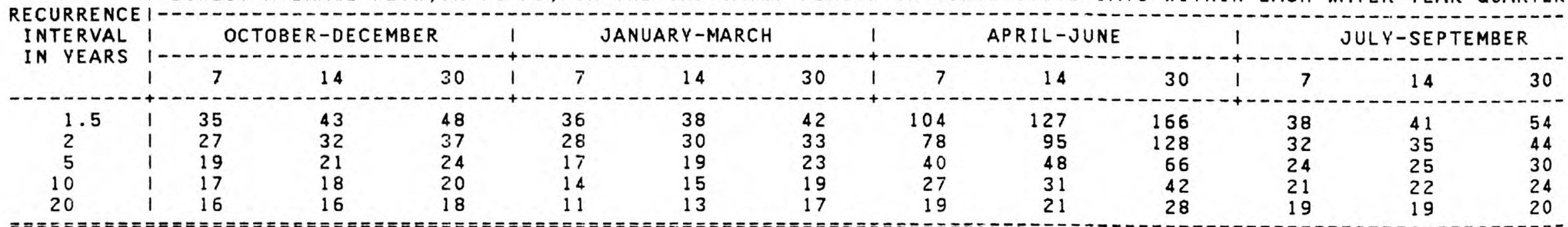

DURATION OF DAILY DISCHARGES FOR ANNUAL AND SEASONAL PERIODS

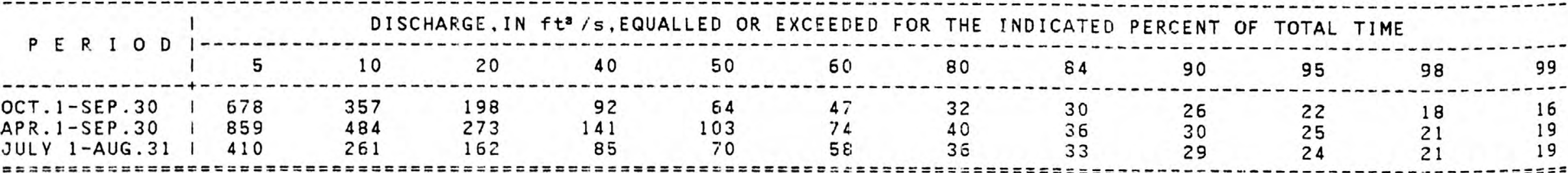


LOW FLOW DISCHARGES, IN VEAR ENDING MARCH 31

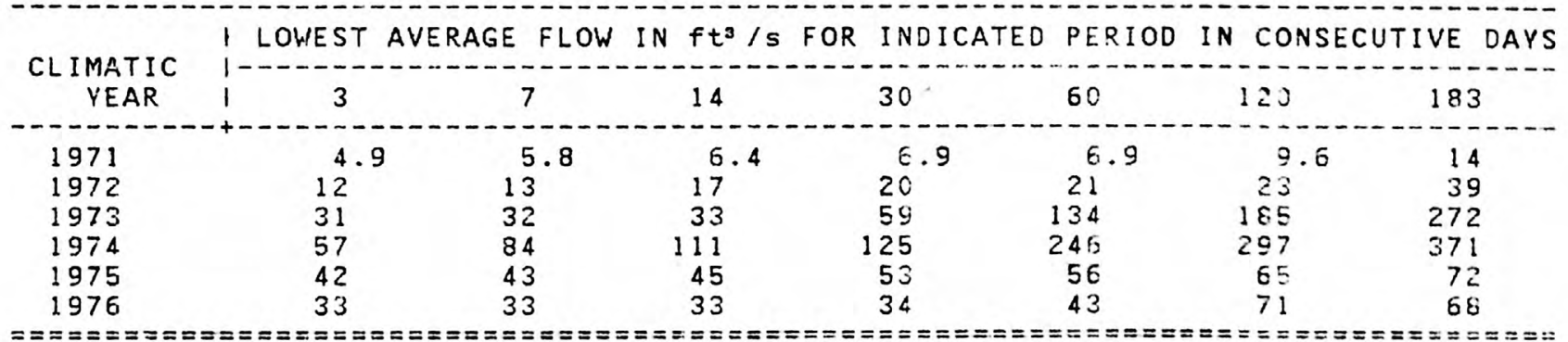




DRAINAGE AREA: $440 \mathrm{~m}^{2} \quad$ PERIOD OF RECORD: 6 VEARS AVERAGE DISCHARGE: 3O3 fta /s
PERIOD OF CONSECUTIVE DAYS I

MAGNITUDE AND FREQUENCY OF ANNUAL LOW FLOWS

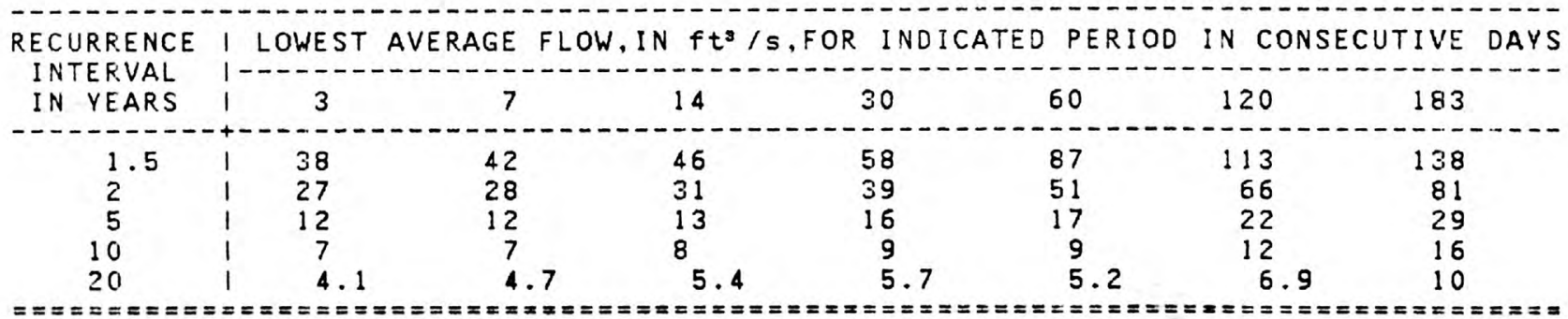

MAGNITUDE AND FREQUENCY OF SEASONAL LOW FLOWS

I LOWEST AVERAGE FLOW, IN $\mathrm{ft}^{\mathrm{s}} / \mathrm{s}, \mathrm{FOR}$ THE INDICATED PERIOD IN CONSECUTIVE DAYS WITHIN EACH WATER-YEAR QUARTER

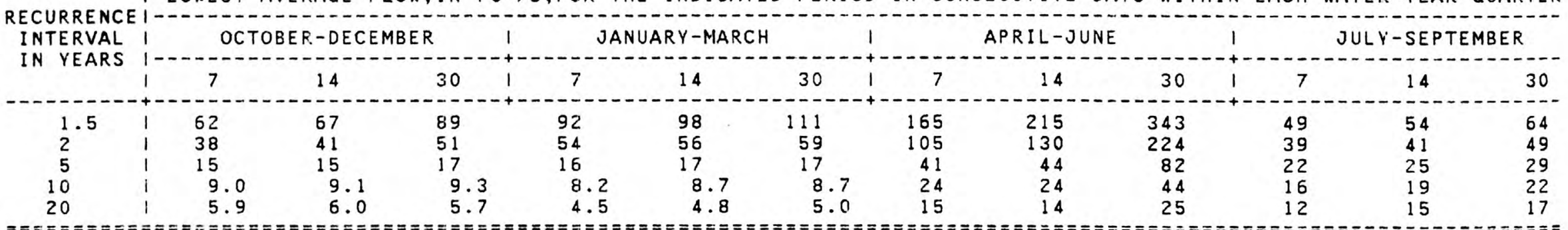

DURATION OF DAILY DISCHARGES FOR ANNUAL AND SEASONAL PERIODS

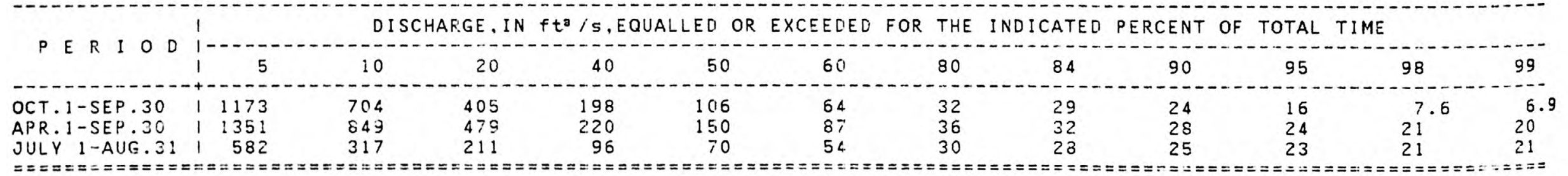




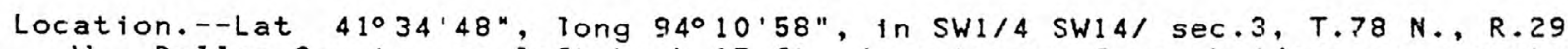
W. Dallas County, on left bank $15 \mathrm{ft}$ downstream from bridge on county highway at Redfleid, 0.8 mile downstream from bridge on U.S. Highway $6,1.0$ mile downstream from Middle Raccoon River, and 15.6 miles upstream from mouth.

LOW FLOW DISCHARGES, IN YEAR ENDING MARCH 31

\begin{tabular}{|c|c|c|c|c|c|c|c|}
\hline \multirow{2}{*}{$\begin{array}{c}\text { CLIMATIC } \\
\text { YEAR }\end{array}$} & I LOWEST & AGE & \multicolumn{2}{|c|}{ IN $\mathrm{ft}^{3} / \mathrm{s}$ FOR II } & PERIOD & \multicolumn{2}{|c|}{ IN CONSECUTIVE DAYS } \\
\hline & 3 & 7 & 14 & 30 & 60 & 120 & 183 \\
\hline $\begin{array}{l}1942 \\
1943 \\
1944 \\
1945 \\
1946 \\
1947 \\
1948 \\
1949\end{array}$ & $\begin{array}{r}31 \\
26 \\
32 \\
55 \\
40 \\
102 \\
30 \\
29\end{array}$ & $\begin{array}{r}33 \\
27 \\
36 \\
63 \\
41 \\
106 \\
31 \\
34\end{array}$ & $\begin{array}{r}48 \\
33 \\
38 \\
71 \\
46 \\
121 \\
37 \\
37\end{array}$ & $\begin{array}{r}54 \\
49 \\
46 \\
76 \\
60 \\
129 \\
48 \\
41\end{array}$ & $\begin{array}{r}92 \\
64 \\
66 \\
91 \\
78 \\
165 \\
71 \\
45\end{array}$ & $\begin{array}{r}140 \\
84 \\
82 \\
98 \\
103 \\
266 \\
109 \\
65\end{array}$ & $\begin{array}{r}175 \\
178 \\
117 \\
138 \\
192 \\
348 \\
100 \\
91\end{array}$ \\
\hline $\begin{array}{l}1950 \\
1951 \\
1952 \\
1953 \\
1954 \\
1955 \\
1956 \\
1957 \\
1958 \\
1959\end{array}$ & $\begin{array}{r}25 \\
36 \\
140 \\
67 \\
20 \\
35 \\
21 \\
31 \\
44 \\
45\end{array}$ & $\begin{array}{r}25 \\
37 \\
144 \\
79 \\
20 \\
36 \\
23 \\
36 \\
46 \\
45\end{array}$ & $\begin{array}{r}26 \\
38 \\
154 \\
87 \\
21 \\
37 \\
24 \\
36 \\
51 \\
45\end{array}$ & $\begin{array}{r}29 \\
43 \\
185 \\
91 \\
31 \\
42 \\
27 \\
44 \\
68 \\
48\end{array}$ & $\begin{array}{r}36 \\
44 \\
203 \\
100 \\
40 \\
81 \\
33 \\
54 \\
84 \\
51\end{array}$ & $\begin{array}{r}52 \\
49 \\
275 \\
109 \\
50 \\
133 \\
33 \\
75 \\
111 \\
65\end{array}$ & $\begin{array}{r}77 \\
58 \\
375 \\
183 \\
53 \\
202 \\
34 \\
79 \\
156 \\
139\end{array}$ \\
\hline $\begin{array}{l}1960 \\
1961 \\
1962 \\
1963 \\
1964 \\
1965 \\
1966 \\
1967 \\
1968 \\
1969\end{array}$ & $\begin{array}{r}49 \\
46 \\
104 \\
39 \\
47 \\
48 \\
53 \\
42 \\
23 \\
26\end{array}$ & $\begin{array}{r}50 \\
47 \\
117 \\
48 \\
48 \\
53 \\
54 \\
46 \\
24 \\
37\end{array}$ & $\begin{array}{r}57 \\
50 \\
125 \\
60 \\
48 \\
64 \\
57 \\
50 \\
26 \\
41\end{array}$ & $\begin{array}{r}74 \\
63 \\
180 \\
68 \\
57 \\
69 \\
70 \\
51 \\
34 \\
56\end{array}$ & $\begin{array}{r}75 \\
63 \\
231 \\
71 \\
71 \\
80 \\
102 \\
57 \\
47 \\
79\end{array}$ & $\begin{array}{r}89 \\
83 \\
320 \\
92 \\
80 \\
87 \\
250 \\
59 \\
54 \\
110\end{array}$ & $\begin{array}{r}102 \\
119 \\
329 \\
114 \\
87 \\
136 \\
337 \\
60 \\
60 \\
120\end{array}$ \\
\hline $\begin{array}{l}1970 \\
1971 \\
1972 \\
1973 \\
1974 \\
1975 \\
1976\end{array}$ & $\begin{array}{r}62 \\
26 \\
36 \\
74 \\
197 \\
73 \\
99\end{array}$ & $\begin{array}{r}63 \\
27 \\
36 \\
75 \\
240 \\
74 \\
101\end{array}$ & $\begin{array}{r}65 \\
28 \\
38 \\
77 \\
266 \\
75 \\
105\end{array}$ & $\begin{array}{r}66 \\
31 \\
43 \\
158 \\
282 \\
80 \\
108\end{array}$ & $\begin{array}{r}77 \\
46 \\
50 \\
413 \\
501 \\
103 \\
141\end{array}$ & $\begin{array}{r}101 \\
83 \\
64 \\
456 \\
595 \\
125 \\
170\end{array}$ & $\begin{array}{r}120 \\
79 \\
74 \\
533 \\
728 \\
143 \\
188\end{array}$ \\
\hline
\end{tabular}




DRAINAGE AREA: $988 \mathrm{mi}^{2}$
MINIMUM AVERAGE FLOWS FOR PERIOD OF RECORD

MAGNITUDE AND FREQUENCY OF ANNUAL LOW FLOWS

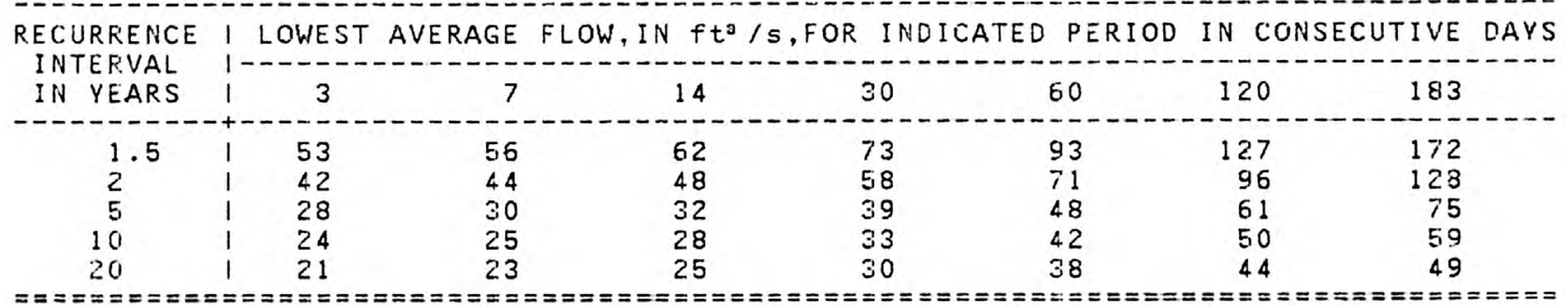

MAGNITUDE AND FREQUENCY OF SEASONAL LOW FLOWS

RECURRENCE I LOWEST AVERAGE FLOW, IN Ft / S,FOR THE INDICATED PERIOD IN CONSECUTIVE DAYS WITHIN EACH WATER-YEAR QUARTER
INTERVAL
IN YEARS

DURATION OF DAILY DISCHARGES FOR ANNUAL AND SEASONAL PERIODS

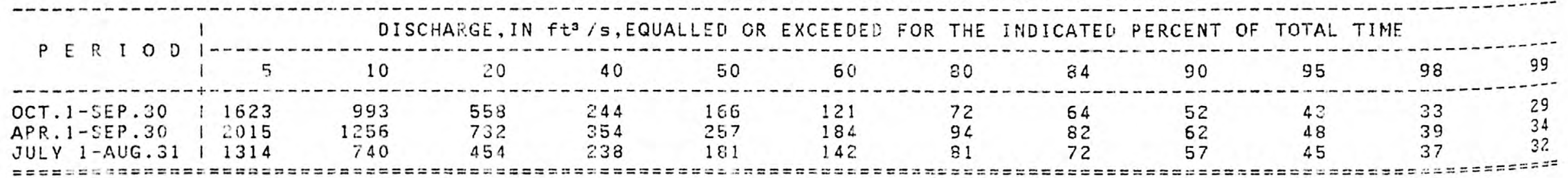




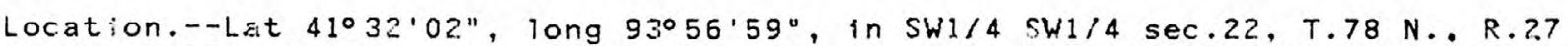
W., Dallas County, on right bank $10 \mathrm{ft}$ downstream from bridge on county highway R.16, $0.3 \mathrm{mile}$ northeast of Van Meter, $0.7 \mathrm{mile}$ upstream from small left bank tributary, 1.2 miles downstream from confluence of North and South Raccoon River, and $30 \mathrm{miles}$ upstream from mouth.

LOW FLOW DISCHARGES, IN YEAR. ENDING MARCH 31

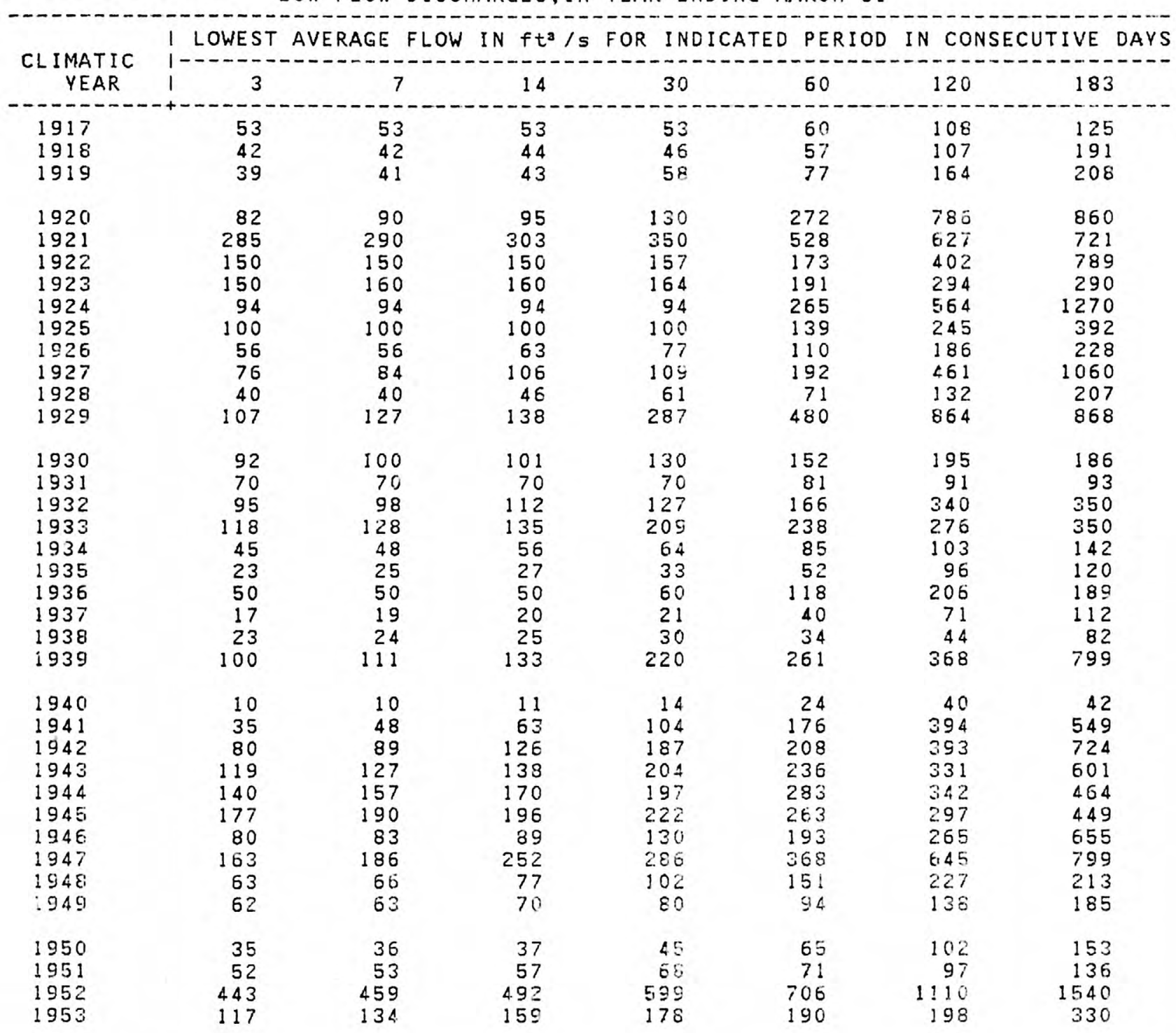


204

$05-4845.00$

RACCOON RIVER AT VAN METER--Cont inued

LOW FLOW OISCHARGES, IN YEAR ENDING MARCH 31--Cont inued

\begin{tabular}{|c|c|c|c|c|c|c|c|}
\hline \multirow{2}{*}{$\begin{array}{c}\text { CLIMATIC } \\
\text { YEAR }\end{array}$} & LOWEST & AGE & IN $f t^{3}$ & OR IND & ED PEF & \multicolumn{2}{|c|}{ IN CONSECUTIVE DAYS } \\
\hline & i & 7 & 14 & 30 & 60 & 120 & 183 \\
\hline $\begin{array}{l}1954 \\
1955 \\
1956 \\
1957 \\
1958 \\
1959\end{array}$ & $\begin{array}{r}45 \\
190 \\
42 \\
35 \\
99 \\
70\end{array}$ & $\begin{array}{r}45 \\
190 \\
44 \\
36 \\
105 \\
71\end{array}$ & $\begin{array}{r}47 \\
192 \\
46 \\
38 \\
116 \\
73\end{array}$ & $\begin{array}{r}56 \\
217 \\
49 \\
58 \\
152 \\
79\end{array}$ & $\begin{array}{r}76 \\
340 \\
52 \\
85 \\
165 \\
92\end{array}$ & $\begin{array}{r}98 \\
686 \\
57 \\
112 \\
226 \\
125\end{array}$ & $\begin{array}{r}114 \\
1110 \\
61 \\
123 \\
333 \\
283\end{array}$ \\
\hline $\begin{array}{l}1960 \\
1961 \\
1962 \\
1963 \\
1964 \\
1965 \\
1966 \\
1967 \\
1968 \\
1969\end{array}$ & $\begin{array}{r}111 \\
100 \\
320 \\
170 \\
91 \\
91 \\
139 \\
71 \\
50 \\
78\end{array}$ & $\begin{array}{r}117 \\
101 \\
343 \\
170 \\
93 \\
107 \\
143 \\
72 \\
51 \\
109\end{array}$ & $\begin{array}{r}134 \\
106 \\
360 \\
174 \\
98 \\
152 \\
145 \\
76 \\
55 \\
142\end{array}$ & $\begin{array}{r}165 \\
126 \\
479 \\
203 \\
107 \\
166 \\
165 \\
83 \\
70 \\
212\end{array}$ & $\begin{array}{r}166 \\
132 \\
706 \\
226 \\
125 \\
189 \\
244 \\
94 \\
94 \\
286\end{array}$ & $\begin{array}{l}221 \\
195 \\
934 \\
319 \\
150 \\
190 \\
726 \\
108 \\
107 \\
475\end{array}$ & $\begin{array}{r}279 \\
299 \\
917 \\
766 \\
171 \\
259 \\
1160 \\
113 \\
125 \\
441\end{array}$ \\
\hline $\begin{array}{l}1970 \\
1971 \\
1972 \\
1973 \\
1974 \\
1975 \\
1976\end{array}$ & $\begin{array}{r}189 \\
100 \\
83 \\
351 \\
939 \\
178 \\
143\end{array}$ & $\begin{array}{r}193 \\
104 \\
87 \\
365 \\
1050 \\
179 \\
199\end{array}$ & $\begin{array}{r}202 \\
109 \\
89 \\
393 \\
1050 \\
180 \\
205\end{array}$ & $\begin{array}{r}212 \\
123 \\
95 \\
531 \\
1400 \\
187 \\
211\end{array}$ & $\begin{array}{r}242 \\
162 \\
99 \\
1590 \\
1520 \\
209 \\
266\end{array}$ & $\begin{array}{r}335 \\
231 \\
158 \\
2000 \\
2280 \\
246 \\
309\end{array}$ & $\begin{array}{r}385 \\
311 \\
151 \\
2100 \\
3060 \\
269 \\
368\end{array}$ \\
\hline
\end{tabular}


DRAINAGE AREA: $3441 \mathrm{~m}^{2}$ PERIOD OF RECORD: 61 YEARS AVERAGE DISCHARGE: 1309 ft3/s MINIMUM AVERAGE FLOWS FOR PERIOD OF RECORD

\begin{tabular}{|c|c|c|c|c|c|c|c|}
\hline PERIOD OF CONSECUTIVE DAYS & 1 & 7 & 14 & 30 & 60 & 120 & 183 \\
\hline $\begin{array}{l}\text { DISCHARGE, IN } \mathrm{ft}^{3} / \mathrm{s} \\
\text { CLIMATIC YEAR }\end{array}$ & $\begin{array}{l}10 \\
1940\end{array}$ & $\begin{array}{l}10 \\
1940\end{array}$ & $\begin{array}{l}11 \\
1940\end{array}$ & $\begin{array}{l}14 \\
1940\end{array}$ & $\begin{array}{l}24 \\
1940\end{array}$ & $\begin{array}{l}40 \\
1940\end{array}$ & $\begin{array}{l}42 \\
1940\end{array}$ \\
\hline
\end{tabular}

MAGNITUDE AND FREQUENCY OF ANNUAL LOW FLOWS

$\begin{aligned} & \text { RECURRENCE } \\ & \text { INTERVAL }\end{aligned}$
IN YOWEARS

MAGNITUDE AND FREQUENCY OF SEASONAL LOW FLOWS

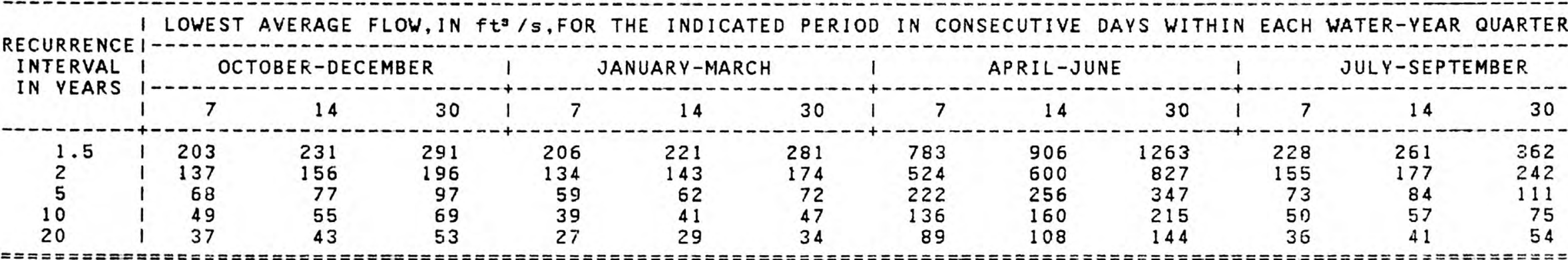

DURATION OF DAILY DISCHARGES FOR ANNUAL AND SEASONAL PERIODS

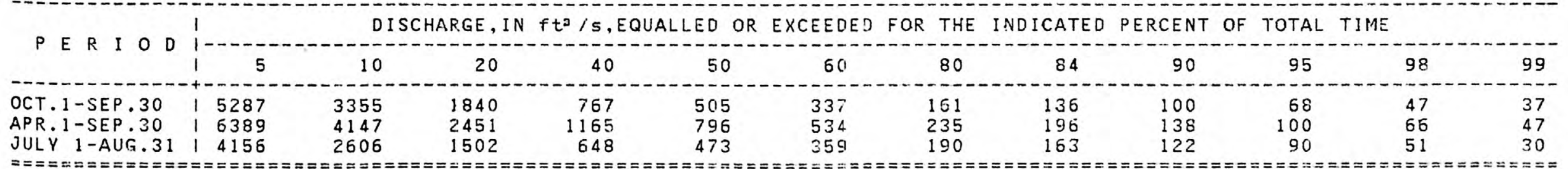


Location.--Lat $41^{\circ} 35^{\prime} 14^{\prime \prime}$, long $93^{\circ} 42^{\prime} 11^{\prime \prime}$, in SWl/4 SE1/4 sec.2, T.78 N., R.25 W. Polk County, on left bank, $25 \mathrm{ft}$ downstream from bridge on $63 \mathrm{rd}$ Street in Des Molnes, and 2.2 miles upstream from Raccoon River.

Dralnage area. $--78.4 \mathrm{mi}^{2}$.

LOW FLOW DISCHARGES, IN YEAR. ENDING MARCH 31

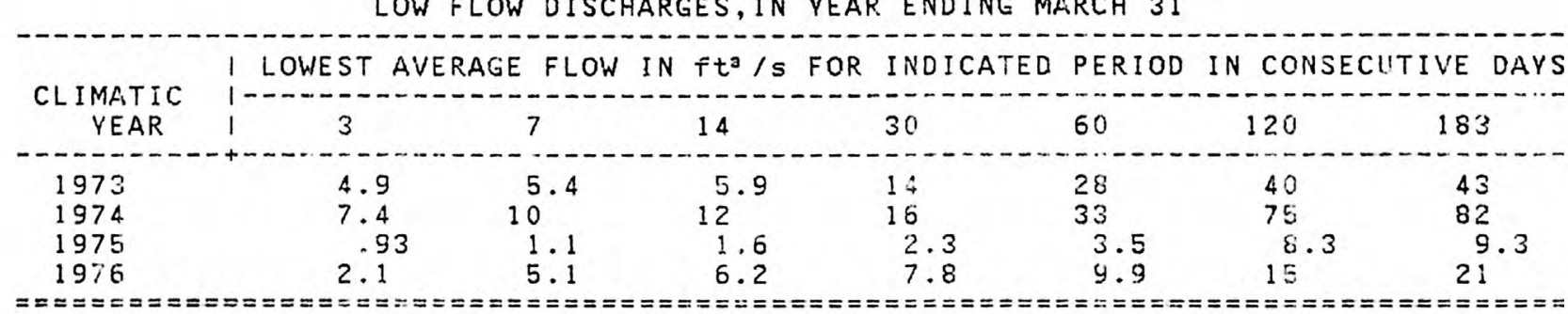

$Q \mathbf{a}=$

$Q(84)=0.4$

$Q\{7,2\}=0.2$

$Q\{7,10\}=0$ 
Location.--Lat $41^{\circ} 34^{\prime} 30^{\prime \prime}$, long $93^{\circ} 35^{\prime} 48^{\prime \prime}$, In NE1/4 SE1/4 sec.10, T.78 N., R.24 W. , Polk County, on right bank $10 \mathrm{ft}$ downstream from bridge on Southeast 14 th Street at Des Moines, $0.8 \mathrm{~m} 1$ le downst.ream from Raccoon River and Scott Street Dam, and at mile $200 . \overline{7}$.

Remarks.--Des Molnes muntcipal water supply is taken from infiltration galleries on Raccoon River, $3.5 \mathrm{mlles}$ above station. Average daily pumpage since 1963 was about $50 \mathrm{cfs}$. At times, water is pumped from Raccoon River into recharge basins, or into Waterworks Reservoir (capacity, 4,800 acre$\mathrm{ft}$. Effluent from sewage treatment plant enters the river $2.3 \mathrm{miles}$ downstream from station. Net effect of diversions not known.

LOW FLOW DISCHARGES, IN YEAR ENDING MARCH 31

\begin{tabular}{|c|c|c|c|c|c|c|c|}
\hline \multirow{2}{*}{$\underset{\text { YEAR }}{\text { CLIMATIC }}$} & 1 LOWES & RAGE & IN $f t^{3}$ & OR IN & ED PEF & IN CON & TIVE \\
\hline & 13 & 7 & 14 & 30 & 60 & 120 & 183 \\
\hline $\begin{array}{l}1941 \\
1942 \\
1943 \\
1944 \\
1945 \\
1946 \\
1947 \\
1948 \\
1949\end{array}$ & $\begin{array}{r}85 \\
227 \\
393 \\
480 \\
400 \\
272 \\
483 \\
149 \\
152\end{array}$ & $\begin{array}{l}103 \\
260 \\
410 \\
542 \\
407 \\
283 \\
557 \\
157 \\
156\end{array}$ & $\begin{array}{l}144 \\
345 \\
463 \\
562 \\
425 \\
308 \\
757 \\
163 \\
162\end{array}$ & $\begin{array}{l}275 \\
496 \\
550 \\
614 \\
452 \\
369 \\
887 \\
218 \\
173\end{array}$ & $\begin{array}{r}516 \\
720 \\
675 \\
872 \\
528 \\
492 \\
1010 \\
346 \\
210\end{array}$ & $\begin{array}{r}953 \\
1910 \\
1260 \\
1080 \\
705 \\
713 \\
1720 \\
532 \\
310\end{array}$ & $\begin{array}{r}1450 \\
2830 \\
2220 \\
1430 \\
1240 \\
1600 \\
1990 \\
501 \\
461\end{array}$ \\
\hline $\begin{array}{l}1950 \\
1951 \\
1952 \\
1953 \\
1954 \\
1955 \\
1956 \\
1957 \\
1958 \\
1959\end{array}$ & $\begin{array}{r}80 \\
120 \\
920 \\
215 \\
110 \\
500 \\
77 \\
56 \\
217 \\
109\end{array}$ & $\begin{array}{r}84 \\
120 \\
977 \\
261 \\
110 \\
506 \\
80 \\
60 \\
239 \\
110\end{array}$ & $\begin{array}{r}91 \\
126 \\
1090 \\
320 \\
113 \\
519 \\
83 \\
64 \\
288 \\
110\end{array}$ & $\begin{array}{r}108 \\
155 \\
1480 \\
366 \\
132 \\
582 \\
88 \\
85 \\
354 \\
115\end{array}$ & $\begin{array}{r}134 \\
174 \\
1730 \\
378 \\
175 \\
894 \\
95 \\
125 \\
436 \\
138\end{array}$ & $\begin{array}{r}215 \\
270 \\
2420 \\
392 \\
246 \\
1620 \\
108 \\
143 \\
535 \\
215\end{array}$ & $\begin{array}{r}306 \\
468 \\
3550 \\
685 \\
318 \\
2540 \\
127 \\
170 \\
672 \\
455\end{array}$ \\
\hline $\begin{array}{l}1960 \\
1961 \\
1962 \\
1963 \\
1964 \\
1965 \\
1966 \\
1967 \\
1968 \\
1969\end{array}$ & $\begin{array}{r}287 \\
245 \\
707 \\
300 \\
190 \\
320 \\
456 \\
145 \\
70 \\
252\end{array}$ & $\begin{array}{r}333 \\
249 \\
898 \\
304 \\
190 \\
340 \\
466 \\
160 \\
74 \\
279\end{array}$ & $\begin{array}{r}373 \\
260 \\
977 \\
316 \\
194 \\
459 \\
483 \\
168 \\
83 \\
320\end{array}$ & $\begin{array}{r}556 \\
305 \\
1240 \\
371 \\
221 \\
555 \\
541 \\
184 \\
115 \\
510\end{array}$ & $\begin{array}{r}562 \\
317 \\
1470 \\
459 \\
269 \\
639 \\
959 \\
214 \\
176 \\
746\end{array}$ & $\begin{array}{r}700 \\
526 \\
2130 \\
814 \\
356 \\
865 \\
2060 \\
245 \\
210 \\
1450\end{array}$ & $\begin{array}{r}896 \\
754 \\
2310 \\
1670 \\
439 \\
1600 \\
3470 \\
259 \\
269 \\
1280\end{array}$ \\
\hline $\begin{array}{l}1970 \\
1971 \\
1972 \\
1973\end{array}$ & $\begin{array}{r}421 \\
346 \\
211 \\
1490\end{array}$ & $\begin{array}{r}444 \\
565 \\
223 \\
1550\end{array}$ & $\begin{array}{r}481 \\
376 \\
234 \\
1630\end{array}$ & $\begin{array}{r}523 \\
4.44 \\
251 \\
1860\end{array}$ & $\begin{array}{r}576 \\
534 \\
272 \\
3560\end{array}$ & $\begin{array}{r}818 \\
809 \\
571 \\
4870\end{array}$ & $\begin{array}{r}1040 \\
955 \\
567 \\
5290\end{array}$ \\
\hline
\end{tabular}


DES MOINES RIVER BELOW RACCOON RIVER AT DES MOINES--Cont inued

LOW FLOW DISCHARGES, IN YEAR ENDING MARCH 31--Cont tnued

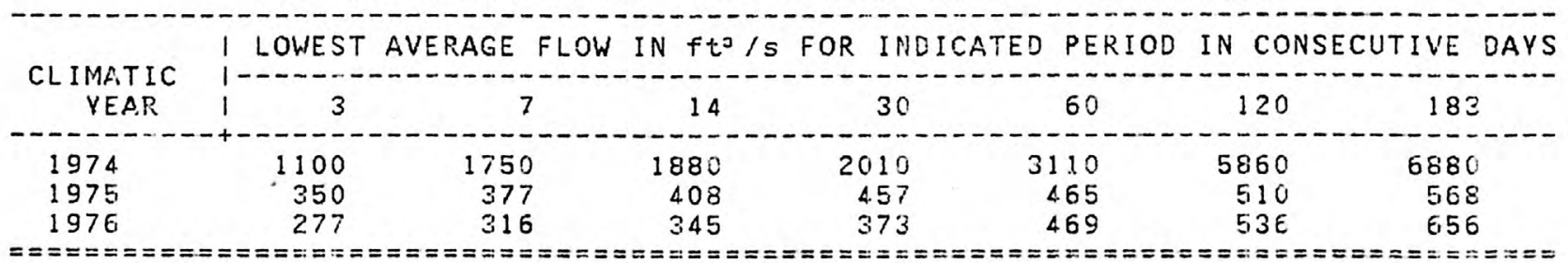




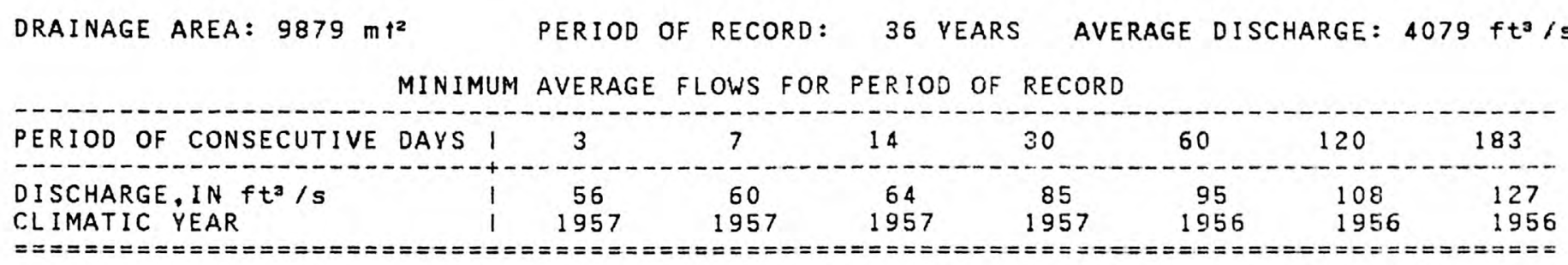

MAGNITUDE AND FREQUENCY OF ANNUAL LOW FLOWS

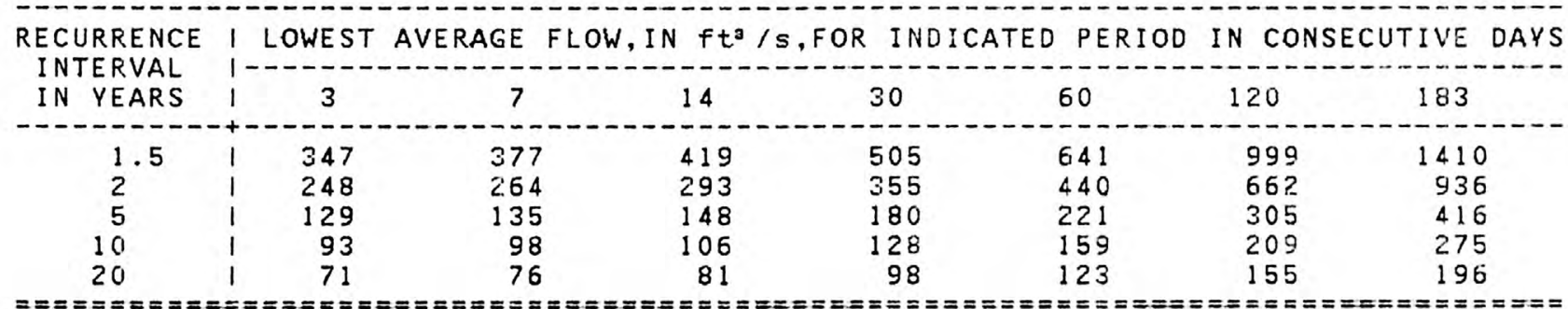

MAGNITUDE AND FREQUENCY OF SEASONAL LOW FLOWS

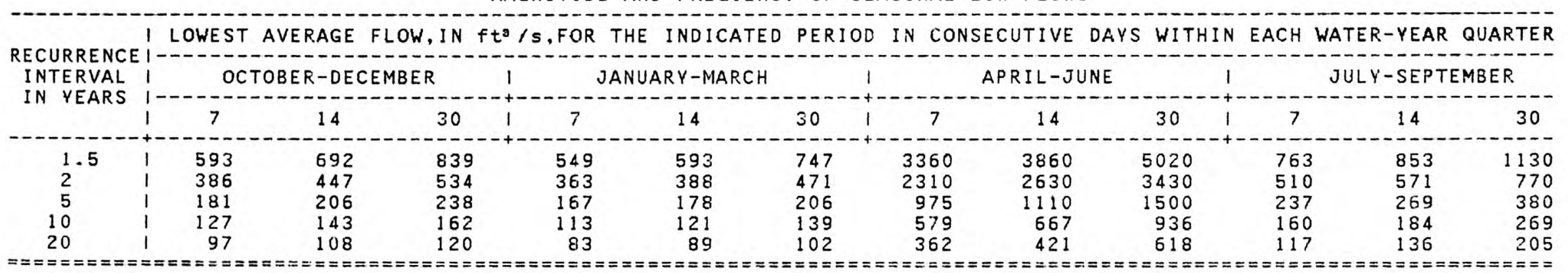

DURATION OF DAILY DISCHARGES FOR ANNUAL ANO SEASONAL FERIODS

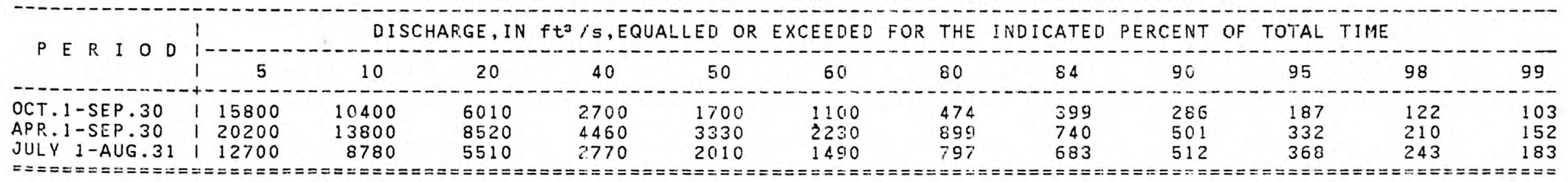




\section{FOURMILE CREEK AT DES MOINES}

Location.--Lat 41036'50", long 93032'43", in NE1/4 NE1/4 sec.32. T.79 N., R.23 W. Polk County, on right bank $20 \mathrm{ft}$ downstream from bridge on Easton Bivd., 4.4 miles downstream from Muchikinock Creek and $5.0 \mathrm{miles}$ upstream from Des Moines River.

Drainage area. $--92.6 \mathrm{~m}^{2}$.

LOW FLOW DISCHARGES, IN YEAR. ENDING MARCH 31

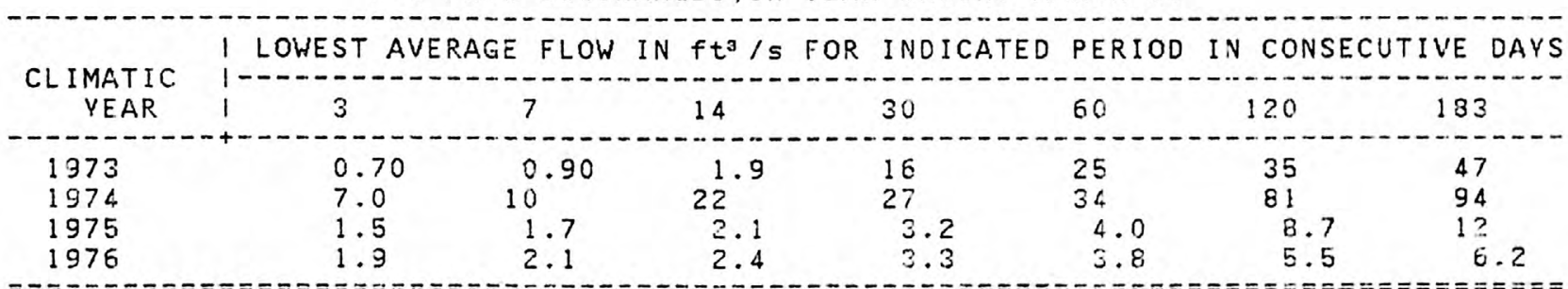

$0 a=48$

$Q(84)=0.5$

$7 Q 2=0.3$

$7010=*$ 
Location.--Lat 41027'25", long 93039'10", In NW1/4 SW1/4 sec.20, T.77 N.,

R. 24 W., Warren County, on left bank io $\mathrm{ft}$ downstream from bridge on county highway R57, 1.7 miles southeast of Norwalk, $5.2 \mathrm{miles}$ upstream from Pliddle Creek, and $6.2 \mathrm{miles}$ downstream from Badger Creek.

LOW FLOW DISCHARGES, IN VEAR. ENDING MARCH 31

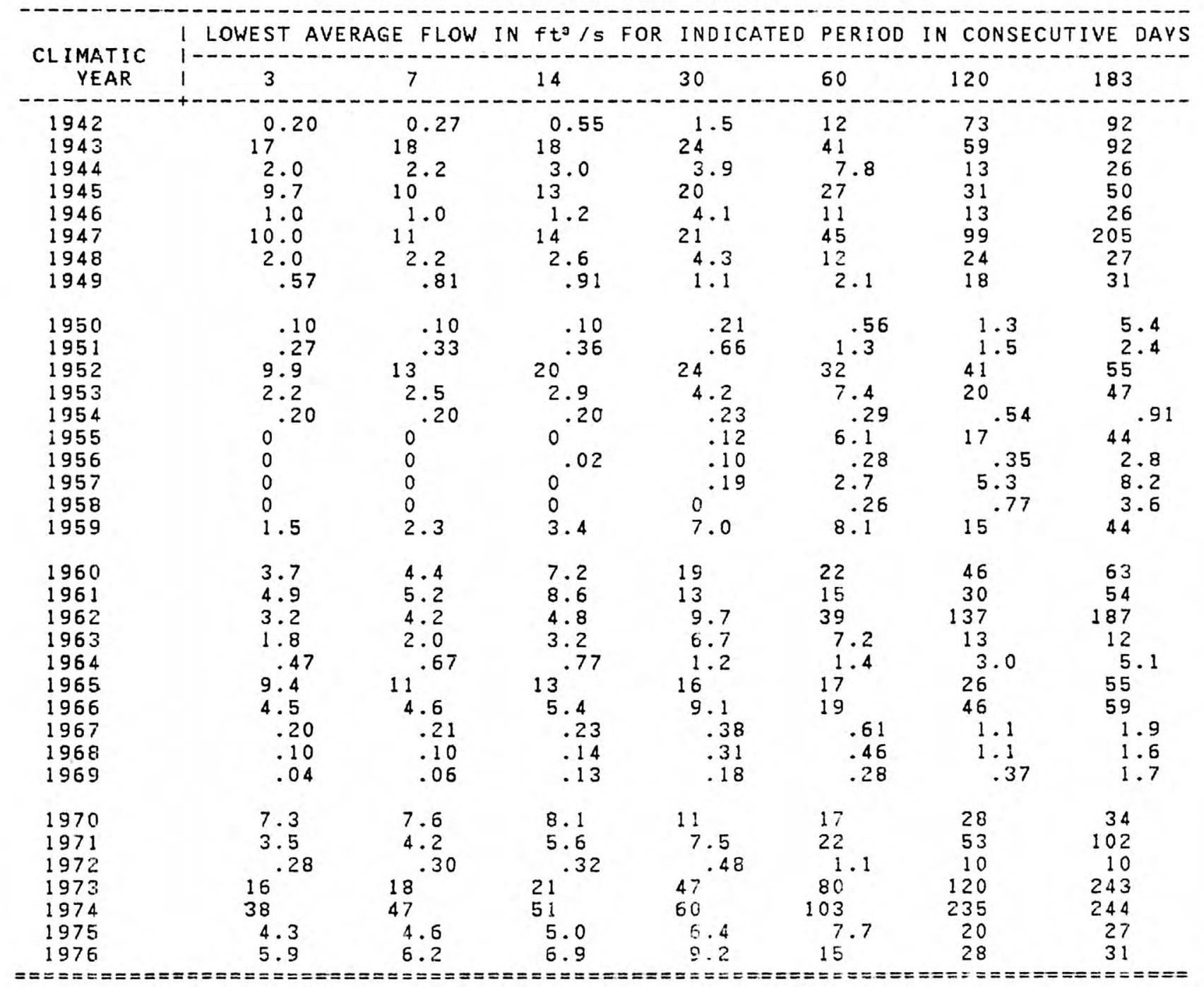


MINIMUM AVERAGE FLOWS FOR PERIOD OF RECORD

\begin{tabular}{|c|c|c|c|c|c|c|c|}
\hline D OF CONSECUTIVE DAYS I & 3 & 7 & 14 & 30 & 60 & 120 & 183 \\
\hline $\begin{array}{l}\text { RGE, IN } f^{3} / \mathrm{s} \\
\text { IC YEAR }\end{array}$ & $\begin{array}{c}0 \\
1 . \quad 1958 \\
\end{array}$ & $\begin{array}{c}0 \\
1958 \\
=====\end{array}$ & $\begin{array}{l}0 \\
1958\end{array}$ & $\begin{array}{l}0 \\
1958 \\
=====\end{array}$ & $\begin{array}{c}1.26 \\
1958 \\
=====\end{array}$ & $\begin{array}{c}0.36 \\
1956 \\
===-==\end{array}$ & \\
\hline
\end{tabular}

MAGNITUDE AND FREQUENCY OF ANNUAL LOW FLOWS

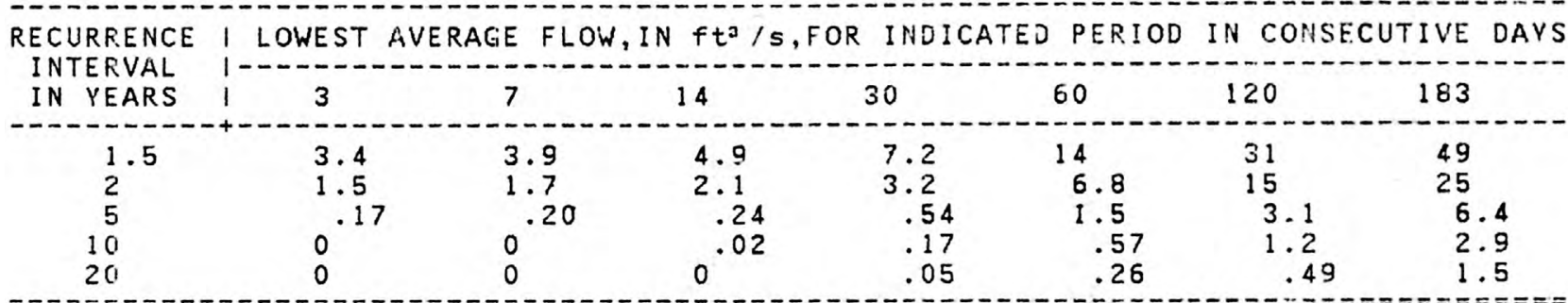

MAGNITUDE AND FREQUENCY OF SEASONAL LOW FLOWS

LOWEST AVERAGE FLOW, IN $\mathrm{ft}^{3} / \mathrm{s}, \mathrm{FOR}$ THE INDICATED PERIOD IN CONSECUTIVE DAYS WITHIN EACH WATER-YEAR QUARTER

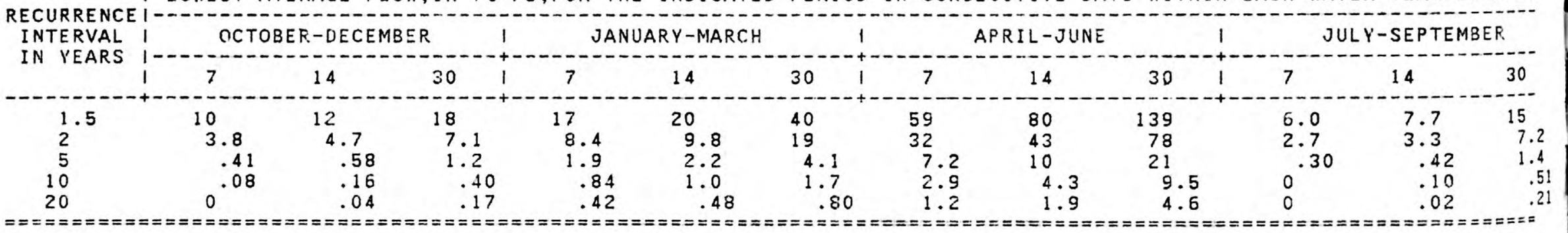

DURATION OF DAILY DISCHARGES FOR ANHUAL AND SEASONAL PERIODS

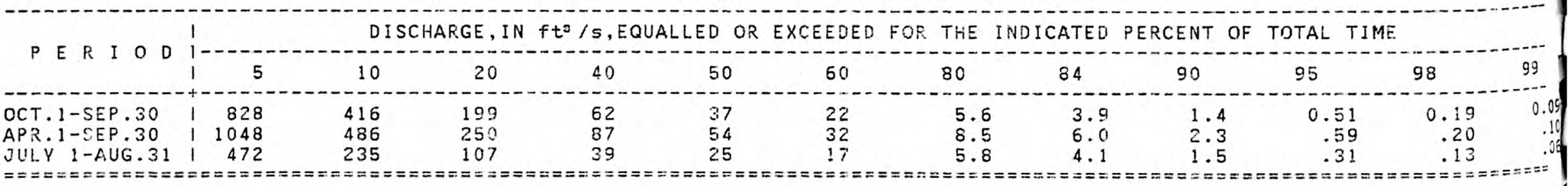




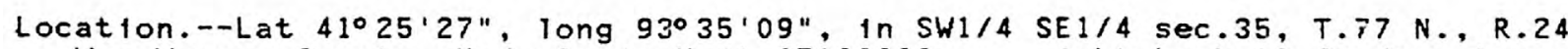
W. Warren County, Hydrologlc Unit 07100008 , on right bank 10 ft downstream from bridge on county highway, 0.4 mile upstream from Cavitt Creek, 1.5 miles upstream from bridge on U.S. Highway 69 , and 4.6 miles northwest of Indianola.

LOW FLOW DISCHARGES, IN YEAR. ENDING MARCH 31

\begin{tabular}{|c|c|c|c|c|c|c|c|}
\hline \multirow{2}{*}{$\begin{array}{c}\text { CLIMATIC } \\
\text { YEAR }\end{array}$} & \multicolumn{2}{|c|}{ I LOWEST AVERAGE FLOW } & $f t^{3} / s$ FOR & INDICATED & PERIOD & \multicolumn{2}{|c|}{ IN CONSECUTIVE DAVS } \\
\hline & 13 & 7 & 14 & 30 & 60 & 120 & 183 \\
\hline $\begin{array}{l}1942 \\
1943 \\
1944 \\
1945 \\
1946 \\
1947 \\
1948 \\
1949\end{array}$ & $\begin{array}{r}11 \\
27 \\
3.7 \\
13 \\
2.0 \\
30 \\
4.7 \\
3.0\end{array}$ & $\begin{array}{c}12 \\
32 \\
6.3 \\
17 \\
2.2 \\
31 \\
5.0 \\
3.1\end{array}$ & $\begin{array}{c}13 \\
39 \\
8.9 \\
24 \\
2.5 \\
34 \\
5.5 \\
4.3\end{array}$ & $\begin{array}{c}17 \\
44 \\
14 \\
35 \\
6.4 \\
38 \\
6.9 \\
7.3\end{array}$ & $\begin{array}{l}32 \\
58 \\
18 \\
45 \\
16 \\
71 \\
17 \\
15\end{array}$ & $\begin{array}{r}178 \\
74 \\
27 \\
55 \\
20 \\
137 \\
33 \\
33\end{array}$ & $\begin{array}{r}222 \\
110 \\
39 \\
117 \\
47 \\
224 \\
38 \\
43\end{array}$ \\
\hline $\begin{array}{l}1950 \\
1951 \\
1952 \\
1953 \\
1954 \\
1955 \\
1956 \\
1957 \\
1958 \\
1959\end{array}$ & $\begin{array}{l}4.6 \\
3.4 \\
15 \\
16 \\
1.7 \\
8.0 \\
1.2 \\
1.0 \\
1.6 \\
11\end{array}$ & $\begin{array}{l}4.8 \\
3.4 \\
19 \\
16 \\
1.8 \\
9.0 \\
1.3 \\
1.1 \\
1.8 \\
12\end{array}$ & $\begin{array}{r}5.2 \\
3.5 \\
29 \\
17 \\
1.9 \\
9.3 \\
1.4 \\
1.2 \\
2.3 \\
13\end{array}$ & $\begin{array}{l}6.5 \\
3.7 \\
42 \\
19 \\
2.2 \\
10 \\
1.6 \\
2.3 \\
4.4 \\
14\end{array}$ & $\begin{array}{l}8.5 \\
3.9 \\
52 \\
20 \\
3.1 \\
14 \\
1.8 \\
5.9 \\
7.6 \\
15\end{array}$ & $\begin{array}{l}9.8 \\
5.5 \\
61 \\
42 \\
4.5 \\
27 \\
2.2 \\
7.9 \\
9.3 \\
29\end{array}$ & $\begin{array}{c}19 \\
9.2 \\
74 \\
83 \\
5.5 \\
51 \\
6.9 \\
9.1 \\
14 \\
101\end{array}$ \\
\hline $\begin{array}{l}1960 \\
1961 \\
1962 \\
1963 \\
1964 \\
1965 \\
1966 \\
1967 \\
1968 \\
1969\end{array}$ & $\begin{array}{r}17 \\
11 \\
23 \\
8.0 \\
1.8 \\
26 \\
17 \\
3.4 \\
.80 \\
.74\end{array}$ & $\begin{array}{r}18 \\
11 \\
25 \\
8.1 \\
1.9 \\
28 \\
18 \\
3.6 \\
.88 \\
.85\end{array}$ & $\begin{array}{l}20 \\
13 \\
29 \\
8.3 \\
2.1 \\
31 \\
20 \\
3.8 \\
1.7 \\
1.1\end{array}$ & $\begin{array}{c}34 \\
16 \\
33 \\
9.3 \\
2.7 \\
34 \\
27 \\
3.8 \\
4.5 \\
1.7\end{array}$ & $\begin{array}{l}48 \\
17 \\
52 \\
10 \\
3.9 \\
37 \\
51 \\
5.2 \\
5.1 \\
3.0\end{array}$ & $\begin{array}{c}56 \\
36 \\
148 \\
17 \\
6.2 \\
62 \\
121 \\
6.2 \\
5.9 \\
4.7\end{array}$ & $\begin{array}{r}83 \\
72 \\
225 \\
20 \\
9.3 \\
155 \\
135 \\
7.5 \\
8.7 \\
6.7\end{array}$ \\
\hline $\begin{array}{l}1970 \\
1971 \\
1972 \\
1973 \\
1974 \\
1975 \\
1976\end{array}$ & $\begin{array}{l}13 \\
12 \\
3.3 \\
26 \\
46 \\
12 \\
11\end{array}$ & $\begin{array}{l}13 \\
13 \\
3.6 \\
27 \\
57 \\
13 \\
11\end{array}$ & $\begin{array}{l}14 \\
15 \\
3.7 \\
29 \\
63 \\
13 \\
12\end{array}$ & $\begin{array}{l}20 \\
18 \\
4 \cdot 4 \\
74 \\
80 \\
15 \\
12\end{array}$ & $\begin{array}{c}39 \\
60 \\
7.0 \\
179 \\
133 \\
16 \\
17\end{array}$ & $\begin{array}{r}69 \\
142 \\
20 \\
228 \\
284 \\
27 \\
24\end{array}$ & $\begin{array}{r}80 \\
134 \\
21 \\
438 \\
395 \\
33 \\
27\end{array}$ \\
\hline
\end{tabular}




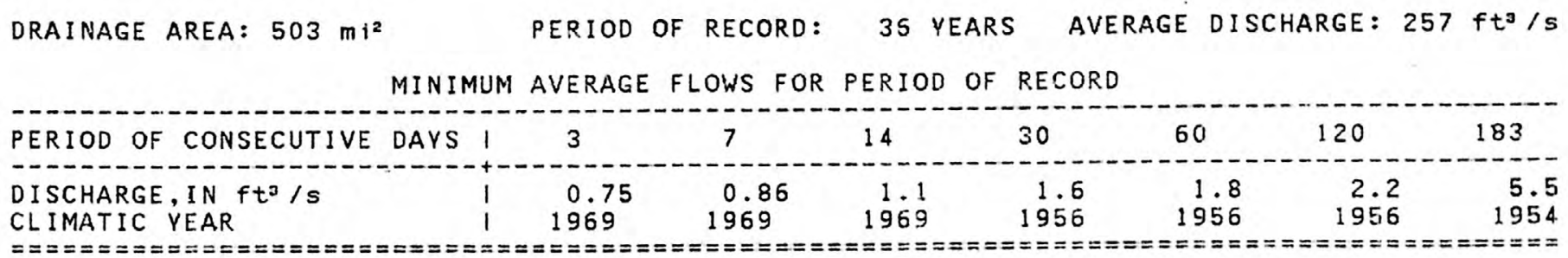

MAGNITUDE AND FREQUENCY OF ANNUAL LOW FLOWS

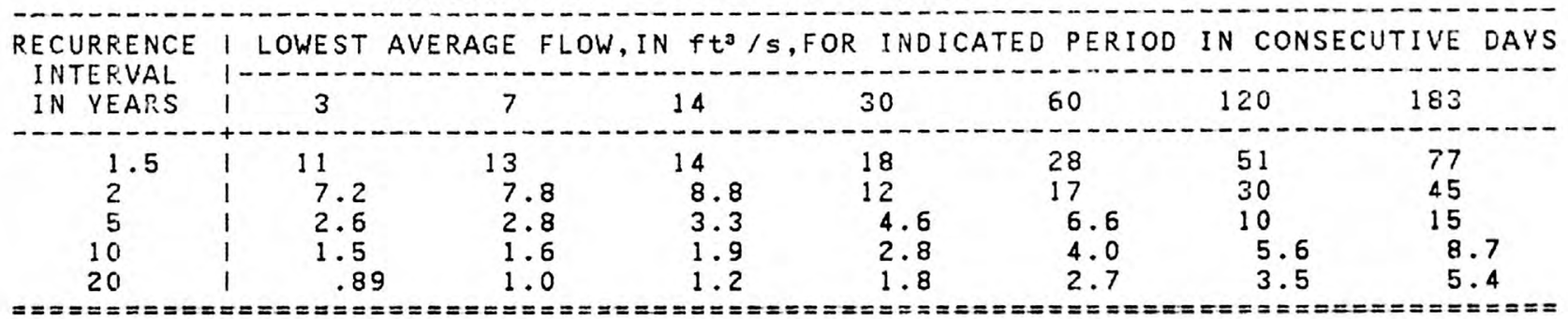

MAGNITUDE AND FREQUENCY OF SEASONAL LOW FLOWS

I LOWEST AVERAGE FLOW, IN $\mathrm{ft}^{3} / \mathrm{s}, \mathrm{FOR}$ THE INDICATED PERIOD IN CONSECUTIVE DAYS WITHIN EACH WATER-YEAR QUARTER

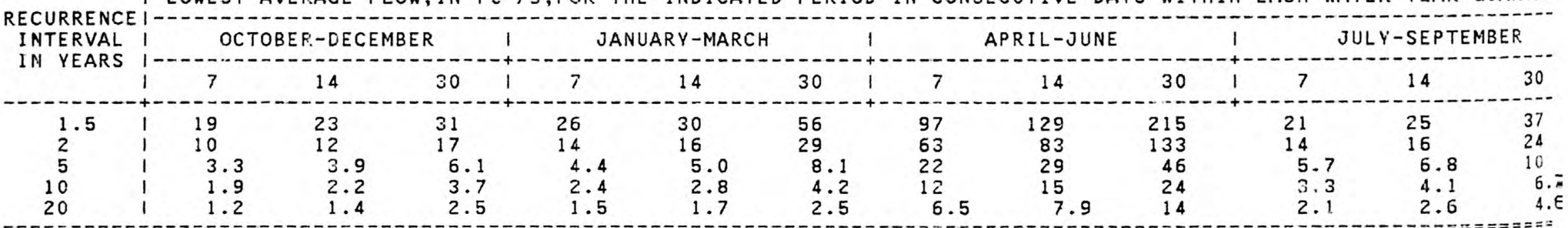

$$
\text { DURATION OF DAILY DISCHARGES FOR ANNUAL AND SEASONAL PERIOOS }
$$

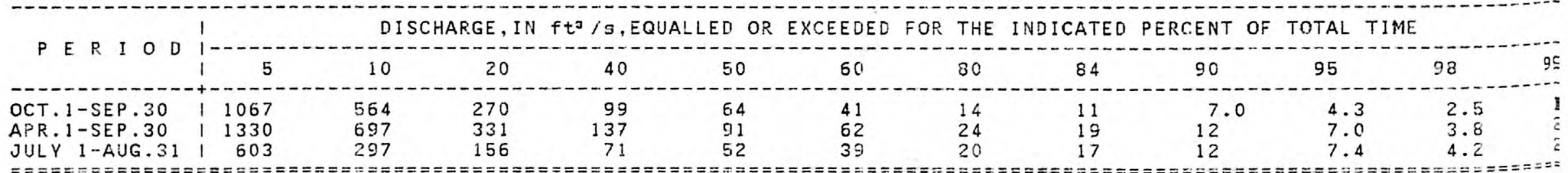


Location.--Lat $41^{\circ} 20^{\prime} 14^{\prime \prime}$, long $93^{\circ} 29^{\prime} 10^{\prime \prime}$, in SE1/4 SE1/4 sec.34, T.76 N., R.23 W. Warren County, on right bank $15 \mathrm{ft}$ downstream from bridge on county
highway, $0.5 \mathrm{~m} l i$ downstream from Otter Creek, and $2.2 \mathrm{miles}$ southwest of Ackworth.

LOW FLOW DISCHARGES, IN YEAR. ENDING MARCH 3

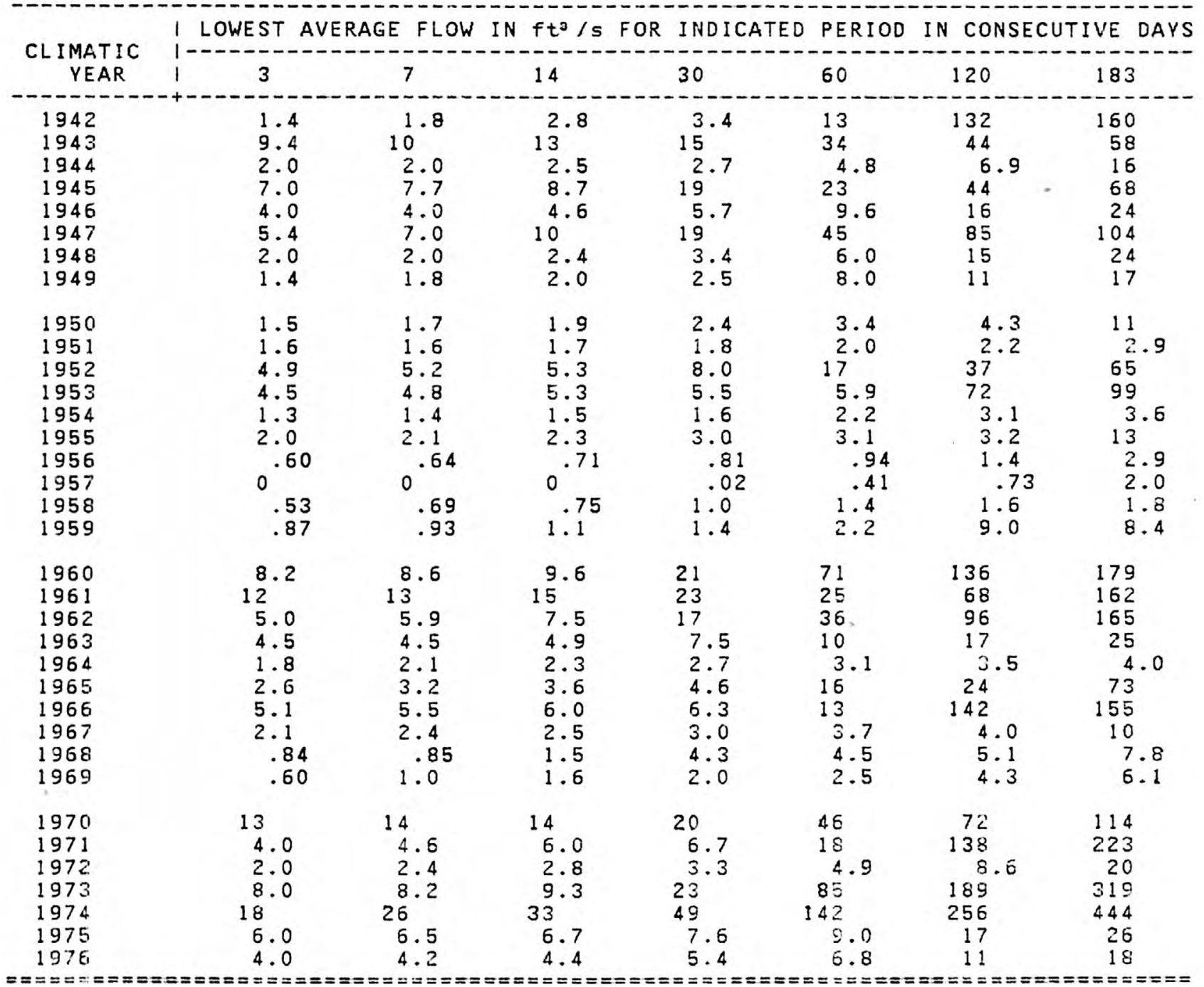


DRAINAGE AREA: $460 \mathrm{mi}^{2}$ PERIOD OF RECORD: 36 YEARS AVERAGE DISCHARGE: 244 ft $3 / \mathrm{s}$ MINIMUM AVERAGE FLOWS FOR PERIOD OF RECORD

\begin{tabular}{|c|c|c|c|c|c|c|c|}
\hline PERIOD OF CONSECUTIVE DAYS & 1 & 7 & 14 & 30 & 60 & 120 & 183 \\
\hline $\begin{array}{l}\text { DISCHARGE, IN } \mathrm{ft}^{3} / \mathrm{s} \\
\text { CLIMATIC YEAR }\end{array}$ & $\begin{array}{l}0 \\
1957\end{array}$ & $\begin{array}{l}0 \\
1957\end{array}$ & $\begin{array}{l}0 \\
1957\end{array}$ & $\begin{array}{l}0.02 \\
1957\end{array}$ & $\begin{array}{l}0.42 \\
195 \%\end{array}$ & $\begin{array}{l}0.74 \\
1957\end{array}$ & \\
\hline
\end{tabular}

MAGNITUDE AND FREQUENCY OF ANNUAL LOW FLOWS

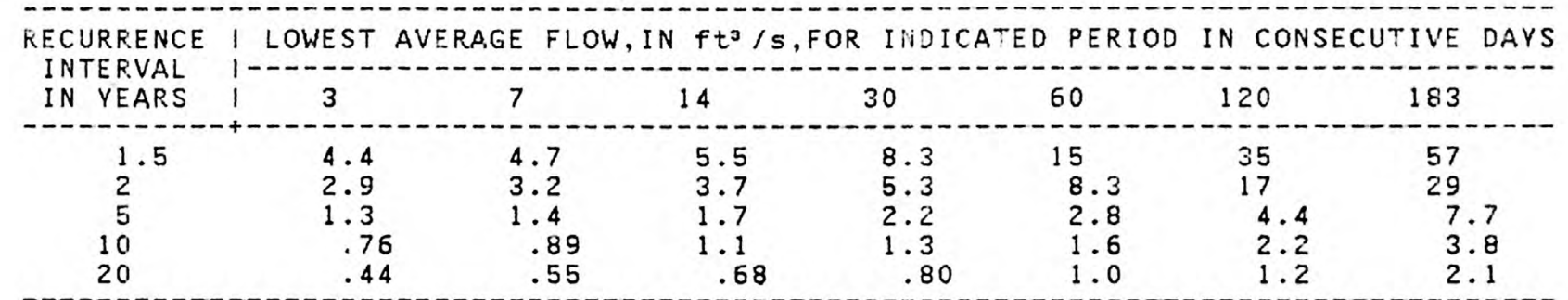

MAGNITUDE AND FREQUENCY OF SEASONAL LOW FLOWS

RECURRENCE | LOWEST AVERAGE FLOW, IN ft3/S,FOR THE INDICATED PERIOD IN CONSECUTIVE DAYS WITHIN EACH WATER-YEAR QUARTER
INTERVAL |
IN YEARS

DON, CHECK THESE COLUMNS TO MAKE SURE NONE OF THE OTHER NUMBERS GOT CHANGED ACCIDENTALLY.

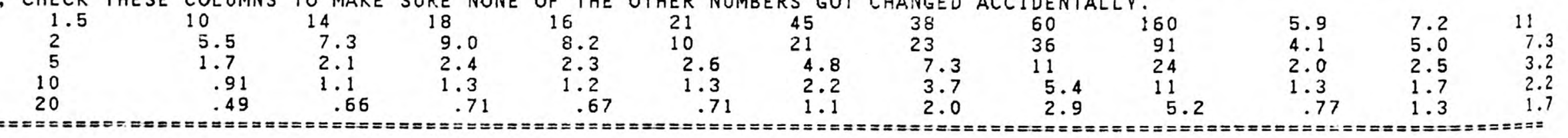

DURATION OF DAILY DISCHARGES FOR ANNUAL AND SEASONAL FERIODS

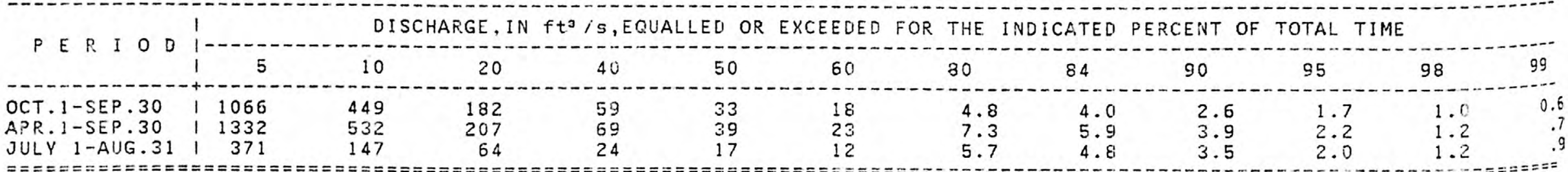




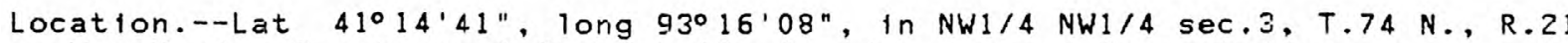
W., Marion County on ieft bank $15 \mathrm{ft}$ downstream from bridge on county highway, 0.5 mile downstream from Klrk Branch, and $1.7 \mathrm{~m} f l e s$ northwest of Dallas.

LOW FLOW DISCHARGES, IN YEAR. ENDING MARCH 31

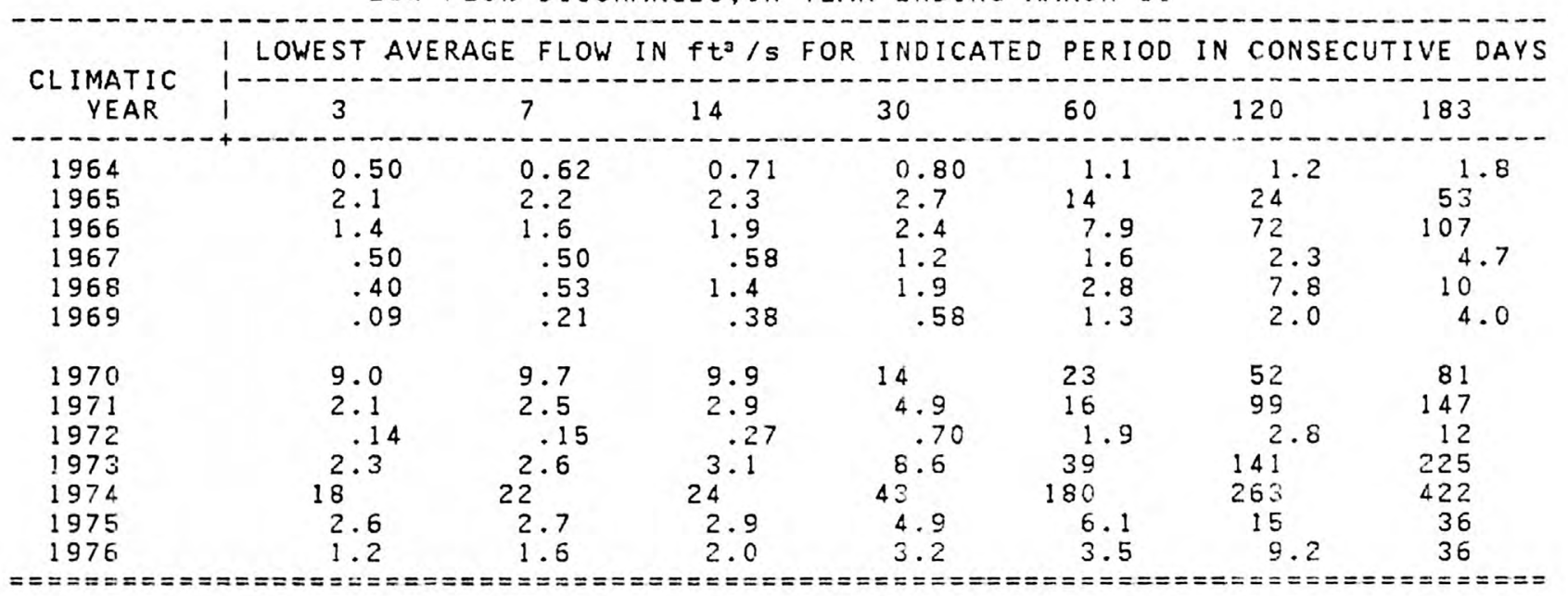




DRAINAGE AREA: $342 \mathrm{~m}^{2} \quad$ PERIOD OF RECORD: 14 YEARS AVERAGE DISCHARGE: $189 \mathrm{ft} / \mathrm{s}$
PERIOD OF CONSECUTIVE DAVS I

MAGNITUDE AND FREQUENCY OF ANNUAL LOW FLOWS

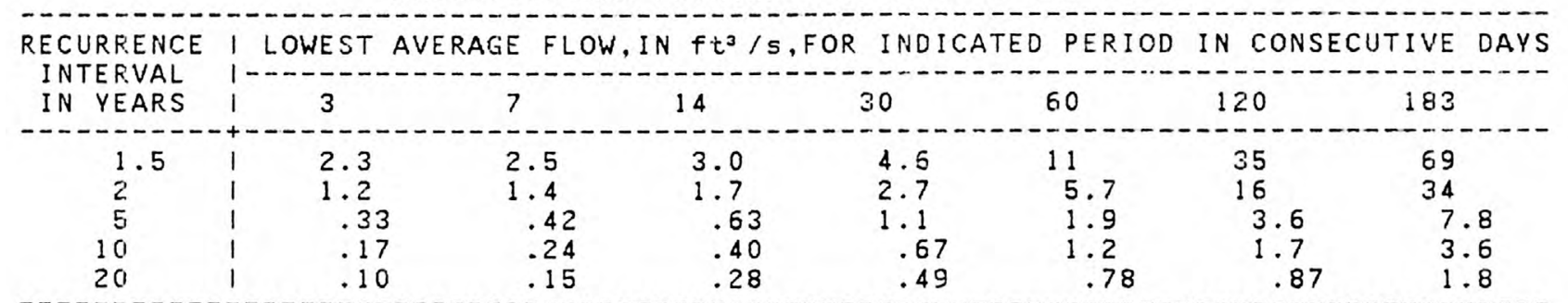

MAGNITUDE AND FREQUENCY OF SEASONAL LOW FLOWS

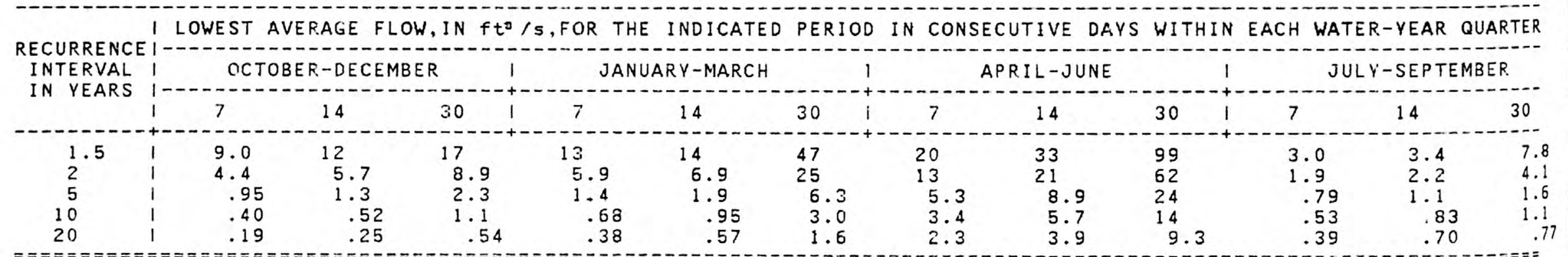

DURATION OF DAILY DISCHARGES FOR ANNUAL AND SEASONAL PERIODS

\begin{tabular}{|c|c|c|c|c|c|c|c|c|c|c|c|c|}
\hline$P E R I O D$ & 15 & 10 & $\frac{G E, I}{20}$ & 40 & ED OR & EDED & THE & ATED & $\begin{array}{l}\text { ENT } \\
90\end{array}$ & TAL & 98 & 99 \\
\hline $\begin{array}{l}T .1-S E P \cdot 30 \\
R .1-S E P .30 \\
i V 1-A \cup G .31\end{array}$ & $\begin{array}{r}1 \\
1 \quad 1252 \\
1 \quad 330\end{array}$ & $\begin{array}{l}375 \\
508 \\
135\end{array}$ & $\begin{array}{r}157 \\
177 \\
43\end{array}$ & $\begin{array}{l}51 \\
57 \\
14\end{array}$ & $\begin{array}{l}30 \\
34 \\
8.9\end{array}$ & $\begin{array}{l}17 \\
19 \\
5.7\end{array}$ & $\begin{array}{l}4.4 \\
4.5 \\
2.8\end{array}$ & $\begin{array}{l}3.4 \\
3.6 \\
2.5\end{array}$ & $\begin{array}{l}1.9 \\
2.3 \\
2.0\end{array}$ & $\begin{array}{l}1.2 \\
1.6 \\
1.5\end{array}$ & $\begin{array}{l}0.74 \\
1.0 \\
.93\end{array}$ & $\begin{array}{r}0.55 \\
.7 ! \\
.75\end{array}$ \\
\hline
\end{tabular}




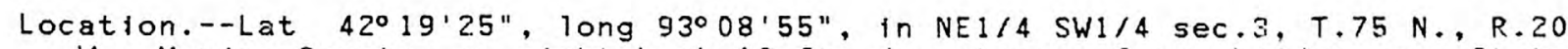
W. Marian county, on right bank $10 \mathrm{ft}$ ' downstream from bridge on State Highway 92, 1.1 miles upstream from Butcher Creek, $2.2 \mathrm{miles}$ west of Knoxvilie, and 11.1 miles upstream from mouth.

LOW FLOW DISCHARGES, IN YEAR ENDING MARCH 31

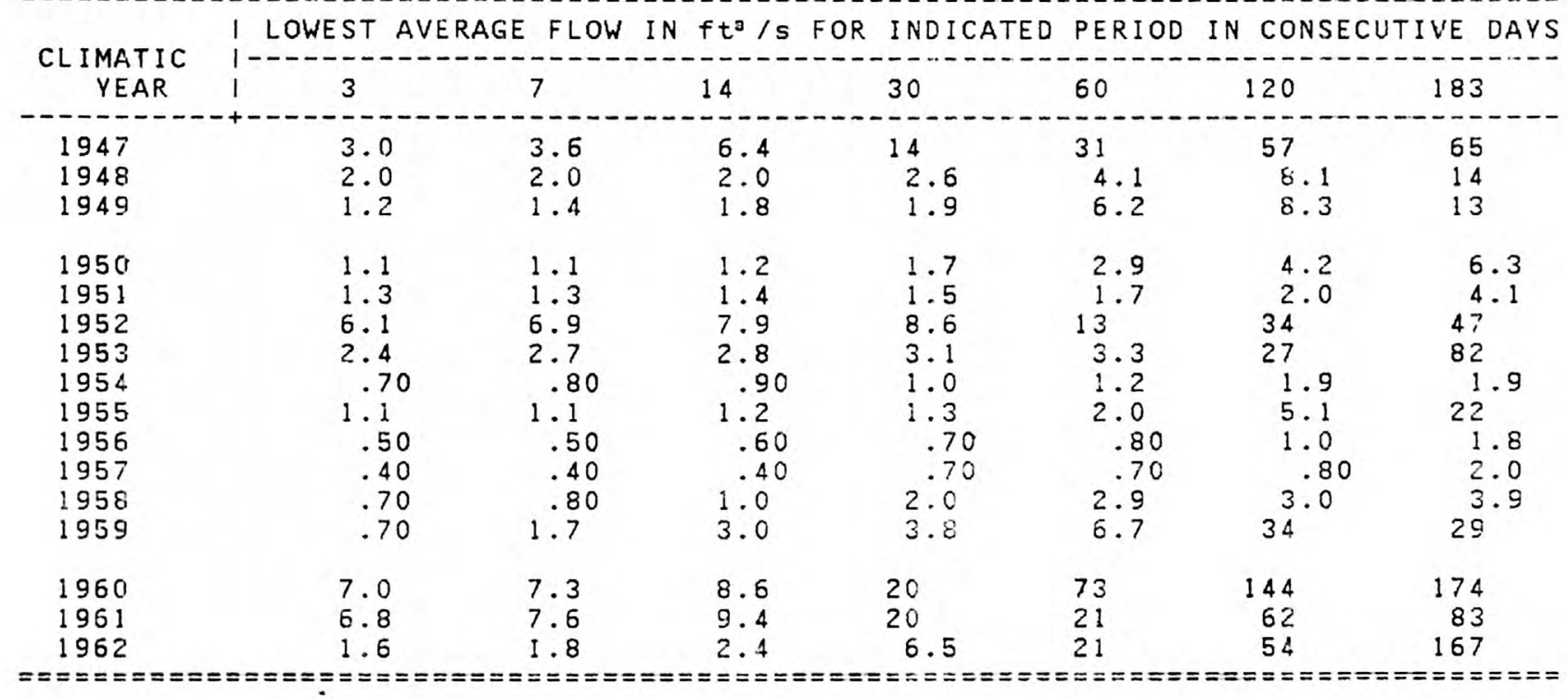




\section{DRAINAGE AREA: $380 \mathrm{~m}^{2}$ PERIOD OF RECORD: 17 YEARS AVERAGE DISCHARGE: $198 \mathrm{ft}^{3} / \mathrm{s}$}

MINIMUM AVERAGE FLOWS FOR PERIOD OF RECORD

\begin{tabular}{|c|c|c|c|c|c|c|c|}
\hline PERIOD OF CONSECUTIVE DAYS & 3 & 7 & 14 & 30 & 60 & 120 & 183 \\
\hline $\begin{array}{l}\text { RGE, IN } \mathrm{ft}^{3} / \mathrm{s} \\
\text { IC YEAR }\end{array}$ & $\begin{array}{l}0.40 \\
1957\end{array}$ & $\begin{array}{r}0.40 \\
1957\end{array}$ & $\begin{array}{l}0.40 \\
1957\end{array}$ & $\begin{array}{r}0.70 \\
1957\end{array}$ & $\begin{array}{l}0.70 \\
1957\end{array}$ & $\begin{array}{l}0.81 \\
1957\end{array}$ & \\
\hline
\end{tabular}

MAGNITUDE AND FREQUENCY OF ANNUAL LOW FLOWS

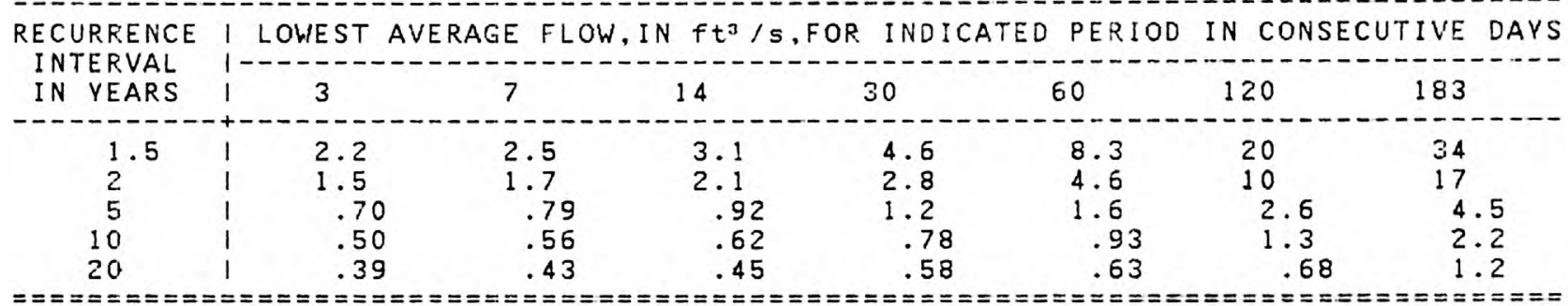

MAGNITUDE AND FREQUENCY OF SEASONAL LOW FLOWS

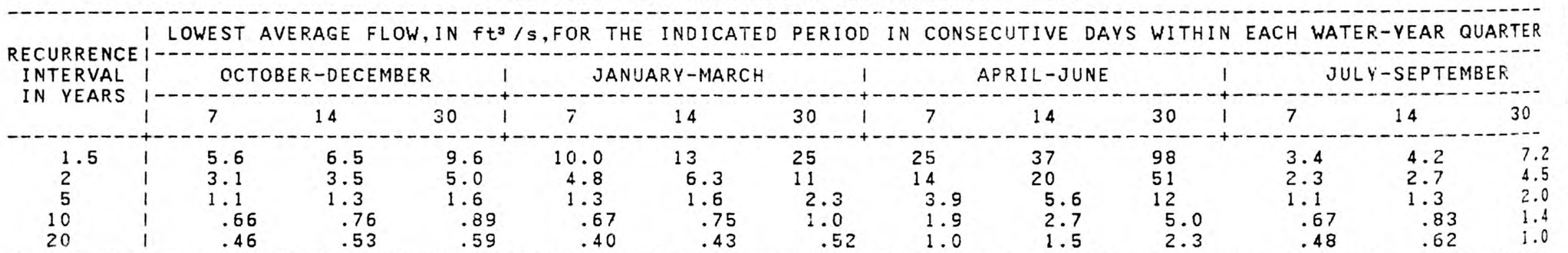

DURATION OF DAILY DISCHARGES FOR ANNUAL AND SEASONAL PERIODS

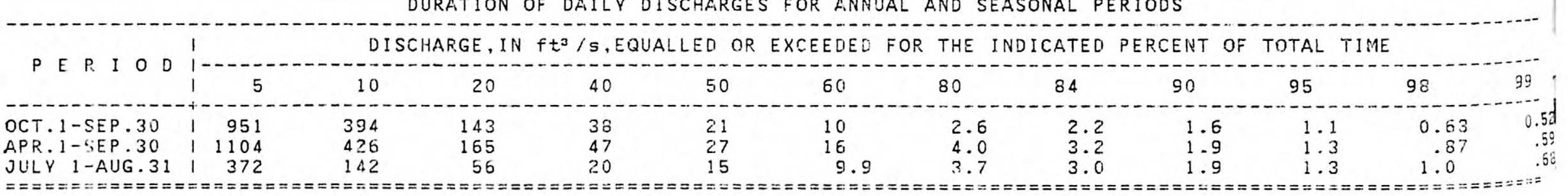


Location.--Lat 41016'53", long 92051'34", in NW1/4 SE1/4 sec.19, T.75 N., R.17 W. Mahaska County, on right bank $250 \mathrm{ft}$ upstream from abandoned Beilefountaine Bridge, 0.5 mile downstream from bridge on State Highway 92 , $0.8 \mathrm{mile}$ east of Tracy, $3.1 \mathrm{miles}$ upstream from Cedar Creek, 6.4 miles downstream from English Creek, and at mile 130.4 .

Remarks.--Flow regulated by Lake Red Rock 11.9 miles upstream since March 12 , 1969.

LOW FLOW DISCHARGES, IN YEAR. ENDING MARCH 31

\begin{tabular}{|c|c|c|c|c|c|c|c|c|}
\hline \multirow{2}{*}{$\begin{array}{c}\text { CLIMATIC } \\
\text { YEAR }\end{array}$} & I LOWEST & RAGE & IN $f t^{3}$ & FOR IN & $\begin{array}{l}\text { ED } P E F \\
-\end{array}$ & IN $\mathrm{CO}$ & TIVE & DAYS \\
\hline & 13 & 7 & 14 & 30 & 60 & 120 & 183 & \\
\hline $\begin{array}{l}1921 \\
1922 \\
1923 \\
1924 \\
1925 \\
1926 \\
1927 \\
1928 \\
1929\end{array}$ & $\begin{array}{r}840 \\
560 \\
400 \\
550 \\
310 \\
435 \\
409 \\
140 \\
1130\end{array}$ & $\begin{array}{r}840 \\
590 \\
400 \\
550 \\
310 \\
445 \\
438 \\
140 \\
1170\end{array}$ & $\begin{array}{r}1160 \\
680 \\
503 \\
550 \\
310 \\
458 \\
482 \\
157 \\
1170\end{array}$ & $\begin{array}{r}1660 \\
924 \\
569 \\
550 \\
325 \\
485 \\
552 \\
215 \\
1230\end{array}$ & $\begin{array}{r}1940 \\
1170 \\
634 \\
843 \\
438 \\
689 \\
800 \\
247 \\
1590\end{array}$ & $\begin{array}{r}2430 \\
1500 \\
885 \\
1540 \\
758 \\
1020 \\
1540 \\
591 \\
2790\end{array}$ & $\begin{array}{r}2960 \\
2710 \\
1080 \\
2330 \\
1140 \\
1070 \\
3380 \\
741 \\
3270\end{array}$ & \\
\hline $\begin{array}{l}1930 \\
1931 \\
1932 \\
1933 \\
1934 \\
1935 \\
1936 \\
1937 \\
1938 \\
1939\end{array}$ & $\begin{array}{l}375 \\
195 \\
160 \\
375 \\
170 \\
127 \\
442 \\
164 \\
125 \\
610\end{array}$ & $\begin{array}{l}375 \\
230 \\
179 \\
375 \\
170 \\
143 \\
465 \\
164 \\
125 \\
707\end{array}$ & $\begin{array}{l}385 \\
232 \\
202 \\
532 \\
204 \\
160 \\
481 \\
170 \\
139 \\
909\end{array}$ & $\begin{array}{r}450 \\
236 \\
481 \\
749 \\
232 \\
189 \\
676 \\
191 \\
164 \\
1070\end{array}$ & $\begin{array}{r}543 \\
290 \\
552 \\
995 \\
284 \\
296 \\
800 \\
263 \\
196 \\
1070\end{array}$ & $\begin{array}{r}570 \\
330 \\
955 \\
1260 \\
382 \\
391 \\
1520 \\
564 \\
311 \\
1590\end{array}$ & $\begin{array}{r}696 \\
357 \\
1080 \\
1560 \\
419 \\
482 \\
1430 \\
792 \\
524 \\
3590\end{array}$ & \\
\hline $\begin{array}{l}1940 \\
1941 \\
1942 \\
1943 \\
1944 \\
1945 \\
1946 \\
1947 \\
1948 \\
1949\end{array}$ & $\begin{array}{r}45 \\
238 \\
433 \\
640 \\
580 \\
583 \\
453 \\
460 \\
270 \\
239\end{array}$ & $\begin{array}{r}45 \\
251 \\
455 \\
733 \\
659 \\
618 \\
480 \\
561 \\
291 \\
248\end{array}$ & $\begin{array}{r}46 \\
281 \\
609 \\
814 \\
705 \\
641 \\
488 \\
856 \\
305 \\
251\end{array}$ & $\begin{array}{r}55 \\
427 \\
772 \\
861 \\
793 \\
688 \\
545 \\
1180 \\
377 \\
280\end{array}$ & $\begin{array}{r}75 \\
591 \\
1120 \\
1250 \\
1100 \\
751 \\
740 \\
1370 \\
533 \\
557\end{array}$ & $\begin{array}{r}134 \\
1160 \\
3190 \\
1760 \\
1290 \\
965 \\
994 \\
2290 \\
767 \\
462\end{array}$ & $\begin{array}{r}180 \\
1830 \\
3640 \\
2720 \\
1770 \\
1720 \\
2480 \\
2830 \\
732 \\
689\end{array}$ & \\
\hline $\begin{array}{l}1950 \\
1951 \\
1952 \\
1953\end{array}$ & $\begin{array}{l}155 \\
170 \\
983 \\
459\end{array}$ & $\begin{array}{r}166 \\
170 \\
1250 \\
464\end{array}$ & $\begin{array}{r}176 \\
177 \\
1410 \\
485\end{array}$ & $\begin{array}{r}209 \\
204 \\
1670 \\
52 !\end{array}$ & $\begin{array}{r}252 \\
227 \\
2100 \\
640\end{array}$ & $\begin{array}{r}336 \\
362 \\
3080 \\
948\end{array}$ & $\begin{array}{r}462 \\
553 \\
4160 \\
1350\end{array}$ & \\
\hline
\end{tabular}


DES MOINES RIVER NEAR TRACY--Cont inued

LOW FLOW DISCHARGES, IN YEAR ENDING MAR.CH 31--Continued

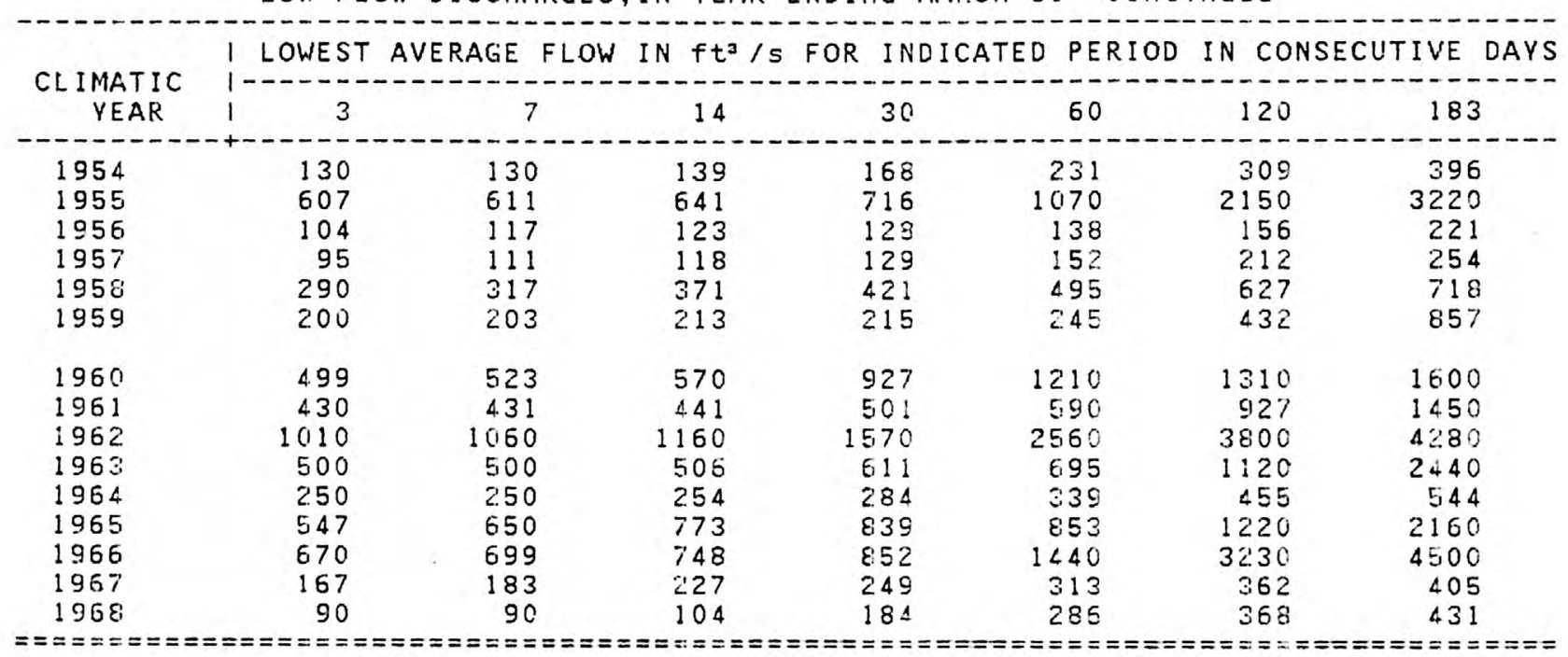


05-4885.00 DES MOINES RIVER NEAR TRACY--Continued

DRAINAGE AREA: $12,479 \mathrm{~m}^{2}$ PERIOD OF RECORD: 48 YEARS AVERAGE DISCHARGE: 4230 Ft $/ \mathrm{s}$

MINIMUM AVERAGE FLOWS FOR PERIOD OF RECORD

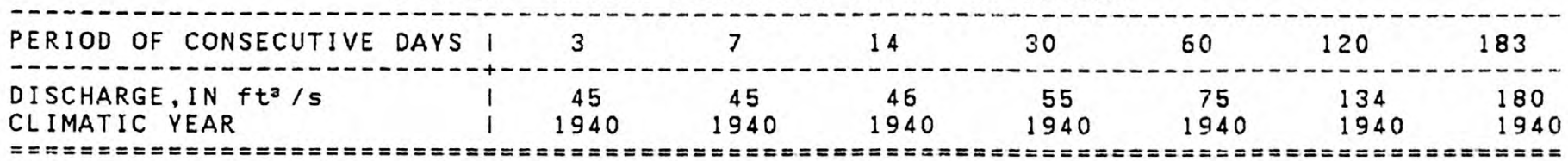

MAGNITUDE AND FREQUENCY OF ANNUAL LOW FLOWS

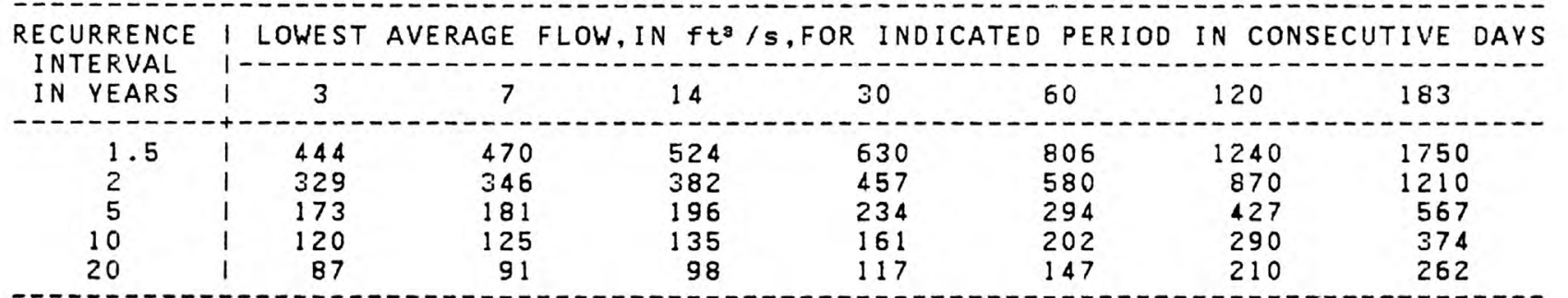

MAGNITUDE AND FREQUENCY OF SEASONAL LOW FLOWS

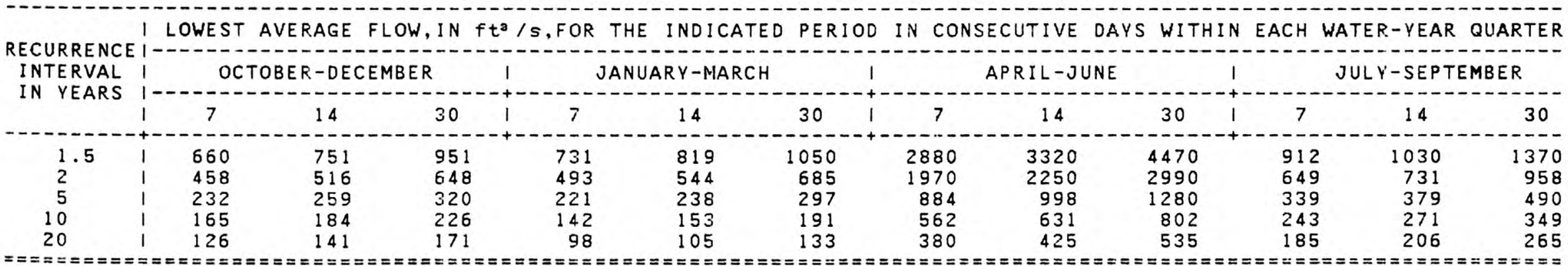

DURATION OF DAILY DISCHARGES FOR ANNUAL AND SEASONAL PERIODS

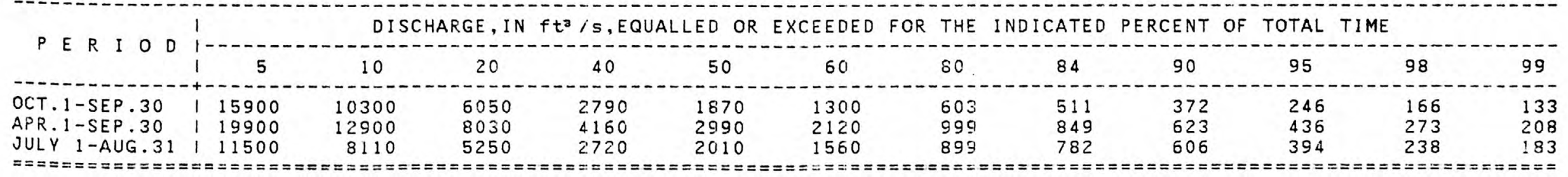


DES MOINES RIVER NEAR TRACY--Cont inued, regulated period

LOW FLOW DISCHARGES, IN YEAR ENDING MARCH 31

\begin{tabular}{|c|c|c|c|c|c|c|c|}
\hline \multirow{2}{*}{$\begin{array}{c}\text { CL IMAT IC } \\
\text { YEAR }\end{array}$} & \multicolumn{7}{|c|}{ I LOWEST AVERAGE } \\
\hline & 13 & 7 & 14 & 30 & 60 & 120 & 183 \\
\hline 1969 & 450 & 4.83 & 541 & 723 & 928 & 1610 & 1540 \\
\hline $\begin{array}{l}1970 \\
1971 \\
1972 \\
1973 \\
1974 \\
1975 \\
1976\end{array}$ & $\begin{array}{r}513 \\
372 \\
342 \\
1250 \\
2990 \\
513 \\
411\end{array}$ & $\begin{array}{r}533 \\
376 \\
345 \\
1610 \\
3050 \\
514 \\
474\end{array}$ & $\begin{array}{r}538 \\
446 \\
353 \\
1760 \\
3130 \\
517 \\
500\end{array}$ & $\begin{array}{r}635 \\
730 \\
357 \\
2540 \\
5860 \\
521 \\
532\end{array}$ & $\begin{array}{r}839 \\
1130 \\
384 \\
5760 \\
7320 \\
662 \\
653\end{array}$ & $\begin{array}{r}1230 \\
1930 \\
776 \\
6650 \\
9040 \\
1110 \\
859\end{array}$ & $\begin{array}{r}1990 \\
2050 \\
792 \\
7220 \\
10100 \\
1100 \\
1090\end{array}$ \\
\hline
\end{tabular}


05-4885.00 DES MOINES RIVER NEAR TRACY--Continued

DRAINAGE AREA: $12,479 \mathrm{~m}^{2}$ PERIOD OF RECORD: 8 YEARS AVERAGE DISCHARGE: 7100 ft? $/ \mathrm{S}$

MINIMUM AVERAGE FLOWS FOR PERIOD OF RECORD

\begin{tabular}{|c|c|c|c|c|c|c|c|}
\hline PERIOD OF CONSECUTIVE DAYS & 3 & 7 & 14 & 30 & 60 & 120 & 183 \\
\hline $\begin{array}{l}\text { RGE, IN } f t^{3} / s \\
\text { IC YEAR }\end{array}$ & $\begin{array}{l}342 \\
1972\end{array}$ & $\begin{array}{l}345 \\
1972\end{array}$ & $\begin{array}{l}353 \\
1972\end{array}$ & $\begin{array}{l}357 \\
1972\end{array}$ & $\begin{array}{l}384 \\
1972\end{array}$ & $\begin{array}{l}776 \\
1972\end{array}$ & $\begin{array}{l}792 \\
1972\end{array}$ \\
\hline
\end{tabular}

MAGNITUDE AND FREQUENCY OF ANNUAL LOW FLOWS

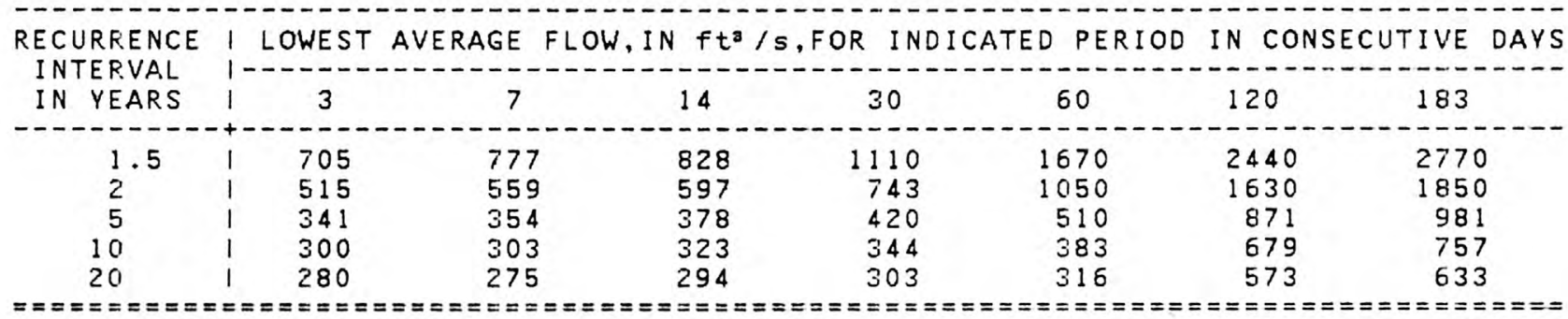

MAGNITUDE AND FREQUENCY OF SEASONAL LOW FLOWS

TSOR THE INDICATED PERIOD IN CONSECUTIVE DAY

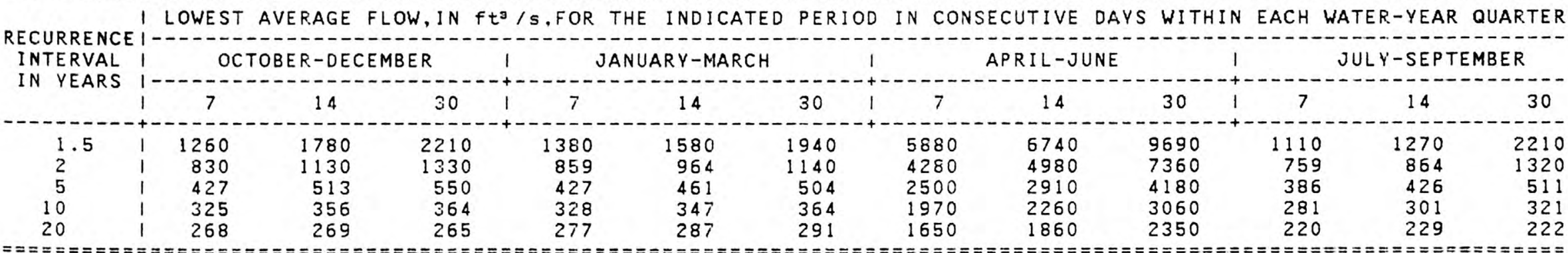

DURATION OF DAILY DISCHARGES FOR ANNUAL AND SEASONAL PERIODS

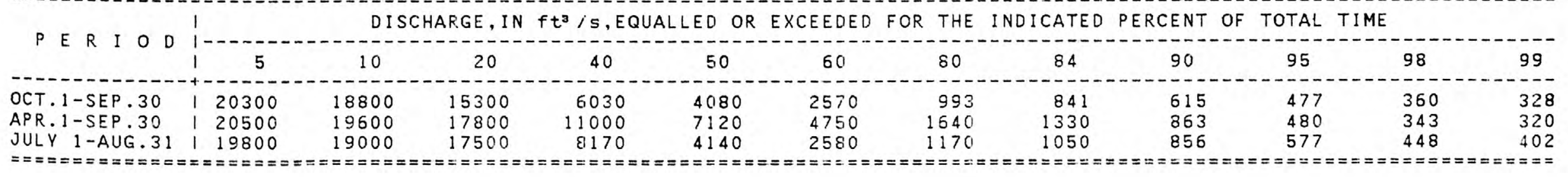


Location.--Lat $41^{\circ} 13^{\prime} 09^{\prime \prime}$, long 92054'38", at SW corner sec.11, T.74 N., R.18 W. Marion County, on left bank $10 \mathrm{ft}$ downstream from bridge on State Highway 156, 0.8 mile downstream from North Cedar Creek, $1.6 \mathrm{miles}$ northwest of Bussey, 3.0 miles upstream from Honey Creek, and 8.9 miles upstream from mouth.

LOW $r$ LOW DISCHARGES, IN YEAR ENDING MARCH 31

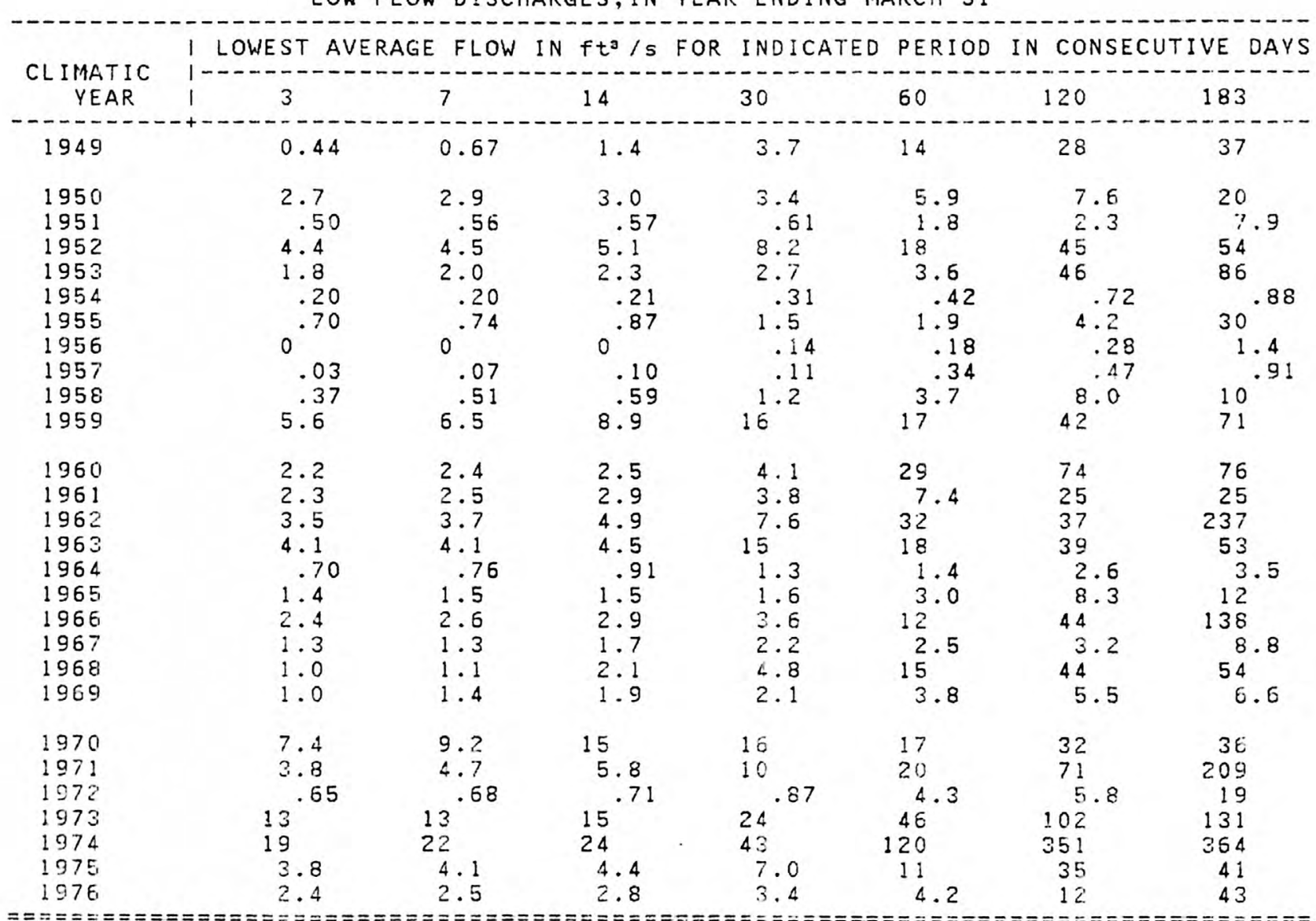


DRAINAGE AREA: $374 \mathrm{mi}^{2}$ PERIOD OF RECORD: 29 YEARS AVERAGE DISCHARGE: 196 Ft $3 / \mathrm{s}$

MINIMUM AVERAGE FLOWS FOR PERIOD OF RECORD

\begin{tabular}{|c|c|c|c|c|c|c|c|}
\hline RIOD OF CONSECUTIVE DAYS I & 3 & 7 & 14 & 30 & 60 & 120 & 183 \\
\hline $\begin{array}{l}\text { AARGE, IN } \mathrm{ft}^{3} / \mathrm{s} \\
\text { ITIC YEAR }\end{array}$ & $\begin{array}{r}0 \\
1956 \\
\end{array}$ & $\begin{array}{l}0 \\
1956\end{array}$ & $\stackrel{0}{1956}$ & $\begin{array}{l}0.12 \\
1957\end{array}$ & 19.19 & $\begin{array}{l}0.28 \\
1956\end{array}$ & $\begin{array}{l}0.88 \\
1954\end{array}$ \\
\hline
\end{tabular}

MAGNITUDE AND FREQUENCY OF ANNUAL LOW FLOWS

RECURRENCE
INTERVAL
IN YOWEARS

MAGNITUDE AND FREQUENCY OF SEASONAL LOW FLOWS

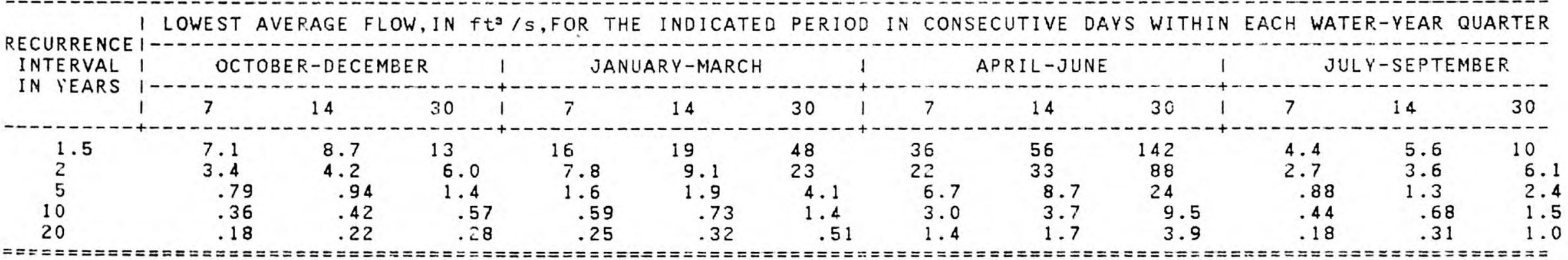

DURATION OF DAILY DISCHARGES FOR ANNUAL AND SEASONAL FERIODS

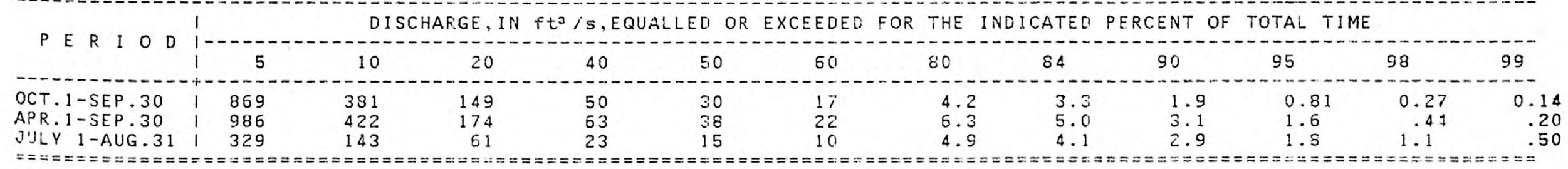


Location.--Lat $41000^{\prime} 39^{\prime \prime}$, long $92^{\circ} 24^{\prime} 40^{\prime \prime}$, In SE1/4 NE1/4 sec.25, T.72 N., R.14 W. Wapello County, on $r$ ight bank $15 \mathrm{ft}$ downstream from Wabash Rallroad Bridge at Ottumwa, $0.4 \mathrm{mile}$ downstream from Ottumwa powerplant, $6.5 \mathrm{miles}$ upstream from Village Creek, $9.5 \mathrm{miles}$ downstream from South Avery Creek, and at mile 94.1 .

Remarks.--Prior to Dec. 12, 1958 and since Nov. 30,1960, diurnal fluctuation at low flow caused by powerplant upstream of station. Flow regulated by Lake Red Rock, 48.2 miles upstream, since March 12, 1969. Fublished as "at Eldon", $1930-35$.

LOW FLOW DISCHARGES, IN YEAR. ENDING MARCH 31

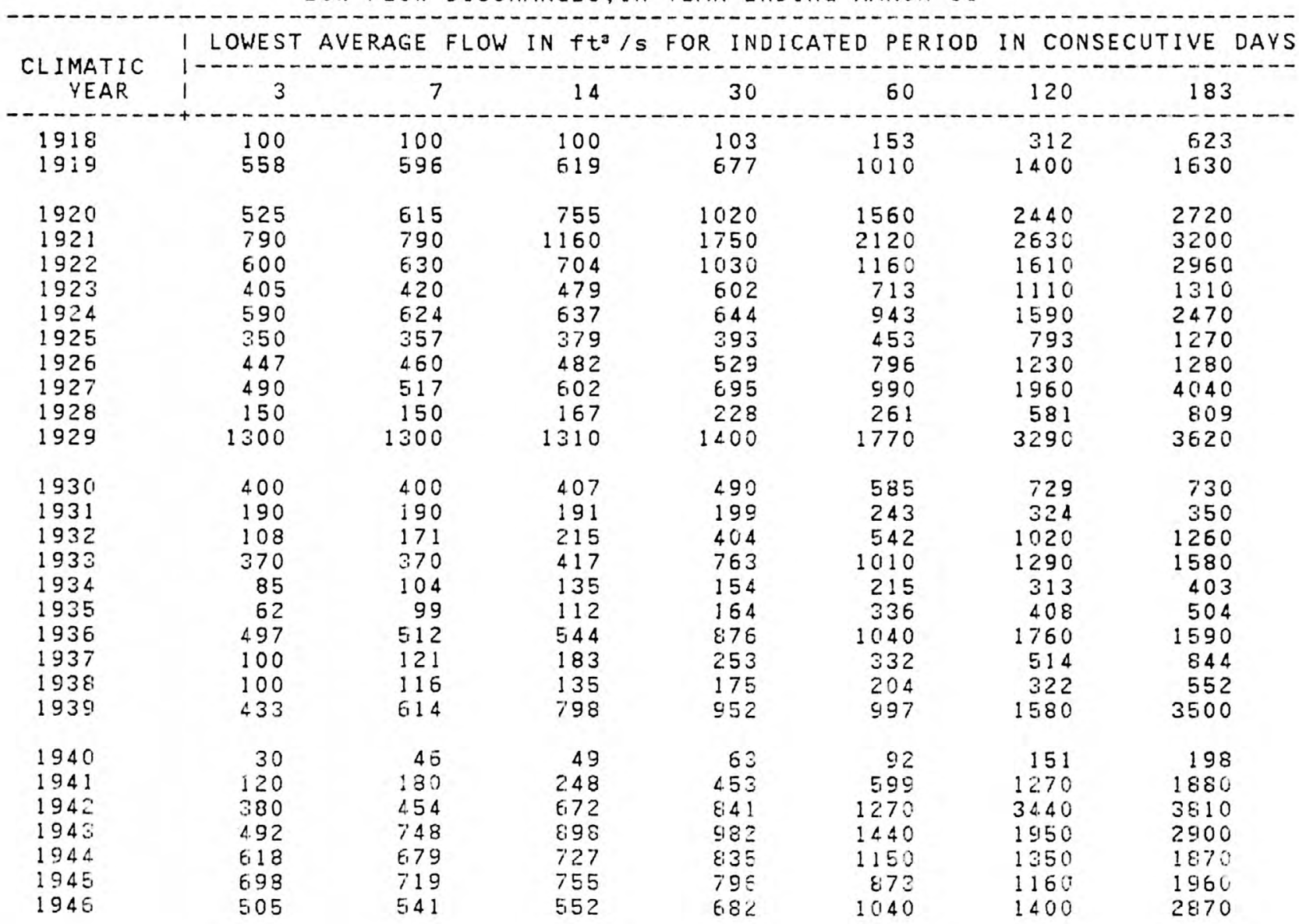


LOW FLOW DISCHARGES, IN YEAR ENDING MARCH 31--Cont inued

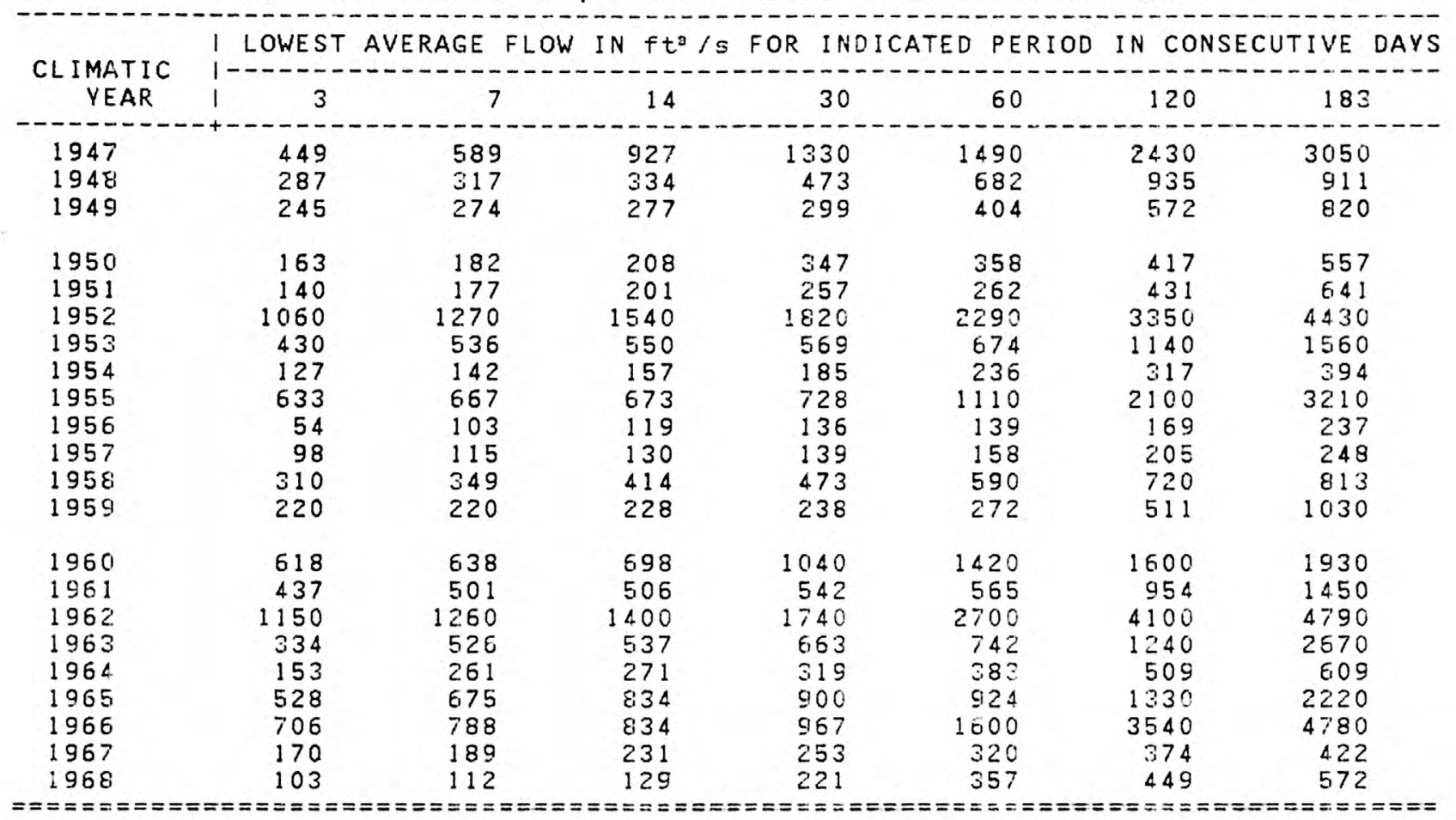


MINIMUM AVERAGE FLOWS FOR PERIOD OF RECORD

\begin{tabular}{|c|c|c|c|c|c|c|c|}
\hline PERIOD OF CONSECUTIVE DAYS & 3 & 7 & 14 & 30 & 60 & 120 & 183 \\
\hline $\begin{array}{l}\text { AARGE, IN } \mathrm{ft}^{3} / \mathrm{s} \\
\text { ITIC YEAR }\end{array}$ & $\begin{array}{l}30 \\
1940\end{array}$ & $\begin{array}{c}46 \\
1940\end{array}$ & $\begin{array}{l}49 \\
1940\end{array}$ & $\begin{array}{l}63 \\
1940\end{array}$ & $\begin{array}{c}92 \\
1940\end{array}$ & $\begin{array}{l}151 \\
1940\end{array}$ & \\
\hline
\end{tabular}

MAGNITUDE AND FREQUENCY OF ANNUAL LOW FLOWS

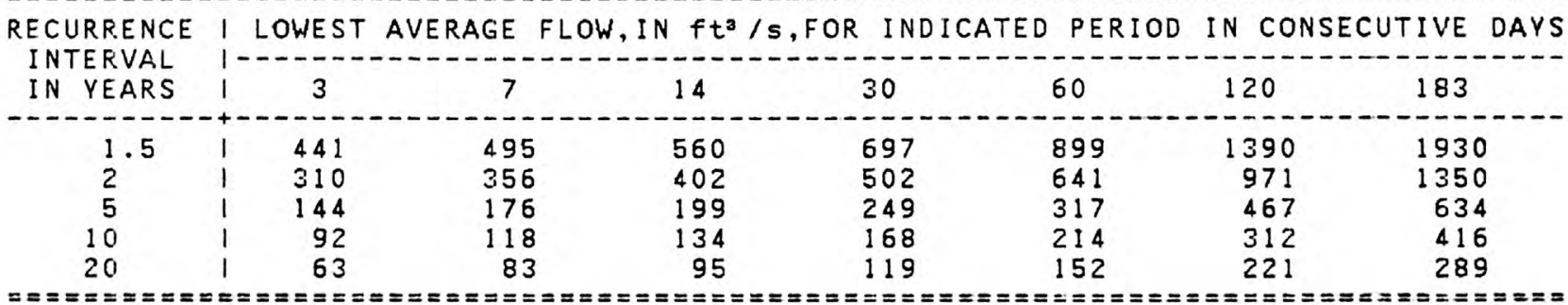

MAGNITUDE AND FREQUENCY OF SEASONAL LOW FLOWS

LOWEST AVERAGE FLOW IN ft3/S,FOR THE INDICATED PERIOD IN CONSECUTIVE DAYS WITHIN EACH WATER-YEAR QUARTER RECURRENCE LOWEST AVERAGE FLOW, IN $\mathrm{ft}^{3} / \mathrm{s}, F O R$ THE INDICATED PERIOD IN CONSECUTIVE DAYS WITHIN EACH WATER-YEAR QUARTER

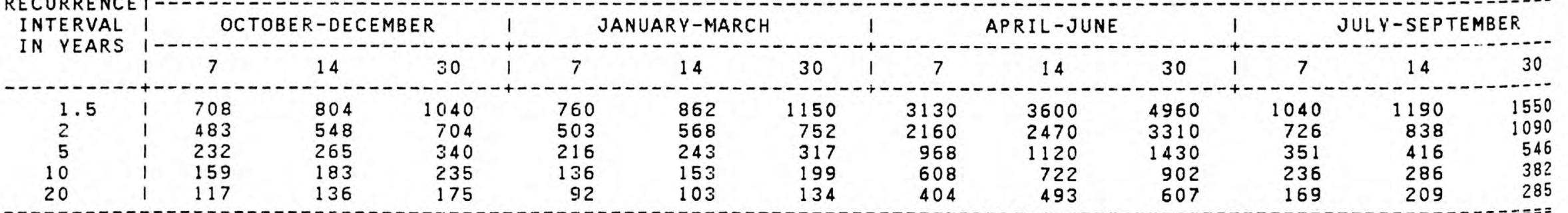

DURATION OF DAILY DISCHARGES FOR ANNUAL AND SEASONAL PERIODS DISCHARGE, IN $\mathrm{ft}^{3} / \mathrm{s}$, EQUALLED OR EXCEEDED FOR THE INDICATED PERCENT OF TOTAL TIME

\begin{tabular}{|c|c|c|c|c|c|c|c|c|c|c|c|c|}
\hline 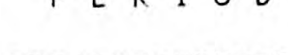 & 5 & 10 & 20 & 40 & 50 & 60 & 80 & 84 & 90 & 95 & 98 & 99 \\
\hline $\begin{array}{l}\text { OCT. } 1 \text {-SEP. } 30 \\
A P R .1-S E P .30 \\
\text { JULY } 1 \text {-AUG. } 31\end{array}$ & $\begin{array}{l}18500 \\
23000 \\
112400\end{array}$ & $\begin{array}{r}11600 \\
15100 \\
9200\end{array}$ & $\begin{array}{l}6760 \\
9110 \\
5840\end{array}$ & $\begin{array}{l}3060 \\
4620 \\
3020\end{array}$ & $\begin{array}{l}2110 \\
3330 \\
2300\end{array}$ & $\begin{array}{l}1500 \\
2410 \\
1820\end{array}$ & $\begin{array}{r}661 \\
1160 \\
1030\end{array}$ & $\begin{array}{l}553 \\
975 \\
872\end{array}$ & $\begin{array}{l}391 \\
696 \\
628\end{array}$ & $\begin{array}{l}248 \\
471 \\
422\end{array}$ & $\begin{array}{l}159 \\
288 \\
238\end{array}$ & $\begin{array}{l}108 \\
207 \\
156\end{array}$ \\
\hline
\end{tabular}


LOW FLOW DISCHARGES, IN YEAR. ENDING MARCH 31

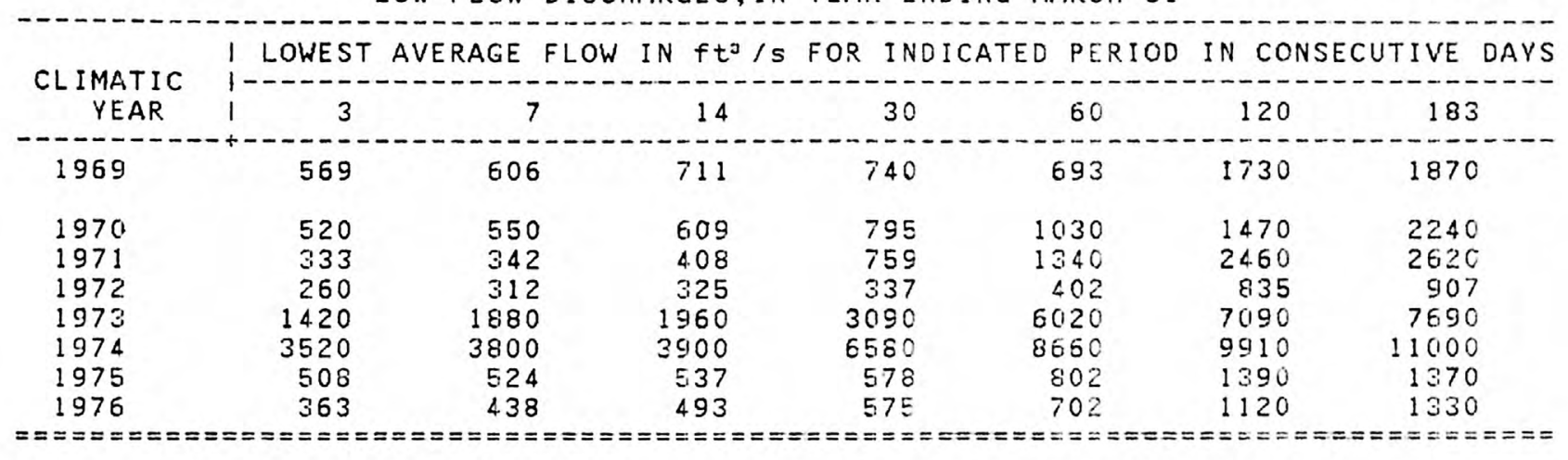


MINIMUM AVERAGE FLOWS FOR PERIOD OF RECORD

\begin{tabular}{|c|c|c|c|c|c|c|c|}
\hline DERIOD OF CONSECUTIVE DAYS & 1 & 7 & 14 & 30 & 60 & 120 & 183 \\
\hline $\begin{array}{l}\text { DISCHARGE, IN } \mathrm{ft}^{\mathrm{s}} / \mathrm{s} \\
\text { CLIMATIC YEAR }\end{array}$ & $\begin{array}{l}260 \\
1972\end{array}$ & $\begin{array}{l}312 \\
1972\end{array}$ & $\begin{array}{l}325 \\
1972\end{array}$ & $\begin{array}{l}337 \\
1972\end{array}$ & $\begin{array}{l}402 \\
1972\end{array}$ & $\begin{array}{l}835 \\
1972\end{array}$ & \\
\hline
\end{tabular}

MAGNITUDE AND FREQUENCY OF ANNUAL LOW FLOWS

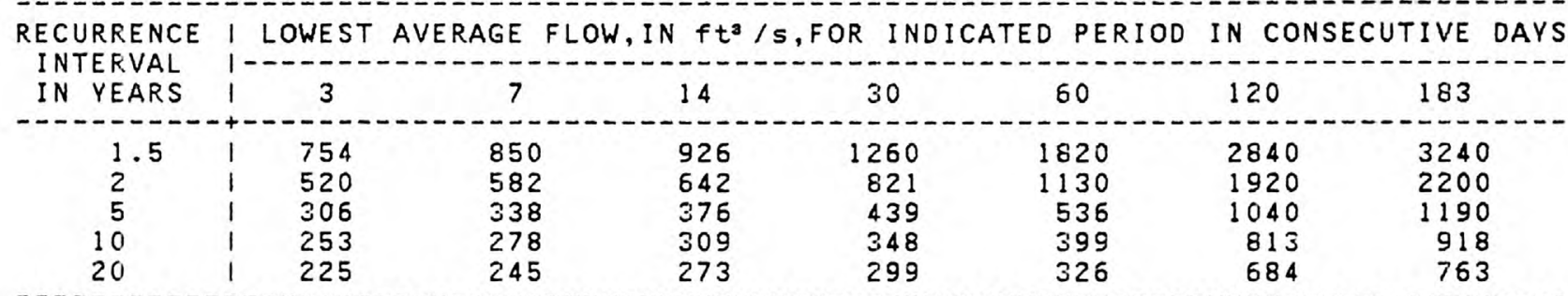

MAGNITUDE AND FREQUENCY OF SEASONAL LOW FLOWS

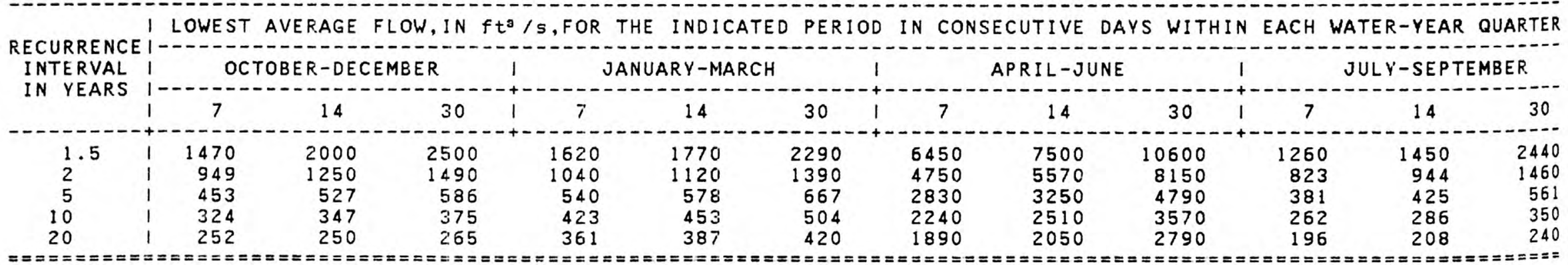

DURATION OF DAILY DISCHARGES FOR ANNUAL AND SEASONAL PERIODS

\begin{tabular}{|c|c|c|c|c|c|c|c|c|c|c|c|c|}
\hline D & 1 & DI & ARGE, II & $t^{2}$ is, $E$ & ED OP & ZEEDED & R THE & ICATE & RCENT & OTAL & & \\
\hline & 5 & 10 & 20 & 40 & 50 & 60 & 80 & 84 & 90 & 95 & 98 & 99 \\
\hline $\begin{array}{l}\text { OCT. } 1 \text {-SEP. } 30 \\
\text { APR. } 1 \text {-SEP. } 30 \\
\text { JULY } 1 \text {-AUG. } 31\end{array}$ & $\begin{array}{l}20700 \\
22700 \\
20000\end{array}$ & $\begin{array}{l}19000 \\
19900 \\
19100\end{array}$ & $\begin{array}{l}16000 \\
18000 \\
17400\end{array}$ & $\begin{array}{r}6900 \\
11900 \\
8950\end{array}$ & $\begin{array}{l}4460 \\
8140 \\
4550\end{array}$ & $\begin{array}{l}2930 \\
5420 \\
29010\end{array}$ & $\begin{array}{l}1180 \\
1950 \\
1260\end{array}$ & $\begin{array}{l}1010 \\
1570 \\
1120\end{array}$ & $\begin{array}{r}748 \\
1000 \\
912\end{array}$ & $\begin{array}{l}534 \\
511 \\
603\end{array}$ & $\begin{array}{l}377 \\
346 \\
441\end{array}$ & $\begin{array}{l}323 \\
308 \\
356\end{array}$ \\
\hline
\end{tabular}


Location.--Lat 40043'40", long 91057'34", in SW1/4 SW1/4 sec.36, T.69 N., R.10 W. Van Buren County, on right bank $10 \mathrm{ft}$ upstream from bridge on State Highway 1 at Keosauqua, 4.0 miles downstream from Chequest Creek, and at mile 51.3 .

Remarks.--Prior to Dec. 21, 1958, and since Nov. 30, 1960, some diurnal fluctuation at medium and low stages caused by powerplant at ottumwa. Flow regulated by Lake Red Rock, 91.0 miles upstream, since March 12, 1969.

LOW FLOW DISCHARGES, IN YEAR. ENDING MARCH 31

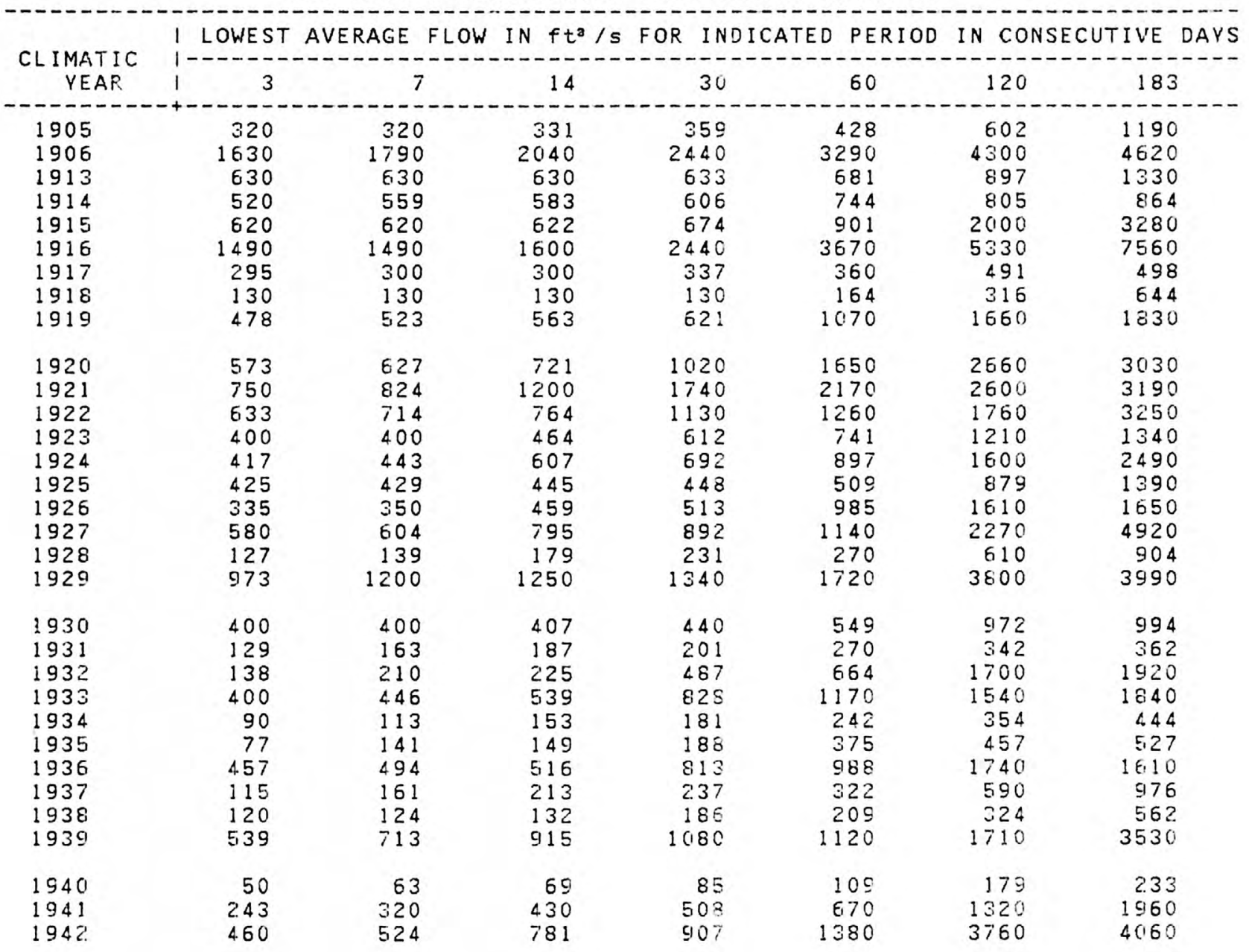


DES MOINES RIVER AT KEOSAUQUA--Cont 1 nued

LOW FLOW DISCHARGES, IN YEAR ENDING MARCH 31--Cont inued

\begin{tabular}{|c|c|c|c|c|c|c|c|c|}
\hline \multirow{2}{*}{$\begin{array}{c}\text { CLIMATIC } \\
\text { YEAR }\end{array}$} & 1 LOWEST & $A G E$ & In $f t^{a}$ & FOR IN & ED PE & IN CO & IVE & DAYS \\
\hline & 13 & 7 & 14 & 30 & 60 & 120 & 183 & \\
\hline $\begin{array}{l}1943 \\
1944 \\
1945 \\
1946 \\
1947 \\
1948 \\
1949\end{array}$ & $\begin{array}{l}567 \\
657 \\
795 \\
573 \\
550 \\
360 \\
269\end{array}$ & $\begin{array}{l}784 \\
714 \\
840 \\
590 \\
679 \\
390 \\
284\end{array}$ & $\begin{array}{r}988 \\
761 \\
856 \\
601 \\
1030 \\
401 \\
289\end{array}$ & $\begin{array}{r}1120 \\
876 \\
919 \\
820 \\
1440 \\
536 \\
335\end{array}$ & $\begin{array}{l}1730 \\
1200 \\
1040 \\
1220 \\
1590 \\
770 \\
482\end{array}$ & $\begin{array}{r}2190 \\
1410 \\
1370 \\
1740 \\
2540 \\
1050 \\
616\end{array}$ & $\begin{array}{r}3170 \\
1960 \\
2230 \\
3210 \\
3200 \\
1010 \\
916\end{array}$ & \\
\hline $\begin{array}{l}1950 \\
1951 \\
1952 \\
1953 \\
1954 \\
1955 \\
1956 \\
1957 \\
1958 \\
1959\end{array}$ & $\begin{array}{r}178 \\
193 \\
1220 \\
443 \\
153 \\
890 \\
101 \\
98 \\
303 \\
200\end{array}$ & $\begin{array}{r}241 \\
199 \\
1400 \\
545 \\
166 \\
716 \\
114 \\
110 \\
324 \\
204\end{array}$ & $\begin{array}{r}296 \\
209 \\
1620 \\
567 \\
183 \\
724 \\
134 \\
117 \\
389 \\
211\end{array}$ & $\begin{array}{r}366 \\
250 \\
1940 \\
597 \\
210 \\
770 \\
151 \\
126 \\
475 \\
217\end{array}$ & $\begin{array}{r}388 \\
270 \\
2450 \\
721 \\
273 \\
1330 \\
161 \\
140 \\
582 \\
275\end{array}$ & $\begin{array}{r}454 \\
442 \\
3620 \\
1200 \\
364 \\
2360 \\
192 \\
203 \\
718 \\
595\end{array}$ & $\begin{array}{r}636 \\
665 \\
4690 \\
1680 \\
450 \\
3680 \\
278 \\
251 \\
831 \\
1160\end{array}$ & \\
\hline $\begin{array}{l}1960 \\
1961 \\
1962 \\
1963 \\
1964 \\
1965 \\
1966 \\
1967 \\
1968\end{array}$ & $\begin{array}{r}687 \\
454 \\
1220 \\
430 \\
123 \\
503 \\
756 \\
190 \\
110\end{array}$ & $\begin{array}{r}743 \\
499 \\
1370 \\
553 \\
236 \\
624 \\
809 \\
217 \\
123\end{array}$ & $\begin{array}{r}856 \\
507 \\
1450 \\
671 \\
291 \\
788 \\
849 \\
258 \\
141\end{array}$ & $\begin{array}{r}1350 \\
543 \\
1800 \\
769 \\
328 \\
919 \\
978 \\
271 \\
270\end{array}$ & $\begin{array}{r}1480 \\
585 \\
3020 \\
781 \\
401 \\
938 \\
1520 \\
344 \\
447\end{array}$ & $\begin{array}{r}2070 \\
1000 \\
4240 \\
1310 \\
529 \\
1370 \\
3700 \\
410 \\
548\end{array}$ & $\begin{array}{r}2470 \\
1520 \\
5100 \\
2840 \\
521 \\
2220 \\
4930 \\
464 \\
858\end{array}$ & $\begin{array}{l}0 \\
0 \\
0 \\
0 \\
1 \\
0 \\
0 \\
4 \\
8\end{array}$ \\
\hline
\end{tabular}


05-4905.00 DES MOINES RIVER AT KEOSAUQUA--Continued

DRAINAGE AREA: $14,038 \mathrm{~m}^{2}$ PERIOD OF RECORD: 59 YEARS AVERAGE DISCHARGE: 5160 ft' /s

MINIMUM AVERAGE FLOWS FOR PERIOD OF RECORD

\begin{tabular}{|c|c|c|c|c|c|c|c|}
\hline PERIOD OF CONSECUTIVE DAYS I & 3 & 7 & 14 & 30 & 60 & 120 & 183 \\
\hline $\begin{array}{l}\text { D I SCHARGE, IN } \mathrm{ft}^{3} / \mathrm{s} \\
\text { CLIMATIC YEAR } \\
====================\end{array}$ & $\begin{aligned} & 50 \\
1940 & =====\end{aligned}$ & $\begin{array}{l}63 \\
1940 \\
====2\end{array}$ & $\begin{array}{l}69 \\
1940 \\
=====\end{array}$ & $\begin{aligned} & 85 \\
& 1940 \\
&=====\end{aligned}$ & $\begin{array}{l}109 \\
1940 \\
=====\end{array}$ & $\begin{array}{l}179 \\
1940 \\
=====\end{array}$ & $\begin{array}{l}233 \\
1940 \\
=====\end{array}$ \\
\hline
\end{tabular}

MAGNITUDE AND FREQUENCY OF ANNUAL LOW FLOWS

\begin{tabular}{l} 
RECURRENCE \\
INTERVAL \\
IN YEARS \\
\hline
\end{tabular}

MAGNITUDE AND FREQUENCY OF SEASONAL LOW FLOWS

RECURRENCE | LOWEST AVERAGE FLOW,IN ft /s, FOR THE INDICATED PERIOD IN CONSECUTIVE DAYS WITHIN EACH WATER-YEAR QUARTER
INTERVAL I
IN VEARS

DURATION OF DAILY DISCHARGES FOR ANNUAL AND SEASONAL PERIODS

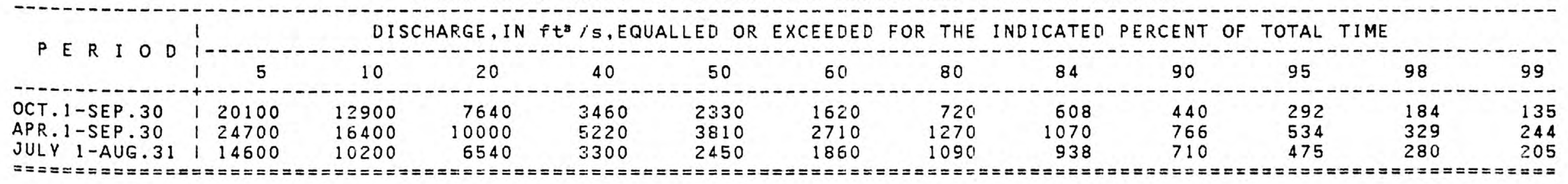


DES MOINES RIVER AT KEOSAUQUA--Continued, regulated period

LOW FLOW DISCHARGES, IN YEAR. ENDINC MARCH 31

\begin{tabular}{|c|c|c|c|c|c|c|c|}
\hline \multirow{2}{*}{$\begin{array}{c}\text { CLIMATIC } \\
\text { YEAR }\end{array}$} & LOWEST & RAGE & IN $\mathrm{ft}^{3}$ & OR IN & D PE & \multicolumn{2}{|c|}{ IN CONSECUTIVE } \\
\hline & 13 & 7 & 14 & 30 & 60 & 120 & 183 \\
\hline 1969 & 619 & 668 & 747 & $79:$ & 1000 & 1680 & 1880 \\
\hline $\begin{array}{l}1970 \\
1971 \\
1972 \\
1973 \\
1974 \\
1975 \\
1976\end{array}$ & $\begin{array}{r}510 \\
531 \\
328 \\
1430 \\
3720 \\
579 \\
421\end{array}$ & $\begin{array}{r}534 \\
551 \\
340 \\
1840 \\
4180 \\
596 \\
499\end{array}$ & $\begin{array}{r}508 \\
625 \\
353 \\
2230 \\
4710 \\
613 \\
537\end{array}$ & $\begin{array}{r}817 \\
1060 \\
371 \\
3720 \\
6970 \\
630 \\
61\end{array}$ & $\begin{array}{r}1010 \\
1490 \\
438 \\
6220 \\
9550 \\
846 \\
748\end{array}$ & $\begin{array}{r}1570 \\
2790 \\
902 \\
7220 \\
10400 \\
1460 \\
1160\end{array}$ & $\begin{array}{r}2350 \\
3310 \\
1170 \\
7940 \\
11700 \\
1460 \\
1400\end{array}$ \\
\hline$:====:=$ & & & & & & & \\
\hline
\end{tabular}


MINIMUM AVERAGE FLOWS FOR PERIOD OF RECORD

\begin{tabular}{|c|c|c|c|c|c|c|c|}
\hline PERIOD OF CONSECUTIVE DAYS & 3 & 7 & 14 & 30 & 60 & 120 & 183 \\
\hline $\begin{array}{l}\text { ISCHARGE, IN } \mathrm{ft}^{3} / \mathrm{s} \\
\text { IMATIC YEAR } \\
==================\end{array}$ & $\begin{array}{ll}1 & 328 \\
1 & 1972\end{array}$ & $\begin{array}{l}340 \\
1972\end{array}$ & $\begin{array}{l}353 \\
1972\end{array}$ & $\begin{array}{l}371 \\
1972\end{array}$ & $\begin{array}{l}438 \\
1972\end{array}$ & $\begin{array}{l}902 \\
1972\end{array}$ & $\begin{array}{l}0 \\
72\end{array}$ \\
\hline
\end{tabular}

MAGNITUDE AND FREQUENCY OF ANNUAL LOW FLOWS

RECURRENCE | LOWEST AVERAGE FLOW, IN $\mathrm{ft}^{3} / \mathrm{s}, \mathrm{FOR}$ INDICATED PERIOD IN CONSECUTIVE DAYS

\begin{tabular}{|c|c|c|c|c|c|c|c|c|}
\hline N YEARS & 1 & 3 & 7 & 14 & 30 & 60 & 120 & 183 \\
\hline $\begin{array}{l}1.5 \\
2 \\
5 \\
10 \\
20\end{array}$ & $\begin{array}{l}1 \\
1 \\
1 \\
1 \\
1\end{array}$ & $\begin{array}{l}828 \\
593 \\
378 \\
326 \\
299\end{array}$ & $\begin{array}{l}930 \\
653 \\
400 \\
338 \\
305\end{array}$ & $\begin{array}{c}1050 \\
727 \\
429 \\
356 \\
316\end{array}$ & $\begin{array}{r}1440 \\
936 \\
487 \\
378 \\
319\end{array}$ & $\begin{array}{r}1990 \\
1250 \\
602 \\
450 \\
367\end{array}$ & $\begin{array}{r}2980 \\
2020 \\
1100 \\
858 \\
721\end{array}$ & $\begin{array}{r}3500 \\
2410 \\
1340 \\
1050 \\
884\end{array}$ \\
\hline
\end{tabular}

319
316

MAGNITUDE AND FREQUENCY OF SEASONAL LOW FLOWS

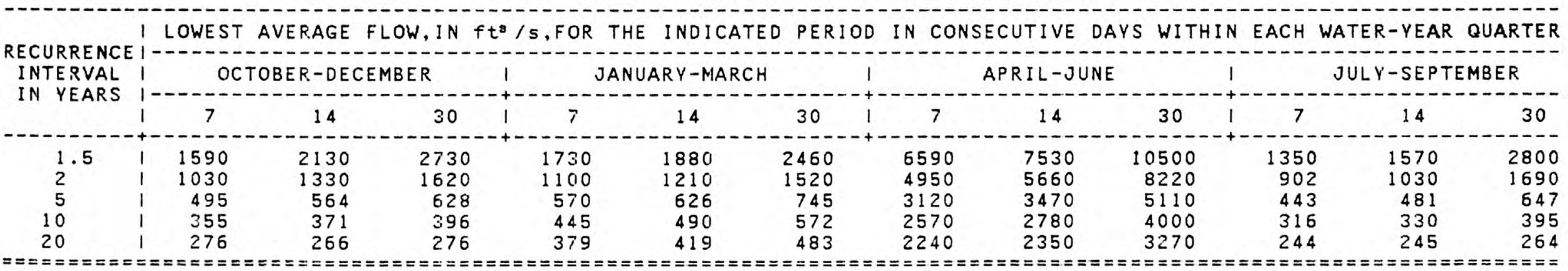

DURATION OF DAILY DISCHARGES FOR ANNUAL AND SEASONAL PERIODS

\begin{tabular}{|c|c|c|c|c|c|c|c|c|c|c|c|c|}
\hline $\mathrm{p}=$ & 1 & DIS & RGE, II & $=3$ is, E & ED OR & CEEDED & FOR THE & INDICATED & PERCENT & OF TOTAL & TIME & \\
\hline & 1 & 10 & 20 & 40 & 50 & 60 & 80 & 84 & 90 & 95 & 98 & 99 \\
\hline $\begin{array}{l}\text { OCT. } 1 \text {-SEP. } 30 \\
\text { APR. } 1-\text { SEP. } 30 \\
\text { JULY } 1 \text {-AUG. } 31\end{array}$ & $\begin{array}{l}123200 \\
125400 \\
1 \quad 20800\end{array}$ & $\begin{array}{l}19900 \\
20700 \\
19900\end{array}$ & $\begin{array}{l}16600 \\
18600 \\
18100\end{array}$ & $\begin{array}{r}7150 \\
12300 \\
9540\end{array}$ & $\begin{array}{l}4870 \\
8310 \\
5040\end{array}$ & $\begin{array}{l}3220 \\
5790 \\
3060\end{array}$ & $\begin{array}{l}1310 \\
2230 \\
1380\end{array}$ & $\begin{array}{l}1120 \\
1760 \\
1230\end{array}$ & $\begin{array}{r}847 \\
1050 \\
1010\end{array}$ & $\begin{array}{l}607 \\
593 \\
747\end{array}$ & $\begin{array}{l}409 \\
379 \\
568\end{array}$ & $\begin{array}{l}353 \\
322 \\
430\end{array}$ \\
\hline
\end{tabular}


Location.--Lat $40^{\circ} 26^{\prime} 33^{\prime \prime}$, long 91028'24", in NW1/4 SE1/4 sec.7. T.65 N., R.5 W. Lee County, on left bank 13 ft downstream from bridge on county highway W62, 2.8 miles downstream from Earlean Creek, 4.6 miles upstream from mouth, and 6.0 miles northwest of post office in Keokuk.

Remarks.--Discontinued September 1973.

LOW FLOW DISCHARGES, IN YEAR. ENDING MARCH 31

\begin{tabular}{|c|c|c|c|c|c|c|c|}
\hline CLIMATIC & I LOWEST A & FLOh & $\mathrm{ft}^{3} / \mathrm{s}$ & INOI & PERIOD & IN CONSE & VE DAYS \\
\hline YEAR & 13 & 7 & 14 & 30 & 60 & 120 & 183 \\
\hline $\begin{array}{l}1924 \\
1925 \\
1926 \\
1927 \\
1928 \\
1929\end{array}$ & $\begin{array}{r}0 \\
0 \\
.37 \\
.83 \\
0 \quad \\
.73\end{array}$ & $\begin{array}{l}0 \\
0 \\
.46 \\
1.2 \\
0 \\
2.2\end{array}$ & $\begin{array}{l}0 \\
0 \\
1.0 \\
3.8 \\
0 \\
3.2\end{array}$ & $\begin{array}{l}0 \\
.30 \\
9.6 \\
6.4 \\
.27 \\
5.9\end{array}$ & $\begin{array}{l}0.15 \\
2.3 \\
27 \\
20 \\
1.3 \\
13\end{array}$ & $\begin{array}{l}0.56 \\
5.7 \\
52 \\
52 \\
28 \\
18\end{array}$ & $\begin{array}{l}0.74 \\
15 \\
60 \\
123 \\
55 \\
48\end{array}$ \\
\hline $\begin{array}{l}1930 \\
1931 \\
1959\end{array}$ & $0^{.90} .20$ & $\begin{array}{l}1.1 \\
0.20\end{array}$ & $\begin{array}{l}3.0 \\
0.26\end{array}$ & $\begin{array}{l}4.5 \\
0.45\end{array}$ & $\begin{array}{l}10 \\
3.2 \\
.72\end{array}$ & $\begin{array}{l}27 \\
17 \\
4.6\end{array}$ & $\begin{array}{l}25 \\
17 \\
13\end{array}$ \\
\hline $\begin{array}{l}1960 \\
1961 \\
1962 \\
1963 \\
1964 \\
1965 \\
1966 \\
1967 \\
1968 \\
1969\end{array}$ & $\begin{array}{l}.30 \\
0 \\
0 \\
0 \\
0 \\
0 \\
.50 \\
0.0 \\
1.0\end{array}$ & $\begin{array}{l}0^{.66} \\
0 \\
0 \\
0 \\
0 \\
0 \\
0.70 \\
1.1 \\
0\end{array}$ & $\begin{array}{l}0 \\
0 \\
0 \\
0 \\
0 \\
0 \\
.72 \\
0.79 \\
\vdots .5\end{array}$ & $\begin{array}{l}5.6 \\
0 \\
.09 \\
.06 \\
0 \\
0 \\
1.6 \\
.18 \\
5.5 \\
0\end{array}$ & $\begin{array}{c}7.8 \\
.17 \\
4.7 \\
.31 \\
0.14 \\
2.6 \\
.66 \\
11.3 \\
2.3\end{array}$ & $\begin{array}{c}41 \\
4.7 \\
13 \\
5.5 \\
0 \\
1.6 \\
16 \\
3.2 \\
14 \\
2.6\end{array}$ & $\begin{array}{c}89 \\
3.4 \\
54 \\
4.9 \\
0 \\
3.1 \\
23 \\
2.9 \\
38 \\
5.9\end{array}$ \\
\hline $\begin{array}{l}1970 \\
1971 \\
1972 \\
1973\end{array}$ & $\begin{array}{l}.66 \\
1.5 \\
0.06\end{array}$ & $\begin{array}{l}.75 \\
1.8 \\
0.13\end{array}$ & $\begin{array}{l}.94 \\
2.4 \\
0 \\
.30\end{array}$ & $\begin{array}{r}1.8 \\
3.4 \\
.51 \\
.42\end{array}$ & $\begin{array}{l}3.2 \\
9.1 \\
2.2 \\
3.1\end{array}$ & $\begin{array}{l}12 \\
41 \\
3.9 \\
7.8\end{array}$ & $\begin{array}{c}22 \\
71 \\
8.7 \\
10\end{array}$ \\
\hline
\end{tabular}


MINIMUM AVERAGE FLOWS FOR PERIOD OF RECORD

\begin{tabular}{|c|c|c|c|c|c|c|c|}
\hline PERIOD OF CONSECUTIVE DAYS & 3 & 7 & 14 & 30 & 60 & 120 & 183 \\
\hline $\begin{array}{l}\text { HARGE, IN } f t^{3} / s \\
\text { ATIC YEAR }\end{array}$ & $\begin{array}{c}0 \\
1972\end{array}$ & $\begin{array}{l}0 \\
1972\end{array}$ & $\stackrel{0}{1972}$ & $\begin{array}{c}0 \\
1969\end{array}$ & $\begin{array}{c}0 \\
1964\end{array}$ & $\begin{array}{l}0 \\
1964\end{array}$ & $\begin{array}{l}0 \\
1964\end{array}$ \\
\hline
\end{tabular}

MAGNITUDE AND FREQUENCY OF ANNUAL LOW FLOWS

RECURRENCE
INTERVAL
IN Y LEWEST AVERAGE FLOW, IN $\mathrm{ft} / \mathrm{s}, \mathrm{FOR}$

MAGNITUDE AND FREQUENCY OF SEASONAL LOW FLOWS

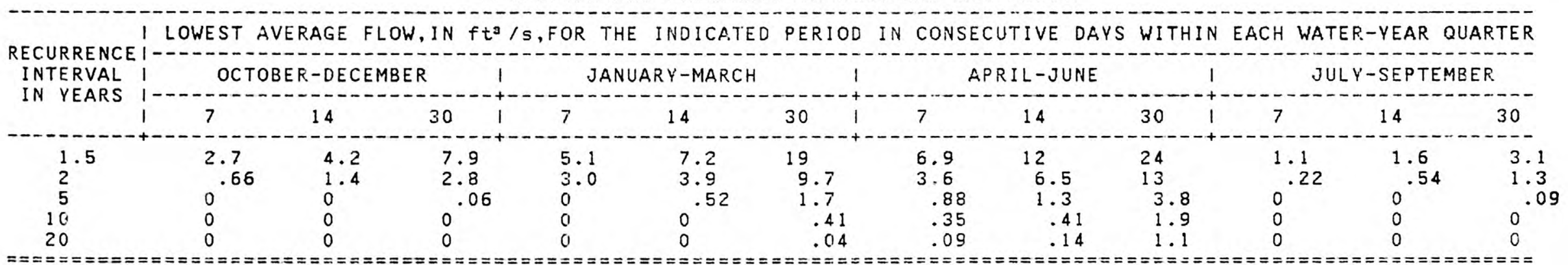

DURATION OF DAILY DISCHARGES FOR ANNUAL AND SEASONAL FERIODS

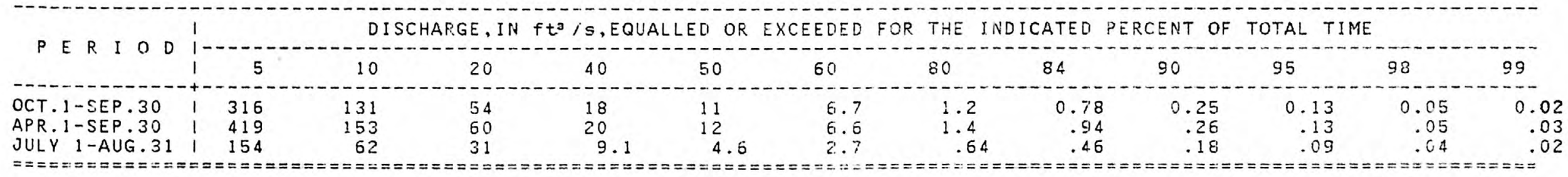


Location.--Lat $40^{\circ} 46^{\prime} 10^{\prime \prime}$, long 92025'05", in SW1/4 SE1/4 sec.13, T.69 N., R.14 W. Davis County, on left bank $15 \mathrm{ft}$ downstream from bridge on county highway $v 20,1.3$ miles northwest of county courthouse at bloomfieid, and $8.6 \mathrm{mi}$ ies downstream from North Fox Creek.

Remarks.--Discontinued September 1973.

LOW FLOW DISCHARGES, IN VEAR. ENDING MARCH 31

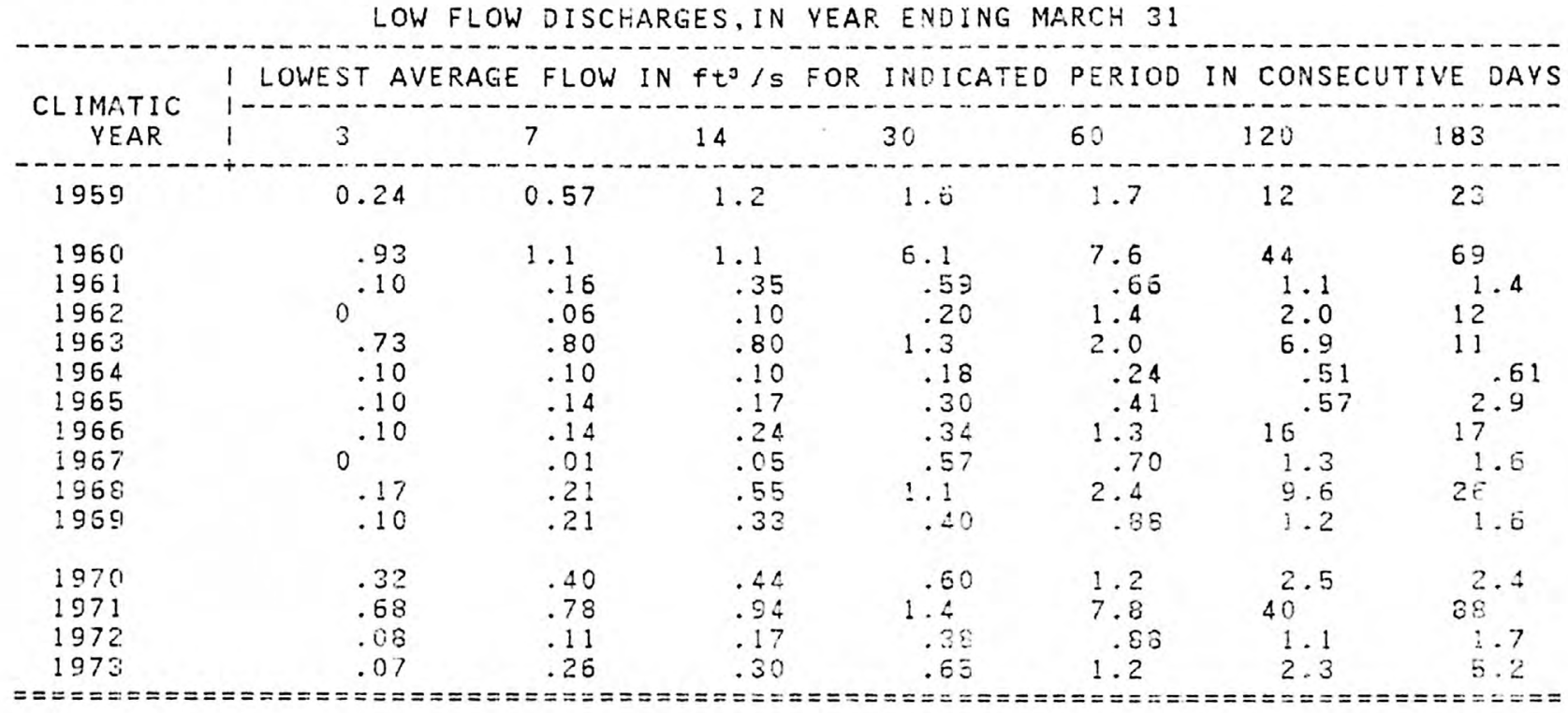


DRAINAGE AREA: $87.7 \mathrm{~m}^{2} \quad$ PERIOD OF RECORD: 16 YEARS AVERAGE DISCHARGE: 49.4 ft3/s

MINIMUM AVERAGE FLOWS FOR PERIOD OF RECORD

\begin{tabular}{|c|c|c|c|c|c|c|c|c|}
\hline ERIOD OF CONSECUTIVE DAYS & 1 & 3 & 7 & 14 & 30 & 60 & 120 & 183 \\
\hline $\begin{array}{l}\text { RGE, IN } f t^{3} / s \\
\text { IC VEAR }\end{array}$ & 1 & $\begin{array}{c}0 \\
1967\end{array}$ & $\begin{array}{l}0.01 \\
1967\end{array}$ & $\begin{array}{l}0.05 \\
1967\end{array}$ & $\begin{array}{l}0.19 \\
1964\end{array}$ & $\begin{array}{l}0.25 \\
1964\end{array}$ & $\begin{array}{r}0.51 \\
1964\end{array}$ & $\begin{array}{l}0.62 \\
1964\end{array}$ \\
\hline
\end{tabular}

MAGNITUDE AND FREQUENCY OF ANNUAL LOW FLOWS

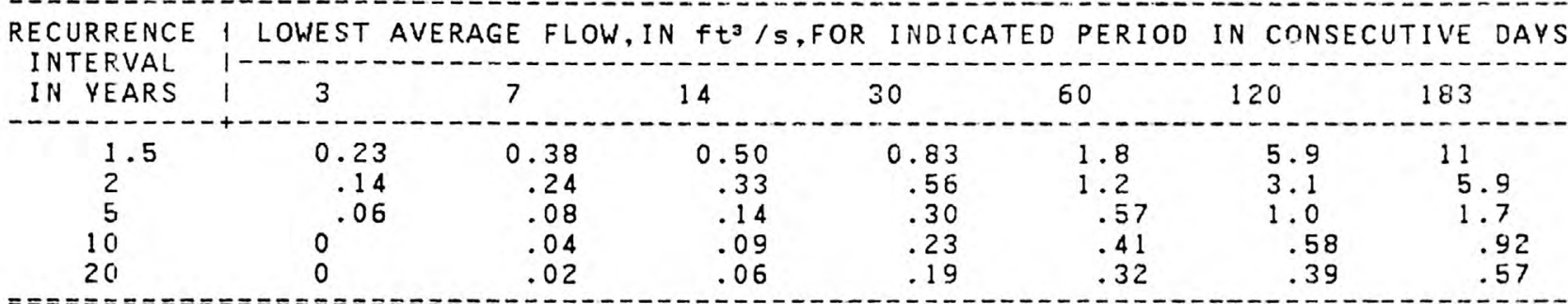

MAGNITUDE AND FREQUENCY OF SEASONAL LOW FLOWS

I LOWEST AVERAGE FLOW, IN $\mathrm{ft}^{3} / \mathrm{s}, \mathrm{FOR}$ THE INDICATED PERIOD IN CONSECUTIVE DAYS WITHIN EACH WATER-YEAR QUARTER

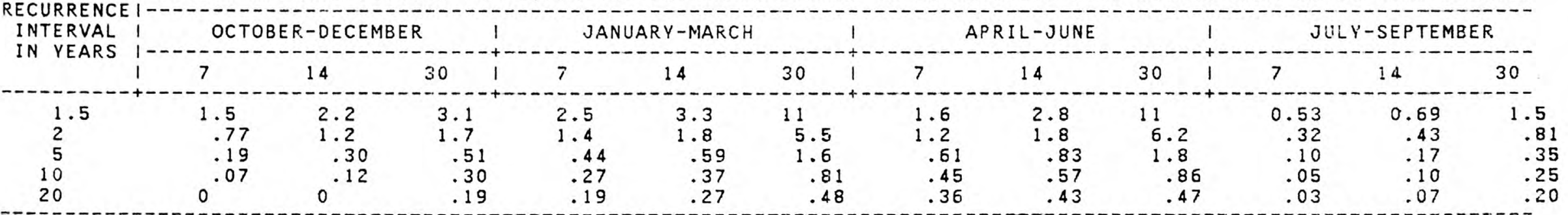

DURATION OF DAILY DISCHARGES FOR ANNUAL AND SEASONAL PERIODS

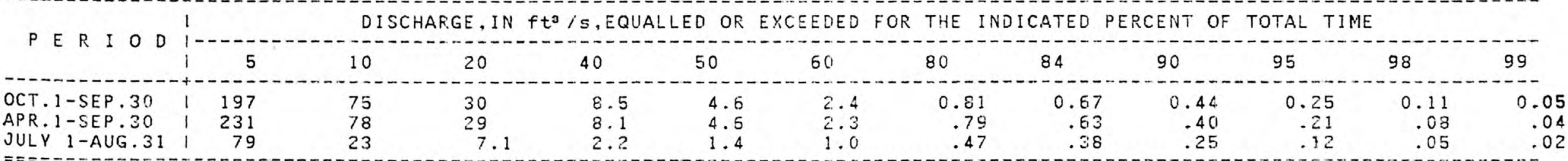


Location.--Lat 40039'35", long 92003'46", in NW1/4 SW1/4 sec.30, T.68 N., R.10 W. Van Buren County, on left bank $5 \mathrm{ft}$ dowristream from bridge on State Highway 2 , 0.25 mile upstream from Bone Run, and $1 \mathrm{mile}$ northeast of Cantrit.

Remarks.--Discontinued September 1951.

LOW FLOW DISCHARGES, IN YEAR. ENDING MARCH 31

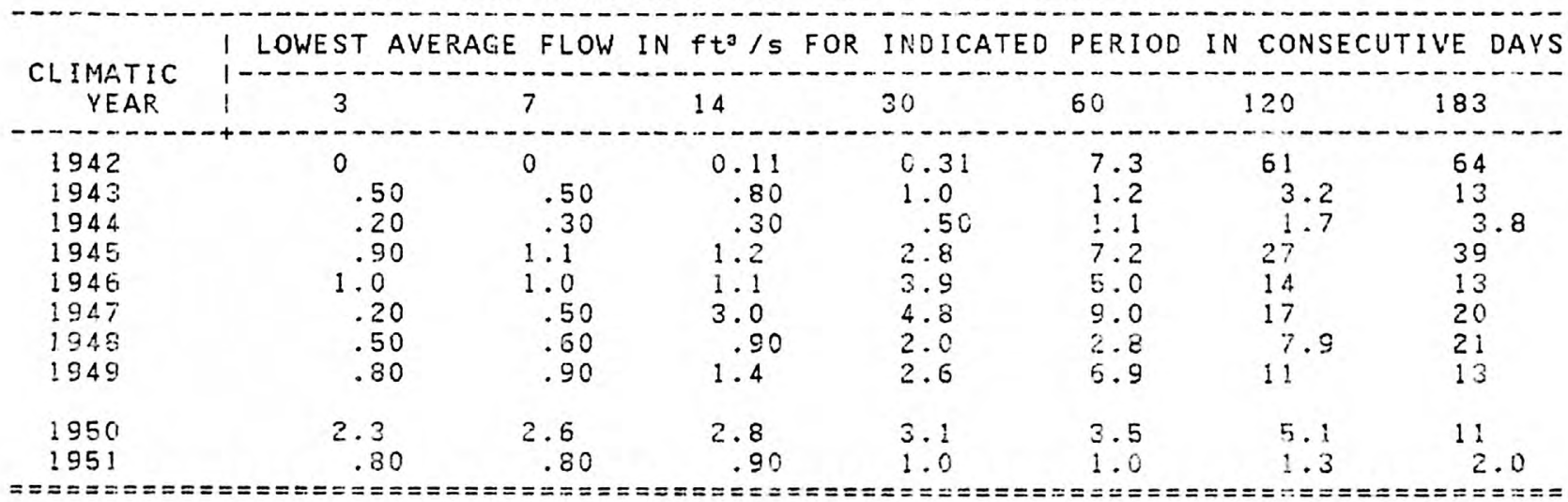


MINIMUM AVERAGE FLOWS FOR PERIOD OF RECORD

\begin{tabular}{|c|c|c|c|c|c|c|c|}
\hline PERIOD OF CONSECUTIVE DAYS & 3 & 7 & 14 & 30 & 60 & 120 & 133 \\
\hline $\begin{array}{l}\text { DISCHARGE, IN } \mathrm{ft}^{3} / \mathrm{s} \\
\text { CL IMATIC YEAR }\end{array}$ & $\begin{array}{c}0 \\
1942\end{array}$ & $\begin{array}{c}0 \\
1942\end{array}$ & $\begin{array}{l}0.11 \\
1942\end{array}$ & $\begin{array}{l}0.31 \\
1942\end{array}$ & $\begin{array}{r}1.0 \\
1951\end{array}$ & $\begin{array}{r}1.3 \\
1951\end{array}$ & \\
\hline
\end{tabular}

MAGNITUDE AND FREQUENCY OF ANNUAL LOW FLOWS

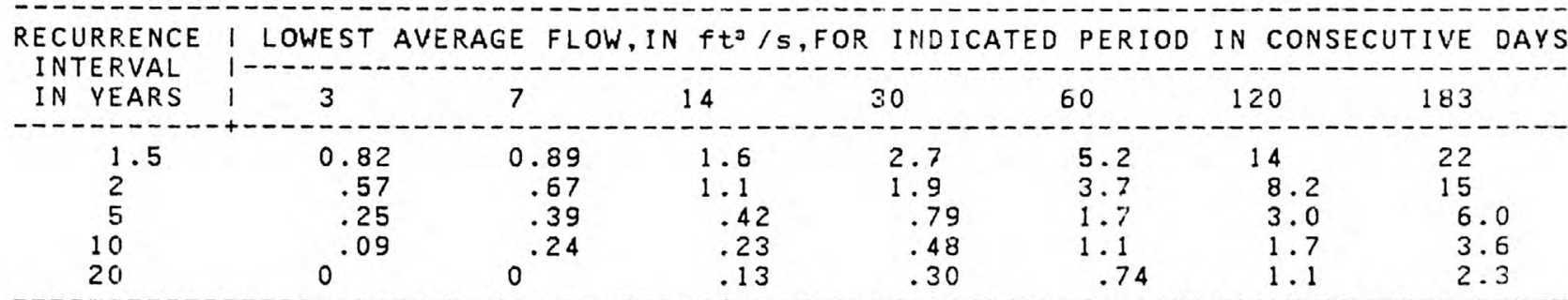

MAGNITUDE AND FREQUENCY OF SEASONAL LOW FLOWS

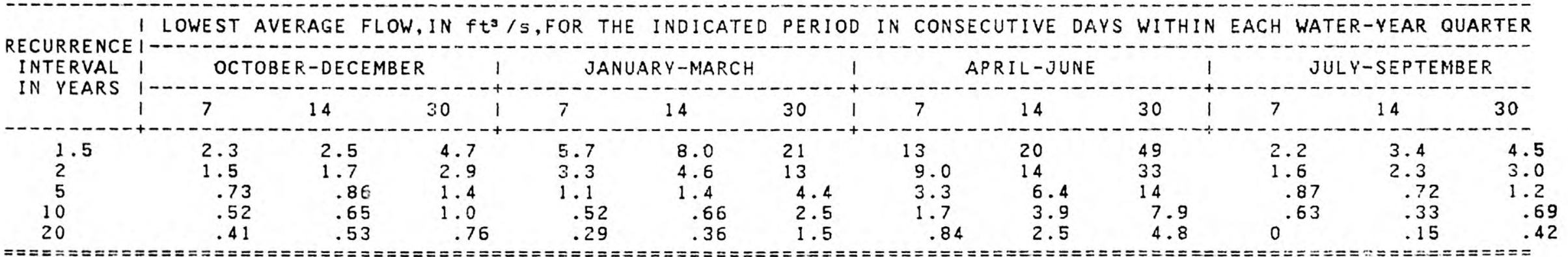

DURATION OF DAILY OISCHARGES FOR ANNUAL AND SEASONAL PERIODS

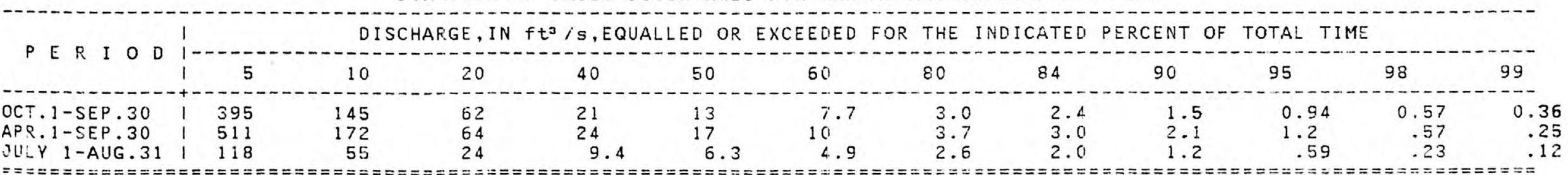




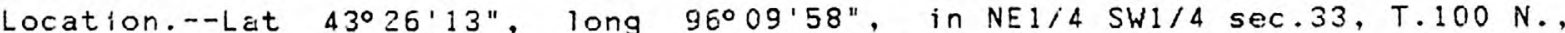
R. 45 W. L Lyon County, on right bank at dam on north side of city park in Rock Rapids, 0.3 mile upstream from Tom Creek, $0.5 \mathrm{mile}$ northeast of junction of U.S. Highways 75 and 9 , and at mile 42.8 .

Remarks.--Discontinued September 1974.

LOW FLOW DISCHARGES, IN YEAR: ENDING MARCH 31

\begin{tabular}{|c|c|c|c|c|c|c|c|}
\hline \multirow{2}{*}{$\begin{array}{c}\text { CLIMATIC } \\
\text { YEAR }\end{array}$} & \multicolumn{7}{|c|}{ E PERIOD IN CONSECUTIVE DAYS } \\
\hline & 13 & 7 & 14 & 30 & 60 & 120 & 183 \\
\hline $\begin{array}{l}1962 \\
1963 \\
1964 \\
1965 \\
1968 \\
1967- \\
1968 \\
1969\end{array}$ & $\begin{array}{l}8.3 \\
3.6 \\
4.7 \\
.80 \\
7.0 \\
4.3 \\
1.3 \\
4.2\end{array}$ & $\begin{array}{l}9.0 \\
3.6 \\
5.0 \\
1.0 \\
7.0 \\
5.4 \\
1.3 \\
5.8\end{array}$ & $\begin{array}{l}11 \\
4.0 \\
5.2 \\
1.3 \\
7.4 \\
5.5 \\
1.3 \\
6.3\end{array}$ & $\begin{array}{r}14 \\
6.2 \\
5.8 \\
1.6 \\
11 \\
5.6 \\
2.0 \\
7.6\end{array}$ & $\begin{array}{r}18 \\
8.4 \\
6.1 \\
6.6 \\
2 ! \\
6.1 \\
3.0 \\
15\end{array}$ & $\begin{array}{l}2.5 \\
17 \\
8.9 \\
5.6 \\
35 \\
14 \\
5.8 \\
22\end{array}$ & $\begin{array}{l}44 \\
24 \\
13 \\
18 \\
43 \\
20 \\
5.7 \\
29\end{array}$ \\
\hline $\begin{array}{l}1970 \\
1971 \\
1972 \\
1973 \\
1974\end{array}$ & $\begin{array}{r}16 \\
3.9 \\
2.1 \\
14.1 \\
6.8\end{array}$ & $\begin{array}{r}16 \\
3.9 \\
2.3 \\
17 \\
7.9\end{array}$ & $\begin{array}{r}16 \\
6.1 \\
2.6 \\
18 \\
9.0\end{array}$ & $\begin{array}{l}15 \\
11 \\
3.8 \\
25 \\
9.1\end{array}$ & $\begin{array}{l}13 \\
17 \\
5.3 \\
31 \\
11\end{array}$ & $\begin{array}{l}25 \\
55 \\
17 \\
58 \\
28\end{array}$ & $\begin{array}{l}3: \\
54 \\
17 \\
65 \\
27\end{array}$ \\
\hline
\end{tabular}


06-4832.70 ROCK RIVER AT ROCK RAPIDS--Cont1nued

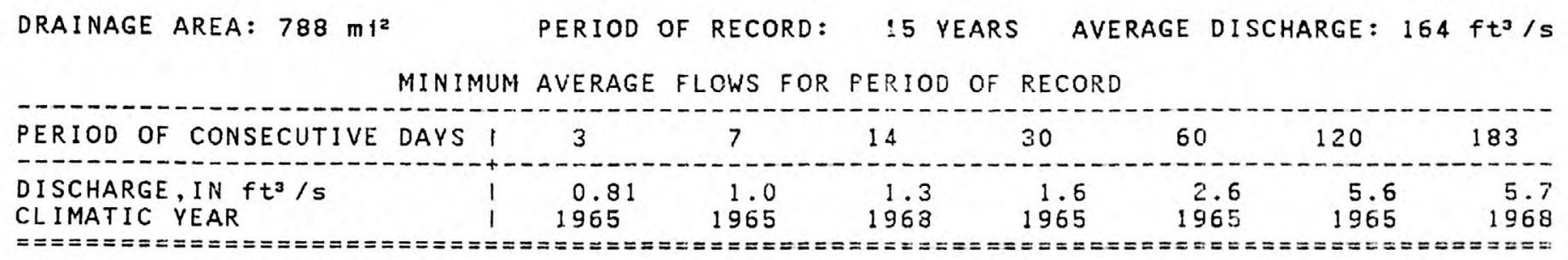

MAGNITUDE AND FREQUENCY OF ANNUAL LOW FLOWS

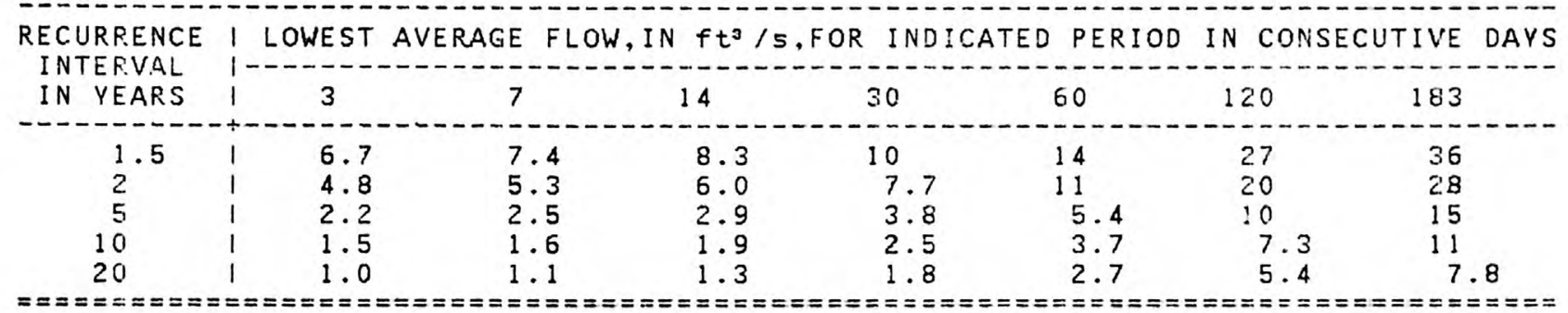

MAGNITUDE AND FREQUENCY OF SEASONAL LOW FLOWS

I LOWEST AVERAGE FLOW, IN $\mathrm{ft}^{3} / \mathrm{s}$, FOR THE INDICATED PERIOD IN CONSECUTIVE DAYS WITHIN EACH WATER-VEAR QUARTER

\begin{tabular}{l}
$\begin{array}{l}\text { RECURRENCE } \\
\text { INTERVAL } \\
\text { IN YEARS }\end{array}$ \\
\hline
\end{tabular}

DURATION OF DAILY DISCHARGES FOR ANNUAL AND SEASONAL FERIODS

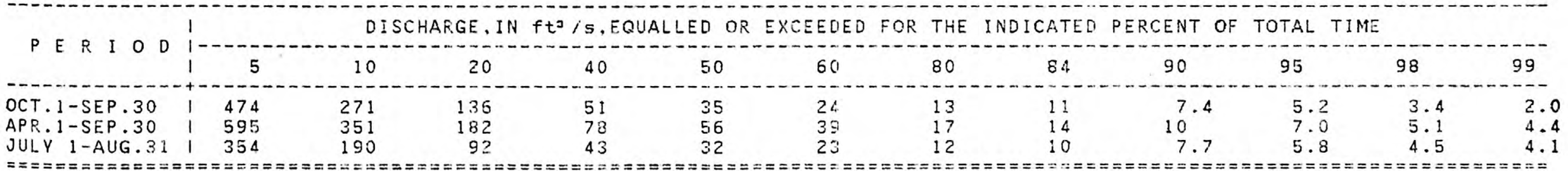


Location.--Lat 43021'52"", long 96017'39", in SW1/4 SWl/4 sec.16. T.97 N., R.46 W.. Sloux County, on $r i g h t$ bark 3 ft upsiream from bridge on county highway K30. 0.3 mile north of Rock Valley and at mile 19.1. Prior to May 5, 1976, at site 3.2 miles downstream.

LOW FLOW DISCHARGES, IN YEAR. ENDING MARCH 31

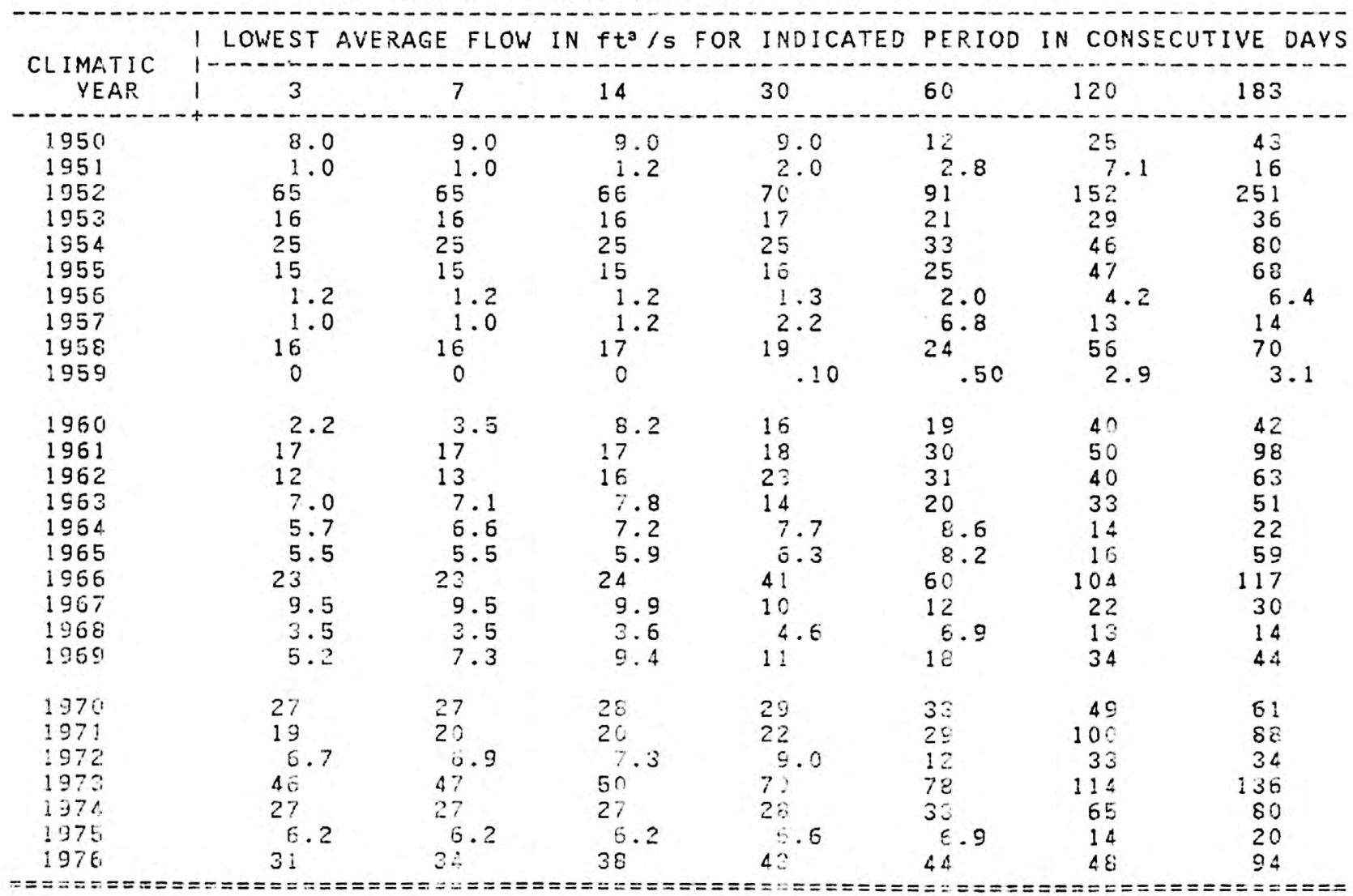




\section{DRAINAGE AREA: $1592 \mathrm{~m}^{2}$ PERIOD OF RECORD: 28 YEARS AVERAGE DISCHARGE: 295 ft? /S}

MINIMUM AVERAGE FLOWS FOR PEPIOD OF RECORD

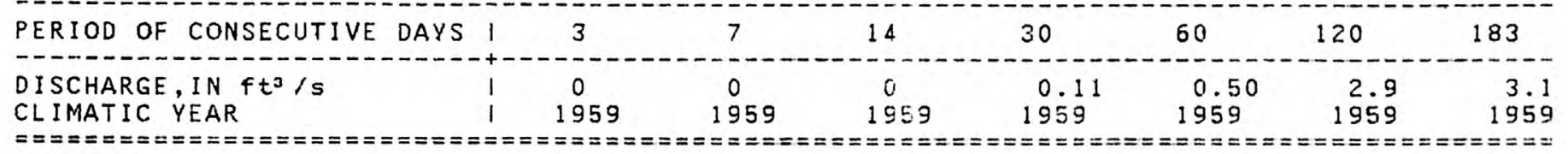

MAGNITUDE AND FREQUENCY OF ANNUAL LOW FLOWS

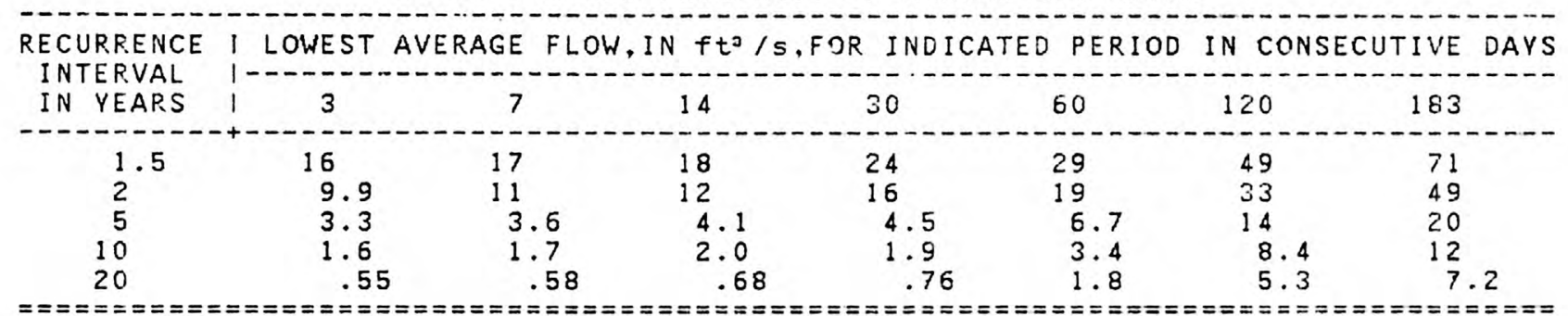

MAGNITUDE AND FREQUENCY OF SEASONAL LOW FLOWS

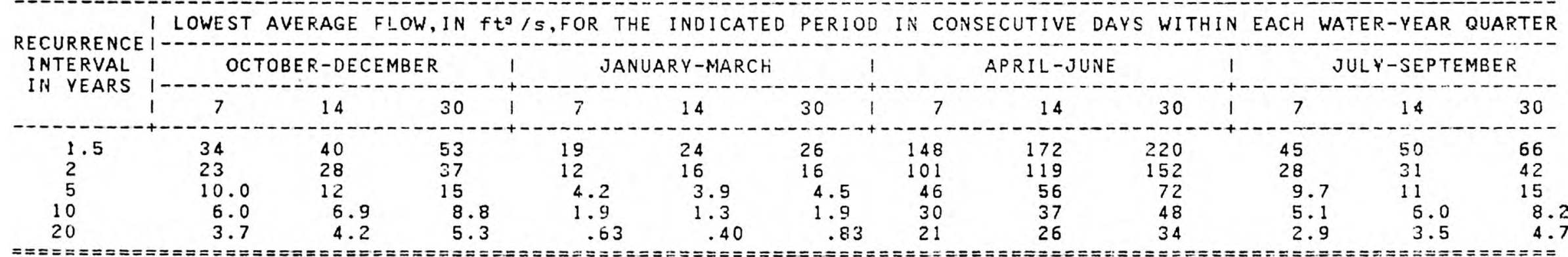

DURATION OF DAILY DISCHARGES FOR ANNUAL AND SERSONAL FERIODS

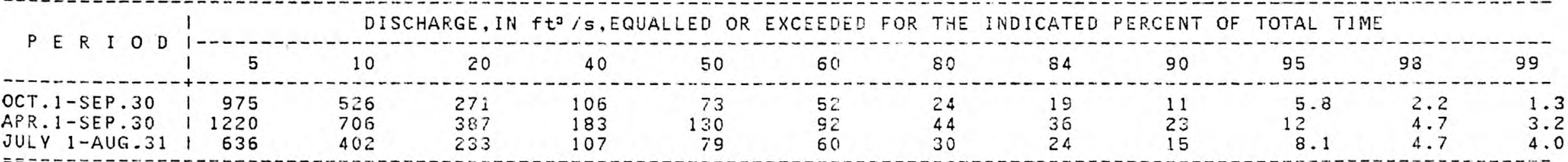


Location.--Lat $42^{\circ} 59^{\prime} 48^{\prime \prime}$, long 96028'10", in NE1/4 NE1/4 sec.ć, T.94 N., R.48 W., Sioux County, on left bank 6 it downstream from bridge on State Highway 10 at east edge of Hawarden ard 2.0 miles upstream from mouth.

Remarks.--Discontinued September 1969.

LOW FLOW DISCHARGES, IN YEAR. ENDING MARCH 31

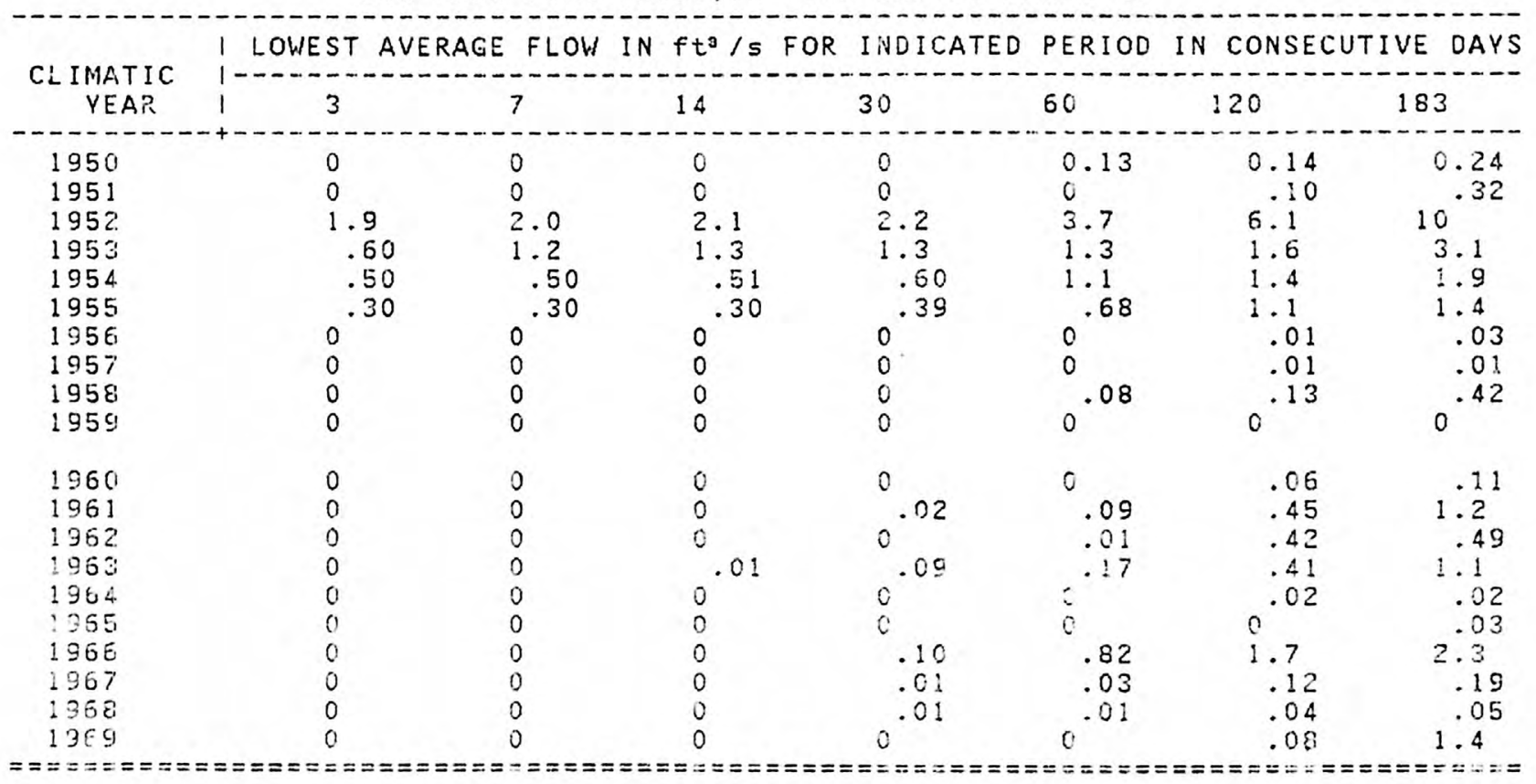


DRAINAGE AREA: $48.4 \mathrm{mi}^{2} \quad$ PERIOD OF RECORD: 20 VEARS AVERAGE DISCHARGE: 7.8 ft $3 / 5$

MINIMUM AVERAGE FLOWS FOR PERIOD OF RECORD

\begin{tabular}{|c|c|c|c|c|c|c|c|}
\hline PERIOD OF CONSECUTIVE DAYS I & 3 & 7 & 14 & 30 & 60 & 120 & 183 \\
\hline $\begin{array}{l}\text { DISCHARGE, IN } \mathrm{ft}^{3} / \mathrm{s} \\
\text { CLIMATIC YEAR }\end{array}$ & $\begin{array}{l}0 \\
1969\end{array}$ & $\stackrel{0}{1969}$ & $\begin{array}{l}0 \\
1959\end{array}$ & $\begin{array}{l}0 \\
1969\end{array}$ & $\begin{array}{l}0 \\
1969\end{array}$ & $\begin{array}{l}0 \\
1965\end{array}$ & 195 \\
\hline
\end{tabular}

MAGNITUDE AND FREQUENCY OF ANNUAL LOW FLOWS

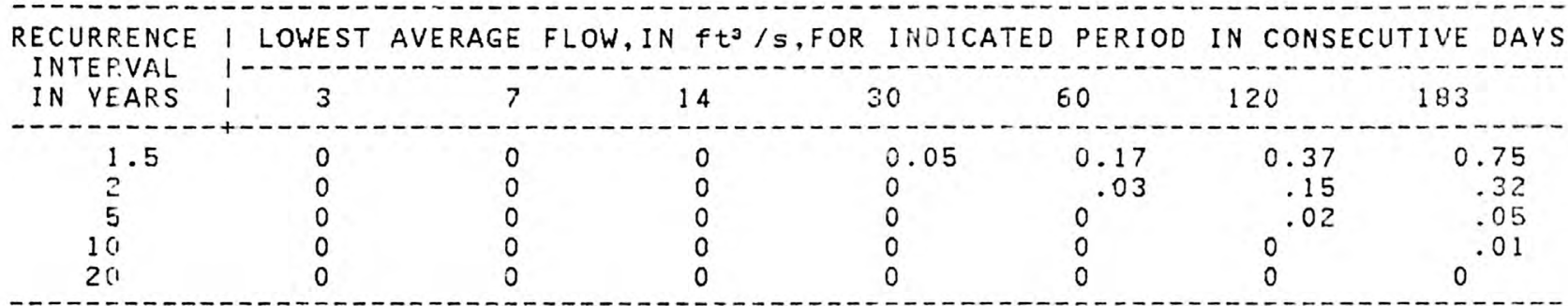

MAGNITUDE AND FREQUENCY OF SEASONAL LOW FLOWS

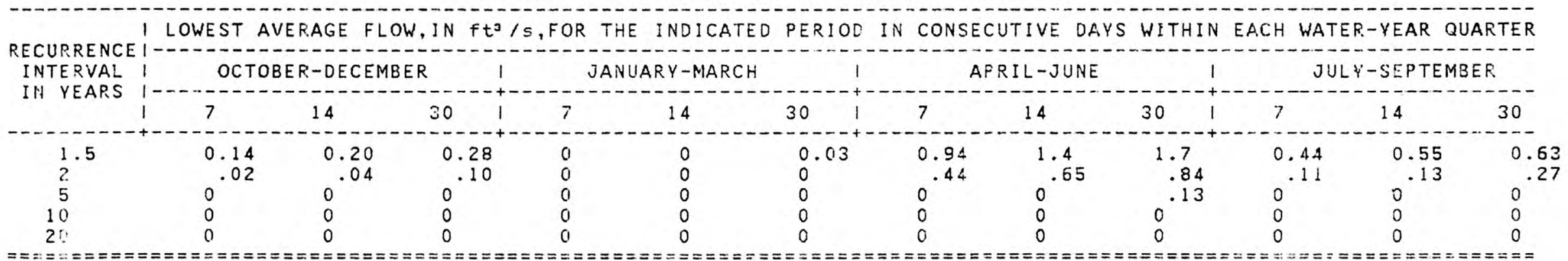

DURATION OF DAILY DISCHARGES FOR ANNUAL AND SEASONAL PERIODS

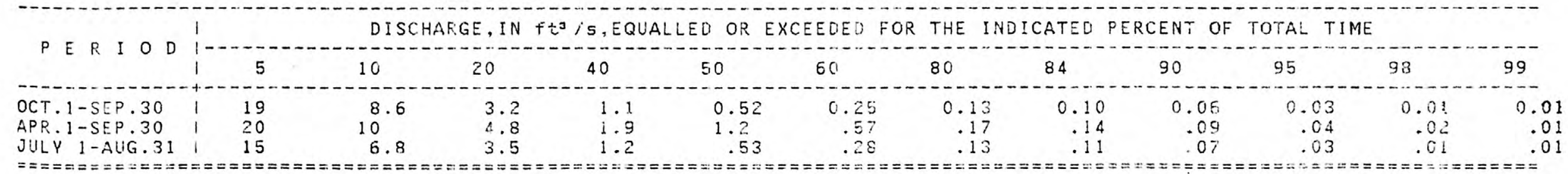


Location.--Lat $42^{\circ} 49^{\prime} 42^{\prime \prime}$, long 98, 33'45", in NW1/4 SW $1 / 4 \mathrm{sec} .31$, T.93 N., R.48 W. Plymouth County, Iowa, on left bank at west $\in$ dge of Akron, 0.6 mile downstream from bridge on State Highway 43 , and 2.3 miles lpstream from Union Creek.

LOW FLOW DISCHARGES, IN YEAR. ENDING MARCH 31

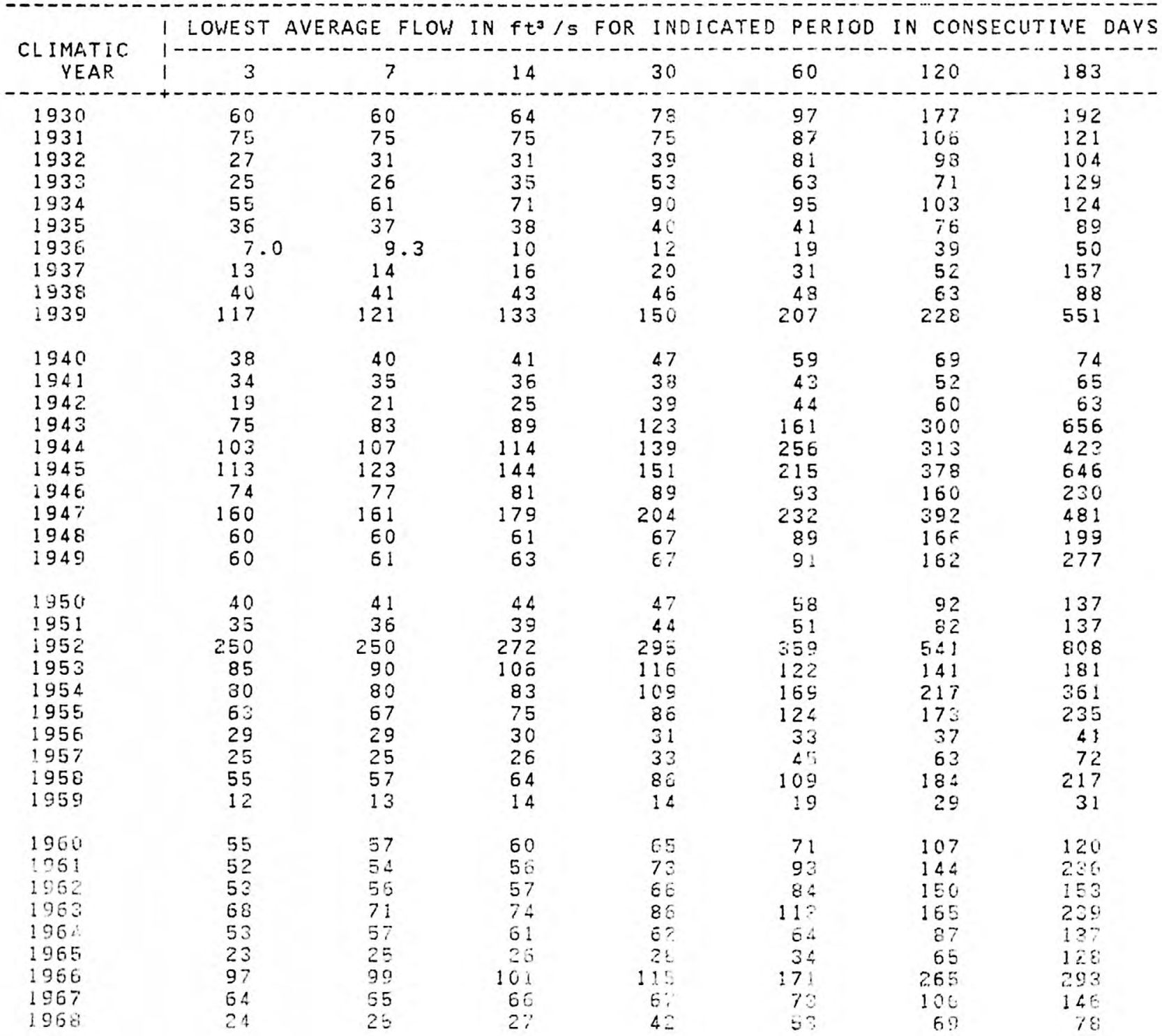


LOW FLOW DISCHARGES, IN YEAR ENDING MARCH 31--Cont1nued

\begin{tabular}{|c|c|c|c|c|c|c|c|}
\hline & 1 LOWES & $A G E$ & $N f t^{3}$ & $R I N$ & $D$ PE & IN $\mathrm{COI}$ & IVE DAYS \\
\hline YEAR & 1 & 7 & 14 & 30 & 60 & 120 & 183 \\
\hline 1969 & 59 & 61 & 61 & 64 & 82 & 134 & 159 \\
\hline $\begin{array}{l}1970 \\
1971 \\
1972 \\
1973 \\
1974 \\
1975 \\
1976\end{array}$ & $\begin{array}{r}103 \\
62 \\
69 \\
223 \\
83 \\
27 \\
62\end{array}$ & $\begin{array}{r}108 \\
64 \\
70 \\
229 \\
88 \\
27 \\
64\end{array}$ & $\begin{array}{r}113 \\
71 \\
72 \\
241 \\
94 \\
30 \\
69\end{array}$ & $\begin{array}{r}118 \\
80 \\
78 \\
256 \\
107 \\
30 \\
71\end{array}$ & $\begin{array}{r}123 \\
112 \\
101 \\
407 \\
131 \\
48 \\
82\end{array}$ & $\begin{array}{r}165 \\
194 \\
151 \\
435 \\
199 \\
63 \\
101\end{array}$ & $\begin{array}{r}218 \\
202 \\
145 \\
501 \\
227 \\
72 \\
161\end{array}$ \\
\hline
\end{tabular}


DRAINAGE AREA: $9030 \mathrm{mi}^{2}$ PERIOD OF RECORD: 48 YEARS AVERAGE DISCHARGE: $832 \mathrm{ft} 3 / \mathrm{s}$

MINIMUM AVERAGE FLOWS FOR PERIOD OF RECORD

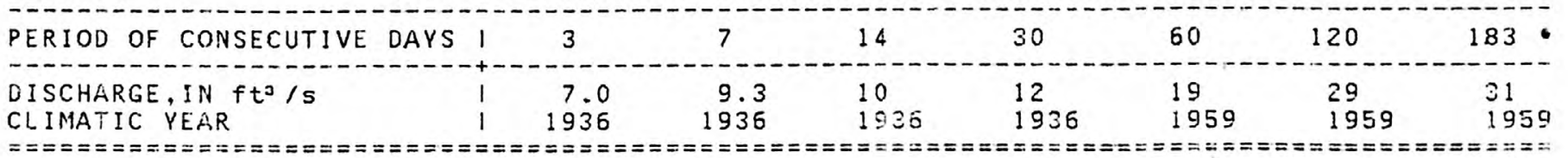

MAGNITUDE AND FREQUENCY OF ANNUAL LOW FLOWS

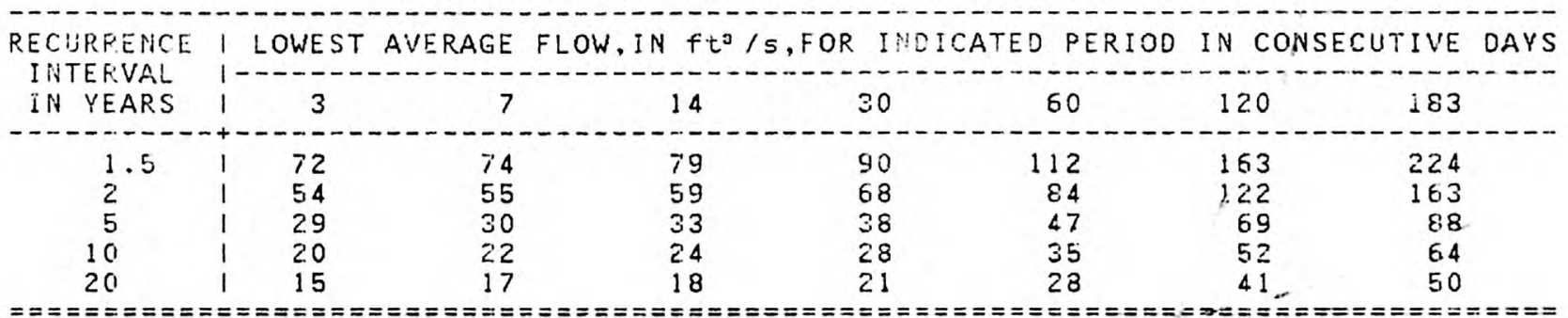

MAGNITUDE AND FREQUENCY OF SEASONAL LOW FLOWS

RECURRENCE
INTERVAL
IN YEARS

DURATION OF DAILY DISCHARGES FOR ANNUAL ANO SEASONAL. PERIUOS

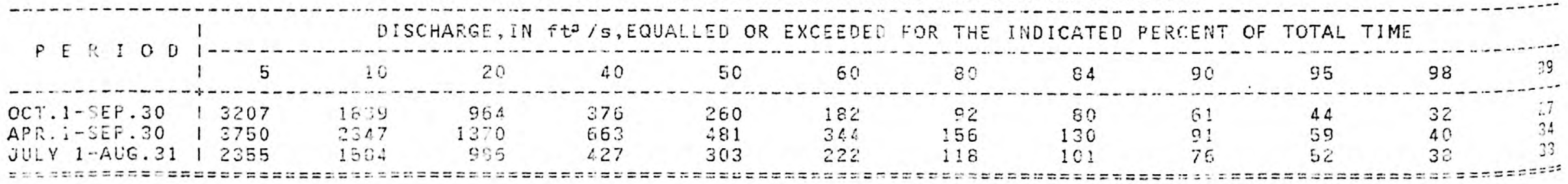


Location.--Lat $42^{\circ} 29^{\prime} 10^{\prime \prime}$, long $96^{\circ} 24^{\prime} 47^{\prime \prime}$, in NW1/4 SE1/4 sec.16, T.29 N., R.9 E., sixth principal meridian, Dakota County, Nebraska, on right bank on upstream 5 lde of bridge on U.S. Highway 77 at South Sloux City, Nebraska, $2.0 \mathrm{~m} l$ lies downstream from Big Sioux River, and at mile 732.3 .

Remarks.--Flow partly regulated by upstream main-stem reservoirs since Nov. 1937 .

LOW FLOW DISCHARGES, IN YEAR. ENDING MARCH 31

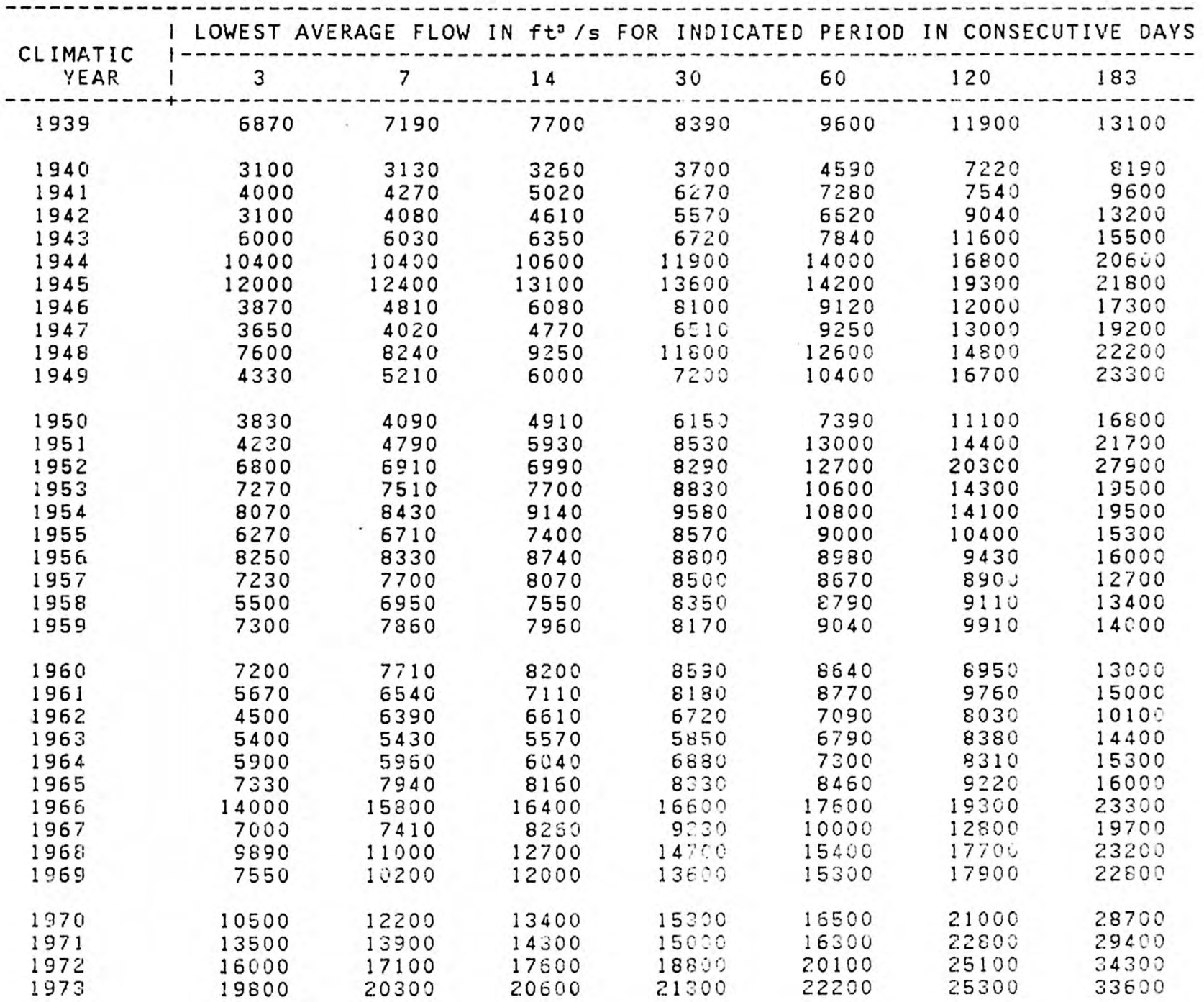


MISSOURI RIVER AT SIOUX CITY--Continued

LOW FLOW DISCHARGES, IN YEAR ENDING MARCH 31--Continued

\begin{tabular}{|c|c|c|c|c|c|c|c|}
\hline CLIMATIC & 1 LOWEST & $A G E F L$ & $\begin{array}{l}n+t^{5} / \\
--n-2-\end{array}$ & R INOI & D PERIC & $N$ CONS & IVE DAY \\
\hline YEAR & 13 & 7 & 14 & 30 & 60 & 120 & 183 \\
\hline $\begin{array}{l}1974 \\
1975 \\
1976\end{array}$ & $\begin{array}{l}13200 \\
10400 \\
13200\end{array}$ & $\begin{array}{l}13900 \\
14700 \\
16800\end{array}$ & $\begin{array}{l}15100 \\
16600 \\
18800\end{array}$ & $\begin{array}{l}16800 \\
17400 \\
21800\end{array}$ & $\begin{array}{l}18000 \\
17800 \\
23600\end{array}$ & $\begin{array}{l}19500 \\
18500 \\
28300\end{array}$ & $\begin{array}{l}23000 \\
23700 \\
40200\end{array}$ \\
\hline
\end{tabular}

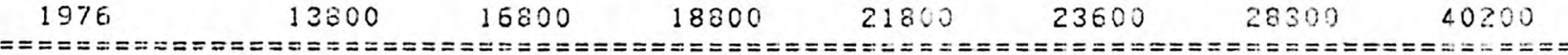


DRAINAGE AREA: $314,600 \mathrm{~m}^{2}$ PERIOD OF RECORD: 39 VEARS AVERAGE DISCHARGE: 32,000 ft $/ \mathrm{s}$

MINIMUM AVERAGE FLOWS FOR PERIOD OF RECORD

\begin{tabular}{|c|c|c|c|c|c|c|c|}
\hline DERIOD OF CONSECUTIVE DAYS I & 3 & 7 & 14 & 30 & 60 & 120 & 183 \\
\hline $\begin{array}{l}\text { RGE, IN } \mathrm{ft}^{3} / \mathrm{s} \\
\text { IC YEAR }\end{array}$ & $\begin{array}{l}3100 \\
1942\end{array}$ & $\begin{array}{l}3130 \\
1940\end{array}$ & $\begin{array}{l}3260 \\
1940\end{array}$ & $\begin{array}{l}3700 \\
1940\end{array}$ & $\begin{array}{l}4590 \\
1940\end{array}$ & $\begin{array}{l}7220 \\
1940\end{array}$ & $\begin{array}{r}8190 \\
1940\end{array}$ \\
\hline
\end{tabular}

MAGNITUDE AND FREQUENCY OF ANNUAL LOW FLOWS

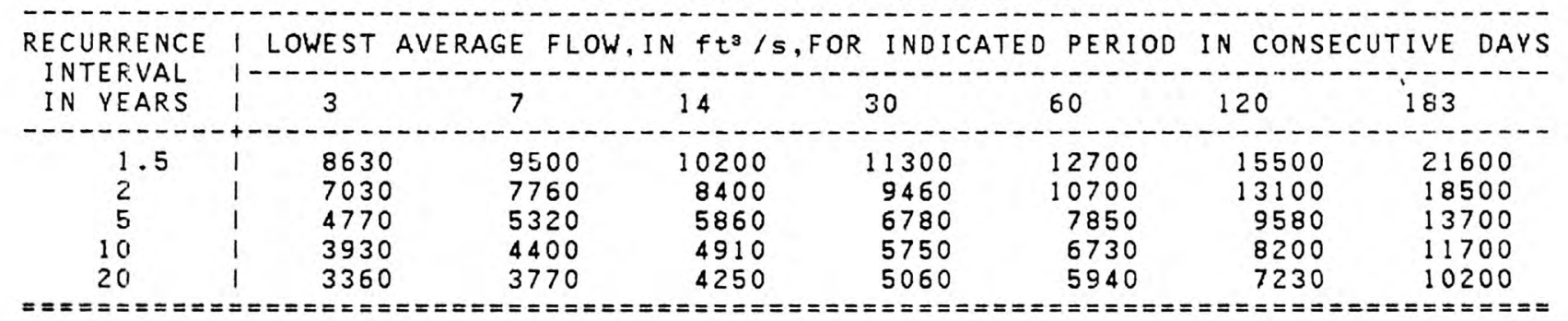

MAGNITUDE AND FREQUENCY OF SEASONAL LOW FLOWS

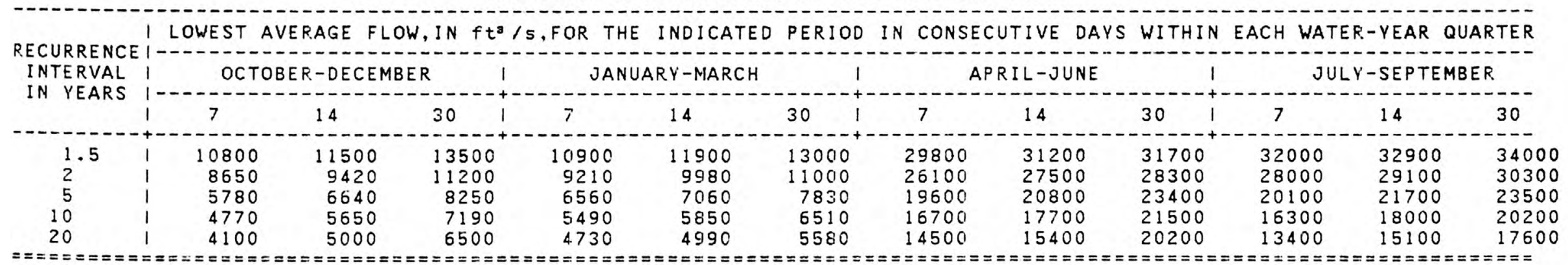

DURATION OF DAILY DISCHARGES FOR ANNUAL AND SEASONAL PERIODS

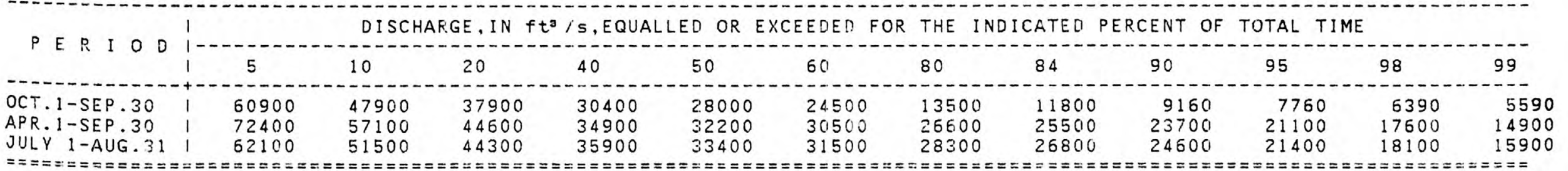


PERRY CREEK AT 38TH ST, SIOUX CITY

Location.--Lat42032.05", long 96024'35", in SE1/4SEl/4 sec.8. T.89 N., R.47 $W$, on right upstream abutment of bridge on 38 th street in Sioux City, 3.6 miles upstream from mouth.

$$
\text { LOW FLOW DISCHARGES, IN YEAR ENDING MARCH } 31
$$

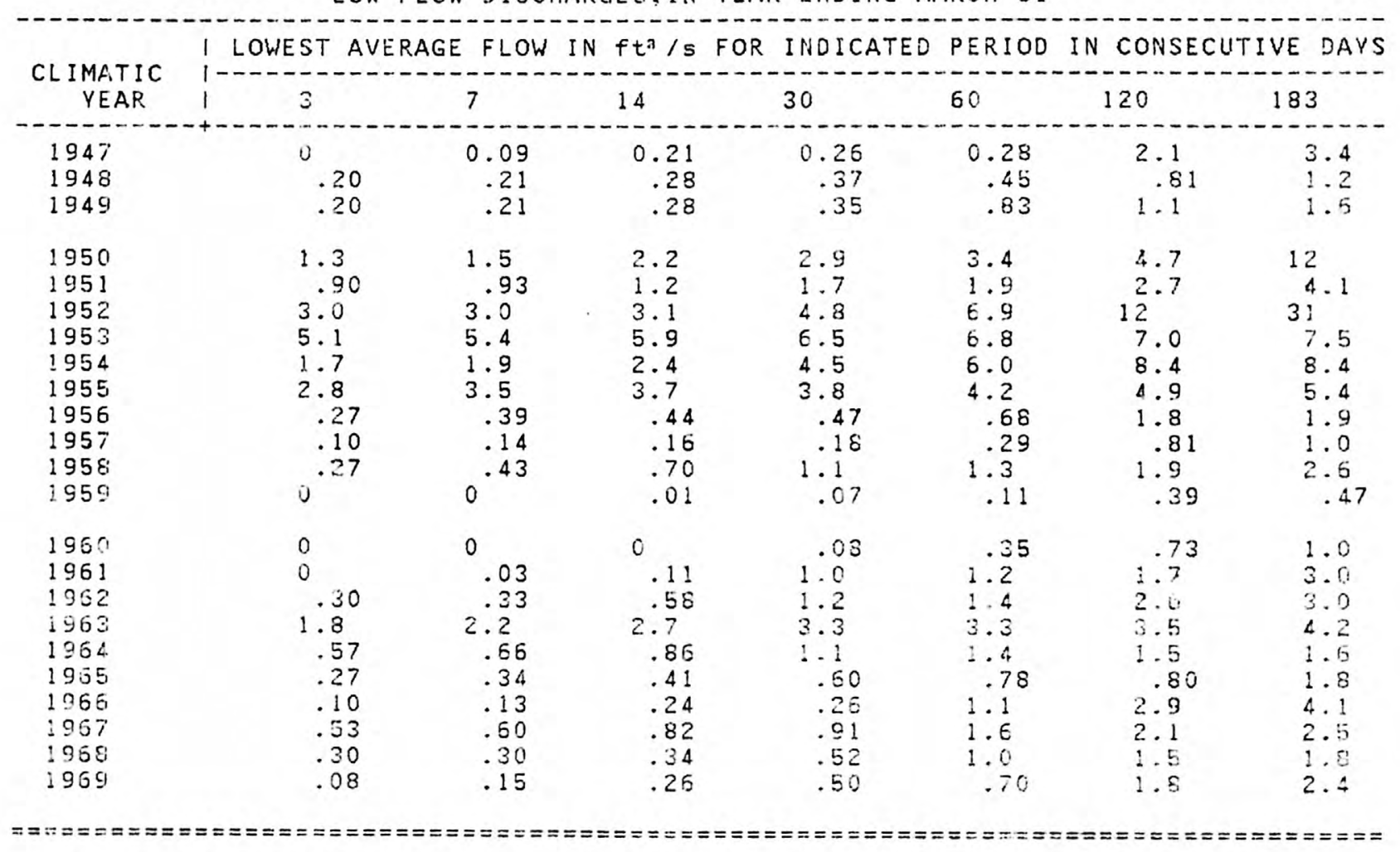


06-6000.00 PERRY CREEK AT 38TH ST, SIOUX CITY--Continued

DRAINAGE AREA: $65.1 \mathrm{~m}^{2}$ PERIOD OF RECORD: 23 YEARS AVERAGE DISCHARGE: $15.6 \mathrm{ft}^{3} / \mathrm{s}$

MINIMUM AVERAGE FLOWS FOR PERIOD OF RECORD

\begin{tabular}{|c|c|c|c|c|c|c|c|}
\hline PERIOD OF CONSECUTIVE DAYS & 3 & 7 & 14 & 30 & 60 & 120 & 183 \\
\hline $\begin{array}{l}\text { DISCHARGE, IN } \mathrm{ft}^{3} / \mathrm{s} \\
\text { CLIMATIC YEAR }\end{array}$ & $\begin{array}{c}0 \\
1961\end{array}$ & $\begin{array}{l}0 \\
1960\end{array}$ & $\begin{array}{l}0 \\
1960\end{array}$ & $\begin{array}{l}0.07 \\
1959\end{array}$ & $\begin{array}{l}0.12 \\
1959\end{array}$ & $\begin{array}{l}0.39 \\
1959\end{array}$ & $\begin{array}{l}0.48 \\
1959\end{array}$ \\
\hline
\end{tabular}

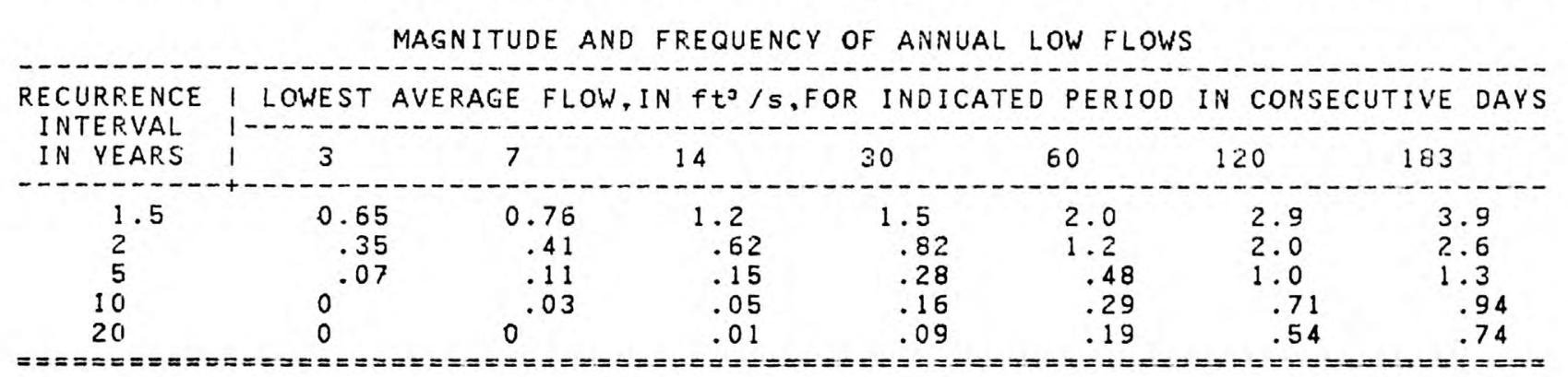

MAGNITUDE AND FREQUENCY OF SEASONAL LOW FLOWS

LOWEST AVERAGE FLOW, IN $\mathrm{ft}^{3} / \mathrm{s}$, FOR THE INDICATED PERIOD IN CONSECUTIVE DAYS WITHIN EACH WATER-VEAR QUARTER

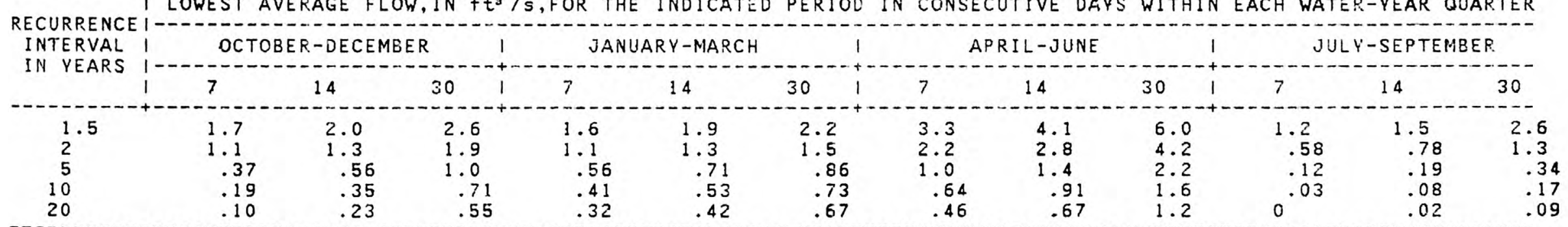

DURATION OF DAILY OISCHARGES FOR ANNUAL AND SEASONAL FERIODS

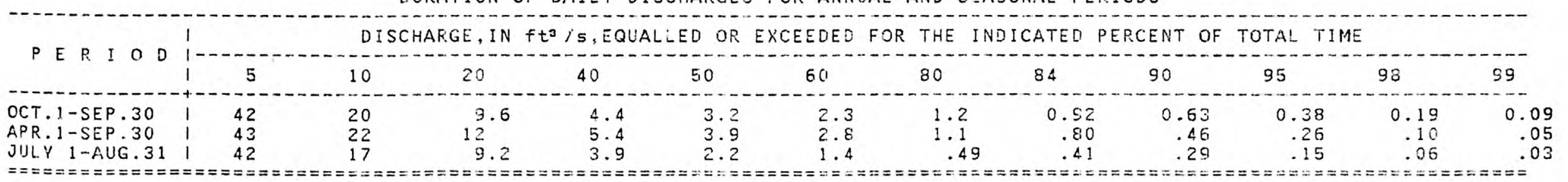


Location.--Lat 42058'55", long 96000'03", in NE1/4 NE1/4 sec.11, T.94 N., R.44 W., Sioux County, on left bank at downstream side of Chicago and Northwestern Railway Company bridge at east edge of Alton, $34.3 \mathrm{miles}$ upstream from West Eranch Floyd River at mile 58.1 .

LOW FLOW DISCHARGES, IN YEAR ENDING MARCH 31

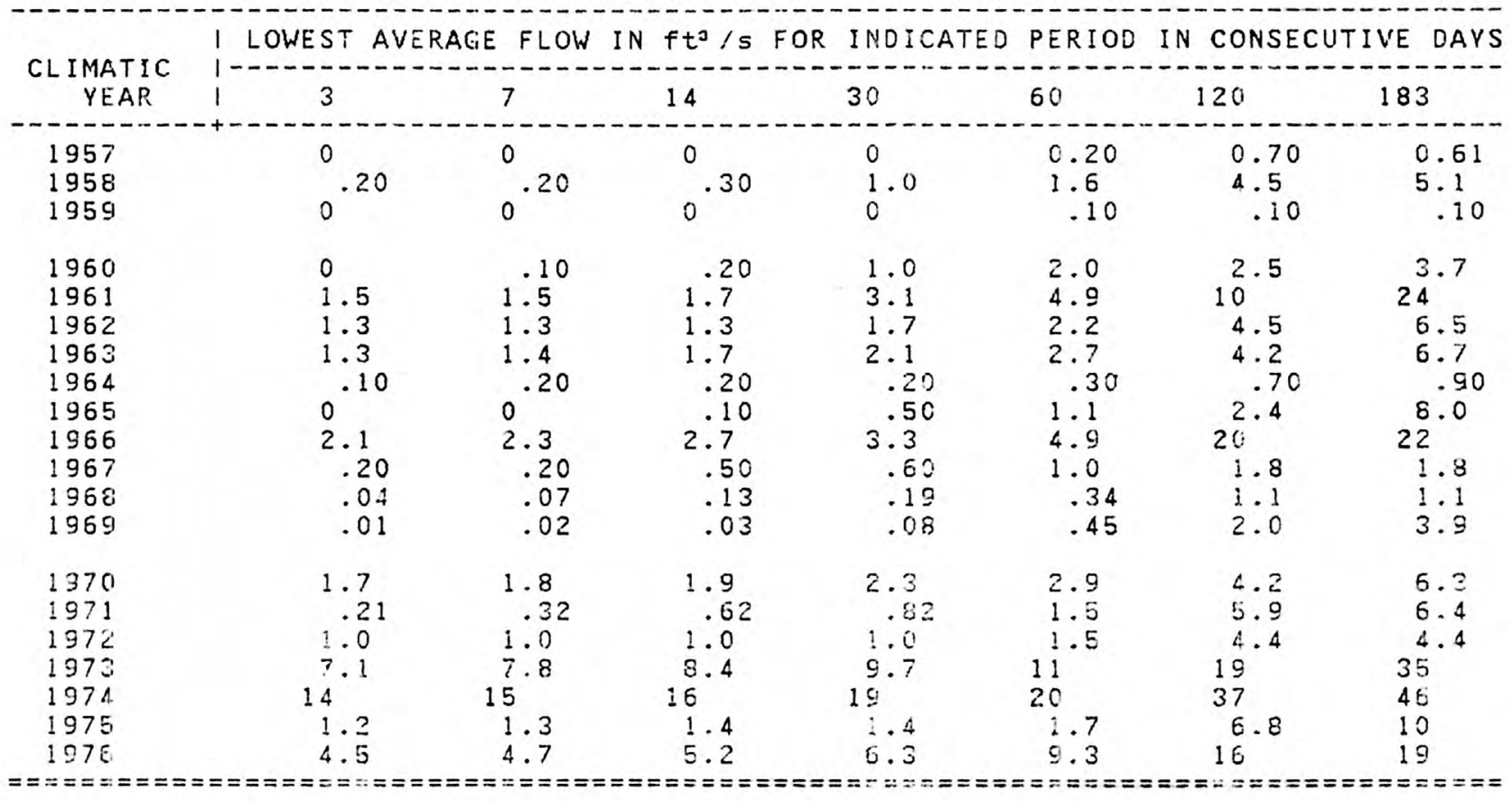


06-6001.00 FLOYD RIVER AT ALTON--Continued

DRAINAGE AREA: $265 \mathrm{~m}^{2}$ PERIOD OF RECORD: 21 YEARS AVERAGE DISCHARGE: 46.3 ft $3 / \mathrm{s}$

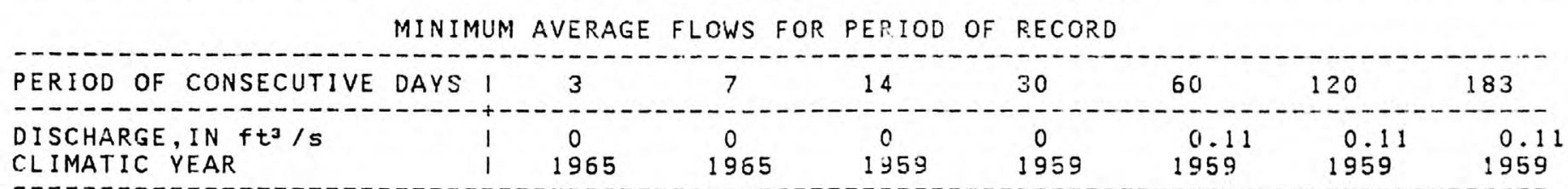

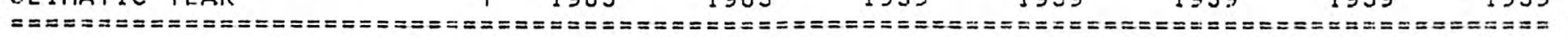

MAGNITUDE AND FREQUENCY OF ANNUAL LOW FLOWS

RECURRENCE I LOWEST AVERAGE FLOW, IN $\mathrm{ft}^{3} / \mathrm{s}$, FOR INDICATED PERIOD IN CONSECUTIVE DAVS

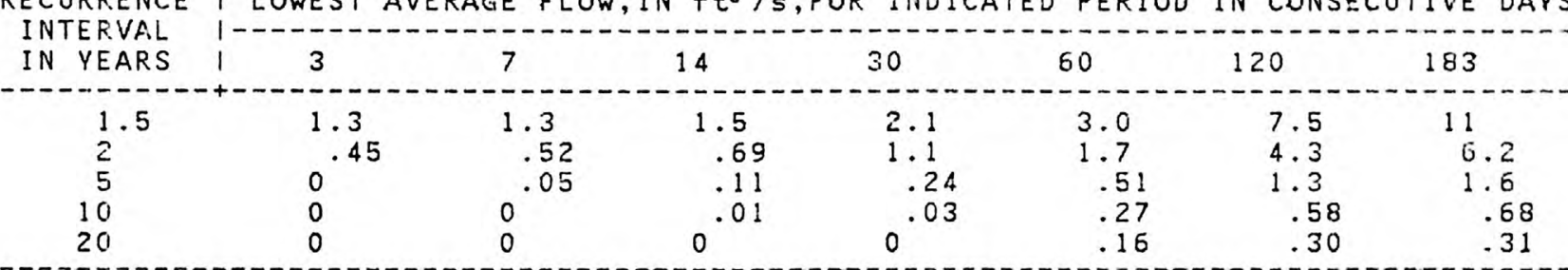

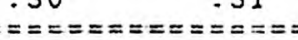

MAGNITUDE AND FREQUENCY OF SEASONAL LOW FLOWS

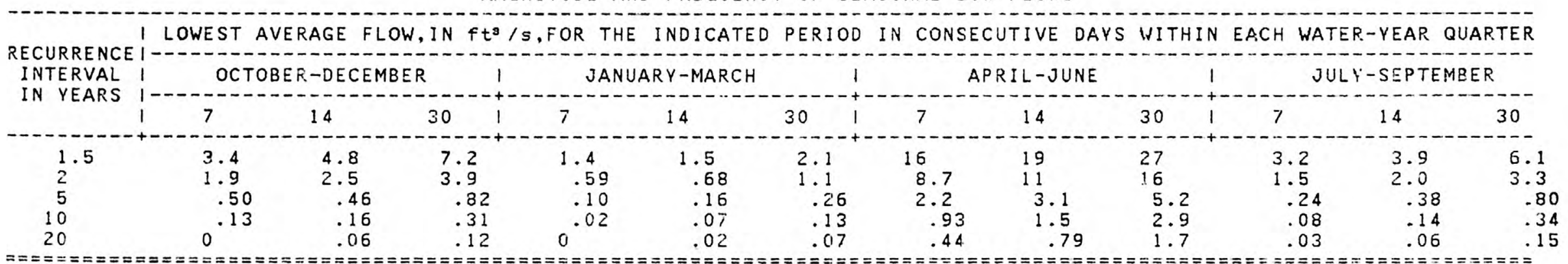

DURATION OF DAILY OISCHARGES FOR ANNUAL AND SEASONAL PERIODS

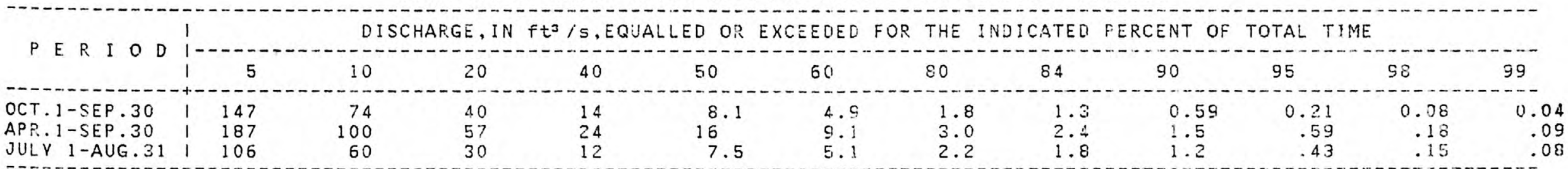


Location.--Lat 42055'15", iong $95010^{\prime} 30^{\prime \prime}$, In NE1/4 NE1/4 sec.32, T.94 N.. R.45 W. Sioux County, on right bank at downstream side of bridge on county highway B.62, 0.2 mile west of U.S. Hilghway $75,0.8 \mathrm{mile}$ downstream from Orange City slough, 2.2 miles northeast of Sirubie, 14 miles upstream from Floyd River, and at mile 39.3 .

LOW FLOW DISCHARGES, IN YEAR. ENDING MARCH 31

\begin{tabular}{|c|c|c|c|c|c|c|c|}
\hline \multirow{2}{*}{$\begin{array}{c}\text { CLIMATIC } \\
\text { YEAR }\end{array}$} & I LOWEST AV & FLOK & $f t^{3} / s$ & INDICA & $\begin{array}{l}\text { PERIOD } \\
-\end{array}$ & N CONSEC & VE DAYS \\
\hline & 13 & 7 & 14 & 30 & 60 & 120 & 183 \\
\hline $\begin{array}{l}1957 \\
1958 \\
1959\end{array}$ & $0_{0}^{0} .10$ & $0^{0} .14$ & $0_{0}^{0} .23$ & $\begin{array}{l}0.02 \\
.54 \\
0\end{array}$ & $\begin{array}{l}0.30 \\
1.1 \\
0\end{array}$ & $\begin{array}{l}0.61 \\
2.9 \\
.07\end{array}$ & $\begin{array}{c}0.62 \\
2.9 \\
.13\end{array}$ \\
\hline $\begin{array}{l}1960 \\
1961 \\
1962 \\
1963 \\
1964 \\
1965 \\
1966 \\
1967 \\
1968 \\
1969\end{array}$ & $\begin{array}{l}0.10 \\
0.60 \\
0 \\
0 \\
0 \\
0 \\
0 \\
0 \\
.13\end{array}$ & $\begin{array}{l}0.10 \\
0 \\
0 \\
0 \\
0 \\
0 \\
0 \\
0 \\
0 \\
.40\end{array}$ & $\begin{array}{l}0.10 \\
.61 \\
.04 \\
0 \\
0 \\
0_{0}^{.04} \\
.45\end{array}$ & $\begin{array}{l}.16 \\
.34 \\
1.3 \\
.12 \\
0 \\
0 \\
.80 \\
0.07 \\
.67\end{array}$ & $\begin{array}{l}.30 \\
.79 \\
2.3 \\
1.2 \\
0 \\
.01 \\
5.2 \\
.03 \\
.15 \\
.75\end{array}$ & $\begin{array}{c}.93 \\
2.6 \\
3.7 \\
3.5 \\
.31 \\
.21 \\
11.01 \\
.61 \\
.49 \\
1.7\end{array}$ & $\begin{array}{c}1.1 \\
7.4 \\
7.1 \\
6.0 \\
.84 \\
1.3 \\
24 \\
1.2 \\
.76 \\
7.4\end{array}$ \\
\hline $\begin{array}{l}1970 \\
1971 \\
1972 \\
1973 \\
1974 \\
1975 \\
1976\end{array}$ & $\begin{array}{l}1.3 \\
1.2 \\
0 \\
3.8 \\
2.8 \\
.40 \\
.75\end{array}$ & $\begin{array}{l}1.4 \\
1.3 \\
.24 \\
4.5 \\
2.8 \\
.43 \\
.79\end{array}$ & $\begin{array}{l}1.5 \\
1.5 \\
.24 \\
5.5 \\
2.8 \\
.45 \\
.83\end{array}$ & $\begin{array}{l}1.7 \\
1.7 \\
.24 \\
5.0 \\
3.0 \\
.53 \\
.85\end{array}$ & $\begin{array}{l}1.9 \\
3.4 \\
.25 \\
7.4 \\
4.6 \\
.63 \\
1.4\end{array}$ & $\begin{array}{c}3.4 \\
6.2 \\
.53 \\
8.8 \\
11 . \\
1.5 \\
2.8\end{array}$ & $\begin{array}{l}4.4 \\
13 \\
2.3 \\
18 \\
15 \\
2.7 \\
3.3\end{array}$ \\
\hline
\end{tabular}




\section{DRAINAGE AREA: $181 \mathrm{~m}^{2}$ PERIOD OF RECORD: 21 YEARS AVERAGE DISCHARGE: 29.7 ft $3 / \mathrm{s}$}

MINIMUM AVERAGE FLOWS FOR PERIOD OF RECORD

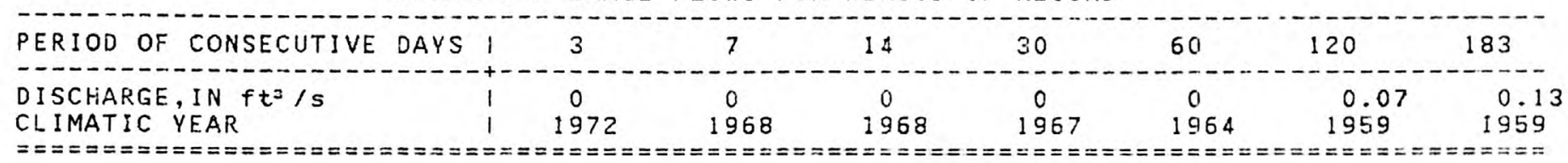

MAGNITUDE AND FREQUENCY OF ANNUAL LOW FLOWS

\begin{tabular}{l|cccccc} 
RECURRENCE \\
INTERVAL \\
IN YNEARS
\end{tabular}

MAGNITUDE AND FREQUENCY OF SEASONAL LOW FLOWS

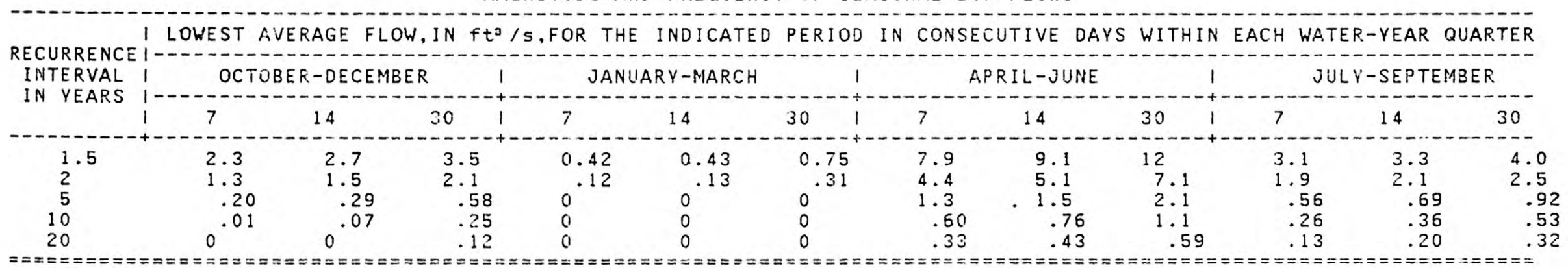

DURATION OF DAILY DISCHARGES FOR ANNUAL ANO SEASONAL FERIODS

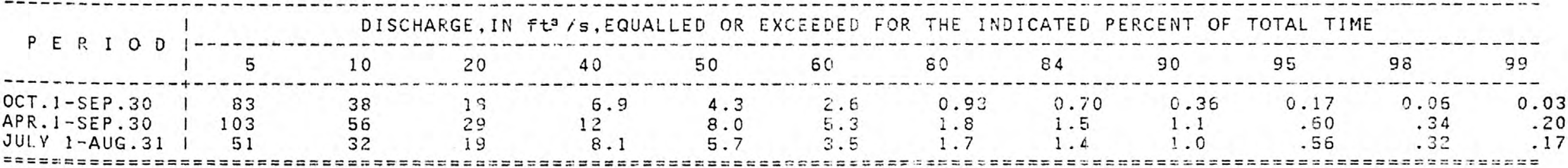




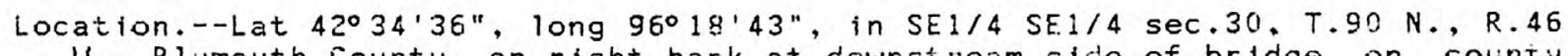
W., Plymouth Eounty, on right bank at downstieam side of bridge on county highway C70, 0.2 mile east of James, $14.3 \mathrm{mlles}$ downstream from West Eranch Floyd River, and at mile 9.5 .

LOW FLOW DISCHARGES, IN YEAR ENDING MARCH 31

\begin{tabular}{|c|c|c|c|c|c|c|c|}
\hline \multirow{2}{*}{$\begin{array}{c}\text { CLIMATIC } \\
\text { YEAR }\end{array}$} & LOWEST & AGE FLO & 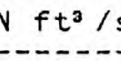 & INDIC & PERIO & \multicolumn{2}{|c|}{ N CONSECUTIVE OAYS } \\
\hline & 13 & 7 & 14 & 30 & 60 & 120 & 183 \\
\hline $\begin{array}{l}1937 \\
1938 \\
1939\end{array}$ & $\begin{array}{l}1.7 \\
6.4 \\
10\end{array}$ & $\begin{array}{r}1.9 \\
9.6 \\
11\end{array}$ & $\begin{array}{l}2.0 \\
10 \\
12\end{array}$ & $\begin{array}{l}2.5 \\
11 \\
22\end{array}$ & $\begin{array}{l}5.0 \\
13 \\
34\end{array}$ & $\begin{array}{l}8.2 \\
20 \\
39\end{array}$ & $\begin{array}{l}30 \\
30 \\
95\end{array}$ \\
\hline $\begin{array}{l}1940 \\
1941 \\
1942 \\
1943 \\
1944 \\
1945 \\
1946 \\
1947 \\
1948 \\
1949\end{array}$ & $\begin{array}{l}2.0 \\
9.4 \\
6.7 \\
15 \\
10 \\
21 \\
21 \\
8.4 \\
8.0 \\
9.0\end{array}$ & $\begin{array}{r}2.2 \\
9.8 \\
7.2 \\
15 \\
10 \\
21 \\
21 \\
9.2 \\
8.3 \\
9.5\end{array}$ & $\begin{array}{l}2.5 \\
10 \\
8.8 \\
15 \\
11 \\
23 \\
21 \\
12 \\
9.0 \\
9.8\end{array}$ & $\begin{array}{l}2.6 \\
12 \\
15 \\
16 \\
13 \\
27 \\
23 \\
15 \\
10 \\
12\end{array}$ & $\begin{array}{l}3.0 \\
12 \\
22 \\
18 \\
24 \\
37 \\
26 \\
23 \\
17 \\
15\end{array}$ & $\begin{array}{l}4.7 \\
14 \\
29 \\
33 \\
28 \\
68 \\
34 \\
37 \\
23 \\
18\end{array}$ & $\begin{array}{c}5.3 \\
25 \\
34 \\
80 \\
42 \\
132 \\
53 \\
57 \\
23 \\
31\end{array}$ \\
\hline $\begin{array}{l}1950 \\
1951 \\
1952 \\
1953 \\
1954 \\
1955 \\
1956 \\
1957 \\
1958 \\
1959\end{array}$ & $\begin{array}{r}10 \\
13 \\
135 \\
15 \\
29 \\
23 \\
5.3 \\
1.7 \\
8.0 \\
1.0\end{array}$ & $\begin{array}{r}11 \\
13 \\
136 \\
21 \\
30 \\
23 \\
5.4 \\
1.7 \\
8.0 \\
1.0\end{array}$ & $\begin{array}{r}13 \\
13 \\
138 \\
30 \\
31 \\
23 \\
5.7 \\
1.8 \\
8.3 \\
1.0\end{array}$ & $\begin{array}{r}13 \\
14 \\
145 \\
36 \\
33 \\
24 \\
5.9 \\
2.3 \\
0.3 \\
1.2\end{array}$ & $\begin{array}{r}17 \\
16 \\
190 \\
37 \\
44 \\
32 \\
5.1 \\
3.6 \\
10 \\
1.6\end{array}$ & $\begin{array}{r}28 \\
22 \\
261 \\
40 \\
58 \\
41 \\
7.8 \\
5.6 \\
21 \\
2.7\end{array}$ & $\begin{array}{r}72 \\
39 \\
466 \\
47 \\
64 \\
53 \\
9.8 \\
6.2 \\
24 \\
3.2\end{array}$ \\
\hline $\begin{array}{l}1950 \\
1961 \\
1962 \\
1963 \\
1964 \\
1965 \\
1966 \\
1967 \\
1968 \\
1969\end{array}$ & $\begin{array}{r}7.0 \\
12 \\
10 \\
10 \\
4.0 \\
4.5 \\
15 \\
9.5 \\
6.0 \\
6.6\end{array}$ & $\begin{array}{r}7.0 \\
12 \\
10 \\
10 \\
4.0 \\
4.5 \\
15 \\
9.5 \\
6.1 \\
7.6\end{array}$ & $\begin{array}{l}7.0 \\
12 \\
10 \\
11 \\
4.2 \\
4.8 \\
17 \\
9.5 \\
6.1 \\
9.5\end{array}$ & $\begin{array}{l}7.2 \\
15 \\
16 \\
17 \\
4.4 \\
5.9 \\
23 \\
9.5 \\
7.0 \\
12\end{array}$ & $\begin{array}{l}7 \frac{7}{17} \\
17 \\
22 \\
6 \cdot 2 \\
6 \cdot 9 \\
35 \\
10 \cdot 0 \\
9.3 \\
14\end{array}$ & $\begin{array}{l}12 \\
27 \\
28 \\
32 \\
9.3 \\
10 \\
69 \\
12 \\
14 \\
22\end{array}$ & $\begin{array}{l}13 \\
60 \\
35 \\
51 \\
11 \\
27 \\
77 \\
14 \\
15 \\
27\end{array}$ \\
\hline $\begin{array}{l}1970 \\
1971 \\
1972 \\
1973 \\
1974\end{array}$ & $\begin{array}{r}12 \\
14 \\
9.2 \\
40 \\
41\end{array}$ & $\begin{array}{l}12 \\
14 \\
9.2 \\
41 \\
4 !\end{array}$ & $\begin{array}{c}13 \\
16 \\
9.3 \\
43 \\
41\end{array}$ & $\begin{array}{l}15 \\
18 \\
5.5 \\
50 \\
42\end{array}$ & $\begin{array}{l}23 \\
20 \\
12 \\
54 \\
50\end{array}$ & $\begin{array}{l}38 \\
42 \\
28 \\
70 \\
75\end{array}$ & $\begin{array}{r}52 \\
47 \\
25 \\
112 \\
94\end{array}$ \\
\hline
\end{tabular}



LOW FLOW DISCHARGES, IN YEAR ENDING MARCH 31--Continued

\begin{tabular}{|c|c|c|c|c|c|c|c|}
\hline CLIMATIC & LOWEST & GE FLC & $\mathrm{ftg}^{3} / \mathrm{s}$ & INOIO & PERI & N CO & VE \\
\hline VEAR & 13 & 7 & 14 & 30 & 60 & 120 & 183 \\
\hline $\begin{array}{l}1975 \\
1976\end{array}$ & $\frac{5.2}{15}$ & $16^{6.2}$ & $\begin{array}{r}6.4 \\
18\end{array}$ & $22^{7.2}$ & $3^{8.4}$ & $\begin{array}{l}17 \\
43\end{array}$ & $\begin{array}{l}26 \\
55\end{array}$ \\
\hline
\end{tabular}


DRAINAGE AREA: $882 \mathrm{mi}^{2}$ PERIOD OF RECORD: 41 VEARS AVERAGE QISCHARGE: 177 ft $3 / 5$

MINIMUM AVERAGE FLOWS FOR PERIOD OF RECORD

\begin{tabular}{|c|c|c|c|c|c|c|c|}
\hline PERIOD OF CONSECUTIVE DAYS I & 3 & 7 & 14 & 30 & 60 & 120 & 183 \\
\hline $\begin{array}{l}\text { ISCHARGE, IN } \mathrm{ft}^{3} / \mathrm{s} \\
\text { LLIMATIC YEAR }\end{array}$ & $\begin{array}{r}1.0 \\
1959\end{array}$ & $\begin{aligned} 1.0 \\
1959\end{aligned}$ & $\begin{array}{r}1.0 \\
1959\end{array}$ & $\begin{array}{r}1.2 \\
1959\end{array}$ & $\begin{array}{r}1.6 \\
1959\end{array}$ & $\begin{array}{r}2.7 \\
1959\end{array}$ & $\begin{array}{r}3.2 \\
1959\end{array}$ \\
\hline
\end{tabular}

MAGNITUDE AND FREQUENCY OF ANNUAL LOW FLOWS

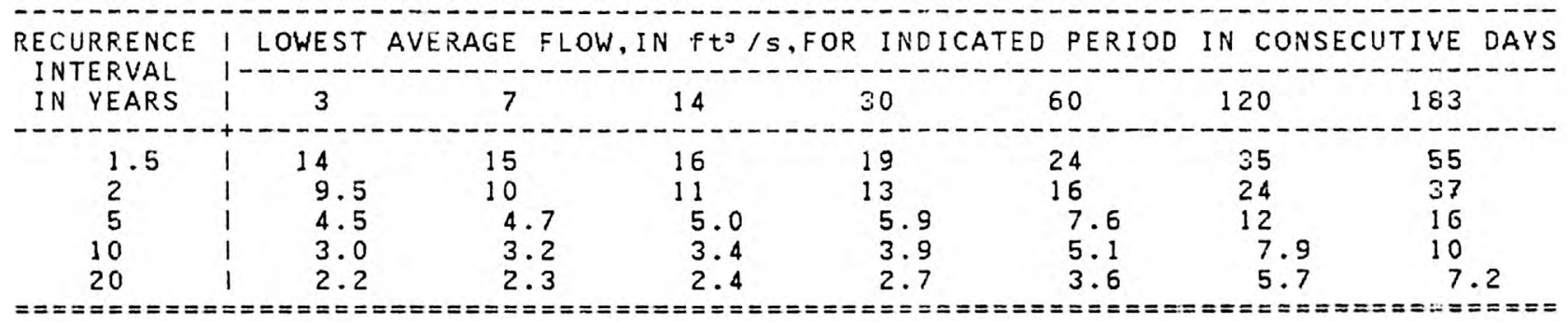

MAGNITUDE AND FREQUENCY OF SEASONAL LON FLOW'S

I LOWEST AVERAGE FLOW, IN $\mathrm{ft}^{3} / \mathrm{s}$, FOR THE INDICATED PERIOD IN CONSECUTIVE DAYS WITHIN EACH WATER-YEAR QUARTER RECURRENCE | OCTOBER-DECEMBER JANUARYMARCH JPRY

INTERVAL
IN YEARS

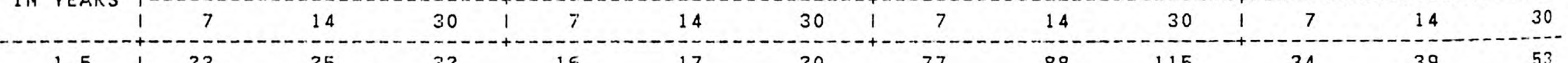

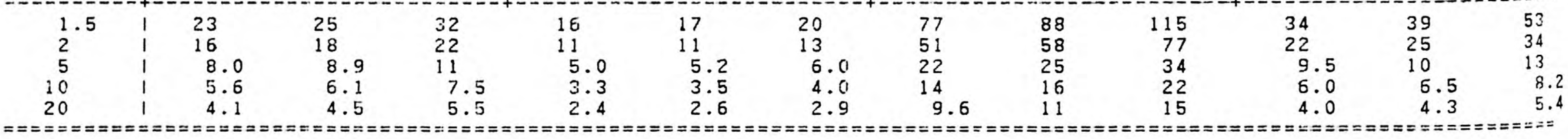

DURATION OF DAILY DISCHARGES FOR ARNUAL AND SEASONAL PERIODS

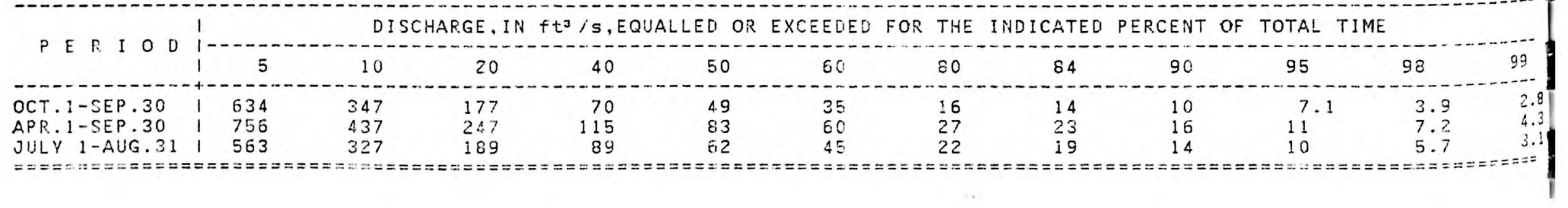


Location.--Lat 42015'34", long 96,04'41", in SE1/4 SE1/4 sec.16, T.86 N., R.45

W. Woodbury County, on $r i g h t$ bank 10 ft downstream from bridge on county road, three-quarters of a mile south of Holly Springs, 11.4 mi ies upstream from Wolf Creek, $15.7 \mathrm{miles}$ north of Onawa and $22 \mathrm{miles}$ southeast of Sioux City.

LOW FLOW DISCHARGES, IN YEAR. ENDING MARCH 31

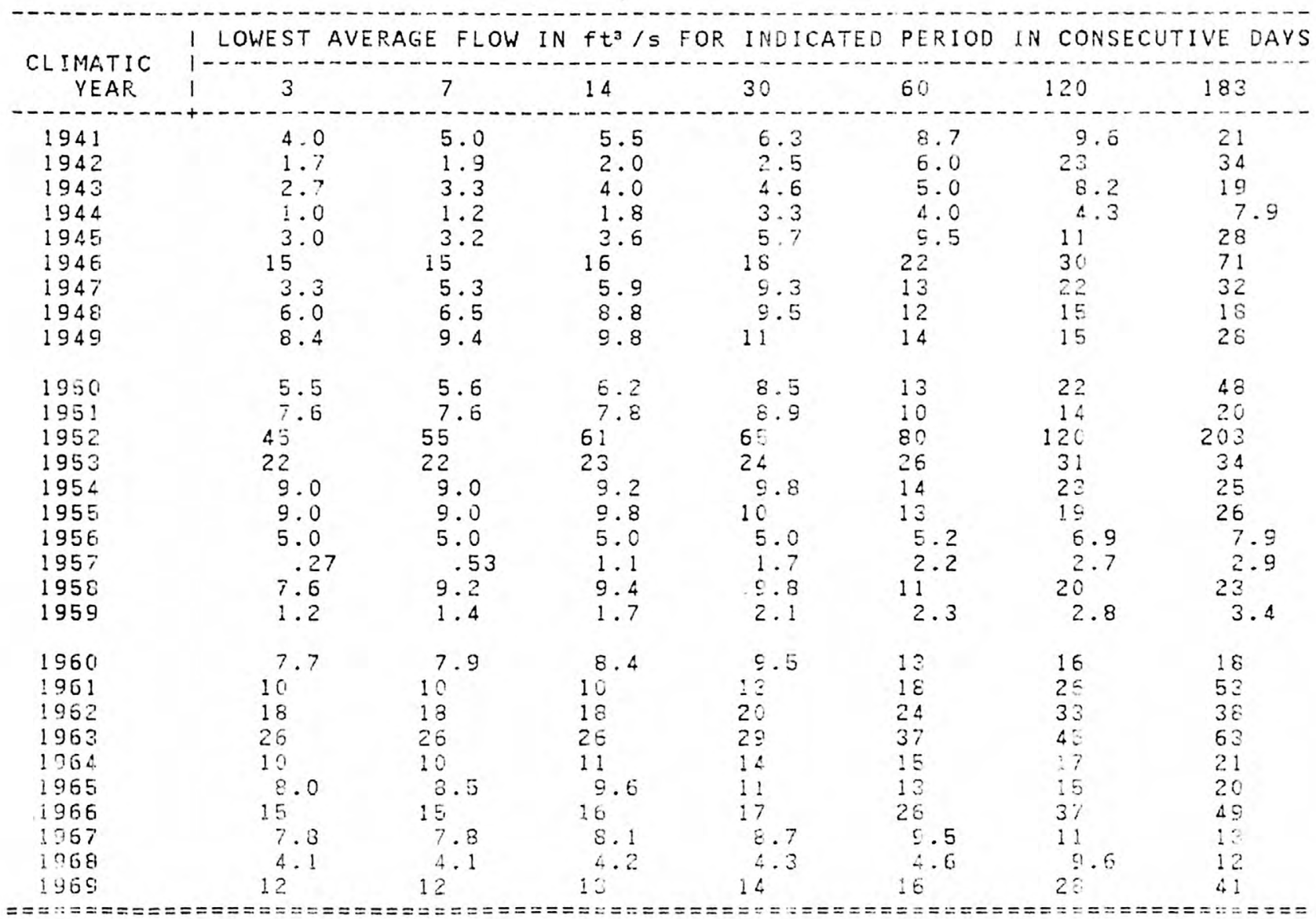


DRAINAGE AREA: $399 \mathrm{~m}^{2} \quad$ PERIOD OF RECORD: 29 YEARS AVERAGE DISCHARGE: 95.8 ft3/s MINIMUM AVERAGE FLOWS FOR PERIOD OF RECORD

\begin{tabular}{|c|c|c|c|c|c|c|c|}
\hline PERIOD OF CONSECUTIVE DAYS & 3 & 7 & 14 & 30 & 60 & 120 & 183 \\
\hline $\begin{array}{l}\text { DISCHARGE, IN } \mathrm{ft}^{3} / \mathrm{s} \\
\text { CLIMATIC YEAR }\end{array}$ & $\begin{array}{l}0.27 \\
1957\end{array}$ & $\begin{array}{l}0.53 \\
1957\end{array}$ & $\begin{array}{r}1.1 \\
1957\end{array}$ & $\begin{array}{r}1.7 \\
1957\end{array}$ & $\begin{array}{r}2.2 \\
1957\end{array}$ & $\begin{array}{r}2.7 \\
1957 \\
\end{array}$ & $\begin{array}{r}2.9 \\
1957 \\
\end{array}$ \\
\hline
\end{tabular}

MAGNITUDE AND FREQUENCY OF ANMUAL LOW FLOWS

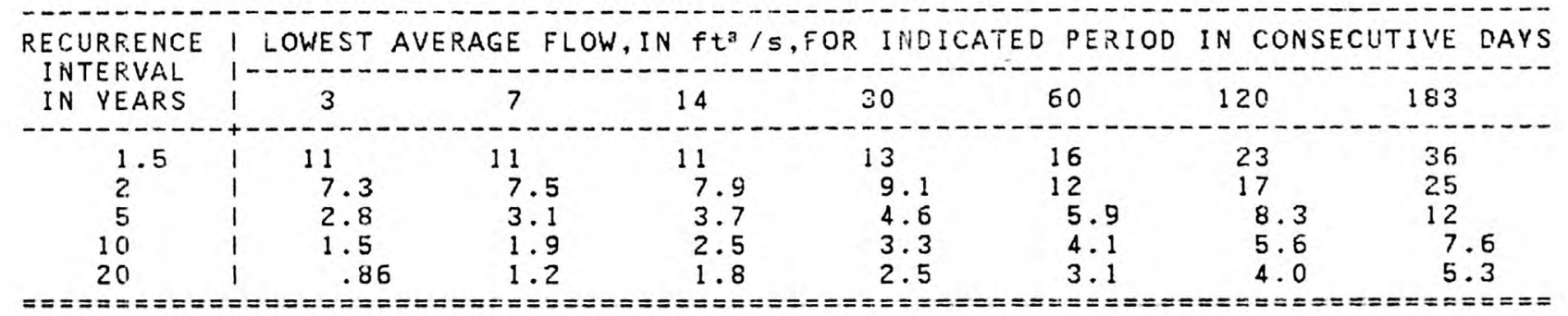

MAGNITUDE AND FREQUENCY OF SEASONAL LOW FLOWS

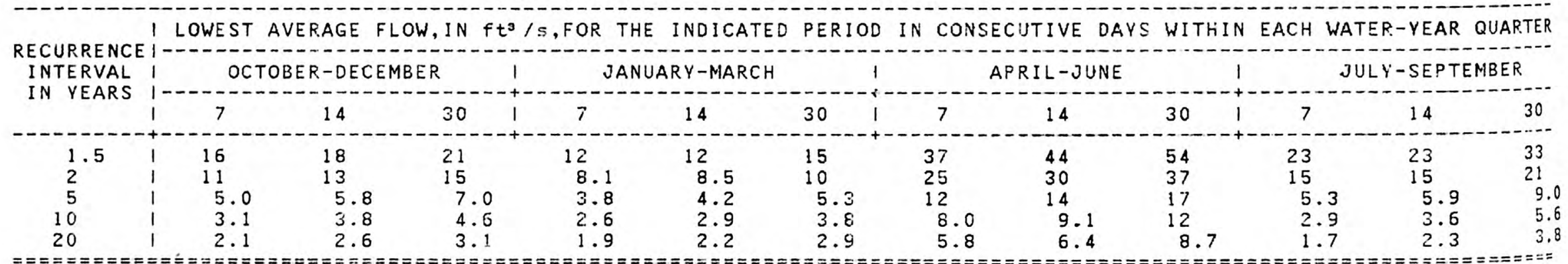

DURATION OF DAILY DISCHARGES FOR ANNUAL AND SEASONAL PERIODS

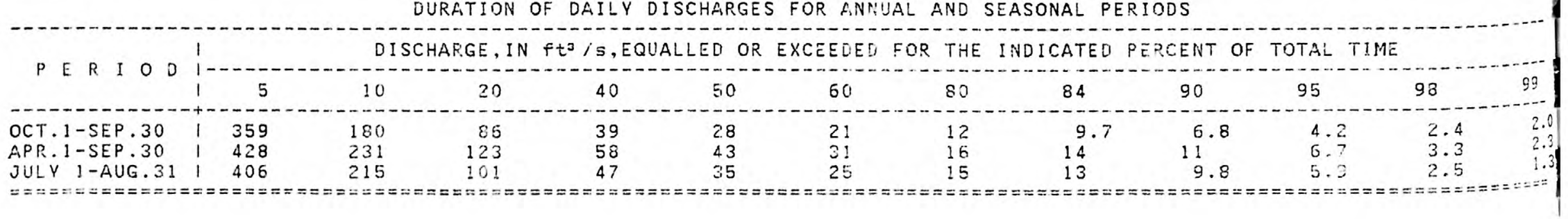


Location.--Lat 41057'52", long 95059'30", In NW1/4 NE1/4 sec.32, T.83 N., R.44 W. Monona County, on left pier at downstream side of bridge on county highway E54, 1.j mile west of gaging station on Little Sicux River near Turin. 4 miles southwest of Turin, 5.2 miles northeast of Blencoe, and 12.5 miles upstream from mouth.

LOW FLOW DISCHARGES, IN YEAR. ENDING MARCH 31

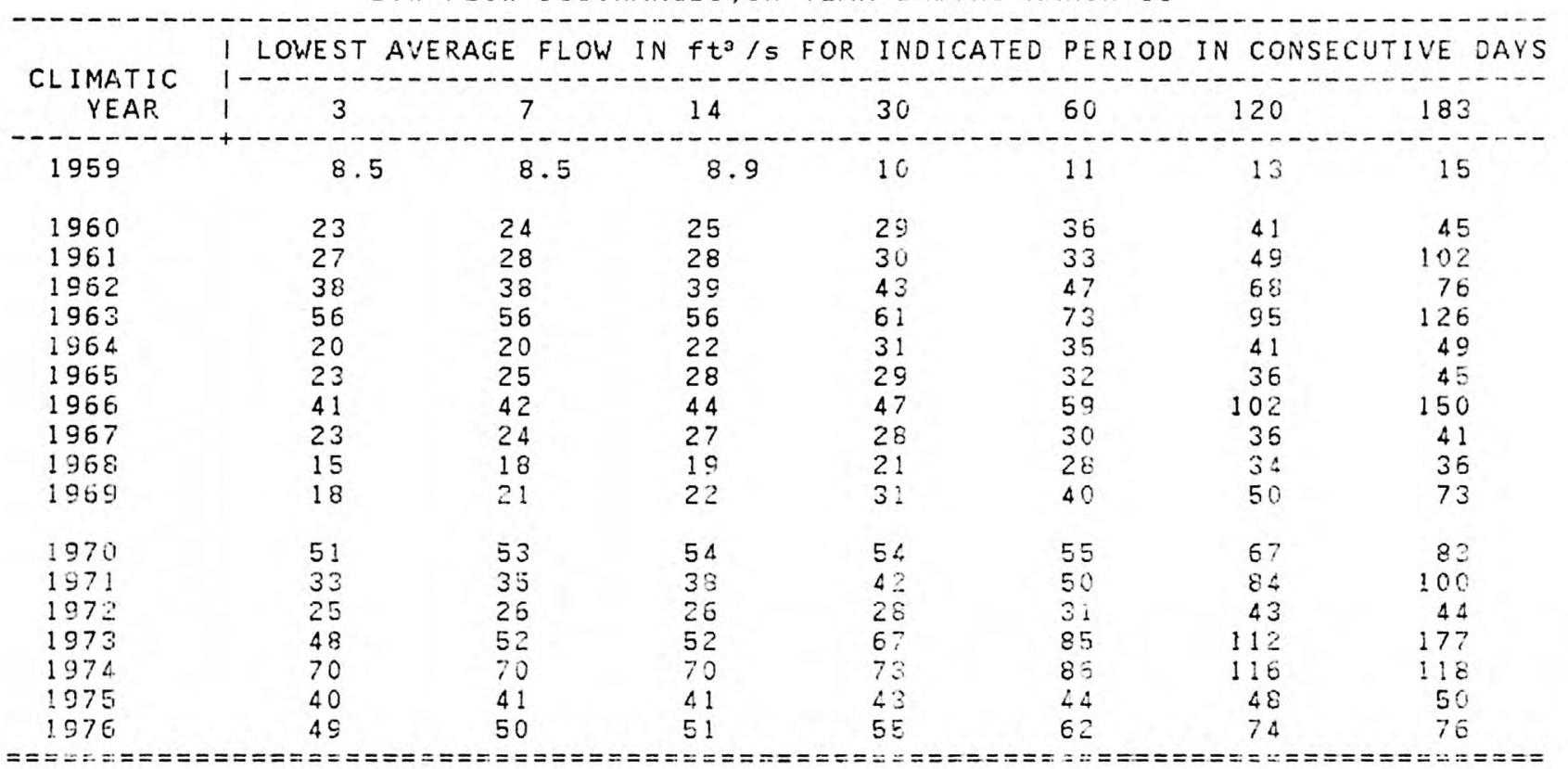


DRAINAGE AREA: $900 \mathrm{ml}=$ PERIOD OF RECORD: 18 YEARS AVERAGE DISCHARGE: 213 ft $3 / 5$

MINIMUM AVERAGE FLOWS FOR PERIOD OF RECORD

\begin{tabular}{|c|c|c|c|c|c|c|c|}
\hline PERIOD OF CONSECUTIVE DAYS I & 3 & 7 & 14 & 30 & 60 & 120 & 183 \\
\hline $\begin{array}{l}\text { ARGE, IN } \mathrm{ft}^{3} / \mathrm{s} \\
\text { TIC YEAR }\end{array}$ & $\begin{array}{r}8.5 \\
1959\end{array}$ & $\begin{array}{r}8.5 \\
1959\end{array}$ & $\begin{array}{r}8.9 \\
1959\end{array}$ & $\begin{array}{l}10 \\
1959\end{array}$ & $\begin{array}{l}11 \\
1959\end{array}$ & $\begin{array}{l}13 \\
1959\end{array}$ & \\
\hline
\end{tabular}

MAGNITUDE AND FREQUENCY OF ANNUAL LOW FLOWS

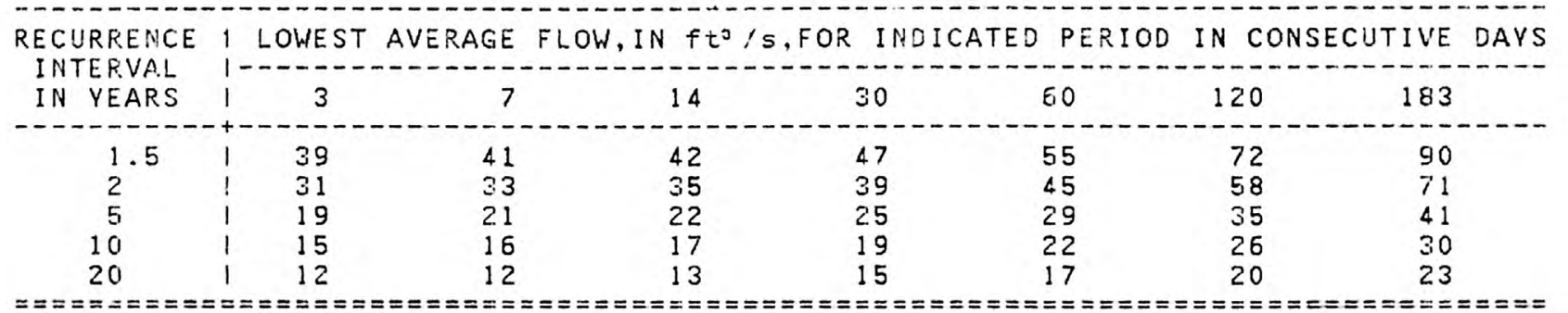

MAGNITUDE AND FREQUENCY OF SEASONAL LOW FLOWS

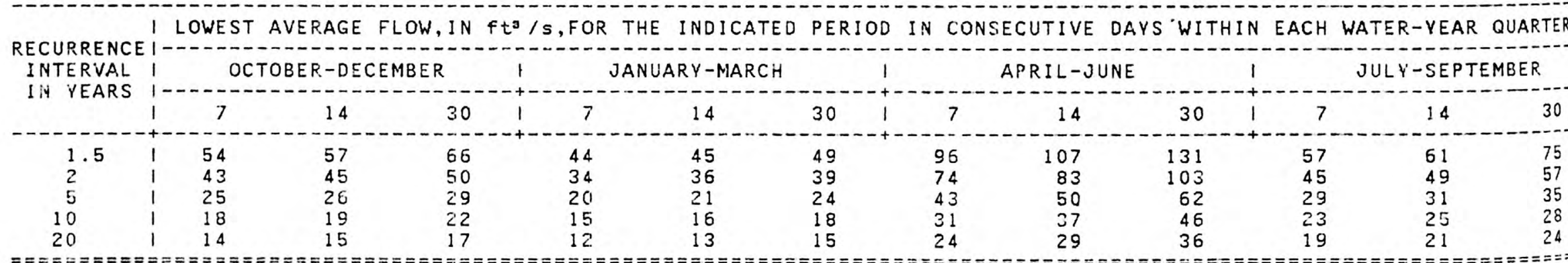

DURATION OF DAILY DISCHARGES FOR ANNUAL AND SEASONAL PERIODS

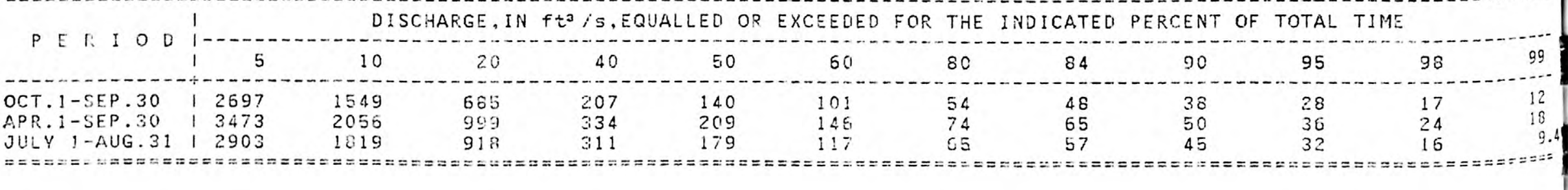


Location.--Lat $43^{\circ} 01^{\prime} 0 E_{1}^{\prime \prime}$, long $95^{\circ} 02^{\prime} 34^{\prime \prime}$, in SWl/4 NE1/4 sec.25, T.95 N., R.36 W. , Clay County, on left bank $5 \mathrm{ft}$ downstream from bridge on county highway B53, $0.4 \mathrm{mile}$ northwest of Gillett Grove, $0.9 \mathrm{mile}$ above Elk Creek, and at mile 145.1 .

Remarks.--Discontinued September 1973.

LOW FLOW DISCHARGES, IN YEAR. ENDING MARCH 31

\begin{tabular}{|c|c|c|c|c|c|c|c|}
\hline \multirow{2}{*}{$\begin{array}{c}\text { CL IMATIC } \\
\text { YEAR }\end{array}$} & I LOWEST & GE $F L$ & $f t^{2} /$ & IND I & PERI & $\mathrm{NCO}$ & I VE \\
\hline & 3 & 7 & 14 & 30 & 60 & 120 & 183 \\
\hline $\begin{array}{l}1960 \\
1961 \\
1962 \\
1963 \\
1964 \\
1965 \\
1966 \\
1967 \\
1968 \\
1969\end{array}$ & $\begin{array}{c}13 \\
30 \\
14 \\
20 \\
6.9 \\
31 \\
33 \\
11 \\
5.3 \\
6.6\end{array}$ & $\begin{array}{c}14 \\
30 \\
15 \\
20 \\
7.0 \\
33 \\
35 \\
13 \\
5.4 \\
7.7\end{array}$ & $\begin{array}{c}16 \\
31 \\
15 \\
21 \\
7.1 \\
40 \\
39 \\
14 \\
5.5 \\
9.9\end{array}$ & $\begin{array}{c}22 \\
35 \\
21 \\
25 \\
8.2 \\
47 \\
54 \\
15 \\
8.4 \\
13\end{array}$ & $\begin{array}{l}28 \\
50 \\
26 \\
29 \\
9.8 \\
56 \\
68 \\
18 \\
10 \\
25\end{array}$ & $\begin{array}{r}31 \\
83 \\
33 \\
46 \\
17 \\
75 \\
169 \\
23 \\
18 \\
30\end{array}$ & $\begin{array}{r}49 \\
150 \\
43 \\
82 \\
21 \\
123 \\
186 \\
26 \\
20 \\
47\end{array}$ \\
\hline $\begin{array}{l}1970 \\
1971 \\
1972 \\
1973\end{array}$ & $\begin{array}{l}46 \\
39 \\
14 \\
68\end{array}$ & $\begin{array}{l}46 \\
42 \\
14 \\
71\end{array}$ & $\begin{array}{l}47 \\
48 \\
14 \\
79\end{array}$ & $\begin{array}{l}50 \\
55 \\
18 \\
91\end{array}$ & $\begin{array}{l}58 \\
80 \\
33 \\
98\end{array}$ & $\begin{array}{r}87 \\
124 \\
80 \\
165\end{array}$ & $\begin{array}{r}137 \\
2.17 \\
74 \\
188\end{array}$ \\
\hline
\end{tabular}




\section{DRAINAGE AREA: $1334 \mathrm{mi}^{2}$ PERIOD OF RECORD: 15 YEARS AVERAGE DISCHARGE: $380 \mathrm{ft} \mathrm{f}^{3} / \mathrm{s}$}

MINIMUM AVERAGE FLOWS FOR PERIOD OF RECORD

\begin{tabular}{|c|c|c|c|c|c|c|c|}
\hline PERIOD OF CONSECUTIVE DAYS & 3 & 7 & 14 & 30 & 60 & 120 & 183 \\
\hline $\begin{array}{l}\text { DISCHARGE, IN } \mathrm{ft}^{3} / \mathrm{s} \\
\text { CL IMATIC YEAR }\end{array}$ & $\begin{array}{r}5.3 \\
1968\end{array}$ & $\begin{array}{r}5.4 \\
1968\end{array}$ & $\begin{array}{r}5.8 \\
1968\end{array}$ & $\begin{array}{r}8 \cdot 2 \\
1964\end{array}$ & $\begin{array}{r}9.8 \\
1964\end{array}$ & $\begin{array}{l}17 \\
1964\end{array}$ & $\begin{array}{l}20 \\
1968\end{array}$ \\
\hline
\end{tabular}

MAGNITUDE AND FREQUENCY OF ANNUAL LOW FLOWS

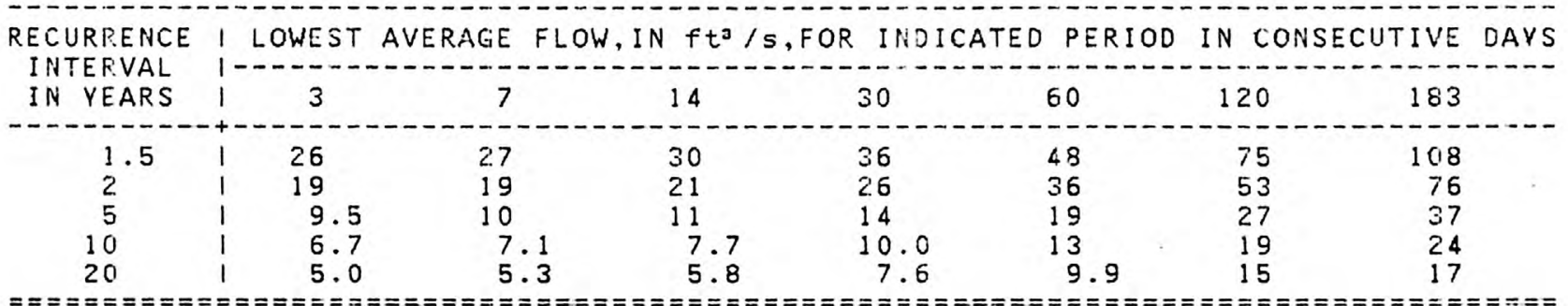

MAGNITUDE AND FREQUENCY OF SEASONAL LOW FLOWS

\begin{tabular}{l} 
RECURRENCE \\
$\begin{array}{l}\text { INTERVAL } \\
\text { IN }\end{array}$ IOWERS \\
\hline
\end{tabular}

DURATION OF DAILY DISCHARGES FOR ANNUAL ANO SEASONAL FERICDS

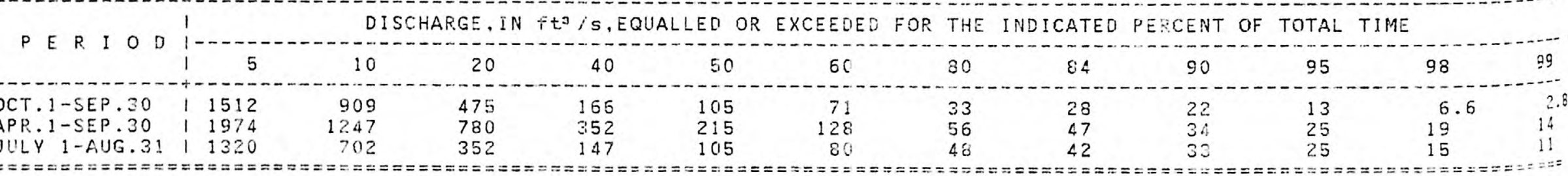




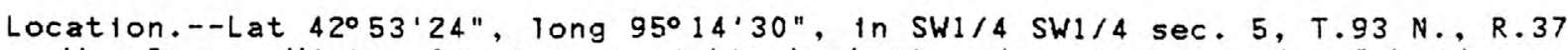
W. Buena Vista County, on right bank at downstieam side of bridge on State Highway 284 , in Linn Grove, Iowa, and at mile 123.7 .

Dránage area. $--1548 \mathrm{~m}^{2}$.

LOW FLOW DISCHARGES, IN YEAR ENDING MARCH 31.

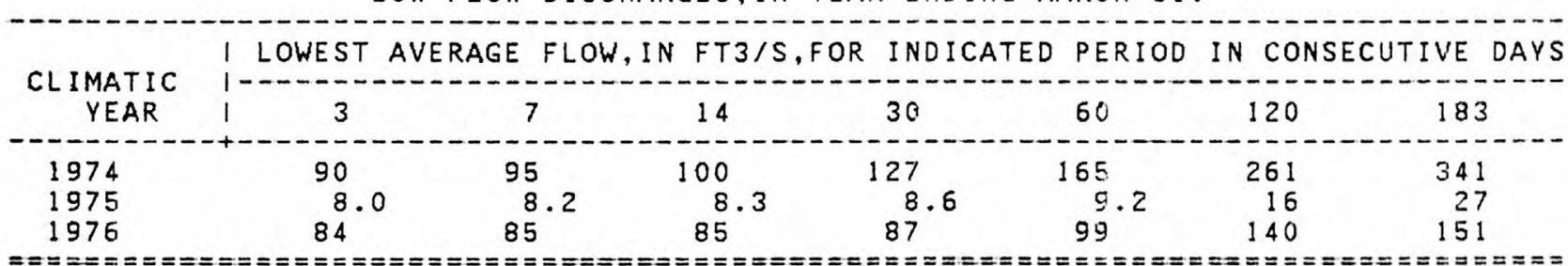

$Q_{a}=425$

$Q(84)=54$

$702=23$

$7 Q 10=8.1$ 
Location.--Lat $42^{\circ} 28^{\prime} 20^{\prime \prime}$, long $95^{\circ} 47^{\prime} 49^{\prime \prime}$, in NE.1/4 NWI/4 sec.1, T.88 N., R.43 W. Woodbury County, on right bank $10 \mathrm{ft}$ upstream from bridge on state Highway $31,0.3$ mile upstream from Eacon Creek, $0.5 \mathrm{mile}$ west of Correctionvili le, $0.8 \mathrm{mile}$ downstream from Pierson Creek, and at mile 56.0 .

LOW FLOW DISCHARGES, IN YEAR. ENDING MARCH 31

\begin{tabular}{|c|c|c|c|c|c|c|c|}
\hline \multirow{2}{*}{$\begin{array}{c}\text { CLIMAT IC } \\
\text { YEAR }\end{array}$} & \multicolumn{2}{|c|}{ LOWEST AVERAGE FLOW } & 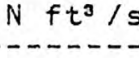 & INDICATED & PERIOD & \multicolumn{2}{|c|}{$\begin{array}{l}\text { N CONSECUTIVE } \\
\text { n }\end{array}$} \\
\hline & 3 & 7 & 14 & 30 & 60 & 120 & 183 \\
\hline $\begin{array}{l}1920 \\
1921 \\
1922 \\
1923 \\
1924 \\
1925 \\
1930 \\
1931 \\
1932 \\
1938 \\
1939\end{array}$ & $\begin{array}{c}130 \\
234 \\
47 \\
6.4 \\
28 \\
119 \\
15 \\
18 \\
9.4 \\
31 \\
97\end{array}$ & $\begin{array}{r}130 \\
247 \\
50 \\
12 \\
31 \\
121 \\
15 \\
19 \\
10 \\
31 \\
99\end{array}$ & $\begin{array}{r}130 \\
250 \\
50 \\
23 \\
41 \\
123 \\
18 \\
20 \\
13 \\
32 \\
105\end{array}$ & $\begin{array}{r}136 \\
264 \\
50 \\
32 \\
52 \\
124 \\
23 \\
22 \\
15 \\
34 \\
121\end{array}$ & $\begin{array}{r}159 \\
307 \\
57 \\
45 \\
83 \\
135 \\
30 \\
24 \\
21 \\
40 \\
171\end{array}$ & $\begin{array}{r}329 \\
353 \\
105 \\
82 \\
234 \\
184 \\
71 \\
36 \\
49 \\
74 \\
274\end{array}$ & $\begin{array}{r}334 \\
396 \\
166 \\
98 \\
222 \\
319 \\
67 \\
35 \\
65 \\
112 \\
547\end{array}$ \\
\hline $\begin{array}{l}1940 \\
1941 \\
1942 \\
1943 \\
1944 \\
1945 \\
1946 \\
1947 \\
1948 \\
1949\end{array}$ & $\begin{array}{r}18 \\
31 \\
33 \\
83 \\
113 \\
107 \\
82 \\
98 \\
47 \\
45\end{array}$ & $\begin{array}{r}18 \\
32 \\
36 \\
89 \\
117 \\
109 \\
83 \\
101 \\
48 \\
46\end{array}$ & $\begin{array}{r}18 \\
33 \\
38 \\
94 \\
119 \\
113 \\
87 \\
107 \\
48 \\
48\end{array}$ & $\begin{array}{r}20 \\
36 \\
44 \\
95 \\
140 \\
120 \\
110 \\
128 \\
61 \\
52\end{array}$ & $\begin{array}{r}22 \\
48 \\
74 \\
107 \\
236 \\
140 \\
122 \\
150 \\
103 \\
63\end{array}$ & $\begin{array}{r}35 \\
65 \\
228 \\
221 \\
271 \\
297 \\
175 \\
266 \\
138 \\
73\end{array}$ & $\begin{array}{r}42 \\
99 \\
286 \\
382 \\
349 \\
521 \\
347 \\
304 \\
132 \\
98\end{array}$ \\
\hline $\begin{array}{l}1950 \\
1951 \\
1952 \\
1953 \\
1954 \\
1955 \\
1956 \\
1957 \\
1958 \\
1959\end{array}$ & $\begin{array}{r}24 \\
27 \\
346 \\
71 \\
40 \\
60 \\
8.0 \\
4.2 \\
50 \\
6.5\end{array}$ & $\begin{array}{r}25 \\
27 \\
370 \\
72 \\
40 \\
60 \\
10 \\
4.6 \\
50 \\
5.7\end{array}$ & $\begin{array}{r}25 \\
28 \\
374 \\
74 \\
41 \\
61 \\
11 \\
5.5 \\
58 \\
6.9\end{array}$ & $\begin{array}{r}29 \\
30 \\
428 \\
78 \\
47 \\
71 \\
12 \\
6.3 \\
81 \\
6.9\end{array}$ & $\begin{array}{r}37 \\
35 \\
523 \\
83 \\
72 \\
102 \\
13 \\
15 \\
110 \\
7.8\end{array}$ & $\begin{array}{r}61 \\
55 \\
669 \\
98 \\
105 \\
183 \\
20 \\
23 \\
198 \\
14\end{array}$ & $\begin{array}{r}85 \\
93 \\
1060 \\
113 \\
146 \\
276 \\
23 \\
23 \\
199 \\
14\end{array}$ \\
\hline $\begin{array}{l}1960 \\
1961 \\
1962 \\
1963 \\
1964 \\
1965 \\
1966 \\
1967\end{array}$ & $\begin{array}{r}47 \\
56 \\
115 \\
110 \\
30 \\
57 \\
83 \\
30\end{array}$ & $\begin{array}{r}50 \\
56 \\
115 \\
110 \\
30 \\
58 \\
87 \\
30\end{array}$ & $\begin{array}{r}57 \\
57 \\
117 \\
114 \\
31 \\
65 \\
89 \\
31\end{array}$ & $\begin{array}{r}78 \\
73 \\
128 \\
135 \\
35 \\
77 \\
99 \\
33\end{array}$ & $\begin{array}{r}110 \\
93 \\
141 \\
146 \\
40 \\
98 \\
143 \\
35\end{array}$ & $\begin{array}{r}125 \\
154 \\
170 \\
184 \\
51 \\
145 \\
362 \\
46\end{array}$ & $\begin{array}{r}176 \\
251 \\
186 \\
309 \\
60 \\
298 \\
360 \\
51\end{array}$ \\
\hline
\end{tabular}


LOW FLOW DISCHARGES, IN YEAR ENDING MARCH 31--Continued

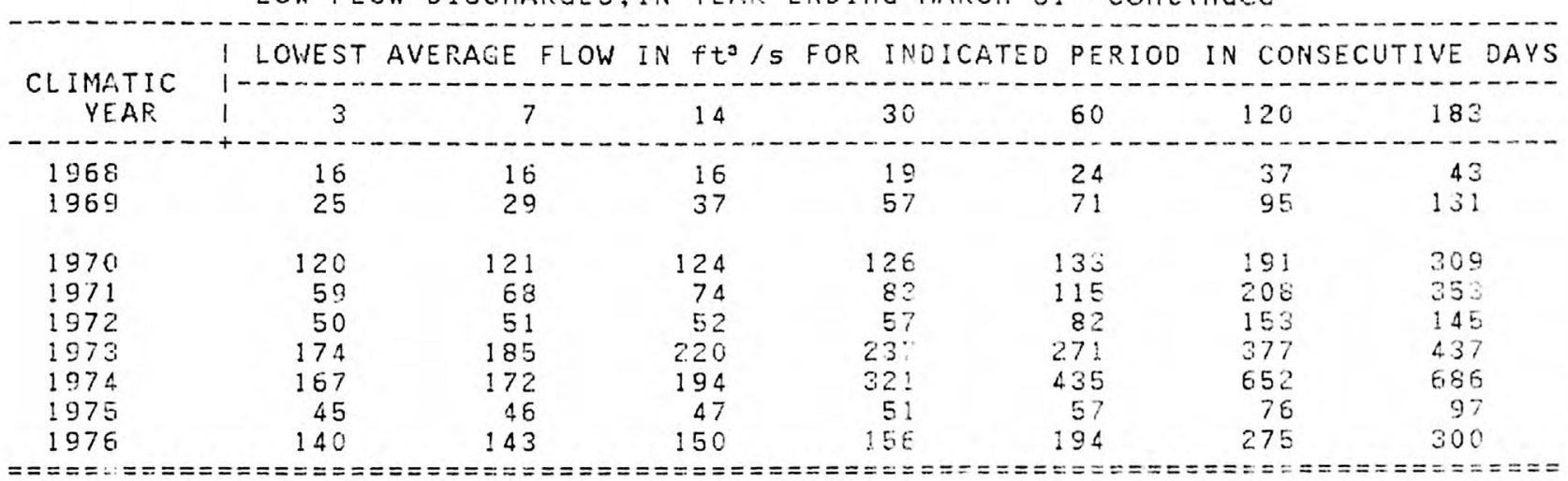


DRAINAGE AREA: $2500 \mathrm{mi}^{2}$ PERIOD OF RECORD: 49 YEARS AVERAGE DISCHARGE: 696 ft3/S

MINIMUM AVERAGE FLOWS FOR PERIOD OF RECORD

\begin{tabular}{|c|c|c|c|c|c|c|c|}
\hline PERIOD OF CONSECUTIVE DAYS & 3 & 7 & 14 & 30 & 60 & 120 & 183 \\
\hline $\begin{array}{l}\text { DISCHARGE, IN } \mathrm{ft}^{3} / \mathrm{s} \\
\text { CLIMATIC YEAR }\end{array}$ & $\begin{array}{r}4.2 \\
1957\end{array}$ & $\begin{array}{r}4.6 \\
1957\end{array}$ & $\begin{array}{r}5.5 \\
1957\end{array}$ & $\begin{array}{r}6.3 \\
1957\end{array}$ & $\begin{array}{r}7.8 \\
1959\end{array}$ & $\begin{array}{l}14 \\
1959\end{array}$ & $\begin{array}{l}14 \\
1959\end{array}$ \\
\hline
\end{tabular}

MAGNITUDE AND FREQUENCY OF ANNUAL LOW FLOWS

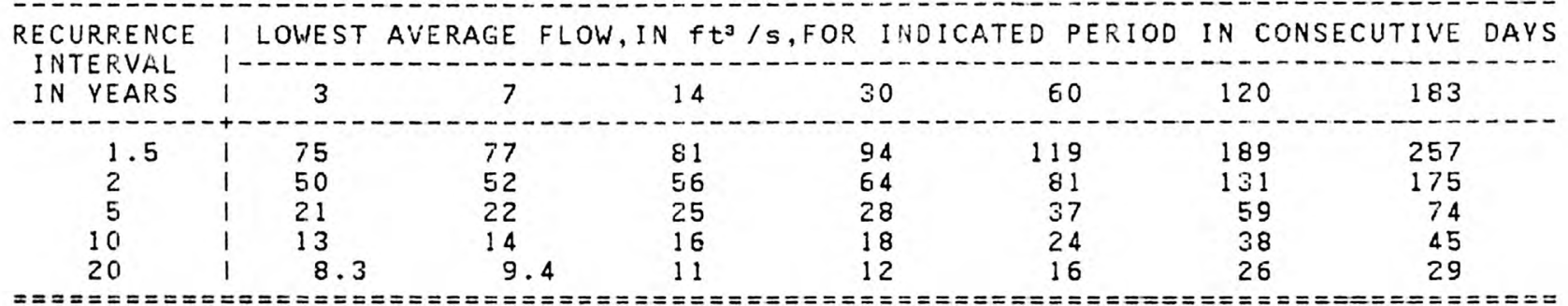

MAGNITUDE AND FREQUENCY OF SEASONAL LOW FLOWS

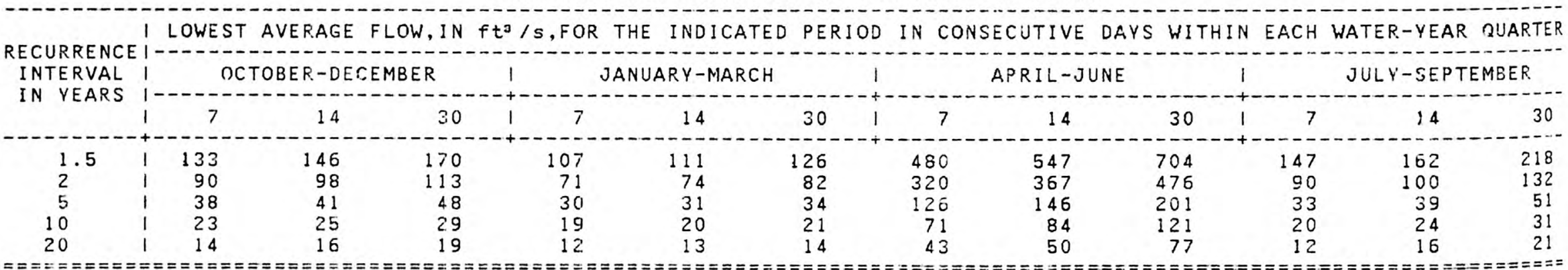

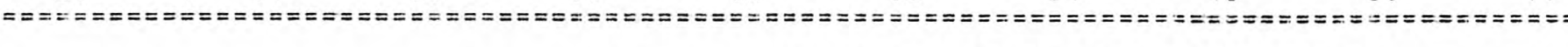

DURATION OF DAILY DISCHARGES FOR ANNUAL AND SEASONAL FERIODS

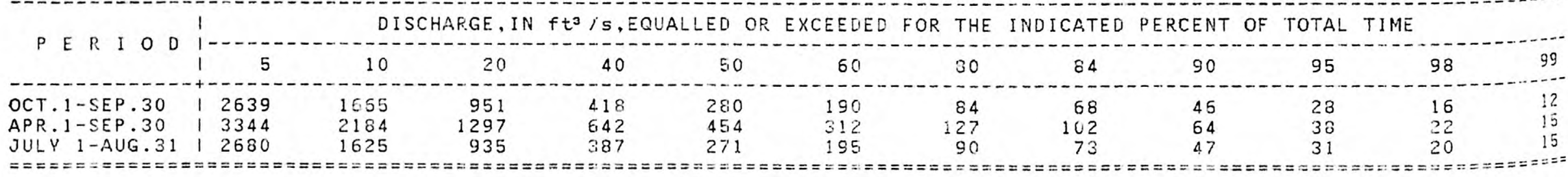


Location.--Lat 42004'55", long 96000'50", in SEi/4 SW1/4 sec.18, T.84 N., R.44 W., Monona County, near left bank on downstream slde of pier of bridge on Monona county highway A, 1.1 miles south of Kennebec, 5.5 miles northeast of Onawa, $6.0 \mathrm{miles}$ upstream from Maple River and at $\mathrm{m} 1 \mathrm{le} 22.0$.

Remarks.--Discontinued sept. $30,1969$.

LOW FLOW DISCHARGES, IN YEAR. ENDING MARCH 31

\begin{tabular}{|c|c|c|c|c|c|c|c|c|}
\hline \multirow{2}{*}{$\begin{array}{c}\text { CLIMATIC } \\
\text { YEAR }\end{array}$} & I LOWEST & $G E$ & $N f t^{3}$ & IND & D PE & I $\mathrm{COI}$ & TIVE & DAYS \\
\hline & 1 3 & 7 & 14 & 30 & 60 & 120 & 183 & \\
\hline $\begin{array}{l}1941 \\
1942 \\
1943 \\
1944 \\
1945 \\
1946 \\
1947 \\
1948 \\
1949\end{array}$ & $\begin{array}{r}44 \\
40 \\
92 \\
120 \\
138 \\
92 \\
127 \\
67 \\
56\end{array}$ & $\begin{array}{r}45 \\
44 \\
96 \\
123 \\
144 \\
92 \\
133 \\
68 \\
57\end{array}$ & $\begin{array}{r}48 \\
46 \\
99 \\
127 \\
150 \\
97 \\
139 \\
69 \\
61\end{array}$ & $\begin{array}{r}52 \\
57 \\
103 \\
152 \\
160 \\
141 \\
156 \\
85 \\
66\end{array}$ & $\begin{array}{r}65 \\
100 \\
119 \\
249 \\
190 \\
156 \\
178 \\
130 \\
74\end{array}$ & $\begin{array}{r}87 \\
266 \\
243 \\
287 \\
346 \\
201 \\
286 \\
165 \\
84\end{array}$ & $\begin{array}{l}139 \\
339 \\
430 \\
364 \\
579 \\
424 \\
333 \\
162 \\
115\end{array}$ & \\
\hline $\begin{array}{l}1950 \\
1951 \\
1952 \\
1953 \\
1954 \\
1955 \\
1956 \\
1957 \\
1958 \\
1959\end{array}$ & $\begin{array}{r}27 \\
38 \\
400 \\
110 \\
50 \\
82 \\
24 \\
12 \\
65 \\
17\end{array}$ & $\begin{array}{r}27 \\
38 \\
401 \\
110 \\
51 \\
83 \\
25 \\
12 \\
65 \\
18\end{array}$ & $\begin{array}{r}28 \\
40 \\
409 \\
113 \\
53 \\
86 \\
25 \\
13 \\
68 \\
18\end{array}$ & $\begin{array}{r}31 \\
43 \\
462 \\
116 \\
64 \\
95 \\
27 \\
15 \\
92 \\
19\end{array}$ & $\begin{array}{r}44 \\
51 \\
577 \\
123 \\
90 \\
128 \\
30 \\
24 \\
133 \\
19\end{array}$ & $\begin{array}{r}72 \\
76 \\
741 \\
134 \\
126 \\
216 \\
35 \\
33 \\
238 \\
26\end{array}$ & $\begin{array}{r}103 \\
125 \\
1170 \\
155 \\
168 \\
327 \\
36 \\
33 \\
223 \\
26\end{array}$ & \\
\hline $\begin{array}{l}1960 \\
1961 \\
1962 \\
1963 \\
1964 \\
1965 \\
1966 \\
1967 \\
1968 \\
1969\end{array}$ & $\begin{array}{r}70 \\
90 \\
120 \\
140 \\
45 \\
70 \\
107 \\
45 \\
20 \\
26\end{array}$ & $\begin{array}{r}72 \\
90 \\
120 \\
140 \\
45 \\
73 \\
109 \\
46 \\
20 \\
32\end{array}$ & $\begin{array}{r}80 \\
90 \\
124 \\
142 \\
47 \\
86 \\
111 \\
47 \\
21 \\
41\end{array}$ & $\begin{array}{r}110 \\
108 \\
138 \\
170 \\
51 \\
108 \\
124 \\
48 \\
25 \\
68\end{array}$ & $\begin{array}{r}133 \\
127 \\
153 \\
183 \\
60 \\
122 \\
190 \\
54 \\
31 \\
85\end{array}$ & $\begin{array}{r}143 \\
196 \\
197 \\
223 \\
76 \\
171 \\
424 \\
69 \\
52 \\
122\end{array}$ & $\begin{array}{r}188 \\
315 \\
215 \\
357 \\
89 \\
401 \\
423 \\
78 \\
60 \\
156\end{array}$ & \\
\hline
\end{tabular}


DRAINAGE AREA: $2738 \mathrm{mi}^{2}$ PERIOD OF RECORD: 29 YEARS AVERAGE DISCHARGE: 779 ft? $/ \mathrm{s}$ MINIMUM AVERAGE FLOWS FOR PERIOD OF RECORD

\begin{tabular}{|c|c|c|c|c|c|c|c|}
\hline PERIOD OF CONSECUTIVE DAYS & 3 & 7 & 14 & 30 & 60 & 120 & 183 \\
\hline $\begin{array}{l}\text { ISCHARGE, IN } \mathrm{ft}^{3} / \mathrm{s} \\
\text { LIMATIC YEAR }\end{array}$ & $\begin{array}{ll}1 & 12 \\
1 & 1957\end{array}$ & $\begin{array}{l}12 \\
1957\end{array}$ & $\begin{array}{l}13 \\
1957\end{array}$ & $\begin{array}{l}15 \\
1957\end{array}$ & $\begin{array}{l}19 \\
1959\end{array}$ & $\begin{array}{l}26 \\
1959\end{array}$ & $\begin{array}{l}26 \\
1959\end{array}$ \\
\hline
\end{tabular}

MAGNITUDE AND FREQUENCY OF ANNUAL LOW FLOWS

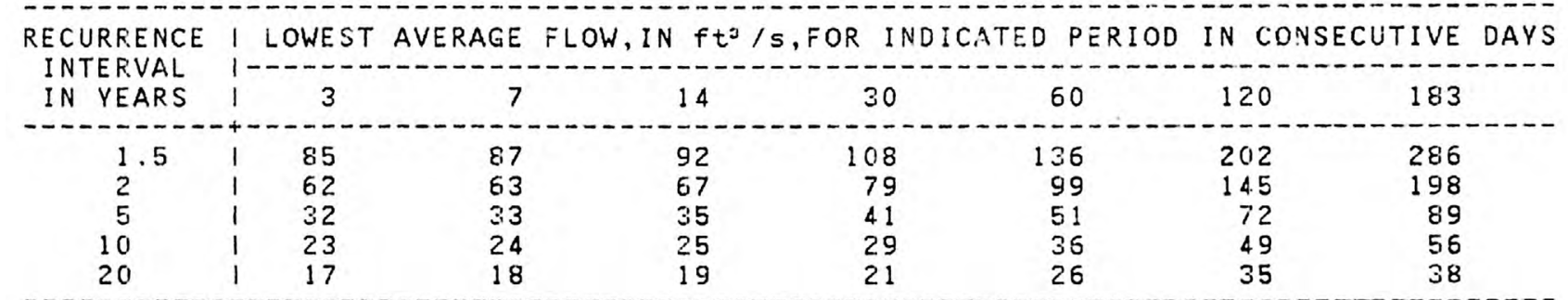

MAGNITUDE AND FREQUENCY OF SEASONAL LOW FLOWS

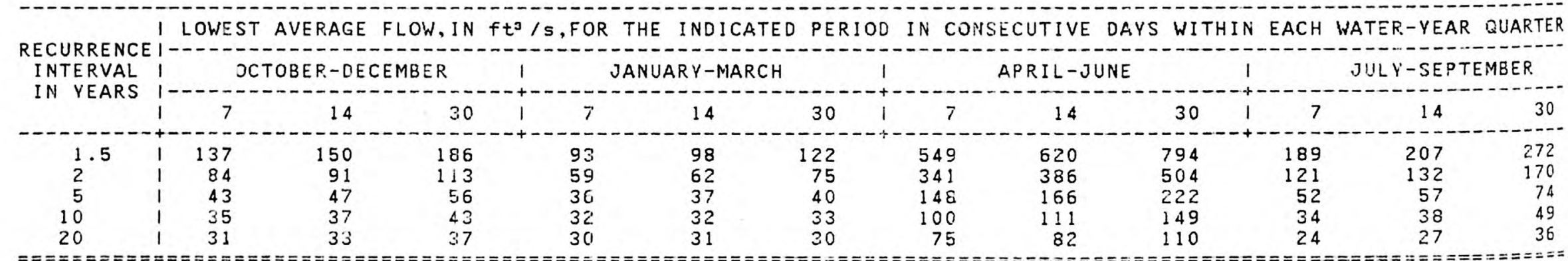

DURATION OF DAILY DISCHARGES FOR RINUAL ANO SEASONAL FERIODS

\begin{tabular}{|c|c|c|c|c|c|c|c|c|c|c|c|c|}
\hline & 1 & & $G E, I$ & $/ 5, E$ & $E D$ & EEDE & THE & CATE & ENT & TAL & & \\
\hline $\begin{array}{c}510 \\
0\end{array}$ & 1 & 10 & 20 & 40 & 50 & 60 & 80 & 84 & 90 & 95 & 98 & 99 \\
\hline $\begin{array}{l}\text {-SEP. } 30 \\
\text {-SEP. } 30 \\
1 \text {-AUG. } 31\end{array}$ & $\begin{array}{l}2990 \\
13991 \\
13386\end{array}$ & $\begin{array}{l}1876 \\
2593 \\
2273\end{array}$ & $\begin{array}{l}1049 \\
1502 \\
1192\end{array}$ & $\begin{array}{l}435 \\
745 \\
512\end{array}$ & $\begin{array}{l}295 \\
518 \\
357\end{array}$ & $\begin{array}{l}205 \\
356 \\
25 \%\end{array}$ & $\begin{array}{l}103 \\
161 \\
133\end{array}$ & $\begin{array}{r}86 \\
135 \\
113\end{array}$ & $\begin{array}{l}60 \\
95 \\
83\end{array}$ & $\begin{array}{l}38 \\
60 \\
54\end{array}$ & $\begin{array}{l}25 \\
37 \\
41\end{array}$ & $\begin{array}{l}21 \\
30 \\
37\end{array}$ \\
\hline
\end{tabular}


Location.--Lat $42^{\circ} 20^{\prime} 10^{\prime \prime}$, long $95^{\circ} 22^{\prime} 52^{\prime \prime}$, In SE1/4 NE1/4 sec.21, T.87 N., R.39 W. Ida County, near center of span on downstream side of bridge on county highway M27, $700 \mathrm{ft}$ south of State Highway 175, 1.0 mile downstream from Hoskins Creek, $1.8 \mathrm{~m} 1 \mathrm{les}$ west of Arthur, $4.5 \mathrm{miles}$ southeast of Ida Grove. and 6.5 miles upstream from mouth.

Remarks.--Discontinued September 1975.

LOW FLOW DISCHARGES, IN YEAR ENDING MARCH 31

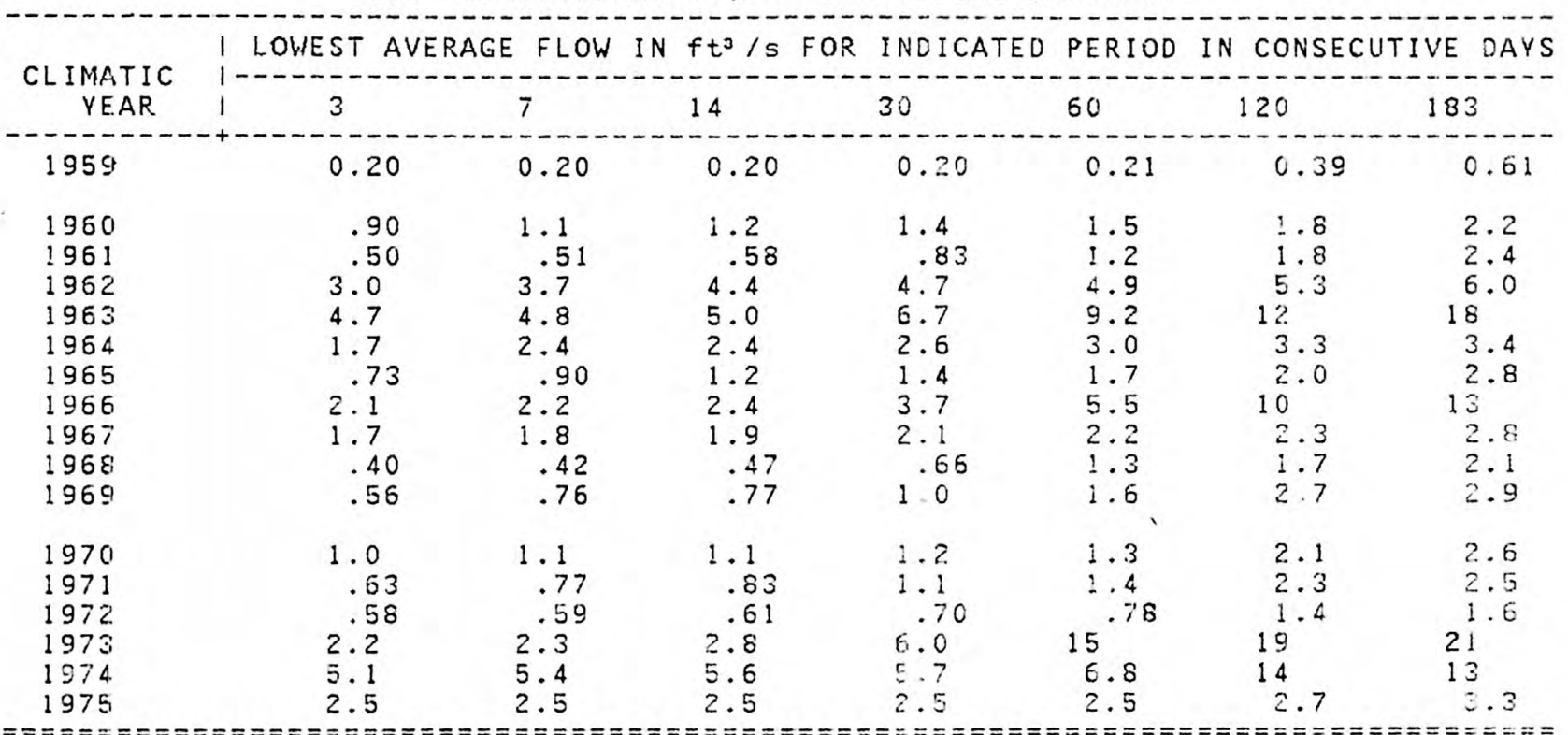


DRAINAGE AREA: $39.3 \mathrm{mi}^{2}$ PERIOD OF RECORD: 18 VEARS AVERAGE OISCHARGE: 15.7 ft $/ 5$

MINIMUM AVERAGE FLOWS FOR PERIOD OF RECORD

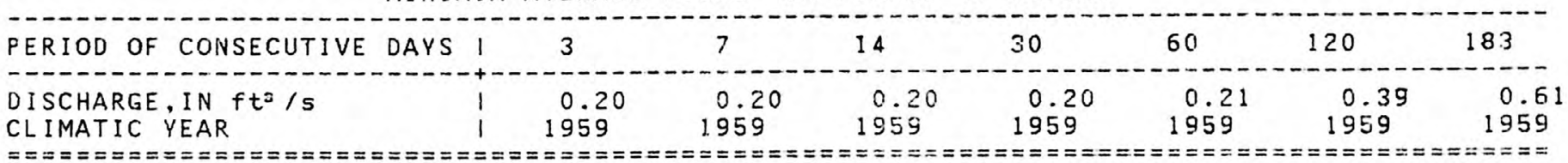

MAGNITUDE AND FREQUENCY OF ANNUAL LOW FLOWS

RECURRENCE I LOWEST AVERAGE FLOW, IN $\mathrm{f} \mathrm{t}^{3} / 5, \mathrm{FOR}$ INDICATED PERIOD IN CONSECUTIVE DAYS

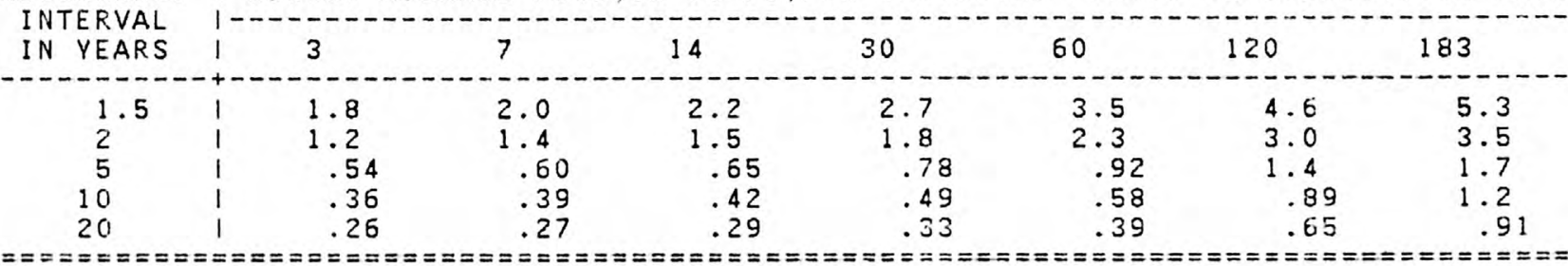

MAGNITUDE AND FREQUENCY OF SEASONAL LOW FLOWS

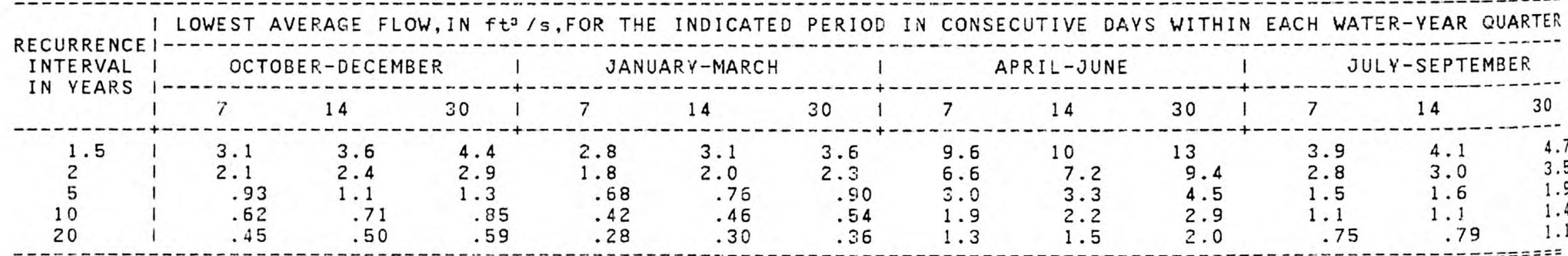

DURATION OF DAILY DISCHARGES FOR ANNUAL AND SEASONAL PERIODS

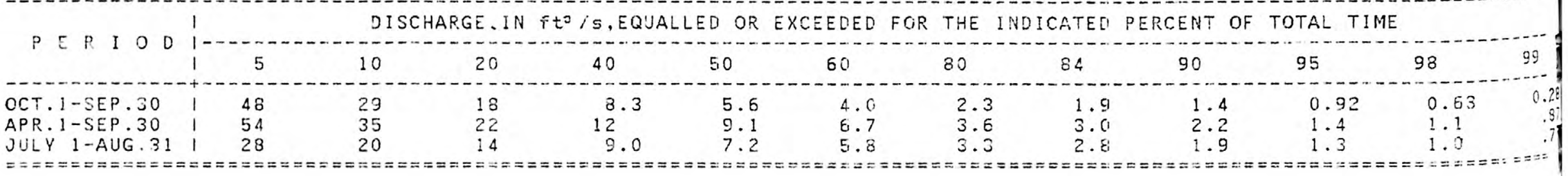


Location.--Lat $42^{\circ} 09^{\prime} 28^{\prime \prime}$, long 95०48'27", in SE1/4 SE1/4 sec.23, T.85 N., R.43 W., Monona County, on right bank on downstream side of bridge on State Highway 175,80 ft downstream from Chicago \& Northwestern Rallway Company bridge, 0.5 miles southwest of Mapleton, $0.5 \mathrm{mile}$ downstream from Wilsey Creek, 2.0 miles upstream from McClarey Creek, and 16 miles upstream from mouth.

LOW FLOW DISCHARGES, IN YEAR. ENDING MARCH 31

\begin{tabular}{|c|c|c|c|c|c|c|c|}
\hline \multirow{2}{*}{$\begin{array}{c}\text { CLIMATIC } \\
\text { YEAR }\end{array}$} & LOWEST & GE FL & $\begin{array}{l}N f t^{3} / s \\
-\end{array}$ & R INOI & D PERIO & IN CONSI & IVE DAYS \\
\hline & 13 & 7 & 14 & 30 & 60 & 120 & 183 \\
\hline $\begin{array}{l}1943 \\
1944 \\
1945 \\
1946 \\
1947 \\
1948 \\
1949\end{array}$ & $\begin{array}{l}18 \\
18 \\
38 \\
25 \\
37 \\
26 \\
13\end{array}$ & $\begin{array}{l}18 \\
21 \\
42 \\
26 \\
37 \\
27 \\
13\end{array}$ & $\begin{array}{l}19 \\
21 \\
44 \\
29 \\
41 \\
28 \\
13\end{array}$ & $\begin{array}{l}22 \\
23 \\
47 \\
43 \\
49 \\
31 \\
14\end{array}$ & $\begin{array}{l}23 \\
31 \\
57 \\
69 \\
53 \\
37 \\
21\end{array}$ & $\begin{array}{l}32 \\
38 \\
80 \\
87 \\
73 \\
47 \\
23\end{array}$ & $\begin{array}{r}56 \\
49 \\
128 \\
160 \\
86 \\
46 \\
32\end{array}$ \\
\hline $\begin{array}{l}1950 \\
1951 \\
1952 \\
1953 \\
1954 \\
1955 \\
1956 \\
1957 \\
1958 \\
1959\end{array}$ & $\begin{array}{r}8.0 \\
14 \\
133 \\
65 \\
14 \\
35 \\
9.8 \\
3.5 \\
6.2 \\
2.5\end{array}$ & $\begin{array}{r}8.1 \\
14 \\
160 \\
70 \\
19 \\
35 \\
10.0 \\
3.5 \\
7.0 \\
2.6\end{array}$ & $\begin{array}{r}8.8 \\
14 \\
166 \\
71 \\
21 \\
35 \\
10 \\
3.7 \\
8.4 \\
2.7\end{array}$ & $\begin{array}{r}12 \\
15 \\
175 \\
73 \\
26 \\
36 \\
11 \\
4.2 \\
17 \\
2.8\end{array}$ & $\begin{array}{c}23 \\
19 \\
204 \\
77 \\
31 \\
46 \\
12 \\
5.3 \\
27 \\
3.1\end{array}$ & $\begin{array}{r}32 \\
27 \\
278 \\
85 \\
43 \\
72 \\
15 \\
7.9 \\
54 \\
6.8\end{array}$ & $\begin{array}{c}51 \\
39 \\
439 \\
105 \\
50 \\
108 \\
18 \\
8.4 \\
54 \\
10\end{array}$ \\
\hline $\begin{array}{l}1960 \\
1961 \\
1962 \\
1963 \\
1964 \\
1965 \\
1966 \\
1967 \\
1968 \\
1969\end{array}$ & $\begin{array}{l}20 \\
22 \\
42 \\
65 \\
30 \\
10 \\
22 \\
17 \\
6.0 \\
19\end{array}$ & $\begin{array}{l}21 \\
22 \\
42 \\
56 \\
34 \\
11 \\
36 \\
17 \\
6.0 \\
21\end{array}$ & $\begin{array}{l}21 \\
23 \\
44 \\
70 \\
34 \\
13 \\
41 \\
17 \\
6.2 \\
27\end{array}$ & $\begin{array}{l}22 \\
27 \\
51 \\
85 \\
37 \\
16 \\
45 \\
19 \\
8.1 \\
3 !\end{array}$ & $\begin{array}{l}30 \\
35 \\
60 \\
98 \\
42 \\
22 \\
60 \\
21 \\
15 \\
39\end{array}$ & $\begin{array}{r}49 \\
54 \\
78 \\
124 \\
51 \\
28 \\
116 \\
29 \\
25 \\
50\end{array}$ & $\begin{array}{r}50 \\
100 \\
96 \\
178 \\
55 \\
40 \\
146 \\
37 \\
34 \\
57\end{array}$ \\
\hline $\begin{array}{l}1970 \\
1971 \\
1972 \\
1973 \\
1974 \\
1975 \\
1976\end{array}$ & $\begin{array}{r}33 \\
24 \\
11 \\
66 \\
117 \\
13 \\
50\end{array}$ & $\begin{array}{r}34 \\
25 \\
11 \\
58 \\
122 \\
19 \\
51\end{array}$ & $\begin{array}{r}34 \\
27 \\
12 \\
70 \\
129 \\
21 \\
52\end{array}$ & $\begin{array}{r}34 \\
31 \\
12 \\
9 \\
136 \\
25 \\
5\end{array}$ & $\begin{array}{r}37 \\
34 \\
16 \\
136 \\
178 \\
36 \\
60\end{array}$ & $\begin{array}{r}57 \\
55 \\
33 \\
173 \\
234 \\
47 \\
86\end{array}$ & $\begin{array}{r}82 \\
68 \\
35 \\
195 \\
233 \\
59 \\
52\end{array}$ \\
\hline
\end{tabular}



MINIMUN AVERAGE FLOWS FOR PERIOD OF RECORD

\begin{tabular}{|c|c|c|c|c|c|c|c|}
\hline PERIOD OF CONSECUTIVE DAYS & 3 & 7 & 14 & 30 & 60 & 120 & 183 \\
\hline $\begin{array}{l}\text { DISCHARGE, IN } \mathrm{ft}^{3} / \mathrm{s} \\
\text { CLIMATIC YEAR }\end{array}$ & $\begin{array}{r}2.5 \\
1959\end{array}$ & $\begin{array}{r}2.6 \\
1959\end{array}$ & $\begin{array}{r}2.7 \\
1959\end{array}$ & $\begin{array}{r}2.8 \\
1959\end{array}$ & $\begin{array}{r}3.1 \\
1959\end{array}$ & $\begin{array}{r}6.8 \\
1959\end{array}$ & $\begin{array}{r}8.4 \\
1957\end{array}$ \\
\hline
\end{tabular}

MAGNITUDE AND FREQUENCY OF ANNUAL LOW FLOWS

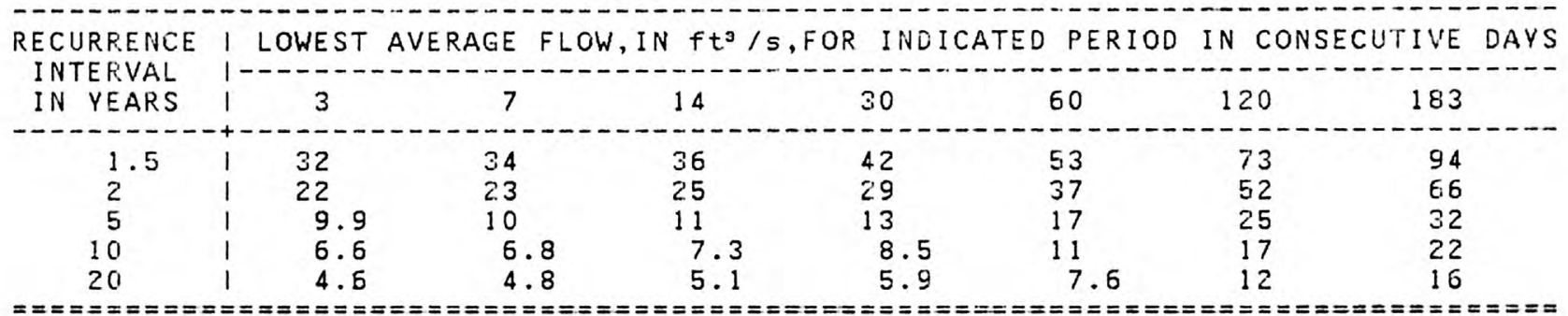

MAGNITUDE AND FREQUENCY OF SEASONAL LOW FLOWS

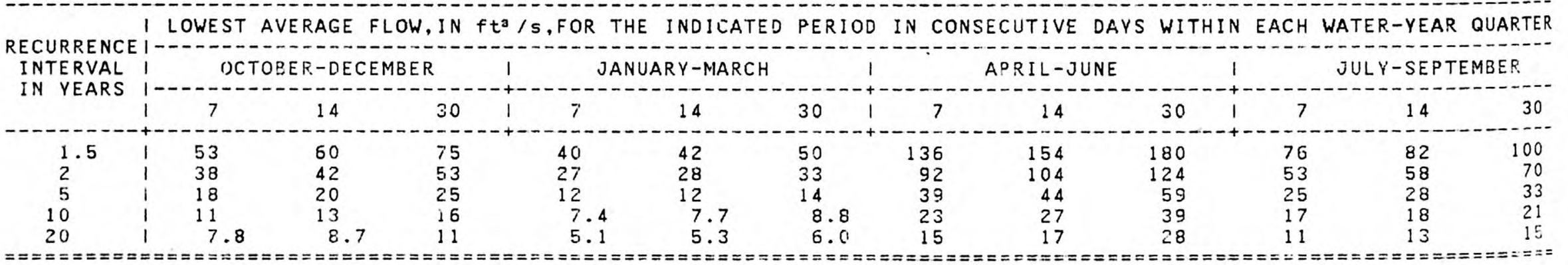

DURATION OF DAILY DISCHARGES FOR ANNUAL AND SEASONAL PERIODS

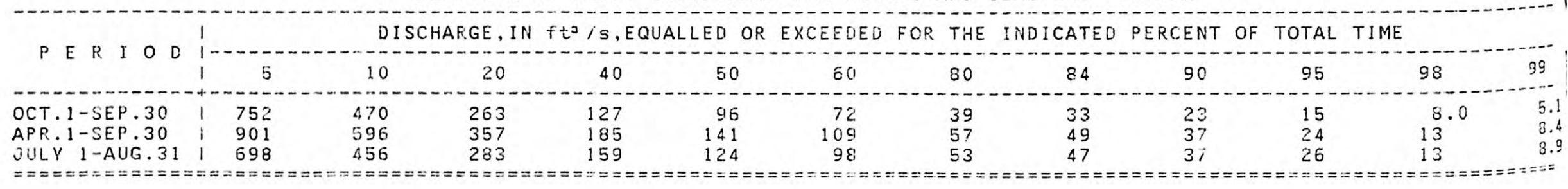




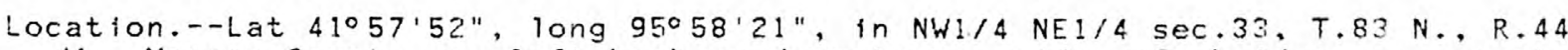
W. Monona County, on left bank on downstream side of bridge on county highway E54, $1.0 \mathrm{~m} l 1$ e east of gaging station on Nonona-Harrison ditch near Turin, 2.5 miles downstream from Maple River, 3.8 miles south of Turin, 6.2

miles northeast of Eilencoe, and at mile 13.5 .

LOW FLOW DISCHARGES, IN YEAR. ENDING MARCH 31

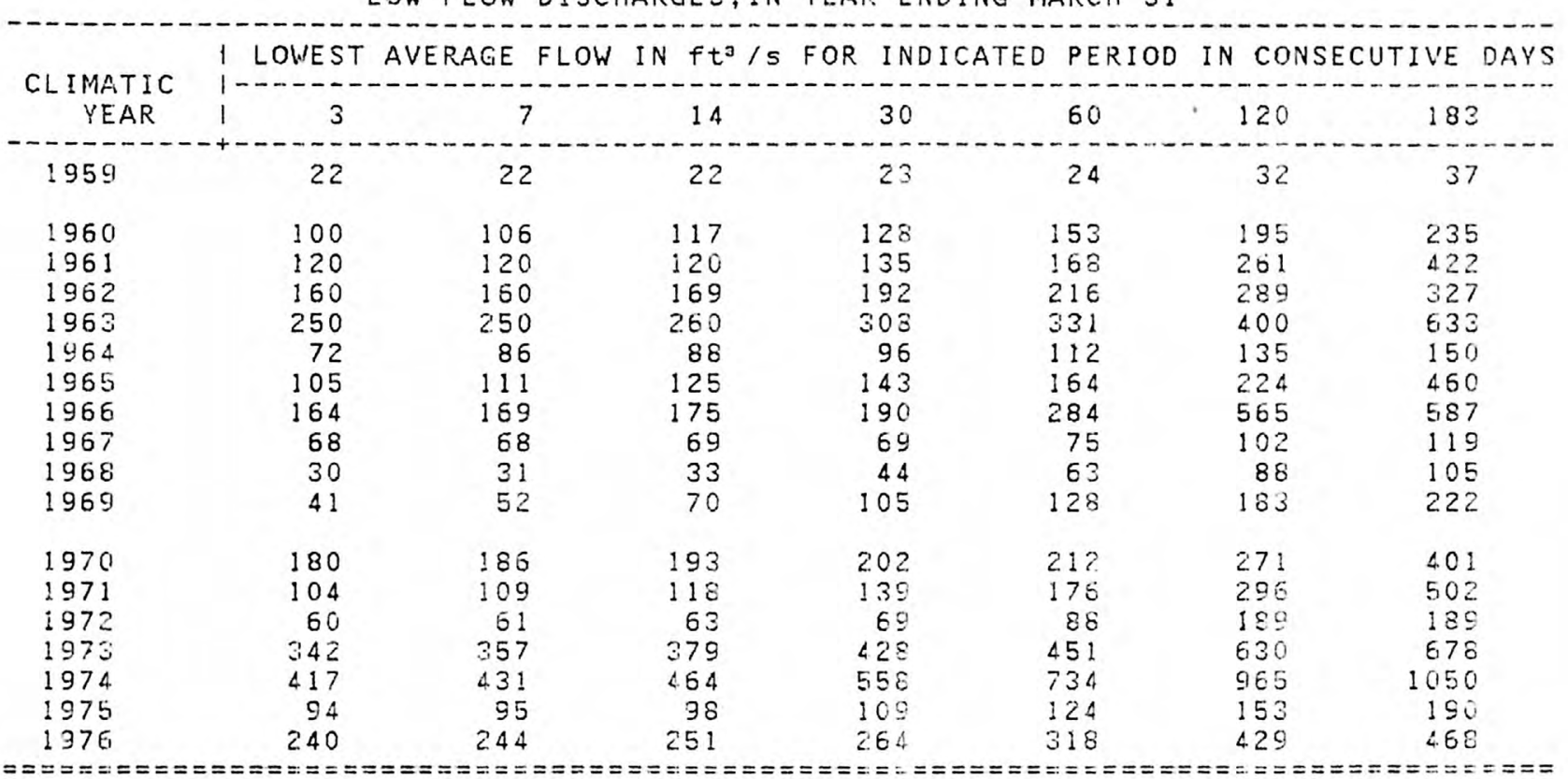


MINIMUM AVERAGE FLOWS FOR PERIOD OF RECORD

\begin{tabular}{|c|c|c|c|c|c|c|c|}
\hline PERIOD OF CONSECUTIVE DAYS 1 & 3 & 7 & 14 & 30 & 60 & 120 & 183 \\
\hline $\begin{array}{l}\text { DISCHARGE, IN } \mathrm{ft}^{3} / \mathrm{s} \\
\text { CLIMATIC YEAR }\end{array}$ & $\begin{array}{l}22 \\
1959\end{array}$ & $\begin{array}{l}22 \\
1959\end{array}$ & $\begin{array}{l}22 \\
1959\end{array}$ & $\begin{array}{l}23 \\
1959\end{array}$ & $\begin{array}{l}24 \\
1959\end{array}$ & $\begin{array}{l}32 \\
1959\end{array}$ & $\begin{array}{l}37 \\
1959\end{array}$ \\
\hline
\end{tabular}

MAGNITUDE AND FREQUENCY OF ANNUAL LOW FLOWS

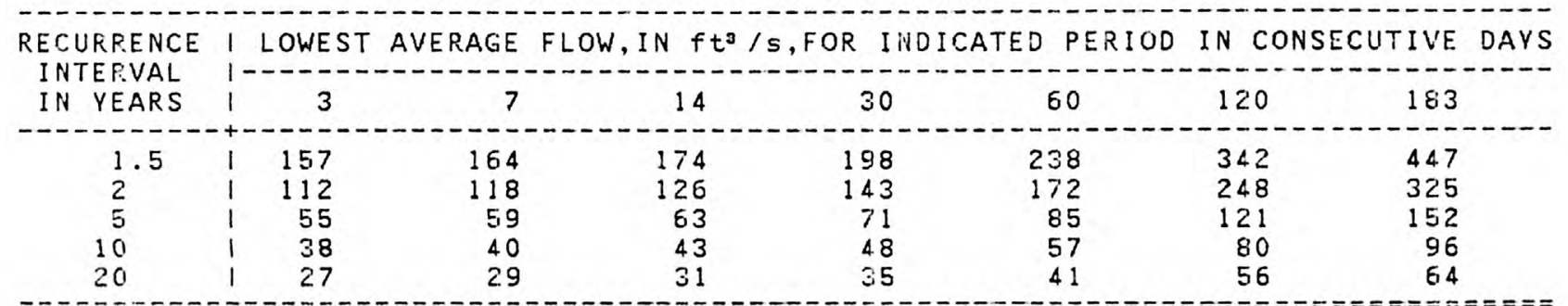

MAGNITUDE AND FREQUENCY OF SEASONAL LOW FLOWS

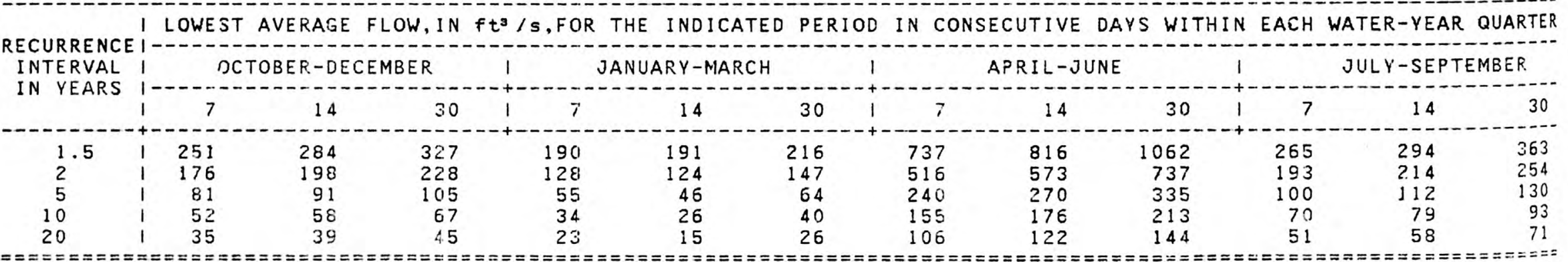

DURATION OF DAILY DISCHARGES FOR ANNUAL AND SEASONAL FERIODS

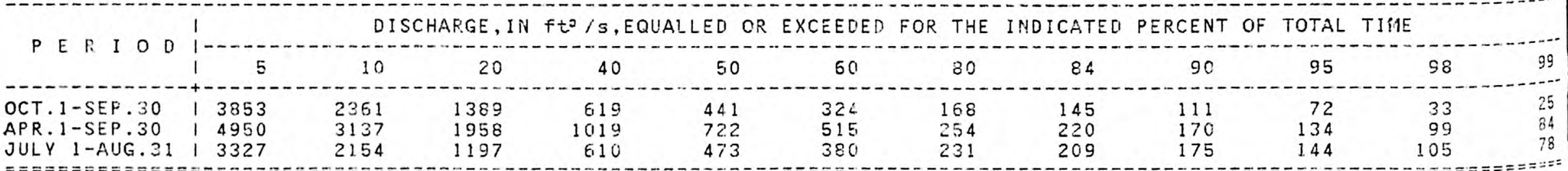

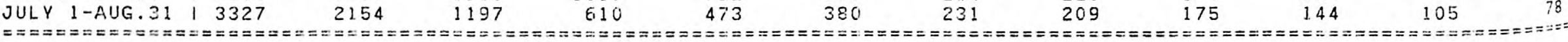




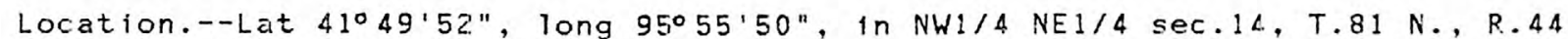
W. Harrison County, on left bank on downstream side of bricige on county highway $\mathrm{F} 20$, at west edge of $\mathrm{Pisgah}, 0.4 \mathrm{mile}$ downstream from Cobb Creek, $0.5 \mathrm{~m} f$ le upstream from Mogger Ditch, and 13.1 niles upstream from mouth.

LOW FLOW DISCHARGES, IN VEAR ENDING MARCH 31

\begin{tabular}{|c|c|c|c|c|c|c|c|}
\hline \multirow{2}{*}{$\begin{array}{c}\text { CLIMATIC } \\
\text { YEAR }\end{array}$} & LOWEST A & E FL & $f t^{2} / s$ & INDI & PERIC & IN CONSE & IVE DAYS \\
\hline & 13 & 7 & 14 & 30 & 60 & 120 & 183 \\
\hline $\begin{array}{l}1942 \\
1943 \\
1944 \\
1945 \\
1946 \\
1947 \\
1948 \\
1949\end{array}$ & $\begin{array}{l}4.7 \\
8.4 \\
6.0 \\
2.0 \\
30 \\
23 \\
11 \\
13\end{array}$ & $\begin{array}{l}4.9 \\
8.9 \\
6.2 \\
2.0 \\
30 \\
24 \\
11 \\
13\end{array}$ & $\begin{array}{l}6.0 \\
11 \\
6.4 \\
2.4 \\
31 \\
26 \\
14 \\
15\end{array}$ & $\begin{array}{l}14 \\
11 \\
8 \cdot 1 \\
3 \cdot 9 \\
36 \\
32 \\
27 \\
16\end{array}$ & $\begin{array}{l}21 \\
13 \\
10 \\
7.2 \\
39 \\
42 \\
36 \\
18\end{array}$ & $\begin{array}{l}35 \\
16 \\
13 \\
19 \\
50 \\
63 \\
44 \\
25\end{array}$ & $\begin{array}{l}44 \\
35 \\
22 \\
28 \\
74 \\
80 \\
44 \\
34\end{array}$ \\
\hline $\begin{array}{l}1950 \\
1951 \\
1952 \\
1953 \\
1954 \\
1955 \\
1956 \\
1957 \\
1958 \\
1959\end{array}$ & $\begin{array}{l}4.0 \\
8.0 \\
62 \\
59 \\
7.4 \\
18 \\
6.0 \\
3.3 \\
2.9 \\
2.5\end{array}$ & $\begin{array}{c}4.0 \\
9.2 \\
65 \\
59 \\
8.0 \\
18 \\
6.0 \\
3.3 \\
3.2 \\
2.5\end{array}$ & $\begin{array}{l}4.0 \\
9.6 \\
76 \\
59 \\
11 \\
19 \\
6.2 \\
3.4 \\
3.9 \\
2.9\end{array}$ & $\begin{array}{l}5.5 \\
12 \\
82 \\
59 \\
18 \\
21 \\
6.5 \\
4.0 \\
5.5 \\
3.3\end{array}$ & $\begin{array}{r}14 \\
14 \\
116 \\
64 \\
30 \\
27 \\
6.7 \\
6.8 \\
11 \\
3.9\end{array}$ & $\begin{array}{r}23 \\
17 \\
161 \\
72 \\
38 \\
35 \\
10 \\
10 \\
30 \\
7.0\end{array}$ & $\begin{array}{r}40 \\
28 \\
237 \\
81 \\
39 \\
46 \\
12 \\
11 \\
35 \\
9.7\end{array}$ \\
\hline $\begin{array}{l}1960 \\
1961 \\
1962 \\
1963 \\
1964 \\
1965 \\
1966 \\
1967 \\
1968 \\
1969\end{array}$ & $\begin{array}{l}7.9 \\
20 \\
24 \\
58 \\
30 \\
20 \\
30 \\
13 \\
10 \\
11\end{array}$ & $\begin{array}{l}8 \cdot 9 \\
21 \\
28 \\
53 \\
32 \\
20 \\
30 \\
14 \\
10 \\
12\end{array}$ & $\begin{array}{l}13 \\
23 \\
31 \\
59 \\
33 \\
23 \\
31 \\
15 \\
10 \\
15\end{array}$ & $\begin{array}{l}17 \\
25 \\
34 \\
65 \\
34 \\
30 \\
34 \\
17 \\
12 \\
20\end{array}$ & $\begin{array}{l}20 \\
27 \\
39 \\
71 \\
39 \\
31 \\
61 \\
18 \\
22 \\
23\end{array}$ & $\begin{array}{l}26 \\
36 \\
49 \\
88 \\
46 \\
37 \\
72 \\
25 \\
29 \\
41\end{array}$ & $\begin{array}{r}27 \\
51 \\
53 \\
114 \\
50 \\
53 \\
91 \\
29 \\
32 \\
58\end{array}$ \\
\hline $\begin{array}{l}1970 \\
1971 \\
1972 \\
1973 \\
1974 \\
1975 \\
1976\end{array}$ & $\begin{array}{r}27 \\
7.4 \\
5.1 \\
30 \\
71 \\
45 \\
33\end{array}$ & $\begin{array}{c}30 \\
7.8 \\
5.1 \\
31 \\
73 \\
47 \\
34\end{array}$ & $\begin{array}{c}30 \\
7.9 \\
5.3 \\
33 \\
77 \\
52 \\
36\end{array}$ & $\begin{array}{l}30 \\
5.5 \\
5.8 \\
42 \\
88 \\
55 \\
39\end{array}$ & $\begin{array}{c}32 \\
13 \\
8.3 \\
75 \\
99 \\
56 \\
44\end{array}$ & $\begin{array}{r}38 \\
23 \\
19 \\
98 \\
118 \\
59 \\
51\end{array}$ & $\begin{array}{r}46 \\
24 \\
17 \\
116 \\
135 \\
80 \\
57\end{array}$ \\
\hline
\end{tabular}



MINIMUM AVERAGE FLOWS FOR PERIOD OF RECORD

\begin{tabular}{|c|c|c|c|c|c|c|c|c|}
\hline PERIOD OF CONSECUTIVE DAYS & 1 & 3 & 7 & 14 & 30 & 60 & 120 & 183 \\
\hline $\begin{array}{l}\text { DISCHARGE, IN } \mathrm{ft}^{\mathrm{o}} / \mathrm{s} \\
\text { CLIMATIC VEAR }\end{array}$ & $\begin{array}{l}1 \\
1\end{array}$ & $\begin{array}{r}2.0 \\
1945\end{array}$ & $\begin{array}{r}2.0 \\
1945\end{array}$ & $\begin{array}{r}2.4 \\
1945\end{array}$ & $\begin{array}{r}3.3 \\
1959\end{array}$ & $\begin{aligned} 3.5 \\
1959\end{aligned}$ & $\begin{array}{r}7.0 \\
1959\end{array}$ & $\begin{array}{r}9.7 \\
1959\end{array}$ \\
\hline
\end{tabular}

MAGNITUDE AND FREQUENCY OF ANNUAL LOW FLOWS

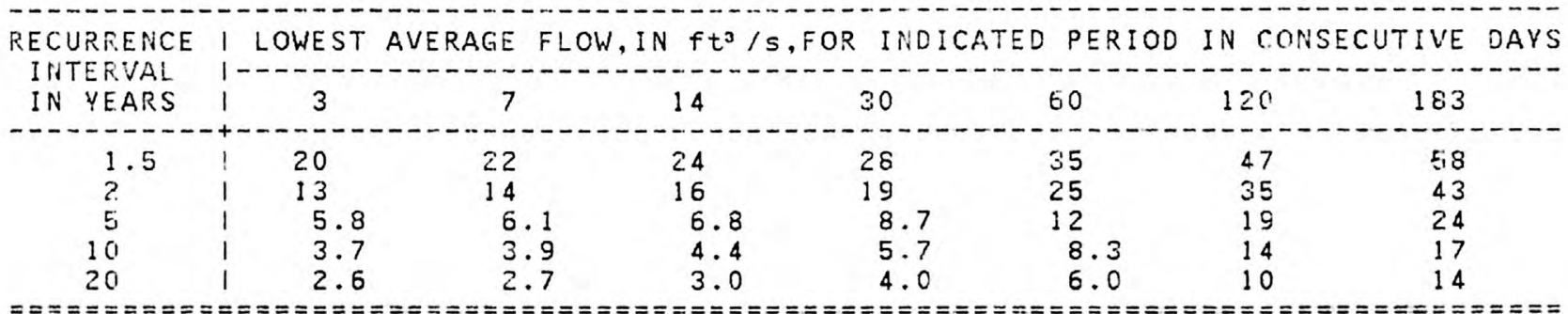

MAGNITUIDE AND FREQUENCY OF SEASONAL LOW FLOWS

I LOWEST AVERAGE FLOW, IN $\mathrm{ft}^{3} / \mathrm{s}, F O R$ THE INDICATED PERIOD IN CONSECUTIVE DAYS WITHIN EACH WATER-YEAR QUARTER

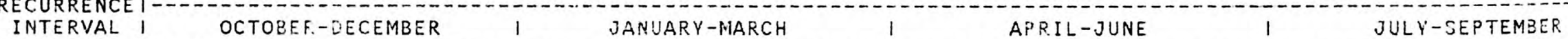

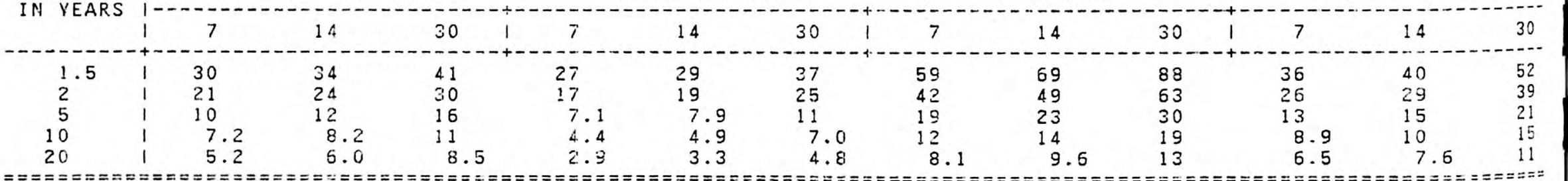

DURATION OF DAILY DISCHARGES FOR ANNUAL AND SEASONAL FERIODS

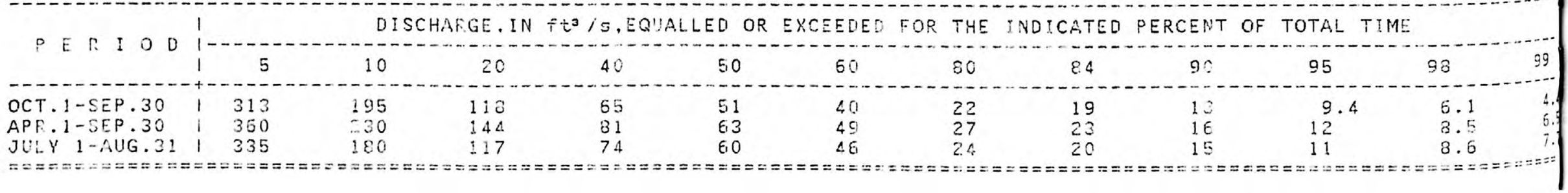


Location.--Lat $41^{\circ} 45^{\prime} 10^{\prime \prime}$, long $95^{\circ} 56^{\prime} 15^{\prime \prime}$, in NW1/4SE1/4 sec. 11, T.80 N., R.44 W. , on upstream side of right wingwali of highway bridge, 5.6 miles upstream from Alien Creek ditch and 5 miles northwest of Magnolia.

Dralnage area. $--9.26 \mathrm{~m}^{2}$.

LOW FLOW DISCHARGES, IN YEAR ENDING MARCH 31.

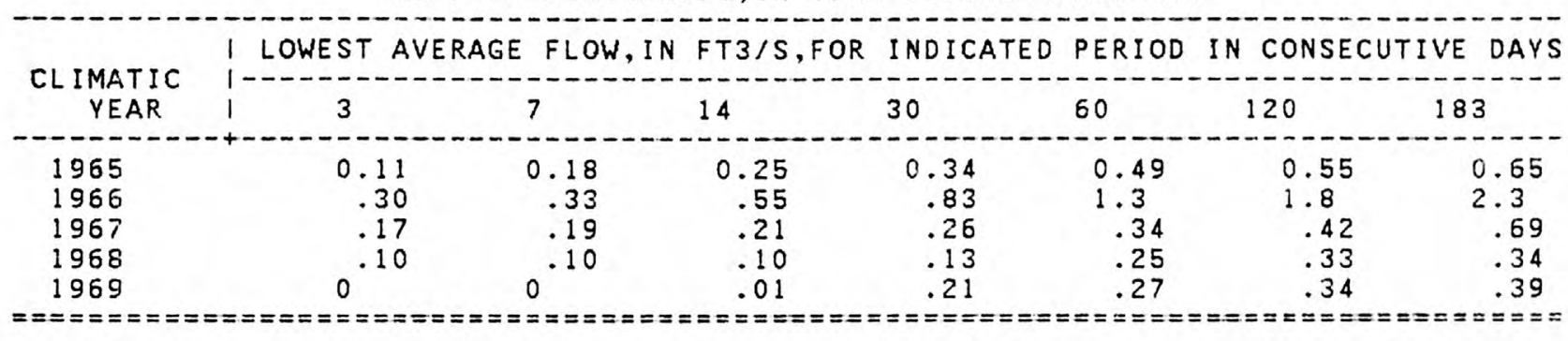

$Q_{a}=1.8$

$Q(84)=0.6$

$702=0.3$

$7 Q 10=0.1$ 


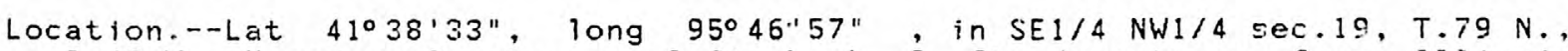
R. 42 W. Harrison County, on left bank 9 ft downstream fron Iirinois Centrai Railroad bridge at Logan, $0.4 \mathrm{~m} l$ le downstream from Elk Grove Creek, 10.5 miles upstream from Willow Creek, and 15.8 miles upstream from mouth. LOW FLOW DISCHARGES, IN YEAR. ENDING MARCH 31

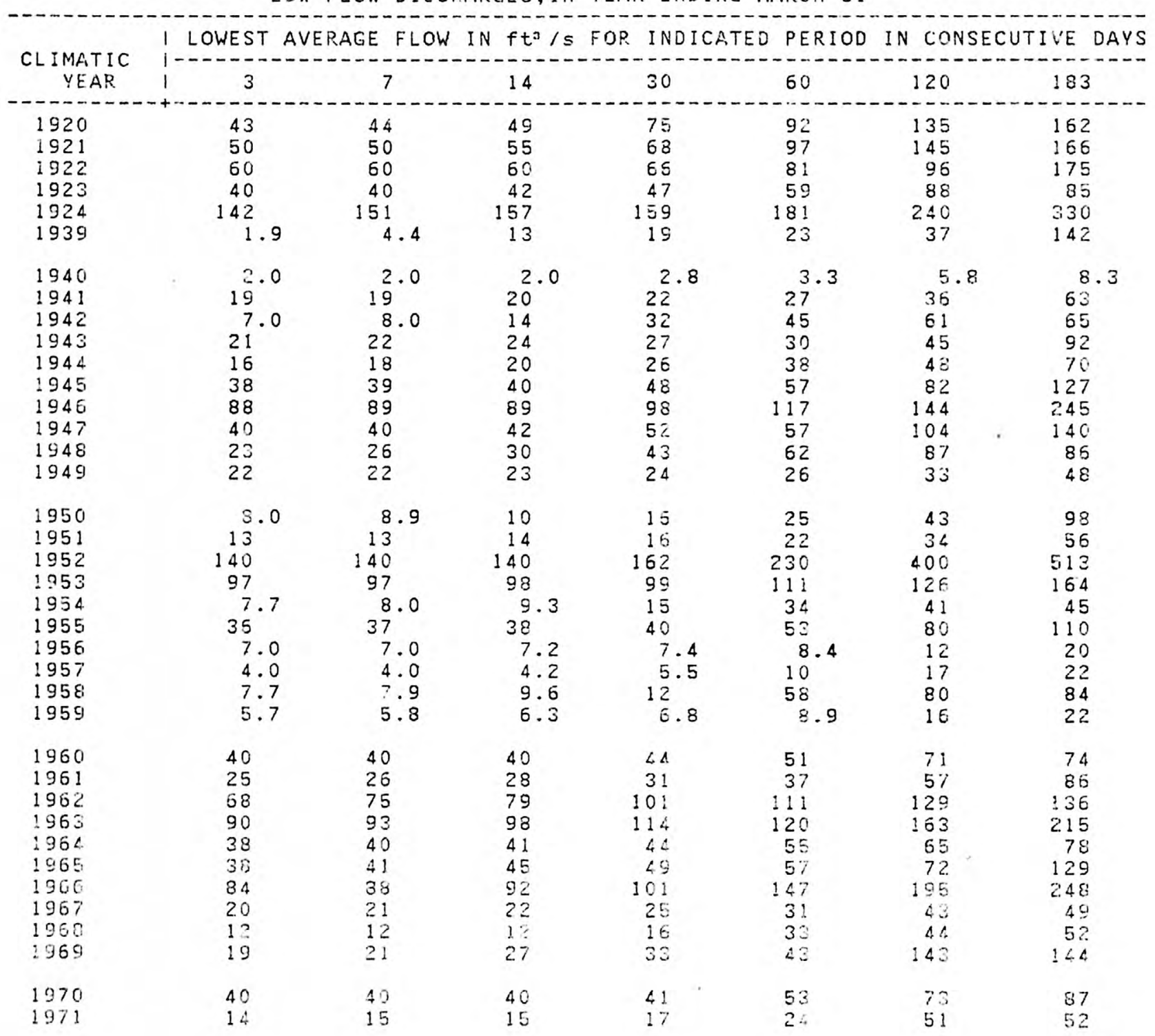


LOW FLOW DISCHARGES, IN YEAR ENDING MARCH 31--Cont inued

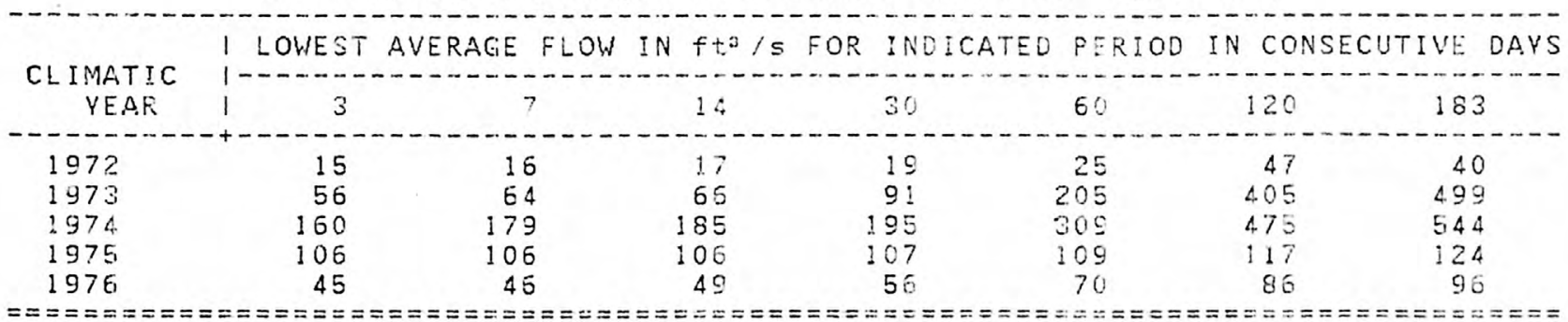


DRAINAGE AREA: $871 \mathrm{~m}^{2}$ PERIOD OF RECORD: 44 YEARS AVERAGE DISCHARGE: 308 ft $3 / \mathrm{S}$ MINIMUM AVERAGE FLOWS FOR PERIOD OF RECORD

\begin{tabular}{|c|c|c|c|c|c|c|c|}
\hline PERIOD OF CONSECUTIVE DAYS & 3 & 7 & 14 & 30 & 60 & 120 & 183 \\
\hline $\begin{array}{l}\text { DISCHARGE, IN } \mathrm{ft}^{3} / \mathrm{s} \\
\text { CLIMATIC YEA.R }\end{array}$ & $\begin{array}{r}1.9 \\
1939\end{array}$ & $\begin{array}{r}2.0 \\
1940\end{array}$ & $\begin{array}{r}2.0 \\
1940\end{array}$ & $\begin{array}{r}2.8 \\
1940\end{array}$ & $\begin{array}{r}3.3 \\
1940\end{array}$ & $\begin{array}{r}5.8 \\
1940\end{array}$ & \\
\hline
\end{tabular}

MAGNITUDE AND FREQUENCY OF ANNUAL LOW FLOWS

\begin{tabular}{|c|c|c|c|c|c|c|c|c|}
\hline $\begin{array}{l}\text { RECURRENCE } \\
\text { INTERVAL }\end{array}$ & 1 & WEST & $A G E \quad F L$ & $\begin{array}{l}\mathrm{N} f \mathrm{t}^{3} / \\
-\end{array}$ & $\begin{array}{l}R \text { IND. } \\
-\end{array}$ & $\begin{array}{l}D \text { PERI } \\
-\end{array}$ & IN Ci & TIVE \\
\hline IN YEARS & 1 & 3 & 7 & 14 & 30 & GO & 120 & 183 \\
\hline $\begin{array}{l}1.5 \\
2 \\
5 \\
10 \\
20\end{array}$ & $\begin{array}{l}1 \\
1 \\
1 \\
1 \\
1\end{array}$ & $\begin{array}{r}44 \\
28 \\
10 \\
6.0 \\
3.7\end{array}$ & $\begin{array}{l}45 \\
29 \\
11 \\
6.8 \\
4.3\end{array}$ & $\begin{array}{r}49 \\
32 \\
13 \\
8.0 \\
5.2\end{array}$ & $\begin{array}{l}58 \\
39 \\
17 \\
10 \\
E .7\end{array}$ & $\begin{array}{c}77 \\
53 \\
23 \\
14 \\
9.5\end{array}$ & $\begin{array}{r}109 \\
75 \\
34 \\
22 \\
15\end{array}$ & $\begin{array}{r}143 \\
100 \\
47 \\
31 \\
22\end{array}$ \\
\hline
\end{tabular}

MAGNITUDE AND FREQUENCY OF SEASONAL LOW FLOWS

\begin{tabular}{l} 
RECURRENCE \\
INTERVAL \\
$\begin{array}{l}\text { IN } \\
\text { YEARS }\end{array}$ \\
\hline
\end{tabular}

DURATION OF DAILY DISCHARGES FOR ANNUAL ANO SEASONAL PERIODS

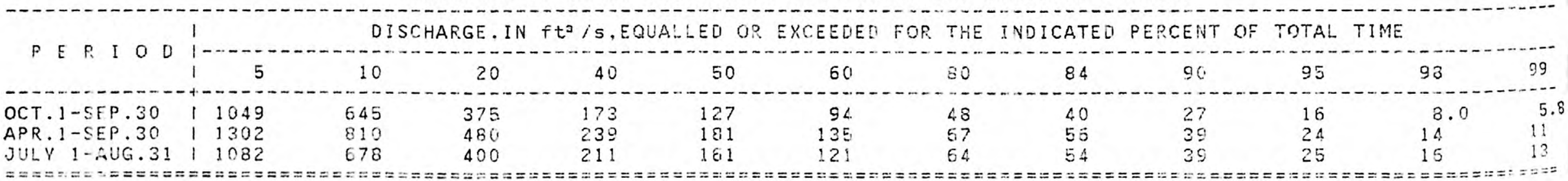


Location.--Lat $41^{\circ} 44^{\prime} 20^{\prime \prime}$, long 95048'20", In SW1/4SW1/4 sec. 13, T.80 N., R.43 W., on left bank $225 \mathrm{ft}$ upstream from county highway brldge, $0.5 \mathrm{mlles}$ upstream from Willow Creek, and $6.0 \mathrm{miles}$ northeast of Magnolia, Iowa.

Remarks.--Discontinued Sept. 30, 1969.

Drainage area. $--6.97 \mathrm{ml}^{2}$.

LOW FLOW DISCHARGES, IN YEAR ENDING MARCH 31

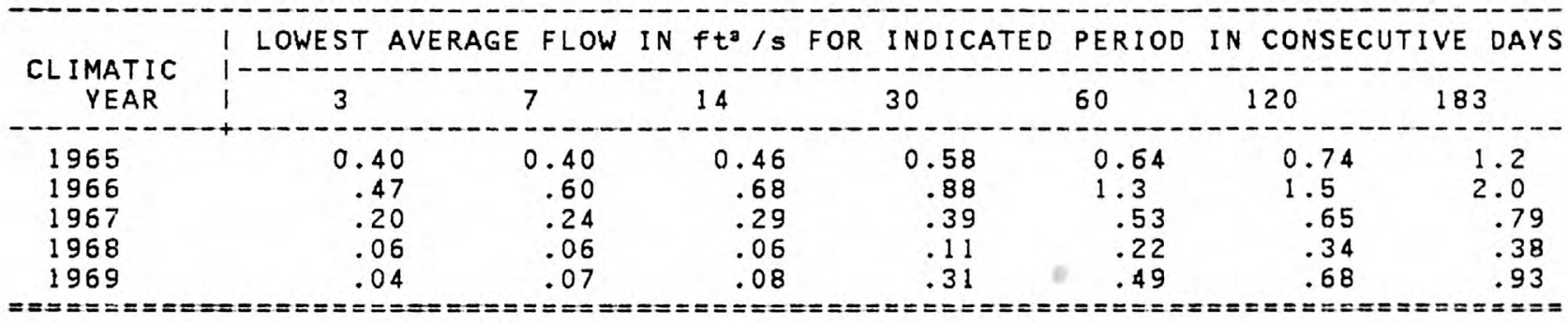

$Q \mathrm{a}=1.3$

$Q(84)=0.4$

$7 Q 2=0.2$

$7010=0$ 
Location.--Lat 41037'54", long 95053'27", in NW1/4 NE1/4 sec.30, T.79 N., R.43 W. Harrison County, on right bank on downstream side of bridge on county highway F50, 5.5 miles west of Logan, and $7.5 \mathrm{miles}$ upstream from mouth.

Remarks.--Discontinued September $30,1975$.

Drainage area. $--129 \mathrm{~m}^{2}$.

LOW FLOW DISCHARGES, IN YEAR. ENDING MARCH 31

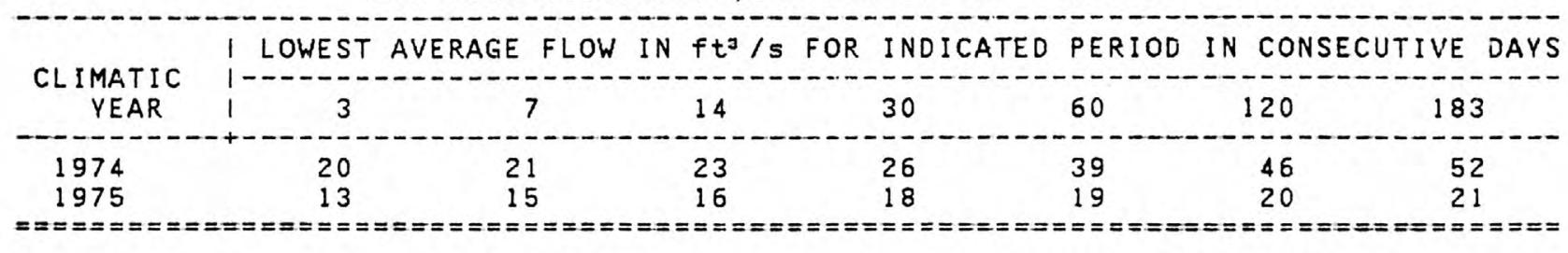

$Q \mathrm{Q}=47$

$Q(84)=8.6$

$7 Q 2=4.4$

$7 Q 10=1 . I$ 


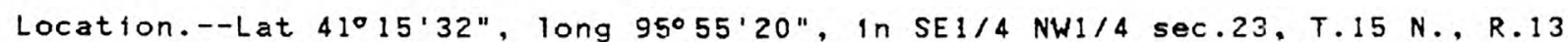
E., Douglas County, on right bank on left side of concrete floodwall, at foot of Douglas Street, $275 \mathrm{ft}$ downstream from Interstate 480 Highway bridge in Omaha, and at mile 615.9.

Remarks.--Flow partly regulated by upstream main-stem reservolrs since Nov. 1937.

LOW FLOW DISCHARGES, IN YEAR ENDING MARCH 31

\begin{tabular}{|c|c|c|c|c|c|c|c|}
\hline CLIMATIC & I LOWEST & E FL & $f t^{3} / s$ & INDI & PERIC & IN CONSE & IVE $\quad$ D \\
\hline YEAR & 13 & 7 & 14 & 30 & 60 & 120 & 183 \\
\hline $\begin{array}{l}1930 \\
1931 \\
1932 \\
1933 \\
1934 \\
1935 \\
1936 \\
1937\end{array}$ & $\begin{array}{l}5810 \\
6030 \\
5010 \\
4000 \\
3500 \\
3700 \\
4430 \\
2270\end{array}$ & $\begin{array}{l}5950 \\
6590 \\
5440 \\
4140 \\
3690 \\
4120 \\
4830 \\
2880\end{array}$ & $\begin{array}{l}6530 \\
7550 \\
5580 \\
4320 \\
3960 \\
4150 \\
5090 \\
4190\end{array}$ & $\begin{array}{l}7780 \\
8120 \\
6030 \\
5330 \\
4930 \\
5080 \\
5610 \\
5350\end{array}$ & $\begin{array}{l}8670 \\
8380 \\
6830 \\
7100 \\
7610 \\
5580 \\
6470 \\
6300\end{array}$ & $\begin{array}{r}11900 \\
12100 \\
8540 \\
8900 \\
10100 \\
7590 \\
7000 \\
7840\end{array}$ & $\begin{array}{r}13100 \\
13600 \\
9120 \\
10600 \\
12400 \\
7750 \\
7920 \\
8830\end{array}$ \\
\hline
\end{tabular}


MINIMUM AVERAGE FLOWS FOR FERIOD OF RECORD

\begin{tabular}{|c|c|c|c|c|c|c|c|}
\hline PERIOD OF CONSECUTIVE DAYS & 3 & 7 & 14 & 30 & 60 & 20 & 183 \\
\hline $\begin{array}{l}\text { HARGE, IN } \mathrm{ft}^{3} / \mathrm{s} \\
\text { ATIC YEAR }\end{array}$ & $\begin{array}{r}2270 \\
1937\end{array}$ & $\begin{array}{l}2880 \\
1937\end{array}$ & $\begin{array}{l}3960 \\
1934\end{array}$ & $\begin{array}{l}4930 \\
1934\end{array}$ & $\begin{array}{l}5580 \\
1935\end{array}$ & $\begin{array}{l}7000 \\
1936\end{array}$ & $\begin{array}{r}7750 \\
1935\end{array}$ \\
\hline
\end{tabular}

MAGNITUDE AND FREQUENCY OF ANNUAL LOW FLOWS

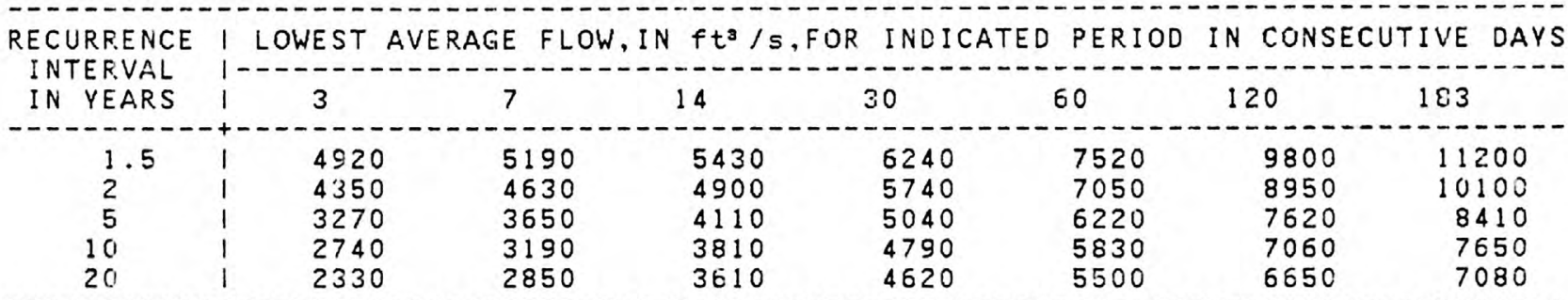

MAGNITUDE AND FREQUENCY OF SEASONAL LOW FLOWS

I LOWEST AVERAGE FLOW, IN $\mathrm{ft}^{3} / \mathrm{s}, \mathrm{FOR}$ THE INDICATED PERIOD IN CONSECUTIVE DAYS WITHIN EACH WATER-YEAR QUARTER RECURRENCE I - OCTOBEF - DECEMBER J J INUAY-MARCH

IN YEARS 1

\begin{tabular}{|c|c|c|c|c|c|c|c|c|c|c|c|c|c|}
\hline & i & 7 & 14 & I & 7 & 14 & $30 \quad 1$ & 7 & 14 & $30 \quad 1$ & 7 & 14 & 30 \\
\hline $\begin{array}{l}1.5 \\
2 \\
5 \\
10 \\
20\end{array}$ & $\begin{array}{l}1 \\
1 \\
1 \\
1\end{array}$ & $\begin{array}{l}5450 \\
5040 \\
4290 \\
3920 \\
3630\end{array}$ & $\begin{array}{l}6110 \\
5660 \\
4860 \\
4480 \\
4180\end{array}$ & $\begin{array}{l}7830 \\
7300 \\
6180 \\
5580 \\
5080\end{array}$ & $\begin{array}{l}6500 \\
5480 \\
3890 \\
3230 \\
2760\end{array}$ & $\begin{array}{l}6860 \\
6020 \\
4730 \\
4180 \\
3800\end{array}$ & $\begin{array}{l}8270 \\
7600 \\
6500 \\
6000 \\
5620\end{array}$ & $\begin{array}{l}20700 \\
18500 \\
14900 \\
13400 \\
12300\end{array}$ & $\begin{array}{l}22100 \\
19700 \\
16100 \\
14500 \\
13400\end{array}$ & $\begin{array}{l}27100 \\
23700 \\
18200 \\
15800 \\
14000\end{array}$ & $\begin{array}{r}11200 \\
9820 \\
7580 \\
6640 \\
5970\end{array}$ & $\begin{array}{r}12200 \\
10600 \\
8040 \\
6930 \\
6110\end{array}$ & $\begin{array}{r}13700 \\
11900 \\
8980 \\
7740 \\
6840\end{array}$ \\
\hline
\end{tabular}

DURATION OF DAILY DISCHARGES FOR ANNUAL AND SEASONAL PERIODS

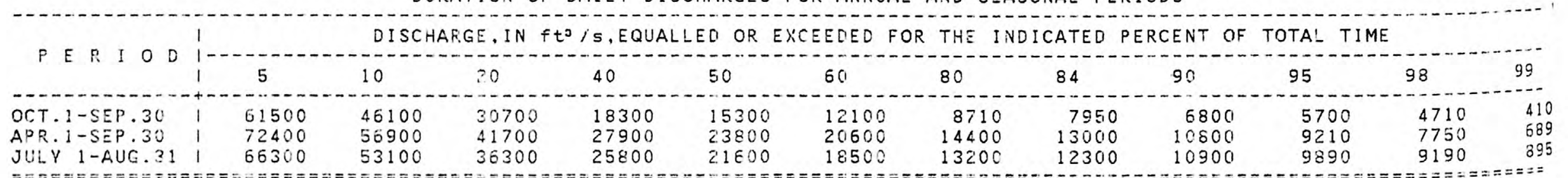


LOW FLOW DISCHARGES, IN YEAR. ENDING MARCH 31

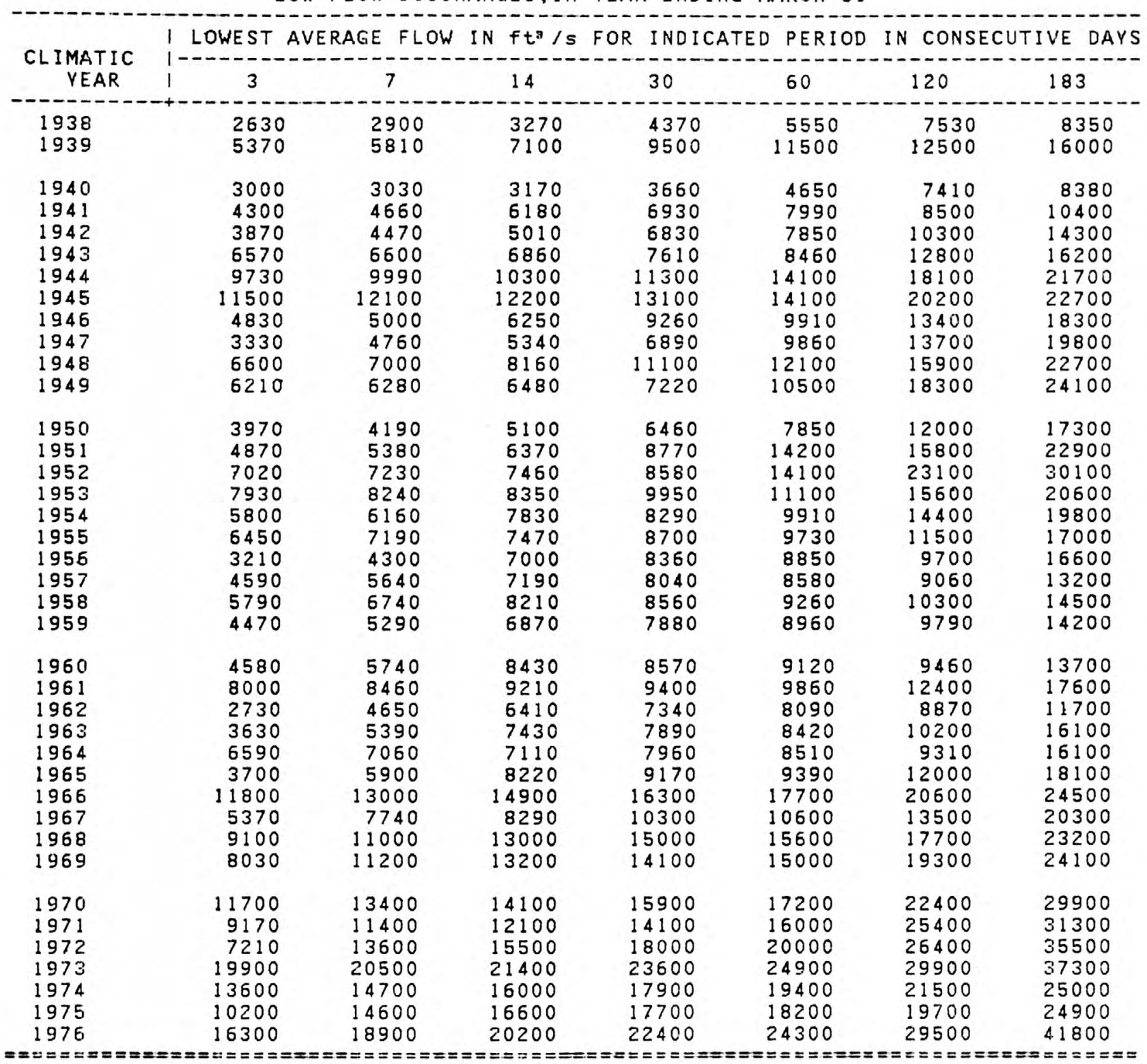


MINIMUM AVERAGE FLOWS FOR PERIOD OF RECORD

\begin{tabular}{l} 
PERIOD OF CONSECUTIVE DAYS \\
\hline IISHARGE, IN fts /s
\end{tabular}

MAGNITUDE AND FREQUENCY OF ANNUAL LOW FLOWS

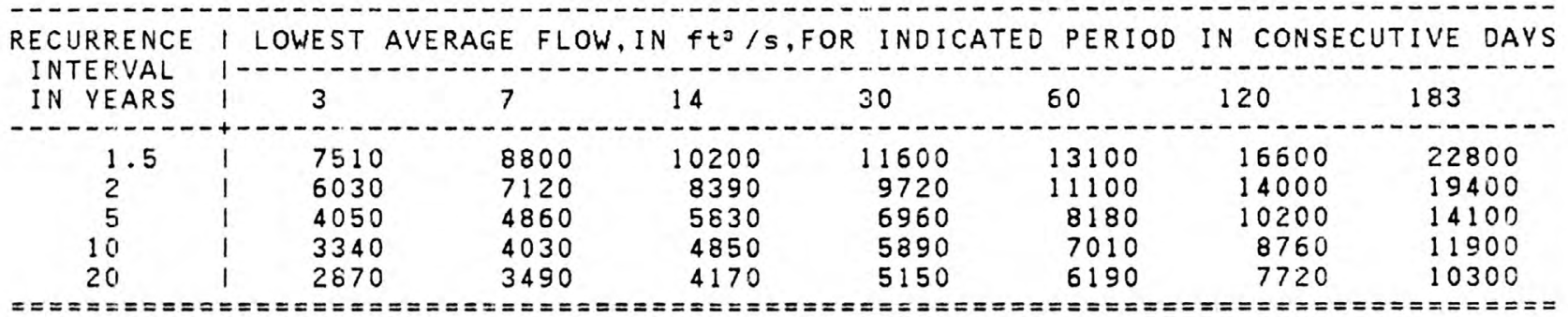

MAGNITUDE AND FREQUENCY OF SEASONAL LOW FLOWS

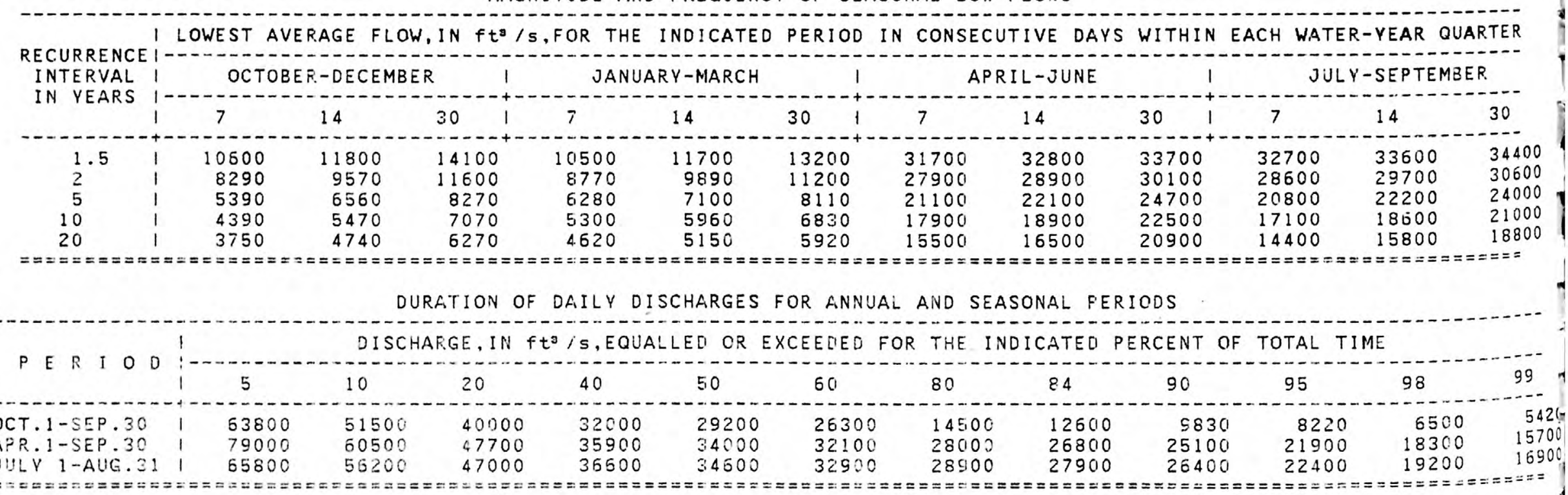


Location.--Lat 41017'32", long 95049'59", in SE1/4 SW1/4 sec.18, T.75 N., R.43 W. Pottawattamie County, on left bank at downstream side of first bridge off State Hlghway 183 , on Mud Hollow Road at north edge of Council Bluffs, and $8.8 \mathrm{~m} 1$ les upstream from mouth.

Remarks.--Discontinued September 1976.

LOW FLOW DISCHARGES, IN YEAR. ENDING MARCH 31

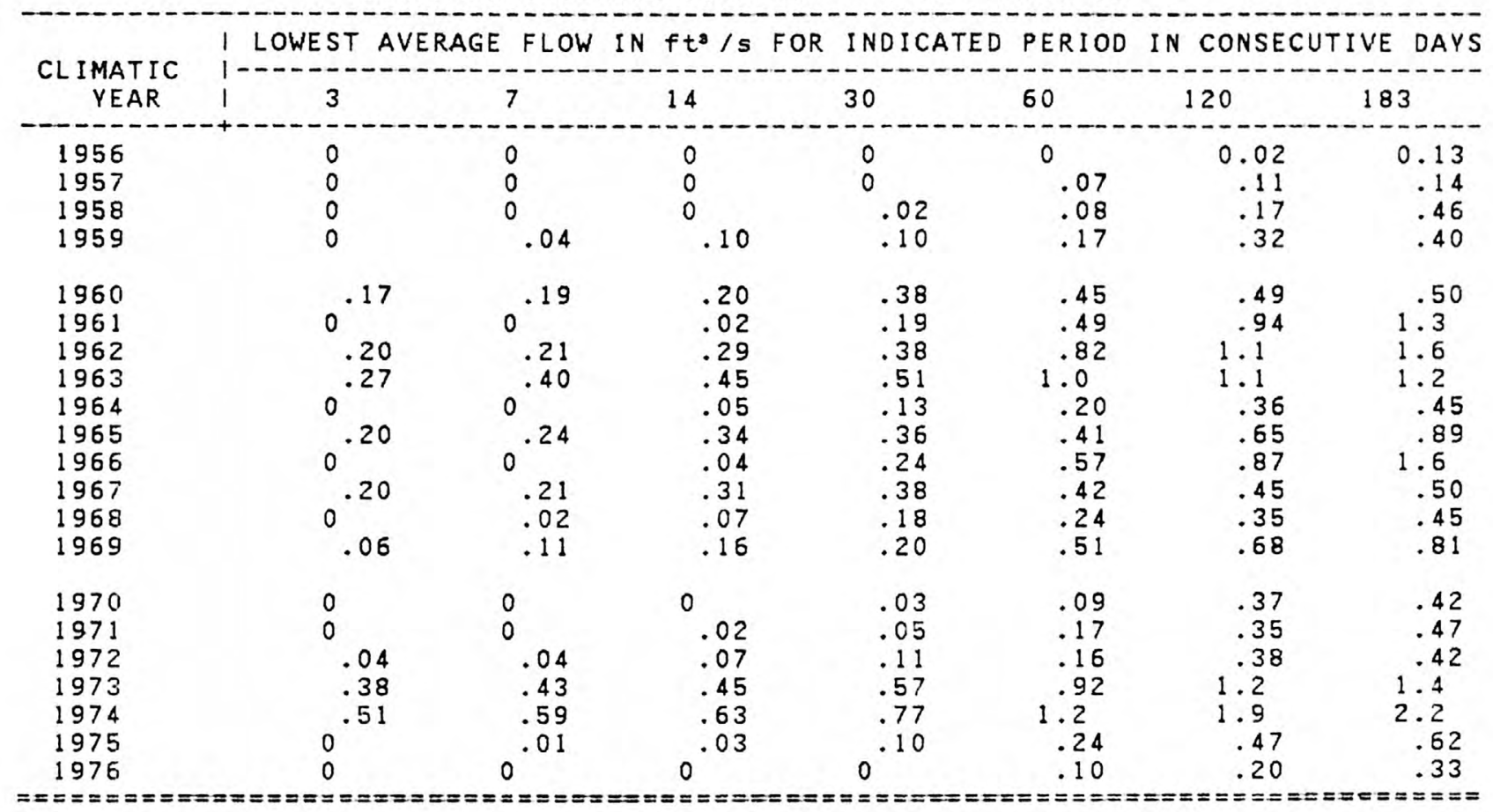




\begin{tabular}{l} 
DRAINAGE AREA: $7.99 \mathrm{~m}^{2} \quad$ PERIOD OF RECORD: 22 YEARS AVERAGE DISCHARGE: 1.5 fts /s \\
MINIMUM AVERAGE FLOWS FOR PERIOD OF RECORD \\
\hline PERIOD OF CONSECUTIVE DAYS I
\end{tabular}

MAGNITUDE ANB FREQUENCY OF ANNUAL LOW FLOWS

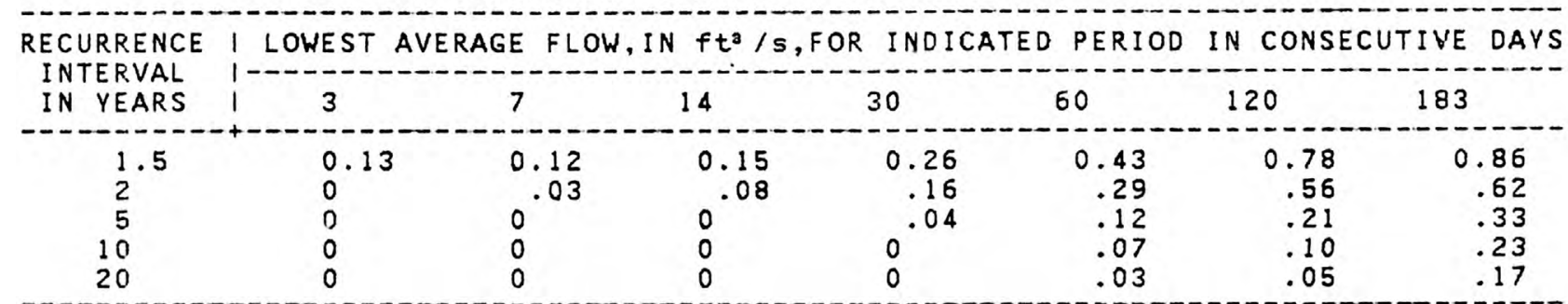

MAGNITUDE AND FREQUENCY OF SEASONAL LOW FLOWS

LOWEST AVERAGE FLOW, IN $\mathrm{ft} / \mathrm{s}$, FOR THE INDICATED PERIOD IN CONSECUTIVE DAYS WITHIN EACH WATER-YEAR QUARTER

\begin{tabular}{|c|c|c|c|c|c|c|c|c|c|c|c|c|}
\hline \multirow{2}{*}{$\begin{array}{l}\text { INTERVAL } \\
\text { IN YEARS }\end{array}$} & \multicolumn{3}{|c|}{ OCTOBER-DECEMBER } & \multicolumn{2}{|c|}{ JANUARY - MARCH } & 1 & \multicolumn{2}{|c|}{ APRIL-JUNE } & 1 & \multicolumn{3}{|c|}{ JULY - SEPTEMBER } \\
\hline & 7 & 14 & 30 & 7 & 14 & 30 & 7 & 14 & 30 & 7 & 14 & 30 \\
\hline $\begin{array}{r}1.5 \\
2 \\
5 \\
10 \\
20\end{array}$ & $\begin{array}{l}0.46 \\
.28 \\
0 \\
0 \\
0\end{array}$ & $\begin{array}{l}0.52 \\
.33 \\
.09 \\
0 \\
0\end{array}$ & $\begin{array}{r}0.68 \\
.46 \\
.18 \\
.06 \\
0 .\end{array}$ & $\begin{array}{l}0.27 \\
.10 \\
0 \\
0 \\
0\end{array}$ & $\begin{array}{l}0.30 \\
.15 \\
.03 \\
0 \\
0\end{array}$ & $\begin{array}{r}0.56 \\
.33 \\
.10 \\
.05 \\
.01\end{array}$ & $\begin{array}{r}0.65 \\
.40 \\
.12 \\
.03 \\
0\end{array}$ & $\begin{array}{r}0.78 \\
.52 \\
.22 \\
.12 \\
.05\end{array}$ & $\begin{array}{r}1.3 \\
.86 \\
.29 \\
.14 \\
.07\end{array}$ & $\begin{array}{l}0.32 \\
.21 \\
0 \\
0 \\
0\end{array}$ & $\begin{array}{l}0.36 \\
.21 \\
.02 \\
0 \\
0\end{array}$ & $\begin{array}{r}0.58 \\
.40 \\
.12 \\
.02 \\
0 .\end{array}$ \\
\hline
\end{tabular}

DURATION OF DAILY DISCHARGES FOR ANNUAL AND SEASONAL PERIODS

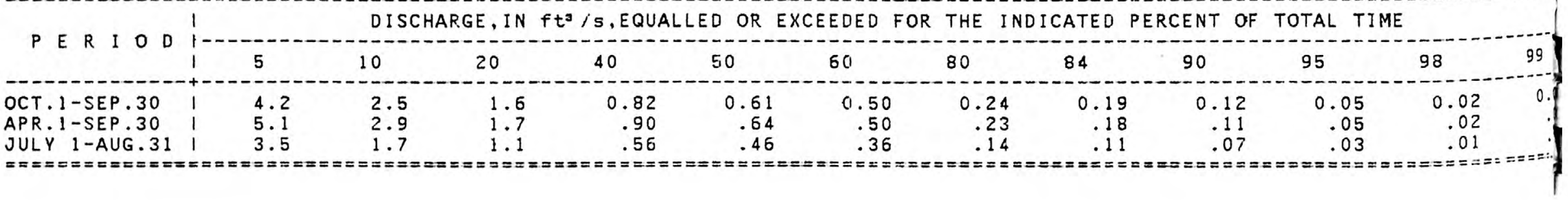


Location.--Lat 41045'10", long 95027'50", in N1/2 SE1/4 sec.11, T.80 N., R.40 W. Shelby County, on right bank at stream-stabllization structure $1,300 \mathrm{ft}$ downstream from bridge on State Highway 191, $0.5 \mathrm{mlle}$ downstream from small left-bank tributary and $2.3 \mathrm{mlles}$ southwest of Earling.

LOW FLOW DISCHARGES, IN YEAR ENDING MARCH 31

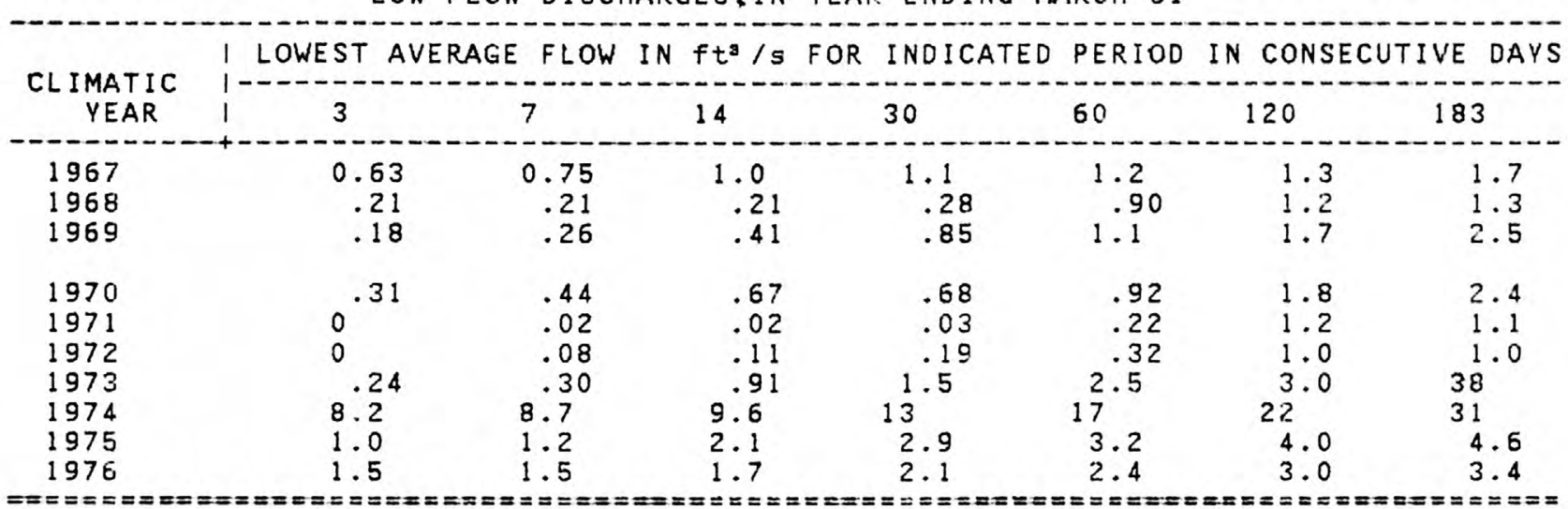




\section{DRAINAGE AREA: $32.0 \mathrm{~m}^{2}$ PERIOD OF RECORD: 11 YEARS AVERAGE DISCHARGE: $15.9 \mathrm{ft}^{3} / \mathrm{s}$}

MINIMUM AVERAGE FLOWS FOR PERIOD OF RECORD

\begin{tabular}{|c|c|c|c|c|c|c|c|}
\hline ERIOD OF CONSECUTIVE DAYS I & 3 & 7 & 14 & 30 & 60 & 120 & 183 \\
\hline $\begin{array}{l}\text { RGE, IN } f t^{2} / \mathrm{s} \\
\text { IC YEAR }\end{array}$ & $\begin{array}{l}0 \\
1972\end{array}$ & $\begin{array}{l}0.02 \\
1971\end{array}$ & $\begin{array}{c}0.02 \\
1971 \\
======\end{array}$ & $\begin{array}{c}0.03 \\
1971 \\
=====\end{array}$ & $\begin{array}{l}0.23 \\
1971 \\
=====\end{array}$ & $\begin{array}{r}1.0 \\
1972 \\
====\end{array}$ & \\
\hline
\end{tabular}

MAGNITUDE AND FREQUENCY OF ANNUAL LOW FLOWS

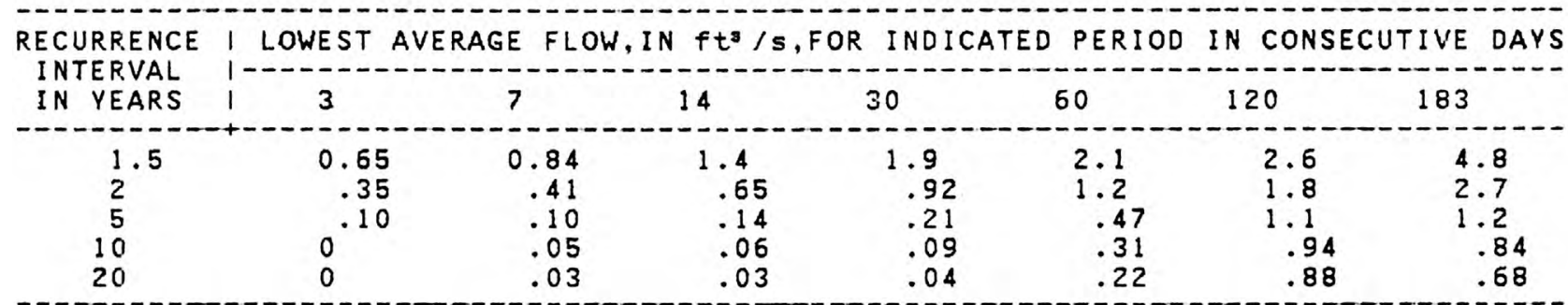

MAGNITUDE AND FREQUENCY OF SEASONAL LOW FLOWS

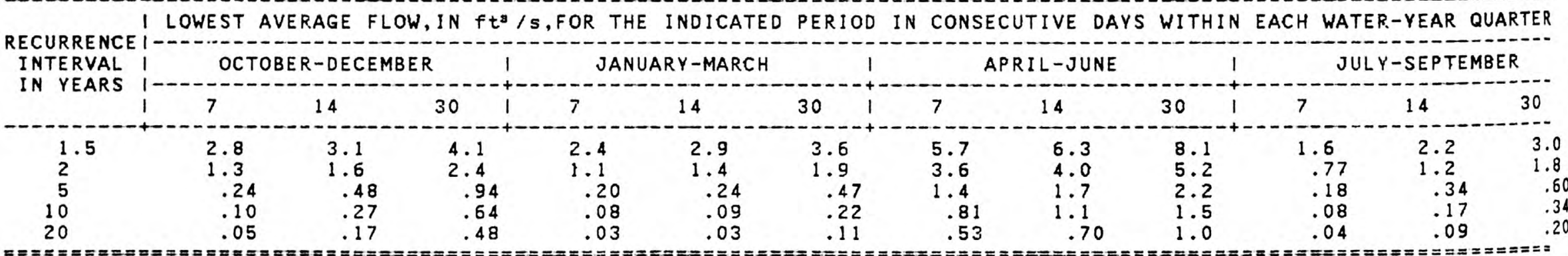

DURATION OF DAILY DISCHARGES FOR ANNUAL AND SEASONAL PERIODS

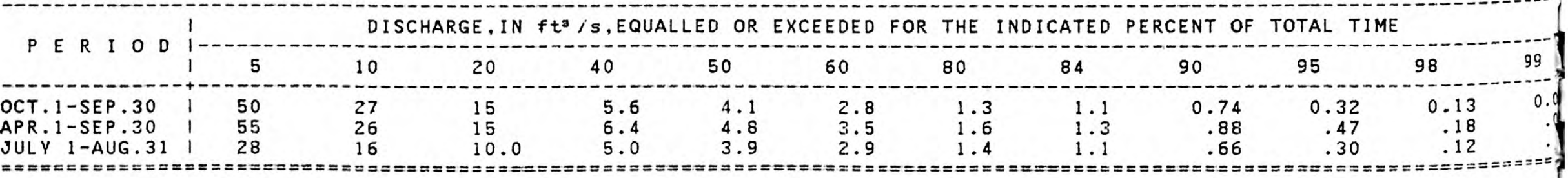


Location.--Lat $40^{\circ} 53^{\prime} 04^{\prime \prime}$, long $95^{\circ} 44^{\prime} 47^{\prime \prime}$, In NE1/4 NE1/4 sec.11, T.70 N., R.43

W., Fremont County, on left pler on downstream side of highway bridge, 2.5

miles east of Bartlett, $3.5 \mathrm{miles}$ west of Tabor, and $3.6 \mathrm{~m} f$ les upstream from mouth.

Remarks.--D1scontinued Sept. 30, 1969.

LOW FLOW DISCHARGES, IN YEAR ENDING MARCH 31

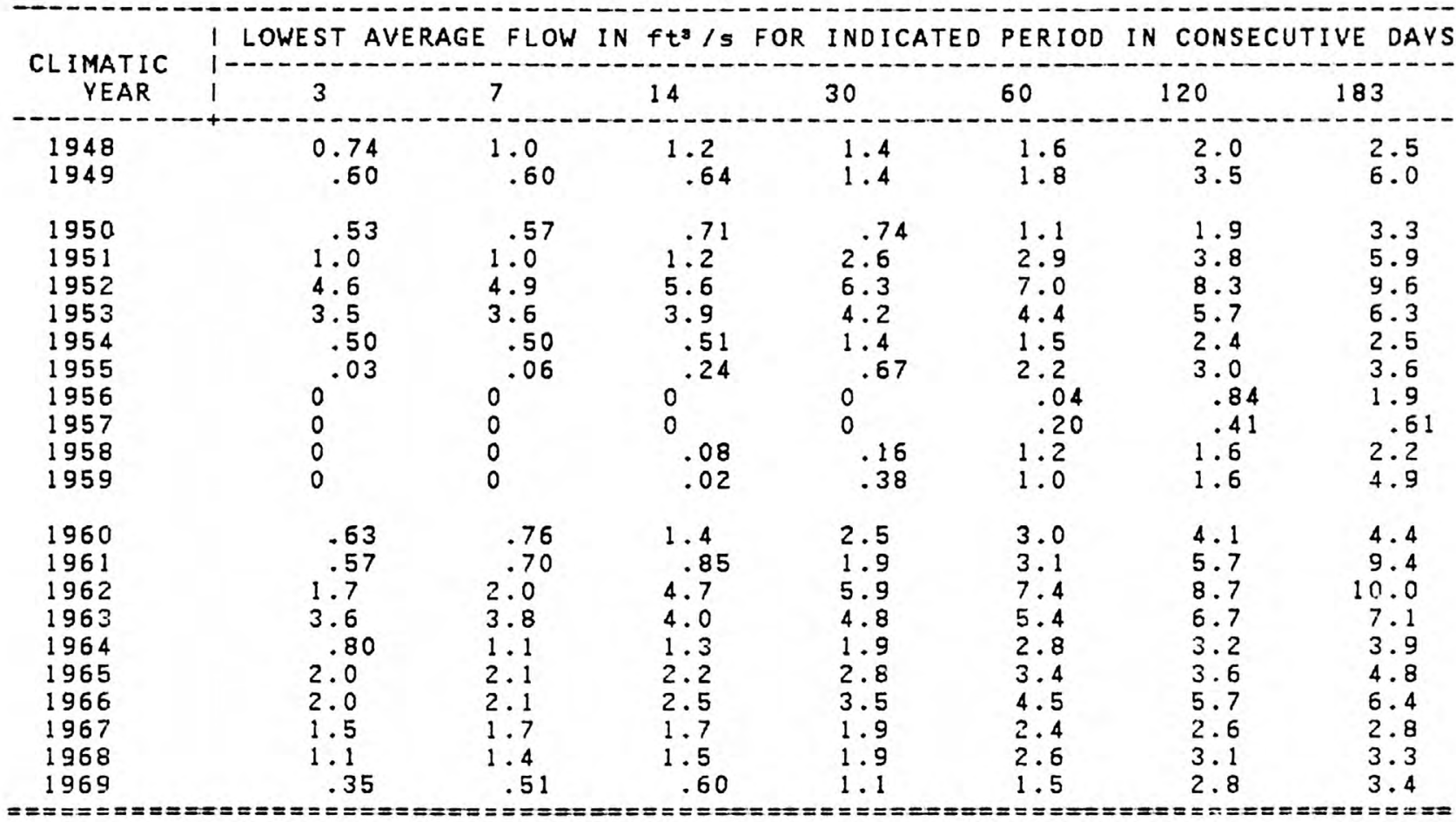


DRAINAGE AREA: $30.4 \mathrm{~m}^{2}$ PERIOD OF RECORD: 22 YEARS AVERAGE DISCHARGE: $11.6 \mathrm{ft}^{3} / \mathrm{s}$

MINIMUM AVERAGE FLOWS FOR PERIOD OF RECORD

\begin{tabular}{|c|c|c|c|c|c|c|c|}
\hline PERIOD OF CONSECUTIVE DAYS & 3 & 7 & 14 & 30 & 60 & 120 & 183 \\
\hline $\begin{array}{l}\text { RGE, IN } \mathrm{ft}^{2} / \mathrm{s} \\
\text { IC YEAR }\end{array}$ & $\begin{array}{l}1 \\
1 \\
1959\end{array}$ & $\begin{array}{c}0 \\
1959\end{array}$ & $\begin{array}{c}0 \\
1957\end{array}$ & $\underbrace{0}_{1957}$ & $\begin{array}{l}0.04 \\
1956 \\
=====\end{array}$ & $\begin{array}{l}0.42 \\
1957 \\
======\end{array}$ & $\begin{array}{l}.62 \\
57 \\
==\end{array}$ \\
\hline
\end{tabular}

MAGNITUDE AND FREQUENCY OF ANNUAL LOW FLOWS

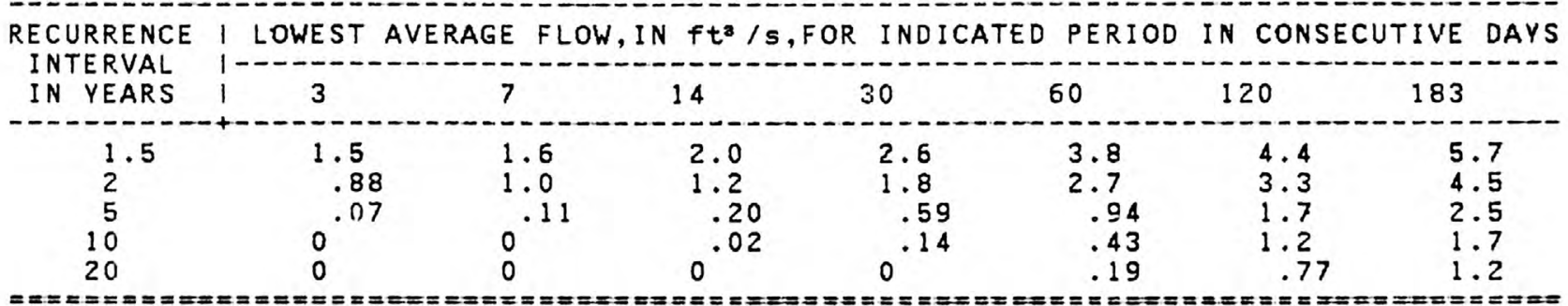

MAGNITUDE AND FREQUENCY OF SEASONAL LOW FLOWS

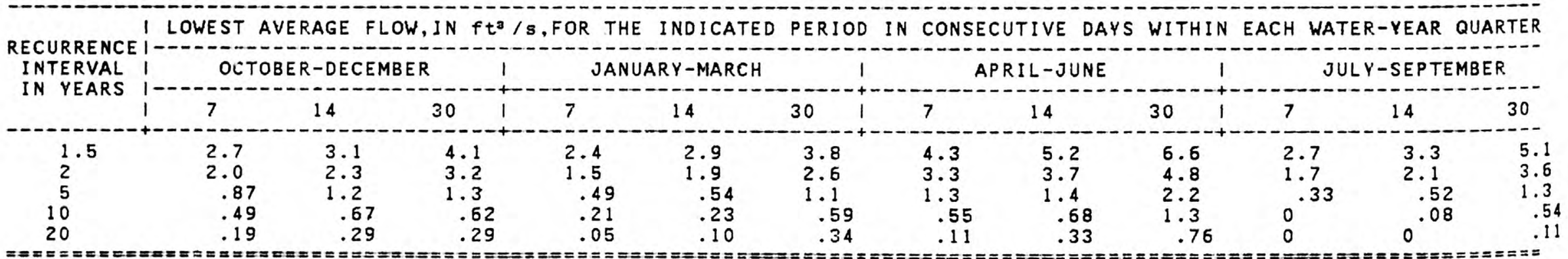

DURATION OF DAILY DISCHARGES FOR ANNUAL AND SEASONAL PERIODS

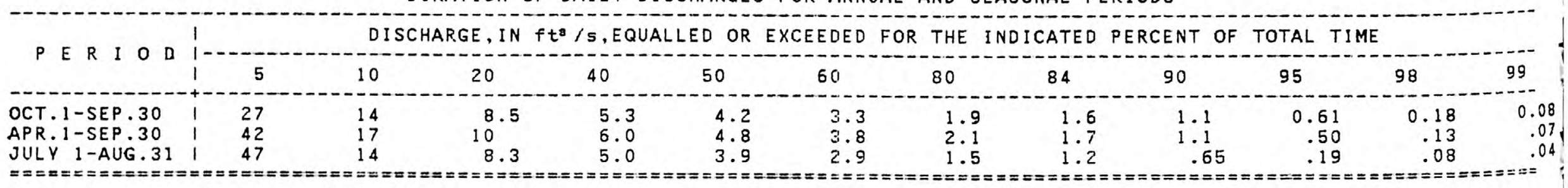




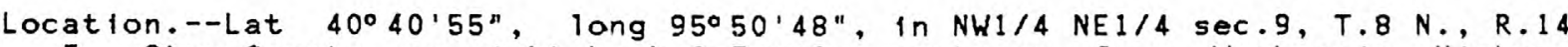
E., Oteo County, on right bank $0.7 \mathrm{mile}$ upstream from Waubonsie Highway Bridge at Nebraska City, and at mile 562.6.

Remarks.--Flow regulated by upstream main-stem reservoirs since Nov. 1937.

LOW FLOW DISCHARGES, IN YEAR ENDING MARCH 31

\begin{tabular}{|c|c|c|c|c|c|c|c|}
\hline \multirow{2}{*}{$\underset{\text { VEAR }}{C L, I M A T I C}$} & I LOWEST & \multicolumn{2}{|c|}{ GE FLOW IN $f t^{\mathrm{s}} / \mathrm{s}$} & $R$ INDICATED & PERIOD & \multicolumn{2}{|c|}{ IN CONSECUTIVE DAYS } \\
\hline & 3 & 7 & 14 & 30 & 60 & 120 & 183 \\
\hline $\begin{array}{l}1931 \\
1932 \\
1933 \\
1934 \\
1935 \\
1936 \\
1937\end{array}$ & $\begin{array}{l}9900 \\
8300 \\
3500 \\
4500 \\
4260 \\
5120 \\
3700\end{array}$ & $\begin{array}{r}10200 \\
8570 \\
3840 \\
4810 \\
4850 \\
5310 \\
4030\end{array}$ & $\begin{array}{r}11000 \\
8760 \\
4550 \\
5220 \\
6440 \\
5810 \\
4820\end{array}$ & $\begin{array}{l}12900 \\
10500 \\
7040 \\
7560 \\
7000 \\
6430 \\
6620\end{array}$ & $\begin{array}{r}14200 \\
11600 \\
10700 \\
12500 \\
7540 \\
7600 \\
7430\end{array}$ & $\begin{array}{l}19700 \\
13000 \\
12900 \\
14600 \\
10100 \\
9460 \\
9880\end{array}$ & $\begin{array}{l}21400 \\
12900 \\
15000 \\
17100 \\
10300 \\
10600 \\
11000\end{array}$ \\
\hline
\end{tabular}


DRAINAGE AREA: $414400 \mathrm{mi}^{2}$ PERIOD OF RECORD: 8 YEARS AVERAGE DISCHARGE: $27,000 \mathrm{ft}^{3} / \mathrm{s}$

MINIMUM AVERAGE FLOWS FOR PERIOD OF RECORD

\begin{tabular}{|c|c|c|c|c|c|c|c|}
\hline PERIOD OF CONSECUTIVE DAYS I & 3 & 7 & 14 & 30 & 60 & 120 & 183 \\
\hline $\begin{array}{l}\text { RGE;IN } f^{3} / s \\
\text { IC YEAR }\end{array}$ & $\begin{array}{l}3500 \\
1933\end{array}$ & $\begin{array}{l}3840 \\
1933\end{array}$ & $\begin{aligned} 4550 \\
1933\end{aligned}$ & $\begin{array}{l}6430 \\
1936\end{array}$ & $\begin{array}{r}7430 \\
1937\end{array}$ & $\begin{array}{l}9460 \\
1936\end{array}$ & $\begin{array}{l}10300 \\
1935\end{array}$ \\
\hline
\end{tabular}

MAGNITUDE AND FREQUENCY OF ANNUAL LOW FLOWS

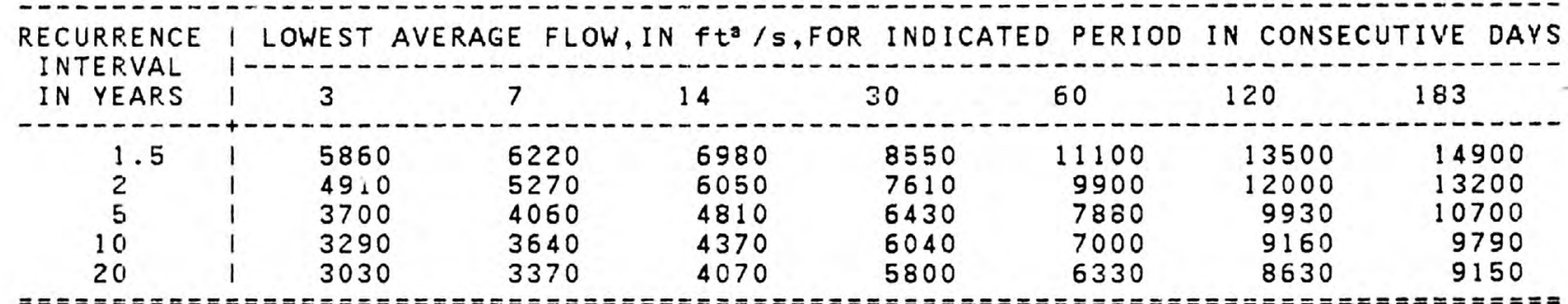

MAGNITUDE AND FREQUENCY OF SEASONAL LOW FLOWS

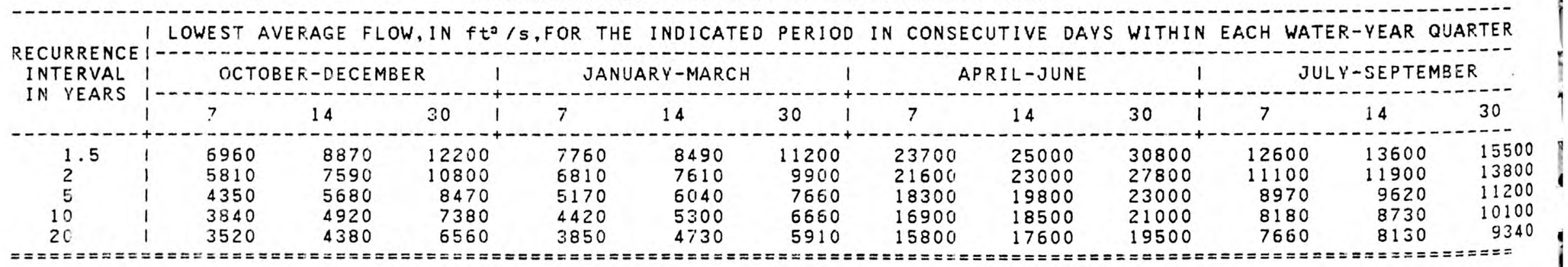

DURATION OF DAILY DISCHARGES FOR ANNUAL AND SEASONAL PERIODS

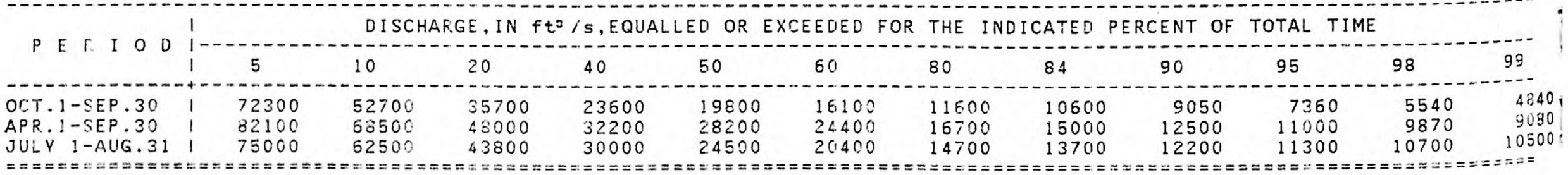


LOW FLOW DISCHARGES, IN YEAR ENDING MARCH 31

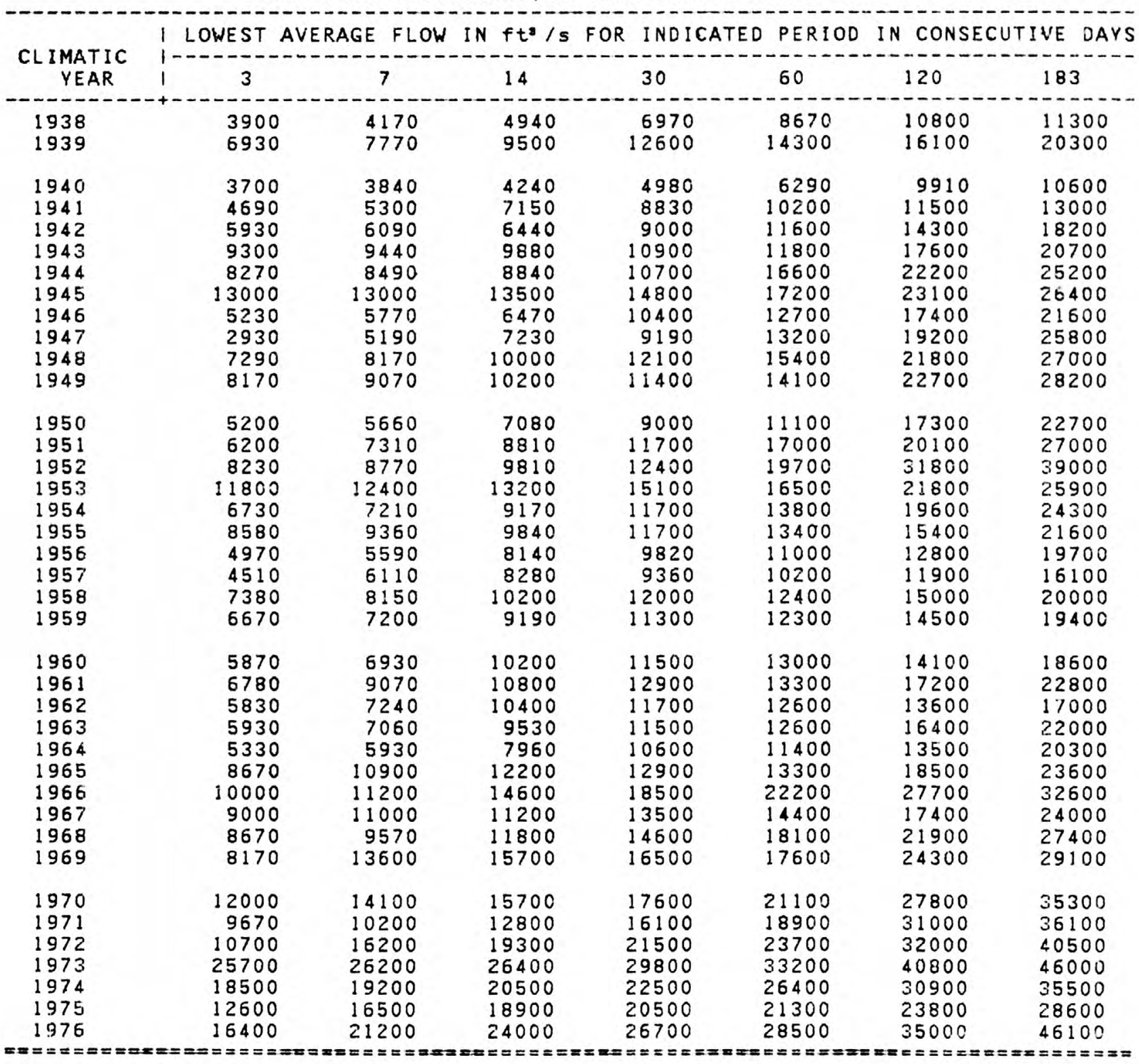


MINIMUM AVERAGE FLOWS FOR PERIOD OF RECORD

\begin{tabular}{|c|c|c|c|c|c|c|c|}
\hline PERIOD OF CONSECUTIVE DAYS & 3 & 7 & 14 & 30 & 60 & 120 & 183 \\
\hline $\begin{array}{l}\text { DISCHARGE, IN } \mathrm{ft}^{3} / \mathrm{s} \\
\text { CLIMATIC YEAR }\end{array}$ & $\begin{array}{l}2930 \\
1947\end{array}$ & $\begin{array}{l}3840 \\
1940\end{array}$ & $\begin{array}{l}4240 \\
1940\end{array}$ & $\begin{array}{l}4980 \\
1940\end{array}$ & $\begin{array}{l}6290 \\
1940\end{array}$ & $\begin{array}{l}9910 \\
1940\end{array}$ & $\begin{array}{l}10600 \\
1940\end{array}$ \\
\hline
\end{tabular}

MAGNITUDE AND FREQUENCY OF ANNUAL LOW FLOWS

\begin{tabular}{|c|c|c|c|c|c|c|c|c|}
\hline $\begin{array}{l}\text { ECURRENCE } \\
\text { INTERVAL }\end{array}$ & 1 & WEST & GE & $\begin{array}{r}f t^{3} \\
--n-1\end{array}$ & $1 N 01$ & PERIS & $\begin{array}{l}-- \\
14 \\
-\end{array}$ & IVE 0 \\
\hline IN YEARS & 1 & 3 & 7 & 14 & 30 & 60 & 120 & 183 \\
\hline $\begin{array}{l}1.5 \\
2 \\
5 \\
10 \\
20\end{array}$ & $\begin{array}{l}1 \\
1 \\
1 \\
1 \\
1\end{array}$ & $\begin{array}{l}8980 \\
7380 \\
5210 \\
4420 \\
3890\end{array}$ & $\begin{array}{r}10300 \\
8530 \\
6070 \\
5180 \\
4590\end{array}$ & $\begin{array}{r}12300 \\
10300 \\
7510 \\
6420 \\
5660\end{array}$ & $\begin{array}{r}14500 \\
12500 \\
9400 \\
8180 \\
7300\end{array}$ & $\begin{array}{r}16900 \\
14700 \\
11200 \\
9780 \\
8780\end{array}$ & $\begin{array}{l}22000 \\
18900 \\
14300 \\
12400 \\
11100\end{array}$ & $\begin{array}{l}28100 \\
24400 \\
18200 \\
15500 \\
13500\end{array}$ \\
\hline
\end{tabular}

MAGNITUDE AND FREQUENCY OF SEASONAL LOW FLOWS

LOWEST AVERAGE FLOW, IN $\mathrm{ft}^{\mathrm{a}} / \mathrm{s}, \mathrm{FOR}$ THE INDICATED PERIOD IN CONSECUTIVE DAYS WITHIN EACH WATER-YEAR QUARTER RECURRENCE IINTERVAL
IN YEARS

7

$13200 \quad 14800 \quad 18200$

$12400 \quad 14200$

16800

$7 \quad 14$

30

364

$\begin{array}{rr}10500 & 1220 \\ 7060 & 855\end{array}$

15300

9010

14400

37300

38800

40300

36000

30100

10800
9260

$25900 \quad 27100$

23900

$\begin{array}{ll}27900 & 19500 \\ 26500 & 16200\end{array}$

$\begin{array}{ll}5830 & 7310 \\ 5020 & 6410\end{array}$

8940

5530
5740

6760

8190

22800
20500

21700

16200

OURATION OF DAILY DISCHARGES FOR ANNUAL AND SEASONAL PERIODS

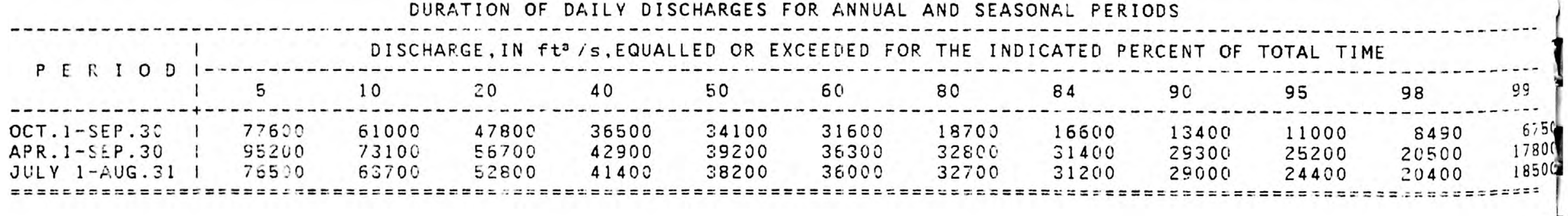




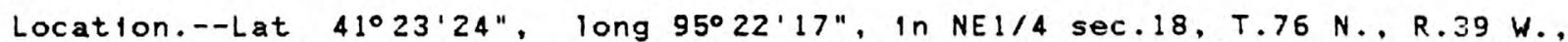
Pottawattamie County, on downstream end of right pler of bridge on county highway G30,0.6 mile west of Hancock school, and $3.0 \mathrm{miles}$ downstream from Jim Creek.

LOW FLOW DISCHARGES, IN YEAR ENDING MARCH 31

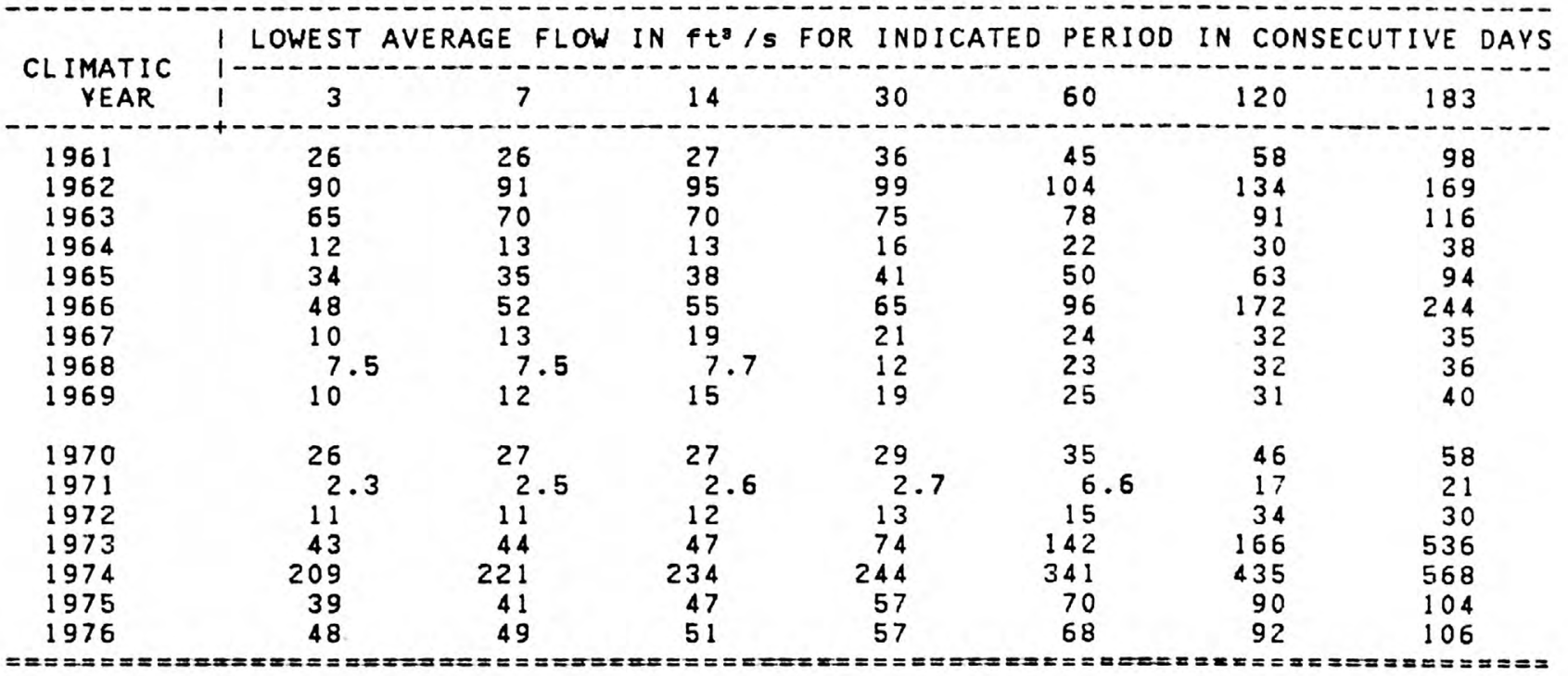




DRAINAGE AREA: $609 \mathrm{~m}^{2} \quad$ PERIOD OF RECORD: 17 YEARS AVERAGE DISCHARGE: $274 \mathrm{ft} / \mathrm{s}$
PINIMUM AVERAGE FLOWS FOR PERIOD OF RECORD
PERIOD OF CONSECUTIVE DAYS I

MAGNITUDE AND FREQUENCY OF ANNUAL LOW FLOWS

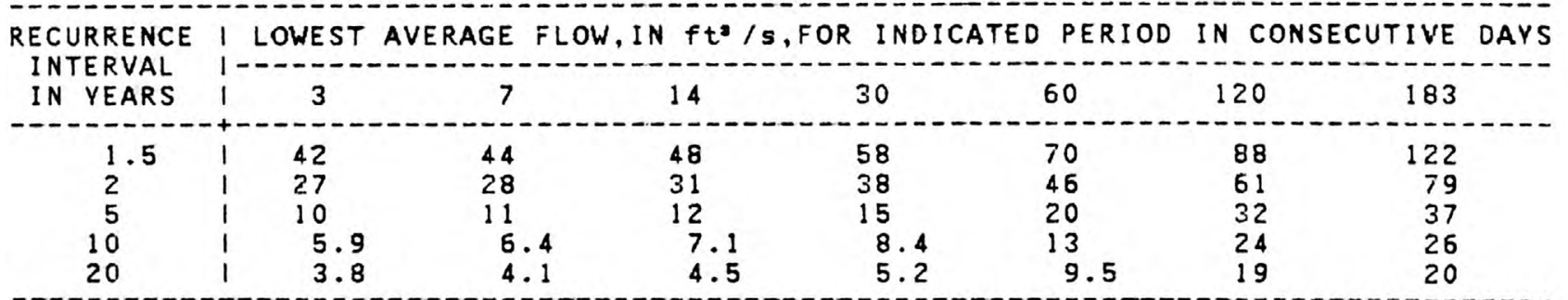

MAGNITUDE AND FREQUENCY OF SEASONAL LOW FLOWS

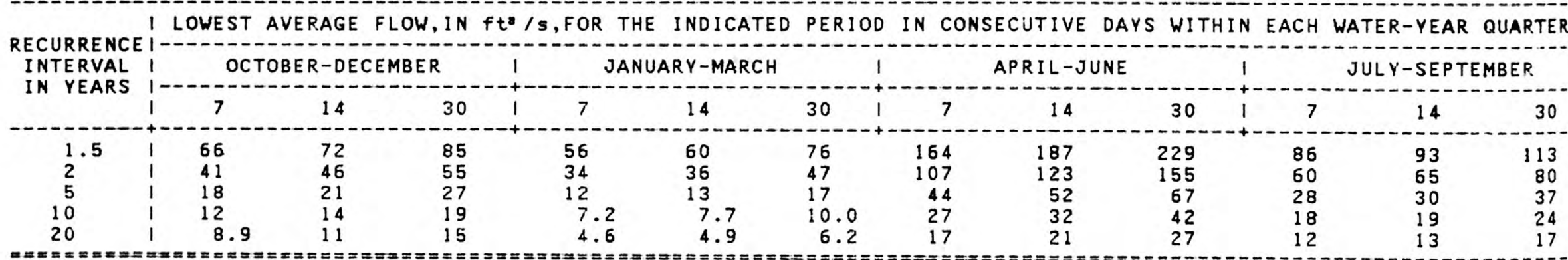

DURATION OF DAILY DISCHARGES FOR ANNUAL AND SEASONAL PERIODS

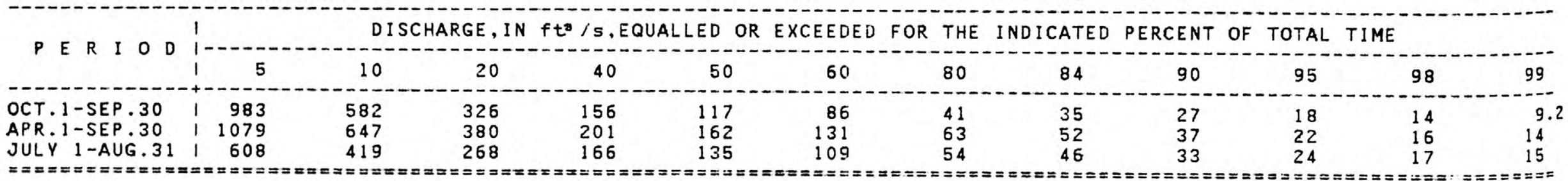


Location.--Lat 40056'36", long 95035'42", In NE1/4 NE1/4 sec.19, T.71 N.. R.41 W., Mills County, on right bank $170 \mathrm{ft}$ upstream from culvert on county highway L63, $0.2 \mathrm{mlle}$ downstream from unnamed tributary, $1.8 \mathrm{~m} 1 \mathrm{les}$ upstream from mouth, and $4.3 \mathrm{mll}$ es south of Malvern.

Remarks.--Discontinued Sept. 30, 1969.

LOW FLOW DISCHARGES, IN YEAR ENDING MARCH 31

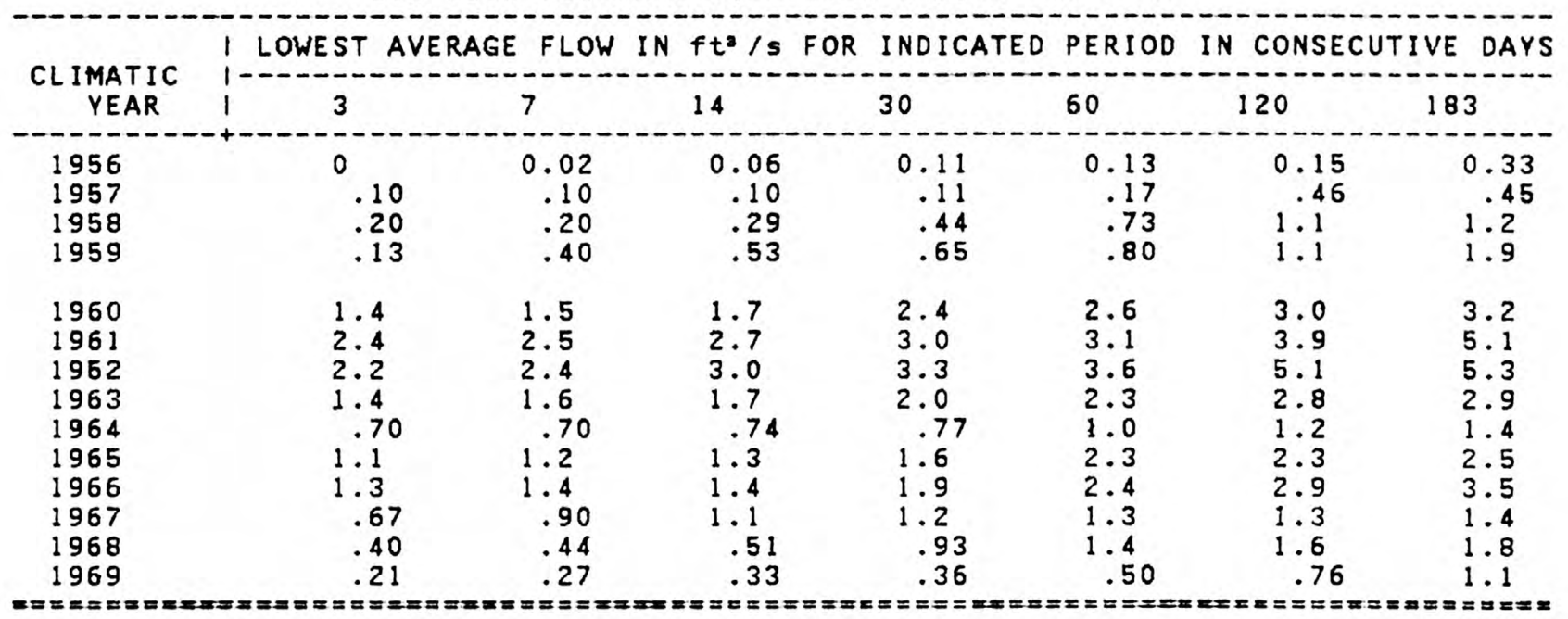




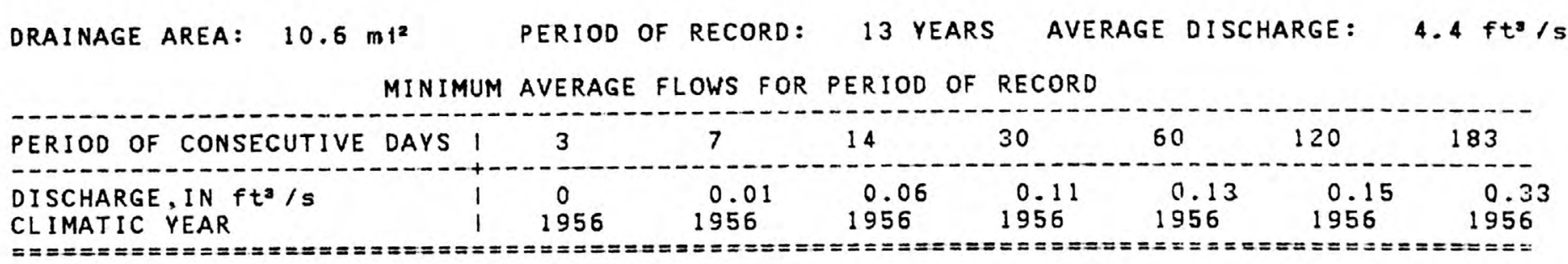

MAGNITUDE AND FREQUENCY OF ANNUAL LOW FLOWS

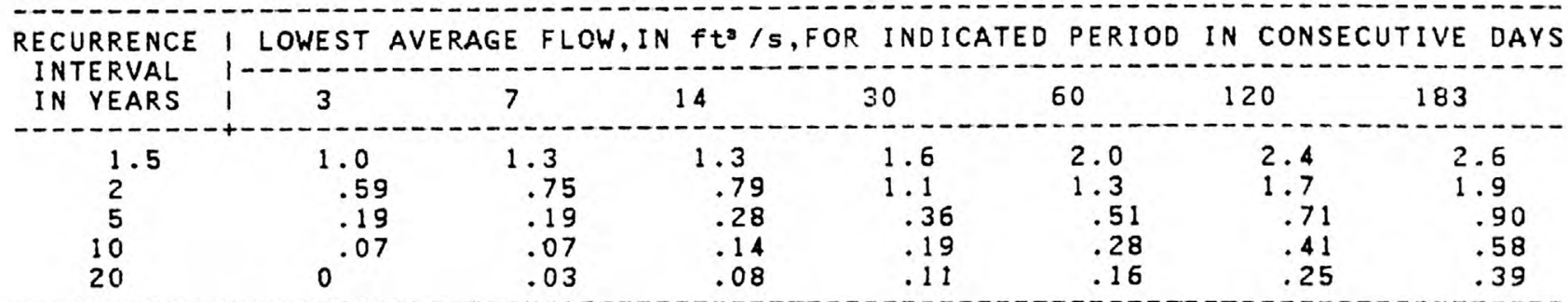

MAGNITUDE AND FREQUENCY OF SEASONAL LOW FLOWS

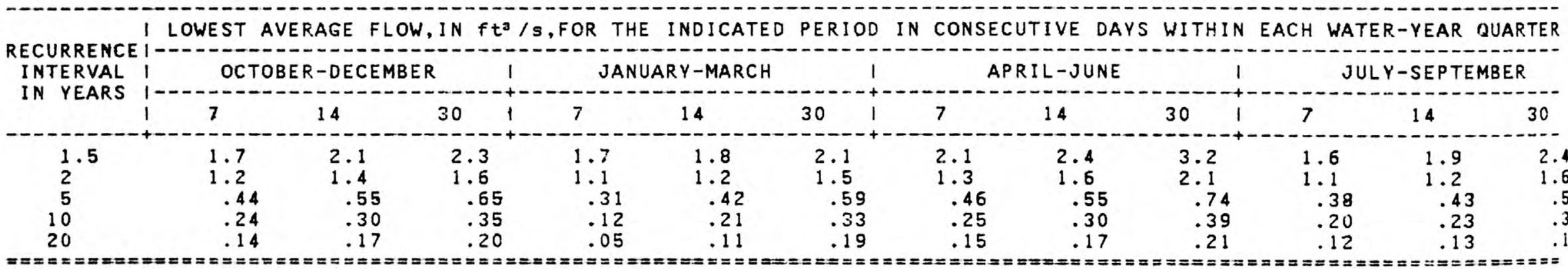

DURATION OF DAILY DISCHARGES FOR ANNUAL AND SEASONAL PERIOOS

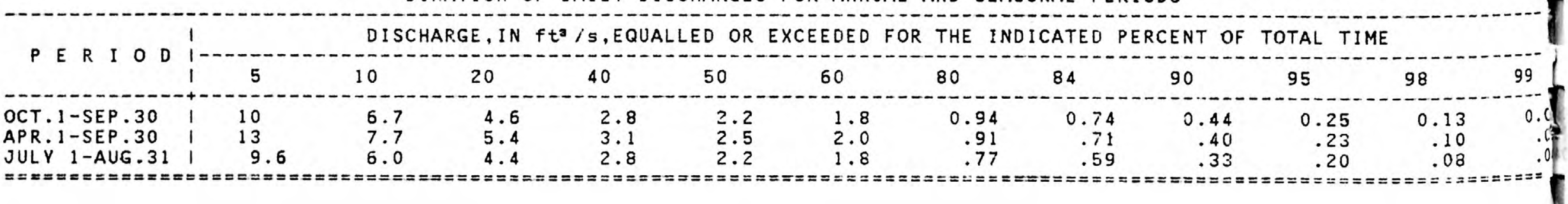


Location.--Lat 40054'35", long 95036'00", In SW1/4 NE1/4 sec.31, T.71 N., R.41

W., Mills County, on left bank $20 \mathrm{ft}$ downstream from hlghway bridge, 1.5 miles upstream from mouth and $4.0 \mathrm{miles}$ northeast of Tabor.

Remarks.--Discontinued Sept. 30, 1964.

Drainage area. $--7.65 \mathrm{mi}^{2}$.

LOW FLOW DISCHARGES, IN YEAR ENDING MARCH 31

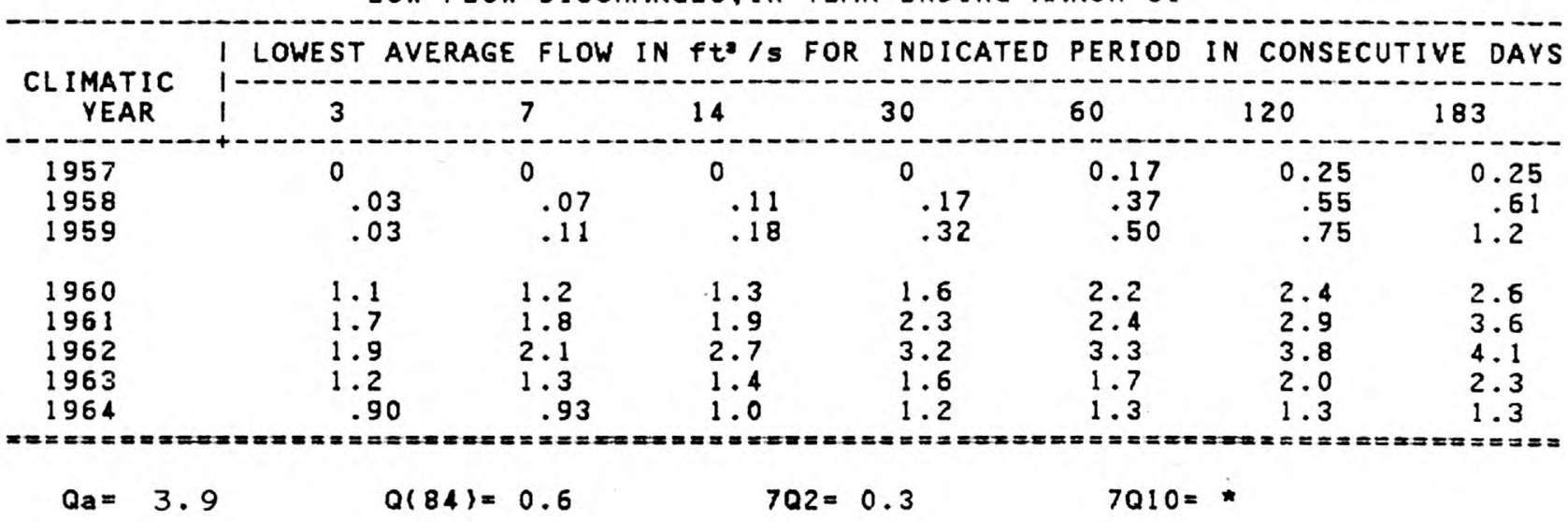


Location.--Lat $40^{\circ} 52^{\prime} 23^{\prime \prime}$, long $95^{\circ} 34^{\prime} 48^{\prime \prime}$, In NE1/4 NE1/4 sec.17. T.70 N., R.41 W. Fremont county, on right bank $30 \mathrm{ft}$ upstream from bridge on State Highway $134,0.3 \mathrm{mlle}$ downstream from Deer Creek, 0.5 mire west of Randolph, and 16.2 miles upstream from confluence with East Nishnabotna River.

LOW FLOW DISCHARGES, IN YEAR ENDING MARCH 31

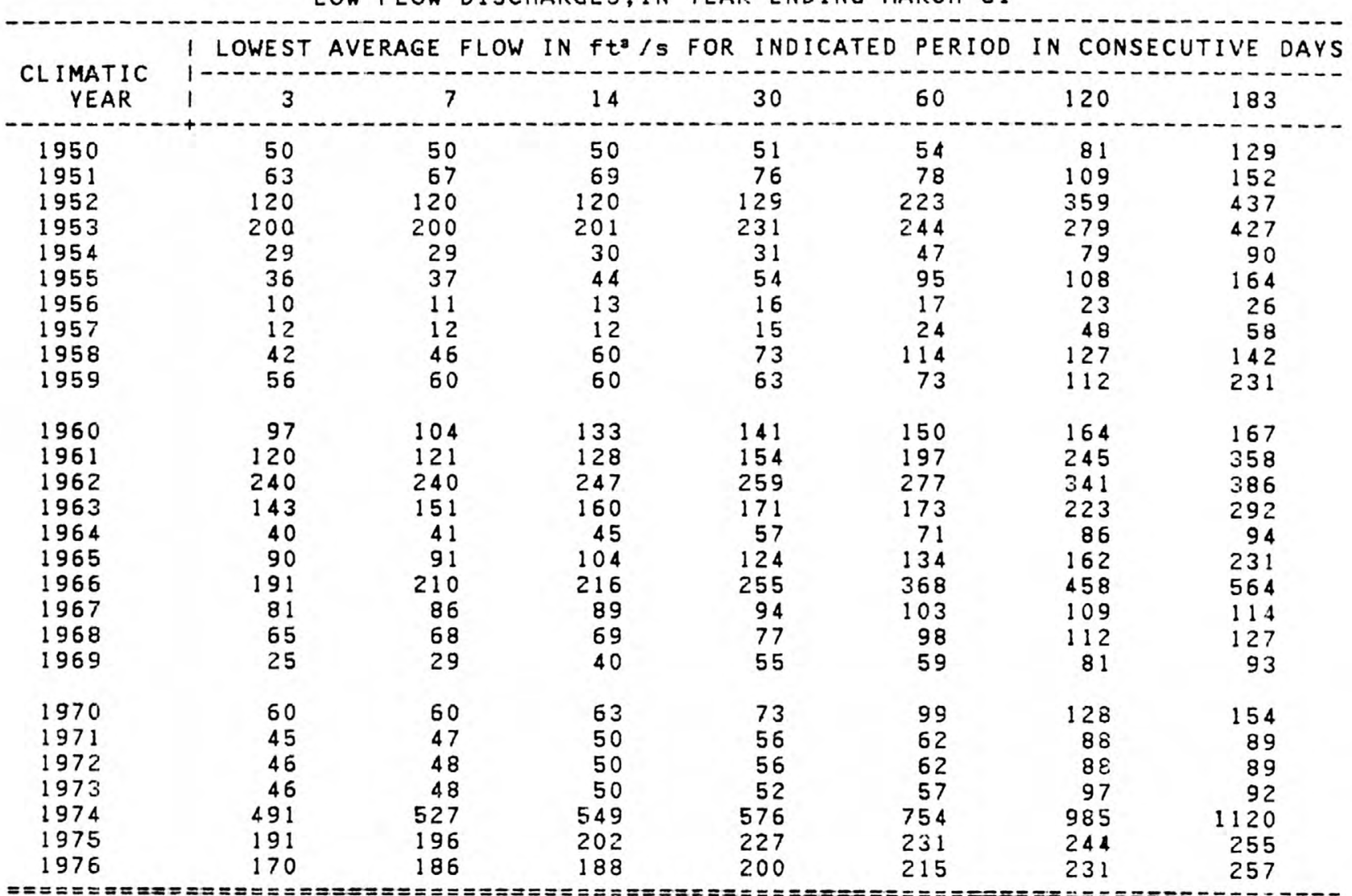


06-8035.00 WEST NISHNABOTNA RIVER AT RANDOLPH--Cont inued

\section{DRAINAGE AREA: $1326 \mathrm{~m}^{2}$ PERIOD OF RECORD: 28 VEARS AVERAGE DISCHARGE: $545 \mathrm{ft} / \mathrm{s}$} MINIMUM AVERAGE FLOWS FOR PERIOD OF RECORD

\begin{tabular}{|c|c|c|c|c|c|c|c|}
\hline PERIOD OF CONSECUTIVE DAYS I & 3 & 7 & 14 & 30 & 60 & 120 & 183 \\
\hline 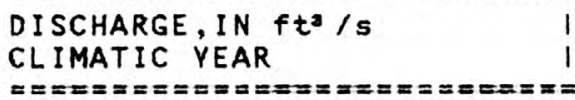 & $\begin{array}{ll}1 & 10 \\
1 & 1956 \\
=======\end{array}$ & $\begin{array}{l}11 \\
1956 \\
===x=\end{array}$ & $\begin{array}{l}12 \\
1957 \\
=====\end{array}$ & $\begin{array}{l}15 \\
1957 \\
=====x\end{array}$ & $\begin{array}{l}17 \\
1956 \\
x===\end{array}$ & $\begin{array}{l}23 \\
1956 \\
== \pm==\end{array}$ & $\begin{array}{l}26 \\
1956 \\
=====\end{array}$ \\
\hline
\end{tabular}

MAGNITUDE AND FREQUENCY OF ANNUAL LOW FLOWS

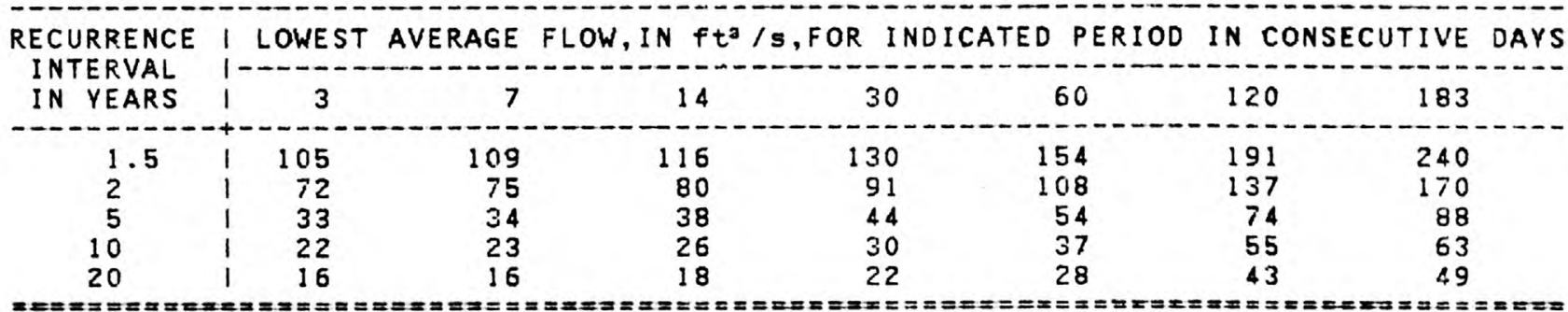

MAGNITUDE AND FREQUENCY OF SEASONAL LOW FLOWS

I LOWEST AVERAGE FLOW, IN $\mathrm{ft}^{\mathrm{s}} / \mathrm{s}, F O R$ THE INDICATED PERIOD IN CONSECUTIVE DAYS WITHIN EACH WATER-YEAR QUARTER

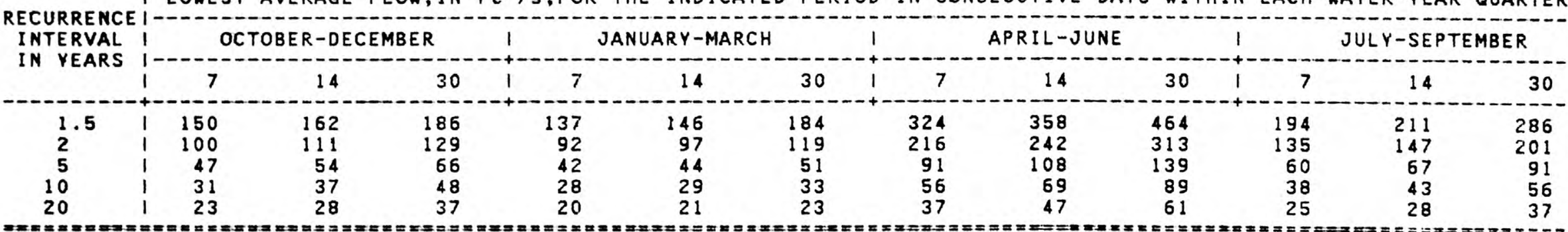

DURATION OF DAILY DISCHARGES FOR ANNUAL AND SEASONAL PERIODS

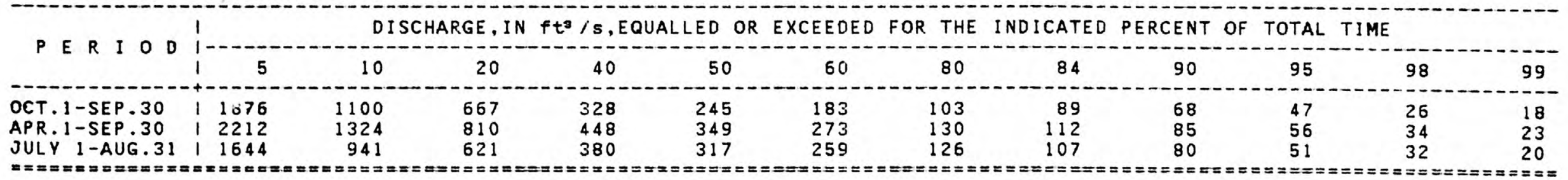




\section{DAVIDS CREEK NEAR HAMLIN}

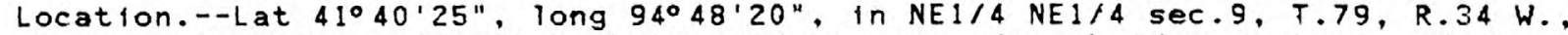
Audubon County, on left bank $20 \mathrm{ft}$ downstream from bridge on State Highway $64,5.2$ miles east of Hamlin, and $8 \mathrm{mll}$ les upstream from mouth.

Remarks.--01scontinued Sept. 30, 1973.

LOW FLOW DISCHARGES, IN YEAR. ENDING MARCH 31

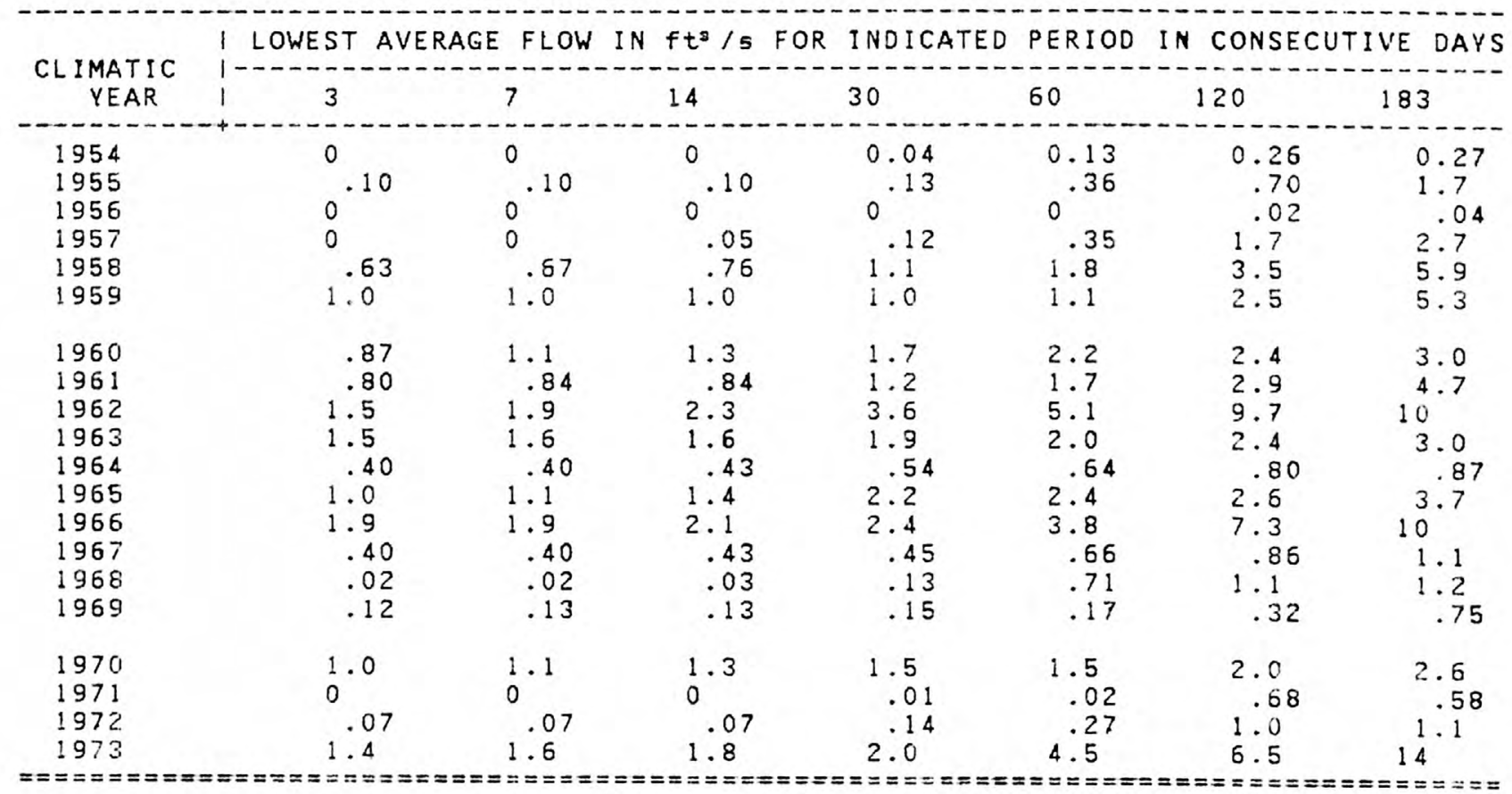




\section{DRAINAGE AREA: $26.0 \mathrm{~m}^{2}$ PERIOD OF RECORD: 21 YEARS AVERAGE DISCHARGE: 11.1 ft? /s}

MINIMUM AVERAGE FLOWS FOR PERIOD OF RECORD

\begin{tabular}{|c|c|c|c|c|c|c|c|}
\hline ERIOD OF CONSECUTIVE DAYS & 3 & 7 & 14 & 30 & 60 & 120 & 183 \\
\hline $\begin{array}{l}\text { RGE, IN fts } / s \\
\text { IC YEAR }\end{array}$ & $\begin{array}{c}0 \\
1971\end{array}$ & $\begin{array}{l}0 \\
1971\end{array}$ & $\begin{array}{l}0 \\
1971\end{array}$ & $\begin{array}{l}0 \\
1956\end{array}$ & $\begin{array}{l}0 \\
1956\end{array}$ & $\begin{array}{l}0.02 \\
1956\end{array}$ & $\begin{array}{l}0.04 \\
1956\end{array}$ \\
\hline
\end{tabular}

MAGNITUDE AND FREQUENCY OF ANNUAL LOW FLOWS

RECURRENCE
INTERVAL
IN YEARS
IN

MAGNITUDE AND FREQUENCY OF SEASONAL LOW FLOWS

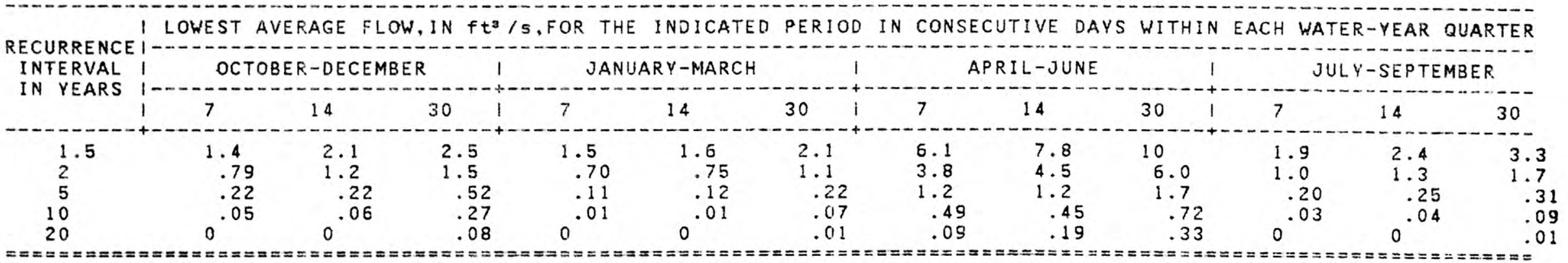

DURATION OF DAILY DISCHARGES FOR ANNUAL AND SEASONAL PERIODS

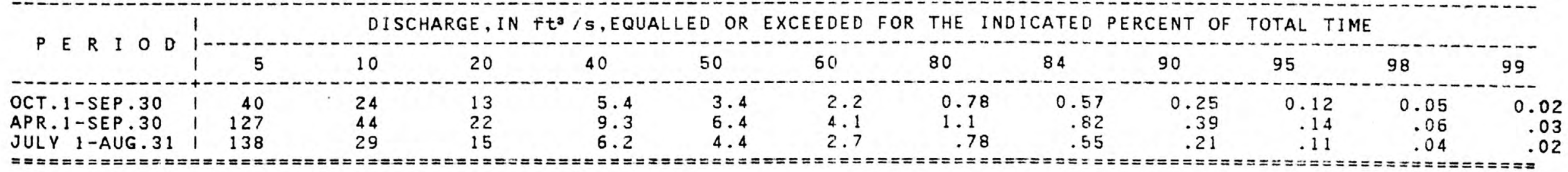


Location.--Lat $37^{\circ} 41^{\circ} 20^{\prime} 37^{\prime \prime}$, long $95^{\circ} 04^{\prime 3} 31^{\prime \prime}$, In NW1/4 NW1/4 sec.35, T.76 N., R.67 W., Cass County, on left bank at downstream side of bridge on county hlghway, 1.9 miles upstream from Turkey Creek, and $5.4 \mathrm{mlles}$ southwest of junction of U.S. Highway 6 and State Highway 83 in Atlantic.

LOW FLOW DISCHARGES, IN YEAR. ENDING MARCH 31

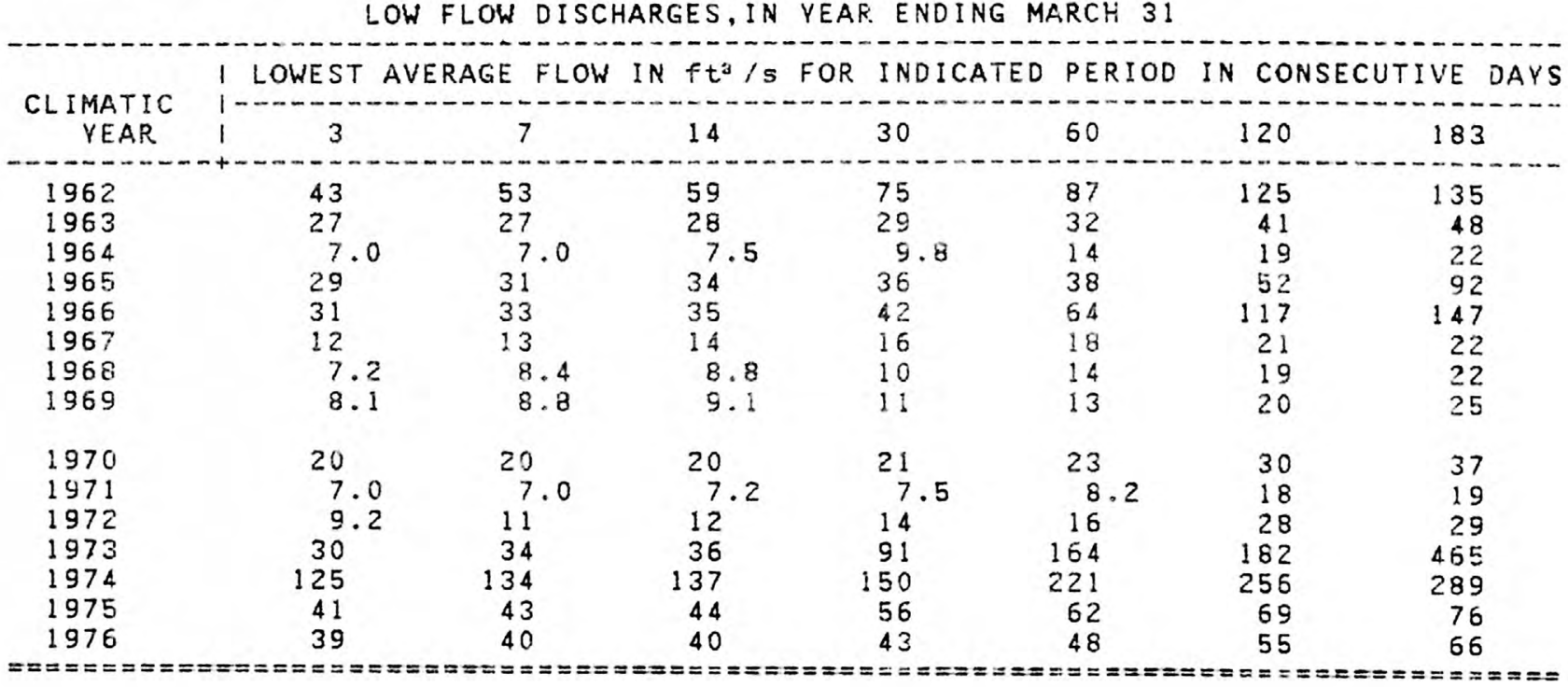




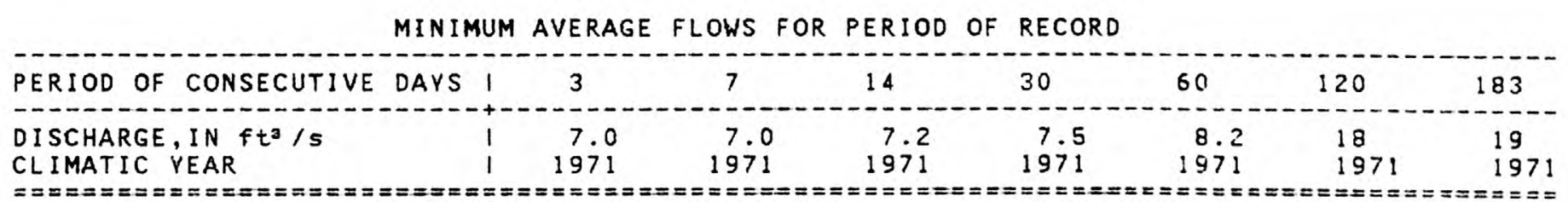

MAGNITUDE AND FREQUENCY OF ANNUAL LOW FLOWS

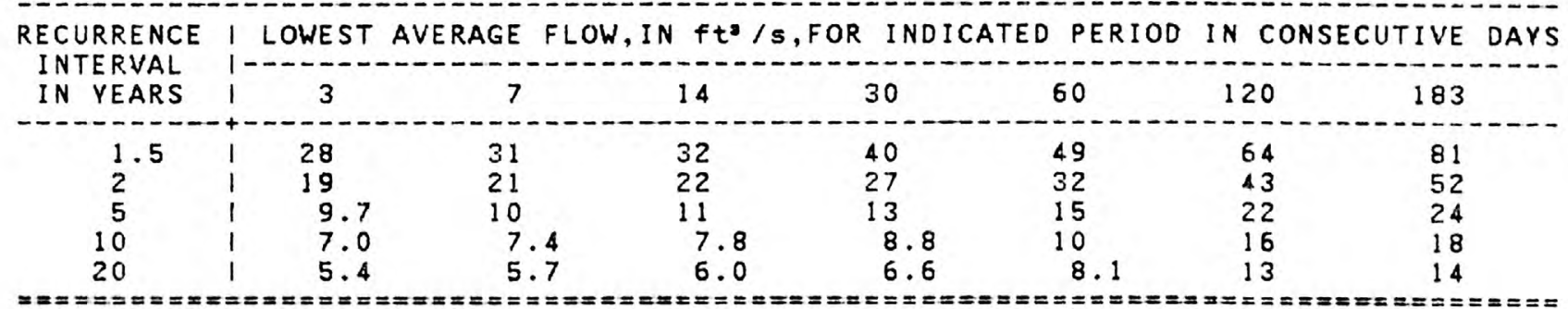

MAGNITUDE AND FREQUENCY OF SEASONAL LOW FLOWS

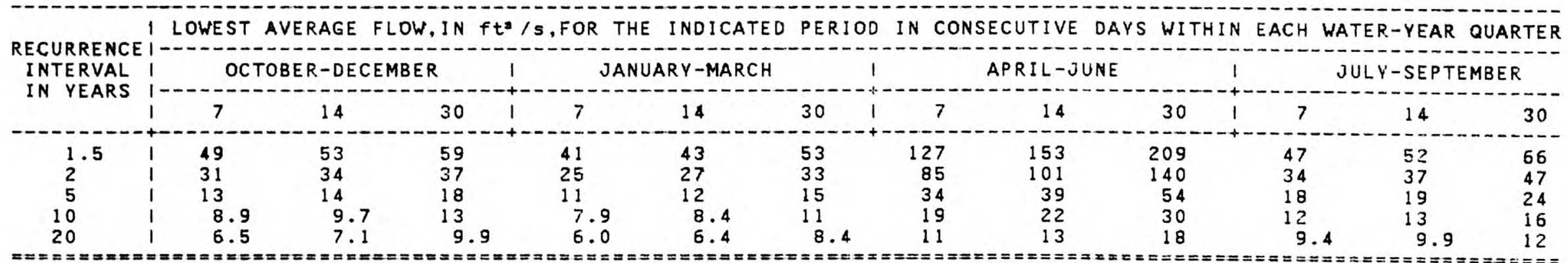

DURATION OF DAILY DISCHARGES FOR ANNUAL AND SEASONAL PERIODS

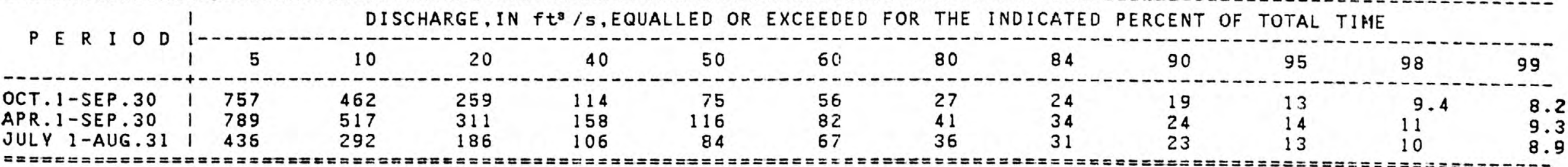


Location.--Lat 41000'41", long $95^{\circ} 14^{\prime} 07^{n}$, In NW1/4 SE1/4 sec.29, T.72 N., R.38 W. Montgomery County, on left bank on downstream side of Coolbaugh Street bridge in Red Oak. and $0.2 \mathrm{~m} t$ le upstream from Red Oak Creek.

LOW FLOW DISCHARGES, IN YEAR. ENDING MARCH 31

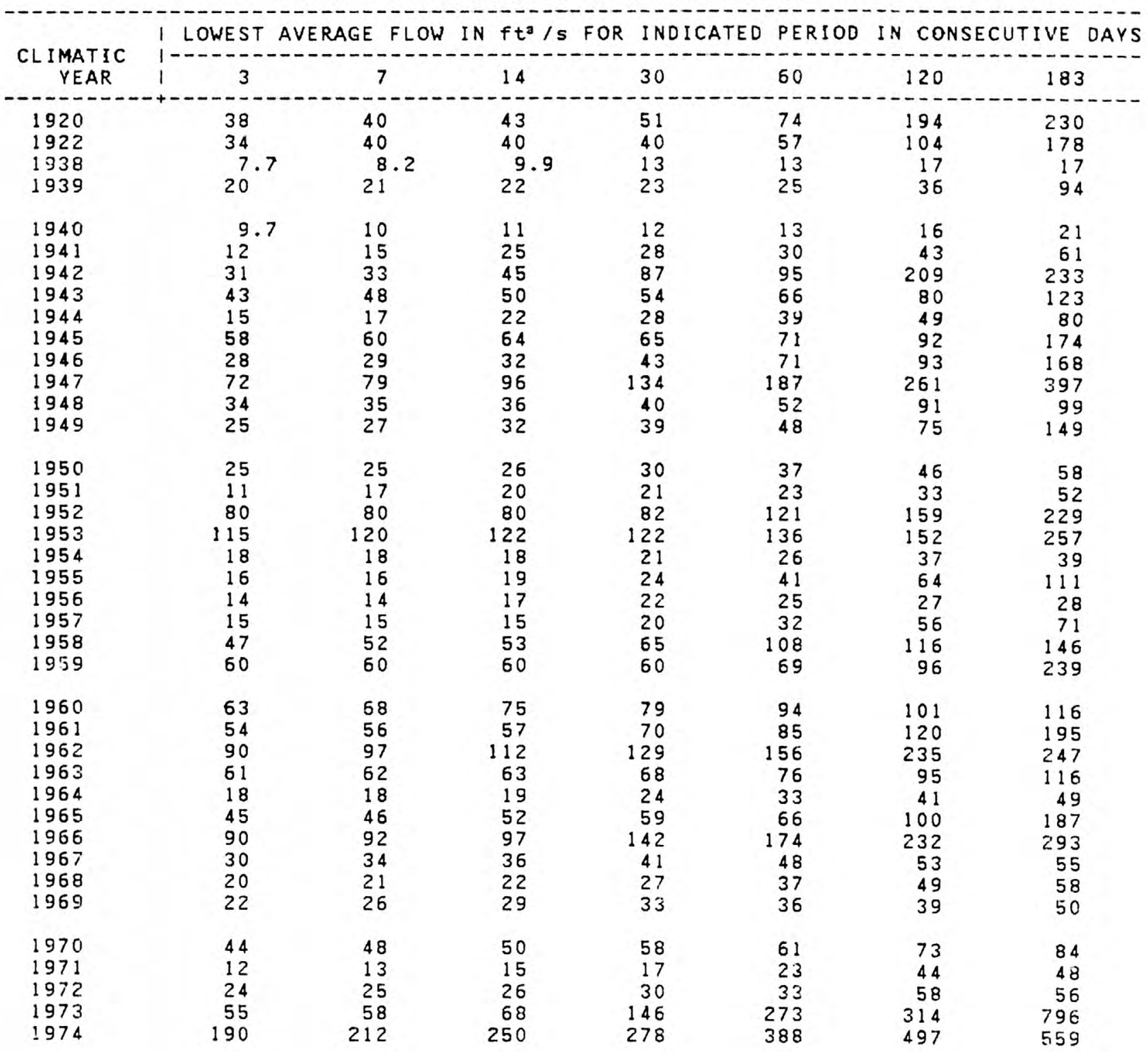


LOW FLOW DISCHARGES, IN YEAR ENDING MARCH 31--Continued

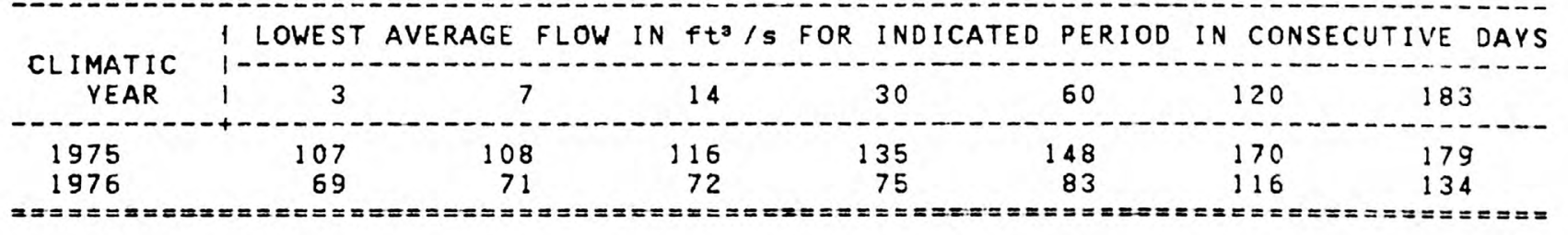




DRAINAGE AREA: $894 \mathrm{~m} \mathbf{1}^{2}$
MINIMUM AVERAGE FLOWS FOR PERIOD OF RECORD

MAGNITUDE AND FREQUENCY OF ANNUAL LOW FLOWS

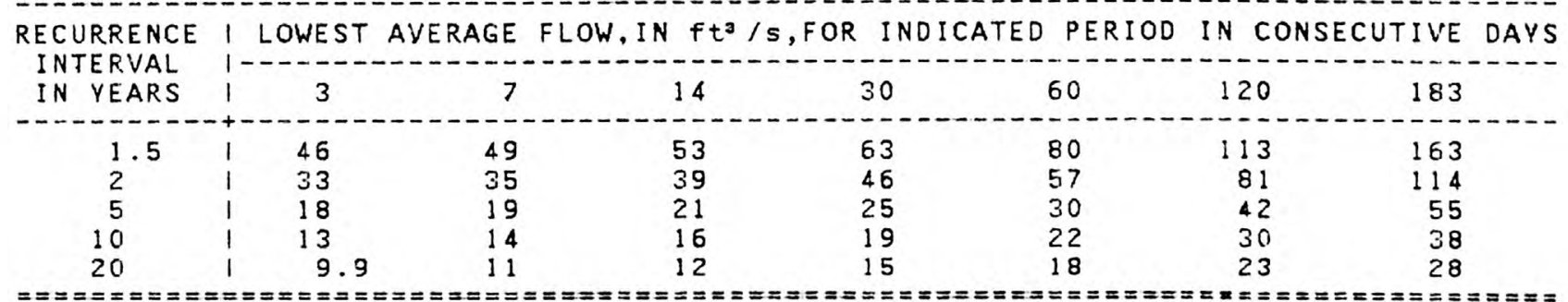

MAGNITUDE AND FREQUENCY OF SEASONAL LOW FLOWS

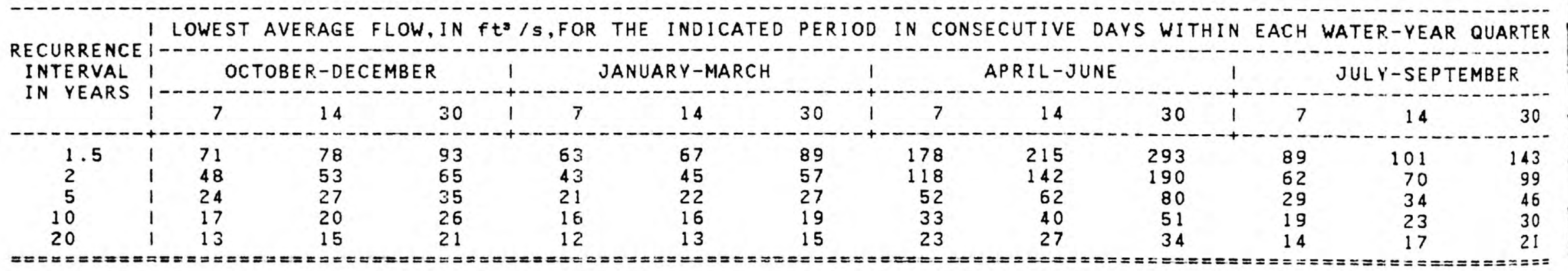

DURATION OF DAILY DISCHARGES FOR ANNUAL ANO SEASONAL PERIODS

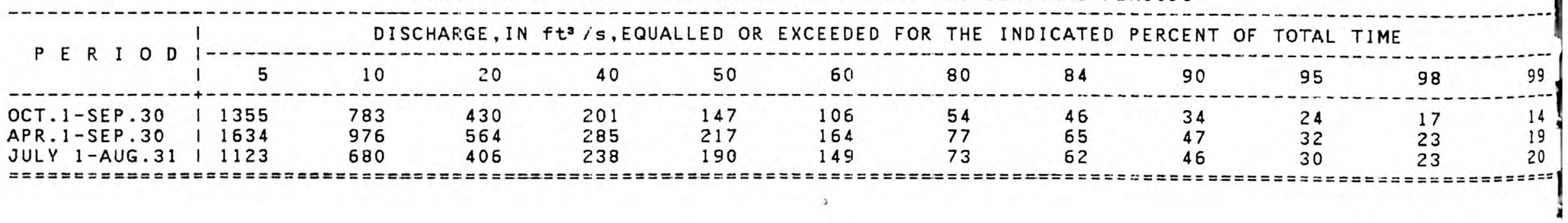


Location.--Lat $40^{\circ} 37^{\prime} 57^{\prime \prime}$, long $95^{\circ} 37^{\prime} 32^{\prime \prime}$, in SW1/4 SE1/4 sec.11, T.67 N., R.42

W. Fremont County, on left bank $1.6 \mathrm{~m}$ lites downstream from confluence of East Nishnabotna and West Nishnabotna Rivers, 2 miles northeast of Hamburg, and at $m i l e 13.2$.

LOW FLOW DISCHARGES, IN YEAR. ENDING MARCH 31

\begin{tabular}{|c|c|c|c|c|c|c|c|}
\hline \multirow{2}{*}{$\underset{\text { YEAR }}{\text { CLIMATIC }}$} & \multicolumn{2}{|c|}{ I LOWEST AVERAGE FL } & $N+t^{3} /$ & R INDICATED & PERIOD & \multicolumn{2}{|c|}{ IN CONSECUTIVE } \\
\hline & 13 & 7 & 14 & 30 & 60 & 120 & 183 \\
\hline $\begin{array}{l}1923 \\
1930 \\
1931 \\
1932 \\
1933 \\
1934 \\
1935 \\
1936 \\
1937 \\
1938 \\
1939\end{array}$ & $\begin{array}{r}165 \\
224 \\
54 \\
72 \\
193 \\
42 \\
7.2 \\
11 \\
13 \\
16 \\
41\end{array}$ & $\begin{aligned} 166 \\
229 \\
56 \\
74 \\
193 \\
49 \\
10.0 \\
13 \\
17 \\
17 \\
48\end{aligned}$ & $\begin{array}{r}172 \\
231 \\
69 \\
91 \\
207 \\
82 \\
14 \\
19 \\
19 \\
19 \\
59\end{array}$ & $\begin{array}{r}191 \\
253 \\
76 \\
110 \\
320 \\
106 \\
15 \\
38 \\
25 \\
24 \\
65\end{array}$ & $\begin{array}{r}235 \\
316 \\
98 \\
165 \\
407 \\
116 \\
36 \\
64 \\
40 \\
28 \\
68\end{array}$ & $\begin{array}{r}273 \\
426 \\
112 \\
559 \\
529 \\
145 \\
144 \\
143 \\
100 \\
35 \\
90\end{array}$ & $\begin{array}{l}319 \\
396 \\
113 \\
551 \\
538 \\
165 \\
159 \\
132 \\
342 \\
40 \\
329\end{array}$ \\
\hline $\begin{array}{l}1940 \\
1941 \\
1942 \\
1943 \\
1944 \\
1945 \\
1946 \\
1947 \\
1948 \\
1949\end{array}$ & $\begin{array}{r}15 \\
52 \\
59 \\
163 \\
47 \\
97 \\
222 \\
329 \\
133 \\
118\end{array}$ & $\begin{array}{r}15 \\
64 \\
64 \\
170 \\
57 \\
113 \\
225 \\
339 \\
139 \\
126\end{array}$ & $\begin{array}{r}17 \\
74 \\
97 \\
172 \\
79 \\
149 \\
235 \\
372 \\
150 \\
130\end{array}$ & $\begin{array}{r}20 \\
83 \\
137 \\
185 \\
82 \\
184 \\
276 \\
458 \\
164 \\
148\end{array}$ & $\begin{array}{r}25 \\
94 \\
198 \\
216 \\
109 \\
215 \\
319 \\
508 \\
260 \\
179\end{array}$ & $\begin{array}{r}37 \\
123 \\
776 \\
259 \\
127 \\
270 \\
386 \\
707 \\
336 \\
261\end{array}$ & $\begin{array}{r}47 \\
168 \\
854 \\
419 \\
191 \\
432 \\
690 \\
948 \\
367 \\
453\end{array}$ \\
\hline $\begin{array}{l}1950 \\
1951 \\
1952 \\
1953 \\
1954 \\
1955 \\
1956 \\
1957 \\
1958 \\
1959\end{array}$ & $\begin{array}{r}80 \\
150 \\
230 \\
285 \\
48 \\
59 \\
23 \\
27 \\
62 \\
170\end{array}$ & $\begin{array}{r}90 \\
150 \\
230 \\
380 \\
54 \\
72 \\
23 \\
27 \\
82 \\
171\end{array}$ & $\begin{array}{r}100 \\
151 \\
236 \\
384 \\
57 \\
82 \\
28 \\
27 \\
98 \\
174\end{array}$ & $\begin{array}{r}107 \\
158 \\
269 \\
403 \\
64 \\
98 \\
39 \\
36 \\
141 \\
175\end{array}$ & $\begin{array}{r}153 \\
168 \\
498 \\
465 \\
80 \\
163 \\
46 \\
62 \\
253 \\
190\end{array}$ & $\begin{array}{r}186 \\
226 \\
701 \\
488 \\
131 \\
209 \\
53 \\
118 \\
290 \\
266\end{array}$ & $\begin{array}{r}232 \\
322 \\
858 \\
754 \\
149 \\
337 \\
59 \\
140 \\
331 \\
601\end{array}$ \\
\hline $\begin{array}{l}1960 \\
1961 \\
1962 \\
1963 \\
1964 \\
1965 \\
1966 \\
1967\end{array}$ & $\begin{array}{l}240 \\
240 \\
420 \\
250 \\
77 \\
210 \\
417 \\
135\end{array}$ & $\begin{array}{r}291 \\
241 \\
505 \\
300 \\
85 \\
216 \\
444 \\
140\end{array}$ & $\begin{array}{l}334 \\
250 \\
578 \\
300 \\
100 \\
241 \\
454 \\
140\end{array}$ & $\begin{array}{l}352 \\
280 \\
669 \\
325 \\
117 \\
250 \\
523 \\
145\end{array}$ & $\begin{array}{l}378 \\
351 \\
789 \\
332 \\
138 \\
278 \\
813 \\
180\end{array}$ & $\begin{array}{l}452 \\
486 \\
958 \\
391 \\
160 \\
326 \\
922 \\
195\end{array}$ & $\begin{array}{r}487 \\
721 \\
962 \\
487 \\
179 \\
497 \\
1090 \\
206\end{array}$ \\
\hline
\end{tabular}


LOW FLOW DISCHARGES, IN YEAR ENDING MARCH 31--Continued

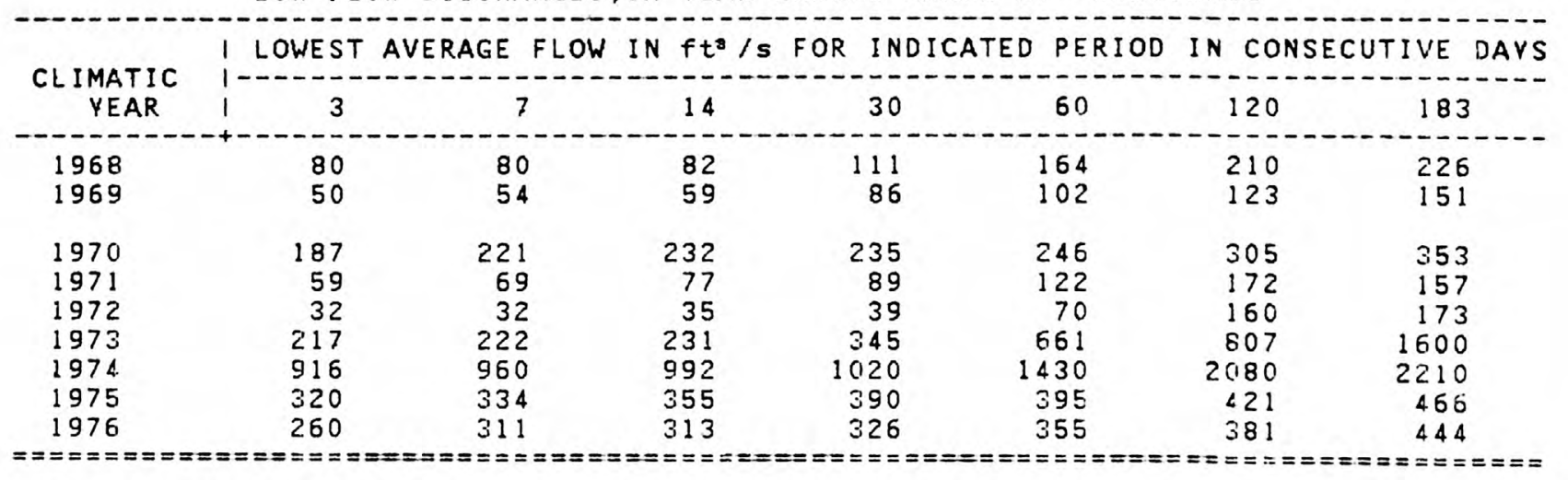


MINIMUM AVERAGE FLOWS FOR PERIOD OF RECORD

\begin{tabular}{|c|c|c|c|c|c|c|c|}
\hline ERIOD OF CONSECUTIVE DAYS & 3 & 7 & 14 & 30 & 60 & 120 & 183 \\
\hline $\begin{array}{l}\text { DISCHARGE, IN } \mathrm{ft}^{\mathrm{s}} / \mathrm{s} \\
\text { CLIMATIC YEAR }\end{array}$ & $\begin{array}{r}7.2 \\
1935\end{array}$ & $\begin{array}{l}10.0 \\
1935\end{array}$ & $\begin{array}{l}14 \\
1935\end{array}$ & $\begin{array}{l}15 \\
1935\end{array}$ & $\begin{array}{l}25 \\
1940\end{array}$ & $\begin{array}{l}35 \\
1938\end{array}$ & $\begin{array}{l}40 \\
1938\end{array}$ \\
\hline
\end{tabular}

MAGNITUDE AND FREQUENCY OF ANNUAL LOW FLOWS

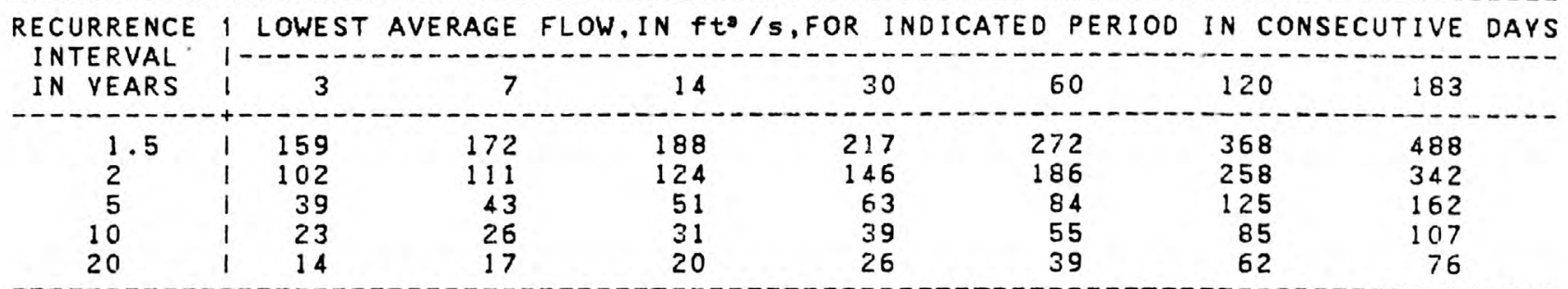

MAGNITUDE AND FREQUENCY OF SEASONAL LOW FLOWS

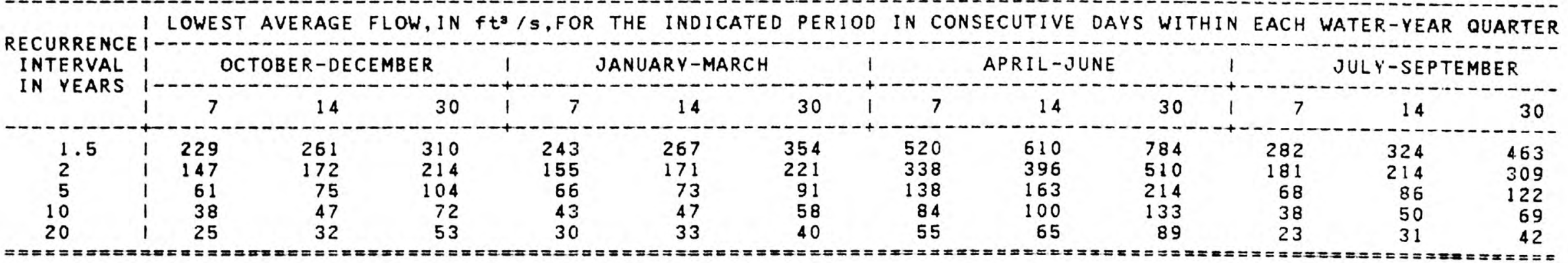

DURATION OF DAILY DISCHARGES FOR ANNUAL AND SEASONAL PERIODS

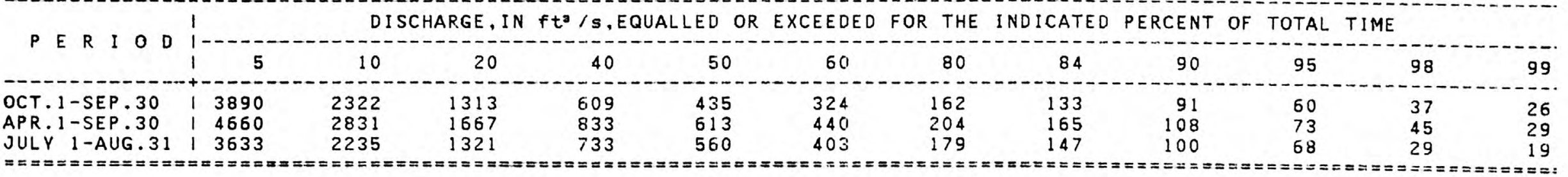




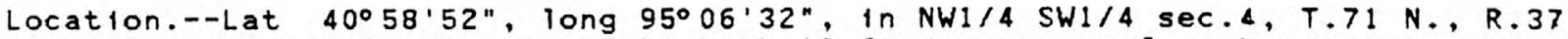

W., Montgomery County, on right bank $10 \mathrm{ft}$ downstream from bridge on county highway, $0.1 \mathrm{~m} l$ le downstream from Little Tarkfo Creek, and $0.5 \mathrm{mlle}$ west of Stanton.

LOW FLOW DISCHARGES, IN YEAR. ENDING MARCH 31

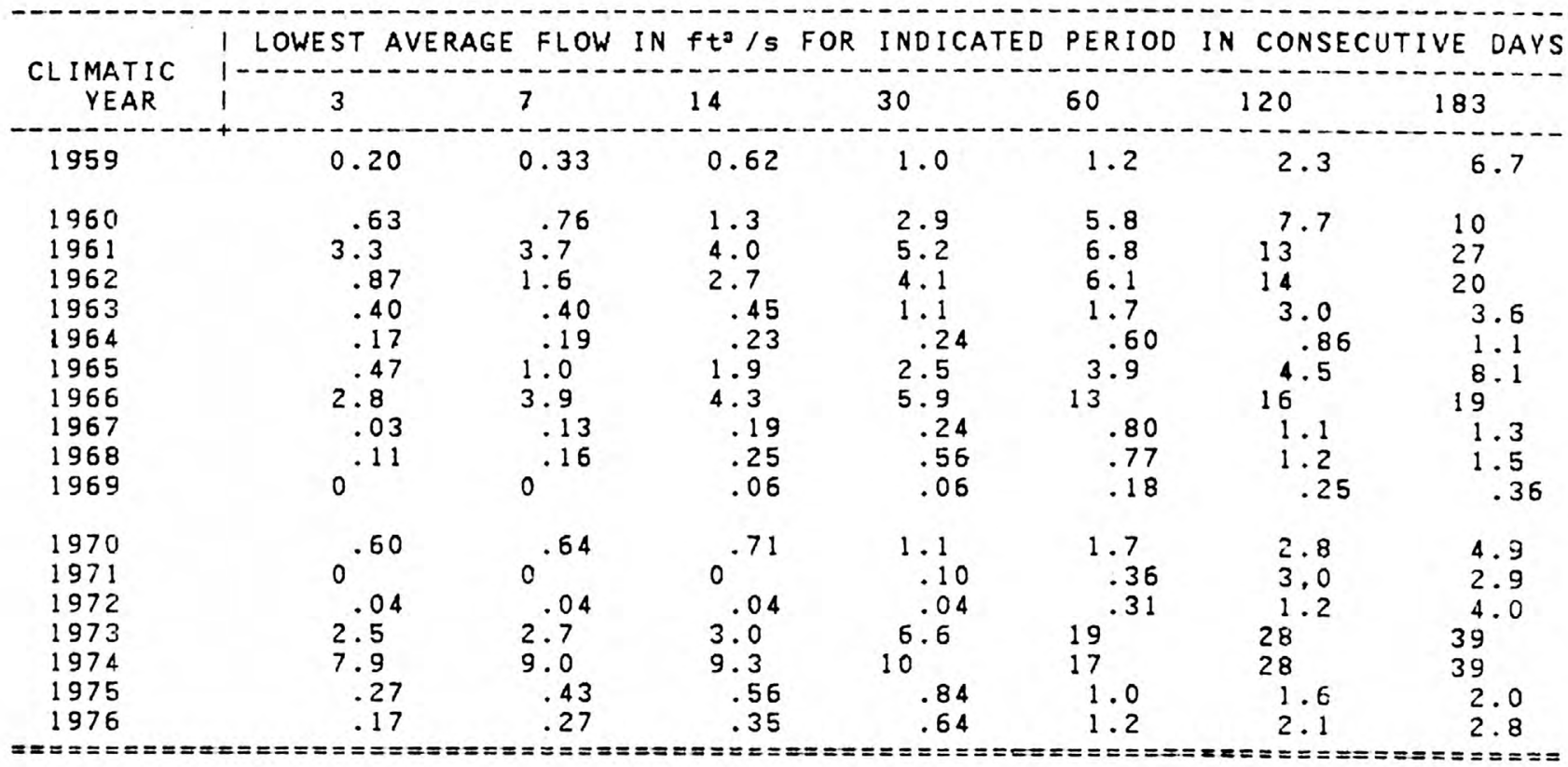


DRAINAGE AREA: $49.3 \mathrm{~m}^{2} \quad$ PERIOD OF RECORD: 19 YEARS AVERAGE DISCHARGE: 25.8 ft=/S MINIMUM AVERAGE FLOWS FOR PERIOD OF RECORD

\begin{tabular}{|c|c|c|c|c|c|c|c|}
\hline PERIOD OF CONSECUTIVE DAYS & 3 & 7 & 14 & 30 & 60 & 120 & 183 \\
\hline 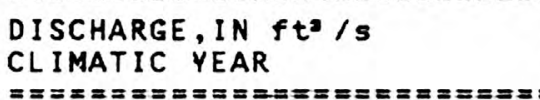 & 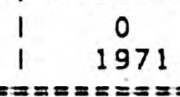 & $\begin{array}{l}0 \\
1971 \\
===2\end{array}$ & $\begin{array}{l}0 \\
1971 \\
==2==\end{array}$ & $\begin{array}{l}0.04 \\
1972 \\
====\pi\end{array}$ & $\begin{array}{l}0.19 \\
1969 \\
:=====\end{array}$ & $\begin{array}{l}0.25 \\
1969\end{array}$ & $\begin{array}{l}0.37 \\
1969\end{array}$ \\
\hline
\end{tabular}

MAGNITUDE AND FREQUENCY OF ANNUAL LOW FLOWS

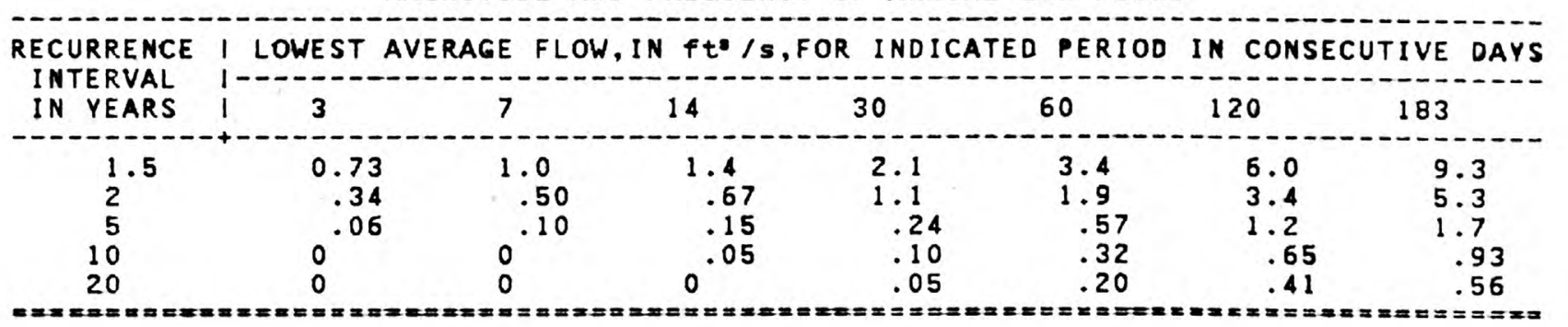

MAGNITUDE AND FREQUENCY OF SEASONAL LOW FLOWS

I LOWEST AVERAGE FLOW, IN f' $/ \mathrm{s}$, FOR THE INDICATED PERIOD IN CONSECUTIVE DAYS WITHIN EACH WATER-YEAR QUARTER

\begin{tabular}{|c|c|c|c|c|c|c|c|c|c|c|c|c|}
\hline \multirow{2}{*}{$\begin{array}{l}\text { INTERVAL } \\
\text { IN YEARS }\end{array}$} & \multicolumn{3}{|c|}{ OCTOBER-DECEMBER } & \multicolumn{2}{|c|}{ JANUARY - MARCH } & 1 & \multicolumn{2}{|c|}{ APRIL-JUNE } & I & \multicolumn{3}{|c|}{ JULY-SEPTEMBER } \\
\hline & 7 & 14 & 30 & 7 & 14 & 30 & 7 & 14 & 30 & 7 & 14 & 30 \\
\hline $\begin{array}{l}1.5 \\
2 \\
5 \\
10 \\
20\end{array}$ & $\begin{array}{r}2.2 \\
.89 \\
.15 \\
.05 \\
.01\end{array}$ & $\begin{array}{l}4.0 \\
1.9 \\
.34 \\
.10 \\
.01\end{array}$ & $\begin{array}{r}4.8 \\
2.5 \\
.67 \\
.30 \\
.06\end{array}$ & $\begin{array}{l}2.4 \\
1.1 \\
.24 \\
.10 \\
.02\end{array}$ & $\begin{array}{l}2.9 \\
1.4 \\
.30 \\
.12 \\
.02\end{array}$ & $\begin{array}{l}4.6 \\
2.6 \\
.92 \\
.56 \\
.27\end{array}$ & $\begin{array}{r}8.2 \\
4.4 \\
.85 \\
.29 \\
.10\end{array}$ & $\begin{array}{r}11 \\
6.4 \\
1.6 \\
.58 \\
.23\end{array}$ & $\begin{array}{c}18 \\
10 \\
2.7 \\
1.2 \\
.50\end{array}$ & $\begin{array}{l}2.2 \\
1.0 \\
.10 \\
0 \\
0\end{array}$ & $\begin{array}{c}2.6 \\
1.2 \\
.18 \\
.04 \\
0\end{array}$ & $\begin{array}{l}3.8 \\
1.9 \\
.40 \\
.16 \\
.07\end{array}$ \\
\hline
\end{tabular}

DURATION OF DAILY DISCHARGES FOR ANNUAL AND SEASONAL PERIODS

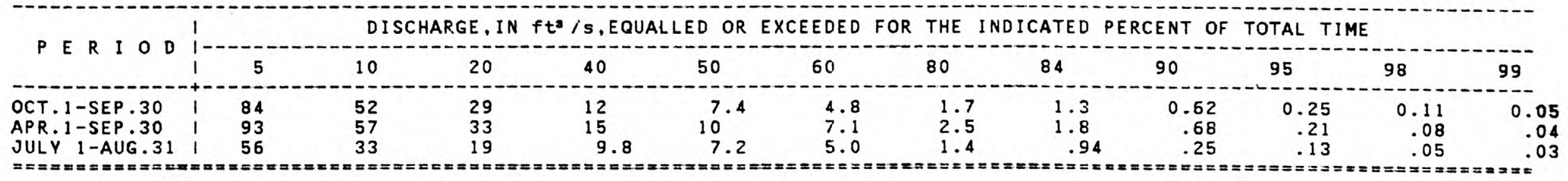


Location.--Lat 40003'14", long 95025'12", In NW1/4 NW1/4 sec.17, T.1 N., R.18 E., Richardson County, on downstream end of middlepler of bridge on U.S. Highway 159 at Rulo, 3.2 miles upstream from Nemaha River, and at mile 498.0 .

Remarks.--Flow regulated by upstream main-stem reservoirs since Nov. 1937. LOW FLOW DISCHARGES, IN YEAR ENDING MARCH 31

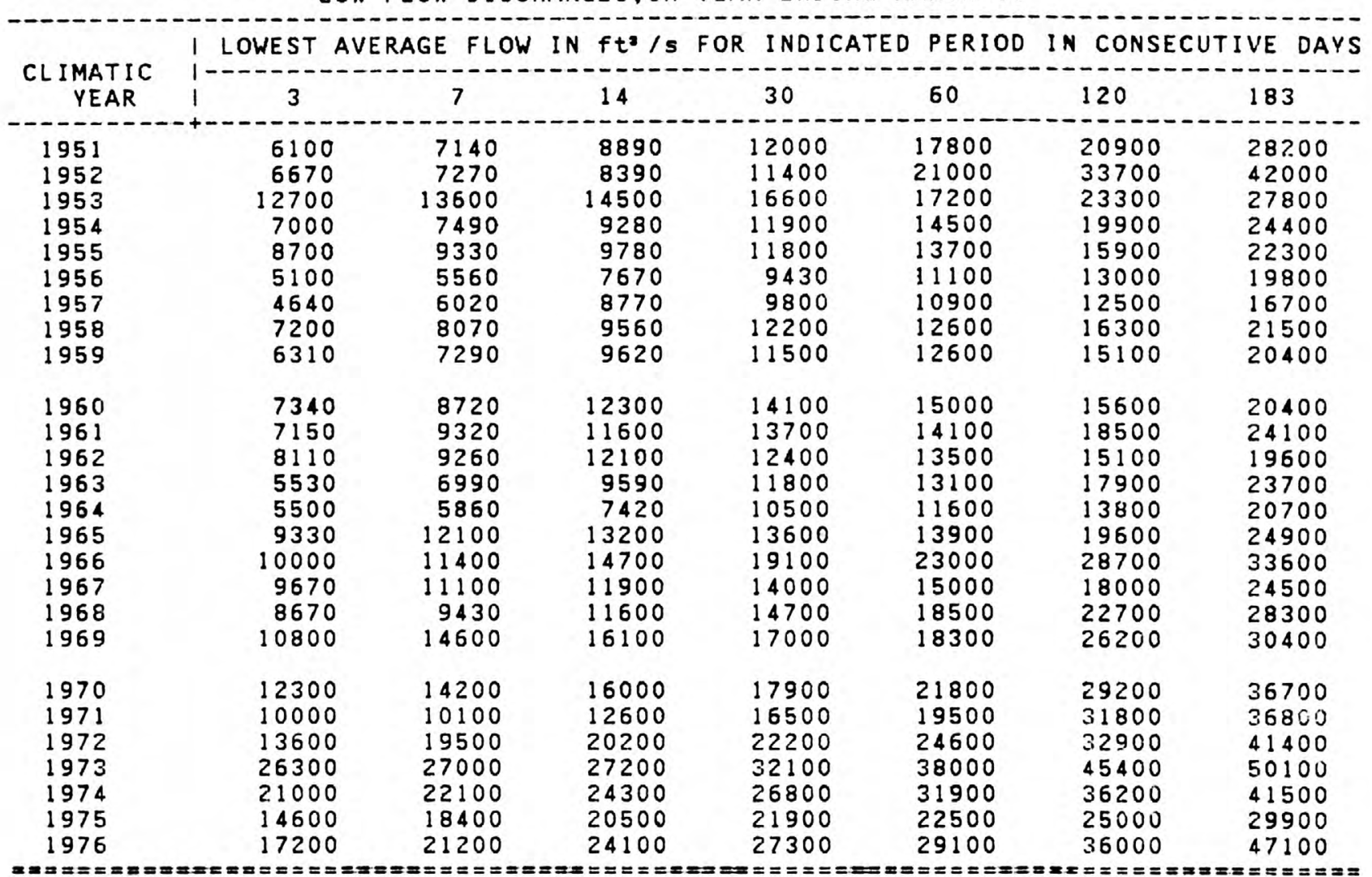


MINIMUM AVERAGE FLOWS FOR PERIOD OF RECORD

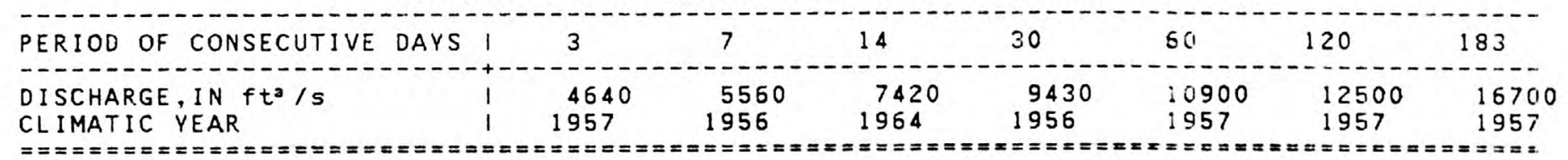

MAGNITUDE AND FREQUENCY OF ANNUAL LOW FLOWS

\begin{tabular}{|c|c|c|c|c|c|c|c|c|}
\hline \multirow{2}{*}{$\begin{array}{l}\text { RECIJRRENCE } \\
\text { INTERVAL } \\
\text { IN YEARS }\end{array}$} & \multicolumn{2}{|c|}{1 LOWEST } & $G E$ & $N t^{3} / s, F O R$ & INDICATED & PERIOD & \multicolumn{2}{|c|}{ IN CONSECUTIVE DAY } \\
\hline & । & 3 & 7 & 14 & 30 & 60 & 120 & 183 \\
\hline $\begin{array}{l}1.5 \\
2 \\
5 \\
10 \\
20\end{array}$ & $\begin{array}{l}1 \\
1 \\
1 \\
1\end{array}$ & $\begin{array}{r}10500 \\
8660 \\
6260 \\
5420 \\
4870\end{array}$ & $\begin{array}{r}12300 \\
10100 \\
7250 \\
6210 \\
5530\end{array}$ & $\begin{array}{r}14300 \\
12200 \\
9200 \\
8110 \\
7370\end{array}$ & $\begin{array}{r}16600 \\
14400 \\
11300 \\
10200 \\
9500\end{array}$ & $\begin{array}{l}19300 \\
16600 \\
13000 \\
11600 \\
10700\end{array}$ & $\begin{array}{l}25100 \\
21400 \\
16000 \\
13900 \\
12400\end{array}$ & $\begin{array}{l}200 \\
300 \\
500 \\
100 \\
500\end{array}$ \\
\hline
\end{tabular}

MAGNITUDE AND FREQUENCY OF SEASONAL LOW FLOWS

I LOWEST AVERAGE FLOW, IN $\mathrm{ft} / \mathrm{s}, \mathrm{FOR}$ THE INDICATED PERIOD IN CONSECUTIVE DAYS WITHIN EACH WATER-YEAR QUARTER

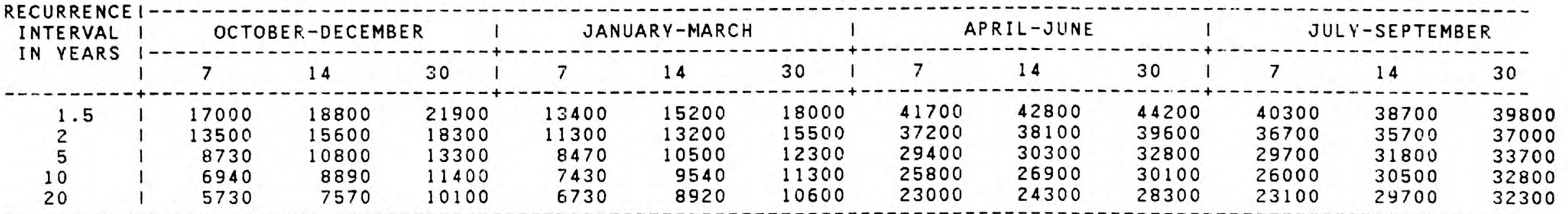

DURATION OF DAILY DISCHARGES FOR ANNUAL AND SEASONAL PERIODS

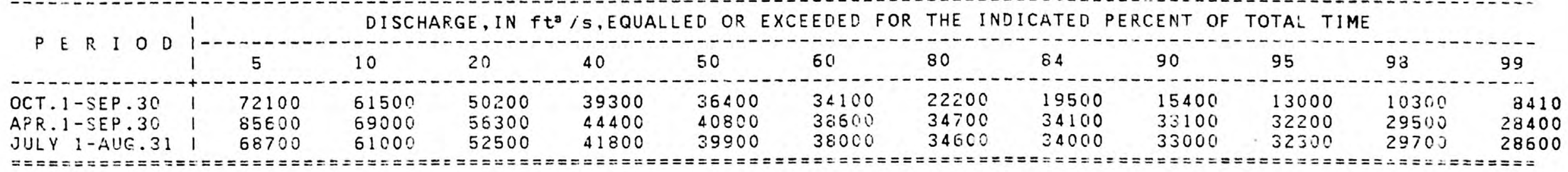


Location.--Lat 40044'19", long 95000'47", in SW1/4 NE1/4 sec.32, T.69 N., R.36 W. Page County, near left abutment on downstream 5 ide of bridge on State Highway 2 (City Route), $0.5 \mathrm{mlle}$ downstream from North 8 ranch, $1.2 \mathrm{mi}$ ies east of city square of Clarinda, and $7.5 \mathrm{~m} 1 \mathrm{les}$ upstream from East Nodaway River.

Remarks.--Clarinda munlcipal water supply is taken from Nodaway Rlver, $500 \mathrm{ft}$ upstream from station.

LOW FLOW DISCHARGES, IN YEAR ENDING MARCH 31

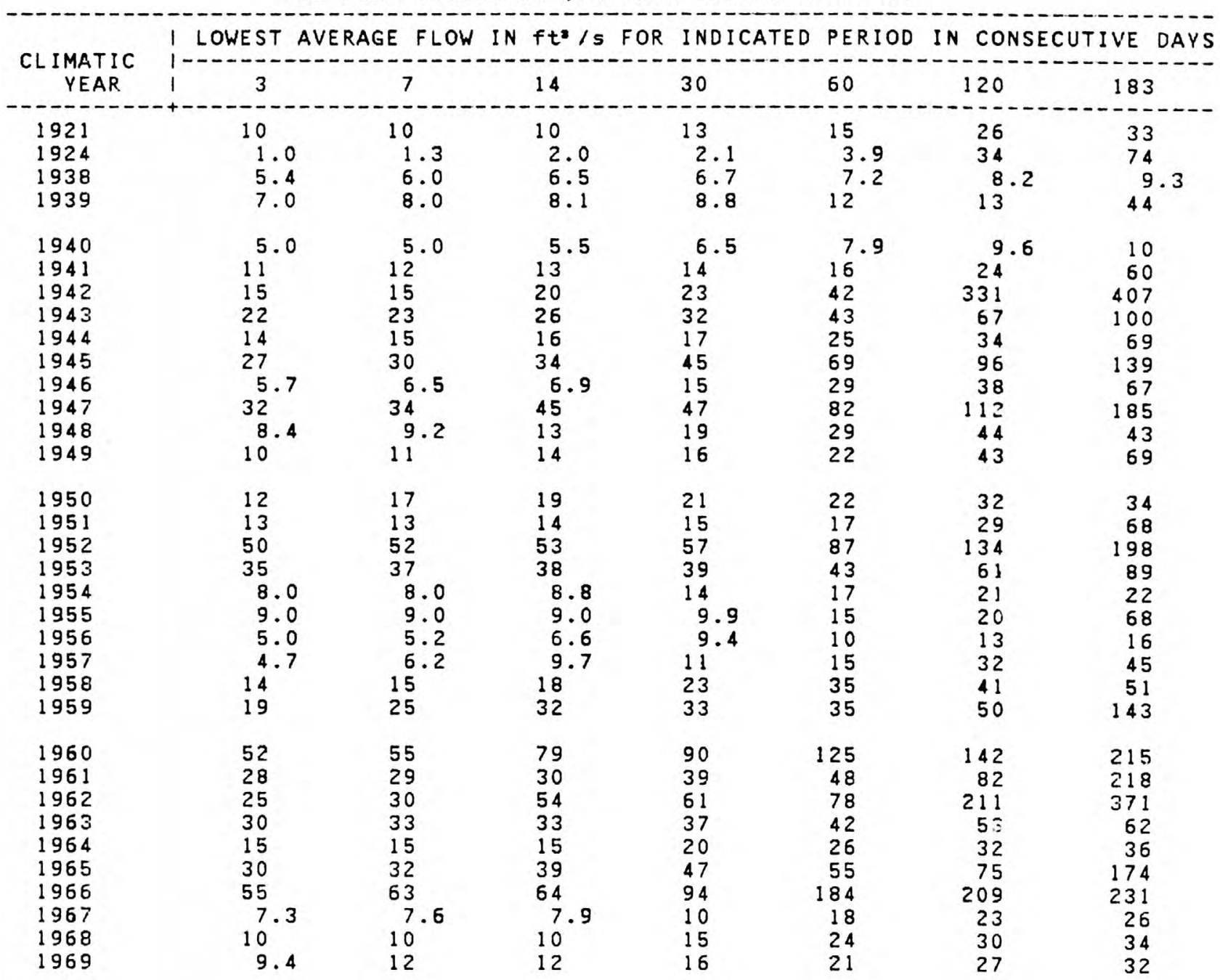


LOW FLOW DISCHARGES, IN VEAR ENDING MARCH 31--Cont 1nued

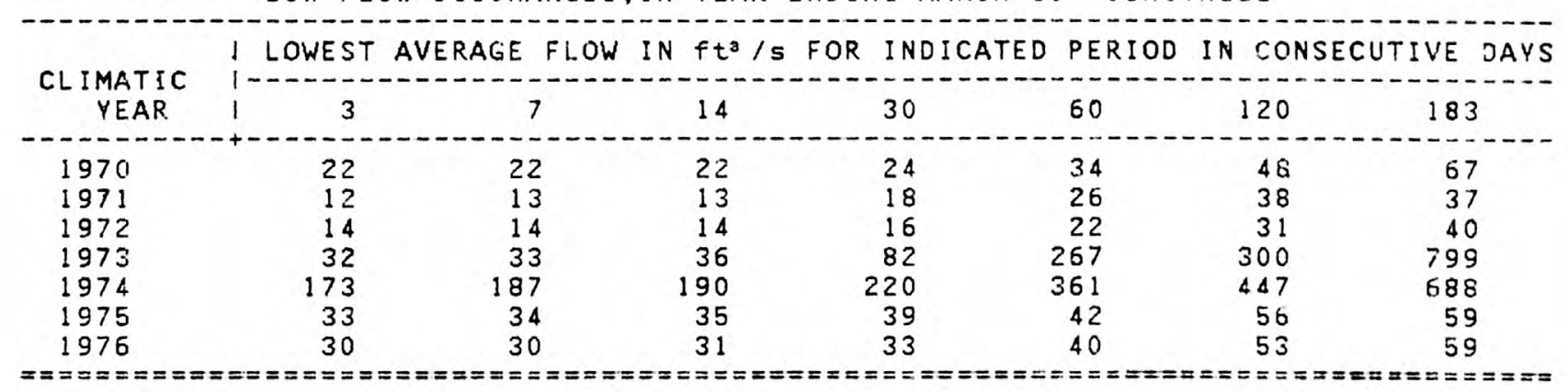


06-8170.00 NODAWAY RIVER AT CLARINDA--CONt1 InUEd

DRAINAGE AREA: $762 \mathrm{~m} \mathbf{m}^{2} \quad$ PERIOD OF RECORD: 46 YEARS AVERAGE DISCHARGE: 322 ft3/s
MINIMUM AVERAGE FLOWS FOR PERIOD OF RECORO

MAGNITUDE AND FREQUENCY OF ANNUAL LOW FLOWS

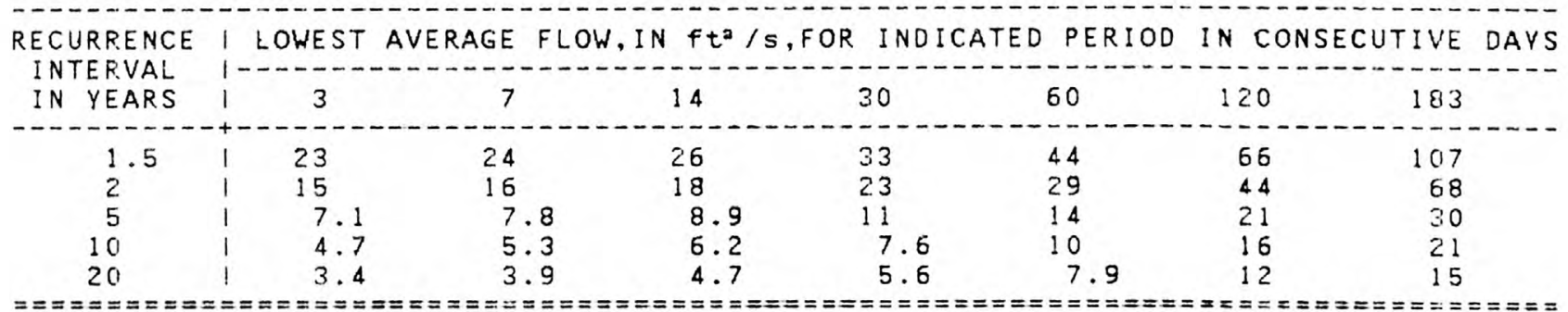

MAGNITUDE AND FREQUENCY OF SEASONAL LOW FLOWS

I LOWEST AVERAGE FLOW, IN $\mathrm{ft}^{3} / 5, F O R$ THE INDICATED PERIOD IN CONSECUTIVE DAYS WITHIN EACH WATER-YEAR QUARTER

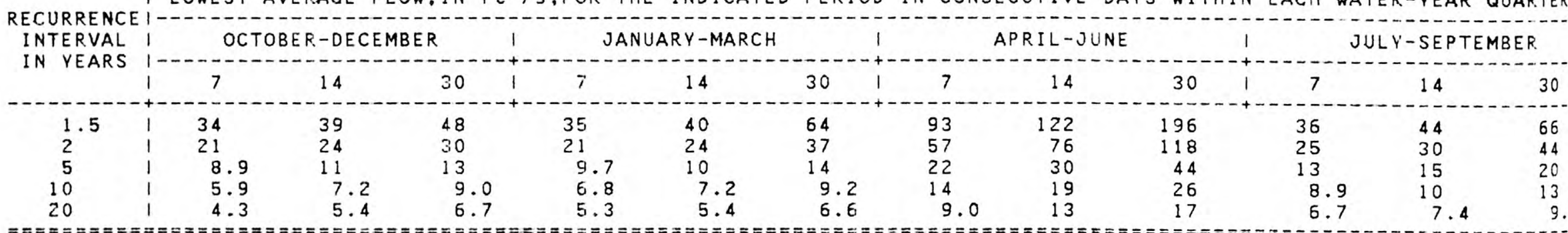

DURATION OF DAILY DISCHARGES FOR ANNUAL AND SEASONAL PERIODS

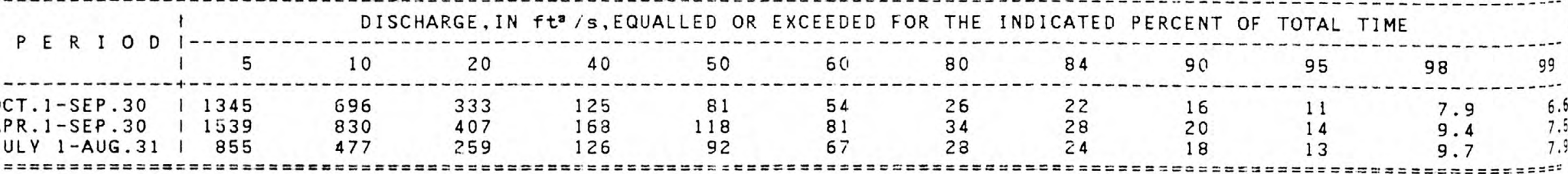


Location.--Lat $40^{\circ} 46^{\prime} 10^{\prime \prime}$, long $94^{\circ} 24^{\prime} 30^{\prime \prime}$, in NEl/4 NW1/4 sec.22, T.69 N., R.31 W., on left bank at downstream slde of hlghway bridge, 2.2 mlles upstream from Turkey Creek, $31 / 2$ miles southwest of Diagonal, and $5.1 \mathrm{mlles}$ downstream from Gard Creek.

Drainage area. $--217 \mathrm{mi}^{2}$.

LOW FLOW DISCHARGES, IN YEAR ENDING MARCH 31

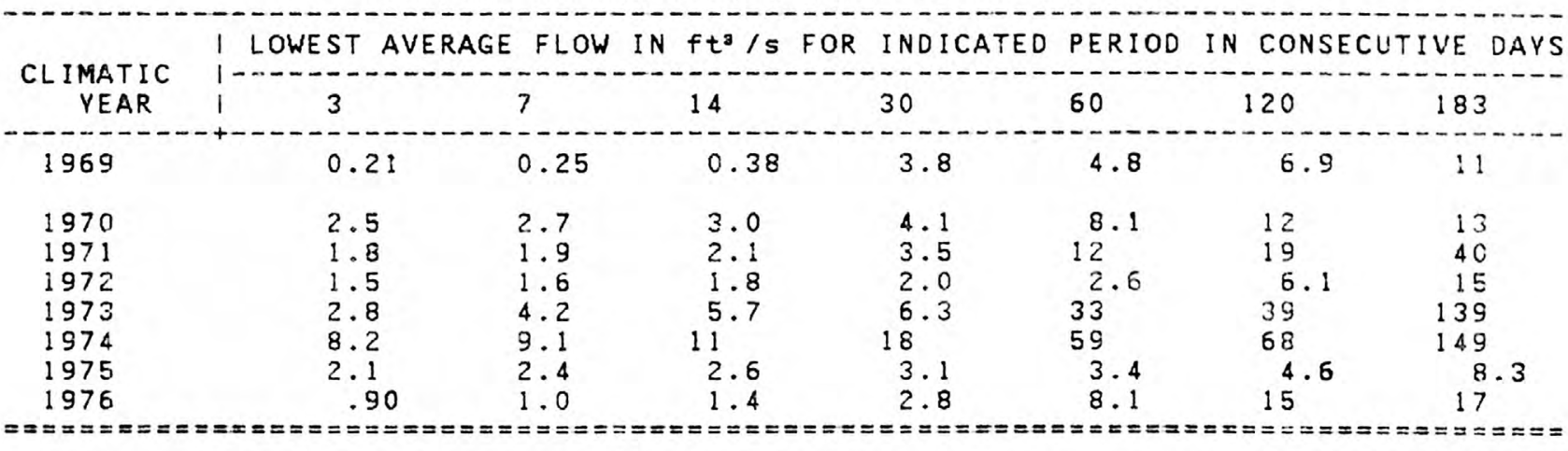

$Q \mathbf{a}=111$

$Q(84)=3.0$

$7 Q 2=1.7$

$7 Q 10=0.2$ 
Location.--Lat $40^{\circ} 38^{\prime} 01^{\prime \prime}$, long $94^{\circ} 44^{\prime} 41^{\prime \prime}$, In NE1/4 NE1/4 sec.9, T.67 N., R.34 W. Taylor County, on left bank at downstream slde of bridge of county highway J55, 0.4 mile upstream from Daugherty Creek, and $2.8 \mathrm{miles}$ southwest of Junction of U.S. Highways 2 and 148 in Bedford.

Remarks.--Silght regulation at low flow by low dam used for water supply in Bedford.

LOW FLOW DISCHARGES, IN YEAR. ENDING MARCH 31

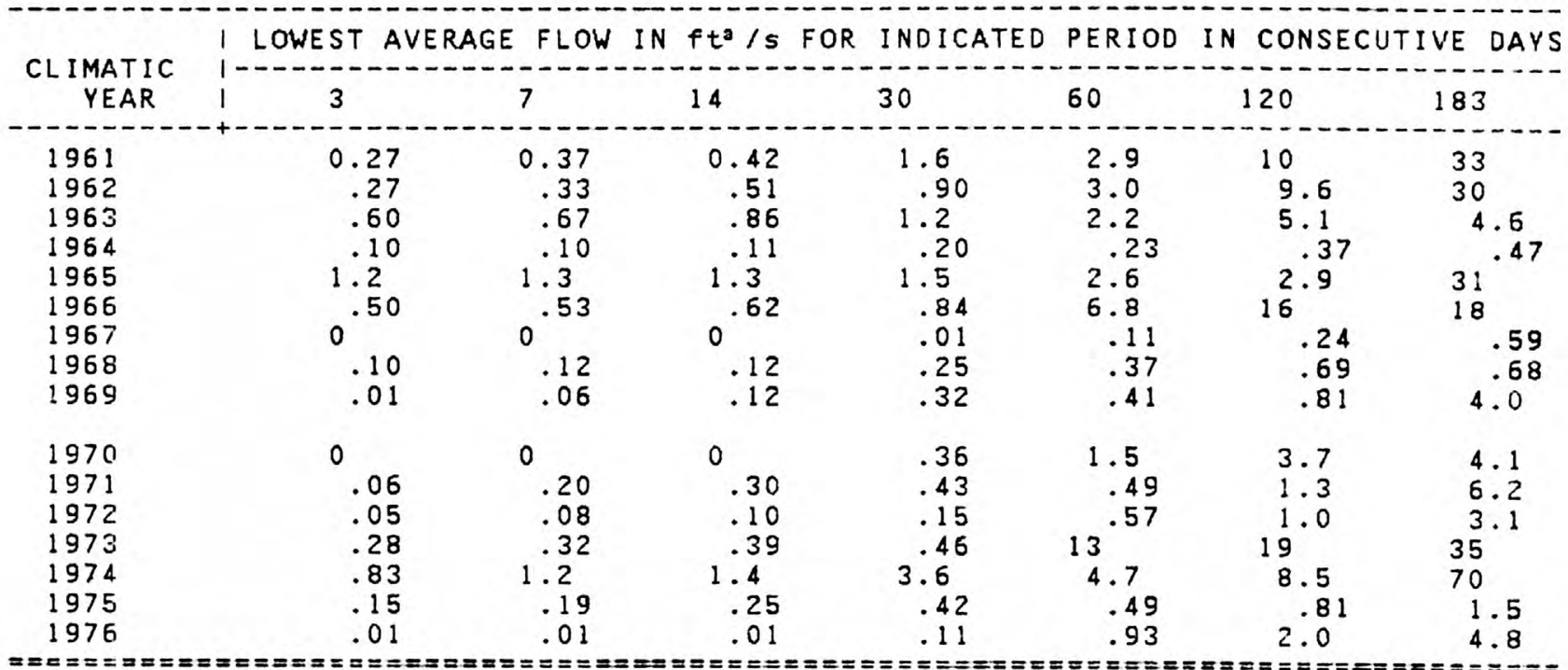




\section{DRAINAGE AREA: $92.1 \mathrm{~m}^{2}$ PERIOD OF RECORD: 17 YEARS AVERAGE DISCHARGE: 50.4 ft $3 / 5$}

MINIMUM AVERAGE FLOWS FOR PERIOD OF RECORD

\begin{tabular}{|c|c|c|c|c|c|c|c|}
\hline RIOD OF CONSECUTIVE DAYS & 3 & 7 & 14 & 30 & 60 & 120 & 183 \\
\hline $\begin{array}{l}\text { HARGE, IN } \mathrm{ft}^{3} / \mathrm{s} \\
\text { ATIC YEAR }\end{array}$ & $\begin{array}{c}0 \\
1970\end{array}$ & $\begin{array}{c}0 \\
1970\end{array}$ & $\begin{array}{l}0 \\
1970\end{array}$ & $\begin{array}{r}0.01 \\
1967\end{array}$ & $\begin{array}{l}0.12 \\
1967\end{array}$ & $\begin{array}{c}0.25 \\
1967\end{array}$ & \\
\hline
\end{tabular}

MAGNITUDE AND FREQUENCY OF ANNUAL LOW FLOWS

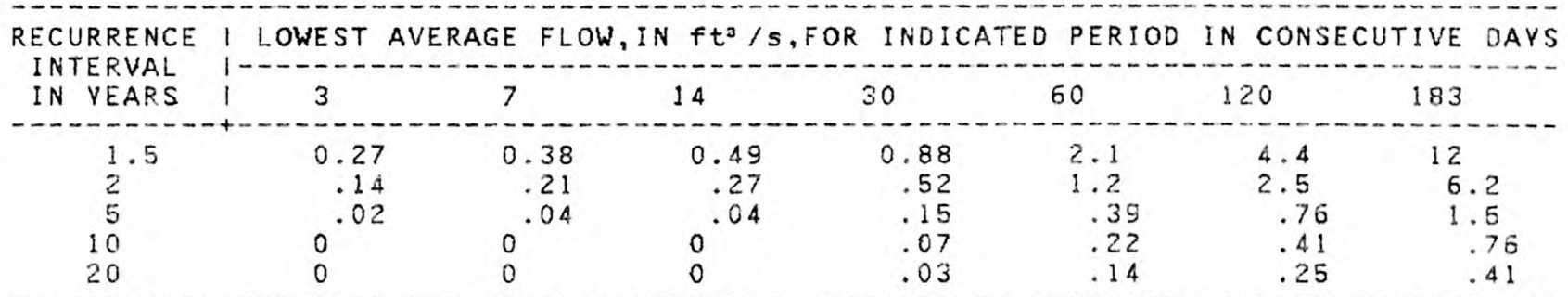

MAGNITUDE AND FREQUENCY OF SEASONAL LOW FLOWS

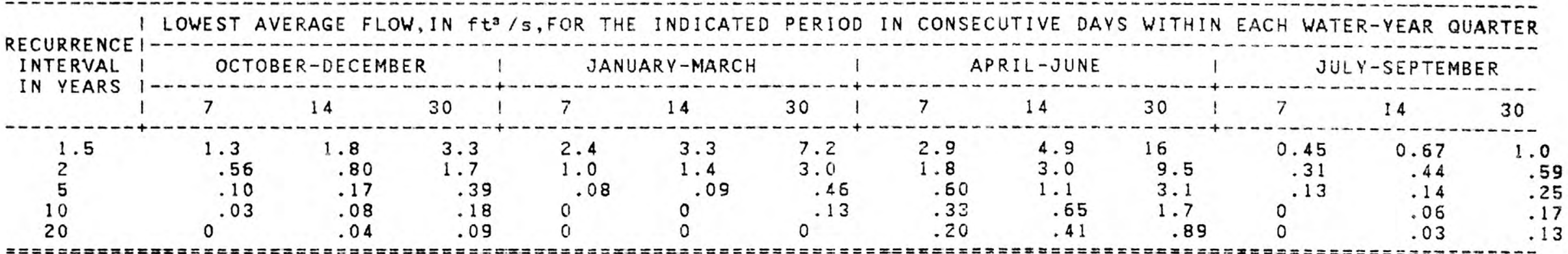

DURATION OF DAILY DISCHARGES FOR ANNUAL AND SEASONAL PERIODS

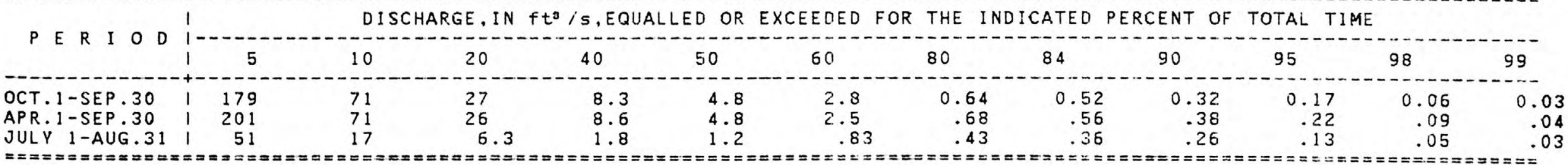


Location.--Lat $40^{\circ} 43^{\prime} 18^{\prime \prime}$, long $93^{\circ} 56^{\prime} 12^{n}$, near the SE corner sec.34, T.69 N., R. 27 W., Decatur County, at right downstream corner of bridge on county highway, 1,000 ft downstream from West Elk Creek, $5.2 \mathrm{miles}$ upstream from mouth, and $5.7 \mathrm{miles}$ southwest of Decatur City.

Dralnage area. $--52.5 \mathrm{ml}^{2}$.

LOW FLOW DISCHARGES, IN YEAR ENDING MARCH 31

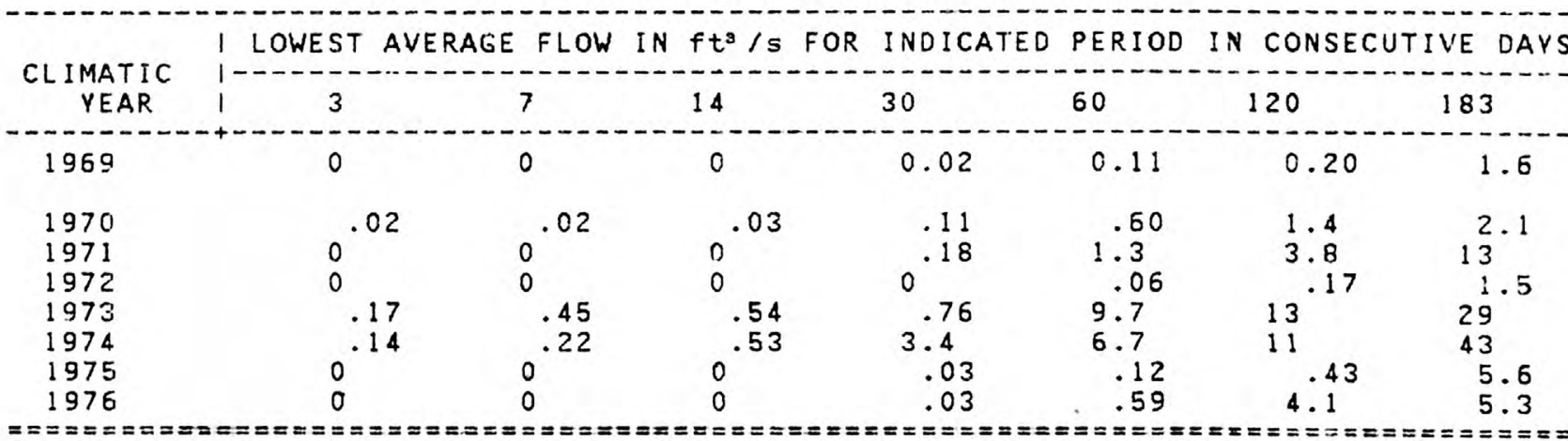

$Q \mathrm{Q}=29$

$Q(84)=0.4$

$7 Q 2=0.2$

$7 Q 10=0$ 
Location.--Lat $40^{\circ} 38^{\prime} 25^{\prime \prime}$, long $93^{\circ} 48^{\prime} 29^{\prime \prime}$, in SE1/4 SE1/4 sec.35. T.68 N., R.26 W. Decatur County, on right bank 15 feet downstream from bridge on U.S. Highway 69 at Davis City, 2.6 miles upstream from Dickersons Branch, and 5.2 miles upstream from Iowa-Missouri State line.

Remarks.--Published as "Grand River" prior to 1918.

LOW FLOW DISCHARGES, IN YEAR ENDING MARCH 31

\begin{tabular}{|c|c|c|c|c|c|c|c|}
\hline \multirow{2}{*}{$\begin{array}{c}\text { CLIMATIC } \\
\text { YEAR }\end{array}$} & \multicolumn{2}{|c|}{ I LOWEST AVERAGE FLOW } & $f t^{2} / s$ & INDICATED & PERIOD & \multicolumn{2}{|c|}{ IN CONSECUTIVE DAVS } \\
\hline & 13 & 7 & 14 & 30 & 60 & 120 & 183 \\
\hline $\begin{array}{l}1920 \\
1921 \\
1923 \\
1924 \\
1925 \\
1943 \\
1944 \\
1945 \\
1946 \\
1947 \\
1948 \\
1949\end{array}$ & $\begin{array}{l}8.4 \\
13 \\
13 \\
15 \\
19 \\
25 \\
6.0 \\
22 \\
11 \\
25 \\
6.2 \\
3.6\end{array}$ & $\begin{array}{l}9.0 \\
15 \\
15 \\
21 \\
19 \\
28 \\
6.6 \\
24 \\
12 \\
27 \\
6.7 \\
4.3\end{array}$ & $\begin{array}{l}9.3 \\
19 \\
18 \\
24 \\
19 \\
30 \\
7.3 \\
26 \\
21 \\
29 \\
10 \\
4.6\end{array}$ & $\begin{array}{l}14 \\
20 \\
28 \\
26 \\
19 \\
38 \\
7.6 \\
53 \\
26 \\
39 \\
12 \\
4.9\end{array}$ & $\begin{array}{l}18 \\
23 \\
45 \\
28 \\
27 \\
73 \\
12 \\
76 \\
45 \\
55 \\
17 \\
9.1\end{array}$ & $\begin{array}{r}95 \\
32 \\
90 \\
42 \\
33 \\
83 \\
16 \\
107 \\
73 \\
89 \\
29 \\
16\end{array}$ & $\begin{array}{r}142 \\
63 \\
225 \\
76 \\
72 \\
105 \\
30 \\
166 \\
78 \\
123 \\
45 \\
27\end{array}$ \\
\hline $\begin{array}{l}1950 \\
1951 \\
1952 \\
1953 \\
1954 \\
1955 \\
1956 \\
1957 \\
1958 \\
1959\end{array}$ & $\begin{array}{c}10 \\
2.7 \\
6.1 \\
6.9 \\
.87 \\
1.1 \\
.60 \\
.20 \\
2.4 \\
7.1\end{array}$ & $\begin{array}{c}11 \\
2.8 \\
8.8 \\
7.3 \\
1.2 \\
1.4 \\
.60 \\
.36 \\
2.5 \\
8.2\end{array}$ & $\begin{array}{c}12 \\
3.1 \\
19 \\
7.6 \\
1.2 \\
1.7 \\
.60 \\
.51 \\
3.0 \\
9.1\end{array}$ & $\begin{array}{c}16 \\
4.4 \\
51 . \\
7.9 \\
1.5 \\
3.8 \\
.61 \\
.90 \\
4.7 \\
10\end{array}$ & $\begin{array}{c}19 \\
5.4 \\
98 \\
9.5 \\
2.1 \\
6.1 \\
.71 \\
1.5 \\
7.9 \\
16\end{array}$ & $\begin{array}{c}24 \\
8.1 \\
158 \\
31 \\
2.9 \\
11 \\
1.2 \\
2.7 \\
13 \\
45\end{array}$ & $\begin{array}{c}46 \\
15 \\
183 \\
64 \\
3.4 \\
40 \\
2.9 \\
3.1 \\
13 \\
50\end{array}$ \\
\hline $\begin{array}{l}1960 \\
1961 \\
1962 \\
1963 \\
1964 \\
1965 \\
1966 \\
1967 \\
1968 \\
1969\end{array}$ & $\begin{array}{l}32 \\
23 \\
20 \\
11 \\
3.9 \\
34 \\
16 \\
5.6 \\
3.4 \\
3.2\end{array}$ & $\begin{array}{l}36 \\
23 \\
22 \\
11 \\
3.9 \\
36 \\
17 \\
6.3 \\
3.5 \\
3.5\end{array}$ & $\begin{array}{l}47 \\
24 \\
24 \\
12 \\
4.0 \\
39 \\
19 \\
6.8 \\
3.7 \\
3.7\end{array}$ & $\begin{array}{l}76 \\
35 \\
28 \\
22 \\
4.4 \\
52 \\
26 \\
7.4 \\
6.1 \\
5.0\end{array}$ & $\begin{array}{l}111 \\
40 \\
81 \\
26 \\
6.9 \\
81 \\
61 \\
10 \\
10.0 \\
5.8\end{array}$ & $\begin{array}{r}289 \\
81 \\
176 \\
49 \\
9.3 \\
115 \\
208 \\
12 \\
13 \\
8.7\end{array}$ & $\begin{array}{r}461 \\
152 \\
348 \\
59 \\
16 \\
295 \\
231 \\
23 \\
21 \\
39\end{array}$ \\
\hline $\begin{array}{l}1970 \\
1971 \\
1972 \\
1973\end{array}$ & $\begin{array}{l}28 \\
12 \\
2.5 \\
15\end{array}$ & $\begin{array}{l}31 \\
12 \\
2.9 \\
17\end{array}$ & $\begin{array}{c}35 \\
14 \\
3.1 \\
25\end{array}$ & $\begin{array}{c}36 \\
19 \\
4.3 \\
39\end{array}$ & $\begin{array}{r}48 \\
42 \\
10 \\
132\end{array}$ & $\begin{array}{r}94 \\
95 \\
18 \\
154\end{array}$ & $\begin{array}{r}118 \\
215 \\
49 \\
434\end{array}$ \\
\hline
\end{tabular}


LOW FLOW OISCHARGES, IN YEAR ENDING MARCH 31--Continued

\begin{tabular}{|c|c|c|c|c|c|c|c|}
\hline CLIMATIC & 1 LOWEST & GE FL & $N \mathrm{ft}^{3} / \mathrm{s}$ & $R$ INDI & D PEF & Y col & VE \\
\hline YEAR & 13 & 7 & 14 & 30 & 60 & 120 & 183 \\
\hline $\begin{array}{l}1974 \\
1975 \\
1976\end{array}$ & $\begin{array}{l}78 \\
6.4 \\
11\end{array}$ & $\begin{array}{r}98 \\
6.7 \\
11\end{array}$ & $\begin{array}{c}116 \\
6.8 \\
12\end{array}$ & $\begin{array}{c}143 \\
8.6 \\
14\end{array}$ & $\begin{array}{r}364 \\
1 i \\
18\end{array}$ & $\begin{array}{r}390 \\
27 \\
45\end{array}$ & $\begin{array}{r}742 \\
47 \\
72\end{array}$ \\
\hline
\end{tabular}




\section{DRAINAGE AREA: $701 \mathrm{~m}^{2}$ PERIOD OF RECORD: 41 VEARS AVERAGE DISCHARGE: 368 ft $3 / \mathrm{s}$} MINIMUM AVERAGE FLOWS FOR PERIOD OF RECORD

\begin{tabular}{|c|c|c|c|c|c|c|c|}
\hline PERIOD OF CONSECUTIVE DAYS & 3 & 7 & 14 & 30 & 60 & 120 & 183 \\
\hline $\begin{array}{l}\text { DISCHARGE, IN } \mathrm{ft}^{\mathrm{s}} / \mathrm{s} \\
\text { CLIMATIC VEAR }\end{array}$ & $\begin{array}{l}0.20 \\
1957\end{array}$ & $\begin{array}{l}0.37 \\
1957\end{array}$ & $\begin{array}{l}0.51 \\
1957\end{array}$ & $\begin{array}{l}0.62 \\
1956\end{array}$ & $\begin{array}{l}0.71 \\
1956\end{array}$ & $\begin{array}{r}1.2 \\
1956\end{array}$ & $\begin{array}{r}2.9 \\
1956\end{array}$ \\
\hline
\end{tabular}

MAGNITUDE AND FREQUENCY OF ANNUAL LOW FLOWS

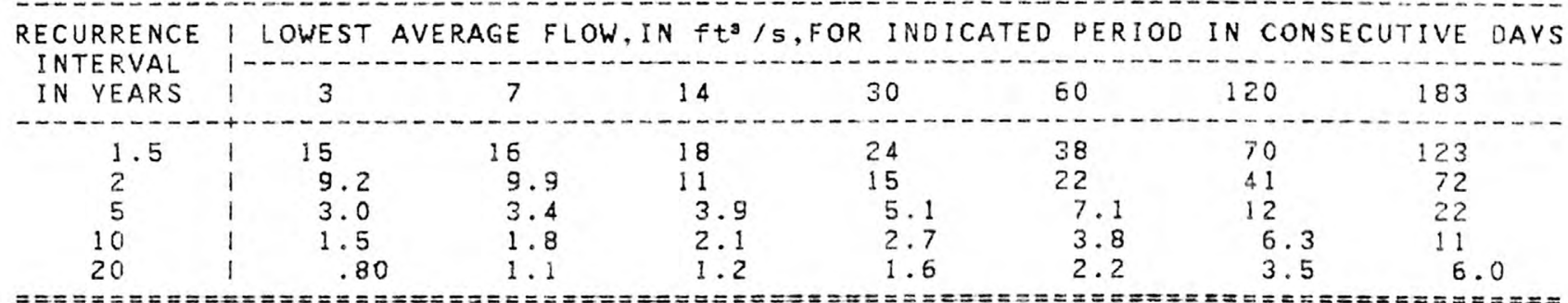

MAGNITUDE AND FREQUENCY OF SEASONAL LOW FLOWS

I LOWEST AVERAGE FLOW, IN $\mathrm{ft}^{\mathrm{s}} / \mathrm{s}, \mathrm{FOR}$ THE INDICATED PERIOD IN CONSECUTIVE OAYS WITHIN EACH WATER-YEAR QUARTER

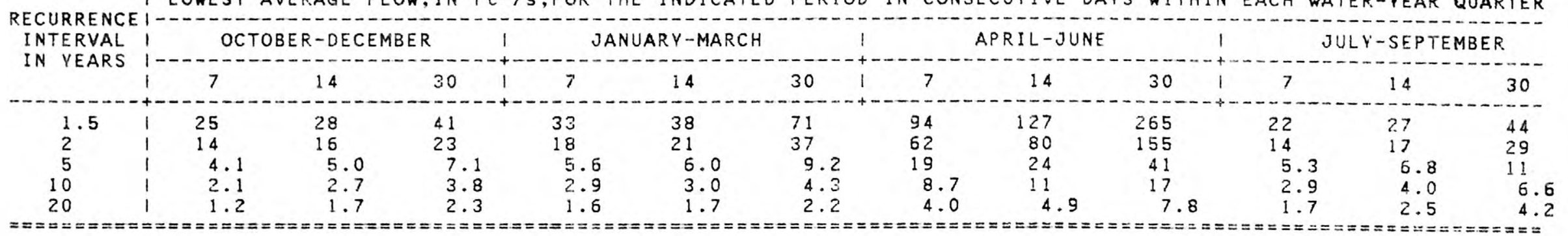

DURATION OF DAILY DISCHARGES FOR ANNUAL AND SEASONAL PERIODS

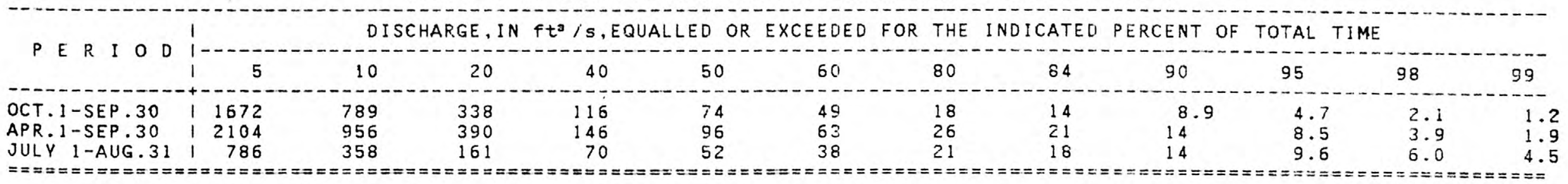




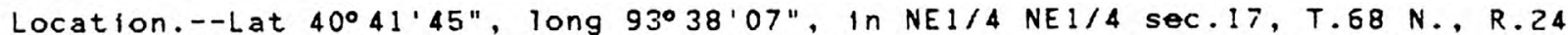
W. , Decatur County, on left bank $10 \mathrm{ft}$ downstream from bridge on county highway A, 200 ft upstream from unnamed creek, $1.3 \mathrm{~m} 1$ les downstream from Brush Creek, and $6.5 \mathrm{mlles}$ southeast of post office at Leon.

LOW FLOW DISCHARGES, IN YEAR ENDING MARCH 31

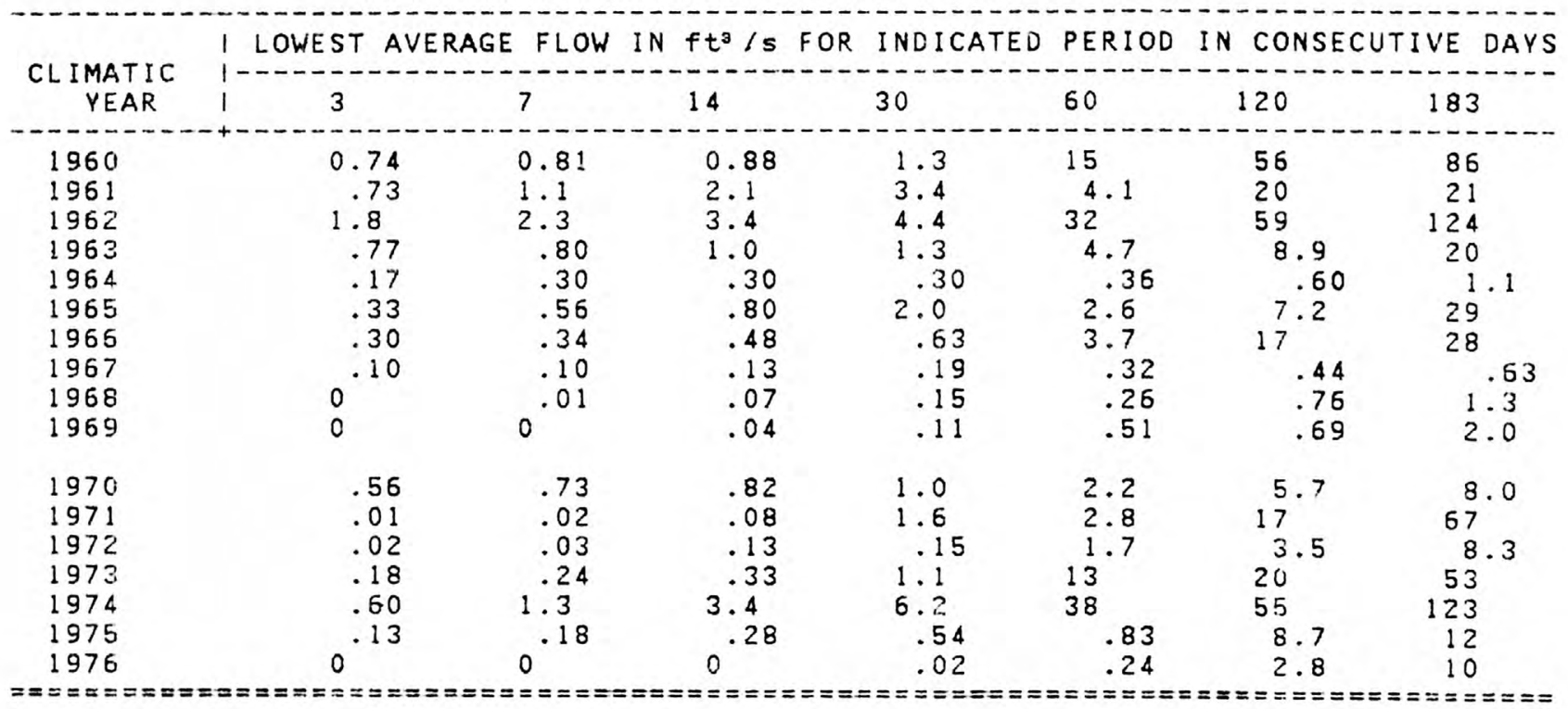


ORAINAGE AREA: $104 \mathrm{~m}^{2}$ PERIOD OF RECORD: 18 VEARS AVERAGE DISCHARGE: 72.3 ft $3 / \mathrm{S}$ MINIMUM AVERAGE FLOWS FOR PERIOD OF RECORD

\begin{tabular}{|c|c|c|c|c|c|c|c|}
\hline PERIOD OF CONSECUTIVE DAYS & 3 & 7 & 14 & 30 & 60 & 120 & 183 \\
\hline $\begin{array}{l}\text { ISCHARGE, IN } \mathrm{ft}^{3} / \mathrm{s} \\
\text { LIIMATIC YEAR }\end{array}$ & $\begin{array}{l}0 \\
1976\end{array}$ & $\begin{array}{l}0 \\
1976\end{array}$ & $\begin{array}{l}0 \\
1976\end{array}$ & $\begin{array}{l}0.02 \\
1976\end{array}$ & $\begin{array}{l}0.25 \\
1976\end{array}$ & $\begin{array}{r}0.44 \\
1967\end{array}$ & $\begin{array}{r}0.63 \\
1967\end{array}$ \\
\hline
\end{tabular}

MAGNITUDE AND FREQUENCY OF ANNUAL LOW FLOWS

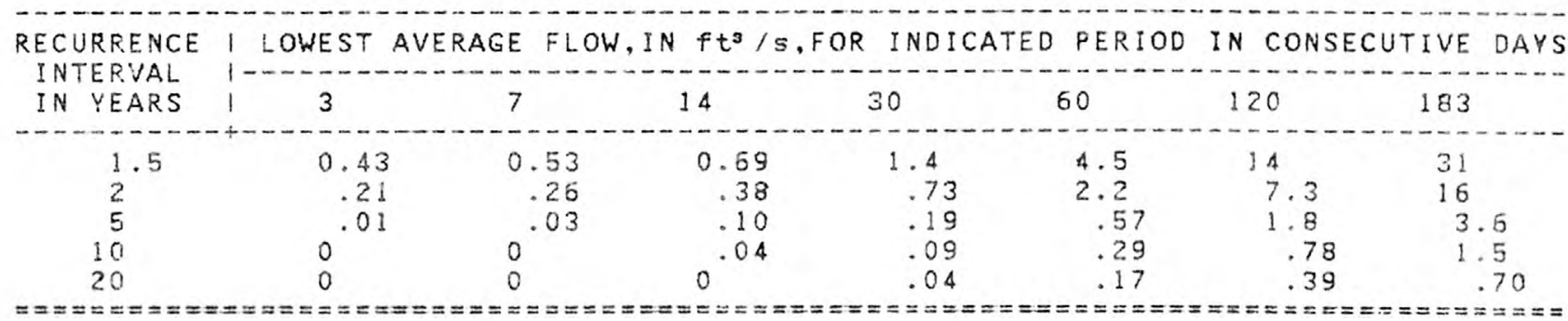

MAGNITUDE AND FREQUENCY OF SEASONAL LOW FLOWS

I LOWEST AVERAGE FLOW, IN $f t^{\circ} / s, F O R$ THE INDICATED PERIOD IN CONSECUTIVE DAYS WITHIN EACH WATER-YEAR QUARTER

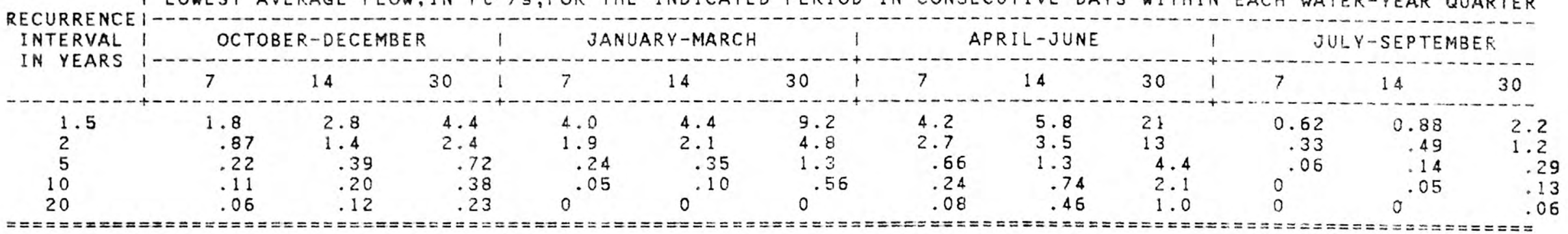

DURATION OF DAILY DISCHARGES FOR ANNUAL AND SEASONAL PERIODS

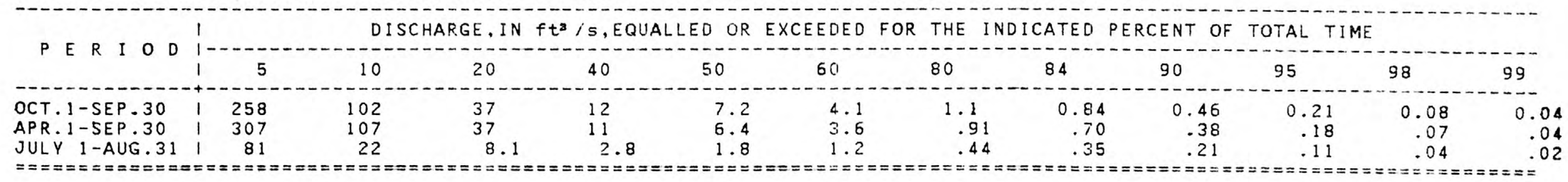




\section{CHARITON RIVER NEAR CHARITON}

Location.--Lat $40^{\circ} 57^{\prime} 12^{\prime \prime}$, long $93^{\circ} 15^{\prime} 27^{\prime \prime}$, In SW1/4 NE1/4 sec.15, T.71 N., R.2 W., Lucas County, on right bank $15 \mathrm{ft}$ downstream from bridge on county highway $S 43,0.4 \mathrm{~m} 1$ le downstream from Wolf Creek, and $5.0 \mathrm{~m} 1 \mathrm{les}$ southeast of Chariton.

LOW FLOW DISCHARGES, IN YEAR ENDING MARCH 31

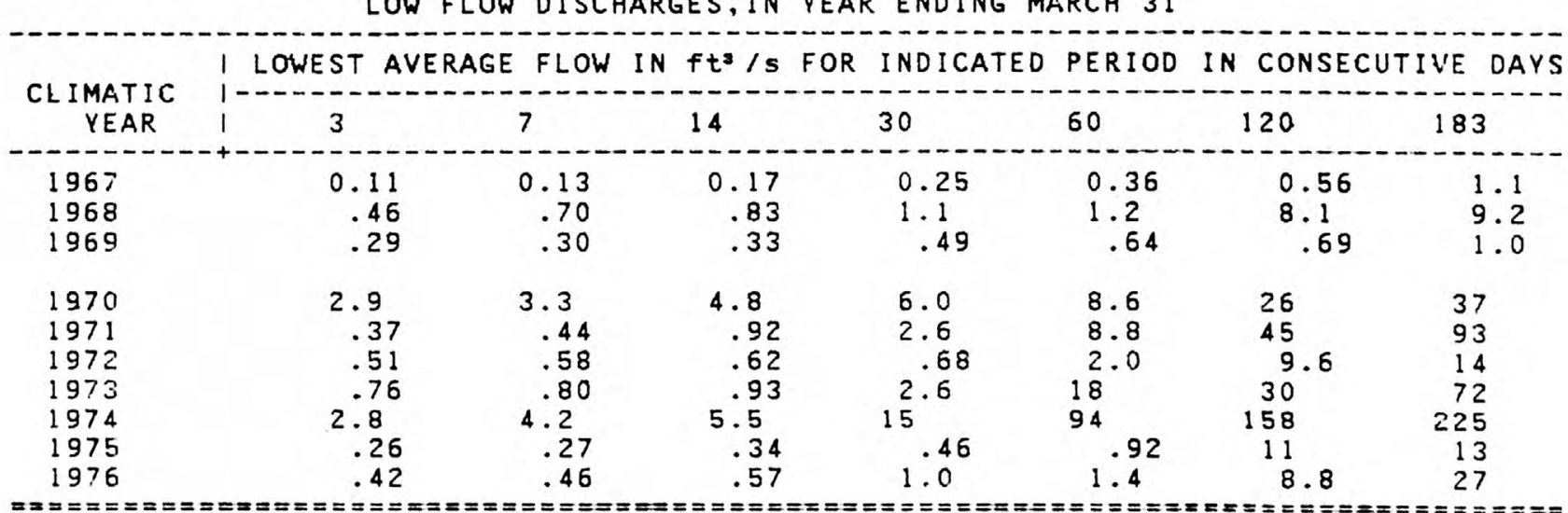


DRAINAGE AREA: $182 \mathrm{~m}^{2}$ PERIOD OF RECORD: 11 YEARS AVERAGE DISCHARGE: 102 ft /s

MINIMUM AVERAGE FLOWS FOR PERIOD OF RECORD

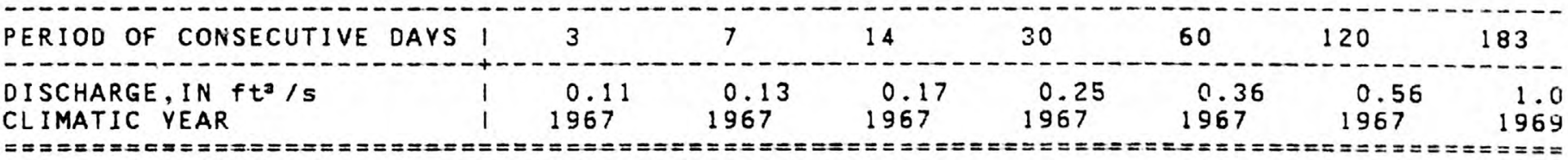

MAGNITUDE AND FREQUENCY OF ANNUAL LOW FLOWS

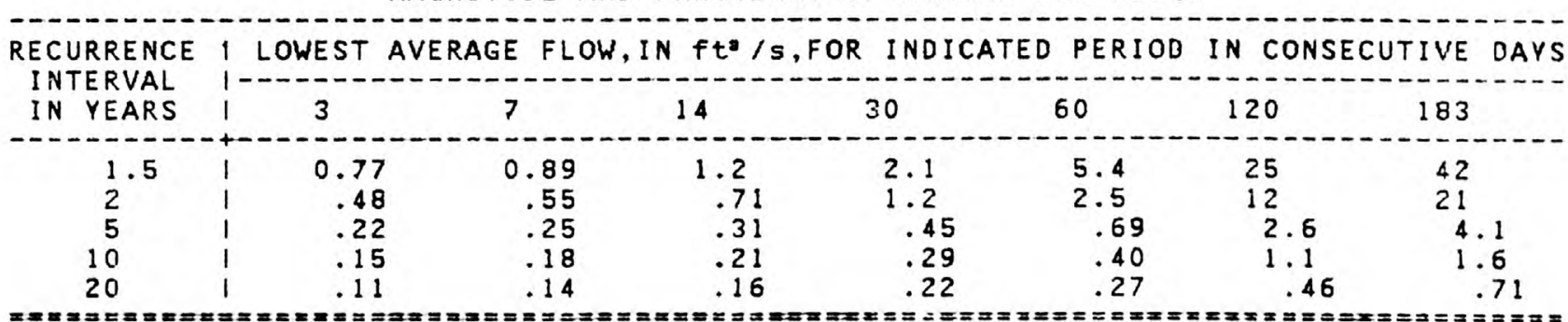

MAGNITUDE AND FREQUENCY OF SEASONAL LOW FLOWS

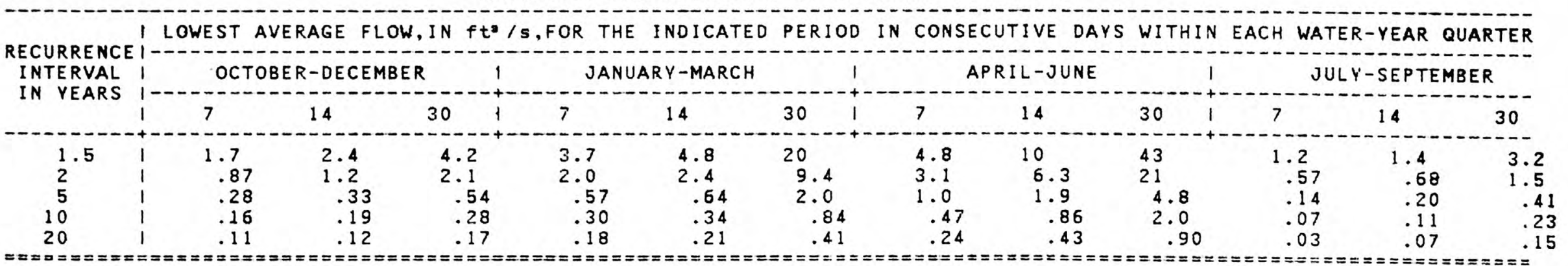

DURATION OF DAILY DISCHARGES FOR ANNUAL AND SEASONAL PERIODS

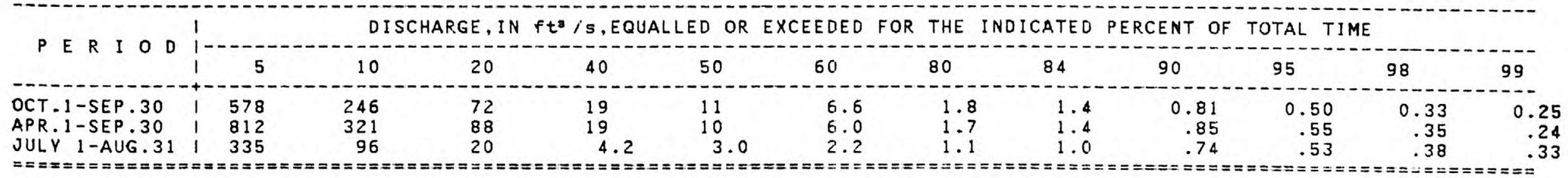


Location.--Lat $40^{\circ} 55^{\prime} 25^{\prime \prime}$, long 93007'55", in SW1/4 NW1/4 sec.26, T.71 N., R.20 W. Lucas County, on left bank $15 \mathrm{ft}$ downstream from highway bridge, 0.7 mile upstream from Chariton River, and $5.5 \mathrm{miles}$ southeast of Russell.

Remarks.--Discontinued Sept. 30, 1962.

LOW FLOW DISCHARGES, IN YEAR. ENDING MARCH 31

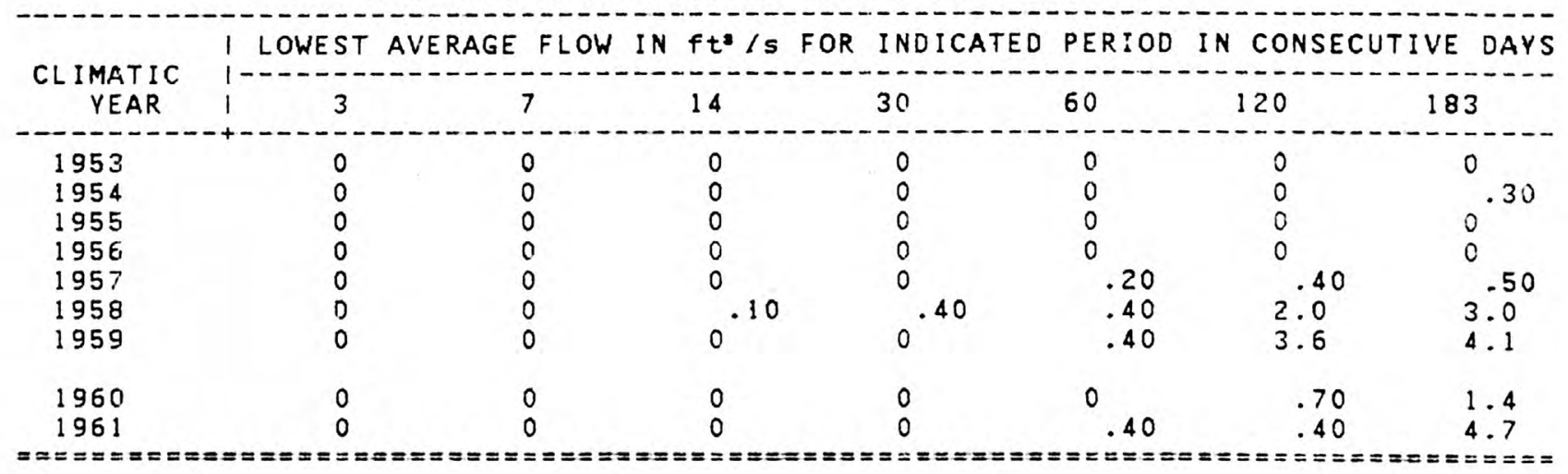




DRAINAGE AREA: $13.2 \mathrm{~m}^{2} \quad$ PERIOD OF RECORD: 10 VEARS AVERAGE DISCHARGE: 7.7 fts Is
MINIMUM AVERAGE FLOWS FOR PERIOD OF RECORD
PERIOD OF CONSECUTIVE DAYS I

MAGNITUDE AND FREQUENCY OF ANNUAL LOW FLOWS

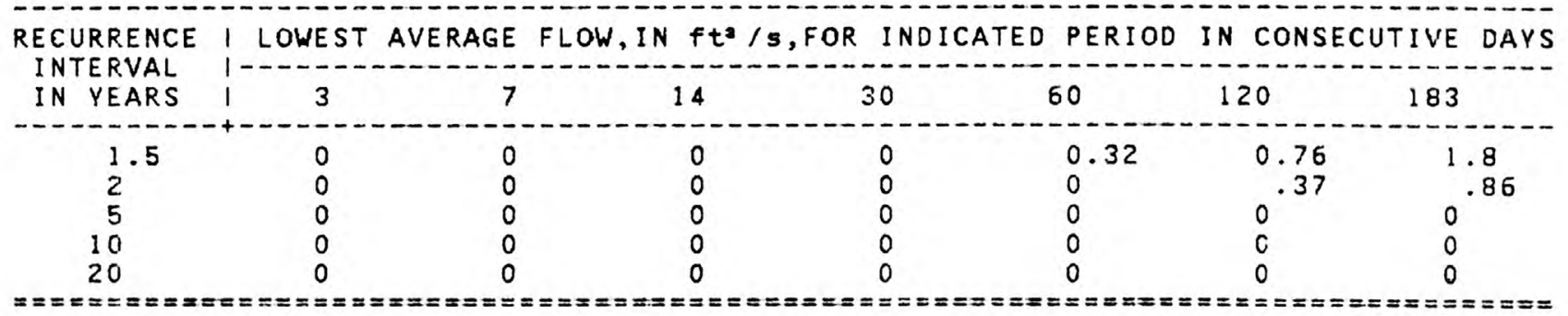

MAGNITUDE AND FREQUENCY OF SEASONAL LOW FLOWS

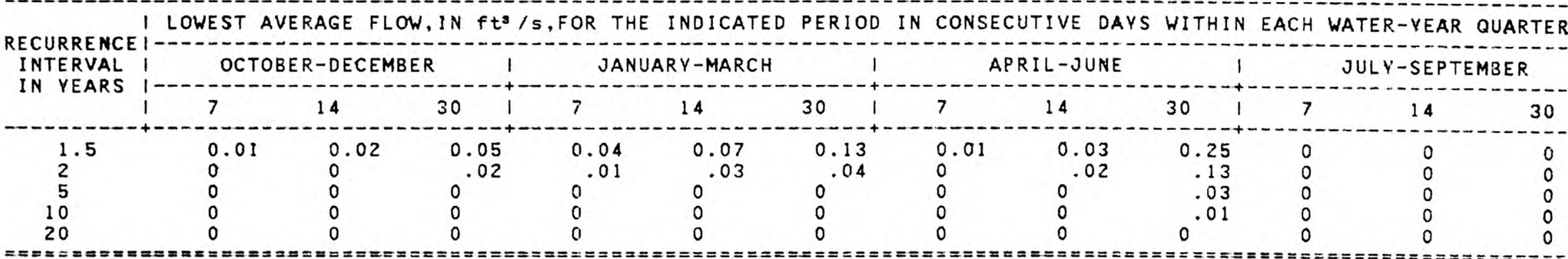

DURATION OF DAILY DISCHARGES FOR ANNUAL AND SEASONAL PERIODS

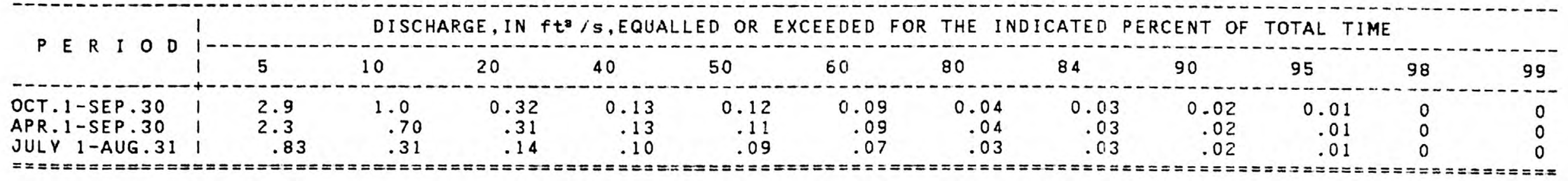




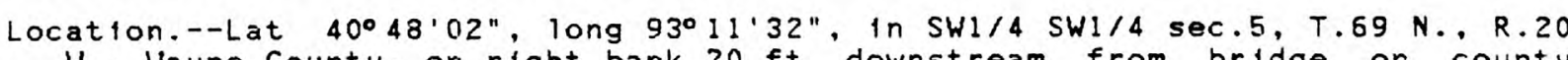
W. Wayne County, on right bank $20 \mathrm{ft}$ downstream from bridge on county highway $550,1.3 \mathrm{miles}$ downstream from Jordan Creek and $4.3 \mathrm{miles}$ northwest of Promise City.

Drainage area. $--168 \mathrm{~m}^{2}$.

LOW FLOW DISCHARGES, IN YEAR. ENDING MARCH 31

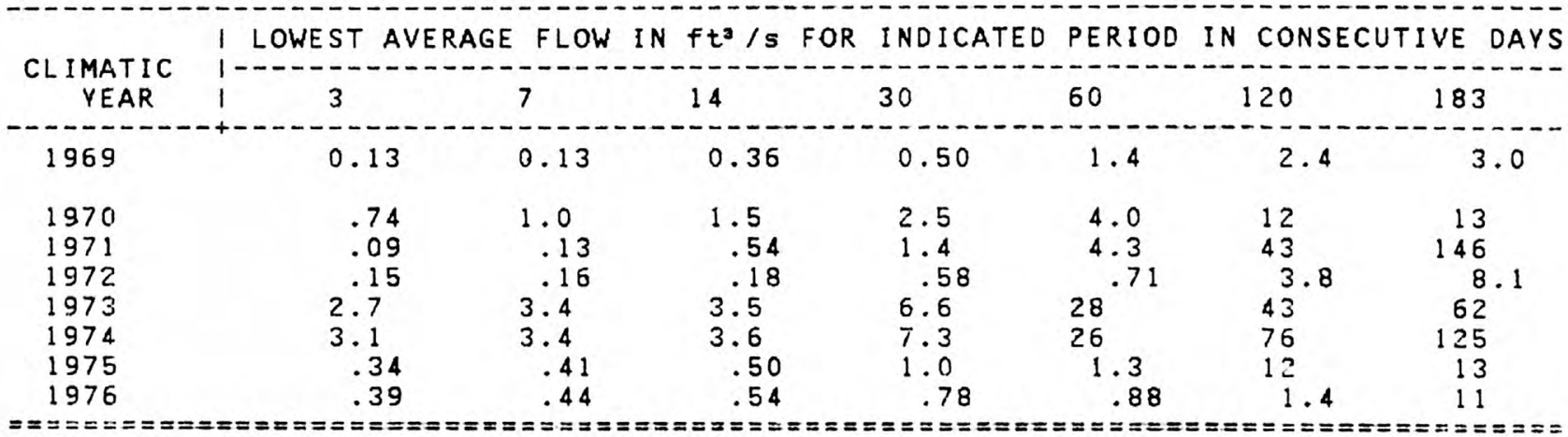

$Q A=96$

$Q(84)=0.6$

$7 Q 2=0.3$

$7 Q 10=*$ 
Location.--Lat $40^{\circ} 49^{\prime} 22^{\prime \prime}$, long $92^{\circ} 53^{\prime} 22^{\prime \prime}$, In SE1/4 NE1/4 sec.35, T.70 N., R. 18 W. Appanoose County, on left bank $600 \mathrm{ft}$ downstream from out let of Rathbun Dam, 1.8 miles north of Rathbun, 3.7 miles upstream from Walnut Creek and at mile 142.1

Remarks.--Flow regulated by Rathbun Lake since Nov. 21, 1969.

LOW FLOW DISCHARGES, IN YEAR. ENDING MARCH 31

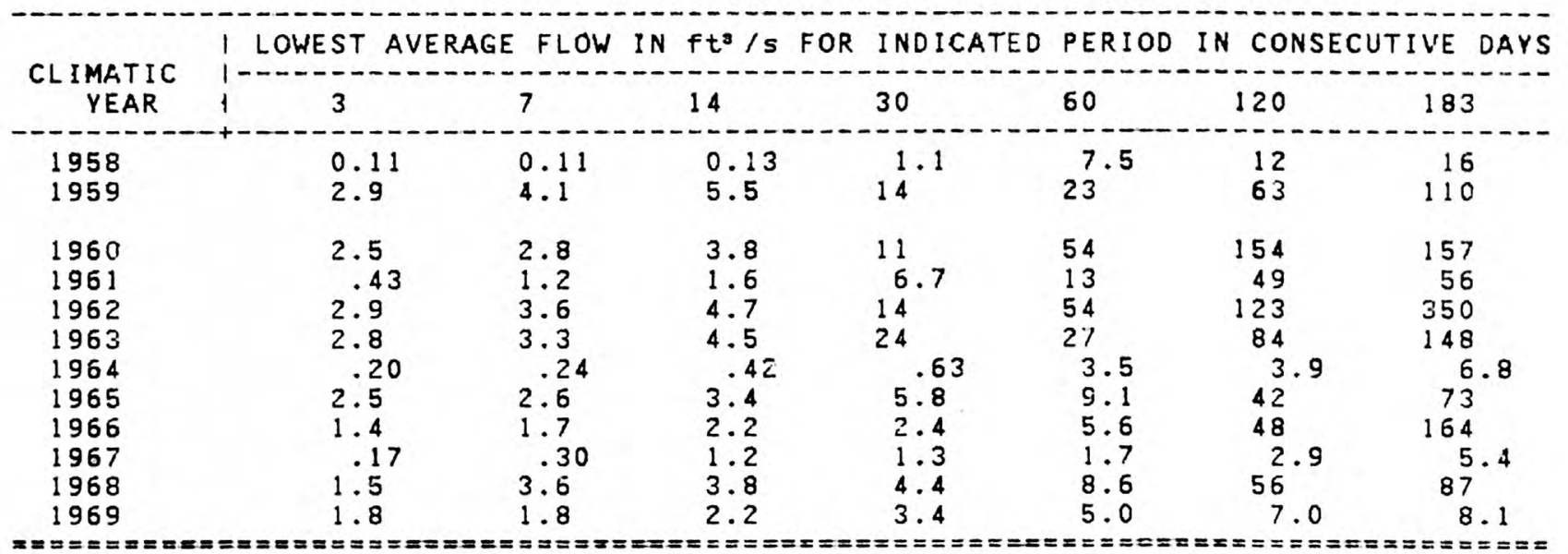


MINIMUM AVERAGE FLOWS FOR PERIOD OF RECORD

\begin{tabular}{|c|c|c|c|c|c|c|c|}
\hline PERIOD OF CONSECUTIVE DAYS I & 3 & 7 & 14 & 30 & 60 & 20 & 183 \\
\hline $\begin{array}{l}\text { ARGE, IN } \mathrm{ft}^{3} / \mathrm{s} \\
\text { TIC YEAR }\end{array}$ & $\begin{array}{l}0.11 \\
1958\end{array}$ & $\begin{array}{r}0.11 \\
1958\end{array}$ & $\begin{array}{l}0.13 \\
1958\end{array}$ & $\begin{array}{l}0.63 \\
1964\end{array}$ & $\begin{array}{r}1.7 \\
967\end{array}$ & $\begin{array}{r}2.9 \\
1967\end{array}$ & $\begin{array}{r}5.4 \\
1967\end{array}$ \\
\hline
\end{tabular}

MAGNITUDE AND FREQUENCY OF ANNUAL LOW FLOWS

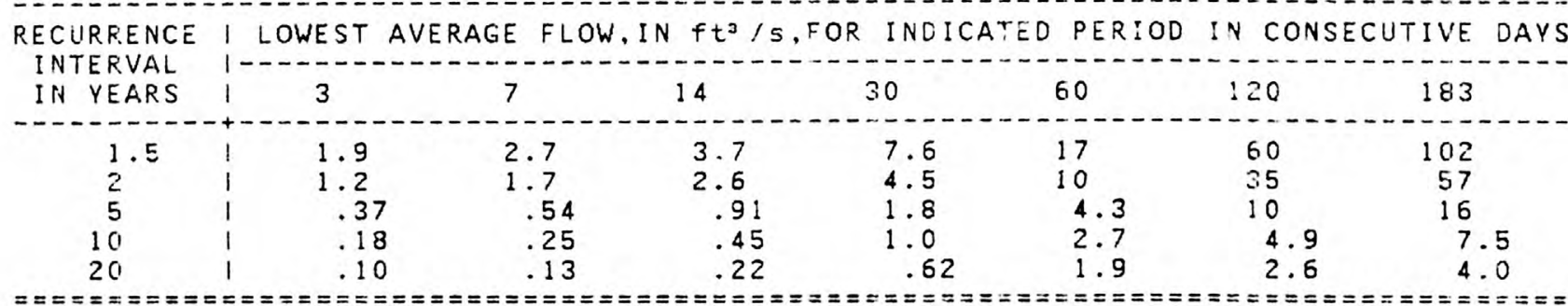

MAGNITUDE AND FREQUENCY OF SEASONAL LOW FLOWS

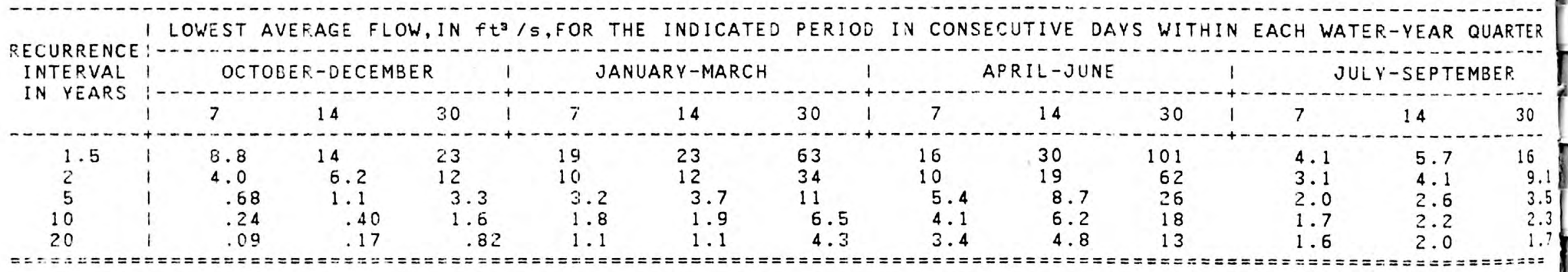

DURATION OF DAILY DISCHARGES FOR ANNUAL AND SEASONAL FERIODS

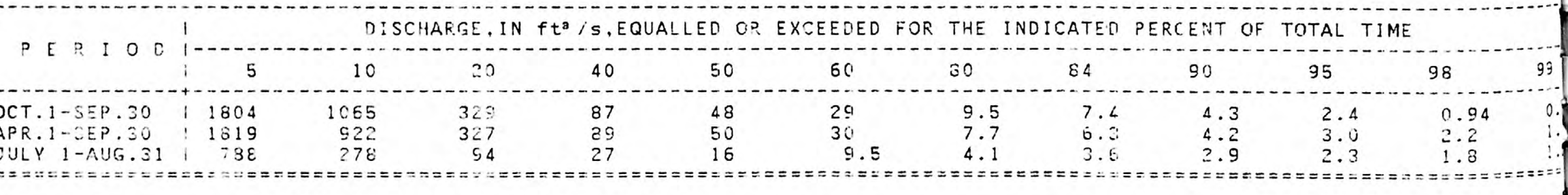


LOW FLOW DISCHARGES, IN YEAR ENDING MARCH 31

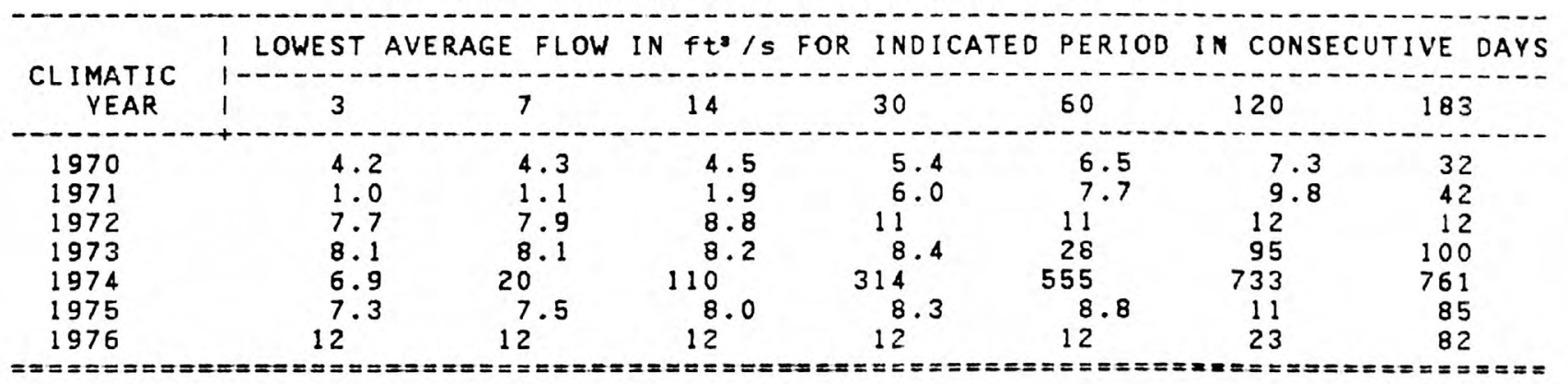




\section{DRAINAGE AREA: $549 \mathrm{~m}^{2} \quad$ PERIOD OF RECORD: 7 YEARS AVERAGE DISCHARGE: $317 \mathrm{ft}$ /s}

MINIMUM AVERAGE FLOWS FOR FERIOD OF RECORD

\begin{tabular}{|c|c|c|c|c|c|c|c|}
\hline PERIOD OF CONSECUTIVE DAYS & 3 & 7 & 14 & 30 & 50 & 120 & 183 \\
\hline $\begin{array}{l}\text { CHARGE, IN } f t^{2} / s \\
\text { MATIC YEAR }\end{array}$ & $\begin{aligned} 1.0 \\
1971\end{aligned}$ & $\begin{aligned} 191 \\
1971\end{aligned}$ & $\begin{array}{r}1.9 \\
1971\end{array}$ & $\begin{array}{r}5.4 \\
1970\end{array}$ & $\begin{array}{r}5.5 \\
: 970\end{array}$ & $\begin{array}{r}793 \\
1970\end{array}$ & \\
\hline
\end{tabular}

MAGNITUDE AND FREQUENCY OF ANNUAL LOW FLOWS

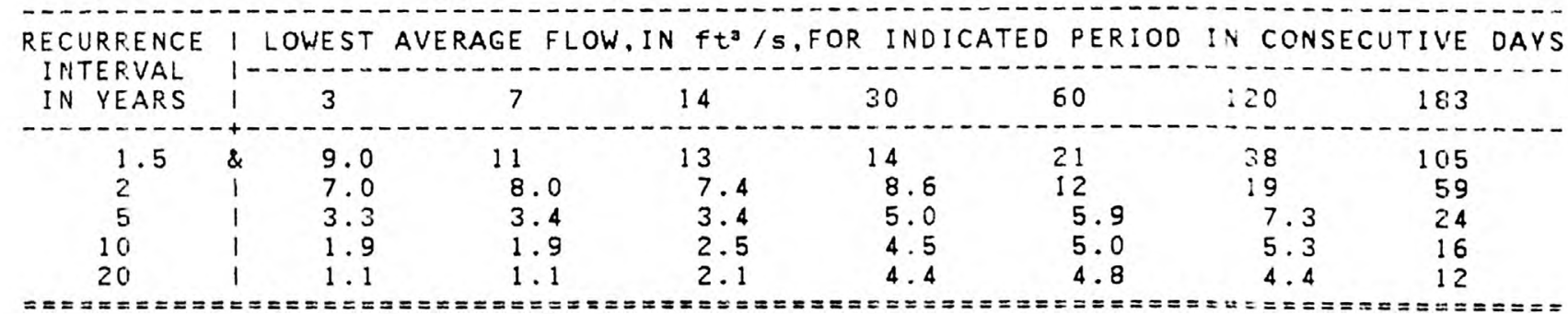

MAGNITUDE AND FREQUENCY OF SEASONAL LOW FLOWS

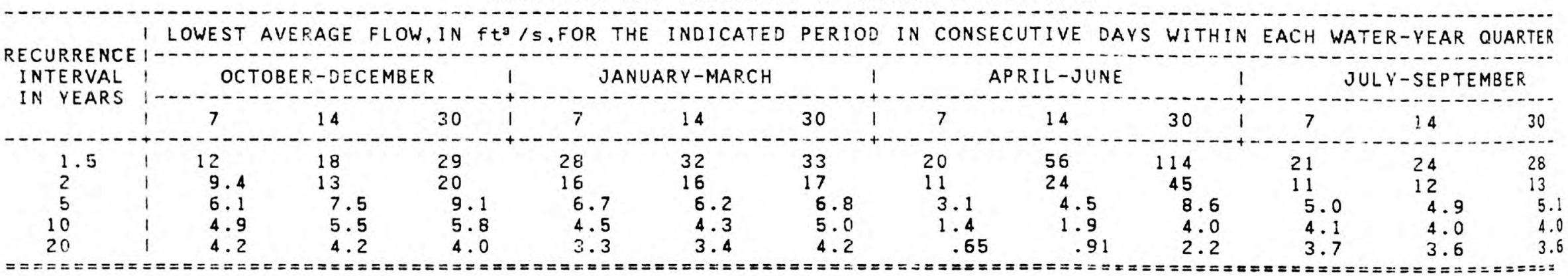

DURATION OF DAILY DISCHARGES FOR ANNUAL ANO SEASONAL PERIODS

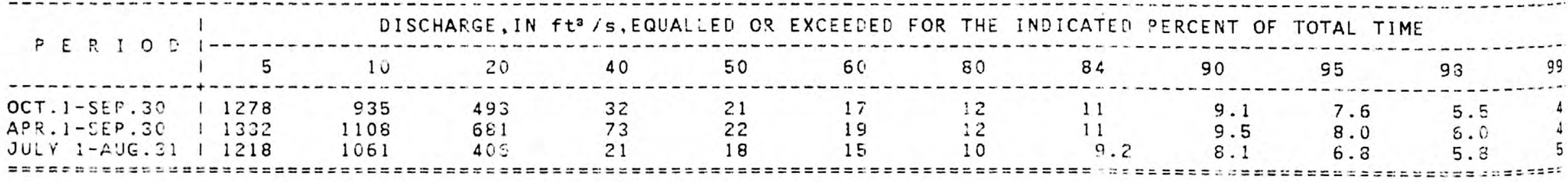




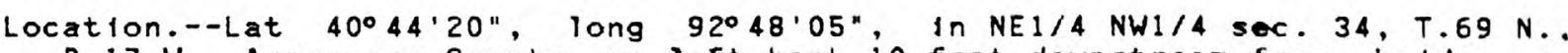
R.17 W. Appanoose County, on left bank 10 feet downstream from bridge on State Highway $2,3.5 \mathrm{mlles}$ downstream from Cooper Creek, and $3 \mathrm{~m} 1 \mathrm{les}$ east of Centerville.

Remarks.--Discontinued Sept. 30, 1959.

LOW FLOW DISCHARGES, IN YEAR ENDING MARCH 31

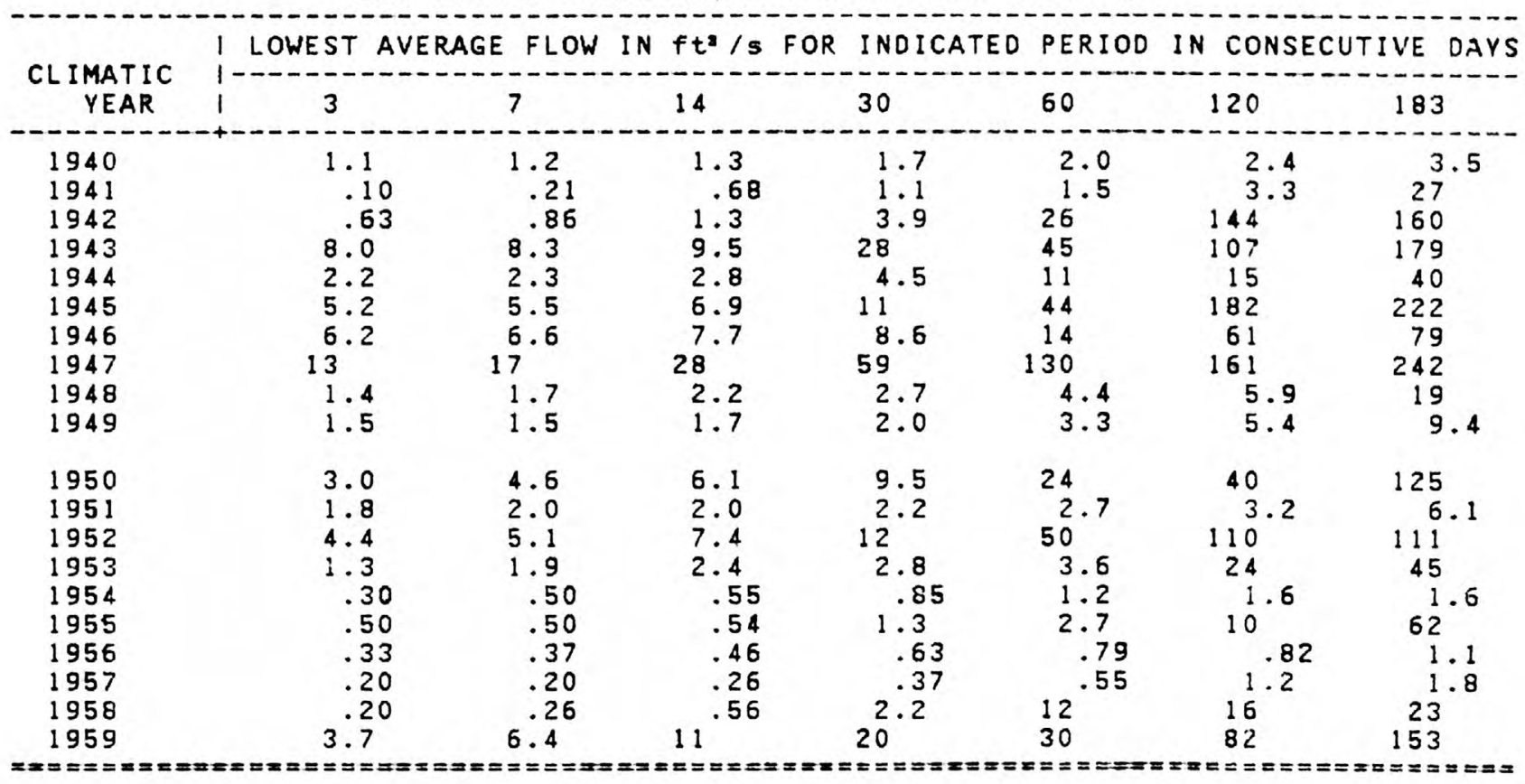




DRAINAGE AREA: $708 \mathrm{~m}^{2} \quad$ PERIOD OF RECORD: 20 YEARS AVERAGE DISCHARGE: 336 ft= /s
MINIMUM AVERAGE FLOWS FOR PERIOD OF RECORD

MAGNITUDE ANO FREQUENCY OF ANNUAL LOW FLOWS

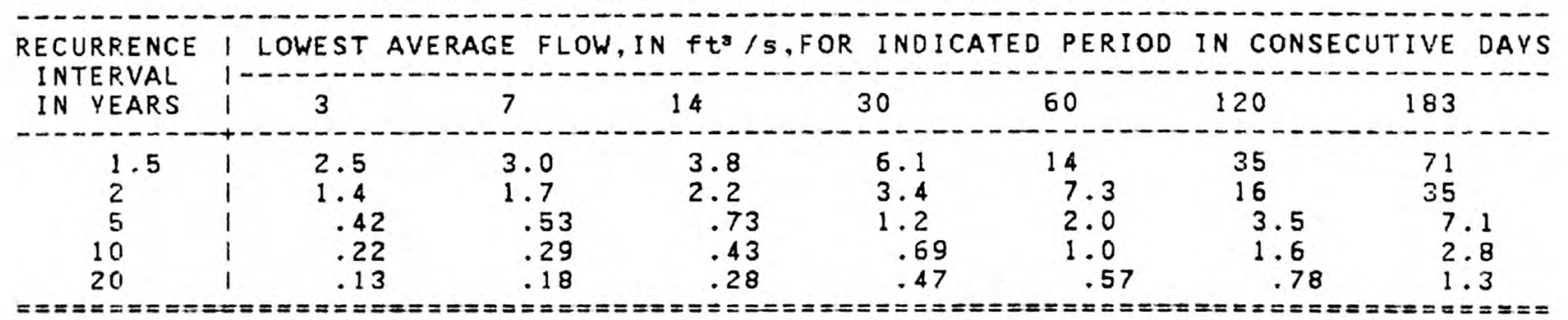

MAGNITUDE AND FREQUENCY OF SEASONAL LOW FLOWS

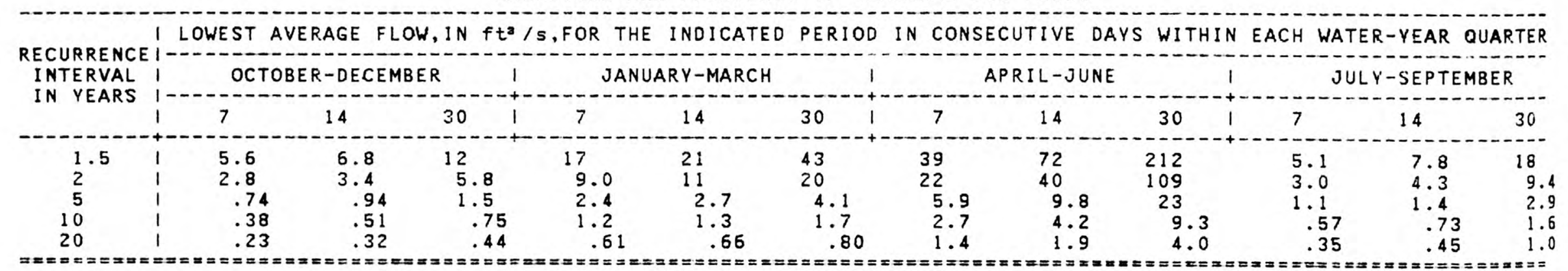

DURATION OF DAILY DISCHARGES FOR ANNUAL AND SEASONAL PERIODS

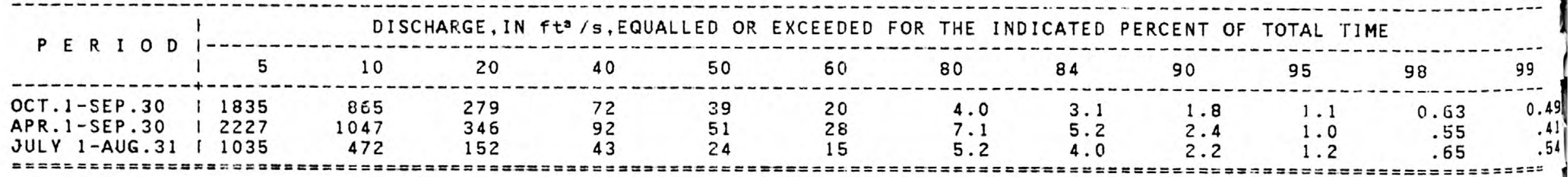


LOW-FLOW PARTIAL-RECORD STATION DATA 

MINNESOTA RIVER BASIN

5-3176.50 BLUE EARTH R NR LAKOTA, IOWA

LAT $4330 \times X$, LONG $9409 \times X$, NEAR SE CORNER

OF SEC.31, T.100 N., R.27 W., KOSSUTH CO.

(55), AT BRIDGE, 4 MILES NE OF LAKOTA.

\section{DRAINAGE AREA $64.6 \mathrm{MI}^{2}$}

$\begin{array}{cr}\text { DATE } & F T / S \\ 09-24-57 & 0.75 \\ 05-13-58 & 5.37 \\ 10-06-58 & 0.39 \\ 10-20-58 & 0.40\end{array}$

$\begin{array}{cr}\text { DATE } & F T / S \\ 08-17-59 & 0.89 \\ 08-16-60 & 1.14 \\ 09-28-61 & 7.26 \\ 10-27-64 & 12.9\end{array}$

$\begin{array}{cr}\text { DATE } & F T / S \\ 08-30-66 & 2.61 \\ 09-22-69 & 2.85 \\ 08-26-70 & 1.50\end{array}$

\section{DATE}

$F T / S$

$10-13-710.94$

0.44

DATE

$08-13-75$

$08-10-76$

$F T / S$

$09-25-74 \quad 0.77$

1.4
0.48

$08-26-70 \quad 1.50$

$7 Q 2=0.9$

$7010=*$

5-3177.00 UNION SLOUGH OUTLET NR LAKOTA, IOWA

LAT $4324 X X$, LONG $9407 X X$, NEAR S $1 / 4$

CORNER OF SEC.11, T.99 N., R.28 W. , KOSSUTH CO.

( 55 ), AT BRIDGE, 2 MILES NW OF LAKOTA.

DRAINAGE AREA $86.4 \mathrm{MI}^{2}$

\begin{tabular}{|c|c|c|c|c|c|c|c|c|c|}
\hline $\begin{array}{c}\text { DATE } \\
09-24-57 \\
05-13-58 \\
10-06-58 \\
10-20-58\end{array}$ & $\begin{array}{r}\mathrm{FT}^{3} / \mathrm{S} \\
0.55 \\
4.24 \\
0.22 \\
0.23\end{array}$ & $\begin{array}{c}\text { DATE } \\
08-17-59 \\
08-16-60 \\
09-28-61 \\
10-27-64\end{array}$ & $\begin{array}{c}\mathrm{FT} / \mathrm{S} \\
1.22 \\
1.37 \\
7.92 \\
25.3\end{array}$ & $\begin{array}{c}\text { DATE } \\
08-30-66 \\
09-22-69 \\
08-26-70\end{array}$ & $\begin{array}{r}F T / S \\
2.22 \\
2.06 \\
0.88\end{array}$ & $\begin{array}{c}\text { DATE } \\
08-23-71 \\
10-13-71 \\
09-25-74\end{array}$ & $\begin{array}{c}F T \cdot / S \\
1.1 \\
0.49 \\
0.76\end{array}$ & $\begin{array}{c}\text { DATE } \\
08-13-75 \\
11-04-75 \\
08-10-76\end{array}$ & $\begin{array}{l}F T / S \\
0 . \\
0 . \\
0.23\end{array}$ \\
\hline Q a & $=19$ & $0<8$ & $=2.2$ & $7 Q 2$ & $=0.8$ & 7010 & * & & \\
\hline
\end{tabular}

5-3178.10 WF BLUE EARTH R BL MINN.-IOWA STATE LINE.

LAT $4326 \times X$, LONG $9404 \times X$, NEAR W $1 / 4$
CORNER OF SEC.36, T.101 N.. R. 28 W., FARIBAULT

CO., MINN., AT BRIDGE, 9 MILES NW OF LAKOTA.

DRAINAGE AREA $154 \quad \mathrm{MI}^{2}$

$\begin{array}{rr}\text { DATE } & \text { FT } / S \\ 09-24-57 & 0.93 \\ 05-13-58 & 8.27 \\ 10-06-58 & 0.53 \\ 10-20-58 & 1.47 \\ Q \mathbf{Q} & =35\end{array}$

$\begin{array}{cc}\text { DATE } & F T^{2} / S \\ 08-17-59 & 1.78 \\ 08-16-60 & 3.26 \\ 09-28-61 & 17.2 \\ 10-27-64 & 36.8 \\ \text { Q(84) } & =4.5\end{array}$

$\begin{array}{rr}\text { DATE } & \mathrm{FT}^{3} / \mathrm{S} \\ 08-30-66 & 4.95 \\ 09-22-69 & 3.94 \\ 08-26-70 & 2.0 \\ 7 Q 2=2.0\end{array}$

DATE
$08-26-71$
$10-13-71$
$09-25-74$

$F T^{2} / S$

1.5

$11-04-75$

$11-04-75$
$08-10-76$

$F T^{3} / \mathrm{S}$

2.5

1.7

$Q_{a}=35$

$Q(84)=4.5$

$7 Q 2=2.0$

$7 Q 10=0.6$ 
UPPER IOWA RIVER BASIN

5-3873.00 UPPER IOWA R AT CHESTER, IOWA

LAT $4330 \times X$, LONG $9222 \times X$, IN SE $1 / 4$ SEC.

10, T. 100 N., R.13 W., HOWARD CO. $(45)$,

AT BRIDGE AT' NORTH CITY LIMITS OF CHESTER.

DRAINAGE AREA I $41 \quad \mathrm{MI}^{2}$

\begin{tabular}{|c|c|c|c|c|c|c|c|c|c|}
\hline $\begin{array}{c}\text { DATE } \\
09-19-57 \\
04-11-58 \\
10-08-58\end{array}$ & $\begin{array}{l}F T^{3} / \mathrm{S} \\
18.8 \\
49.1 \\
5.78\end{array}$ & $\begin{array}{c}\text { DATE } \\
09-15-59 \\
08-09-60 \\
08-30-61\end{array}$ & $\begin{array}{l}F T^{3} / \mathrm{S} \\
17.1 \\
17.8 \\
11.1\end{array}$ & $\begin{array}{c}\text { DATE } \\
08-13-64 \\
09-11-67\end{array}$ & $\begin{array}{c}F T^{2} / S \\
4.28 \\
12.1\end{array}$ & $\begin{array}{c}\text { DATE } \\
09-22-69 \\
09-13-73\end{array}$ & $\begin{array}{l}F T^{3} / \mathrm{S} \\
16.1 \\
26 .\end{array}$ & $\begin{array}{c}\text { DATE } \\
10-01-74 \\
08-31-76\end{array}$ & $\begin{array}{l}\mathrm{FT} / \mathrm{S} \\
20 . \\
7.5\end{array}$ \\
\hline Qa & $=84$ & $Q<\varepsilon$ & $=13$ & $7 Q 2$ & $=7.5$ & 7010 & 4.3 & & \\
\hline
\end{tabular}

5-3874.00 UPPER IOWA $R$ NR KENDALVILLE, IOWA

LAT $4328 \times X$, LONG $9202 \times X$, NEAR CENTER OF

SEC.21, T. 100 N., R.10 W., WINNESHIEK CO. (96),

AT BRIQGE I MILE NORTH OF KENDALVILLE.

DRAINAGE AREA $273 \quad \mathrm{MI}^{2}$

\begin{tabular}{|c|c|c|c|c|c|c|c|c|c|}
\hline $\begin{array}{c}\text { DA.TE } \\
09-18-57 \\
04-11-58 \\
10-07-58\end{array}$ & $\begin{array}{l}F T^{3} / \mathrm{S} \\
44.8 \\
107 . \\
13.3\end{array}$ & $\begin{array}{c}\text { DATE } \\
09-15-59 \\
08-09-60 \\
08-31-61\end{array}$ & $\begin{array}{l}F T^{3} / \mathrm{S} \\
40.4 \\
67.2 \\
29.2\end{array}$ & $\begin{array}{c}\text { DATE } \\
08-12-64 \\
09-12-67\end{array}$ & $\begin{array}{l}F T^{3} / \mathrm{S} \\
16.4 \\
34.3\end{array}$ & $\begin{array}{c}\text { DATE } \\
09-22-69 \\
09-13-73\end{array}$ & $\begin{array}{l}F T^{2} / \mathrm{S} \\
63.4 \\
76 .\end{array}$ & $\begin{array}{c}\text { DATE } \\
10-01-74 \\
08-31-76\end{array}$ & $\begin{array}{l}\mathrm{FT}^{3} / \mathrm{S} \\
62 . \\
31\end{array}$ \\
\hline & $=164$ & $Q<8$ & $\gamma=39$ & 702 & $=22$ & $7 Q 10$ & 13 & & \\
\hline
\end{tabular}

5-3881.00 CANOE CR NR DECORAH, IONA

LAT $4321 \times X$. LONG $9141 \times X$, IN NE $1 / 4$ SEC.

33 , T.99 N., R.7 W., WINNESHIEK CO.(96),

AT BRIDGE, 7 MILES NE OF DECORAH.

DRAINAGE AREA $58.9 \mathrm{MI}^{2}$

\begin{tabular}{|c|c|c|c|c|c|c|c|c|c|}
\hline $\begin{array}{c}\text { DATE } \\
09-18-57 \\
10-07-58 \\
09-15-59\end{array}$ & $\begin{array}{c}\mathrm{FT}^{\circ} / \mathrm{S} \\
12.0 \\
5.00 \\
9.02\end{array}$ & $\begin{array}{c}\text { DATE } \\
08-09-60 \\
08-31-61\end{array}$ & $\begin{array}{l}F T^{3} / \mathrm{S} \\
20.7 \\
7.63\end{array}$ & $\begin{array}{c}\text { DATE } \\
08-12-64 \\
09-12-67\end{array}$ & $\begin{array}{r}\mathrm{FT}^{3} / \mathrm{S} \\
7.56 \\
5.55\end{array}$ & $\begin{array}{c}\text { DATE } \\
\text { O9-23-69 } \\
09-13-73\end{array}$ & $\begin{array}{l}F T^{3} / \mathrm{S} \\
20.1 \\
26 .\end{array}$ & $\begin{array}{c}\text { DATE } \\
10-01-74 \\
08-31-76\end{array}$ & $\begin{array}{l}\mathrm{FT}^{9} / \mathrm{S} \\
23 . \\
14 .\end{array}$ \\
\hline Qa & $=35$ & $O<8$ & $=12$ & $7 Q 2$ & $=7.0$ & 7010 & 4.3 & & \\
\hline
\end{tabular}


UPPER IOWA RIVER BASIN--Cont Inued

5-3883.00 BEAR CR NR HIGHLANDVILLE, IOWA

LAT $4327 X X$. LONG $9137 X X$, IN SE $1 / 4$ SEC.

$25, T .100$ N., R.7 W., WINNESHIEK CO. (95),

AT'BRIDGE, 3 'MILES EAST OF HIGHLANDVILLE.

DRAINAGE AREA $53.4 \quad \mathrm{MI}^{2}$

$\begin{array}{cl}\text { DATE } & F T^{3} / S \\ 09-18-57 & 16.4 \\ 10-07-58 & 11.3 \\ 09-15-59 & 12.6\end{array}$

$Q_{a}=31$

$\begin{array}{rl}\text { DATE } & F T / S \\ 08-09-60 & 21.2 \\ 08-31-61 & 17.6 \\ & \\ 0(84) & =16\end{array}$

$Q(84)=16$

\section{DATE}

$09-23-69$

$08-12-64 \quad 17.9$

$7 Q 2=15$
FT /S

23.1

42 .

$7010=11$

\section{VILLAGE CREEK BASIN}

5-3883.50 VILLAGE CR AT VILLAGE CREEK, IOWA

LAT $4319 \times X$, LONG $9114 \times X$, IN NW $1 / 4$ SEC.

$18, T .98$ N., R.3 W. ALLAMAKEE CO. 13$\}$

AT BRIDGE IN VILLAGE CREEK.

DRAINAGE AREA $58.5 \mathrm{MI}^{2}$

$\begin{array}{rl}\text { DATE } & F T^{3} / S \\ 09-17-57 & 19.2 \\ 04-10-58 & 23.5 \\ 10-07-58 & 18.0 \\ Q \mathbf{Q} & =34\end{array}$

$\begin{array}{cl}\text { DATE } & F T^{T} / S \\ 09-14-59 & 18.5 \\ 08-10-60 & 24.0 \\ 08-31-61 & 19.4 \\ Q(84) & =20\end{array}$

\section{DATE \\ $08-12-64$}

$09-12-67$

$\mathrm{FT}^{\mathrm{3}} / \mathrm{S}$

19.9

$7 Q 2=19$

YELLOW RIVER BASIN

5-3888.00 YELLOW R AT MYRON, IOWA

LAT $4310 \times X$, LONG $9132 \times X$, IN NE $1 / 4$ SEC.

3 , T.96 N., R. 6 W., ALLAMAKEE CO. $(3)$

AT BRIDGE, 0.5 MILE SOUTH OF MYRON.

DRAINAGE AREA $59.5 \mathrm{MI}^{2}$

$\begin{array}{cc}\text { DATE } & \mathrm{FT}^{3} / \mathrm{S} \\ 09-18-57 & 10.2 \\ 10-07-58 & 3.76 \\ 09-14-59 & 7.83\end{array}$

$\begin{array}{cc}\text { DATE } & F T^{3} / S \\ 08-10-60 & 19.8 \\ 09-01-61 & 6.67\end{array}$

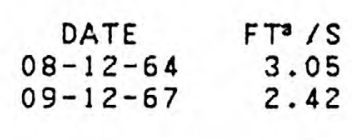

$7 Q 2=4.1$

$Q_{\mathbf{a}}=35$

$Q(84)=7.0$

DATE

$09-01-76$

$F T^{\circ} / \mathrm{S}$

39.

26. 
YELLOW RIVER BASIN--Cont inued

5-3890.00 YELLOW R AT ION, IOWA

LAT $4307 \times X$, LONG $9115 \times X$, IN SW $1 / 4$ SEC.

24, T.96 N., R.4 W. ALLAMAKEE CO.13\},

AT BRIDGE, 7.5 MILES NW OF MCGREGOR.

DRAINAGE AREA $22.1 \quad \mathrm{MI}^{2}$

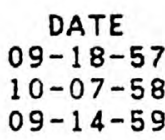

$F T^{3} / \mathrm{S}$
51.6
19.4

$\begin{array}{cc}\text { DATE } & F T^{3} / \mathrm{S} \\ 08-10-50 & 113 . \\ 08-31-61 & 49.7\end{array}$

$\begin{array}{ll}\text { DATE } & F T / S \\ 08-12-64 & 28.6 \\ 09-12-67 & 20.9\end{array}$

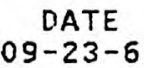

$09-23-69$

$F T^{3} / S$

63.1

84.1

DATE

$10-03-74$

$09-02-76$

$F T \cdot / S$

46.3

$Q(84)=45$

$7 Q 2=28$

$7010=14$

\section{TUR.KEY RIVER BASIN}

5-4115.50 NB TURKEY R NR VEFNON SPRINGS, IOWA

LAT $4321 \times X$, LONG $9211 \times X$, IN SW $1 / 4$ SEC.

31 , T.99 N., R.11 W., HOWARD CO. ( 45 ),

AT BRIDGE, 3 MILES WEST OF VERNON SPRINGS.

DRAINAGE AREA $40.1 \mathrm{MI}^{2}$

$\begin{array}{rc}\text { DATE } & F T / S \\ 09-19-57 & 3.47 \\ 04-11-58 & 10.7 \\ 10-08-58 & 1.42 \\ \text { Oa } & =23\end{array}$

DATE
$09-15-59$
$08-09-60$

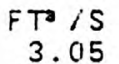
DATE
$08-30-61$
$08-13-64$
$\mathrm{FT}^{3} / \mathrm{S}$
1.73
.00

6.59

$Q(84)=3.0$

$7 Q 2=1.6$

$\begin{array}{cc}\text { DATE } & \text { FTO } / S \\ 09-11-67 & 2.08 \\ 09-13-73 & 5.9\end{array}$

DATE

$10-01-74$
$08-31-76$

F to $/ 2$

3.4

$7010=0.8$

5-4115.60 TURKEY R. NR VERNON SPRINGS, IOWA

LAT $4320 \times X$, LONG $9207 \times X$. IN NW $1 / 4$ SEC.

2. T.9\& N., R.11 W. HDWARD CO. $(45)$

AT BRIDGE, 2.5 MILES SOUTH OF VERNON'SPRINGS.

DRAINAGE AREA $87.0 \mathrm{MI}^{2}$

$\begin{array}{cr}\text { DATE } & F^{T} / S \\ 09-19-57 & 6.94 \\ 04-11-58 & 2.96 \\ 10-08-58 & 1.62 \\ & =51\end{array}$

$\begin{array}{cr}\text { DATE } & \text { FT' } / S \\ 09-15-59 & 7.04 \\ 08-09-60 & 16.10 \\ & \end{array}$

$\begin{array}{cr}\text { DATE } & F T^{3} / S \\ 08-30-61 & 4.44 \\ 08-13-64 & 1.43 \\ 7 Q 2= & 2.5\end{array}$

$\begin{array}{cc}\text { DATE } & F T / S \\ 09-11-67 & 3.69 \\ 09-13-73 & 11 .\end{array}$

DATE
$10-01-7$
$08-31-7$

$F T / S$

$08-31-76 \quad 11.3$
$Q(84)=5.0$

$7 Q 2=2.5$ 
TURKEY RIVER BASIN--Cont inued

5-4116.20 L TURKEY R NR WAUCOMA, IOWA

LAT $4301 \times X$, LONG $9159 \times X$, IN NW $1 / 4$ SEC.

25, T.95 N., R.10 W., FAYETTE CO. (33),

AT BRIDGE, 4 MILES SÉ OF WAUCOMA.

DRAINAGE AREA 102 MI2

$\begin{array}{rl}\text { DATE } & F T^{3} / \mathrm{S} \\ 09-23-57 & 12.5 \\ 10-09-58 & 10.10 \\ 09-16-59 & 21.00 \\ & \mathbf{Q a}=60\end{array}$

DATE FT /S

$08-11-60 \quad 23.80$

$\begin{array}{cl}\text { DATE } & F T^{\mathrm{a}} / \mathrm{S} \\ 08-11-64 & 10.8 \\ 09-11-67 & 10.4\end{array}$

OATE

$06-11-68$

$F T^{2} / S$

29.1

DATE

$09-30-74$

$F T^{3} / S$

$08-29-61 \quad 31.30$

$7 Q 2=13$

$7010=5.2$

5-4117.00 CRANE CR NR LOURDES, IOWA

LAT $4315 X X$, LONG $9219 \times X$, IN NW $1 / 4$ SEC.

$6, T .97 \mathrm{~N} .$, R.12 W., HOWARD CO. (45),

AT BRIDGE ON STATE HIGHWAY 272, 1 MILE

$S W$ OF LOURDES.

DRAINAGE AREA $75.8 \mathrm{MI}^{2}$

$\begin{array}{rc}\text { DATE } & \text { FT }^{3} / \mathrm{S} \\ 09-20-57 & 4.19 \\ 04-11-58 & 19.1 \\ 10-08-58 & 1.30 \\ Q a & =45\end{array}$

$\begin{array}{rr}\text { DATE } & F T \text { iS } \\ 09-15-59 & 4.76 \\ 08-09-60 & 7.17 \\ & \\ Q(84)= & 4.0\end{array}$

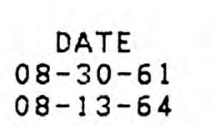

$F T^{3} / S$

5.47

$08-13-64$

$7 Q 2=2.0$

\section{DATE
$09-11-67$ \\ $09-13-73$}

$F T^{3} / S$

2.01

$7010=0.5$

5-4118.00 L TURKEY R NR ALPHA, IOWA

LAT $4301 \times X$, LONG $9157 \times X$, IN SW $1 / 4$ SEC.

30, T.95 N., R. 9 W. , FAYETTE CO. $(33)$,

AT BRIDGE, 3 MILES NE OF ALPHA.

DRAINAGE AREA $319 \quad \mathrm{MI}^{2}$

$\begin{array}{cl}\text { DATE } & \mathrm{FT}^{3} / \mathrm{S} \\ 09-23-57 & 28.9 \\ 10-09-58 & 21.6 \\ 09-16-59 & 53.9\end{array}$

$Q \mathbf{a}=193$

$\begin{array}{rl}\text { DATE } & F T^{2} / S \\ 08-11-60 & 59.5 \\ 08-29-61 & 90.8 \\ Q(84) & =46\end{array}$

$Q(84)=46$

\section{DATE}

$08-11-64$

$\mathrm{FT}^{\mathrm{a}} / \mathrm{S}$

22.4

22.2
DATE

06-11-68

$09-13-73$

$F T / S$

96.2

54.

DATE

$09-30-74$

08-31-76

FTP /S

45

28 . 
TURKEY RIVER BASIN--Continued

5-4121.00 ROBERTS CR AB ST. OLAF, IOWA

LAT 425549, LONG 912303 , IN NW $1 / 4$ SEC.

25, T.94 N., R.5 W., CLAYTON CO. (22),

AT BRIDGE NEAR NORTH CITY LIMITS OF ST. OLAF.

DRAINAGE AREA $70.7 \quad \mathrm{MI}^{2}$

\begin{tabular}{|c|c|c|c|c|c|c|c|c|c|}
\hline $\begin{array}{c}\text { DATE } \\
09-17-57 \\
10-06-58\end{array}$ & $\begin{array}{l}F T / S \\
0.07 \\
0.03\end{array}$ & $\begin{array}{c}\text { DATE } \\
09-14-59 \\
08-11-60\end{array}$ & $\begin{array}{l}\mathrm{FT}^{3} / \mathrm{S} \\
0.52 \\
4.77\end{array}$ & $\begin{array}{c}\text { DATE } \\
09-01-61 \\
08-11-64\end{array}$ & $\begin{array}{l}\mathrm{FT} / \mathrm{S} \\
0.22 \\
0.01\end{array}$ & $\begin{array}{c}\text { DATE } \\
09-12-73 \\
09-30-74\end{array}$ & $\begin{array}{c}F T / S \\
9.8 \\
6.3\end{array}$ & $\begin{array}{c}\text { DATE } \\
08-31-76\end{array}$ & $\begin{array}{r}\mathrm{FT}^{2} / \mathrm{S} \\
0.02\end{array}$ \\
\hline & $=42$ & Q & $=0.1$ & $7 Q$ & $=\star$ & 7010 & 0 & & \\
\hline
\end{tabular}

5-4121.50 ROBERTS CR AT ST. OLAF, IOWA

LAT 425542, LONG 912301, IN SW $1 / 4$ SEC.

AT BRIDGE NEAR EAST' CITY LIMITS OF ST: OLAF.

DRAINAGE AREA $101 \quad \mathrm{MI}^{2}$

\begin{tabular}{|c|c|c|c|c|c|c|c|c|c|}
\hline $\begin{array}{c}\text { DATE } \\
09-17-57 \\
10-06-58\end{array}$ & $\begin{array}{r}\mathrm{FT}^{3} / \mathrm{S} \\
0.07 \\
0.11\end{array}$ & $\begin{array}{c}\text { DATE } \\
09-14-59 \\
08-11-60\end{array}$ & $\begin{array}{r}\mathrm{FT}^{3} \text { is } \\
1.74 \\
7.61\end{array}$ & $\begin{array}{c}\text { DATE } \\
09-01-61 \\
08-11-64\end{array}$ & $\begin{array}{r}\mathrm{FT}^{3} / \mathrm{S} \\
0.41 \\
0.06\end{array}$ & $\begin{array}{c}\text { DATE } \\
09-12-73 \\
09-30-74\end{array}$ & $\begin{array}{l}F T / S \\
10 . \\
9.7\end{array}$ & $\begin{array}{c}\text { DATE } \\
08-31-76\end{array}$ & $\begin{array}{r}\mathrm{FT}^{3} / \mathrm{S} \\
0.85\end{array}$ \\
\hline Qa & $=60$ & $Q<8$ & $=0.1$ & $7 Q 2$ & $=*$ & 7010 & $=0$ & & \\
\hline
\end{tabular}

5-4122.00 VOLGA $R$ NR FAYETTE, IOWA

LAT $4249 \times X$, LONG $9153 \times X$. IN SW $1 / 4$ SEC.

$35, T .93 \mathrm{~N} ., \mathrm{R} .9 \mathrm{~W}$. FAYETTE CO, $\{33$ \},

AT BRIDIE, 4.5 MILES SW OF FAYETTE.

DRAINAGE AREA $53.0 \mathrm{MI}^{2}$

\begin{tabular}{|c|c|c|c|c|c|c|c|c|c|}
\hline $\begin{array}{c}\text { DATE } \\
09-23-57 \\
10-09-58 \\
09-16-59\end{array}$ & $\begin{array}{r}F T^{3} / S \\
3.16 \\
4.98 \\
5.18\end{array}$ & $\begin{array}{c}\text { DATE } \\
08-08-60 \\
08-28-61\end{array}$ & $\begin{array}{c}F T^{3} \text { is } \\
8 . E_{1} 8 \\
10 . \varepsilon\end{array}$ & $\begin{array}{c}\text { DATE } \\
08-11-64 \\
09-11-67\end{array}$ & $\begin{array}{r}F T^{0} / \mathrm{S} \\
1.54 \\
2.18\end{array}$ & $\begin{array}{c}\text { DATE } \\
06-10-68 \\
09-13-73\end{array}$ & $\begin{array}{l}\mathrm{FT}^{\mathrm{S}} / \mathrm{S} \\
7.40 \\
5.7\end{array}$ & $\begin{array}{c}\text { DATE } \\
09-30-74 \\
08-30-76\end{array}$ & $\begin{array}{c}F T / / S \\
5.0 \\
3.1\end{array}$ \\
\hline & $=31$ & $Q<$ & $\gamma=3.9$ & $7 Q 2$ & $=2.8$ & 7010 & 1.6 & & \\
\hline
\end{tabular}


TURKEY RIVER BASIN--Cont inued

5-4123.00 L VOLGA R NR FAYETTE, IOWA

LAT $4249 \times X$, LONG $9153 \times X$, NEAR S $1 / 4$

CORNER OF SEC. $35, T .93 \mathrm{~N}$., R.9 W., FAYETTE CO.

(33), AT BRIDGE, 4 MILES SW OF FAYETTE.

DRAINAGE AREA $31.0 \quad \mathrm{MI}^{2}$

\begin{tabular}{|c|c|c|c|c|c|c|c|c|c|}
\hline $\begin{array}{c}\text { DATE } \\
09-23-57 \\
10-09-58 \\
09-16-59\end{array}$ & $\begin{array}{l}\mathrm{FT}^{3} / \mathrm{S} \\
2.06 \\
1.40 \\
2.08\end{array}$ & $\begin{array}{c}\text { DATE } \\
08-08-60 \\
08-28-61\end{array}$ & $\begin{array}{r}\mathrm{FT} \text { /S } \\
3.06 \\
6.95\end{array}$ & $\begin{array}{c}\text { DATE } \\
08-11-64 \\
09-11-67\end{array}$ & $\begin{array}{r}\mathrm{FT}^{2} / \mathrm{S} \\
0.65 \\
0.97\end{array}$ & $\begin{array}{c}\text { DATE } \\
06-10-68 \\
09-13-73\end{array}$ & $\begin{array}{c}\mathrm{FT}^{\mathrm{T}} / \mathrm{S} \\
8.24 \\
3.3\end{array}$ & $\begin{array}{c}\text { DATE } \\
09-30-74 \\
08-30-76\end{array}$ & $\begin{array}{l}\mathrm{FT} / \mathrm{S} \\
2.4 \\
2.1\end{array}$ \\
\hline$Q$ & $=18$ & $Q(8$ & $\gamma=2.4$ & $7 Q 2$ & $=1.6$ & $7 Q 10$ & 0.4 & & \\
\hline
\end{tabular}

5-4124.00 VOLGA R AT LITTLEPORT, IOWA

LAT 424514, LONG 912208 , IN SE $1 / 4$ SEC.

25, T.92 N., R.5 W., CLAYTON CO. $\{22\}$,

AT BRIDGE IN LITTLEPORT.

DRAINAGE AREA $348 \quad \mathrm{MI}^{2}$

\begin{tabular}{|c|c|c|c|c|c|c|c|c|c|}
\hline $\begin{array}{c}\text { DATE } \\
09-17-57 \\
10-06-58\end{array}$ & $\begin{array}{l}\mathrm{FT}^{3} / \mathrm{S} \\
35.9 \\
21.1\end{array}$ & $\begin{array}{c}\text { DATE } \\
09-14-59 \\
08-11-60\end{array}$ & $\begin{array}{l}F T^{2} / \mathrm{S} \\
47.1 \\
88.3\end{array}$ & $\begin{array}{c}\text { DATE } \\
08-28-61 \\
08-11-64\end{array}$ & $\begin{array}{l}\mathrm{FT}^{2} / \mathrm{S} \\
103 . \\
21.3\end{array}$ & $\begin{array}{c}\text { DATE } \\
09-12-73 \\
09-30-74\end{array}$ & $\begin{array}{l}F T^{\circ} / \mathrm{S} \\
127 . \\
93 .\end{array}$ & $\begin{array}{c}\text { DATE } \\
08-31-76\end{array}$ & $\begin{array}{l}F T^{3} / S \\
46 .\end{array}$ \\
\hline$Q_{z}$ & $=211$ & QC & $=52$ & 702 & $=34$ & 7010 & $=17$ & & \\
\hline
\end{tabular}

LITTLE MAQUOKETA RIVER BASIN

5-4144.50 NF LITTLE MAQUOKETA RIVER NR RICKARDSVILLE, IOWA

LAT 423509, LONG 905120, NEAR NW CORNER

SEC. 28, T.90 N., R.1 E., DUBUQUE CO. (31), AT BRIDGE, 1 MILE NE OF RICKARDSVILLE.

DRAINAGE AREA $21.6 \mathrm{MI}^{2}$

$\begin{array}{rr}\text { DATE } & F T^{2} / S \\ 09-25-57 & 0.47 \\ 10-06-58 & 0.29 \\ 09-16-59 & 0.59 \\ Q \mathbf{Q} & =13\end{array}$

$\begin{array}{cr}\text { DATE } & F T / S \\ 08-17-60 & 2.68 \\ 08-28-61 & 0.93 \\ 08-11-64 & 0.26\end{array}$

$\begin{array}{cr}\text { DATE } & \text { FT } / S \\ 10-05-66 & 0.56 \\ 09-23-69 & 2.19 \\ & \end{array}$

$\begin{array}{cc}\text { DATE } & F T / S \\ 09-12-73 & 1.7 \\ 09-30-74 & 2.3\end{array}$

DATE

$10-07-75$

$F T^{2} / S$

$08-31-76$

1.1

$Q(84)=0.8$

$7 Q 2=0.6$

$7 Q 10=0.3$ 
MAQUOKETA RIVER BASIN

5-4163.00 MAQUOKETA RIVER NR DUNDEE, IOWA

$$
\begin{aligned}
& \text { LAT } 423655, \text { LONG } 913344 \text {, IN SW } 1 / 4 \text { SEC } \\
& 9 \text { T T } 90 \mathrm{~N} \% \text { R.6 W. DELAWARE CO. } 128 \% \text {, } \\
& \text { AT BRIDGE, 2.5 MILES NORTH OF DUNDEE. }
\end{aligned}
$$

DRAINAGE AREA $61.1 \mathrm{MI}^{2}$

$Q(84)=13$

$7 Q 2=11$

5-4164.00 SF MAQUOKETA R NR DUNDEE, IOWA

LAT 423608, LONG 913513, IN SW $1 / 4$ SEC. 17 , T.90 N., R.6 W., DELAWARE CO. (28), AT BRILGE, 2.5 MILES NW OF DUNDEE.

DRAINAGE AREA $54.8 \mathrm{MI}^{2}$
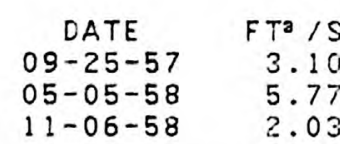

3.10

2.03

DATE
$09-1 E-59$
$08-17-60$

$\mathrm{FT}^{3}$ /S
4.54
8.62

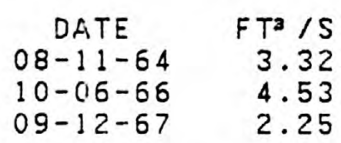

Q $=32$

$Q(84)=5.6$

$702=4.1$

5-4175.40 PLUM CR NR EARLVILLE, IOWA

LAT 422.604, LONG 911358 , IN NE $1 / 4$ SEC.

$18, T .88$ N., R.3 W., DELAWARE CO. $(28)$,

AT BRIIIGE, 4 MILES SE OF EARLVILLE.

DRAINAGE AREA $65.7 \mathrm{MI}^{2}$

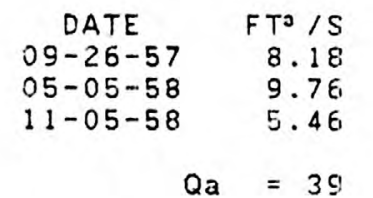

$$
\begin{array}{cc}
\text { DATE } & F T^{3} / S \\
09-16-59 & 7.86 \\
08-17-60 & 17.5 \\
08-29-61 & 11.5
\end{array}
$$$$
Q(84)=12
$$

$Q \mathbf{a}=39$

$Q(84)=12$

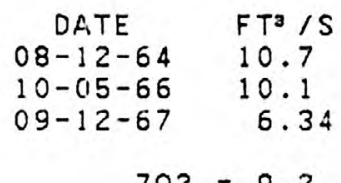

$\begin{array}{rl}\text { DATE } & F T / S \\ 09-22-69 & 20.1 \\ 09-12-73 & 22 . \\ 09-30-74 & 15 . \\ 7 Q 10 & =6.7\end{array}$

DAT $10-07-75$ $08-31-76$
$F^{3} / S$

12 .

DATE

09-23-69

$09-12-73$

10-01-74

$F T^{3} / S$

25.3

27.

$7 Q 10=5.0$ 
MAQUOKETA RIVER BASIN--COnt inued

5-4175.60 MAQUOKETA R NR HOPKINTON, IOWA

LAT $4222 \times X$, LONG $9116 \times X$, IN NE $1 / 4$ SEC.

$11, \mathrm{~T} .87 \mathrm{~N} ., \mathrm{R} .4 \mathrm{~W}$. , DELAWARE CO. (28),

AT BRIDGE, 2 MILES NW OF HOPKINTON.

DRAINAGE AREA $454 \mathrm{MI}^{2}$

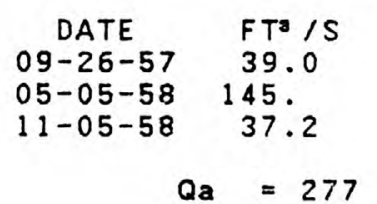

$Q_{\mathbf{a}}=277$

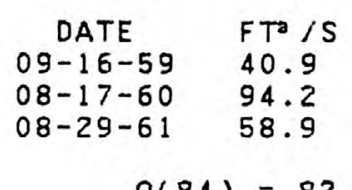

$Q(84)=82$

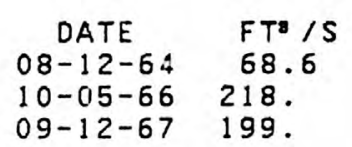

$09-12-67 \quad 199$.

5-4175.80 BUCK CR NR HOPKINTON, IOWA

LAT $4221 \times X$, LONG $9117 \times X$, IN SE $1 / 4$ SEC.

10, T.87 N., R. 4 W., DELAWARE CO. (28),

AT BRIDGE, 2.5 MILES NW OF HOPKINTON.

\begin{abstract}
DRAINAGE AREA $50.7 \mathrm{MI}^{2}$
\end{abstract}

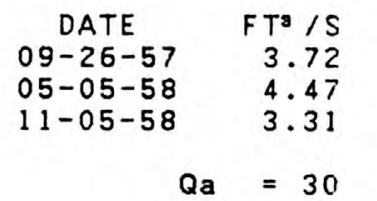

$\begin{array}{cc}\text { DATE } & F T^{3} / S \\ 09-16-59 & 6.38 \\ 08-17-60 & 13.4 \\ 08-29-61 & 6.49 \\ Q(84)=7.3\end{array}$

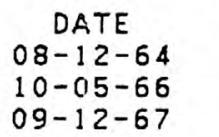

$\mathrm{FT}^{\mathrm{a}} / \mathrm{S}$

8.21
7.74

$09-12-67$

5.43

$Q \mathbf{a}=30$

$Q(84)=7.3$

$7 Q 2=5.9$

5-4176.00 MAQUOKETA R NR SCOTCH GROVE, IOWA

LAT $4212 \times X$, LONG $9101 \times X$, NEAR CENTER OF SEC. 6, T.85 N., R. $1 \mathrm{~W}$., JONES CO. (53), AT BRIDGE ON STATE HIGHWAY 136, 6 MILES NE OF SCOTCH GROVE.

DRAINAGE AREA $704 \quad \mathrm{MI}^{2}$

$\begin{array}{rc}\text { DATE } & F T^{3} / S \\ 09-24-57 & 131 . \\ 05-06-58 & 63.1 \\ 11-05-58 & 91.1 \\ Q a & =433\end{array}$

$\begin{array}{ll}\text { DATE } & F^{3} / S \\ 09-17-59 & 111 \\ 08-18-60 & 307 \\ 08-30-61 & 206\end{array}$

$\begin{array}{cl}\text { DATE } & F T^{\circ} / \mathrm{S} \\ 08-12-64 & 179 \\ 09-13-66 & 210 \\ 10-05-66 & 213\end{array}$

$Q(84)=139$

$702=107$

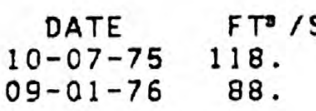

$09-22-69 \quad 216$

$09-12-73 \quad 151$.

$09-01-76$

88 .

$7010=34$

$\begin{array}{rlcc}\text { DATE } & \text { FT /S } & \text { DATE } & F^{3} / S \\ 09-22-69 & 16.2 & 10-07-75 & 7.1 \\ 09-12-73 & 12 . & 09-01-76 & 5.7 \\ 10-01-74 & 13 . & & \\ 7 Q 10 & =3.5 & & \end{array}$

$\begin{array}{clcc}\text { DATE } & F T^{3} / S & \text { DATE } & F T / S \\ 09-23-69 & 310 . & 10-07-75 & 141 . \\ 09-30-74 & 246 . & 08-30-76 & 128 .\end{array}$

$7010=59$ 
MAQUOKETA RIVER BASIN--Continued

5-4181.00 NF MAQUOKETA R AT DYERSVILLE, IOWA

LAT 422905, LONG 910726 , IN NW $1 / 4$ SEC.

$31, T .89$ N., R.2 W., DUBUQUE CO. $\{31\rangle$,

AT BRIDGE, IN DYERSVILLE.

DRAINAGE AREA $80.2 \mathrm{MI}^{2}$

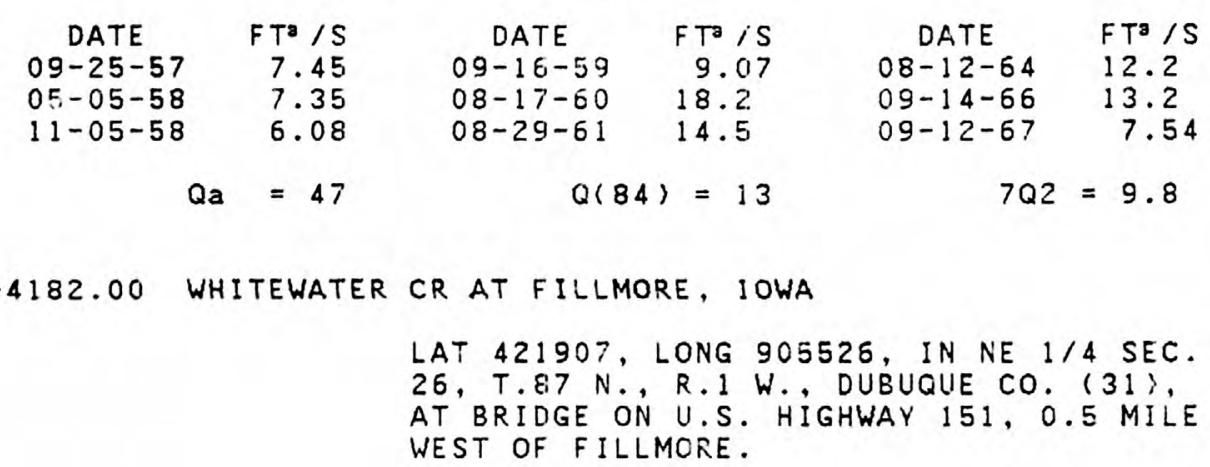

DRAINAGE AREA $91.9 \mathrm{MI}^{2}$

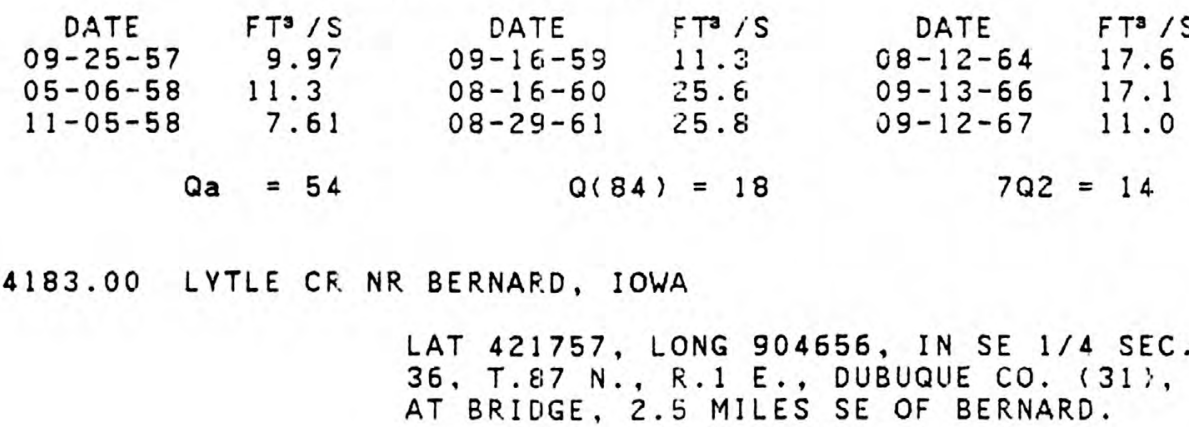

DRAINAGE AREA $62.7 \quad \mathrm{MI}^{2}$

$\begin{array}{rr}\text { DATE } & F T^{3} / S \\ 09-25-57 & 6.72 \\ 05-06-58 & 8.61 \\ 11-05-58 & 5.56 \\ Q a & =37\end{array}$

$\begin{array}{cc}\text { DATE } & F T^{3} / S \\ 09-16-59 & 7.610 \\ 08-16-60 & 18.2 \\ 08-29-61 & 17.8 \\ a(84)=13\end{array}$

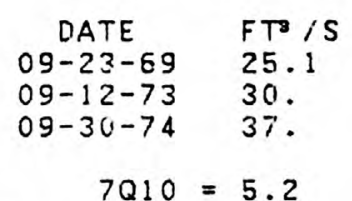

DATE
$10-07-75$

$10-07-75$
$08-31-76$

$F T^{2} / S$

17 .

11 .

$$
7 Q 10=5.2
$$


MAQUOKETA RIVER BASIN--Continued

5-4183.50 LYTLE CR NR FULTON, IOWA

LAT $4212 \times X$, LONG $9045 \times X$, NEAR CENTER OF SEC. 5, T. 85 N., R.2 E., JACKSON CO. (49), AT BRIDGE, 5 MILES NW OF FULTON.

DRAINAGE AREA $114 \quad \mathrm{MI}^{2}$

\begin{tabular}{|c|c|c|c|c|c|c|c|c|c|}
\hline $\begin{array}{c}\text { DATE } \\
09-24-57 \\
05-06-58 \\
11-04-58\end{array}$ & $\begin{array}{l}F T^{\mathrm{a}} / \mathrm{S} \\
15.5 \\
17.8 \\
12.1\end{array}$ & $\begin{array}{c}\text { DATE } \\
09-17-59 \\
08-16-60 \\
08-29-61\end{array}$ & $\begin{array}{l}\mathrm{FT}^{3} / \mathrm{S} \\
14.6 \\
38.6 \\
35.4\end{array}$ & $\begin{array}{c}\text { DATE } \\
08-11-64 \\
09-13-66 \\
09-11-67\end{array}$ & $\begin{array}{l}F T^{3} / \mathrm{S} \\
30.8 \\
32.0 \\
21.0\end{array}$ & $\begin{array}{c}\text { DATE } \\
09-24-69 \\
09-13-73 \\
10-01-74\end{array}$ & $\begin{array}{l}\mathrm{FT} / \mathrm{S} \\
44.3 \\
23 . \\
73 .\end{array}$ & $\begin{array}{c}\text { DATE } \\
10-07-75 \\
08-31-76\end{array}$ & $\begin{array}{l}\mathrm{FT}^{3} / \mathrm{S} \\
47 . \\
28 .\end{array}$ \\
\hline$Q$ & $=68$ & $Q<\varepsilon$ & $=25$ & 702 & $=18$ & $7 Q 10$ & 8.2 & & \\
\hline
\end{tabular}

5-4184.00 NF MAQUOKETA R NR FULTON, IOWA

LAT $4211 X X$, LONG $9044 \times X$, IN SE $1 / 4$ SEC.

9 , T.85 N., R.2 E., JACKSON CO. ( 49$)$,

AT BRIDGE, 3 MILES NW OF FULTON.

\begin{tabular}{|c|c|c|c|}
\hline & DRA I NAGE & AREA & $M i^{2}$ \\
\hline $\begin{array}{c}\text { DATE } \\
09-24-57 \\
05-06-58 \\
11-04-58\end{array}$ & $\begin{array}{l}F T^{3} / \mathrm{S} \\
74.7 \\
75.8 \\
61.6\end{array}$ & $\begin{array}{c}\text { DATE } \\
09-17-59 \\
08-16-60 \\
08-29-61\end{array}$ & $\begin{array}{l}F T^{\circ} / \mathrm{S} \\
78.7 \\
148 . \\
151 .\end{array}$ \\
\hline Qa & $=305$ & $Q<8$ & 4) $=122$ \\
\hline
\end{tabular}

\begin{tabular}{rl} 
DATE & \multicolumn{1}{c}{$F T^{3} / \mathrm{S}$} \\
$08-11-64$ & 119. \\
$09-13-66$ & 120. \\
$09-12-67$ & 78.2 \\
$7 Q 2$ & $=94$
\end{tabular}
DATE $F T^{3} / \mathrm{S}$ 09-24-69 206 . $10-01-74 \quad 254$. $10-07-75 \quad 170$ 08-31-76 112. $Q_{a}=305$ $Q(84)=122$

$7010=52$

5-4186.50 DEEP CR NR CHARLOTTE, IOWA

LAT $4200 \times X$, LONG $9024 \times X$, NEAR CENTER OF SEC.17, T.83 N., R.5 E., CLINTON CO. (23), AT BRIDGE, 4 MILES NE OF CHARLOTTE.

DRAINAGE AREA $67.7 \mathrm{MI}^{2}$

$\begin{array}{rc}\text { DATE } & F^{3} / S \\ 09-24-57 & 3.52 \\ 05-07-58 & 11.5 \\ 11-04-58 & 2.39 \\ \text { Qa } & =40\end{array}$

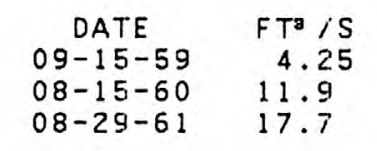

$Q(84)=7.3$

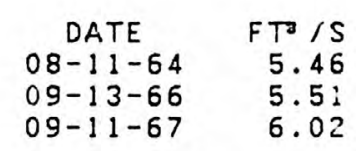

$702=5.2$

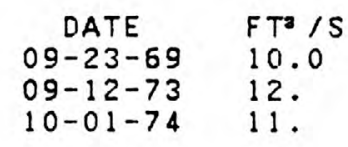

$7 Q 10=2.4$ 
MAQUOKETA RIVER BASIN--Continued

5-4187.00 DEEP CR NR PRESTON, IOWA

LAT $4203 \times X$, LONG $9026 \times X$. NEAR $N 1 / 4$

CORNER OF SEC.31, T.84 N., R.5 E., JACKSON CO.

(49), AT BRIDGE, 2 MILES WEST OF PRESTON.

DRAINAGE AREA $91.9 \mathrm{MI}^{2}$

\begin{tabular}{|c|c|c|c|c|c|c|c|c|c|}
\hline $\begin{array}{c}\text { DATE } \\
09-24-57 \\
03-07-58 \\
11-04-58\end{array}$ & $\begin{array}{c}F T^{3} / S \\
4.72 \\
14.8 \\
3.79\end{array}$ & $\begin{array}{c}\text { DATE } \\
09-15-59 \\
08-16-60 \\
08-29-61\end{array}$ & $\begin{array}{l}\mathrm{FT}^{3} \text { is } \\
6.77 \\
18.2 \\
26.0\end{array}$ & $\begin{array}{c}\text { DATE } \\
08-11-64 \\
09-13-66 \\
09-11-67\end{array}$ & $\begin{array}{r}\mathrm{FT}^{3} / \mathrm{S} \\
8.62 \\
7.95 \\
10.0\end{array}$ & $\begin{array}{c}\text { DATE } \\
09-23-69 \\
09-12-73 \\
10-01-74\end{array}$ & $\begin{array}{l}\mathrm{FT}^{3} / \mathrm{S} \\
15.0 \\
0 . \\
19 .\end{array}$ & $\begin{array}{c}\text { DATE } \\
10-07-75 \\
09-01-76\end{array}$ & $\begin{array}{l}F T^{\circ} / S \\
11.0 \\
5.0\end{array}$ \\
\hline Qa & $=54$ & $Q<8$ & $y=11$ & $7 Q 2$ & $=8.2$ & $7 Q 10$ & $=4.1$ & & \\
\hline
\end{tabular}

ELK. RIVER BASIN

5-4203.00 ELK R NR ALMONT, IOWA

LAT 420039 , LONG 901205, NEAR CENTER OF SEC.12, T.83 N., R.6 E., CLINTON CO. (23), AT BRILGE, 2.5 MILES NORTH OF ALMONT.

DRAINAGE AREA $55.9 \mathrm{MI}^{2}$

\begin{tabular}{|c|c|c|c|c|c|c|c|c|c|}
\hline $\begin{array}{c}\text { DATE } \\
09-24-57 \\
05-07-58 \\
11-04-58\end{array}$ & $\begin{array}{c}\mathrm{FT}^{3} / \mathrm{S} \\
5.15 \\
11.8 \\
3.75\end{array}$ & $\begin{array}{c}\text { DATE } \\
\text { O9-15-59 } \\
08-15-60 \\
08-31-61\end{array}$ & $\begin{array}{c}F T^{3} \text { is } \\
4.93 \\
16.1 \\
26.2\end{array}$ & $\begin{array}{c}\text { DATE } \\
08-11-64 \\
09-13-66 \\
09-23-69\end{array}$ & $\begin{array}{c}F T^{3} / S \\
9.50 \\
10.3 \\
15.3\end{array}$ & $\begin{array}{c}\text { DATE } \\
09-12-73 \\
10-01-74\end{array}$ & $\begin{array}{l}\mathrm{FT}^{3} / \mathrm{S} \\
21 . \\
26 .\end{array}$ & $\begin{array}{c}\text { DATE } \\
10-07-75 \\
09-01-76\end{array}$ & $\begin{array}{l}\mathrm{FT}^{3} / \mathrm{S} \\
11 . \\
6.6\end{array}$ \\
\hline$Q$ & $=33$ & $Q($ & $=12$ & 702 & $=9.7$ & 7010 & 6.1 & & \\
\hline
\end{tabular}

WAFSIPINICON RIVER BASIN

5-4205.40 WAPSIPINICON R NR RICEVILLE, IOWA

LAT $4320 \times X$, LONG $9234 \times X$, IN NE $1 / 4$ SEC.

12, T.98 N., R.15 W. MITCHELL CO. (E6),

AT BRIOGE, 2.5 MILES' SOUTH OF RICEVILLE,

DRAINAGE AREA $72.3 \mathrm{MI}^{2}$

\begin{tabular}{|c|c|c|c|c|c|c|c|c|c|}
\hline $\begin{array}{c}\text { DATE } \\
09-19-57 \\
04-11-58 \\
10-08-58\end{array}$ & $\begin{array}{c}F T^{3} / \mathrm{S} \\
6.41 \\
18.8 \\
7.50\end{array}$ & $\begin{array}{c}\text { DATE } \\
0 S-15-59 \\
08-09-60 \\
08-30-61\end{array}$ & $\begin{array}{r}\mathrm{FT}^{3} \text { iS } \\
4.86 \\
7.41 \\
5.59\end{array}$ & $\begin{array}{c}\text { DATE } \\
\text { OS-13-64 } \\
10-05-66\end{array}$ & $\begin{array}{r}\mathrm{FT}^{0} 1 \mathrm{~S} \\
4.70 \\
7.14\end{array}$ & $\begin{array}{c}\text { DATE } \\
09-02-71 \\
09-14-73\end{array}$ & $\begin{array}{l}\mathrm{FT}^{3} / \mathrm{S} \\
6.8 \\
19 .\end{array}$ & $\begin{array}{c}\text { DATE } \\
10-01-74 \\
08-31-76\end{array}$ & $\begin{array}{l}\mathrm{FT}^{3} / \mathrm{S} \\
10 . \\
4.8\end{array}$ \\
\hline$Q$ & $=43$ & $Q 1$ & $\gamma=7.2$ & $7 Q 2$ & $=6.1$ & $7 Q 10$ & $=4.0$ & & \\
\hline
\end{tabular}


WAPSIPINICON RIVER BASIN--Continued

5-4205.80 WAPSIPINICON R NR IONIA, IOWA

LAT $4301 \times X$, LONG $9223 \times X$, IN NW $1 / 4$ SEC.

33 , T.95 N., R.13 W., CHICKASAW CO. (19), AT BRIDGE, 4 MILES SE OF IONIA.

DRAINAGE AREA $161 \mathrm{MI}^{2}$

\begin{tabular}{|c|c|c|c|c|c|c|c|c|c|}
\hline $\begin{array}{c}\text { DATE } \\
09-20-57 \\
05-09-58 \\
10-09-58\end{array}$ & $\begin{array}{l}\mathrm{FT}^{\mathrm{3}} / \mathrm{S} \\
12.0 \\
17.2 \\
8.98\end{array}$ & $\begin{array}{c}\text { DATE } \\
09-16-59 \\
08-08-60 \\
08-30-61\end{array}$ & $\begin{array}{l}F T^{2} / S \\
10.8 \\
13.3 \\
15.7\end{array}$ & $\begin{array}{c}\text { DATE } \\
08-13-64 \\
10-015-66 \\
06-10-68\end{array}$ & $\begin{array}{l}\mathrm{FT}^{3} / \mathrm{S} \\
5.10 \\
9.47 \\
22.6\end{array}$ & $\begin{array}{c}\text { DATE } \\
09-02-71 \\
09-13-73\end{array}$ & $\begin{array}{l}F T=/ S \\
11.0 \\
28 .\end{array}$ & $\begin{array}{c}\text { DATE } \\
09-30-74 \\
08-30-76\end{array}$ & $\begin{array}{l}F T \cdot 15 \\
14 . \\
4.9\end{array}$ \\
\hline 0 & $=96$ & QS & $=12$ & $7 Q$ & $=9.5$ & 7010 & 4.7 & & \\
\hline
\end{tabular}

5-4206.40 L WAPSIPINICON R AT ELMA, IOWA

LAT $4314 X X$, LONG $9227 \times X$, IN NW $1 / 4$ SEC

$12, T .97 \mathrm{~N} .$, R.14 W. , HOWARD CO. $\{45\}$,

AT BRIDGE ON COUNTY ROAD A NEAR WEST

CITY LIMITS OF ELMA.

DRAINAGE AREA $37.3 \mathrm{MI}^{2}$

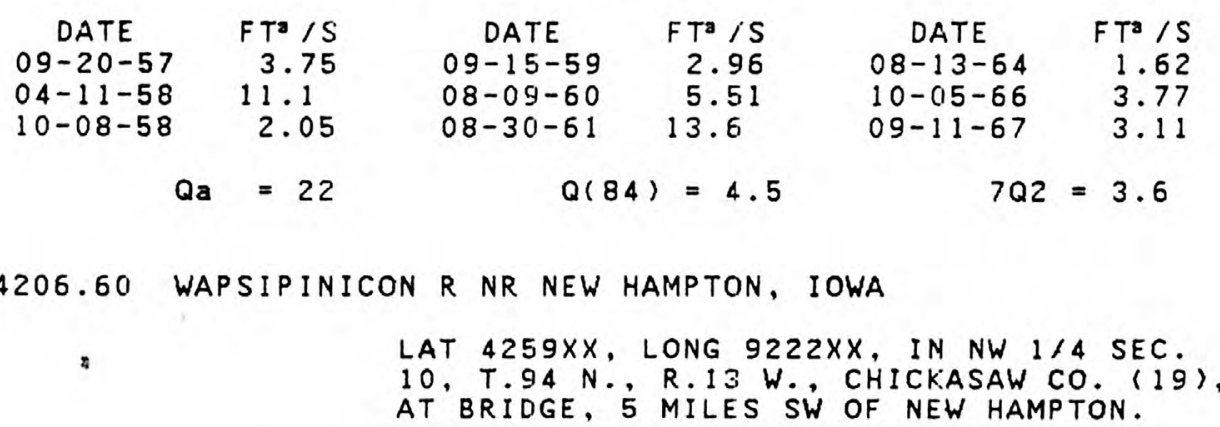

DRAINAGE AREA $291 \quad \mathrm{MI}^{2}$

\begin{tabular}{|c|c|c|c|c|c|c|c|c|c|}
\hline $\begin{array}{c}\text { DATE } \\
09-20-57 \\
05-09-58 \\
10-09-58\end{array}$ & $\begin{array}{l}\mathrm{FT}^{\mathrm{3}} / \mathrm{S} \\
27.2 \\
29.3 \\
18.4\end{array}$ & $\begin{array}{c}\text { DATE } \\
09-16-59 \\
08-08-60 \\
08-30-61\end{array}$ & $\begin{array}{l}\mathrm{FT}^{3} / \mathrm{S} \\
23.6 \\
28.1 \\
46.3\end{array}$ & $\begin{array}{c}\text { DATE } \\
08-13-64 \\
10-05-66 \\
06-10-68\end{array}$ & $\begin{array}{c}F T^{3} / \mathrm{S} \\
9.28 \\
21.1 \\
39.8\end{array}$ & $\begin{array}{c}\text { DATE } \\
09-02-71 \\
09-13-73\end{array}$ & $\begin{array}{l}\mathrm{FT}^{2} / \mathrm{S} \\
23.0 \\
44 .\end{array}$ & $\begin{array}{c}\text { DATE } \\
09-30-74 \\
08-30-76\end{array}$ & $\begin{array}{l}\mathrm{FT}^{\mathrm{T} / \mathrm{S}} \\
26 . \\
8.6\end{array}$ \\
\hline Qa & $=176$ & $Q 18$ & $=23$ & $7 Q 2$ & $=13$ & $7 Q 10$ & 5.0 & & \\
\hline
\end{tabular}


WAPSIPINICON RIVER BASIN--Cont inued

5-4206.80 WAPSIPIMICON R NR TRIPOLI, IOWA

LAT $4250 \times X$, LONG $9215 \times X$, IN SW $1 / 4$ SEC. 27, T.93 N., R.12 W., BREMER CO. (09)

AT BRIDGE ON STATE HIGHWAY 93, 2 MILES

NORTH OF TRIPOLI.

DRAINAGE AREA $343 \quad \mathrm{MI}^{2}$

$\begin{array}{rl}\text { DATE } & F T^{3} / \mathrm{S} \\ 09-24-57 & 23.7 \\ 05-09-58 & 38.6 \\ 10-09-58 & 13.4 \\ Q a & =208\end{array}$

$\begin{array}{ll}\text { DATE } & F T^{3} / S \\ 09-16-59 & 26.3 \\ 08-08-60 & 30.7 \\ 08-29-61 & 52.1\end{array}$

$\begin{array}{cc}\text { DATE } & F T^{3} / S \\ 08-14-64 & 9.02 \\ 10-05-66 & 16.4 \\ 06-10-68 & 57.5 \\ 7 Q 2= & 13\end{array}$

DATE
$09-01-71$
$09-13-73$

$F T \cdot 1 S$

$09-13-73$

21.0

$D A T E$
$10-01-7$

$10-01-74$

$T^{3} / S^{2}$

$Q=208$

$Q(84)=23$

$702=13$

$7010=5.0$

5-4207.00 EF WAPSIPINICON R NR FREDERICKSBURG, IOWA

LAT $4301 \times X$, LONG $9213 \times X$, IN NW $1 / 4$ SEC.

36 T.95 N. R.12 W. CHICKASAW CO (19)

AT ERIDGE, 3 MILES NORTH OF FREDERICKSBURG.

DRAINAGE AREA $62.2 \quad \mathrm{MI}^{2}$

$\begin{array}{cccccr}\text { DATE } & F^{3} / S & \text { DATE } & F T^{3} / S & \text { DATE } & F^{3} / \\ 09-20-57 & 5.29 & 09-16-59 & 6.79 & 08-13-64 & 2.0 \\ 05-09-58 & 4.97 & 08-08-60 & 6.70 & 10-05-66 & 5.3 \\ 10-09-58 & 4.30 & 08-29-61 & 12.5 & 06-10-68 & 9.1 \\ & & & & \end{array}$

5-4207.20 EF WAPSIPINICON R NR TRIPOLI, IOWA

LAT $4251 \mathrm{XX}$, LONG $9214 \times \mathrm{X}$, IN NW $1 / 4$ SEC.

$26, T .93$ N., R.12 W.. BREMER CO. (09\},

AT BRIDGE ON STATE HIGHWAY 93,3 MILES

NORTH OF TRIPOLI.

DRAINAGE AREA $144 \quad \mathrm{MI}^{2}$

$\begin{array}{cl}\text { DATE } & F T^{2} / S \\ 09-24-57 & 14.4 \\ 05-09-58 & 16.5 \\ 10-09-58 & 9.05\end{array}$

$\begin{array}{cl}\text { DATE } & F T^{3} / S \\ 09-16-59 & 17.6 \\ 08-08-60 & 18.1 \\ 08-29-61 & 27.1\end{array}$

$\begin{array}{cc}\text { DATE } & \mathrm{FT}^{2} / \mathrm{S} \\ 08-14-64 & 4.53 \\ 10-015-66 & 10.8 \\ 10-12-66 & 8.91\end{array}$

D.ATE
$06-10-68$

$09-01-7$

$09-13-73$

$F T^{2} / S$
15.4

13.0

14.

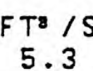

DATE

$09-30-74$

$\mathrm{FT}^{\mathrm{T}} / \mathrm{S}$

5.4
1.9

$Q \mathrm{a}=86$

$a(84)=11$

$702=6.6$

$7 Q 10=2.6$ 
WAPSIPINICON RIVER BASIN--Cont 1 nued

5-4207.40 WAPSIPINICON R AT TRIPOLI, IOWA

LAT $4248 \times X$, LONG $9214 \times X$, IN SW $1 / 4$ SEC.
$2, T .92$ N., R.12 W., BREMER CO. $(09)$,

AT BRIDGE, 1.5 MILES EAST OF TRIPOLI:

DRAINAGE AREA $498 \quad \mathrm{MI}^{2}$

\begin{tabular}{|c|c|c|c|c|c|c|c|c|c|}
\hline $\begin{array}{c}\text { DATE } \\
09-24-57 \\
05-09-58 \\
10-09-58\end{array}$ & $\begin{array}{l}F T^{3} / \mathrm{S} \\
39.0 \\
55.6 \\
22.7\end{array}$ & $\begin{array}{c}\text { DATE } \\
09-16-59 \\
08-08-50 \\
08-29-61\end{array}$ & $\begin{array}{l}\text { FT/S } \\
48.4 \\
32.8 \\
65.6\end{array}$ & $\begin{array}{c}\text { DATE } \\
08-14-64 \\
10-05-66 \\
10-13-66\end{array}$ & $\begin{array}{c}\mathrm{FT}^{\mathrm{a}} / \mathrm{S} \\
8.76 \\
18.2 \\
20.0\end{array}$ & $\begin{array}{c}\text { DATE } \\
06-10-68 \\
09-01-71 \\
09-14-73\end{array}$ & $\begin{array}{l}F T^{. / S} \\
64.5 \\
23.0 \\
53 .\end{array}$ & $\begin{array}{c}\text { DATE } \\
10-01-74 \\
08-30-76\end{array}$ & $\begin{array}{l}\mathrm{FT}^{3} / \mathrm{S} \\
35 . \\
3.9\end{array}$ \\
\hline Qa & $=304$ & $Q<\varepsilon$ & $\gamma=28$ & 702 & $=14$ & 7010 & 4.1 & & \\
\hline
\end{tabular}

5-4208.00 CRANE CR NR DENVER, IOWA

LAT 423832, LONG 921521 , IN NW $1 / 4$ SEC.

3, T.90 N., R.12 W., BLACK HAWK CO. ( 07 ), AT BRIDGE, 5 MILES SE OF DENVER.

DRAINAGE AREA $63.6 \mathrm{MI}^{2}$

$\begin{array}{rc}\text { DATE } & \mathrm{FT}^{a} / \mathrm{S} \\ 09-25-57 & 0 . \\ 05-09-58 & 2.09 \\ 10-10-58 & 0.17 \\ Q a & =37\end{array}$

$\begin{array}{rc}\text { DATE } & F T^{\mathrm{a}} / \mathrm{S} \\ 09-17-59 & 1.95 \\ 08-11-60 & 0.64 \\ 08-28-61 & 0.51 \\ Q(84) & =0.4\end{array}$
DATE $F T^{3} / \mathrm{S}$
$08-13-64 \quad 0.01$
$10-06-66 \quad 0.34$
$06-11-68$
6.87
$7 Q 2=\star$

$\begin{array}{rccc}\text { DATE } & F T^{3} / \mathrm{S} & \text { DATE } & F T / S \\ 10-22-68 & 29.9 & 10-01-74 & 0.56 \\ 09-01-71 & 0.9 & 08-30-76 & 0 . \\ 09-14-73 & 1.7 & & \\ 7 Q 10 & =0\end{array}$

5-4208.20 CRANE CR AT DUNKERTON, IOWA

LAT $4234 \times X$, LONG $9210 \times X$, IN SW $1 / 4$ SEC.

29, T.90 N., R. 11 W. BLACK HAWK CO. ( 07 ),

DRAINAGE AREA $101 \quad \mathrm{MI}^{2}$

\begin{tabular}{|c|c|c|c|c|c|c|c|c|c|}
\hline $\begin{array}{c}\text { DATE } \\
09-25-57 \\
05-08-58 \\
10-10-58\end{array}$ & $\begin{array}{r}\mathrm{FT}^{\mathrm{a}} / \mathrm{S} \\
0.38 \\
6.12 \\
1.16\end{array}$ & $\begin{array}{c}\text { DATE } \\
09-17-59 \\
08-11-60 \\
08-28-61\end{array}$ & $\begin{array}{l}\mathrm{FT}^{\mathrm{a}} / \mathrm{S} \\
1.77 \\
0.78 \\
0.95\end{array}$ & $\begin{array}{c}\text { DATE } \\
08-13-64 \\
10-06-66 \\
06-11-68\end{array}$ & $\begin{array}{l}F T^{2} / S \\
0 . \\
0.82 \\
11.1\end{array}$ & $\begin{array}{c}\text { DATE } \\
09-01-71 \\
07-12-73\end{array}$ & $\begin{array}{l}\mathrm{FT}^{2} / \mathrm{S} \\
0 . \\
2.3\end{array}$ & $\begin{array}{c}\text { DATE } \\
09-30-74 \\
08-30-76\end{array}$ & $\begin{array}{l}\mathrm{FT} / \mathrm{S} \\
0.88 \\
0 .\end{array}$ \\
\hline$Q=$ & $=60$ & $Q($ & $1=0.7$ & 702 & $=\star *$ & 7010 & 0 & & \\
\hline
\end{tabular}


WAPSIPINICON RIVER BASIN--Cont inued

5-4208.40 L WAPSIPINICON R NR WESTGATE, IOWA

LAT $4247 \times X$, LONG $9205 \times X$, IN NE $1 / 4$ SEC.

13, T.92 N., R.11 W., BREMER CO. (09),

AT BRIDGE, 4.5 MILES'NW OF WESTGATE.

DRAINAGE AREA $57.4 \mathrm{MI}^{2}$
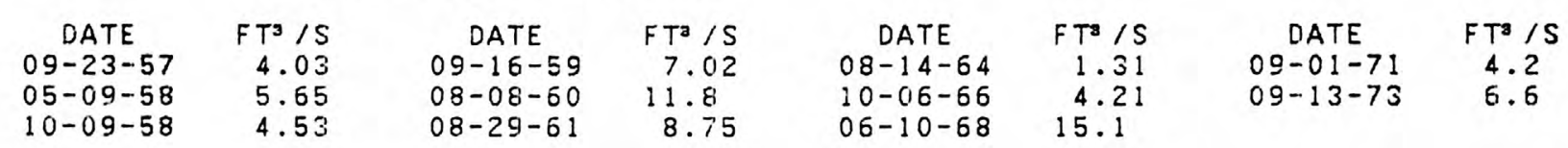

DATE

$08-29-61$

8.75

$06-10-68$

15.1

$Q \mathbf{a}=34$

$Q(84)=5.2$

$7 Q 2=2.6$

$7 Q 10=0.6$

5-4208.60 BUCK CR NR LITTLETON, IOWA

LAT $4235 \times X$, LONG $9203 \times X$, NEAR CENTER OF SEC.29, T.90 N., R.10 W., EUCHANAN CO. (10), AT BRIDGE, 3 MILES NW OF LITTLETON.

DRAINAGE AREA $57.0 \quad \mathrm{MI}^{2}$

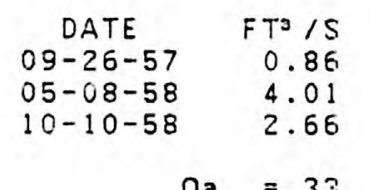

$Q_{a}=33$

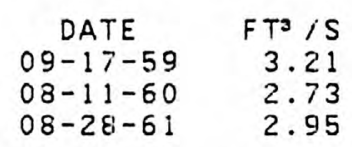

$Q(84)=1.6$

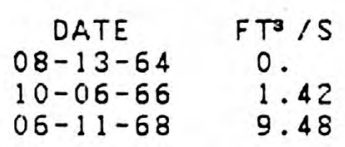

$7 Q 2=0.6$

$\begin{array}{cc}\text { DATE } & \text { FT /S } \\ 09-01-71 & 0.06 \\ 09-12-73 & 3.1 \\ 7010= & 0.1\end{array}$

$7 Q 10=0.1$

5-4209.00 L WAPSIPINICON R AT LITTLETON, IOWA

LAT $4233 \times X$. LONG $9202 \times X$. IN NE CORNER

SEC.9, T.89 N., R.10 W., BUICHANAN CO. (10),

AT BRIDGE, 0.5 MILE NORTH OF LITTLETON.

DRAINAGE AREA $205 \quad \mathrm{MI}^{2}$

$\begin{array}{rc}\text { DATE } & F T^{3} / S \\ 09-26-57 & 9.56 \\ 05-08-58 & 9.74 \\ 10-26-58 & 1 . .3 \\ Q a & =12.3\end{array}$

$\begin{array}{cc}\text { DATE } & F T^{J} / S \\ 09-17-59 & 18.6 \\ 08-11-60 & 20.8 \\ 08-28-61 & 26.9 \\ Q(84) & =17\end{array}$

$\begin{array}{rr}\text { DATE } & F T^{3} / \mathrm{S} \\ 08-13-64 & 3.27 \\ 10-06-66 & 13.9 \\ 10-13-66 & 25.7 \\ 702= & 10\end{array}$

$\begin{array}{rl}\text { DATE } & F T / S \\ 06-11-68 & 53.1 \\ 09-01-71 & 10.0 \\ 09-12-73 & 29 . \\ 7010= & 3.9\end{array}$

DATE

$09-30-74$

$F T^{3} / S$

$08-30-76$ 


\section{DISCHARGE MEASUREMENTS AT \\ LOW-FLOW PARTIAL-RECORD STATIONS}

WAPSIPINICON RIVER BASIN--COnt inued

5-4209.40 OTTER CR NR OTTERVILLE, IOWA

LAT $4233 \times X$, LONG $9157 \times X$, NEAR SW CORNER

OF SEC.5, T.89 N., R.9 W., BUCHANAN CO. $(10)$,

AT BRIDGE, 2 MILES NORTH OF OTTERVILLE.

DRAINAGE AREA $101 \quad \mathrm{MI}^{2}$

\begin{tabular}{|c|c|c|c|c|c|c|c|c|c|}
\hline $\begin{array}{c}\text { DATE } \\
09-26-57 \\
05-08-58 \\
10-20-58\end{array}$ & $\begin{array}{l}\mathrm{FT}^{3} / \mathrm{S} \\
9.83 \\
11.9 \\
8.34\end{array}$ & $\begin{array}{c}\text { DATE } \\
09-17-59 \\
08-11-60 \\
08-28-61\end{array}$ & $\begin{array}{l}F T^{3} / \mathrm{S} \\
17.0 \\
24.3 \\
18.9\end{array}$ & $\begin{array}{c}\text { DATE } \\
08-13-64 \\
10-06-66 \\
06-11-68\end{array}$ & $\begin{array}{l}F T / S \\
9.26 \\
17.7 \\
52.6\end{array}$ & $\begin{array}{c}\text { DATE } \\
09-01-71 \\
09-12-73\end{array}$ & $\begin{array}{l}\mathrm{FT}^{2} / \mathrm{S} \\
12.0 \\
25 .\end{array}$ & $\begin{array}{c}\text { DATE } \\
09-30-74 \\
08-30-76\end{array}$ & $\begin{array}{l}\mathrm{FT}^{3} / \mathrm{S} \\
20 . \\
8.0\end{array}$ \\
\hline$Q$ & $=60$ & $Q($ & $=17$ & $7 Q$ & $=9.4$ & 7010 & 3.1 & & \\
\hline
\end{tabular}

5-4215.00 WAPSIPINICON R AT STONE CITY, IOWA

LAT $4207 X X$. LONG $9121 X X$, IN NE $1 / 4$ SEC.

$6, T .84$ N., R. 4 W. , JONES CO. $(53)$,

AT BRIDGE, IN STONE CITY.

DRAINAGE AREA $1324 \quad \mathrm{MI}^{2}$

\begin{tabular}{|c|c|c|c|c|c|c|c|c|c|}
\hline $\begin{array}{c}\text { DATE } \\
09-27-57 \\
05-01-58 \\
11-05-58\end{array}$ & $\begin{array}{l}\mathrm{FT}^{3} / \mathrm{S} \\
111 . \\
229 . \\
74.4\end{array}$ & $\begin{array}{c}\text { DATE } \\
09-17-59 \\
08-18-60 \\
08-30-61\end{array}$ & $\begin{array}{l}F T^{\circ} / \mathrm{S} \\
172 . \\
209 . \\
286 .\end{array}$ & $\begin{array}{c}\text { DATE } \\
08-13-64 \\
09-12-66 \\
10-13-66\end{array}$ & $\begin{array}{l}F^{2} / \mathrm{S} \\
75.6 \\
232 . \\
152 .\end{array}$ & $\begin{array}{c}\text { DATE } \\
09-01-71 \\
09-12-73 \\
09-30-74\end{array}$ & $\begin{array}{l}F T^{\circ} / \mathrm{S} \\
115.0 \\
321 . \\
174 .\end{array}$ & $\begin{array}{c}\text { DATE } \\
10-07-75 \\
08-30-76\end{array}$ & $\begin{array}{l}\mathrm{FT} / \mathrm{S} \\
91 . \\
83 .\end{array}$ \\
\hline & $=825$ & $Q($ & 4) $=164$ & 702 & $=96$ & $7 Q 10$ & $=42$ & & \\
\hline
\end{tabular}

5-4215.50 BUFFALO CR ABOVE WINTHROP, IOWA

LAT $4230 \times X$, LONG $9144 \times X$, NEAR NE CORNER

SEC. $25, T .89 \mathrm{~N}$, R. 8 W., BUCHANAN CO. (10),

AT BRIDGE, 1.5 MILES NE OF WINTHROP.

DRAINAGE AREA $68.2 \mathrm{MI}^{2}$

\begin{tabular}{|c|c|c|c|c|c|c|c|c|c|}
\hline $\begin{array}{c}\text { DATE } \\
09-26-57 \\
05-08-58 \\
10-20-58\end{array}$ & $\begin{array}{r}\mathrm{FT}^{3} / \mathrm{S} \\
2.61 \\
3.90 \\
1.70\end{array}$ & $\begin{array}{c}\text { DATE } \\
09-17-59 \\
08-12-60 \\
08-28-61\end{array}$ & $\begin{array}{c}\mathrm{FT}^{3} / \mathrm{S} \\
3.90 \\
10.5 \\
5.99\end{array}$ & $\begin{array}{c}\text { DATE } \\
08-13-64 \\
10-06-66 \\
06-11-68\end{array}$ & $\begin{array}{l}F T^{3} / \mathrm{S} \\
2.52 \\
3.99 \\
63.4\end{array}$ & $\begin{array}{c}\text { DATE } \\
09-01-71 \\
09-12-73\end{array}$ & $\begin{array}{c}\mathrm{FT} / \mathrm{S} \\
4.4 \\
8.6\end{array}$ & $\begin{array}{c}\text { DATE } \\
09-30-74 \\
08-30-76\end{array}$ & $\begin{array}{l}\mathrm{FT}^{3} / \mathrm{S} \\
6.7 \\
4.3\end{array}$ \\
\hline$Q_{c}$ & $=40$ & QR & $=3.7$ & $7 Q_{2}$ & $=2.7$ & 7010 & 1.1 & & \\
\hline
\end{tabular}


WAPSIPINICON RIVER BASIN--Cont inued

5-4217.00 BUFFALO CR NR STONE CITY, IOWA

LAT $4208 \times X$, LONG $9121 \times X$, NEAR E $1 / 4$

CORNER SEC. 30 , T.85 N., R. 4 W., JONES CO. (53),

AT BRIDGE, 2 MILES NORTH OF STONE CITY.

DRAINAGE AREA $217 \quad \mathrm{MI}^{2}$

\begin{tabular}{|c|c|c|c|c|c|c|c|c|c|}
\hline $\begin{array}{c}\text { DATE } \\
09-27-57 \\
05-01-58 \\
11-05-58\end{array}$ & $\begin{array}{l}\mathrm{FT}^{\mathrm{J}} / \mathrm{S} \\
12.0 \\
2 \mathrm{i} .3 \\
9.88\end{array}$ & $\begin{array}{c}\text { DATE } \\
09-17-59 \\
08-18-60 \\
08-12-64\end{array}$ & $\begin{array}{l}F T^{3} / \mathrm{S} \\
20.8 \\
48.1 \\
21.8\end{array}$ & $\begin{array}{c}\text { DATE } \\
09-12-66 \\
10-05-66 \\
09-01-71\end{array}$ & $\begin{array}{l}\mathrm{FT}^{\mathrm{a}} / \mathrm{S} \\
34.0 \\
20.6 \\
24.0\end{array}$ & $\begin{array}{c}\text { DATE } \\
09-12-73 \\
09-30-74\end{array}$ & $\begin{array}{l}F T^{3} / \mathrm{S} \\
55 . \\
37 .\end{array}$ & $\begin{array}{c}\text { DATE } \\
10-07-75 \\
08-30-76\end{array}$ & $\begin{array}{l}\mathrm{FT} / \mathrm{s} \\
22 . \\
28 .\end{array}$ \\
\hline Qa & $=130$ & $Q<\varepsilon$ & $=29$ & $7 Q$ & $=15$ & 7010 & 5.3 & & \\
\hline
\end{tabular}

5-4218.00 YANKEE RUN CR AT WHEATLAND, IOWA

LAT 414934 , LONG 905025 , IN NE $1 / 4$ SEC.

16, T. EI N. RR. 1 E., CLINTON CO. ( 23$\rangle$,

RAINAGE AREA $52.2 \quad \mathrm{MI}^{2}$

\begin{tabular}{|c|c|c|c|c|c|c|c|c|c|}
\hline $\begin{array}{c}\text { DATE } \\
09-23-57 \\
05-01-58 \\
11-04-58\end{array}$ & $\begin{array}{c}\mathrm{FT}^{3} / \mathrm{S} \\
3.91 \\
12.7 \\
2.87\end{array}$ & $\begin{array}{c}\text { DATE } \\
09-15-59 \\
08-15-60 \\
08-3(1-61\end{array}$ & $\begin{array}{c}\mathrm{FT}^{3} / \mathrm{S} \\
3.73 \\
11.9 \\
9.23\end{array}$ & $\begin{array}{c}\text { DATE } \\
08-10-64 \\
09-12-66 \\
09-02-71\end{array}$ & $\begin{array}{c}\mathrm{FT}^{3} / \mathrm{S} \\
4.51 \\
3.61 \\
4.1\end{array}$ & $\begin{array}{c}\text { DATE } \\
09-12-73 \\
10-02-74\end{array}$ & $\begin{array}{c}\mathrm{FT}^{2} / \mathrm{S} \\
5.9 \\
4.9\end{array}$ & $\begin{array}{c}\text { DATE } \\
10-07-75 \\
09-01-76\end{array}$ & $\begin{array}{l}\mathrm{FT}^{\mathrm{S}} / \mathrm{S} \\
2.8 \\
1.7\end{array}$ \\
\hline Qa & $=31$ & $Q(84)$ & $=5.4$ & $7 Q 2$ & $=3.4$ & $7 Q 10$ & $=1.6$ & & \\
\hline
\end{tabular}

5-4218.50 MUD CR NR PLAINVIEW, IOWA

LAT 414202, LONG 904526, IN SW $1 / 4$ SEC.

29, T.EO N., R.2 E., SCOTT CO. (82),

DRAINAGE AREA $109 \quad \mathrm{MI}^{2}$

$\begin{array}{rr}\text { DATE } & F T^{\top} / S \\ 09-23-57 & 1.66 \\ 05-01-58 & 9.45 \\ 11-04-58 & 2.71 \\ & =65\end{array}$

$\begin{array}{cc}\text { DATE } & \text { FT }^{3} / \mathrm{S} \\ 09-15-59 & 2.01 \\ 08-15-60 & 10.7 \\ 08-301-51 & 7.15\end{array}$

$\begin{array}{cc}\text { DATE } & F T^{a} / \mathrm{S} \\ 08-10-64 & 3.32 \\ 09-12-66 & 5.50 \\ 09-02-71 & 5.4\end{array}$

D.ATE
$09-12-73$
$10-02-7$

$F^{3} / S$
9.5

DATE
$10-07-75$

$09-01-76$

FT / $/ \mathrm{S}$

$7 Q 2=4.0$

$7 Q 10=2.1$ 
WAPSIPINICON RIVER BASIN--Cont Inued

5-4219.00 SILVER CR NR DE WITT, IOWA

LAT 414709, LONG 903313, IN SE 1/4 SEC

$25, T .81$ N., R.3 E., CLINTON CO. \{23\},

AT BRIDGE, 2.5 MILES SOUTH OF DE WITT.

DRAINAGE AREA $60.8 \mathrm{MI}^{2}$

$\begin{array}{rc}\text { DATE } & F T^{3} / \mathrm{S} \\ 09-23-57 & 8.06 \\ 05-01-58 & 19.5 \\ 11-04-58 & 6.44 \\ \text { Qa } & =36\end{array}$

$\begin{array}{rc}\text { DATE } & F T^{2} / S \\ 09-15-59 & 7.50 \\ 08-15-60 & 17.6 \\ 08-30-61 & 13.2 \\ Q(84) & =11\end{array}$

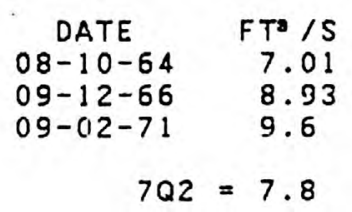
DATE FT /S
$09-12-73 \quad 15$
$10-02-74 \quad 15$.

DATE
$10-07-75$

$F T^{2} / S$

$09-01-76$

5.0

$7010=4.7$

5-4221.00 BROPHY CR NR LOW MOOR, IOWA

LAT 414856, LONG 902414 , NEAR N 1/4 CORNER SEC. $20, T .81$ N., R.5 E., CLINTON CO. ( 23 ), AT BRIDGE ON U.'S. HIGHWAY 30,3 MILES

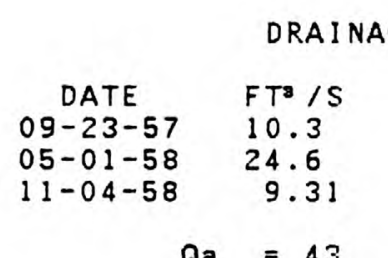

$Q \mathrm{a}=43$
NW OF LOW MOOR.

RAINAGE AREA $72.8 \mathrm{MI}^{2}$

$\begin{array}{ll}\text { DATE } & F T^{3} / S \\ 09-15-59 & 10.0 \\ 08-15-60 & 21.6 \\ 08-30-61 & 26.4\end{array}$

$\begin{array}{cc}\text { DATE } & F T^{3} / S \\ 08-10-64 & 11.5 \\ 09-12-66 & 8.58 \\ 09-02-71 & 9.9\end{array}$

$\begin{array}{ll}\text { DATE } & F T / S \\ \text { O9-12-73 } & 19 . \\ 10-01-74 & 18 .\end{array}$

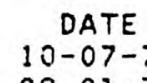

$10-07-75$

F T / $/ S$

6.2

$7 Q 10=6.2$

IOWA RIVER BASIN

5-4483.00 WB IOWA R NR BRITT, IOWA

LAT $4306 \times \mathrm{X}$, LONG $9345 \times \mathrm{X}$, NEAR CENTER OF

SEC. 25, T.96 N., R.25 W., HANCOCK CO. (41),

AT BRIDGE ON U. S. HIGHWAY 1.8, 3 MILES EAST'

OF BRITT.

DRAINAGE AREA $61.5 \mathrm{MI}^{2}$

$\begin{array}{rr}\text { DATE } & \mathrm{FT}^{3} / \mathrm{S} \\ 09-25-57 & 1.86 \\ 04-03-58 & 8.57 \\ 10-23-58 & 1.19 \\ & \mathbf{Q a}=29\end{array}$

$Q_{a}=29$

$\begin{array}{cr}\text { DATE } & \text { FT is } \\ 05-13-59 & 6.18 \\ 10-04-60 & 2.13 \\ 08-03-61 & 4.44\end{array}$

$Q(84)=2.8$

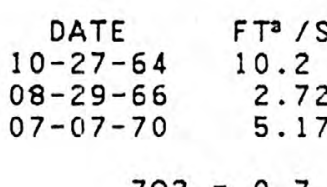

$702=0.7$

$\begin{array}{cc}\text { DATE } & F T / S \\ 09-01-71 & 1.2 \\ 09-05-73 & 2.1\end{array}$

DATE $08-04-75$

F T / S

0.21 
IOWA R.IVER BASIN--Cont inued

5-4484.00 WESTMAIN DRAINAGE DITCH 1 \& 2 NR BRITT, IOWA.

LAT $4306 \times X$, LONG $9347 \times X$, IN SW $1 / 4$ SEC. $27, T .96$ N., R.25 W., HANCOCK CO. (41), AT BRIDGE ON U.S. HIGHWAY 18 NEAR EAST

CITY LIMITS OF BRITT.

DRAINAGE AREA $21.2 \quad \mathrm{MI}^{2}$

\begin{tabular}{|c|c|c|c|c|c|c|c|c|c|}
\hline $\begin{array}{c}\text { DATE } \\
04-03-58 \\
10-23-58 \\
05-13-59\end{array}$ & $\begin{array}{l}\mathrm{FT}^{\mathrm{a}} / \mathrm{S} \\
2.21 \\
0.76 \\
1.56\end{array}$ & $\begin{array}{c}\text { DATE } \\
10-04-60 \\
08-30-61 \\
10-27-64\end{array}$ & $\begin{array}{r}\mathrm{FT}^{3} / \mathrm{S} \\
0.75 \\
1.74 \\
3.613\end{array}$ & $\begin{array}{c}\text { DATE } \\
08-29-66 \\
07-07-70\end{array}$ & $\begin{array}{r}\mathrm{FT}^{3} / \mathrm{S} \\
2.26 \\
2.82\end{array}$ & $\begin{array}{c}\text { DATE } \\
10-13-70 \\
09-05-73\end{array}$ & $\begin{array}{c}\mathrm{FT}^{\mathrm{T}} / \mathrm{S} \\
2.3 \\
2.5\end{array}$ & $\begin{array}{c}\text { DATE } \\
08-04-75 \\
09-07-76\end{array}$ & $\begin{array}{l}\mathrm{FT} / \mathrm{S} \\
2.4 \\
0.66\end{array}$ \\
\hline 0 & $=10$ & $Q C$ & $=1.5$ & 702 & $=0.5$ & 7010 & 0.1 & & \\
\hline
\end{tabular}

5-4511.00 SF IOWA R NR ALDEN, IOWA.

LAT $4228 \times X$, LONG $9327 \times X$, NEAR NW CORNER

OF SEC.5, T. 38 N., R.22 W., HARDIN CO. ( 42$)$,

AT BRIDGE, 5 MILES SW OF ALDEN.

DRAINAGE AREA $79.5 \mathrm{MI}^{2}$

\begin{tabular}{|c|c|c|c|c|c|c|c|c|c|}
\hline $\begin{array}{c}\text { DATE } \\
09-25-57 \\
05-08-58 \\
10-23-58\end{array}$ & $\begin{array}{l}\mathrm{FT}^{3} / \mathrm{S} \\
0.44 \\
11.5 \\
0.65\end{array}$ & $\begin{array}{c}\text { DATE } \\
05-15-59 \\
10-04-50 \\
09-05-61\end{array}$ & $\begin{array}{l}F T \cdot i S \\
11.2 \\
7.81 \\
4.02\end{array}$ & $\begin{array}{c}\text { DATE } \\
10-26-64 \\
09-018-66 \\
09-13-67\end{array}$ & $\begin{array}{l}\mathrm{FT}^{3} / \mathrm{S} \\
0.9 \mathrm{C} \\
0.13 \\
2.15\end{array}$ & $\begin{array}{c}\text { DATE } \\
07-07-70 \\
09-02-71 \\
09-05-73\end{array}$ & $\begin{array}{l}F T / S \\
10.8 \\
0.12 \\
1.3\end{array}$ & $\begin{array}{c}\text { DATE } \\
08-06-75 \\
09-08-76\end{array}$ & $\begin{array}{l}\mathrm{FT}^{3} / \mathrm{S} \\
2.0 \\
0 .\end{array}$ \\
\hline & $=37$ & Q 6 & $=1.2$ & & 0.7 & 7010 & 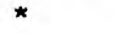 & & \\
\hline
\end{tabular}

5-4511.50 TIPTON CR NR NEW FROVIDENCE, IOWA

LAT $4220 X X$, LONG $9312 \times X$, IN SW $1 / 4$ SEC.

21, T.E 7 N., R. 20 W., HARDIN CO. $\{42\}$,

AT BRIDGE, 3 MILES NW OF NEW PROVIDENCE.

DRAINAGE AREA $81.4 \mathrm{MI}^{2}$

$\begin{array}{cc}\text { DATE } & F T^{2} / S \\ 05-25-57 & 1.64 \\ 05-08-58 & 15.9 \\ 10-24-58 & 2.87\end{array}$

$Q \mathbf{a}=4 \mathrm{C}$

$\begin{array}{rl}\text { DATE } & F T / S \\ 05-14-59 & 21.3 \\ 10-03-60 & 27.4 \\ 09-05-61 & 5.512 \\ \text { Q(84) } & =1.4\end{array}$

$Q(84)=1.4$

$\begin{array}{cr}\text { DATE } & F T / S \\ 10-26-64 & 0.05 \\ 09-08-56 & 1.42 \\ 07-07-70 & 3.34\end{array}$

$702=0.4$

\begin{abstract}
DATE
$09-02-71$

$09-05-73$
\end{abstract}

$\mathrm{FT} / \mathrm{S}$

0.08

5 .

$7010=\star$
DATE

$09-08-76$

$F^{3} / S$

5.8

0 . 
IOWA RIVER BASIN--Cont Inued

5-4512.00 SF IOWA R NR NEW PROVIDENCE, IOWA

LAT $4219 \times X$, LONG $9310 \times X$, NEAR N $1 / 4$ CORNER

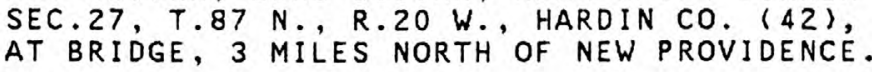

DRAINAGE AREA $223 \quad \mathrm{MI}^{2}$

\begin{tabular}{|c|c|c|c|c|c|c|c|c|c|}
\hline $\begin{array}{c}\text { DATE } \\
09-25-57 \\
05-08-58 \\
10-24-58\end{array}$ & $\begin{array}{c}\mathrm{FT}^{3} / \mathrm{S} \\
5.36 \\
41.2 \\
7.94\end{array}$ & $\begin{array}{c}\text { DATE } \\
05-14-59 \\
10-03-60 \\
09-05-61\end{array}$ & $\begin{array}{l}F T^{3} / \mathrm{S} \\
53.6 \\
71.7 \\
19.8\end{array}$ & $\begin{array}{c}\text { DATE } \\
10-26-64 \\
09-08-66 \\
07-07-70\end{array}$ & $\begin{array}{r}\mathrm{FT}^{3} / \mathrm{S} \\
3.87 \\
5.86 \\
9.97\end{array}$ & $\begin{array}{c}\text { DATE } \\
09-02-71 \\
09-05-73\end{array}$ & $\begin{array}{l}\mathrm{FT} / \mathrm{S} \\
3.4 \\
13 .\end{array}$ & $\begin{array}{c}\text { DATE } \\
08-06-75 \\
09-08-76\end{array}$ & $\begin{array}{l}F T / 1 S \\
12 . \\
0.95\end{array}$ \\
\hline Qa & $=108$ & $Q C$ & $=5.8$ & $7 Q 2$ & $=2.2$ & 7010 & $0.2 * \star$ & & \\
\hline
\end{tabular}

5-4512.50 BEAVER CR NR ELDORA, IOWA

LAT $4221 \times X$, LONG 9308XX, NEAR CENTER OF

SEC.13, T.87 N., R. 20 W., HARDIN CO. (42), AT BRIDGE, 2 MILES SW OF ELDORA.

DRAINAGE AREA $69.4 \mathrm{MI}^{2}$

$\begin{array}{rc}\text { DATE } & F^{3} / S \\ 09-24-57 & 3.51 \\ 05-08-58 & 11.1 \\ 10-24-58 & 4.64 \\ & \text { Qa }=35\end{array}$

$\begin{array}{cc}\text { DATE } & F T^{3} / S \\ 05-14-59 & 17.0 \\ 10-04-60 & 36.4 \\ 09-05-61 & 5.2 .9\end{array}$

$\begin{array}{rr}\text { DATE } & F T^{3} / S \\ 10-26-64 & 2.14 \\ 09-08-66 & 5.62 \\ 07-07-70 & 9.94 \\ 7 Q 2= & 1.9\end{array}$

$\begin{array}{cc}\text { DATE } & \text { FTs /S } \\ 09-02-71 & 1.7 \\ 09-05-73 & 3.3 \\ 7 Q 10=0 * *\end{array}$

5-4513.00 HONEY CR NR NEW PROVIDENCE, IOWA

LAT $4216 \times X$, LONG $9311 \times X$, AT E $1 / 4$ CORNER

SEC.16, T.86 N., R.20 W., HARDIN CO. (42),

AT BRIDGE, 1.5 MILES SOUTH OF NEW PROVIDENCE.

DRAINAGE AREA $66.5 \mathrm{MI}^{2}$

\begin{tabular}{|c|c|c|c|c|c|c|c|c|c|}
\hline $\begin{array}{c}\text { DATE } \\
09-25-57 \\
05-08-58 \\
10-24-58\end{array}$ & $\begin{array}{c}\mathrm{FT}^{3} / \mathrm{S} \\
1.96 \\
13.7 \\
2.94\end{array}$ & $\begin{array}{c}\text { DATE } \\
05-15-59 \\
10-03-60 \\
09-05-61\end{array}$ & $\begin{array}{l}F T^{a} / S \\
23.2 \\
34.6 \\
3.97\end{array}$ & $\begin{array}{c}\text { DATE } \\
10-2.6-64 \\
09-018-66 \\
07-07-70\end{array}$ & $\begin{array}{r}F T^{3} / S \\
0.34 \\
0.65 \\
4.05\end{array}$ & $\begin{array}{c}\text { DATE } \\
09-02-71 \\
09-05-73\end{array}$ & $\begin{array}{c}\mathrm{FT}^{\mathrm{a}} / \mathrm{S} \\
0.10 \\
6.4\end{array}$ & $\begin{array}{c}\text { DATE } \\
08-06-75 \\
09-08-76\end{array}$ & $\begin{array}{l}F T / S \\
4.1 \\
0.18\end{array}$ \\
\hline$Q$ & $=33$ & $Q($ & $=2.3$ & $7 Q$ & $=0.7$ & $7 Q 10$ & * & & \\
\hline
\end{tabular}


IOWA R.IVER BASIN--Cont inued

5-4513.50 HONEY CR. AT BANGOR, IOWA

LAT $4210 X X$, LONG $9305 \times X$, NEAR $W 1 / 4$ CORNER

SEC.16, T.85 N., R.19 W., MARSHALL CO. (64), AT BRIDGE, I MILE EAST OF BANGOR.

DRAINAGE AREA $95.6 \quad \mathrm{MI}^{2}$

\begin{tabular}{|c|c|c|c|c|c|c|c|c|c|}
\hline $\begin{array}{c}\text { DATE } \\
09-20-57 \\
05-08-58 \\
10-24-58\end{array}$ & $\begin{array}{c}F T^{2} / S \\
4.86 \\
18.6 \\
5.97\end{array}$ & $\begin{array}{c}\text { DATE } \\
05-15-59 \\
10-03-60 \\
09-05-61\end{array}$ & $\begin{array}{l}F T^{2} / \mathrm{S} \\
38.3 \\
40.4 \\
7.48\end{array}$ & $\begin{array}{c}\text { DATE } \\
10-26-64 \\
09-08-66 \\
07-018-70\end{array}$ & $\begin{array}{l}F T^{3} / S \\
0.60 \\
2.04 \\
11.2\end{array}$ & $\begin{array}{c}\text { DATE } \\
09-02-71 \\
09-05-73\end{array}$ & $\begin{array}{c}\mathrm{FT}^{3} / \mathrm{S} \\
1.1 \\
13 .\end{array}$ & $\begin{array}{c}\text { DATE } \\
08-06-75 \\
09-08-76\end{array}$ & $\begin{array}{l}\mathrm{FT}^{3} / \mathrm{S} \\
11 . \\
0.78\end{array}$ \\
\hline$Q_{a}$ & $=48$ & $Q(84)$ & $=7.1$ & 702 & $=2.5$ & $7 Q 10$ & 0.4 & & \\
\hline
\end{tabular}

5-4514.00 MINERVA CR AT CLEMONS, IOWA

LAT $4208 \times X$, LONG $9309 \times X$, NEAR CENTER OF SEC. $35, T .85$ N., R. 20 W., MARSHALL CO. (64), AT BRIDGE, I MILE NE OF CLEMONS.

URAINAGE AREA $69.6 \mathrm{MI}^{2}$

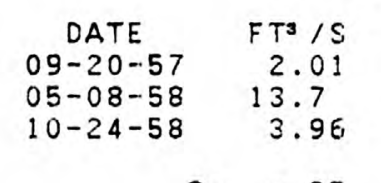

$\mathrm{Qa}_{\mathbf{a}}=35$

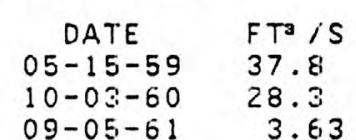

$\begin{array}{cc}10-03-60 & 28.3 \\ 09-05-61 & 3.6,3\end{array}$

$Q(84)=3.2$

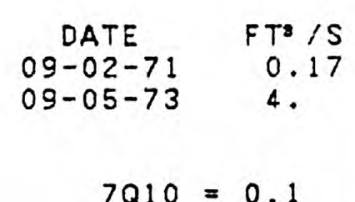

$7010=0.1$

5-4514.50 MINERVA CR NR CLEMONS, IOWA

$$
\begin{aligned}
& \text { LAT } 4207 \times X \text {, LONG } 9305 \times X \text {, NEAR CENTER OF } \\
& \text { SEC. } 5, \text { T. } 84 \text { N., R. } 19 \text { W. MARSHALL CO. ( } 64) \text {, } \\
& \text { AT BRIOGE, } 3.5 \text { MILES EAST OF CLEMONS }
\end{aligned}
$$

DRAINAGE AREA $148 \quad \mathrm{MI}^{2}$

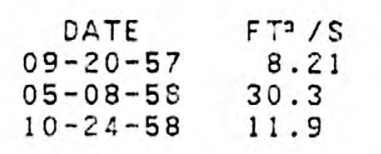

$Q_{\mathbf{a}}=75$

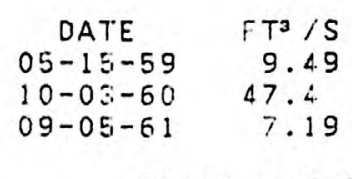

$Q(84)=\epsilon_{i} .8$

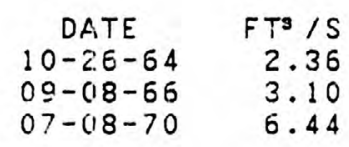

$7 Q 2=4.4$

$\begin{array}{cc}\text { DATE } & \mathrm{FT}^{3} / \mathrm{S} \\ 09-02-71 & 3.7 \\ 09-05-73 & 13 .\end{array}$

DATE
$08-06-75$ $08-06-75$
$09-08-76$

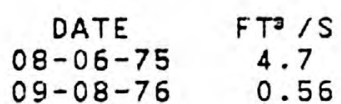

$09-08-76 \quad 0.56$ 
IOWA RIVER BASIN--Cont inued

5-4516.00 LINN CR AT MARSHALLTOWN, IOWA

LAT 420222, LONG 925440 , IN SW $1 / 4$ SEC.

35, T.84 N., R.18 W., MARSHALL CO. (64)

AT BRIDGE ON STATE HIGHWAY 14 IN MARSHALLTOWN.

DRAINAGE AREA $60.5 \mathrm{MI}^{2}$

\begin{tabular}{|c|c|c|c|c|c|c|c|c|c|}
\hline $\begin{array}{c}\text { DATE } \\
09-20-57 \\
04-23-58 \\
10-06-58\end{array}$ & $\begin{array}{c}F T / S \\
3.81 \\
16.3 \\
6.13\end{array}$ & $\begin{array}{c}\text { DATE } \\
10-21-59 \\
08-17-60 \\
09-05-61\end{array}$ & $\begin{array}{c}\mathrm{FT}^{3} / \mathrm{S} \\
2.52 \\
10.8 \\
6.96\end{array}$ & $\begin{array}{c}\text { DATE } \\
10-26-64 \\
09-12-66 \\
07-08-70\end{array}$ & $\begin{array}{l}F T^{2} / \mathrm{S} \\
3.92 \\
2.78 \\
11.4\end{array}$ & $\begin{array}{c}\text { DATE } \\
09-06-73 \\
09-26-74\end{array}$ & $\begin{array}{c}F T / S \\
8.1 \\
11 .\end{array}$ & $\begin{array}{c}\text { DATE } \\
08-05-75 \\
09-09-76\end{array}$ & $\begin{array}{l}\mathrm{FT}^{3} / \mathrm{s} \\
16 . \\
1.3\end{array}$ \\
\hline Qa & $=33$ & $Q<\varepsilon$ & $=4.8$ & 702 & $=3.0$ & $7 Q 10$ & $0.6 * * *$ & & \\
\hline
\end{tabular}

5-4516.50 S TIMBER CR NR LE GRAND, IOWA

LAT $4159 \times X$, LONG $9250 \times X$, IN SW $1 / 4$ SEC.

21, T.83 N., R.17 W., MARSHALL CO. (64)
AT BRIDGE, 4 MILES SW OF LE GRAND.

DRAINAGE AREA $62.0 \quad \mathrm{MI}^{2}$

$\begin{array}{rr}\text { DATE } & F T / S \\ 09-20-57 & 1.96 \\ 04-23-58 & 9.32 \\ 10-06-58 & 1.36 \\ Q a & =34\end{array}$

$\begin{array}{cc}\text { DATE } & F T^{3} / S \\ .10-21-59 & 2.48 \\ 08-17-60 & 9.18 \\ 09-05-61 & 8.45\end{array}$

$\begin{array}{cr}\text { DATE } & F T^{2} / S \\ 10-27-64 & 3.09 \\ 09-12-65 & 2.81 \\ 07-08-70 & 14.1\end{array}$
DATE
$09-06-73$
$\mathrm{FT}^{\mathrm{a}} / \mathrm{S}$
6.7
$09-26-74 \quad 13$

DATE

$09-09-76$

$F T^{3} / S$

2.1

$7010=0.2 * \star \star$

5-4518.00 DEER CR AT TOLEDO, IOWA

LAT $4159 \times X$, LONG $9235 \times X$, NEAR W $1 / 4$

CORNER SEC.15, T.83 N., R.15 W., TAMA CO. (85),

AT BRIDGE NEAR NW CITY LIMITS OF TOLEDO.

DRAINAGE AREA $76.4 \mathrm{MI}^{2}$

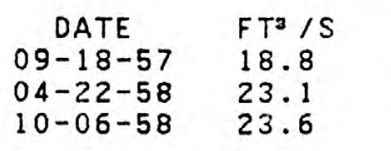

$Q_{a}=42$

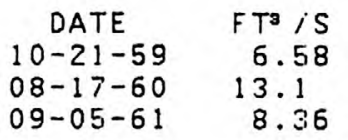

$Q(84)=6.3$

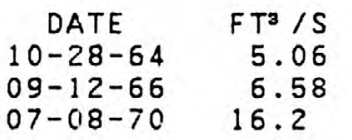

$7 Q 2=2.5$

$\begin{array}{cl}\text { DATE } & F T^{\mathrm{a}} / \mathrm{S} \\ 09-06-73 & 11 . \\ 09-27-74 & 24 .\end{array}$

$7010=0.7 * * *$

$\begin{array}{cc}\text { DATE } & F T^{3} / \mathrm{S} \\ 08-05-75 & 12 . \\ 09-09-76 & 3.2\end{array}$


IOWA R.IVER BASIN--Cont inued

5-4519.30 SALT CR NR CLUTIER, IOWA

$$
\begin{aligned}
& \text { LAT } 4203 \times X, \text { LONG } 9222 \times X \text {, NEAR E } 1 / 4 \\
& \text { CORNER SEC.33, T.84 N., R. } 13 \text { W. TAMA CO. (86), } \\
& \text { AT BRIDGE, 3.5 MILES SE OF CLUTIER. }
\end{aligned}
$$

DRAINAGE AREA $85.2 \quad \mathrm{MI}^{2}$

$\begin{array}{rl}\text { DATE } & F T^{3} / S \\ 09-18-57 & 10.9 \\ 04-22-58 & 21.3 \\ 10-06-58 & 24.0 \\ \text { Oa } & =48\end{array}$

$Q a=48$

$\begin{array}{cc}\text { DATE } & F T^{3} / S \\ 10-21-59 & 8.28 \\ 08-17-60 & 13.2 \\ 09-05-61 & 8.82 \\ Q(84) & =7.4\end{array}$

$\begin{array}{cr}\text { DATE } & \mathrm{FT}^{3} / \mathrm{S} \\ 10-28-64 & 5.25 \\ 09-12-66 & 8.41 \\ 07-018-70 & 18.5 \\ 7 Q 2= & 4.0\end{array}$

DATE $08-05-75$ $09-10-76$ $09-06-73 \quad 11$. $09-27-74 \quad 22$.

$\mathrm{FT} / \mathrm{S}$

11.

$7010=1.2 * * *$

5-4519.50 EB SALT CR NR ELBERON, IOWA

LAT $4204 \times X$, LONG 9220XX, NEAR E $1 / 4$

CORNER SEC. 27, T.84 N., R.13 W., TAMA CO. (86),

\begin{tabular}{|c|c|c|c|c|c|c|c|c|c|}
\hline $\begin{array}{c}\text { DATE } \\
09-13-57 \\
04-22-58 \\
10-06-58\end{array}$ & $\begin{array}{l}\mathrm{FT}^{3} / \mathrm{S} \\
4.16 \\
12.0 \\
11.6\end{array}$ & $\begin{array}{c}\text { DATE } \\
10-21-59 \\
08-17-60 \\
09-05-61\end{array}$ & $\begin{array}{l}\mathrm{FT}^{3} \text { is } \\
8.25 \\
9.39 \\
8.55\end{array}$ & $\begin{array}{c}\text { DATE } \\
10-28-64 \\
09-12-66 \\
07-08-70\end{array}$ & $\begin{array}{l}F T / S \\
3.68 \\
6.26 \\
16.1\end{array}$ & $\begin{array}{c}\text { DATE } \\
09-05-73 \\
09-27-74\end{array}$ & $\begin{array}{l}F T^{3} / S \\
6.3 \\
13 .\end{array}$ & $\begin{array}{c}\text { DATE } \\
08-05-75 \\
09-10-76\end{array}$ & $\begin{array}{l}\mathrm{FT}^{3} / \mathrm{S} \\
8.8 \\
1.7\end{array}$ \\
\hline Qa & $=40$ & $Q(84)$ & $=5.2$ & 702 & $=2.6$ & $7 Q 10$ & $=0.9 * * *$ & & \\
\hline
\end{tabular}

AT BRIDGE, 4 MILES NW OF ELBERON.

DRAINAGE AREA $71.3 \quad \mathrm{MI}^{2}$

5-4527.00 BIG BEAR. CR AT BROOKLYN, IOWA

LAT $4145 \times X$, LONG $9226 \times X$, NEAR NE CORNER

OF SEC.14, T.80 N., R.14 W., POWESHIEK CO. $179 \%$,

\begin{tabular}{|c|c|c|c|c|c|c|c|c|c|}
\hline $\begin{array}{c}\text { DATE } \\
09-17-57 \\
04-22-58 \\
10-05-58\end{array}$ & $\begin{array}{l}F T^{3} / S \\
2.87 \\
11.3 \\
10.4\end{array}$ & $\begin{array}{c}\text { DATE } \\
10-21-59 \\
09-06-60 \\
09-06-61\end{array}$ & $\begin{array}{l}\mathrm{FT}^{3} / \mathrm{S} \\
5.00 \\
4.48 \\
6.4 .2\end{array}$ & $\begin{array}{c}\text { DATE } \\
\text { 10-28-64 } \\
10-015-66 \\
07-018-70\end{array}$ & $\begin{array}{l}\mathrm{FT}^{3} / \mathrm{S} \\
3.32 \\
2.67 \\
15.6\end{array}$ & $\begin{array}{c}\text { DATE } \\
09-07-73 \\
09-26-74\end{array}$ & $\begin{array}{l}\mathrm{FT}^{2} / \mathrm{S} \\
5.8 \\
9.4\end{array}$ & $\begin{array}{c}\text { DATE } \\
08-06-75 \\
09-07-76\end{array}$ & $\begin{array}{l}\mathrm{FT}^{3} / \mathrm{S} \\
9.0 \\
1.3\end{array}$ \\
\hline Qa & $=45$ & Q & $=3.8$ & $7 Q 2$ & $=2.0$ & $7 Q 10$ & $0.2 * \star \star *$ & & \\
\hline
\end{tabular}

AT BRILIGE, 1 MILE NORTH OF BROOKLYN.

DRAINAGE AREA $77.9 \quad \mathrm{MI}^{2}$ 
IOWA RIVER BASIN--Continued

5-4542.00 CLEAR CR NR OXFORD, IOWA

LAT $4143 X X$, LONG $9147 X X$, IN NE $1 / 4$ SEC

$28, T .80 \mathrm{~N} .$, R.8 W., JOHNSON CO. $\{52\}$,

AT BRIDGE, I MILE SE OF OXFORD.

DRAINAGE AREA $55.0 \mathrm{MI}^{2}$

$\begin{array}{rr}\text { DATE } & F T / S \\ 09-23-57 & 0.10 \\ 04-22-58 & 2.19 \\ 10-06-58 & 2.27 \\ Q \mathbf{Q} & =32\end{array}$

$\begin{array}{cc}\text { DATE } & F T / S \\ 10-23-59 & 5.29 \\ 08-15-60 & 11.1 \\ 09-05-61 & 1.56\end{array}$

$\begin{array}{rr}\text { DATE } & F^{3} / \mathrm{S} \\ 10-29-64 & 1.86 \\ 10-06-66 & 0.99 \\ 07-08-70 & 16.1 \\ 702= & 0.7\end{array}$

$\begin{array}{cc}\text { DATE } & F T^{3} / S \\ 09-06-73 & 5.4 \\ 09-27-74 & 12 . \\ 7010= & \end{array}$

DATE
$08-07-75$

$F T / S$

$08-07-75 \quad 0.43$

$Q(84)=1.2$

$7 Q 2=0.7$

5-4550.50 OLD MANS CR NR PARNELL, IOWA

LAT $4136 \times X$, LONG $9157 \times X$, NEAR SW CORNER

OF SEC.31, T.79 N., R.9 W., IOWA CO. (48),

AT BRIDGE, 3 MILES NE OF PARNELL.

\begin{abstract}
DRAINAGE AREA $81.2 \mathrm{MI}^{2}$
\end{abstract}

\begin{tabular}{|c|c|c|c|c|c|c|c|c|c|}
\hline $\begin{array}{c}\text { DATE } \\
09-25-57 \\
04-21-58 \\
10-22-58\end{array}$ & $\begin{array}{l}F T^{2} / \mathrm{S} \\
0.14 \\
2.57 \\
9.29\end{array}$ & $\begin{array}{c}\text { DATE } \\
10-23-59 \\
09-06-60 \\
09-07-61\end{array}$ & $\begin{array}{l}\mathrm{FT}^{\mathrm{a}} \text { iS } \\
4.27 \\
1.57 \\
0.99\end{array}$ & $\begin{array}{c}\text { DATE } \\
10-28-64 \\
09-13-66 \\
10-05-66\end{array}$ & $\begin{array}{l}F^{3} / \mathrm{S} \\
0.52 \\
1.50 \\
0.57\end{array}$ & $\begin{array}{c}\text { DATE } \\
07-08-70 \\
09-07-73 \\
09-26-74\end{array}$ & $\begin{array}{l}F T / S \\
21.6 \\
1.0 \\
13 .\end{array}$ & $\begin{array}{c}\text { DATE } \\
08-07-75 \\
09-10-76\end{array}$ & $\begin{array}{r}\mathrm{FT}^{\mathrm{T}} / \mathrm{S} \\
0.21 \\
0.20\end{array}$ \\
\hline Q & $=47$ & QR & $\Rightarrow=2.0$ & 702 & $=0.6$ & 7010 & * & & \\
\hline
\end{tabular}

5-4552.00 N ENGLISH R NR GUERNSEY, IOWA

LAT $4138 \times X$, LONG $9224 \times X$, NEAR SW CORNER

SEC. $17, T .79 \mathrm{~N}$., R.13 W., POWESHIEK CO. (79),

AT BRIDGE, 2.5 MILES WEST OF GUERNSEY.

DRAINAGE AREA $68.7 \mathrm{MI}^{2}$

\begin{tabular}{|c|c|c|c|c|c|c|c|c|c|}
\hline $\begin{array}{c}\text { DATE } \\
09-18-57 \\
04-22-58 \\
10-03-58\end{array}$ & $\begin{array}{c}\mathrm{FT}^{\mathrm{a}} / \mathrm{S} \\
5.44 \\
10.0 \\
9.94\end{array}$ & $\begin{array}{c}\text { DATE } \\
10-21-59 \\
09-06-60 \\
09-06-61\end{array}$ & $\begin{array}{c}\mathrm{FT}^{3} / \mathrm{S} \\
5.28 \\
4.11 \\
10.1\end{array}$ & $\begin{array}{c}\text { DATE } \\
04-15-63 \\
07-01-63 \\
11-06-63\end{array}$ & $\begin{array}{r}\mathrm{FT}^{3} / \mathrm{S} \\
8.75 \\
2.45 \\
2.58\end{array}$ & $\begin{array}{c}\text { DATE } \\
10-05-66 \\
07-08-70 \\
09-07-73\end{array}$ & $\begin{array}{l}F T / S \\
1.16 \\
9.80 \\
2.2\end{array}$ & $\begin{array}{c}\text { DATE } \\
09-26-74 \\
08-07-75 \\
09-08-76\end{array}$ & $\begin{array}{l}\mathrm{FT} / \mathrm{S} \\
6.2 \\
2.7 \\
0.7\end{array}$ \\
\hline & $=40$ & QR & $=3.2$ & $7 Q 2$ & $=1.8$ & 7010 & $0.6 * * *$ & & \\
\hline
\end{tabular}


IOWA R.IVER BASIN--Continued

5-4552.50 N ENGLISH R NR NORTH ENGLISH, IOWA

LAT $4133 \times X$, LONG $9203 \times X$, NEAR SW CORNER

SEC 17 T.78 N. R.10 W. IOWA CO. (48),

AT BRIDGE, 3.25 MILES NE OF NORTH ENGLISH.

DRAINAGE AREA $221 \quad . M I^{2}$

\begin{tabular}{|c|c|c|c|c|c|c|c|c|c|}
\hline $\begin{array}{c}\text { DATE } \\
09-25-57 \\
04-21-58 \\
10-22-58 \\
10-22-59\end{array}$ & $\begin{array}{l}\mathrm{FT}^{3} / \mathrm{S} \\
8.47 \\
23.3 \\
38.0 \\
30.0\end{array}$ & $\begin{array}{c}\text { DATE } \\
09-06-60 \\
09-07-61 \\
04-16-63 \\
06-26-63\end{array}$ & $\begin{array}{c}\mathrm{FT}^{\mathrm{a}} / \mathrm{S} \\
9.46 \\
16.7 \\
31.8 \\
9.78\end{array}$ & $\begin{array}{c}\text { DATE } \\
07-01-63 \\
11-06-63 \\
09-13-66\end{array}$ & $\begin{array}{r}F T^{3} / S \\
9.78 \\
6.17 \\
8.68\end{array}$ & $\begin{array}{c}\text { DATE } \\
10-05-66 \\
07-08-70 \\
09-07-73\end{array}$ & $\begin{array}{c}F T \cdot / S \\
3.85 \\
38.7 \\
7.3\end{array}$ & $\begin{array}{c}\text { DATE } \\
09-26-74 \\
08-07-75 \\
09-10-76\end{array}$ & $\begin{array}{l}\mathrm{FT}^{3 / \mathrm{S}} \\
26 . \\
7.4 \\
7.7\end{array}$ \\
\hline$Q$ & $=126$ & $Q<\varepsilon$ & )$=10$ & $7 Q 2$ & $=5.1$ & 7010 & $=1.5 * * \star$ & & \\
\hline
\end{tabular}

5-4552.60 M ENGLISH R NR NORTH ENGLISH, IOWA

LAT $4132 \times X$, LONG $9204 \times X$, NEAR NE CORNER SEC. 25, T.78 N., R.11 W., IOWA CO. (48). AT BRIDGE, 2 MILES NE OF' NORTH ENGLISH. DRAINAGE AREA $66.7 \mathrm{MI}^{2}$

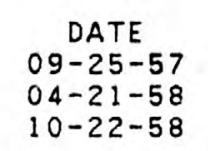

$\mathrm{FT}^{3} / \mathrm{S}$
0.16
2.69
0.87

$\begin{array}{cr}\text { DATE } & \text { FT } / S \\ 10-22-59 & 3.40 \\ 09-06-60 & 0.52 \\ 04-16-63 & 7.58\end{array}$

$\begin{array}{cr}\text { DATE } & F T / S \\ 06-26-63 & 1.40 \\ 07-01-63 & 0.80 \\ 11-016-63 & 1.35\end{array}$

DATE
$09-13-6$

$07-08-70$

$09-07-73$

$F T^{3} / S$
0.23
11.0
0.45

$7 Q 10=0$

5-4554.00 S ENGLISH R NR KESWICK, IOWA

LAT 412813, LONG 921531 , IN SW $1 / 4$ SEC.

$16, T .77$ N., R.12 W., KEOKUK CO. (54),

AT BRIDGE, 1.5 MILES NW OF KESWICK.

DRAINAGE AREA $66.2 \quad \mathrm{MI}^{2}$

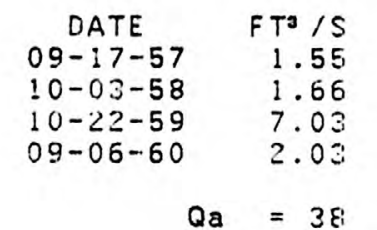

$\begin{array}{lr}D A T E & F T^{3} / S \\ 09-07-61 & 3.47 \\ 04-15-63 & 5.58 \\ 07-01-63 & 1.33 \\ 11-06-63 & 1.30\end{array}$

$\begin{array}{cc}\text { DATE } & F T^{9} / S \\ 06-013-66 & 39.0 \\ 07-2.5-66 & 4.69 \\ 09-13-66 & 0.25\end{array}$

$Q(84)=0.6$

$7 Q 2=$

$Q \mathbf{a}=3 \varepsilon_{i}$

$\begin{array}{cr}\text { DATE } & \text { FT } / S \\ 11-03-66 & 0.18 \\ 07-08-70 & 9.41 \\ 09-07-73 & 0.38\end{array}$

DATE
$09-26-74$
$08-07-75$
$08-10-76$

$F T \cdot / S$

4.7

0.03

0.03

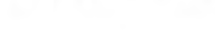

$7010=0$ 
IOWA RIVER BASIN--Cont inued

5-4554.50 S ENGLISH R NR KINROSS, IOWA

LAT $4130 \times X$, LONG $9157 \times X$, IN NW $1 / 4$ SEC.

7 , T.77 N., R.9 W., WASHINGTON CO. ( 92 ),

AT BRIDGE, 3 MILES NE OF KINROSS.

$\begin{array}{rc} & \text { DRAINA } \\ \text { DATE } & \text { FT } / S \\ 09-25-57 & 5.68 \\ 04-22-58 & 7.20 \\ 10-03-58 & 1.64 \\ 10-22-59 & 11.0 \\ \text { Qa } & =72\end{array}$

$\begin{array}{cc}\text { AREA } 125 & M^{2} \\ \text { DATE } & F T a / S \\ 09-06-60 & 3.23 \\ 09-07-61 & 5.76 \\ 04-16-63 & 15.3 \\ 06-26-63 & 4.34 \\ & \end{array}$

$\begin{array}{rr}\text { DATE } & F T / S \\ 07-01-63 & 4.05 \\ 11-06-63 & 2.13 \\ 06-02-66 & 70.7 \\ 07-25-66 & 11.8 \\ 702 & =1.0\end{array}$

$\begin{array}{cc}\text { DATE } & F T^{3} / S \\ 09-13-66 & 1.95 \\ 07-08-70 & 22.2 \\ 09-07-73 & 2.5\end{array}$

DATE
O9-26-74
$08-07-75$ $08-07-75$

$\mathrm{FT}^{\mathrm{O}} / \mathrm{S}$

$7010=0.2 * * *$

5-4573.00 OTTER CR NR OTRANTO, IOWA

LAT $4328 \times X$, LONG $9258 \times X$, IN NW $1 / 4$ SEC. $22, T .100$ N., R. 18 W., MITCHELL CO. (66), AT BRIDGE, 1.5 MILES NE OF OTRANTO.

DRAINAGE AREA $60.3 \mathrm{MI}^{2}$

\begin{tabular}{|c|c|c|c|c|c|c|c|c|c|}
\hline $\begin{array}{c}\text { DATE } \\
09-19-57 \\
05-06-58 \\
10-22-58\end{array}$ & $\begin{array}{r}F^{3} / S \\
6.04 \\
7.25 \\
3.11\end{array}$ & $\begin{array}{c}\text { DATE } \\
05-13-59 \\
10-05-60 \\
08-29-61\end{array}$ & $\begin{array}{c}F T=\text { iS } \\
8.60 \\
11.7 \\
8.02\end{array}$ & $\begin{array}{c}\text { DATE } \\
08-19-64 \\
09-08-66 \\
09-12-67\end{array}$ & $\begin{array}{r}\mathrm{FT}^{\circ} / \mathrm{S} \\
3.95 \\
7.22 \\
6.14\end{array}$ & $\begin{array}{c}\text { DATE } \\
07-07-70 \\
09-02-71 \\
09-05-73\end{array}$ & $\begin{array}{l}F^{\circ} / \mathrm{S} \\
8.87 \\
5.3 \\
16 .\end{array}$ & $\begin{array}{c}\text { DATE } \\
08-04-75 \\
09-08-76\end{array}$ & $\begin{array}{l}\mathrm{FT}^{\mathrm{T}} / \mathrm{S} \\
10 . \\
2.5\end{array}$ \\
\hline Q & $=30$ & $Q(84)$ & $1=6.8$ & 702 & $=4.7$ & $7 Q 10$ & $=2.3$ & & \\
\hline
\end{tabular}

5-4573.50 CEDAR R AT OTRANTO, IOWA

LAT $4327 \times X$, LONG 9259XX, IN NW $1 / 4$ SEC.

AT, BRIDGE NEAR EAST CITY LIMITS OF OTRANTO.

DRAINAGE AREA $656 \quad \mathrm{MI}^{2}$

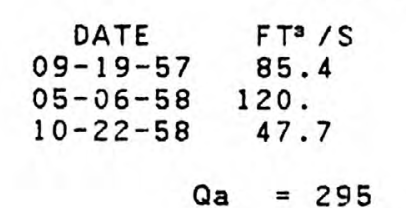

$Q_{a}=295$

$\begin{array}{rl}\text { DATE } & F T^{3} / \mathrm{S} \\ 07-21-64 & 81.8 \\ 08-19-64 & 61.5 \\ 09-08-66 & 74.4 \\ 7 Q 2 & =61\end{array}$

$7 Q 2=61$

$\begin{array}{cc}\text { DATE } & F T^{3} / S \\ 09-12-67 & 66.9 \\ 07-07-70 & 120 . \\ 09-02-71 & 73 . \\ & \end{array}$

$7 Q 10=32 * * *$

$\begin{array}{cc}\text { DATE } & F T / S \\ 09-05-73 & 193 . \\ 08-04-75 & 102 . \\ 09-08-76 & 51 .\end{array}$


IOWA RIVER BASIN--Cont inued

5-4574.00 DEER CR NR MELTONVILLE, IOWA

LAT $4326 \times X$, LONG $9305 \times X$, IN SW $1 / 4$ SEC.

27, T.100 N., R.19 W. WORTH CO. (98),

AT'BRIDGE, 2.5 MILES WEST OF MELTONVILLE.

DRAINAGE AREA $67.5 \quad \mathrm{MI}^{2}$

$\begin{array}{rc}\text { DATE } & F T^{3} / S \\ 09-26-57 & 1.85 \\ 05-06-58 & 10.0 \\ 10-22-58 & 0.65 \\ Q a & =33\end{array}$

$\begin{array}{cr}\text { DATE } & F^{3} / S \\ 05-13-59 & 4.28 \\ 10-04-60 & 4.20 \\ 08-29-61 & 2.84\end{array}$

$\begin{array}{rc}\text { DATE } & F T^{3} / \mathrm{S} \\ 08-19-64 & 0 . \\ 09-08-66 & 1.48 \\ 09-11-67 & 0.83 \\ 702= & 0.9\end{array}$

$\begin{array}{cc}\text { DATE } & \mathrm{FT}^{3} / \mathrm{S} \\ 07-07-70 & 4.47 \\ 09-01-71 & 2.3 \\ 09-05-73 & 4.0 \\ 7010= & 0\end{array}$

DATE

$\begin{array}{ll}08-04-75 & 5.9 \\ 09-07-76 & 1.8\end{array}$

$F T^{3} / \mathrm{S}$

$Q(84)=2.3$

5-4574.50 DEER CR AT ST. ANSGAR, IOWA

LAT $4323 \times X$, LONG $9258 \times X$, IN SW $1 / 4$ SEC.

15. T.99 N., R.18 W., MITCHELL CO. ( 66 )

AT BRIDGE, 2.5 MILES NW OF ST. ANSGAR.

DRAINAGE AREA $97.5 \mathrm{MI}^{2}$

\begin{tabular}{|c|c|c|c|c|c|c|c|c|c|}
\hline $\begin{array}{c}\text { DATE } \\
09-19-57 \\
05-07-58 \\
10-22-58\end{array}$ & $\begin{array}{c}\mathrm{FT}^{3} / \mathrm{S} \\
3.10 \\
14.8 \\
1.22\end{array}$ & $\begin{array}{c}\text { DATE } \\
05-14-59 \\
10-05-60 \\
08-28-61\end{array}$ & $\begin{array}{l}\mathrm{FT}^{\mathrm{a}} / \mathrm{S} \\
7.28 \\
8.72 \\
5.00\end{array}$ & $\begin{array}{c}\text { DATE } \\
08-19-64 \\
09-08-66 \\
09-12-67\end{array}$ & $\begin{array}{l}F^{3} / \mathrm{S} \\
0.29 \\
3.76 \\
1.40\end{array}$ & $\begin{array}{c}\text { DATE } \\
07-07-70 \\
09-02-71 \\
09-05-73\end{array}$ & $\begin{array}{c}\mathrm{FT}^{3} / \mathrm{S} \\
9.08 \\
4.2 \\
6.8\end{array}$ & $\begin{array}{c}\text { DA.TE } \\
08-04-75 \\
09-08-76\end{array}$ & $\begin{array}{l}F T^{3} / \mathrm{S} \\
10 . \\
2.1\end{array}$ \\
\hline Qa & $=47$ & $Q(84)$ & $=3.4$ & $7 Q 2$ & $=1.1$ & $7 Q 10$ & 0.1 & & \\
\hline
\end{tabular}

5-4576.00 ROCK CR NR FLOYD, IO'NA

LAT $4313 \times X$, LONG $9249 \times X$, IN NW $1 / 4$ SEC.

24, T. 97 N., R.17 W., FLOYO CO. (34),

AT BRIDIGE, 6 MILES NW OF FLOYD.

DRAINAGE AREA $69.7 \mathrm{MI}^{2}$

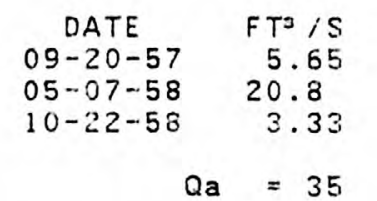
DATE
OE- $14-59$
$10-05-60$
$08-28-61$
$\mathrm{FT}^{3}$ is
9.73
20.9
6.31
DATE
$09-07-66$
$09-12-67$
07-07-70
$F T^{3} / S$
13.5
5.73
17.9

$Q(84)=\xi .4$ $\begin{array}{cc}\text { DATE } & F T^{3} / \mathrm{S} \\ 09-02-71 & 9.4 \\ 09-06-73 & 11 .\end{array}$

DATE
$08-05-75$

$09-08-76$

FTo / S

11. 4 
IOWA RIVER BASIN--Continued

5-4578.00 L CEDAR R NR STACEYVILLE, IOWA

LAT $4328 \times X$, LONG $9247 \times X$, IN NE $1 / 4$ SEC.

19, T.100 N., R.16 W., MITCHELL CO. (66),

AT BRIDGE, 2 MILES NORTH OF STACEYVILLE.

DRAINAGE AREA $77.3 \mathrm{MI}^{2}$

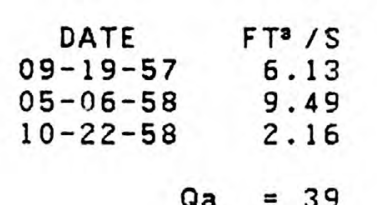

$Q_{\mathbf{a}}=39$

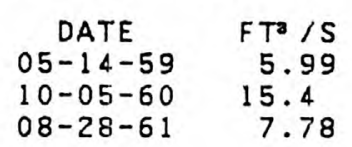

$Q(84)=6.1$

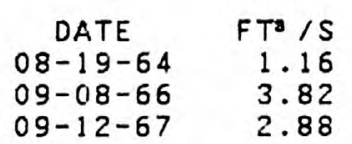

$702=3.1$

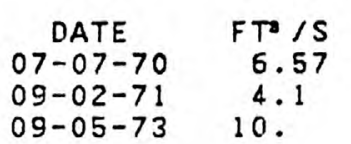

$7 Q 10=0.8$

5-4584.00 QUARTER SECTION RUN NR DENVER, IOWA

LAT 423951, LONG 922346, IN NE $1 / 4$ SEC.

29, T.91 N., R.13 W., BREMER CO. $\{09\}$,

AT BRIDGE, 3 MILES SW OF DENVER.

DRAINAGE AREA $83.5 \mathrm{MI}^{2}$

\begin{tabular}{|c|c|c|c|c|c|c|c|c|c|}
\hline $\begin{array}{c}\text { DATE } \\
09-25-57 \\
06-07-58 \\
10-21-58\end{array}$ & $\begin{array}{l}\mathrm{FT}^{3} / \mathrm{S} \\
0 . \\
0 . \\
0 .\end{array}$ & $\begin{array}{c}\text { DATE } \\
05-12-59 \\
10-03-60 \\
08-28-61\end{array}$ & $\begin{array}{l}\mathrm{FT}^{3} \text { iS } \\
0 . \\
0 . \\
0 .\end{array}$ & $\begin{array}{c}\text { DATE } \\
08-18-64 \\
09-08-66 \\
09-12-67\end{array}$ & $\begin{array}{l}\mathrm{FT} / \mathrm{S} \\
0 . \\
0.26 \\
0 .\end{array}$ & $\begin{array}{c}\text { DATE } \\
07-07-70 \\
09-01-71 \\
09-06-73\end{array}$ & $\begin{array}{l}\mathrm{FT}^{3} / \mathrm{S} \\
2.93 \\
0 . \\
0 .\end{array}$ & $\begin{array}{c}\text { DATE } \\
08-05-75 \\
09-08-76\end{array}$ & $\begin{array}{c}F T^{3} \\
0 . \\
0 .\end{array}$ \\
\hline & & Q1 & $y=0$ & 702 & $=0$ & 7010 & 0 & & \\
\hline
\end{tabular}

5-4585.50 BEAVERDAM CR NR ROCKWELL, IOWA

LAT $4258 \times X$, LONG $9315 \times X$, NEAR EAST $1 / 4$

CORNER SEC.18, T.94 N., R.20 W., CERRO GORDO CO.

(17), AT BRIDGE, 3 MILES SW OF ROCKWELL.

DRAINAGE AREA $72.4 \mathrm{MI}^{2}$

$\begin{array}{lr}\text { DATE } & F T^{3} / S \\ 09-24-57 & 1.35 \\ 04-03-58 & 7.43 \\ 10-23-58 & 0.96 \\ 05-13-59 & 5.76\end{array}$

$\begin{array}{cr}\text { DATE } & \mathrm{FT}^{3} / \mathrm{S} \\ 10-03-60 & 6.31 \\ 08-30-61 & 1.41 \\ 10-08-63 & 3.50 \\ 08-17-64 & 2.16\end{array}$

$\begin{array}{cc}\text { DATE } & F T^{3} / \mathrm{S} \\ 10-07-64 & 11.4 \\ 09-06-66 & 3.49 \\ 09-11-67 & 2.66\end{array}$

$\begin{array}{cc}\text { DATE } & F T / S \\ 06-10-68 & 23.3 \\ 07-09-70 & 7.40 \\ 09-02-71 & 4.3\end{array}$


IOWA RIVER BASIN--Cont inued

5-4586.00 BAILEY CR NR SHEFFIELD, IOWA

LAT $4254 \times X$, LONG $9316 \times X$, IN NW $1 / 4$ SEC.

$1, T .93$ N., R.21 W., FRANKLIN CO. (35).

AT BRIDGE, 4 MILES NW OF SHEFFIELD.

DRAINAGE AREA $75.2 \mathrm{MI}^{2}$

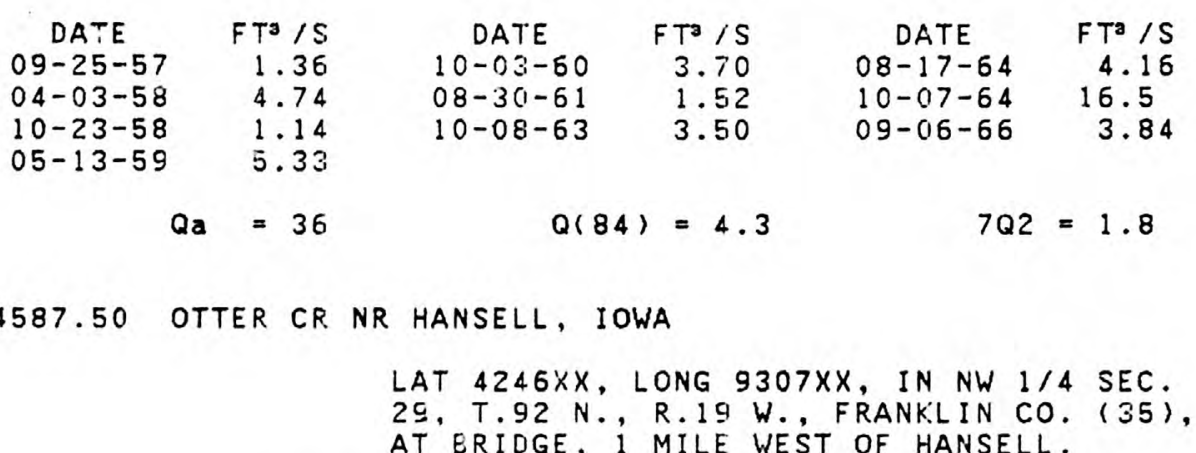

DRAINAGE AREA $92.0 \quad \mathrm{MI}^{2}$

$\begin{array}{cc}\text { CATE } & \mathrm{FT}^{3} / \mathrm{S} \\ 09-24-57 & 3.42 \\ 05-07-58 & 11.3 \\ 10-21-58 & 3.01 \\ 05-12-59 & 11.4\end{array}$

$Q_{a}=46$

$\begin{array}{rr}\text { DATE } & F T^{T} / S \\ 10-04-60 & 14.6 \\ 08-3(1-61 & 3.69 \\ 10-081-63 & 9.614 \\ Q(84)=8.4\end{array}$

$\begin{array}{cc}\text { DATE } & F T^{2} / S \\ 08-17-64 & 5.72 \\ 10-08-64 & 18.9 \\ 09-06-66 & 10.4\end{array}$

$7 Q 2=4 \cdot 1$

DATE
$09-11-67$
$07-08-70$
$09-23-71$

$F T / 5$
2.74
8.99
5.4

DATE
$09-08-73$
$08-05-75$

$09-07-76$

$7010=0.4 * * *$

5-4587.70 SQUAW CR. NR HANSELL, IOWA

LAT $4244 \times X$, LONG $9307 \times X$, NEAR CENTER OF

SEC.32, T.92 N., R.19 W., FRANKLIN CO. (35),

AT BRIUGE, 1.5 MILES SW OF HANSELL.

DRAINAGE AREA $24.2 \quad \mathrm{MI}^{2}$

$\begin{array}{cr}\text { DATE } & \text { FTO/S } \\ 09-24-57 & 1.23 \\ 05-07-58 & 2.27 \\ 10-21-58 & 1.36 \\ 05-12-59 & 3.27\end{array}$

$Q_{a}=12$

$\begin{array}{cr}\text { DATE } & F T^{3} / S \\ 10-04-60 & 6.22 \\ 08-30-61 & 2.04 \\ 10-08-63 & 2.83\end{array}$

$Q(84)=3.0$

$\begin{array}{cr}\text { DATE } & F^{2} / S \\ 08-17-64 & 2.38 \\ 10-08-64 & 5.75 \\ 09-016-66 & 4.55\end{array}$

$7 Q 2=1.6$

$\begin{array}{cc}\text { DATE } & F T^{3} / S \\ 09-12-67 & 7.47 \\ 07-08-70 & 17.0 \\ 09-01-71 & 8.4\end{array}$

DATE
O9-06-

$09-06-73$

$08-04-75$

$09-07-76$

$7010=1.3 * \star \star$
$F T^{3} / \mathrm{S}$

5.5
6.7

2. 0 
IOWA RIVER BASIN--Cont inued

5-4587.80 HARTGRAVE CR NR HANSELL, IOWA

LAT $4244 X X$, LONG $9305 X X$, IN NW $1 / 4$ SEC.

$34, T .92$ N., R.19 W., FRANKLIN CO. (35),

AT BRIDGE, 1.5 MILES SE OF HANSELL.

DRAINAGE AREA $161 \mathrm{MI}^{2}$

$\begin{array}{rc}\text { DATE } & F T^{3} / S \\ 09-24-57 & 8.69 \\ 05-07-58 & 22.6 \\ 10-21-58 & 8.58 \\ 05-12-59 & 23.0 \\ \text { Qa } & =80\end{array}$

$\begin{array}{rl}\text { DATE } & F T^{a} / S \\ 10-04-60 & 30.4 \\ 08-30-61 & 8.60 \\ 10-08-63 & 17.2 \\ 07-29-64 & 11.6 \\ Q(84) & =18\end{array}$

$\begin{array}{cc}\text { DATE } & F T^{3} / \\ 08-17-64 & 13.8 \\ 10-08-64 & 38.5 \\ 09-06-66 & 24.8\end{array}$

$\begin{array}{cl}\text { DATE } & F T^{2} / S \\ 09-12-67 & 17.4 \\ 07-08-70 & 37.9 \\ 09-01-71 & 18.0\end{array}$

DATE

$09-07-73$

$08-04-75$

$09-07-76$

$F T^{3} / \mathrm{S}$

31.

$09-06-66-24.8$

$702=10$

$7010=3 \cdot 6^{* * *}$

5-4587.90 BOYLAN CR NR BRISTOW, IOWA

LAT $4246 \times X$, LONG $9256 \times X$, IN NE $1 / 4$ SEC.

23 , T.92 N., R.18 W., BUTLER CO. (12),

AT BRIDGE, I MILE WEST OF BRISTOW.

DRAINAGE AREA $55.7 \mathrm{MI}^{2}$

DATE
$09-24-57$
$05-07-58$
$10-21-58$
$05-12-59$

$F T^{3} / S$

$\begin{array}{cc}\text { DATE } & F T^{3} \text { is } \\ 10-04-60 & 14.7 \\ 08-30-61 & 0.17 \\ 10-08-63 & 0.88\end{array}$

$\begin{array}{cc}\text { DATE } & F T^{2} / \mathrm{S} \\ 08-18-64 & 0 . \\ 10-08-64 & 2.76 \\ 09-07-66 & 1.11\end{array}$

DATE
$09-12-67$

$09-12-67$
$07-07-70$

$09-22-71$

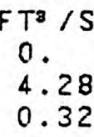

DATE

$09-06-73$

$08-05-75$

$F T^{a} / S$

1.8

2.9.
0.

$a(84)=0.8$

$7 Q 2=0$

$7 Q 10=0$

5-4588.00 MAYNES CR NR HAMPTON, IOWA

LAT $4241 \times X$, LONG $9312 \times X$, IN NW $1 / 4$ SEC. $22, T .91 \mathrm{~N} .$, R. $20 \mathrm{~W}$. , FRANKL IN CO. (35), AT BRIDGE ON U.S. HIGHWAY 65, 4 MILES

SOUTH OF HAMPTON.

DRAINAGE AREA $71.0 \mathrm{MI}^{2}$

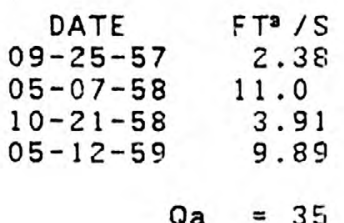

Qa

$\begin{array}{cc}\text { DATE } & F T^{3} / \mathrm{S} \\ 10-04-60 & 14.6 \\ 08-30-61 & 2.68 \\ 10-09-63 & 3.20 \\ 08-17-64 & 4.38\end{array}$

$Q(84)=5.2$

$\begin{array}{cc}\text { DATE } & F T a / S \\ 10-08-64 & 12.2 \\ 09-07-66 & 6.61 \\ 09-12-67 & 3.25 \\ & \\ & \end{array}$

$7 Q 2=2.2$

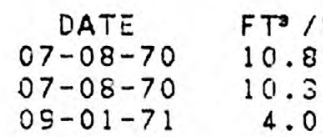

DATE
$09-07-73$
$08-04-75$
$09-08-76$

$\mathrm{FT} / \mathrm{S}$

$7 \cdot 2$

1.6 
IOWA RIVER BASIN--Cont inued

5-4588.50 MAYNES CR NR DUMONT, IOWA

LAT $4242 \times X$, LONG 9258XX, IN SW $1 / 4$ SEC.

$15, T .91$ N., R. $1 \&$ W., BUTLER CO. $(12\}$,

AT BRIDGE, 4 MILES SOUTH OF DUMONT.

DRAINAGE AREA $121 \quad \mathrm{MI}^{2}$

\begin{tabular}{|c|c|c|c|c|c|c|c|c|c|}
\hline $\begin{array}{c}\text { DATE } \\
09-24-57 \\
05-07-58 \\
10-21-58 \\
05-12-59\end{array}$ & $\begin{array}{c}\mathrm{FT}^{3} / \mathrm{S} \\
3.72 \\
19.4 \\
8.00 \\
30.1\end{array}$ & $\begin{array}{c}\text { DATE } \\
10-04-60 \\
08-30-61 \\
10-08-63\end{array}$ & $\begin{array}{c}\mathrm{FT}^{3} \text { is } \\
34.1 \\
6.59 \\
7.71\end{array}$ & $\begin{array}{c}\text { DATE } \\
08-18-64 \\
10-018-64 \\
09-017-66\end{array}$ & $\begin{array}{c}\mathrm{FT}^{3} / \mathrm{S} \\
7.61 \\
28.3 \\
16.2\end{array}$ & $\begin{array}{c}\text { DATE } \\
09-12-67 \\
07-07-70 \\
09-02-71\end{array}$ & $\begin{array}{c}F^{3} / S \\
8.88 \\
25.2 \\
9.4\end{array}$ & $\begin{array}{c}\text { DATE } \\
09-05-73 \\
08-05-75 \\
09-08-76\end{array}$ & $\begin{array}{l}F T / S \\
17 . \\
14 . \\
3.1\end{array}$ \\
\hline$Q \mathbf{a}$ & $=60$ & $Q C$ & $=11$ & $7 Q 2$ & $=5.4$ & 7010 & $1.6 * * *$ & & \\
\hline
\end{tabular}

5-4590.50 LIME CR NR SCARVILLE, IOWA

LAT $4327 \times X$, LONG $9335 \times X$. IN SW $1 / 4$ SEC.

28, T. 100 N., R.23 W., WINNEBAGO CO. (95),

AT BRIDGE, 3.5 MILES SE OF SCARVILLE.

DRAINAGE AREA $113 \quad \mathrm{MI}^{2}$

$\begin{array}{rccccc}\text { DATE } & F T=/ S & \text { DATE } & F T^{3} / S & \text { DATE } & F T / S \\ 09-25-57 & 6.11 & 05-13-59 & 75.9 & 08-28-63 & 17.4 \\ 04-33-58 & 24.5 & 10-04-60 & 7.25 & 08-19-64 & 2.05 \\ 10-22-58 & 3.00 & 08-29-61 & 6.611 & 09-07-66 & 8.85 \\ Q a & =53 & & Q(84)=7.2 & 7 Q 2=2.6\end{array}$

$\begin{array}{cc}\text { DATE } & F T O I S \\ 09-11-67 & 6.45 \\ 07-07-70 & 9.32 \\ 09-01-71 & 9.5\end{array}$

DATE

$09-05-73$

$\mathbf{a}=53$

$Q(84)=7.2$

$7010=0.6 * * *$

5-4592.00 WINNEBAGO R NR FOFEST CITY, IOWA

LAT 4318XX, LONG 9339XX, IN NW $1 / 4$ SEC.

AT BRIUGE, 2.5 MILES NORTH OF FOREST CITY.

DRAINAGE AREA $2.25 \quad \mathrm{MI}^{2}$

$\begin{array}{cc}\text { DATE } & F T^{3} / S \\ 09-25-57 & 9.05 \\ 04-03-58 & 45.5 \\ 10-22-58 & 3.92\end{array}$

$Q_{\mathbf{a}}=93$

$\begin{array}{cl}\text { DATE } & F T / S \\ 05-13-59 & 70.8 \\ 10-04-60 & 11.4 \\ 08-29-61 & 11.7\end{array}$

$Q(84)=10$

$\begin{array}{cc}\text { DATE } & F T^{3} / S \\ 08-19-64 & 2.85 \\ 09-017-66 & 20.0 \\ 09-11-67 & 7.10\end{array}$

$7 Q 2=2.9$

$\begin{array}{cl}\text { DATE } & F T^{3} / S \\ 07-07-70 & 18.0 \\ 09-01-71 & 10.0 \\ 09-05-73 & 18 .\end{array}$

$7010=0.7 * \star \star *$ 
IOWA R.IVER BASIN--Continued

5-4593.00 WINNEBAGO R NR FERTILE, IOWA

LAT $4315 \times X$, LONG $9326 \times X$, NEAR WEST $1 / 4$

CORNER SEC.3, T.97 N., R.22 W., CERRO GORDO CO.

(17), AT BRIDGE, 1.5 MILES SW OF FERTILE.

\begin{tabular}{|c|c|c|c|c|c|c|c|c|c|}
\hline & DRAINAGE & AREA & $M I^{2}$ & & & & & & \\
\hline $\begin{array}{c}\text { DATE } \\
09-26-57 \\
04-03-58 \\
10-23-58\end{array}$ & $\begin{array}{l}F T=/ S \\
15.1 \\
59.0 \\
8.47\end{array}$ & $\begin{array}{c}\text { DATE } \\
05-13-59 \\
10-04-60 \\
08-29-61\end{array}$ & $\begin{array}{l}\mathrm{FT}^{3} \text { is } \\
83.7 \\
20.9 \\
23.4\end{array}$ & $\begin{array}{c}\text { DATE } \\
08-19-64 \\
09-08-66 \\
09-11-67\end{array}$ & $\begin{array}{l}\mathrm{FT}^{3} / \mathrm{S} \\
12.6 \\
37.8 \\
17.6\end{array}$ & $\begin{array}{c}\text { DATE } \\
07-07-70 \\
09-01-71 \\
09-05-73\end{array}$ & $\begin{array}{l}F T \cdot / S \\
33.0 \\
21.0 \\
33 .\end{array}$ & $\begin{array}{c}\text { DATE } \\
08-05-75 \\
09-07-76\end{array}$ & $\begin{array}{l}\mathrm{FT}^{\mathrm{T}} / \mathrm{S} \\
46 . \\
3.9\end{array}$ \\
\hline Qa & $=137$ & $Q<84$ & $=23$ & $7 Q 2$ & $=9.6$ & $7 Q 10$ & $2.8 * \star *$ & & \\
\hline
\end{tabular}

5-4594.00 BEAVER CR NR FERTILE, IOWA

LAT $4316 \times X$, LONG $9327 \times X$, IN SW $1 / 4$ SEC.

28 , T.98 N., R.22 W., WORTH CO. ( 98 ),

AT BRIDGE, 2 MILES NW OF FERTILE.

DRAINAGE AREA $54.9 \mathrm{MI}^{2}$

\begin{tabular}{|c|c|c|c|c|c|c|c|c|c|}
\hline $\begin{array}{c}\text { DATE } \\
09-26-57 \\
04-03-58 \\
10-23-58\end{array}$ & $\begin{array}{c}\mathrm{FT}^{3} / \mathrm{S} \\
1.42 \\
11.6 \\
0.47\end{array}$ & $\begin{array}{c}\text { DATE } \\
05-13-59 \\
10-04-60 \\
08-29-61\end{array}$ & $\begin{array}{l}\mathrm{FT}^{\mathrm{a}} / \mathrm{S} \\
7.84 \\
2.67 \\
2.015\end{array}$ & $\begin{array}{c}\text { DATE } \\
08-19-64 \\
09-07-66 \\
09-11-67\end{array}$ & $\begin{array}{r}\mathrm{FT}^{\mathrm{a}} / \mathrm{S} \\
1.68 \\
5.60 \\
1.82\end{array}$ & $\begin{array}{c}\text { DATE } \\
07-07-70 \\
09-01-71 \\
09-05-73\end{array}$ & $\begin{array}{l}F T / S \\
10.7 \\
4.8 \\
5.4\end{array}$ & $\begin{array}{c}\text { DATE } \\
08-05-75 \\
09-07-76\end{array}$ & $\begin{array}{l}\mathrm{FT}^{\mathrm{S}} / \mathrm{S} \\
6.6 \\
1.2\end{array}$ \\
\hline Qa & $=26$ & $Q(84)$ & $=2.7$ & $7 Q 2$ & $=0.8$ & $7 Q 10$ & 0.1 & & \\
\hline
\end{tabular}

5-4602.00 WILLOW CR AT MASON CITY, IOWA

LAT 430946, LONG 931420 , NEAR WEST $1 / 4$

CORNER SEC. 5 , T. $96 \mathrm{~N} . . \mathrm{R} .20 \mathrm{~W}$., CERRO GORDO CO.

(17), AT BRIDGE NEAR WEST CITY'LIMITS OF

MASON CITY.

DRAINAGE AREA $86.0 \quad \mathrm{MI}^{2}$

\begin{tabular}{|c|c|c|c|c|c|c|c|c|c|}
\hline $\begin{array}{c}\text { DATE } \\
09-26-57 \\
04-03-58 \\
10-23-58\end{array}$ & $\begin{array}{c}\text { FT } / \mathrm{S} \\
3.77 \\
13.3 \\
2.77\end{array}$ & $\begin{array}{c}\text { DATE } \\
05-13-59 \\
10-04-60 \\
08-29-61\end{array}$ & $\begin{array}{l}\mathrm{FT}^{3} / \mathrm{S} \\
7.10 \\
8.71 \\
4.84\end{array}$ & $\begin{array}{c}\text { DATE } \\
08-19-64 \\
09-08-66 \\
09-11-67\end{array}$ & $\begin{array}{l}\mathrm{FT}^{3} / \mathrm{S} \\
4.70 \\
7.75 \\
4.73\end{array}$ & $\begin{array}{c}\text { DATE } \\
07-08-70 \\
09-02-71 \\
09-06-73\end{array}$ & $\begin{array}{c}F T^{a} / S \\
12.1 \\
6.8 \\
7.8\end{array}$ & $\begin{array}{c}\text { DATE } \\
08-05-75 \\
09-08-76\end{array}$ & $\begin{array}{c}\mathrm{FT}^{3} / \mathrm{S} \\
9.8 \\
3.0\end{array}$ \\
\hline & $=41$ & Q & $=5.9$ & $7 Q 2$ & $=3.3$ & $7 Q 10$ & 1. $4 * \star \star \star$ & & \\
\hline
\end{tabular}


IOWA RIVER BASIN--Cont inued

5-4611.00 COLD WATER CR NR GREENE, IOWA

LAT $4253 \times X$, LONG $9251 \times X$, IN SW $1 / 4$ SEC

$10, T .93 \mathrm{~N}$. , R.17 W., BUTLER CO. \{12\},

AT BRIDGE, 2.5 MILES' SW OF GREENE.

DRAINAGE AREA $56.8 \mathrm{MI}^{2}$

$\begin{array}{rlcccc}\text { DATE } & \mathrm{FT}^{3} / \mathrm{S} & \text { DATE } & \mathrm{FT}^{3} / \mathrm{S} & \text { DATE } & \mathrm{FT}^{3} / \mathrm{S} \\ 09-24-57 & 0.05 & 05-14-59 & 0 . & 08-19-64 & 0.03 \\ 05-07-58 & 0.09 & 10-03-60 & 8.75 & 09-07-66 & 0.03 \\ 10-21-58 & 0 . & 08-28-61 & 0.01 & 09-12-67 & 0.05 \\ & & & & \end{array}$

$\begin{array}{cc}\text { DATE } & F^{3} / S \\ 07-08-70 & 0.04 \\ 09-02-71 & 0.01\end{array}$

DATE
$08-05-75$

O8-05-75

$F T=1 S$

$09-02-71 \quad 0.01$

$09-08-76$

0.

$Q \mathbf{a}=29$

$Q(84)=0.3$

$7 Q 10=0$

5-4513.00 FLOOD CR. NR ROCKFORD, IOWA

LAT $4303 \times X$, LONG $9251 \times X$, IN NW $1 / 4$ SEC.

15. T.95 N., R. 17 W. FLOYO CO. (34)

AT BRIUGE, 5 MILES EAST OF ROCKFORD.

DRAINAGE AREA $59.3 \mathrm{MI}^{2}$

$\begin{array}{cccccc}\text { DATE } & F T^{3} / S & \text { DATE } & F T^{3} / S & \text { DATE } & F T^{3} / S \\ 09-20-57 & 0 . & 05-14-53 & 0 . & 08-19-64 & 0 . \\ 05-07-58 & 0 . & 10-05-60 & 11.5 & 09-07-66 & 0 . \\ 10-22-58 & 0 . & 08-28-61 & 0 . & 09-12-67 & 0 . \\ & & 0(84)=0 & & 702=0\end{array}$

$\begin{array}{ll}\text { DATE } & F T / S \\ 07-08-70 & 1.37 \\ 09-02-71 & 0 . \\ 09-06-73 & 0 .\end{array}$

DATE

$08-05-75$ T T

$09-06-73$

$09-08-76 \quad 0$.

$Q_{\mathbf{a}}=30$

$Q(84)=0$

$7 Q 10=0$

5-4614.00 FLOOD CR NR PACKAR.D, IOWA

LAT $4253 \times X$, LONG $9242 \times X$, IN NE $1 / 4$ SEC.

$23, T .93 \mathrm{~N},$, R.16 W. BLTLER CO. (12)

AT BRIDGE, 2 MILES NE OF PACKARD.

DRAINAGE AREA $145 \quad \mathrm{MI}^{2}$

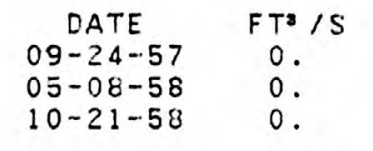

$Q_{a}=73$

\begin{abstract}
DATE
$05-14-509$
O5-14-59

$10-03-60$

$08-28-61$

$\mathrm{FT}^{\mathrm{T}}$ /S

11.5
\end{abstract}

$Q(84)=0$

$\begin{array}{cc}\text { DATE } & \mathrm{FT}^{3} / \mathrm{S} \\ 03-19-64 & 0.01 \\ 09-07-66 & 5.91 \\ 09-12-67 & 0 .\end{array}$

$7 Q 2=$
DATE
$07-08-70$

$07-08-70$
$09-02-71$

$09-06-73$

$F T=/ S$
6.12
0.20
2.8

DATE

O 8-05-75

$09-08-76$

$F T a / S$

$7010=0$ 
IOWA R.IVER BASIN--Cont inued

5-4627.00 BEAVER CR NR ACKLEY, IOWA

LAT $4234 \times X$, LONG $9302 \times X$. IN SW $1 / 4$ SEC.

36, T.90 N., R.19 W., FRANKLIN CO. $\{35\}$,

AT BRIDGE NEAR EAST CITY LIMITS OF ACKLEY.

DRAINAGE AREA $55.5 \mathrm{MI}^{2}$

\begin{tabular}{|c|c|c|c|c|c|c|c|c|c|}
\hline $\begin{array}{c}\text { DATE } \\
09-25-57 \\
05-07-58 \\
10-21-58\end{array}$ & $\begin{array}{l}F T^{3} / \mathrm{S} \\
1.59 \\
7.45 \\
4.06\end{array}$ & $\begin{array}{c}\text { DATE } \\
05-12-59 \\
10-04-60 \\
08-30-61\end{array}$ & $\begin{array}{l}\mathrm{FT}^{3} / \mathrm{S} \\
16.5 \\
33.1 \\
3.53\end{array}$ & $\begin{array}{c}\text { DATE } \\
08-18-64 \\
09-07-66 \\
09-13-67\end{array}$ & $\begin{array}{l}F T^{3} / S \\
2.96 \\
4.76 \\
1.88\end{array}$ & $\begin{array}{c}\text { DATE } \\
07-08-70 \\
09-01-71 \\
09-07-73\end{array}$ & $\begin{array}{l}F T .1 S \\
5.26 \\
2.9 \\
5.0\end{array}$ & $\begin{array}{c}\text { DATE } \\
08-04-75 \\
09-10-76\end{array}$ & $\begin{array}{c}\mathrm{FT}^{\mathrm{T}} / \mathrm{S} \\
3.4 \\
1.0\end{array}$ \\
\hline & $=28$ & QR & $=3.4$ & $7 Q_{2}$ & $=1.6$ & $7 Q 10$ & $0.4 * * x$ & & \\
\hline
\end{tabular}

5-4628.00 S BEAVER CR NR PARKERSBURG, IOWA

LAT $4234 \times X$, LONG $9249 \times X$. IN SE $1 / 4$ SEC.

AT, CULVERT, 2 MILES SW OF PARKERSBURG.

DRAINAGE AREA $114 \quad \mathrm{MI}^{2}$

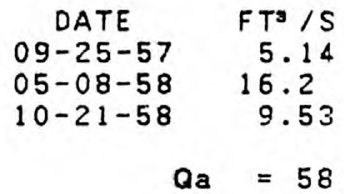

$\begin{array}{cl}\text { DATE } & F T^{3} / S \\ 05-12-59 & 26.2 \\ 10-04-60 & 43.5\end{array}$

$10-04-60 \quad 43.5$

$08-30-61$

$Q(84)=9.5$

$\begin{array}{cc}\text { DATE } & F T / S \\ 07-08-70 & 6.10 \\ 09-01-71 & 5.6 \\ 09-07-73 & 17 .\end{array}$

$7010=1.5 * \star *$

5-4631.00 BLACK HAWK CR NR GRUNDY CENTER, IOWA

LAT $4222 X X$, LONG $9244 X X$, NEAR E $1 / 4$

CORNER SEC.8, T.87 N., R.16 W., GRUNDY CO. $\{38\}$,

AT BRIDGE, 2 MILES EAST OF GRUNDY CENTER.

DRAINAGE AREA $71.0 \mathrm{MI}^{2}$

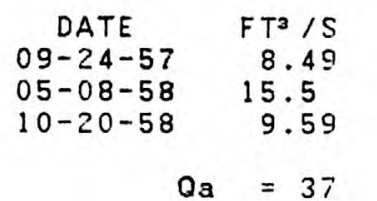

$Q_{a}=3 T$

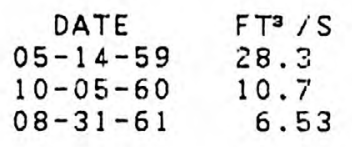

$Q(84)=6.8$

$\begin{array}{cc}\text { DATE } & F T^{3} / S \\ 08-19-64 & 3.23 \\ 09-018-66 & 11.7 \\ 07-07-70 & 10.2\end{array}$

$7 Q 2=3.9$

$\begin{array}{cc}\text { DATE } & F T^{\circ} / \mathrm{S} \\ 09-02-71 & 4.0 \\ 09-07-73 & 14 .\end{array}$

4.0

$09-07-73$

$7010=1.3 * * *$
DATE
$08-05-75$

$09-10-76 \quad 6.1$ 
IOWA RIVER BASIN--Continued

5-4632.00 MOSQUITO CR AT REINBECK, IOWA

LAT $4220 \times X$, LONG $9237 \times X$, IN SE $1 / 4$ SEC.

20, T.87 N., R.15 W., GRUNDY CO. 138 ; ,

AT BRIDGE, i MILE WEST OF REINBECK.

DRAINAGE AREA $24.0 \mathrm{MI}^{2}$

\begin{tabular}{|c|c|c|c|c|c|c|c|c|c|}
\hline $\begin{array}{c}\text { DATE } \\
09-24-57 \\
05-08-58 \\
10-20-58\end{array}$ & $\begin{array}{r}\mathrm{FT}^{3} / \mathrm{S} \\
2.27 \\
4.68 \\
2.06\end{array}$ & $\begin{array}{c}\text { DATE } \\
05-14-59 \\
10-05-60 \\
08-31-61\end{array}$ & $\begin{array}{l}\mathrm{FT}^{3} \text { is } \\
9.66 \\
4.72 \\
3.23\end{array}$ & $\begin{array}{c}\text { DATE } \\
08-19-64 \\
09-018-66 \\
07-017-70\end{array}$ & $\begin{array}{l}\mathrm{FT}^{3} / \mathrm{S} \\
0.40 \\
1.89 \\
6.14\end{array}$ & $\begin{array}{c}\text { DATE } \\
09-02-71 \\
09-07-73\end{array}$ & $\begin{array}{l}\mathrm{FT}^{\mathrm{T}} / \mathrm{S} \\
0.92 \\
2 .\end{array}$ & $\begin{array}{c}\text { DATE } \\
08-05-75 \\
09-09-76\end{array}$ & $\begin{array}{l}\mathrm{FT}^{9} / \mathrm{S} \\
5.8 \\
0.66\end{array}$ \\
\hline & $=13$ & ar & $\gamma=1.8$ & $7 Q^{2}$ & $=0.6$ & $70: 10$ & * & & \\
\hline
\end{tabular}

5-4633.00 BLACK HAWK CR AT REINBECK, IOWA

LAT $4220 \times X$, LONG $9236 \times X$, NEAR E $1 / 4$

CORNER SEC.21. T.87 N., R.15 W., GRUNDY CO. (38), AT BRIDGE, 1 MILE. NORTH OF REINBECK.

ORAINAGE AREA $135 \quad \mathrm{MI}^{2}$

\begin{tabular}{|c|c|c|c|c|c|c|c|c|c|}
\hline $\begin{array}{c}\text { DATE } \\
09-24-57 \\
05-08-58 \\
10-20-58\end{array}$ & $\begin{array}{l}F^{3} / \mathrm{S} \\
16.6 \\
28.3 \\
21.4\end{array}$ & $\begin{array}{c}\text { DATE } \\
05-14-59 \\
10-05-60 \\
08-31-61\end{array}$ & $\begin{array}{l}\mathrm{FT}^{3} \text { iS } \\
51.8 \\
25.9 \\
16.8\end{array}$ & $\begin{array}{c}\text { DATE } \\
08-19-64 \\
09-018-55 \\
07-017-70\end{array}$ & $\begin{array}{l}\mathrm{FT}^{3} / \mathrm{S} \\
6.30 \\
22.6 \\
10.6\end{array}$ & $\begin{array}{c}\text { DATE } \\
09-02-71 \\
09-07-73\end{array}$ & $\begin{array}{l}\mathrm{FT}^{3} / \mathrm{S} \\
9.0 \\
24 .\end{array}$ & $\begin{array}{c}\text { DATE } \\
08-05-75 \\
09-09-76\end{array}$ & $\begin{array}{l}\mathrm{FT}^{3} / \mathrm{S} \\
20 . \\
8.7\end{array}$ \\
\hline Qa & $=71$ & Q & )$=19$ & 702 & $=7.4$ & $7 Q 10$ & $2.2^{* \star *}$ & & \\
\hline
\end{tabular}

5-4634.00 N BLACK HAWK CR AT DIKE, IOWA

LAT $4227 \times X$, LONG $9237 \times X$, NEAR $N$ I/4

CORNER SEC.8, T.8B N., R.15 W., GRINNOY CO. (38:,

AT BRILIGE NEAR SE CITY LIMITS OF DIKE.

DRAINAGE AREA $75.3 \mathrm{MI}^{2}$

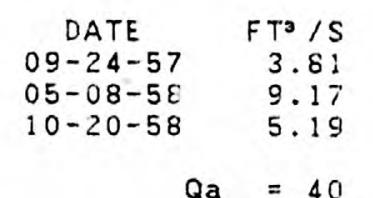

$Q \mathbf{a}=40$

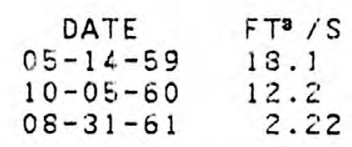

$Q(84)=3.7$

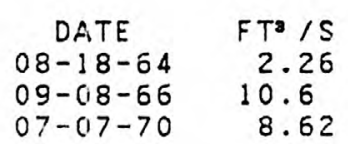

$7 Q 2=1.6$

$\begin{array}{cc}\text { DATE } & F^{3} / S \\ 09-02-71 & 2.0 \\ 09-07-73 & 6.2\end{array}$

$7010=0.3 * * t$ 
IOWA R.IVER BASIN--Cont inued

5-4640.50 MILLERS CR NR LAPORTE CITY, IOWA

LAT $4223 \times X$, LONG $9215 \times X$, IN SE $1 / 4$ SEC.

33, T.88 N., R.12 W., BLACK HAWK CO. (07),

AT BRIDGE ON U. S. HIGHWAY 218,6 MILES

NW OF LAPORTE CITY.

DRAINAGE AREA $54.8 \mathrm{MI}^{2}$

$\begin{array}{rr}\text { DATE } & F T^{3} / S \\ 09-26-57 & 3.68 \\ 05-09-58 & 7.54 \\ 10-08-58 & 4.50 \\ 09-18-59 & 2.98 \\ Q a & =30\end{array}$

$\begin{array}{rr}D A T E & F T^{3} / S \\ 10-21-59 & 4.59 \\ 08-17-60 & 9.78 \\ 08-28-61 & 8.70 \\ 10-27-64 & 1.53 \\ Q(84) & =3.7\end{array}$

$\begin{array}{rc}\text { DATE } & F T^{3} / \mathrm{S} \\ 09-13-66 & 8.18 \\ 07-07-70 & 10.3 \\ 09-01-71 & 6.5 \\ 09-06-73 & 9.0 \\ 702= & 0.9\end{array}$

DATE
$09-26-74$
$10-01-74$

$\mathrm{FT}^{\mathrm{a}} / \mathrm{S}$

3.9

DATE

$09-24-75$

$10-29-75$

$\mathrm{FT}^{\mathrm{T}} / \mathrm{S}$

$\begin{array}{rrr}10-01-74 & 12.1 & 10-29-75 \\ 08-04-75 & 5.18 & 09-08-76\end{array}$

4.14

3.3

$Q \mathbf{a}=30$

$Q(84)=3.7$

$7 Q 10=*$

5-4641.00 WOLF CR NR BEAMAN, IOWA

LAT 421247, LONG 924712 , IN SW 1/4 SEC

$36, T .86$ N., R.17 W., GRUNDY CO. $\{38\}$,

AT BRIUGE, 2 MILES SE OF BEAMAN.

\section{DRAINAGE AREA $63.2 \mathrm{MI}^{2}$}

$\begin{array}{cccc}\text { DATE } & F T^{3} / \mathrm{S} & \text { DATE } & F T^{3} / S \\ 09-20-57 & 6.44 & 10-21-59 & 3.53 \\ 05-09-58 & 12.8 & 08-17-60 & 5.59 \\ 10-08-58 & 13.5 & 08-28-61 & 4.16 \\ 09-18-59 & 3.17 & 10-27-64 & 1.77\end{array}$

DATE
$09-12-66$
$07-07-70$
$09-01-71$
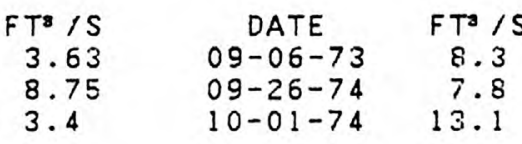

$09-01-71$

3.4

$09-26-74$
$10-01-74$

8.3
7.8

$08-05-75$

$Q_{a}=33$

$Q(84)=4.6$

$702=2.2$

$7010=0.8 * * *$

5-4641.50 TWELVE MILE CR NR BUCKINGHAM, IOWA

LAT $4214 X X$, LONG $9226 \times X$, IN SW $1 / 4$ SEC.

$24, T .86$ N., R.14 W., TAMA CO. (86),

AT BRIOGE, 1.5 MILES SOUTH OF BUCKINGHAM.

DRAINAGE AREA $76.8 \mathrm{MI}^{2}$

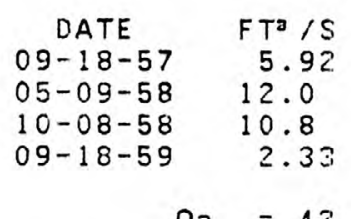

$Q_{a}=42$

$\begin{array}{cr}\text { DATE } & F T^{3} / S \\ 10-21-59 & 4.44 \\ 08-17-60 & 8.41 \\ 08-25-61 & 8.21 \\ 10-27-64 & 2.41\end{array}$

$Q(84)=4.1$

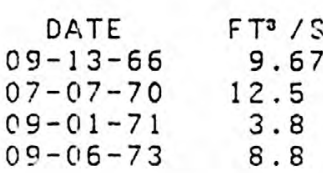

$7 Q 2=1.1$

$\begin{array}{cc}\text { DATE } & \mathrm{FT}^{3} / \mathrm{S} \\ 09-26-74 & 9.2 \\ 10-21-74 & 21.1 \\ 08-05-75 & 10.8\end{array}$

DATE
$09-24-75$
$10-29-75$
$09-09-76$

$\mathrm{FT}^{3} / \mathrm{S}$

5.11

1.3

$7 Q 10=0.1 * \star \star *$ 
IOWA R.IVER BASIN--Cont inued

5-4642.00 WOLF CR NR BUCKINGHAM, IOWA

LAT 421533, LONG 922142, IN NE $1 / 4$ SEC.

$21, T .86$ N., R.13 W., TAMA CO. $(86)$,

AT BRIDGE, 4.5 MILES SE OF BUCKINGHAM.

\section{DRAINAGE AREA $287 \quad \mathrm{MI}^{2}$}

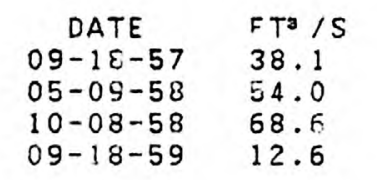

$Q \mathbf{a}=152$

$\begin{array}{ll}\text { DATE } & F T^{3} / 5 \\ 10-21-59 & 18.8 \\ 08-17-60 & 39.4 \\ 08-28-61 & 28.8 \\ 10-27-64 & 13.5\end{array}$

$Q(84)=26$

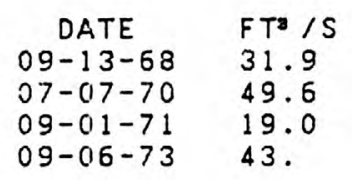

$7 Q 2=9.5$

$\begin{array}{clcc}\text { DATE } & F T^{3} / \mathrm{S} & \text { DATE } & F T^{3} / \mathrm{S} \\ 09-26-74 & 42 . & 09-24-75 & 21.7 \\ 10-21-74 & 89 . & -10-29-75 & 19 . \\ 08-05-75 & 45.2 & 09-09-76 & 9.5\end{array}$

$7 Q 10=2.2 * \star \star *$

5-4642.50 WOLF CR AT LAPORTE CITY, IOWA

LAT $4219 \times X$, LONG $9212 \times X$, IN SW $1 / 4$ SEC.

25, T.87 N., R.12 W., BLACK HAWK CO. (07),

AT BRIDGE ON U. S. HIGHWAY 218 IN LAPORTE CITY.

DRAINAGE AREA $327 \quad \mathrm{MI}^{2}$

$\begin{array}{clclcl}\text { DATE } & F T^{3} / S & \text { DATE } & F T^{3} / S & \text { DATE } & F T^{3} / S \\ 09-26-57 & 33.2 & 08-17-60 & 62.0 & 07-07-70 & 68.4 \\ 10-20-58 & 65.8 & 08-28-61 & 44.7 & 09-01-71 & 31.0 \\ 09-18-59 & 18.4 & 10-27-64 & 17.1 & 09-016-73 & 60 . \\ 10-21-59 & 27.2 & 09-13-66 & 46.7 & 09-26-74 & 54 .\end{array}$

$Q_{a}=175$

$Q(84)=37$

$7 Q 2=16$

$\begin{array}{cc}\text { DATE } & \mathrm{FT}^{3} / \mathrm{S} \\ 10-21-74 & 93.8 \\ 06-24-75 & 986 . \\ 08-04-75 & 61.7\end{array}$

DATE

$09-24-75$
$10-29-75$

$10-29-75$
$09-08-76$

$F T / S$

37.3

17 .

5-4643.00 SPRING CR NR LAPORTE CITY, IOWA

LAT $4220 \times X$, LONG 9206XX. IN NW $1 / 4$ SEC.

$23, T .57$ N., R.11 W. B BLACK. HAWK CO. (07),

AT BRIDGE, 5 MILES NE OF LAPORTE CITY.

DRAINAGE AREA $57.5 \mathrm{MI}^{2}$

DATE
$09-25-57$
$10-08-58$
$09-18-59$
$10-21-59$

$\begin{array}{cc}\text { DATE } & F T^{3} / S \\ 08-17-60 & 15.4 \\ 08-28-61 & 9.12 \\ 10-27-64 & 3.33 \\ 09-13-66 & 15.6\end{array}$

$\begin{array}{cc}\text { DATE } & F T^{3} / S \\ 07-07-70 & 17.9 \\ 09-01-71 & 8.3 \\ 09-016-73 & 9.6 \\ 09-2.6-74 & 9.8\end{array}$

DATE
$10-21-74$

$10-21-74$
$08-04-75$

$08-04-75$
$09-24-75$

$F T^{3} / 5$
12.4

12.4

6.84

DATE

$10-29-75$

$07-19-76$

09-08-76

$F T^{3} / S$

5.15

$Q(84)=4.5 * \star \star$

$7 Q 2=2.9 * *$

$7010=0.9 * *$ 
IOWA RIVER BASIN--Continued

5-4543.20 E BLUE CR NR CENTER POINT, IOWA

LAT 421141, LONG 914828, IN NW $1 / 4$ SEC.

$8, T .85$ N., R.8 W., LINN CO. (57),

AT BRIDGE, 1 MILE WEST OF CENTER POINT.

DRAINAGE AREA $27.1 \mathrm{MI}^{2}$

$\begin{array}{cr}\text { DATE } & F^{3} / S \\ 09-26-57 & 1.51 \\ 04-29-58 & 1.84 \\ 10-20-58 & 1.30 \\ 10-20-59 & 5.00\end{array}$

$Q_{a}=16$

$\begin{array}{cr}\text { DATE } & F T^{3} / S \\ 08-16-60 & 3.73 \\ 08-29-61 & 3.49 \\ 10-27-64 & 1.49 \\ 09-13-66 & 3.17\end{array}$

$Q(84)=2.0 * \star *$

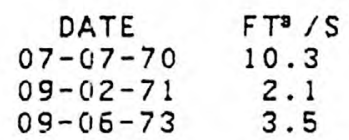

$702=1.4 * *$

$\begin{array}{cc}\text { DATE } & F T^{\circ} / S \\ 09-26-74 & 3.2 \\ 10-21-74 & 5.63 \\ 08-04-75 & 3.32\end{array}$

DATE
$09-24-75$
$10-29-75$
$09-07-76$

$F T^{3} / S$

1.62

1.7

5-4643.50 BEAR CR AT SHELLSEURG, IOWA

LAT 420539. LONG 915334. IN NW 1/4 SEC.

$15, T .84$ N., R.9 W., BENTON CO. (OE),

CRAINAGE AREA $55.8 \mathrm{MI}^{2}$

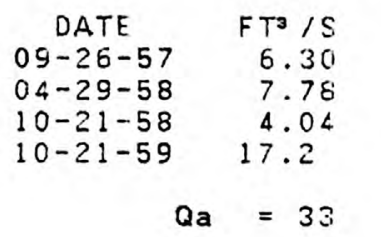

$\begin{array}{cr}\text { DATE } & F T^{3} / S \\ 08-16-60 & 7.41 \\ 08-29-61 & 5.48 \\ 10-27-64 & 1.44 \\ 09-13-66 & 2.84\end{array}$

$\begin{array}{cr}\text { DATE } & F^{3} / S \\ 07-07-70 & 16.1 \\ 09-012-71 & 1.8 \\ 09-016-73 & 4.0\end{array}$

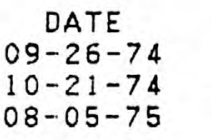

$F T^{2} / S$
6.9

6.9
10.6

DATE

$09-24-75-F T^{3} / S$

$7 Q 10=0.4 * *$

$Q(84)=4.4 * * *$

$7 Q 2=2.8 * x$

$7 Q 10=0.8 * *$

5-4644.00 BEAR CR NR PALO, IOWA

LAT 420455, LONG 914740 , IN SE $1 / 4$ SEC.

17. T.84 N., R.8 W. LINN CO. (57),

AT BRIDGE, I MILE NORTH OF PALO.

DRAINAGE AREA $\$ 5.9 \mathrm{MI}^{2}$

$\begin{array}{rl}\text { DATE } & \mathrm{FT}^{3} / \mathrm{S} \\ 09-26-57 & 12.0 \\ 04-29-58 & 12.0 \\ 10-21-58 & 7.75 \\ 09-17-59 & 5.09 \\ Q a & =56\end{array}$

$\begin{array}{rl}\text { DATE } & F T^{0} \text { is } \\ 10-20-59 & 28.6 \\ 08-16-60 & 14.3 \\ 08-29-61 & 12 . \hat{2} \\ 10-27-64 & 2.50 \\ Q(84) & =8.5 * *\end{array}$

$\begin{array}{rc}\text { DATE } & F T^{3} / S \\ 09-13-66 & 7.43 \\ 07-017-70 & 27.9 \\ 09-012-71 & 5.0 \\ 06-(12-72 & 42.2 \\ 7 Q 2 & =4.8 * *\end{array}$

DATE
$09-05-73$
$09-26-74$
$10-21-74$
$08-05-75$

$F T^{3} / S$

9.0

14.

16.4
$\varepsilon .41$

DATE

$09-24-75$

$09-07-76$

$\mathrm{FT}^{3} / \mathrm{S}$

4. 01

4.2
1.6

$7 Q 10=1.4 * *$ 
IOWA R.IVER BASIN--Continued

5-4544.60 OTTER CR NR CEDAR RAPIDS, IOWA

$$
\begin{aligned}
& \text { LAT } 420357 \text {, LONG } 914427 \text {, IN SE } 1 / 4 \text { SEC. } \\
& 24, \text { T.84 N., R.8 W., LINN CO. (57), }
\end{aligned}
$$

AT BRIDGE, 7 MILES NW OF CEDAR RAPIDS.

\begin{tabular}{|c|c|c|c|c|c|c|c|}
\hline $\begin{array}{c}\text { DATE } \\
09-26-57 \\
04-29-58 \\
10-21-58 \\
09-17-59\end{array}$ & $\begin{array}{r}\mathrm{FT}^{2} / \mathrm{S} \\
4.11 \\
6.11 \\
2.85 \\
4.89\end{array}$ & $\begin{array}{c}\text { DATE } \\
10-20-59 \\
08-16-60 \\
08-29-61 \\
10-27-64\end{array}$ & $\begin{array}{l}\mathrm{FT}^{3} / \mathrm{S} \\
14.4 \\
11.1 \\
6.06 \\
3.21\end{array}$ & $\begin{array}{c}\text { DATE } \\
09-13-66 \\
07-017-70 \\
09-012-71 \\
06-012-72\end{array}$ & $\begin{array}{c}\mathrm{FT}^{3} / \mathrm{S} \\
5.71 \\
20.5 \\
6.8 \\
32.3\end{array}$ & $\begin{array}{c}\text { DATE } \\
09-06-73 \\
09-26-74 \\
10-21-74 \\
06-24-75\end{array}$ & $\begin{array}{l}\mathrm{FT}^{\mathrm{T}} / \mathrm{S} \\
9.3 \\
8.2 \\
11 . \\
119 .\end{array}$ \\
\hline Qa & $=38$ & $Q(84)$ & $=5.0 * * *$ & 702 & $=3 \cdot 3^{\star \star}$ & 7010 & $=1 \cdot 0 * *$ \\
\hline
\end{tabular}

DRAINAGE AREA $65.1 \mathrm{MI}^{2}$

5-4645.50 PRAIRIE CR NR BLAIRSTOWN, IOWA

LAT 415606, LONG 920751, NEAR NORTH $1 / 4$ CORNER SEC.9, T.82 N., R.11 W., BENTON CO. $(06\}$,

AT BRIDGE, 3 MILES NW OF BLAIRSTOWN.

\begin{tabular}{|c|c|c|c|c|c|c|c|c|c|}
\hline $\begin{array}{c}\text { DATE } \\
09-26-57 \\
10-21-58 \\
10-21-59\end{array}$ & $\begin{array}{l}F T^{3} / S \\
4.73 \\
11.8 \\
11.4\end{array}$ & $\begin{array}{c}\text { DATE } \\
08-16-60 \\
08-29-61 \\
10-28-64\end{array}$ & $\begin{array}{l}\mathrm{FT}^{3} \text { iS } \\
6.83 \\
3.22 \\
2.06\end{array}$ & $\begin{array}{c}\text { DATE } \\
10-06-66 \\
07-07-70 \\
09-02-71\end{array}$ & $\begin{array}{c}\mathrm{FT}^{3} / \mathrm{S} \\
1.43 \\
12.6 \\
1.9\end{array}$ & $\begin{array}{c}\text { DATE } \\
09-06-73 \\
09-26-74\end{array}$ & $\begin{array}{l}\mathrm{FT}^{3} / \mathrm{S} \\
3.4 \\
8 . \ddot{2}\end{array}$ & $\begin{array}{c}\text { DATE } \\
08-06-75 \\
09-10-76\end{array}$ & $\begin{array}{l}\mathrm{FT}^{3} / \mathrm{S} \\
3.8 \\
0.2^{7}\end{array}$ \\
\hline 0 & $=37$ & Q6 & $=4.7$ & $7 Q 2$ & $=2.6$ & $7 Q 10$ & 0.8 & & \\
\hline
\end{tabular}

\begin{abstract}
DRAINAGE AREA $64.2 \mathrm{MI}^{2}$
\end{abstract}
5-4646.00 PRAIRIE CR AT NORWAY, IOWA

LAT 415335 , LONG 915543, NEAR SW CORNER SEC.19, T.82 N., R.9 W., BENTON CO. (O6), AT BRILGE, I MILE SW OF' NORWAY.

\begin{tabular}{|c|c|c|c|c|c|c|c|c|c|}
\hline $\begin{array}{c}\text { DATE } \\
09-26-57 \\
10-22-58 \\
10-21-59\end{array}$ & $\begin{array}{l}F T=/ S \\
12.0 \\
27.0 \\
28.5\end{array}$ & $\begin{array}{c}\text { DATE } \\
08-16-60 \\
08-29-61 \\
10-28-64\end{array}$ & $\begin{array}{l}F^{3} / \mathrm{S} \\
17.8 \\
7.65 \\
4.50\end{array}$ & $\begin{array}{c}\text { DATE } \\
09-12-66 \\
10-06-66 \\
09-012-71\end{array}$ & $\begin{array}{l}\mathrm{FT}^{3} / \mathrm{S} \\
6.94 \\
4.90 \\
5.9\end{array}$ & $\begin{array}{c}\text { DATE } \\
09-06-73 \\
09-26-74\end{array}$ & $\begin{array}{l}\mathrm{FT}^{3} / \mathrm{S} \\
8.1 \\
25 .\end{array}$ & $\begin{array}{c}\text { DATE } \\
08-06-75 \\
09-10-76\end{array}$ & $\begin{array}{l}\mathrm{FT}^{3} / 3 \\
9.4 \\
2.0\end{array}$ \\
\hline$Q_{a}$ & $=72$ & QC & $=13$ & $7 Q 2$ & $=8.6$ & $7 Q 10$ & $=3.5$ & & \\
\hline
\end{tabular}

DRAINAGE AREA $126 \quad \mathrm{MI}^{2}$ 
IOWA R.IVER BASIN--Continued

5-4645.50 PRAIRIE CR AT CEDAR RAPIDS, IOWA

LAT 415549 , LONG 914034 , IN NW $1 / 4$ SEC.

9, T.82 N., R.7 W., LINN CO. (57),

AT BRIDGE, 3 MILES SOUTH OF CEDAR RAPIDS.

DRAINAGE AREA $208 \quad \mathrm{MI}^{2}$

\begin{tabular}{|c|c|c|c|c|c|c|c|c|c|}
\hline $\begin{array}{c}\text { DATE } \\
09-26-57 \\
04-29-58 \\
10-21-58\end{array}$ & $\begin{array}{l}F T^{3} / \mathrm{S} \\
20.7 \\
24.6 \\
45.1\end{array}$ & $\begin{array}{c}\text { DATE } \\
10-20-59 \\
08-16-60 \\
08-29-61\end{array}$ & $\begin{array}{l}F T^{3} / \mathrm{S} \\
65.0 \\
40.1 \\
24.8\end{array}$ & $\begin{array}{c}\text { DATE } \\
10-28-64 \\
10-06-66 \\
07-07-70\end{array}$ & $\begin{array}{l}\mathrm{FT} / \mathrm{S} \\
10.5 \\
13.9 \\
65.2\end{array}$ & $\begin{array}{c}\text { DATE } \\
09-02-71 \\
09-07-73 \\
09-27-74\end{array}$ & $\begin{array}{l}F T^{2} / S \\
16.0 \\
23 . \\
47 .\end{array}$ & $\begin{array}{c}\text { DATE } \\
08-04-75 \\
09-07-76\end{array}$ & $\begin{array}{l}F T^{3} / \\
25 . \\
14 .\end{array}$ \\
\hline$Q_{a}$ & $=118$ & $Q<\varepsilon$ & $=32$ & 702 & $=21$ & 7010 & 8.9 & & \\
\hline
\end{tabular}

5-4647.00 INDIAN CR AT CEDAR RAPIDS, IOWA

LAT 415942, LONG 913703 , IN SW 1/4 SEC.

AT, TRIDGE, NEAR NE CITY LIMITS OF CEDAR RAPIDS.

DRAINAGE AREA $72.0 \quad \mathrm{MI}^{2}$

\begin{tabular}{|c|c|c|c|c|c|c|c|c|c|}
\hline $\begin{array}{c}\text { DATE } \\
09-26-57 \\
04-29-58 \\
10-21-58\end{array}$ & $\begin{array}{l}\mathrm{FT}^{3} / \mathrm{S} \\
3.47 \\
4.50 \\
2.91\end{array}$ & $\begin{array}{c}\text { DATE } \\
10-20-59 \\
08-16-60 \\
08-30-61\end{array}$ & $\begin{array}{l}\mathrm{FT}^{3} \text { is } \\
20.2 \\
10.4 \\
7.12\end{array}$ & $\begin{array}{c}\text { DATE } \\
10-28-64 \\
10-06-66 \\
07-017-70\end{array}$ & $\begin{array}{l}\mathrm{FT}^{3} / \mathrm{S} \\
3.00 \\
4.00 \\
16.4\end{array}$ & $\begin{array}{c}\text { DATE } \\
09-02-71 \\
09-07-73 \\
09-27-74\end{array}$ & $\begin{array}{l}F T / S \\
6.9 \\
8.2 \\
8.3\end{array}$ & $\begin{array}{c}\text { DATE } \\
08-04-75 \\
09-07-76\end{array}$ & $\begin{array}{c}\mathrm{FT}^{3} / \mathrm{S} \\
6.3 \\
1.4\end{array}$ \\
\hline$Q$ & $=43$ & $Q<\varepsilon$ & $=4.9$ & 702 & $=3.1$ & $7 Q 10$ & $=1.5$ & & \\
\hline
\end{tabular}

5-4647.50 BIG CR AT BERTRAM, IOWA

LAT 415723, LONG 913135, NEAR EAST $1 / 4$

CORNER SEC.34, T.83 N., R.6 W., LINN CO. (57),

AT BRIUGE NEAR EAST CITY LIMITS OF BERTRAM.

DRAINAGE AREA $81.2 \quad \mathrm{MI}^{2}$

\begin{tabular}{|c|c|c|c|c|c|c|c|c|c|}
\hline $\begin{array}{c}\text { DATE } \\
09-27-57 \\
04-29-58 \\
10-21-58\end{array}$ & $\begin{array}{c}F T=/ S \\
6.18 \\
10.9 \\
7.57\end{array}$ & $\begin{array}{c}\text { DATE } \\
10-2(1-59 \\
08-15-50 \\
03-30-61\end{array}$ & $\begin{array}{l}F T \cdot i S \\
22.6 \\
22.0 \\
19.01\end{array}$ & $\begin{array}{c}\text { DATE } \\
10-28-64 \\
10-016-66 \\
07-017-70\end{array}$ & $\begin{array}{l}\mathrm{FT}^{3} / \mathrm{S} \\
8.60 \\
14.6 \\
27.5\end{array}$ & $\begin{array}{c}\text { DATE } \\
09-02-71 \\
09-07-73 \\
09-27-74\end{array}$ & $\begin{array}{l}\mathrm{FT}^{3} / \mathrm{S} \\
18.0 \\
26 . \\
35 .\end{array}$ & $\begin{array}{c}\text { DATE } \\
08-04-75 \\
09-07-76\end{array}$ & $\begin{array}{l}\mathrm{FT} / \mathrm{S} \\
15 . \\
7.7\end{array}$ \\
\hline & $=48$ & Qces & $=13$ & $7 Q 2$ & $=10$ & $7 Q 10$ & 5.2 & & \\
\hline
\end{tabular}


IOWA RIVER BASIN--Cont inued

5-4648.00 ROCK CR AT ROCHESTER, IOWA

LAT 414040, LONG 910952 , IN NW $1 / 4$ SEC.
$2, T .79$ N., R. 3 W., CEDAR CO. (16),

AT BRIDGE, 0.5 MILE NW OF ROCHESTER.

DRAINAGE AREA $63.4 \mathrm{MI}^{2}$

\begin{tabular}{|c|c|c|c|c|c|c|c|c|c|}
\hline $\begin{array}{c}\text { DATE } \\
09-23-57 \\
05-01-58 \\
11-03-58\end{array}$ & $\begin{array}{r}F^{2} / S \\
5.16 \\
9.95 \\
3.10\end{array}$ & $\begin{array}{c}\text { DATE } \\
10-22-59 \\
08-15-60 \\
08-301-61\end{array}$ & $\begin{array}{l}F T^{0} / \mathrm{S} \\
14.0 \\
15.7 \\
13.2\end{array}$ & $\begin{array}{c}\text { DATE } \\
10-29-64 \\
09-12-65 \\
07-07-70\end{array}$ & $\begin{array}{r}F T^{3} / \mathrm{S} \\
2.77 \\
4.87 \\
26.2\end{array}$ & $\begin{array}{c}\text { DATE } \\
09-01-71 \\
09-07-73 \\
09-26-74\end{array}$ & $\begin{array}{c}F T^{3} / S \\
6.3 \\
9.1 \\
10 .\end{array}$ & $\begin{array}{c}\text { DATE } \\
08-05-75 \\
09-09-76\end{array}$ & $\begin{array}{l}\mathrm{FT}^{\mathrm{T}} / \mathrm{S} \\
4 . \mathrm{S} \\
2.0\end{array}$ \\
\hline Qa & $=38$ & $Q<8$ & $=7.0$ & $7 Q^{2}$ & $=5.2$ & $7 Q 10$ & 2.8 & & \\
\hline
\end{tabular}

5-4648.50 SUGAR CR NR BENNETT, IOWA

LAT 414156 , LONG 910243 , NEAR S $1 / 4$
CORNER OF SEC.26, T.80 N., R. 2 W. C CEDAR CO. (16),

AT BRIDGE, 4.5 MILES SW OF BENNETT.

DRAINAGE AREA $80.7 \mathrm{MI}^{2}$

\begin{tabular}{|c|c|c|c|c|c|c|c|c|c|}
\hline $\begin{array}{c}\text { DATE } \\
09-23-57 \\
05-01-58 \\
11-03-58\end{array}$ & $\begin{array}{c}F T / S \\
5.88 \\
12.5 \\
2.89\end{array}$ & $\begin{array}{c}\text { DATE } \\
10-22-59 \\
08-15-60 \\
08-3(1-61\end{array}$ & $\begin{array}{l}F T \text { is } \\
22.6 \\
28.0 \\
16.9\end{array}$ & $\begin{array}{c}\text { DATE } \\
10-29-64 \\
09-12-66 \\
07-07-70\end{array}$ & $\begin{array}{l}F T \cdot / S \\
2.15 \\
3.29 \\
52.4\end{array}$ & $\begin{array}{c}\text { DATE } \\
09-01-71 \\
09-07-73 \\
09-26-74\end{array}$ & $\begin{array}{l}\mathrm{FT}^{3} / \mathrm{S} \\
4.1 \\
6.4 \\
7.1\end{array}$ & $\begin{array}{c}\text { DATE } \\
08-06-75 \\
09-09-76\end{array}$ & $\begin{array}{l}\mathrm{FT} / \mathrm{S} \\
2.5 \\
0.71\end{array}$ \\
\hline & $=49$ & $Q<\varepsilon$ & $1=5.7$ & 702 & $=3.2$ & 7010 & $0.8 * * *$ & & \\
\hline
\end{tabular}

5-4549.00 MUD CR NR WILTON, IOWA

LAT 413445, LONG 910217 , IN NW $1 / 4$ SEC.

12. T.78 N., R.2 W., MUSCATINE CO. (70).

AT BRIDGE, I MILE. SW OF W'ILTON.

DRAINAGE AREA $102 \quad \mathrm{MI}^{2}$

\begin{tabular}{|c|c|c|c|c|c|c|c|c|c|}
\hline $\begin{array}{c}\text { DATE } \\
09-23-57 \\
05-01-58 \\
11-03-58\end{array}$ & $\begin{array}{l}F T^{0} / \mathrm{S} \\
=.48 \\
3.09 \\
4.58\end{array}$ & $\begin{array}{c}\text { DATE } \\
10-22-59 \\
08-15-60 \\
08-30-61\end{array}$ & $\begin{array}{l}F T^{3} / S \\
25.9 \\
11.2 \\
9.14\end{array}$ & $\begin{array}{c}\text { DATE } \\
10-29-64 \\
09-12-66 \\
07-07-70\end{array}$ & $\begin{array}{c}F T^{3} / \mathrm{S} \\
3.13 \\
6.10 \\
36.7\end{array}$ & $\begin{array}{c}\text { DATE } \\
09-01-71 \\
09-07-73 \\
09-25-74\end{array}$ & $\begin{array}{l}\mathrm{FT}^{3} / \mathrm{S} \\
5.3 \\
10 . \\
13 .\end{array}$ & $\begin{array}{c}\text { DATE } \\
08-06-75 \\
09-09-70\end{array}$ & $\begin{array}{l}\mathrm{FT}^{3} / \mathrm{S} \\
6.9 \\
3.8\end{array}$ \\
\hline & $=60$ & or & $=7.1$ & $7 Q 2$ & $=5.4$ & $7 Q 10$ & 2.9 & & \\
\hline
\end{tabular}


IOWA RIVER BASIN--Cont I nued

5-4649.20 SUGAR CR. NR MOSCOW, IOWA

LAT 413400 , LONG 910409 , NEAR N $1 / 4$

CORNER OF SEC.15, T.78 N., R.2 W., MUSCATINE CO.

(70), AT BRIDGE, 1 MILE SE OF MOSCOW.

DRAINAGE AREA $218 \quad \mathrm{MI}^{2}$

\begin{tabular}{|c|c|c|c|c|c|c|c|c|c|}
\hline $\begin{array}{c}\text { DATE } \\
09-23-57 \\
05-01-58 \\
11-03-58\end{array}$ & $\begin{array}{c}\mathrm{FT}^{9} / \mathrm{S} \\
9.71 \\
26.0 \\
8.09\end{array}$ & $\begin{array}{c}\text { DATE } \\
10-22-59 \\
08-15-60 \\
08-30-61\end{array}$ & $\begin{array}{l}F T^{3} / \mathrm{S} \\
51.3 \\
42.0 \\
32.8\end{array}$ & $\begin{array}{c}\text { DATE } \\
10-29-64 \\
09-12-66 \\
07-07-70\end{array}$ & $\begin{array}{l}F T^{3 / S} \\
7.36 \\
14.5 \\
104 .\end{array}$ & $\begin{array}{c}\text { DATE } \\
09-01-71 \\
09-07-73 \\
09-26-74\end{array}$ & $\begin{array}{l}\mathrm{FT}^{3} / \mathrm{S} \\
15.0 \\
24 . \\
28 .\end{array}$ & $\begin{array}{c}\text { DATE } \\
06-23-75 \\
08-06-75 \\
09-09-76\end{array}$ & $\begin{array}{l}F T^{3} / \mathrm{S} \\
55 . \\
14 . \\
8.4\end{array}$ \\
\hline$Q$ & $=126$ & $Q<\varepsilon$ & $=19$ & $7 Q^{2}$ & $=14$ & $7 Q 10$ & 7.2 & & \\
\hline
\end{tabular}

5-4649.40 WAPSINONOC CR AT WEST LIVERTY, IOWA

LAT 413326, LONG 911519 , IN SE $1 / 4$ SEC.

$13, T .78$ N., R. 4 W., MUSCATINE CO. $(70)$,

AT BRIDGE ON STATE HIGHWAY $76,1 / 2$ MILE

SE OF WEST LIBERTY.

DRAINAGE AREA $51.7 \quad \mathrm{MI}^{2}$

\begin{tabular}{|c|c|c|c|c|c|c|c|c|c|}
\hline $\begin{array}{c}\text { DATE } \\
09-23-57 \\
05-01-58 \\
11-03-58\end{array}$ & $\begin{array}{r}F T^{3} / \mathrm{S} \\
0.94 \\
3.37 \\
2.03\end{array}$ & $\begin{array}{c}\text { DATE } \\
10-22-59 \\
08-15-60 \\
08-30-61\end{array}$ & $\begin{array}{l}F T^{3} / S \\
4.52 \\
7.50 \\
7.13\end{array}$ & $\begin{array}{c}\text { DATE } \\
09-12-66 \\
07-07-70 \\
09-01-71\end{array}$ & $\begin{array}{c}\mathrm{FT}^{3} / \mathrm{S} \\
2.48 \\
26.7 \\
2.7\end{array}$ & $\begin{array}{c}\text { DATE } \\
09-07-73 \\
09-26-74\end{array}$ & $\begin{array}{c}\mathrm{FT}^{3} / \mathrm{S} \\
3.33 \\
4.7\end{array}$ & $\begin{array}{c}\text { DATE } \\
08-06-75 \\
09-08-76\end{array}$ & $\begin{array}{l}\mathrm{FT}^{3} / \mathrm{S} \\
2.1 \\
2.2\end{array}$ \\
\hline Qa & $=30$ & $Q(84)$ & $=3.0$ & 702 & $=2.2$ & 7010 & $=0.7$ & & \\
\hline
\end{tabular}

5-4649.50 WB WAPSINONOC CR AT WEST LIBERTY, IOWA

LAT 413348 , LONG 911613 , NEAR E $1 / 4$

CORNER OF SEC.14, T.78 N., R. 4 W. MUSCATINE CO.

(70), AT BRIDGE, 1 MILE SOUTH OF WEST LIBERTY.

DRAINAGE AREA $52.5 \quad \mathrm{MI}^{2}$

\begin{tabular}{|c|c|c|c|c|c|c|c|c|c|}
\hline $\begin{array}{c}\text { DATE } \\
09-23-57 \\
05-01-58 \\
11-03-58\end{array}$ & $\begin{array}{l}\mathrm{FT}^{3} / \mathrm{S} \\
0 . \\
0 . \\
2.28\end{array}$ & $\begin{array}{c}\text { DATE } \\
10-22-59 \\
10-22-59 \\
08-15-60\end{array}$ & $\begin{array}{l}F^{a} / S \\
7.04 \\
7.21 \\
6.38\end{array}$ & $\begin{array}{c}\text { DATE } \\
08-30-61 \\
10-29-64 \\
09-12-66\end{array}$ & $\begin{array}{l}\mathrm{FT}^{3} / \mathrm{S} \\
7.21 \\
0.1 \\
0.27\end{array}$ & $\begin{array}{c}\text { DATE } \\
07-07-70 \\
09-01-71 \\
09-07-73\end{array}$ & $\begin{array}{c}\mathrm{FT}^{3} / \mathrm{S} \\
24.5 \\
0.99 \\
1.4\end{array}$ & $\begin{array}{c}\text { DATE } \\
09-26-74 \\
08-06-75 \\
09-08-76\end{array}$ & $\begin{array}{l}\mathrm{FT}^{3} / \mathrm{S} \\
7.7 \\
0.32 \\
0.07\end{array}$ \\
\hline Qa & $=31$ & $Q<8$ & $=0.8$ & $7 Q 2$ & 0.3 & $7 Q 10$ & 0 & & \\
\hline
\end{tabular}


IOWA RIVER BASIN--Cont inued

5-4652.00 LONG CR NR AINSWORTH, IOWA

LAT $4116 \times X$, LONG $9130 \times X$, IN SE $1 / 4$ SEC.

26, T. 75 N., R. 6 W., WASHINGTON CO. (92),

AT BRIDGE, 2.5 MILES SE OF AINSWORTH.

DRAINAGE AREA $68.4 \mathrm{MI}^{2}$

\begin{tabular}{|c|c|c|c|c|c|c|c|c|c|}
\hline $\begin{array}{c}\text { DATE } \\
09-24-57 \\
04-28-58 \\
10-02-58 \\
10-22-59\end{array}$ & $\begin{array}{c}\mathrm{FT}^{\mathrm{a}} / \mathrm{S} \\
0 . \\
1.75 \\
1.12 \\
15.9\end{array}$ & $\begin{array}{c}\text { DATE } \\
09-06-60 \\
08-31-61 \\
11-04-63 \\
10-29-64\end{array}$ & $\begin{array}{l}\mathrm{FT}^{3} / \mathrm{S} \\
0.02 \\
0.12 \\
0.22 \\
0.18\end{array}$ & $\begin{array}{c}\text { DATE } \\
05-10-66 \\
07-26-66 \\
09-13-66\end{array}$ & $\begin{array}{l}\mathrm{FT}^{2} / \mathrm{S} \\
24.1 \\
2.18 \\
0 .\end{array}$ & $\begin{array}{c}\text { DATE } \\
10-31-66 \\
07-10-70 \\
09-06-73\end{array}$ & $\begin{array}{l}\mathrm{FT} / \mathrm{1S} \\
1.31 \\
8.97 \\
0.26\end{array}$ & $\begin{array}{c}\text { DATE } \\
09-27-74 \\
08-06-75 \\
09-08-76\end{array}$ & $\begin{array}{l}\mathrm{FT}^{3} / \mathrm{S} \\
0 . \\
0.39 \\
0.14\end{array}$ \\
\hline & $=40$ & $Q($ & \}$=0.3$ & 702 & $=0.3 * *$ & 7010 & * & & \\
\hline
\end{tabular}

5-4653.00 LONG CR NR WAPELLO, IOWA

LAT $4112 \times X$, LONG $9117 \times X$, NEAR SOUTH $1 / 4$

CORNER SEC.23, T.74 N., R. 4 W., LOUISA CO. 158 ;,

AT ERIDGE, 5 MILES NW OF WAPELLO.

DRAINAGE AREA $146 \quad \mathrm{MI}^{2}$

\begin{tabular}{|c|c|c|c|c|c|c|c|}
\hline $\begin{array}{c}\text { DATE } \\
09-24-57 \\
04-28-58 \\
10-02-58 \\
10-22-59\end{array}$ & $\begin{array}{c}\mathrm{FT} / \mathrm{S} \\
0.03 \\
5.95 \\
3.53 \\
39.7\end{array}$ & $\begin{array}{c}\text { DATE } \\
09-0 E_{1}-60 \\
08-31-61 \\
11-04-63 \\
10-29-64\end{array}$ & $\begin{array}{r}\mathrm{FT}^{3} . \mathrm{S} \\
1.26 \\
0.58 \\
0.37 \\
0.20\end{array}$ & $\begin{array}{c}\text { DATE } \\
06-(13-66 \\
07-26-66 \\
09-12-66 \\
10-31-66\end{array}$ & $\begin{array}{l}\mathrm{FT}^{3} / \mathrm{S} \\
87.6 \\
6.77 \\
1.30 \\
0.98\end{array}$ & $\begin{array}{c}\text { DATE } \\
04-17-67 \\
06-12-67 \\
09-11-67 \\
07-10-70\end{array}$ & $\begin{array}{c}F T^{2} / S \\
91.4 \\
247.0 \\
0.16 \\
15.8\end{array}$ \\
\hline & $=85$ & & $y=0$. & 70 & $=0.7 *$ & 7010 & $=0.1 * *$ \\
\hline
\end{tabular}

5-4656.00 OTTER CR. NR WAPELLO, IOWA

LAT 410720 , LONG 910900 , NEAR CENTER OF SEC.13, T.73 N., R. 3 W. LOUISA CO. (58), AT BRIDIGE, 4 MILES SE ÖF WAPELLO.

DRAINAGE AREA $64.7 \mathrm{MI}^{2}$

$\begin{array}{cr}\text { CATE } & F^{3} / S \\ 09-24-57 & 0.65 \\ 04-28-58 & 9.81 \\ 10-02-58 & 0.04 \\ 10-22-59 & 17.6 \\ Q a & =40\end{array}$

$\begin{array}{rr}\text { DATE } & F T^{J} \text {;S } \\ 09-0 E_{1}-50 & 7.39 \\ 08-31-61 & 5.53 \\ 11-02-63 & 3.72 \\ 10-29-64 & 3.48 \\ Q(84) & =5.0\end{array}$

$\begin{array}{rc}\text { DATE } & F T^{3} / S \\ 06-(13-66 & 32.0 \\ 07-26-66 & 7.89 \\ 09-12-66 & 5.30 \\ 7 Q 2= & 3.4\end{array}$

DATE
$10-31-66$
$07-10-70$

$F^{3} / S$

5.70

8.60

$09-06-73$

36 .

DATE
$09-06-73$
$09-27-74$
$08-06-75$
$09-08-76$

$F T^{3} / S$

9.8

8.0

1.1
0.44

$Q a=40$

$Q(84)=5.0$

$7010=1.7 * * *$ 
FLINT CREEK EASIN

5-4697.00 FLINT CR NR BURLINGTON, IOWA

LAT 405200 , LONG 911203 , IN NE $1 / 4$ SEC.

$16, T .70$ N., R.3 W., DES MOINES CO. (29), AT BRIDGE, 6 MILES NW OF BURLINGTON.

DRAINAGE AREA $107 \quad \mathrm{MI}^{2}$

$\begin{array}{rc}\text { DATE } & F^{2} / S \\ 10-02-57 & 0 . \\ 03-25-58 & 12.0 \\ 10-28-58 & 1.06 \\ 09-07-60 & 1.07 \\ Q a & =68\end{array}$

$\begin{array}{cc}\text { DATE } & F T / S \\ 09-06-61 & 1.02 \\ 11-04-63 & 0.78 \\ 10-15-64 & 0.28 \\ 06-06-65 & 44.5 \\ Q(84) & =1.8\end{array}$

$\begin{array}{rc}\text { DATE } & \text { FT /S } \\ 08-18-66 & 0 . \\ 11-02-66 & 0.85 \\ 04-17-67 & 126 . \\ 09-11-67 & 1.05 \\ 7 Q 2= & 0.5 * \star\end{array}$

$\begin{array}{cc}\text { DATE } & F T^{3} / S \\ 06-20-68 & 13.2 \\ 07-14-70 & 4.32 \\ 08-28-71 & 1.9 \\ & \\ 7010=*\end{array}$

DATE

$09-20-74$

$09-16-76$

$Q_{a}=68$

SKUNK RIVER EASIN

5-4698.00 S SKUNK R NR ELLSWORTH, IOWA

LAT $4219 \times X$, LONG $9335 X X$, NEAR $N 1 / 4$

CORNER OF SEC. $36, T .87 \mathrm{~N} ., \mathrm{R} .24 \mathrm{~W}$., HAMILTON CO.

(40). AT BRIDGE ON STATE HIGHWAY 175 , NEAR

WEST' CITY LIMITS OF ELLSWORTH.

DRAINAGE AREA $54.9 \mathrm{MI}^{2}$

$\begin{array}{rc}\text { DATE } & F T^{3} / S \\ 09-23-57 & 0.81 \\ 04-22-58 & 15.0 \\ 10-21-58 & 0.37 \\ Q \mathbf{a} & =27\end{array}$

$\begin{array}{cr}\text { DATE } & \mathrm{FT}^{3} / \mathrm{S} \\ 07-28-59 & 3.13 \\ 09-07-60 & 1.01 \\ 09-05-61 & 3.50\end{array}$

$\begin{array}{cr}\text { DATE } & F T^{2} / S \\ 10-12-64 & 1.15 \\ 09-08-66 & 1.30 \\ 07-13-70 & 1.28\end{array}$

$\begin{array}{cc}\text { DATE } & F T / S \\ 08-24-71 & 0.19 \\ 09-20-74 & 2.7\end{array}$

DATE

$09-29-75$

$F T^{3} / S$ $07-13-70 \quad 1.28$

$7 Q 10=0$

5-4698.50 MUD LAKE DRAINAGE DITCH 71 AT JEWELL, IOWA

LAT $4219 \times X$, LONG $9338 \times X$, IN NW $1 / 4$ SEC.

28, T.87 N.. R.24 W. HAMILTON CO. $(\triangle 0)$,

AT BRIDGE, I MILE NORTH OF JEWELL.

DRAINAGE AREA $64.1 \mathrm{MI}^{2}$

$\begin{array}{cc}\text { DATE } & F T^{3} / S \\ 09-23-57 & 0.77 \\ 04-22-58 & 16.8 \\ 10-21-58 & 0.33 \\ & =31\end{array}$

$\begin{array}{cr}\text { DATE } & F^{3} / S \\ 07-28-59 & 5.06 \\ 09-07-60 & 2.14 \\ 09-05-51 & 3.210\end{array}$

$\begin{array}{cr}\text { DATE } & F T=/ S \\ 10-12-54 & 0.46 \\ 09-018-66 & 0.35 \\ 07-13-70 & 2.47\end{array}$

$\begin{array}{cc}\text { DATE } & F T^{S} / S \\ 08-24-71 & 0.78 \\ 09-20-74 & 3.9\end{array}$

$09-05 i-61-3.20$

$7 Q 2=0.1$

$7 Q 10=0$ 
SKUNK RIVER SASIN--Cont iniled

5-4699.50 S SKUNK R AT RANDALL, IOWA

LAT $4214 \times X$, LONG $9335 \times X$. IN NE $1 / 4$ SEC.

$25, T .86$ N., R.24 W., HAMILTON CO. $(40)$,

AT BRIDGE, I MILE EAST OF RANDALL.

DRAINAGE AREA $160 \quad \mathrm{MI}^{2}$

\begin{tabular}{|c|c|c|c|c|c|c|c|c|c|}
\hline $\begin{array}{c}\text { DATE } \\
09-23-57 \\
04-22-58 \\
10-21-58\end{array}$ & $\begin{array}{c}\mathrm{FT}^{3} / \mathrm{S} \\
2.90 \\
45.4 \\
3.08\end{array}$ & $\begin{array}{c}\text { DATE } \\
07-2 \varepsilon-59 \\
09-07-60 \\
09-05-51\end{array}$ & $\begin{array}{l}\mathrm{FT}^{3} \text { is } \\
9.85 \\
5.71 \\
8.79\end{array}$ & $\begin{array}{c}\text { DATE } \\
10-12-64 \\
09-018-66 \\
07-13-70\end{array}$ & $\begin{array}{l}F T^{\circ} / \mathrm{S} \\
0.82 \\
1.23 \\
6.69\end{array}$ & $\begin{array}{c}\text { DATE } \\
08-24-71 \\
08-20-74\end{array}$ & $\begin{array}{l}F T \cdot / S \\
0.72 \\
11 .\end{array}$ & $\begin{array}{c}\text { DATE } \\
09-29-75 \\
09-15-76\end{array}$ & $\begin{array}{c}\mathrm{FT}^{\mathrm{a}} / \mathrm{S} \\
3.3 \\
0.23\end{array}$ \\
\hline$Q$ & $=78$ & $Q<\varepsilon$ & $=2.7$ & 702 & $=0.6$ & 7010 & 0 & & \\
\hline
\end{tabular}

5-4702.00 SQUAW CR. NR STANHOPE, IOWA

LAT 421234, LONG 934707, NEAR N 1/4

CORNER OF SEC.5, T.85 N., R. 25 W., BOONE CO. (08),

AT BRIDGE, 5 MILES SOUTH OF STANHOPE.

DRAINAGE AREA $62.6 \quad \mathrm{MI}^{2}$

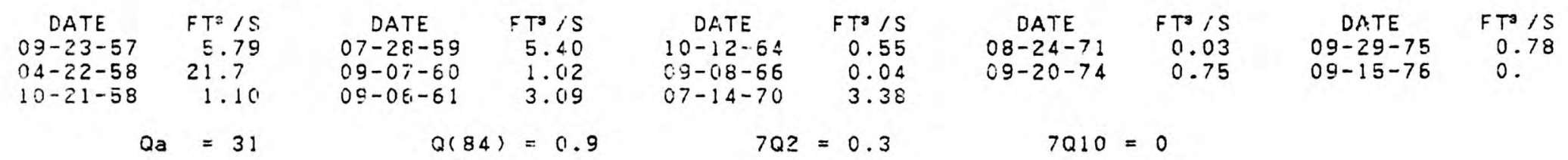

5-4710.50 S SKUNK R AT COLFAX, IOWA

LAT 414055, LONG 931447 , IN NW $1 / 4$ SEC.

AT BRIDGE ON STATE HIGHWAY 117 , AT NORTH CITY

AT BRIDGE ON STAT
LIMITS OF COLFAX.

DIRAINAGE AREA $803 \quad \mathrm{MI}^{2}$

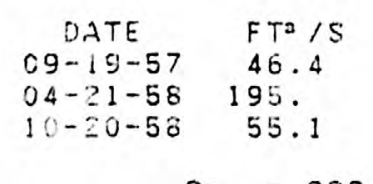

$Q \mathbf{Q}=392$
DATE
$07-28-59$
075

$07-28-59 \quad 115$

$\begin{array}{rr}05-07-60 & 115 . \\ 09-07-51 & 80.2\end{array}$

$Q(84)=53$

$\begin{array}{cc}\text { DATE } & F T 0 / S \\ 10-13-64 & 33.2 \\ 07-2.9-66 & 125 . \\ 09-017-66 & 32.3\end{array}$

$7 Q 2=22$

$\begin{array}{cl}\text { DATE } & F T^{3} / \mathrm{S} \\ 07-13-70 & 95.5 \\ 08-23-71 & 33.0 \\ 09-19-74 & 98 .\end{array}$

$7 Q 10=3.2$ 
SKUNK RIVER BASIN--Cont inued

5-4711.00 EB INDIAN CR NR NEVADA, IOWA

LAT $4102 \times X$, LONG $9322 \times X$, NEAR N $1 / 4$ CORNER

OF SEC.2, T.83 N., R.22 W., STORY CO. (85), AT BRIDGE, 4 MILES NE OF NEVAOA.

DRAINAGE AREA $65.7 \mathrm{MI}^{2}$

\begin{tabular}{|c|c|c|c|c|c|c|c|c|c|}
\hline $\begin{array}{c}\text { DATE } \\
09-25-57 \\
09-26-57 \\
04-22-58\end{array}$ & $\begin{aligned} \mathrm{FT}^{2} / \mathrm{S} \\
1.82 \\
1.53 \\
15.1\end{aligned}$ & $\begin{array}{c}\text { DATE } \\
10-20-58 \\
07-28-59 \\
09-07-60\end{array}$ & $\begin{array}{l}F T / S \\
3.84 \\
2.39 \\
5.64\end{array}$ & $\begin{array}{c}\text { DATE } \\
09-06-61 \\
10-13-64 \\
09-07-66\end{array}$ & $\begin{array}{l}\mathrm{FT}^{3} / \mathrm{S} \\
1.78 \\
0.38 \\
0 .\end{array}$ & $\begin{array}{c}\text { DATE } \\
07-13-70 \\
08-23-71 \\
09-19-74\end{array}$ & $\begin{array}{l}F T / S \\
1.29 \\
0.05 \\
4.0\end{array}$ & $\begin{array}{c}\text { DATE } \\
09-29-75 \\
09-14-76\end{array}$ & $\begin{array}{l}F T^{3} / \mathrm{S} \\
1.6 \\
0 .\end{array}$ \\
\hline Qa & $=34$ & $Q(84)$ & $=2.1$ & $7 Q 2$ & $=0.2$ & 7010 & 0 & & \\
\hline
\end{tabular}

5-4711.50 WB INDIAN CR NR IOWA CENTER, IOWA

LAT $4156 \times X$, LONG $9326 \times X$, IN NW $1 / 4$ SEC.

8, T.82 N.) R.22 W. STORY CO. (85),

AT BRILGE, 2 MILES NW OF IOWA CENTER.

DRAINAGE AREA $65.9 \mathrm{MI}^{2}$

\begin{tabular}{|c|c|c|c|c|c|c|c|c|c|}
\hline $\begin{array}{c}\text { DATE } \\
09-25-57 \\
09-26-57 \\
04-22-58\end{array}$ & $\begin{array}{c}\mathrm{FT}^{3} / \mathrm{S} \\
0.16 \\
0.11 \\
7.96\end{array}$ & $\begin{array}{c}\text { DATE } \\
10-20-58 \\
07-28-59 \\
09-07-60\end{array}$ & $\begin{array}{r}F T \text { is } \\
5.07 \\
3.38 \\
7.56\end{array}$ & $\begin{array}{c}\text { DATE } \\
09-016-61 \\
10-13-64 \\
09-017-66\end{array}$ & $\begin{array}{l}\mathrm{FT}^{3} / \mathrm{S} \\
2.41 \\
2.11 \\
0.09\end{array}$ & $\begin{array}{c}\text { DATE } \\
07-13-70 \\
08-23-71 \\
09-19-74\end{array}$ & $\begin{array}{l}\mathrm{FT}^{3} / \mathrm{S} \\
2.75 \\
0.82 \\
2.3\end{array}$ & $\begin{array}{c}\text { DATE } \\
09-29-75 \\
09-14-76\end{array}$ & $\begin{array}{l}\mathrm{FT}^{\mathrm{s}} / \mathrm{S} \\
2.4 \\
0.06\end{array}$ \\
\hline & $=34$ & $Q<8$ & $=2.9$ & 702 & $=0.3$ & $7 Q 10$ & 0 & & \\
\hline
\end{tabular}

5-4711.80 INDIAN CR NR IOWA CENTER, IOWA

LAT $4155 \times X$, LONG $9325 \times X$, NEAR CENTER OF SEC.16, T.82 N., R.22 W. , STORY CO. (85), AT BRIDGE, 1 MILE SW OF IOWA CENTER.

DRAINAGE AREA $203 \quad$ MI2

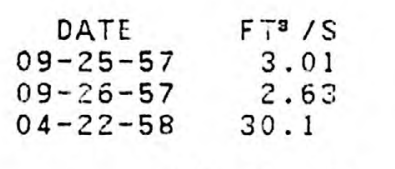

$Q_{a}=104$

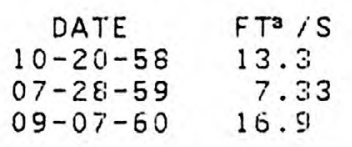

$Q(84)=7.3$

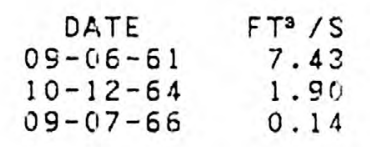

$7 Q 2=0.8$

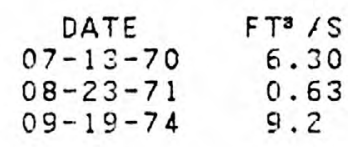

$09-19-74$

9.2

$7 Q 10=0$

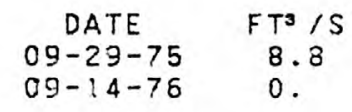


SKUNK RIVER BASIN--Cont inued

5-4713.50 CLEAR CR NR MINGO, IOWA

LAT $4147 \times X$, LONG $9316 \times X$, IN SW $1 / 4$ SEC.

$35, T .81 \mathrm{~N} .$, R.21 W., JASPER CO. $\langle 50\rangle$,

AT BRIDGE, I MILE NE OF MINGO.

DRAINAGE AREA $84.1 \mathrm{MI}^{2}$

\begin{tabular}{|c|c|c|c|c|c|c|}
\hline $\begin{array}{c}\text { DATE } \\
09-19-5 \\
04-21-5 \\
10-20-5\end{array}$ & & $\begin{array}{l}\mathrm{FT}^{3} / \mathrm{S} \\
0.33 \\
9.98 \\
2.25\end{array}$ & $\begin{array}{c}\text { DATE } \\
07-28-59 \\
09-07-60 \\
09-07-61\end{array}$ & $\begin{array}{r}F T^{3} / S \\
6.82 \\
3.16 \\
4.72\end{array}$ & $\begin{array}{c}\text { DATE } \\
: 0-12-64 \\
09-017-66 \\
07-13-70\end{array}$ & $\begin{array}{r}\mathrm{FT}^{3} / \mathrm{S} \\
5.51 \\
1.81 \\
3.80\end{array}$ \\
\hline & $Q \mathbf{a}$ & $=45$ & $Q(84)$ & $=5.0$ & $7 Q 2$ & $=2.1$ \\
\hline 4714.00 & ELK & CR NR & TAINTOR, IOWA & & & \\
\hline
\end{tabular}

DRAINAGE AREA $59.9 \mathrm{MI}^{2}$

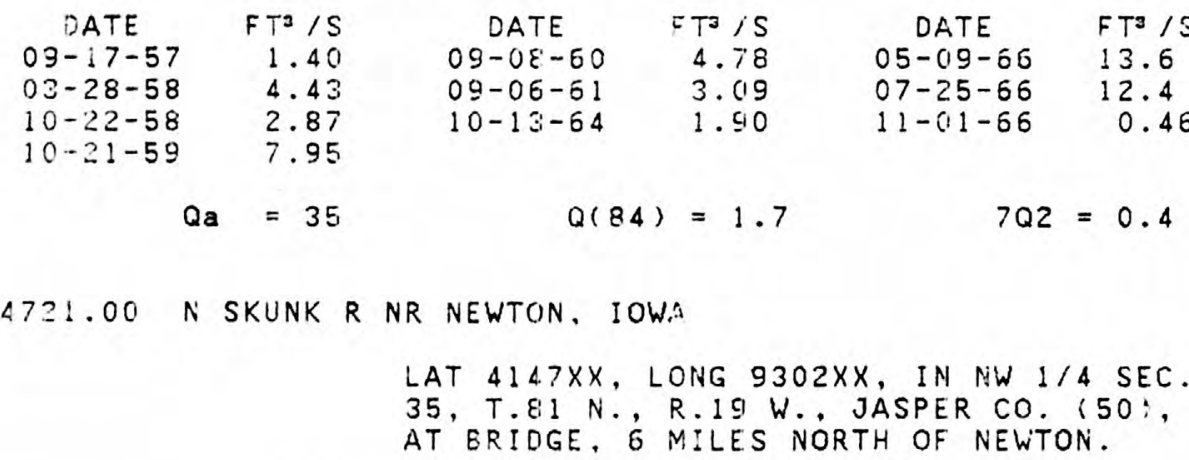

ORAINAGE AREA 101 MI=

\begin{tabular}{|c|c|c|c|c|c|c|c|c|c|}
\hline $\begin{array}{c}\text { DATE } \\
09-19-57 \\
03-28-58 \\
10-20-58\end{array}$ & $\begin{array}{c}F T^{3} / S \\
\vdots .54 \\
11.8 \\
3.60\end{array}$ & $\begin{array}{c}\text { DATE } \\
07-28-59 \\
09-07-60 \\
09-0 E-61\end{array}$ & $\begin{array}{l}F T^{0} / \mathrm{S} \\
18.9 \\
4.95 \\
15.1\end{array}$ & $\begin{array}{c}\text { DATE } \\
10-13-64 \\
10-015-66 \\
06-10-68\end{array}$ & $\begin{array}{l}\mathrm{FT}^{3} / \mathrm{S} \\
8.65 \\
1.27 \\
0.27\end{array}$ & $\begin{array}{c}\text { DATE } \\
07-13-70 \\
08-23-71 \\
09-19-74\end{array}$ & $\begin{array}{l}F T^{3} / \mathrm{S} \\
10.4 \\
1.5 \\
12 .\end{array}$ & $\begin{array}{c}\text { DATE } \\
09-29-75 \\
09-14-76\end{array}$ & $\begin{array}{l}F T=/ S \\
16 . \\
0.94\end{array}$ \\
\hline & $=55$ & & $=3.5$ & $7 Q 2$ & $=1.4$ & $7 Q 10$ & * & & \\
\hline
\end{tabular}


SKUNK RIVER BASIN--Cont inued

5-4723.00 N SKUNK R NR SEARSBORO, IOWA

LAT $4132 \times X$, LONG $9242 \times X$. NEAR CENTER OF SEC.27, T.78 N., R.16 W. POWESHIEK CO. (79), AT BRIDGE, 3.5 MILES SOUTH OF SEARSBORO.

DRAINAGE AREA $358 \quad \mathrm{MI}^{2}$

\begin{tabular}{rlclcr} 
DATE & $F T^{3} / S$ & DATE & $F T^{3} / S$ & DATE & $F T^{3} / S$ \\
$09-18-57$ & 12.4 & $10-21-59$ & 31.6 & $10-13-64$ & 21.2 \\
$03-28-58$ & 41.5 & $09-08-60$ & 20.8 & $10-05-66$ & 5.76 \\
$10-22-58$ & 23.3 & $09-06-61$ & 47.0 & $06-10-68$ & 9.63 \\
& \\
$Q a$ & $=196$ & \multicolumn{2}{c}{$0(84)=20$} & $7 Q 2=11$
\end{tabular}

$\begin{array}{rl}\text { DATE } & F T / S \\ 07-13-70 & 31.2 \\ 08-23-71 & 13.0 \\ 09-19-74 & 40 \\ 7010= & 2.0 * * *\end{array}$
DATE $09-30-75$ $09-13-76$

$F T^{3} / S$ 6.4 Q $\mathbf{a}=196$ $Q(84)=20$ $7 Q 2=11$

$7010=2.0 * * *$

5-4724.00 MIDDLE CR NR ROSE HILL, IOWA

LAT 412042 , LONG 922825, IN NE $1 / 4$ SEC. 33. T. $76 \mathrm{~N}$. R. 14 W.. MAHASKA CO. (62). AT BRIOUGE, 2 MILES NW OF ROSE HILL.

DRAINAGE AREA $58.5 \mathrm{MI}^{2}$

$\begin{array}{cc}\text { DATE } & F T^{3} / S \\ 09-17-57 & 1.52 \\ 03-27-58 & 3.07 \\ 10-22-58 & 0.14 \\ 08-25-59 & 0.76 \\ & =34\end{array}$

$\begin{array}{rr}\text { DATE } & F^{3} / S \\ 10-21-59 & 4.73 \\ 09-09-60 & 1.12 \\ 09-06-61 & 2.016 \\ 10-13-64 & 0.05 \\ Q(84) & =0.5\end{array}$

$\begin{array}{cc}\text { DATE } & F T^{3} / S \\ 05-09-66 & 20.9 \\ 07-25-66 & 11.2 \\ 11-03-66 & 0 .\end{array}$

$\begin{array}{cr}\text { DATE } & \text { FT } / S \\ 06-10-68 & 0.81 \\ 07-13-70 & 3.78 \\ 08-23-71 & 0.15 \\ & \\ 7 Q 10= & 0\end{array}$

$Q_{\mathbf{a}}=34$

$7 Q 2=0.1$

5-4724.50 CEDAR CR NR SIGOURNEY, IOWA

LAT 411842 , LCNG 921333 , IN SE $1 / 4$ SEC. 10. T.75 N.. R.12 W.. KEOKUIK CO. (54),

AT BRILGE, 2 MILES SW OF SIGOURNEY.

\begin{abstract}
DRAINAGE AREA $92.5 \mathrm{MI}^{2}$
\end{abstract}
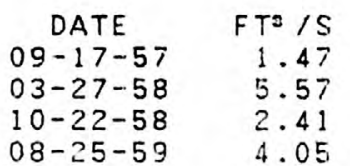

DATE
$09-09-60$
$09-0 E-61$
$10-13-64$
$05-09-66$

$\mathrm{FT}^{2}$ is

2.17

0.48

26.3

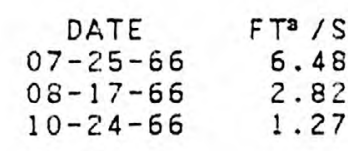

$T^{3} / 5$
6.48
2.82

$05-09-66$

$Q(84)=2.2$

$7 Q 2=0.5$

$\begin{array}{ll}\text { DATE } & \text { FT } / S \\ 06-10-68 & 3.91 \\ 07-13-70 & 4.28 \\ 08-23-71 & 1.2\end{array}$

DATE

$09-19-74$
$09-30-75$

$09-13-76$

$\mathrm{FT}^{3} / \mathrm{S}$

$8-23-71+1.2$

$7 Q 10=0.1$ 
SKUNK RIVER BASIN--Continued

5-4730.20 EF CROOKED CR NR WINFIELD, IOWA

LAT $4109 \times X$, LONG $9126 \times X$, IN NE $1 / 4$ SEC.

9 , T.73 N., R.5 W., HENRY CO. ( 44 ),

AT BRIDGE, 2 MILES NORTH OF WINFIELD.

DRAINAGE AREA $65.3 \mathrm{MI}^{2}$

\begin{tabular}{|c|c|c|c|c|c|c|c|c|c|}
\hline $\begin{array}{c}\text { DATE } \\
10-02-57 \\
03-25-58 \\
10-27-58 \\
09-06-60\end{array}$ & $\begin{array}{l}F T^{3} / S \\
0.10 \\
9.54 \\
3.52 \\
0.80\end{array}$ & $\begin{array}{c}\text { DATE } \\
09-65_{1}-61 \\
11-04-53 \\
10-13-64\end{array}$ & $\begin{array}{l}F^{3} \text { is } \\
0.50 \\
0.015 \\
0.05\end{array}$ & $\begin{array}{c}\text { DATE } \\
07-26-66 \\
08-16-66 \\
10-31-66\end{array}$ & $\begin{array}{l}F T^{a} / \mathrm{S} \\
1.54 \\
0.60 \\
0.46\end{array}$ & $\begin{array}{c}\text { DATE } \\
06-20-68 \\
07-13-70 \\
08-24-71\end{array}$ & $\begin{array}{l}\mathrm{FT}^{3} / \mathrm{S} \\
5.56 \\
2.67 \\
0.96\end{array}$ & $\begin{array}{c}\text { DATE } \\
09-19-74 \\
09-29-75 \\
09-16-75\end{array}$ & $\begin{array}{l}\mathrm{FT}^{3} / \mathrm{S} \\
0.56 \\
3.5 \\
0.33\end{array}$ \\
\hline & $=41$ & Q: & $=0.6$ & $7 Q 2$ & $=0.1$ & 7010 & 0 & & \\
\hline
\end{tabular}

5-4730.50 CROOKED CR NR COPFOCK, IOWA

LAT $4112 \times X$, LONG $9142 \times X$. IN NE $1 / 4$ SEC. $30,7.74 \mathrm{~N}$., R.7 W. WASHINGTON CO. ( 92 ), AT BRIUGE, 2 MILES NE OF COPFOCK.

DRAINAGE AREA $259 \quad \mathrm{MI}^{2}$

$\begin{array}{cccccc}\text { DATE } & F^{3} / S & \text { DATE } & F T^{3} / S & \text { DATE } & F T^{3} / S \\ 09-24-57 & 2.30 & 09-06-60 & 2.36 & 05-10-66 & 40.8 \\ 03-25-58 & 24.5 & 09-05-61 & 3.26 & 08-16-66 & 6.58 \\ 10-27-58 & 11.2 & 11-04-63 & 0.80 & 11-(11-66 & 0.56 \\ 10-20-59 & 76.5 & 10-15-64 & 0.27 & 09-11-67 & 1.4 E\end{array}$

$Q \mathbf{a}=152$

$Q(84)=2.5$

$7 Q 2=0.7$

5-4731.00 WALNUT CR AT GERMANVILLE, IOWA

LAT $41016 \times X$, LONG $9146 \times X$. IN SW $1 / 4$ SEC. 27, T.73 N., R. 8 W. J JEFFER.SON CO. (51), AT BRIDGE, I MILE WEST OF GERMANVILLE.

DRAINAGE AREA $66.3 \mathrm{MI}^{2}$

$\begin{array}{rr}\text { DATE } & F T^{2} / S \\ 09-17-57 & 0 . \\ 03-25-58 & 3.81 \\ 10-27-58 & 1.35 \\ 10-20-59 & 1.0 .0 \\ Q a & =41\end{array}$

$\begin{array}{rr}D A T E & F T^{3} / S \\ 09-06-60 & 0.96 \\ 09-05-61 & 1.41 \\ 11-02-63 & 0.43 \\ 10-13-64 & 0.37 \\ 0(84) & =1.0\end{array}$

$\begin{array}{rr}\text { DATE } & F T^{3} / \mathrm{S} \\ 05-10-66 & 10.1 \\ 08-16-66 & 0.86 \\ 10-31-66 & 0.52 \\ 7 Q 2= & 0.4\end{array}$

DATE
$06-20-68$
$07-13-70$
$08-24-71$

$\mathrm{FT}^{\mathrm{a}} / \mathrm{S}$

1.15

0.93
DATE $\quad F T^{3} / S$
$06-20-68 \quad 41.3$

$\begin{array}{rr}07-13-70 & 21.1 \\ 08-24-71 & 3.7\end{array}$

DA.TE

$09-20-74$

$09-30-75$

FTa is

$09-13-76$

12.0

0.16

$7010=*$

$7010=*$ 
SKUNK RIVER BASIN--Cont inued

5-4732.00 CEDAR CR NR HIGHLAND CENTER, IOWA

LAT 410630 , LONG 922158 , IN SW $1 / 4$ SEC.

$21, T .73$ N., R.13 W., WAPELLO CO. (90),

AT BRIDGE, I MILE SW' OF HIGHLAND CENTER.

DRAINAGE AREA $73.6 \mathrm{MI}^{2}$

\begin{tabular}{|c|c|c|c|c|c|c|c|c|c|}
\hline $\begin{array}{c}\text { DATE } \\
09-18-57 \\
03-27-58 \\
10-22-58 \\
08-25-59\end{array}$ & $\begin{array}{l}\mathrm{FT}^{3} / \mathrm{S} \\
0.50 \\
4.18 \\
7.44 \\
0.63\end{array}$ & $\begin{array}{l}\text { DATE } \\
09-08-60 \\
09-07-61 \\
11-04-63 \\
10-14-64\end{array}$ & $\begin{array}{r}\mathrm{FT}^{3} / \mathrm{S} \\
0.30 \\
0.36 \\
0.40 \\
0.12\end{array}$ & $\begin{array}{c}\text { DATE } \\
05-019-66 \\
08-17-66 \\
11-01-66\end{array}$ & $\begin{array}{l}\mathrm{FT}^{3} / \mathrm{S} \\
16.5 \\
0.35 \\
0.16\end{array}$ & $\begin{array}{c}\text { DATE } \\
06-20-68 \\
07-13-70 \\
08-25-71\end{array}$ & $\begin{array}{l}\mathrm{FT}^{2} / \mathrm{S} \\
2.33 \\
1.14 \\
0.28\end{array}$ & $\begin{array}{c}\text { DATE } \\
09-19-74 \\
09-30-75 \\
09-14-76\end{array}$ & $\begin{array}{l}\mathrm{FT}^{\mathrm{T}} / \mathrm{S} \\
4.2 \\
5.0 \\
0.06\end{array}$ \\
\hline & $=44$ & $Q<\varepsilon$ & $=0.4$ & $7 Q 2$ & $=0.1$ & $7 Q 10$ & 0 & & \\
\hline
\end{tabular}

5-4732.50 COMPETINE CR BELOW FORKS NR BATAVIA, IOWA

LAT $4102 \times X$, LONG $9207 \times X$. IN NE $1 / 4$ SEC.

$21, T .72$ N., R.11 W. JEFFERSON CO. ( 51 ), AT BRIOGE. 3 MILES NE OF BATAVIA.

DRAINAGE AREA $68.8 \mathrm{MI}^{2}$

\begin{tabular}{|c|c|c|c|c|c|}
\hline $\begin{array}{c}\text { DATE } \\
09-18-57 \\
03-27-58 \\
10-29-58 \\
09-08-60\end{array}$ & $\begin{array}{l}F T^{3} / S \\
0.0 \\
4.25 \\
0.88 \\
0 .\end{array}$ & $\begin{array}{c}\text { DATE } \\
09-07-61 \\
11-05-63 \\
10-14-64\end{array}$ & $\begin{array}{l}F T^{3} / S \\
0.62 \\
0 . \\
0 .\end{array}$ & $\begin{array}{c}\text { DATE } \\
05-10-66 \\
08-17-66 \\
11-01-66\end{array}$ & $\begin{array}{l}\mathrm{FT}^{3} / \mathrm{S} \\
9.13 \\
0 . \\
0 .\end{array}$ \\
\hline$Q$ & $=42$. & Qr & $=0.2$ & $7 Q 2$ & $=0$ \\
\hline
\end{tabular}

$\begin{array}{cccc}\text { DATE } & \text { FT /S } & \text { DATE } & F T^{3} / S \\ 06-20-68 & 1.57 & 09-20-74 & 0.35 \\ 07-13-70 & 0.09 & 09-30-75 & 4.2 \\ 08-25-71 & 0.01 & 09-14-76 & 0.00 \\ & & & \\ 7 Q 10=0 & & \end{array}$

5-4733.00 CEDAR CR. NR BATAVIA, IOWA

LAT $4101 \times X$, LONG $9207 X X$, IN NW $1 / 4$ SEC.

27, T. T2 N., R.11 W., JEFFERSON CO. ( 5 i), AT BRIIIGE ON U.S. HIGHWAY $30,2.5$ MILES NE OF EATAVIA.

DRAINAEE AREA $252 \quad \mathrm{MI}^{2}$

$\begin{array}{rc}\text { DATE } & F T^{3} / S \\ 09-18-57 & 0.4 \varepsilon \\ 03-27-58 & 11.8 \\ 10-22-58 & 9.27 \\ 10-29-58 & 5.23 \\ Q a & =153\end{array}$

$\begin{array}{cc}\text { DATE } & F T \text { IS } \\ 10-23-59 & 50.5 \\ 09-06-60 & 0.76 \\ 09-07-51 & 2.16 \\ 11-05-63 & 0.81\end{array}$
DATE
$10-14-64$
$10-14-64$
$05-10-66$
$08-17-66$
$08-17-66$
$F T^{3} / S$
0.08
35.7
0.54
0.28
DATE
$06-20-68$
$07-13-70$
$08-25-71$

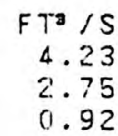

DATE

$09-20-74$
$09-30-75$

$09-14-76$

$7010=0$ 
SKUNK RIVER EASIN--Cont inued

5-4733.50 L CEDAR CR NR SALEM, IOWA

LAT $4051 \times X$, LONG $9141 \times X$, IN SW $1 / 4$ SEC.

17, T.70 N., R.7 W. HENRY CO. (44),

AT BRIDGE, 4 MILES WEST OF SALEM.

DRAINAGE AREA $55.0 \quad \mathrm{MI}^{2}$

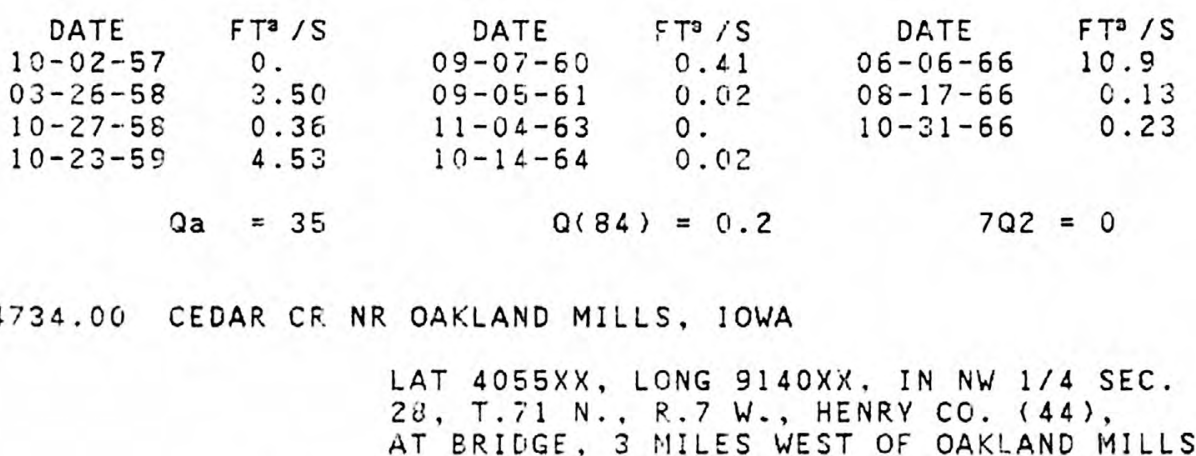

DRAINAGE AREA $522 \quad \mathrm{MI}^{2}$

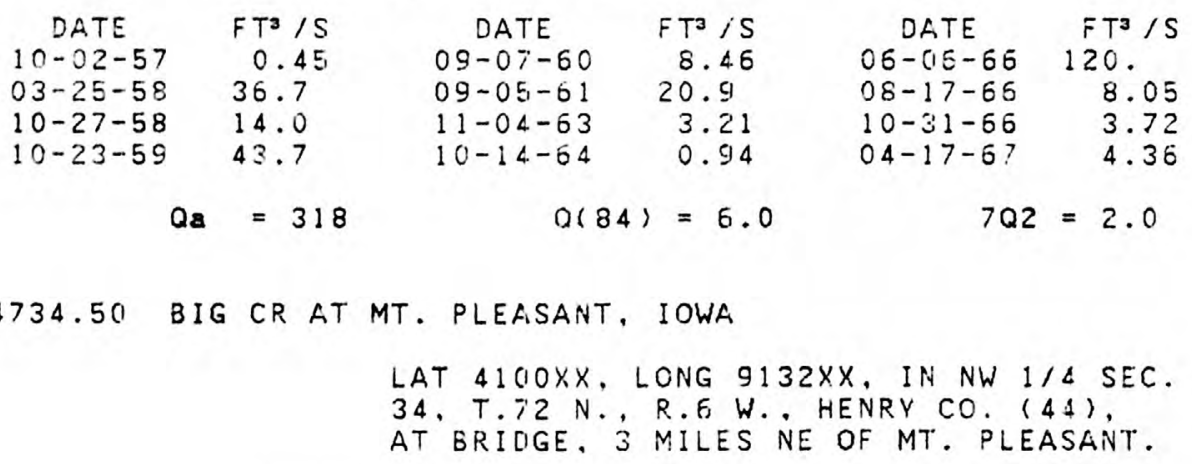

DRAINAGE AREA $58.0 \mathrm{MI}^{2}$

$\begin{array}{rc}\text { DATE } & \text { FT }=/ S \\ 10-02-57 & 0.6 \\ 03-25-58 & 3.67 \\ 10-27-58 & 0.52 \\ 10-20-59 & 17.2 \\ 2 a & =37\end{array}$

$\begin{array}{cl}\text { DATE } & F T^{3} / S \\ 09-07-60 & 0.06 \\ 09-0 E-61 & 0.2 .3 \\ 11-04-63 & 0 . \\ 10-1<-64 & 0 .\end{array}$

$\begin{array}{rl}\text { DATE } & F^{3} / S \\ \text { OS- } 16-66 & 19.8 \\ 08-17-66 & 0 . \\ 10-31-66 & 0 . \\ 7 Q 2= & 0\end{array}$

DATE
$06-20-68$
$07-13-70$

$T^{3} / S$

0.04

$07-13-70$

0.03

DATE

$09-19-74$

$09-29-75$

$09-14-76$

$F T^{3} / S$

0.7

0.

$7 Q 10=0$ 
DEVILS CREEK BASIN

5-4741.90 DEVILS CR NR VIELE, IOWA

LAT 403703, LONG 912534, IN SW 1/4 SEC.

10, T.67 N., R. 5 W., LEE CO. (56),

AT BRIDGE, 1 MILE NE OF VIELE.

DRAINAGE AREA $20.0 \mathrm{MI}=$

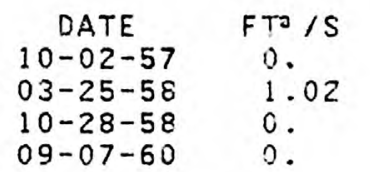

$Q_{a}=13$

$\begin{array}{cl}\text { DATE } & \mathrm{FT}^{3} / \mathrm{S} \\ 09-06-61 & 0 . \\ 11-05-63 & 0 . \\ 10-15-64 & 0 .\end{array}$

$Q(84)=0$

$\begin{array}{cc}\text { DATE } & F^{3} / S \\ 06-07-66 & 0.36 \\ 08-22-65 & 0 . \\ 11-012-66 & 0 .\end{array}$

$7 Q 2=0$

$\begin{array}{cl}\text { DATE } & \mathrm{FT}^{3} / \mathrm{S} \\ 06-13-68 & 0 . \\ 07-14-70 & 0 . \\ 08-25-71 & 0 .\end{array}$

$7010=0$

5-4742.00 SUGAR CR. NR FRANKLIN, IOWA

LAT 403954 , LONG 912839 , IN NE $1 / 4$ SEC. 30 , T. 6.8 N., R. 5 W. , LEE CO. $(56)$,

AT BRIUGE, 2 MILES EAST OF FRANKLIN.

DRAINAGE AREA $75.6 \mathrm{MI}^{2}$

\begin{tabular}{|c|c|c|c|c|c|c|c|c|c|}
\hline $\begin{array}{c}\text { DATE } \\
10-02-57 \\
03-25-58 \\
10-28-58 \\
09-07-60\end{array}$ & $\begin{array}{c}F T^{3} / S \\
0 . \\
7.32 \\
1.33 \\
0.85\end{array}$ & $\begin{array}{c}\text { DATE } \\
09-05-61 \\
11-05-63 \\
10-15-64\end{array}$ & $\begin{array}{l}\mathrm{FT}^{3} / \mathrm{S} \\
0.17 \\
0.17 \\
0.02\end{array}$ & $\begin{array}{c}\text { DATE } \\
08-07-66 \\
08-22-66 \\
11-012-65\end{array}$ & $\begin{array}{l}\mathrm{FT}^{3} / \mathrm{S} \\
10.4 \\
0.1 \\
0.11\end{array}$ & $\begin{array}{c}\text { DATE } \\
06-13-68 \\
07-14-70 \\
08-25-71\end{array}$ & $\begin{array}{l}F T^{3} / \mathrm{S} \\
1.16 \\
0.66 \\
0.01\end{array}$ & $\begin{array}{c}\text { DATE } \\
09-20-74 \\
09-30-75 \\
09-15-76\end{array}$ & $\begin{array}{l}\mathrm{FT}^{3} / \mathrm{S} \\
1.2 \\
0.76 \\
0.01\end{array}$ \\
\hline & & Q: & $=0.3$ & 1Q2 & $\star$ & 1010 & 0 & & \\
\hline
\end{tabular}

5-4743.00 SUGAR CR NR VIELE, IOWA

LAT 403639 , LONG 912624, IN SE $1 / 4$ SEC.

9, T.6\% N.. R.5 W., LEE CO. (56),

AT BRICIGE. 0.5 MILE WEST OF VIELE.

DRAINAGE AREA $109 \mathrm{MI}^{2}$

$\begin{array}{cc}\text { DATE } & F T^{0} / S \\ 10-02-57 & 0 . \\ 03-25-58 & 12.6 \\ 10-28-58 & 1.76 \\ 09-07-60 & 2.10\end{array}$

$\begin{array}{cr}\text { DATE } & F T^{3} / S \\ 09-06-61 & 0.58 \\ 11-05-53 & 0.08 \\ 10-15-64 & 0.15\end{array}$

$\begin{array}{cc}\text { DATE } & F^{3} / \mathrm{S} \\ 06-017-66 & 13.6 \\ 08-2.2-66 & 1.78 \\ 11-012-66 & 0.65\end{array}$

DATE

OE-13-68

$07-14-70$

$08-25-71$

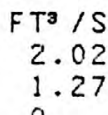

2.02

1.2
DATE
09-20-74 $09-30-75$ $09-15-76$

$F T / S$

0 .

0 .

$\mathbf{Q}=70$

$Q(84)=0.4$

$7 Q 2=*$

$7 Q 10=0$ 
DES MIOINES RIVER BASIN

5-4765.50 JACK CR NR RINGSTED, IOWA

LAT $4316 \times X$, LONG $9438 \times X$, NEAR $S 1 / 4$ CORNER

OF SEC.28, T.98 N., R.32 W., EMMET CO. (32),

AT BRIDGE, 6 MILES SW OF RINGSTED. DRAINAGE AREA $74.8 \quad \mathrm{MI}^{2}$

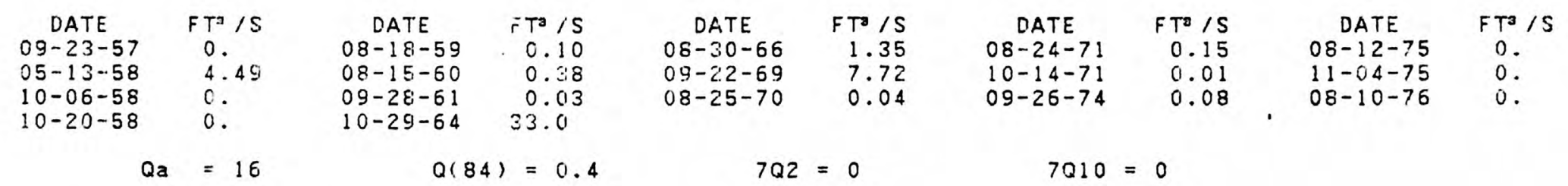

5-4765.00 SILVER CR NR EMMETSBURG, IOWA

LAT 4306XX. LONG $9443 \times X$, NEAR N $1 / 4$

CORNER SEC.34, T.96 N., R.33 W., PALO ALTO CO.

(74), AT BRIDGE, 3 MILES SW OF EMMETTSBURG.

ORAINAGE AREA $61.8 \mathrm{MI}^{2}$

\begin{tabular}{|c|c|c|c|c|c|c|c|c|c|}
\hline $\begin{array}{l}\text { DATE } \\
09-23-57 \\
05-13-58 \\
10-07-58 \\
10-20-58\end{array}$ & $\begin{array}{l}F T^{3} / S \\
0.57 \\
5.22 \\
0.82 \\
0.43\end{array}$ & $\begin{array}{l}\text { DATE } \\
08-18-59 \\
08-15-60 \\
09-28-61 \\
10-29-64\end{array}$ & $\begin{array}{l}F T 3 \text { is } \\
0.95 \\
1.06 \\
1.76 \\
13.0\end{array}$ & $\begin{array}{c}\text { DATE } \\
08-30-66 \\
09-23-69 \\
08-25-70\end{array}$ & $\begin{array}{c}F T^{\mathrm{a}} / \mathrm{S} \\
0.48 \\
16.8 \\
0.74\end{array}$ & $\begin{array}{c}\text { DATE } \\
08-25-71 \\
10-14-71 \\
09-26-74\end{array}$ & $\begin{array}{l}\mathrm{FT} / \mathrm{S} \\
3.6 \\
1.0 \\
0.99\end{array}$ & $\begin{array}{c}\text { DATE } \\
08-12-75 \\
11-04-75 \\
08-10-76\end{array}$ & $\begin{array}{l}F T \text { S } \\
1.1 \\
0.93 \\
0.34\end{array}$ \\
\hline & $=13$ & $Q<\varepsilon$ & 2.0 & 702 & 0.9 & 7010 & 0.4 & & \\
\hline
\end{tabular}

5-4756.50 CYLINDER CR NR RODMAN, IOWA

LAT $4302 X X$, LONG $9434 X X$, NEAR S $1 / 4$

CORNER SEC.13, T.95 N., R.32 W., PALO ALTO CD.

$(74)$, AT BRIDGE, 2.5 MILES NW OF RODMAN.

DRAINAGE AREA $88.6 \mathrm{MI}^{2}$

$\begin{array}{cr}\text { DATE } & \text { FTa/S } \\ 09-23-57 & 1.19 \\ 05-23-58 & 4.08 \\ 10-08-58 & 1.07 \\ 10-20-58 & 1.04\end{array}$

$Q \mathrm{Q}=20$

$\begin{array}{cr}\text { DATE } & F T / S \\ 08-18-59 & 2.85 \\ 08-15-60 & 2.55 \\ 09-28-61 & 3.74 \\ 10-29-64 & 37.9 \\ Q(84)=4.0\end{array}$

$Q(84)=4.0$

$\begin{array}{cc}\text { DATE } & F T^{3} / S \\ 08-31-66 & 2.51 \\ 09-23-69 & 11.2 \\ 08-25-70 & 1.94\end{array}$

$7 Q 2=1.8$

DATE
$08-24-71$
$10-14-71$
$09-26-74$

2.8
1.9
2.8

$7 Q 10=0.9$ 
DES MOINES RIVER. BASIN--Cont inued

5-4767.00 PRAIRIE CR NR WEST BEND, IOWA

LAT $4255 X X$, LONG $9427 X X$, NEAR $N 1 / 4$

CORNER SEC. $36, T .94 \mathrm{~N} .$, R.31 W., PALO ALTO CO.

( 74 ), BRIDGE, 2.5 MILES SW OF WEST BEND.

DRAINAGE AREA $61.1 \mathrm{MI}^{2}$

\begin{tabular}{|c|c|c|c|c|c|c|c|c|c|}
\hline $\begin{array}{c}\text { DATE } \\
09-23-57 \\
05-13-58 \\
10-08-58 \\
10-20-58\end{array}$ & $\begin{array}{l}\mathrm{FT}^{3} / \mathrm{S} \\
0.15 \\
1.17 \\
0 . \\
0 .\end{array}$ & $\begin{array}{c}\text { DATE } \\
08-18-59 \\
08-15-60 \\
09-28-61 \\
10-29-64\end{array}$ & $\begin{array}{c}\mathrm{FT} / \mathrm{S} \\
0.2 .1 \\
0.07 \\
0.79 \\
14.2\end{array}$ & $\begin{array}{c}\text { DATE } \\
08-31-66 \\
09-23-59 \\
08-25-70\end{array}$ & $\begin{array}{l}F T^{2} / S \\
0.18 \\
3.33 \\
0 .\end{array}$ & $\begin{array}{c}\text { DATE } \\
08-24-71 \\
10-14-71 \\
09-26-74\end{array}$ & $\begin{array}{l}\mathrm{FT}^{3} / \mathrm{S} \\
0.18 \\
0.09 \\
0 .\end{array}$ & $\begin{array}{c}\text { DATE } \\
08-12-75 \\
11-04-75 \\
08-11-76\end{array}$ & $\begin{array}{c}F T^{3} / \\
0 . \\
0 . \\
0 .\end{array}$ \\
\hline Qa & $=13$ & Qr & $=0.4$ & 702 & $=0$ & 7010 & 0 & & \\
\hline
\end{tabular}

5-4767.20 BEAVER CR NR ROLFE, IOWA

LAT $4250 \times X$, LONG $9428 \times X$. NEAR CENTER OF SEC.

$35, T .93$ N., R.31 W., POCAHONTAS CO. $(76)$,

AT BRIDGE, 3 MILES NE OF ROLFE.

DRAINAGE AREA $62.2 \mathrm{MI}^{2}$

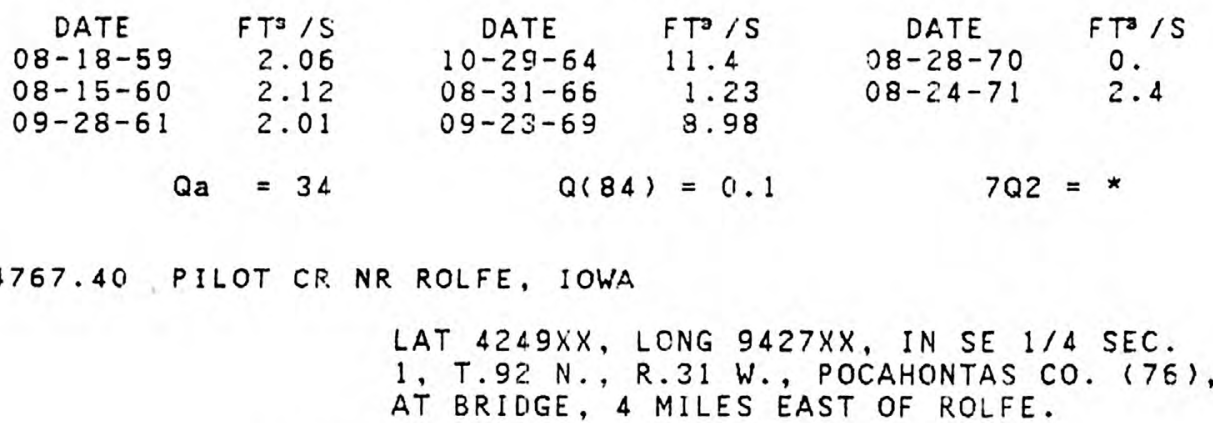

DRAINAGE AREA $97.0 \mathrm{MI}^{2}$

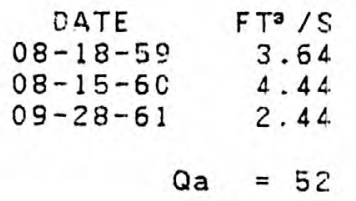

$\begin{array}{cc}\text { DATE } & F T^{3} / S \\ 10-29-64 & 9.32 \\ 08-31-66 & 1.77 \\ 09-23-69 & 10.3\end{array}$

$\begin{array}{cc}\text { DA.TE } & \text { FT'/S } \\ 08-27-70 & 1.29 \\ 08-2.4-71 & 4.9\end{array}$

$\begin{array}{cc}\text { DATE } & F T / S \\ 10-15-71 & 1.6 \\ 09-24-74 & 2 .\end{array}$

DATE

$11-06-75$

$\mathrm{FT}^{\mathrm{a}} / \mathrm{S}$

$09-23-69 \quad 30.3$

$7 Q 2=1.6$

$7 Q 10=0.3$ 
DES MOINES RIVER. BASIN--Cont inued

5-4776.00 EF DES MOINES R NR. DOLLIVER, IOWA

LAT $4328 \times X$. LONG $9435 \times K$, IN SW $1 / 4$ SEC.

13, T.100 N., R. 32 W. EMMET CO. $\{32\}$,

AT BRIDGE, 2 MILES NE OF DOLLIVER.

DRAINAGE AREA $196 \quad \mathrm{MI}^{2}$

\begin{tabular}{|c|c|c|c|c|c|c|c|c|c|}
\hline $\begin{array}{c}\text { QATE } \\
09-23-57 \\
05-13-58 \\
10-06-58 \\
10-20-58\end{array}$ & $\begin{array}{l}\mathrm{F} T^{2} / \mathrm{S} \\
0.65 \\
10.3 \\
0 . \\
0 .\end{array}$ & $\begin{array}{c}\text { DATE } \\
08-17-59 \\
08-15-60 \\
09-28-61 \\
10-27-64\end{array}$ & $\begin{array}{l}\mathrm{FT}^{3} \text { is } \\
0 . \\
2.53 \\
0.51 \\
27.7\end{array}$ & $\begin{array}{c}\text { DATE } \\
08-30-66 \\
09-22-69 \\
08-25-70\end{array}$ & $\begin{array}{l}\mathrm{FT}^{3} / \mathrm{S} \\
0 . \\
0.10 \\
0.01\end{array}$ & $\begin{array}{c}\text { DATE } \\
08-24-71 \\
10-13-71 \\
09-25-74\end{array}$ & $\begin{array}{l}\mathrm{FT}^{3} / \mathrm{S} \\
0 . \\
0 . \\
0 .\end{array}$ & $\begin{array}{c}\text { DATE } \\
08-12-75 \\
11-04-75 \\
00-10-76\end{array}$ & $\begin{array}{l}\mathrm{FT}^{3} / \mathrm{S} \\
0 . \\
0 . \\
0 .\end{array}$ \\
\hline$Q$ & $=4 E_{1}$ & Qr & $\gamma=2.5$ & 702 & $=0$ & 7010 & 0 & & \\
\hline
\end{tabular}

5-4777.00 EF DES MOINES R NR SWEA CITY, IOWA

LAT $4319 \times X$, LONG $9425 \times X$, NEAR CENTER OF SEC. 8, T. 38 N., F. 30 W. . KOSSUTH CO. (55), AT BRIQIGE. 7 MILES SW OF SWEA CITY.

DRAINAGE AREA $314 \quad \mathrm{MI}^{2}$

\begin{tabular}{|c|c|c|c|c|c|c|c|c|c|}
\hline $\begin{array}{l}\text { DATE } \\
09-23-57 \\
05-13-58 \\
10-06-58 \\
10-20-58\end{array}$ & $\begin{array}{l}\mathrm{FT}^{3} / \mathrm{S} \\
0 . \\
21.4 \\
0 . \\
0 .\end{array}$ & $\begin{array}{c}\text { DATE } \\
08-17-59 \\
08-16-60 \\
09-2 \varepsilon_{i}-61\end{array}$ & $\begin{array}{l}\mathrm{FT}^{3} / \mathrm{S} \\
0 . \\
3.54 \\
0.72\end{array}$ & $\begin{array}{c}\text { DATE } \\
10-27-64 \\
08-30-66 \\
09-22-69\end{array}$ & $\begin{array}{l}\text { FTO /S } \\
46.5 \\
0.27 \\
3.03\end{array}$ & $\begin{array}{c}\text { DATE } \\
08-25-70 \\
08-24-71 \\
10-13-71\end{array}$ & $\begin{array}{l}\mathrm{FT}^{3} / \mathrm{S} \\
0 . \\
0 . \\
0 .\end{array}$ & $\begin{array}{c}\text { DATE } \\
09-25-74 \\
08-12-75 \\
08-10-76\end{array}$ & $\begin{array}{l}\mathrm{FT}^{\mathrm{S}} / \mathrm{S} \\
0.58 \\
0.88 \\
0 .\end{array}$ \\
\hline & 75 & Q1 & $=5.0$ & 702 & 0 & 7010 & 0 & & \\
\hline
\end{tabular}

5-4778.00 MUD CR AT BANCROFT, IOWA

LAT $4318 \times X$. LONG $9412 \times X$, NEAR CENTER OF SEC.19, T.98 N., R.28W., KOSSUTH CO. (55), AT BRILIGE, 1 MILE EAST OF EANCROFT.

ORAINAGE AREA EB.1 MI2

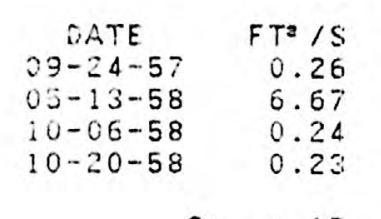

$Q \mathrm{a}=15$

$\begin{array}{cc}\text { DATE } & F T=/ S \\ 08-17-50 & 0.51 \\ 08-16_{1}-60 & 0.66 \\ 09-28-61 & 5.2 .8 \\ 10-27-66 & 27.61 \\ 0(84)=1.6\end{array}$

$Q(84)=1.6$

$\begin{array}{rr}\text { DATE } & F^{3} / 5 \\ 08-30-66 & 2.77 \\ 09-2.2-69 & 1.82 \\ 68-25-70 & 0.46 \\ 702= & 0.4\end{array}$

$\mathrm{FT}^{\mathrm{a}} / \mathrm{S}$

(1. E6

0.15

0.42
DATE

$08-13-75$

$11-04-75$

$08-10-76$
$F T^{2} / S$

0 .

0.05 
DES MOINES RIVER. BASIN--Cont inued

5-4780.50 BUFFALO CR NR TITONKA, IOWA

LAT $4314 \times X$, LONG $9359 \times X$, IN NW $1 / 4$ SEC.

12, T.97 N., R.2T W., KOSSUTH CO. (55),

AT BRIDGE, 3 MILES EAST OF TITONKA.

DRAINAGE AREA $47.9 \mathrm{MI}^{2}$

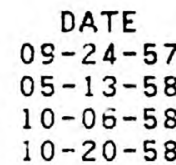

$\begin{array}{lcc}F T / S & \text { DATE } & F T / S \\ 0.0 & 08-17-59 & 0.09 \\ 2.40 & 08-16-60 & 0.4 \\ 0 . & 09-28-61 & 3.59 \\ 0 . & 10-29-64 & 7.43\end{array}$

$\begin{array}{cc}\text { DATE } & F T / S \\ 08-30-66 & 1.08 \\ 09-22-69 & 0.17 \\ 08-26-70 & 0 .\end{array}$

DATE
$08-23-71$
$10-13-71$
$09-25-74$

FTo $/ S$

.

DATE

$08-13-75$

$r T^{3} / S$

$09-25-74 \quad 0.04 \quad 08-10-76$

0 .

$Q_{a}=10$

$Q(84)=0.3$

$7 Q 2=0$

$7 Q 10=0$

5-4781.00 N BUFFALO CR NR BUFFALO CENTER, IOWA

LAT $4319 \times X$, LONG $9358 \times X$, IN NW $1 / 4$ SEC.

$18, T .98$ N., R.26 W. WINNESAGO CO. (95),

AT BRIDGE, 5 MILES SOUTH OF BUFFALO CENTER.

DRAINAGE AREA $62.5 \mathrm{MI}^{2}$

$\begin{array}{cr}\text { DATE } & F T^{3} / S \\ 09-24-57 & 0.22 \\ 05-14-58 & 4.23 \\ 10-06-58 & 0.03 \\ 10-20-58 & 0.10\end{array}$

$Q_{\mathbf{a}}=14$

$\begin{array}{cr}\text { DATE } & F T^{3} / S \\ 08-17-59 & 0.41 \\ 08-16-60 & 0.68 \\ 09-28-61 & 5.96 \\ 10-29-64 & 10.0\end{array}$

$Q(84)=1.6$

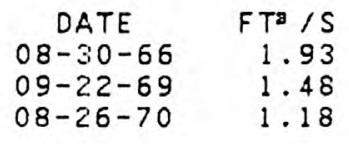

$7 Q 2=0.2$

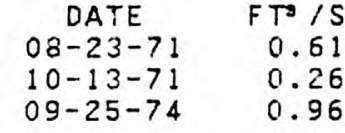

DATE

$08-13-75$
$11-04-75$

$11-04-75$
$08-10-76$

$\mathrm{FT}^{3}$ is

0.89

0.34

0.16

5-4781.50 BLACK CAT CR NR LONE ROCK, IOWA

LAT $4312 \times \%$, LONG $9420 X X$, NEAR S $1 / 4$ CORNER SEC. 24 , T.97 N., R. 30 W., KOSSUTH CO. (55), AT BRILIGE, 2 MILES SW OF LONE ROCK.

\section{DRAINAGE AREA $58.2 \mathrm{MI}^{2}$}

$\begin{array}{rl}\text { DATE } & F T^{5} / S \\ 09-24-57 & 0.03 \\ 05-13-58 & 3.93 \\ 10-06-58 & 0 . \\ 10-20-58 & 0 . \\ \text { Qa } & =13\end{array}$

DATE
$08-17-59$
$08-16-60$
$09-2 \varepsilon-61$

$\mathrm{FT}^{3} / \mathrm{S}$
0.29
0.31

$09-28-61$

2.89

$\begin{array}{cc}\text { DATE } & F^{3} / S \\ 10-2.6-64 & 21.2 \\ 08-30-66 & 0.93 \\ 09-23-69 & 3.60 \\ 7 Q 2=*\end{array}$

$$
\begin{gathered}
\text { DATE } \\
08-25-70 \\
08-23-71 \\
10-13-71
\end{gathered}
$$
F TO is
0.02
(1. 10
(1.0!

DATE

$09-26-74$

$08-12-75$

$08-10-76$

$\mathrm{FT}^{3} / \mathrm{S}$

0.06

0.75

0 .

$7 Q 10=0$ 
DES MOINES RIVER. BASIN--Cont inued

5-4782.00 BLACK CAT CR NR ALGONA, IOWA

LAT $4308 \times X$, LONG $9414 \times X$, NEAR S $1 / 4$ CORNER SEC.11, T.96 N.. R.29 W., KOSSUTH CO. (55), AT BRIDGE ON U. $S$. HIGHWAY 169, 5 MILES NORTH OF ALGONA.

DRAINAGE AREA $112 \quad \mathrm{MI}^{2}$

$\begin{array}{rl}\text { DATE } & \text { FT } / S \\ 09-24-57 & 0 . \\ 05-14-58 & 8.46 \\ 10-06-58 & 0 . \\ 10-20-58 & 0 . \\ \text { Qa } & =25\end{array}$

$\begin{array}{cr}\text { DATE } & F^{3} / S \\ 08-17-59 & 0.43 \\ 08-18-60 & 0.02 \\ 09-28-61 & 9.47 \\ 10-26-64 & 41.6 \\ Q(84)= & 0.8\end{array}$

$\begin{array}{cc}\text { DATE } & F^{3} / S \\ 08-30-66 & 1.63 \\ 09-23-69 & 7.29 \\ 08-25-70 & 0 . \\ 7 Q 2= & \star\end{array}$

$\begin{array}{cl}\text { DATE } & F T / S \\ 08-23-71 & 0.02 \\ 10-13-71 & 0 . \\ 09-26-74 & 0 . \\ 7010= & 0\end{array}$

DATE
$08-13-75$

$11-04-75$

$11-04-75$
$08-08-76$

$F T^{3} / S$

0.45

0.

Qa $=25$

$Q(84)=0.8$

5-4783.50 LOTTS CR NR WEST EEND, IOWA

LAT $4258 \times \times$. LONG $9423 \times X$, NEAR S $1 / 4$ CORNER

SEC.9, T.94 N., R. 30 W., KOSSUTH CO. (55),

AT BRIOGE, 3 MILES EAST OF WEST BEND.

DRAINAGE AREA $66.2 \mathrm{MI}^{2}$

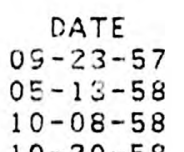

DATE
$08-18-53$
$08-15-60$
$09-28-61$

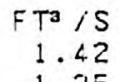

1.42
1.35
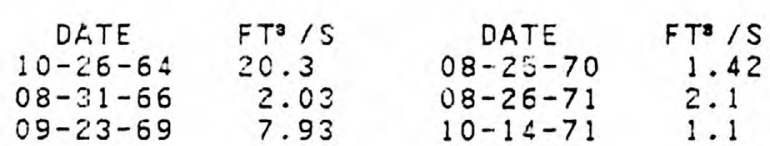

DATE
$09-26-74$

$08-12-75$

$\mathrm{FT}^{\mathrm{3}} / \mathrm{S}$

4.44
0.61

6.96

$09-23-69$

$10-14-71$

2.1

.3

$$
0.41
$$

$Q(84)=3.0$

$7 Q 2=1.2$

$7 Q 10=0.5$

5-4784.00 LOTTS CF. AT LIVERMORE, IOWA

LAT 4252XX, LONG $9411 \times X$. IN NE $1 / 4$ SEC;

$18, T .93$ N., R. 28 W., HUMBOLDT CO. (4E),

AT BRIQIGE NEAR NW CITTY LIMITS OF LIVERMORE.

DRAINAGE AREA $165 \mathrm{MI}^{2}$

$\begin{array}{cc}\text { DATE } & F T^{3} / S \\ 09-24-57 & 0.32 \\ 05-14-58 & 10.5 \\ 10-08-58 & 0.60 \\ 10-21-58 & 0.24 \\ & \end{array}$

$\begin{array}{cc}\text { UATE } & \text { FT /S } \\ 05-12-59 & 36.4 \\ 08-17-59 & 8.26 \\ 08-18-59 & 3.22 \\ 08-15-50 & 26.8\end{array}$

$\begin{array}{cc}\text { DATE } & F T^{3} / S \\ 09-2.8-61 & 8.14 \\ 10-26-64 & 32.1 \\ 08-31-66 & 2.62 \\ 09-23-69 & 11.9\end{array}$

DATE
$08-25-70$
$08-23-71$
$10-14-71$

$F T^{2} / S$
1.37
5.5
1.9

DATE

$09-25-74$

$08-13-75$

$F^{\mathrm{a}} / \mathrm{S}$

2.0
4.4

$08-15-50 \quad 26.8$

$7 Q 2=1.6$

$7 Q 10=0.5$ 
DES MOINES RIVER. BASIN--Cont inued

5-4796.00 LIZARD CR NR PALMER, IOWA

LAT $4239 \times X$, LONG $9430 \times X$, IN NW $1 / 4$ SEC.

3 , T. 90 N., R. 31 W., POCAHONTAS CO. (76),

AT BRIDGE, 5 MILES NE OF PALMER.

DRAINAGE AREA $66.4 \mathrm{MI}^{2}$

$\begin{array}{rr}\text { DATE } & \mathrm{FT}^{3} / \mathrm{S} \\ 09-24-57 & 1.91 \\ 05-12-58 & 8.12 \\ 10-08-58 & 2.06 \\ 10-22-58 & 0.31 \\ Q a & =26\end{array}$

$\begin{array}{cr}\text { DATE } & \text { FT iS } \\ 08-13-59 & 1.09 \\ 08-15-60 & 1.42 \\ 09-28-61 & 1.10\end{array}$

$\begin{array}{cr}\text { DATE } & F^{3} / S \\ 10-29-64 & 1.97 \\ 08-31-66 & 1.13 \\ 09-23-69 & 3.03\end{array}$

DATE
$08-27-70$
$08-24-71$
$10-15-71$

$F T^{3} / \mathrm{S}$
0.58

1.58
1.0

DATE

$11-06-75$

$10-15-71 \quad 0.46$

$08-11-76$

1.3

0.57

$7010=0.1$

5-4798.00 NB LIZARD CR NR HAVELOCK, IOWA

LAT $4248 \times X$, LONG $9440 \times K$, IN NE $1 / 4$ SEC.

18, T. 92 N., R.32 W., POCAHONTAS CO. (76), AT BRIUGE, 4 MILES SÉ OF HAVELOCK.

DRAINAGE AREA $79.4 \mathrm{MI}^{2}$

$\begin{array}{rr}\text { DATE } & F^{3} / S \\ 09-25-57 & 0.41 \\ 05-13-58 & 5.76 \\ 10-08-58 & 5.96 \\ 10-22-58 & 0.25 \\ \text { Qa } & =30\end{array}$

$\begin{array}{rr}\text { DATE } & \mathrm{FT}^{3} \text { is } \\ 08-13-59 & 1.03 \\ 08-15-60 & 1.62 \\ 09-28-61 & 0.59 \\ Q(84) & =1.4\end{array}$

$\begin{array}{cr}\text { DATE } & F^{3} / S \\ 10-29-64 & 4.70 \\ 08-31-66 & 0.81 \\ 09-23-69 & 6.58\end{array}$

DATE
$08-27-70$
$08-24-71$
$10-15-71$

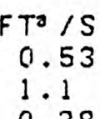

5-4799.00 LIZARD CR NR GILMORE CITY, IOWA

LAT $4238 \times X$, LONG $9428 \times X$, IN NW $1 / 4$ SEC.

1 , T.90 N., R.31 W., POCAHONTAS CO. (76),

AT BRIDGE, 6 MILES SW OF GILMORE CITY.

DRAINAGE AREA $219 \quad M^{2}$

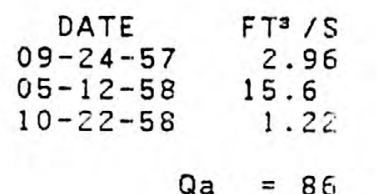

$Q a=8 \varepsilon_{1}$

$\begin{array}{cr}\text { DATE } & F T^{3} / S \\ 08-13-59 & 4.65 \\ 08-15-60 & 5.19 \\ 09-28-61 & 3.79\end{array}$

$Q(84)=4.4$

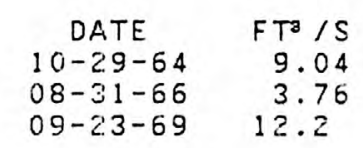

$7 Q 2=1.1$

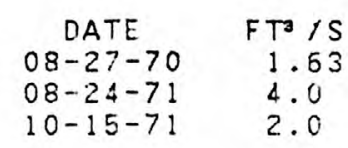

DATE
O9-24-74
$11-06-75$
$08-11-76$

$\mathrm{FT}^{3} / \mathrm{S}$

1.3

1. 5 
DES MOINES RIVER. BASIN--Cont inued

5-4801.00 SE LIZAR.D CR NR PALMER, IOWA

LAT $4235 \times X$, LONG $9432 \times X$, IN SW $1 / 4$ SEC.

29. T.90 N., R.31 W., POCAHONTAS CO. (76), AT BRIDGE, 4.5 MILES'SE OF PALMER.

DRAINAGE AREA $66.4 \mathrm{MI}^{2}$

$\begin{array}{rr}\text { DATE } & \text { FT /S } \\ 09-24-57 & 0.40 \\ 05-12-58 & 5.49 \\ 10-22-58 & 0.09 \\ Q a & =28\end{array}$

$\begin{array}{cr}\text { DATE } & F^{3} / S \\ 08-13-59 & 0.14 \\ 08-15-60 & 0.49 \\ 09-28-61 & 0.49\end{array}$

$\begin{array}{cr}\text { DATE } & F T^{3} / S \\ 10-29-64 & 0.10 \\ 08-31-66 & 0.03 \\ 09-23-69 & 1.36 \\ 702= & \star\end{array}$

$\begin{array}{cc}\text { DATE } & \text { FT /S } \\ 08-28-70 & 0.07 \\ 08-24-71 & 0.06 \\ 10-15-71 & 0 . \\ 7010= & 0 * *\end{array}$

$5-4803.00$ SB LIZAR.D CR NR FORT DODGE, IOWA

LAT 422950, LONG 941359. IN NE $1 / 4$ SEC. $26, T .89 \mathrm{~N} ., \mathrm{R} .29 \mathrm{~W}$. WEBSTER CO. (94), AT BRIUGE, 3 MILES WEST OF FORT DODGE.

DRAINAGE AREA $154 \quad \mathrm{MI}^{2}$

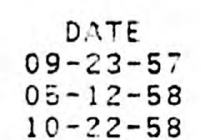

$\begin{array}{ccr}F T^{2} / S & \text { DATE } & F T^{\circ} \text { is } \\ 2.19 & 08-13-59 & 2.23 \\ 14.4 & 08-15-60 & 1.95 \\ 0.46 & 09-28-61 & 4.60\end{array}$

$$
\begin{gathered}
\text { DATE } \\
10-29-64 \\
08-31-66 \\
09-23-69
\end{gathered}
$$

$\mathrm{FT}^{\mathrm{a}} / \mathrm{S}$

$09-2 \varepsilon-61$

4.60

$09-23-69$

1.05

8.25

$Q_{a}=64$

$Q(84)=1.6$

$7 Q 2=0.5$

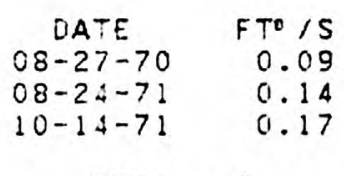

DATE

$08-11-76$

$\mathrm{FT}^{3} / \mathrm{s}$

0.34

5-4806.20 BRUSHY CR NR HOMER, IOWA

LAT $4223 X X$. LONG $9359 \times X$, IN SE $1 / 4$ SEC.

34. T.88 N.. R.27 W., WEBSTER CO. (94),

AT BRILIGE, 3 MILES NW OF HOMER.

DRAINAGE AREA $88.5 \mathrm{MI}^{2}$
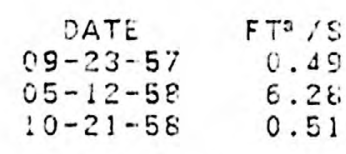

DATE
$08-14-59$
$08-16-63$
$09-28-61$

$\mathrm{FT}^{3}$ is

C. 49

0.51

0.01

0.63
6.512

DATE
$10-26-64$
$08-29-66$
$09-22-69$

$F T^{3} / S$
0.44
0.28
6.52

$Q(84)=0.7$

$702=0.2$

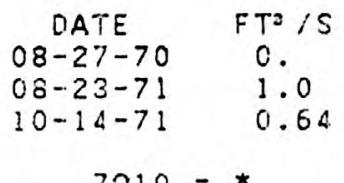

DATE
$09-24-74$
$11-06-75$
$08-10-76$

$F T^{3} / S$

0.36

$Q_{a}=4 \mathrm{O}$

$7010=$ * 
DES MOINES RIVER. BASIN--Cant inued

5-4805.60 BOONE R NR KANAWHA, IOWA

LAT $4255 \times X$, LONG $9353 \times X$, NEAR NORTH $1 / 4$

CORNER SEC.35, T.94 N., R.26 W., HANCOCK CO.

(41), AT BRIDGE, 4 MILES SW OF KANAWHA.

\section{DRAINAGE AREA $71.4 \quad \mathrm{MI}^{2}$}

$\begin{array}{cl}\text { DATE } & F^{3} / S \\ 09-25-57 & 0 . \\ 05-14-58 & 2.29 \\ 10-21-58 & 0 . \\ 08-14-59 & 0.88\end{array}$

$\begin{array}{cc}\text { DATE } & \text { FT } / S \\ 08-16-60 & 0 . \\ 09-28-61 & 5.68 \\ 10-27-64 & 5.61 \\ 08-29-66 & 1.42\end{array}$

$\begin{array}{cr}\text { DATE } & \mathrm{FT}^{2} / \mathrm{S} \\ 08-07-67 & 2.09 \\ 09-22-69 & 1.98 \\ 08-26-70 & 0.32\end{array}$

DATE
$08-23-71$
$10-13-71$
$09-25-74$

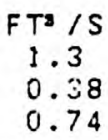

DATE
$08-14-75$
$11-05-75$
$08-11-76$

$F T^{3} / S$

$08-26-70-0.32$

$702=0.1$

$7010=0 * \star$

5-4807.00 BOONE R NR RENWICK, IOWA

LAT $4253 \times x$, LONG $9355 \times X$. IN SW $1 / 4$ SEC

$3, T .93 \mathrm{~N} ., \mathrm{R} .26 \mathrm{~W}, \mathrm{WRIGHT}$ CO. (99).

AT BRIDGE, 6 MILES NE OF RENWICK.

DRAINAGE AREA $134 \quad \mathrm{MI}^{2}$

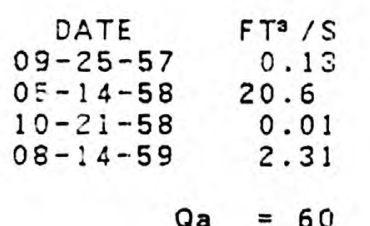

$Q_{a}=60$

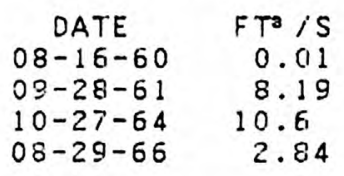

$Q(84)=3.2$
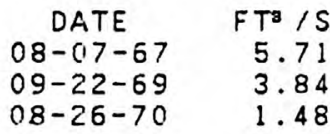

5.71
3.84

1.48

$7 Q 2=0.2$

$\begin{array}{cc}\text { DATE } & F T^{3} / S \\ 08-23-71 & 2.5 \\ 10-13-71 & 1.2 \\ 09-25-74 & 2.1\end{array}$

DATE

$08-14-75$
$11-05-75$

$11-05-75$
$08-11-76$

$\mathrm{FT}^{3} / \mathrm{S}$

$09-25-74 \quad 2$.

$7 Q 10=*$

5-4807.20 PRAIRIE CR NR LUVERNE, IOWA

LAT $4257 \times X$, LONG $9405 \times X$, IN SW $1 / 4$ SEC.

$18, T .94$ N., R.27 W. , KOSSUTH CO. (55).

AT BRIDIGE. 3 MILES NORTH OF LUVERNE.

DRAINAGE AREA $68.6 \mathrm{MI}^{2}$

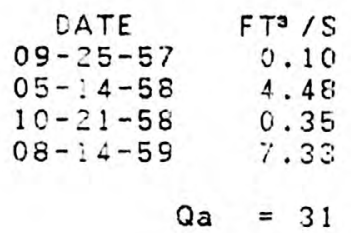

DATE
$03-1 E-60$
$03-2 E-61$
$10-2 E-64$
$\mathrm{FT}^{\mathrm{T}} / \mathrm{S}$
1.78

$09-28-6$

9.17

$\begin{array}{cr}\text { DATE } & \mathrm{FT}^{3} / \mathrm{S} \\ 08-2.9-66 & 2.46 \\ 09-2.2-69 & 2.82 \\ 08-2.6-70 & 0.87\end{array}$
DATE
$08-23-71$
$10-13-7$
$09-25-74$

$F T^{3} / S$

2.4

1.2

1.1

$\begin{array}{cc}\text { DATE } & F T^{3} / S \\ 08-14-75 & 2.3 \\ 11-04-75 & 6.1 \\ 08-11-76 & 1.1\end{array}$

$7 Q 10=*$ 
DES MOINES RIVER. BASIN--Continued

5-4807.60 PRAIRIE CR NR RENWICK, IOWA

LAT $4252 \times X$, LONG $9359 \times X$, IN NE $1 / 4$ SEC.

$23, T .93$ N., R.27 W., HUMBOLDT CO. (46),

AT BRILGE, 3 MILES NW OF RENWICK

DRAINAGE AREA $118 \quad \mathrm{MI}=$

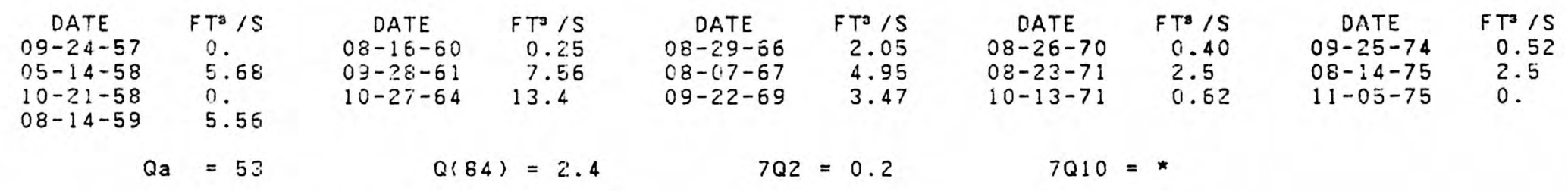

5-4808.00 OTTER CR. NR GOLDFIELD, IOWA

LA.T $4247 X X$, LONG $9353 \times X$, IN NE $1 / 4$ SEC.

15, T. 22 N., R.2E W. W WRIGHT CO. $199 ;$,

AT BRIDGE, 4 MILES NE OF GOLDFIELD.

DRAINAGE AREA $\quad 75.5 \mathrm{MI}^{2}$

\begin{tabular}{|c|c|c|c|c|c|c|}
\hline \multirow[t]{2}{*}{$\begin{array}{c}\text { DATE } \\
09-27-57 \\
05-14-58 \\
10-21-58 \\
08-14-59\end{array}$} & & $\begin{array}{l}\mathrm{FT}^{3} / \mathrm{S} \\
0.16 \\
2.15 \\
0.38 \\
0.48\end{array}$ & $\begin{array}{c}\text { DATE } \\
08-16-60 \\
09-28-61 \\
10-27-64 \\
08-29-66\end{array}$ & $\begin{array}{r}\mathrm{FT}^{3} / \mathrm{S} \\
0.12 \\
0.26 \\
5.24 \\
0.56\end{array}$ & $\begin{array}{c}\text { DATE } \\
08-07-67 \\
09-22-69 \\
08-27-70\end{array}$ & $\begin{array}{l}\mathrm{FT}^{3} / \mathrm{S} \\
2.37 \\
1.36 \\
0.51\end{array}$ \\
\hline & Qa & $=35$ & 0184 & 4) $=0.9$ & 7.22 & $=0.2$ \\
\hline 08.20 & BOON & $N E R \quad N R$ & GOLDFIELD, I & I OWA & & \\
\hline
\end{tabular}

DRAINAGE AREA $419 \quad \mathrm{MI}^{2}$

\begin{tabular}{|c|c|c|c|c|c|c|c|c|c|}
\hline $\begin{array}{c}\text { DATE } \\
03-27-57 \\
05-14-58 \\
10-21-58 \\
03-14-59\end{array}$ & $\begin{array}{c}F T^{3} / \mathrm{S} \\
0.33 \\
16.2 \\
0.50 \\
4.41\end{array}$ & $\begin{array}{c}\text { DATE } \\
03-1 \epsilon_{1}-60 \\
09-2 \varepsilon_{i}-61 \\
1 C-2 \varepsilon_{1}-64 \\
08-2 \varepsilon_{1}-66\end{array}$ & $\begin{array}{c}F T^{3} / \mathrm{S} \\
0.72 \\
18.3 \\
35.7 \\
6.812\end{array}$ & $\begin{array}{c}\text { DATE } \\
08-017-67 \\
09-22-69 \\
08-27-70\end{array}$ & $\begin{array}{l}F T^{3} / \mathrm{S} \\
17.1 \\
10.3 \\
2.54\end{array}$ & $\begin{array}{c}\text { DATE } \\
08-23-71 \\
10-13-71 \\
09-25-74\end{array}$ & $\begin{array}{l}F T^{\circ} / \mathrm{S} \\
11.0 \\
4.0 \\
1.9\end{array}$ & $\begin{array}{c}\text { DATE } \\
08-14-75 \\
11-05-75 \\
08-11-76\end{array}$ & $\begin{array}{l}\mathrm{FT}^{\mathrm{a}} / \mathrm{S} \\
9.4 \\
3.8 \\
3.3\end{array}$ \\
\hline Qa & $=186$ & $Q(84)$ & $=6.4$ & 702 & $=1.3$ & 7010 & $=0.2$ & & \\
\hline
\end{tabular}


DES MOINES RIVER. BASIN--Cont 1 nued

5-4808.60 EAGLE CR. NR EAGLE GROVE, IOWA

LAT $4242 \times X$, LONG $9349 \times X$, IN SE $1 / 4$ SEC.

8, T.91 N. . R.25 W., WRIGHT CO. (99)

AT BRIDGE, 5 MILES NE OF EAGLE GROVE.

DRAINAGE AREA $62.8 \quad \mathrm{MI}^{2}$

$\begin{array}{rr}\text { DATE } & F T^{3} / S \\ 09-27-57 & 0.85 \\ 05-14-58 & 3.06 \\ 10-21-58 & 0.60 \\ 08-14-59 & 1.01 \\ Q a & =29\end{array}$

$\begin{array}{rr}D A T E & F T^{3} / S \\ 08-16-60 & 0.53 \\ 09-28-61 & 0.46 \\ 10-26-64 & 4.74 \\ 08-29-66 & 0.80 \\ Q(84) & =1.4\end{array}$

$\begin{array}{cr}\text { DATE } & F T^{3} / \mathrm{S} \\ 08-017-67 & 1.74 \\ 09-22-69 & 2.27 \\ 08-27-70 & 1.28\end{array}$

DATE
$08-23-71$
$10-13-71$
$09-25-74$

$\mathrm{FT}^{3} / \mathrm{S}$

1.5
0.97
0.95

$09-25-74 \quad 0.95 \quad 08-11-76$

DATE

$08-14-75$

$11-05-75$

$09-25-74 \quad 0.95 \quad 08-11-76$

$\mathrm{FT}^{\mathrm{T}} / \mathrm{S}$

0.93

$7 Q 10=0.3$

5-4809.00 EAGLE CR NR WOOLSTOCK, IOWA

LAT $4234 \times X$, LONG $9351 \times X$, NEAR CENTER OF SEC.36, T.90 N., R.26 W., WRIGHT CO. (99), AT BRIDGE, 0.5 MILE WEST OF WOOLSTOCK.

DRAINAGE AREA $105 \quad \mathrm{MI}^{2}$

$\begin{array}{rrcrrr}\text { DATE } & \text { FT }^{3} / \mathrm{S} & \text { DATE } & \mathrm{FT}^{3} / \mathrm{S} & \text { DATE } & \mathrm{FT}^{3} / \mathrm{S} \\ 09-27-57 & 1.40 & 08-16-60 & 2.55 & 08-29-66 & 1.70 \\ 10-21-58 & 2.56 & 09-28-61 & 2.18 & 08-07-67 & 3.50 \\ 08-14-59 & 1.52 & 10-26-64 & 9.23 & 09-22-69 & 3.58 \\ & & & & \end{array}$

$\begin{array}{cc}\text { DATE } & \mathrm{FT}^{3} / \mathrm{S} \\ 08-27-70 & 2.39 \\ 08-22-71 & 3.9 \\ 10-14-71 & 2.0\end{array}$

DATE
$09-25-74$

2.1

$10-14-71 \quad 2.0$

$11-06-75$

2.1

$Q_{a}=48$

$Q(84)=2.9$

$7 Q 10=0.6 * * *$

5-4809.40 WHITE FOX CR NR WOOLSTOCK, JOWA

LAT $4236 \times x$, LONG $9345 \times X$, IN SW $1 / 4$ SEC.

13. T.90 N., R.25 W. WRIGHT CO (99:

AT BRIDGE, 5 MILES NE OF WOOLSTOCK.

DRAINAGE AREA $62.0 \mathrm{MI}^{2}$

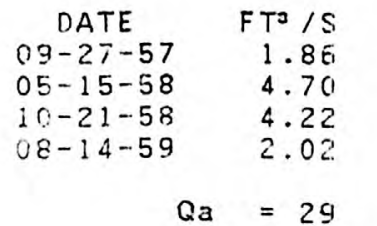

$Q_{a}=29$

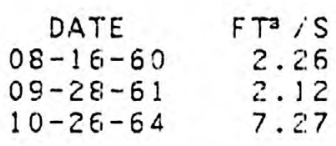

$Q(84)=3.5$

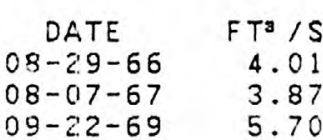

4.01
3.87

5.70

$7 Q 2=1.6$

$\begin{array}{cc}\text { DATE } & F T^{a} / S \\ 08-27-70 & 2.49 \\ 08-23-71 & 3.1 \\ 10-14-71 & 2.0\end{array}$

DATE
$09-25-74$
$11-06-75$
$08-10-76$

$F T^{3} / S$

2.3

2.1
1.4 
DES MOINES RIVER. BASIN--Cont inued

5-4809.80 WHITE FOX CR AT WEBSTER CI,Y, IOWA

LAT $4230 \times X$, LONG $9348 \times X$, IN NW $1 / 4$ SEC.

28 , T. 89 N., R.25 W., HAMILTON CO. $(40)$,

AT BRIDGE, 2 MILES NORTH OF WEBSTER C.ITY.

DRAINAGE AREA $111 \quad \mathrm{MI}^{2}$

\begin{tabular}{|c|c|c|c|c|c|c|c|c|c|}
\hline $\begin{array}{c}\text { DATE } \\
09-23-57 \\
05-15-58 \\
10-21-58 \\
08-14-59\end{array}$ & $\begin{array}{r}\mathrm{FT}^{0} / \mathrm{S} \\
2.39 \\
8.29 \\
5.07 \\
1.68\end{array}$ & $\begin{array}{c}\text { DATE } \\
08-1 E-60 \\
09-28-61 \\
10-26-64\end{array}$ & $\begin{array}{l}\mathrm{FT}^{3} / \mathrm{S} \\
2.79 \\
3.91 \\
9.31\end{array}$ & $\begin{array}{c}\text { DATE } \\
08-29-66 \\
08-017-67 \\
09-22-69\end{array}$ & $\begin{array}{c}\mathrm{FT}^{3} / \mathrm{S} \\
5.19 \\
4.73 \\
10.1\end{array}$ & $\begin{array}{c}\text { DATE } \\
08-27-70 \\
08-23-71 \\
10-14-71\end{array}$ & $\begin{array}{l}\mathrm{FT}^{8} / \mathrm{S} \\
2.27 \\
3.7 \\
2.7\end{array}$ & $\begin{array}{c}\text { DATE } \\
09-24-74 \\
11-0 E-75 \\
08-10-76\end{array}$ & $\begin{array}{l}\mathrm{FT}^{3} / \mathrm{S} \\
4.0 \\
2.9 \\
1.2\end{array}$ \\
\hline Qa & $=52$. & $Q(84)$ & $=4.8$ & $7 Q 2$ & $=1.8$ & $7 Q 10$ & $0.7 * * *$ & & \\
\hline
\end{tabular}

5-4817.00 BEAVER CR NR BEAVER. IOWA

LAT $4202 \times X$, LONG $9409 \times X$, NEAR S $1 / 4$

CORNER SEC.6, T.83 N., R.28 W., BOONE CO.

(08). A.T BRIDGE, 1 MILE SW OF BEAVER.

DRAINAGE AREA $84.5 \mathrm{MI}^{2}$

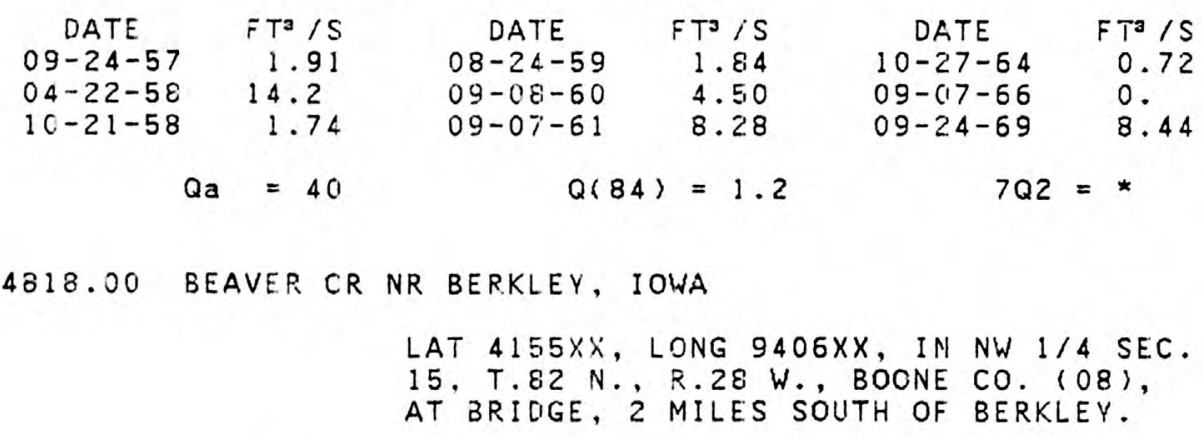

DRAINAGE AREA $175 \quad \mathrm{MI}^{2}$

$\begin{array}{rc}\text { DATE } & F T / S \\ 09-24-57 & 2.74 \\ 04-22-58 & 23.8 \\ 10-21-58 & 4.89 \\ 2 a & =83\end{array}$

$\begin{array}{cc}\text { DATE } & F T^{3} / S \\ 08-24-59 & 8.4 .6 \\ 09-08-60 & 15.5 \\ 09-07-61 & 14.0 \\ 0(84) & =2.4\end{array}$

$\begin{array}{cc}\text { DATE } & F T^{3} / S \\ 10-27-64 & 2.28 \\ 09-017-65 & 0.0 \\ 09-24-69 & 14.9 \\ 7 Q 2= & *\end{array}$
DATE
$07-14-70$
$08-24-71$
$09-23-74$
$\mathrm{FT}^{3}$ is
1.
$7 Q 10=0$

DATE

$09-30-75$
$08-10-76$

$\mathrm{T}^{3} / \mathrm{S}$

0.51

0.02

$2 a=83$

$Q(84)=2.4$

$Q_{2}=$

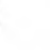


DES MOINES RIVER. BASIN--Cont inued

5-4819.00 BEAVER CR AT GRANGER, IOWA

$$
\begin{aligned}
& \text { LAT } 414539, \text { LONG } 935101 \text {, IN SW } 1 / 4 \text { SEC. } \\
& 2, \text { T.80 N., R.26 W. DALLAS CO. ( } 25), \\
& \text { AT BRIDGE, 1.5 MILES WEST OF GRANGER. }
\end{aligned}
$$

\begin{tabular}{|c|c|c|c|c|c|c|c|c|c|}
\hline $\begin{array}{c}\text { DATE } \\
09-26-57 \\
04-23-58 \\
10-21-58\end{array}$ & $\begin{array}{c}F^{3} / S \\
2.25 \\
41.0 \\
15.3\end{array}$ & $\begin{array}{c}\text { DATE } \\
09-08-60 \\
09-07-61 \\
10-26-64\end{array}$ & $\begin{array}{l}F T^{0} / S \\
35.6 \\
23.6 \\
9.34\end{array}$ & $\begin{array}{c}\text { DATE } \\
09-07-66 \\
09-24-69 \\
07-14-70\end{array}$ & $\begin{array}{l}F T^{9} / S \\
1.08 \\
22.1 \\
18.2\end{array}$ & $\begin{array}{c}\text { DATE } \\
08-24-71 \\
09-23-74\end{array}$ & $\begin{array}{c}\mathrm{FT}^{\mathrm{g}} / \mathrm{S} \\
0.61 \\
2.9\end{array}$ & $\begin{array}{c}\text { DATE } \\
09-30-75 \\
10-14-76\end{array}$ & $\begin{array}{c}\mathrm{FT}^{\mathrm{S}} / \mathrm{S} \\
2.1 \\
0.26\end{array}$ \\
\hline$Q$ & $=152$ & Q18 & $1=7.8$ & $7 Q$ & $=1.5$ & 7010 & * & & \\
\hline
\end{tabular}

DRAINAGE AREA $314 \quad \mathrm{MI}^{2}$

5-4821.00 N RACCOON R NR REMBRANDT, IOWA

LAT $4247 \times X$, LONG $9506 \times X$, IN NE $1 / 4$ SEC.

$21, T .92$ N., R. 36 W., BUENA VISTA CO. (11), AT BRIDGE, 5 MILES SE OF REMBRANDT.

DRAINAGE AREA $77.4 \quad \mathrm{MI}^{2}$

$\begin{array}{cccccc}\text { DATE } & \text { FT'/S } & \text { DATE } & F T^{3} / S & \text { DATE } & F^{3} / S \\ 0.9-25-57 & 0.84 & 07-29-59 & 2.91 & 10-17-61 & 3.88 \\ 04-15-58 & 26.4 & 10-04-60 & 7.94 & 10-28-64 & 4.70 \\ 10-21-58 & 0.71 & 10-09-61 & 4.11 & 09-13-66 & 0.63\end{array}$

$Q_{a}=28$

$Q(84)=3.3$

$7 Q 2=1.0$

$\begin{array}{cccr}\text { DATE } & \mathrm{FT}^{3} / \mathrm{S} & \text { DATE } & \mathrm{FT}^{3} / \mathrm{S} \\ 03-19-68 & 1.01 & 09-23-74 & 0.79 \\ 07-13-70 & 3.84 & 09-15-76 & 0.12 \\ 08-03-71 & 5.9 & & \\ 7010= & & & \end{array}$

5-4821.20 N RACCOON R NR TRUESDALE, IOWA

LAT $4242 \times X$, LONG $9505 \times X$, IN NE $1 / 4$ SEC.

15, T. 91 N., R. 36 W., BUENA VISTA CO. (11),

AT BRIDGE, 6 MILES SE OF TRUESDALE.

DRAINAGE AREA $164 \quad \mathrm{MI}^{2}$

$\begin{array}{rc}\text { DATE } & F T^{3} / S \\ 09-25-57 & 2.37 \\ 04-15-58 & 43.5 \\ 10-21-58 & 0.90 \\ Q a & =59\end{array}$

$$
\begin{array}{cc}
\text { DATE } & F T^{3} / S \\
07-29-59 & 4.09 \\
10-04-60 & 15.1 \\
10-09-61 & 4.914
\end{array}
$$$$
\begin{array}{cr}
\text { DATE } & F T^{3} / S \\
10-17-61 & 7.39 \\
10-28-64 & 9.05 \\
09-13-66 & 3.74
\end{array}
$$

DATE

03-19-68

$07-13-70$

$08-03-71$

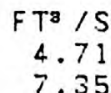

4.71
7.35

11.0

$7 Q 2=1.7$

$7010=0.5$

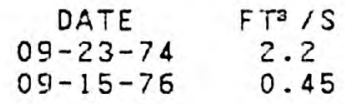


DES MOINES RIVER. BASIN--Cont inued

5-4821.80 L CEDAR CR NR FONDA, IOWA

AT $4237 \times X$, LONG $9451 \times X$, IN NW $1 / 4$ SEC.

15, T.90 N., R.34 W., POCAHONTAS CO. (76),

AT BRIDGE, 2 MILES NORTH OF FONDA.

DRAINAGE AREA $83.5 \mathrm{MI}^{2}$

$\begin{array}{cccccr}\text { DATE } & F T^{3} / S & \text { DATE } & F T \text { IS } & \text { DATE } & F T / S \\ 09-25-57 & 1.46 & 05-12-59 & 53.2 & 10-09-61 & 1.75 \\ 04-15-58 & 19.7 & 07-29-59 & 1.05 & 10-17-61 & 3.24 \\ 10-21-58 & 0.19 & 10-04-60 & 2.39 & 10-28-64 & 2.92 \\ & & & & \end{array}$

$\begin{array}{cr}\text { DATE } & F T \text { T } \\ 09-13-66 & 0.55 \\ 03-19-68 & 0.98 \\ 07-13-70 & 3.43\end{array}$

DATE
$08-04-71$
$09-23-74$
$09-15-76$

$\mathrm{FT}^{\mathrm{T}} / \mathrm{S}$

$a=31$

$Q(84)=1.7$

$7 Q 10=0.1$

5-4822.00 B CEDAR CR AT FONDA, IOWA

LAT $4235 \times X$, LONG $9451 \times X$, IN SW $1 / 4$ SEC.

22, T.90 N., R. 34 W. POCAHONTAS CO. ( 76 ),

AT BRIUGE ON STATE HIGHWAY 7, AT NORTH CITY

LIMITS OF FONDA.

URAINAGE AREA $196 \quad \mathrm{MI}^{2}$

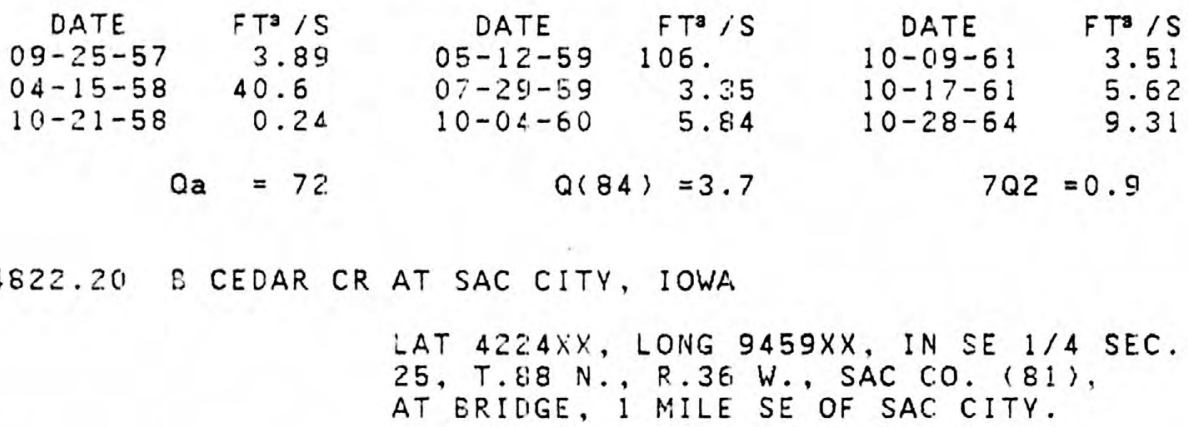

DRAINAGE AREA $342 \quad \mathrm{MI}^{2}$

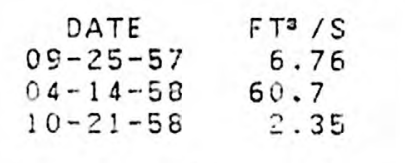

Qa

$\begin{array}{cc}\text { DATE } & \text { FT } / S \\ 07-30-59 & 8.43 \\ 10-03-60 & 10.2 \\ 10-17-61 & 16.9\end{array}$

$Q(84)=8.8$

$\begin{array}{cc}\text { DATE } & F T^{2} / S \\ 10-2.8-64 & 13.3 \\ 09-13-66 & 3.13 \\ 03-19-68 & 10.6\end{array}$

$7 Q 2=4.1$

$\begin{array}{cr}\text { DATE } & \mathrm{FT}^{3} / \mathrm{S} \\ 09-13-66 & 0.78 \\ 03-19-68 & 3.63 \\ 07-13-70 & 8.90\end{array}$

DATE

$08-24-71$
$09-23-74$

$09-23-74$
$09-15-76$

$\mathrm{FT}^{3} / \mathrm{S}$ 7.4

0.69

$7010=0.1$ 
DES MOINES RIVER. BASIN--Cont inued

5-4823.20 INDIAN CR NR LAKE VIEW, IOWA

LAT $4220 \times X$, LONG $9500 \times X$, IN NW $1 / 4$ SEC.

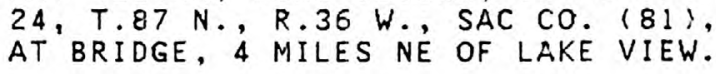

DRAINAGE AREA $90.2 \mathrm{MI}^{2}$

\begin{tabular}{|c|c|c|c|c|c|c|c|c|c|}
\hline $\begin{array}{c}\text { DATE } \\
\text { O9-25-57 } \\
04-16-58 \\
10-21-58\end{array}$ & $\begin{array}{c}\mathrm{FT}^{3} / \mathrm{S} \\
1.69 \\
10.9 \\
1.16\end{array}$ & $\begin{array}{c}\text { DATE } \\
07-30-59 \\
10-03-60 \\
10-17-61\end{array}$ & $\begin{array}{c}\mathrm{FT}^{3} / \mathrm{S} \\
4.41 \\
4.37 \\
18.7\end{array}$ & $\begin{array}{c}\text { DATE } \\
10-28-64 \\
09-13-66 \\
03-19-68\end{array}$ & $\begin{array}{r}\mathrm{FT}^{\mathrm{a}} / \mathrm{S} \\
2.18 \\
1.82 \\
5.65\end{array}$ & $\begin{array}{c}\text { DATE } \\
07-13-70 \\
08-03-71\end{array}$ & $\begin{array}{c}\mathrm{FT}^{2} / \mathrm{S} \\
4.28 \\
1.2\end{array}$ & $\begin{array}{c}\text { DATE } \\
09-23-74 \\
09-15-76\end{array}$ & $\begin{array}{l}F T \cdot 1 \mathrm{~S} \\
4.1 \\
1.2\end{array}$ \\
\hline$Q_{a}$ & $=33$ & 0,8 & $=3.6$ & 7Q2 & $=1.9$ & $7 Q 10$ & $=0.7$ & & \\
\hline
\end{tabular}

5-4823.60 CAMP CR NR LYTTON, IOWA

LAT $4223 \times X$. LONG $9450 \times X$, IN NW $1 / 4$ SEC.

5 , T.8T N., R. 34 W., CALHOUN CO. (13:,

AT BRIDGE, 3 MILES SE OF LYTTON.

DRAINAGE AREA $62.0 \quad \mathrm{MI}^{2}$

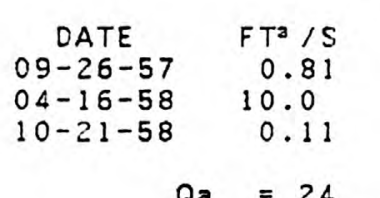

$Q_{a}=24$

$\begin{array}{cr}\text { DATE } & F T 3 / S \\ 07-30-59 & 2.015 \\ 10-03-60 & 3.17 \\ 10-18-61 & 15.8\end{array}$

$10-18-61 \quad 15.8$

$Q(84)=1.5$

$\begin{array}{cr}\text { DATE } & F T^{2} / S \\ 10-28-64 & 0.05 \\ 09-13-66 & 0.69 \\ 03-19-68 & 0.94\end{array}$

$7 Q 2=0.4$

\section{DATE
$07-13-70$ \\ $08-03-71$ \\ $F^{3}$
2.05 \\ 0.22}

$7010=*$

5-4823.30 CAMP CR NR LAKE CITY, IOWA

LAT $4217 \times X$, LONG $9450 \times X$, IN NW $1 / 4$ SEC.

5, T.86 N., R. 34 W. CALHOUN CO. $\{13\}$.

AT BRIDGE, 5 MILES NW OF LAKE CITV.

DRAINAGE AREA $147 \quad \mathrm{MI}^{2}$

\begin{tabular}{|c|c|c|c|c|c|c|c|c|c|}
\hline $\begin{array}{c}\text { DATE } \\
09-2 E-57 \\
04-17-58 \\
10-21-58\end{array}$ & $\begin{array}{c}\mathrm{FT}^{3} / \mathrm{S} \\
1.29 \\
22.7 \\
0.19\end{array}$ & $\begin{array}{c}\text { DATE } \\
07-30-59 \\
10-04-60 \\
10-18-61\end{array}$ & $\begin{array}{r}\mathrm{FT}^{3} / \mathrm{S} \\
3.58 \\
4.62 \\
45.6\end{array}$ & $\begin{array}{c}\text { DATE } \\
10-27-64 \\
09-13-66 \\
03-19-68\end{array}$ & $\begin{array}{r}\mathrm{FT}^{3} / \mathrm{S} \\
0.18 \\
1.63 \\
3.34\end{array}$ & $\begin{array}{c}\text { DATE } \\
07-13-70 \\
08-03-71 \\
09-23-74\end{array}$ & $\begin{array}{l}\mathrm{FT}^{3} / \mathrm{S} \\
7.34 \\
1.2 \\
0.81\end{array}$ & $\begin{array}{c}\text { DATE } \\
11-07-75 \\
09-15-76\end{array}$ & $\begin{array}{r}\mathrm{FT}^{3} / \mathrm{S} \\
0.49 \\
0.01\end{array}$ \\
\hline$Q$ & $=57$ & Qr & $=2.2$ & $7 Q 2$ & $=0.4$ & 7010 & * & & \\
\hline
\end{tabular}


DES MOINES RIVER. BASIN--Cont inued

5-4824.00 N RACCOON R NR LAKE CITY, IOWA

LAT $4216 X X$, LONG 9450XX, NEAR E $1 / 4$

CORNER SEC.17, T.86 N., R.34 W., CALHOUN CO.

(13), AT BRIDGE ON STATE HIGHWAY 175, 4 MILES

WEST OF LAKE CITY.

DRAINAGE AREA $1003 \quad \mathrm{MI}^{2}$

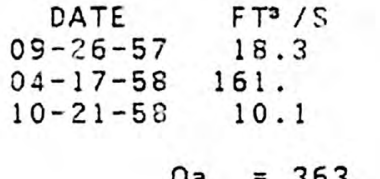

$Q_{\mathbf{a}}=363$

$\begin{array}{cc}\text { DATE } & \text { FTO/S } \\ 07-30-59 & 32.3 \\ 10-04-60 & 52.1 \\ 10-18-61 & 116 .\end{array}$

$Q(84)=37$

$\begin{array}{rl}\text { DATE } & F T / S \\ 10-28-64 & 38.3 \\ 09-13-66 & 20.2 \\ 03-19-68 & 50.1 \\ 7 Q 2 & =18\end{array}$

$07-13-7$

$07-13-70$

$08-03-71$

$09-23-74$

$\mathrm{FT}^{3} / \mathrm{S}$

51.7

57.0

$7 Q 10=5.3$

5-4824.10 LAKE CR NR ROCKWELL CITY, IOWA

LAT $4224 \times X$, LONG $9436 \times X$, IN SW $1 / 4$ SEC. 29, T.88 N., R.32 W., CALHOUN CO. (13), AT BRIDGE ON U. S. HIGHWAY 20, 1 MILE EAST OF ROCKWELL CITY.

DRAINAGE AREA $71.5 \mathrm{MI}^{2}$

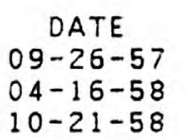

$\mathrm{FT}^{3} / \mathrm{S}$

0.96

13.4

DATE
$07-29-59$

$F T^{2}$ is

$10-04-60$

$10-18-61$

9.34

$Q(84)=0.8$

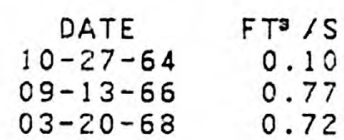

$7 Q 2=0.2$

$$
Q \mathbf{a}=30
$$

DATE

$11-07-75$ $09-15-76$

$F^{3} / S^{2}$

28 .

6.8

LAT $4216 \times X$, LCNG $9447 X X$, IN SW $1 / 4$ SEC.

$14, T .86$ N., R.34. W. CALHOUN CO. (13)

AT BRIDGE, 3 MILES WEST OF LAKE CITY.

DRAINAGE AREA $128 \quad \mathrm{MI}^{2}$

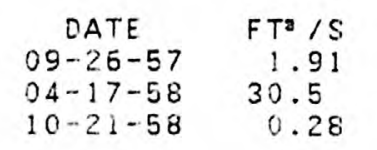

$Q_{a}=52$

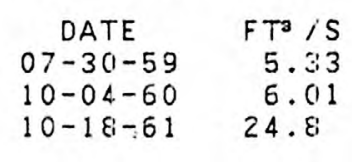

$Q(84)=1.1$

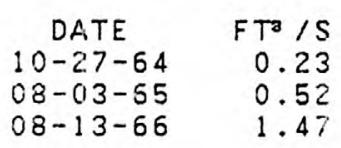

$7 Q 2=0.3$

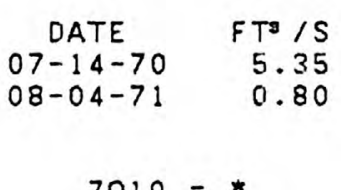

$7010=*$
DATE

$09-15-76$

$F^{3} / S$
0.57

0 .

$\begin{array}{cc}\text { DATE } & \text { FT } / S \\ 03-20-68 & 2.10 \\ 07-14-70 & 7.97 \\ 08-02-71 & 1.7 \\ 7010=*\end{array}$

DATE
$09-23-74$

$09-23-74$
$11-07-75$

$09-15-76$

FT: /S

1.1

0. 
DES MOINES RIVER. BASIN--COntinued

5-4824.40 PURGATORY CR NR LANESBORO, IOWA

LAT $4210 \times X$, LONG $9438 \times X$, IN NE $1 / 4$ SEC.
24, T.85 N., R. 33 W., CARROLL CO. (14), 24, T. 85 N. , R. 33 W. , CARROLL CO. (14),
AT BRIDGE, 3 MILES SE OF LANESBORO.

DRAINAGE AREA $65.0 \mathrm{MI}^{2}$

\begin{tabular}{|c|c|c|c|c|c|c|c|c|c|}
\hline $\begin{array}{c}\text { DATE } \\
09-23-57 \\
04-17-58 \\
10-22-58\end{array}$ & $\begin{array}{c}\mathrm{FT}^{3} / \mathrm{S} \\
1.83 \\
12.7 \\
0.63\end{array}$ & $\begin{array}{c}\text { DATE } \\
07-29-59 \\
10-05-60 \\
10-18-61\end{array}$ & $\begin{array}{l}\mathrm{FT}^{3} / \mathrm{S} \\
1.93 \\
2.56 \\
16.2\end{array}$ & $\begin{array}{c}\text { DATE } \\
10-27-64 \\
09-14-66 \\
03-20-68\end{array}$ & $\begin{array}{l}\mathrm{FT}^{\mathrm{B}} / \mathrm{S} \\
0.50 \\
0.10 \\
1.99\end{array}$ & $\begin{array}{c}\text { DATE } \\
07-14-70 \\
08-02-71 \\
09-25-74\end{array}$ & $\begin{array}{l}\mathrm{FT}^{\mathrm{T}} / \mathrm{S} \\
4.20 \\
0.44 \\
1.1\end{array}$ & $\begin{array}{c}\text { DATE } \\
11-07-75 \\
09-15-76\end{array}$ & $\begin{array}{r}F T^{2} / \mathrm{S} \\
0.85 \\
0.06\end{array}$ \\
\hline & $=28$ & Q 1 & $=0.9$ & $7 Q 2$ & $=0.2$ & $7 Q 10$ & * & & \\
\hline
\end{tabular}

5-4824.60 E CEDAR CR NR SOMERS, IOWA

LAT 422207, LONG 942703, IN NW $1 / 4$ SEC. $10, T .87$ N., R.31 W., CALHOUN CO. (13), AT BRIDGE, I MILE SW'OF SOMERS.

DRAINAGE AREA $62.4 \mathrm{MI}^{2}$

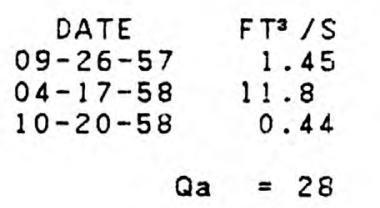
$\begin{array}{cr}\text { DATE } & \mathrm{FT}^{3} / \mathrm{S} \\ 07-23-59 & 2.77\end{array}$
$10-04-60 \quad 3.56$
DATE
$10-27-64$
$F T^{3} / S$
$10-27-64$
$09-13-66$
0.31

$10-18-61$

9.70

$Q(84)=1.3$

$7 Q 2=0.2$
DATE
$08-04-71$
$F T^{3} / S$
4.66
$7010=$

DATE

$09-15-7$

$F T^{3} / S$

0.19

5-4824.80 CEDAR CR NR CHURDAN, IOWA

LAT $4208 \times X$, LONG $9435 \times X$, NEAR S $1 / 4$

CORNER SEC.28, T.85 N., R.32 W., GREENE

CO. $(3 \%)$. AT BRIDGE, 5 MILES SW OF CHURDAN.

DRAINAGE AREA $151 \quad \mathrm{MI}^{2}$

\begin{tabular}{|c|c|c|c|c|c|c|c|c|c|}
\hline $\begin{array}{c}\text { DATE } \\
09-23-57 \\
04-17-58 \\
10-22-58\end{array}$ & $\begin{array}{c}\mathrm{FT}^{3} / \mathrm{S} \\
2.64 \\
25.2 \\
0.75\end{array}$ & $\begin{array}{c}\text { DATE } \\
07-29-59 \\
10-05-60 \\
10-18-61\end{array}$ & $\begin{array}{c}\mathrm{FT}^{3} / \mathrm{S} \\
4.35 \\
10.8 \\
31.1\end{array}$ & $\begin{array}{c}\text { DATE } \\
10-27-64 \\
09-14-66 \\
03-20-68\end{array}$ & $\begin{array}{l}\mathrm{FT}^{3} / \mathrm{S} \\
0.11 \\
0.1 \\
4.49\end{array}$ & $\begin{array}{c}\text { DATE } \\
07-14-70 \\
08-02-71\end{array}$ & $\begin{array}{l}F T^{3} / \mathrm{S} \\
9.98 \\
0.71\end{array}$ & $\begin{array}{c}\text { DATE } \\
09-25-74 \\
09-15-76\end{array}$ & $\begin{array}{l}F T^{3} / S \\
0 . \\
0 .\end{array}$ \\
\hline$Q$ & $=65$ & QR & $=2.2$ & $7 Q 2$ & * & $7 Q 10$ & 0 & & \\
\hline
\end{tabular}


DES MOINES RIVEF. BASIN--Continued

5-4827.00 HARDIN CR NR CHURDAN, IOWA

LAT $4210 \times X$, LONG $9426 \times X$, IN SW $1 / 4$ SEC.

$14, T .85$ N., R. 31 W., GREENE CO. $\{37\}$,

AT BRIDGE, 2 MILES EAST OF CHURDAN.

DRAINAGE AREA $74.0 \quad \mathrm{MI}^{2}$

$\begin{array}{rc}D A T E & F T^{3} / S \\ 09-24-57 & 0.85 \\ 04-17-58 & 13.5 \\ 10-20-58 & 1.04 \\ Q \mathbf{a} & =33\end{array}$

$\begin{array}{rrrr}\text { DATE } & F T^{3} / S & \text { DATE } & F T^{3} / S \\ 07-29-59 & 1.57 & 10-27-64 & 0.01 \\ 10-05-60 & 6.21 & 09-14-66 & 0.06 \\ 10-18-61 & 33.0 & 03-20-68 & 0.72 \\ Q(84)=0.6 & 7 Q 2=0.1\end{array}$

DATE
$07-14-70 \quad 3.56$
$08-02-71 \quad 0.43$
$7010=$

HARDIN CR NR JEFFERSON, IOWA

LAT $4201 \times X$, LONG $9420 \times X$, IN NW $1 / 4$ SEC.

10, T.83 N., R. 30 W., GREENE CO. (37):

AT BRIDGE, 2 MILES EAST OF JEFFERSON.

DRAINAGE AREA $161 \quad \mathrm{MI}^{2}$

$\begin{array}{cccccr}\text { DATE } & F T^{3} / S & \text { DATE } & F T^{3} / S & \text { DATE } & F^{S} / S \\ 0 S-24-57 & 2.44 & 07-28 i-59 & 6.617 & 10-27-64 & 1.31 \\ 04-17-58 & 31.7 & 10-04-60 & 12.1 & 09-14-66 & 1.13 \\ 10-20-58 & 2.26 & 10-18-61 & 72.5 & 03-20-68 & 4.18\end{array}$

DATE
$07-14-70$
$08-02-71$

5-4831.00 W BUTTRICK CR NR FARNHAMVILLE, IOWA

LAT $4213 \times X$, LONG 9422XX. IN NW $1 / 4$ SEC.

4$, T. 85 N., R. 30 W., GREENE CO. ( 37$)$,

DRAINAGE AREA $80.1 \mathrm{MI}^{2}$

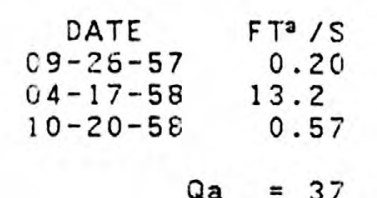

$Q_{\mathbf{a}}=37$

$$
\begin{array}{cr}
\text { DATE } & F^{3} / S \\
07-29-59 & 0.85 \\
10-05-60 & 2.22 \\
10-18-51 & 37.8
\end{array}
$$$$
Q(84)=0.9
$$

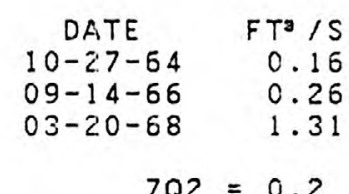

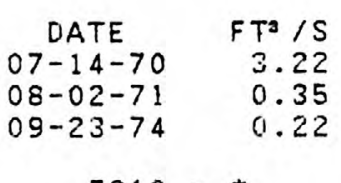

$7010=\star *$

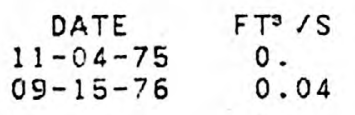


DES MOINES RIVER. BASIN--Cont inued

$5-4831.50$ E BUTTRICK CR NR GRAND JUNCTION, IOWA

LAT $4204 X X$, LONG $9416 \times X$, IN NE $1 / 4$ SEC.

$30, T .84 \mathrm{~N} .$, R.29 W., GREENE CO. $(37$ )

AT BRIDGE, 2.5 MILES NW OF GRAND JUNCTION.

DRAINAGE AREA $79.6 \mathrm{MI}^{2}$

$\begin{array}{cccccr}\text { DATE } & \mathrm{FT}^{3} / \mathrm{S} & \text { DATE } & \mathrm{FT}^{3} / \mathrm{S} & \text { DATE } & F T^{\mathbf{3}} / \mathrm{S} \\ 09-24-57 & 1.76 & 07-28-59 & 1.69 & 10-27-64 & 0.48 \\ 04-17-58 & 20.7 & 10-04-60 & 2.78 & 09-14-66 & 0.69 \\ 10-20-58 & 1.20 & 10-18-61 & 32.1 & 03-20-68 & 1.55\end{array}$

$\begin{array}{cr}\text { DATE } & \text { FT. } 1 S \\ 07-14-70 & 2.18 \\ 08-02-71 & 0.25\end{array}$

DATE

$09-23-74$
$09-15-76$

$F T^{3} / \mathrm{S}$

$09-15-76 \quad 0.53$

$\mathrm{Qa}=37$

$Q(84)=0.9$

$7 Q 2=0.2$

5-4832.00 BUTTRICK CR NR GRAND JUNCTION, IOWA

LAT $4202 \times X$, LONG $9417 \times X$, AT $S 1 / 4$

CORNER SEC.36. T.84 N., R. $30 \mathrm{~W}$., GREENE CO.

(37), AT BRIDGE, 2.5 MILES WEST OF GRAND

JUNCTION.

DRAINAGE AREA $202 \quad \mathrm{MI}^{2}$
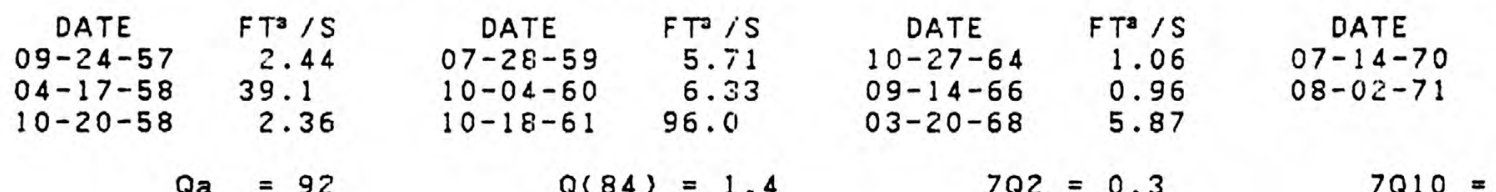

$\mathrm{FT}^{3} / \mathrm{S}$
6.80

DATE
$09-23-74$

$\mathrm{FT} / \mathrm{S}$

$08-02-71 \quad 0.45$

$09-15-76$

0.86

$Q_{a}=92$

$Q(84)=1.4$

$7 Q 2=0.3$

$7010=*$

5-4832.50 GREEN BRIER CR NR JAMAICA, IOWA

LAT $4151 X X$, LONG $9417 \times X$, NEAR CENTER OF

SEC. 1 , T.81 N., R. 30 W., GUTHRIE CO. (39), AT BRIDGE, 1.5 MILES NE OF JAMAICA.

DRAINAGE AREA $65.8 \mathrm{MI}^{2}$

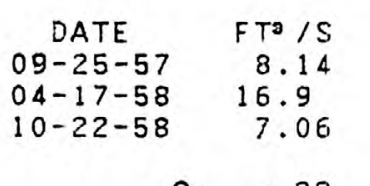

$Q_{\mathbf{a}}=32$

$\begin{array}{cc}\text { DATE } & \mathrm{FT}^{3} / \mathrm{S} \\ 10-26-64 & 2.38 \\ 09-13-66 & 0 . \\ 03-19-68 & 1.83\end{array}$

$7 Q 2=0.2$

\begin{tabular}{|c|c|}
\hline $\begin{array}{c}\text { DATE } \\
07-14-70 \\
08-02-71\end{array}$ & $\begin{array}{c}\mathrm{FT}^{3} / \mathrm{S} \\
3.99 \\
3.0\end{array}$ \\
\hline $7 Q 10$ & 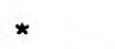 \\
\hline
\end{tabular}

DATE

$09-23-74 \quad 0.36$

$09-15-76 \quad 0$. 
DES MOINES RIVER. BASIN--Cont inued

5-4833.00 N RACCOON R NR PERRY, IOWA

LAT $4150 \times X$, LONG $9408 \times X$, NEAR CENTER OF

SEC. $8, T .81$ N., R. 28 W., DALLAS CO. (25),

AT BRIDGE ON STATE HIGHWAY 141, I MILE WEST

OF PERRY.

DRAINAGE AREA $2169 \quad \mathrm{MI}^{2}$

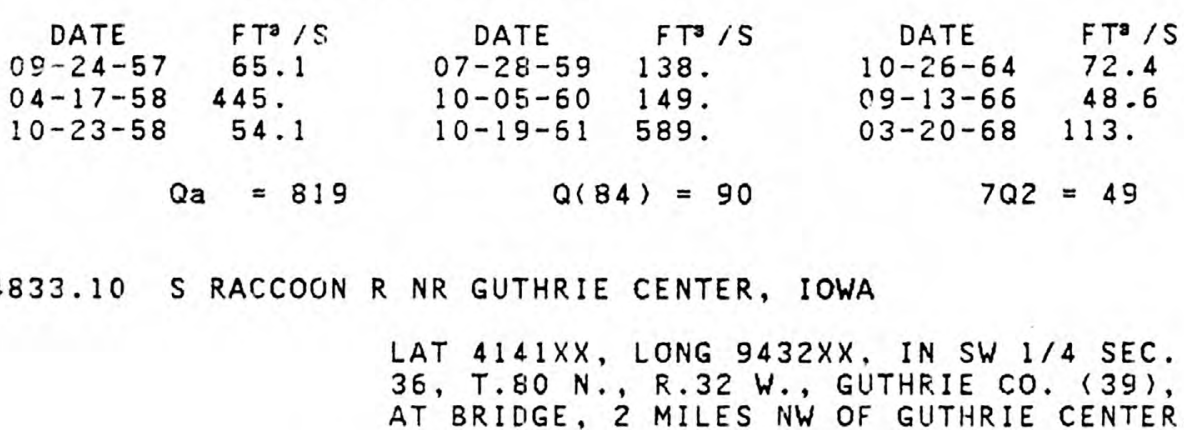

DRAINAGE AREA $77.2 \quad \mathrm{MI}^{2}$

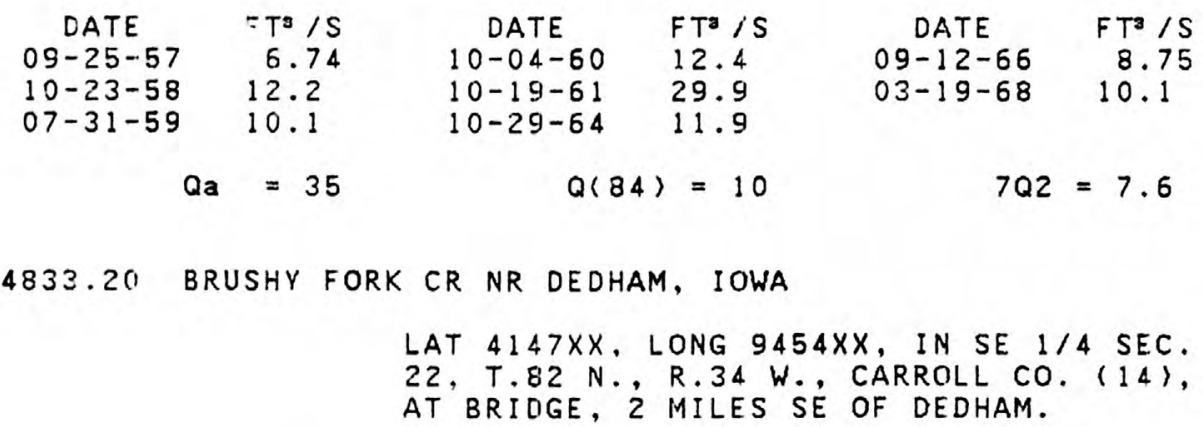

DRAINAGE AREA $68.1 \mathrm{MI}^{2}$

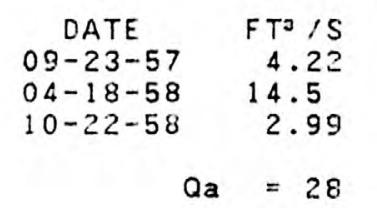
$\begin{array}{rr}\text { DATE } & F T^{3} / S \\ 07-30-59 & 7.92\end{array}$
$10-04-60 \quad 8.02$
16.9
$Q(84)=5.7$
DATE $\quad F T^{3} / S$
$10-28-64$
$09-12-65$

$Q_{a}=28$

$7 Q 2=3.6$

\begin{tabular}{cl} 
DATE & \multicolumn{1}{c}{$\mathrm{FT}^{2} / \mathrm{S}$} \\
$07-14-70$ & 165. \\
$08-02-71$ & 132.0 \\
& \\
&
\end{tabular}

DATE

$09-23-74$
$09-14-76$

$\mathrm{FT} / \mathrm{S}$

89.

$7 Q 10=9.0 * * \star$

$\begin{array}{cccl}\text { DATE } & \text { FT } / S & \text { DATE } & F T^{3} / S \\ 07-13-70 & 8.90 & 09-24-74 & 16 . \\ 08-03-71 & 11.0 & 09-14-76 & 13 .\end{array}$

$7010=4.4 * * *$

$\begin{array}{cc}\text { DATE } & F T^{3} / \mathrm{S} \\ 07-14-70 & 8.66 \\ 08-02-71 & 5.4 \\ 09-25-74 & 12 .\end{array}$

$7010=1.8 * * \star$ 
DES MOINES RIVER. BASIN--Cont inued

5-4833.30 BRUSHY FORK CR NR GUTHRIE CENTER, IOWA

LAT $4139 \times X$, LONG $9427 \times X$, NEAR CENTER OF

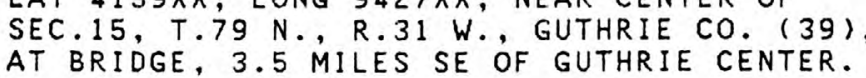

DRAINAGE AREA $142 \quad \mathrm{MI}^{2}$

\begin{tabular}{|c|c|c|c|c|c|c|c|c|c|}
\hline $\begin{array}{c}\text { DATE } \\
09-25-57 \\
10-23-58 \\
07-31-59\end{array}$ & $\begin{array}{l}\mathrm{FT}^{3} / \mathrm{S} \\
12.5 \\
16.7 \\
21.7\end{array}$ & $\begin{array}{c}\text { DATE } \\
10-03-60 \\
10-18-61 \\
10-29-64\end{array}$ & $\begin{array}{l}F T / S \\
21.7 \\
44.4 \\
17.4\end{array}$ & $\begin{array}{c}\text { DATE } \\
09-12-66 \\
03-19-68\end{array}$ & $\begin{array}{l}\mathrm{FT}^{3} / \mathrm{S} \\
17.0 \\
19.3\end{array}$ & $\begin{array}{c}\text { DATE } \\
07-13-70 \\
08-03-71\end{array}$ & $\begin{array}{l}F T / S \\
18.4 \\
21.0\end{array}$ & $\begin{array}{c}\text { DATE } \\
09-24-74 \\
09-14-76\end{array}$ & $\begin{array}{l}\mathrm{FT}^{3} / \mathrm{S} \\
34 . \\
19 .\end{array}$ \\
\hline$Q$ & $=62$ & $Q<\varepsilon$ & $y=19$ & 702 & $=15$ & 7010 & $9.6 * \star \star *$ & & \\
\hline
\end{tabular}

5-4833.40 S RACCOON R NR MONTEITH, IOWA

LAT $4138 \times X$, LONG $9425 \times X$, IN SE $1 / 4$ SEC. 23, T. 79 N., R. 31 W. GUTHRIE CO. ( 39 )
AT BRIDGE, O. 5 MILE EAST OF MONTEITH.

DRAINAGE AREA $267 \quad M=$

\begin{tabular}{|c|c|c|c|c|c|c|c|c|c|}
\hline $\begin{array}{c}\text { DATE } \\
09-25-57 \\
10-23-58 \\
07-31-59\end{array}$ & $\begin{array}{l}F T^{3} / \mathrm{S} \\
23.2 \\
32.9 \\
36.0\end{array}$ & $\begin{array}{c}\text { DATE } \\
10-03-60 \\
10-18-61 \\
10-29-64\end{array}$ & $\begin{array}{l}F T^{3} / S \\
47.4 \\
80.1 \\
39.7\end{array}$ & $\begin{array}{c}\text { DATE } \\
09-12-66 \\
03-19-68\end{array}$ & $\begin{array}{l}F T^{3} / \mathrm{S} \\
29.5 \\
32.7\end{array}$ & $\begin{array}{c}\text { DATE } \\
07-13-70 \\
08-03-71\end{array}$ & $\begin{array}{l}\mathrm{FT}^{3} / \mathrm{S} \\
30.1 \\
41.0\end{array}$ & $\begin{array}{c}\text { DATE } \\
09-24-74 \\
09-14-76\end{array}$ & $\begin{array}{l}\mathrm{FT}^{3} / \mathrm{S} \\
57 . \\
36 .\end{array}$ \\
\hline Qa & $=117$ & $Q<8$ & $1=30$ & $7 Q 2$ & $=22$ & $7 Q 10$ & $16 * * *$ & & \\
\hline
\end{tabular}

5-4833.50 M RACCOON R NR CARROLL, IOWA

LAT $4203 \times X$, LONG $9449 \times X$, IN SE 1/4 SEC. 29 , T. 14 N., R. 34 W. CARROLL CO. (14), AT BRIDGE, 2 MILES SE OF CARROLL.

DRAINAGE AREA $74.3 \mathrm{MI}^{2}$

\begin{tabular}{|c|c|c|c|c|c|c|c|c|c|}
\hline $\begin{array}{c}\text { DATE } \\
\text { O9-23-57 } \\
04-17-58 \\
10-22-58\end{array}$ & $\begin{array}{l}\mathrm{FT}^{\mathrm{O}} / \mathrm{S} \\
3.45 \\
15.2 \\
2.01\end{array}$ & $\begin{array}{c}\text { DATE } \\
07-30-59 \\
10-04-60 \\
10-19-61\end{array}$ & $\begin{array}{l}\mathrm{FT}^{3} / \mathrm{S} \\
7.69 \\
5.00 \\
14.8\end{array}$ & $\begin{array}{c}\text { DATE } \\
10-28-64 \\
09-12-66 \\
03-19-68\end{array}$ & $\begin{array}{r}\mathrm{FT}^{\mathrm{a}} / \mathrm{S} \\
4.18 \\
2.60 \\
7.18\end{array}$ & $\begin{array}{c}\text { DATE } \\
07-14-70 \\
08-02-71 \\
09-25-74\end{array}$ & $\begin{array}{c}F^{3} / \mathrm{S} \\
7.58 \\
3.1 \\
10 .\end{array}$ & $\begin{array}{c}\text { DATE } \\
11-07-75 \\
09-14-76\end{array}$ & $\begin{array}{c}\mathrm{FT}^{3} / \mathrm{S} \\
6.2 \\
3.3\end{array}$ \\
\hline$Q$ & $=28$ & $Q<\varepsilon$ & $=4.0$ & $7 Q 2$ & $=2.5$ & $7 Q 10$ & $1.2 * * *$ & & \\
\hline
\end{tabular}


DES MOINES RIVER. BASIN--Cont inued

5-4833.60 M RACCOON R NR GLIDDEN, IOWA

LAT $4203 \times X$, LONG $9446 \times X$, NEAR CENTER OF SEC. 35, T.84 N., R. 34 W., CARROLL CO. (14), AT BRIDGE, 2.5 MILES SW OF GLIDDEN.

DRAINAGE AREA $138 \quad \mathrm{MI}^{2}$

$\begin{array}{rc}\text { DATE } & F T^{3} / S \\ 09-23-57 & 4.51 \\ 04-17-58 & 30.1 \\ 10-22-58 & 3.29 \\ Q a & =53\end{array}$

$\begin{array}{cc}\text { DATE } & F T / S \\ 07-30-59 & 8.07 \\ 10-04-60 & 4.23 \\ 10-19-61 & 36.2 \\ Q(84)=4.8\end{array}$

$\begin{array}{rr}\text { DATE } & F^{2} / 5 \\ 10-28-64 & 4.80 \\ 09-12-66 & 4.41 \\ 03-19-68 & 9.74 \\ 7 Q 2= & 3.0\end{array}$

$\begin{array}{cc}\text { DATE } & F T^{2} / S \\ 07-14-70 & 9.39 \\ 08-02-71 & 4.0 \\ 09-25-74 & 9.8\end{array}$

$F T^{3} / S$

$6 \cdot \frac{1}{3}$

$Q(84)=4.8$

$7010=1.2 * * *$

5-4833.80 WILLOW CR NR SCRANTON, IOWA

LAT $4154 \times X$, LONG $9435 \times X$, IN SW $1 / 4$ SEC.

$21, T .82$ N., R.32 W., GREENE CO. (37).

AT BRIDGE, 9 MILES SW OF SCRANTON.

DRAINAGE AREA $51.8 \quad \mathrm{MI}^{2}$

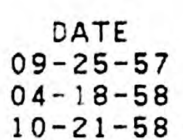

$\begin{array}{cr}\text { DATE } & F T^{2} / S \\ 07-31-59 & 3.32 \\ 10-04-60 & 2.55 \\ 10-19-61 & 22.2\end{array}$
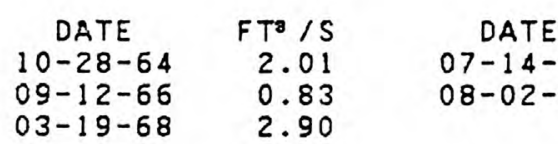

$07-14-70$

FT $/ \mathrm{S}$

3.83
0.83

DATE

$09-25-74$

$F T^{3} / S$

$03-19-68$

$7 Q 2=0.8$

$7010=0.1 * * *$

5-4834.00 WILLOW CR NR BAYARD. IOWA

LAT $4149 \times X$, LONG $9433 \times X$, IN SE $1 / 4$ SEC.

15, T.81 N., R.32 W., GUTHRIE CO. (39),

AT BRIDGE, 2 MILES SOUTH OF BAYARD.

DRAINAGE AREA $112 \quad \mathrm{MI}^{2}$

$\begin{array}{cccc}\text { DATE } & \text { FT /S } & \text { DATE } & F T / S \\ 09-25-57 & 7.20 & 10-04-60 & 10.3 \\ 04-18-58 & 26.5 & 10-19-61 & 47.1 \\ 10-22-58 & 9.34 & 10-28-64 & 6.96\end{array}$

$\begin{array}{cr}\text { DATE } & F T^{2} / S \\ 09-12-66 & 2.73\end{array}$

$T^{2} / S$
2.73
8.16

DATE
$07-14-70$

$F T^{3} / S$

$03-19-68$

$08-02-71$

2.6

DATE

$09-24-74$

$09-14-76$

$F T=/ S$

6.3

$Q \mathbf{a}=49$

$Q(84)=5.1$

$7 Q 2=2.8$

$7 Q 10=0.7 * * \star$ 
DISCHARGE MEASUREMENTS AT

DES MOINES RIVER. BASIN--Continued

5-4834.50 M RACCOON R NR BAYARD, IOWA

LAT $4147 X X$, LONG $9430 X X$, IN SE $1 / 4$ SEC.

31 , T.81 N., R.31 W., GUTHRIE CO. (39),

AT BRIDGE ON STATE HIGHWAY 25,6 MILES'SE OF BA.YARD.

\begin{tabular}{|c|c|c|c|c|c|c|c|c|c|}
\hline & DRAINAGE & AREA & $M I^{2}$ & & & & & & \\
\hline $\begin{array}{c}\text { DATE } \\
09-25-57 \\
04-18-58 \\
10-22-58\end{array}$ & $\begin{array}{l}\mathrm{FT}^{3} / \mathrm{S} \\
22.6 \\
85.5 \\
23.7\end{array}$ & $\begin{array}{c}\text { DATE } \\
07-31-59 \\
10-04-60 \\
10-19-61\end{array}$ & $\begin{array}{l}F T^{3} / S \\
28.9 \\
33.2 \\
128 .\end{array}$ & $\begin{array}{c}\text { DATE } \\
10-28-64 \\
09-12-66 \\
03-19-68\end{array}$ & $\begin{array}{l}F T^{2} / \mathrm{S} \\
28.6 \\
22.5 \\
33.5\end{array}$ & $\begin{array}{c}\text { DATE } \\
07-13-70 \\
08-02-71 \\
09-22-71\end{array}$ & $\begin{array}{l}F T^{3} / \mathrm{S} \\
30.7 \\
22.0 \\
10.0\end{array}$ & $\begin{array}{c}\text { DATE } \\
09-24-74 \\
09-14-76\end{array}$ & $\begin{array}{l}\mathrm{FT}^{3} / \mathrm{S} \\
42 . \\
21 .\end{array}$ \\
\hline$Q \mathbf{a}$ & $=152$ & $Q<8$ & 4) $=28$ & $7 Q 2$ & $=18$ & 7010 & $6.1 * * x$ & & \\
\hline
\end{tabular}

5-4836.20 MOSQUITO CR NR LINDEN, IOWA

LAT $4143 \times X$, LONG $9415 \times X$, NEAR S $1 / 4$

CORNER SEC.20, T.80 N., R. 29 W., DALLAS CO. (25),

AT BRIDGE, 5 MILES NE OF LINDEN.

DRAINAGE AREA $67.4 \mathrm{MI}^{2}$

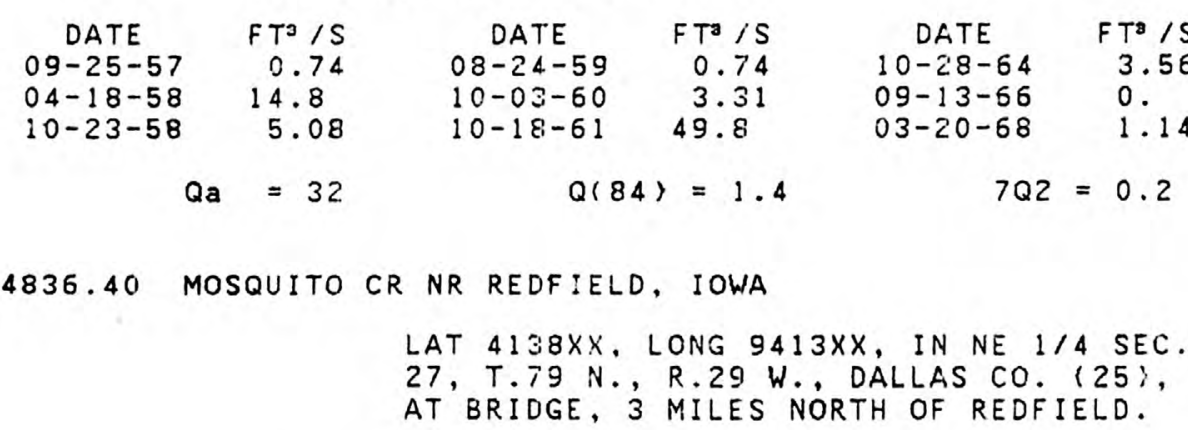

DRAINAGE AREA $110 \quad \mathrm{MI}^{2}$

\begin{tabular}{|c|c|c|c|c|c|c|c|c|c|}
\hline $\begin{array}{c}\text { DATE } \\
09-26-57 \\
04-18-58 \\
10-23-58\end{array}$ & $\begin{array}{l}F T^{3} / \mathrm{S} \\
0.53 \\
23.0 \\
10.3\end{array}$ & $\begin{array}{c}\text { DATE } \\
08-25-59 \\
10-03-60 \\
10-18-61\end{array}$ & $\begin{array}{c}\mathrm{FT}^{3} / \mathrm{S} \\
8.16 \\
12 . \mathrm{E}_{1} \\
77.8\end{array}$ & $\begin{array}{c}\text { DATE } \\
10-29-64 \\
09-13-66 \\
03-20-68\end{array}$ & $\begin{array}{l}F T / S \\
5.86 \\
0 . \\
2.10\end{array}$ & $\begin{array}{c}\text { DATE } \\
07-13-70 \\
08-03-71 \\
09-24-74\end{array}$ & $\begin{array}{l}\mathrm{FT}^{3} / \mathrm{S} \\
3.40 \\
2.5 \\
0.53\end{array}$ & $\begin{array}{c}\text { DATE } \\
11-07-75 \\
09-14-76\end{array}$ & $\begin{array}{l}\mathrm{FT}^{\mathrm{T}} / \mathrm{S} \\
5.6 \\
0 .\end{array}$ \\
\hline Qa & $=52$ & $Q<$ & $=2.2$ & $7 Q^{2}$ & $=0.4$ & 7210 & $\star$ & & \\
\hline
\end{tabular}


DES MGINES RIVEF. BASIN--Continued

5-4836.60 M RACCOON R AT REDFIELD, IOWA

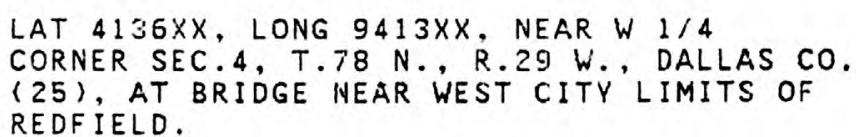

\begin{tabular}{|c|c|c|c|c|c|c|c|c|c|}
\hline $\begin{array}{c}\text { DATE } \\
09-26-57 \\
04-18-58 \\
10-23-58\end{array}$ & $\begin{array}{l}F T^{3} / \mathrm{S} \\
28.5 \\
123 . \\
47.8\end{array}$ & $\begin{array}{c}\text { DATE } \\
08-25_{1}-59 \\
10-03-60 \\
10-18-61\end{array}$ & $\begin{array}{l}F^{3} \text { is } \\
43.0 \\
55.0 \\
263 .\end{array}$ & $\begin{array}{c}\text { DATE } \\
10-2.9-64 \\
09-13-66 \\
03-20-68\end{array}$ & $\begin{array}{l}F T^{3} / S \\
45.0 \\
27.2 \\
35.5\end{array}$ & $\begin{array}{c}\text { DATE } \\
07-13-70 \\
08-03-71 \\
09-24-74\end{array}$ & $\begin{array}{l}5 T^{3} / \mathrm{S} \\
38.1 \\
30.0 \\
48 .\end{array}$ & $\begin{array}{c}\text { DATE } \\
11-07-75 \\
09-14-76\end{array}$ & $\begin{array}{l}\mathrm{FT}^{3} / \mathrm{S} \\
68 . \\
32 .\end{array}$ \\
\hline & $=257$ & Q( & 1) $=42$ & 702 & $=29$ & $7 Q 10$ & 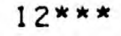 & & \\
\hline
\end{tabular}

5-4842.00 PANTHER CR NR ACEL, IOWA

LAT $4136 \times X$, LONG $9406 \times X$, NEAR N $1 / 4$

CORNER SEC. 5, T.78 N., R. 28 W., DALLAS CO. (25;,

AT BRILIGE, 4 MILES SW OF ADIEL.

ORAINAGE AREA $56.0 \mathrm{MI}^{2}$

\begin{tabular}{|c|c|c|c|c|c|c|c|c|c|}
\hline $\begin{array}{c}\text { DATE } \\
09-26-57 \\
04-18-58 \\
10-24-58\end{array}$ & $\begin{array}{l}F T^{3} / \mathrm{S} \\
0 . \\
10.0 \\
5.87\end{array}$ & $\begin{array}{c}\text { DATE } \\
08-25-59 \\
10-03-60 \\
10-18-61\end{array}$ & $\begin{array}{l}F T \cdot \text { iS } \\
2.55 \\
12.6 \\
62.1\end{array}$ & $\begin{array}{c}\text { DATE } \\
10-29-64 \\
09-13-56 \\
03-20-68\end{array}$ & $\begin{array}{l}F T^{3} / S \\
5.10 \\
0.47 \\
1.05\end{array}$ & $\begin{array}{c}\text { DATE } \\
07-13-70 \\
08-03-71 \\
09-24-74\end{array}$ & $\begin{array}{l}F T / S \\
1.85 \\
8.3 \\
1.1\end{array}$ & $\begin{array}{c}\text { DATE } \\
11-07-75 \\
09-14-76\end{array}$ & $\begin{array}{c}F T / S \\
4.8 \\
0.51\end{array}$ \\
\hline & $=29$ & $Q<\varepsilon$ & $=1.7$ & 702 & $=0.2$ & $7 Q 10$ & 0 & & \\
\hline
\end{tabular}

5-4856.00 FOLRMILE CR NP. ANKENY. IOWA

LAT 414354 , LONG 933421 , NEAR S $1 / 4$ CORNER SEC.18, T.80 N.. R.23 W., POLK CO. 177 , AT BRIUGE, 1.5 MILES EAST OF ANKENY.

DRAINAGE AREA $59.3 \mathrm{MI}^{2}$

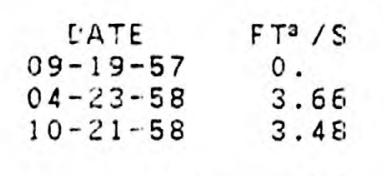

$Q_{\mathbf{a}}=31$

$\begin{array}{cc}\text { DATE } & F^{3} / S \\ 08-24-59 & 6.39 \\ 09-0 E-60 & 2.34 \\ 09-0 E_{1}-61 & 0.617 \\ 0<84 ?= & 0.2\end{array}$

$a\langle 84\}=0.2$

$\begin{array}{cr}D A_{1} T E & F T^{3} / S \\ 10-2.6-64 & 0.77 \\ 09-07-66 & 0.02 \\ 09-2.4-59 & 1.43 \\ 702= & \star\end{array}$

$70,2=*$

$$
\begin{gathered}
\text { DATE } \\
07-14-70 \\
08-23-71
\end{gathered}
$$
$\mathrm{FT}^{3} / \mathrm{S}$
2.86
0.

$7010=0$ 
DES MOINES RIVER BASIN--Cont I nued

5-4857.00 NORTH R NR EARLHAM, IOWA

LAT $4124 K X$, LONG $9411 X X$, IN NE $1 / 4$ SEC.
9, T.76 N., R. 29 W. , MADISON CO. (61),

$9, T .76 \mathrm{~N}, 1$ R. 29 W., MADISON CO.
AT BRIDGE, 7 MILES SW OF EARLHAM.

DRAINAGE AREA $68.9 \mathrm{MI}^{2}$

\begin{tabular}{|c|c|c|c|c|c|c|c|c|c|}
\hline $\begin{array}{c}\text { DATE } \\
09-26-57 \\
03-28-58 \\
10-27-58\end{array}$ & $\begin{array}{l}\mathrm{FT}^{3} / \mathrm{S} \\
0 . \\
10.7 \\
8.11\end{array}$ & $\begin{array}{c}\text { DATE } \\
10-23-59 \\
09-13-60 \\
10-10-61\end{array}$ & $\begin{array}{r}\mathrm{FT}^{3} / \mathrm{S} \\
5.33 \\
4.19 \\
12.9\end{array}$ & $\begin{array}{c}\text { DATE } \\
10-17-61 \\
10-29-63 \\
10-12-64\end{array}$ & $\begin{array}{l}\mathrm{FT}^{3} / \mathrm{S} \\
23.0 \\
0 . \\
17.1\end{array}$ & $\begin{array}{c}\text { DATE } \\
08-29-66 \\
03-12-68\end{array}$ & $\begin{array}{r}\mathrm{FT}^{3} / \mathrm{S} \\
0.40 \\
1.09\end{array}$ & $\begin{array}{c}\text { DATE } \\
08-24-71 \\
09-24-74\end{array}$ & $\begin{array}{l}F T^{3} / \mathrm{S} \\
0.67 \\
0.56\end{array}$ \\
\hline Qa & $=35$ & $Q<\varepsilon$ & $=0.7$ & 702 & $=0.1$ & $7 Q 10$ & 0 & & \\
\hline
\end{tabular}

5-4858.50 NB NORTH R NR WINTERSET, IOWA

LAT $4126 \times X$, LONG $9356 \times X$, IN NE $1 / 4$ SEC. $34, T .77 \mathrm{~N} .$, R.27 W., MADISON CO. (61), AT BRIUIGE, 7 MILES NE OF WINTERSET.

DRAINAGE AREA $74.7 \quad \mathrm{MI}^{2}$

$\begin{array}{rr}\text { DATE } & F T / S \\ 09-26-57 & 0 \\ 03-27-58 & 6.80 \\ 10-28-58 & 4.88 \\ Q a & =38\end{array}$

$\begin{array}{cc}\text { DATE } & F T^{3} / S \\ 10-23-59 & 3.78 \\ 09-13-60 & 5.88 \\ 10-10-61 & 14.0 \\ Q(84) & =0.8\end{array}$

$\begin{array}{rl}\text { DATE } & F T / S \\ 10-17-61 & 39.9 \\ 10-29-63 & 0.9 \\ 10-12-64 & 7.53 \\ 7 Q Z= & 0.1\end{array}$
DATE $F T^{3} / S$
$08-29-66 \quad 0.43$
$03-13-68 \quad 3.44$
$07-29-70 \quad 0.31$

DATE

$09-24-74 \quad 0.77$

$08-12-75 \quad 2.6$

5-4859.00 NORTH R NR WINTERSET, IOWA

LAT $4126 \times X$, LONG $9355 \times X$, IN NW $1 / 4$ SEC.
$36, T .77$. R. R. 27 W. MADISON CO. (6I),

AT BRIUGE, 8 MILES NE OF WINTERSET.

DRAINAGE AREA $203 \quad \mathrm{MI}^{2}$

$\begin{array}{cc}\text { DATE } & F T / S \\ 09-26-57 & 0 . \\ 03-27-58 & 21.6 \\ 10-28-58 & 12.3\end{array}$

$\begin{array}{cl}\text { DATE } & F T^{3} / S \\ 10-23-59 & 10.9 \\ 09-13-60 & 13.0 \\ 10-10-61 & 37.5\end{array}$

$\begin{array}{cc}\text { DATE } & F T^{3} / S \\ 10-29-63 & 0 . \\ 10-12-64 & 29.0 \\ 08-29-66 & 1.57\end{array}$

DATE
$03-13-68$
$07-29-70$
$08-24-71$

$F T^{3} / S$

20.1

1.60

$7 Q 10=0$
$702=0.4$

$Q_{a}=102$

\begin{abstract}
$Q(84)=2.6$
\end{abstract}


DES MOINES RIVEF: BASIN--Cont inued

5-4861.00 MIDDLE R. NR CASEY, IOWA

LAT $4130 \times X$, LONG $9429 \times X$, IN SW $1 / 4$ SEC.

$36, T .78$ N., R.32 W., GUTHRIE CO. (39),

AT BRIDGE, 1.5 MILES EAST OF CASEY.

DRAINAGE AREA $72.8 \mathrm{MI}^{2}$

\begin{tabular}{|c|c|c|c|c|c|c|c|c|c|}
\hline $\begin{array}{c}\text { DATE } \\
09-26-57 \\
03-27-58 \\
10-27-58\end{array}$ & $\begin{array}{c}F T / S \\
1.94 \\
29.9 \\
16.9\end{array}$ & $\begin{array}{c}\text { DATE } \\
10-23-59 \\
09-13-60 \\
10-10-61\end{array}$ & $\begin{array}{c}\mathrm{FT}^{\mathrm{a}} / \mathrm{S} \\
2.6,2 \\
3.63 \\
26.0\end{array}$ & $\begin{array}{c}\text { DATE } \\
10-18-61 \\
10-2.9-63 \\
10-12-64\end{array}$ & $\begin{array}{c}F T^{3} / \mathrm{S} \\
18.0 \\
0.94 \\
13.7\end{array}$ & $\begin{array}{c}\text { DATE } \\
08-29-66 \\
03-13-68 \\
07-28-70\end{array}$ & $\begin{array}{r}\mathrm{FT}^{3} / \mathrm{S} \\
0.76 \\
1.11 \\
1.75\end{array}$ & $\begin{array}{c}\text { DATE } \\
08-24-71 \\
09-24-74 \\
08-12-75\end{array}$ & $\begin{array}{l}\mathrm{FT}^{3} / \mathrm{S} \\
1.1 \\
1.8 \\
4.7\end{array}$ \\
\hline & $=35$ & QC & $=2.5$ & 702 & $=0.7$ & $7 Q 10$ & * & & \\
\hline
\end{tabular}

5-4861.50 MIDDLE F. AT MIDDLE RIVER, IOWA

LAT $4120 X X$, LONG $9414 X X$, NEAR CENTER OF SEC. $6, T .75$ N., R. 29 W., MADISON CO. ( 61 ), AT ERIOGE NEAR SOUTH CITY LIMITS OF MIDDLE RIVER.

DRAINAGE AREA $164 \quad \mathrm{MI}^{2}$

$\begin{array}{rr}\text { DATE } & F T^{3} / S \\ 09-20-57 & 1.81 \\ 03-26-58 & 54.2 \\ 10-28-58 & 30.4 \\ & =80\end{array}$

$\begin{array}{cc}\text { DATE } & F T^{3} / S \\ 10-23-59 & 21.8 \\ 09-13-60 & 8.17 \\ 10-10-61 & 37.6\end{array}$

$\begin{array}{cc}\text { DATE } & F T^{3} / \mathrm{S} \\ 10-17-61 & 55.9 \\ 10-29-63 & 1.67 \\ 10-12-64 & 48.0\end{array}$

$\begin{array}{cc}\text { DATE } & F T^{3} / S \\ 08-29-66 & 3.14 \\ 03-12-68 & 18.0 \\ 07-28-70 & 8.55\end{array}$

5-4863.00 CLANTON CR AT EAST PERU, IOWA

LAT $4114 \times X$, LONG $9355 \times X$, IN NE $1 / 4$ SEC.

AT BRIDGE NEAR EAST CITY LIMITS OF EAST PERU.

DRAINAGE AREA $84.5 \quad \mathrm{MI}^{2}$

\begin{tabular}{|c|c|c|c|c|c|c|c|c|c|}
\hline $\begin{array}{c}\text { DATE } \\
03-26-57 \\
10-28-58 \\
10-22-59\end{array}$ & $\begin{array}{l}F T 0 / S \\
0.01 \\
0.30 \\
6.38\end{array}$ & $\begin{array}{c}\text { DATE } \\
O S-13-60 \\
10-05-61 \\
10-17-61\end{array}$ & $\begin{array}{l}F T^{3} / S \\
0.2 .4 \\
10.1 \\
40.6\end{array}$ & $\begin{array}{c}\text { DATE } \\
10-2.9-63 \\
10-12-64 \\
08-2.9-60\end{array}$ & $\begin{array}{l}F T^{3} / S \\
0 . \\
14.3 \\
0.16\end{array}$ & $\begin{array}{c}\text { DATE } \\
03-12-68 \\
07-09-70 \\
08-23-71\end{array}$ & $\begin{array}{r}F T^{2} / S \\
2.17 \\
0.02 \\
0.02\end{array}$ & $\begin{array}{c}\text { DATE } \\
09-24-74 \\
08-12-75\end{array}$ & $\begin{array}{l}\mathrm{FT}^{3} / \mathrm{S} \\
0.01 \\
0.46\end{array}$ \\
\hline 0 & $=45$ & $Q C$ & $=*$ & 70 & $=0$ & 7010 & 0 & & \\
\hline
\end{tabular}


DES MOINES RIVER. BASIN--Cont inued

5-4863.50 CLANTON CR NR MARTENSDALE, IOWA

LAT $4121 \times X$, LONG $9345 \times X$, IN NE $1 / 4$ SEC.

32 , T. 76 N., R. 25 W., WARREN CO. $(91$ ),

AT BRIDGE, 2 MILES SW OF MARTENSDALE.

DRAINAGE AREA $159 \quad \mathrm{MI}^{2}$

\begin{tabular}{|c|c|c|c|c|c|c|c|c|c|}
\hline $\begin{array}{c}\text { DATE } \\
09-25-57 \\
03-27-58 \\
10-28-58\end{array}$ & $\begin{array}{l}F T=/ S \\
0.24 \\
3.52 \\
0.55\end{array}$ & $\begin{array}{c}\text { DATE } \\
10-23-59 \\
09-14-60 \\
10-10-61\end{array}$ & $\begin{array}{l}\mathrm{FT}^{3} / \mathrm{S} \\
5.66 \\
2.13 \\
17.6\end{array}$ & $\begin{array}{c}\text { DATE } \\
10-17-61 \\
10-2.9-63 \\
10-13-64\end{array}$ & $\begin{array}{c}\mathrm{FT}^{3} / \mathrm{S} \\
72.0 \\
0.40 \\
20.0\end{array}$ & $\begin{array}{c}\text { DATE } \\
08-29-66 \\
03-12-68 \\
07-28-70\end{array}$ & $\begin{array}{r}\mathrm{FT}^{3} / \mathrm{S} \\
1.48 \\
3.79 \\
1.62\end{array}$ & $\begin{array}{c}\text { DATE } \\
08-24-71 \\
09-23-74 \\
08-13-75\end{array}$ & $\begin{array}{l}\mathrm{FT}^{3} / \mathrm{S} \\
0.99 \\
1.0 \\
2.9\end{array}$ \\
\hline & $=84$ & $Q<8$ & $=2.1$ & $7 Q 2$ & $=0.6$ & 7010 & * & & \\
\hline
\end{tabular}

5-4864.00 MIDDLE R AT MARTENSDALE, IOWA

LAT $4122 \times X$, LONG $9344 \times X$. IN SE $1 / 4$ SEC.

21 , T.76 N., R.25 W., WARREN CO. (91),

AT BRIDGE ON STATE HIGHWAY $92,0.5$ MILE SE OF

MARTENSDALE

ORAINAGE AREA $451 \quad \mathrm{MI}^{2}$

$\begin{array}{cccc}\text { DATE } & \text { FT: } / S & \text { DATE } & F T / S \\ 09-25-57 & 2.50 & 10-23-59 & 17.0 \\ 03-27-58 & 64.2 & 09-14-60 & 17.5 \\ 10-28-58 & 39.2 & 10-10-61 & 74.1\end{array}$

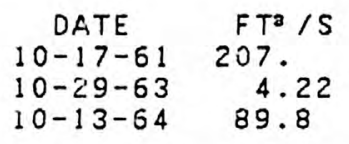

$Q(84)=14$

$7 Q 2=5.4$

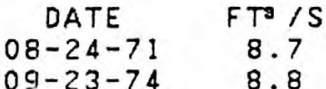

$09-23-74 \quad 8$.

$08-13-75 \quad 23.0$

5-4867.00 SOUTH R NR NEW VIRGINIA, IOWA

LAT $4113 \times X$, LONG $9344 \times X$, IN NE $1 / 4$ SEC

16, T.74 N., R.25 W., WARREN CO. ( 91 )

AT BRIUGE, 2.5 MILES NORTH OF NEW VIRGINIA.

DRAINAGE AREA $65.4 \mathrm{MI}^{2}$

$\begin{array}{cl}\text { DATE } & F T^{3} / 5 \\ 09-25-57 & 0 . \\ 03-26-58 & 0.53 \\ 10-28-58 & 0 . \\ & =35\end{array}$

$\begin{array}{cr}\text { DATE } & F T^{3} / S \\ 10-22-59 & 5.912 \\ 09-13-60 & 0.014 \\ 10-09-61 & 6.47\end{array}$

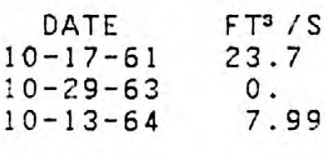
DATE
$08-29-6$
$03-14-6$

$F T^{3} / S$

0.02

0.89

DATE

$09-23-74$

$08-13-75$

$T^{3} / S$

$\mathrm{a} \mathbf{a}=3 \bar{E}_{\mathbf{1}}$

$Q(84)=$ *

$7 Q 2=0$

$7 Q 10=0$ 
DES MOINES RIVER. BASIN--Cont inued

5-4859.00 SQUAW CR. NR JAMISON, IOWA

LAT $4108 \times X$. LONG $9344 \times X$, IN NE $1 / 4$ SEC.

16. T.73 N., R.25 W., CLARKE CO. $\{20\}$,

AT BRIDGE, 0.5 MILE NW OF JAMISON.

DRAINAGE AREA $60.8 \quad \mathrm{MI}^{2}$

\begin{tabular}{|c|c|c|c|c|c|}
\hline $\begin{array}{c}\text { DATE } \\
09-25-57 \\
03-26-58 \\
10-28-58\end{array}$ & $\begin{array}{l}F T^{-/ S} \\
0 . \\
0.81 \\
0 .\end{array}$ & $\begin{array}{c}\text { DATE } \\
10-22-59 \\
09-13-60 \\
10-09-61\end{array}$ & $\begin{array}{l}\mathrm{FT}^{3} / \mathrm{S} \\
4.16 \\
0.02 \\
2.89\end{array}$ & $\begin{array}{c}\text { DATE } \\
10-17-61 \\
10-29-63 \\
10-13-64\end{array}$ & $\begin{array}{l}F T^{2} / S \\
9.88 \\
0 . \\
4.18\end{array}$ \\
\hline & 34 & & $=$ & $7 Q 2$ & $=0$ \\
\hline
\end{tabular}

$\begin{array}{rl}\text { DATE } & F T^{3} / S \\ 08-29-56 & 0.07 \\ 03-14-68 & 0.98 \\ 07-29-70 & 0 . \\ 7 Q 10= & 0\end{array}$

DATE

$08-23-71$
$09-23-74$

$08-13-75$

$\mathrm{FT}^{3} / \mathrm{S}$

0 .

0.00

Qa $=34$

$Q(84)=*$

$7 Q 2=0$

$Q 10=0$

5-4871.00 SQUAW CR. NR INDIANOLA, IOWA

LAT $4118 \times X$, LONG $9336 \times X$, IN NE $1 / 4$ SEC.

$15, T .75$ N.. R.24 W., WARREN CO. (31),

AT BRIDGE, 4 MILES SW OF INDIANOLA.

DRAINAGE AREA $134 \quad \mathrm{MI}^{2}$

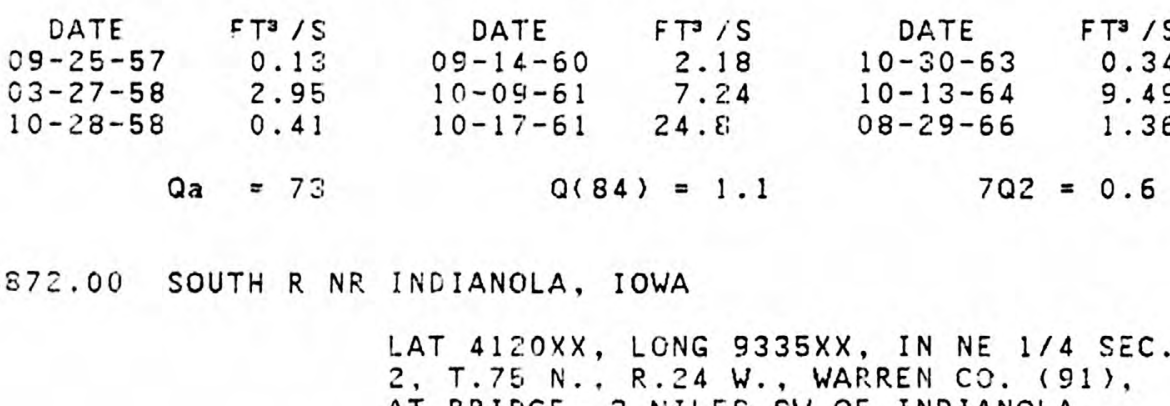

AT BRIN.. R.24 W. WARREN CO. ( 91 ).

DRAINAGE AREA $278 \quad \mathrm{MI}^{2}$

$\begin{array}{cccccc}\text { DATE } & \text { FTO/S } & \text { DATE } & \text { FT } \text { iS } & \text { DATE } & F^{3} / S \\ 09-25-57 & 0.32 & 09-14-60 & 5.4 .7 & 10-30-63 & 1.27 \\ 03-27-58 & 3.92 & 10-09-61 & 24.5 & 10-13-64 & 14.6 \\ 10-28-58 & 1.06 & 10-17-61 & 75.0 & 08-29-66 & 3.41\end{array}$

$\begin{array}{ll}\text { DATE } & F T^{3} / S \\ 03-14-68 & 6.25 \\ 08-24-71 & 2.4\end{array}$

DATE
$09-23-74$

$09-23-74$
$08-13-75$

$\mathrm{FT}^{3} / \mathrm{S}$

O3-13-

$F^{3} / \mathrm{S}$
2.68

DATE

$09-23-74$
$08-13-75$

$\mathrm{FT}^{3} / \mathrm{S}$

$08-24-71$

0.40

$8-13-75$

1

$7010=0.2$

$Q \mathbf{a}=1: 9$

$Q(84)=4.0$

$7 Q 2=1.6$

$7010=0.5$ 
DES MOINES RIVER BASIN--Cont inued

5-4874.00 OTTER CR NR NORWOOD, IOWA

LAT $4109 \times X$, LONG $9332 \times X$. IN SW $1 / 4$ SEC.

$5, T .73 \mathrm{~N} .$, R.23 W., LUCAS CO. (59),

AT BRIDGE, 3 MILES NW OF NORWOOD.

DRAINAGE AREA $102 \quad \mathrm{MI}^{2}$

\begin{tabular}{|c|c|c|c|c|c|}
\hline $\begin{array}{c}\text { DATE } \\
09-19-57 \\
03-25-58 \\
10-29-58\end{array}$ & $\begin{array}{l}\mathrm{FT}^{\mathrm{T}} / \mathrm{S} \\
0 . \\
4.07 \\
0 .\end{array}$ & $\begin{array}{c}\text { DATE } \\
10-22-59 \\
09-12-60 \\
10-09-61\end{array}$ & $\begin{array}{l}F T \text { iS } \\
10.21 \\
0.818 \\
6.61\end{array}$ & $\begin{array}{c}\text { DATE } \\
10-17-61 \\
10-30-63 \\
10-13-64\end{array}$ & $\begin{array}{l}\mathrm{FT}^{3} / \mathrm{S} \\
20.6 \\
0.0 \\
4.77\end{array}$ \\
\hline$Q$ & $=57$ & $Q<8$ & $=\star$ & $7 Q 2$ & 0 \\
\hline
\end{tabular}

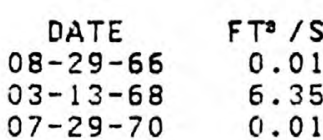

$7 Q 10=0$

5-4874.50 OTTER CR NR MILO, IOWA

LAT 411702, LONG 932909, IN NE $1 / 4$ SEC. $22, T .75 \mathrm{~N} ., \mathrm{R} .23 \mathrm{~W}$.. WARREN CO. ( 91 ), AT ERIDGE ON STATE HIGHWAY 205, 2 MILES WEST OF MILO.

DRAINAGE AREA $155 \quad \mathrm{MI}^{2}$

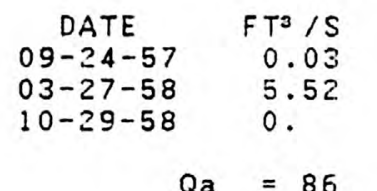

$$
\begin{array}{cc}
\text { DATE } & F T^{3} / S \\
09-14-60 & 2.48 \\
10-09-61 & 7.87 \\
10-17-61 & 31.4 \\
Q(84)=0.7
\end{array}
$$

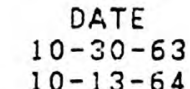

$10-30-63$
$10-13-64$

F 19

$Q a=86$

$7 Q 2=0.3$

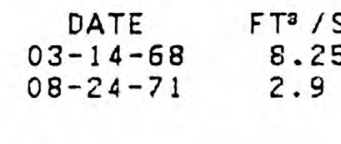

$7010=*$

5-4877.00 WHITE BREAST CR NR. WOODBURN, IOWA

LAT 405836, LONG 933514 , IN SE $1 / 4$ SEC $2, T .71 \mathrm{~N} ., 2 \mathrm{R} .24 \mathrm{~W}$. CLARKE CO. $(20)$,
AT BRIDGE, 2 MILES SOUTH OF WOODBURN.

\begin{abstract}
DRAINAGE AREA $82.9 \mathrm{MI}^{2}$
\end{abstract}

$\begin{array}{rr}\text { DATE } & F T=1 S \\ 03-26-58 & 2.96 \\ 10-29-58 & 0 . \\ 10-22-59 & 7.84 \\ Q a & =46\end{array}$

$\begin{array}{cc}\text { DATE } & F T^{3} / S \\ 09-12-60 & 0.51 \\ 10-18-61 & 17.7 \\ 10-29-63 & 0.01\end{array}$

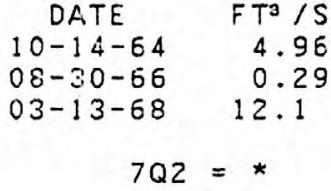

$Q(84)=0.1$

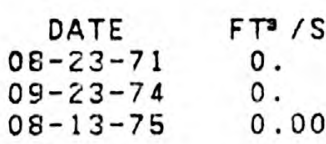

$08-13-75$

$\begin{array}{cl}\text { DATE } & F^{3} / \mathrm{S} \\ 07-28-70 & 0.05 \\ 08-24-71 & 0 .\end{array}$

$7010=0$ 
DES MOINES RIVEF: BASIN--Cont inued

5-4878.00 WHITE BREAST CR AT LUCAS, IOWA

LAT $4101 \times X$, LONG $9328 \times X$, IN NE $1 / 4$ SEC.

23, T.72 N., R.23 W., LUCAS CO. (59),

AT BRIDGE ON U.S. HIGHWAY 65, NEAR SOUTH CITY

LIMITS OF LUCAS.

DRAINAGE AREA $128 \quad \mathrm{Ml}^{2}$

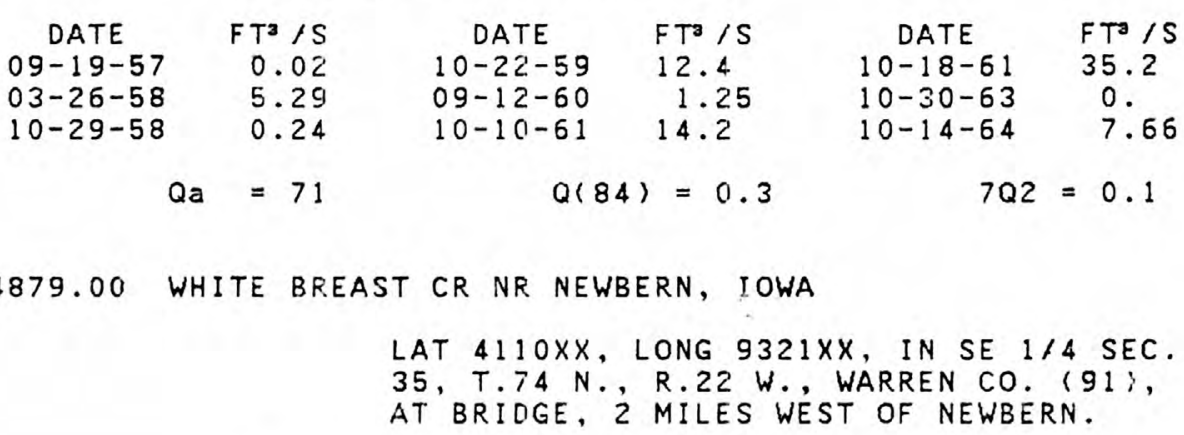

DRAINAGE AREA $243 \quad \mathrm{MI}{ }^{2}$

\begin{tabular}{|c|c|c|c|c|c|c|c|c|c|}
\hline $\begin{array}{c}\text { DATE } \\
09-19-57 \\
03-27-58 \\
10-29-58\end{array}$ & $\begin{array}{c}F T^{3} / S \\
0.15 \\
13.3 \\
0 .\end{array}$ & $\begin{array}{c}\text { DATE } \\
10-22-59 \\
09-12-60 \\
10-10-61\end{array}$ & $\begin{array}{l}\mathrm{FT}^{\mathrm{a}} / \mathrm{S} \\
23.5 \\
2.41 \\
37.3\end{array}$ & $\begin{array}{c}\text { DATE } \\
10-18-61 \\
10-30-63 \\
10-15-64\end{array}$ & $\begin{array}{l}\mathrm{FT}^{3} / \mathrm{S} \\
76.2 \\
0.20 \\
11.2\end{array}$ & $\begin{array}{c}\text { DATE } \\
08-30-66 \\
03-13-68 \\
07-28-70\end{array}$ & $\begin{array}{c}F T^{\circ} / \mathrm{S} \\
1.03 \\
23.2 \\
1.69\end{array}$ & $\begin{array}{c}\text { DATE } \\
08-23-71 \\
09-23-74 \\
08-13-75\end{array}$ & $\begin{array}{l}F T^{3} / \mathrm{S} \\
0.13 \\
0.80 \\
0.47\end{array}$ \\
\hline Qa & $=134$ & $Q<\varepsilon$ & $=1.4$ & 7Q2 & $=0.4$ & 7010 & * & & \\
\hline
\end{tabular}

5-4882.00 ENGLISH CR NR KNOXVILLE, IOWA

LAT 411615, LONG 930526, NEAR CENTER OF SEC. $30, T .75 \mathrm{~N} .$, R. 19 W., MARION CO. (63), AT BRIDGE, 3 MILES SOUTH OF KNOXVILLE.

DRAINAGE AREA $73.0 \mathrm{MI}^{2}$

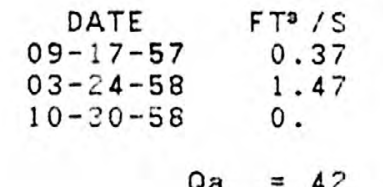

$Q_{a}=42$

$$
\begin{array}{cc}
\text { DATE } & F T^{3} / S \\
10-20-59 & 0.03 \\
09-14-60 & 0.01 \\
09-11-61 & 0 .
\end{array}
$$

$Q(84)=$ *

$\begin{array}{cc}\text { DATE } & F T^{3} / S \\ 10-09-61 & 8.27 \\ 10-17-61 & 24.1 \\ 10-30-63 & 0 .\end{array}$

$7 Q 2=0$

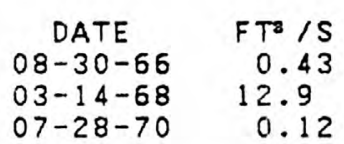

DATE
$08-24-71$
$09-24-74$
$08-12-75$

$F T^{3} / S$

0.07

0.13

\section{$7 Q 10=0$}


DES MOINES RIVER, BASIN--Continued

5-4883.00 ENGLISH CR NR HARVEY, IOWA

LAT $4120 \times X$, LONG $9257 \times X$, NEAR E $1 / 4$

CORNER SEC.5, T.75 N., R. 18 W., MARION CO.

(63), AT BRIDGE, 1.5 MILES NW OF HARVEY.

DRAINAGE AREA $108 \quad \mathrm{MI}^{2}$

\begin{tabular}{|c|c|c|c|c|c|c|c|c|c|}
\hline $\begin{array}{c}\text { DATE } \\
09-17-57 \\
03-24-58 \\
10-30-58\end{array}$ & $\begin{array}{r}\mathrm{FT}^{3} / \mathrm{S} \\
2.84 \\
2.86 \\
0.63\end{array}$ & $\begin{array}{c}\text { DATE } \\
10-20-59 \\
09-14-60 \\
09-11-61\end{array}$ & $\begin{array}{l}\mathrm{FT}^{3} / \mathrm{S} \\
0.82 \\
0.27 \\
0.19\end{array}$ & $\begin{array}{c}\text { DATE } \\
10-09-61 \\
10-17-61 \\
10-30-63\end{array}$ & $\begin{array}{l}\mathrm{FT}^{3} / \mathrm{S} \\
14.7 \\
35.9 \\
0 .\end{array}$ & $\begin{array}{c}\text { DATE } \\
10-15-64 \\
C 8-29-66 \\
03-13-68\end{array}$ & $\begin{array}{l}\mathrm{FT}^{3} / \mathrm{S} \\
0.53 \\
0.16 \\
4.57\end{array}$ & $\begin{array}{c}\text { DATE } \\
08-23-71 \\
09-23-74 \\
08-12-75\end{array}$ & $\begin{array}{l}\mathrm{FT}^{3} / \mathrm{S} \\
0.03 \\
2.3 \\
2.5\end{array}$ \\
\hline$Q_{a}$ & $=62$ & $Q(84)$ & $=0.4$ & $7 Q 2$ & $=0.1$ & $7 Q 10$ & 0 & & \\
\hline
\end{tabular}

5-4885.50 CEDAR CR AT MELROSE, IOWA

LAT $4058 \times X$, LONG $9303 \times X$, IN SW $1 / 4$ SEC

4, T.71 N., R.19 W., MONROE CO. (68).

AT BRIDGE NEAR SOUTH CITY LIMITS OF MELROSE.

DRAINAGE AREA $23.9 \mathrm{MI}^{2}$

\begin{tabular}{|c|c|c|c|c|c|c|c|c|c|}
\hline $\begin{array}{c}\text { DATE } \\
0 S-18-57 \\
03-28-58 \\
10-29-58\end{array}$ & $\begin{array}{l}F T=/ S \\
0.01 \\
3.75 \\
2.27\end{array}$ & $\begin{array}{c}\text { DATE } \\
10-20-59 \\
09-12-60 \\
09-11-61\end{array}$ & $\begin{array}{l}\mathrm{FT}^{3} \text { jS } \\
2.20 \\
0 . \\
0.01\end{array}$ & $\begin{array}{c}\text { DATE } \\
10-19-61 \\
10-29-63 \\
10-15-64\end{array}$ & $\begin{array}{l}\mathrm{FT}^{3} / \mathrm{S} \\
3.97 \\
0.01 \\
0.05\end{array}$ & $\begin{array}{c}\text { DATE } \\
08-29-66 \\
03-12-68 \\
07-29-70\end{array}$ & $\begin{array}{l}\mathrm{FT}^{3} / \mathrm{S} \\
0.02 \\
4.42 \\
0.01\end{array}$ & $\begin{array}{c}\text { DATE } \\
08-23-71 \\
09-24-74 \\
08-12-75\end{array}$ & $\begin{array}{l}\mathrm{FT}^{3} / \mathrm{S} \\
0.02 \\
0.02 \\
0.00\end{array}$ \\
\hline$Q$ & $=14$ & $Q 1 \varepsilon$ & $=$ & 702 & $=0$ & 7010 & 0 & & \\
\hline
\end{tabular}

5-4886.00 CEDAR CR. NR ALBIA, IOWA

LAT $4101 \times X$, LONG $9253 \times X$, IN NE $1 / 4$ SEC. $26, T .72$ N., R.18 W., MONROE CO. (68) AT BRILIGE ON U.S. HIGHWAY 34,4 MILES WEST OF ALBIA.

DRAINAGE AREA $102 \quad \mathrm{MI}^{2}$

$\begin{array}{ll}\text { DATE } & F T 3 / S \\ 03-28-58 & 11.5 \\ 10-29-58 & 8.13 \\ 10-20-59 & 11.4\end{array}$

$Q \mathrm{a}=60$

DATE $\mathrm{FT}^{3} / \mathrm{S}$

$09-12-60 \quad 0.27$

$09-11-61 \quad 0.14$

$10-19-61$

12.14

$Q(84)=0.4$ $\begin{array}{cc}\text { DATE } & F^{3} / \mathrm{S} \\ 03-12-68 & 13.4 \\ 07-29-70 & 0.18 \\ 08-23-71 & 0.18\end{array}$

$7010=0$ 
DES MOINES RIVER. BASIN--Cont inued

5-4887.00 CEDAR CR NR LOVILLA, IOWA

LAT $4107 X X$, LONG $9256 \times X$, NEAR S $1 / 4$

CORNER SEC.16, T.73 N., R.18 W., MONROE CO. (68),

AT BRIDGE, 2 MILES SW OF LOVILIA.

DRAINAGE AREA $211 \quad \mathrm{MI}^{2}$

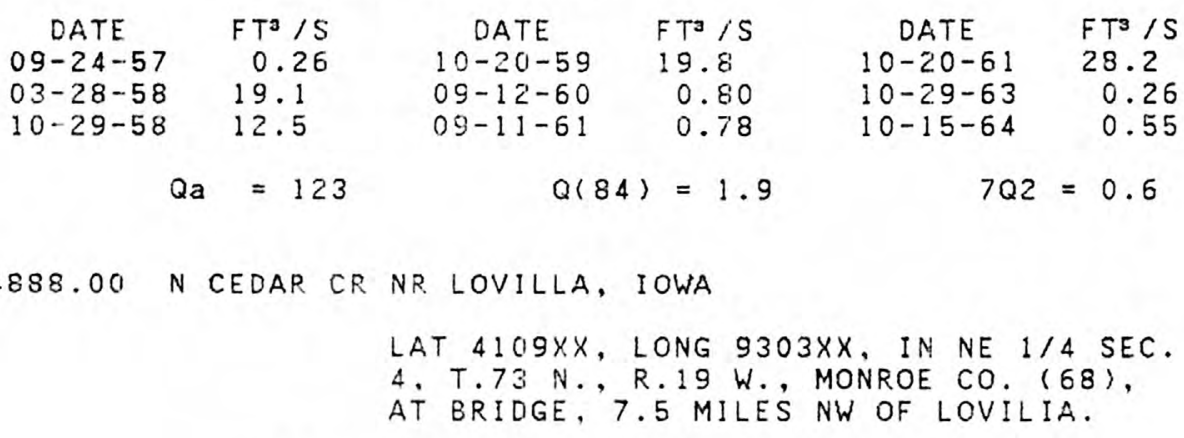

ORAINAGE AREA $61.3 \mathrm{MI}^{2}$

$\begin{array}{rccccc}\text { DATE } & F T^{3} / S & \text { DATE } & F T \text { IS } & \text { DATE } & F T^{3} / S \\ 09-23-57 & 0.07 & 10-20-59 & 3.15 & 10-20-61 & 12.1 \\ 03-24-58 & 5.61 & 09-12-60 & 0.11 & 10-29-63 & 0 . \\ 10-30-58 & 0.80 & 09-11-61 & 0.01 & 10-15-64 & 0.05 \\ Q a & =36 & 0(84)=0.1 & 702=\star\end{array}$

$\begin{array}{cc}\text { OATE } & F T^{T} / S \\ 08-29-65 & 0.01 \\ 03-12-68 & 11.8 \\ 08-23-71 & 0 \\ 7010= & 0\end{array}$

DATE

$09-23-74$

$F T^{2} / S$
0.04

$08-12-75$

0.04

5-4889.00 N CEDAR CR NR. MARYSVILLE, IOWA

$$
\begin{aligned}
& \text { LAT } 4111 X X \text {, LONG } 9301 X X \text {, IN SE } 1 / 4 \text { SEC. } \\
& 26, T .74 N \text {. R. }
\end{aligned}
$$

DRAINAGE AREA $111 \mathrm{MI}^{2}$

$\begin{array}{rrcccr}\text { DATE } & F^{3} / S & \text { DATE } & F T^{3} / S & \text { DATE } & F T^{3} / S \\ 03-24-58 & 8.20 & 09-12-60 & 0.18 & 10-29-63 & 0.03 \\ 10-30-58 & 1.27 & 09-11-61 & 0.14 & 10-15-64 & 0.10 \\ 10-20-59 & 2.22 & 10-20-61 & 23.1 & 08-29-66 & 0.01 \\ & 0 a=65 & & 0(84)=0.3 & 702=*\end{array}$

$\begin{array}{ll}\text { DATE } & F^{3} / \mathrm{S} \\ 03-13-68 & 7.03 \\ 08-23-71 & 0 .\end{array}$

DATE

$09-23-74$

$\mathrm{FT}^{3} / \mathrm{S}$

$08-12-75$

0.18

$Q(84)=0.3$

$7 Q 2=x$ 
DES MOINES RIVEF. BASIN--Cont inued

5-4893.00 N AVERY CR NR CHILLICOTHE, IOWA

LAT $4106 \times X$, LONG $9233 \times X$, IN SE $1 / 4$ SEC.

26, T.73 N., R.15 W. WAPELLO CO. (90),

AT ERIOGE, I MILE NW OF CHILLICOTHE.

DRAINAGE AREA $60.1 \mathrm{MI}^{2}$

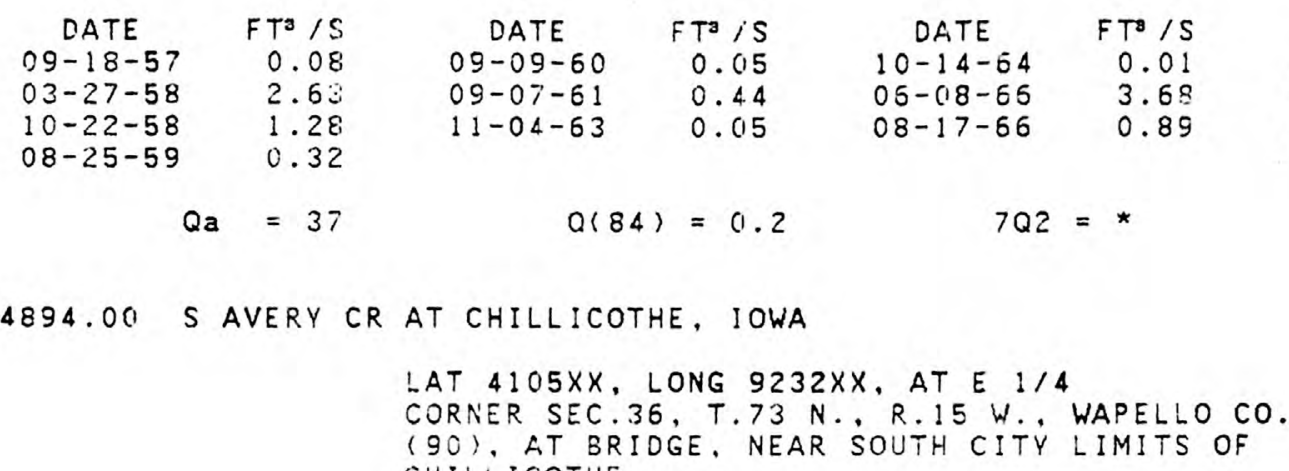

(90). AT BRIDGE. NEAR SOUTH CITY LIMITS OF

CHILLICOTHE.

$\begin{array}{cl}\text { DATE } & F T / S \\ 11-03-66 & 0 . \\ 06-20-68 & 0.67 \\ 07-14-70 & 0 . \\ & \end{array}$

DATE
$08-25-71$
$09-24-74$
$09-30-75$

$F T^{3} / S$

$09-30-75 \quad 2.7$

DRAINAGE AREA $51.6 \mathrm{MI}^{2}$
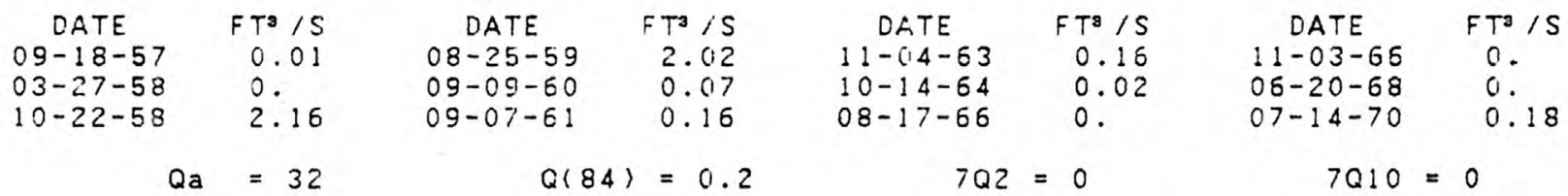

DATE
$08-25-71$
$09-24-74$
$09-30-75$

$F T^{3} / S$

$09-30-75 \quad 4.3$

$Q \mathbf{a}=32$

$Q(84)=0.2$

$702=0$

$7010=0$

5-4899.00 SOAP CR NR ASH GROVE, IOWA

LAT $4051 \times X$, LONG $9236 \times X$, IN SW $1 / 4$ SEC

$21, T .70$ N., R.15 W. DAVIS CO. (26),

AT BRIUGE, 3 MILES SW OF ASH GROVE.

DRAINAGE AREA $97.3 \mathrm{MI}^{2}$

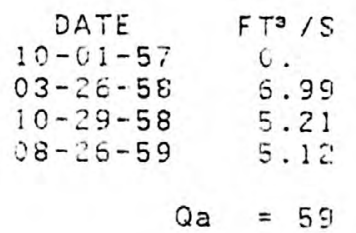

$\begin{array}{cr}\text { DATE } & F T^{3} / S \\ 09-081-60 & 0.38 \\ 09-0 E_{1}-61 & 0.03 \\ 11-04-63 & 0.18\end{array}$

$\begin{array}{cr}\text { DATE } & F T^{3} / S \\ 10-14-54 & 0.28 \\ 06-018-65 & 6.39 \\ 08-2.2-66 & 1.04\end{array}$
DATE
$0.5-12-6$
$11-03-6$
$05-12-6$

$\mathrm{FT}^{3} / \mathrm{S}$

4.41

4.41 
DES MOINES RIVER. BASIN--Cont inued

5-4901.00 SOAP CR NR FLORIS, IOWA

LAT 405337 , LONG 921553, NEAR CENTER OF SEC. $5, T .70 \mathrm{~N} ., \mathrm{R} .12 \mathrm{~W}$., DAVIS CO. (26), AT BRIDGE, 4 MILES NE OF FLORIS.

DRAINAGE AREA $243 \mathrm{MI}^{2}$

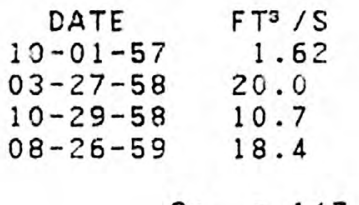

$Q_{a}=14.7$

$\begin{array}{rc}\text { DATE } & F T^{3} \text { iS } \\ 09-08-60 & 23.1 \\ 09-07-61 & 2.63 \\ 11-04-63 & 1.67 \\ 10-14-64 & 1.93 \\ \text { Q(84) } & =5.6\end{array}$

$\begin{array}{rr}\text { DATE } & F T^{3} / S \\ 06-018-66 & 24.1 \\ 08-22-66 & 5.99 \\ 11-03-66 & 2.40 \\ 04-18-67 & 103 . \\ 702= & 2.1\end{array}$

\section{DATE
$09-12-67$ \\ $09-12-67$
$06-12-68$}

$06-12-68$
$07-14-70$

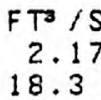

18.3

18.3
6.21

$7010=0.2 * *$

5-4902.00 LICK CR A.T KILBOUR.N, IOWA

LAT $4048 \times X$, LONG $9158 \times X$, IN SH $1 / 4$ SEC.

1, T.69 N., R.10 W., VAN BUREN CO. ( 89 )

AT BRIDGE NEAR EAST'CITY LIMITS OF KILBOURN.

DRAINAGE AREA $82.7 \quad \mathrm{MI}^{2}$

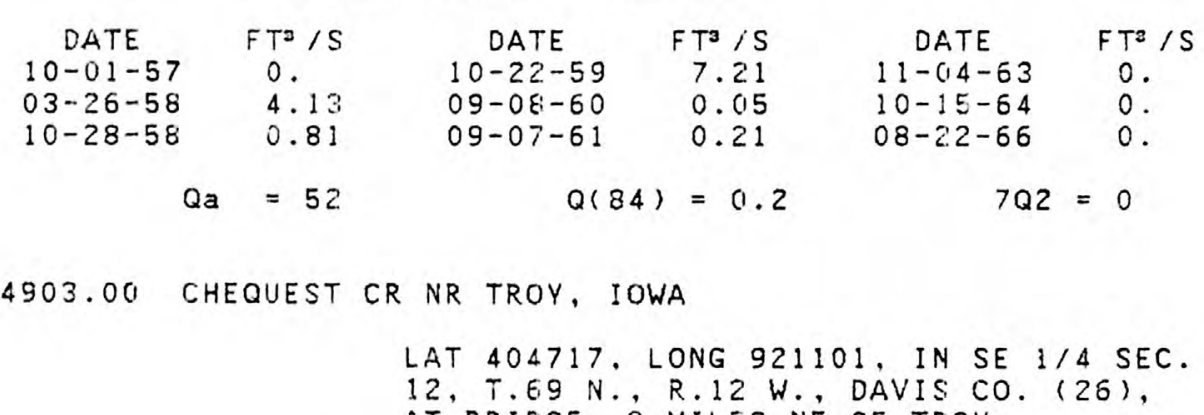

AT, BRIOGE. 3 MILES NE OF TROY.

DRAINAGE AREA $85.0 \mathrm{MI}^{2}$

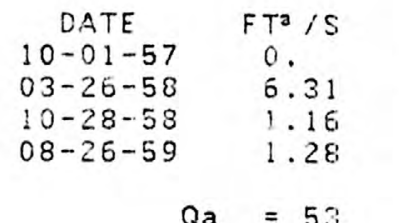

$Q_{a}=53$

$\begin{array}{cl}\text { DATE } & \text { FTa/S } \\ 09-08-60 & 0.09 \\ 09-07-61 & 0.36 \\ 11-04-63 & 0 .\end{array}$

$Q(84)=0.2$

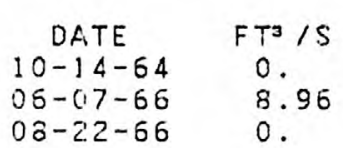

8. 96

0 .

$7 Q 2=0$

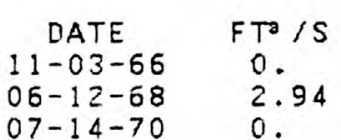

DATE

OB-25-71

$09-25-74$

$F T^{2} / \ddot{s}$ 0 .

0.36

$7 Q 10=0$
$08-25-71$

$09-25-74$

$09-29-75$

FTh

4.7

3.2

Q
DATE
$11-03-66$
$11-03-66$
$06-12-68$
$07-14-70$

$\mathrm{FT}^{3} / \mathrm{S}$

0.80

0.09

DATE

$08-25-71$

$09-25-74$

$\mathrm{FT}^{3} / \mathrm{S}$

$09-29-75$

0.06

0.52

$7210=0$ 
DES MOINES RIVER. BASIN--Continued

5-4904.00 CHEQUEST CR NR PITTSBURG, IOWA

LAT 404541 , LONG 920057 , NEAR CENTER OF SEC.21, T.69 N., R.10 W., DAVIS CO. (26), AT BRIDGE, 1.5 MILES NW OF PITTSBURG.

DRAINAGE AREA $123 \quad \mathrm{MI}^{2}$

\begin{tabular}{|c|c|c|c|c|c|c|c|c|c|}
\hline $\begin{array}{c}\text { DATE } \\
10-01-57 \\
03-26-58 \\
10-28-58 \\
10-22-59\end{array}$ & $\begin{array}{c}\mathrm{FT}^{3} / \mathrm{S} \\
0 . \\
8.35 \\
2.16 \\
13.5\end{array}$ & $\begin{array}{c}\text { DATE } \\
09-08-60 \\
09-07-61 \\
11-04-63\end{array}$ & $\begin{array}{r}F T^{3} / S \\
0.44 \\
1.32 \\
0.11\end{array}$ & $\begin{array}{c}\text { DATE } \\
10-14-64 \\
06-017-66 \\
08-2.2-66\end{array}$ & $\begin{array}{c}\mathrm{FT}^{3} / \mathrm{S} \\
0.01 \\
31.6 \\
1.02\end{array}$ & $\begin{array}{c}\text { DATE } \\
11-03-66 \\
06-12-68 \\
07-14-70\end{array}$ & $\begin{array}{l}F T / S \\
0.24 \\
2.46 \\
0.55\end{array}$ & $\begin{array}{c}\text { DATE } \\
08-24-71 \\
09-25-74 \\
09-29-75\end{array}$ & $\begin{array}{l}\mathrm{FT}^{2} / \mathrm{S} \\
0.03 \\
1.2 \\
1.2\end{array}$ \\
\hline$Q$ & $=77$ & $Q 1 \varepsilon$ & $=0.4$ & 702 & $=0$ & 7010 & 0 & & \\
\hline
\end{tabular}

5-4907.00 SUGAR CR. NR CHARLESTON, IOWA.

LAT 403353 , LONG 913343 , IN NW $1 / 4$ SEC.

$33, T .67$ N., R.6 W., LEE CO. (56),

AT BRIUGE, 2 MILES SW OF CHARLESTON.

DRAINAGE AREA $62.3 \quad \mathrm{MI}^{2}$

$\begin{array}{rr}\text { DATE } & F T^{3} / S \\ 10-01-57 & 0 . \\ 03-26-58 & 3.92 \\ 10-28-58 & 0.49 \\ & =40\end{array}$

$\begin{array}{cr}\text { DATE } & F^{3} / S \\ 09-06-60 & 0.08 \\ 09-06-61 & 0.02 \\ 11-05-63 & 0.01\end{array}$

$\begin{array}{cc}\text { DATE } & \mathrm{FT}^{3} / \mathrm{S} \\ 10-15-64 & 0.01 \\ 06-07-66 & 17.9 \\ 08-22-66 & 0 .\end{array}$

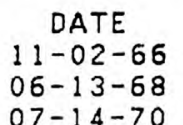

$F T / S$

1.02
0.64

$08-22-66 \quad 0$.

$07-14-70$

0.02

DATE
$08-25-71$
$09-20-74$

$09-30-75$

$\mathrm{FT}^{\mathrm{s}} / \mathrm{S}$

0.02

0.06

$Q_{a}=40$

$Q(84)=0.2$

$702=0$

$7010=0$

FOX RIVER BASIN

5-4945.00 FOX R AT CANTRIL, IOWA

LAT $4039 \times X$, LONG $9203 \times X$, IN SW $1 / 4$ SEC.

30 , T. 68 N., R. 10 W., VAN EUREN CO. (89),

AT BRIUGE ON STATE HIGHWAY 2, 1 MILE NE OF CANTRIL.

DRAINAGE AREA $161 \mathrm{MI}^{2}$

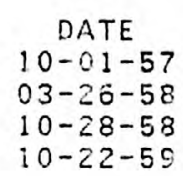

$\mathrm{FT}^{3} / \mathrm{S}$
0.12
11.2
7.14
21.2

DATE
$09-08-60$
$09-06-61$
$11-04-63$
$10-15-64$

$\mathrm{FT}^{3} / \mathrm{S}$
2.85
2.65
1.35

21.2

$10-15-64$

1.35

DATE
O6-07-66
$08-22-66$
$11-02-66$

$F T a / S$
16.9
4.27
2.01

DATE
$09-12-67$
$06-12-68$
$07-14-70$

$F^{3} / S$
0.67

0.67
5.90

DATE
$08-25-71$

$08-25-71$
$09-25-74$

$09-29-75$

$F^{3} / \mathrm{S}$

6.2

4. 2

$C a=100$

$Q(84)=\hat{2} .0$

$7 Q 2=0.8$

$7010=*$ 
BIG SIOUJX RIVER. BASIN

6-483:.00 ROCK R NR ROCK RAFIDS, IOWA

LAT 433001, LONG 961103, IN NE $1 / 4$ SEC.

8, T. 100 N., R. 45 W., LYON CO. (60),

AT BRIDGE, 5 MILES NORTH OF ROCK RAPIDS.

DRAINAGE AREA $558 \quad \mathrm{MI}^{2}$

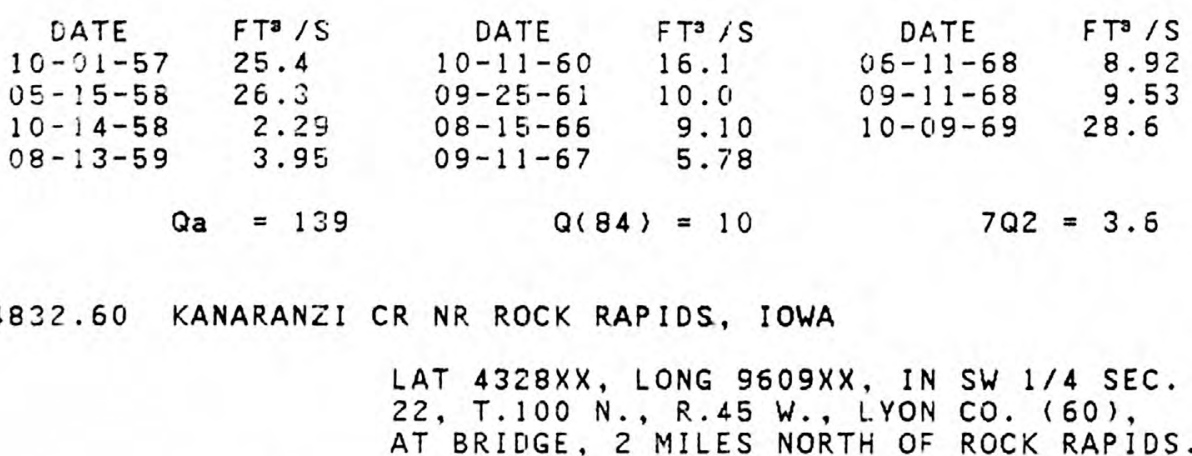

DRAINAGE AREA $203 \quad \mathrm{MI}^{2}$

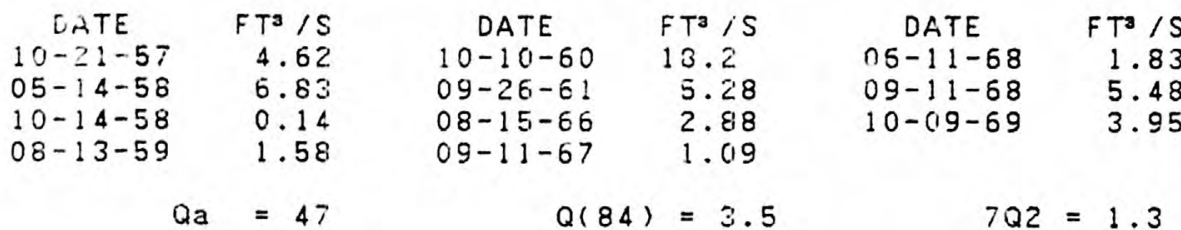

$\begin{array}{cccc}\text { DATE } & F T^{3} / S & \text { DATE } & F T^{3} / S \\ 09-01-70 & 2.46 & 09-25-74 & 1.6 \\ 09-01-71 & 3.5 & 10-21-75 & 3.2\end{array}$

$Q \mathbf{a}=47$

$Q(84)=3.5$

$\begin{array}{cc}\text { DATE } & F T / S \\ 09-01-70 & 12.7 \\ 09-01-71 & 11.0 \\ 09-11-73 & 9.4\end{array}$

DATE
$09-25-74$

$09-25-74$
$10-21-75$

$08-24-76$

$\mathrm{FT}^{3} / \mathrm{S}$

7.6

12.

$7 Q 10=0.7$

6-4832.80 TOM CR AT ROCK RAFIDS, ICWA

LAT $4326 \times X$, LONG 9609XX, IN SW $1 / 4$ SEC.

$34, T .100$ N., R. 45 W., LYON CO. $(60)$,

AT BRIOGE IN'NE CORNER OF ROCK RAPIDS.

DRAINAGE AREA $61.9 \quad \mathrm{MI}^{2}$

$\begin{array}{rr}\text { DATE } & F T^{3} / S \\ 10-01-57 & 0.48 \\ C 5-14-58 & 0.46 \\ 10-14-58 & 0 . \\ C 8-13-59 & 0.03 \\ Q a & =13\end{array}$

$\begin{array}{cl}\text { DATE } & \text { FT } / S \\ 10-10-60 & 0.29 \\ 09-26-61 & 0.06 \\ 08-15-66 & 0 . \\ 09-11-67 & 0.02 \\ 0(84)=*\end{array}$

$\begin{array}{cl}\text { DATE } & \text { FT / S } \\ 06-11-68 & 0.01 \\ 09-11-68 & 0.1 \\ 10-09-69 & 0.24 \\ 09-01-70 & 0 . \\ 702= & 0\end{array}$

$\begin{array}{cr}\text { DATE } & F T / S \\ 09-01-71 & 0.06 \\ 10-16-72 & 0.88 \\ 09-11-73 & 0.24 \\ 7010= & 0\end{array}$

DATE

$09-25-74$

$101-21-75$

$08-24-76$ 
DISCHARGE MEASUREMENTS AT

BIG SIOUX RIVER BASIN--Continued

6-4833.00 ROCK R BELOW ROCK RAPIDS, IOWA

LAT $4324 X X$, LONG $9609 \times X$, NEAR N $1 / 4$

CORNER SEC.15, T.99 N., R.45 W., LYON CO. (50),

AT BRIDGE, 2 MILES SOUTH OF ROCK RAPIDS.

DRAINAGE AREA $859 \quad \mathrm{MI}^{2}$

\begin{tabular}{|c|c|c|c|c|c|c|c|c|c|}
\hline $\begin{array}{c}\text { DATE } \\
10-01-57 \\
05-14-58 \\
10-14-58 \\
08-12-59\end{array}$ & $\begin{array}{l}\mathrm{FT}^{3} / \mathrm{S} \\
33.6 \\
38.2 \\
0.46 \\
7.92\end{array}$ & $\begin{array}{c}\text { DATE } \\
10-10-60 \\
09-26-61 \\
08-15-66 \\
09-11-67\end{array}$ & $\begin{array}{l}\mathrm{FT}^{3} / \mathrm{S} \\
36.3 \\
14.8 \\
13.5 \\
6.60\end{array}$ & $\begin{array}{c}\text { DATE } \\
\text { O6-11-68 } \\
09-11-68 \\
10-09-69 \\
09-01-70\end{array}$ & $\begin{array}{l}\mathrm{FT}^{3} / \mathrm{S} \\
10.5 \\
14.8 \\
33.5 \\
17.0\end{array}$ & $\begin{array}{c}\text { DATE } \\
09-01-71 \\
10-15-72 \\
09-11-73\end{array}$ & $\begin{array}{l}\mathrm{FT}^{3} / \mathrm{S} \\
16.0 \\
41 . \\
13 .\end{array}$ & $\begin{array}{c}\text { DATE } \\
09-25-74 \\
10-21-75 \\
08-24-76\end{array}$ & $\begin{array}{l}F T / S \\
8.2 \\
14 . \\
0.70\end{array}$ \\
\hline$Q=$ & $=219$ & $Q(84)$ & $=14$ & $7 Q 2$ & $=2.9$ & $7 Q 10$ & 0.2 & & \\
\hline
\end{tabular}

6-4833.20 MUD CR AT LESTER, IOWA

LAT $4327 \mathrm{XX}$, LONG $9620 \times \mathrm{X}$, IN NW $1 / 4$ SEC.

36, T.J00 N., R. 47 W., LYON CO. $(60)$,

AT ERIDIGE NEAR NW CITY LIMITS OF LESTER. DRAINAGE AREA $63.7 \mathrm{MI}^{2}$

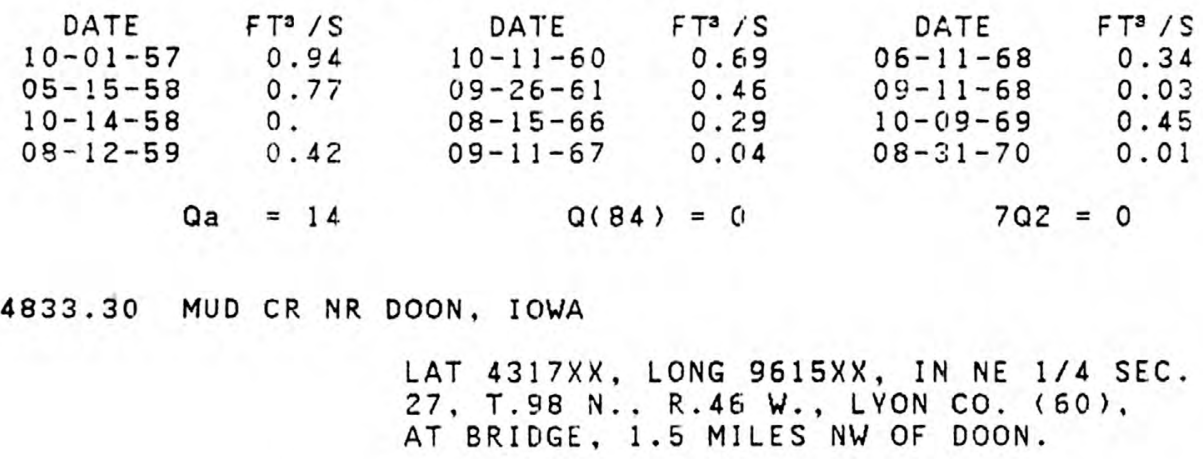

DRAINAGE AREA $138 \quad \mathrm{MI}^{2}$

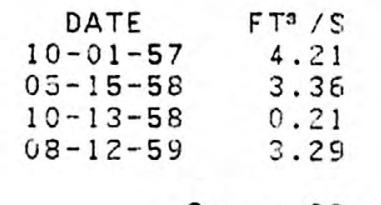

$Q \mathbf{a}=32$

$\begin{array}{ll}D A T E & F T^{3} / S \\ 10-11-60 & 3.41 \\ 09-26-61 & 2.56 \\ 05-15-66 & 0.87 \\ 09-11-67 & 0.94 \\ Q(84) & =1.0\end{array}$

$Q(84)=1.0$

$\begin{array}{rr}\text { DATE } & F T^{3} / S \\ 06-11-68 & 0.91 \\ 09-11-63 & 0.07 \\ 10-09-59 & 2.09 \\ 08-31-70 & 0.65 \\ 702= & 0.3\end{array}$

$702=0.3$

$\begin{array}{cc}\text { DATE } & F T^{3} / \mathrm{S} \\ 09-01-71 & 0 . \\ 10-16-72 & 0.20 \\ 09-11-73 & 0.12 \\ 7 Q 10= & 0\end{array}$

DATE

$09-25-74$
$10-21-75$

$\mathrm{FT}^{\mathrm{T}} / \mathrm{S}$

0.01

$08-24-76 \quad 0.00$ 
BIG SIOUX RIVER BASIN--Continued

6-4833.40 ROCK R NR DOON, IOWA

LAT $4316 \times X$, LONG $9615 X X$, IN NW $1 / 4$ SEC.

$35, T .98$ N., R.46 W., LYON CO. $(60)$,

AT BRIDGE, 1 MILE SW OF DOON.

DRAINAGE AREA $1050 \quad \mathrm{MI}^{2}$

\begin{tabular}{|c|c|c|c|c|c|c|}
\hline $\begin{array}{c}\text { DATE } \\
10-01-57 \\
05-15-58 \\
10-13-58 \\
08-12-58\end{array}$ & & $\begin{array}{l}\mathrm{FT}^{\mathrm{a}} / \mathrm{S} \\
41.4 \\
48.2 \\
1.05 \\
15.2\end{array}$ & $\begin{array}{c}\text { DATE } \\
10-11-60 \\
09-2 \varepsilon-61 \\
08-15-66 \\
09-11-67\end{array}$ & $\begin{array}{l}F T^{3} / \mathrm{S} \\
46.5 \\
21.8 \\
15.6 \\
11.6\end{array}$ & $\begin{array}{c}\text { DATE } \\
06-11-68 \\
09-11-68 \\
10-09-69 \\
08-31-70\end{array}$ & $\begin{array}{l}\mathrm{FT} / \mathrm{S} \\
13.4 \\
14.5 \\
44.2 \\
18.9\end{array}$ \\
\hline & Qa & $=271$ & Q 184 & $1=19$ & $7 Q 2$ & $=6.1$ \\
\hline 4833.60 & $L$ & ROCK R NR & LITTLE ROCK. & , IOWA & & \\
\hline
\end{tabular}

DRAINAGE AREA $92.0 \mathrm{MI}^{2}$

\begin{tabular}{|c|c|c|c|c|c|c|c|c|c|}
\hline $\begin{array}{c}\text { DATE } \\
10-02-57 \\
05-14-58 \\
10-14-58 \\
05-19-59\end{array}$ & $\begin{array}{l}F T^{3} / S \\
0.42 \\
0.79 \\
0.4 \\
0.28\end{array}$ & $\begin{array}{c}\text { DATE } \\
08-13-59 \\
10-10-60 \\
09-25-61 \\
08-15-66\end{array}$ & $\begin{array}{l}F T^{3} / S \\
0 . \\
7.90 \\
0.42 \\
0.11\end{array}$ & $\begin{array}{c}\text { DATE } \\
09-11-67 \\
06-11-68 \\
09-11-68 \\
10-09-69\end{array}$ & $\begin{array}{l}\mathrm{FT}^{3} / \mathrm{S} \\
0.01 \\
0.01 \\
0.0 \\
0.57\end{array}$ & $\begin{array}{c}\text { DATE } \\
09-01-70 \\
09-01-71 \\
10-16-72 \\
09-11-73\end{array}$ & $\begin{array}{r}\mathrm{FT}^{\mathrm{S}} / \mathrm{S} \\
0.71 \\
0.11 \\
0.94 \\
0.05\end{array}$ & $\begin{array}{c}\text { DATE } \\
09-25-74 \\
10-20-75 \\
08-25-76\end{array}$ & $\begin{array}{l}F T^{3} / S \\
0.18 \\
0.13 \\
0 .\end{array}$ \\
\hline & $=21$ & Qr & $=*$ & $7 Q_{2}$ & $=0$ & 7010 & 0 & & \\
\hline
\end{tabular}

6-4833.80 L ROCK R. AT LITTLE ROCK, IOWA

LAT $4326 \times X$, LONG $9554 \times X$, IN NE $1 / 4$ SEC.

$3, T .99 \mathrm{~N} .$, R. 43 W. LYON CO. $\{60\}$,

AT BRIDGE, 1 MILE SW OF LITTLE ROCK.

DRAINAGE AREA $: 34 \quad \mathrm{MI}^{2}$

$\begin{array}{cr}\text { DATE } & \text { FT } / S \\ 10-02-57 & 1.67 \\ 05-14-58 & 2.83 \\ 10-14-58 & 0.26 \\ 05-19-59 & 1.44\end{array}$

$\begin{array}{cccc}\text { DATE } & \text { FT } / S & \text { DATE } & F T^{3} / S \\ 08-13-59 & 0.10 & 09-11-67 & 0.53 \\ 10-10-60 & 10.4 & 05-11-68 & 0.87 \\ 05-25-61 & 2.37 & 09-11-68 & 0.23 \\ 08-15-66 & 1.25 & 10-09-69 & 1.66 \\ & & & \\ \text { Q } 84)=1.1 & \end{array}$

$\begin{array}{cc}\text { DATE } & F T / S \\ 09-01-70 & 1.13 \\ 09-01-71 & 1.1 \\ 10-16-72 & 2.9 \\ 09-11-73 & 0.86 \\ 7010=*\end{array}$
DATE
$09-25-74$
$10-20-75$
$08-25-76$

$F T^{3} / S$

2. 6

0.02

$Q(84)=1.1$

$702=0$ 
BIG SIOUX RIVER BASIN--Continued

6-4834.00 L ROCK R. NR GEORGE, IOWA

LAT $4319 \times X$, LONG $9602 \times X$, IN NE $1 / 4$ SEC.

15, T.98 N., R.44 W., LYON CO. $(60)$,

AT BRIDGE, 2 MILES SW OF GEORGE.

\section{DRAINAGE AREA I99 MI²}

$\begin{array}{cr}\text { DATE } & F T^{3} / S \\ 10-01-57 & 6.34 \\ 05-15-58 & 7.81 \\ 10-14-58 & 1.13 \\ 05-19-59 & 4.89\end{array}$

$\begin{array}{cc}\text { DATE } & F T^{3} / S \\ 08-12-59 & 3.43 \\ 10-11-60 & 19.7 \\ 09-25-61 & 7.37 \\ 08-15-66 & 7.21\end{array}$

DATE
$09-11-67$
$06-11-68$
$10-09-69$

$06-11-68$

$10-09-69$

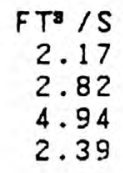

$Q(84)=3.3$

$7 Q 2=0.6$

6-4834.60 OTTER CR. NR ASHTON, IOWA

LAT $4320 X X$, LONG $9546 \times X$. IN SE $1 / 4$ SEC.

2 , T.98 N., R. 42 W., OSCEOLA CO. $(72)$,

AT BRILIGE, 2 MILES NE OF ASHTON.

\section{DRAINAGE AREA $88.0 \quad \mathrm{MI}^{2}$}

$\begin{array}{cr}\text { DATE } & F T^{3} / S \\ 10-02-57 & 2.12 \\ 05-14-58 & 6.90 \\ 10-14-58 & 0.60 \\ 05-19-59 & 2.77 \\ Q_{a} & =20\end{array}$

$\begin{array}{rc}\text { DATE } & F T^{3} / S \\ 08-12-59 & 0.98 \\ 10-11-60 & 12.8 \\ 09-25-61 & 1.48 \\ 08-15-66 & 6.75 \\ Q(84) & =1.8\end{array}$

DATE
$09-11-67$
$06-11-68$
$09-11-68$
$10-09-69$

$\mathrm{FT}^{3} / \mathrm{S}$
2.14
1.16
0.62

2.14
1.16

0.62
1.39

$Q_{a}=20$
$702=0.3$
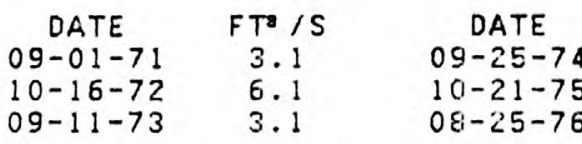

$\mathrm{T} / \mathrm{S}$

3.0

6.9

0.13

6-4834.70 OTTER CR NR MATLOCK, IOWA

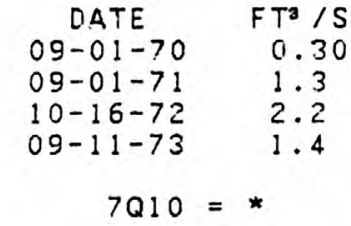

$7010=*$

LAT $4316 \times X$, LONG 9555XX, NEAR W 1/4

CORNER SEC.34, T.98 N., R.43 W., LYOM CO.

(GO), AT BRIDGE, 2 MILES NE OF MATLOCK.

\begin{abstract}
DR.AINAGE AREA $129 \quad \mathrm{MI}^{2}$
\end{abstract}

$\begin{array}{rr}\text { DATE } & F T^{3} / S \\ 10-01-57 & 5.20 \\ 05-14-58 & 9.90 \\ 10-14-58 & 0.23 \\ 05-19-59 & 4.81 \\ Q a & =29\end{array}$

$\begin{array}{rc}\text { DATE } & F^{3} \text { is } \\ 08-12-59 & 1.85 \\ 10-11-60 & 21.6 \\ 09-25-61 & 2.82 \\ 08-15-66 & 4.76 \\ Q(84)=2.6\end{array}$

$\begin{array}{rr}\text { DATE } & F^{3} / S \\ 09-11-67 & 1.20 \\ 06-11-68 & 1.94 \\ 09-11-68 & 0 . \\ 10-019-69 & 2.81 \\ 7 Q 2= & 0.4\end{array}$

$\begin{array}{cc}\text { DATE } & \mathrm{FT}^{3} / \mathrm{S} \\ \text { O9-01-70 } & 0.84 \\ 09-01-71 & 2.0 \\ 10-16-72 & 4.0 \\ 09-11-73 & 2.3 \\ 7010=\star\end{array}$
DATE
$10-21-75 \quad 3.8$
$08-25-76 \quad 1.2$


BIG SIOUX RIVER BASIN--Cont inued

6-4834.80 OTTER CR NR GEORGE, IOWA

LAT $4317 \times X$, LONG $9603 \times X$, IN NW $1 / 4$ SEC.

28, T.98 N., R.44 W., LYON CO. (60),

AT BRIDGE, 5 MILES SW OF GEORGE.

DRAINAGE AREA $208 \quad \mathrm{MI}^{2}$

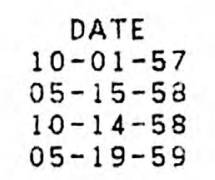

$F T^{3} / \mathrm{S}$
5.53
10.9
0.9
7.16

DATE
$08-12-59$
$10-11-60$
$09-25-61$
$08-15-66$

$\mathrm{FT}^{3}$ is

4.36

30.5

4.38
7.79
DATE
$09-11-67$
$09-11-67$
$06-11-68$
$09-11-68$

$10-09-69$

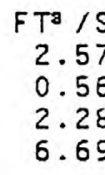

$702=0.6$
$Q(84)=3.7$

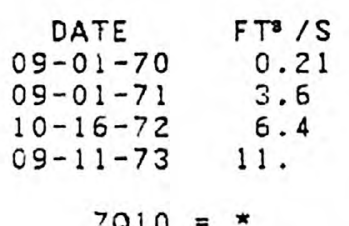

$7 Q 10=\star$

6-4834.90 L ROCK R NR DOON, IOWA

LAT $4316 \times X$, LONG $9614 \times X$, NEAR $W 1 / 4$ CORNER SEC.36, T.98 N., R.46 W., LYON CO. (60), AT BRIDGE, 1 MILE SOUTH OF DOON.

\section{DRAINAGE AREA $474 \quad \mathrm{MI}^{2}$}

$\begin{array}{cc}\text { DATE } & F T^{3} / \mathrm{S} \\ 10-01-57 & 14.4 \\ 05-15-58 & 22.5 \\ 10-13-58 & 0.48 \\ 08-12-59 & 13.7\end{array}$

$Q_{a}=117$

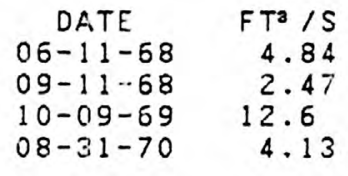

$7 Q 2=2.3$

$\begin{array}{cl}\text { DATE } & \text { FT' } / \mathrm{S} \\ 09-01-71 & 9.3 \\ 10-16-72 & 16 . \\ 09-11-73 & 17 . \\ 7010= & 0.2\end{array}$

DATE
$09-25-74$

$09-25-74$

$10-20-75$
$08-24-76$

$\mathrm{FT}^{\mathrm{a}} / \mathrm{S}$

8.2

27.

3.0

6-4841.00 SIXMILE CR NR HAWARDEN, IOWA

$$
\begin{aligned}
& \text { LAT } 4302 \times X, \text { LONG } 9624 \times X \text {, IN NW } 1 / 4 \text { SEC. } \\
& 28 \text { T.95 N., R. } 47 \text { W. S IOUX CO. }(84), \\
& \text { AT BRIDGE, } 5 \text { MILES NE OF HAWARDEN. }
\end{aligned}
$$

DRAINAGE AREA $68.8 \mathrm{MI}^{2}$

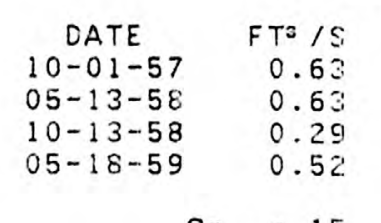

$Q \mathrm{a}=15$

$\begin{array}{cr}\text { DATE } & \text { FT }^{3} / S \\ 08-11-59 & 0.35 \\ 10-11-60 & 1.014 \\ 09-26-61 & 1.55 \\ 08-15-66 & 1.56\end{array}$

$Q(84)=0.6$

$\begin{array}{rr}\text { DATE } & \text { FT'/S } \\ 09-11-67 & 0.69 \\ 06-11-68 & 0.26 \\ 09-11-68 & 0.39 \\ 10-09-69 & 1.59 \\ 7 Q 2= & 0.1\end{array}$

$\begin{array}{cc}\text { DATE } & F^{3} / S \\ \text { O9-01-70 } & 1.62 \\ 09-01-71 & 2.2 \\ 10-16-72 & 1.8 \\ 09-11-73 & 1.9 \\ 7010=\star\end{array}$

DATE

$09-25-74$

$10-20-75$

$08-24-7 \varepsilon$

$F T^{3} / S$

$1 \cdot 1$

0.39 
BIG SIOUX RIVER BASIN--Continued

6-4841.50 SIXMILE CR NR CHATSWORTH, IOWA

LAT $4256 \times X$, LONG $9629 \times X$, IN SW $1 / 4$ SEC.

$26, T .94$ N., R.48 W., SIOUX CO. ( 84 ),

AT BRIDGE, i. 5 MILES NE OF CHATSWORTH.

DRAINAGE AREA $104 \quad \mathrm{MI}^{2}$

$\begin{array}{rrcccr}\text { DATE } & \mathrm{FT}^{3} / \mathrm{S} & \text { DATE } & \mathrm{FT}^{\mathrm{a}} / \mathrm{S} & \text { DATE } & \mathrm{FT}^{3} / \mathrm{S} \\ 09-30-57 & 1.72 & 08-11-59 & 1.10 & 09-11-67 & 2.04 \\ 05-13-58 & 1.87 & 10-11-60 & 2.97 & 06-11-68 & 1.26 \\ 10-13-58 & 0.25 & 09-26-61 & 2.32 & 09-11-68 & 1.16 \\ 05-18-59 & 2.77 & 08-15-66 & 3.98 & 10-09-69 & 3.66 \\ & \mathrm{Qa}=23 & \mathrm{Q}=(84)=1.6 & 702=0.4\end{array}$

$\begin{array}{cc}\text { DATE } & F T / S \\ 09-01-70 & 4.03 \\ 09-01-71 & 5.0 \\ 10-16-72 & 6.6 \\ 09-11-73 & 4.0 \\ 7010\end{array}$

$7010=*$

6-4842.00 INDIAN CR NR CHATSWORTH, IOWA

LAT $4253 \times X$, LONG $9630 \times X$, IN NW $1 / 4$ SEC.

$10, T .93$ N., R. 48 W., PLYMOUTH CO. (75),

AT BRIDGE, 1.5 MILES SOUTH OF CHATSWORTH.

DRAINAGE AREA $62.2 \quad \mathrm{MI}^{2}$

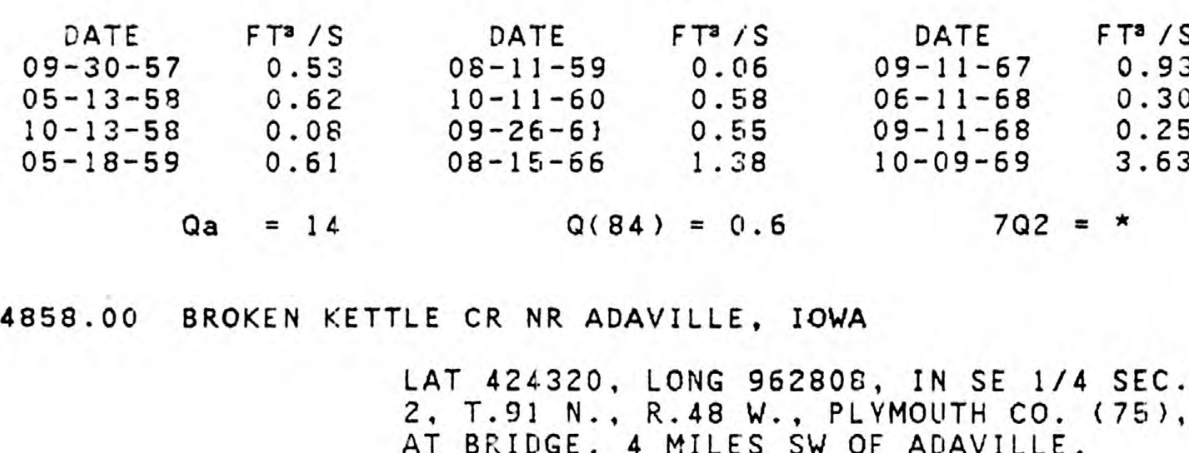

DRAINAGE AREA $60.7 \mathrm{MI}^{2}$

\begin{tabular}{|c|c|c|c|c|c|c|c|c|c|}
\hline $\begin{array}{c}\text { DATE } \\
09-27-57 \\
05-12-58 \\
10-13-58 \\
05-18-59\end{array}$ & $\begin{array}{r}\mathrm{FT}^{3} / \mathrm{S} \\
2.03 \\
1.43 \\
1.54 \\
1.99\end{array}$ & $\begin{array}{c}\text { DATE } \\
08-11-59 \\
10-11-60 \\
09-26-61 \\
08-16-66\end{array}$ & $\begin{array}{r}\mathrm{FT}^{3} / \mathrm{S} \\
1.38 \\
2.09 \\
1.87 \\
3.84\end{array}$ & $\begin{array}{c}\text { DATE } \\
09-12-67 \\
06-12-68 \\
09-12-68 \\
10-10-69\end{array}$ & $\begin{array}{r}\mathrm{FT}^{3} / \mathrm{S} \\
2.92 \\
1.93 \\
1.63 \\
4.23\end{array}$ & $\begin{array}{c}\text { DATE } \\
09-02-70 \\
09-02-71 \\
10-17-72 \\
09-12-73\end{array}$ & $\begin{array}{c}\mathrm{FT}^{3} / \mathrm{S} \\
3.03 \\
3.2 \\
4.7 \\
3.3\end{array}$ & $\begin{array}{c}\text { DATE } \\
09-25-74 \\
10-21-76 \\
08-26-76\end{array}$ & $\begin{array}{l}\mathrm{FT}^{3} . \mathrm{S} \\
2.4 \\
2.9 \\
2.1\end{array}$ \\
\hline
\end{tabular}


BIG SIOUX RIVER BASIN--Continued

6-4859.00 BROKEN KETTLE CR NR SIOUX CITY, IOWA

$$
\begin{aligned}
& \text { LAT } 423816, \text { LONG } 963028 \text {, IN SW } 1 / 4 \text { SEC. } \\
& 3 \text {, T.9O N., R.48 W. PLYMOUTH CO. ( } 75) \text {, } \\
& \text { AT BRIDGE, } 9 \text { MILES NW OF SIOUX CITY. }
\end{aligned}
$$

\section{DRAINAGE AREA $\quad 97.4 \quad \mathrm{MI}^{2}$}

$\begin{array}{rr}\text { DATE } & F T^{3} / S \\ 09-27-57 & 2.73 \\ 05-12-57 & 3.31 \\ 10-13-58 & 1.73 \\ 05-18-59 & 2.78 \\ \text { Qa } & =22\end{array}$

$\begin{array}{cc}\text { DATE } & F T^{3} / S \\ 08-11-59 & 1.72 \\ 10-11-60 & 2.74 \\ 09-26-61 & 2.97 \\ 08-16-66 & 5.58 \\ Q(84) & =3.0\end{array}$

DATE
$09-12-67$
$05-12-68$
$09-12-68$

$10-10-69$

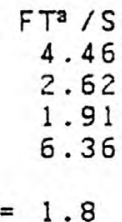

$7.22=1.8$

6-6000.20 FLOYD R NR SHELDON, IOWA LAT 431219 , LONG 954922 , IN SW $1 / 4$ SEC. AT BRIDGE, 2 MILES NE OF SHELDON.

DRAINAGE AREA $64.0 \quad \mathrm{MI}^{2}$

$\begin{array}{cccc}\text { DATE } & \mathrm{FT}^{3} / S & \text { DATE } & F T^{3} / S \\ 10-02-57 & 0.79 & 10-10-60 & 5.53 \\ 05-14-58 & 1.89 & 10-10-60 & 5.31 \\ 10-14-58 & 0.46 & 09-26-61 & 1.45 \\ 05-19-59 & 2.34 & 10-01-63 & 1.12 \\ 08-12-59 & 0.97 & 10-12-64 & 2.58\end{array}$

$\begin{array}{cr}\text { DATE } & F T^{3} / S \\ 08-15-66 & 1.52 \\ 09-11-67 & 0.65 \\ 06-11-68 & 0.88\end{array}$

$Q_{a}=14$

$$
Q(84)=0.9
$$

DATE
$09-02-70$
$09-02-71$
$10-17-72$
$09-12-73$

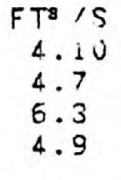

$7 Q 10=0.7 * *$
DATE

$-74$

$10-21-75$

$08-26-76$

$\begin{array}{cc}\text { DATE } & \mathrm{FT}^{3} / \mathrm{S} \\ 10-09-59 & 1.39 \\ 09-01-70 & 0.33 \\ 09-02-71 & 1.2 \\ 10-16-72 & 1.2 \\ & \\ 7 Q 10= & 0\end{array}$

$702=0.3$

\section{DATE}

OS-11-73

$09-26-74$

$10-21-75$

$08-25-76$

$T^{3} / \mathrm{S}$

$2 \cdot 3$

$2 \cdot 8$

$4 \cdot 8$
0.25

6-6000.40 L FLOYD R NR SHELDON, IOWA

LAT 430925, LONG 955202 , IN SE $1 / 4$ SEC.
1, T.96 N., R. 43 W., SIOUX CO. (84),

$1, T .96 \mathrm{~N}, 2 \mathrm{R} .43 \mathrm{~W} ., \mathrm{SIOUX}$ CO. ( 84 )
AT BRIDGE, 2 MILES SW OF SHELDON.

DRAINAGE AREA $59.3 \mathrm{MI}^{2}$

$\begin{array}{rr}\text { DATE } & F \Gamma / S \\ 10-01-57 & 0.13 \\ 05-14-58 & 1.06 \\ 10-15-58 & 0.0 \\ 05-19-59 & 1.92 \\ 08-12-59 & 0.39 \\ Q a & =13\end{array}$

$\begin{array}{rr}\text { DATE } & \mathrm{FT}^{3} / \mathrm{S} \\ 10-10-60 & 2.64 \\ 09-26-61 & 0.75 \\ 10-01-63 & 0.35 \\ 10-12-64 & 1.78 \\ Q(84) & =0.3\end{array}$

DATE O8-15-66 $08-15-66$
$09-11-67$ $06-11-68$ $09-11-68$

$F T^{T 0} / S$ 0.44 0.01

0.04
0.25

$7 Q 2=*$

\section{DATE}

$10-09-69$

$09-01-70$

$09-02-71$

$10-16-72$

$\mathrm{FT}^{\mathrm{s}} / \mathrm{S}$

0.80

0.

0.48

2.0

DATE

09-11-73

$09-26-74$

$10-21-75$

$08-25-76$

$\mathrm{FT}^{\mathrm{S}} / \mathrm{S}$

3.1

4.2
3.7

3.7
0.04 
FLOYD RIVER BASIN--Cont inuled

6-6000.60 ELOYD R BELOW SHELDON, IOWA

LAT 430738 , LONG 955327 , IN N $1 / 2$ SEC.
$23, T .96 \mathrm{~N}$., R. 43 W., SIOUX CO. (84),

AT BRIDGE, 4 MILES SW OF SHELDON.

DRAINAGE AREA $165 \mathrm{MI}^{2}$

\begin{tabular}{|c|c|c|c|c|c|c|c|c|c|}
\hline $\begin{array}{c}\text { DATE } \\
10-01-57 \\
05-14-58 \\
10-15-58 \\
05-19-59 \\
08-12-59 \\
10-10-60\end{array}$ & $\begin{array}{l}\mathrm{FT} / \mathrm{S} \\
1.31 \\
3.87 \\
0 . \\
6.42 \\
1.67 \\
15.1\end{array}$ & $\begin{array}{c}\text { DATE } \\
10-10-60 \\
09-2 E_{1}-61 \\
10-01-63 \\
10-13-64 \\
08-15-66 \\
09-11-67\end{array}$ & $\begin{array}{l}F T / S \\
14.1 \\
2.05 \\
0.30 \\
6.46 \\
4.812 \\
0.45\end{array}$ & $\begin{array}{c}\text { EATE } \\
06-11-68 \\
09-11-68 \\
10-09-69 \\
09-01-70 \\
09-012-71 \\
08-17-72\end{array}$ & $\begin{array}{c}\mathrm{FT}^{\mathrm{S}} / \mathrm{S} \\
0.87 \\
0.50 \\
1.96 \\
0.56 \\
0.73 \\
11.6\end{array}$ & $\begin{array}{c}\text { DATE } \\
10-16-72 \\
09-11-73 \\
08-06-74 \\
09-26-74 \\
10-01-75 \\
10-21-75\end{array}$ & $\begin{array}{c}\mathrm{FT} / \mathrm{S} \\
4.93 \\
4.97 \\
2.26 \\
8.16 \\
13 . \\
9.4\end{array}$ & $\begin{array}{c}\text { DATE } \\
05-05-76 \\
07-06-76 \\
07-27-76 \\
08-25-76 \\
09-27-76\end{array}$ & $\begin{array}{l}F T^{3} / S \\
31 . \\
11 . \\
5.9 \\
1.6 \\
3.0\end{array}$ \\
\hline & $=38$ & Qr & $=1.2$ & 702 & $=0.3$ & 7010 & 0 & & \\
\hline
\end{tabular}

6-6001.20 DEEP CR NR OYENS, IOWA

LAT 424926, LONG 960653, IN SW $1 / 4$ SEC.

36, T. 93 N., R. 45 W., PLYMOUTH CO. $(75)$,

AT BRIDGE, 3 MILES NW OF OYENS.

DRAINAGE AREA $82.7 \quad \mathrm{MI}^{2}$

\begin{tabular}{|c|c|c|c|c|c|c|c|c|c|}
\hline $\begin{array}{c}\text { DATE } \\
09-30-57 \\
05-13-58 \\
10-15-58 \\
05-18-59 \\
08-11-59\end{array}$ & $\begin{array}{l}\mathrm{FT}^{3} / \mathrm{S} \\
1.14 \\
1.95 \\
0.01 \\
0.40 \\
2.66\end{array}$ & $\begin{array}{c}\text { DATE } \\
10-10-60 \\
09-27-61 \\
10-01-63 \\
10-13-64\end{array}$ & $\begin{array}{c}F T / S \\
3.55 \\
11.1 \\
0.93 \\
2.68\end{array}$ & $\begin{array}{c}\text { DATE } \\
08-16-66 \\
09-12-67 \\
06-12-68 \\
09-12-68\end{array}$ & $\begin{array}{r}\mathrm{FT}^{\mathrm{S}} / \mathrm{S} \\
2.38 \\
1.49 \\
0.49 \\
1.82\end{array}$ & $\begin{array}{c}\text { DATE } \\
10-10-69 \\
09-02-70 \\
09-02-71 \\
10-17-72\end{array}$ & $\begin{array}{l}\mathrm{FT}^{3} / \mathrm{S} \\
6.55 \\
2.23 \\
3.0 \\
3.0\end{array}$ & $\begin{array}{c}\text { DATE } \\
09-12-73 \\
09-26-74 \\
10-20-75 \\
08-25-76\end{array}$ & $\begin{array}{l}\mathrm{FT}^{3} / \mathrm{S} \\
2.8 \\
2.2 \\
2.4 \\
0.66\end{array}$ \\
\hline Qa & $=18$ & $Q C$ & $=1.6$ & $7 Q 2$ & $=0.5$ & 7010 & * & & \\
\hline
\end{tabular}

6-5001.40 WILLOW CR NR OYENS, IOWA

LAT 424942, LONG 960654, NEAR W 1/4

CORNER SEC. $36, \mathrm{~T} .93 \mathrm{~N} .$, R. 45 W., PLYMOUTH CO.

(75), A.T BRIDGE, 3 MILES NW OF OVENS.

DRAINAGE AREA $65.2 \quad \mathrm{MI}^{2}$

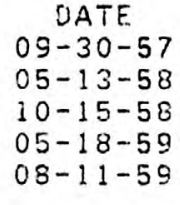

$\begin{array}{lcl}\text { FT } / S & \text { DATE } & F T^{3} / S \\ 0.06 & 10-10-60 & 1.36 \\ 0.12 & 09-27-61 & 2.03 \\ 0.1 & 10-01-63 & 0.12 \\ 0.02 & 10-12-64 & 0.42\end{array}$

$\begin{array}{cc}\text { DATE } & \text { FT } 0 / S \\ 08-16-66 & 0.09 \\ 09-12-67 & 0.04 \\ 06-12-68 & 0.0 \\ 09-12-68 & 0.02\end{array}$

$\begin{array}{cr}\text { DATE } & F T^{3} / S \\ 10-10-69 & 1.95 \\ 09-02-70 & 0.05 \\ 09-02-71 & 0.78\end{array}$

1.95
0.05 $09-02-71 \quad 0.78$

0.20
$7 Q 2=0$
$7010=0$
$Q_{a}=14$
$Q(84)=*$ 
FLOYD RIVER BASIN--Cont inued

6-6001.60 DEEP CR AT LE MARS, IOWA

LAT 424815, LONG 960928, IN NE $1 / 4$ SEC.

9, T.92 N., R.45 W., PLYMOUTH CO. $\{75\}$,

AT BRIDGE NEAR NORTH CITY LIMITS OF LE MARS.

\begin{abstract}
DRAINAGE AREA $156 \quad \mathrm{MI}^{2}$
\end{abstract}

\begin{tabular}{|c|c|c|c|c|c|c|c|c|c|}
\hline $\begin{array}{c}\text { DATE } \\
09-30-57 \\
05-13-58 \\
10-15-58 \\
05-18-59 \\
08-11-59\end{array}$ & $\begin{array}{l}F T=/ S \\
1.15 \\
2.22 \\
0 . \\
0.01 \\
3.57\end{array}$ & $\begin{array}{c}\text { DATE } \\
10-10-60 \\
0 S-27-61 \\
10-01-63 \\
10-14-64\end{array}$ & $\begin{array}{c}\mathrm{FT}^{\mathrm{a}} \text { is } \\
4.83 \\
13.5 \\
0.65 \\
2.95\end{array}$ & $\begin{array}{c}\text { DATE } \\
08-16-66 \\
09-12-67 \\
05-12-68 \\
09-12-68\end{array}$ & $\begin{array}{l}\mathrm{FT}^{3} / \mathrm{S} \\
2.62 \\
1.35 \\
0.31 \\
2.01\end{array}$ & $\begin{array}{c}\text { DATE } \\
10-10-69 \\
09-02-70 \\
09-02-71 \\
10-17-72\end{array}$ & $\begin{array}{l}\mathrm{FT}^{3} / \mathrm{S} \\
9.79 \\
2.05 \\
4.5 \\
0.8\end{array}$ & $\begin{array}{c}\text { DATE } \\
09-12-73 \\
09-26-74 \\
10-20-75 \\
08-25=76\end{array}$ & $\begin{array}{l}\mathrm{FT}^{3} / \mathrm{S} \\
4.7 \\
3.2 \\
3.7 \\
0.21\end{array}$ \\
\hline & $=36$ & $Q(\varepsilon$ & $y=1.6$ & $7 ?$ & $=0.3$ & 7010 & * & & \\
\hline
\end{tabular}

6-6001.80 FLOYD R AT LE MARS, IOWA

LAT 424802, LONG 961026, IN NW $1 / 4$ SEC.

9 , T.92 N., R. 45 W.., PLYMOUTH CO. (75),

AT BRIDGE NEAR NORTH CITY LIMITS OF LE MARS.

DRAINAGE AREA $478 \quad \mathrm{MI}^{2}$

\begin{tabular}{|c|c|c|c|c|c|c|c|c|c|}
\hline $\begin{array}{c}\text { DATE } \\
05-13-58 \\
10-15-58 \\
05-18-59 \\
08-11-59\end{array}$ & $\begin{array}{l}F T=/ S \\
13.8 \\
1.57 \\
15.6 \\
17.0\end{array}$ & $\begin{array}{c}\text { DATE } \\
10-10-60 \\
09-27-61 \\
10-01-63 \\
10-14-64\end{array}$ & $\begin{array}{l}\mathrm{FT}^{3} / \mathrm{S} \\
35.1 \\
21.1 \\
2.77 \\
12.1\end{array}$ & $\begin{array}{c}\text { DATE } \\
22-15-65 \\
09-12-67 \\
06-12-68 \\
09-12-68\end{array}$ & $\begin{array}{l}F T^{3} / S \\
2.56 \\
4.46 \\
2.03 \\
2.89\end{array}$ & $\begin{array}{c}\text { OATE } \\
10-10-59 \\
09-02-70 \\
09-02-71 \\
10-17-72\end{array}$ & $\begin{array}{l}\mathrm{FT}^{3} / \mathrm{S} \\
20.5 \\
5.36 \\
13.0 \\
18 .\end{array}$ & $\begin{array}{c}\text { DATE } \\
09-12-73 \\
09-26-74 \\
10-21-75 \\
08-25-76\end{array}$ & $\begin{array}{l}\mathrm{FT}^{3} / \mathrm{S} \\
2.9 . \\
18 . \\
2.1 . \\
4.1\end{array}$ \\
\hline Qa & $=118$ & $Q<8$ & $=7.6$ & 702 & $=2.2$ & 7010 & $0.4 * * *$ & & \\
\hline
\end{tabular}

6-6002.00 FLOYD R NR MERRILL, IDWA

LAT 424459 , LONG 961232 , IN NW $1 / 4$ SEC.
31, T.92 N., R. 45 W., PLYMOUTH CO. (75), AT, T. 92 N., R.45 W. PLYMOUTH CO. (75),

DRAINAGE AREA $489 \quad \mathrm{MI}^{2}$

$\begin{array}{cc}\text { DATE } & F T=/ S \\ 09-30-57 & 7.12 \\ 05-18-58 & 14.3 \\ 10-15-58 & 2.07 \\ 05-18-59 & 18.5 \\ 08-11-59 & 17.9\end{array}$

$Q_{\mathbf{a}}=121$

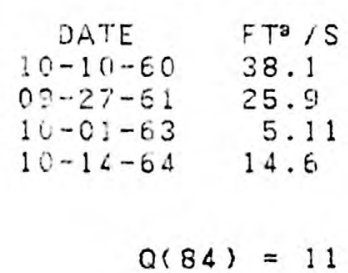

$Q(84)=11$

DATE
$08-16-66$
$09-12-57$
$06-12-68$
$09-12-68$
$F T^{3}, \bar{S}$
9.18
6.89
4.04
4.49

$7 Q 2$

4.0

$\begin{array}{cl}\text { DATE } & F T^{3} / S \\ 10-10-69 & 22.6 \\ 09-02-70 & 8.81 \\ 09-02-71 & 19.0 \\ 10-17-72 & 22 .\end{array}$

DATE
$09-12-73$
$09-26-74$
$10-21-75$
$08-25-76$

FT: is

36.

2.2 .

6.7 
FLOYD RIVER BASIN--Cont inued

6-6002.50 WB FLOYD R NR MIDLLEBURG, IOWA

LAT 430649 , LONG 960452 , IN $N E 1 / 4$ SEC.

30, T.96 N., R. 44 W. SIOUX CO. (84),

AT BRIDGE, ; MILE WEST OF MIDDLEBURG.

DRAINAGE AREA $59.7 \mathrm{MI}^{2}$

\begin{tabular}{|c|c|c|c|c|c|c|c|c|c|}
\hline $\begin{array}{c}\text { DATE } \\
10-01-57 \\
05-13-58 \\
10-15-58 \\
05-19-59 \\
08-12-59\end{array}$ & $\begin{array}{l}F T^{3 / S} \\
0 . \\
0.36 \\
0 . \\
0.08 \\
0.06\end{array}$ & $\begin{array}{c}\text { DATE } \\
10-10-60 \\
09-2 E-51 \\
10-01-63 \\
10-13-64\end{array}$ & $\begin{array}{l}\mathrm{FT}^{3} / \mathrm{S} \\
2.71 \\
0.93 \\
0.09 \\
0.14\end{array}$ & $\begin{array}{c}\text { DATE } \\
08-15-66 \\
09-11-67 \\
06-11-68 \\
09-11-68\end{array}$ & $\begin{array}{l}\mathrm{FT}^{\mathrm{S}} / \mathrm{S} \\
1.21 \\
0.07 \\
0.01 \\
0 .\end{array}$ & $\begin{array}{c}\text { DATE } \\
10-09-69 \\
09-01-70 \\
09-01-71 \\
10-16-72\end{array}$ & $\begin{array}{l}\mathrm{FT}^{3} / \mathrm{S} \\
0.15 \\
0.04 \\
0.14 \\
0.38\end{array}$ & $\begin{array}{c}\text { DATE } \\
09-11-73 \\
09-25-74 \\
10-20-75 \\
08-25-76\end{array}$ & $\begin{array}{l}F T^{n} / \mathrm{S} \\
2.4 \\
0.08 \\
0.05 \\
0.04\end{array}$ \\
\hline & $=13$ & $Q 1$ & $1=\star$ & $7 Q 2$ & $=0$ & $7 Q 10$ & 0 & & \\
\hline
\end{tabular}

6-6004.00 WB FLOYD R NR MERRILL, IOWA

LAT 424459, LONG 961426, IN NE $1 / 4$ SEC.

35, T. 92 N., R. 46 W. . PLYMOUTH CO. (75), AT BRIOGE, 2 MILES NORTH OF MERRILL.

DRAINAGE AREA $232 \quad M I^{2}$

$\begin{array}{cr}\text { DATE } & F^{3} / S \\ 09-30-57 & 5.65 \\ 05-13-58 & 6.26 \\ 10-15-58 & 0.92 \\ 05-18-59 & 2.65 \\ 08-11-59 & 4.99\end{array}$

$\begin{array}{cc}\text { DATE } & F^{3} / \mathrm{S} \\ 10-10-60 & 11.5 \\ 09-27-61 & 9.91 \\ 10-01-63 & 2.85 \\ 10-14-64 & 3.94\end{array}$

$\begin{array}{cr}\text { DATE } & F Y^{3} / S \\ 08-16-66 & 4.60 \\ 09-12-67 & 3.09 \\ 06-12-68 & 1.76 \\ 09-12-68 & 1.40\end{array}$

$\begin{array}{cc}\text { DATE } & F T / S \\ 10-10-69 & 9.68 \\ 09-02-70 & 5.24 \\ 09-02-71 & 8.1 \\ 10-17-72 & 10.1\end{array}$

DATE $09-12-73$ $09-26-74$ $10-21-75$ $\mathrm{FT}^{3} / \mathrm{S}$

$Q_{\mathbf{a}}=55$

$$
Q(84)=4.3
$$$$
7 Q 2=1.8
$$

$7 Q 10=0.5^{* \star *}$

MONONA-HARRISON DITCH BASIN

6-6015.00 BIG WHISKEY SLOUGH NR KINGSLEY, IOWA

LAT $4240 \times X$, LONG $9552 \times X$. NEAR S $1 / 4$

CORNER SEC.25, T.91 N.. R. 43 W., PLYMOUTH CO.

(75), AT BRIDGE. 7 MILES NE OF KINGSLEY.

DRAINAGE AREA $55.3 \mathrm{MI}^{2}$

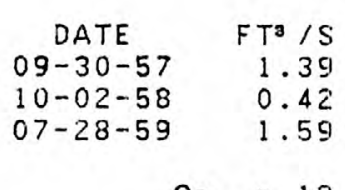

$\begin{array}{cr}\text { DATE } & \text { FT } / S \\ 10-04-60 & 3.52 \\ 08-30-61 & 8.59 \\ 10-15-64 & 2.04\end{array}$

$\begin{array}{cr}\text { DATE } & F T^{3} / 5 \\ 09-08-66 & 0.87 \\ 05-2.4-57 & 0.94 \\ 03-26-68 & 1.14\end{array}$
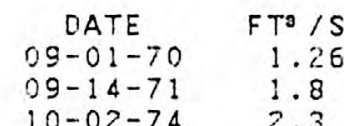

$10-15-64 \quad 2.04$

$7 Q 2=0.8$

$7 Q 10=0.2 * \star \star$ 
MONONA-HARRISON DITCH BASIN--COntinued

6-6016.00 WF L SIOUX R NR FIELDING, IOWA

LAT $4239 \times X$, LONG $9552 \times X$, IN NW $1 / 4$ SEC.

1, T. 90 N., R. 43 W. PLYMOUTH CO. (75),

AT BRIDGE, 4 MILES SW OF FIELDING.

DRAINAGE AREA $135 \quad \mathrm{MI}^{2}$

$\begin{array}{rrll}\text { DATE } & F T=i S & \text { DATE } & F T^{3} / S \\ 09-20-57 & 3.79 & 10-04-60 & 10.2 \\ 10-02-58 & 2.09 & 08-30-61 & 27.1 \\ 07-23-59 & 3.91 & 10-15-64 & 7.03 \\ Q a=31 & Q(84)=4.4\end{array}$

$\begin{array}{cr}\text { DATE } & \text { FT } 3 / S \\ 09-018-66 & 3.19 \\ 05-24-67 & 3.49 \\ 03-26-68 & 3.11\end{array}$

$\begin{array}{cc}\text { DATE } & F T^{3} / S \\ 09-01-70 & 3.34 \\ 09-14-71 & 3.9 \\ 10-02-74 & 6.2\end{array}$

DATE

$10-08-75$

$F T^{3} / S$

6-6017.00 WF L SIOUX R NR KINGSLEY, IOWA

LAT $4235 \times X$, LONG $9600 \times X$, IN NW $1 / 4$ SEC. 25, T.90 N., R. 44 W., PLYMOUTH CO. (75) AT BRIUGE, I MILE WEST OF KINGSLEY.

DRAINAGE AREA $219 \quad \mathrm{MI}^{2}$

$\begin{array}{cccc}=-T E & F T^{3} / S & \text { OATE } & F T^{3} \text { iS } \\ 09-30-57 & 7.17 & 10-04-60 & 21.2 \\ 10-02-58 & 2.12 & 08-30-61 & 32.5 \\ 07-25-59 & 11.7 & 10-15-64 & 10.6\end{array}$

$\begin{array}{cr}\text { DATE } & F T^{3} / S \\ 09-0.6-66 & 5.20 \\ 05-24-67 & 7.77 \\ 03-2.6-68 & 5.24\end{array}$

$\begin{array}{cc}\text { DATE } & F T / S \\ 09-01-70 & 5.62 \\ 09-14-71 & 8.5 \\ : 002-74 & : 2 .\end{array}$

6-6012.20 MUD CR AT MOVILLE, IOWA

LAT 422928, LONG 960524 , IN SW $1 / 4$ SEC.

30, T.E 9 N., R, 44. W. WOODEURY CO. 〈97;

AT BRIGIGE, I MILE WEST OF MOVILLE.

DRAINAGE AREA $68.7 \mathrm{MI}^{2}$

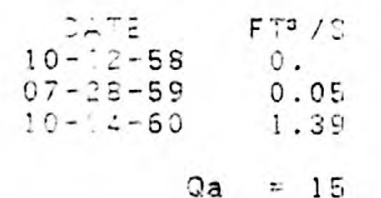

$2 a=15$

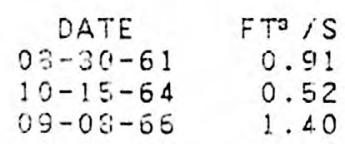

$09-03-66$

$Q(84)=0.1$

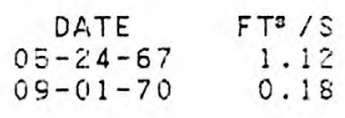

$7 Q 2$

\section{OATE
O9-14-7
$10-02-74$}

$10-02-74$

$F T^{3} / S$
0.74
0.65

DAT

$10-07-75$

$08-12-76$

$F T^{2} / S$
0.77

0.06

$7010=0$ 
MONONA-HARRISON DITCH EASIN--Cont inuEd

6-6019.00 WF L SIOUX R AT MOVILLE, IOWA

LAT 422830, LONG 960439 , IN SE $1 / 4$ SEC.

$31, T .89$ N., R. 44. W., WOODEURY CO. ( 97 ),

AT BRIDGE ON U. S. HIGHWAY $20,1 / 2$ MILE

SW OF MOVILLE.

DRAINAGE AREA $344 \quad \mathrm{MI}^{2}$

\begin{tabular}{|c|c|c|c|c|c|c|c|c|c|}
\hline $\begin{array}{c}\text { DATE } \\
09-25-57 \\
10-02-58 \\
07-28-59\end{array}$ & $\begin{array}{l}F T^{3} / S \\
11.1 \\
3.25 \\
12.2\end{array}$ & $\begin{array}{c}\text { DATE } \\
10-04-60 \\
08-30-61 \\
10-15-64\end{array}$ & $\begin{array}{l}\mathrm{FT}^{3} / \mathrm{S} \\
32.3 \\
41.0 \\
15.4\end{array}$ & $\begin{array}{c}\text { DATE } \\
09-08-66 \\
05-24-67 \\
03-26-68\end{array}$ & $\begin{array}{l}F T^{8 / S} \\
10.2 \\
10.1 \\
10.2\end{array}$ & $\begin{array}{c}\text { DATE } \\
09-01-70 \\
09-14-71 \\
10-02-74\end{array}$ & $\begin{array}{l}F T=1 \mathrm{~S} \\
10.6 \\
12.0 \\
14 .\end{array}$ & $\begin{array}{c}\text { DATE } \\
10-07-75 \\
08-13-76\end{array}$ & $\begin{array}{l}\mathrm{FT}^{3} / \mathrm{s} \\
22 . \\
8.1\end{array}$ \\
\hline Qa & $=83$ & $Q 18$ & $=11$ & $7 Q 2$ & $=6.2$ & $7 Q 10$ & $2.4 * \star$ & & \\
\hline
\end{tabular}

6-6022.00 ELLIOT CR NR BRONSON, IOWA

LAT 422353, LONG 961405, IN NE $1 / 4$ SEC.

31. T.88 N., R.4E W., WOODEURY CO. (97),

AT BRIDGE, 1.5 MILES SW OF BRONSON.

DRAINAGE AREA $58.6 \mathrm{MI}^{2}$

\begin{tabular}{|c|c|c|c|c|c|c|c|c|c|}
\hline $\begin{array}{c}\text { DATE } \\
09-23-57 \\
10-02-58 \\
07-28-59\end{array}$ & $\begin{array}{l}\mathrm{FT}^{3} / \mathrm{S} \\
1.01 \\
0.39 \\
0.39\end{array}$ & $\begin{array}{c}\text { DATE } \\
10-05-60 \\
08-31-61 \\
10-15-64\end{array}$ & $\begin{array}{l}F T^{3} / S \\
2.95 \\
1.06 \\
1.42\end{array}$ & $\begin{array}{c}\text { DATE } \\
09-(18-66 \\
05-24-67 \\
03-2.6-68\end{array}$ & $\begin{array}{r}F T^{3} / S \\
0.87 \\
0.95 \\
1.07\end{array}$ & $\begin{array}{c}\text { DATE } \\
09-02-70 \\
09-14-71 \\
10-02-74\end{array}$ & $\begin{array}{l}F^{3} / S \\
0.64 \\
0.62 \\
0.62\end{array}$ & $\begin{array}{c}\text { DATE } \\
10-08-75 \\
0 \mathrm{~S}-12-76\end{array}$ & $\begin{array}{l}\mathrm{FT}, 1 \mathrm{~S} \\
2.7 \\
0.81\end{array}$ \\
\hline & $=13$ & Q & $=1.1$ & $7 Q 2$ & $=0.7$ & 7010 & $=0.4 * \star$ & & \\
\hline
\end{tabular}

6-6022.50 BIG WHISKEY CR NR BRONSON, IOWA

LAT 422404, LONG 961429. IN NE $1 / 4$ SEC.

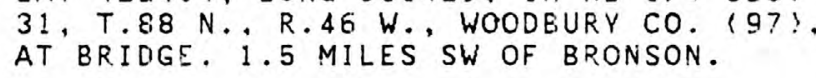

DRAINAGE AREA $62.4 \mathrm{MI}^{2}$

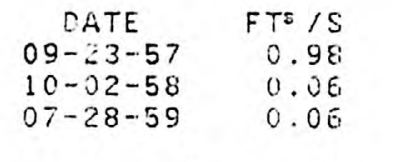

$Q_{a}=14$

$\begin{array}{cc}D A T E & F T^{3} / S \\ 10-05-60 & 2.614 \\ 08-31-61 & 0.98 \\ 10-15-64 & 0.618\end{array}$

$Q(84)=0.7$

$\begin{array}{cc}\text { DA.TE } & F T=/ S \\ 09-08-66 & 1.37 \\ 05-24-67 & 1.27 \\ 03-2.6-68 & 1.34\end{array}$

$T Q 2=0.3$

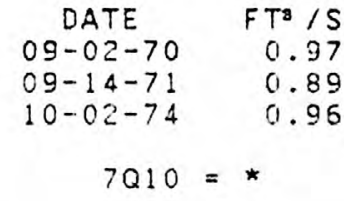

$\begin{array}{cc}\text { DATE } & F T^{3} / S \\ 10-08-75 & 3.8 \\ 08-12-76 & 0.90\end{array}$


MONONA-HARRISON DITCH BASIN--Continued

5-6023.00 WOLF CR NR HOLLY SPRINGS, IOWA

LAT 421806, LONG 960110 , IN SN $1 / 4$ SEC.

31 , T.87 N., R.44. W., WOODEURY CO. (97),

AT BRIDGE, 4 MILES NE OF HOLLY SPRINGS.

DRAINAGE AREA $99.2 \mathrm{MI}^{2}$

\begin{tabular}{|c|c|c|c|c|c|c|c|c|c|}
\hline $\begin{array}{c}\text { DATE } \\
09-25-57 \\
10-02-58 \\
07-28-59\end{array}$ & $\begin{array}{r}\mathrm{FT}^{3} / \mathrm{S} \\
3.61 \\
1.11 \\
1.76\end{array}$ & $\begin{array}{c}\text { DATE } \\
10-04-60 \\
08-31-61 \\
10-15-64\end{array}$ & $\begin{array}{c}\mathrm{FT}^{3} / \mathrm{S} \\
11.7 \\
3.29 \\
6.83\end{array}$ & $\begin{array}{c}\text { DATE } \\
09-08-66 \\
05-24-67 \\
03-26-58\end{array}$ & $\begin{array}{r}\mathrm{FT}^{3} / \mathrm{S} \\
3.61 \\
1.77 \\
3.05\end{array}$ & $\begin{array}{c}\text { DATE } \\
09-01-70 \\
09-14-71 \\
10-02-74\end{array}$ & $\begin{array}{c}F T^{3} / \mathrm{S} \\
1.06 \\
1.3 \\
6.0\end{array}$ & $\begin{array}{c}\text { DATE } \\
10-08-75 \\
08-12-76\end{array}$ & $\begin{array}{c}\mathrm{FT}^{3} / \mathrm{S} \\
9.6 \\
3.5\end{array}$ \\
\hline
\end{tabular}

LITTLE SIOUX RIVER BASIN

6-6036.00 L SIOUX R NR MONTGOMERY, IOWA

LAT $4326 \times X$, LCNG $9515 \times X$, IN NE $1 / 4$ SEC.

$6, T .99 \mathrm{~N} .$, R.37 W., DICKINSON CO. (30)

AT BRILIGE ON STATE HIGHWAY $9,2.5$ MILES

SW OF MONTGOMERY.

DRAINAGE AREA $118 \quad \mathrm{MI}^{2}$

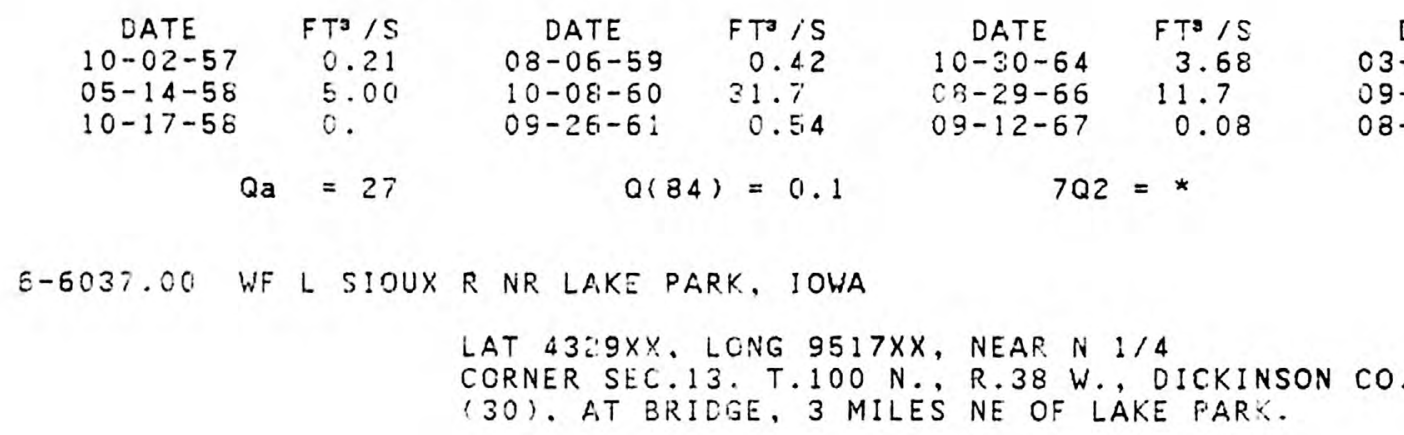

E-6037.00 WF L SIOUX R NR LAKE PARK, IOWA

LAT 43:9XX. LONG $9517 \times X$, NEAR N $1 / 4$

CORNER SEC.13. T.100 N., R.38 W., DICKINSON CO

(30). AT BRIDGE, 3 MILES NE OF LAKE FARK.

ORAINAGE AREA $116 \quad \mathrm{MI}^{2}$

\begin{tabular}{|c|c|}
\hline $\begin{array}{c}\text { DATE } \\
10-02-57 \\
05-15-58 \\
10-17-58\end{array}$ & $\begin{array}{c}F / S \\
0.77 \\
4.92 \\
0 .\end{array}$ \\
\hline & $=26$ \\
\hline
\end{tabular}

$\begin{array}{rc}\text { DATE } & F^{3} / S \\ 08-0 E_{i}-50 & 0 . \\ 10-0 \varepsilon_{1}-60 & 41.8 \\ 09-26_{1}-61 & 0.11 \\ C(34) & =0.2\end{array}$

$\begin{array}{rl}\text { DATE } & F T O / S \\ 10-30-64 & 2.26 \\ 03-29-66 & 1.85 \\ 09-12-67 & 0 . \\ 7 Q 2= & 0\end{array}$

$\begin{array}{cc}\text { DATE } & \text { FT }^{3} / \mathrm{S} \\ 03-26-58 & 0.05 \\ 09-01-70 & 5.93 \\ 08-09-71 & 1.9 \\ 7 Q 10= & 0\end{array}$

DATE

$10-C 1-74$

$101-08-75$

O $8-12-76$

$T^{2} / 5$

0 .

0 . 
LITTLE SIOUX RIVER BASIN--Cont inued

6-6038.00 WF L SIOUX R NR MONTGOMERY, IOWA

LAT $4325 \times X$, LONG $9516 \times X$, IN SW $1 / 4$ SEC.

$6, T .99 \mathrm{~N} .$, R.37 W., DICKINSON CO. (30),

AT BRIUGE, 4 MILES SW OF MONTGOMERY.

\begin{abstract}
DRAINAGE AREA $173 \quad \mathrm{MI}^{2}$
\end{abstract}

$\begin{array}{rr}\text { DATE } & F T^{3} / S \\ 10-02-57 & 1.32 \\ 05-14-58 & 7.17 \\ 10-17-58 & 0 . \\ Q a & =40\end{array}$

$\begin{array}{rc}\text { DATE } & F T^{3} / S \\ \text { O8-06-59 } & 0.01 \\ 10-08-60 & 63.1 \\ 09-26-61 & 0.54 \\ \text { Q(84) } & =0.7\end{array}$

$\begin{array}{rr}\text { DATE } & F T^{3} / S \\ 10-30-64 & 6.11 \\ 08-29-66 & 2.56 \\ 09-12-67 & 0.01 \\ 7 Q 2= & 0.1\end{array}$

$\begin{array}{cc}\text { DATE } & \mathrm{FT}^{3} / \mathrm{S} \\ 03-26-68 & 0.78 \\ 09-01-70 & 5.88 \\ 08-09-71 & 2.3\end{array}$

DATE

$10-08-75 \quad 0.85$

$Q_{a}=40$

$Q(84)=0.7$

$7 Q 10=0.1 * \star$

6-6039.00 L SIOUX R NR MILFORD, IOWA

LAT $4319 \times X$, LONG $9511 \times X$, NEAR CENTER OF

SEC.11, T.98 N., R.37 W., DICKINSON CO.

(30), AT BRIDGE, 1.5 MILES SW OF MILFORO.

DRAINAGE AREA $333 \quad \mathrm{MI}^{2}$

$\begin{array}{cc}\text { DATE } & F T^{3} / S \\ 10-01-57 & 3.92 \\ 05-15-58 & 16.3 \\ 10-17-58 & 0 .\end{array}$

$\begin{array}{cc}\text { DATE } & F T^{3} / S \\ 08-06-59 & 0.68 \\ 10-08-60 & 1: 6.5 \\ 09-26-61 & 2.52\end{array}$

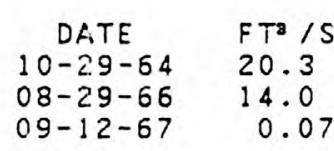

$a\langle 84\rangle=1.5$

$702=0.2$

$\begin{array}{cc}\text { DATE } & F T^{3} / S \\ 03-26-68 & 2.80 \\ 09-01-70 & 8.97 \\ 08-09-71 & 7.0\end{array}$

DATE
$10-02-74$

$10-08-75 \quad 3.5$

$Q_{a}=80$

\begin{abstract}
ORD, IOWA
\end{abstract}
LAT 431914, LONG 950841 , IN SW $1 / 4$ SEC.

7, T.98 N., R.36 W., DICKINSON CO. (30),

AT BRIQIGE AT EAST EDGE OF MILFORD.

\begin{abstract}
ORAINAGE AREA $146 \quad \mathrm{MI}^{2}$
\end{abstract}

$\begin{array}{rr}\text { DATE } & F^{3} / S \\ 10-01-57 & 0.79 \\ 05-15-58 & 2.58 \\ 10-17-58 & 0.90 \\ \text { Qa } & =35\end{array}$

$$
\begin{array}{cr}
\text { DATE } & \text { FT } / S \\
08-06-59 & 1.46 \\
10-28-60 & 2.31 \\
09-26-61 & 1.95
\end{array}
$$

$\begin{array}{cr}\text { DATE } & F T^{3} / S \\ 10-2.9-64 & 4.62 \\ 08-2.9-66 & 1.97 \\ 09-12-67 & 3.81\end{array}$

$0(84)=2.0$

$7 Q 2=1.5$

$\begin{array}{cc}\text { DATE } & \mathrm{FT}^{3} / \mathrm{S} \\ 08-09-71 & 6.9 \\ 08-12-76 & 2.5\end{array}$


LITTLE SIOUX RIVER BASIN--Cont i nued

6-6045.00 OCHEYEDAN R NR BIGELOW, MINN.

LAT $4327 \times X$, LONG $9537 \times X$, IN SE $1 / 4$ SEC.

$24, T .100$
AT BRIDGE IN IOWA, 4.5 MILES SE OF BIGELOW.

DRAINAGE AREA $68.7 \mathrm{MI}^{2}$

\begin{tabular}{|c|c|c|c|c|c|c|c|c|c|}
\hline $\begin{array}{c}\text { DATE } \\
10-02-57 \\
05-14-58 \\
10-17-58 \\
08-05-59\end{array}$ & $\begin{array}{l}\mathrm{FT}^{3} / \mathrm{S} \\
0.23 \\
0.69 \\
0.0 \\
0.05\end{array}$ & $\begin{array}{c}\text { DATE } \\
10-07-60 \\
09-26-61 \\
10-30-64 \\
06-15-66\end{array}$ & $\begin{array}{l}\mathrm{FT}^{3} / \mathrm{S} \\
17.3 \\
0.15 \\
0.54 \\
1.56\end{array}$ & $\begin{array}{c}\text { DATE } \\
07-12-66 \\
08-29-66 \\
09-12-67\end{array}$ & $\begin{array}{l}F^{3} / S \\
0.27 \\
0.60 \\
0.03\end{array}$ & $\begin{array}{c}\text { DATE } \\
03-26-68 \\
09-01-70 \\
08-09-71\end{array}$ & $\begin{array}{c}F T^{3} / \mathrm{S} \\
0.01 \\
10.2 \\
0.86\end{array}$ & $\begin{array}{c}\text { DATE } \\
10-01-74 \\
10-08-75 \\
08-12-76\end{array}$ & $\begin{array}{l}\mathrm{FT}^{3} / \mathrm{S} \\
0.04 \\
0 . \\
0 .\end{array}$ \\
\hline$Q$ & $=15$ & $Q<\varepsilon$ & $=0.1$ & $7 Q 2$ & $=*$ & $7 Q 10$ & $0 * *$ & & \\
\hline
\end{tabular}

6-6046.00 L OCHEYEDAN R NR MAY CITY, IOWA

LAT $4317 \times X$, LONG $9528 \times X$, IN NE $1 / 4$ SEC.

29, T.98 N., R.39 W., OSCEOLA CO. (72),

AT BRIDGE, 3 MILES SOUTH OF MAY CITY.

DRAINAGE AREA $54.2 \mathrm{MI}^{2}$

\begin{tabular}{|c|c|c|c|c|c|c|c|c|c|}
\hline $\begin{array}{c}\text { DATE } \\
10-02-57 \\
05-14-58 \\
10-17-58\end{array}$ & $\begin{array}{l}\mathrm{FT}^{\mathrm{a}} / \mathrm{S} \\
1.16 \\
4.75 \\
0 .\end{array}$ & $\begin{array}{c}\text { DATE } \\
08-06-59 \\
10-07-60 \\
09-26-61\end{array}$ & $\begin{array}{c}\mathrm{FT}^{\mathrm{a}} / \mathrm{S} \\
0.34 \\
12.8 \\
0.20\end{array}$ & $\begin{array}{c}\text { DATE } \\
10-30-64 \\
08-30-66 \\
09-12-67\end{array}$ & $\begin{array}{r}F T / S \\
1.86 \\
1.45 \\
0.01\end{array}$ & $\begin{array}{c}\text { DATE } \\
03-26-68 \\
09-01-70 \\
08-10-71\end{array}$ & $\begin{array}{l}F T^{2} / \mathrm{S} \\
0.04 \\
0.02 \\
0.52\end{array}$ & $\begin{array}{c}\text { DATE } \\
10-01-74 \\
10-08-75 \\
08-12-76\end{array}$ & $\begin{array}{l}\mathrm{FT}^{\mathrm{a}} / \mathrm{S} \\
1.5 \\
4.8 \\
0.14\end{array}$ \\
\hline & $=12$ & Qr & $=0.1$ & 702 & $=\star *$ & $7 Q 10$ & $0 * *$ & & \\
\hline
\end{tabular}

6-6047.00 OCHEYEDAN R NR MAY CITY, IOWA

LAT $4316 \times X$, LONG $9527 \times X$, NEAR $N 1 / 4$ CORNER SEC.34, T.98 N., R.39 W. OSCEOLA CO.

(72), AT BRIDGE, 4 MILES SE OF MAY CITY.

DRAINAGE AREA $226 \quad \mathrm{MI}^{2}$

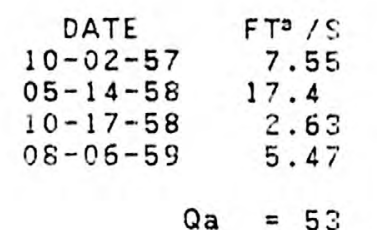

$Q_{a}=53$

$\begin{array}{cc}\text { DATE } & F T^{3} / S \\ 10-07-60 & 60.9 \\ 09-26-61 & 5.26 \\ 10-30-64 & 11.0 \\ 06-15-66 & 28.4\end{array}$

$Q(84)=6.4$

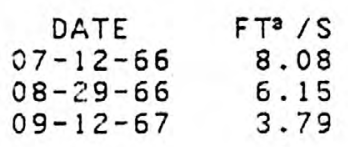

$7 Q 2=3.7$

$\begin{array}{cc}\text { DATE } & F T^{3} / S \\ 03-26-68 & 2.71 \\ 09-01-70 & 18.2 \\ 08-10-71 & 12.0\end{array}$

DATE
$10-01-74$
$10-08-75$

$10-08-75$

$08-12-76$

$\mathrm{FT}^{3}$ is

8.6

20.8 
LITTLE SIOUX RIVER BASIN--Continued

6-6048.00 STONEY CR NR FOSTORIA, IOWA

LAT $4314 \times X$, LONG $9520 \times X$, IN NW $1 / 4$ SEC.
10, T.97 N., R. 38 W., CLAY CO. (21),

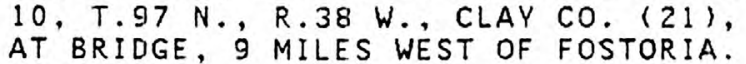

DRAINAGE AREA $65.4 \mathrm{MI}=$

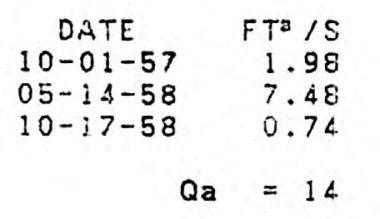

$\begin{array}{rccr}\text { DATE } & \text { FT } / S & \text { DATE } & F^{3} / S \\ 08-06-59 & 1.17 & 10-30-64 & 8.40 \\ 10-07-60 & 16.6 & 08-30-66 & 2.26 \\ 09-26-61 & 4.16 & 09-12-67 & 0.97 \\ \text { Q(84) }=2.4 & 702=1.1\end{array}$

$\begin{array}{rr}\text { DATE } & F T^{2} / S \\ 03-2 E-68 & 1.49 \\ 09-02-70 & 1.08 \\ 08-10-71 & 4.1 \\ 7010= & 0.2\end{array}$

$\begin{array}{cc}\text { DATE } & F T / S \\ 10-02-74 & 1.5 \\ 10-07-75 & 6.6 \\ 08-12-76 & 1.2\end{array}$

6-6049.00 STONEY CR NR EVERLY, IOWA

LAT 430922 , LONG 951458 , IN NE $1 / 4$ SEC.
7, T.96 N., R.37 W., CLAY CO. (21),

$7, T .96 \mathrm{~N} .$, R. $37 \mathrm{~W} .$, CLAY CO. (21)
AT BRIDGE, 4 MILES SE OF EVERLY.

DRAINAGE AREA $81.6 \mathrm{MI}^{2}$

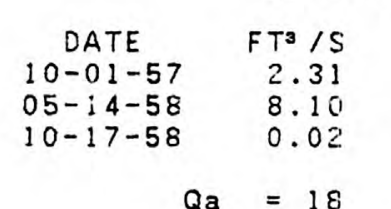

$Q_{a}=18$

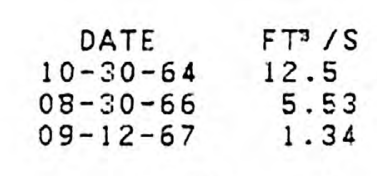

$7 Q 2=1.2$

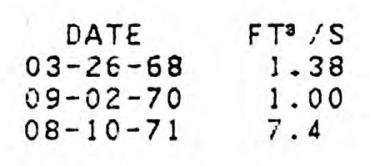

$7 Q 10=0.2$

6-6050.00 OCHEYEDAN R NR SPENCER, IOWA

LAT 430744, LONG 951237, IN SW $1 / 4$ SEC. 15, T. 96 N., R.37 W., CLAY CO. (21), AT BRIDGE, 3 MILES SW OF SFENCER.

\begin{abstract}
URAINAGE AREA $426 \quad \mathrm{MI}^{2}$
\end{abstract}

$\begin{array}{cl}\text { DATE } & F T / S \\ 10-01-57 & 13.0 \\ 05-14-58 & 40.0 \\ 10-17-58 & 2.95\end{array}$

$\begin{array}{cl}\text { DATE } & F T^{3} \text { is } \\ 08-05-59 & 14.9 \\ 10-07-60 & 98.8 \\ 09-26-61 & 16.0\end{array}$

$Q \mathbf{Q}=1014$

\begin{abstract}
$Q(84)=12$
\end{abstract}

\begin{abstract}
$7 Q 2=5.2$
\end{abstract}
$\begin{array}{cc}\text { DATE } & F T^{3} / \mathrm{S} \\ 03-26-58 & 8.70 \\ 09-02-70 & 19.8 \\ 08-10-71 & 31.0\end{array}$ DATE
$10-02-74$
$10-07-75$
$08-12-76$

$F T^{3} / S$

12 .

4.5.

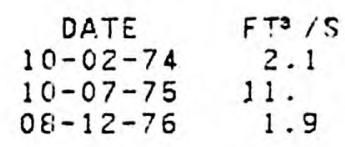

$08-12-76 \quad 1.9$

Q

$7 Q 10=0.4 * *$ 
LITTLE SIOUX RIVER BASIN--Continued

6-6051.00 L SIOUX R AT SPENCER, IOWA

LAT 430813 , LONG 950839 , IN N $1 / 2$ SEC.

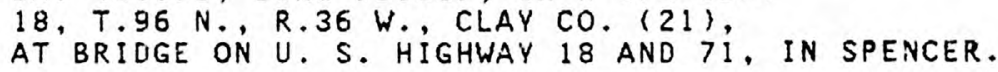

DRAINAGE AREA $990 \quad \mathrm{MI}^{2}$

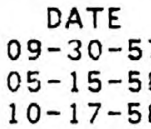

$\begin{array}{lcc}\mathrm{FT}^{3} / \mathrm{S} & \mathrm{DATE} & \mathrm{FT}^{3} / \mathrm{S} \\ 29.5 & 08-05-59 & 28.8 \\ 67.3 & 10-07-60 & 246 . \\ 7.44 & 09-26-61 & 32.7\end{array}$

$\begin{array}{rl}\text { DATE } & F^{3} / \mathrm{S} \\ 10-30-64 & 75.2 \\ 08-30-66 & 22.8 \\ 09-12-67 & 14.4 \\ 7 Q 2= & 14\end{array}$

DATE
$03-26-68$
$09-02-70$
$09-02-70 \quad 37.2$ $08-10-71 \quad 60.0$

$\mathrm{FT}^{3} / \mathrm{S}$

25.9

DATE $10-02-74$
$10-07-75$

$7010=5.8$

6-6052.00 BIG MUDDY CR NR LANGDON, IOWA

LAT 431149 , LONG 950411 , IN NW $1 / 4$ SEC.

26, T.97 N., R.36 W., CLAY CO. (21),

AT BRIDGE, I.5 MILES SE OF LANGDON.

DRAINAGE AREA. $59.7 \mathrm{MI}^{2}$

$\begin{array}{cc}\text { DATE } & F T^{3} / \\ 09-30-57 & 0.6 \\ 05-15-58 & 4.3 \\ 10-17-58 & 0.30\end{array}$

$\begin{array}{cr}\text { DATE } & F T^{3} / S \\ 08-05-59 & 0.88 \\ 10-07-60 & 7.92 \\ 09-26-61 & 1.07\end{array}$
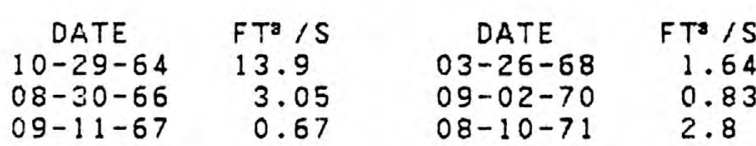

DATE

$10-02-74$

$10-07-75$

$\mathrm{FT}$ is

$08-10-71$

2.8

$08-12-76$

$Q_{\mathbf{a}}=13$

$Q(84)=1.2$

$7 Q 2=0.5$

$7 Q 10=0 * *$

6-6053.00 BIG MUDDY CR NR SPENCER, IOWA

LAT 430828 , LONG 950514 , IN NW $1 / 4$ SEC.

15, T.96 N., R. 36 W. , CLAY CO. (21),

AT BRIDGE, 3 MILES EAST OF SPENCER.

DRAINAGE AREA $102 \quad \mathrm{MI}^{2}$

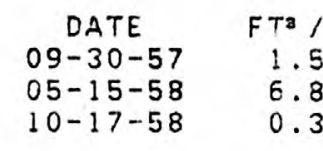

$Q_{a}=23$

$$
\begin{array}{cc}
\text { DATE } & F^{3} / S \\
08-05-59 & 1.75 \\
10-07-60 & 10.3 \\
09-26-61 & 2.94
\end{array}
$$

$Q(84)=2.5$

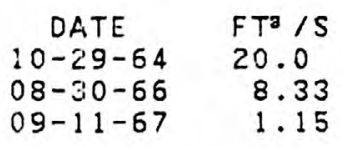

$09-11-67 \quad 1.15$

$702=1.1$

$\begin{array}{cc}\text { DATE } & \mathrm{FT}^{3} / \mathrm{S} \\ 03-25-68 & 3.43 \\ 09-02-70 & 1.97 \\ 08-10-71 & 6.5\end{array}$

DATE

$10-02-74$

$10-07-75$

$08-12-76$

$\mathrm{FT}^{3} / \mathrm{S}$

$08-10-71$

6.5

$7 Q 10=0.1 * *$
8.2

1.2 
LITTLE SIOUX RIVER BASIN--Cont inued

6-6054.00 PICKEREL RUN NR SPENCER, IOWA

$$
\begin{aligned}
& \text { LAT } 4312 \times X, \text { LONG } 9458 \times X \text {, IN NW } 1 / 4 \text { SEC. } \\
& 27,97 N \text {. R. } 35 \text { W. CLAY CO. (21), } \\
& \text { AT BRIOGE, S MILES NE OF SPENCER. }
\end{aligned}
$$

\begin{tabular}{|c|c|c|c|c|c|}
\hline $\begin{array}{c}\text { DATE } \\
09-30-57 \\
05-15-58 \\
10-17-58\end{array}$ & $\begin{array}{l}\mathrm{FT}^{3} / \mathrm{S} \\
0.18 \\
0.18 \\
0 .\end{array}$ & $\begin{array}{c}\text { DATE } \\
08-05-59 \\
10-07-60 \\
09-26-61\end{array}$ & $\begin{array}{l}\mathrm{FT}^{2} / \mathrm{S} \\
0.10 \\
0.70 \\
0.05\end{array}$ & $\begin{array}{c}\text { DATE } \\
10-29-64 \\
08-30-66 \\
09-11-67\end{array}$ & $\begin{array}{l}\mathrm{FT} / \mathrm{S} \\
18.4 \\
1.32 \\
0.04\end{array}$ \\
\hline Qa & $=17$ & & $=*$ & $7 Q 2$ & 0 \\
\hline
\end{tabular}

ORAINAGE AREA $75.7 \mathrm{MI}^{2}$

$\begin{array}{cc}\text { DATE } & F T^{0} / S \\ 03-26-68 & 0.66 \\ 09-02-70 & 0.03 \\ 08-10-71 & 8.7 \\ 7010= & 0\end{array}$

DATE

$10-02-74$
$10-07-75$

$10-07-75$
$08-12-76$

$\mathrm{FT}^{3} / \mathrm{S}$

0.47

0.47

$\mathrm{Q} \mathbf{a}=17$

$Q(84)=*$

$7 Q 10=0$

6-6055.00 LOST ISLAND OUTI.ET NR DICKENS, IOWA

LAT 430707 , LONG 950158 , AT W $1 / 4$

CORNER SEC.19, T. $96 \mathrm{~N}$., R. 35 W. O CLAY CO.
(21), AT ORIDGE, 1 MILE SOUTH OF DICKENS.

DRAINAGE AREA $151 \quad \mathrm{MI}^{2}$

$\begin{array}{rr}\text { DATE } & F T=/ S \\ 09-30-57 & 1.32 \\ 05-15-58 & 3.43 \\ 10-17-58 & 1.09 \\ \text { Qa } & =35\end{array}$

$\begin{array}{cr}\text { DATE } & F T 3 / S \\ 08-05-59 & 3.38 \\ 10-07-60 & 3.92 \\ 09-26-61 & 3.11\end{array}$
$\begin{array}{cl}\text { DATE } & F T^{3} / S \\ 10-30-64 & 45.1\end{array}$
$\begin{array}{rr}10-30-64 & 45.1 \\ 08-30-66 & 4.30 \\ 09-11-67 & 1.56\end{array}$
4.30
1.56

$7 Q 2=1.5$

$\begin{array}{cr}\text { DATE } & F^{3} / S \\ 03-28-68 & 2.08 \\ 09-02-70 & 2.54 \\ 08-10-71 & 28.0\end{array}$

DATE

$10-02-74$

$101-07-75$

$F T^{2} / S$

$F T^{2} / S$
2.7
6.5

$08-12-76 \quad 1.3$

6-6057.00 WILLOW CR NR ROSSI, IOWA

LAT $4259 \times X$, LONG $9510 X X$, IN SE $1 / 4$ SEC.

$4, T .94$ N., R.37 W. CLAY CO. $(21)$.

AT BRIUGE, 2 MILES SE OF ROSSI.

\begin{abstract}
DRAINAGE AREA $62.6 \quad \mathrm{MI}^{2}$
\end{abstract}

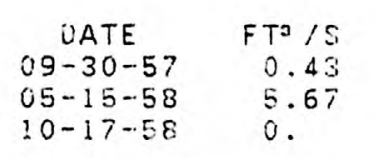

$Q \mathbf{a}=12$ $\begin{array}{cr}\text { DATE } & \text { FTJ /S } \\ 08-05-59 & 2.31 \\ 10-05-60 & 1.23 \\ 09-25-61 & 0.58\end{array}$

$Q(84)=x$

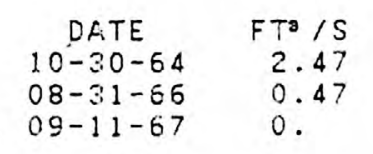

$7 Q 2=0$ 
LITTLE SIOUX RIVER BASIN--Cont I nued

6-6058.00 WILLOW CR NR GREENVILLE, IOWA

LAT $4259 \times X$, LONG $9509 \times X$, NEAR CENTER OF SEC.7, T.94 N., R. 36 W., CLAY CO. (21), AT BRIDGE, 3 MILES SOUTH OF GREENVILLE.

DRAINAGE AREA $30.3 \mathrm{MI}^{2}$

$\begin{array}{rccccr}\text { DATE } & F T^{3} / S & \text { DATE } & F T^{3} / S & \text { DATE } & F T^{3} / S \\ 09-30-57 & 0.29 & 08-06-59 & 3.89 & 10-30-64 & 4.60 \\ 05-15-58 & 8.69 & 10-06-60 & 2.34 & 08-31-66 & 4.24 \\ 10-17-58 & 0 . & 09-25-61 & 1.04 & 09-11-67 & 0.04 \\ & Q=20 & Q(84)=0.3 & 702=\star\end{array}$

6-6059.00 WATERMAN CR NR HARTLEY, IOWA

LAT $4305 \times X$, LONG $9527 \times X$, IN NE $1 / 4$ SEC.

4, T:95 N., R.39 W., O'BRIEN CO. (71),

AT BRIDGE, 6.5 MILES SE OF HARTLEY.

DRAINAGE AREA $58.4 \mathrm{MI}^{2}$

$\begin{array}{crcccr}\text { DATE } & \text { FT }^{3} / S & \text { DATE } & \text { FT /S } & \text { DATE } & F T^{3} / S \\ 10-02-57 & 0.18 & 08-13-59 & 0.16 & 10-30-64 & 1.40 \\ 05-14-58 & 1.47 & 10-06-60 & 3.74 & 08-31-66 & 0.59 \\ 10-16-58 & 0.01 & 09-25-61 & 0.66 & 09-11-67 & 0.01\end{array}$

$Q \mathbf{a}=13$

$Q(84)=0.1$

$7 Q 2=0$

6-6060.00 WATERMAN CR NR SUTHERLAND, IOWA

LAT $4257 \times X$, LONG $9525 \times X$, NEAR CENTER OF SEC. 23, T. 94 N., R. 39 W., O'BRIEN CO. (71), AT BRIDGE, 4.5 MILES SE OF SUTHERLAND.

ORAINAGE AREA $139 \mathrm{MI}^{2}$

$\begin{array}{rr}\text { DATE } & F T^{T} / S \\ 10-03-57 & 1.10 \\ 05-14-58 & 8.79 \\ 10-16-58 & 0.15 \\ Q a & =32\end{array}$

$\begin{array}{rr}D A T E & F T / S \\ 08-13-59 & 1.17 \\ 10-06-60 & 6.87 \\ 09-25-61 & 2.16 \\ Q(84) & =1.8\end{array}$

$\begin{array}{rr}\text { DATE } & F^{3} / S \\ 10-30-64 & 4.18 \\ 08-31-66 & 9.34 \\ 09-11-67 & 0.45 \\ 702= & 0.2\end{array}$

$Q a=32$

$Q(84)=1.8$
$F T^{3} / S$

0.31

0.18

5.1
DATE

$10-02-74$

$10-08-75$

08-12-76

$09-01-70$

0.02

7.7

$7010=0 * \star$
$F T=/ S$

1.0

$09-01-70$
$08-11-71$

DATE
$10-03-74$
$10-07-75$

$10-03-74$

$10-07-75$

$F T^{a} / S$

$7 Q 10=$ * 
LITTLE SIOUX RIVER BASIN--Cont Inued

6-6061.00 L SIOUX R NR SUTHERLAND, IOWA

LAT $4256 \times X$, LONG $9525 \times X$, IN NW $1 / 4$ SEC.

26, T. 94 N.. R. 39 W., O'BRIEN CO. ( 71 ),

AT BRIOGE, 5 MILES SE OF SUTHERLAND.

DRAINAGE AREA $1803 \quad \mathrm{MI}^{2}$

$\begin{array}{rc}\text { DATE } & F T^{3} / S \\ 10-03-57 & 37.2 \\ 05-14-58 & 152 . \\ 10-16-58 & 1.83 \\ Q a & =481\end{array}$

$\begin{array}{cc}\text { DATE } & F T^{3} / \mathrm{S} \\ 08-13-59 & 55.9 \\ 10-0 E_{1}-60 & 390 . \\ 09-25-61 & 79.6\end{array}$

$\begin{array}{cc}\text { DATE } & F T^{3} / \mathrm{S} \\ 10-29-64 & 216 . \\ 08-31-66 & 42.7 \\ 09-11-67 & 26.9 \\ 7 Q 2= & 29\end{array}$

$\begin{array}{cc}\text { DATE } & F T^{\mathrm{a}} / \mathrm{S} \\ 03-26-68 & 47.5 \\ 09-01-70 & 59.6 \\ 08-11-71 & 185.0 \\ 7 Q 10= & 9.4\end{array}$

DATE

$10-03-10.000$

$10-07-75 \quad 153$

Qa $=481 \quad Q(84)=64$

$08-13-76$

24.

6-6062.00 MILL CR NR PAULINA, IOWA

LAT 430134 , LONG 954237, NEAR N $1 / 4$

CORNER SEC.29, T.95 N., R.41 W., O'BRIEN CO.

(71). AT BRIDGE, 3 MILES NW OF PAULINA.

DRAINAGE AR.EA $61.6 \quad \mathrm{MI}^{2}$

\begin{tabular}{|c|c|c|c|c|c|c|}
\hline $\begin{array}{c}\text { DATE } \\
10-02-5 \\
05-14-5 \\
10-16-5\end{array}$ & & $\begin{array}{l}\mathrm{FT}^{3} / \mathrm{S} \\
0.19 \\
1.74 \\
0 .\end{array}$ & $\begin{array}{c}\text { DATE } \\
08-13-59 \\
10-06-60 \\
09-25-61\end{array}$ & $\begin{array}{l}\mathrm{FT}^{3} \text { is } \\
0.06 \\
6.13 \\
1.78\end{array}$ & $\begin{array}{c}\text { DATE } \\
10-30-64 \\
08-31-66 \\
09-11-67\end{array}$ & $\begin{array}{l}\mathrm{FT}^{3} / \mathrm{S} \\
0.71 \\
0.15 \\
0 .\end{array}$ \\
\hline & Qa & $=13$ & $Q(84)$ & $1=1.9$ & $7 Q 2$ & $=0$ \\
\hline 063.00 & MIL & $-L \quad C R \quad N R$ & \multicolumn{4}{|l|}{ CHEROKEE, IOWA } \\
\hline & & & $\begin{array}{l}\text { LAT } 4247 \times X, L \\
\text { SEC. } 15, \text { T. } 92 \\
(18), \text { AT BRID }\end{array}$ & $\begin{array}{l}\text { ONG } 953 \\
N ., R .4 \\
\text { DEE ON U }\end{array}$ & $\begin{array}{l}X \text {, NEAR CEN } \\
\text { W. CHEROKE } \\
\text { S. HIGHWAY }\end{array}$ & $\begin{array}{l}\text { NTER OF } \\
\text { EE CO. } \\
59,2\end{array}$ \\
\hline
\end{tabular}

(18) AT BRIDGE ON U. S. HIGHWAY 59, 2

MILES MORTH Ö̈ CHEROKEE.

DRAINAGE AREA $292 \quad \mathrm{MI}^{2}$

$\begin{array}{cc}\text { DATE } & F T^{2} / S \\ 04-15-58 & 41.0 \\ 05-14-58 & 12.9 \\ 10-16-58 & 0.89\end{array}$

$\begin{array}{ll}\text { DATE } & F T^{3} / \mathrm{S} \\ 08-13-59 & 16.3 \\ 10-06-6.0 & 28.0 \\ 09-25-61 & 47.6\end{array}$

$\begin{array}{cc}\text { DATE } & F T / S \\ 10-29-64 & 13.6 \\ 08-31-66 & 9.41 \\ 09-11-67 & 2.31\end{array}$

DATE
$03-26-68$
$09-01-70$
$08-11-71$

$\mathrm{FT}^{3} / \mathrm{S}$

$6 \cdot 24$

$08-11-71-13.0$

DATE
$10-03-74$
$10-07-75$
$08-13-76$

$\mathrm{FT}^{3} / \mathrm{S}$

3.

$a_{a}=70$

$Q(84)=9.0$

$702=2.8$

$7010=0.5$ 
LITTLE SIOUX RIVER BASIN--Cont Inued

6-6064.00 L SIOUX R AT CHEROKEE, IOWA

LAT $4245 \times X$, LONG $9532 \times X$, IN E $1 / 2$ SEC.

26, T.92 N., T.40 W., CHEROKEE CO. (18),

AT BRIDGE NEAR EAST CITY LIMITS OF CHEROKEE.

DRAINAGE AREA $2173 \quad \mathrm{MI}^{2}$

\begin{tabular}{|c|c|c|c|c|c|c|c|c|c|}
\hline $\begin{array}{c}\text { DATE } \\
04-15-58 \\
05-13-58 \\
10-16-58 \\
08-13-59\end{array}$ & $\begin{array}{c}F T^{3} / S \\
411 . \\
212 . \\
3.63 \\
98.9\end{array}$ & $\begin{array}{c}\text { DATE } \\
10-06-60 \\
09-25-61 \\
10-01-63\end{array}$ & $\begin{array}{l}F T^{\circ} / S \\
441 . \\
131 . \\
54.8\end{array}$ & $\begin{array}{c}\text { DATE } \\
10-29-64 \\
08-31-66 \\
09-11-67\end{array}$ & $\begin{array}{l}F T^{\circ} / \mathrm{S} \\
244 . \\
83.9 \\
35.0\end{array}$ & $\begin{array}{c}\text { DATE } \\
03-2 E-68 \\
09-01-70 \\
08-11-71\end{array}$ & $\begin{array}{l}F T^{3} / S \\
55.7 \\
73.6 \\
240.0\end{array}$ & $\begin{array}{c}\text { DATE } \\
10-03-74 \\
10-07-75 \\
08-13-76\end{array}$ & $\begin{array}{l}\mathrm{FT}^{3} / \mathrm{S} \\
75 . \\
202 . \\
27 .\end{array}$ \\
\hline$Q=$ & $=586$ & Qr & 4) $=81$ & $7 Q 2$ & $=39$ & $7 Q 10$ & $=13$ & & \\
\hline
\end{tabular}

6-6065.00 PIERSON CR NR CORRECTIONVILLE, IOWA

LAT $4229 \times X$, LONG $9548 \times X$, IN NE $1 / 4$ SEC.

33, T.89 N., R. 42 W. WOODBURY CO. (97),

AT BRIDGE, I MILE NW OF CORRECTIONVILLE.

DRAINAGE AREA $55.1 \mathrm{MI}^{2}$

\begin{tabular}{|c|c|c|c|c|c|c|c|c|c|}
\hline $\begin{array}{c}\text { DATE } \\
09-25-57 \\
10-02-58 \\
07-28-59\end{array}$ & $\begin{array}{l}\mathrm{FT}^{3} / \mathrm{S} \\
3.74 \\
0.01 \\
2.68\end{array}$ & $\begin{array}{c}\text { DATE } \\
10-04-60 \\
03-30-61 \\
10-15-64\end{array}$ & $\begin{array}{l}F T / S \\
7.27 \\
4.64 \\
3.02\end{array}$ & $\begin{array}{c}\text { DATE } \\
09-08-66 \\
05-24-67 \\
03-26-68\end{array}$ & $\begin{array}{l}\mathrm{FT}^{3} / \mathrm{S} \\
1.57 \\
0.99 \\
2.10\end{array}$ & $\begin{array}{c}\text { DATE } \\
09-01-70 \\
10-02-74\end{array}$ & $\begin{array}{c}\mathrm{FT}^{3} / \mathrm{S} \\
0.35 \\
3.5\end{array}$ & $\begin{array}{c}\text { DATE } \\
10-07-75 \\
08-12-76\end{array}$ & $\begin{array}{c}\mathrm{FT}^{3} / \mathrm{S} \\
4.6 \\
1.4\end{array}$ \\
\hline$Q$ & $=12$ & $Q(84)$ & $=2.0$ & 702 & $=0.2$ & $7 Q 10$ & * & & \\
\hline
\end{tabular}

6-6068.00 MAPLE R NR AURELIA, IOWA

LAT $4243 \times X$, LONG $9529 \times X$, IN NW $1 / 4$ SEC.

8, T.91 N., R.39 W. CHEROKEE CO. (18),

AT BRIDGE, 2 MILES NW OF AURELIA.

DRAINAGE AREA $85.2 \mathrm{MI}^{2}$

$\begin{array}{rr}D A T E & F T^{3} / S \\ 04-15-58 & 17.4 \\ 05-13-58 & 5.75 \\ 10-16-58 & 0.03 \\ Q a & =19\end{array}$

$\begin{array}{cr}\text { DATE } & \mathrm{FT}^{3} / \mathrm{S} \\ 08-13-59 & 5.71 \\ 10-06-60 & 5.41 \\ 09-25-61 & 6.45\end{array}$

$\begin{array}{cr}\text { DATE } & \mathrm{FT}^{3} / \mathrm{S} \\ 10-29-64 & 0.29 \\ 08-31-66 & 0.65 \\ 09-11-67 & 0.21 \\ 7 Q 2= & \star\end{array}$

$\begin{array}{rr}\text { DATE } & \mathrm{FT}^{3} / \mathrm{S} \\ 03-26-68 & 0.29 \\ 09-01-70 & 0.15 \\ 08-11-71 & 2.6 \\ 7010= & 0.6 * *\end{array}$

$\begin{array}{cc}\text { DATE } & \mathrm{FT}^{3} / \mathrm{S} \\ 10-03-74 & 1.4 \\ 10-07-75 & 1.4 \\ 08-13-76 & 0.26\end{array}$


LITTLE SIOUX RIVER BASIN--Cont inued

6-6069.00 MAPLE R NR IDA GROVE, IOWA

LAT 422155, LONG 352727 , IN NW $1 / 4$ SEC.

12, T. 87 N., R. 40 W., IDA CO. (47).

AT BRIDGE, I MILE NE OF IDA GROVE.

DRAINAGE AREA $364 \quad M I^{2}$

$\begin{array}{rlclcl}\text { DATE } & \text { FTO/S } & \text { DATE } & F T^{3} / S & \text { DATE } & F T^{3} / S \\ 09-25-57 & 12.6 & 08-31-61 & 45.1 & 09-08-66 & 22.4 \\ 10-02-58 & 5.22 & 10-29-63 & 20.8 & 05-24-67 & 12.2 \\ 07-28-59 & 27.9 & 10-15-64 & 14.3 & 09-11-67 & 21.0 \\ 10-04-60 & 39.8 & & & & \\ \text { Qa }=88 & & & & & \end{array}$

6-6071.00 ODEBOLT CR AT IDA GROVE, IOWA

LAT 422:049, LONG 952803, NEAR CENTER OF SEC.14, T.87 N., R. 40 W., IDA CO. (47), AT BRIDGE IN IDA GROVE.

DRAINAGE AREA $61.1 \mathrm{M}]^{2}$

$\begin{array}{rr}\text { CATE } & F T^{3} / S \\ 09-25-57 & 2.10 \\ 10-02-58 & 2.18 \\ 07-28-59 & 6.47 \\ Q a & =13\end{array}$

$\begin{array}{rr}\text { DATE } & \mathrm{FT}^{3} \text { is } \\ 10-04-60 & 6.34 \\ 08-31-61 & 7.00 \\ 10-15-64 & 5.16 \\ \text { Q(84) } & =5.7\end{array}$

$\begin{array}{rr}\text { DATE } & F T^{3} / S \\ 09-08-56 & 7.76 \\ 05-2.4-67 & 2.57 \\ 09-11-67 & 6.69 \\ 7 Q 2= & 2.7\end{array}$
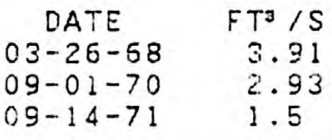

3.91

2.93
1.5

$7010=0.9 * * *$

6-6074.00 MAPLE R NR TURIN, IOWA

LAT $4201 X X$, LONG $9558 \times X$, IN SW $1 / 4$ SEC.

10, T. \& 3 N., R. 44 W., MONONA CO. $157 \%$.

AT BRIDGE, i MILE SE' OF TURIN.

DRAINAGE AREA 741 MI $=$

$\begin{array}{cl}\text { CATE } & F T^{2} / \mathrm{S} \\ 09-24-57 & 31.0 \\ 09-30-58 & 16.2 \\ 07-27-59 & 37.4\end{array}$

\begin{tabular}{cl} 
DATE & \multicolumn{1}{c}{$F T^{3} / S$} \\
$09-07-60$ & 121. \\
$10-04-60$ & 105. \\
$08-30-61$ & 100.
\end{tabular}

$Q_{3}=187$

$Q(84)=\epsilon_{1} 1$
$10-15-64 \quad 5 T^{3} / S$ $00-018-65 \quad 51.2$ $05-24-67 \quad 34.9$

$702=28$

$\begin{array}{cl}\text { IATE } & F T^{3} / S \\ 03-2 E-68 & 43.4 \\ 09-0 Z-70 & 31.5 \\ 09-14-7 ! & 3 E .0\end{array}$

DATE $F T=/ S$ $10-01-74$ G. $08-12-76 \quad 46$.

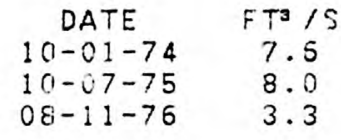

8.0
3.3

Q

$70: 0=7.6 * * *$ 
SOLDIER RIVER BASIN

6-6083.00 SOLDIER R NR RICKETTS, IOWA

LAT $4212 \times X$, LONG $9535 \times X$, IN SW $1 / 4$ SEC.
$1, T .85$ N., R.41 W., CRAWFORD CO. (24),

$1, T .85 \mathrm{~N}$.
$A T$ R.
BRIDGE,

DRAINAGE AREA $90.5 \mathrm{MI}^{2}$

\begin{tabular}{|c|c|c|c|c|c|c|c|c|c|}
\hline $\begin{array}{c}\text { DATE } \\
09-25-57 \\
10-02-58 \\
07-28-59\end{array}$ & $\begin{array}{r}F T^{3} / S \\
2.04 \\
1.89 \\
7.61\end{array}$ & $\begin{array}{c}\text { DATE } \\
10-04-60 \\
08-30-61 \\
10-15-64\end{array}$ & $\begin{array}{l}\mathrm{FT}^{\circ} / \mathrm{S} \\
9.62 \\
6.62 \\
13.4\end{array}$ & $\begin{array}{c}\text { DATE } \\
\text { O9-08-66 } \\
05-24-67 \\
03-2.6-68\end{array}$ & $\begin{array}{l}\text { FTa /S } \\
13.0 \\
3.24 \\
7.53\end{array}$ & $\begin{array}{c}\text { DATE } \\
07-22-70 \\
09-01-70 \\
08-11-71\end{array}$ & $\begin{array}{c}\mathrm{FT}^{\mathrm{J}} / \mathrm{S} \\
8.43 \\
4.57 \\
3.8\end{array}$ & $\begin{array}{c}\text { DATE } \\
10-01-74 \\
10-07-75 \\
08-11-76\end{array}$ & $\begin{array}{l}\mathrm{FT}^{3} / \mathrm{S} \\
15 . \\
15 . \\
6.7\end{array}$ \\
\hline Q & $=20$ & $Q 18$ & $=6.3$ & 702 & $=3.5$ & 7010 & $=0.8 * * *$ & & \\
\hline
\end{tabular}

6-6083.50 SOLDIER R NR UTE, IOWA

LAT $4203 \times X$, LONG $9543 \times X$, IN SE $1 / 4$ SEC.

$34, T .84$ N., R.42 W., MONONA CO. $\{67\}$, AT

BRIDGE ON STATE HIGHWAY 183,1 MILE SW OF UTE.

DRAINAGE AREA $155 \quad \mathrm{MI}^{2}$

\begin{tabular}{|c|c|c|c|c|c|c|c|c|c|}
\hline $\begin{array}{c}\text { DATE } \\
09-24-57 \\
10-03-58 \\
07-27-59\end{array}$ & $\begin{array}{l}\mathrm{FT}^{\mathrm{a}} / \mathrm{S} \\
5.77 \\
3.73 \\
6.47\end{array}$ & $\begin{array}{c}\text { CATE } \\
10-05-60 \\
08-3(1-61 \\
10-15-64\end{array}$ & $\begin{array}{l}\mathrm{FT}^{3} / \mathrm{S} \\
22.7 \\
12.9 \\
22.9\end{array}$ & $\begin{array}{c}\text { DATE } \\
09-08-66 \\
05-24-67 \\
03-26-68\end{array}$ & $\begin{array}{l}F T^{3} / \mathrm{S} \\
18.3 \\
8.70 \\
13.5\end{array}$ & $\begin{array}{c}\text { DATE } \\
07-22-70 \\
09-01-70 \\
08-11-71\end{array}$ & $\begin{array}{c}F T^{3} / \mathrm{S} \\
11.1 \\
7.30 \\
6.1\end{array}$ & $\begin{array}{c}\text { DATE } \\
10-01-74 \\
10-08-75 \\
08-11-76\end{array}$ & $\begin{array}{l}F T^{3} / S \\
25 . \\
22 . \\
11 .\end{array}$ \\
\hline Q & $=36$ & $Q<\varepsilon$ & )$=10$ & 702 & $=6.0$ & 7010 & $1.4 * * *$ & & \\
\hline
\end{tabular}

6-6084.00 E SOLDIER R NR UTE, IOWA

LAT $4203 \times X$, LONG $9542 \times X$. IN SW $1 / 4$ SEC.

$35, T .84$ N., R. 42 W., MONONA CO. (67),

AT BRIOGE NEAR SW CITY LIMITS OF UTE.

DRAINAGE AREA $97.8 \mathrm{MI}^{2}$

\begin{tabular}{|c|c|c|c|c|c|c|c|c|c|}
\hline $\begin{array}{c}\text { DATE } \\
09-24-57 \\
10-03-58 \\
07-27-59\end{array}$ & $\begin{array}{r}F T^{3} / S \\
6.29 \\
3.05 \\
4.09\end{array}$ & $\begin{array}{c}\text { DATE } \\
10-05-60 \\
08-30-61 \\
10-15-64\end{array}$ & $\begin{array}{l}F T / S \\
15.8 \\
8.68 \\
16.4\end{array}$ & $\begin{array}{c}\text { DATE } \\
\text { C9-018-66 } \\
05-24-67 \\
03-2.6-68\end{array}$ & $\begin{array}{l}F T / S \\
5.24 \\
3.0 E \\
7.20\end{array}$ & $\begin{array}{c}\text { DATE } \\
07-22-70 \\
09-01-70 \\
08-11-71\end{array}$ & $\begin{array}{c}\mathrm{FT}^{\mathrm{I}} / \mathrm{S} \\
4.98 \\
2.95 \\
3.1\end{array}$ & $\begin{array}{c}\text { DATE } \\
10-01-74 \\
10-08-75 \\
08-11-76\end{array}$ & $\begin{array}{l}\mathrm{FT}^{3} / \mathrm{S} \\
12 . \\
9.9 \\
3.2\end{array}$ \\
\hline$Q_{a}$ & $=22$ & Qr & $\gamma=5.8$ & $7 Q 2$ & $=3.4$ & 7010 & $0.8 * * *$ & & \\
\hline
\end{tabular}


ALLEN CREEK EASIN

6-6092.20 ALLEN C.FEEK NR LOVELAND, IOWA

LAT $4129 \times X$, LONG $9555 \times X$, IN NE $1 / 4$ SEC.

$17, T .77$ N., R.44 W. POTTAWATAMIE CO.
$(78)$, AT BRIDGE, 2 MILES SW OF LOVELANO.

DRAINAGE AREA $92.1 \mathrm{MI}^{2}$

$\begin{array}{rr}\text { DATE } & F T^{3} / S \\ 09-23-57 & 0.70 \\ 10-01-58 & 0.7 \\ 07-28-59 & 5.76 \\ Q a & =21\end{array}$

$\begin{array}{rr}\text { DATE } & F T^{3} / S \\ 10-04-80 & 6.03 \\ 08-30-61 & 2.07 \\ 10-14-64 & 8.70 \\ Q(84) & =1.1\end{array}$

$\begin{array}{cr}\text { DATE } & F T^{3} / S \\ 09-08-66 & 4.59 \\ 05-25-67 & 1.62 \\ 03-27-68 & 3.57\end{array}$

$\begin{array}{cc}\text { DATE } & \text { FT /S } \\ 08-26-70 & 0 . \\ 08-18-71 & 0 . \\ 10-03-74 & 2.5 \\ 7010= & *\end{array}$

DATE

$10-07-75$

$08-12-76$

$\mathrm{FT}^{\mathrm{a}} / \mathrm{S}$

7.1

$702=0.3$

$7010=*$

BOYER RIVER EASIN

6-6092.60 BOYER R NR EARLY, IOWA

LAT 4228XX, LONG 9511XX. IN NE $1 / 4$ SEC.

5. T.88 N.. R.37 W., SAC CO. (81), AT

BRIDGE ON U.S. HIGHWAY 20,2 MILES NW OF EARLY.

DRAINAGE AREA $67.5 \mathrm{MI}^{2}$

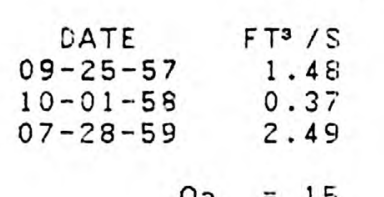

$\mathrm{Qa}=15$

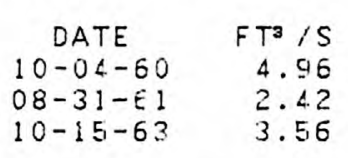

$Q(84)=4.0$

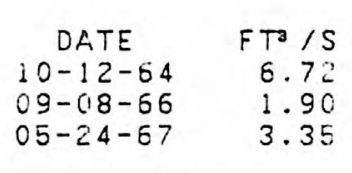

$7 Q 2=0.9$

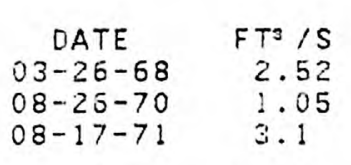

$7 Q 10=*$

6-6093.00 E BOYER R AT VAIL, IOWA

LAT $4204 \times X$, LONG $9512 \times X$. IN E $1 / 2$ SEC.

30, T. $\$ 4$ N., R.37 W., CRAWFORD CO. ( 24 ),

AT BRIUGE NEAR EA.ST CITY LIMITS OF VAIL.

\begin{abstract}
DRAINAGE AREA $65.4 \mathrm{MJ}^{2}$
\end{abstract}

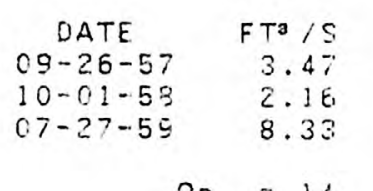

$\begin{array}{cr}\text { DATE } & \text { FT is } \\ 10-04-60 & 6.02 \\ 08-30-61 & 9.23 \\ 10-15-62 & 9.15\end{array}$
$\begin{array}{cc}\text { DATE } & \text { FTO IS } \\ 10-13-64 & 7.4 \\ 03-08-66 & 4.5\end{array}$
$05-24-67 \quad 1.07$

$2(84)=5.1$
$\mathrm{TQ2}=3.1$

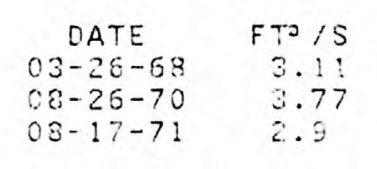

$\begin{array}{cc}\text { DATE } & F T^{3} / 5 \\ 10-01-74 & 11 . \\ 10-07-75 & 5.9 \\ 06-11-76 & 3.2\end{array}$

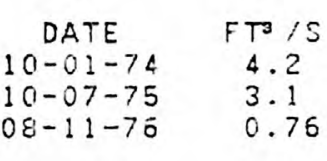

4.2
3.1

0.76 
BOYER RIVER BASIN--Cont inued

6-6093.50 E BOYER R AT DENISON, IOWA

LAT $4201 \times X$, LONG $9522 \times X$, IN SE $1 / 4$ SEC.

10, T.83 N., R.39 W., CRAWFORD CO. (24),

AT BRIDGE ON U.S, HIGHWAY 30 , NEAR WEST

CITY LIMITS OF DENISON.

DRAINAGE AREA $130 \quad \mathrm{MI}^{2}$

\begin{tabular}{|c|c|c|c|c|c|c|c|c|c|}
\hline $\begin{array}{c}\text { DATE } \\
09-25-57 \\
10-01-58 \\
07-27-59\end{array}$ & $\begin{array}{l}F T / S \\
8.04 \\
4.54 \\
13.0\end{array}$ & $\begin{array}{c}\text { DATE } \\
10-04-60 \\
08-30-61 \\
10-15-63\end{array}$ & $\begin{array}{l}F T . / S \\
13.9 \\
22.0 \\
16.4\end{array}$ & $\begin{array}{c}\text { DATE } \\
10-13-64 \\
09-08-66 \\
05-24-67\end{array}$ & $\begin{array}{l}F T^{3} / \mathrm{S} \\
16.4 \\
10.9 \\
3.05\end{array}$ & $\begin{array}{c}\text { DATE } \\
03-26-68 \\
08-26-70 \\
08-17-71\end{array}$ & $\begin{array}{l}F T \cdot S \\
7.33 \\
5.47 \\
6.2\end{array}$ & $\begin{array}{c}\text { DATE } \\
10-01-74 \\
10-07-75 \\
08-11-76\end{array}$ & $\begin{array}{l}F T^{3} \text { is } \\
24 . \\
13 . \\
6.7\end{array}$ \\
\hline $\mathbf{Q}$ & $=30$ & $Q<\varepsilon$ & $=11$ & $7 Q 2$ & $=6.2$ & 7010 & I. $8 * * *$ & & \\
\hline
\end{tabular}

6-6094.00 BOYER R NR DENISON, IOWA

LAT $4200 \times X$, LONG $9523 \times X$, IN NE $1 / 4$ SEC.

$16, T .83$ N., R.39 W., CRAWFORD CO. ( 24 ).

AT BRIDGE, 2 MILES SW OF DENISON.

DRAINAGE AREA $517 \quad \mathrm{MI}^{2}$

\begin{tabular}{|c|c|c|c|c|c|c|c|c|c|}
\hline $\begin{array}{c}\text { DATE } \\
09-25-57 \\
10-01-58 \\
07-27-59\end{array}$ & $\begin{array}{c}\mathrm{FT}^{\mathrm{3}} / \mathrm{S} \\
20.2 \\
7.79 \\
29.4\end{array}$ & $\begin{array}{c}\text { DATE } \\
10-04-60 \\
08-30-61 \\
10-15-63\end{array}$ & $\begin{array}{l}\mathrm{FT}^{3} / \mathrm{S} \\
58.2 \\
71.5 \\
48.4\end{array}$ & $\begin{array}{c}\text { DATE } \\
10-13-64 \\
09-08-66 \\
05-24-67\end{array}$ & $\begin{array}{l}F T^{8} / \mathrm{S} \\
52.2 \\
40.8 \\
12.9\end{array}$ & $\begin{array}{c}\text { DATE } \\
03-26-68 \\
08-26-70 \\
08-17-71\end{array}$ & $\begin{array}{l}F T^{3} / S \\
26.9 \\
17.9 \\
20.0\end{array}$ & $\begin{array}{c}\text { DATE } \\
10-01-74 \\
10-09-75 \\
08-11-76\end{array}$ & $\begin{array}{l}\mathrm{FT}^{\circ} / \mathrm{S} \\
66 . \\
36 . \\
17 .\end{array}$ \\
\hline & $=128$ & $Q 18$ & $=35$ & 70 & 16 & 7010 & $2.2 * \star$ & & \\
\hline
\end{tabular}

6-6095.50 BOYER R NR MISSOURI VALLEY, IOWA

LAT $4131 X X$, LONG $9554 X X$. IN SE $1 / 4$ SEC.

28, T.78 N., R. 44 W., HARRISON CO. (43)

AT BRIDGE, 2 MILES SOUTH OF MISSOURI VALLEY.

DRAINAGE AREA $935 \mathrm{MI}^{2}$

\begin{tabular}{|c|c|c|c|}
\hline $\begin{array}{c}\text { DATE } \\
09-24-57 \\
09-30-58 \\
07-28-59\end{array}$ & $\begin{array}{l}F T^{2} / S \\
43.3 \\
27.6 \\
78.9\end{array}$ & $\begin{array}{c}\text { DATE } \\
10-04-60 \\
08-30-61 \\
10-14-64\end{array}$ & $\begin{array}{l}\mathrm{FT}^{3} / \mathrm{S} \\
98.2 \\
90.8 \\
108 .\end{array}$ \\
\hline Qa & $=240$ & $Q<8$ & $=62$ \\
\hline
\end{tabular}

$\begin{array}{rl}\text { DATE } & F T^{3} / S \\ 09-08-66 & 63.0 \\ 05-25-67 & 26.2 \\ 03-27-68 & 67.8 \\ 7 Q 2 & =32\end{array}$
DATE $F T^{2} / S$
$08-26-70 \quad 35.8$
$08-18-71 \quad 31.0$
10-03-74 123 .

$\begin{array}{cl}\text { DATE } & F T^{3} / S \\ 10-07-75 & 80 . \\ 08-12-76 & 36 .\end{array}$


BOYER RIVER BASIN--Cont inued.

6-6095.80 WILLOW CR NR WOODEINE, IOWA

LAT $4148 \% X$, LONG $9545 \times X$, IN NE $1 / 4$ SEC. 29, T. \&1 N.. R.42 W., HARRISON CO. (43), AT BRIDGE, 5.5 MILES NW OF WOODBINE.

DRAINAGE AREA $67.0 \quad \mathrm{MI}^{2}$

\begin{tabular}{|c|c|c|c|c|c|c|c|c|c|}
\hline $\begin{array}{c}\text { DATE } \\
09-24-57 \\
10-01-58 \\
07-28-59\end{array}$ & $\begin{array}{l}\mathrm{FT}^{3} / \mathrm{S} \\
1.26 \\
2.42 \\
6.21\end{array}$ & $\begin{array}{c}\text { DATE } \\
10-04-60 \\
08-3(1-61 \\
10-14-64\end{array}$ & $\begin{array}{l}F T \text { is } \\
9.73 \\
6.28 \\
10.1\end{array}$ & $\begin{array}{c}\text { DATE } \\
09-018-66 \\
05-25-67 \\
03-2.7-68\end{array}$ & $\begin{array}{r}\mathrm{FT}^{3}: \mathrm{S} \\
3.59 \\
3.37 \\
8.29\end{array}$ & $\begin{array}{c}\text { DATE } \\
08-26-70 \\
08-17-71 \\
10-02-74\end{array}$ & $\begin{array}{c}\mathrm{FT}^{3} / \mathrm{S} \\
1.10 \\
1.4 \\
7.5\end{array}$ & $\begin{array}{c}\text { DATE } \\
10-07-75 \\
08-11-76\end{array}$ & $\begin{array}{c}\mathrm{FT}^{3} / \mathrm{S} \\
5.1 \\
2.1\end{array}$ \\
\hline Qa & $=15$ & QR & $=3.2$ & $7 Q 2$ & $=1.4$ & 7010 & $=0.2 * \star \star *$ & & \\
\hline
\end{tabular}

E-6095.00 WILLOW CR NR LOGAN, IOWA

LAT $4138 \times X$, LONG $9553 \times X$, IN NE $1 / 4$ SEC.

30 , T.T9 N., R. 43 W., HARRISON CO. (43), AT BRILGE, 5 NILES WEST OF LOGAN.

DRAINAGE AREA $129 \quad \mathrm{MI}^{2}$

\begin{tabular}{|c|c|c|c|c|c|c|c|c|c|}
\hline $\begin{array}{c}\text { DATE } \\
09-24-57 \\
09-30-58 \\
07-28-59\end{array}$ & $\begin{array}{c}F T=1 S \\
3.04 \\
4.69 \\
12.6\end{array}$ & $\begin{array}{c}\text { DATE } \\
10-0 \angle-50 \\
08-30-61 \\
10-1 \angle-6:\end{array}$ & $\begin{array}{l}F T^{3} \text { is } \\
18.0 \\
11.5 \\
16.7\end{array}$ & $\begin{array}{c}\text { DATE } \\
\text { OS-0.8-65 } \\
05-25-67\end{array}$ & $\begin{array}{r}F^{3} / S \\
7.66 \\
5.39\end{array}$ & $\begin{array}{c}\text { DATE } \\
03-2 E-68 \\
08-2 E-70\end{array}$ & $\begin{array}{l}\mathrm{FT}^{3} / \mathrm{S} \\
17.6 \\
3.11\end{array}$ & $\begin{array}{c}\text { DATE } \\
08-17-71 \\
08-12-76\end{array}$ & $\begin{array}{l}\mathrm{FT}^{\mathrm{S}} / \mathrm{S} \\
3.3 \\
5.7\end{array}$ \\
\hline & $=29$ & $Q<\varepsilon$ & $=\varepsilon .5$ & $7 Q 2$ & $=4.4$ & $7 Q 10$ & 1. $0 * * *$ & & \\
\hline
\end{tabular}

6-6096.20 WILLOW C.R NR MISSOURI VALLEY, IOWA

LAT $4131 \times X$, LONG $9554 \times X$, IN SE $1 / 4$ SEC.

28, T. 78 N. 2 $2.4 L$ W. HARRISON CO. ( $\angle 3)$,

DRAINAGE AREA $146 \quad \mathrm{MI}^{2}$

\begin{tabular}{|c|c|c|c|c|c|c|c|c|c|}
\hline $\begin{array}{c}\text { DATE } \\
09-24-57 \\
09-30-58 \\
0 \%-28-59\end{array}$ & $\begin{array}{l}F T^{3} / 5 \\
4.63 \\
6.18 \\
13.5\end{array}$ & $\begin{array}{c}\text { DATE } \\
10-0 C-50 \\
08-30-61\end{array}$ & $\begin{array}{l}\text { FTO is } \\
\text { is. } 9 \\
11.1\end{array}$ & $\begin{array}{c}\text { DATE } \\
10-14-64 \\
09-08-66\end{array}$ & $\begin{array}{l}F T^{3} / \mathrm{S} \\
17.2 \\
9.5 \mathrm{~S}\end{array}$ & $\begin{array}{c}\text { QATE } \\
05-25-67 \\
03-27-68\end{array}$ & $\begin{array}{l}F^{3} \cdot 15 \\
5.55 \\
15.7\end{array}$ & $\begin{array}{c}\text { DATE } \\
08-26-70 \\
08-18-71\end{array}$ & $\begin{array}{l}\mathrm{FT}^{3} / \mathrm{S} \\
2.01 \\
1.9\end{array}$ \\
\hline 0 & $=35$ & 018 & $=9.2$ & 702 & $=4.6$ & 7010 & 1. $1 * * *$ & & \\
\hline
\end{tabular}


BOYER RIVER BASIN--Continued

6-6096.70 BOYER R NR LOVELAND, IOWA

LAT 412758 , LONG 955437, IN CENTER OF

SEC. $4, T .77 \mathrm{~N}$. R. R.44 W.., POTTAWATAMIE CO.

(78). AT BRIDGE, 1 MILE WEST OF LOVELAND.

DRAINAGE AREA $1084 \quad \mathrm{MI}^{2}$

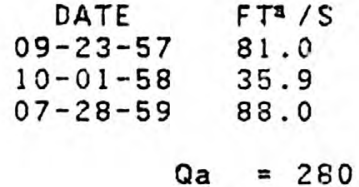

$Q \mathbf{a}=280$

\begin{tabular}{cl} 
DATE & \multicolumn{1}{c}{$F T / S$} \\
$10-04-60$ & 128. \\
$08-30-61$ & 112. \\
$10-14-64$ & 120.
\end{tabular}

$Q(84)=78$

$\begin{array}{cc}\text { DATE } & F T / S \\ 09-08-66 & 72.3 \\ 05-25-67 & 38.2 \\ 03-27-68 & 84.7 \\ 702= & 44\end{array}$

DATE

$08-12-76$
FT / $/ S$

0.

\section{PIGEON CREEK BASIN}

6-6099.00 PIGEON CR EAST OF LOVELAND, IOWA

$$
\begin{aligned}
& \text { LAT } 412838, \text { LOMG } 954213 \text {, IN SW } 1 / 4 \text { SEC } \\
& 8, T .77 \text { N., R. } 42 \text { W., POTTAWATAMIE CO. }(78), \\
& \text { AT BRIDGE, IO MILES SE OF LOVELAND. }
\end{aligned}
$$

\begin{tabular}{|c|c|c|c|c|c|c|c|c|c|}
\hline $\begin{array}{c}\text { DATE } \\
09-23-57 \\
10-16-58 \\
10-20-59\end{array}$ & $\begin{array}{r}F T=15 \\
2.18 \\
4.97 \\
4.88\end{array}$ & $\begin{array}{c}\text { DATE } \\
09-15-60 \\
08-31-61 \\
11-06-63\end{array}$ & $\begin{array}{l}F T^{3} / S \\
7.06 \\
6.51 \\
3.80\end{array}$ & $\begin{array}{c}\text { DATE } \\
10-28-54 \\
08-30-66 \\
05-24-67\end{array}$ & $\begin{array}{r}F T^{3} / S \\
5.68 \\
7.82 \\
2.11\end{array}$ & $\begin{array}{c}\text { DATE } \\
06-18-68 \\
07-23-70\end{array}$ & $\begin{array}{r}F T^{3} / S \\
0.72 \\
1.76\end{array}$ & $\begin{array}{c}\text { DATE } \\
08-18-71 \\
10-09-75\end{array}$ & $\begin{array}{l}\mathrm{FT}^{3} / \mathrm{S} \\
1.1 \\
4.9\end{array}$ \\
\hline Qa & $=15$ & $Q(84)$ & $=4.4$ & 702 & $=2.2$ & 7010 & $0.7 * *$ & & \\
\hline
\end{tabular}

DRAINAGE AREA $66.6 \mathrm{MI}^{2}$

6-6099.50 PIGEON CR NR CRESCENT, IOWA

$$
\begin{aligned}
& \text { LAT } 411947, \text { LONG } 955319 \text {, IN NE } 1 / 4 \text { SEC. } \\
& 3, \text { T. } 75 \mathrm{~N}, 1 \text { R. } 44 \text { W. POTTAWATAMIE CO. (78), } \\
& \text { AT BRIDGE, } 3 \text { MILES SW OF CRESCENT. }
\end{aligned}
$$

DRAINAGE AREA $163 \quad \mathrm{MI}^{2}$

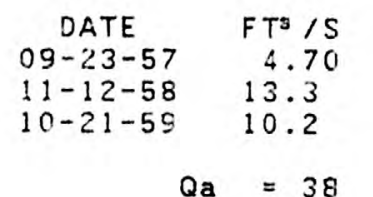

$Q \mathbf{a}=38$

$\begin{array}{cl}\text { DATE } & F T^{3} / S \\ 09-1 E-60 & 28.9 \\ 08-30-61 & 11.0 \\ 11-06-63 & 8.47\end{array}$

$Q(84)=9.7$

$\begin{array}{cc}\text { DATE } & F T^{3} / S \\ 10-28-64 & 14.2 \\ 08-30-66 & 23.0 \\ 05-2.5-67 & 6.58\end{array}$

$7 Q 2=5.4$

$\begin{array}{cr}\text { DATE } & F^{2} / S \\ 06-18-68 & 4.22 \\ 07-23-70 & 2.19\end{array}$

\section{DATE}

$08-18-71$

$F T^{3} / S$

$4 \cdot 1$ 
MOSQUITO CREEK BASII

6-6105.50 MOSQUITO CR AT POR.TSMOUTH, :OWA

LAT $4139 \times X$, LONG $9531 \times X$, IN SW $1 / 4$ SEC.

IE, T.79 N., R. 40 W., SHELEY CO. (83),

AT BRIDGE ON STATE HIGHWAY 64, NEAR

EAST CITY LIMITS OF PORTSMOUTH.

DRAINAGE AREA $63.9 \mathrm{MI}^{2}$

$\begin{array}{cc}\text { DATE } & F T^{3} / S \\ 09-26-57 & 4.37 \\ 10-16-58 & 5.35 \\ 10-20-50 & 4.36\end{array}$

$Q=24$

$\begin{array}{rc}\text { DATE } & F T^{3} / S \\ 09-15-60 & 9.93 \\ 08-31-61 & 10.5 \\ 11-05-63 & 4.00 \\ Q(84) & =4.5\end{array}$

$\begin{array}{cr}\text { DATE } & \text { FT } / 5 \\ 10-26-64 & 8.45 \\ 08-29-66 & 5.63 \\ 05-23-67 & 0.99\end{array}$

$7 Q 2=2.1$

$\begin{array}{cr}\text { DATE } & F T^{3} / S \\ 06-18-68 & 1.16 \\ 07-23-70 & 2.80 \\ & \\ 7010=0.6 \star\end{array}$

$7 Q 10=0.6 \star \star$

6-6106.00 MOSQUITO CR AT NEOLA, IOWA

LAT 412709 , LONG 953637. IN NE $1 / 4$ SEC.

19, T. 77 N.. R. 42 W.. POTTAWATAMIE CO.

(78), F.T BRIDGE ON COUNTY FOAD $S, 0.5$ MILE SOUTH OF NEOLA.

LRAINAGE AREA $131 \mathrm{MI}^{2}$

$\begin{array}{cc}\text { DATE } & F T=/ S \\ 09-23-57 & 8.21 \\ 10-16-58 & 12.6 \\ 10-20-59 & 11.1\end{array}$

$\begin{array}{cl}\text { DATE } & F T^{3} / \mathrm{S} \\ 09-15-60 & 26.1 \\ 08-31-61 & 19.6 \\ 11-06-63 & 8.53\end{array}$
$\begin{array}{ll}\text { UATE } & \mathrm{FT}^{3} / \mathrm{S} \\ 10-2.8-54 & 14.9\end{array}$
$08-30-56 \quad 15 \cdot 9$
$08-30-66 \quad 15.0$

$0(84)=9.8$

$7 Q 2=5.4$

$Q_{a}=49$
DATE
$08-17-71$
$10-08-75$
$T^{2} / \mathrm{s}$
0.88
8.1

NCIL BLUFFS, IOWA

6-6106.50 MOSQUITO CR NR COUNCIL BLUFFS, IOWA

$$
\begin{aligned}
& \text { LAT } \$ 11609, \text { LONG } 954822 \text {. IN E } 1 / 2 \text { SEC. } \\
& 29, \text { T. I5 N. B. } 43 \text { W. POTTAWATAMIE CO. (78), } \\
& \text { AT BRILGE, } 3 \text { MILES EAST OF COUNCIL BLUFFS. }
\end{aligned}
$$

DRAINAGE AREA $211 \mathrm{MI}^{2}$

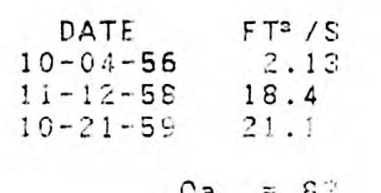

$\mathrm{Sa}=8 \mathrm{a}$

\begin{abstract}
$\begin{array}{cl}\text { DATE } & F T^{3} / S \\ 09-1 E_{1}-E O & 33.2 \\ 09-01-E 1 & 26.3 \\ 11-0 E-62 & 13.5\end{array}$
\end{abstract}

\begin{abstract}
$n(34)=16$
\end{abstract}
DATE
$06-1 \varepsilon-58$
$07-23-70$
$\mathrm{FT}^{\mathrm{a}} / \mathrm{S}$
10.1

DATF

$08-17-71$

$10-9.9-75$

$\mathrm{FT}^{\mathrm{a}} / \mathrm{S}$

10.0

$7210=2.1 * *$ 
KEG CREEK BASIN

6-8057.00 KEG CR AT MINDEN, IOWA

LAT 412757 , LONG 953215 , IN SE $1 / 4$ SEC.

$15, T .77$ N., R. 41 W., POTTAWATAMIE CO. (78),

AT BRIDGE, AT EAST CITY LIMITS OF MINDEN.

DRAINAGE AREA $59.6 \mathrm{MI}^{2}$

\begin{tabular}{|c|c|c|c|c|c|c|c|c|c|}
\hline $\begin{array}{c}\text { DATE } \\
09-23-57 \\
10-16-58 \\
10-20-59\end{array}$ & $\begin{array}{l}F T^{3} / S \\
3.52 \\
7.09 \\
5.87\end{array}$ & $\begin{array}{c}\text { DATE } \\
09-15-60 \\
09-01-61 \\
11-06-63\end{array}$ & $\begin{array}{l}\mathrm{FT}^{\mathrm{3}} / \mathrm{S} \\
14 \cdot 1 \\
8.25 \\
2.15\end{array}$ & $\begin{array}{c}\text { DATE } \\
10-28-64 \\
08-30-66 \\
05-2.3-67\end{array}$ & $\begin{array}{r}\mathrm{FT}^{3} / \mathrm{S} \\
4.15 \\
6.00 \\
1.68\end{array}$ & $\begin{array}{c}\text { DATE } \\
06-19-68 \\
07-23-70\end{array}$ & $\begin{array}{r}F T / S \\
1.32 \\
1.85\end{array}$ & $\begin{array}{c}\text { DATE } \\
08-17-71 \\
09-26-75\end{array}$ & $\begin{array}{l}\mathrm{FT}^{3} / \mathrm{S} \\
1.3 \\
9.7\end{array}$ \\
\hline Qa & $=24$ & $Q<$ & $=4.1$ & 702 & $=2.0$ & 7010 & $=0.9 * *$ & & \\
\hline
\end{tabular}

6-8058.00 KEG CR. NR DUMFRIES, IOWA

LAT 411120, LONG 954059, IN NW $1 / 4$ SEC.

28, T.T4 N., R. 42 W. , POTTAWATAMIE CO.

( 78 ), AT BRIDGE, 3 MILES NE OF DUMFRIES.

DRAINAGE AREA $131 \mathrm{MI}^{2}$

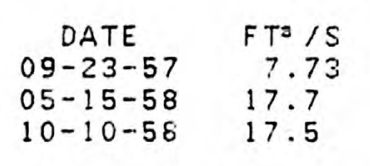

$Q_{a}=54$

$\begin{array}{cl}\text { DATE } & F T \text { is } \\ 10-20-59 & 11.2 \\ 09-16-60 & 33.4 \\ 09-01-61 & 17.8\end{array}$

$Q(84)=14$

$\begin{array}{cc}\text { DATE } & F^{3} / S \\ 11-06-63 & 9.63 \\ 10-27-54 & 10.1 \\ 08-31-66 & 18.0\end{array}$

$7 Q 2=8.5$

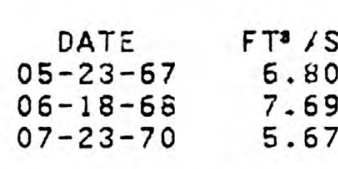

$7010=2.0 * *$

6-8059.00 KEG CR NR GLENWOOD, IOWA

LAT 410056, LONG 954559 . IN NE $1 / 4$ SEC.
27 T. $72 N$. R R 43 W. MILLS CO. $(65)$,
AT BRIDGE, 2 MILES SW OF GLENWODD.

DRAINAGE AREA $190 \quad \mathrm{MI}^{2}$

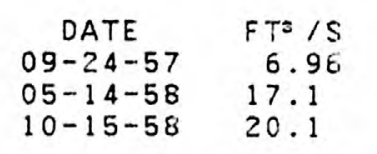

$Q_{a}=80$

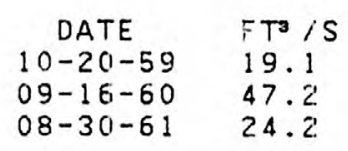

$Q(84)=21$

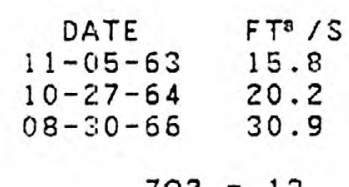

$7 Q 2=13$

$\begin{array}{cc}\text { DATE } & F T^{3} / S \\ 05-23-67 & 9.78 \\ 06-19-68 & 11.2 \\ 07-23-70 & 10.9\end{array}$

DATE

$08-17-71$
$F T^{3} / S$

15.

$\begin{array}{cc}\text { DATE } & F T^{3} / S \\ 08-17-71 & 8.8 \\ 09-26-75 & 21\end{array}$

$7 Q 10=2.8 * *$ 
NISHNABOTNA RIVER BASIN

6-8072.50 W NISHNABOTNA R NR. MANNING, IOWA

LAT $4153 \times X$, LONG $9505 \times X$, IN NW $1 / 4$ SEC.

31, T. 12 N., R. 2E W. CARROLL CO. (14).

AT BRIOGE, 3 MILES SW OF MANNING.

DRAINAGE AREA $58.5 \mathrm{MI}$

\begin{tabular}{|c|c|c|c|c|c|c|c|c|c|}
\hline $\begin{array}{c}\text { DATE } \\
09-2.3-57 \\
10-23-58 \\
10-20-59\end{array}$ & $\begin{array}{r}F T^{3} / S \\
5.27 \\
4.96 \\
1.63\end{array}$ & $\begin{array}{c}\text { DATE } \\
09-15-60 \\
08-31-61 \\
11-05-63\end{array}$ & $\begin{array}{c}\mathrm{FT}^{3} ; \mathrm{S} \\
5.56 \\
12.2 \\
3.51\end{array}$ & $\begin{array}{c}\text { DATE } \\
10-26-64 \\
08-29-66\end{array}$ & $\begin{array}{r}\mathrm{FT}^{3} / \mathrm{S} \\
6.42 \\
4.09\end{array}$ & $\begin{array}{c}\text { DATE } \\
05-23-67 \\
06-18-68\end{array}$ & $\begin{array}{r}F T^{3} / 5 \\
0.23 \\
0.32\end{array}$ & $\begin{array}{c}\text { DATE } \\
08-30-71 \\
09-24-75\end{array}$ & $\begin{array}{l}\mathrm{FT}^{3} / \mathrm{S} \\
5.2 \\
5.7\end{array}$ \\
\hline$Q$ & $=22$ & $Q<\varepsilon$ & $y=1.7$ & $7 Q 2$ & $=0.5$ & 7210 & * & & \\
\hline
\end{tabular}

6-8072.80 WF WNISHNABOTNA F. MANILLA, IOWA

LAT 415.2XX, LONG $9515 X X$. NEAR W $1 / 4$

CORNER SEC.35, T.82 N., R.38 W., CRAWFORD CO.

(24). F.T BRIDGE, 1 MILE SOUTH OF MANILLA.

DRAINAGE AREA $64.2 \quad \mathrm{MI}^{2}$

\begin{tabular}{|c|c|c|c|c|c|c|c|c|c|}
\hline $\begin{array}{c}\text { DATE } \\
09-15-60 \\
08-31-61\end{array}$ & $\begin{array}{c}F T^{3} / \mathrm{S} \\
5.60 \\
16.6\end{array}$ & $\begin{array}{c}\text { DATE } \\
11-05-63 \\
10-26-64\end{array}$ & $\begin{array}{l}\mathrm{FT}^{3} \text { is } \\
6.25 \\
7.31\end{array}$ & $\begin{array}{c}\text { DATE } \\
08-29-66 \\
05-23-67\end{array}$ & $\begin{array}{r}\mathrm{FT}^{3} / \mathrm{S} \\
5.53 \\
1.13\end{array}$ & $\begin{array}{c}\text { DATE } \\
06-18-68 \\
08-30-71\end{array}$ & $\begin{array}{l}\mathrm{FT}^{3} / \mathrm{S} \\
1.52 \\
1.4\end{array}$ & $\begin{array}{c}\text { DATE } \\
09-24-75\end{array}$ & $\begin{array}{l}\mathrm{FT}^{3} / \mathrm{S} \\
10 .\end{array}$ \\
\hline 0 & $=24$ & $Q<\varepsilon$ & $=4.2$ & $7 Q 2$ & $=2.0$ & $7 Q 10$ & * & & \\
\hline
\end{tabular}

6-8073.00 WF WNISHNABOTNA F. AT HARLAN, IOWA

LAT $4140 X X$. LONG 9518XX, IN NE $1 / 4$ SEC.

AT ERIUGE NEAR NE CITY LIMITS OF HARLAN.

DRAINAGE AREA $146 \quad \mathrm{MI}^{2}$

\begin{tabular}{|c|c|}
\hline $\begin{array}{c}\text { DATE } \\
09-26-57 \\
10-09-56 \\
10-20-59\end{array}$ & $\begin{array}{r}F T=15 \\
9.97 \\
9.18 \\
8.16\end{array}$ \\
\hline & $=5 L$ \\
\hline
\end{tabular}

$\begin{array}{cl}\text { DATE } & F T J / S \\ 09-151-60 & 25.9 \\ 08-31-61 & 34.3 \\ 11-05-63 & 11.5\end{array}$

$\begin{array}{cl}\text { DATE } & F T^{3} / S \\ 10-28-54 & 18.0 \\ 08-29-56 & 14.7\end{array}$

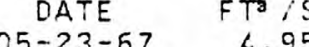
$06-18-68 \quad 3.51$

DATE

$09-24-75$

$5 T^{2}: S$

2.6

$\mathrm{Ca}=5 \mathrm{~L}$

$Q(84)=10$

$7 Q 2=5.6$

$7010=2.2 * *$ 
NISHNABOTNA RIVER BASIN--Continued

6-8073.20 WNISHNABOTNA R AT HARLAN, IOWA

LAT $4138 \times X$, LONG $9518 \times X$, IN NE $1 / 4$ SEC.

$19, T .79$ N., R. 3E W., SHELBY CO. $\{83\}$,

AT BRIDGE ON STATE HIGHWAY 64, NEAR

EAST CITY LIMITS OF HARLAN.

DRAINAGE AREA $316 \quad \mathrm{MI}^{2}$

\begin{tabular}{|c|c|c|c|c|c|c|c|c|c|}
\hline $\begin{array}{c}\text { DATE } \\
09-26-57 \\
10-09-58 \\
10-20-59\end{array}$ & $\begin{array}{l}\mathrm{FT}^{3} / \mathrm{S} \\
20.1 \\
27.7 \\
17.3\end{array}$ & $\begin{array}{c}\text { DATE } \\
09-15-60 \\
08-31-61 \\
11-05-63\end{array}$ & $\begin{array}{l}F T^{3} / S \\
52.0 \\
65.5 \\
25.3\end{array}$ & $\begin{array}{c}\text { DATE } \\
10-28-64 \\
08-29-66\end{array}$ & $\begin{array}{l}\mathrm{FT}^{3} / \mathrm{S} \\
40.3 \\
31.9\end{array}$ & $\begin{array}{c}\text { DATE } \\
05-23-67 \\
05-18-68\end{array}$ & $\begin{array}{l}\mathrm{FT}^{\mathrm{T}} / \mathrm{S} \\
11.8 \\
8.67\end{array}$ & $\begin{array}{c}\text { DATE } \\
08-30-71 \\
09-24-75\end{array}$ & $\begin{array}{l}F T^{2} / S \\
7.1 \\
49 .\end{array}$ \\
\hline Q & $=117$ & $Q<\varepsilon$ & $=28$ & $7 Q_{2}$ & $=15$ & $7 Q 10$ & $4.7 * \star$ & & \\
\hline
\end{tabular}

6-8073.40 WNISHNABOTNA R AT AVOCA, IOWA

LAT 412810 , LONG 952114 , IN NE $1 / 4$ SEC.

$17, T .77$ N., R.39 W., POTTAWATAMIE CO. (78),

AT BRIDGE ON STATE HIGHWAY 83 , NEAR WEST CITY

LIMITS OF AVOCA.

DRAINAGE AREA $357 \quad \mathrm{MI}^{2}$

\begin{tabular}{|c|c|c|c|c|c|c|c|c|c|}
\hline $\begin{array}{c}\text { DATE } \\
05-09-57 \\
09-23-57 \\
10-16-58\end{array}$ & $\begin{array}{c}\mathrm{FT}^{3} / \mathrm{S} \\
8.57 \\
28.0 \\
32.7\end{array}$ & $\begin{array}{c}\text { DATE } \\
10-20-59 \\
09-15-60 \\
09-01-61\end{array}$ & $\begin{array}{l}F T^{3} / \mathrm{S} \\
21.3 \\
70.7 \\
70.2\end{array}$ & $\begin{array}{c}\text { DATE } \\
11-06-63 \\
10-28-64 \\
08-30-66\end{array}$ & $\begin{array}{l}\mathrm{FT}^{3} / \mathrm{S} \\
28.1 \\
50.4 \\
40.3\end{array}$ & $\begin{array}{c}\text { DATE } \\
05-23-67 \\
06-19-68\end{array}$ & $\begin{array}{c}F T^{3} / \mathrm{S} \\
11.7 \\
9.9\end{array}$ & $\begin{array}{c}\text { DATE } \\
08-31-71 \\
09-26-75\end{array}$ & $\begin{array}{l}F T / S \\
9.9 \\
65 .\end{array}$ \\
\hline$Q$ & $=134$ & $Q<8$ & )$=33$ & $7 Q 2$ & $=18$ & 7010 & $4.1 * * *$ & & \\
\hline
\end{tabular}

6-8073.60 EB W NISHNABOTNA R. NR RED LINE, IOWA

LAT $4144 X X$, LONG $9506 \times X$, IN NE $1 / 4$ SEC.

$13, T .80 \mathrm{~N} .$, R.37 W., SHELEY CO. 183 ;

AT BRIDGE 3 MILES NE OF RED LINE.

DRAINAGE AREA $70.3 \mathrm{MI}^{2}$

$\begin{array}{rr}\text { DATE } & F T^{2} / S \\ 09-26-57 & 2.79 \\ 10-09-58 & 8.80 \\ 10-20-59 & 2.96 \\ Q a & =28\end{array}$

$Q \mathbf{Q}=28$

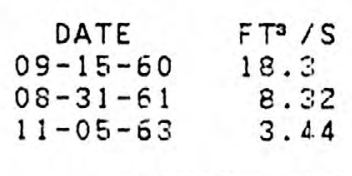

$Q(84)=4.2$

$\begin{array}{rc}\text { DATE } & F T / S \\ 10-26-64 & 10.0 \\ 08-29-66 & 4.25 \\ 7 Q 2= & 1.7\end{array}$

DATE

$08-30-71$ 
NISHNABOTNA PIVER BASIN--Cont inued

6-8073.80 EB W NISHNABOTNA R NR JACKSONVILLE, IOWA

LAT $4139 \times X$, LONG $9514 \times X$, IN NE $1 / 4$ SEC.

$23, T .79$ N., R. 38 W. , SHELEY CO. (83),

AT BRIDGE ON STATE HIGHWAY 44, 4MILES

WEST OF JACKSCNVILLE.

DRAINAGE AREA $151 \quad M^{2}$

\begin{tabular}{|c|c|c|c|c|c|c|c|c|c|}
\hline $\begin{array}{c}\text { DATE } \\
09-26-57 \\
10-09-58 \\
10-20-59\end{array}$ & $\begin{array}{c}F T^{2} / S \\
8.30 \\
20.2 \\
8.63\end{array}$ & $\begin{array}{c}\text { DATE } \\
09-15-60 \\
08-31-61 \\
11-05-63\end{array}$ & $\begin{array}{l}\mathrm{FT}^{3} / \mathrm{S} \\
44.2 \\
20.2 \\
9.49\end{array}$ & $\begin{array}{c}\text { DATE } \\
10-28-64 \\
08-29-66\end{array}$ & $\begin{array}{l}\mathrm{FT}^{3} / \mathrm{S} \\
22.8 \\
11.2\end{array}$ & $\begin{array}{c}\text { DATE } \\
05-23-67 \\
06-18-68\end{array}$ & $\begin{array}{r}\mathrm{FT}^{3} / \mathrm{S} \\
3.16 \\
2.05\end{array}$ & $\begin{array}{c}\text { DATE } \\
08-30-71 \\
09-24-75\end{array}$ & $\begin{array}{l}\mathrm{FT}^{3} / \mathrm{S} \\
4.0 \\
22 .\end{array}$ \\
\hline$Q$ & $=60$ & $Q R$ & $=9.0$ & 702 & 4.6 & 7010 & $0.8 * * *$ & & \\
\hline
\end{tabular}

6-80?4.00 EB W NISHNABOTNA R. AT AVOCA, IOWA

LAT 412835 , LONG 951947 , IN NE $1 / 4$ SEC.

$16, T .77 \mathrm{~N}$., R.39 W., POTTAWATAMIE CO. (78),

AT BRIUGE ON STATE HIGHWAY 83 IN AVOCA.

DRAINAGE AREA $223 \quad \mathrm{MI}^{2}$

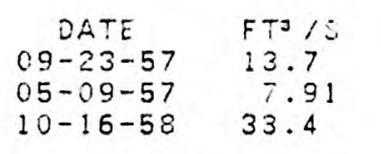

$Q \mathbf{Q}=89$

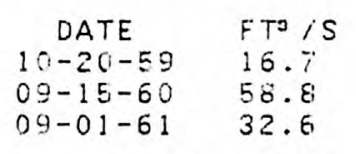

$Q(84)=14$

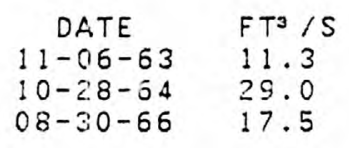

$7 Q 2=5.8$

$\begin{array}{cr}\text { DATE } & F T^{3} / S \\ 05-23-67 & 4.62 \\ 06-19-68 & 2.36\end{array}$

DATE
$08-31-71$

$09-26-75$

$7 Q 10=1.0 * * *$

6-807:.20 GRAYBILL CR NR MACEDONIA, IOWA

LAT $4111 X X$, LONG $9523 \times X$, IN SE $1 / 4$ SEC.

25. T.74 N., R. 40 W.. POTTAWATAMIE CO. $\{78\}$, AT BRIUGE, 2 MILES SE OF MACEDONIA.

DRAINAGE AREA $52.1 \mathrm{MI}^{2}$

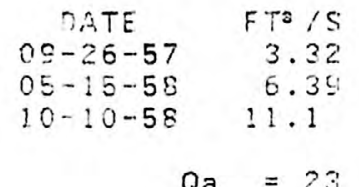

$Q_{a}=23$

$\begin{array}{rl}\text { DATE } & F T^{2} \text { IS } \\ 10-20-59 & 11.7 \\ 05-16-60 & 13.6 \\ 09-01-61 & 5.71\end{array}$

$0: 84 ;=4.6$

$\begin{array}{rr}\text { DA.TE } & F T^{3} / S \\ 11-016-63 & 2.12 \\ 10-2.7-64 & 6.84 \\ 08-30-66 & 5.64 \\ 7 Q 2= & 2.6\end{array}$

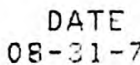

$08-31-7$
$09-26-75$

$F T^{3} / S$

1.6
$F T^{3} / S$

41 . 
NISHNABOTNA RIVER BASIN--Cont inued

6-8074.40 FARM CR NR MACEDONIA, IOWA

LAT $4110 \times X$, LONG $9523 \times X$, IN SE $1 / 4$ SEC.

36, T.74 N., R. 40 W., POTTAWATAMIE CO. (78),

AT BRIDGE, 3 MILES SE OF MACEDONIA.

DRAINAGE AREA $104 \quad \mathrm{MI}^{2}$

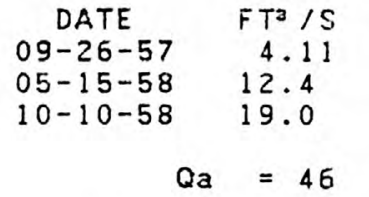

$Q a=46$

$\begin{array}{cc}\text { DATE } & \text { FT }^{3} / S \\ 10-20-59 & 6.10 \\ 09-16-60 & 32.8 \\ 09-01-61 & 11.7\end{array}$

$Q(84)=9.5$

$\begin{array}{cc}\text { DATE } & F T^{3} / S \\ 11-06-63 & 5.66 \\ 08-30-66 & 13.7\end{array}$

$702=6.3$

$\begin{array}{crc}\text { DATE } & F T^{3} / S & \text { DATE } \\ 05-23-67 & 4.80 & 08-31-71 \\ 06-18-68 & 4.84 & 09-26-75\end{array}$

$08-31-71$
$09-26-75$

$F T^{3} / S$

4.4

16.

6-8074.80 INDIAN CR NR HASTINGS, IOWA

LAT 410151 , LONG 953004 , IN SE $1 / 4$ SEC. $13, T .72$ N., R.41 W., MILLS CO. (65),

AT BRIDGE, 0.5 MILE NORTH OF HASTINGS.

DRAINAGE AREA $67.9 \mathrm{MI}^{2}$

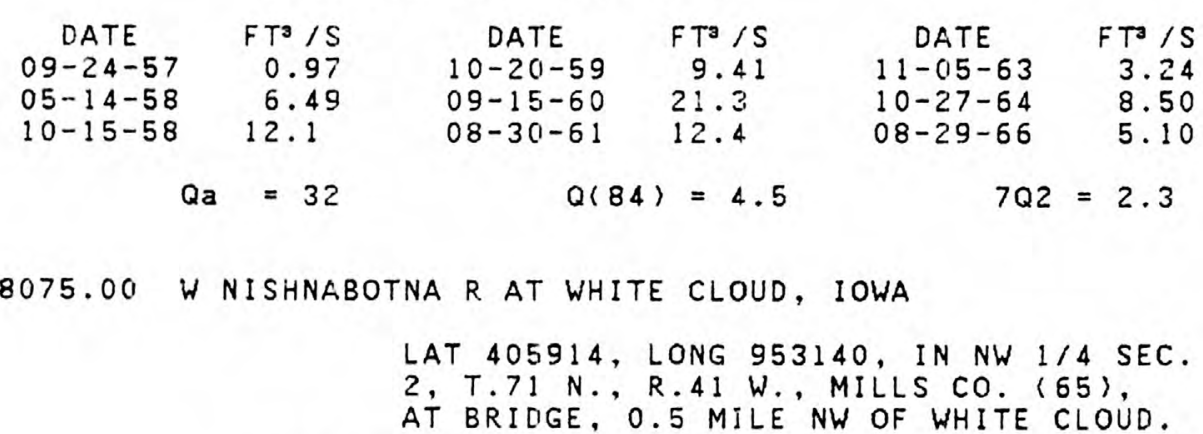

DRAINAGE AREA $967 \quad \mathrm{MI}^{2}$

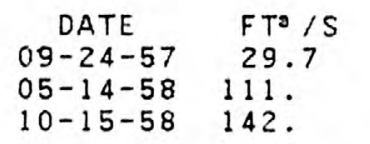

$Q \mathbf{a}=400$

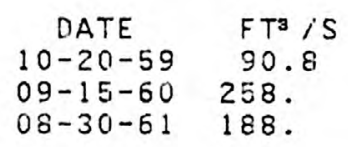

$\begin{array}{ll}9-15-60 & 258 \\ 08-30-61 & 188\end{array}$

$Q(84)=87$

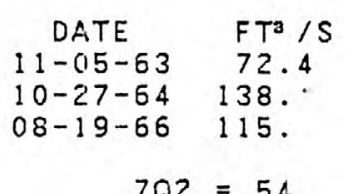

$7 Q 2=54$

$\begin{array}{cccc}\text { DATE } & \text { FT }^{3} / \mathrm{S} & \text { DATE } & F^{3} / \mathrm{S} \\ 05-23-67 & 1.92 & 08-31-71 & 0.75 \\ 06-18-68 & 1.89 & 09-25-75 & 7.6 \\ & & & \\ 7010=0.4 * * * & \end{array}$

$\begin{array}{ll}\text { DATE } & F T^{3} / S \\ 08-31-71 & 41.0\end{array}$ $09-26-75 \quad 204$. 
NISHNABOTNA RIVER BASIN--Continued

6-8075.50 WNISHNABOTNA $\approx$ NF. MALVERN, IOWA

LAT 405730 , LONG 953322 , IN NW $1 / 4$ SEC.

$15, T .71$ N., R. 41 W., MILLS CO. (65),

AT BRIDGE, 3.5 MILES SE OF MALVERN.

DRAINAGE AREA $974 \quad M I^{2}$

$\begin{array}{rl}\text { DATE } & \text { FTO } / S \\ 09-24-57 & 30.4 \\ 05-14-53 & 113 . \\ 10-15-58 & 153 . \\ Q a & =403\end{array}$

$\begin{array}{cc}\text { DATE } & F^{3} / S \\ 10-20-59 & 94.6 \\ 09-15-60 & 264 . \\ 08-30-61 & 185 .\end{array}$

$\begin{array}{cc}\text { DATE } & F T^{3} / S \\ 11-05-63 & 76.9 \\ 10-27-64 & 142 .\end{array}$

$7 Q 2=55$

$\begin{array}{cc}\text { DATE } & F T^{3} / S \\ 08-29-66 & 120 . \\ 05-23-67 & 45.1\end{array}$

$7010=16 * * *$

6-8076.00 SILVER CR NR AVOCA, IOWA

LAT 412507 , LONG 952653. IN NE 1/4 SEC

A. T.7G N., R. 40 W., POTTAWATAMIE CO.

( $>8)$, AT BRIDGE, 7 MILES SW OF AVOCA.

\section{DRAINAGE AREA $59.2 \mathrm{MI}^{2}$}

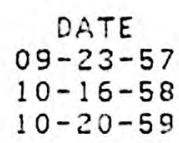

$\begin{array}{cc}\text { DATE } & F T^{3} / S \\ 09-15-60 & 15.9 \\ 09-01-61 & 8.818 \\ 11-06-53 & 2.20\end{array}$

$\begin{array}{cr}\text { DATE } & F T / 5 \\ 10-28-64 & 5.76 \\ 08-30-66 & 5.56\end{array}$

DATE

$05-23-67$

$F T=/ S$

0.82
1.59

DATE

$08-31-71$

$=T / S$

$06-19-68 \quad 1.59$

12 .

$7010=0.2 * * *$

$Q(84)=3.6$

$7 Q 2=1.5$

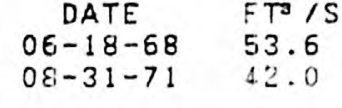

3.6

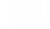

6-8076.50 SILVER CR NR TREYNOR, IOWA

LAT 411042, LONG 953434, IN SW 1/4 SEC.

28, T.74 N.. R. 41 W., POTTAWATAMIE CO.

(78), AT BRIDGE, 4 MILES SE OF TREYNOR.

DRAINAGE AREA $115 \quad \mathrm{MI}^{2}$

\begin{tabular}{|c|c|c|c|c|c|c|c|c|c|}
\hline $\begin{array}{c}\text { DATE } \\
09-23-57 \\
05-15-58 \\
10-10-58\end{array}$ & $\begin{array}{l}F T=/ S \\
8.20 \\
25.6 \\
22.4\end{array}$ & $\begin{array}{c}\text { DATE } \\
10-20-59 \\
09-1 E_{1}-60 \\
09-01-61\end{array}$ & $\begin{array}{l}F T^{3} \text { is } \\
1.8 .8 \\
36.7 \\
16.5\end{array}$ & $\begin{array}{c}\text { DATE } \\
11-0.6-63 \\
10-2.7-64 \\
08-30-66\end{array}$ & $\begin{array}{l}F^{3} / S \\
6.43 \\
13.6 \\
13.6\end{array}$ & $\begin{array}{c}\text { DATE } \\
05-23-67 \\
06-18-68\end{array}$ & $\begin{array}{l}\mathrm{FT}^{3} / \mathrm{S} \\
5.82 \\
4.75\end{array}$ & $\begin{array}{c}\text { DATE } \\
\text { OE-31-71 } \\
09-26-75\end{array}$ & $\begin{array}{l}F T^{3} / S \\
6.9 \\
28 .\end{array}$ \\
\hline Qa & $=47$ & $Q<8$ & $=12$ & $7 Q 2$ & $=7.0$ & $7 Q 10$ & 1. $8 * * *$ & & \\
\hline
\end{tabular}


NISHNABOTNA RIVER BASIN--Continued

6-8078.00 M SILVER CR NR TREYNOR, IOWA

LAT 411041 , LONG 953600 , IN SE $1 / 4$ SEC.

30, T.74 N., R.41 W., POTTAWATAMIE CO. (78),

AT BRIDGE, 4 MILES SOUTH OF TREYNOR.

DRAINAGE AREA $74.3 \mathrm{MI}^{2}$

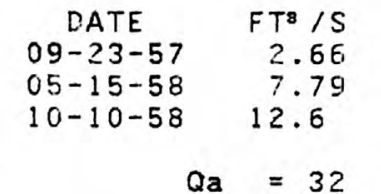

$Q_{\mathbf{a}}=32$

$\begin{array}{rc}\text { DATE } & F T^{3} / S \\ 10-2(1-59 & 9.31 \\ 09-161-60 & 27.7 \\ 09-02-61 & 8.77 \\ Q(84) & =7.9\end{array}$

$\begin{array}{cr}\text { DATE } & F^{3} / S \\ 05-23-67 & 3.32 \\ 05-18-68 & E .30 \\ & \\ 7010=0.8 * * *\end{array}$

$7010=0.8 * \star *$

6-8079.00 SILVER CR NR MALVERN, IOWA

LAT 405656, LONG 953420, IN SW $1 / 4$ SEC.

$16, T .71$ N., R.41 W., MILLS CO. (65),

AT BRIDGE, 4 MILES SOUTH OF MALVERN.

DRAINAGE AREA $282 \quad M^{2}$

\begin{tabular}{|c|c|c|c|c|c|c|c|c|c|}
\hline $\begin{array}{c}\text { DATE } \\
09-24-57 \\
05-14-58 \\
10-15-58\end{array}$ & $\begin{array}{l}\mathrm{FT}^{3} / \mathrm{S} \\
12.6 \\
26.3 \\
44.6\end{array}$ & $\begin{array}{c}\text { DATE } \\
10-20-59 \\
09-15-60 \\
08-30-61\end{array}$ & $\begin{array}{l}F T \cdot / S \\
39.1 \\
96.8 \\
42.3\end{array}$ & $\begin{array}{c}\text { DATE } \\
11-05-63 \\
10-27-64 \\
08-29-66\end{array}$ & $\begin{array}{l}F T^{3} / \mathrm{S} \\
23.0 \\
34.8 \\
35.9\end{array}$ & $\begin{array}{c}\text { DATE } \\
05-23-67 \\
06-18-68\end{array}$ & $\begin{array}{l}\mathrm{FT}^{3} / \mathrm{S} \\
17.1 \\
17.9\end{array}$ & $\begin{array}{c}\text { DATE } \\
08-31-71 \\
09-26-75\end{array}$ & $\begin{array}{l}\mathrm{FT}^{\mathrm{T}} / \mathrm{S} \\
21.0 \\
61 .\end{array}$ \\
\hline Qa & $=117$ & $Q<8$ & $\gamma=30$ & 702 & $=20$ & 7010 & $7.2 * \star *$ & & \\
\hline
\end{tabular}

6-8086.00 WALNUT CR NR GRISWOLD, IOWA

LAT $4117 \times X$, LONG $9513 \times X$, IN NW $1 / 4$ SEC.

$22, T .74$ N., R. 38 W., POTTAWATAMIE CO. (78),

AT BRIDGE, 5 MILES NW OF GRISWOLD.

DRAINAGE AREA $61.3 \mathrm{MI}^{2}$

\begin{tabular}{|c|c|c|c|c|c|c|c|c|c|}
\hline $\begin{array}{c}\text { DATE } \\
09-26-57 \\
05-15-58 \\
10-16-58\end{array}$ & $\begin{array}{c}F T^{2} / \mathrm{S} \\
5.91 \\
12.1 \\
17.3\end{array}$ & $\begin{array}{c}\text { DATE } \\
10-20-59 \\
09-16-60 \\
09-01-60\end{array}$ & $\begin{array}{c}\mathrm{FT}^{3} / \mathrm{S} \\
5.15 \\
13.2 \\
4.41\end{array}$ & $\begin{array}{c}\text { DA.TE } \\
11-0.6-63 \\
10-28-64 \\
08-30-66\end{array}$ & $\begin{array}{l}F T^{0} / S \\
0.68 \\
6.00 \\
4.76\end{array}$ & $\begin{array}{c}\text { DATE } \\
05-23-67 \\
06-19-68\end{array}$ & $\begin{array}{r}F T / S \\
1.18 \\
1.20\end{array}$ & $\begin{array}{c}\text { DATE } \\
08-31-71 \\
09-25-75\end{array}$ & $\begin{array}{l}\mathrm{FT}=/ \mathrm{S} \\
0.65 \\
7.6\end{array}$ \\
\hline$Q$ & $=28$ & $Q<8$ & $=3.2$ & 702 & $=1.4$ & 7010 & $=0.2 * * *$ & & \\
\hline
\end{tabular}


NISHNABOTNA RIVER BASIN--Cont inued

6-8087.00 WALNUT CR NR HAWTHORNE, IOWA

LAT $4058 \times x$, LONG $9522 \times X$, IN NW $1 / 4$ SEC.

$17, T .71$ N., R.39 W., MONTGOMERY CO. (69),

AT BRIDGE, 3 MILES SW OF HAWTHORNE.

DRAINAGE AREA $140 \quad \mathrm{MI}^{2}$

$\begin{array}{rc}\text { DATE } & F T^{3} / 5 \\ 09-25-57 & 8.10 \\ 05-14-58 & 20.5 \\ 10-14-58 & 29.6 \\ Q a & =65\end{array}$

$\begin{array}{cl}\text { DATE } & F T^{3} / S \\ 10-20-59 & 14.8 \\ 0 S-15-60 & 34.2 \\ 08-30-61 & 17.7\end{array}$

$\begin{array}{rc}\text { DATE } & F T^{J} / \mathrm{S} \\ 11-05-63 & 2.92 \\ 10-2.6-64 & 16.3 \\ 08-2.9-66 & 12.7 \\ 7 Q 2 & =3.4\end{array}$

$\begin{array}{cr}\text { DATE } & \text { FT /S } \\ 05-23-67 & 2.53 \\ 06-18-68 & 3.15 \\ 7 Q 10= & 0.4 * \star \star\end{array}$
DATE
$08-31-71$

$09-25-75$

$\mathrm{FT}^{3} / \mathrm{S}$

$7 Q 10=0.4 * \star \star$

6-8088.00 WALNUT CR NR RANDOLPH, IOWA

LAT 404739, LONG 953325, NEAR E $1 / 4$

CORNER SEC.9, T.69 N., R.41 W., FREMONT CO.

(36), AT BRIDGE, 5.5 MILES SOUTH OF RANDOLPH.

DRAINAGE AREA 222 MI2

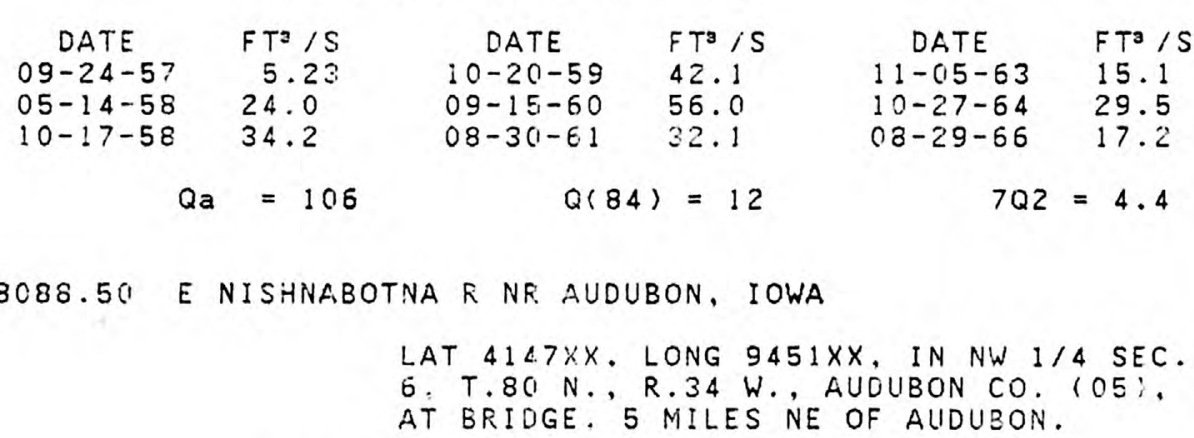

DRAINAGE AREA $66.7 \mathrm{MI}^{2}$

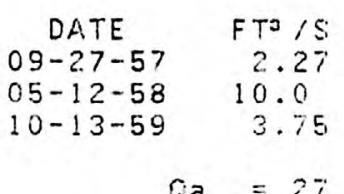

$\mathrm{Qa}=27$

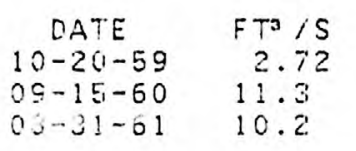

$(2(84)=2.4$

$\begin{array}{cr}\text { DA.TE } & F T^{3} / S \\ 11-05-63 & 3.25 \\ 10-2.6-64 & 7.15 \\ 08-29-66 & 3.99\end{array}$

$7 Q 2=0.9$

$\begin{array}{cr}\text { DATE } & F T^{3} / S \\ 05-23-67 & 5.89 \\ 06-18-68 & 5.17 \\ & \\ & \end{array}$

$7 Q 10=1.1 * * *$

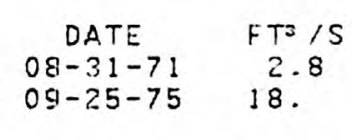

8 . 
NISHNABOTNA RIVER BASIN--Continued

6-8089.00 E NISHNABOTNA R AT EXIRA, IOWA

LAT $4135 \times X$, LONG $9454 X X$, IN NW $1 / 4$ SEC.

$\begin{array}{lll}4 & T .78 & N \\ \text { AT BRIDGE AT WEST CITY LIMITS OF EXIRA. }\end{array}$

DRAINAGE AREA $195 \mathrm{MI}^{2}$

$\begin{array}{rc}\text { DATE } & F T^{3} / S \\ 09-27-57 & 7.46 \\ 05-12-58 & 19.6 \\ 10-13-58 & 15.9 \\ & \\ & =79\end{array}$

$\begin{array}{cc}\text { DATE } & F T / S \\ 10-20-59 & 7.29 \\ 09-15-60 & 31.5 \\ 08-31-61 & 19.6\end{array}$

$\begin{array}{cc}\text { DATE } & \mathrm{FT}^{3} / \mathrm{S} \\ 11-06-63 & 7.35 \\ 10-2.7-64 & 15.8 \\ 08-29-66 & 13.9\end{array}$

DATE
$05-23-67$
$06-18-68$
$\mathrm{FT}^{3} / \mathrm{S}$
2.38
2.38

$06-18-68$

2.99

DATE

$08-30-71$
$09-24-75$

$09-24-75$

$F T^{3} / \mathrm{S}$

2.9

22.

6-8090.50 DAVIDS CR AT EXIRA, IOWA

LAT $4135 \times X$, LONG $9453 \times X$, IN NE $1 / 4$ SEC.

$4, T .78$ N., R.35 W., AUDUBON CO. $\{05\}$,

AT BRIDGE NEAR EAST CITY LIMITS OF EXIRA.

DRAINAGE AREA $56.7 \mathrm{MI}^{2}$

\begin{tabular}{|c|c|c|c|c|c|c|c|c|c|}
\hline $\begin{array}{c}\text { DATE } \\
09-27-57 \\
05-12-58 \\
10-13-58\end{array}$ & $\begin{array}{l}\mathrm{FT}^{5} / \mathrm{S} \\
2.84 \\
9.55 \\
2.41\end{array}$ & $\begin{array}{c}\text { DATE } \\
10-20-59 \\
09-15-60 \\
08-31-61\end{array}$ & $\begin{array}{c}F T^{3} / \mathrm{S} \\
4.43 \\
17.0 \\
6.38\end{array}$ & $\begin{array}{c}\text { DATE } \\
11-05-63 \\
10-27-64 \\
08-29-66\end{array}$ & $\begin{array}{l}\mathrm{FT}^{3} / \mathrm{S} \\
2.09 \\
9.12 \\
5.38\end{array}$ & $\begin{array}{c}\text { DATE } \\
05-23-67 \\
06-18-68\end{array}$ & $\begin{array}{r}F T / S \\
0.99 \\
0.82\end{array}$ & $\begin{array}{c}\text { DATE } \\
08-30-71 \\
09-24-75\end{array}$ & $\begin{array}{l}\mathrm{FT}^{3} / \mathrm{S} \\
1.5 \\
10 .\end{array}$ \\
\hline$Q$ & $=25$ & $Q 6$ & $=2.7$ & $7 Q 2$ & $=1.0$ & 7010 & $0.2 * \star \star \star$ & & \\
\hline
\end{tabular}

6-809:.00 TROUBLESOME CR NR WIOTA, IOWA

LAT $4130 \times X$, LONG $9451 \times X$, IN NW $1 / 4$ SEC.

2, T.77 N., R. 35 W., CASS CO. (15),

AT BRIUGE, 7.5 MILES NE OF WIOTA.

DRAINAGE AREA $68.4 \mathrm{MI}^{2}$

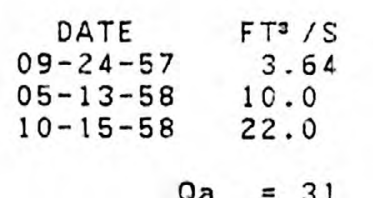

$Q_{a}=31$

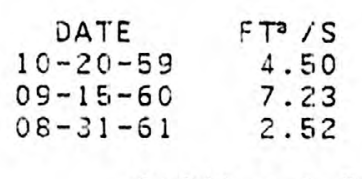

$Q(84)=1.7$

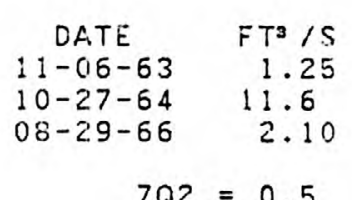

$7 Q 2=0.5$

$\begin{array}{cr}\text { DATE } & F T / 5 \\ 05-23-67 & 0.35 \\ 06-18-68 & 0.48 \\ & \\ 7010=*\end{array}$


NISHNABOTNA RIVER BASIN--cont inued

5-8091.50 TROUBLESOME CR MR ATLANTIC, IOWA

LAT $4125 \times \times$, LONG $9458 \times X$. IN NE $1 / 4$ SEC.

$3, T .76$ N.. R. 36 W., CASS CO. $\{15\}$,

AT BRIDGE, 2 MILES NE OF ATLANTIC.

DRAINAGE AREA $128 \quad \mathrm{MI}^{2}$

$\begin{array}{cc}\text { DATE } & F T^{3} / S \\ 09-24-57 & 8.07 \\ 05-13-58 & 17.8 \\ 10-15-58 & 39.9\end{array}$

$\begin{array}{cc}\text { DATE } & F T^{3} / S \\ 10-21-59 & 9.58 \\ 09-15-60 & 18.5 \\ 08-31-61 & 9 .(16\end{array}$

$Q_{a}=50$

Q(84)

$=6.6$
DATE
$05-23-67$

$05-23-67$
$06-18-68$

$F T^{3} / S$
3.84

3.84
2.56

DATE

$08-30-71$
$09-25-75$

$\mathrm{FT}^{3} / \mathrm{S}$

$10-27-64 \quad 25.3$

$\begin{array}{rr}10-27-64 & 25.3 \\ 08-30-66 & 7.00\end{array}$

$7 Q 2=2.9$

$7010=0.8 * * *$

6-8092.00 E NISHNABOTNA R AT ATLANTIC, IOWA

LAT $4124 X X$, LONG 9502XX. IN SE $1 / 4$ SEC.

O, T.7E N., R. 36 W. CASS CO. $(15)$,

AT SRIDGE ON STATE HIGHWAY 83, NEAR

WEST CITY LIMITS OF ATLANTIC.

DRAINAGE AREA $382 \quad \mathrm{MI}^{2}$

$\begin{array}{cl}\text { DATE } & F T^{2} / S \\ 09-24-57 & 27.5 \\ 05-13-58 & 58.1 \\ 10-15-58 & 95.7\end{array}$

$\begin{array}{cl}\text { DATE } & F T^{3} / \mathrm{S} \\ 10-21-59 & 35.1 \\ 09-15-60 & 77.0 \\ 08-31-61 & 42.1\end{array}$

$\begin{array}{cl}\text { DATE } & F T^{3} / \mathrm{S} \\ 1 \mathrm{i}-06-63 & 19.5 \\ 10-27-54 & 59.5 \\ 08-30-66 & 29.7\end{array}$

$\begin{array}{ll}\text { DATE } & F T^{3} / 5 \\ 05-23-67 & 12.2 \\ 06-18-68 & 10.8\end{array}$

DATE
OB $-30-7$
$09-25-75$

$08-30-7$

$\mathrm{FT}^{3} / \mathrm{S}$

$06-18-68 \quad 10.8$

$09-25-75$

15

$7 Q 10=4 \cdot 3 * * *$

6-8092.50 TURKEY CR EAST OF ATLANTIC, IOWA

LAT 4123XX, LONG $9455 \times X$, IN SE $1 / 4$ SEC.

$7, T .7 \epsilon_{1} N ., R .35 \mathrm{~W}$. CASS CO. $\{15\}$,

AT BRIUGE, 3 MILES SE OF ATLANTIC.

DRAINAGE AREA $69.5 \mathrm{MI}^{2}$

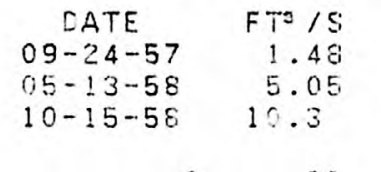

$\mathrm{Oa}_{\mathrm{a}}=33$

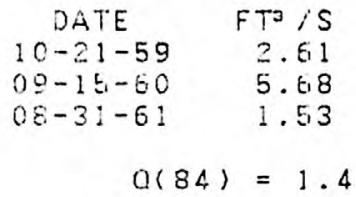

$a(84)=1.4$

$\begin{array}{rr}\text { DATE } & F T^{3} / S \\ 11-016-83 & 0.86 \\ 10-27-64 & 7.32 \\ 08-30-66 & 1.26 \\ 702= & 0.6\end{array}$

$702=0.6$

$\begin{array}{cccc}\text { DATE } & \mathrm{FT}^{3} / \mathrm{S} & \text { DATE } & \mathrm{FT}^{3} / \mathrm{S} \\ 05-23-67 & 0.83 & 08-30-71 & 0.48 \\ 06-18-68 & 0.67 & 09-25-75 & 2.0 \\ & & & \\ 7010=0.1 * * * & & \end{array}$


NISHNABOTNA RIVER BASIN--Continued

6-8093.00 TURKEY CR NR ATLANTIC, IOWA

LAT $4119 \times X$, LONG $9404 \times X$, NEAR CENTER OF SEC. 2, T.75 N., R. $37 \mathrm{~W}$., CASS CO. (15), AT BRIDGE, 6 MILES SW OF ATLANTIC.

DRAINAGE AREA $133 \quad \mathrm{MI}^{2}$

$\begin{array}{cr}\text { DATE } & F T^{3} / \mathrm{S} \\ 09-24-57 & 3.69 \\ 05-13-58 & 6.55 \\ 10-15-58 & 19.8\end{array}$

$\begin{array}{cc}\text { DATE } & F T^{3} / S \\ 10-20-59 & 6.30 \\ 09-15-60 & 14.0 \\ 09-01-61 & 3.22\end{array}$

$\begin{array}{cc}\text { DATE } & F T^{3 / S} \\ 11-06-63 & 1.56 \\ 10-27-64 & 12.8 \\ 08-30-66 & 4.92\end{array}$

$Q_{a}=62$

\section{DATE}

$08-31-71$
$09-25-75$

$\mathrm{FT}^{\mathrm{3}} / \mathrm{S}$

1.2

6-8093.30 E NISHNABOTNA R NR LEWIS, IOWA

LAT $4119 \times X$, LONG $9505 \times X$, IN NE $1 / 4$ SEC.

10 , T.75 N., R.37 W., CASS CO. (15), AT ERIDGE ON U.S. HIGHWAY 6, I MILE NORTH OF LEWIS.

DRAINAGE AREA $574 \quad \mathrm{MI}^{2}$

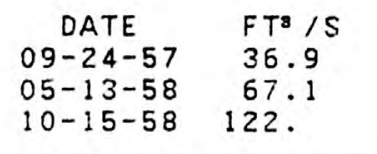

$Q_{a}=251$

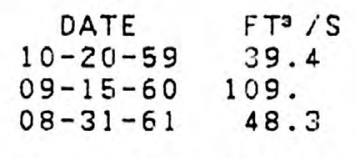

$Q(84)=30$

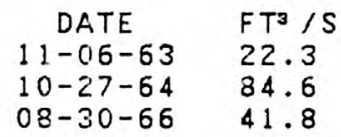

$7 Q 2=14$

6-8093.50 INDIAN CR NR ELKHORN, IOWA

LAT $4133 \times X$, LONG $9508 \times X$, IN N $1 / 2$ SEC.

20, T.78 N., R.37 W. SHELEY CO. ( 83 ; ,

AT BRIUGE, 5 MILES SW OF ELKHORN.

ORAINAGE AREA $67.4 \mathrm{MI}^{2}$

$\begin{array}{rc}\text { DATE } & \mathrm{FT}^{3} / S \\ 09-26-57 & 1.16 \\ 10-09-58 & 18.0 \\ 10-20-59 & 5.35 \\ Q a & =29\end{array}$

$\begin{array}{rl}\text { DATE } & \text { FT iS } \\ 09-15-60 & 16.1 \\ 08-3(i-61 & 11.3 \\ 11-06-63 & 2.94 \\ 0(84) & =2.5\end{array}$

$\begin{array}{cr}\text { DATE } & \mathrm{FT}^{3} / \mathrm{S} \\ 10-28-64 & 9.11 \\ 08-29-66 & 6.91\end{array}$

DATE
$05-23-67$
$06-18-68$

$F T^{3} / 2$

0.80

DATE

$08-30-71$

$09-24-75$

$F T^{3} / S$

$1 \cdot 5$

$7 Q 10=0.2 * * *$ 
NISHNABOTNA RIVER BASIN--Cont inued

6-8079.00 INDIAN CR NR LEWIS, IOWA

LAT $4118 \times X$, LONG $9508 \times X$. IN SW $1 / 4$ SEC.

8. T.75 N., R.37 W., CASS CO. $(15)$.

AT BRIDGE, 2 MILES WEST OF LEWIS.

DRAINAGE AREA $183 \quad \mathrm{MI}^{2}$

$\begin{array}{rlclcr}\text { DATE } & F^{3} / \mathrm{S} & \text { DATE } & \text { FT } / \mathrm{S} & \text { DATE } & F^{3} / S \\ 09-24-57 & 15.2 & 10-20-59 & 16.5 & 11-06-63 & 7.36 \\ 05-13-58 & 25.9 & 09-16-60 & 31.9 & 10-28-64 & 21.0 \\ 10-15-58 & 52.5 & 09-01-61 & 17.3 & 08-31-66 & 15.0 \\ Q \text { Q } & =79 & 0(84)=9.1 & 702=3.6\end{array}$

$\begin{array}{cccc}\text { DATE } & \text { FT /S } & \text { DATE } & F T^{3} / S \\ 05-23-67 & 3.71 & 08-31-71 & 4.5 \\ 06-19-68 & 2.60 & 09-25-75 & 24 . \\ & & \end{array}$

6-8094.50 E NISHNABOTNA R NR. GRISWOLD, IOWA

LAT $4117 \times X$, LONG $9508 \times X$, IN SE $1 / 4$ SEC, 18, T.75 N., R.37 W., CASS CO. (15), AT BRIÓGE ON STATE HIGHWAY 48,4 MILES NORTH OF GRISWOLD.

ORAINAGE AREA $778 \quad \mathrm{MI}^{2}$

\begin{tabular}{|c|c|c|c|c|c|}
\hline $\begin{array}{c}\text { DATE } \\
09-24-57 \\
05-13-58 \\
10-15-58\end{array}$ & $\begin{array}{l}\mathrm{FT}^{3} / \mathrm{S} \\
54.5 \\
103 . \\
190 .\end{array}$ & $\begin{array}{c}\text { DATE } \\
10-20-59 \\
09-16-60 \\
09-01-61\end{array}$ & $\begin{array}{l}F T^{3} \text { is } \\
64.7 \\
135 . \\
60.9\end{array}$ & $\begin{array}{c}\text { DATE } \\
11-06-63 \\
10-28-64 \\
08-31-66\end{array}$ & $\begin{array}{l}\mathrm{FT}^{3} / \mathrm{S} \\
37.6 \\
102 . \\
58.8\end{array}$ \\
\hline & $=334$ & $Q<$ & 43 & $7 Q 2$ & \\
\hline
\end{tabular}

$\begin{array}{rl}\text { DATE } & F T / S \\ 05-23-67 & 20.2 \\ 06-19-68 & 15.7 \\ 7010=6.2 \star \star \star\end{array}$

6-8098.00 E NISHNABOTNA R NR FARRAGUT, IOWA

I.AT $4045 \times X$, LONG $9529 \times X$, IN SE $1 / 4$ SEC.

$30, T .69$ N., R. 40 W., FREMONT CO. (36),

AT BRIDGE ON STATE HIGHWAY $174,1.5$

MILES NORTH OF FARRAGUT.

DRAINAGE AREA $1082 \quad \mathrm{MI}^{2}$

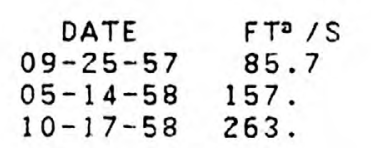

Qa $=472$

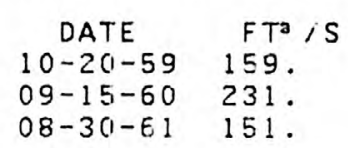

$Q(84)=85$

\begin{tabular}{cl} 
DATE & \multicolumn{1}{c}{$F T^{3} / \mathrm{S}$} \\
$11-05-63$ & 64.7 \\
$10-27-64$ & 169. \\
$08-30-66$ & 103.
\end{tabular}

$7 Q 2=48$

$\begin{array}{cl}\text { DATE } & F T^{\prime} / S \\ 05-23-67 & 52.8 \\ 06-18-68 & 51.7\end{array}$

DATE

$08-31-71$ ( 18 (3)

09-25-75 123. 
TARKIO RIVER BASIN

6-8118.60 TARKIO R NR COBURG, IOWA

LAT $4054 \times X$, LONG $9508 \times X$, IN NW $1 / 4$ SEC.

5, T.70 N., R.37 W., PAGE CO. (73),

AT BRIDGE, 6 MILES SE OF COBURG.

DRAINAGE AREA $66.6 \mathrm{MI}^{2}$

$\begin{array}{ccclcr}\text { DATE } & \text { FTa/S } & \text { DATE } & F T \text { IS } & \text { DATE } & F T / S \\ 09-24-57 & 0.50 & 09-12-60 & 18.9 & 10-27-64 & 6.34 \\ 05-13-58 & 5.72 & 09-13-60 & 16.8 & 08-10-56 & 6.47 \\ 10-14-58 & 9.58 & 10-25-61 & 19.8 & 12-12-67 & 3.54 \\ 07-27-59 & 6.65 & 10-31-63 & 1.08 & 03-12-68 & 1.53 \\ & & & & & \end{array}$

DATE
$09-23-69$
$07-09-70$
$08-24-71$

$F T=/ S$

.02

0.82

DATE
$09-24-74$

$09-24-75$

$F T^{3} / S$

$08-24-71 \quad 0.40 \quad 09-21-76$

0.51

$Q \mathbf{a}=33$

$Q(84)=2.8$

$7010=*$

6-8118.80 E TARKIO CR NR YOR.KTOWN, IOWA

LAT $4043 \times X$, LONG $9512 \times X$, IN SW $1 / 4$ SEC.

$10, T .68$ N., R.38 W., PAGE CO. (73),

AT BRIUGE, 2.5 MILES SW OF YORKTOWN.

DRAINAGE AREA $58.0 \quad \mathrm{MI}^{2}$

$\begin{array}{cccc}\text { DATE } & F T^{3} / S & \text { DATE } & F T^{3} / S \\ 09-24-57 & 0.72 & 09-12-60 & 13.6 \\ 05-13-58 & 5.82 & 09-13-60 & 11.8 \\ 10-13-58 & 11.7 & 10-25-61 & 24.8 \\ 07-27-59 & 10.1 & 10-31-63 & 6.01\end{array}$

$\begin{array}{cr}\text { DATE } & F T^{3} / S \\ 10-27-64 & 6.22 \\ 08-09-66 & 3.49 \\ 12-13-67 & 0.62 \\ & \end{array}$

$\begin{array}{cr}\text { DATE } & F T / S \\ 03-12-68 & 2.26 \\ 07-09-70 & 0.41 \\ 08-24-71 & 0.70 \\ & \\ 7010=* & \end{array}$

DATE

$09-23-74$

$09-24-75$

$F T^{3} / S$

$09-21-76 \quad 0.4$

$Q_{a}=30$

$Q(84)=2.1$

$7 Q 2=0.5$

6-8119.00 TARKIO R NR YORKTOWN, IOWA

LAT $4043 \times X$, LONG $9513 \times X$, IN $N 1 / 2$ SEC.

$16, T .68 \mathrm{~N} .$, R.38 W., PAGE CO. $(73)$,

AT BRIDGE, 3 MILES SW OF YORKTOWN.

DRAINAGE AREA $155 \quad \mathrm{MI}^{2}$

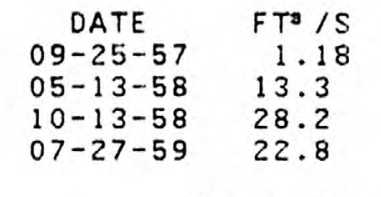

$Q_{a}=78$

$\begin{array}{cl}\text { DATE } & F T^{3} / S \\ 09-12-60 & 37.5 \\ 09-13-60 & 33.3 \\ 10-25-61 & 59.8 \\ 10-31-63 & 11.0\end{array}$

$Q(84)=6.1$

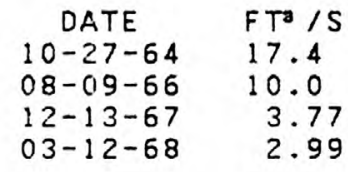

$702=1.8$

$\begin{array}{cc}\text { DATE } & F T^{3} / \mathrm{S} \\ 09-22-69 & 12.3 \\ 07-09-70 & 1.38 \\ 08-24-71 & 1.5 \\ 7010= & \end{array}$

$\begin{array}{cc}\text { DATE } & F T^{2} / S \\ 09-23-74 & 2.4 \\ 09-24-75 & 2.4 \\ 09-21-76 & 2.3\end{array}$


TARKIO RIVER BASIN--Cont inued

6-8120.00 TARKIO R. AT BLANCHARD, IOWA

LAT $4036 \times X$, LONG $9514 \times X$, IN NE $1 / 4$ SEC.

29. T.67 N., R.38 W. PAGE CO. (73),

DRAINAGE AREA $200 \quad \mathrm{MI}^{2}$

\begin{tabular}{|c|c|c|c|c|c|c|c|c|c|}
\hline $\begin{array}{c}\text { DATE } \\
09-24-57 \\
05-14-58 \\
10-13-58 \\
07-27-59\end{array}$ & $\begin{array}{l}F T^{3} / \mathrm{S} \\
2.02 \\
23.5 \\
36.0 \\
32.7\end{array}$ & $\begin{array}{c}\text { DATE } \\
09-12-60 \\
09-13-60 \\
10-25-61 \\
10-31-63\end{array}$ & $\begin{array}{l}\mathrm{FT}^{\mathrm{a}} / \mathrm{S} \\
56.1 \\
49.8 \\
89.9 \\
13.6\end{array}$ & $\begin{array}{c}\text { DATE } \\
10-27-64 \\
08-09-66 \\
12-12-67 \\
03-12-68\end{array}$ & $\begin{array}{l}F T \cdot / S \\
19.7 \\
12.9 \\
10.2 \\
11.2\end{array}$ & $\begin{array}{c}\text { DATE } \\
09-22-69 \\
07-09-70 \\
08-24-71\end{array}$ & $\begin{array}{l}F T \cdot / S \\
14.8 \\
2.28 \\
2.8\end{array}$ & $\begin{array}{c}\text { DATE } \\
09-23-74 \\
09-24-75 \\
09-21-76\end{array}$ & $\begin{array}{l}\mathrm{FT}^{3} / \mathrm{S} \\
4.6 \\
4.3 \\
3.9\end{array}$ \\
\hline & $=102$ & $Q<$ & $=10$ & $7 Q^{2}$ & $=3.2$ & 7010 & $0.5^{\star \star \star}$ & & \\
\hline
\end{tabular}

6-8123.00 W TARKIO CR NR COIN, IOWA

LAT $4041 X X$, LONG $9518 \times X$, NEAR S $1 / 2$

CORNER SEC.2Z, T.68 N., R.39 W., PAGE CO.

(73), AT BRIDGE, 4 MILES NW OF COIN.

DRAINAGE AREA $66.9 \mathrm{MI}^{2}$

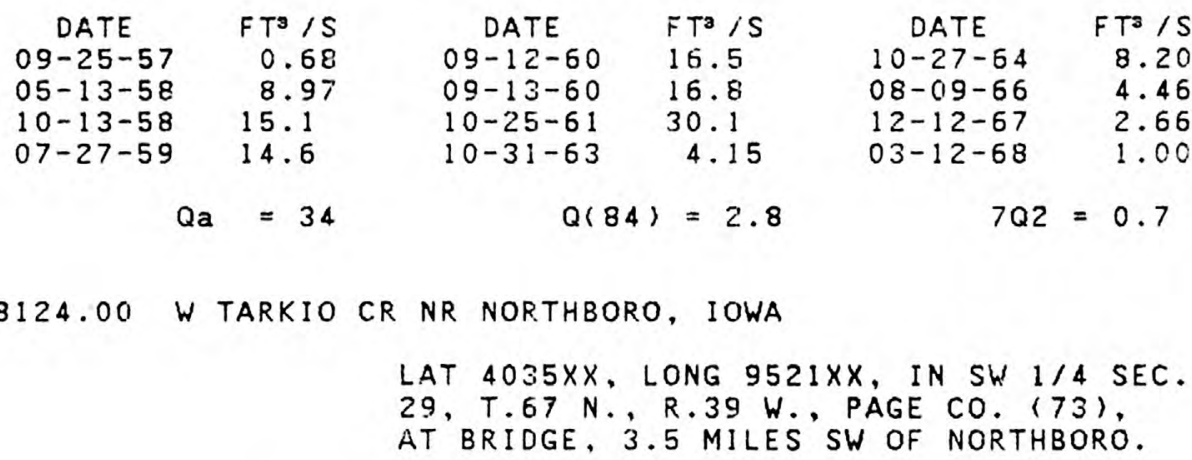

DRAINAGE AREA $87.7 \mathrm{MI}^{2}$

$\begin{array}{rl}D A T E & F T / S \\ 09-25-57 & 2.57 \\ 05-14-58 & 17.1 \\ 10-13-58 & 26.5 \\ 07-27-59 & 28.5 \\ Q a & =46\end{array}$

$Q \mathrm{a}=46$

DATE
$09-12-60$
$09-13-60$
$10-25-61$

$10-31-63$

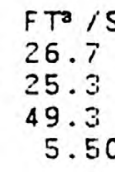

$Q(84)=6.4$

$\begin{array}{rc}\text { DATE } & F T^{3} / \mathrm{S} \\ 10-27-64 & 10.9 \\ 08-09-66 & 5.96 \\ 12-12-67 & 5.45 \\ 03-12-68 & 3.18 \\ 702= & 2.3\end{array}$

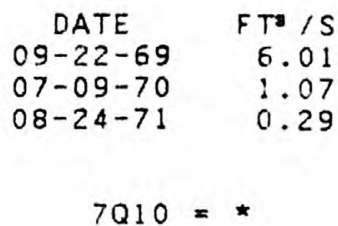

$\begin{array}{cr}\text { DATE } & F T / S \\ 09-22-69 & 9.75 \\ 07-09-70 & 1.88 \\ 08-24-71 & 0.88 \\ & \\ 7010= & \end{array}$

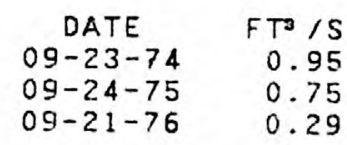

$09-21-76 \quad 0.29$ 
NODAWAY RIVER. BASIN

6-8163.00 W NODAWAY $R$ NR CUMBERLAND, IOWA

LAT $4112 \times X$, LONG $9452 \times X$, IN SW $1 / 4$ SEC.

$15, T .74 \mathrm{~N}, \mathrm{R}, 35 \mathrm{~W}$, CASS CO. (15),

AT BRIOGE, 4 MILES SOUTH OF CUMBERLAND.

DRAINAGE AREA $65.1 \mathrm{MI}^{2}$

\begin{tabular}{|c|c|c|c|c|c|}
\hline $\begin{array}{c}\text { DATE } \\
09-24-57 \\
05-13-58 \\
10-14-58 \\
07-27-59\end{array}$ & $\begin{array}{l}\mathrm{FT}^{\mathrm{T}} / \mathrm{S} \\
0.25 \\
2.71 \\
3.73 \\
3.47\end{array}$ & $\begin{array}{c}\text { DATE } \\
09-12-60 \\
09-13-50 \\
10-24-61 \\
10-31-63\end{array}$ & $\begin{array}{c}\mathrm{FT}^{3} / \mathrm{S} \\
3.01 \\
3.04 \\
28.0 \\
0.50\end{array}$ & $\begin{array}{c}\text { DATE } \\
10-26-64 \\
08-09-66 \\
12-12-67 \\
03-12-68\end{array}$ & $\begin{array}{r}\mathrm{FT}^{3} / \mathrm{S} \\
7.16 \\
2.65 \\
1.13 \\
1.25\end{array}$ \\
\hline$Q z$ & $=32$ & $Q($ & $=0.6$ & $7 Q 2$ & 0.2 \\
\hline
\end{tabular}

$\begin{array}{cr}\text { OATE } & F T^{3} / 5 \\ 09-22-69 & 1.09 \\ 07-07-70 & 0.96 \\ 09-14-71 & 0.26 \\ 7210=*\end{array}$

DATE $09-24-74$ $09-23-75$ $09-21-76$

$\mathrm{FT}^{\mathrm{s}} / \mathrm{S}$

1. 0

0.38 2.0

6-8163.50 SEVENMILE CR NR LYMAN, IOWA

LAT $4115 \times X$, LONG 9459XX, IN SE $1 / 4$ SEC.

33, T.75 N., R.36 W., CASS CO. (15),

AT BRIDGE ON U. S. HIGHWAY $71,1.5$

MILES NORTH OF LYMAN.

DRAINAGE AREA $60.8 \quad \mathrm{MI}^{2}$

DATE
$09-24-57$
$05-13-58$
$10-14-58$
$07-27-59$

$\begin{array}{ccc}\mathrm{FT}^{3} / 5 & \mathrm{DATE} & \mathrm{FT}^{3} / \mathrm{S} \\ 1.58 & 09-12-60 & 7.99 \\ 6.18 & 09-13-60 & 7.65 \\ 8.39 & 10-24-61 & 19.1 \\ 6.88 & 10-31-63 & 1.21\end{array}$

$\begin{array}{cc}\text { DATE } & F T / S \\ 10-26-64 & 11.5 \\ 08-09-66 & 5.49 \\ 12-12-67 & 1.75 \\ 03-12-68 & 1.41\end{array}$

DATE
$09-22-69$
$07-07-70$
$08-24-71$

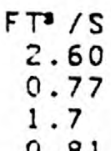

DATE

$09-24-74$

$09-23-75$

$09-22-76$

$F T / S$

$\mathrm{Q} \mathbf{a}=30$

$Q(84)=1.6$

$702=0.7$

$7 Q 10=0.2 * * *$

6-8164.00 SEVENMILE CR NR MORTON MILL, IOWA

LAT $4106 \times X$, LONG $9500 X X$, IN NW $1 / 4$ SEC.

$33, T .73$ N., R.36 W., MONTGOMERY CO. (69),

AT BRIUGE, 1 MILE NW OF MORTON MILL.

DRAINAGE AREA $124 \quad \mathrm{MI}^{2}$

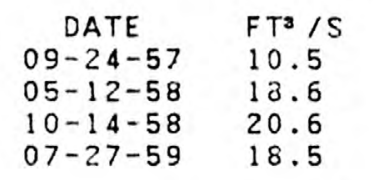

$Q \mathbf{a}=59$

$\begin{array}{cl}\text { DATE } & F T^{3} / \mathrm{S} \\ 09-13-60 & 27.5 \\ 09-13-60 & 31.2 \\ 10-24-61 & 43.4 \\ 10-31-63 & 11.0\end{array}$

$Q(84)=13$

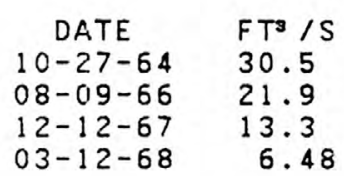

$702=7.9$

$\begin{array}{cc}\text { DATE } & F T / S \\ 09-22-69 & 13.6 \\ 07-08-70 & 8.70 \\ 09-14-71 & 11.0\end{array}$


NOBAWAY RIVER BASIN--Cont Inued

6-8165.50 W NODAWAY R NR VILLISCA, IOWA

LAT $4055 \times X$, LONG $9500 X X$. NEAR CENTER OF

SEC. 28, T.71 N., R.36 W., MONTGOMERY CO. (69),

AT BRIDGE NEAR WEST CITY LIMITS OF VILLISCA.

DRAINAGE AREA $344 \quad \mathrm{MI}^{2}$

\begin{tabular}{|c|c|c|c|c|c|c|c|c|c|}
\hline $\begin{array}{c}\text { DATE } \\
09-24-57 \\
05-12-58 \\
10-14-58 \\
07-27-59\end{array}$ & $\begin{array}{l}F T^{3} / \mathrm{S} \\
17.0 \\
38.2 \\
37.0 \\
38.7\end{array}$ & $\begin{array}{c}\text { DATE } \\
09-12-60 \\
09-13-60 \\
10-24-61 \\
10-31-63\end{array}$ & $\begin{array}{l}F T \text { IS } \\
70.1 \\
61.7 \\
150 . \\
16.9\end{array}$ & $\begin{array}{c}\text { DATE } \\
10-27-64 \\
08-10-66 \\
12-12-67 \\
03-12-68\end{array}$ & $\begin{array}{l}\mathrm{FT}^{3} / \mathrm{S} \\
54.5 \\
38.6 \\
24.6 \\
14.2\end{array}$ & $\begin{array}{c}\text { DATE } \\
09-22-69 \\
07-08-70 \\
08-24-71 \\
09-14-71\end{array}$ & $\begin{array}{l}F T / S \\
30.2 \\
13.3 \\
14.0 \\
14.0\end{array}$ & $\begin{array}{c}\text { DATE } \\
09-24-74 \\
09-24-75 \\
09-21-76\end{array}$ & $\begin{array}{l}\mathrm{FT}^{3} / \mathrm{S} \\
26 . \\
2.2 . \\
30 .\end{array}$ \\
\hline$Q a$ & $=165$ & QR & $1=$ & 702 & $=12$ & $7 Q 10$ & $4.6 * \star \star \star$ & & \\
\hline
\end{tabular}

6-8166.00 M NODAWAY R NR BRIDGEWATER, IOWA

LAT $4110 \times X$, LONG $9439 \times X$, IN NE $1 / 4$ SEC.

$33, T .74$ N.. R.33 W., ADAIR CO. $(01)$,

DRAINAGE AREA $89.3 \mathrm{MI}^{2}$

\begin{tabular}{|c|c|c|c|c|c|c|c|c|c|}
\hline $\begin{array}{c}\text { DATE } \\
09-26-57 \\
10-13-58 \\
07-31-59 \\
09-12-60\end{array}$ & $\begin{array}{l}\mathrm{FT}^{3} / \mathrm{S} \\
0 . \\
7.56 \\
9.83 \\
4.86\end{array}$ & $\begin{array}{c}\text { DATE } \\
09-13-60 \\
10-25-61 \\
10-31-63\end{array}$ & $\begin{array}{c}F T \cdot / S \\
1.79 \\
25.3 \\
0.80\end{array}$ & $\begin{array}{c}\text { DATE } \\
10-26-64 \\
08-09-66 \\
12-12-67\end{array}$ & $\begin{array}{l}F T / S \\
15.0 \\
4.96 \\
0.44\end{array}$ & $\begin{array}{c}\text { DATE } \\
03-12-68 \\
07-07-70 \\
09-14-71\end{array}$ & $\begin{array}{l}\mathrm{FT}^{3} / \mathrm{S} \\
1.32 \\
0.38 \\
0.02\end{array}$ & $\begin{array}{c}\text { DATE } \\
\text { O9-24-74 } \\
09-23-75 \\
09-22-76\end{array}$ & $\begin{array}{l}\mathrm{FT} / \mathrm{S} \\
0.62 \\
1.2 \\
1.1\end{array}$ \\
\hline & $=45$ & $Q<\varepsilon$ & $=0.3$ & $7 Q 2$ & $=\star *$ & 7010 & 0 & & \\
\hline
\end{tabular}

6-8167.00 WF M NODAWAY R NR FONTANELLE, IOWA

LAT $4119 \times X$, LONG $9439 \times X$. NEAR CENTER OF SEC. $4, T .75$ N., R. 33 W. ADAIR CO. (01), AT BRIDGE, 5 MILES NW OF FONTANELLE.

DRAINAGE AREA $67.9 \mathrm{MI}^{2}$

$\begin{array}{rc}\text { DATE } & \mathrm{FT}^{3} / \mathrm{S} \\ 09-26-57 & 0.40 \\ 10-13-58 & 14.1 \\ 07-31-59 & 5.69 \\ 09-12-60 & 5.89 \\ \text { Qa } & =33\end{array}$

$\begin{array}{rc}\text { DATE } & F T^{3} / S \\ 09-13-60 & 5.03 \\ 10-25-61 & 21.6 \\ 10-31-63 & 0.47 \\ 10-26-64 & 7.55 \\ Q(84) & =0.7\end{array}$

$\begin{array}{rr}\text { DATE } & \text { FT } / S \\ 08-09-66 & 1.84 \\ 12-12-67 & 0.45 \\ 03-12-68 & 1.23 \\ 702= & 0.3\end{array}$

$\begin{array}{cr}\text { DATE } & \text { FT3/S } \\ 09-22-69 & 2.35 \\ 07-07-70 & 1.25 \\ 09-14-71 & 0.02 \\ 7010=\star\end{array}$
$\begin{array}{cc}\text { DATE } & F T^{3} / S \\ 09-24-74 & 0.55\end{array}$
$09-24-74 \quad 0.55$
$09-23-75 \quad 1.2$
$09-22-76 \quad 0.53$


NODAWAY RIVER BASIN--Cont inued

6-8168.00 WF M NODAWAY R NR BRIDGEWATER, IOWA

LAT $4111 \% X$, LONG $9439 \times X$, NEAR CENTER OF SEC. $28, T .74$ N., R.33 W. , ADAIR CO. (01),

AT BRIDGE, 4.5 MILES SOUTH OF BRIDGEWATER.

DRAINAGE AREA $128 \quad \mathrm{MI}^{2}$

\begin{tabular}{|c|c|c|c|c|c|c|c|}
\hline $\begin{array}{l}\text { DATE } \\
09-26-57 \\
10-13-58 \\
07-31-59 \\
09-12-50\end{array}$ & $\begin{array}{l}\mathrm{FT}^{5} / \mathrm{S} \\
0.94 \\
18.7 \\
16.6 \\
9.77\end{array}$ & $\begin{array}{c}\text { DATE } \\
09-13-60 \\
10-25-61 \\
10-31-63\end{array}$ & $\begin{array}{l}\mathrm{FT}^{\mathrm{T}} \mathrm{iS} \\
10.0 \\
34.6 \\
3.39\end{array}$ & $\begin{array}{c}\text { DATE } \\
10-26-64 \\
08-09-66 \\
12-12-67\end{array}$ & $\begin{array}{l}\mathrm{FT}^{3} / \mathrm{S} \\
24.5 \\
3.88 \\
2.46\end{array}$ & $\begin{array}{c}\text { DATE } \\
03-12-68 \\
07-07-70 \\
09-14-71\end{array}$ & $\begin{array}{l}F T^{3} / S \\
2.09 \\
2.38 \\
0.88\end{array}$ \\
\hline 0 & $=62$ & & $=2.1$ & 702 & $=0.9$ & 7010 & * \\
\hline
\end{tabular}

6-8169.00 M NODAWAY R NR VILLISCA, IOWA

LAT $4055 \times X$, LONG $9459 \times X$, IN NW $1 / 4$ SEC.

34, T.71 N., R.36 W. MONTGOMERY CO. (69), AT BRIDGE ON U. S. HIGHWAY 71, 1 MILE SOUTH OF VILLISCA.

$\begin{array}{rccc} & \text { DRAINAGE } & \text { AREA } 341 & \mathrm{MI}^{2} \\ \text { DATE } & \mathrm{FT}^{3} / \mathrm{S} & \text { DATE } & \mathrm{FT}^{3} / \mathrm{S} \\ 09-24-57 & 2.74 & 09-12-60 & 36.2 \\ 05-12-58 & 21.4 & 09-13-60 & 29.8 \\ 10-14-58 & 38.7 & 10-24-51 & 112 . \\ 07-27-59 & 36.0 & 10-31-63 & 7.01 \\ \mathrm{Qa}=166 & \mathrm{Q}(84)=10\end{array}$

$\begin{array}{rl}\text { DATE } & F T^{3} / S \\ 10-27-64 & 53.0 \\ 08-10-66 & 19.1 \\ 12-12-67 & 9.24 \\ 03-12-68 & 12.5 \\ 702 & =5.4\end{array}$

$\begin{array}{cc}\text { DATE } & \text { FT./S } \\ 09-22-69 & 14.8 \\ 07-08-70 & 6.51 \\ 08-24-71 & 11.0 \\ 09-14-71 & 5.4\end{array}$

DATE $09-24-74$ $09-24-75$ $09-21-76 \quad 22$.

6-8170.50 E NODAWAY R NR WILLIAMSON, IOWA

LAT $4106 \times X$, LONG $9433 \times X$, IN NW $1 / 4$ SEC. 28, T.73 N. R. 32 W. ADAMS CO. $\{02\}$, AT BRIDGE, 3 MILES SE OF WILLIAMSON.

DRAINAGE AREA $54.2 \mathrm{MI}^{2}$

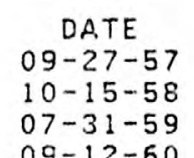

$\mathrm{FT}^{3} / \mathrm{S}$
0.
0.43
6.08
1.83

$\begin{array}{cc}\text { DATE } & F T^{3} / S \\ 09-13-60 & 1.23 \\ 10-26-61 & 19.7 \\ 10-31-63 & 0.07\end{array}$

$\begin{array}{cr}\text { DATE } & F^{3} / S \\ 10-26-64 & 9.30 \\ 08-09-66 & 2.41 \\ 12-12-67 & 0.47 \\ 7 Q 2=*\end{array}$

$\begin{array}{cr}\text { DATE } & F^{3} / S \\ 03-12-68 & 0.37 \\ 07-08-70 & 0.39 \\ 09-14-71 & 0.02\end{array}$


NODAWAY RIVER BASIN--Cont inued

6-8171.00 E NODAWAY R NR SHAMBAUGH, IOWA

LAT $4038 \times X$, LONG $9501 X X$, IN NE $1 / 4$ SEC.

G, T.6T N., R.36 W., PAGE CO. ( 73 ),

DRAINAGE AREA $333 \quad \mathrm{MI}^{2}$

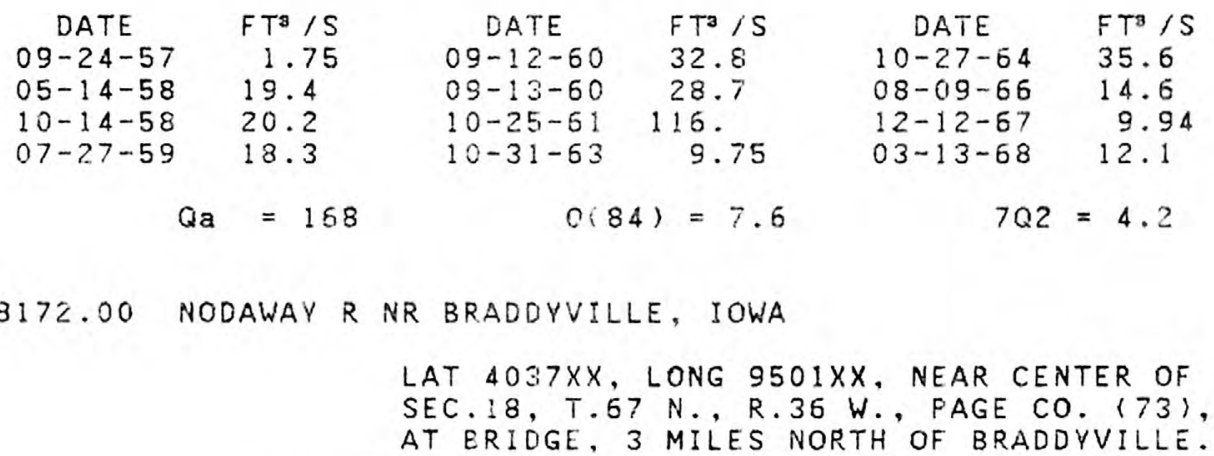

DRAINAGE AREA $1135 \quad \mathrm{MI}^{2}$

\begin{tabular}{cl} 
DATE & \multicolumn{1}{c}{$\mathrm{FT}^{3} / \mathrm{S}$} \\
$09-24-57$ & 23.5 \\
$05-14-58$ & 80.9 \\
$10-14-58$ & 105. \\
$07-27-59$ & 107.
\end{tabular}

$Q=541$

\begin{tabular}{cl} 
DATE & \multicolumn{1}{c}{$F T^{3} / S$} \\
$09-12-60$ & 189. \\
$09-13-60$ & 145. \\
$10-25-61$ & 401. \\
$10-31-63$ & 47.2
\end{tabular}

$Q(84)=41$

$\begin{array}{cc}\text { DATE } & F T^{3} / S \\ 10-27-64 & 140 . \\ 08-09-66 & 77.4 \\ 12-12-67 & 46.4 \\ 03-13-68 & 41.5\end{array}$

$7 Q 2=24$

$\begin{array}{cc}\text { DATE } & F T^{3} / S \\ 07-08-70 & 6.50 \\ 08-24-71 & 5.9 \\ 09-14-71 & 4.1 \\ & \\ 7010= & 0.8\end{array}$

DATE
$09-23-74$

$09-24-75$

$09-21-76$

$F T^{3} / S$

5.8

8. 0

\section{PLATTE RIVER BASIN}

6-8186.00 PLATTE R. NR KENT, IOWA

LAT $4057 \times X$, LONG $9429 \times X$. IN SW $1 / 4$ SEC.

13. T. 71 N., R.32 W., ADAMS CO. $(02)$,

AT BRIDGE, 2 MILES WEST OF KENT.

DRAINAGE AREA $77.9 \quad \mathrm{MI}^{2}$

$\begin{array}{rr}\text { DATE } & \mathrm{FT}^{3} / \mathrm{S} \\ 09-27-57 & 0.60 \\ 10-15-58 & 2.50 \\ 07-28-59 & 1.90 \\ 09-13-60 & 2.16 \\ Q \mathrm{Qa} & =41\end{array}$

$\begin{array}{rc}\text { DATE } & F^{3} / S \\ 09-13-60 & 4.96 \\ 10-26-61 & 21.3 \\ 10-31-63 & 1.41 \\ 10-26-64 & 6.13 \\ & \\ \text { Q(84) } & =2.1\end{array}$

$\begin{array}{rr}\text { DATE } & \mathrm{FT}^{3} / \mathrm{S} \\ 08-10-66 & 2.16 \\ 12-13-67 & 1.52 \\ 03-12-68 & 1.96 \\ & \\ & \end{array}$

$\begin{array}{cr}\text { DATE } & F^{3} / 5 \\ 10-16-68 & 1.76 \\ 07-08-70 & 1.33 \\ 08-24-71 & 0.90 \\ & \\ 7010=*\end{array}$

DATE
$09-23-7$
$09-23-75$

FTo / S

DATE $\quad F T^{3} / S$

30.2

$09-14-71 \quad 25.0$

DATE

$09-2.3-74$

$09-24-75$

$09-21-76$

$\mathrm{FT}^{\mathrm{T}} / \mathrm{S}$

47.

76. 
PLATTE RIVER BASIN--Continued

6-8186.50 E PLATTE R NR KNOWLTON, IOWA

LAT $4054 \times X$, LONG $9426 \times X$, IN NW $1 / 4$ SEC.

$4, T .70$ N., R.31 W. RINGGOLD CO. (80),

AT BRIDGE, 7 MILES NW OF KNOWLTON.

DRAINAGE AREA $66.8 \mathrm{MI}^{2}$

\begin{tabular}{|c|c|c|c|c|c|c|c|c|c|}
\hline $\begin{array}{c}\text { DATE } \\
09-27-57 \\
10-14-58 \\
07-28-59 \\
09-12-60\end{array}$ & $\begin{array}{l}\mathrm{FT}^{\mathrm{a}} / \mathrm{S} \\
0 . \\
2.58 \\
0.55 \\
1.99\end{array}$ & $\begin{array}{c}\text { DATE } \\
09-13-60 \\
10-25-61 \\
10-31-63 \\
10-26-64\end{array}$ & $\begin{array}{c}\mathrm{FT}^{\mathrm{a}} \text { iS } \\
1.85 \\
12.4 \\
0.02 \\
4.87\end{array}$ & $\begin{array}{c}\text { DATE } \\
08-10-66 \\
12-13-67 \\
03-12-68\end{array}$ & $\begin{array}{l}F T^{3} / \mathrm{S} \\
0.66 \\
0 . \\
1.69\end{array}$ & $\begin{array}{c}\text { DATE } \\
09-22-69 \\
07-08-70 \\
08-24-71\end{array}$ & $\begin{array}{l}F T / S \\
0.17 \\
0.60 \\
0.02\end{array}$ & $\begin{array}{c}\text { DATE } \\
09-23-74 \\
09-23-75 \\
09-21-76\end{array}$ & $\begin{array}{l}\mathrm{FT}^{0} / \mathrm{S} \\
0.01 \\
0.04 \\
0 .\end{array}$ \\
\hline & $=36$ & $Q<8$ & $=*$ & 702 & $=0$ & 7010 & 0 & & \\
\hline
\end{tabular}

6-8187.00 PLATTE R. NR KNOWLTON, IOWA

LAT $4052 \times X$, LONG $9426 \times X$, IN NW $1 / 4$ SEC.

$16, T .70$ N., R.31 W. RINGGOLD CO. (80),

AT BRIDGE, 6 MILES NW OF KNOWLTON.

DRAINAGE AREA $179 \quad \mathrm{MI}^{2}$

\begin{tabular}{|c|c|c|c|c|c|c|c|}
\hline $\begin{array}{c}\text { DATE } \\
10-14-58 \\
07-28-59 \\
09-12-60 \\
09-13-60\end{array}$ & & $\begin{array}{l}\mathrm{FT}^{3} / \mathrm{S} \\
6.11 \\
3.18 \\
9.07 \\
8.67\end{array}$ & & $\begin{array}{c}\text { DATE } \\
10-25-61 \\
10-31-63 \\
10-26-64\end{array}$ & $\begin{array}{c}\mathrm{FT}^{\mathrm{a}} / \mathrm{S} \\
33.2 \\
1.59 \\
14.0\end{array}$ & $\begin{array}{c}\text { DATE } \\
08-10-66 \\
12-13-67 \\
03-12-68\end{array}$ & $\begin{array}{r}\mathrm{FT}^{3} / \mathrm{S} \\
6.19 \\
3.13 \\
8.25\end{array}$ \\
\hline & Qa & $=94$ & & $Q(84)$ & $1=2.4$ & $7 Q 2$ & $=1.6$ \\
\hline 3191.00 & WB & $102 \mathrm{R}$ & NR & GRAVITY, IOW & & & \\
\hline
\end{tabular}

DRAINAGE AREA $52.2 \mathrm{MI}^{2}$

\begin{tabular}{|c|c|c|c|c|c|c|c|c|c|}
\hline $\begin{array}{c}\text { DATE } \\
09-27-57 \\
10-15-58 \\
07-28-59 \\
09-12-60\end{array}$ & $\begin{array}{l}\mathrm{FT}^{2} / \mathrm{S} \\
0 . \\
2.28 \\
0.14 \\
1.44\end{array}$ & $\begin{array}{c}\text { DATE } \\
09-14-60 \\
10-26-61 \\
10-31-63\end{array}$ & $\begin{array}{l}\mathrm{FT}^{3} / \mathrm{S} \\
1.08 \\
6.95 \\
0.07\end{array}$ & $\begin{array}{c}\text { DATE } \\
10-27-64 \\
08-10-66 \\
12-12-67\end{array}$ & $\begin{array}{r}F T^{2} / \mathrm{S} \\
3.78 \\
0.71 \\
0.28\end{array}$ & $\begin{array}{c}\text { DATE } \\
03-12-68 \\
07-08-70 \\
08-24-71\end{array}$ & $\begin{array}{l}F T^{2} / S \\
0.12 \\
0.02 \\
0.01\end{array}$ & $\begin{array}{c}\text { DATE } \\
09-23-74 \\
09-23-75 \\
09-21-76\end{array}$ & $\begin{array}{l}F T^{3} / \mathrm{S} \\
0.0 \\
0.59 \\
0.00\end{array}$ \\
\hline $\mathrm{Qa}$ & $=27$ & $Q<\varepsilon$ & $=*$ & 702 & $=0$ & $7 Q 10$ & 0 & & \\
\hline
\end{tabular}


PLATTE RIVER BASIN--Cont inued

6-8191.20 WB 102 R BELOW MB NR GRAVITY, IOWA

LAT $4048 \times X$, LONG $9449 \times X$, IN NW $1 / 4$ SEC.

7, T.69 N., R.34 W., TAYLOR. CO. (87),

AT BRIDGE, 4.5 MILES NW OF GRAVITY.

DRAINAGE AREA $106 \quad \mathrm{MI}^{2}$

\begin{tabular}{|c|c|c|c|c|c|c|c|c|c|}
\hline $\begin{array}{c}\text { DATE } \\
09-27-57 \\
10-15-58 \\
07-28-59 \\
09-12-60\end{array}$ & $\begin{array}{l}\mathrm{FT}^{3} / \mathrm{S} \\
0 . \\
3.35 \\
0.68 \\
3.55\end{array}$ & $\begin{array}{c}\text { DATE } \\
09-14-60 \\
10-26-61 \\
10-31-63\end{array}$ & $\begin{array}{c}\mathrm{FT}^{3} \text { is } \\
3.05 \\
12.4 \\
0 .\end{array}$ & $\begin{array}{c}\text { DATE } \\
10-27-64 \\
08-10-66 \\
12-12-67\end{array}$ & $\begin{array}{r}F T^{2} / \mathrm{S} \\
7.95 \\
1.00 \\
1.08\end{array}$ & $\begin{array}{c}\text { DATE } \\
03-12-68 \\
07-08-70 \\
08-24-71\end{array}$ & $\begin{array}{l}F T / S \\
1.63 \\
0.04 \\
0.18\end{array}$ & $\begin{array}{c}\text { DATE } \\
09-23-74 \\
09-23-75 \\
09-21-76\end{array}$ & $\begin{array}{l}\text { FTP / S } \\
0.00 \\
1.2 \\
0.04\end{array}$ \\
\hline & $=55$ & Qr & )$=0.3$ & $7 Q^{2}$ & * & $7 Q 10$ & 0 & & \\
\hline
\end{tabular}

6-8191.40 WB 102 R. NR NEW MARKET, IOWA

LAT $4044 \times X$, LONG $9451 \times X$, IN SW $1 / 4$ SEC.

35, T.69 N., R.35 W., TAYLOR CO. (87),

AT BRIDGE, 2.75 MILES EAST OF NEW MARKET.

DRAINAGE AREA $123 \quad \mathrm{MI}^{2}$

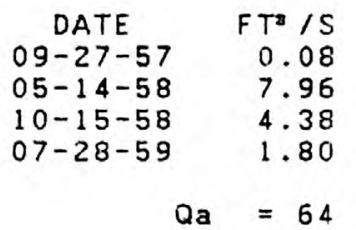

$\begin{array}{rr}\text { DATE } & F T^{3} / S \\ 09-12-60 & 5.23 \\ 09-14-60 & 4.57 \\ 10-26-61 & 12.7 \\ 10-31-63 & 0.46 \\ Q(84) & =0.9\end{array}$

$\begin{array}{cr}\text { DATE } & F T^{3} / S \\ 10-26-64 & 9.60 \\ 03-10-66 & 1.57 \\ 12-12-67 & 0.72 \\ 03-12-68 & 2.27\end{array}$

$09-23-69$

$07-08-70$

0.45

DATE

$09-23-75 \quad 0.36$

$09-21-76 \quad 0.78$

$Q \mathbf{a}=64$

$702=0.2$

$7010=*$

6-8191.50 WF 102 R. NR NEW MARKET, IOWA

LAT $4043 \times X$, LONG $9551 \times X$, IN NW $1 / 4$ SEC.

10, T.68 N., R.35 W., TAYLOR CO. (87),

AT BRIDGE, 3 MILES SE OF NEW MARKET.

DRAINAGE AREA $183 \mathrm{MI}^{2}$

$\begin{array}{rc}\text { DATE } & F T^{3} / S \\ 09-27-57 & 0.16 \\ 05-14-58 & 11.0 \\ 10-15-58 & 7.96 \\ 07-28-59 & 2.76 \\ Q a & =95\end{array}$

$\begin{array}{rc}\text { DATE } & F T^{3} / \mathrm{S} \\ 09-12-60 & 8.19 \\ 09-14-60 & 6.77 \\ 10-26-61 & 23.3 \\ 10-31-63 & 1.03 \\ Q(84) & =1.3\end{array}$

$\begin{array}{rc}\text { DATE } & F T^{3} / \mathrm{S} \\ 10-26-64 & 12.9 \\ 08-10-66 & 2.19 \\ 12-12-67 & 1.37 \\ 03-12-68 & 2.91 \\ 7 Q 2= & 0.3\end{array}$
DATE
$\begin{array}{ll}09-23-69 & 2.41\end{array}$
O. O. E8
$08-24-71 \quad 0.51$


PLATTE RIVER BASIN--Cont inued

5-8191.80 EF $102 R$ NR BEDFORD, IOWA

LAT $4044 \times X$, LONG $9439 \times X$, IN NE $1 / 4$ SEC.

4, T.68 N., R.33 W., TAYLOR CO. $(87)$,

AT BRIDGE, 5 MILES NE OF BEDFORD.

DRAINAGE AREA $60.4 \quad \mathrm{MI}^{2}$

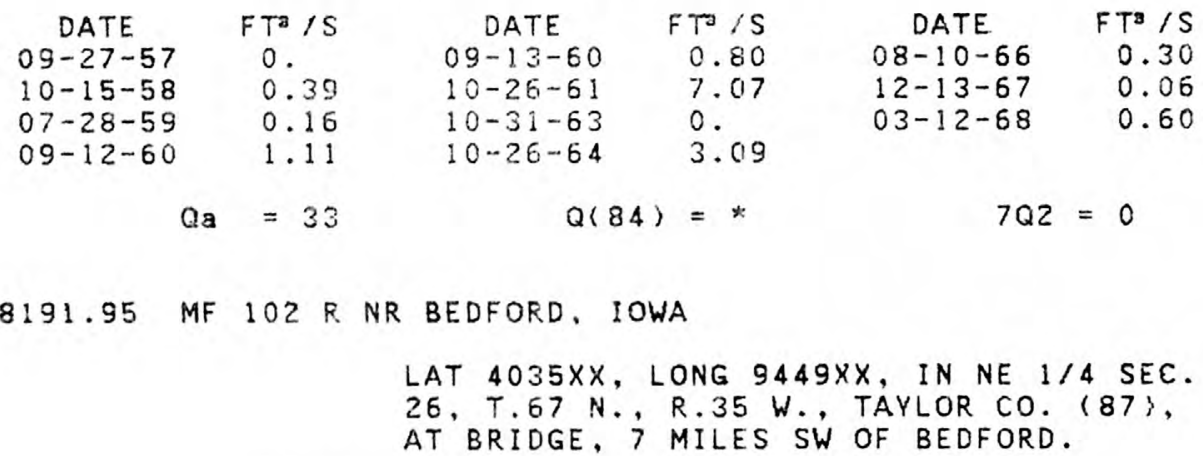

DRAINAGE AREA $59.8 \mathrm{MI}^{2}$

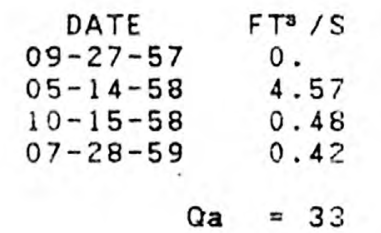

$Q_{a}=33$

$\begin{array}{rr}\text { DATE } & F^{3} / S \\ 09-12-60 & 2.85 \\ 09-13-60 & 2.21 \\ 10-26-61 & 5.93 \\ 10-31-63 & 0.02 \\ Q(84)=*\end{array}$

$\begin{array}{cr}\text { DATE } & F T^{3} / 5 \\ 10-26-64 & 1.80 \\ 08-10-66 & 0.13 \\ 12-13-67 & 0.23 \\ 03-13-68 & 0.48 \\ 7 Q 2= & 0\end{array}$

$09-23-69$

$07-08-70$

$08-24-71$

$\mathrm{FT}^{3} / \mathrm{S}$

0.30

0.07

0.14

DATE

$3-7$

$09-23-75$

$09-21-76$

$\mathrm{FT}^{3} / \mathrm{S}$

0.00

0.04

0.00

\section{GRAND RIVER BASIN}

6-8961.00 GRAND R AT KNOWLTON, IOWA

LAT $4050 X X$, LONG $9420 X X$, IN SE $1 / 4$ SEC.

29 , T.70 N., R. 30 W., RINGGOLD CO. $(80$ )

AT BRIDGE NEAR EAST CITY LIMITS OF KNOWLTON.

\section{DRAINAGE AREA $67.5 \mathrm{MI}^{2}$}

$\begin{array}{cccccr}\text { DATE } & F^{3} / \mathrm{S} & \text { DATE } & \mathrm{FT}^{3} / \mathrm{S} & \mathrm{DATE} & \mathrm{FT}^{\mathrm{a}} / \mathrm{S} \\ 09-26-57 & 0 . & 09-13-60 & 1.20 & 08-10-66 & 1.29 \\ 10-14-58 & 1.72 & 10-25-61 & 11.8 & 12-13-67 & 0.35 \\ 07-28-59 & 1.40 & 10-31-63 & 0.17 & 03-12-68 & 0.97 \\ 09-12-60 & 0.91 & 10-27-64 & 6.35 & 10-17-68 & 0.45\end{array}$

$Q_{a}=37$

$Q(84)=0.4$

$7 Q 2=0.1$

DATE
$09-22-69$
$07-28-70$
$09-20-71$

$F T^{2} / S$

0.40

0.17

0.10

DATE

$09-24-74$
$08-13-75$

$09-21-76$

$\mathrm{FT}^{\mathrm{a}} / \mathrm{S}$

0.10

0.23
0.19 
GRAND RIVER BASIN--Cont inued

6-8961.50 GRAND R NR BLOCKTON, IOWA

LAT $4034 X X$, LONG $9427 \times X$, IN SW $1 / 4$ SEC.

29, T.67 N., R.31 W., RINGGOLD CO. (80),

AT BRIDGE, 3 MILES SE OF BLOCKTON.

\begin{tabular}{|c|c|c|c|c|c|c|c|c|c|}
\hline & DRAINAGE & AREA & $M I^{2}$ & & & & & & \\
\hline $\begin{array}{c}\text { DATE } \\
09-26-57 \\
10-15-58 \\
07-28-59 \\
09-12-60\end{array}$ & $\begin{array}{c}\mathrm{FT}^{3} / \mathrm{S} \\
0.87 \\
5.61 \\
10.0 \\
12.1\end{array}$ & $\begin{array}{c}\text { DATE } \\
09-12-60 \\
10-25-61 \\
10-31-63 \\
10-27-64\end{array}$ & $\begin{array}{l}F T \text { is } \\
13.1 \\
45.9 \\
4.10 \\
17.2\end{array}$ & $\begin{array}{c}\text { DATE } \\
12-12-67 \\
03-12-68 \\
10-16-68\end{array}$ & $\begin{array}{l}F T^{3} / \mathrm{S} \\
3.40 \\
2.45 \\
3.10\end{array}$ & $\begin{array}{c}\text { DATE } \\
09-22-69 \\
07-28-70 \\
09-20-71\end{array}$ & $\begin{array}{l}F T^{3} / \mathrm{S} \\
7.01 \\
2.76 \\
2.5\end{array}$ & $\begin{array}{c}\text { DATE } \\
09-24-74 \\
08-13-75 \\
09-21-76\end{array}$ & $\begin{array}{l}\text { FTo } / S \\
2.7 \\
5.9 \\
2.4\end{array}$ \\
\hline Qa & $=113$ & $Q<8$ & $=5.0$ & $7 Q 2$ & $=2.2$ & 7010 & $0.2 * \star$ & & \\
\hline
\end{tabular}

6-8962.00 EF GRAND R NR MT. AYR, IOWA

LAT $4043 \times X$, LONG $9410 X X$, IN SE 1/4 SEC. $3, T .68 \mathrm{~N} .$, R.29 W., RINGGOLD CO. (80), AT BRIDGE ON STATE HIGHWAY 2, 3 MILES EAST OF MT. AYR.

DRAINAGE AREA $64.7 \mathrm{MI}^{2}$

$\begin{array}{rc}\text { DATE } & F^{a} / S \\ 09-26-57 & 0 . \\ 10-14-58 & 1.57 \\ 07-28-59 & 0.54 \\ 09-12-60 & 1.58 \\ Q a & =36\end{array}$

$\begin{array}{cc}\text { DATE } & \text { FT /S } \\ 09-12-60 & 0.17 \\ 10-25-61 & 11.9 \\ 10-31-63 & 0.18\end{array}$

DATE
$10-27-64$
$08-10-66$
$03-12-68$

13
3.90
0.64

0.64

$Q(84)=0.2$

$7 Q 2=$

\section{DATE \\ $07-28-70$}

$F T / S$
0.29
0.08
0.07

$7 Q 10=0$

6-8962.50 EF GRAND $R$ SOUTH OF MT. AYR, IOWA

LAT $4035 \mathrm{XX}$, LONG $9414 \mathrm{XX}$, IN SW $1 / 4$ SEC.

$19, T .67$ N., R.29 W., RINGGOLD CO. (80), AT BRIDGE, 9 MILES SOUTH OF MT. AYR.

DRAINAGE AREA $95.9 \mathrm{MI}^{2}$

$\begin{array}{rc}\text { DATE } & F^{3} / S \\ 09-26-57 & 0 . \\ 10-15-58 & 2.47 \\ 07-28-59 & 1.66 \\ 09-12-60 & 2.29 \\ & Q_{a}=53\end{array}$

$\begin{array}{cc}\text { DATE } & F T \text { is } \\ 09-12-60 & 2.64 \\ 10-25-61 & 18.8 \\ 10-31-63 & 1.58 \\ 10-27-64 & 10.1\end{array}$

$\begin{array}{cr}\text { DATE } & \mathrm{FT}^{s / S} / \mathrm{S} \\ 08-10-66 & 3.35 \\ 12-12-67 & 0.41 \\ 03-12-68 & 1.48 \\ 10-16-68 & 0.51\end{array}$

$\begin{array}{cr}\text { DATE } & F^{3} / S \\ 09-22-69 & 3.63 \\ 07-28-70 & 0.32 \\ 09-20-71 & 0.05 \\ 7010= & \end{array}$


GRAND RIVER BASIN--Cont inued

6-8977.70 THOMPSON R NR HEBRON, IOWA

LAT $4114 X X$, LONG $9416 X X$, IN SW $1 / 4$ SEC.

$1, T .74$ N., R.30 W. ADAIR CO. $(01)$,

AT BRIDGE, 2 MILES SE OF HEBRON.

DRAINAGE AREA $80.0 \mathrm{MI}^{2}$

\begin{tabular}{|c|c|c|c|c|c|c|c|c|c|}
\hline $\begin{array}{c}\text { DATE } \\
09-26-57 \\
10-13-58 \\
10-23-59 \\
09-13-60\end{array}$ & $\begin{array}{l}\mathrm{FT}^{3} / \mathrm{S} \\
0.11 \\
4.77 \\
7.18 \\
2.03\end{array}$ & $\begin{array}{c}\text { DATE } \\
09-13-60 \\
10-26-61 \\
10-31-63 \\
10-26-64\end{array}$ & $\begin{array}{c}\mathrm{FT}^{3} / \mathrm{S} \\
2.48 \\
31.5 \\
0.26 \\
18.0\end{array}$ & $\begin{array}{c}\text { DATE } \\
08-09-66 \\
12-12-57 \\
03-13-68 \\
10-16-68\end{array}$ & $\begin{array}{r}\mathrm{FT}^{3} / \mathrm{S} \\
2.55 \\
2.27 \\
1.34 \\
0.57\end{array}$ & $\begin{array}{c}\text { DATE } \\
09-22-69 \\
07-27-70 \\
09-20-71\end{array}$ & $\begin{array}{c}F T .15 \\
4.80 \\
0.17 \\
0.31\end{array}$ & $\begin{array}{c}\text { DATE } \\
09-23-74 \\
08-12-75 \\
09-20-76\end{array}$ & $\begin{array}{l}F T^{3} / \mathrm{S} \\
0.23 \\
2.6 \\
4.2\end{array}$ \\
\hline$Q \mathbf{a}$ & $=41$ & $Q<\varepsilon$ & $=1.5$ & $7 Q 2$ & $=0.4$ & $7 Q 10$ & * & & \\
\hline
\end{tabular}

6-8978.00 THREEMILE CR NR AFTON, IOWA

LAT $4102 \times X$, LONG $9408 \times X$, NEAR CENTER OF SEC.13, T.72 N., R.29 W., UNION CO. (88), AT BRIDGE 3 MILES EAST OF AFTON.

DRAINAGE AREA $54.8 \quad \mathrm{MI}^{2}$

$\begin{array}{rccccr}\text { DATE } & F^{3} / S & \text { DATE } & F T^{3} / S & \text { DATE } & F^{3} / S \\ 09-26-57 & 0 . & 09-13-60 & 0.70 & 08-09-66 & 5.55 \\ 10-13-58 & 0.40 & 10-27-61 & 14.1 & 12-12-67 & 1.03 \\ 10-21-59 & 3.44 & 10-31-63 & 0.13 & 03-13-68 & 2.60 \\ 09-12-60 & 0.96 & 10-26-64 & 6.62 & 10-16-68 & 0.08 \\ Q a & =29 & Q(84)=0.4 & 7 Q 2=0.1\end{array}$

DATE
$09-22-69$
$07-27-70$
$09-21-71$

$T \cdot / S$

0.8

DATE $09-23-74$

$08-12-75$

$\mathrm{FT}^{\mathrm{T}} / \mathrm{S}$

$10-13-5$

$Q_{a}=29$

$Q(84)=0.4$

$7 Q 10=$

6-8978.20 THOMPSON R NR AFTON, IOWA

LAT 4 I0 $2 \times X$, LONG $9406 \times X$, IN SW $1 / 4$ SEC.

17, T.T2 N., R.28 W. UNION CO. (88),

AT BRIDGE ON U. S. HIGHWAY 34 AND 169,5

MILES EAST OF AFTON.

DRAINAGE AR.EA $231 \mathrm{MI}^{2}$

\begin{tabular}{|c|c|c|c|c|c|c|c|c|c|}
\hline $\begin{array}{c}\text { DATE } \\
09-26-57 \\
10-13-58 \\
10-21-59 \\
09-12-60\end{array}$ & $\begin{array}{c}\mathrm{FT}^{3} / \mathrm{S} \\
0 . \\
7.40 \\
15.5 \\
5.73\end{array}$ & $\begin{array}{c}\text { DATE } \\
09-13-60 \\
10-26-61 \\
10-31-63 \\
10-26-64\end{array}$ & $\begin{array}{c}\mathrm{FT}^{3} / \mathrm{S} \\
5.81 \\
76.2 \\
1.06 \\
39.8\end{array}$ & $\begin{array}{c}\text { DATE } \\
08-09-66 \\
12-12-67 \\
03-13-68\end{array}$ & $\begin{array}{l}\mathrm{FT}^{3} / \mathrm{S} \\
18.7 \\
4.79 \\
8.55\end{array}$ & $\begin{array}{c}\text { DATE } \\
10-16-68 \\
07-27-70 \\
09-21-71\end{array}$ & $\begin{array}{l}F T^{2} / S \\
1.32 \\
1.36 \\
0.88\end{array}$ & $\begin{array}{c}\text { DATE } \\
09-23-74 \\
08-12-75 \\
09-22-76\end{array}$ & $\begin{array}{l}F T^{3} / \mathrm{S} \\
1.2 \\
4.4 \\
8.4\end{array}$ \\
\hline Qa & $=120$ & $Q($ & $=3.6$ & 702 & $=1.3$ & 7010 & $0.1 * * *$ & & \\
\hline
\end{tabular}


GRAND RIVER BASIN--Cont inued

8-8978.80 TWELVEMILE CR NR ARISPE, IOWA

LAT $4056 \times X$, LONG $9406 \times X$, IN SE $1 / 4 \mathrm{SEC}$.

$17, T .71$ N., R. 28 W. , UNION CO. ( 88 ),

AT BRIOGE, 6 MILES EAST OF ARISPE.

DRAINAGE AREA $68.0 \quad \mathrm{MI}^{2}$

\begin{tabular}{|c|c|c|c|c|c|c|c|c|c|}
\hline $\begin{array}{c}\text { DATE } \\
09-26-57 \\
10-13-58 \\
09-12-60 \\
09-13-60\end{array}$ & $\begin{array}{l}\mathrm{FT}^{\mathrm{a}} / \mathrm{S} \\
0 . \\
0.66 \\
1.78 \\
1.32\end{array}$ & $\begin{array}{c}\text { DATE } \\
10-27-61 \\
10-31-63 \\
10-26-64 \\
08-09-66\end{array}$ & $\begin{array}{l}\mathrm{FT}^{\mathrm{a}} / \mathrm{S} \\
13.5 \\
0.28 \\
8.39 \\
4.59\end{array}$ & $\begin{array}{c}\text { DATE } \\
12-13-67 \\
03-13-68 \\
10-16-68\end{array}$ & $\begin{array}{l}F T^{2} / S \\
1.88 \\
4.55 \\
0.35\end{array}$ & $\begin{array}{c}\text { DATE } \\
09-22-69 \\
07-27-70 \\
09-21-71\end{array}$ & $\begin{array}{l}F T / S \\
1.74 \\
0.21 \\
0 .\end{array}$ & $\begin{array}{c}\text { DATE } \\
09-23-74 \\
08-12-75 \\
09-21-76\end{array}$ & $\begin{array}{r}\mathrm{FT}^{3} \text { is } \\
0.05 \\
0.08 \\
0.38\end{array}$ \\
\hline & $=36$ & Q: & $\gamma=0.5$ & $7 Q 2$ & $=0.1$ & $7 Q 10$ & $\star$ & & \\
\hline
\end{tabular}

6-8979.00 THOMPSON R NR GRAND RIVER, IOWA

LAT $4052 \times X$, LONG $9358 \times X$. IN NE $1 / 4$ SEC.

$16, T .70$ N., R.27 W., DECATUR CO. (27).

AT BRIDGE, 3.5 MILES NORTH OF GRAND RIVER.

DRAINAGE AREA $401 \quad \mathrm{MI}^{2}$

\begin{tabular}{|c|c|c|c|c|c|c|c|c|c|}
\hline $\begin{array}{c}\text { DATE } \\
09-26-57 \\
10-14-58 \\
10-21-59 \\
09-12-60\end{array}$ & $\begin{array}{c}\mathrm{FT}^{\mathrm{a}} / \mathrm{S} \\
0.83 \\
8.40 \\
38.8 \\
11.7\end{array}$ & $\begin{array}{c}\text { DATE } \\
09-13-60 \\
10-24-61 \\
10-31-63 \\
10-26-64\end{array}$ & $\begin{array}{c}\mathrm{FT}^{3} \text { is } \\
10.1 \\
140 . \\
2.25 \\
59.8\end{array}$ & $\begin{array}{c}\text { DATE } \\
08-09-66 \\
12-12-67 \\
03-12-68 \\
10-16-68\end{array}$ & $\begin{array}{l}\mathrm{FT}^{3} / \mathrm{S} \\
43.1 \\
11.4 \\
27.2 \\
4.95\end{array}$ & $\begin{array}{c}\text { DATE } \\
09-22-69 \\
07-27-70 \\
09-20-71\end{array}$ & $\begin{array}{l}F T^{3} / \mathrm{S} \\
25.5 \\
4.87 \\
2.4\end{array}$ & $\begin{array}{c}\text { DATE } \\
09-24-74 \\
08-12-75 \\
09-20-76\end{array}$ & $\begin{array}{l}\mathrm{FT}^{3} / \mathrm{S} \\
2.4 \\
11 . \\
5.9\end{array}$ \\
\hline$Q$ & $=207$ & $Q<8$ & $=7.8$ & $7 Q 2$ & 2.9 & 7010 & $0.3 * \star *$ & & \\
\hline
\end{tabular}

6-8979.40 LONG CR NR VAN WERT, IOWA

LAT $4049 \times X$, LONG 9352XX, IN NE $1 / 4$ SEC.

$32, T .70$ N., R.26 W., DECATUR CO. (27),

AT BRIDGE, 5 MILES SE OF VAN WERT.

DRAINAGE AREA $117 \quad \mathrm{MI}^{2}$

$\begin{array}{rc}\text { DATE } & F T^{3} / S \\ 09-26-57 & 0 . \\ 10-14-58 & 0.22 \\ 10-21-59 & 13.6 \\ 09-12-60 & 1.78 \\ \text { Qa } & =65\end{array}$

$\begin{array}{rc}\text { DATE } & F T^{2} \text { iS } \\ 09-13-60 & 1.58 \\ 10-24-61 & 16.0 \\ 10-31-E 3 & 0.13 \\ 10-26-64 & 4.99 \\ Q(84) & =0.4\end{array}$

$\begin{array}{rr}\text { DATE } & \text { FT } / S \\ 08-09-66 & 6.24 \\ 12-12-67 & 3.22 \\ 03-12-68 & 15.9 \\ 702= & 0.1\end{array}$
DATE
$10-16-58$
$07-27-70$
$09-20-71$

$F T / S$

0.27

0.21

0.05

DATE

O9-24-74

$08-12-75$
$09-20-76$

$\mathrm{FT}^{3} / \mathrm{S}$

0.04

0.06

$7010=$ 
GRAND RIVER BASIN--Cont Inued

6-8983.00 WELDON R EAST OF LEON, IOWA

LAT 404518 , LONG 933805 , IN SE $1 / 4$ SEC. $20, T .69$ N. R.24 W. DECATUR CO. (27), AT BRIDGE ON STATE HIGHWAY 2, 6 MILES AT BRIDGE ON
EAST OF LEON.

DRAINAGE AREA $72.4 \mathrm{MI}^{2}$

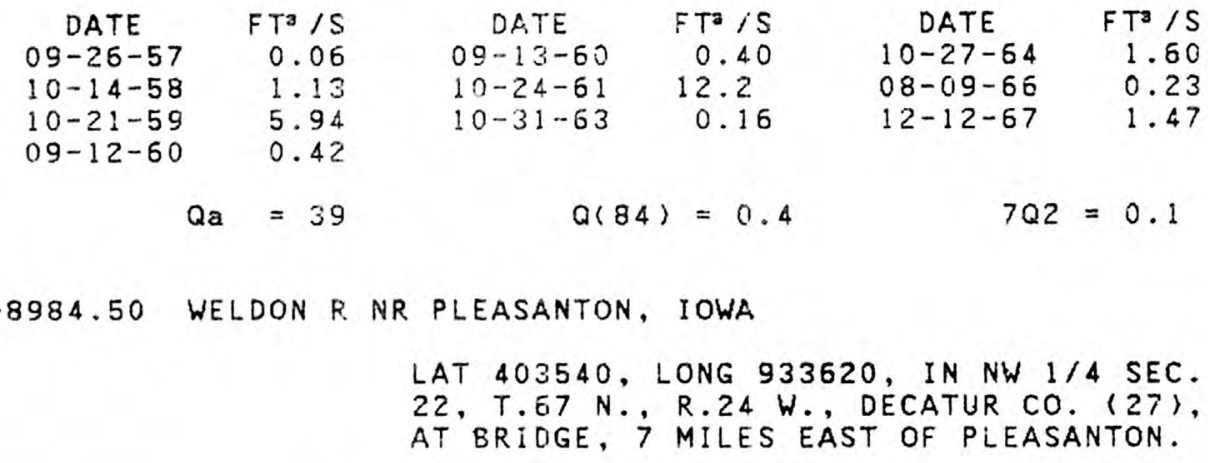

DRAINAGE AREA $228 \quad \mathrm{MI}^{2}$

\begin{tabular}{|c|c|c|c|c|c|}
\hline $\begin{array}{c}\text { DATE } \\
09-26-57 \\
10-14-58 \\
10-21-59 \\
09-12-60\end{array}$ & $\begin{array}{c}\mathrm{FT}^{3} / \mathrm{S} \\
0 . \\
7.57 \\
24.7 \\
2.55\end{array}$ & $\begin{array}{c}\text { DATE } \\
09-13-60 \\
10-24-61 \\
10-31-63\end{array}$ & $\begin{array}{c}\mathrm{FT}^{3} / \mathrm{S} \\
2.71 \\
42.9 \\
1.26\end{array}$ & $\begin{array}{c}\text { DATE } \\
10-27-64 \\
08-09-66 \\
12-12-67\end{array}$ & $\begin{array}{r}F T^{3} / \\
9.1 \\
2.5 \\
12.5\end{array}$ \\
\hline & $=130$ & Q & $\gamma=2.5$ & $7 Q 2$ & 1. \\
\hline
\end{tabular}

DATE
$03-13-68$
$07-28-70$

$F T^{2} / S$

$3-28-70 \quad 20.8$
$09-21-71$

0.67

DATE

$09-25-74$

$08-13-75$

$\mathrm{FT}^{3} / \mathrm{S}$

$09-21-71$

$09-21-76$

1.6

8.5

$Q \mathbf{a}=130$

$Q(84)=2.5$

$7010=$

6-8984.70 LITTLE R NR LEON, IOWA

LAT 403936, LONG 934459 , IN SE $1 / 4$ SEC.

$29, T .68$ N., R.25 W., DECATUR CO. (27),

AT BRIDGE, 6 MILES SOUTH OF LEON.

DRAINAGE AREA $69.2 \mathrm{MI}^{2}$

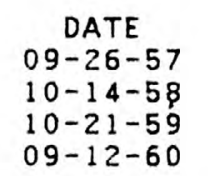

$F T / S$
0.16
2.04
6.27
0.57

DATE
$09-13-60$
$10-24-61$
$10-31-63$

$\mathrm{FT}^{\mathrm{a}} / \mathrm{S}$
0.48

$10-31-63$

11.4

DATE
$10-27-64$
$08-09-66$
$12-12-67$
$\mathrm{FT}^{\mathrm{T}} / \mathrm{S}$
0.19

0.79

DATE
$03-13-68$
$07-28-70$
$09-21-71$

F T / S

4.50

0.07 
CHARITON RIVER BASIN

6-9033.00 CHARITON R NR DERBY, IOWA

LAT $4057 \times X$, LONG $9328 \times X$, IN NW $1 / 4$ SEC.

$13, T .71$ N., R. 23 W. LUCAS CO. ( 59 ),

DRAINAGE AREA $71.0 \mathrm{MI}^{2}$

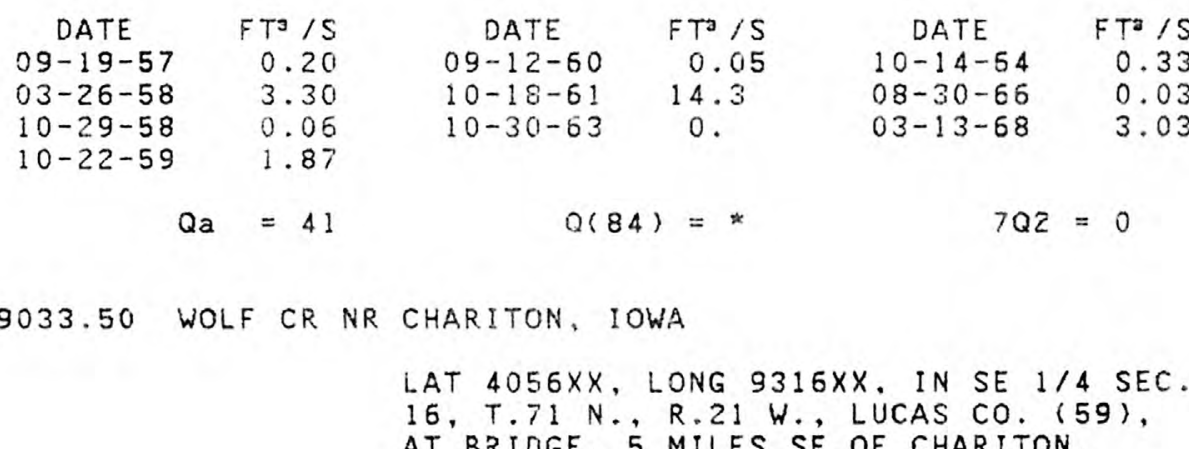

DRAINAGE AREA $65.0 \quad \mathrm{MI}^{2}$

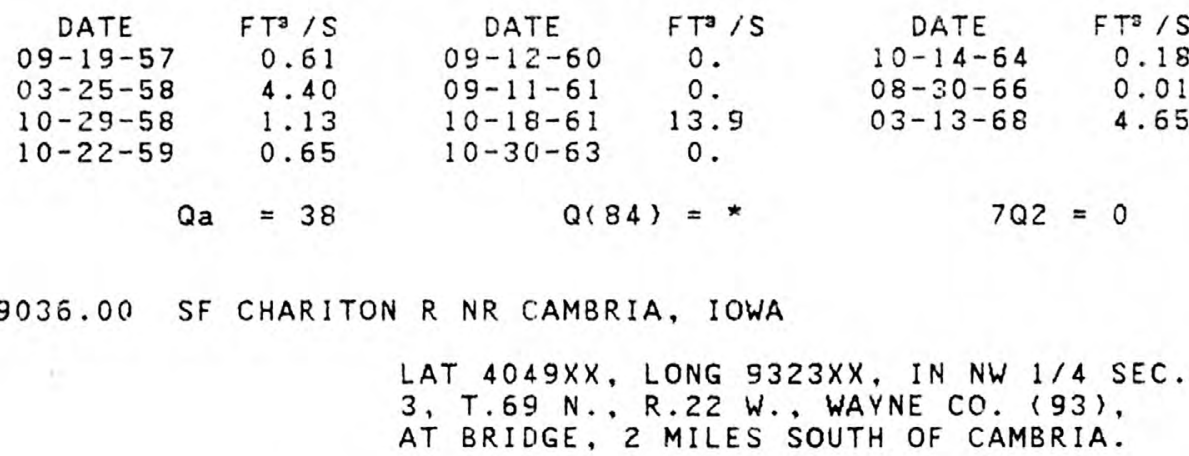

DRAINAGE AREA $58.0 \mathrm{MI}=$

$\begin{array}{rr}\text { DATE } & \text { FTa } / S \\ 03-25-58 & 2.82 \\ 10-30-58 & 0.17 \\ 10-21-59 & 4.41 \\ 09-14-60 & 0.59 \\ \text { Qa } & =34\end{array}$

$\begin{array}{cccc}\text { DATE } & \text { FT3 } / S & \text { DATE } & F^{3} / S \\ 09-11-61 & 0.26 & 10-14-64 & 1.65 \\ 10-19-61 & 9.97 & 08-30-66 & 0.03 \\ 10-30-63 & 0 . & 03-13-68 & 2.73\end{array}$

$\begin{array}{cr}\text { DATE } & F T=15 \\ 09-22-69 & 0.22 \\ 07-28-70 & 0.08 \\ 08-24-71 & 0.01 \\ 7010= & 0\end{array}$

DATE

$09-24-74 \quad F$ IS

$08-12-75 \quad 0.28$

$09-21-76 \quad 0.04$

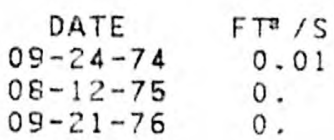

0 . 
CHARITON RIVER BASIN--COnt inued

6-9036.50 SF CHARITON R NR CORYDON, IOWA

LAT $4049 \times X$, LONG $9319 \times X$, IN NW $1 / 4$ SEC.

$6, T .69$ N., R.21 W. , WAYNE CO. (93),

AT BRIDGE ON STATE HIGHWAY 14,4 MILES

NORTH OF CORYDON.

DRAINAGE AREA $68.1 \quad \mathrm{MI}^{2}$

\begin{tabular}{|c|c|c|c|c|c|c|c|c|c|}
\hline $\begin{array}{c}\text { DATE } \\
09-26-57 \\
03-25-58 \\
10-30-58 \\
10-21-59\end{array}$ & $\begin{array}{l}F T^{3} / S \\
0 . \\
3.92 \\
0.33 \\
3.20\end{array}$ & $\begin{array}{c}\text { DATE } \\
09-14-60 \\
09-11-61 \\
10-19-61 \\
10-30-63\end{array}$ & $\begin{array}{c}F T^{2} / S \\
0.08 \\
0.45 \\
12.5 \\
0.05\end{array}$ & $\begin{array}{c}\text { DATE } \\
10-14-64 \\
08-30-66 \\
03-13-68\end{array}$ & $\begin{array}{l}\mathrm{FT}^{3} / \mathrm{S} \\
1.96 \\
0.07 \\
2.66\end{array}$ & $\begin{array}{c}\text { DATE } \\
09-22-69 \\
07-28-70 \\
08-24-71\end{array}$ & $\begin{array}{l}F T=/ S \\
0.40 \\
0.21 \\
0.10\end{array}$ & $\begin{array}{c}\text { DATE } \\
09-24-74 \\
08-12-75 \\
09-21-76\end{array}$ & $\begin{array}{l}F^{3} / S \\
0.04 \\
0.43 \\
0.10\end{array}$ \\
\hline & $=40$ & $Q(1$ & $=0.2$ & 702 & $=*$ & 7010 & 0 & & \\
\hline
\end{tabular}

6-9041.50 SHOAL CR NR CINCINNATI, IOWA

LAT $4037 \times X$, LONG $9252 \times X$, IN SW $1 / 4$ SEC.

$6, T .67$ N., R.17 W., APPANOOSE CO. 104 )

AT BRIDGE, 3 MILES EAST OF CINCINNATI.

DRAINAGE AREA $56.6 \mathrm{MI}^{2}$

$\begin{array}{rrcccr}\text { DATE } & \text { FT /S } & \text { DATE } & F T^{3} / S & \text { DATE } & F T^{3} / S \\ 03-25-58 & 4.26 & 09-11-61 & 0 . & 10-14-64 & 0.54 \\ 10-29-58 & 2.26 & 10-19-61 & 7.04 & 08-30-66 & 0 . \\ 10-21-59 & 5.95 & 10-30-63 & 0.03 & 03-12-68 & 4.29 \\ 09-14-60 & 0.02 & & & & \\ \text { Qa }=34 & Q(84)=* & & 702=0\end{array}$

$\begin{array}{cr}\text { DATE } & F T / S \\ 09-22-69 & 0.46 \\ 07-29-70 & 0.04 \\ 08-24-71 & 0.01\end{array}$

DATE

$09-24-74$

$\mathrm{FT} / \mathrm{S}$

$\begin{array}{ll}0.04 & 08-12-75 \\ 0.01 & 09-22-76\end{array}$

0.04

0.02

$Q_{\mathbf{a}}=34$

$Q(84)=$ *

$702=0$

$7 Q 10=0$

* Less than 0.1 cubic foot per second

* * estimated from generalized maP

* * estimated from extrapolated correlation curve 
BEAVER CREEK NR GRIMES

BEAVER CREEK AT NEW HARTFORD

BIG BEAR CREEK AT LADORA

BIG CEDAR CREEK NR VARINA

BIG CREEK NR MOUNT PLEASANT

BIG SIOUX RIVER AT AKRON

BLACK HAWK CREEK AT HUDSON

BOONE RIVER NR WEBSTER CITY

BOYER RIVER AT LOGAN

$$
-\mathrm{C}-
$$

CEDAR CREEK NR BUSSEY

CEDAR RIVER AT CEDAR RAPIDS

CEDAR RIVER AT CHARLES CITY

CEDAR RIVER NR CONESVILLE

CEDAR RIVER AT JANESVILLE

CEDAR RIVER AT WATERLOO

CHARITON RIVER NR CENTERVILLE

CHARITON RIVER NR CHARITON

CHARITON RIVER NR RATHBUN

CLEAR CREEK NR CORALVILLE

$-\mathrm{D}-$

DAVIDS CREEK NR HAMLIN

DES MOINES RIVER AT DES MOINES

DES MOINES RIVER BELOW RACCOON RIVER AT DES MOINES

DES MOINES RIVER AT ESTHERVILLE

DES MOINES RIVER AT FORT DODGE

DES MOINES RIVER AT HUMBOLDT

DES MOINES RIVER AT KEOSAQUA

DES MOINES RIVER AT OTTUMWA

DES MOINES RIVER NR SAYLORVILLE

DES MOINES RIVER NR STRATFORD

DES MOINES RIVER NR TRACY

DRY CREEK AT HAWARDEN

$\begin{array}{lr}05-4177.00 & 41 \\ 05-4819.50 & 184 \\ 05-4630.00 & 120 \\ 05-4530.00 & 78 \\ 05-4821.70 & 189 \\ 05-4735.00 & 155 \\ 06-4855.00 & 250 \\ 05-4635.00 & 122 \\ 05-4810.00 & 177 \\ 06-6095.00 & 286\end{array}$

$05-4890.00$

$05-4645.00$

$05-4577.00$

$05-4650.00$

$05-4585.00$

$05-4640.00$

06-9040.00

06-9034.00

$06-9039.00$

$05-4543.00$

$05-4855.00$ 


$$
-E-
$$

EAST BRANCH IOWA RIVER NEAR KLEMME

EAST FORK DES MOINES RIVER NR BURT

$05-4490.00$

$05-4780.00$ 61

EAST FORK DES MOINES RIVER AT DAKOTA CITY

$05-4790.00$

169

EAST FORK HARDIN CREEK NR CHURDAN

$05-4830.00$

171

EAST FORK ONE HUNDRED AND TWO RIVER NR

$$
\text { BEDFORD }
$$

$06-8191.90$

$06-8092.10$

$06-8095.00$

$06-8079.50$

EAST NISHNABOTNA RIVER AT RED OAK

ELK CREEK NR DECATUR CITY

$05-4555.00$

ENGLISH RIVER AT KALONA

$$
-F-
$$

FLOYD RIVER AT ALTON

FLOYD RIVER AT JAMES

FOURMILE CREEK AT DES MOINES

FOX RIVER AT BLOOMFIELD

FOX RIVER AT CANTRIL

$$
-\mathrm{H}-
$$

HALF MILE CREEK NR GLADBROOK

INDIAN CREEK AT COUNCIL BLUFFS

$06-6105.00$

INDIAN CREEK NR MINGO

IOWA RIVER NR BELLE PLAINE

$05-4712.00$

$05-4525.00$

147

IOWA RIVER AT IOWA CITY

$05-4545.00$

$05-4557.00$

$05-4531.00$

IOWA RIVER AT MARENGO

$05-4515.00$

IOWA RIVER AT MARSHALLTOWN

$05-4495.00$

IOWA RIVER NR ROWAN

$05-4655.00$ 


\section{STATION NAME}

LITTLE SIOUX RIVER AT CORRECTIONVILLE LITTLE SIOUX RIVER AT GILLETT GROVE

LITTLE SIOUX RIVER NR KENNEBEC

LITTLE SIOUX RIVER AT LINN GROVE

LITTLE SIOUX RIVER NR TURIN

LIZARD CREEK NR CLARE

$-M-$

MAPLE RIVER AT MAPLETON

MAQUOKETA RIVER NR MANCHESTER

MAQUOKETA RIVER NR MAQUOKETA

MIDDLE RACCOON RIVER AT PANORA

MIDOLE RIVER NR INDIANOLA

MISSISSIPPI RIVER AT CLINTON

MISSISSIPPI RIVER AT KEOKUK

MISSISSIPPI RIVER AT MCGREGOR

MISSOURI RIVER AT NEBRASKA CITY, NEBRASKA

MISSOURI RIVER AT OMAHA, NEBRASKA

MISSOURI RIVER AT RULO, NEBRASKA

MISSOURI RIVER AT SIOUX CITY

MONONA-HARRISON DITCH NR TURIN

MOSQUITO CREEK NR EARLING

MULE CREEK NR MALVERN

$$
-\mathrm{N}-
$$

NISHNABOTNA RIVER ABOVE HAMBURG

NODAWAY RIVER AT CLARINDA

NORTH RACCOON RIVER NR JEFFERSON

NORTH RACCOON RIVER NR SAC CITY

NORTH RIVER NR NORWALK

NORTH SKUNK RIVER NR SIGOURNEY

$$
-\mathrm{O}-
$$

OOEBOLT CREEK NR ARTHUR

OLD MANS CREEK NR IOWA CITY
STA. NO.

PAGE

$06-6066.00$

06-6056.00

06-6067.00

$06-6058.50$

$06-6075.00$

$05-4800.00$

$06-6072.00$

05-4170.00

05-4185.00

$05-4836.00$

$05-4864.90$

05-4205.00

05-4745.00

05-3895.00

06-8070.00

06-6100.00

$06-8135.00$

06-4860.00

$06-6024.00$

06-6105. 20

06-8080.00

06-8100.00

06-8170.00

05-4825.00

05-4823.00

05-4860.00

05-4725.00

272

269

275

271

281

173

279

38

43

197

213

46

160

25

301

291

324

253

267

297

307

319

326

193

191

211

151

06-6070.00

277

$05-4551.00$

96

$-\mathrm{P}-$ 


\section{STATION NAME}

PERRY CREEK AT 38TH STREET, SIOUX CITY

PLATTE RIVER NR DIAGONAL

PRAIRIE CREEK AT FAIRFAX

$-\mathrm{R}-$

RACCOON RIVER AT VAN METER

RALSTON CREEX AT IOWA CITY

RAFID CREEK NR IOWA GITY

RICHILAND CREEK. NR HAVEN

ROCK RIVER AT ROCK RAPIDS

ROCK RIVER NR ROCK VALLEY

$$
-\mathrm{S}-
$$

SALT CREEK NR ELBERON

SHELL ROCK RIVER AT MARBLE ROCK

SHELL ROCK RIVER NR NORTHWOOD

SHELL ROCK RIVER AT SHELL ROCK

SKUNK RIVER AT AUGUSTA

SKUNK RIVER AT COPPOCK

SOLDIER RIVER AT PISGAH

SOUTH BRANCH RALSTON CREEK AT IOWA CITY

SOUTH FORK CHARITON RIVER NR PROMISE CITY

SOUTH RACCOON RIVER AT REDFIELD

SOUTH RIVER NR ACKWORTH

SOUTH SKUNK RIVER NR AMES

SOUTH SKUNK RIVER BELOW SQUAW CREEK NR AMES

SOUTH SKUNK RIVER NR OSKALOOSA

SPRING VALLEY CREEK NR TABOR

SQUAW CREEK AT AMES

STEER CREEK NEAR MAGNOLIA

SUGAR CREEK NR KEOKUK

$$
-T-
$$

TARKIO RIVER AT STANTON

THOMPSON CREEK NEAR WOODBINE

THOMPSON RIVER AT DAVIS CITY

TIMBER CREEK NR MARSHALLTOWN

TURKEY RIVER AT ELKADER
STA. NO. PAGE

06-6000.00 256

06-8187.50 329

$05-4646.40 \quad 132$

$05-4845.00$

$05-4550.00$

C5-4540.00

C5-4519.00

$06-4832.70$

$06-4835.00$

203

91

82

70

244

246

$05-4520.00$

$05-4605 . \mathrm{CC}$

05-4590.00

$05-4620.00$

$05-4740.00$

$05-4730.00$

06-6085.00

$05-4550.10$

$06-9037.00$

05-4840.00

$05-4874.70$

$05-4700.00$

$05-4710.00$

05-4715.00

$06-8082.00$

05-4705.00

06-6092.00

05-4910.00

72

116

111

118

157

153

283

94

342

201

215

140

145

149

309

143

285

238

$06-8118.40$

$06-6095.90$

289

$06-8980.00$

$05-4517.00$

333

05-4120.00

68 


\section{STATION NAME}

TURKEY RIVER AT GARBER

TURKEY RIVER AT SPILLVILLE

$-U-$

UPPER IOWA RIVER AT DECORAH

UPPER IOWA RIVER NR DECORAH

$-W-$

WALNUT CREEK AT DES MOINES WALNUT CREEK NR HARTWICK WAPSIPINICON RIVER NR DEWITT WAPSIPINICON RIVER NR ELMA WAPSIPINICON RIVER AT INDEPENDENCE WAUBONSIE CREEK NR BARTLETT WELDON RIVER NR LEON WEST BRANCH FLOYD RIVER NR STRUBLE WEST BRANCH IOWA RIVER NR KLEMME WEST FORK CEDAR RIVER AT FINCHFORD WEST FORK DITCH AT HOLLY SPRINGS WEST NISHNABOTNA RIVER AT HANCOCK WEST NISHNABOTNA RIVER AT RANDOLPH WHITE BREAST CREEK NR DALLAS

WHITE BREAST CREEK NR KNOXVILLE WILLOW CREEK NR LOGAN

WINNEBAGO RIVER AT MASON CITY

$$
-Y-
$$

STA. NO.

PAGE

$05-4125.00$

$05-4116.00$

32

28

$05-3875.00$ 05-3880.00

17

19

$05-4848.00$

$05-4522.00$

$05-4222.00$

$05-4205.60$

$05-4210.00$

$06-8060.00$

06-8984.00

06-6003.00

05-4485.00

05-4589.00

$06-6020.00$

06-8074. 10

06-8085.00

$05-4879.80$

$05-4880.00$

06-6096.00

05-4595.00

74

56

51

53

299

336

260

59

109

265

305

310

217

219

290

113

$05-3890.00$ 

B CEDAR CR AT FONDA, IOWA

B CEDAR CR AT SAC CITY, IOWA

BAILEY CR NR SHEFFIELD, IOWA

BEAR CR AT SHELLSBURG, IOWA

BEAR CR NR HIGHLANDVILLE, IOWA

BEAR CR NR PALO, IOWA

BEAVER CR AT GRANGER, IOWA

BEAVER CR NR ACKLEY. IOWA

BEAVER CR NR BEAVER, IOWA

BEAVER CR NR BERKLEY, IOWA

BEAVER CR NR ELDORA, IOWA

BEAVER CR NR FERTILE, IOWA

BEAVER CR NR ROLFE, IOWA

BEAVERDAM CR NR ROCKWELL, IOWA

BIG BEAR CR AT BROOKLYN, IOWA

BIG CR AT BEP.TRAM, IOWA

BIG CR AT MT. PLEASANT, IOWA

$B I G$ MUDDY CR NR LANGDON, IOWA

BIG MUDDY CR NR SPENCER, IOWA

BIG WHISKEY CR NR BRONSON, IOWA

BIG WHISKEY SLOUGH NR KINGSLEY, IOWA

BLACK CAT CR NR ALGONA, IOWA

BLACK CAT CR NR LONE ROCK, IOWA

BLACK HAWK CR AT REINBECK, IOWA

BLACK HAWK CR NR GRUNDY CENTER, IOWA

BLUE EARTH R NR LAKOTA, IOWA

BOONE R NR GOLDFIELD, IOWA

BOONE R NR KANAWHA, IOWA

BOONE R NR RENWICK, IOWA

BOYER R NR DENISON, IOWA

BOYER R NR EARLY IOWA

BOYER R NR LOVELAND, IOWA

BOYER $R$ NR MISSOURI VALLEY, IOWA

BOYLAN CR NR BRISTOW, IOWA

BROKEN KETTLE CR NR ADAVILLE, IOWA

BROKEN KETTLE CR NR SIOUX CITY, IOWA

BROPHY CR NR LOW MOOR, IOWA

BRUSHY CR NR HOMER, IOWA

BRUSHY FORK CR NR DEDHAM, IOWA

BRUSHY FORK CR NR GUTHRIE CENTER, IOWA

BUCK CR NR HOPKINTON, IOWA

BUCK CR NR LITTLETON, IOWA

BUFFALO CR ABOVE WINTHROP, IOWA

BUFFALO CR NR STONE CITY, IOWA

BUFFALO CR NR TITONKA, IOWA

BUTTRICK CR NR GRAND JUNCTION, IOWA

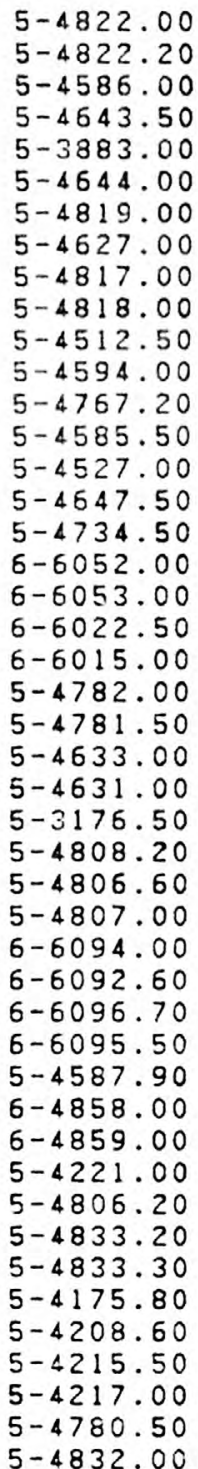




\section{STATION NAME}

CAMP CR NR LYTTON, IOWA

CANOE CR NR DECORAH, IOWA

CEDAR CR AT MELROSE, IOWA

CEDAR CR NR ALBIA, IOWA

CEDAR CR NR BATAVIA, IOWA

CEDAR CR NR CHURDAN, IOWA

CEDAR CR NR HIGHLAND CENTER, IOWA

CEDAR CR NR LOVILLA, IOWA

CEDAR CR NR OAKLAND MILLS, IOWA

CEDAR CR NR SIGOURNEY, IOWA

CEDAR R AT OTRANTO, IOWA

CHARITON R NR. DERBY, IOWA

CHEQUEST CR NR PITTSBURG, IOWA

CHEQUEST CR NR TROY, IOWA

CLANTON CF. AT EAST PERU, IOWA

CLANTON CR NR. MARTENSDALE, IOWA

CLEAR CR NR MINGO, IOWA

ILEAR CR NR OXFORD, IOWA

COLD WATER CR. NR GREENE, IOWA

COMPETINE CR BELOW FORKS NR BATAVIA, IOWA

CRANE CR AT DUNKERTON, IOWA

CRANE CR NR DENVER, IOWA

CRANE CR NR LOURDES, IOWA

CROOKED CR NR. COPPOCK, IOWA

CYLINDER CR NR RODMAN, IOWA

$-0-$

DAVIDS CR AT EXIRA, IOWA

DEEP CR AT LE MARS, I OWA

DEEP CR NR CHARLOTTE, IOWA

DEEP CR NR OYENS, IOWA

DEEP CR NR PRESTON, IOWA

DEER CR AT ST. ANSGAR, IOWA

DEER CR AT TOLEDO, IOWA

DEER CR NR MELTONVILLE, IOWA

DEVILS CR NR VIELE, IOWA

$-E-$

E ELUE CR NR CENTER POINT, IOWA

E BOYER R AT DENISON, IOWA

E BOYER R AT VAIL, IOWA

E BUTTRICK CR. NR GRAND JUNCTION, IOWA

E BUTTRICK CR NR GRAND JUNCT
E CEDAR CR NR SOMERS, IOWA

E NISHNABOTNA R AT ATLANTIC, IOWA

E NISHNABOTNA R AT EXIRA, IOWA

E NISHNABOTNA R NR AUDUBON, IOWA

E NISHNABOTNA R NR FARRAGUT, IOWA

E NISHNABOTNA $R$ NR GRISWOLD, IOWA

E NISHNABOTNA R NR LEWIS, IOWA

$E$ NODAWAY $R$ NR SHAMBAUGH, IOWA

$E$ NODAWAY $R$ NR WILLIAMSON, IOWA

E PLATTE R NR KNOWLTON, IOWA

E SOLDIER R NR UTE, IOWA

E TARKIO CR NR YORKTOWN. IOWA
STA. NO.

Page

$5-4823.60$

17

$5-4885.50$

$5-4886.00$

$5-4733.00$

$5-4824.80$

$5-4732.00$

$5-4887.00$

$5-4734.00$

$5-4724.50$

$5-4573.50$

$6-9033.00$

5-4904.00

5-4903.00

$5-4863.00$

$5-4863.50$

$5-4713.50$

5-4542.00

$5-4611.00$

$5-4732.50$

5-4208. 20

5-4208.00

5-4117.00

$5-4730.50$

$5-4766.50$

401

419

401

434

402

399

377
491

437

436

428

429

398

384

401

365

365

355

400

404

$6-8090.50$

$6-6001.60$

5-4186.50

$6-6001.20$

$5-4187.00$

$5-4574.50$

$5-4518.00$

$5-4574.00$

$5-4741.90$

474

446

361

445

362

378

378

373

378
403

$5-4643.20$

$6-6093.50$

$6-6093.00$

$5-4831.50$

$5-4824.60$

$6-8092.00$

6-8089.00

$6-8088.50$

$6-8098.00$

$6-8094.50$

$6-8093.30$

$6-8171.00$

$6-8170.50$

$6-8186.50$

$6-6084.00$

$6-8118.80$
389

462

461

$42 !$

419

475

474

473

477

477

476

483

482

484

460

473 


\section{STATION NAME}

EAGLE CR NR EAGLE GROVE, IOWA

EAGLE CR NR WOOLSTOCK. IOWA

EB INDIAN CR NR NEVADA, IOWA

EB SALT CR NR. ELBERON, IOWA

EB W NISHNABOTNA R AT AVOCA, IOWA

EB W NISHNABOTNA R NR JACKSONVILLE, IOWA

EB W NISHNABOTNA R NR R.ED LINE, IOWA

EF CROOKED CR NR WINFIELD, IOWA

EF DES MOINES R NR DOLLIVER, IOWA

EF DES MOINES R NR SWEA CITY, IOWA

EF GRAND R NR. MT. AYR, IOWA

EF GRAND $R$ SOUTH OF MT. AYR, IOWA

EF WAPSIPINICON R NR FREDERICKSBURG, IOWA

EF WAPSIPINICON R NR TRIPOLI, IOWA

EF 102 R NR BEDFORD, IOWA

ELK. CR NR TAINTOR, IOWA

ELK. R NR ALMONT, IOWA

ELLIOT CR NR BRONSON, IOWA

ENGL ISH CR NR. HARVEY, I OWA

ENGLISH CR NR KNOXVILLE, IOWA

$-F-$

FARM CR NR MACEDONIA, IOWA

FLINT CR NR BURLINGTON, IOWA

FLOOD CR NR PACKARD, IOWA

FLOOD CR NR R.OCKFORD, IOWA

FLOYD R AT LE MARS, IOWA

FLOYD R BELOW SHELDON, IOWA

FLOYD R NR MERRILL, IOWA

FLOYD R NR SHELDON, IOWA

FOURMILE CR NR ANKENY, IOWA

FOX: R AT CANTRIL, IOWA

$-G-$

GRAND R AT KNOWLTON, IOWA

GRAND R NR BLOCKTON, IOWA

GRAYBILL CR NR MACEDONIA, IOWA

GREEN BRIER CR NR JAMAICA, IOWA

$-\mathrm{H}-$

HARDIN CR NR CHURDAN, IOWA

HAR.D IN CR NR JEFFERSON, IOWA

HARTGRAVE CR NR HANSELL, IOWA

HONEY CR AT EANGOR, IOWA

HONEY CR NR. NEW PROVIDENCE, IOWA

$$
-1-
$$

INDIAN CR AT CEDAR RAPIOS, IOWA

INOIAN CR NR CHATSWORTH, IOWA

INDIAN CR NR ELKHORN, IOWA

INDIAN CR NR HASTINGS, IOWA

INDIAN CR NR IOWA CENTER, IOWA
STA. NO.

PAGE

$5-4808.60$

$5-4809.00$

$5-4711.00$

$5-4519.60$

$6-8074.00$

$6-8073.80$

$6-8073.60$

$5-4730.20$

$5-4730.20$

$5-4776.00$

$5-4777.00$
$6-8962.00$

$6-8962.00$
$6-8962.50$

$6-8962.50$
$5-4207.00$

$5-4207.20$

$6-8191.80$

$5-4714.00$

$5-4203.00$

$5-4203.00$

$6-6022.00$

$5-4883.00$
$5-4882.00$

5-4697.00

5- 4614.00

$5-4613.00$

$6-6001.80$

$6-6000.60$

6- 6002.00

$6-6000.20$

$5-4856.00$

$5-4945.00$

413
413

413
397

374

469

469

468

400

406

406
487

487

364

364

486

398

362

449

433

432

470

395

384

384

446

445

446

444

426

437

$6-8961.00$

$6-8961.50$

$6-8074.20$
$5-4832.50$

485

487

469

421

$5-4827.00$

$5-4830.50$

$5-4587.80$

$5-4513.50$

$5-4513.00$

420

420

381

372

$5-4647.00$

$6-4842.00$

$6-8093.50$

$6-8074.80$

$5-4711.80$

391

443

476

470 
INDIAN CR NR LAKE VIEW, IOWA

$5-4823.20$

$5-4821.80$ $5-4733.50$ $5-4578.00$ $6-6000.40$ $6-6046.00$ $6-4833.80$ $6-4834.90$

$6-4834.00$

$6-4833.60$

$6-4833.60$

$6-6064.00$

$6-6051.00$

$6-6039.00$
$6-6036.00$

$6-6036.00$

$6-6061.00$

$5-4118.00$

$5-4123.00$

$5-4206.40$

$5-4206.40$

$5-4209.00$

$5-4208.40$

$5-4824 \cdot 20$
$5-4824 \cdot 10$

$5-4824.10$

$5-4902.00$

$5-4516.00$

6-8984.70

$5-4799.00$

$5-4796.00$

$5-4652.00$

$6-8979.40$

$5-4653.00$

$6-6055.00$

5- 4784.00

$5-4783.50$

$5-4183.00$

5-4183.50

416

402

379

444

452 


\section{STATION NAME}

$M$ NODAWAY $R$ NR BRIDGEWATER, IOWA

$M$ NODAWAY R NR VILLISCA, IOWA

$M$ RACCOON R AT REDFIELO, IOWA

$M$ RACCOON R NR BAYARD, IOWA

$M$ RACCOON R NR CARROLL, IOWA

$M$ F.ACCOON R NR GLIDDEN, IOWA

M SILVER CR NR TREYNOR, IOWA

MAPLE R NR AURELIA, IOWA

MAPLE R NR IDA GROVE, IOWA

MAPLE R NR TURIN, IOWA

MAQUOKETA R NR HOPKINTON, IOWA

MAQUOKETA R NR SCOTCH GROVE, IOWA

MAQUOKETA RIVER NR DUNDEE, IOWA

MAYNES CR NR DUMONT, IOWA

MAYNES CR NR HAMPTON, IOWA

MF 102 R NR BEDFORD, IOWA

MIODLE CR NR ROSE HILL, IOWA

MIDDLE R AT MARTENSDALE, IOWA

MIDDLE R AT MIDDLE RIVER, IOWA

MIDDLE R NR CASEY, IOWA

MILFORD CR AT MILFORD, IOWA

MILL CR NR CHEROKEE, IOWA

MILL CR NR PAULINA, IOWA

MILLERS CR NR LAPORTE CITY, IOWA

MINERVA CR AT CLEMONS, IOWA

MINERVA CR NR CLEMONS, IOWA

MOSQUITO CR AT NEOLA, IOWA

MOSQUITO CR AT PORTSMOUTH, IOWA

MOSQUITO CR AT REINBECK, IOWA

MOSQUITO CR NR COUNCIL BLUFFS, IOWA

MOSQUITO CR NR LINDEN, IOWA

MOSQUITO CR NR REDFIELD, IOWA

MUD CR AT BANCROFT, IOWA

MUD CR AT LESTER, IOWA

MUD CR AT MOVILLE, IOWA

MUD CR NR DOON, IOWA

MUO CR NR PLAINVIEW, IOWA

MUD CR NR WILTON, IOWA

MUD LAKE IRAINAGE DITCH 71 AT JEWELL, IOWA

$-\mathrm{N}-$

N AVERY CR NR CHILLICOTHE, IOWA

$N$ BLACK HAWK CR AT DIKE, IOWA

$N$ BUFFALO CR NR BUFFALO CENTER, IOWA

N CEDAR CR NR. LOVILLA, IOWA

N CEDAR CR NR MARYSVILLE, IOWA

N ENGLISH R NR GUERNSEY, IOWA

$N$ ENGLISH R NR NORTH ENGLISH, IOWA

$N$ R.ACCOON R NR LAKE CITY, IOWA

$N$ RACCOON R NR PERRY, IOWA

$N$ RACCOON R NR REMBRANDT, IOWA

$N$ F.ACCOON R NR TRUESDALE, IOWA

$N$ SKUNK R NR NEWTON, IOWA

N SKUNK R NR SEARSBORO. IOWA

NB LIZARD CR NR HAVELOCK, IOWA
STA. NO.

$6-8166.00$

$6-8169.00$

$5-4836.60$

$5-4834.50$

$5-4833.50$

$5-4833.60$

$6-8078.00$

$6-6068.00$

$6-6069.00$

$6-6074.00$

$5-4175.60$

5-4176.00

$5-4163.00$

$5-4588.50$

$5-4588.00$

$6-8191.95$

$5-4724.00$

$5-4864.00$

$5-4861.50$

$5-4861.00$

$6-6044.00$

$6-6063.00$

6- 6062.00

$5-4640.50$

5-4514.00

5-4514.50

$6-6106.00$

$6-6105.50$

5- 4632.00

$6-6106.50$

$5-4836.20$

$5-4836.40$

$5-4778.00$

$6-4833.20$

$6-6018.00$

$6-4833.30$

$5-4218.50$

$5-4649.00$

$5-4698.50$

PAGE

481

482

426

425

424

472

472

458

459
359

359

358

382

381

486

(39

429

428

428

451

457

457

387

372

465

465

386

465

425

425

406

439

439

448

439

368

392

395

$5-4893.00$

$5-4634.00$

$5-4781.00$

$5-4888.00$

$5-4889.00$

$5-4552.00$

$5-4552.50$

$5-4824.00$

$5-4833.00$

$5-4821.00$

$5-4821.20$

$5-4721.00$

$5-4723.00$

$5-4798.00$
435

386

407

434

434 
NB NORTH R NR WINTERSET, IOWA

$5-4858.50$

427

NB TURKEY R NR VERNON SPRINGS, IOWA

NF LITTLE MAQUOKETA RIVER NR RICKARDSVILLE, IOWA

NF MAQUOKETA R AT DYERSVILLE, IOWA

NF MAQUOKETA R NR FULTON, IOWA

NODAWAY R NR BRADDYVILLE, IOWA

NOR.TH R NR EARLHAM, IOWA

$5-4115.50$

$5-4144.50$

$5-4181.00$

$5-4184.00$

$6-8172.00$

$5-4857.00$

NORTH R NR WINTERSET, IOWA

$5-4859.00$

OCHEYEDAN R NR BIGELOW. MINN.

OCHEYEDAN R NR MAY CITY, IOWA

OCHEYEDAN R NR SPENCER, IOWA

ODEBOLT CR AT IDA GROVE, IOWA

OLD MANS CR NR PARNELL, IOWA

OTTER CR NR ASHTON, IOWA

OTTER CR NR CEDAR RAPIDS, IOWA

OTTER CR MR GEORGE IOWA

OTTER CR NR. GOLDFIELD, IOWA

OTTER CR NR HANSELL, IOWA

OTTER CR NR MATLOCK, IOWA

OTTER CR NR MILO, IOWA

OTTER CR NR NORWOOD. IOWA

OTTER CR NR OTRANTO, IOWA

OTTER CR NR OTTERVILLE, IOWA

OTTER CR. NR WAPELLO, IOWA

$6-6045.00$

$6-6047.00$

$6-6050.00$

$6-6071.00$

$5-4550.50$

$6-4834.60$

$5-4644.60$

$6-4834.80$

$5-4808.00$

$5-4587.50$

$6-4834.70$

$5-4874.50$

$5-4874.00$

$5-4573.00$

$5-4209.40$

427

$-p-$

PANTHER CR NR, ADEL, IOWA

PICKEREL RUN NR SPENCER, IOWA

PIERSON CR NR CORRECTIONVILLE, IOWA

PIGEON CR EAST OF LOVELAND, IOWA

PIGEON CR NR CRESCENT, IOWA

PILOT CR NR ROLFE, IOWA

PLATTE R NR KENT, IOWA

PLATTE R NR KNOWLTON, IOWA

PLUIM CR NE EARLVILLE, IOWA

PRAIRIE CR AT CEDAR RAPIDS, IOWA

PRAIRIE CR AT NORWAY, IOWA

PRAIRIE CR NR BLAIRSTOWN, IOWA

PRAIRIE CR NR LUVERNE, IOWA

PRAIRIE CR NR RENWICK, IOWA

PRAIRIE CR NR WEST BEND, IOWA

PURGATORY CR NR LANESBORO. IOWA

$-0-$

QUARTER SECTION RUN NR DENVER, IOWA

$5-4842.00$ $6-6054.00$ $6-6065.00$ $6-6099.00$ $6-6099.50$ $5-4767.40$

$6-8186.00$

$6-8187.00$

$5-4175.40$

$5-4646.50$

5-4646.00

$5-4645.50$

$5-4807.20$

$5-4807.60$

$5-4767.00$

$5-4824.40$ 


\section{STATION NAME}

ROCK CR NR FLOYD, IOWA

RCCK R BELOW ROCK RAPIDS, IOWA

ROCK R NR DOON, IOWA

ROCK $R$ NR ROCK RAPIDS, IOWA

$$
-\mathrm{S}-
$$

S AVERY CR AT CHILLICOTHE, IOWA

$S$ EEAVER CR NR PARKERSBURG, IOWA

$S$ ENGLISH R NR KESWICK, IOWA

$S$ ENGLISH R NR KINROSS, IOWA

$S$ R.ACCOON R NR GUTHRIE CENTER, IOWA

$S$ RACCOON R NR MONTEITH, IOWA

$S$ SKUNK R AT COLFAX, IOWA

$S$ SKUNK R AT RANDALL, IOWA

$S$ SKUNK R NR ELLSWORTH, IOWA

S TIMBER CR NR LE GRAND, IOWA

SALT CR NR CLUTIEP., IOWA

SB LIZARO CR NR FORT DODGE, IOWA

SB LIZARD CR NR PALMER, IOWA

SEVENMILE CR NR LYMAN, IOWA

SEVIENMILE CR NR MORTON MILL, IOWA

SF CHARITON R NR CAMBRIA, IOWA

SF CHARITON R NR CORYDON, IOWA

SF IOWA R NR ALDEN, IOWA.

SF IOWA R NR NEW PROVIDENCE, IOWA

SF MAQUOKETA R NR DUNDEE, IOWA

SHOAL CR NR CINCINNATI, IOWA

SILVER CR NR AVOCA. IOWA

SILVER CR NR DE WITT, IOWA

SILVER CR NR EMMETSBURG, IOWA

SILVER CR NR MALVERN, IOWA

SILVER CR NR TREYNOR, IOWA

SIYMILE CR NR. CHATSWORTH, IOWA

SIXMILE CR NR HAWARDEN, IOWA

SOAP CR NR ASH GROVE, IOWA

SOAP CR NR FLORIS IOWA

SOLDIER R NR RICKETTS, IOWA

SOLDIER R NR UTE, IOWA

SOUTH R NR INOIANOLA, IOWA

SOUTH R NR NEW VIRGINIA, IOWA

SPRING CR NR LAPORTE CITY, IOWA

SQUIAW CR NR HANSELL, IOWA

SQUAW CR NR INDIANOLA, IOWA

SQUAW CR NR JAMISON IOWA

SQUAW CR NR STANHOPE, IOWA

STONEY CR NR EVERLY, IOWA

STONEY CR NR FOSTORIA, IOWA

SUGAR CR NR BENNETT, IOWA

SUGAR CR NR CHARLESTON, IOWA

SUGAR CR NR FRANKLIN, IOWA

SUGAR CR NR MOSCOW, IOWA

SUGAR CR NR VIELE, IOWA
STA. NO.

PAGE

$5-4576.00$

$6-4833.00$

$6-4833.40$

$6-4831.00$

378

439

438

$5-4894.00 \quad 435$

$5-4628.00 .385$

$5-4554.60 \quad 376$

$-4554.50 \quad 377$

$5-4833.10 \quad 422$

$\begin{array}{ll}5-4833.40 & 423 \\ 5-4710.50 & 396\end{array}$

$5-4699.50 \quad 396$

$5-4698.00 \quad 395$

$5-4516.50 \quad 373$

$5-4519.30 \quad 374$

$5-4803.00 \quad 410$

$-4801.00 \quad 410$

$-8163.50 \quad 480$

$6-8164.00 \quad 480$

$6-9036.00 \quad 491$

$\begin{array}{ll}6-9036.50 & 492 \\ 5-4511.00 & 370\end{array}$

$5-4512.00$

$5-4164.00$

$6-9041.50$

$6-8076.00 \quad 471$

$5-4219.00 \quad 369$

$5-4766.00 \quad 404$

$6-8079.00 \quad 472$

$6-8076.50 \quad 47$

$6-4841.50$

$6-4841.00 \quad 442$

$5-4899.00 \quad 435$

$5-4901.00 \quad 436$

$6-6083.00 \quad 450$

$6-6083.50 \quad 460$

$5-4872.00 \quad 430$

$5-4867.00 \quad 429$

$5-4643.00 \quad 388$

$5-4587.70$

$5-4871.00$

$5-4869.00 \quad 430$

$5-4702.00 \quad 396$

6-6049.00 453

$6-6048.00 \quad 453$

$5-4648.50 \quad 392$

$5-4907.00 \quad 437$

$5-4742.00 \quad 403$

5-4649.20 393

$5-4743.00 \quad 403$ 
TARKIO R AT BLANCHARD, IOWA

TARKIO R NR COBURG, IOWA

THOMPSON R NR AFTON, IOWA

THOMPSON R NR. GRAND RIVER, IOWA

THOMPSON R NR HEBRON, IOWA

THREEMILE CR NR AFTON, IOWA

TIPTON CR NR NEW PROVIDENCE. IOWA

TOM CR AT ROCK RAPIDS, IOWA

TROUBLESOME CR NR ATLANTIC, IOWA

TROUELESOME CR NR WIOTA. IOWA

TURKEY CR EAST OF ATLANTIC, IOWA

TURKEY CR ITR ATLANTIC, IOWA

TUFKEY R NR VERNON SPRINGS, IOWA

TWELVE MILE CR NR BUCKINGHAM, IOWA

TWELVEMILE CR. NR ARISPE, IOWA

$$
-u-
$$

UNION SLOUGH OUTLET NR LAKOTA, IOWA

UPPER IOWA R AT CHESTER, IOWA

UPFEK IOWA R NR KENDALVILLE, IOWA

$$
-V-
$$

VILLAGE CR AT VILLAGE CREEK, IOWA VOLGA R AT LITTLEPORT, IOWA

VOLGA R NR FAYETTE, IOWA

$$
-w-
$$

W EUTTRICK CR NR FARNHAMVILLE, IOWA

W NISHNABOTNA R AT AVOCA, IOWA

W MISHINABOTNA R AT HARLAN, IOWA

W NISHNABOTNA R AT WHITE CLOUD, IOWA

W NISHNABOTNA R NR MALVERN, IOWA

W NISHNABOTNA R NR MANNING, IOWA

W NODAWAY $R$ NR CUMBERLAND, IOWA

W NODAWAY R NR VILLISCA, IOWA

W TARKIO CR NR COIN, IOWA

W TARKIO CR NR NORTHBORO, IOWA

WALNUT CR AT GERMANVILLE, IOWA

WALNUT CR NR GRISWOLD, IOWA

WALNUT CR NR HAWTHORNE, IOWA

WALNUT CR NR RANDOLPH, IOWA

WAPSINONOC CR AT WEST LIVERTY, IOWA

WAPSIPINICON R AT STONE CITY, IOWA

WAPSIPINICON R AT TRIPOLI, IOWA

WAPSIPINICON R NR IONIA, IOWA

WAPSIPINICON R NR NEW HAMPTON, IOWA

WAPSIPINICON R NR RICEVILLE, IOWA

WAPSIPINICON R NR TRIPOLI, IOWA

\begin{tabular}{|c|c|}
\hline $\begin{array}{l}6-8120.00 \\
6-8118.60 \\
6-8119.00 \\
6-8978.20 \\
6-8979.00 \\
6-8977.70 \\
6-8978.00 \\
5-4511.50 \\
6-4832.80 \\
6-8091.50 \\
6-8091.00 \\
6-8092.50 \\
6-8093.00 \\
5-4115.60 \\
5-4641.50 \\
6-8978.80\end{array}$ & $\begin{array}{l}479 \\
478 \\
478 \\
488 \\
489 \\
488 \\
488 \\
370 \\
438 \\
475 \\
474 \\
475 \\
476 \\
354 \\
387 \\
489\end{array}$ \\
\hline $\begin{array}{l}5-3177.00 \\
5-3873.00 \\
5-3874.00\end{array}$ & $\begin{array}{l}351 \\
352 \\
352\end{array}$ \\
\hline $\begin{array}{l}5-3883.50 \\
5-4124.00 \\
5-4122.00\end{array}$ & $\begin{array}{l}353 \\
357 \\
356\end{array}$ \\
\hline $\begin{array}{l}5-4831.00 \\
6-8073.40 \\
5-8073.20 \\
6-8075.00 \\
6-8075.50 \\
6-8072.60 \\
6-8163.00 \\
6-8165.50 \\
6-8123.00 \\
6-8124.00 \\
5-4731.00 \\
6-8086.00 \\
6-8087.00 \\
6-8088.00 \\
5-4649.40 \\
5-4215.00 \\
5-4207.40 \\
5-4205.80 \\
5-4206.60 \\
5-4205.40 \\
5-4206.80 \\
6-6059.00 \\
5-6060.00\end{array}$ & $\begin{array}{l}420 \\
468 \\
468 \\
470 \\
471 \\
467 \\
480 \\
481 \\
479 \\
479 \\
400 \\
472 \\
473 \\
473 \\
393 \\
367 \\
365 \\
363 \\
363 \\
362 \\
364 \\
456 \\
456\end{array}$ \\
\hline
\end{tabular}

WATERMAN CR NR HARTLEY, IOWA

WATERMAN CR NR SUTHERLAND, IOWA 
STATION NAME

WB FLOYD R NR MERRILL, IOWA

WB FLOYD R NR MIDDLEBURG, IOWA

WB INDIAN CR NR IOWA CENTER, IOWA

WB IOWA R NR BRITT, IOWA

WB WAPSINONOC CR AT WEST LIBERTY, IOWA

WB 102 R BELOW MB NR GRAVITY, IOWA

WB $102 R$ NR GRAVITY, IOWA

WB $102 R$ NR NEW MARKET. IOWA

WELDON R EAST OF LEON, IOWA

WELDON R NR PLEASANTON, IOWA

WESTMAIN DRAINAGE DITCH 1 \& 2 NR BRITT, IOWA.

WF BLUE EARTH $R$ BL MINN. -IOWA STATE LINE.

WF L SIOUX R AT MOVILLE, IOWA

WF $L$ SIOUX R NR FIELDING. IOWA

WF $L$ SIOUX R NR KINGSLEY. IOWA

WF $L$ SIOUX $R$ NR LAKE PARK, IOWA

WF $L$ SIOUX $R$ NR MONTGOMERY, IOWA

WF M NODAWAY R NR BRIDGEWATER, IOWA

WF M NODAWAY R NR FCNTANELLE, IOWA

WF W NISHNABOTNA R AT HARLAN, IOWA

WF $W$ NISHN $\triangle B O T N A$ R MANILLA, IOWA

WF 102 R NR NEW MARKET, IOWA

WHITE BREAST CR AT LUCAS. IOWA

WHITE BREAST CR NR NEWBERN. IOWA

WHITE BREAST CR NR WOODBURN, IOWA

WHITE FOX CR AT WEBSTER CITV, IOWA

WHITE FOX CR NR WOOLSTOCK, IOWA

WHITEWATER CR AT FILLMORE, IOWA

WILLOW CR AT MASON CITY, IOWA

WILLOW CR NR BAYARD, IOWA

WILLOW CR NR GREENVILLE, IOWA

WILLOW CR NR LOGAN, IOWA

WILLOW CR NR MISSOURI VALLEY, IOWA

WI'LLOW CR NR OYENS, IOWA

WILLOW CR NR ROSSI, IOWA

WILLOW CR NR SCRANTON, IOWA

WILLOW CR NR WOODBINE, IOWA

WINNEBAGO R NR FERTILE, IOWA

WINNEBAGO R NR FOREST CITY, IOWA

WOLF CR AT LAPORTE CITY, IOWA

WOLF CR NR BEAMAN, IOWA

WOLF CR NF BUCKINGHAM, IOWA

WOLF CR NR CHARITON, IOWA

WOLF CR NR HOLLY SPRINGS, IOWA

$$
-\gamma-
$$

YANKEE RUN CR. AT WHEATLAND, IOWA YELLOW R AT JON, IOWA

YELLOW R AT MYRON, IOWA
STA. NO.

PAGE

$6-6004.00$

$6-6002.50$

$5-4711.50$

$5-4483.00$

$5-4649.50$

$6-8191.20$

$6-8191.00$

$6-8191.40$

$6-8983.00$

$6-8984.50$

5-4484.00

$5-3178.10$

$6-6019.00$

$6-6016.00$

$6-6017.00$

$6-6037.00$

$6-6038.00$

$6-8168.00$

$6-8167.00$

$6-8073.00$

$6-8072.80$

$6-8191.50$

$5-4878.00$

$5-4879.00$

$5-4877.00$

$5-4809.80$

$5-4809.40$

$5-4182.00$

5-4602.00

5-4834.00

$6-6058.00$

$6-6096.00$

$6-6096.20$

$6-6001.40$

$6-6057.00$

$5-4833.80$

$6-6095.80$

$5-4593.00$

$5-4592.00$

$5-4642.50$

$5-4641.00$

$5-4642.00$

$6-9033.50$

$6-6023.00$ 


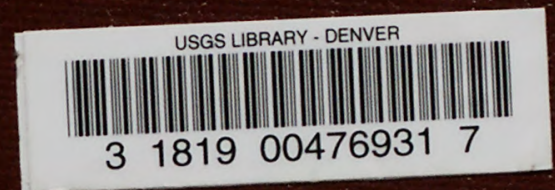

